\title{
Water Resources Data Minnesota Water Year 1991
}

Volume 2. Upper Mississippi and Missouri River Basins

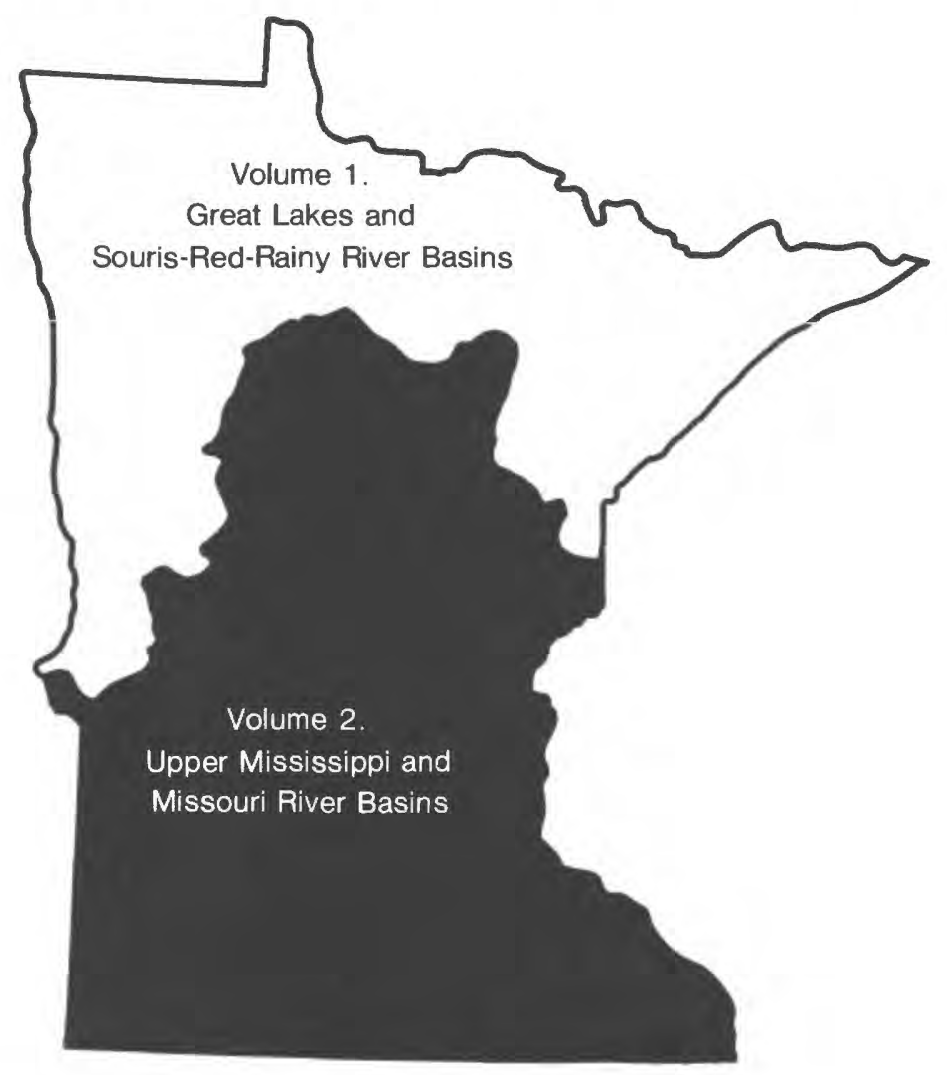

U.S. GEOLOGICAL SURVEY WATER-DATA REPORT MN-91-2

Prepared in cooperation with the Minnesota Department of Natural Resources, Division of Waters; the Minnesota

Department of Transportation; and with other State, municipal, and Federal agencies 


\begin{tabular}{|c|c|c|c|c|c|c|c|c|c|c|c|c|c|c|c|c|c|}
\hline \multicolumn{18}{|c|}{1990} \\
\hline \multicolumn{6}{|c|}{ OCTOBER } & \multicolumn{6}{|c|}{ NOVEMBER } & \multicolumn{6}{|c|}{ DECEMBER } \\
\hline$S$ & M & $T \quad W$ & $T$ & $\mathrm{~F}$ & $S$ & $S$ & $M$ & $T \quad W$ & $T$ & $F$ & $S$ & $S$ & M & $T W$ & $T$ & $F$ & $S$ \\
\hline $\begin{array}{cc}7 & \\
14 & 1 \\
21 & 2 \\
28 & 2\end{array}$ & $\begin{array}{c}1 \\
8 \\
15 \\
22 \\
22 \\
29 \\
29\end{array}$ & 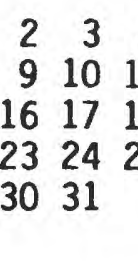 & $\begin{array}{c}4 \\
11 \\
18 \\
25\end{array}$ & $\begin{array}{cc}5 & \\
12 & 1 \\
19 & 2 \\
26 & 2\end{array}$ & & $\begin{array}{cc}4 & \\
11 & 1 \\
18 & 1 \\
25 & 2\end{array}$ & $\begin{array}{r}5 \\
12 \\
19 \\
26\end{array}$ & $\begin{array}{rr}6 & 7 \\
13 & 14 \\
20 & 21 \\
27 & 28\end{array}$ & $\begin{array}{r}1 \\
8 \\
15 \\
22 \\
29\end{array}$ & $\begin{array}{r}2 \\
9 \\
16 \\
23 \\
30\end{array}$ & $\begin{array}{r}3 \\
10 \\
17 \\
24\end{array}$ & $\begin{array}{r}2 \\
9 \\
16 \\
23 \\
30\end{array}$ & $\begin{array}{r}3 \\
10 \\
17 \\
24 \\
31\end{array}$ & $\begin{array}{rr}4 & 5 \\
11 & 12 \\
18 & 19 \\
25 & 26\end{array}$ & $\begin{array}{rr}6 & \\
13 & 1 \\
20 & 2 \\
27 & 2\end{array}$ & & $\begin{array}{r}1 \\
8 \\
15 \\
22 \\
29\end{array}$ \\
\hline \multicolumn{18}{|c|}{1991} \\
\hline \multicolumn{6}{|c|}{ JANUARY } & \multicolumn{6}{|c|}{ FEBRUARY } & \multicolumn{6}{|c|}{ MARCH } \\
\hline$S$ & M & $T W$ & $T$ & F & $s$ & $S$ & $M$ & $T W$ & $T$ & $\mathrm{~F}$ & $S$ & $S$ & M & $T W$ & $T$ & $\mathrm{~F}$ & $S$ \\
\hline $\begin{array}{cc}6 & \\
13 & 1 \\
20 & 2 \\
27 & 2\end{array}$ & $\begin{array}{cc}7 & \\
14 & 1 \\
21 & 2 \\
28 & 2\end{array}$ & 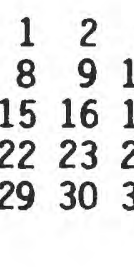 & $\begin{array}{l}3 \\
10 \\
17 \\
24 \\
31\end{array}$ & $\begin{array}{cc}4 & \\
11 & 1 \\
18 & 19 \\
25 & 2\end{array}$ & & $\begin{array}{cc}3 & \\
10 & 1 \\
17 & 1 \\
24 & 2\end{array}$ & $\begin{array}{c}4 \\
11 \\
18 \\
25\end{array}$ & $\begin{array}{rr}5 & 6 \\
12 & 13 \\
19 & 20 \\
26 & 27\end{array}$ & $\begin{array}{r}7 \\
14 \\
21 \\
28\end{array}$ & $\begin{array}{r}1 \\
8 \\
15 \\
22\end{array}$ & $\begin{array}{r}2 \\
9 \\
16 \\
23\end{array}$ & $\begin{array}{c}5 \\
10 \\
17 \\
24 \\
31 \\
31\end{array}$ & $\begin{array}{r}4 \\
11 \\
18 \\
25\end{array}$ & $\begin{array}{rr}5 & 6 \\
12 & 13 \\
19 & 20 \\
26 & 27\end{array}$ & $\begin{array}{cc}7 & \\
14 & 1 \\
21 & 2 \\
28 & 2\end{array}$ & $\begin{array}{cc}1 & \\
8 \\
15 & 1 \\
22 & 2 \\
29 & 3\end{array}$ & $\begin{array}{r}2 \\
9 \\
16 \\
23 \\
30\end{array}$ \\
\hline \multicolumn{6}{|c|}{ APRIL } & \multicolumn{6}{|c|}{ MAY } & \multicolumn{6}{|c|}{ JUNE } \\
\hline$S$ & M & T W & $T$ & $F:$ & $s$ & $S$ & $M$ & $T W$ & $T$ & $F$ & $S$ & $S$ & M & $T W$ & $T$ & $\mathrm{~F}$ & $S$ \\
\hline $\begin{array}{cc}7 & \\
14 & 1 \\
21 & 2 \\
28 & 2\end{array}$ & 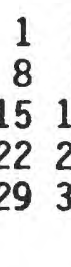 & $\begin{array}{rrr}2 & 3 \\
9 & 10 & 1 \\
16 & 17 & 1 \\
23 & 24 & 2 \\
30 & & \end{array}$ & $\begin{array}{c}4 \\
11 \\
18 \\
25\end{array}$ & $\begin{array}{rr}5 & \\
12 & 1 \\
19 & 2 \\
26 & 2\end{array}$ & & $\begin{array}{c}5 \\
12 \\
19 \\
26 \\
26\end{array}$ & $\begin{array}{r}6 \\
13 \\
20 \\
27\end{array}$ & $\begin{array}{rr} & 1 \\
7 & 8 \\
14 & 15 \\
21 & 22 \\
28 & 29\end{array}$ & $\begin{array}{r}2 \\
9 \\
16 \\
23 \\
30\end{array}$ & $\begin{array}{r}3 \\
10 \\
17 \\
24 \\
31\end{array}$ & $\begin{array}{r}4 \\
11 \\
18 \\
25\end{array}$ & $\begin{array}{rr}2 \\
9 & 1 \\
16 & 1 \\
23 & 2 \\
30 & \end{array}$ & $\begin{array}{r}3 \\
10 \\
17 \\
24\end{array}$ & $\begin{array}{rr}4 & 5 \\
11 & 12 \\
18 & 19 \\
25 & 26\end{array}$ & $\begin{array}{rr}6 & \\
13 & 1 \\
20 & 2 \\
27 & 2\end{array}$ & $\begin{array}{cc}7 & \\
14 & 1 \\
21 & 2 \\
28 & 2\end{array}$ & $\begin{array}{r}1 \\
8 \\
15 \\
22 \\
29\end{array}$ \\
\hline \multicolumn{6}{|c|}{ JULY } & \multicolumn{6}{|c|}{ AUGUST } & \multicolumn{6}{|c|}{ SEPTEMBER } \\
\hline s 1 & M & $T \quad W$ & $T$ & $F:$ & $S$ & $S$ & $M$ & $T W$ & $T$ & $F$ & $S$ & $S$ & M & $T W$ & $T$ & $F$ & $S$ \\
\hline $\begin{array}{cc}7 & \\
14 & 1 \\
21 & 2 \\
28 & 2\end{array}$ & $\begin{array}{rr}1 & \\
8 & \\
15 & 1 \\
22 & 2 \\
29 & 3\end{array}$ & $\begin{array}{rrr}2 & 3 \\
9 & 10 & 1 \\
16 & 17 & 1 \\
23 & 24 & 2 \\
30 & 31 & \end{array}$ & $\begin{array}{c}4 \\
11 \\
18 \\
25\end{array}$ & $\begin{array}{rr}5 & \\
12 & 1 \\
19 & 2 \\
26 & 2\end{array}$ & & $\begin{array}{cc}4 & \\
11 & 1 \\
18 & 1 \\
25 & 2\end{array}$ & $\begin{array}{r}5 \\
12 \\
19 \\
26\end{array}$ & $\begin{array}{rr}6 & 7 \\
13 & 14 \\
20 & 21 \\
27 & 28\end{array}$ & $\begin{array}{r}1 \\
8 \\
15 \\
22 \\
29\end{array}$ & $\begin{array}{r}2 \\
9 \\
16 \\
23 \\
30\end{array}$ & $\begin{array}{r}3 \\
10 \\
17 \\
24 \\
31\end{array}$ & $\begin{array}{c}1 \\
8 \\
15 \\
22 \\
29 \\
29\end{array}$ & $\begin{array}{r}2 \\
9 \\
16 \\
23 \\
30\end{array}$ & $\begin{array}{cc}3 & 4 \\
10 & 11 \\
17 & 18 \\
24 & 25\end{array}$ & $\begin{array}{cc}5 & \\
12 & 1 \\
19 & 2 \\
26 & 2\end{array}$ & $\begin{array}{cc}6 & \\
13 & 1 \\
20 & 2 \\
27 & 2\end{array}$ & $\begin{array}{r}7 \\
14 \\
21 \\
28\end{array}$ \\
\hline
\end{tabular}


DEPARTMENT OF THE INTERIOR

MANUEL LUJAN, JR, Secretary

GEOLOGICAL SURVEY

Dallas L. Peck, Director

For additional information write to:

District Chief, Water Resources Division

U.S. Geological Survey

2280 Woodale Drive

Mounds View, Minnesota 55112 
This volume of the annual hydrologic data report of Minnesota is one of a series of annual reports that document hydrologic data gathered from the U.S. Geological Survey's surface- and ground-water data-collection networks in each State, Puerto Rico, and the Trust Territories. These records of streamflow, ground-water levels, and water quality provide the hydrologic information needed by State, local, and Federal agencies, and the private sector for developing and managing our Nation's land and water resources. Hydrologic data for Minnesota are contained in two volumes:

Volume 1. Great Lakes and Souris-Red-Rainy River Basins Volume 2. Upper Mississippi and Missouri River Basins

This report is the culmination of a concerted effort by dedicated personnel of the U.S. Geological Survey who collected, compiled, analyzed, verified, and organized the data, and who typed, edited, and assembled the report. In addition to the authors, who had primary responsibility for assuring that the information contained herein is accurate, complete, and adheres to Geological Survey policy and established guidelines, the following individuals contributed significantly to the preparation of this report:

Lan H. Tornes, Water-Quality Specialist, Minnesota District

Alex Brietkrietz, Ground-Water Network Project Chief, Minnesota District

Most of the data were collected, processed, and tabulated by the following individuals:

\section{St. Paul District Office}

\begin{tabular}{l} 
Allan D. Arntson \\
George H. Carlson \\
Pierce A. Flanagan \\
Paul E. Hanson \\
Mark R. Have \\
Joan M. Helms \\
Rebecca A. Miller \\
Gregory B. Mitton \\
Grand Rapids Field Head \\
\hline \\
Howard D. Braden \\
William A. Gothar \\
Gregory R. Melhus
\end{tabular}

Shelley L. Morrison

George A. Roach

Chris A. Sanocki

Charles J. Smith

Gregory W. Stratton

Duane A. Wicklund

Thomas A. Winterstein

Montevideo Field Headquarters

Roderick L. Johnson

This report was prepared in cooperation with the State of Minnesota and with other agencies under the general supervision of George Garklavs, District Chief, Minnesota. 


\begin{tabular}{|c|c|c|c|}
\hline \begin{tabular}{|l|l} 
REPORT DOCUMENTATION \\
PAGE
\end{tabular} $\begin{array}{l}\text { 1. Report No. } \\
\text { USGS/WRD/HD-92/308 }\end{array}$ & 2. & \multicolumn{2}{|c|}{ 3. Recipient's Accession No. } \\
\hline \multirow{2}{*}{\multicolumn{2}{|c|}{$\begin{array}{l}\text { 4. Title and Subtitle } \\
\text { Water Resources for Minnesota, Water Year } 1991 \\
\text { Volume 2, Upper Mississippi and Missouri River Basin }\end{array}$}} & \multicolumn{2}{|l|}{$\begin{array}{l}\text { 5. Report } \\
\text { September } 1992\end{array}$} \\
\hline & & \multicolumn{2}{|l|}{6.} \\
\hline \multicolumn{2}{|c|}{ 7. Author(s) Kurt T. Gunard, Joseph H. Hess and James L. Zirbel } & \multicolumn{2}{|c|}{$\begin{array}{l}\text { 8. Performing Organization Rept. No. } \\
\text { USGS-WDR-MH-91-2 }\end{array}$} \\
\hline \multirow{2}{*}{\multicolumn{2}{|c|}{$\begin{array}{l}\text { 9. Performing Organization Mame and Address } \\
\text { U.S. Geological Survey, Water Resources Division } \\
2280 \text { Woodale Drive } \\
\text { Mounds View, MM } 55112\end{array}$}} & \multicolumn{2}{|c|}{ 10. Project/Task/Work Unit No. } \\
\hline & & \multicolumn{2}{|c|}{$\begin{array}{l}\text { 11. Contract(C) or Grant(G) No. } \\
\text { (C) } \\
\text { (G) }\end{array}$} \\
\hline \multirow{2}{*}{\multicolumn{2}{|c|}{$\begin{array}{l}\text { 12. Sponsoring Organization Mame and Address } \\
\text { U.S. Geological Survey, Water Resources Division } \\
2280 \text { Woodale Drive } \\
\text { Mounds View, MN } 55112\end{array}$}} & \multicolumn{2}{|c|}{$\begin{array}{l}\text { 13. Type of Report \& Period Covered } \\
\text { Annual Oct. 1, } 1990 \\
\text { Sept. 30, 1991 }\end{array}$} \\
\hline & & \multicolumn{2}{|l|}{14.} \\
\hline \multicolumn{4}{|c|}{$\begin{array}{l}\text { 15. Supplementary Notes } \\
\text { Prepared in cooperation with the State of Minnesota and with other agencies. }\end{array}$} \\
\hline \multicolumn{4}{|c|}{$\begin{array}{l}\text { 16. Abstract (Limit: } 200 \text { words) } \\
\text { Water-resources data for the } 1991 \text { water year for Minnesota consist of records of stage, } \\
\text { discharge and water quality of streams; stage, contents, and water quality of lakes and reservoirs; } \\
\text { and water levels and water quality in wells and springs. This volume contains discharge records for } \\
60 \text { gaging stations; stage and contents for } 9 \text { lakes and reservoirs; water quality for } 18 \text { stream } \\
\text { stations, } 1 \text { lake station, } 22 \text { partial-record sites, } 1 \text { precipitation station, } 108 \text { wells; and } \\
\text { water levels for } 119 \text { observation wells. Also included are } 59 \text { high-flow partial-record stations. } \\
\text { Additional water data were collected at various sites, not part of the systematic data collection } \\
\text { program and are published as miscellaneous measurements or low-flow investigations. These data, } \\
\text { together with the data in Volume 1, represent that part of the National Water Data System operated } \\
\text { by the U.S. Geological Survey and cooperating State. and Federal Agencies in Minnesota }\end{array}$} \\
\hline \multicolumn{4}{|c|}{$\begin{array}{l}\text { 17. Document Analysis a. Descriptors } \\
\text { *Minnesota, *Hydrologic data, *Surface water, *Ground water, *Water quality, Flow rate, Gaging } \\
\text { stations, Lakes, Reservoirs, Chemical analyses, Precipitation, Sediments, Water temperatures, } \\
\text { Sampling sites, Water Levels, Water analyses, Data collection } \\
\text { b. Ident ifiers/Open-Ended Terms }\end{array}$} \\
\hline \multirow{2}{*}{$\begin{array}{l}\text { 18. Availability Statement: } \\
\text { No restriction on distribution. } \\
\text { This report may be purchased from: } \\
\text { National Technical Information Service } \\
\text { Springfield, va } 22161\end{array}$} & \multicolumn{3}{|c|}{\begin{tabular}{|l|l|} 
19. Security Class (This Report) & 21. No. of Pages \\
UNCLASSIFIED & 285
\end{tabular}} \\
\hline & \multicolumn{2}{|c|}{$\begin{array}{l}\text { 20. Security Class (This Page) } \\
\text { UNCLASSIFIED }\end{array}$} & 22. Price \\
\hline
\end{tabular}


Preface

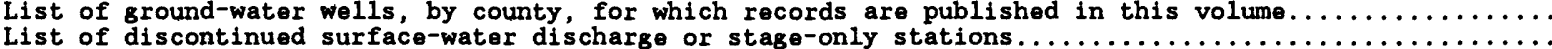

Introduction.

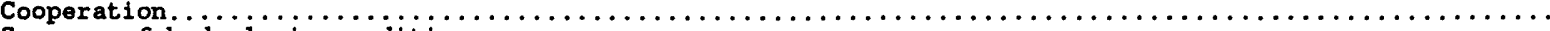

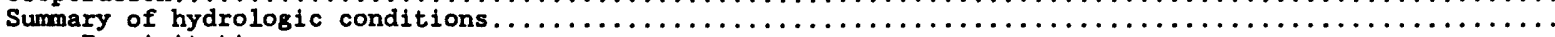

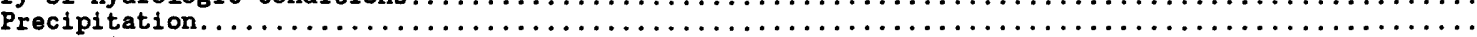

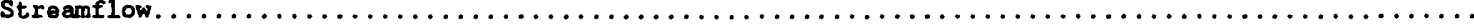

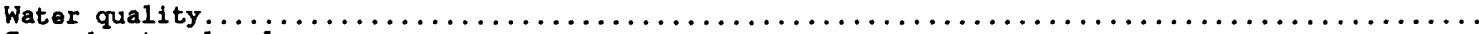

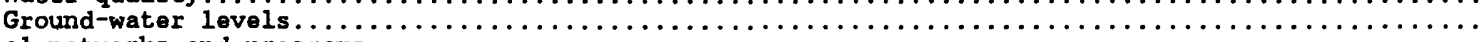

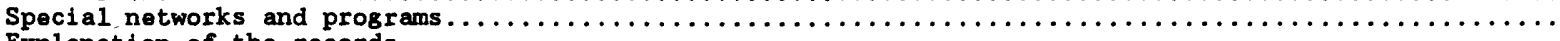

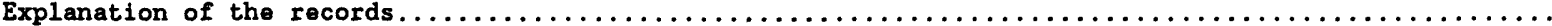

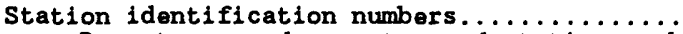

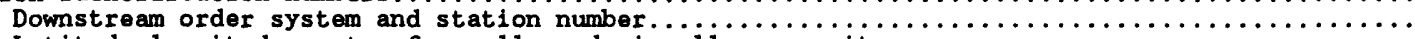

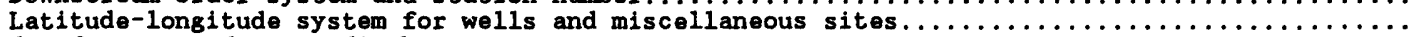

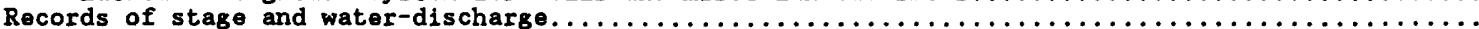

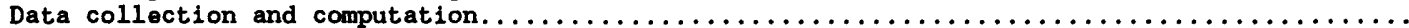

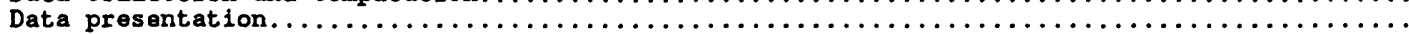

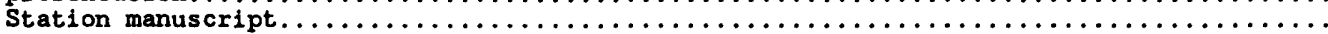

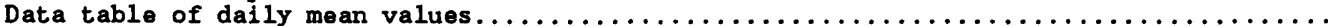

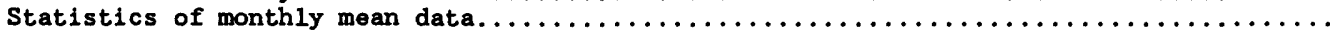

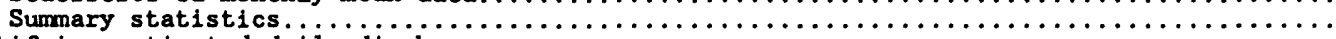

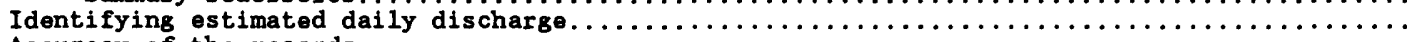

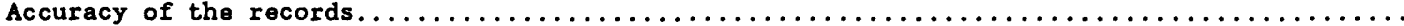

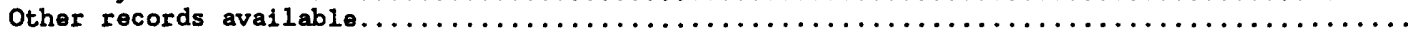

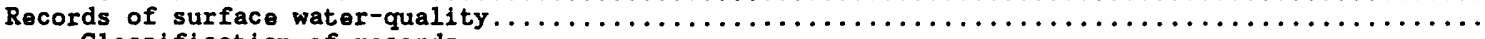

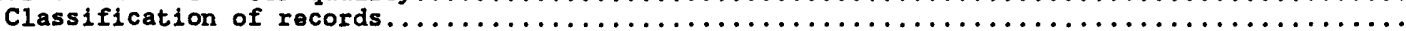

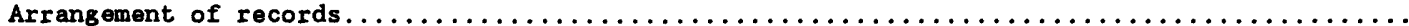

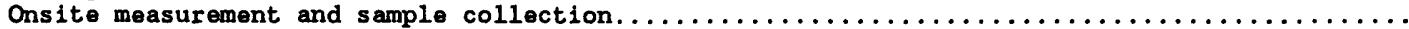

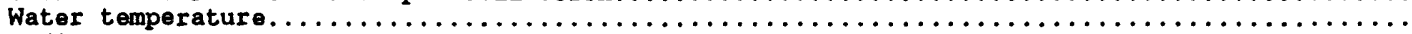

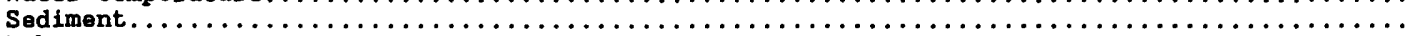

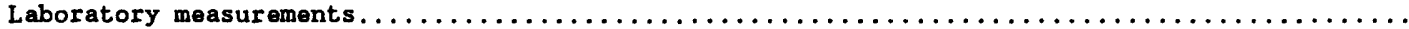

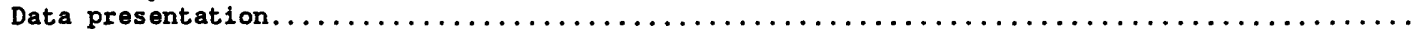

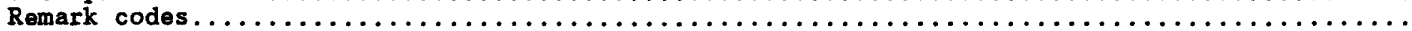

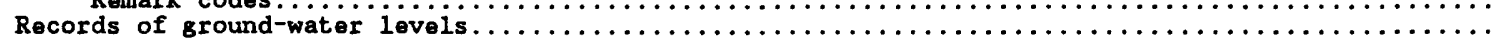

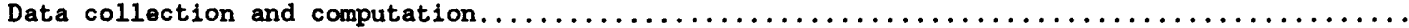
Data presentation.

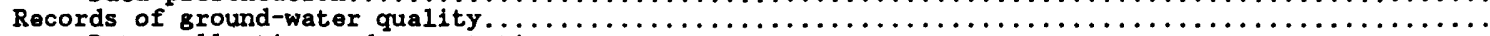

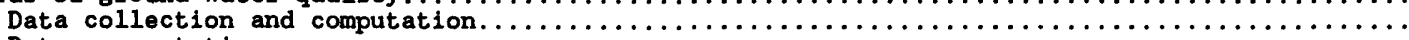
Data presentation.

Access to WATSTORE data.

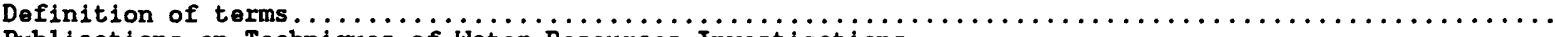

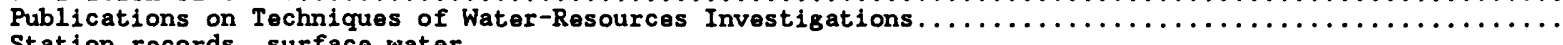

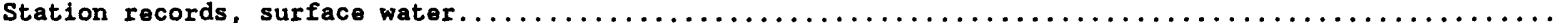

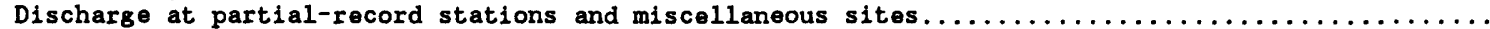

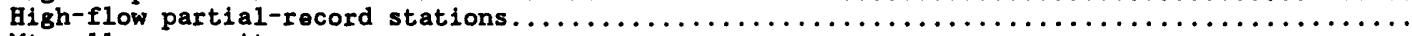

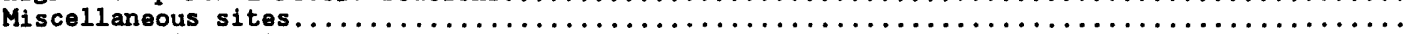

Analyses of samples collected at water-quality partial-record stations...................

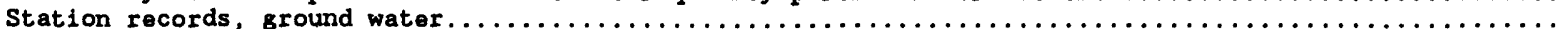

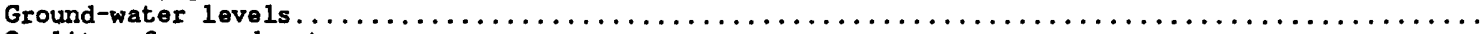

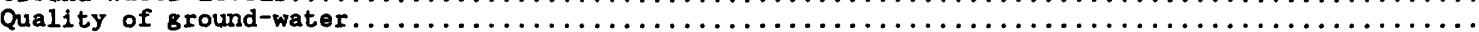

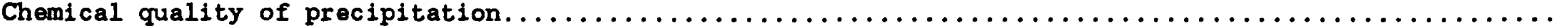

Index

\section{ILLUSTRATIOWS}

Figure 1. Map showing precipitation, in inches, during 1991 water year compared to normal annual

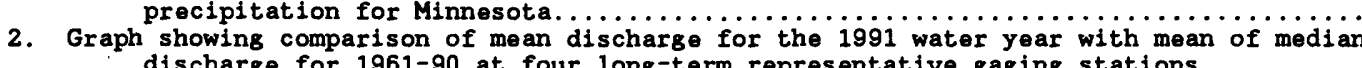

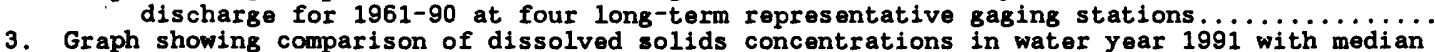
for period of record at representative gaging stations...........

4. Graph showing comparison of nitrite plus nitrate concentrations in water year iggi with

5a. Hydrographs showing comparison of seasonal water levels during 1991 to iong-term ievels

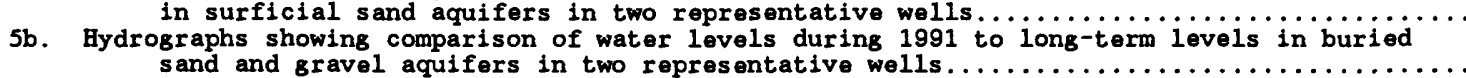

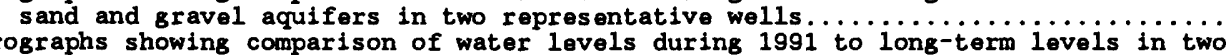

5c. Hydrographs showing comparison of water levels during 1991 to long-term levels in two
5epresentative bedrock wells in the Prairie du Chien-Jordan aquifer .............................. Hydrographs showing comparison of water levels during 1991 to long-term levels in representative bedrock wells in the St. Peter and Franconia-Ironton-Galesville aquifers

5e. Hydrographs showing comparison of water levels during 1991 to long-term levels in two representative bedrock wells in the Mount Simon-Hinckley-Fond du Lac aquifer.......... 


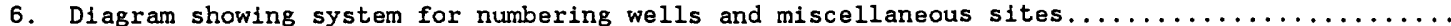

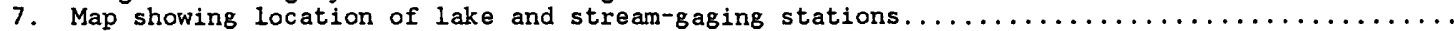

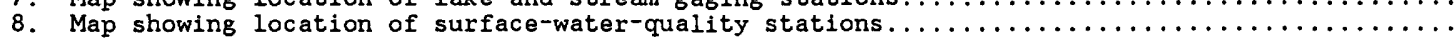

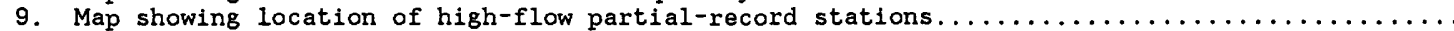

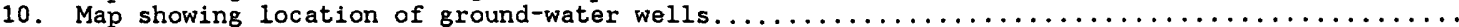

GAGING STATIONS, IN DOWNSTREAM ORDER, FOR WHICH RECORDS ARE PUBLISHED

\begin{abstract}
Note.--Data for partial-record stations and miscellaneous sites for both surface-water quantity and quality are published in separate sections of the data report. See references at the end of this list for page numbers for these sections.
\end{abstract}

[Letters after station name designates type of data: (d) discharge; (e) gage height, elevation, or contents; (c) chemical, radio-chemical, or pesticides; (b) biological or mictro-biological; $(p)$ physical (water temperature, sediment, or specific conductance)]

UPPER MISSISSIPPI RIVER BASIN

Mississippi River near Bemidj

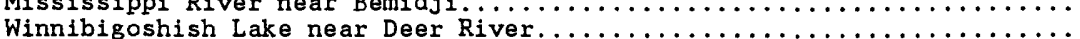

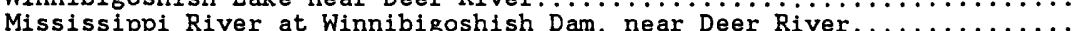
LEECH LAKE RIVER BASIN

Williams Lake near Akeley.

Leech Lake at Federal Dam. . . .

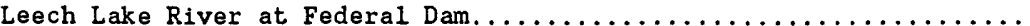

Pokegama Lake near Grand Rapids.

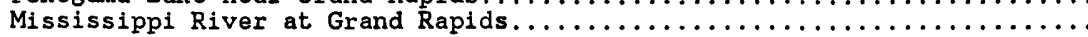
SANDY RIVER BASIN

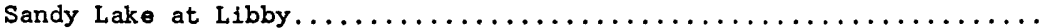

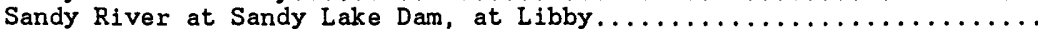

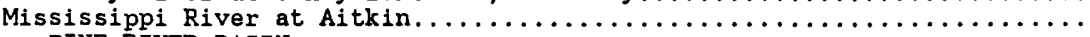
PINE RIVER BASIN

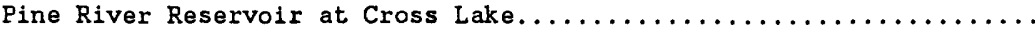

Pine River at Cross Lake Dam, at Cross Lake...................

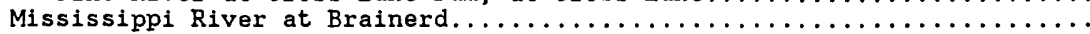
CROW WING RIVER BASIN

Shell River:

Straight River at County Highway 125 near Osage............

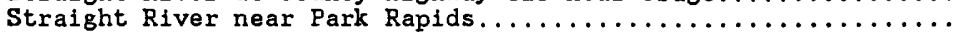

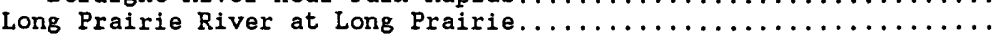
Gull River:

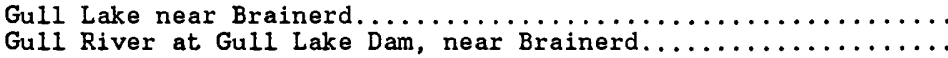

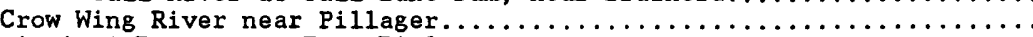

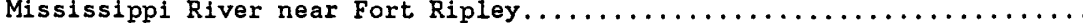

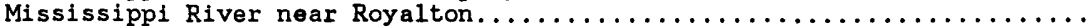
SAUK RIVER BASIN

Sauk River near St. Cloud.

Mississippi River at St. Cloud..............

ELK RIVER BASIN

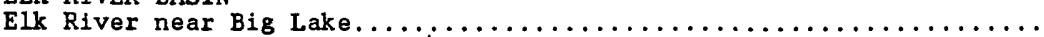

CROW RIVER BASIN

Crow River at Rockford.

RUM RIVER BASIN

Mille Lacs Lake (head of Rum River) at Cove Bay near Onamia........

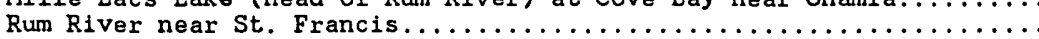

ELM CREEK BASIN

Elm Creek near Champlin.

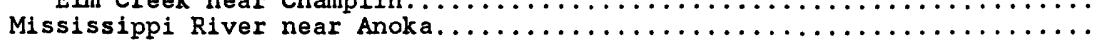

\begin{tabular}{|c|c|c|c|c|}
\hline $\begin{array}{l}\text { ld } \\
l- \\
d\end{array}$ & $\begin{array}{l}- \\
\text { e }\end{array}$ & $\begin{array}{l}- \\
-\end{array}$ & $\begin{array}{l}- \\
-\end{array}$ & $\begin{array}{l}\text { Station number } \\
-\{.05200510 \\
-) \ldots 05201000 \\
-) \ldots 05201500\end{array}$ \\
\hline d & $\begin{array}{l}e \\
e \\
- \\
e \\
-\end{array}$ & $\begin{array}{l}c \\
- \\
- \\
-\end{array}$ & $\begin{array}{l}\text { b } \\
- \\
- \\
-\end{array}$ & $\begin{array}{l}\text { p)..05202000 } \\
\text {-)..05206000 } \\
\text {-)..05206500 } \\
\text {-)..05210500 } \\
\text {-)...05211000 }\end{array}$ \\
\hline d & $\begin{array}{l}e \\
-\end{array}$ & $\begin{array}{l}- \\
-\end{array}$ & $\overline{-}$ & 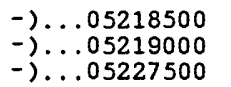 \\
\hline (d & $\begin{array}{l}e \\
-\end{array}$ & $\begin{array}{l}- \\
-\end{array}$ & $\overline{-}$ & $\begin{array}{l}\text {-)..05230500 } \\
\text {-)..05231000 } \\
\text {-)..05242300 }\end{array}$ \\
\hline $\begin{array}{l}(d \\
(d) \\
(d)\end{array}$ & $=$ & $\begin{array}{l}- \\
-\end{array}$ & $\begin{array}{l}- \\
-\end{array}$ & 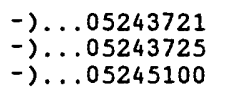 \\
\hline $\begin{array}{l}\text { (d } \\
\text { (d } \\
\text { d }\end{array}$ & $\begin{array}{l}e \\
- \\
- \\
-\end{array}$ & $\begin{array}{l}- \\
- \\
-\end{array}$ & $\begin{array}{l}- \\
\bar{z} \\
\bar{b}\end{array}$ & $\begin{array}{l}\text {-) } \ldots 05246500 \\
\text {-)..05247000 } \\
\text {-) } \ldots .05247500 \\
-\{.05261000 \\
\text { p) } \ldots 05267000\end{array}$ \\
\hline (d) & - & - & - & $\begin{array}{l}-) \ldots 05270500 \\
-) \ldots 05270700\end{array}$ \\
\hline (d & - & - & - & -)...05275000 \\
\hline (d) & - & - & - & -)...05280000 \\
\hline (- & - & - & - & $\begin{array}{l}\text {-) } \ldots .05284000 \\
-) \ldots 05286000\end{array}$ \\
\hline (c & - & c & b & $\begin{array}{l}\text { p) } \ldots .05287890 \\
\text { p) } \ldots 05288500\end{array}$ \\
\hline
\end{tabular}


UPPER MISSISSIPPI RIVER BASIN--Continued

MINNESOTA RIVER BASIN

Little Minnesota River (head of Minnesota River) near Peever, SD..... Whetstone River near Big Stone City, SD....................

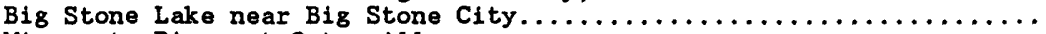

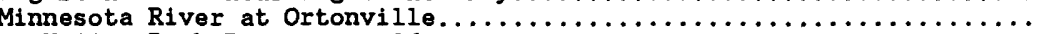

Yellow Bank River near Odessa.............................

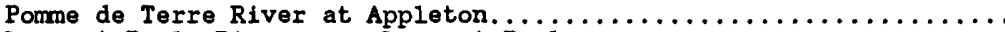

Lac qui Parle River near Lac qui Parle $\ldots \ldots \ldots \ldots \ldots \ldots \ldots \ldots \ldots \ldots \ldots \ldots \ldots \ldots$
Minnesota River near Lac qui Parle. $\ldots \ldots \ldots \ldots \ldots \ldots \ldots \ldots \ldots \ldots$

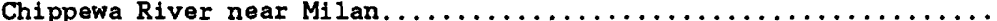

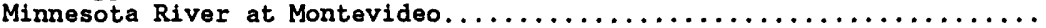

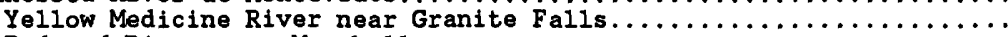

Redwood River near Marshall.

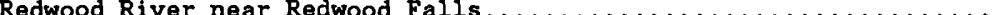

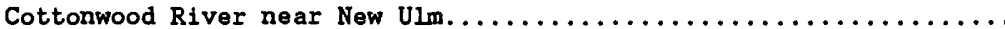

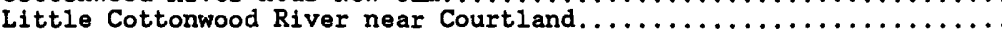

Blue Earth River:

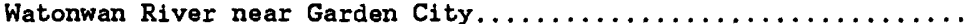

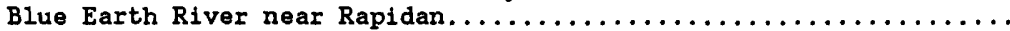

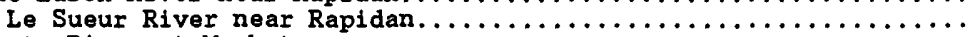

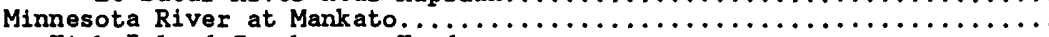

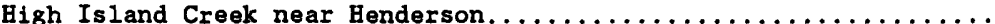

Minnesota River near Jordan. . . . . . . . . . . . . . . . . . . . . .

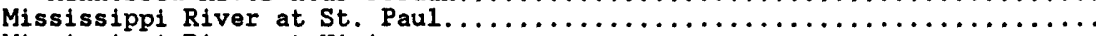

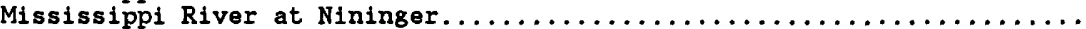

ST. CROIX RIVER BASIN

St. Croix River:

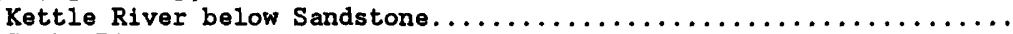
Snake River:

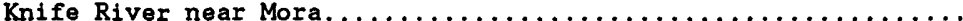

St. Croix River at St. Croix Falls, WI .......................

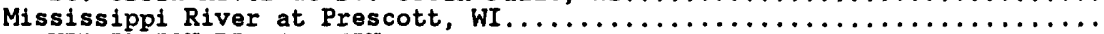

VERMILLION RIVER BASIN

Vermillion River near Empire.

CANNON RIVER BASIN

Cannon River:

Straight River near Faribault.

ZUMBRO RIVER BASIN

South Fork Zumbro River at Rochester.

WHITEWATER RIVER BASIN

North Fork Whitewater River near Elba.

Mide Fork Whitewater River near si

GARVIN BROOK BASIN

Garvin Brook near Minnesota City

Mississippi River at Winona...

ROOT RIVER BASIN

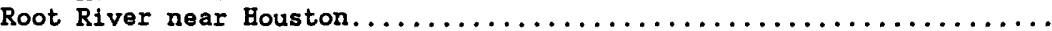

IOWA RIVER BASIN

Iowa River:

Cedar River near Austin.

DES MOINES RIVER BASIN

Des Moines River at Jackson.

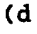

(d

(d

(d

(d

(d
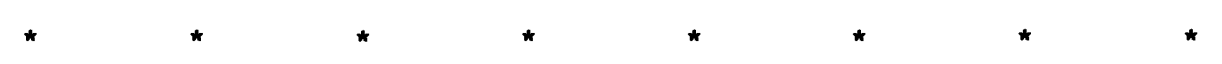

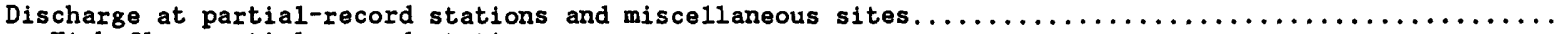

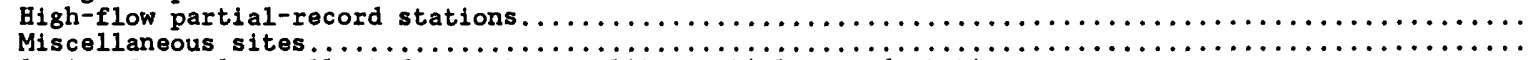


ANOKA

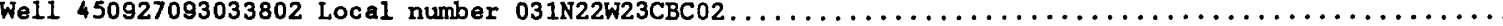

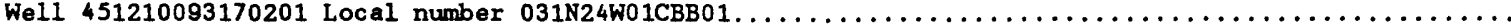

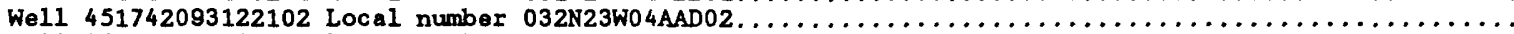

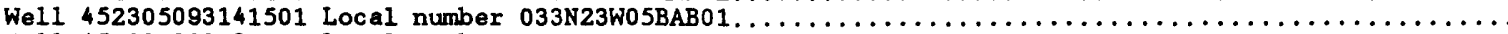

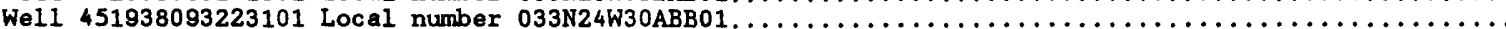
BELTRAMI

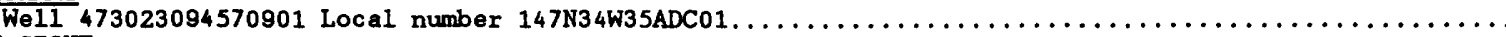
BIG STONE

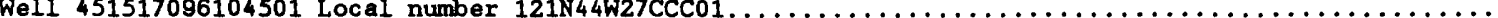

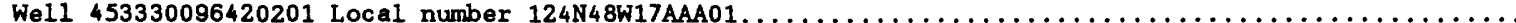

BLUE EARTH

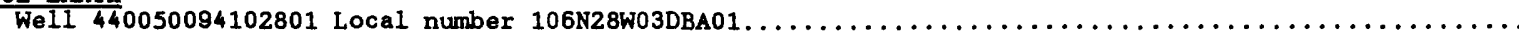

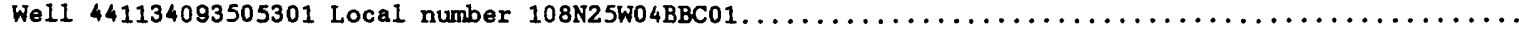
BROWN

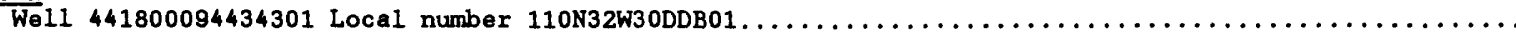
CHIPPEWA

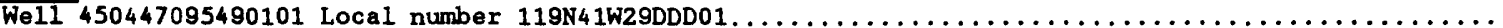

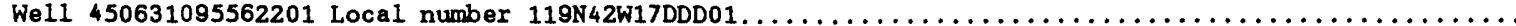

CHISAGO

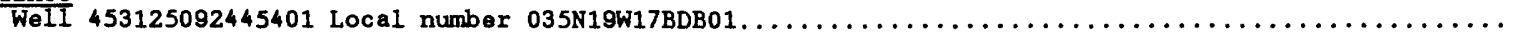
CROW WING

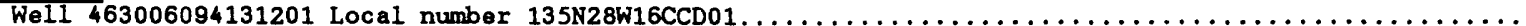

DAKOTA

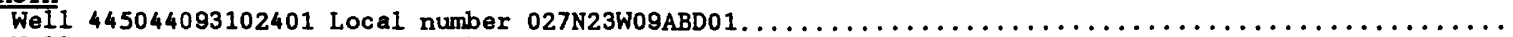

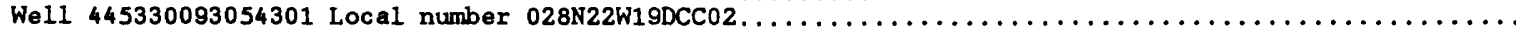

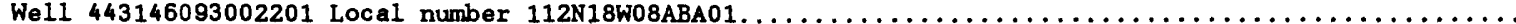

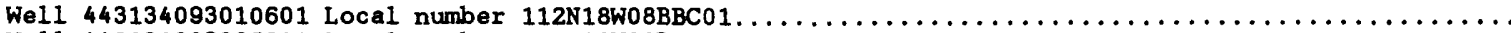

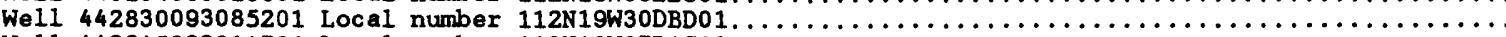

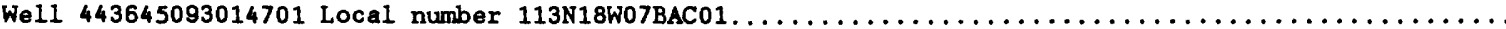

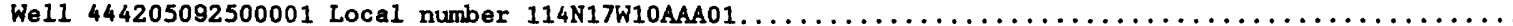

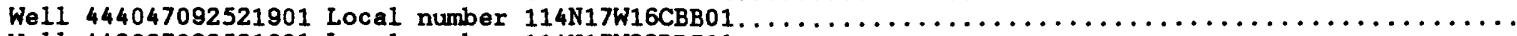

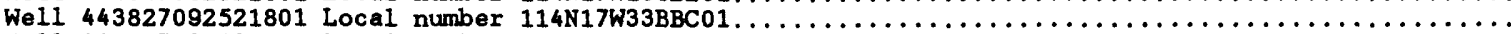

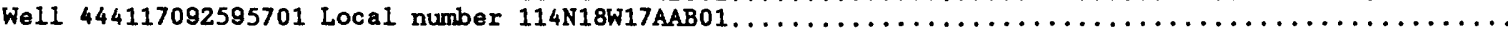

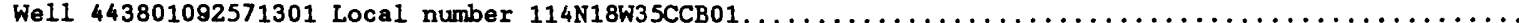

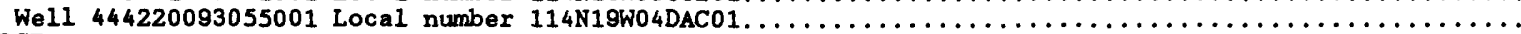
DODGE

We11 440448092485501 Local number 107N17W13BBA01.

FARIBAULT

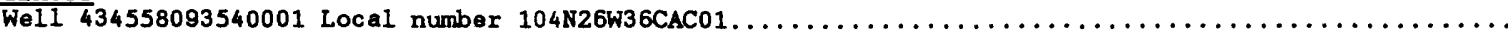
FILLMORE

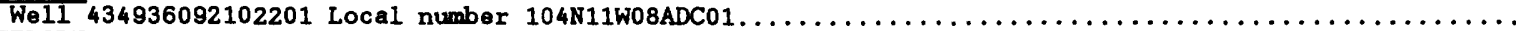
FREEBORN

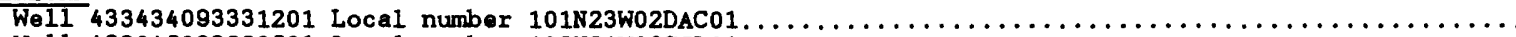

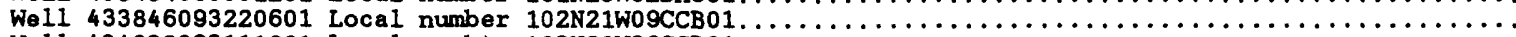

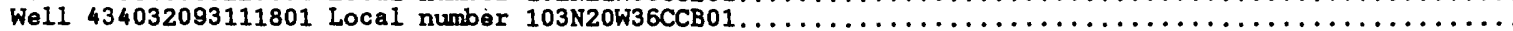

Well 434308093322001 Local number 103N23W13CDA01.

GOODHUE

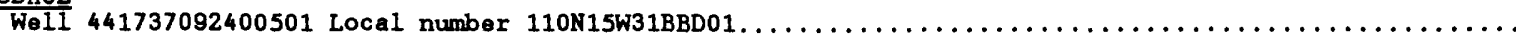

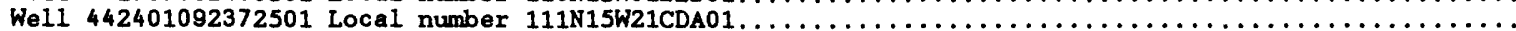

We11 443012092362201 Local number 113N15W27BAB01.

HENNEPIN

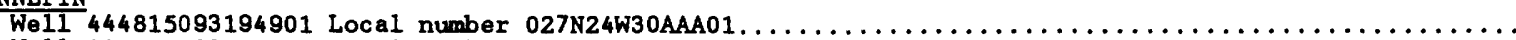

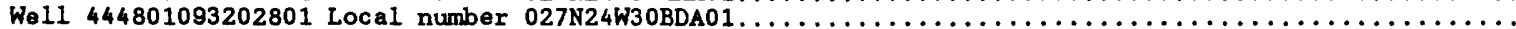

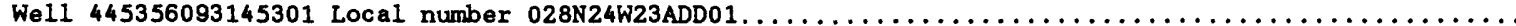

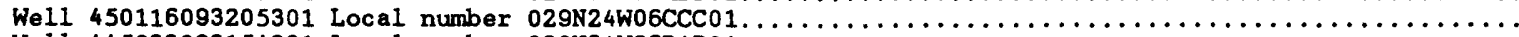

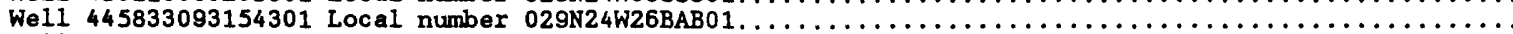

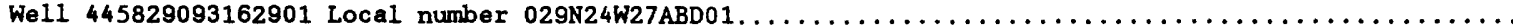

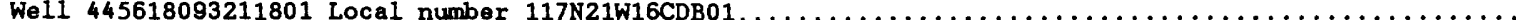

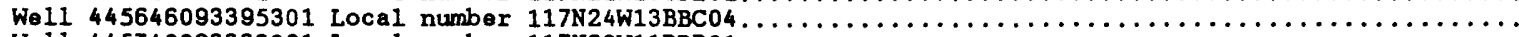

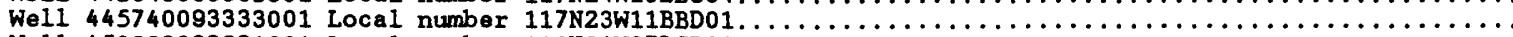

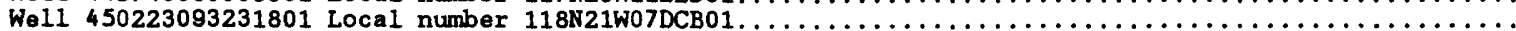

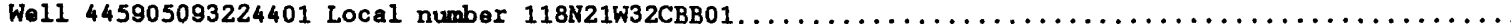

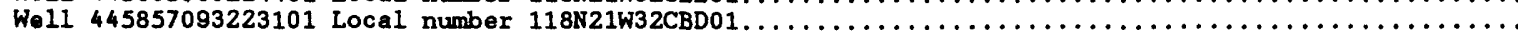

194

194

194

195

195

196

196

197

197

198

199

199

199

200

200

201

202

202

203

203

203

204

204

204

205

205

206

206

207

207

207

208

208

209

209

209

210

210

211

211

212

212

212

213

213

214

215

215

215 
HOUSTON

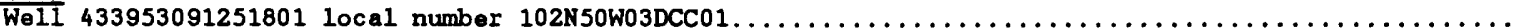

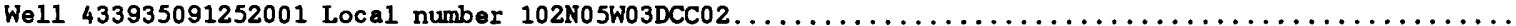

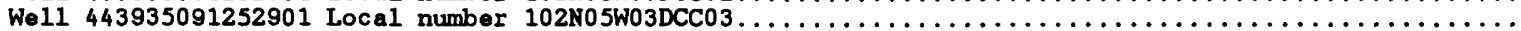
HUBBARD

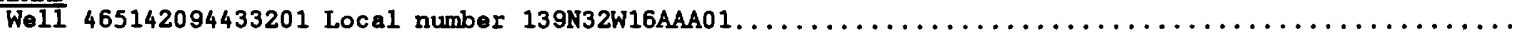

ISANT I

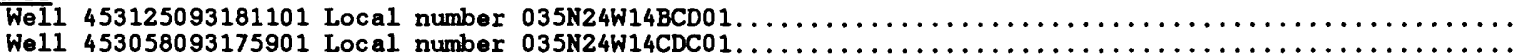

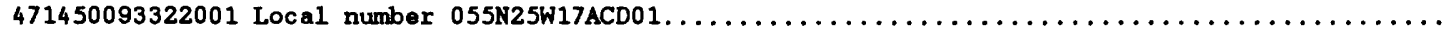

JACKSON

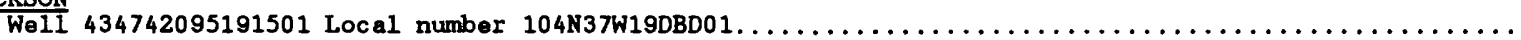

We11 455236093172301 Local number 039N24W11DDC01 $\ldots \ldots \ldots \ldots$

LE SUEUR

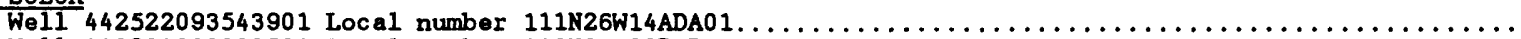

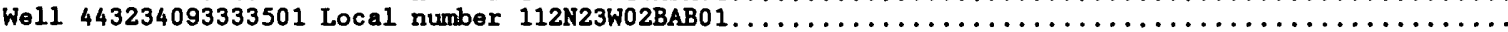

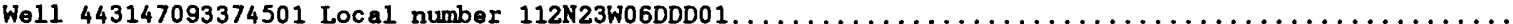

LINCOLN

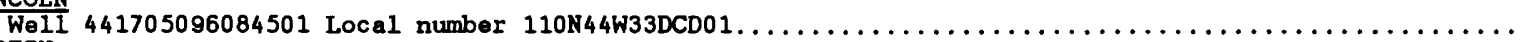

MARTIN

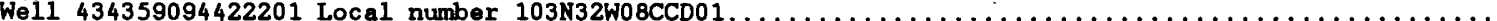

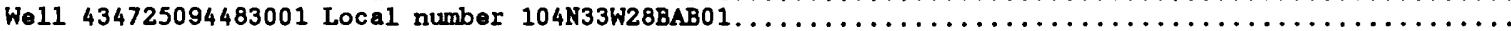

MC LEOD

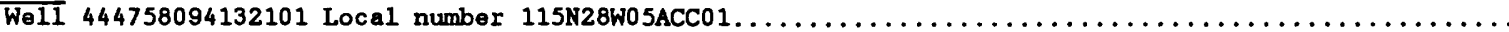

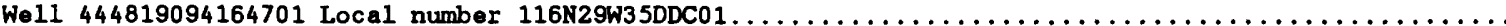

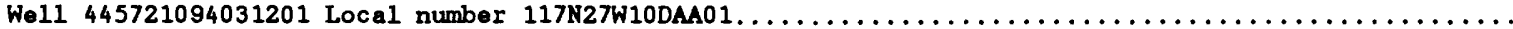
MEEKER

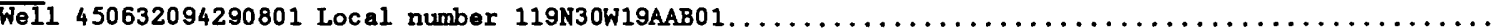

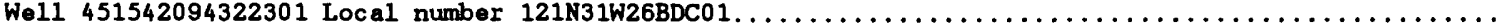

MILLE IACS

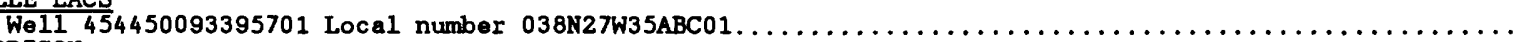
MORRISON

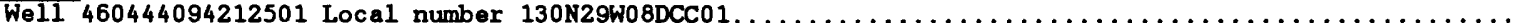

MOWER

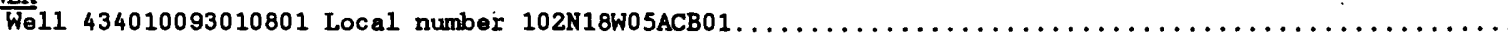

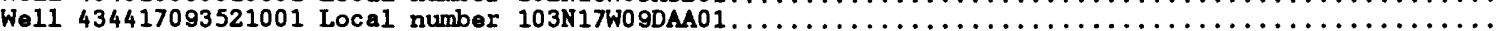
OLMSTED

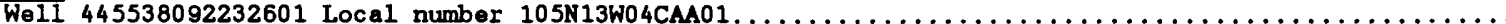

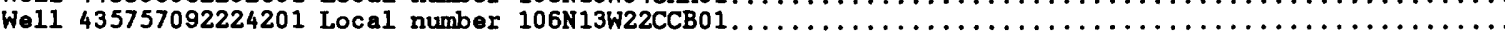
RAMSEY

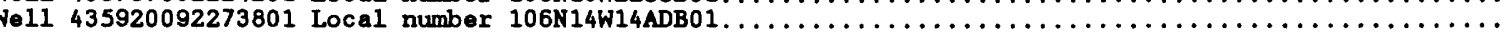

We11 445955093011001 Local number 029N22W14CAB01.

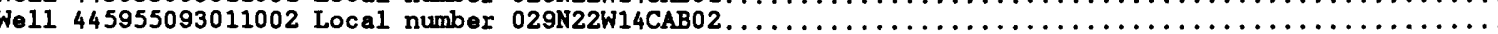

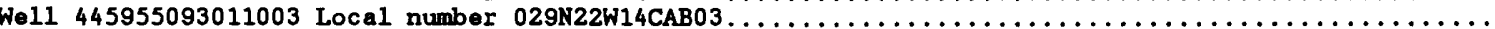

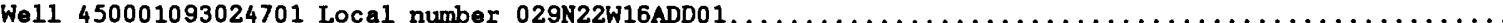

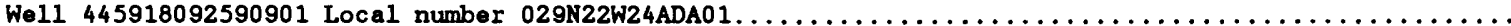

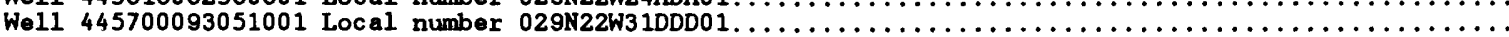

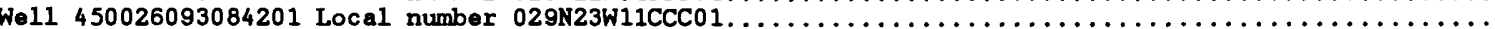

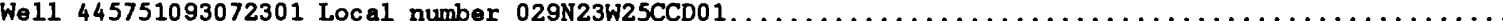

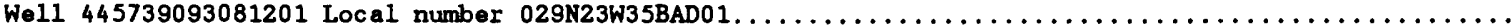

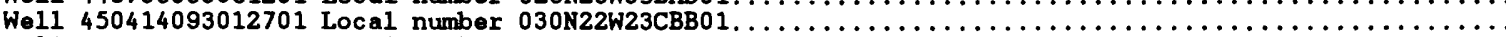

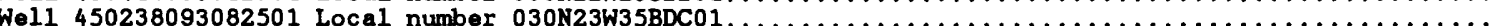
RDWOOD

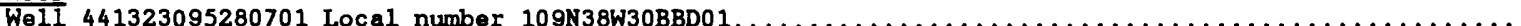

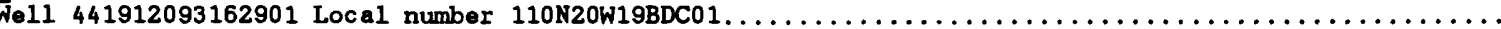

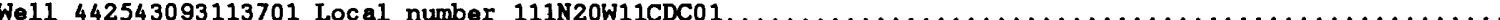

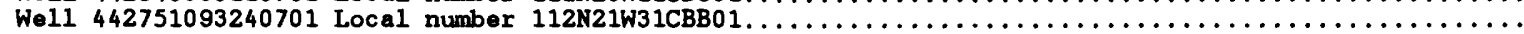

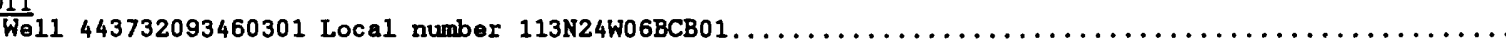

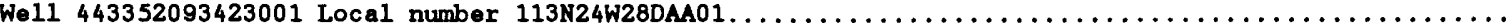

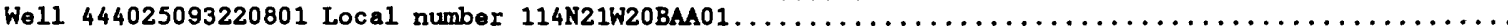

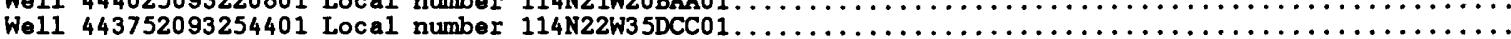

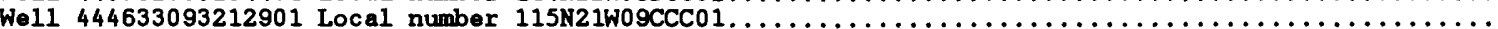

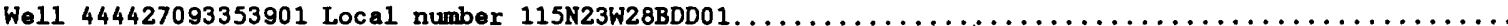

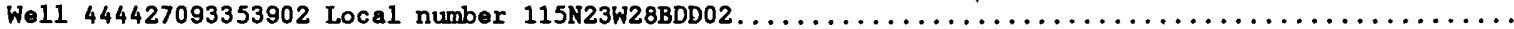

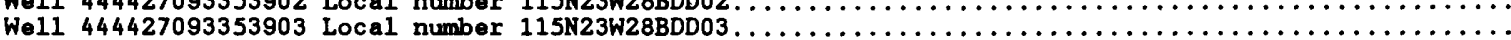


STEELE

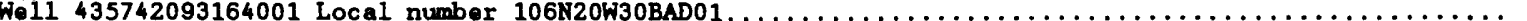

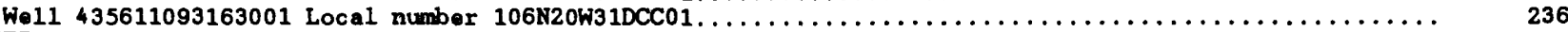
SWIFT

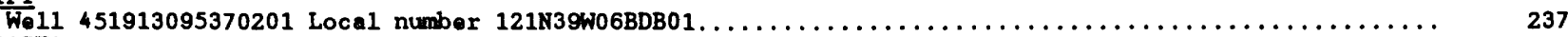
WABASHA

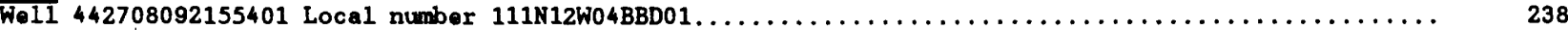

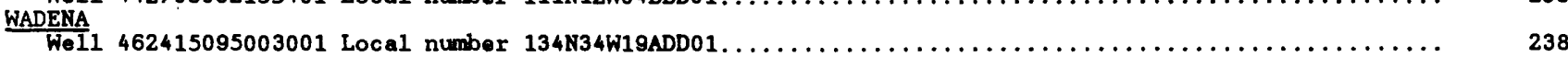
WASHINGTON

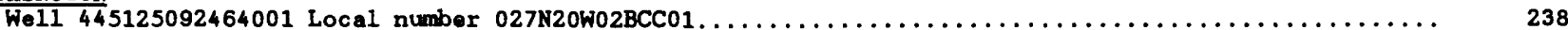

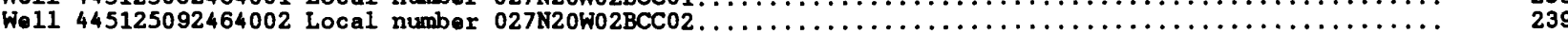

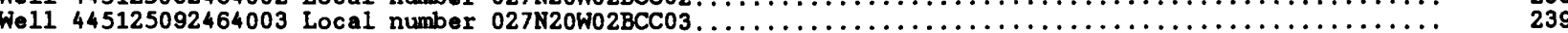

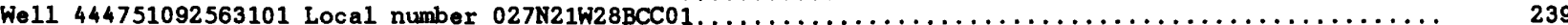

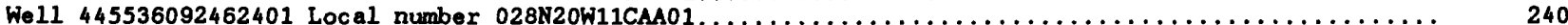

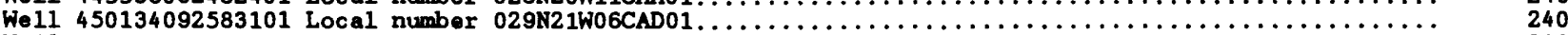

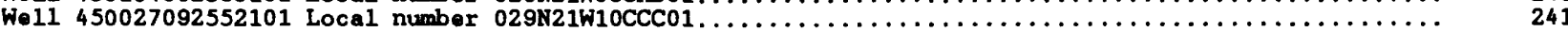

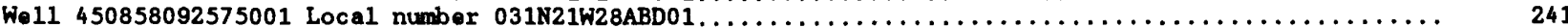

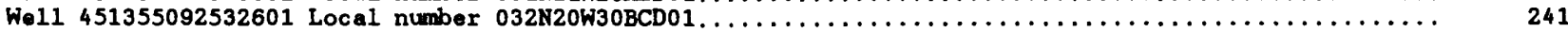
WATONWAN

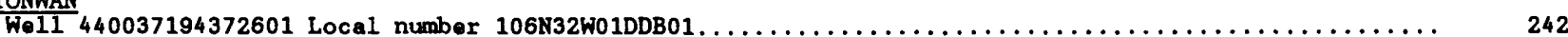

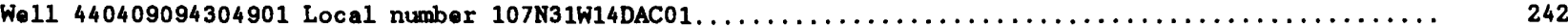

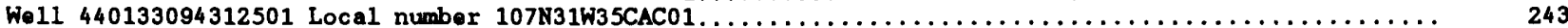
WINONA

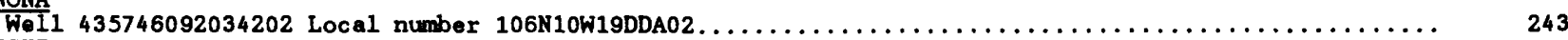
WRIGET

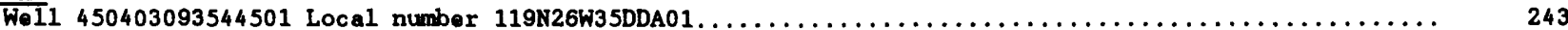

YELLOW MEDICINE

We11 444219096165501 Local number 114N45W04DCDO1.

COUATIES WIIH QUAITI OF GROUID WATER RBCORDS

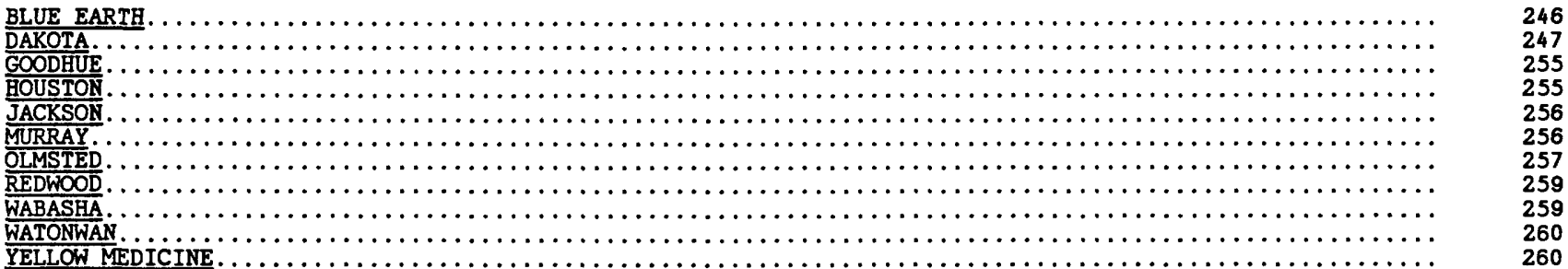

FRECIPITATIOT SITES, POQ KIICH CHOMCAL QUALTY RECORDS ARE PUBLISHFD

Precipitation Station at Camp Riploy 
WATER RESOURCES DATA - MINNESOTA, 1991

DISCONTINUED SURFACE-WATER DISCHARGE OR STAGE-ONLY STATIONS

The following continuous-record surface-water discharge or stage-only stations (gaging stations) in Minnesota have been discontinued. Dally streamflow or stage records were collected and published for the period of record, expressed in water years, shown for each station. Those stations with an asterisk ( $*$ ) after the station number are currently operated as crest-stage partial-record stations. Discontinued project stations with less than 3 years of reccord have not been included. Information regarding these stations may be obtained from the District office at the address given on the back side of the title page of this report.

[Letters after station name designate type of the data collected:

(d) discharge, (e) elevation (stage only)]

\begin{tabular}{|c|c|c|c|}
\hline Station name & $\begin{array}{l}\text { Station } \\
\text { number }\end{array}$ & $\begin{array}{c}\text { Drainage } \\
\operatorname{arga} \\
\left(\mathrm{mi}^{2}\right)\end{array}$ & $\begin{array}{l}\text { Period } \\
\text { of } \\
\text { record }\end{array}$ \\
\hline UPPER MISSISSIPPI RIVER BASIN & & & \\
\hline Mississippi River near Deer River, MN (d) & 05210000 & a3, 190 & $1945-50$ \\
\hline Prairie River near Taconite, MN (d) & 05212700 & a360 & $1967-83$ \\
\hline Pralrie River near Grand Raplds, MN (d) & $05213000 *$ & 485 & $\begin{array}{r}1909+ \\
1925-49\end{array}$ \\
\hline O'Brien Creek near Pengilly, MN (d) & 05216800 & - & $1963-68$ \\
\hline Initial tallings basin outflow near Keewatin, $M$ (d) & 05216820 & 2.5 & $1982-85$ \\
\hline Swan River near Calumet, MN (d) & 05216850 & 114 & $1964-90$ \\
\hline Swan River near Warba, MN (d) & 05217000 & 254 & $1954-69$ \\
\hline Swan River near Swan River, MN (d) & 05217500 & a290 & 1929 \\
\hline $\begin{array}{l}\text { Mississippl River above Sandy River near Libby (above } \\
\text { Sandy River), MN (d) }\end{array}$ & 05218000 & 4,560 & $\begin{array}{l}1895-1915 \\
1925-29\end{array}$ \\
\hline Mississippi River below Sandy River near Libby, MN (d) & 05220500 & a5, 060 & $1930-90$ \\
\hline Willow River near Palisade, MN (d) & 05221000 & 442 & 1929 \\
\hline Ripple (Mud) River near Wealthwood, MN (d) & 05226200 & - & $1937-39$ \\
\hline Pelican Brook (Lons Lake) near Pequot Lakes, MN (d) & 05232000 & - & $\begin{array}{l}1938-42 \\
1943-47\end{array}$ \\
\hline Rabbit River near Crosby, MN (d) & 05241500 & 8.38 & $1945-63$ \\
\hline Little Sand Lake outlet (Sand Lake outlet) near Dorset, MN (d) & 05242700 & a74 & $1930-41$ \\
\hline Crow Wing River at Nimrod, MN (d) & $05244000 *$ & a1, 010 & $\begin{array}{l}1910-14 \\
1930-81\end{array}$ \\
\hline Crow Wing River at Motley, MN (d) & 05244500 & a2, 140 & $\begin{array}{c}1909+ \\
1913-17 \\
1930-31\end{array}$ \\
\hline Diversion from Long Prairle River near Osak1s, MN (d) & 05244980 & - & $1939-47$ \\
\hline Long Prairle River near Osakis, MN (d) & 05245000 & - & $1949-54$ \\
\hline Long Prairie River near Motley, MN (d) & 05245500 & 973 & $\begin{array}{l}1909-17 \\
1930-31\end{array}$ \\
\hline Crow Wing River at Pillager, MN (d) & 05246000 & a3, 230 & $\begin{array}{l}1903\} \\
1909-13 \\
1925-50\end{array}$ \\
\hline Mississippl River near Fort Ripley, MN (d) & $05261000 *$ & a11, 010 & $\begin{array}{c}1906 \\
1909-10 \\
1929\end{array}$ \\
\hline Nokasippi River near Fort Ripley, MN (d) & 05261500 & 210 & 1929 \\
\hline Platte (Platt) River at Royalton, MN (d) & $05268000 *$ & 338 & $1929-36$ \\
\hline Mississippl River near Sauk Rapids, MN (d) & 05269000 & a 12,400 & $1903-06$ \\
\hline Mississippi River at Sartell, MN (d) & 05270000 & a 12,450 & 1929 \\
\hline
\end{tabular}




\begin{tabular}{|c|c|c|c|}
\hline Station name & $\begin{array}{l}\text { Station } \\
\text { number }\end{array}$ & $\begin{array}{c}\text { Drainage } \\
\text { area } \\
\left(m i^{2}\right)\end{array}$ & $\begin{array}{l}\text { Period } \\
\text { of } \\
\text { record }\end{array}$ \\
\hline \multicolumn{4}{|c|}{ UPPER MISSISSIPPI RIVER BASIN--Continued } \\
\hline Clearwater River at Clearwater, MN (d) & 05273500 & - & $\begin{aligned} 1937 \\
1940-42\end{aligned}$ \\
\hline Elk River above St. Francis River near Bis Lake, MN (d) & 05274500 & 384 & 1929 \\
\hline St. Francis River at Santiago, MN (d) & 05274700 & - & $\begin{array}{l}1965-70 \\
1980-81\end{array}$ \\
\hline St. Francis River above Zimmerman, MN (d) & 05274750 & - & $1980-84$ \\
\hline St. Francis River near Big Lake, MN (d) & 05274900 & - & $1965-70$ \\
\hline Mississippi River at Elk River, MN (d) & 05275500 & a14,500 & $1915-56$ \\
\hline North Fork Crow River near Regal, MN (d) & 05276000 & 215 & $1943-54$ \\
\hline Middle Fork Crow River at New London, MN (d) & 05277000 & - & $\begin{array}{l}1939-42 \\
1943-47\end{array}$ \\
\hline $\begin{array}{l}\text { Middle Fork Crow River (Calhoun Lake Diversion) near } \\
\text { Spicer, MN }\end{array}$ & 05277500 & - & $\begin{array}{r}1939 \\
1940-46\end{array}$ \\
\hline Middle Fork Crow River near Spicer, MN (d) & 05278000 & 179 & $1949-87$ \\
\hline North Fork Crow River near Rockford, MN (d) & 05278400 & - & $1909-10$ \\
\hline South Fork Crow River at Cosmos, MN (d) & 05278500 & 221 & $1945-64$ \\
\hline Buffalo Creek near Glencoe, MN (d) & 05278930 & 374 & $1972-80$ \\
\hline South Fork Crow River near Mayer, MN (d) & $05279000 *$ & a1, 170 & $1934-79$ \\
\hline South Fork Crow River near Rockford, MN (d) & 05279500 & a1,250 & $1909-12$ \\
\hline Mississippi River at Anoka, MN (d) & 05283500 & a17, 100 & $1897-13$ \\
\hline Rum River at Onamia, MN (d) & 05284500 & 414 & $1910-12$ \\
\hline Rum River at Spencer Brook MN (d) & 05284750 & - & $1960-64$ \\
\hline Rum River at Cambridge, MN (d) & 05285000 & a1, 160 & $1909-14$ \\
\hline Rum River at St. Francis, MN (d) & 05285500 & - & 1903 \\
\hline Rum River near Anoka, MN (d) & 05286500 & 1,430 & $\begin{array}{l}1905-06 \\
1909\end{array}$ \\
\hline $\begin{array}{l}\text { Minnetonka Lake (head of Minnehaha Creek) near Wayzata } \\
\text { (at Excelsior). MN (d). }\end{array}$ & 05289000 & - & $1938-64$ \\
\hline Minnehaha Creek at Minnetonka Mills, MN (d) & 05289500 & 130 & $1953-64$ \\
\hline \multicolumn{4}{|c|}{ MINNESOTA RIVER BASIN } \\
\hline Minnesota River near Odessa, MN (d) & 05292500 & a1,340 & $\begin{array}{l}1909-12 \\
1944-63\end{array}$ \\
\hline Pomme de Terre River near Morris, MN (d) & 05293500 & - & $\begin{array}{l}1937-39 \\
1940-47\end{array}$ \\
\hline Canby Creek at Canby, MN (d) & 05299500 & - & $\begin{array}{l}1938-39 \\
1940-46\end{array}$ \\
\hline Ten Mile Creek near Boyd, MN (d) & 05300500 & 82.8 & $1949-51$ \\
\hline Little Chippewa River near Lowry, MN (d) & 05302000 & a54 & 1941 \\
\hline Little Chippewa River near Starbuck, MN (d) & $05302500 *$ & 111 & $1938-39$ \\
\hline Chippewa River at diversion dam near Hancock, MN (d) & 05303000 & - & $\begin{array}{l}1930-39 \\
1940-46\end{array}$ \\
\hline Chippewa River at Benson, MN (d) & 05303500 & a1, 270 & $1949-51$ \\
\hline Shakopee Creek near Benson, MN (d) & 05304000 & 352 & $1949-54$ \\
\hline
\end{tabular}


DISCONTINUED SURFACE-WATER DISCHARGE OR STAGE-ONLY STATIONS.--Continued

\begin{tabular}{|c|c|c|c|}
\hline Station name & $\begin{array}{r}\text { Station } \\
\text { number }\end{array}$ & $\begin{array}{c}\text { Drainage } \\
\text { area } \\
\left(\mathrm{mi}^{2}\right)\end{array}$ & $\begin{array}{l}\text { Period } \\
\text { of } \\
\text { record }\end{array}$ \\
\hline \multicolumn{4}{|l|}{ MINNESOTA RIVER BASIN--Continued } \\
\hline Chippewa River near Watson, MN (d) & 05305000 & a2, 050 & $\begin{array}{l}1910-17 \\
1931-36\end{array}$ \\
\hline South Branch Yellow Medicine River at Minneota, MN (d) & 05311400 & 111 & $\begin{array}{l}1960-81 \\
1983-87\end{array}$ \\
\hline Yellow Medicine River near Cottonwood, MN (d) & 05311500 & 465 & $1945-46$ \\
\hline Spring Creek near Clarkfield, MN (d) & 05312000 & a89 & $1945-46$ \\
\hline Spring Creok near Hazel Run, MN (d) & 05312500 & 101 & $1945-48$ \\
\hline Yellow Medicine River near Hanley Falls, MN (d) & 05313000 & 606 & $1945-47$ \\
\hline Hawk Creek at outlet of Eagle Lake near Willmar, MN (d) & 05313521 & - & $1972-73$ \\
\hline Eagle Lake tributary No. 7 near Willmar, MN (d) & 05313560 & - & $1972-73$ \\
\hline Eagle Lake tributary No. 8 near Willmar, MN (d) & 05313570 & - & $1972-73$ \\
\hline Chetomba Creek near Maynard, MN (d) & 05314000 & a200 & $1949-51$ \\
\hline Hawk Creek near Maynard, MN (d) & $05314500 *$ & 474 & $1949-54$ \\
\hline Prairie Ravine near Marshall, MN (d) & $05315200 *$ & 5.63 & $1959-64$ \\
\hline Redwood River near Green Valley, MN (d) & 05315500 & 436 & $1945-57$ \\
\hline Redwood River near Seaforth, MN (d) & 05316000 & 573 & $1945-46$ \\
\hline Minnesota River at New U1m, MN (d) & 05316770 & 9,536 & $1968-76$ \\
\hline Dry Creek near Jeffers, MN (d) & 05316900 & 3.13 & $1982-85$ \\
\hline Minnesota River at Judson, MN (d) & 05317500 & a11,200 & $1938-50$ \\
\hline East Branch (East Fork) Blue Earth River near Bricelyn, MN (d) & $05318000 *$ & 132 & $1951-70$ \\
\hline South Fork Watonwan River at diversion dam near St. James, MN (d) & 05319000 & - & $\begin{array}{l}1939 \\
1940-46\end{array}$ \\
\hline Blue Earth River at Mankato, MN (d) & 05321000 & a3,550 & $\begin{array}{l}1938-39 \\
1940-42\end{array}$ \\
\hline Sand Creek at diversion dam near Jordan, MN (d) & 05330400 & - & $\begin{array}{l}1938-39 \\
1940-46\end{array}$ \\
\hline Purgatory Creek at Eden Prairie, MN (d) & 05330800 & - & $1975-80$ \\
\hline Nine Mile Creek at Bloomington, MN (d) & 05330900 & - & $1963-73$ \\
\hline ST. CROIX RIVER BASIN & & & . \\
\hline Glaisby Brook near Kettle River, MN (d) & $05336200 *$ & 24.2 & $1959-70$ \\
\hline Kettle River near Sandstone, MN (d) & 05336500 & 825 & $1908-16$ \\
\hline Grindstone River at Hinckley, MN (d) & 05337000 & - & $1940-47$ \\
\hline Snake River at Mora, MN (d) & 05337500 & 422 & $1909-13$ \\
\hline Snake River at Sanatorium Bridge near Pine City, MN (d) & 05338000 & - & $1937-38$ \\
\hline St. Croix River near Rush City, MN (d) & 05339500 & a5, 120 & $1923-61$ \\
\hline Sunrise River near Stacy, MN (d) & 05340000 & 167 & $1949-65$ \\
\hline Sunrise River near Lindstrom, MN (d) & 05340050 & 231 & $1965-85$ \\
\hline
\end{tabular}




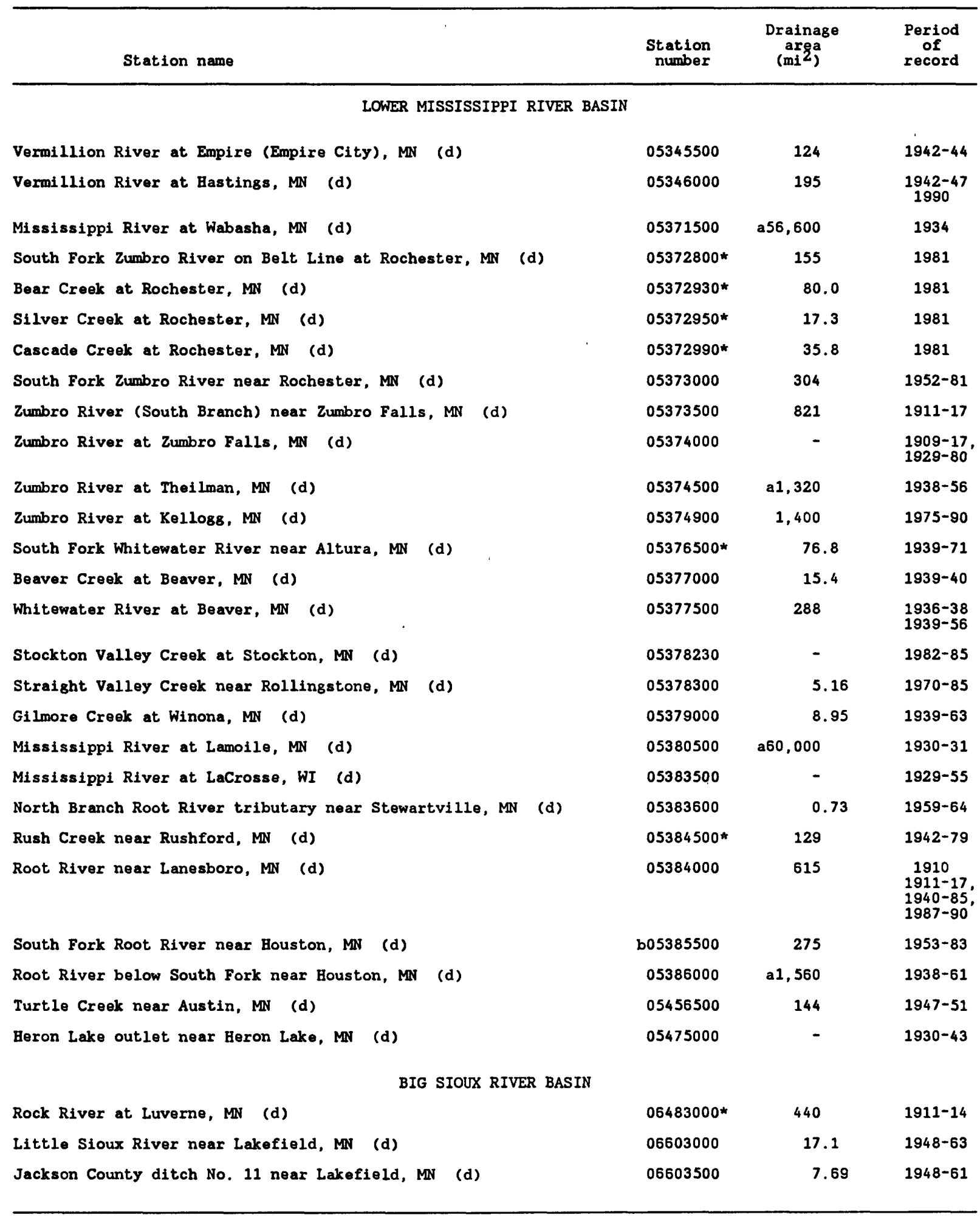




\section{WATER RESOURCES DATA FOR MINNESOTA, 1990}

\section{INTRODUCTION}

The Water Resources Division of the U.S Geological Survey, in cooperation with State agencies, obtains a large amount of data pertaining to the water resources of Minnesota each water year. These data, accumulated during many years, constitute a valuable data base for developing an improved understanding of the water resources of the State. To make these data readily available to interested parties outside the Geological Survey, the data are published annually in this report series entitled "Water Resources Data - Minnesota."

Water resources data for the 1991 water year for Minnesota consist of records of stage, discharge, and water quality of streams; stage, contents, and water quality of lakes and reservoirs; and water levels and water quality of ground water. This volume contains discharge records for 60 gaging stations; stage and contents for 9 lakes and reservoirs; water quality for 18 stream stations, 1 lake station, 22 partial-record sites, 1 precipitation station, 108 wells; and water levels for 119 observation wells. Also included are 59 high-flow partial-record stations. Additional water data were collected at various sites, not involved in the systematic data collection program, and are published as miscellaneous measurements or low-flow investigations. These data, together with the data in Volume 1, represent that part of the National Water Data System collected by the U.S. Geological Survey and cooperating State and Federal agencies in Minnesota.

This series of annual reports for Minnesota began with the 1961 water year with a report that contained only data relating to the quantities of surface water. For the 1964 water year, a similar report was introduced that contained only data relating to water quality. Beginning with the 1975 water year, the report was changed to present, in one volume, data on quantities of surface water, quality of surface and ground water, and ground-water levels.

Prior to introduction of this series and for several water years concurrent with it, water-resources data for Minnesota were published in U.S. Geological Survey Water-Supply Papers. Data on stream discharge and stage and on lake or reservoir contents and stage, through September 1960, were published annually under the title "Surface-Water Supply of the United States, Parts 4, 5 and 6A." For the 1961 through 1970 water years, the data were published in two 5-year reports. Data on chemical quality, temperature, and suspended sediment for the 1941 through 1970 water years were published annually under the title "Quality of Surface Waters of the United States," and water levels for the 1935 through 1974 water years were published under the title "Ground-Water Levels in the United States." The above mentioned Water-Supply papers can be consulted in the libraries of the principal cities of the United States and may be purchased from Distribution Branch, Text Products Section, U.S. Geological Survey, 604 Pickett Street, Alexandria, VA 22304.

Publications similar to this report are published annually by the Geological Survey for all States. These official Survey reports have an identification number consisting of the two-letter State abbreviation, the last two digits of the water year, and volume number. For example, this volume is identified as the "U.S. Geological Survey Water-Data Report MN-91-2. For archiving and general distribution, the reports for 1971-1974 water years also are identified as water-data reports. These water-data reports are for sale in paper copy or in microfiche by the National Technical Information Service, U.S. Department of Commerce, Springfield, VA 22161.
Additional information, including current prices, for ordering specific reports may be obtained from the district chief at the address given on the back of the title page or by telephone (612) 7833101.

\section{COOPERATION}

The U.S. Geological Survey and organizations of the State of Minnesota have had cooperative agreements for the systematic collection of streamflow records since 1909, for ground-water levels since 1948, and for water-quality records since 1952 . Organizations that assisted in collecting data through cooperative agreement with the Survey are:

Minnesota Department of Natural Resources, Division of Waters, Kenneth Lokkesmoe, director.

Minnesota Department of Transportation, Leonard W. Levine, commissioner.

Metropolitan Waste Control Commission of the Twin Cities Area, Louis R. Clark, chairperson.

Beltrami Soil and Water Conservation District, Floyd W. Jorgensen, chairperson.

Elm Creek Conservation Commission, Fred G. Moore, chairperson.

Leech Lake Reservation Business Committee, Daniel Brown, chairperson.

Lower Red River Watershed Management Board, Donald Ogaard, chairman.

Whitewater Joint Powers Board, Eugene Kalmes, chairman.

Assistance in the form of funds or services was given by the U.S. Army Corps of Engineers, in collecting records for 46 gaging stations and 12 water-quality stations published in this report of 2 volumes. Thirteen gaging stations in the Hudson Bay and St. Lawrence River basins were maintained by funds appropriated to the United States Department of State. Eight of these, on water adjacent to the international boundary, are maintained by the United States (or Canada) under agreement with Canada (or the United States), and the records are obtained and compiled in a manner equally acceptable in both countries. These stations are designated herein as "International gaging stations."

\section{SUMMARY OF HYDROLOGIC CONDITIONS}

\section{PRECIPITATION}

Normal annual precipitation in Minnesota ranges from about 19 in. (inches) in the northwest to more than 32 in. in the southeast. Precipitation during water year 1991 ranged from less than $20 \mathrm{in}$. in the northwest to 44 in. in several areas in southern, central, and east-central Minnesota. Precipitation ranged from more than 3 in. below normal (based on record period 1961-90) in small areas of northern, southwestern, and southeastern Minnesota to more than 12 in. above normal in other areas of southern, central, and east-central Minnesota; precipitation in one small area in central 
Minnesota was more than $16 \mathrm{in.}$ above normal during the water year (fig. 1).

The water year began with an 8- to 12 -in. precipitation deficit in parts of northern Minnesota, and a 12- to 16-in. precipitation excess in parts of east-central and southeastern Minnesota. The following is a summary of precipitation during the 1991 water year.

October - above normal statewide except in the northwest and south where it was below normal.

\section{November - below normal statewide.}

December - near normal statewide except in parts of the south where it was considerably above normal.

January - below normal statewide.

February - below normal statewide except in the east-central, west-central, north-central, and northwest where it was above normal.

March - above normal statewide except in the northwest and northeast where it was below normal.

April - above normal statewide except in the northeast where it was below normal.

May - above normal statewide except in west-central region where it was below normal.

June - below normal statewide except in the northwest, westcentral, and southwest where it was above normal.

July - above normal statewide except in the southwest where it was below normal.

August - below normal statewide except in the south-central and southeast where it was above normal.

September - above normal statewide.

Two notable rainfalls occurred during the summer of 1991. The first was on Sunday, July 21, when approximately 6 in. of rain fell in a one-hour period in the Garvin Brook basin in southeastern Minnesota. This is about twice the 100 -year one-hour rainfall for that area. The entire town of Stockton, which lies in the Garvin Brook valley, was flooded to depths as much as 5 feet. Four miles downstream from Stockton, the gage shelter for the gaging station, Garvin Brook near Minnesota City (05378235), was washed away. A survey showed that Garvin Brook had risen 17 feet at the gage site.

The second event was on September 7, when more than 6 in. of rain fell on Saturday evening between $2 \mathrm{pm}$ and $10 \mathrm{pm}$ from south of Breckenridge in west-central Minnesota near the North Dakota border to Paynesville in central Minnesota, and from Hector to Glencoe in central Minnesota. A Soil and Water Conservation District official reported 11 in. of rain just west of Glencoe. An additional 2 or more inches of rain fell over much of Minnesota on Sunday night, September 8 . As a result, flood stages or near flood stages were reached in several tributaries to the Minnesota and Mississippi Rivers during September.

\section{STREAMFLOW}

Average annual runoff in Minnesota ranges from 1 in. in the west to $14 \mathrm{in}$. in the northeast. Annual runoff in water year 1991 ranged from 0.14 in. ( 6 percent of average) in a small part of northwestern Minnesota to 16.90 in. (129 percent of average) in eastcentral Minnesota (table 1, vol. 1). In contrast to the previous year, when runoff in the western one-third of the State was considerably less than one-half the long-term average, runoff in 1991 was less than one-half the long-term average only in the northwest. Runoff in west-central and southwestern Minnesota during 1991 generally exceeded the long-term average and for some streams was almost twice the long-term average. In the remaining eastern two-thirds of the State, runoff ranged from more than one-half the long-term average in the north to greater than twice the long-term average in some areas of the south (table 1, vol. 2).

In 1991, runoff to the Upper Mississippi and Missouri River Basins (Volume 2) ranged from below average in the north to considerably above average in parts of the south. Runoff ranged from as low as 47 percent of average in the Mississippi River at Grand Rapids (05211000) to as high as 222 percent of average in the Minnesota River at Mankato (05325000) in south-central Minnesota.

In east-central Minnesota, runoff in the Mississippi River at Aitkin (05227500) was 4.71 in. - 74 percent of the 46-year average (1946-91) of 6.40 in. and almost the same as in the previous year when runoff was $4.72 \mathrm{in}$. and 73 percent of average.

Runoff in the Crow River at Rockford (05280000), in the southern part of central Minnesota, was 8.54 in. or 216 percent of the station's 66-year average (1910-17, 1931, 1935-91) annual runoff of 3.96 in. reflecting the 8 to 20 in. of above normal precipitation in this basin during the 1991 water year (normal annual precipitation is 28 in.). In contrast, runoff in the previous year was 3.34 in. (86 percent of average).

In west-central Minnesota runoff in the Chippewa River near Milan (05304500) was 2.88 in., 127 percent of the station's 54-year average (1938-91) of 2.27 in. and more than twice the runoff that occurred in $1990-1.03 \mathrm{in}$. (46 percent of average)

In southwestern Minnesota, runoff in the Des Moines River at Jackson (05476000) was 4.84 in., 136 percent of the station's 56-year average (1936-91) of 3.56 in. This is $71 / 2$ times the runoff that occurred in the previous year -0.64 in. (18 percent of average), and is the result of 8 to 16 in. of above normal precipitation in this basin during 1991. Figure 2 shows the 1991 annual- and monthly-mean discharges for the above stations compared to the median of their mean discharge for a 30-year base period.

A new record peak discharge was recorded at Garvin Brook near Minnesota City (05378235) in southeastern Minnesota on July 21, 1991. The entire town of Stockton was inundated as a result of the intense rainfall. The stage rose to $\mathbf{1 7 . 7 9}$ feet at the gage on Garvin Brook, and a discharge of $10,800 \mathrm{ft}^{3} / \mathrm{s}$ was determined by indirect methods. The previous peak of record (1983, 1985-91), 1,580 $\mathrm{ft}^{3} / \mathrm{s}$, occurred on September 21, 1986 at a gage height of $6.63 \mathrm{ft}$. Also, record- and near-record-high monthly volumes occurred in the Minnesota and Mississippi Rivers as well as in several of their larger tributaries during late spring and summer of 1991.

The combined storage in the six Mississippi River Headwater Reservoirs (Winnibigoshish, Leech, Pokegama, Pine, Sandy, and Gull), in northern and central Minnesota, was 1,555,308 acre-feet at the close of the 1991 water year - an increase of 102,640 acre-feet from the close of last year.

\section{WATER QUALITY}

Boxplots for three U.S. Geological Survey National StreamQuality Accounting Network (NASQAN) stations and one benchmark station are used to depict variability in concentrations of dissolved solids and nitrate as nitrogen in the Upper Mississippi River basin (figs. 3 and 4); there are no water-quality stations in the Missouri River basin in Minnesota.

Boxplots are a useful graphical technique because they display the central tendency, variation, and skewness of a data set, as well as the presence or absence of extreme values. A boxplot consists of a centerline (the median) dividing a rectangle defined by the 75 th and 25 th percentiles. Whiskers are drawn from the ends of the box (75th and 25 th percentiles) to the most extreme observation within 1.5 times the interquartile range (the distance from the 25th to the 75th percentile values) beyond the ends of the box. Values more than 1.5 interquartile ranges from the box ends may indicate extreme hydrologic and chemical conditions or sampling and analytical errors. Observations from 1.5 to 3 interquartile ranges from the box in either direction are plotted individually with an asterisk. 


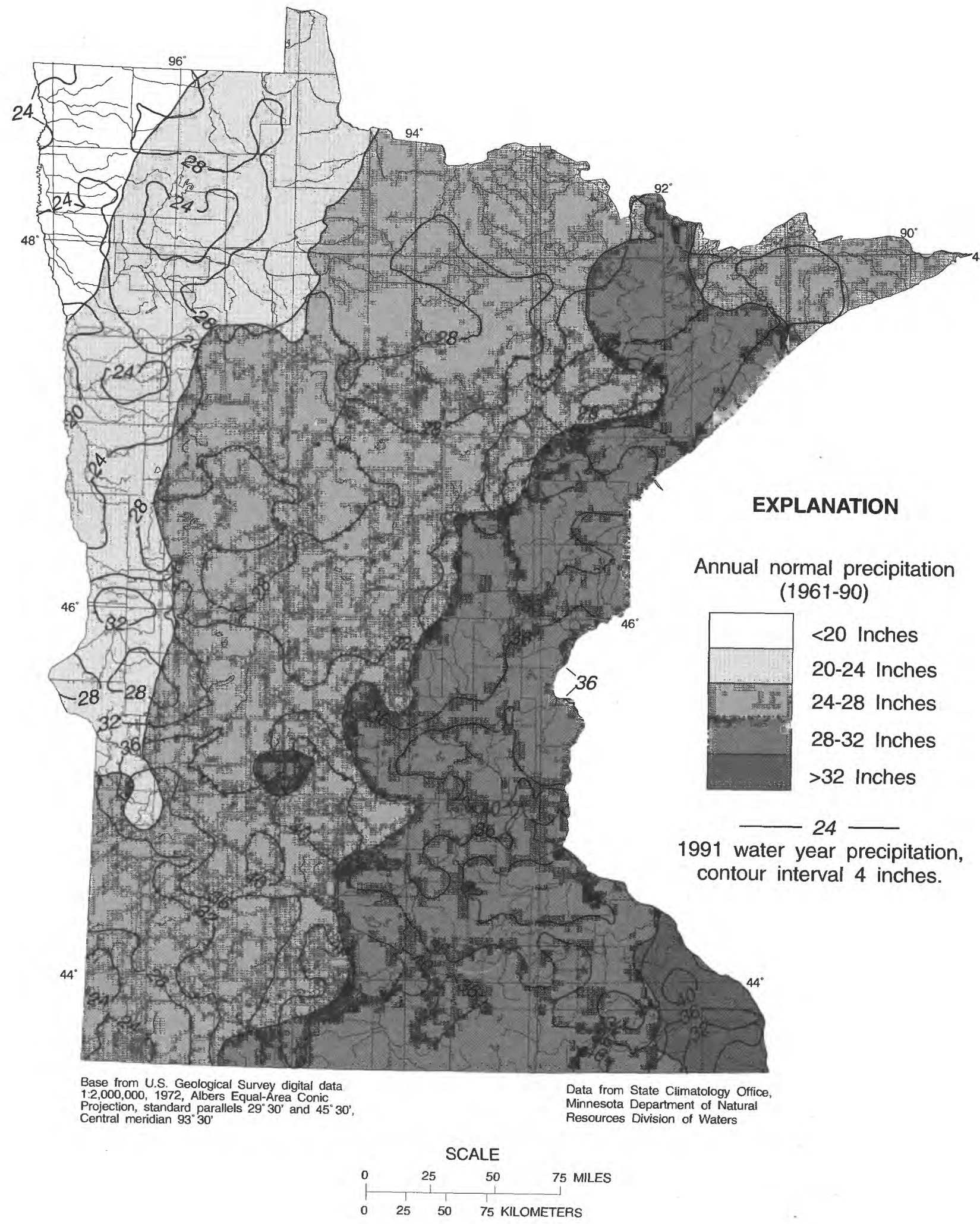

Figure 1.--Precipitation, in inches, during 1991 water year compared to normal annual precipitation in Minnesota. 


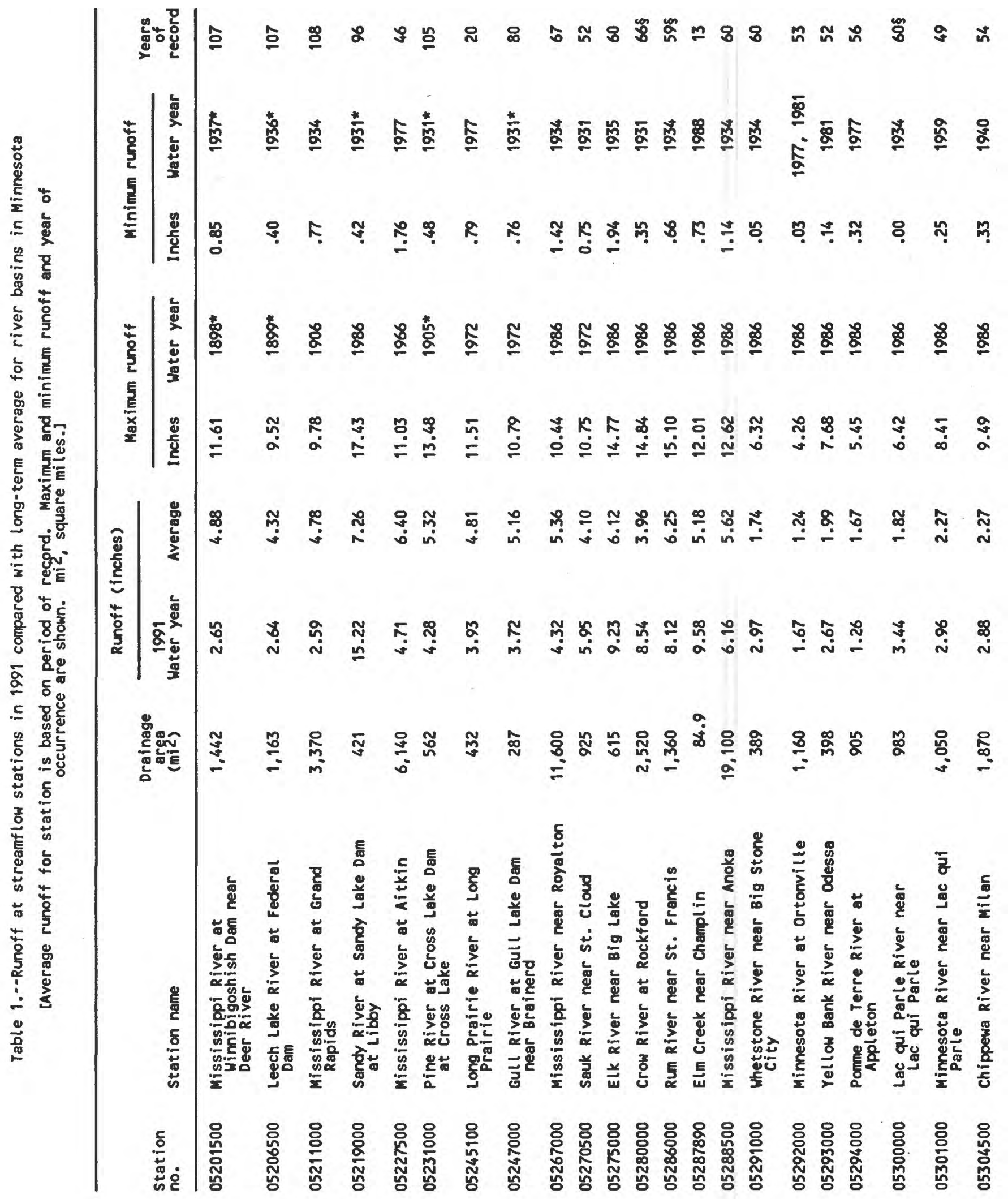




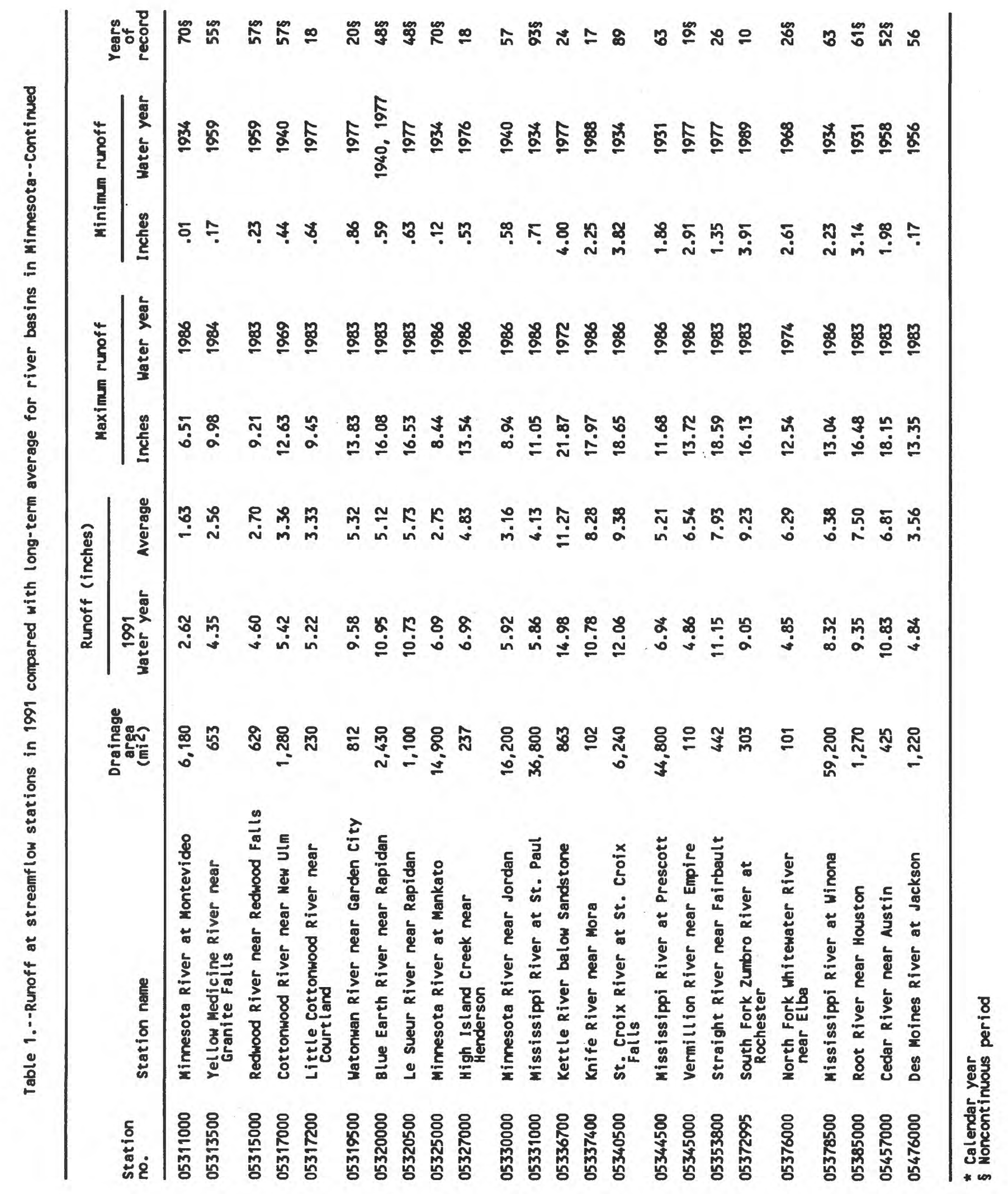




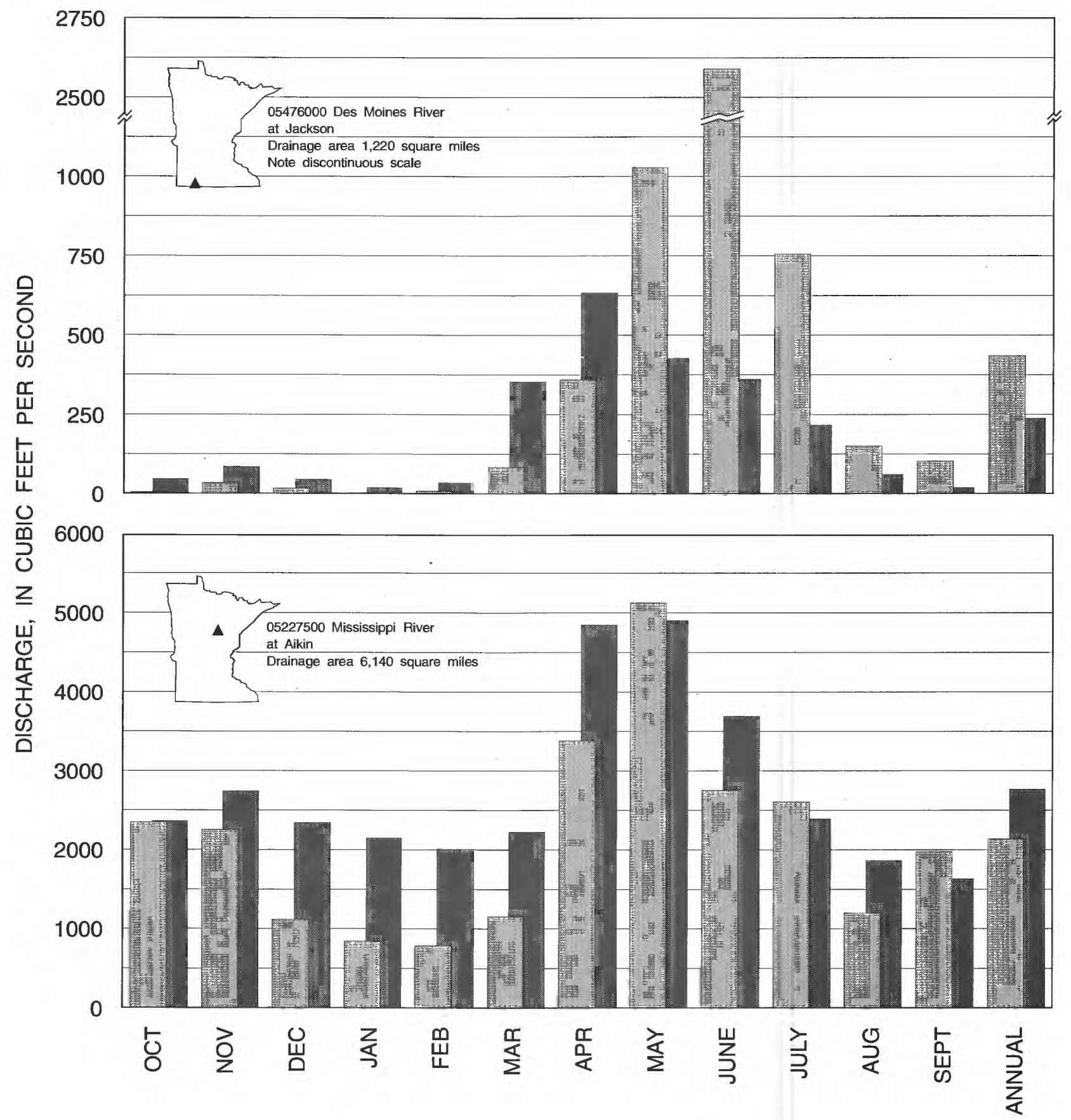

Figure 2.--Comparison of mean discharge for the 1991 water year with median 


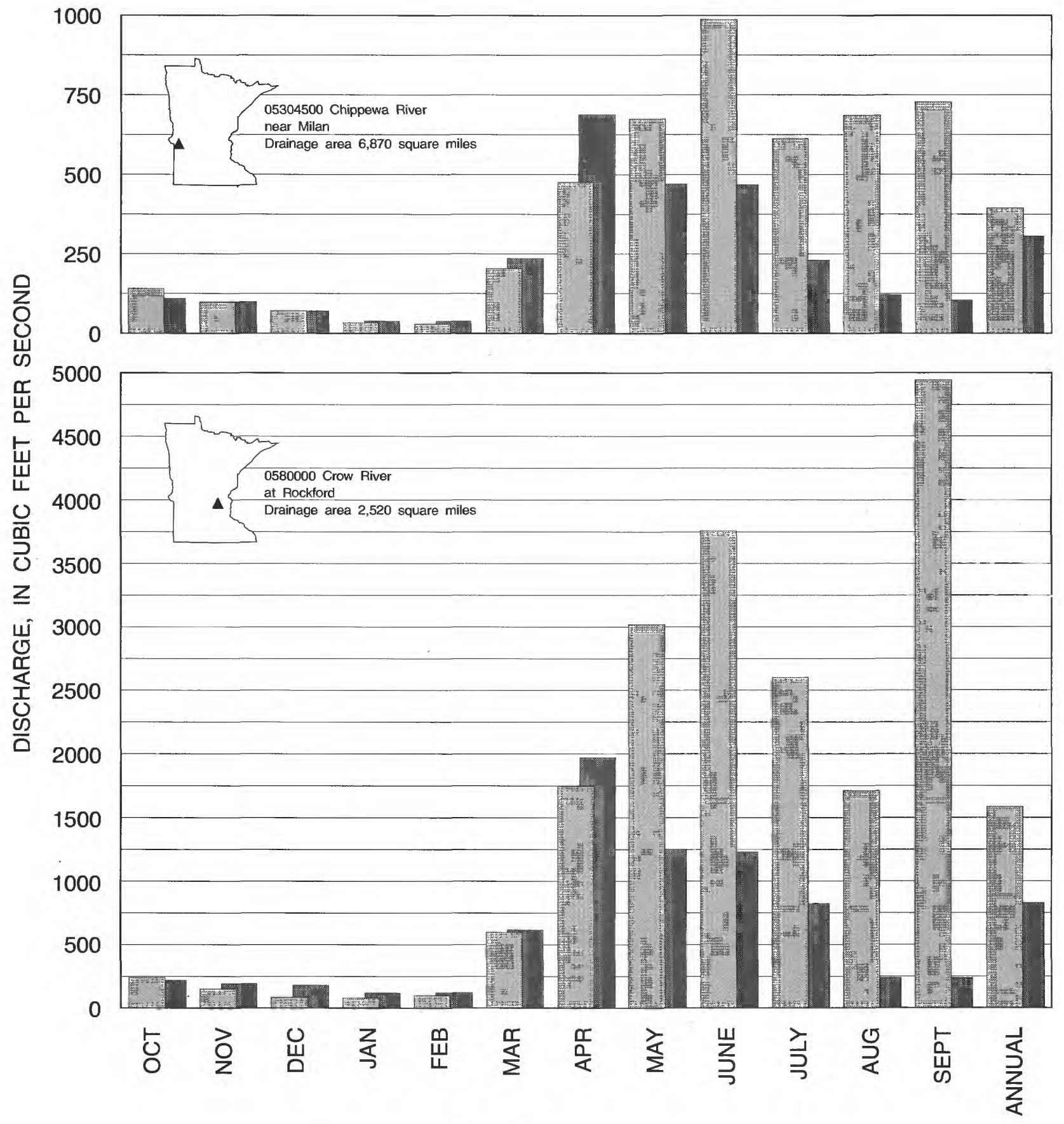

\section{EXPLANATION}

Mean discharge for water year 1991

Median of mean discharge for 1961-1990 

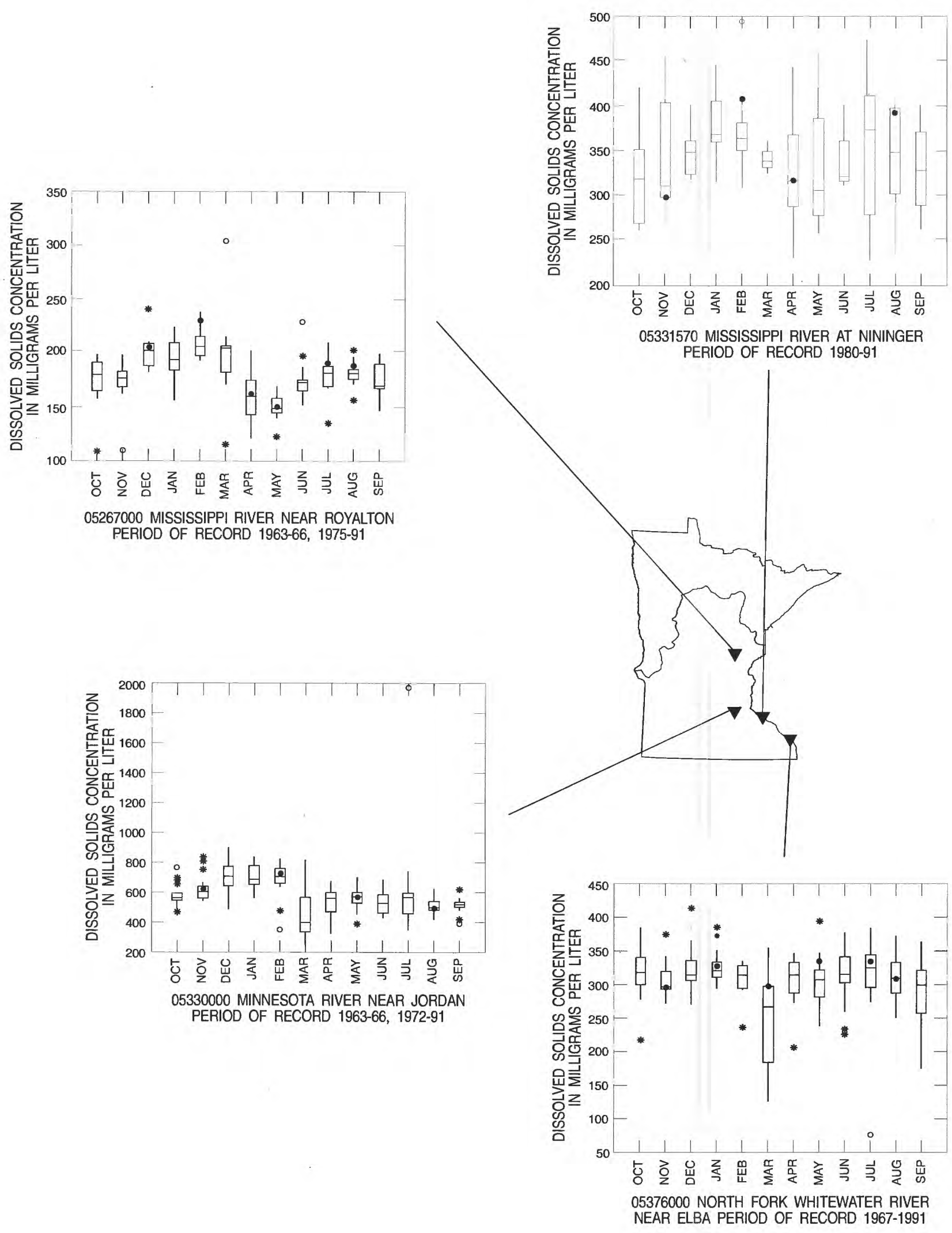

Figure 3--Comparison of dissolved-solids concentrations in water year 1991 with median for period of record at representative gaging stations. 


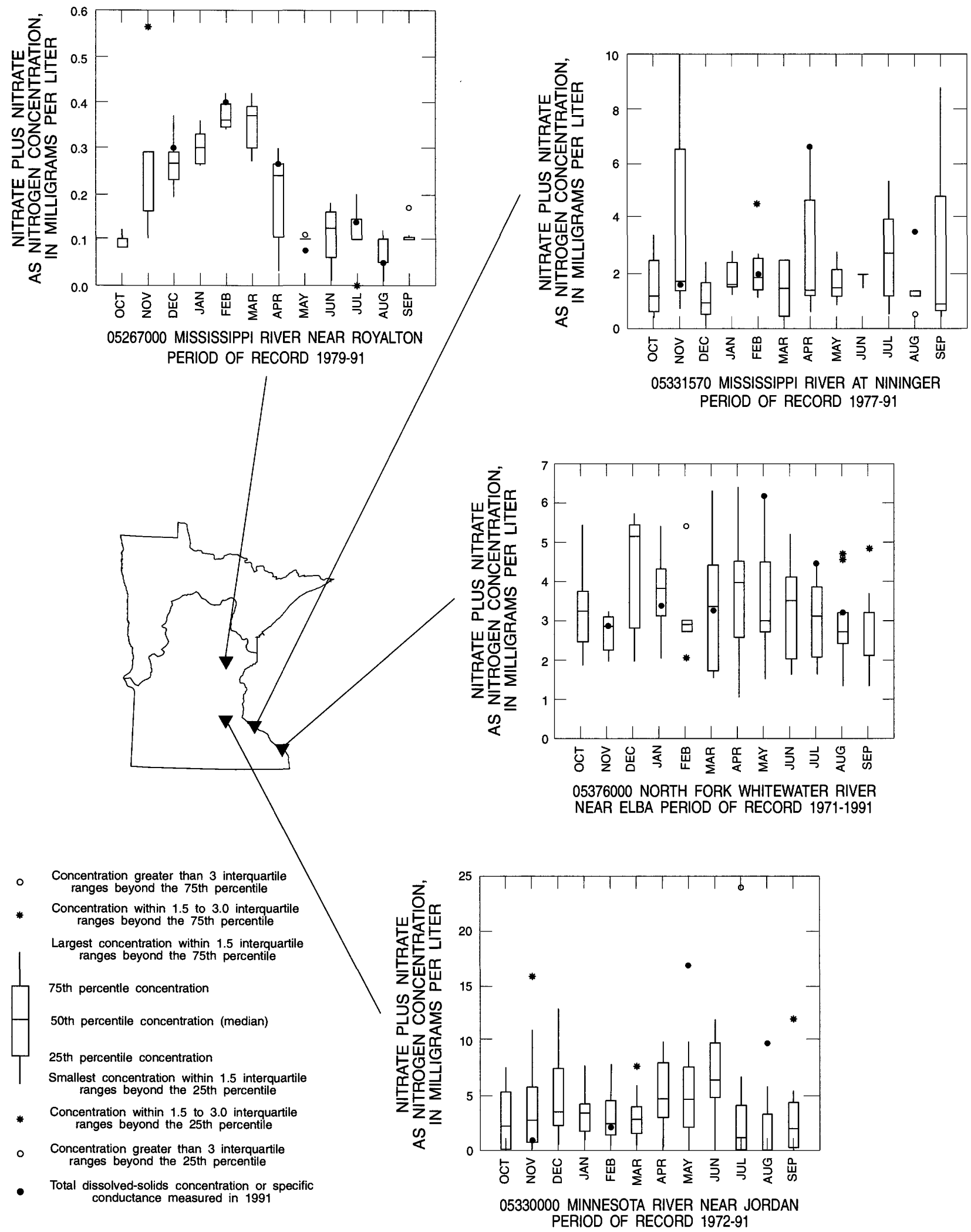

Figure 4--Comparison of nitrate plus nitrate concentrations in water year 1991 with median for period of record at representative gaging stations. 
Observations greater than three interquartile ranges from the ends of the box are plotted with an open circle. Water year 1991 values are plotted with a closed circle to show where these data lie with respect to the historic distribution of data.

Dissolved-solids concentrations determined in 1991 generally were near the monthly medians in the Mississippi River near Royalton, the Minnesota River near Jordan, and the North Fork Whitewater River near Elba. Dissolved-solids concentrations in the Mississippi River at Nininger were near the monthly medians in all quarterly samples except the February sample, which was higher than the median.

Nitrate concentrations reported as nitrogen (analyzed for nitrate plus nitrite, with nitrite concentration assumed to be negligible) were near the median in samples collected in the Mississippi River near Royalton, but were higher in some samples collected at the other three stations. The samples with higher concentrations were collected in the spring and summer. A sample collected in August in the Minnesota River near Jordan had a nitrate concentration of 17 milligrams per liter.

One hundred twenty three water samples were collected from 88 wells. Nitrate concentrations were above the primary drinkingwater standard of $10 \mathrm{mg} / \mathrm{L}$ (Minnesota Pollution Control Agency, 1988 ) in 13 samples. Fourteen samples were above the iron standard of $300 \mathrm{ug} / \mathrm{L}$, and 7 samples were above the manganese standard of 50 ug/L.

\section{GROUND-WATER LEVELS}

Data for 18 wells completed in surficial sand aquifers, 17 in buried sand and gravel aquifers, 6 in the Upper Carbonate Aquifer, 6 in the St. Peter Aquifer, 35 in the Prairie du Chien-Jordan Aquifer, 12 in the Franconia-Ironton-Galesville Aquifer, 14 in the Mount Simon-Hinckley-Fond du Lac Aquifer, 4 in the Cretaceous Aquifer, and 2 in the Sioux Quartzite Aquifer are published in this volume.

\section{Surficial Sand Aquifers}

Water levels were higher in 13 of 18 shallow surficial-sand wells located in central and southern Minnesota at the end of the 1991 water year as compared to the previous year; water levels in 5 were lower. Water levels rose 4 feet in a well (440037094372601) in south-central Minnesota and were above the monthly average from June through September, this was the first time a monthly average had been exceeded in this well since July 1986 (see hydrograph page 242). Rainfall near this well was in excess of 10 inches above average during April-June. In well (46044094212501) in central Minnesota water levels rose 1.1 feet, but levels were below the monthly average during the entire year except for July (fig. 5a). During April and May, 4 inches of above average precipitation fell near this well. In the southern part of west-central Minnesota water levels in well (450631095562201) were almost at record highs the entire year in an area where precipitation was 11 inches above average. Water levels were below average during the entire water year in well (473023094570901) in north-central Minnesota with new monthly low levels from October to July, and an all-time low level in March for a 21-year period of record (see hydrograph page 196). In another well with 21 years of record (465142094433201) in the same part of the State an all-time record low level also occurred in March (fig. 5a and hydrograph page 217). The same well had new monthly low levels from October to May, near-record low levels in June, and new monthly low levels from July to September. In well (462415095003001) in the northern part of central Minnesota, water levels were below average all year. Less than average precipitation is the cause of the lower water levels in this part of the State.

\section{Buried Sand and Gravel Aquifers}

Water levels in 15 of 17 buried sand and gravel wells were higher at the end of the water year than they were at the end of the previous year. The two wells with lower water levels were in local areas of large groundwater withdrawals. One of these wells (444815093194901) with 12 years of record had new all-time monthly low levels for the entire water year (see hydrograph page 210). Ten feet of water level decline (about one foot per year) has been measured in this well. This well, located in Hennepin County, is in an area where pumping from the Prairie du Chien-Jordan aquifer has contributed to a decline in water levels in the overlying buried-drift aquifer. The other well (443801092571301) that had lower water levels is located in Dakota County and is in an area of large withdrawals for irrigation (see hydrograph page 205). This well had a new monthly low level in July, and was below average during August and September. As mentioned previously, most wells completed in the buried-drift aquifer had noticeable water level rises over the previous water year. Water level rises ranged from less than 1 foot to over 6 feet and averaged about 2 feet. For example, monthly water levels were about 3 feet above average from April to September in well 455236093172301 (fig. $5 b$ and hydrograph page 219). Precipitation during this same period was in excess of 8 inches above average.

\section{Upper Carbonate Aquifer}

Two of 6 wells in the Upper Carbonate Aquifer, well (435742093164001) in south-central Minnesota and well (445538092232601) in southeastern Minnesota, about 45 miles apart, had above average levels from October through April, all-time high levels in May, and monthly high levels from June through September. Rainfall amounts during late spring and summer were 5 inches above average. Another well (440448092485501) in the southeast had record monthly high levels only in June, August, and September; the water level in this well was 4 feet higher at the end of September as compared to September 1990 . Precipitation was at least 5 inches above average in the area of this well. Three wells (433846093220601, see hydrograph page 208,434032093111801 , and 434308093322001 ) in south-central Minnesota had record monthly high levels in November. These highs probably reflect the continuation of rising water levels from the wet fourth quarter of the previous water year.

\section{St. Peter Aquifer}

Two (450026093084201 and 450134092583101) of six wells completed in the St. Peter aquifer in the Twin Cities Metropolitan Area had record monthly low levels from October through July and both were below average during August and September (see hydrograph for former well page 229). Both wells have 20 years of record. Another well (445857093223101) had record monthly low levels from October through January, and levels were below average the rest of the year (see hydrograph page 215). Less than average precipitation in past years and increased demand for ground water have affected water levels in the St. Peter aquifer (fig. 5d). A well (434558093540001) in south-central Minnesota had an average monthly water-level rise of 0.3 foot during the water year. In this part of the State, precipitation was 6 inches above the yearly average and pumping of ground water for agricultural use from this well was reduced.

\section{Prairie du Chien-Jordan Aquifer}

Of 35 wells completed in the Prairie du Chien-Jordan aquifer, water levels were higher in 25 and lower in 10 wells compared to water levels in the previous water year. Water levels in 10 wells in the Minneapolis-St. Paul pumping center were, on the average, 3.4 feet higher than last year. This was in marked contrast to 7 wells in areas where irrigation is practiced where water levels were, on the average, about 2 feet lower than last year. In areas of large withdrawals, such as downtown St. Paul and Minneapolis, the monthly fluctuations in measured water levels was 51 feet in St. Paul and 54 feet in Minneapolis. In September, water levels rose 37 feet in St. Paul and 44 feet in Minneapolis. Water levels at the end of September were 11 feet above average in St. Paul and 14 feet above average in Minneapolis. A recorder well (445700093051001) in downtown St. Paul with 20 years of record had record-high levels in February, March, April, and May (fig. 5c). These record-high levels all occurred on the first Monday of each month between the hours of $2 \mathrm{a} . \mathrm{m}$. and $6 \mathrm{a}$.m. It was determined that during the first weekend of each month, most if not all, nearby wells were not pumping, allowing the water level in this well to rise to its maximum natural level. A recorder well (43393509125180) in southeastern Minnesota had new monthly low levels for the entire year. Precipitation in this part of 

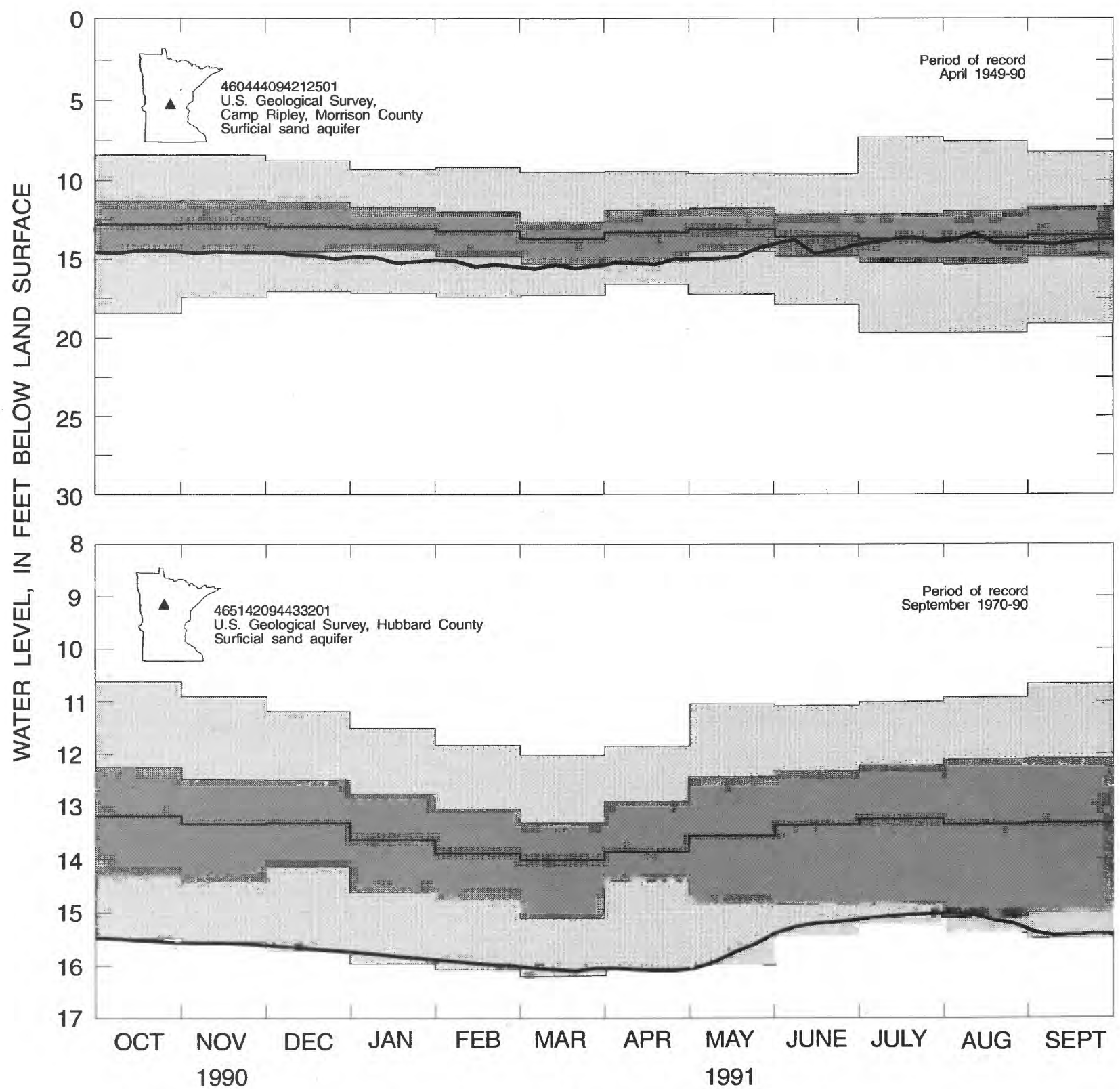

EXPLANATION

1991 water levels

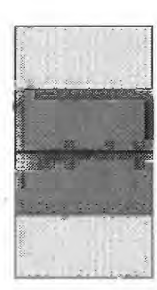

$\left.\begin{array}{l}- \text { Minimum } \\ -25 \% \\ -75 \% \\ - \text { Mean }\end{array}\right\} \begin{aligned} & \text { Monthly water levels } \\ & \text { for period of record }\end{aligned}$

Figure 5a.--Relation of water levels during 1991 to long-term levels in two representative wells in surficial sand aquifers. 


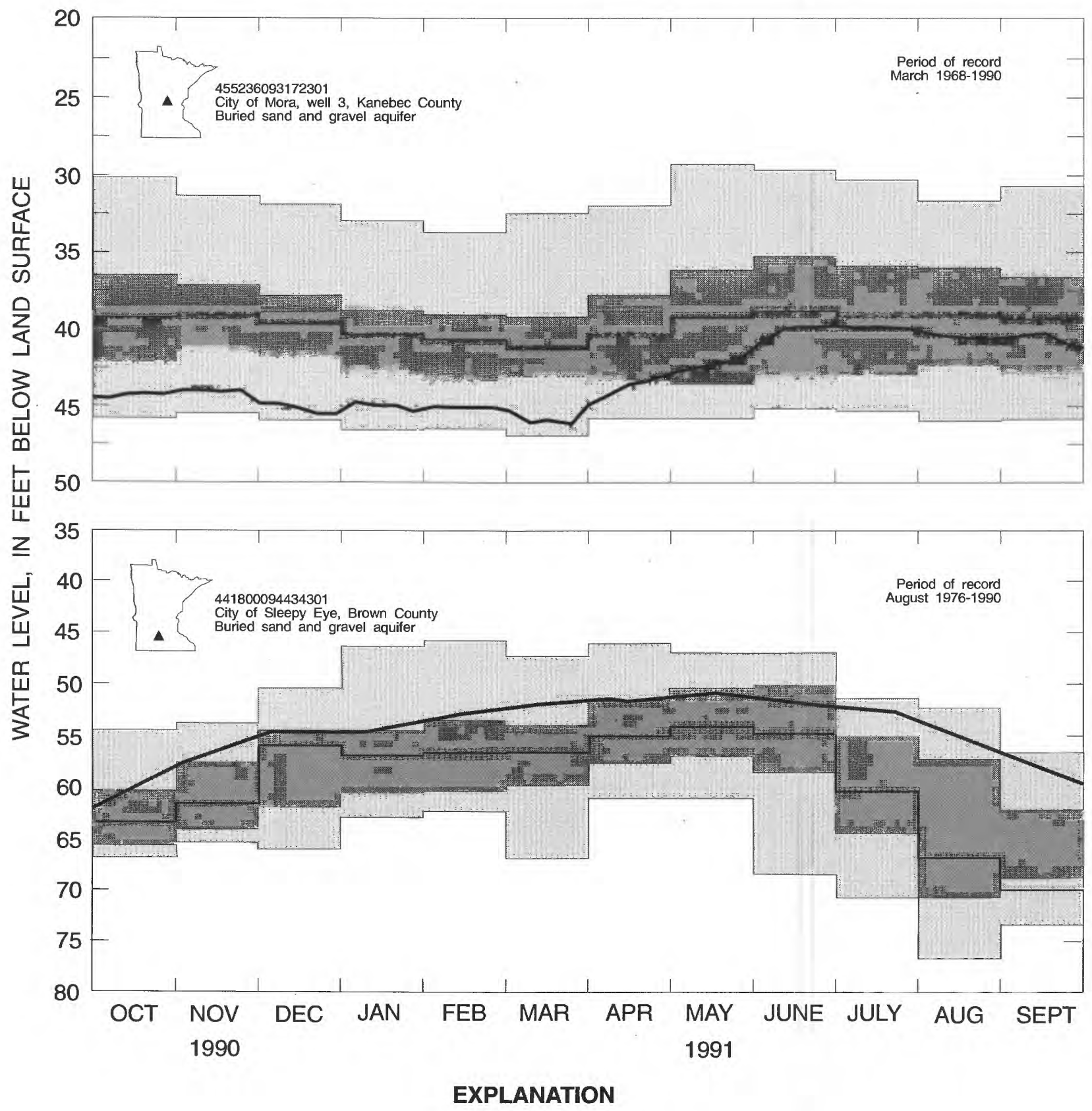

1991 water levels
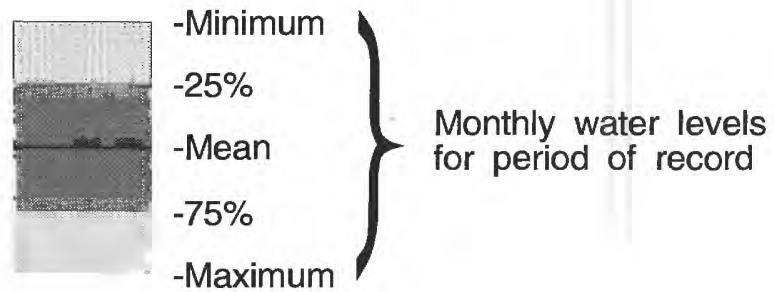

Figure 5b.--Relation of water levels during 1991 to long-term levels in two representative wells in buried sand and gravel aquifers. 

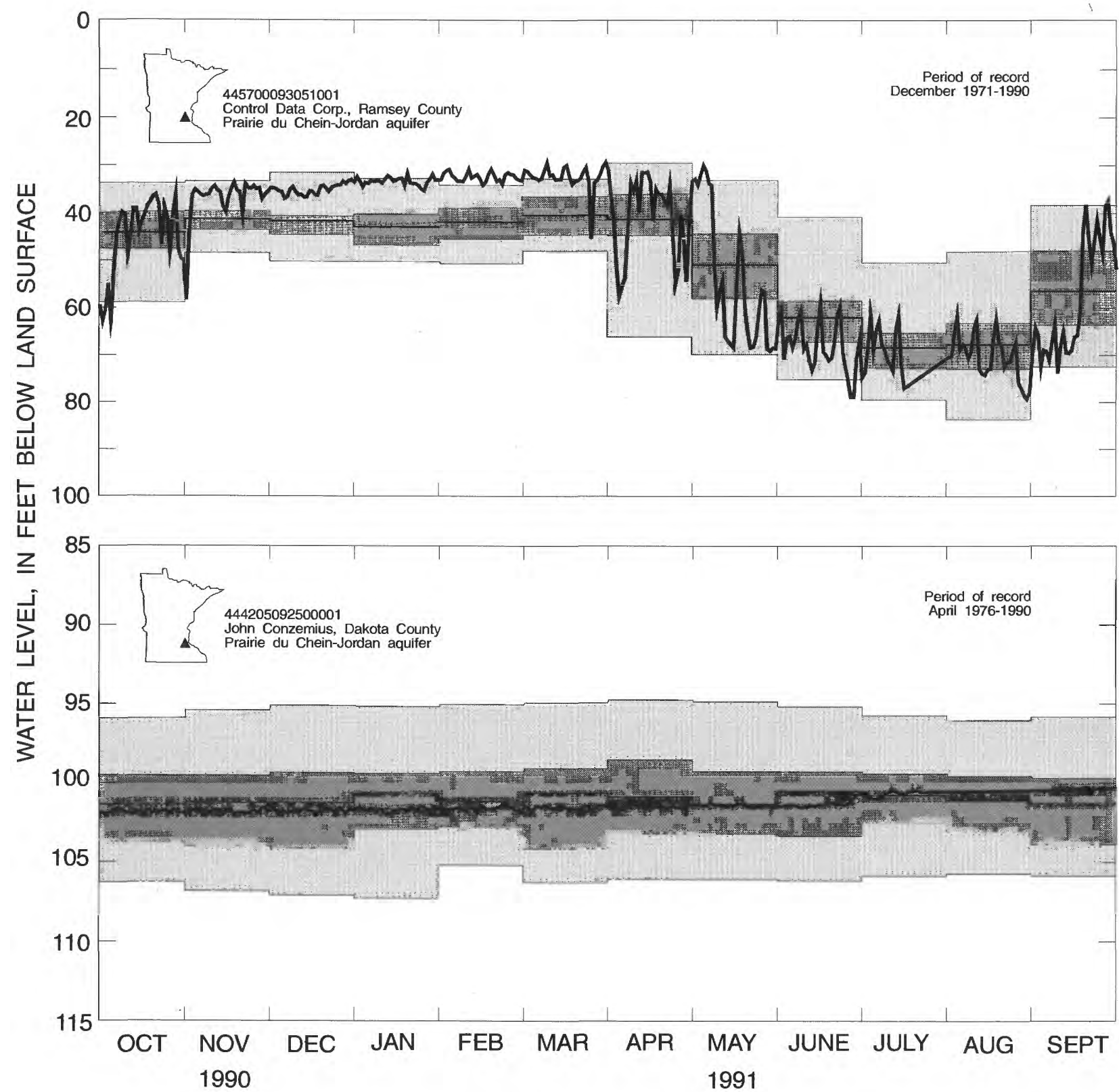

\section{EXPLANATION}

1991 water levels

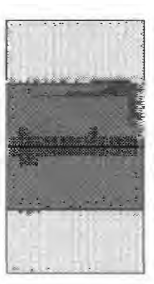

$\left.\begin{array}{l}- \text { Minimum } \\ -25 \% \\ - \text { Mean } \\ -75 \% \\ - \text { Maximum }\end{array}\right\}$

Monthly water levels

for period of record

Figure 5c---Relation of water levels during 1991 to long-term levels in two representative bedrock wells in the Prairie du Chein-Jordan aquifer. 

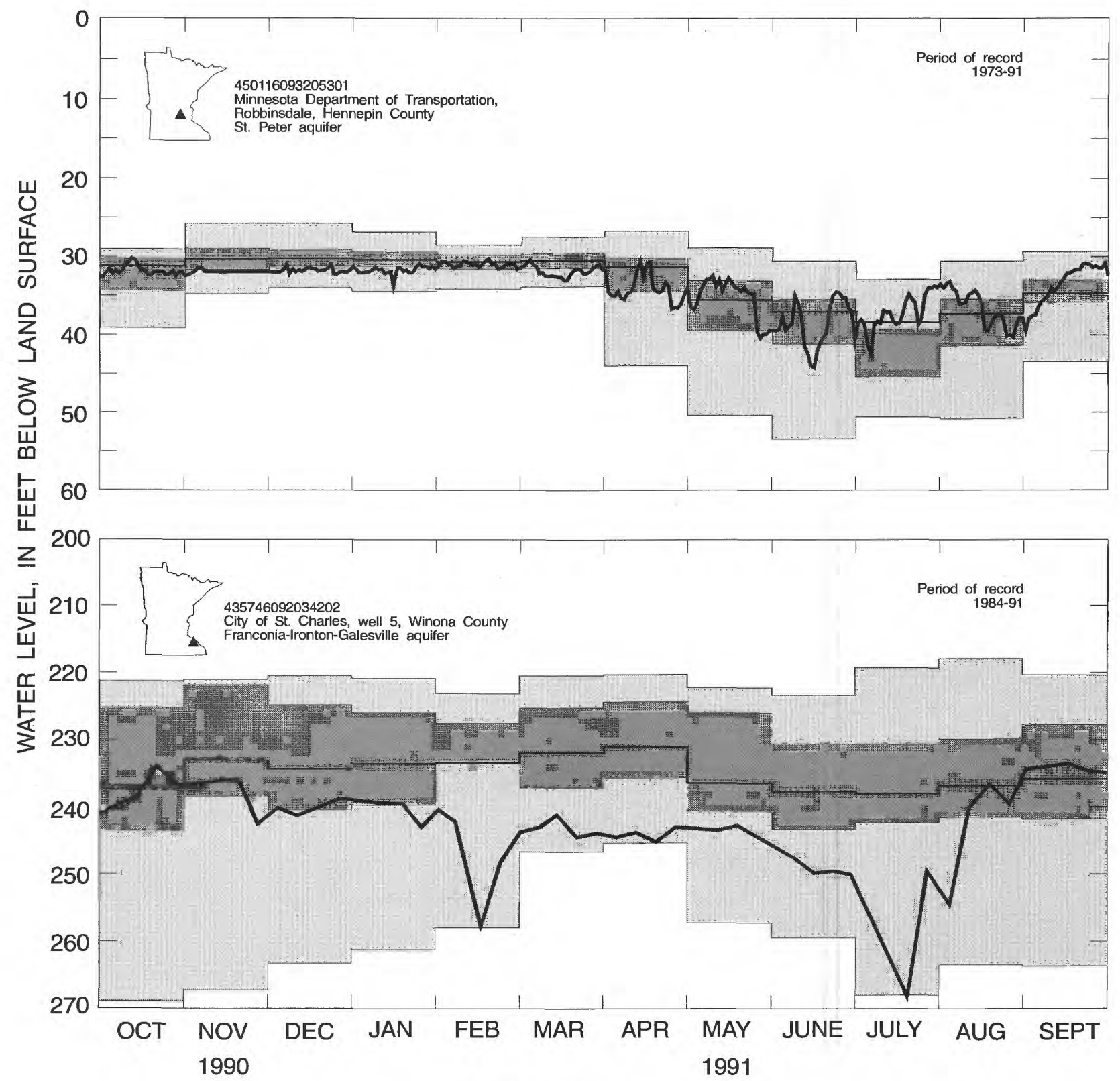

EXPLANATION

1991 water levels

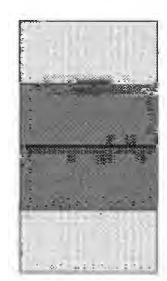

$\left.\begin{array}{l}- \text { Minimum } \\ -25 \% \\ - \text { Mean } \\ -75 \% \\ - \text { Maximum }\end{array}\right\}$

Monthly water levels

for period of record

Figure 5d.-Relation of water levels during 1991 to long-term levels in two representative bedrock wells in the St. Peter and Franconia-Ironton-Galesville aquifer. 
the State has not been adequate to reverse this downward trend in water level initiated by the drought in previous years. For example, from June to September, rainfall near this well was 4.6 inches below average. Another well (441134093505301), in a rural setting in southcentral Minnesota had record monthly lows from October through June (see hydrograph page 198). These new lows are probably due to natural causes, such as less than average precipitation in previous years. Water levels rose in this well from July through September when precipitation was above average. A well (445044093102401) with 14 years of record, located in an urban setting, had record-low levels in November and July and water levels were below average during the entire year except for September. This well is less than 1 mile from several municipal supply wells.

\section{Franconia-Ironton-Galesville Aquifer}

Of 12 wells completed in the Franconia-Ironton-Galesville aquifer, 11 had higher water levels and 1 had lower water levels compared to the previous water year. The well with lower water levels is in a local pumping center; water levels were 3 feet lower than during comparable periods last year. A recorder well (440050094102801) in south-central Minnesota, had water levels below average from October through April and average from May through September. Rainfall was 8 inches above average from May to September in the area of this well. Two wells had record monthly low levels. One well (444427093353902), in central Minnesota, had a new low level in July; it is located near a sandstone quarry where pumping occurs. The other well (445536092462401), with 13 years of record, is located in east-central Minnesota. It had a new low level in March (see hydrograph page 240). In May the water level rose 4 feet as a result of 5 inches of above average rainfall during the month. Recharge to this well is very rapid as sand overlies the aquifer.

\section{Mount Simon-Hinckley-Fond du Lac Aquifer}

Of 14 wells completed in the Mount Simon-Hinckley-Fond du Lac aquifer, one well (444633093212901), with 20 years of record, had an all-time low water level in September. Another well (444427093353903), with 7 years of record, had a new monthly low level in September. Both wells are located in Scott county. Reversal of water level declines from 1989 within the Twin Cities Metropolitan Area has been observed in three wells. At the end of September 1991, water levels in two wells located in St. Paul and Minneapolis were 12 and 10 feet higher, respectively, than levels in September 1990 (fig. Se). Water levels were, however, 13 feet below average in St. Paul and 25 feet below average in Minneapolis at the end of the water year. The third well, located at Lake Minnetonka, had a rise in water level of 6 feet from the lowest level of 1990. A well (443935091252901), in southeastern Minnesota, had a water level range of only 0.5 foot for the entire water year. Most of the wells in this part of the State are in the upper Prairie du Chien-Jordan aquifer and do not affect the Mount Simon-Hinckley Aquifer. Another well (450403093544501) in a rural setting of central Minnesota had a steady monthly water level rise of 1.1 feet over the previous water year.

\section{Cretaceous Aquifer}

Water levels in 4 wells completed in Cretaceous Sandstones were measured during the water year. Two of these wells in southcentral Minnesota had gradual water level rises of $\mathbf{4}$ to 5 feet from their low levels of 1988 which was a drought year. In one well (440409094304901) the water rose 2 feet in response to 6 inches of above average rainfall during April and May of 1991. Water level in the other well (434359094422201) rose only 0.5 foot during this same period; 4 inches of above average rainfall fell in the area of this well. The latter well has a top screen setting of 372 feet, while the former well has a top screen setting of 100 feet. One well (441705096084501) in southwestern Minnesota, completed in the Dakota Sandstone (screened at 900 feet), had very little annual water level change about $\mathbf{0 . 1 0}$ foot for the water year (see hydrograph page 221).

\section{Sioux-Quartzite Aquifer}

Two wells completed in the Sioux Quartzite in southwestern Minnesota were measured during the water year. One of these wells
(434725094483001) with 10 years of record had new record low levels from October through March. This same well also had new monthly low levels for the entire previous water year and September of water year 1989 (19 consecutive months). These low water levels are probably related to deficient precipitation in previous years in this part of the State. The other well (434742095191501), located in southwestern Minnesota, had average water levels from October through March and above average April to September. Water levels in this well were also lower during water year 1990 but did not set any monthly records. It should be noted that the depth to the quartzite aquifer in the former well is $\mathbf{1 2 1}$ feet and in the latter well 225 feet.

\section{SPECIAL NETWORKS AND PROGRAMS}

Hydrologic Bench-Mark Network is a network of 57 sites in small drainage basins around the country whose purpose is to provide consistent data on the hydrology, including water quality, and related factors in representative undeveloped watersheds nationwide, and to provide analyses on a continuing basis to compare and contrast conditions observed in basins more obviously affected by the activities of man.

National Stream Quality Accounting Network (NASQAN) is a national data-collection network designed by the U.S. Geological Survey to meet many of the information needs of government agencies and other groups involved in natural or regional waterquality planning and management. The 500 or so sites in NASQAN are generally located at the downstream ends of the hydrologic accounting units designated by the U.S. Geological Survey Office of Water Data Coordination in consultation with the Water Resources Council. The objectives of NASQAN are (1) to obtain information on the quality and quantity of water moving within and from the United States through a systematic and uniform process of data collection, summarization, analysis, and reporting such that the data may be used for, (2) description of the areal variability of water quality in the Nation's rivers through analysis of data from this and other programs, (3) detection of changes or trends with time in the pattern of occurrence of water-quality characteristics, and (4) providing a nationally consistent data base useful for water quality assessment and hydrologic research.

The National Trends Network (NTN) is a 150-station network for sampling atmospheric deposition in the United States. The purpose of the network is to determine the variability, both in location and in time, of the composition of atmospheric deposition, which includes snow, rain, dust particles, and aerosols, and gases. The core from which the NTN was built was the already-existing deposition-monitoring network of the National Atmospheric Deposition Program (NADP).

Radiochemical program is a network of regularly sampled water-quality stations where samples are collected to be analyzed for radioisotopes. The streams that are sampled represent major drainage basins in the conterminous United States.

Tritium network is a network of stations which has been established to provide baseline information on the occurrence of tritium in the Nation's surface waters. In addition to the surfacewater stations in the network, tritium data are also obtained at a number of precipitation stations. The purpose of the precipitation stations is to provide an estimate sufficient for hydrologic studies of the tritium input to the United States.

\section{EXPLANATION OF THE RECORDS}

The surface-water and ground-water records published in this report are for the 1991 water year that began October 1, 1990, and ended September 30,1991. A calendar of the water year is provided on the inside of the front cover. The records contain streamflow data, stage and content data for lakes and reservoirs, water-quality data for the surface and ground water, and ground-water-level data. 


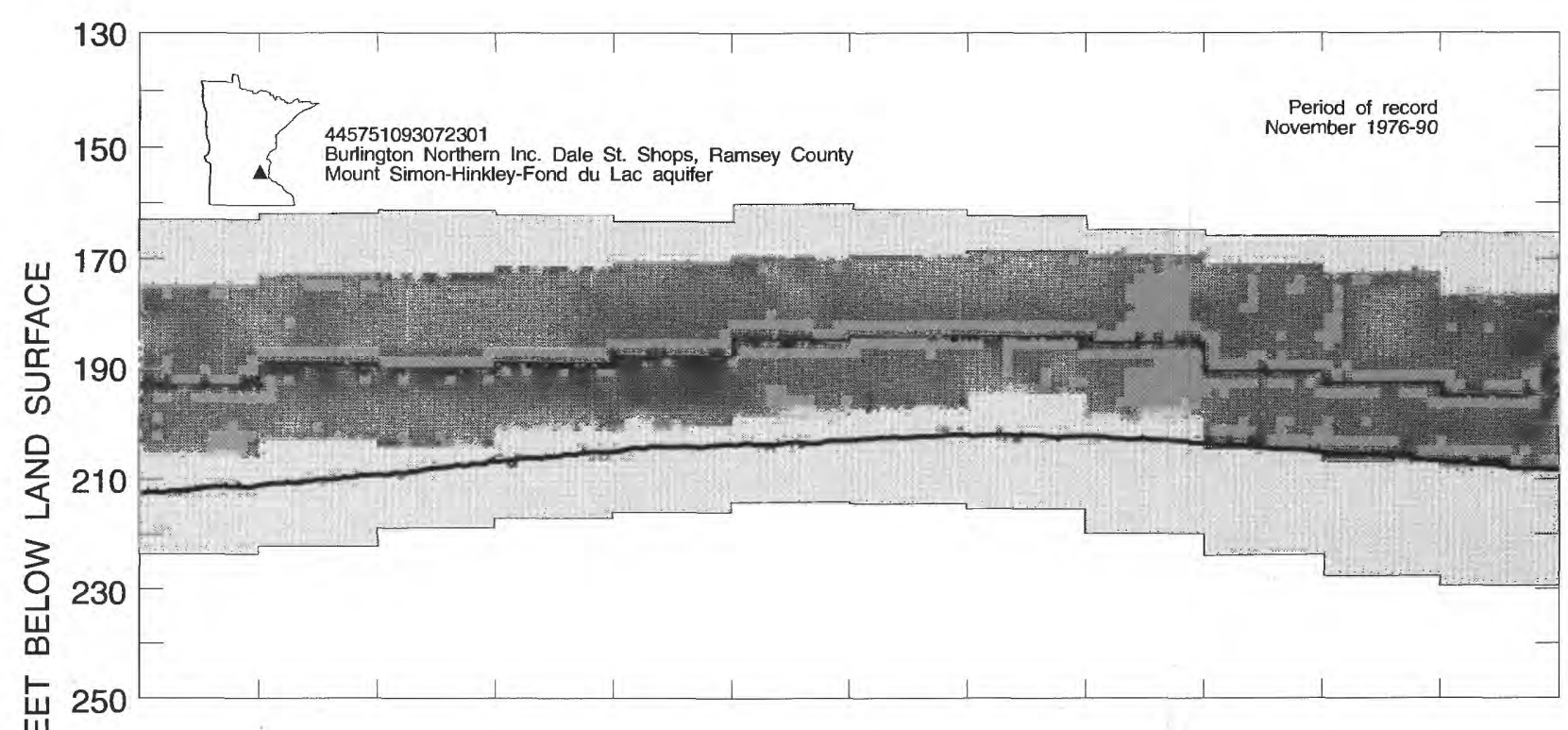

4

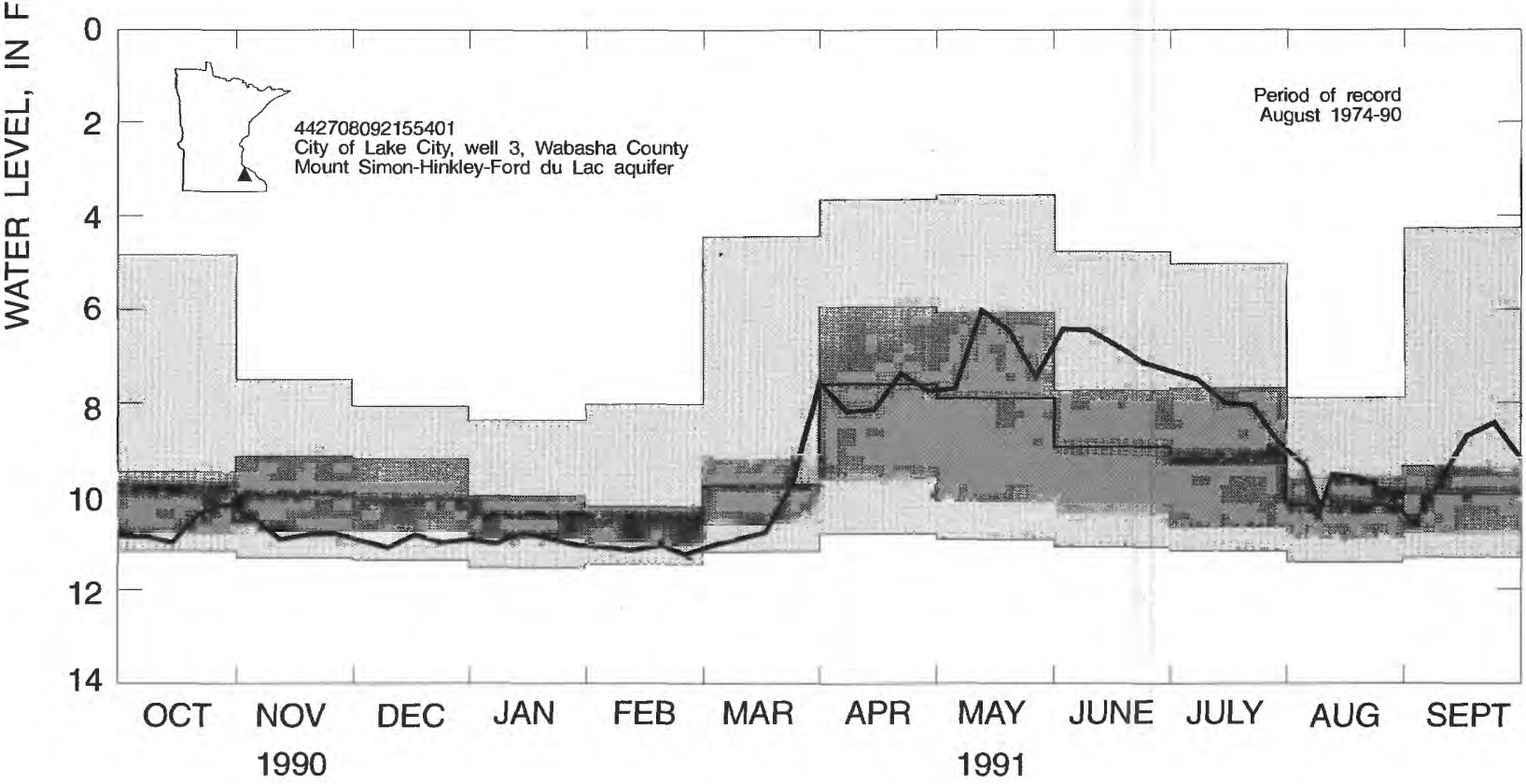

\section{EXPLANATION}

1991 water levels

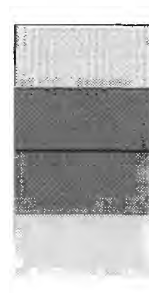

$\left.\begin{array}{l}- \text { Minimum } \\ -25 \% \\ - \text { Mean } \\ -75 \% \\ - \text { Maximum }\end{array}\right\} \begin{aligned} & \text { Monthly water levels } \\ & \text { for period of record }\end{aligned}$

Figure 5e.--Relation of water levels during 1991 to long-term levels in two representative wells in the Mount Simon-Hinkley-Ford du Lac aquifer. 
The locations of the stations and wells where the data were collected are shown in figures $7,8,9$, and 10 . The following sections of the introductory text are presented to provide users with a more detailed explanation of how the hydrologic data published in this report were collected, analyzed, computed, and arranged for presentation.

\section{STATION IDENTIFICATION NUMBERS}

Each data station, whether streamsite or well, in this report is assigned a unique identification number. This number is unique in that it applies specifically to a given station and to no other. The number usually is assigned when a station is first established and is retained for that station indefinitely. The system used by the U.S. Geological Survey to assign identification numbers for surface-water stations and for ground-water well sites differ, but both are based on geographic location. The "downstream order" system is used for regular surface-water stations and the "latitude-longitude" system is used for wells and, in Minnesota, for surface-water stations where only miscellaneous measurements are made.

\section{Downstream Order System and Station Number}

Since October 1, 1950, the order of listing hydrologic-station records in Survey reports is in a downstream direction along the main stream. All stations on a tributary entering upstream from a main-stream station are listed before that station. A station on a tributary that enters between two main-stream sections is listed between them. A similar order is followed by listing stations on first rank, second rank, and other ranks of tributaries. The rank of any tributary on which a station is situated with respect to the stream to which it is immediately tributary is indicated by an indentation in a list of stations in front of the report. Each indention represents one rank. This downstream order and system of indention show which stations are on tributaries between any two stations and the rank of the tributary on which each station is situated.

As an added means of identification, each hydrologic station and partial-record station has been assigned a station number. These are in the same downstream order in this report. In assigning station numbers, no distinction is made between partial-record stations and other stations; therefore, the station number for a partial-record station indicates downstream-order position in a list made up of both types of stations. Gaps are left in the series of numbers to allow for new stations that may be established; hence, the numbers are not consecutive. The complete 8-digit number for each station such as 05041000 , which appears just to the left of the station name, includes the 2-digit part number "05" plus the 6-digit downstream order number "041000."

\section{Latitude-Longitude System for Wells and Miscellaneous Sites}

The 8-digit downstream order station numbers are not assigned to wells and miscellaneous sites where only random waterquality samples or discharge measurements are taken.

The well and miscellaneous site numbering system of the U.S Geological Survey is based on the grid system of latitude and longitude. The system provides the geographic location of the well or miscellaneous site and a unique number for each site. The number consists of 15 digits. The first 6 digits denote the degrees, minutes, and seconds of latitude, the next 7 digits denote degrees, minutes, and seconds of longitude, and the last 2 digits (assigned sequentially) identify the wells or other sites within a 1-second grid. See figure 6. Each well site is also identified by a local well number which consists of township, range, and section numbers, three letters designating $1 / 4,1 / 4,1 / 4$ section location, and a two-digit sequential number.

\section{RECORDS OF STAGE AND WATER DISCHARGE}

Records of stage and water discharge may be complete or partial. Complete records of discharge are those obtained using a continuous stage-recording device through which either

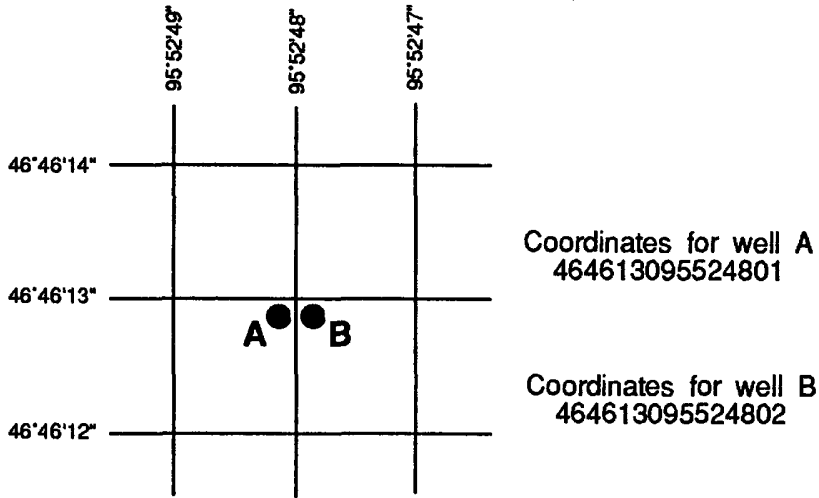

\section{Figure 6.--Example of system for numbering wells and miscellaneous sites.}

instantaneous or mean daily discharge may be computed for any time, or any period of time, during the period of record. Complete records of lake or reservoir content, similarly, are those for which stage or content may be computed or estimated with reasonable accuracy for any time, or period of time. They may be obtained using a continuous stage-recording device, but need not be. Because daily mean discharges and end-of-day contents commonly are published for such stations, they are referred to as "daily stations".

By contrast, partial records are obtained through discrete measurements without using a continuous stage-recording device and pertain only to a few flow characteristics, or perhaps only one. The nature of the partial record is indicated by table titles such as "Highflow partial records," or "Low-flow partial records." Records of miscellaneous discharge measurements or of measurements from special studies, such as low-flow seepage studies, may be considered as partial records, but they are presented separately in this report. Location of all complete-record and high-flow partial-record stations for which data are given in this report are shown in figures 7 and 9.

\section{Data Collection and Computation}

The data obtained at a complete-record gaging station on a stream or canal consist of a continuous record of stage, individual measurements of discharge throughout a range of stages, and notations regarding factors that may affect the relationships between stage and discharge. These data, together with supplemental information, such as weather records, are used to compute daily discharges. The data obtained at a complete-record gaging station on a lake or reservoir consist of a record of stage and of notations regarding factors that may affect the relationship between stage and lake content. These data are used with stage-area and stage-capacity curves or tables to compute water-surface areas and lake storage.

Continuous records of stage are obtained with analog recorders that trace continuous graphs of stage or with digital recorders that punch stage values on paper tapes at selected time intervals. Measurements of discharge are made with current meters using methods adapted by the Geological Survey as a result of experience accumulated since 1880 . These methods are described in standard textbooks, in Water-Supply Paper 2175, and in U.S Geological Survey Techniques of Water-Resources Investigations, Book 3, Chapter A6.

In computing discharge records, results of individual measurements are plotted against the corresponding stages, and stage-discharge relation curves are then constructed. From these curves, rating tables indicating the approximate discharge for any stage within the range of the measurements are prepared. If it is 
necessary to define extremes of discharge outside the range of current-meter measurements, the curves are extended using: (1) logarithmic-plotting; (2) velocity-area studies; (3) results of indirect measurements of peak discharge, such as slope-area or contractedopening measurements, and computations of flow-over-dams or weirs; or (4) step-backwater techniques.

Daily mean discharges are computed by applying the daily mean stages (gage heights) to the stage-discharge curves or tables. If the stage-discharge relation is subject to change because of frequent or continual change in the physical features that form the control, the daily mean discharge is determined by the shifting-control method, in which correction factors based on the individual discharge measurements and notes of the personnel making the measurements are applied to the gage heights before the discharges are determined from the curves or tables. This shifting-control method also is used if the stage-discharge relation is changed temporarily because of aquatic growth or debris on the control. For some stations, formation of ice in the winter may so obscure the stage-discharge relations that daily mean discharges must be estimated from other information such as temperature and precipitation records, notes of observations, and records for other stations in the same or nearby basins for comparable periods.

At some stream-gaging stations the stage-discharge relation is affected by the backwater from reservoirs, tributary streams, or other sources. This necessitates the use of the slope method in which the slope or fall in a reach of the stream is a factor in computing discharge. The slope or fall is obtained by means, of an auxiliary gage set at some distance from the base gage. At some stations the stage-discharge relation is affected by changing stage; at these stations the rate of change in stage is used as a factor in computing discharge.

In computing records of lake or reservoir contents, it is necessary to have available from surveys, curves, or tables defining the relationship of stage and content. The application of stage to the stage-content curves or tables gives the contents from which daily, monthly, or yearly changes then are determined. If the stage-content relationship changes because of deposition of sediment in a lake or reservoir, periodic resurveys may be necessary to redefine the relationship. Even when this is done, the contents computed may become increasingly in error as time since the last survey increases. Discharge over lake or reservoir spillways are computed from stagedischarge relationships much as other stream discharges are computed.

For some gaging stations there are periods when no gageheight record is obtained, or the recorded gage height is so faulty that it cannot be used to compute daily discharge or contents. This happens when the recorder stops or otherwise fails to operate properly, intakes are plugged, the float is frozen in the well, or for various other reasons. For such periods, the daily discharges are estimated from the recorded range in stage, previous or following record, discharge measurements, weather records, and comparison with other station records from the same or nearby basins. Likewise, daily contents may be estimated from operator's logs, previous or following record, inflow-outflow studies, and other information. Information explaining how estimated daily-discharge values are identified in station records is included in the next two sections, "Data Presentation" (REMARKS paragraph) and "Identifying Estimated Daily Discharge."

\section{Data Presentation}

Streamflow data in this report are presented in a new format that is considerably different from the format in data reports prior to the 1991 water year. The major changes are that statistical characteristics of discharge now appear in tabular summaries following the water-year data table and less information is provided in the text or station manuscript above the table. These changes represent the results of a pilot program to reformat the annual water-data report to meet current user needs and data preferences.
The records published for each continuous-record surfacewater discharge station (gaging station) now consist of four parts, the manuscript or station description; the data table of daily mean values of discharge for the current water year with summary data; a tabular statistical summary of monthly mean flow data for a designated period, by water year; and a summary statistics table that includes statistical data of annual, daily and instantaneous flows as well as data pertaining to annual runoff, 7-day low-flow minimums, and flow duration.

\section{Station manuscript}

The manuscript provides, under various headings, descriptive information, such as station location; period of record; historical extremes outside the period of record; record accuracy; and other remarks pertinent to station operation and regulation. The following information, as appropriate, is provided with each continuous record of discharge or lake content. Comments to follow clarify information presented under the various headings of the station description.

LOCATION.-Information on locations is obtained from the most accurate maps available. The location of the gage with respect to the cultural and physical features in the vicinity and with respect to the reference place mentioned in the station name is given. River mileages, given for only a few stations, were determined by methods given in "River Mileage Measurement," Bulletin 14, Revision of October 1968, prepared by the Water Resources Council or were provided by the U.S. Army Corps of Engineers.

DRAINAGE AREA.-Drainage areas are measured using the most accurate maps available. Because the type of maps available varies from one drainage basin to another, the accuracy of drainage areas likewise varies. Drainage areas are updated as better maps become available.

PERIOD OF RECORD.-This indicates the period for which there are published records for the station or for an equivalent station. An equivalent station is one that was in operation at a time when the present station was not, and whose location was such that records from it can reasonably be considered equivalent with records from the present station.

REVISED RECORDS.--Published records, because of new information, occasionally are found to be incorrect, and revisions are printed in later reports. Listed under this heading are all reports in which revisions have been published for the station and water years to which the revisions apply. If a revision did not include daily, monthly, or annual figures of discharge, that fact is noted after the year dates as follows: "(M)" means that only the instantaneous maximum discharge was revised; "( $m)^{n}$ that only the instantaneous minimum was revised; and "(P)" that only peak discharges were revised. If the drainage area has been revised, the report in which the most recently revised figure was first published is given.

GAGE.--The type of gage in current use, the datum of the current gage referred to National Geodetic Vertical Datum of 1929 (see glossary), and a condensed history of the types, locations, and datum of previous gages are given under this heading.

REMARKS.-All periods of estimated daily-discharge record will either be identified by date in this paragraph of the station description for water-discharge stations or flagged in the dailydischarge table. If a remarks statement is used to identify estimated record, the paragraph will begin with this information presented as the first entry. The paragraph is also used to present information relative to the accuracy of the records, to special methods of computation, to conditions that affect natural flow at the station and, possibly, to other pertinent items. For reservoir stations, information is given on the dam forming the reservoir, the capacity, outlet works and spillway, and purpose and use of the reservoir.

COOPERATION.--Records provided by a cooperating organization or obtained for the Geological Survey by a cooperating organization are identified here. 
EXTREMES OUTSIDE PERIOD OF RECORD.-Included here is the information concerning major floods or unusually low flows that occurred outside the stated period of record. The information may or may not have been obtained by the U.S. Geological Survey.

REVISIONS.--If a critical error in published records is discovered, a revision is included in the first report published following discovery of the error.

Although rare, occasionally the records of a discontinued gaging station may need revision. Because, for these stations, there would be no current or, possibly, future station manuscript published to document the revision in a "Revised Records" entry, users of data for these stations who obtained the record from previously published data reports may wish to contact the district office (address given on the back of title page of this report) to determine if the published records were ever revised after the station was discontinued. Of course, if the data were obtained by computer retrieval, the data would be current and there would be no need to check because any published revision of data is always accompanied by revision of the corresponding data in computer storage.

Manuscript information for lake or reservoir stations differs from that for stream stations in the nature of the "Remarks" and to the inclusion of a skeleton stage-capacity table when daily contents are given.

Headings for AVERAGE DISCHARGE, EXTREMES FOR PERIOD OF RECORD, AND EXTREMES FOR CURRENT YEAR have been deleted and the information contained in these paragraphs, except for the listing of secondary instantaneous peak discharges in the EXTREMES FOR CURRENT YEAR paragraph, is now presented in the tabular summaries following the discharge table or in the REMARKS paragraph, as appropriate. No changes have been made to the data presentations of lake contents.

\section{Data table of daily mean values}

The daily table of discharge records for stream-gaging stations gives mean discharge for each day of the water year. In the monthly summary for the table, the line headed "TOTAL" gives the sum of the daily figures for each month; the line headed "MEAN" gives the average flow in cubic feet per second for the month; and the lines headed "MAX" and "MIN" give the maximum and minimum daily mean discharges, respectively, for each month. Discharge for the month also is usually expressed in cubic feet per second per square mile (line headed "CFSM"); or in inches (line headed "IN"); or in acre-feet (line headed "AC-FT). Figures for cubic feet per second per square mile and runoff in inches or in acre-feet may be omitted if there is extensive regulation or diversion or if the drainage area includes large noncontributing areas. At some stations monthly and (or) yearly observed discharges are adjusted for reservoir storage or diversion, or diversion data or reservoir contents are given. These figure are identified by a symbol and corresponding footnote.

\section{Statistics of monthly mean data}

A tabular summary of the mean (line headed "MEAN"), maximum (line headed "MAX"), and minimum (line headed "MIN") of monthly mean flows for each month for a designated period is provided below the mean values table. The water years of the first occurrence of the maximum and minimum monthly flows are provided immediately below those figures. The designated period will be expressed as "FOR WATER YEARS 19 -19, BY WATER YEAR (WY)," and will list the first and last water years of the range of years selected from the PERIOD OF RECORD paragraph in the station manuscript. It will consist of all of the station record within the specified water years, inclusive, including complete months of record for partial water years, if any, and may coincide with the period of record for the station. The water years for which the statistics are computed will be consecutive, unless a break in the station record is indicated in the manuscript.

\section{Summary statistics}

A table titled "SUMMARY STATISTICS" follows the statistics of monthly mean data tabulation. This table consists of four columns, with the first column containing the line headings of the statistics being reported. The table provides a statistical summary of yearly, daily, and instantaneous flows, not only for the current water year but also for the previous calendar year and for a designated period, as appropriate. The designated period selected, "WATER YEARS $19-19$," will consist of all of the station record within the specified water years, inclusive, including complete months of record for partial water years, if any, and may coincide with the period of record for the station. The water years for which the statistics are computed will be consecutive, unless a break in the station record is indicated in the manuscript. All of the calculations for the statistical characteristics designated ANNUAL (see line headings below), except for the ANNUAL 7-DAY MINIMUM" statistic, are calculated for the designated period using complete water years. The other statistical characteristics may be calculated using partial water years.

The date or water year, as appropriate, of the first occurrence of each statistic reporting extreme values of discharge is provided adjacent to the statistic. Repeated occurrences may be noted in the REMARKS paragraph of the manuscript or in footnotes. Because the designated period may not be the same as the station period of record published in the manuscript, occasionally the dates of occurrence listed for the daily and instantaneous extremes in the designated-period column may not be within the selected water years listed in the heading. When this occurs, it will be noted in the REMARKS paragraph or in footnotes. Selected streamflow duration curve statistics and runoff data are also given. Runoff data may be omitted if there is extensive regulation or diversion of flow in the drainage basin.

The following summary statistics data, as appropriate, are provided with each continuous record of discharge. Comments to follow clarify information presented under the various line headings of the summary statistics table.

ANNUAL TOTAL.--The sum of the daily mean values of discharge for the year. At some stations the yearly mean discharge is adjusted for reservoir storage or diversion. The adjusted figures are identified by symbol and corresponding footnotes.

ANNUAL MEAN.--The arithmetic mean of the individual daily mean discharges for the year noted or for the designated period. At some stations the yearly mean discharge is adjusted for reservoir storage or diversion. The adjusted figures are identified by a symbol and corresponding footnotes. At least 5 complete years of record must be available before this statistic is published for the designated period.

HIGHEST ANNUAL MEAN.--The maximum annual mean discharge occurring for the designated period.

LOWEST ANNUAL MEAN.--The minimum annual mean discharge occurring for the designated period.

HIGHEST DAILY MEAN.--The maximum daily mean discharge for the year or for the designated period.

LOWEST DAILY MEAN.--The minimum daily mean discharge for the year or for the designated period.

ANNUAL 7-DAY MINIMUM.--The lowest mean discharge for 7 consecutive days for a calendar year or a water year. Note that most low-flow frequency analyses of annual 7day minimum flows use a climatic year (April 1-March 31). The date shown in the summary statistics table is the 
initial date of the 7-day period. (This value should not be confused with the 7-day 10-year low-flow statistic.)

INSTANTANEOUS PEAK FLOW.--The maximum instantaneous discharge occurring for the water year or for the designated period. Note that secondary instantaneous peak discharges above a selected base discharge are stored in District computer files for stations meeting certain criteria. Those discharge values may be obtained by writing to the District Office. (See address on back of title page of this report.)

INSTANTANEOUS PEAK STAGE.--The maximum instantaneous stage occurring for the water year or for the designated period. If the dates of occurrence for the instantaneous peak flow and instantaneous peak stage differ, the REMARKS paragraph in the manuscript or a footnote may be used to provide further information.

INSTANTANEOUS LOW FLOW.--The minimum instantaneous discharge occurring for the water year or for the designated period.

ANNUAL RUNOFF (AC-FT).-Indicates the depth, in acrefeet, to which the drainage area would be covered if all the runoff for the year were uniformly distributed on it.

ANNUAL RUNOFF (CFSM).--Indicates the average number of cubic feet of water flowing per second from each square mile of area drained, assuming that the runoff is distributed uniformly in time and area for the year.

ANNUAL RUNOFF (INCHES).-Indicates the depth to which the drainage area would be covered if all the runoff for the year were uniformly distributed on it.

10 PERCENT EXCEEDS.-The discharge that is exceeded by 10 percent of the flow for the designated period.

50 PERCENT EXCEEDS.-The discharge that is exceeded by 50 percent of the flow for the designated period.

90 PERCENT EXCEEDS.-The discharge that is exceeded by 90 percent of the flow for the designated period.

Data collected at partial-record stations follow the information for continuous-record sites. Data for partial-record discharge stations are presented in two tables. The first is a table of discharge measurements at low-flow partial-record stations, and the second is a table of annual maximum stage and discharge at crest-stage stations. The tables of partial-record stations are followed by a listing of discharge measurements made at sites other than continuous-record or partial-record stations. These measurements are generally made in times of drought or flood to give better areal coverage to those events. Those measurements and others collected for some special reason are called measurements at miscellaneous sites.

\section{Identifying Estimated Daily Discharge}

Estimated daily-discharge values published in the waterdischarge tables of annual State data reports are identified either by flagging individual daily values with the letter symbol " $e$ " and printing a table footnote, "e Estimated", or by listing the dates of the estimated record in the REMARKS paragraph of the station description.

\section{Accuracy of the Records}

The accuracy of streamflow records depends primarily on: (1) The stability of the stage-discharge relation or, if the control is unstable, the frequency of discharge measurements; and (2) the accuracy of measurements of stage, measurements of discharge, and interpretation of records.

The accuracy attributed to the records is indicated under "REMARKS." "Excellent" means that about 95 percent of the daily discharges are within 5 percent of the true; "good," within 10 percent; and "fair," within 15 percent. Records that do not meet the criteria mentioned, are rated "poor." Different accuracies may be attributed to different parts of a given record.

Daily mean discharges in this report are given to the nearest hundredth of a cubic foot per second for values less than $1 \mathrm{ft}^{3} / \mathrm{s}$; to the nearest tenth between 1.0 and $10 \mathrm{ft}^{3} / \mathrm{s}$; to whole numbers between 10 and $1000 \mathrm{ft}^{3} / \mathrm{s}$; and to 3 significant figures for more than $1000 \mathrm{ft}^{3} / \mathrm{s}$. The number of significant figures used is based solely on the magnitude of the discharge value. The same rounding rules apply to discharges listed for partial-record stations and miscellaneous sites.

Discharge at many stations, as indicated by the monthly mean, may not reflect natural runoff due to the effects of diversion, consumption, regulation by storage, increase or decrease in evaporation due to artificial causes, or to other factors. For such stations, figures of cubic feet per second per square mile and of runoff, in inches, are not published unless satisfactory adjustments can be made for diversions, for changes in contents of reservoirs, or for other changes incident to use and control. Evaporation from a reservoir is not included in the adjustments for changes in reservoir contents, unless it is so stated. Even at those stations where adjustments are made, large errors in computed runoff may occur if adjustments or losses are large in comparison with the observed discharge.

\section{Other Records Available}

Information of a more detailed nature than that published for most of the gaging stations such as observations of water temperatures, discharge measurements, gage-height records, and rating tables is on file in the district office. Also most gaging-station records are available in computer-usable form and many statistical analyses have been made.

Information on the availability of unpublished data or statistical analyses may be obtained from the district office.

The National Water Data Exchange, Water Resources Division, U.S. Geological Survey, National Center, Reston, VA 22092, maintains an index of all discharge measurement sites in the State as well as an index of records of discharge collected by other agencies but not published by the Geological Survey. Information on records available at specific sites can be obtained upon request.

\section{RECORDS OF SURFACE-WATER QUALITY}

Records of surface water quality ordinarily are obtained at or near stream-gaging stations because interpretation of records of surface-water quality nearly always requires corresponding discharge data. Records of surface-water quality in this report may involve a variety of types of data and measurement frequencies.

\section{Classification of Records}

Water-quality data for surface-water sites are grouped into one of three classifications. A continuing-record station is a site where data are collected on a regularly scheduled basis. Frequency may be once or more times daily, weekly, monthly, or quarterly. A partialrecord station is a site where limited water-quality data are collected systematically over a period of years. Frequency of sampling is usually less than quarterly. A miscellaneous sampling site is a location other than a continuing or partial-record station, where random samples are collected to give better areal coverage to define water-quality conditions in the river basin.

A careful distinction needs to be made between "continuing records" as used in this report and "continuous recordings," which refers to a continuous graph or a series of discrete values punched at short intervals on a paper tape. Some records of water quality, such as temperature and specific conductance, may be obtained through continuous recordings; however, because of costs, most data are 
obtained only monthly or less frequently. Locations of stations for which records on the quality of surface water appear in this report are shown in figure 8.

\section{Arrangement of Records}

Water-quality records collected at a surface-water daily record station are published immediately following that record, regardless of the frequency of sample collection. Station number and name are the same for both records. Where a surface-water daily record station is not available or where the water quality differs significantly from that at the nearby surface-water station, the continuing waterquality record is published with its own station number and name in the regular downstream-order sequence. Water-quality data for partial-record stations and for miscellaneous sampling sites appear in separate tables following the table of discharge measurements at miscellaneous sites.

\section{Onsite Measurement and Collection}

In obtaining water quality data, a major concern needs to be assuring that the data obtained represents the in situ quality of water. To assure this, certain measurements, such as water temperature, $\mathrm{pH}$, and dissolved oxygen need to be made onsite when the samples are taken. To assure that measurements made in the laboratory also represent the in situ water, carefully prescribed procedures need to be followed in collecting the samples, in treating the samples to prevent changes in quality pending analysis, and in shipping the samples to the laboratory. Procedures for onsite measurements and for collecting, treating, and shipping samples are given in publications on "Techniques of Water-Resources Investigations ," Book 1, Chap. D2; Book 3, Chap. C2; Book 5 Chap. $A 1, A 3$, and A4. All of these references are listed on p. 17 of this report. Also, detailed information on collecting, treating, and shipping samples may be obtained from the Geological Survey district office.

One sample can define adequately the water quality at a given time if the mixture of solutes throughout the stream cross section is homogeneous. However, the concentration of solutes at different locations in the cross section may vary widely with different rates of water discharge, depending on the source of material and the turbulence and mixing of the stream. Some streams must be sampled through several vertical sections to obtain a representative sample needed for an accurate mean concentration and for use in calculating load. All samples obtained for the National Stream Quality Accounting Network (see definitions) are obtained from at least several verticals. Whether samples are obtained from the centroid of flow or from several verticals, depends on flow conditions and other factors which must be evaluated by the collector.

Chemical-quality data published in this report are considered to be the most representative values available for the stations listed. The values reported represent water-quality conditions at the time of sampling as much as possible, consistent with available sampling techniques and methods of analysis. In the rare case where an apparent inconsistency exists between a reported $\mathrm{pH}$ value and the relative abundance of carbon dioxide species (carbonate and bicarbonate), the inconsistency is the result of a slight uptake of carbon dioxide from the air by the sample between measurement of $\mathrm{pH}$ in the field and determination of carbonate and bicarbonate in the laboratory.

For chemical-quality stations equipped with digital monitors, the records consist of daily maximum, minimum, and mean values for each constituent measured and are based upon hourly punches beginning at 0100 hours and ending at 2400 hours for the day of record. More detailed records (hourly values) may be obtained from the U.S.G.S. district office whose address is given on the back of the title page of this report.

\section{Water Temperature}

Water temperatures are measured at most of the water-quality stations. In addition, water temperatures are taken at time of discharge measurements for water-discharge stations. For stations where water temperatures are taken manually once or twice daily, the water temperatures are taken at about the same time each day. Large streams have a small diurnal temperature change; shallow streams may have a daily range of several degrees and may follow closely the changes in air temperature. Some streams may be affected by waste-heat discharges.

At stations where recording instruments are used, either mean temperatures or maximum and minimum temperatures for each day are published. Water temperatures measured at the time of waterdischarge measurements are on file in the district office.

\section{Sediment}

Suspended-sediment concentrations are determined from samples collected by using depth-integrating samplers. Samples usually are obtained at several verticals in the cross section, or a single sample may be obtained at a fixed point and a coefficient applied to determine the mean concentration in the cross sections.

During periods of rapidly changing flow or rapidly changing concentration, samples may have been collected more frequently (twice daily or, in some instances, hourly). The published sediment discharges for days of rapidly changing flow or concentration were computed by the subdivided-day method (time-discharge weighted average). Therefore, for those days when the published sediment discharge value differs from the value computed as the product of discharge times mean concentration times 0.0027 , the reader can assume that the sediment discharge for that day was computed by the subdivided-day method. For periods when no samples were collected, daily loads of suspended sediment were estimated on the basis of water discharge, sediment concentrations observed immediately before and after the periods, and suspended-sediment loads for other periods of similar discharge.

At other stations, suspended-sediment samples were collected periodically at many verticals in the stream cross section. Although data collected periodically may represent conditions only at the time of observations, such data are useful in establishing seasonal relations between quality and streamflow and in predicting long-term sediment-discharge characteristics of the stream.

In addition to the records of suspended sediment discharge, records of the periodic measurements of the particle-size distribution of the suspended sediment and bed material are included for some stations.

\section{Laboratory Measurements}

Samples for indicator bacteria and specific conductance are analyzed locally. All other samples are analyzed in the Geological Survey laboratories in Arvada, Colo., Doraville, Ga., or Iowa City, Ia. Methods used in analyzing sediment samples and computing sediment records are given in TWRI, Book 5, Chap. C1. Methods used by the Geological Survey laboratories are given in TWRI, Book 1, Chap. D2; Book 3, Chap. C2; Book 5, Chap. A1, A3, and A4.

\section{Data Presentation}

For continuing-record stations, information pertinent to the history of station operation is provided in descriptive headings preceding the tabular data. These descriptive headings give details regarding location, drainage area, period of record, type of data available, instrumentation, general remarks, cooperation, and extremes for parameters currently measured daily. Tables of chemical, physical, biological, radiochemical data, and so forth, obtained at a frequency less than daily are presented first. Tables of "daily values" of specific conductance, $\mathrm{pH}$, water temperature, dissolved, and suspended sediment then follow in sequence.

In the descriptive headings, if the location is identical to that of the discharge gaging station, neither the LOCATION nor the DRAINAGE AREA statements are repeated. The following information, when appropriate, is provided with each continuousrecord station. Comments that follow clarify information presented under the various headings of the station description. 
LOCATION.-See Data Presentation under "Records of stage and Water Discharge"; same comments apply.

DRAINAGE AREA.-See Data Presentation under "Records of stage and Water Discharge"; same comments apply.

PERIOD OF RECORD.-This indicates the periods for which there are published water-quality records for the station. The periods are shown separately for records of parameters measured daily or continuously and those measured less than daily. For those measured daily or continuously, periods of record are given for the parameters individually.

INSTRUMENTATION.-Information on instrumentation is given only if a water-quality monitor, temperature recorder, sediment pumping sampler, or other sampling device is in operation at a station.

REMARKS.-Remarks provide added information pertinent to the collection, analysis, or computation of the records.

COOPERATION.--Records provided by a cooperating organization or obtained for the Geological Survey by a cooperating organization are identified here.

EXTREMES.--Maximums and minimums are given only for parameters measured daily or more frequently. None are given for parameters measured weekly or less frequently, because the true maximums or minimums may not have been sampled. Extremes, when given, are provided for both the period of record and for the current water year.

REVISIONS.-If errors in published water-quality records are discovered after publication, appropriate updates are made to the Water-Quality File in the U.S. Geological Survey's computerized data system, WATSTORE, and subsequently by monthly transfer of update transactions to the U.S. Environmental Protection Agency's STORET system. Because the usual volume of updates makes it impractical to document individual changes in the State data-report series or elsewhere, potential users of U.S. Geological Survey waterquality data are encouraged to obtain all required data from the appropriate computer file to insure the most recent updates.

The surface-water-quality records for partial-record stations and miscellaneous sampling sites are published in separate tables following the table of discharge measurements at miscellaneous sites. No descriptive statements are given for these records. Each station is published with its own station number and name in the regular downstream-order sequence.

\section{Remark Codes}

The following remark codes may appear with the water-quality data in this report:

\section{PRINTED OUTPUT REMARK}

\begin{tabular}{ll} 
E & $\begin{array}{l}\text { Estimated value } \\
>\end{array}$ \\
\hline & $\begin{array}{l}\text { Actual value is known to be } \\
\text { greater than the value shown }\end{array}$ \\
$\mathrm{K}$ & $\begin{array}{l}\text { Actual value is known to be } \\
\text { less than the value shown } \\
\text { Results based on colony count } \\
\text { outside the acceptance range } \\
\text { (non-ideal colony count) }\end{array}$
\end{tabular}

L Biological organisms count less than 0.5 percent (organisms may be observed rather than counted) Biological organism count equal to or greater than 15 percent (dominant)

\section{RECORDS OF GROUND-WATER LEVELS}

Only water-level data from a national network of observation wells are given in this report. These data are intended to provide a sampling and historical record of water-level changes in the Nation's most important aquifers. Locations of the observation wells in this network in Minnesota are shown in figure 10.

Although, in this report, records of water levels are presented for fewer than 200 wells, records are obtained through cooperative efforts of many Federal, State, and local agencies for several hundred observation wells throughout Minnesota and are placed in computer storage. Each spring, the Minnesota Department of Natural Resources, Division of Waters publishes a report for the previous water year entitled "Observation Well Data Summary, Water Year 19 ." This report contains hydrographs of recorder wells, detailed maps showing the location of active observation wells, and other useful items. Information about the availability of the data in the water-level file may be obtained from the District Chief, Minnesota District. (See address on back of front page).

\section{Data Collection and Computation}

Measurements of water levels are made in many types of wells under varying conditions, but the methods of measurement are standardized to the extent possible. The equipment and measuring techniques used at each observation well assure that measurements at each well are of consistent accuracy and reliability.

Tables of water-level data are presented by counties arranged in alphabetical order. The prime identification number for a given well is the 15-digit number that appears in the upper left corner of the table. The secondary identification number is the local well number, an alphanumeric number, derived from the township-range location of the well.

Water-level records are obtained from direct measurements with a steel tape or from the graph or punched tape of a water-stage recorder. The water-level measurements in this report are given in feet with reference to land-surface datum (1sd). Land-surface datum is a datum plane that is approximately at land surface at each well. If nown, the elevation of the land-surface datum is given in the well description. The height of the measuring point (MP) above or below land-surface datum is given in each well description. Water levels in wells equipped with recording gages are reported for every fifth day and the end of each month (eom).

All water-level measurements are reported to the nearest hundredth of a foot. The error of water-level measurements is normally only a hundredth or a few hundredth of a foot.

Hydrographs showing water-level fluctuations are included for 28 representative wells; 7 in surficial-sand aquifers, 6 in buried-sand aquifers, and 15 in bedrock aquifers.

\section{Data Presentation}

Each well consists of two parts, the station description and the data table of water levels observed during the water year. In addition a graph of water levels for the current year or other selected period is included for several representative wells. The description of the well is presented first through use of descriptive headings preceding the tabular data. The comments to follow clarify information presented under the various headings.

LOCATION.-This paragraph follows the well-identification number and reports the latitude and longitude (given in degrees, minutes and seconds); a landline location designation; the hydrologic-unit number; the distance and direction from a geographic point of reference; and the owner's name.

AQUIFER.-- This entry designates by name (if a name exists) and geologic age the aquifer(s) open to the well. 
WELL CHARACTERISTICS.-This entry describes the well in terms of depth, diameter, casing depth and/or screened interval, method of construction, use, and includes additional information such as casing breaks, collapsed screen, and other changes since construction.

INSTRUMENTATION.-This paragraph provides information on both the frequency of measurement and the collection method used, allowing the user to better evaluate the reported water-level extremes by knowing whether they are based on weekly, monthly, or some other frequency of measurement.

DATUM.-This entry describes both the measuring point and the land-surface elevation at the well. The measuring point is described physically (such as top of collar, notch in the top of casing, plug in pump base and so on), and in relation to land surface (such as $1.3 \mathrm{ft}$ above land-surface datum). The elevation of the landsurface datum is described in feet above (or below) National Geodetic Vertical Datum of 1929 (NGVD of 1929); it is reported with a precision depending on the method of determination.

REMARKS.-This entry describes factors that may influence the water level in a well or the measurement of the water level. It should identify wells that are also water-quality observation wells, and may be used to acknowledge the assistance of local (non-Survey) observers.

PERIOD OF RECORD.--This entry indicates the period for which there are published records for the well. It reports the month and year of the start of the publication of water-level records by the U.S. Geological Survey and the words "to current year" if the records are to be continued into the following year. Periods for which waterlevel records are available, but are not published by the Geological Survey, may be noted.

EXTREMES FOR THE PERIOD OF RECORD.-This entry contains the highest and lowest water levels of the period of published record, with respect to land-surface datum, and the dates of their occurrence.

A table of water levels follows the station description for each well. Water levels are reported in feet below land-surface datum and all taped measurements of water level are listed. For wells equipped with recorders, abbreviated tables are published; generally, only water-level lows are listed for every fifth day and at the end of the month (eom). The highest and lowest water levels of the water year and their dates of occurrence are shown on a line below the abbreviated table. Because all values are not published for wells with recorders, the extremes may be values that are not listed in the table. Missing records are indicated by dashes in place of the water level. A hydrograph for a selected period of record follows the water-level table for several representative wells.

\section{RECORDS OF GROUND-WATER QUALITY}

Records of ground-water quality, in this report differ from other types of records in that for most sampling sites they consist of only one set of measurements for the water year. The quality of ground water ordinarily changes only slowly; therefore, for most general purposes one annual sampling, or only a few samples taken at infrequent intervals during the year, is sufficient. Frequent measurement of the same constituents is not necessary unless one is concerned with a particular problem, such as monitoring for trends in nitrate concentration. In the special cases where the quality of ground water may change more rapidly, more frequent measurements are made to identify the nature of the changes.

\section{Data Collection and Computation}

The records of ground-water quality in this report were obtained mostly as a part of special studies in specific areas. Consequently, a number of chemical analyses are presented for some counties but none are presented for others. As a result, the records for this year, by themselves, do not provide a balanced view of ground-water quality statewide. Such a view can be attained only by considering records for this year in context with similar records obtained for these and other counties in earlier years.

Most methods for collecting and analyzing water samples are described in the "U.S. Geological Survey Techniques of WaterResources Investigation" manuals listed on a following page. The values reported in this report represent water-quality conditions at the time of sampling as much as possible, consistent with available sampling techniques and methods of analysis. All samples were obtained by trained personnel. The wells sampled were pumped long enough to assure that the water collected came directly from the aquifer and had not stood for a long time in the well casing where it would have been exposed to the atmosphere and to the material, possibly metal, comprising the casings.

\section{Data Presentation}

The records of ground-water quality are published in a section titled QUALITY OF GROUND WATER immediately following the ground-water-level records. Data for quality of ground water are listed alphabetically by County, and are identified by well number. The prime identification number for wells sampled is the 15-digit number derived from the latitude-longitude locations. No descriptive statements are given for ground-water-quality records; however, the well number, depth of well, date of sampling, and other pertinent data are given in the table containing the chemical analyses of the ground water. The REMARK codes listed for surface-water-quality records are also applicable to ground-water-quality records.

\section{ACCESS TO WATSTORE DATA}

The U.S. Geological Survey is the principal Federal water-data agency and, as such, collects and disseminates about 70 percent of the water data currently being used by numerous State, local, private, and other Federal agencies to develop and manage our water resources. As part of the U.S. Geological Survey's program of releasing water data to the public, a large-scale computerized system has been developed for the storage and retrieval of water data collected through its activities. The National Water Data Storage and Retrieval System (WATSTORE) was established in 1972 to provide an effective and efficient means for the processing and maintenance of water data collected through the activities of the U.S. Geological Survey and to facilitate release of the data to the public. A variety of useful products, ranging from data tables to complex statistical analyses such as Log Pearson Type III, can be produced using WATSTORE. The system resides on the central computer facilities of the U.S. Geological Survey at its National Center in Reston, Virginia, and consists of related files and data bases.

- Station Header File - Contains descriptive information on more than 440,000 sites throughout the United States and its territories where the U.S. Geological Survey collects or has collected data.

- Daily Values File - Contains more than $\mathbf{2 2 0}$ million daily values of stream flows, stages, reservoir contents, water temperature, specific conductances, sediment concentrations, sediment discharges, and ground-water levels.

- Peak Flow File - Contains approximately 500,000 maximum (peak) streamflow and gage-height values at surface-water sites.

* Water Quality File - Contains approximately 2 million analyses of water samples that describe the chemical, physical, biological, and radio-chemical characteristics of both surface and ground water.

- Ground-Water Site Inventory Data Base - Contains inventory data for more than 900,000 wells, springs, and other sources of ground water. The data includes site location, geohydrologic characteristics, well-construction history, and one-time field measurements such as water temperature.

In 1976, the U.S. Geological Survey opened WATSTORE to 
the public for direct access. The signing of a Memorandum of Agreement with the Survey is required to obtain direct access to WATSTORE. The system can be accessed either synchronously or asynchronously. The requester will be expected to pay all computer costs he/she incurs. Direct access may be obtained by contacting:

\section{U.S. Geological Survey \\ National Water Data Exchange \\ 421 USGS National Center \\ Reston, Virginia 22092}

In addition to providing direct access to WATSTORE, data can be provided in various machine-readable formats on magnetic tape or 5-1/4 inch floppy disk; and, as noted in the introduction, on CD-ROM discs. Beginning with the 1990 water year, all water-data reports will also be available on Compact disc - Read Only Memory (CD-ROM). All data reports published for the current water year for the entire Nation, including Puerto Rico and the Trust Territories, will be reproduced on a single CD-ROM disc. Information about the availability of specific types of data or products, and user charges, can be obtained locally from each of the Water Resources Division's District offices. (See address on the back of the title page.) A limited number of CD-ROM discs will be available for sale by the Books and Open-File Reports Section, U.S. Geological Survey, Federal Center, Box 25425, Denver, Colorado 80225.

\section{DEFINITION OF TERMS}

Terms related to streamflow, water-quality, and other hydrologic data, as used in this report, are defined below. See also table for converting inch-pound units to International System of units (SI) on the inside of back cover.

Acre-foot (AC-FT, acre-ft) is the quantity of water required to cover 1 acre to a depth of 1 foot and is equivalent to 43,560 cubic feet or about 326,000 gallons or 1,233 cubic meters.

Adenosine triphosphate (ATP) is the primary energy donor in cellular life process. Its central role in living cells makes it an excellent indicator of the presence of living material in water. A measure of ATP, therefore, provides a sensitive and rapid estimate of biomass. ATP is reported in micrograms per liter of the original water sample.

Algae are mostly aquatic single-celled, colonial, or multi-celled plants, containing chlorophyll and lacking roots, stems, and leaves.

Algal growth potential (AGP) is the maximum algal dry weight biomass that can be produced in a natural water sample under standardized laboratory conditions. The growth potential is the algal biomass present at stationary phase and is expressed as milligrams dry weight of algae produced per liter of sample.

Aquifer is a geologic formation, group of formations, or part of a formation that contains sufficient saturated permeable material to yield significant quantities of water to wells and springs.

Artesian means confined and is used to describe a well in which the water level stands above the top of the aquifer tapped by the well. A flowing artesian well is one in which the water level is above the land surface.

Bacteria are microscopic unicellular organisms, typically spherical, rodlike, or spiral and threadlike in shape, often clumped into colonies. Some bacteria cause disease, others perform an essential role in nature in the recycling of materials; for example, by decomposing organic matter into a form available for reuse by plants.

Total coliform bacteria are a particular group of bacteria that are used as indicators of possible sewage pollution. They are characterized as aerobic or facultative anaerobic, gramnegative, nonspore- forming, rod-shaped bacteria which ferment lactose with gas formation within 48 hours at $35 \circ C$. In the laboratory these bacteria are defined as the organisms which produce colonies with a golden-green metallic sheet within 24 hours when incubated at $35^{\circ} \mathrm{C}+1.0^{\circ} \mathrm{C}$ on $\mathrm{M}$-Endo medium (nutrient medium for bacterial growth). Their concentrations are expressed as number of colonies per 100 $\mathrm{mL}$ of sample.

Fecal coliform bacteria are bacteria that are present in the intestine or feces of warmblooded animals. They are often used as indicators of the sanitary quality of the water. In the laboratory they are defined as all organisms which produce blue colonies within 24 hours when incubated at $44.5^{\circ} \mathrm{C}+0.2^{\circ} \mathrm{C}$ on M-FC medium (nutrient medium for bacterial growth). Their concentrations are expressed as number of colonies per $100 \mathrm{~mL}$ of sample.

Fecal streptococcal bacteria are bacteria also found in the intestine of warmblooded animals. Their presence in water is considered to verify fecal pollution. They are characterized as gram-positive, cocci bacteria which are capable of growth in brain-heart infusion broth. In the laboratory they are defined as all the organisms which produce red or pink colonies within 48 hours at $35^{\circ} \mathrm{C}+1.0^{\circ} \mathrm{C}$ on $\mathrm{M}-\mathrm{FC}$ medium (nutrient medium for bacterial growth). Their concentrations are expressed as number of colonies per $100 \mathrm{~mL}$ of sample.

Bed material is the unconsolidated material of which a streambed, lake, pond, reservoir, or estuary bottom is composed.

Biochemical oxygen demand (BOD) is a measure of the quantity of dissolved oxygen, in milligrams per liter, necessary for the decomposition of organic matter by microorganisms, such as bacteria.

Biomass is the amount of living matter present at any given time, expressed as the mass per unit area or volume of habitat.

Ash mass is the mass or amount of residue present after the residue from the dry mass determination has been ashed in a muffle furnace at a temperature of $500^{\circ} \mathrm{C}$ for 1 hour. The ash mass values of zooplankton and phytoplankton are expressed in grams per cubic meter $\left(\mathrm{g} / \mathrm{m}^{3}\right)$, and periphyton and benthic organisms in grams per square meter $\left(\mathrm{g} / \mathrm{m}^{2}\right)$.

Dry mass refers to the weight of residue present after drying in an oven at $60^{\circ} \mathrm{C}$ for zooplankton and $105^{\circ} \mathrm{C}$ for periphyton, until the mass remains unchanged. This mass represents the total organic matter, ash and sediment, in the sample. Dry mass values are expressed in the same units as ash mass.

Organic mass or volatile mass of the living substance is the difference between the dry mass and the ash mass, and represents the actual mass of the living matter. The organic mass is expressed in the same units as for ash mass and dry mass. water.

Wet mass is the mass of living matter plus contained

\section{Bottom material: See Bed Material.}

Cells/volume refers to the number of cells or any organism which is counted by using a microscope and grid or counting cell. Many planktonic organisms are multicelled and are counted according to the number of contained cells per sample, usually milliliters $(\mathrm{mL})$ or liters $(\mathrm{L})$.

Cfs-day is the volume of water represented by a flow of 1 cubic foot per second for 24 hours. It is equivalent to 86,400 cubic feet, approximately 1.9835 acre-feet, or about 646,000 gallons or 2,447 cubic meters.

Chemical oxygen demand (COD) is a measure of the chemically oxidizable material in the water, and furnishes an approximation of the amount of organic and reducing material present. The determined value may correlate with natural water color or with carbonaceous organic pollution from sewage or 
industrial wastes.

Chlorophyll refers to the green pigments of plants. Chlorophyll $\underline{a}$ and $\underline{b}$ are the two most common pigments in plants.

Color unit is produced by one milligram per liter of platinum in the form of the chloroplatinate ion. Color is expressed in units of the platinum-cobalt scale.

Contents is the volume of water in a reservoir or lake. Unless otherwise indicated, volume is computed on the basis of a level pool and does not include bank storage.

Control designates a feature downstream from the gage that determines the stage-discharge relation at the gage. This feature may be a natural constriction of the channel, an artificial structure, or a uniform cross section over a long reach of the channel.

Cubic feet per second per square mile (CFSM) is the average number of cubic feet of water flowing per second from each square mile of area drained, assuming that the runoff is distributed uniformly in time and area.

Cubic foot per second $\left(\mathrm{FT}^{3} / \mathrm{s}, \mathrm{ft}^{3} / \mathrm{s}\right)$ is the rate of discharge representing a volume of 1 cubic foot passing a given point during 1 second and is equivalent to approximately 7.48 gallons per second or 448.8 gallons per minute or 0.02832 cubic meters per second.

Discharge is the volume of water (or more broadly, volume of fluid pius suspended sediment), that passes a given point within a given period of time.

Mean discharge (MEAN) is the arithmetic mean of individual daily mean discharges during a specific period.

Instantaneous discharge is the discharge at a particular instant of time.

Annual 7-day minimum is the lowest mean discharge for 7 consecutive days for a calender year or a water year. Note that most low-flow frequency analyses of annual 7-day minimum flows use a climatic year (April 1-March 31). The date shown in the summary statistics table is the initial date of the 7-day period. (This value should not be confused with the 7-day 10-year low-flow statistic.)

Dissolved refers to the amount of substance present in true chemical solution. In practice, however, the term includes all forms of substance that will pass through a 0.45 -micrometer membrane filter, and thus may include some very small (colloidal) suspended particles. Analyses are performed on filtered samples.

Dissolved-solids concentration of water is determined either analytically by the "residue-on-evaporation" method, or mathematically by totaling the concentrations of individual constituents reported in a comprehensive chemical analysis. During the analytical determination of dissolved solids, the bicarbonate (generally a major dissolved component of water) is converted to carbonate. Therefore, in the mathematical calculation of dissolvedsolids concentration, the bicarbonate value, in milligrams per liter, is multiplied by 0.492 to reflect the change.

Diversity index is a numerical expression of evenness of distribution of aquatic organisms. The formula for diversity index is:

$$
\bar{d}=-\sum_{i=1}^{s} \frac{n_{i}}{n} \log _{2} \frac{n_{i}}{n}
$$

Where ' $n_{i}$ ' is the number of individuals per taxon, ' $n$ ' is the total number of individuals, and ' $s$ ' is the total number of taxa in the sample of the community. Diversity index values range from zero, when all the organisms in the sample are the same, to some positive number, when some or all of the organisms in the sample are different.

Drainage area of a stream at a specified location is that area, measured in a horizontal plane, enclosed by a topographic divide from which direct surface runoff from precipitation normally drains by gravity into the river above the specified point. Figures of drainage area given herein include all closed basins, or noncontributing areas, within the area unless otherwise noted.

Drainage basin is a part of the surface of the earth that is occupied by a drainage system, which consists of a surface stream or a body of impounded surface water together with all tributary surface streams and bodies of impounded surface water.

Gage height (G.H.) is the water-surface elevation referred to some arbitrary gage datum. Gage height is often used interchangeably with the more general term "stage," although gage height is more appropriate when used with a reading on a gage.

Gaging station is a particular site on a stream, canal, lake, or reservoir where systematic observations of hydrologic data are obtained.

Hardness of water is a physical-chemical characteristic that is commonly recognized by the increased quantity of soap required to produce lather. It is attributable to the presence of alkaline earths (principally calcium and magnesium) and is expressed as equivalent calcium carbonate $\left(\mathrm{CaCO}_{3}\right)$.

Hydrologic unit is a geographic area representing part or all of a surface drainage basin or distinct hydrologic feature as delineated by the Office of Water Data Coordination on the State Hydrologic Unit Maps; each hydrologic unit is identified by an 8-digit number.

Metamorphic stage refers to the stage of development that an organism exhibits during its transformation from an immature form to an adult form. This developmental process exists for most insects, and the degree of difference from the immature stage to the adult form varies from relatively slight to pronounced, with many intermediates. Examples of metamorphic stages of insects are egglarva-adult or egg-nymph-adult.

Methylene blue active substance (MBAS) is a measure of apparent detergents. This determination depends on the formation of a blue color when methylene blue dye reacts with synthetic detergent compounds.

Micrograms per gram (UG/G, ug/g) is a unit expressing the concentration of a chemical element as the mass (micrograms) of the element sorbed per unit mass (gram) of sediment.

Micrograms per kilogram (MG/KG, $\mathrm{mg} / \mathrm{kg}$ ) is a unit expressing the concentration of a chemical element as the mass (micrograms) of the element sorbed per unit mass (kilogram) of sediment.

Micrograms per liter (UG/L, ug/L) is a unit expressing the concentration of chemical constituents in solution as mass (micrograms) of solute per unit volume (liter) of water. One thousand micrograms per liter is equivalent to one milligram per liter.

Milligrams per liter (MG/L, $\mathrm{mg} / \mathrm{L}$ ) is a unit for expressing the concentration of chemical constituents in solution. Milligrams per liter represent the mass of solute per unit volume (liter) of water. Concentration of suspended sediment also is expressed in $\mathrm{mg} / \mathrm{L}$, and is based on the mass of sediment per liter of water-sediment mixture.

National Geodetic Vertical Datum of 1929 (NGVD) is a geodetic datum derived from a general adjustment of the first order level nets of both the United States and Canada. It was formerly called "Sea Level Datum of 1929 " or "mean sea level" in this series of reports. Although the datum was derived from the average sea level 
over a period of many years at 26 tide stations along the Atlantic, Gulf of Mexico, and Pacific Coasts, it does not necessarily represent local mean sea level at any particular place.

National Stream Quality Accounting Network (NASQAN) is a nationwide data-collection network designed by the U.S. Geological Survey to meet many of the information needs of government agencies and other groups involved in natural or regional waterquality planning and management. The 500 or so sites in NASQAN are generally located at the downstream ends of hydrologic accounting units designated by the U.S. Geological Survey Office of Water Data Coordination in consultation with the Water Resources Council. The objectives of NASQAN are (1) to obtain information on the quality and quantity of water moving within and from the United States through a systematic and uniform process of data collection, summarization, analysis, and reporting such that the data may be used for, (2) description of the areal variability of water quality in the Nation's rivers through analysis of data from this and other programs, (3) detection of changes or trends with time in the pattern of occurrence of water-quality characteristics, and (4) providing a nationally consistent data base useful for water-quality assessment and hydrologic research.

The National Trends Network (NTN) is a 150-station network for sampling atmospheric deposition in the United States. The purpose of the network is to determine the variability, both in location and in time, of the composition of atmospheric deposition, which includes snow, rain, dust particles, aerosols, and gases, The core from which the NTN was built was the already-existing deposition-monitoring network of the National Atmospheric Deposition Program (NADP).

Organism is any living entity, such as an insect, phytoplankter, or zooplankter.

Organism count/area refers to the number of organisms collected and enumerated in a sample and adjusted to the number per area habitat, usually square meters $\left(\mathrm{m}^{2}\right)$, acres, or hectares. Periphyton, benthic organisms, and macrophytes are expressed in these terms.

Organism count/volume refers to the number of organisms collected and enumerated in a sample and adjusted to the number per sample volume, usually milliters $(\mathrm{mL})$ or liters $(\mathrm{L})$. Numbers of planktonic organisms can be expressed in these terms.

Total organism count is the total number of organisms collected and enumerated in any particular sample.

Parameter code numbers are unique five-digit code numbers assigned to each parameter placed into storage. These codes are assigned by the Environmental Protection Agency and are also used to identify data exchanged among agencies.

Partial-record station is a particular site where limited streamflow and (or) water-quality data are collected systematically over a period of years for use in hydrologic analyses.

Particle size is the diameter, in millimeters ( $\mathrm{mm}$ ), of suspended sediment or bed material determined by either sieve or sedimentation methods. Sedimentation methods (pipet, bottomwithdrawal tube, visual-accumulation tube) determine fall diameter of particles in distilled water (chemically dispersed).

Particle-size classification used in this report agrees with recommendations made by the American Geophysical Union Subcommittee on Sediment Terminology.

The classification is as follows:

\begin{tabular}{lcc} 
Classification & \multicolumn{2}{c}{ Size $(\mathrm{mm})$} \\
Clay & 0.00024 & -0.004 \\
Silt & .004 & -062 \\
Sand & .062 & -2.0 \\
Gravel & 2.0 & -64.0
\end{tabular}

Method of analysis

Sedimentation

Sedimentation

Sedimentation or sieve

Sieve
The particle-size distributions given in this report are not necessarily representative of all particles in transport in the stream. Most of the organic material is removed and the sample is subjected to mechanical and chemical dispersion before analysis in distilled water.

Percent composition is a unit for expressing the ratio of a particular part of a sample or population to the total sample or population, in terms of types, numbers, mass or volume.

Periphyton is the assemblage of microorganisms attached to and growing upon solid surfaces. While primarily consisting of algae, they also include bacteria, fungi, protozoa, rotifers, and other small organisms. Periphyton is a useful indicator of water quality.

Pesticides are chemical compounds used to control undesirable plants and animals. Major categories of pesticides include insecticides, miticides, fungicides, herbicides, and rodenticides. Insecticides and herbicides, which control insects and plants respectively, are the two categories reported.

Picocurie (PC, pCi) is one trillionth $\left(1 \times 10^{-12}\right)$ of the amount of radioactivity represented by a curie (C1). A curie is the amount of radioactivity that yields $3.7 \times 10^{10}$ radioactive disintegrations per second. A picocurie yields $2.22 \mathrm{dpm}$ (disintegrations per minute).

Plankton is the community of suspended, floating, or weakly swimming organisms that live in the open water of lakes and rivers.

Phytoplankton is the plant part of the plankton. They are usually microscopic and their movement is subject to the water currents. Phytoplankton growth is dependent upon solar radiation and nutrient substances. Because they are able to incorporate as well as release materials to the surrounding water, the phytoplankton have a profound effect upon the quality of the water. They are the primary food producers in the aquatic environment, and are commonly known as algae.

Blue-green algae are a group of phytoplankton organisms having a blue pigment, in addition to the green pigment called chlorophyll. Blue-green algae often cause nuisance conditions in water.

Diatoms are the unicellular or colonial algae having a siliceous shell. Their concentrations are expressed as number of cells/mL of sample.

Green algae have chlorophyll pigments similar in color to those of higher green plants. Some forms produce algal mats or floating "moss" in lakes. Their concentrations are expressed as number of cells $/ \mathrm{mL}$ of sample.

Zooplankton is the animal part of the plankton. Zooplankton are capable of extensive movements within the water column, and are often large enough to be seen with the unaided eye. Zooplankton are secondary consumers feeding upon bacteria, phytoplankton, and detritus. Because they are the grazers in the aquatic environment, the zooplankton are a vital part of the aquatic food web. The zooplankton community is dominated by small crustaceans and rotifers.

Polychlorinated biphenyls (PCBs) are industrial chemicals that are mixtures of chlorinated biphenyl compounds having various percentages of chlorine. They are similar in structure to organochlorine insecticides.

Primary productivity is a measure of the rate at which new organic matter is formed and accumulated through photosynthetic and chemosynthetic activity of producer organisms (chiefly green plants). The rate of primary production is estimated by measuring the amount of oxygen released (oxygen method) or the amount of carbon assimilated by the plants (carbon method).

Milligrams of carbon per area or volume per unit time Img $\mathrm{C} /\left(\mathrm{m}^{2}\right.$. time) for periphyton and macrophytes and $\mathrm{mg}$ $\mathrm{C} /\left(\mathrm{m}^{3}\right.$ - time $)$ for phytoplanktonl are units for expressing 
primary productivity. They define the amount of carbon dioxide consumed as measured by radioactive carbon (carbon 14). The carbon 14 method is of greater sensitivity than the oxygen light and dark bottle method, and is preferred for use in unenriched waters. Unit time may be either the hour or day, depending on the incubation period.

Milligrams of oxygen per area or volume per unit time Img $0_{2} /\left(\mathrm{m}^{2}\right.$ - time) for periphyton and macrophytes and $\mathrm{mg}$ $\underline{0}_{2} /\left(\mathrm{m}^{3}\right.$. time $)$ for phytoplankton] are the units for expressing primary productivity. They define production and respiration rates as estimated from changes in the measured dissolved oxygen concentration. The oxygen light and dark bottle method is preferred if the rate of primary production is sufficient for accurate measurements to be made within 24 hours. Unit time may be either the hour or day, depending on the incubation period.

Radiochemical program is a network of regularly sampled water-quality stations where samples are collected to be analyzed for radioisotypes. The streams that are sampled represent major drainage basins in the conterminous United States.

Recoverable from bottom material is the amount of a given constituent that is in solution after a representative sample of bottom material has been digested by a method (usually using an acid or mixture of acids) that results in dissolution of only readily soluble substances. Complete dissolution of all bottom material is not achieved by the digestion treatment and thus the determination represents less than the total amount (that is, less than 95 percent) of the constituent in the sample. To achieve comparability of analytical data, equivalent digestion procedures would be required of all laboratories performing such analyses because different digestion procedures are likely to produce different analytical results.

Return period is the average time interval between occurrences of a hydrological event of a given or greater magnitude, usually expressed in years. May also be called recurrence interval.

Runoff in inches (IN, in) shows the depth to which the drainage area would be covered if all the runoff for a given time period were uniformly distributed on it.

Sediment is solid material that originates mostly from disintegrated rocks and is transported by, suspended in, or deposited from water; it includes chemical and biochemical precipitates and decomposed organic material, such as humus. The quantity, characteristics, and cause of the occurrence of sediment in streams are influenced by environmental factors. Some major factors are degree of slope, length of slope, soil characteristics, land usage, and precipitation.

Bed load is the sediment that is transported in a stream by rolling, sliding, or skipping along the bed and very close to it. In this report, bed load is considered to consist of particles in transit within $0.25 \mathrm{ft}$ of the streambed.

Bed load discharge (tons per day) is the quantity of bed load measured by dry weight that moves past a section as bed load in a given time.

Suspended sediment is the sediment that at any given time is maintained in suspension by the upward components of turbulent currents or that exists in suspension as a colloid.

Suspended-sediment concentration is the velocityweighted concentration of suspended sediment in the sampled zone (from the water surface to a point approximately $0.3 \mathrm{ft}$ above the bed) expressed as milligrams of dry sediment per liter of water-sediment mixture $(\mathrm{mg} / \mathrm{L})$.

Mean concentration is the time-weighted concentration of suspended sediment passing a stream section during a 24hour day.
Suspended-sediment discharge (tons/day) is the rate at which dry weight of sediment passes a section of a stream or is the quantity sediment, as measured by dry weight or volume, that passes a section in a given time. It is computed by multiplying discharge times $\mathrm{mg} / \mathrm{L}$ times 0.0027 .

Suspended-sediment load is quantity of suspended sediment passing a section in a specified period.

Total sediment discharge (tons/day) is the sum of the suspended-sediment discharge and the bed-load discharge. It is the total quantity of sediment, as measured by dry weight or volume, that passes a section during a given time.

Total-sediment load or total load is a term which refers to the total sediment (bed load plus suspended-sediment load) that is in transport. It is not synonymous with total-sediment discharge.

7-day 10 year low flow $\left(7 Q_{10}\right)$ is the discharge at the 10 -year recurrence interval taken from a frequency curve of annual values of the lowest mean discharge for 7 consecutive days (the 7-day low flow).

Sodium-adsorption-ratio (SAR) is the expression of relative activity of sodium ions in exchange reactions within soil and is an index of sodium or alkali hazard to the soil. Waters range in respect to sodium hazard from those which can be used for irrigation on almost all soils to those which are generally unsatisfactory for irrigation.

Solute is any substance derived from the atmosphere, vegetation, soil, or rocks that is dissolved in water.

Specific conductance is a measure of the ability of a water to conduct an electrical current. It is expressed in micromhos per centimeter at $25^{\circ} \mathrm{C}$. Specific conductance is related to the type and concentration of ions in solution and can be used for approximating the dissolved-solids content of the water. Commonly, the concentration of dissolved solids (in milligrams per liter) is about 65 percent of the specific conductance (in micromhos). This relation is not constant from stream to stream, and it may vary in the same source with changes in the composition of the water.

Stage-discharge relation is the relation between gage height (stage) and volume of water per unit of time, flowing in a channel.

Streamflow is the discharge that occurs in a natural channel. Although the term "discharge" can be applied to the flow of a canal, the word "streamflow" uniquely describes the discharge in a surface stream course. The term "streamflow" is more general than "runoff" as a streamflow may be applied to discharge whether or not it is affected by diversion or regulation.

Substrate is the physical surface upon which an organism lived.

Natural substrates refers to any naturally occurring emersed or submersed solid surface, such as a rock or tree, upon which an organism lived.

Artificial substrate is a device which is purposely placed in a stream or lake for colonization of organisms. The artificial substrate simplifies the community structure by standardizing the substrate from which each sample is taken. Examples of artificial substrates are basket samplers (made of wire cages filled with clean streamside rocks) and miltiplate samplers (made of hardboard) for benthic organism collection, and plexiglass strips for periphyton collection.

Surface area of a lake is that area outlined on the latest USGS topographic map as the boundary of the lake and measured by a planimeter in acres. In localities not covered by topographic maps, the areas are computed from the best maps available at the time planimetered. All areas shown are those for the stage when the 
planimetered map was made. All areas shown are those for the stage when the planimetered map was made.

Surficial bed material is that part $(0.1$ to $0.2 \mathrm{ft})$ of the bed material that is sampled using U.S. Series Bed-Material Samplers.

Suspended (as used in tables of chemical analyses) refers to the amount (concentration) of the total concentration in a watersediment mixture. The water-sediment mixture is associated with (or sorbed on) that material retained on a 0.45 micrometer filter.

Suspended, recoverable is the amount of a given constituent that is in solution after the part of a representative water-suspended sediment sample that is retained on a 0.45 micrometer filter has been digested by a method (usually using a dilute acid solution) that results in dissolution of only readily soluble substances. Complete dissolution of all the particulate matter is not achieved by the digestion treatment and thus the determination represents something less than the "total" amount (that is, less than 95 percent) of the constituent present in the sample. To achieve comparability of analytical data, equivalent digestion procedures would be required of all laboratories performing such analyses because different digestion procedures are likely to produce different analytical results.

Determinations of "suspended, recoverable" constituents are made either by analyzing portions of the material collected on the filter or, more commonly, by difference, based on determinations of (1) dissolved and (2) total recoverable concentrations of the constituent.

Suspended, total is the total amount of a given constituent in the part of a representative water-suspended sediment sample that is retained on a $0.45 \mathrm{um}$ membrane filter. This term is used only when the analytical procedure assures measurement of at least 95 percent of the constituent determined. A knowledge of the expected form of the constituent in the sample, as well as the analytical methodology used, is required to determine when the results should be reported as "suspended, total."

Determinations of "suspended, total" constituents are made either by analyzing portions of the material collected on the filter or, more commonly, by difference, based on determinations of (1) dissolved and (2) total concentrations of the constituent.

Taxonomy is the division of biology concerned with the classification and naming of organisms. The classification of organisms is based upon a hierarchical scheme beginning with Kingdom and ending with Species at the base. The higher the classification level, the fewer features the organisms have in common.

For example, the taxonomy of a particular mayfly, Hexagenia limbata is the following:

Kingdom..................Animal
Phylum................ Irthropoda
Class...................nsects
Order............Ephemeroptera
Family.............Ephermeridae
Genus................ Hexageria
Species....... Hexagenia limbata

Thermograph is an instrument that continuously records variations of temperature on a chart. The more general term "temperature recorder" is used in the table headings and refers to any instrument that records temperature whether on a chart, a tape, or any other medium.

Time-weighted average is computed by multiplying the number of days in the sampling period by the concentrations of individual constituents for the corresponding period and dividing the sum of the products by the total number of days. A time-weighted average represents the composition of water that would be contained in a vessel or reservoir that had received equal quantities of water from the stream each day for the year.
Tons per acre-foot indicates the dry mass of dissolved solids in 1 acre-foot of water. It is computed by multiplying the concentration in milligrams per liter by 0.00136 .

Tons per day is the quantity of substance in solution or suspension that passes a stream section during a 24-hour day.

Total is the total amount of a given constituent in a representative water-suspended sediment sample, regardless of the constituent's physical or chemical form. This term is used only when the analytical procedure assures measurement of at least 95 percent of the constituent present in both the dissolved and suspended phases of the sample. A knowledge of the expected form of the constituent in the sample, as well as the analytical methodology used, is required to judge when the results should be reported as "total." (Note that the word "total" does double duty here, indicating both that the sample consists of a water-suspended sediment mixture and that the analytical method determines all of the constituent in the sample.)

Total in bottom material is the total a mount of a given constituent in a representative sample of bottom material. This term is used only when the analytical procedure assures measurement of at least 95 percent of the constituent determined. A knowledge of the expected form of the constituent in the sample, as well as the analytical methodology used, is required to judge when the results should be reported as "total in bottom material."

Total load (tons) is the total quantity of any individual constituent, as measured by dry mass or volume, that is dissolved in a specific amount of water (discharge) during a given time. It is computed by multiplying the total discharge, times the $\mathrm{mg} / \mathrm{L}$ of the constituent, times the factor 0.0027 , times the number of days.

Total recoverable refers to the amount of a given constituent that is in solution after a representative water-suspended sediment sample has been digested by a method (usually using a dilute acid solution) that results in dissolution of only readily soluble substances. Complete dissolution of all particulate matter is not achieved by the digestion treatment, and thus the determination represents something less than the "total" amount (that is, less than 95 percent) of the constituent percent in the dissolved and suspended phases of the sample. To achieve comparability of analytical data, equivalent digestion procedures would be required of all laboratories performing such analyses because different digestion procedures are likely to produce different analytical results.

Tritium Network is a network of stations which has been established to provide baseline information on the occurrence of tritium in the Nation's surface waters. In addition to the surfacewater stations in the network, tritium data are also obtained at a number of precipitation stations. The purpose of the precipitation stations is to provide an estimate sufficient for hydrologic studies of the tritium input to the United States.

Water year in Geological Survey reports dealing with surfacewater supply is the 12-month period, October 1 through September 30. The water year is designated by the calendar year in which it ends and which includes 9 of the 12 months. Thus, the year ending September 30,1991, is called the "1991 water year."

WDR is used as an abbreviation for "Water-Data Report" in reference to published reports beginning in 1975 .

Weighted average is used in this report to indicate dischargeweighted average. It is computed by multiplying the discharge for a sampling period by the concentrations of individual constituents for the corresponding period and dividing the sum of the products by the sum of the discharges. A discharge- weighted average approximates the composition of water that would be found in a reservoir containing all the water passing a given location during the water year after thorough mixing in the reservoir.

WRD is used as an abbreviation for "Water-Resources Data" 
in the REVISED RECORDS paragraph to refer to State annual basic-data reports published before 1975 .
WSP is used as an abbreviation for "Water-Supply Paper" in references to previously published reports. 


\section{PUBLICATIONS ON TECHNIQUES OF WATER-RESOURCES INVESTIGATIONS}

The U.S. Geological Survey publishes a series of manuals describing procedures for planning and conducting specialized work in water-resources investigations. The material is grouped under major subject headings called books and is further divided into sections and chapters. For example, Section A of Book 3 (Applications of Hydraulics) pertains to surface water. The chapter, the unit of publication, is limited to a narrow field of subject matter. This format permits flexibility in revision and publication as the need arises.

The reports listed below are for sale by the U.S. Geological Survey, Books and Open-File Reports Section, Federal Center, Box 25425 , Denver, Colorado 80225 (authorized agent of the Superintendent of Documents, Government Printing Office). Prepayment is required. Remittance should be sent by check or money order payable to the U.S. Geological Survey. Prices are not included because they are subject to change. Current prices can be obtained by writing to the above address. When ordering or inquiring about prices for any of these publications, please give the title, book number, chapter number, and "U.S. Geological Survey Techniques of Water-Resources Investigations."

1-D1. Water temperature--influential factors, field measurement, and data presentation, by H. H. Stevens, Jr., J. F. Ficke, and G. F. Smoot: USGS--TWRI Book 1, Chapter D1. 1975. 65 pages.

1-D2. Guidelines for collection and field analysis of ground-water samples for selected unstable constituents, by W. W. Wood: USGS-TWRI Book 1, Chapter D2. 1976. 24 pages.

2-D1. Application of surface geophysics to ground-water investigations, by A. A. R. Zohdy, G. P. Eaton, and D. R. Mabey: USGS-TWRI Book 2, Chapter D1. 1974. 116 pages.

2-D2. Application of seismic-refraction techniques to hydrologic studies, by F. P. Haeni: USGS--TWRI Book 2, Chapter D2. 1988.86 pages.

2-E1. Application of borehole geophysics to water-resources investigations, by W. S. Keys and L. M. McCary: USGS-TWRI Book 2, Chapter E1. 1971. 126 pages.

2-E2. Borehole geophysics applied to ground-water investigations, by W. Scott Keys: USGS--TWRI Book 2, Chapter E2. 1990. 150 pages.

2-F1. Application of drilling, coring, and sampling techniques to test holes and wells, by Eugene Shuter and Warren E. Teasdale: USGS--TWRI Book'2, Chapter F1. 1989. 97 pages.

3-A1. General field and office procedures for indirect discharge measurements, by M. A. Benson and Tate Dalrymple: USGS--TWRI Book 3, Chapter A1. 1967. 30 pages.

3-A2. Measurement of peak discharge by the slope-area method, by Tate Dalrymple and M. A. Benson: USGS-- TWRI Book 3, Chapter A2. 1967. 12 pages.

3-A3. Measurement of peak discharge at culverts by indirect methods, by G. L. Bodhaine: USGS--TWRI Book 3, Chapter A3. 1968. 60 pages.

3-A4. Measurement of peak discharge at width contractions by idirect methods, by H. F. Matthai: USGS-TWRI Book 3, Chapter A4. 1967. 44 pages.

3-A5. Measurement of peak discharge at dams by indirect methods, by Harry Hulsing: USGS--TWRI Book 3, Chapter A5. 1967. 29 pages.

3-A6. General procedure for gaging streams, by R. W. Carter and Jacob Davidian: USGS--TWRI Book 3, Chapter A6. 1968. 13 pages.

3-A7. Stage measurements at gaging stations, by T. J. Buchanan and W. P. Somers: USGS--TWRI Book 3. Chapter A7. 1968. 28 pages.

3-A8. Discharge measurements at gaging stations, by T. J. Buchanan and W. P. Somers: USGS--TWRI Book 3, Chapter A8. 1969. 65 pages.

3-A9. Measurement of time of travel in streams by dye tracing, by F. A. Kilpatrick and J. F. Wilson, Jr.: USGS--TWRI Book 3, Chapter A9. 1989. 27 pages.

3-Al0. Discharge ratings at gaging stations, by E. J. Kennedy: USGS--TWRI Book 3, Chapter A 10. 1984. 59 pages. 
3-A11. Measurement of discharge by moving-boat method, by G. F. Smoot and C. E. Novak: USGS--TWRI Book 3, Chapter A11. 1969. 22 pages.

3-A12. Fluorometric procedures for dye tracing, by J. F. Wilson, Jr., E. D. Cobb, and F. A. Kilpatrick: USGS- -TWRI Book 3, Chapter A12. 1986. 41 pages.

3-A13. Computation of continuous records of streamflow, by E. J. Kennedy: USGS--TWRI Book 3, Chapter A13. 1983. 53 pages.

3-A14. Use of flumes in measuring discharge, by F. A. Kilpatrick and V. R. Schneider: USGS--TWRI Book 3, Chapter A14. 1983. 46 pages.

3-A15. Computation of water-surface profiles in open channels, by Jacob Davidian: USGS--TWRI Book 3, Chapter A15. 1984. 48 pages.

3-A16. Measurement of discharge using tracers, by F. A. Kilpatrick and E. D. Cobb: USGS-TWRI Book 3, Chapter A16. 1985. 52 pages.

3-A17. Acoustic velocity meter systems, by Antonius Laenen: USGS--TWRI Book 3, Chapter A17. 1985. 38 pages.

3-A18. Determination of stream reaeration coefficients by use of tracers, by F. A. Kilpatrick, R. E. Rathburn, N. Yotsukura, G. W. Parker, and L. L. DeLong: USGS--TWRI Book 3, Chapter A18. 1989. 52 pages.

3-A19. Levels of streamflow gaging stations, by E.J. Kennedy: USGS-TWRI Book 3, Chapter A19. 1990. 27 pages.

3-B1. Aquifer-test design, observation, and data analysis, by R. W. Stallman: USGS--TWRI Book 3, Chapter B1. 1971. 26 pages.

3-B2. Introduction to ground-water hydraulics, a programmed text for self-instruction, by G. D. Bennett: USGS-- TWRI Book 3, Chapter B2. 1976. 172 pages.

3-B3. Type curves for selected problems of flow to wells in confined aquifers, by J. E. Reed: USGS--TWRI Book 3, Chapter B3. 1980. 106 pages.

3-B4. Regression modeling of ground-water flow, by Richard L. Cooley and Richard L. Naff: USGS-TWRI Book 3, Chapter B4. 1990. 232 pages.

3-B5. Definition of boundary and initial conditions in the analysis of saturated ground-water flow systems--An introduction, by O. L. Franke, T. E. Reilly, and G. D. Bennett: USGS--TWRI Book 3, Chapter B5. 1987. 15 pages.

3-B6. The principle of superposition and its application in ground-water hydraulics, by T. E. Reilly, O. L. Franke, and G. D. Bennett: USGS--TWRI Book 3, Chapter B6. 1987. 28 pages.

3-C1. Fluvial sediment concepts, by H. P. Guy: USGS--TWRI Book 3, Chapter C1. 1970. 55 pages.

3-C2. Field methods for measurement of fluvial sediment, by H. P. Guy and V. W. Norman: USGS--TWRI Book 3, Chapter C2. 1970. 59 pages.

3-C3. Computation of fluvial-sediment discharge, by George Porterfield: USGS--TWRI Book 3, Chapter C3. 1972.66 pages.

4-A1. Some statistical tools in hydrology, by H. C. Riggs: USGS--TWRI Book 4, Chapter A1. 1968. 39 pages.

4-A2. Frequency curves, by H. C. Riggs: USGS--TWRI Book 4, Chapter A2. 1968. 15 pages.

4-B1. Low-flow investigations, by H. C. Riggs: USGS--TWRI Book 4, Chapter B1. 1972. 18 pages

4-B2. Storage analyses for water supply, by H. C. Riggs and C. H. Hardison: USGS--TWRI Book 4, Chapter B2. 1973.20 pages.

4-B3. Regional analyses of streamflow characteristics, by H. C. Riggs: USGS--TWRI Book 4, Chapter B3. 1973.15 pages.

4-D1. Computation of rate and volume of stream depletion by wells, by C. T. Jenkins: USGS--TWRI Book 4, Chapter D1. 1970.17 pages.

5-A1. Methods for determination of inorganic substances in water and fluvial sediments, by M. J. Fishman and L. C. Friedman: USGS--TWRI Book 5, Chapter A1. 1989. 545 pages. 


\section{PUBLICATIONS ON TECHNIQUES OF WATER-RESOURCES INVESTIGATIONS-Continued}

5-A2. Determination of minor elements in water by emission spectroscopy, by P. R. Barnett and E. C. Mallory, Jr.: USGS--TWRI Book 5. Chapter A2. 1971. 31 pages.

5-A3. Methods for the determination of organic substances in water and fluvial sediments, edited by R. L. Wershaw, M. J. Fishman, R. R. Grabbe, and L. E. Lowe: USGS--TWRI Book 5, Chapter A3. 1987. 80 pages.

5-A4. Methods for collection and analysis of aquatic biological and microbiological samples, by L. J. Britton and P. E. Greeson, editors: USGS-TWRI Book 5, Chapter A4. 1989. 363 pages.

5-A5. Methods for determination of radioactive substances in water and fluvial sediments, by L. L. Thatcher, V. J. Janzer, and K. W. Edwards: USGS--TWRI Book 5, Chapter A5. 1977. 95 pages.

5-A6. Quality assurance practices for the chemical and biological analyses of water and fluvial sediments, by $L$. C. Friedman and D. E. Erdmann: USGS--TWRI Book 5, Chapter A6. 1982. 181 pages.

5-C1. Laboratory theory and methods for sediment analysis, by H. P. Guy: USGS-TWRI Book 5, Chapter C1. 1969. 58 pages.

6-A1. A modular three-dimensional finite-difference ground-water flow model, by M. G. McDonald and A. W. Harbaugh: USGS--TWRI Book 6, Chapter A1. 1988. 586 pages.

7-C1. Finite difference model for aquifer simulation in two dimensions with results of numerical experiments, by P. C. Trescott, G. F. Pinder, and S. P. Larson: USGS--TWRI Book 7, Chapter C1. 1976. 116 pages.

7-C2. Computer model of two-dimensional solute transport and dispersion in ground water, by L. F. Konikow and J. D. Bredehoeft: USGS--TWRI Book 7, Chapter C2. 1978. 90 pages.

7-C3. A model for simulation of flow in singular and interconnected channels, by R. W. Schaffrannek, R. A. Baltzer, and D. E. Goldberg: USGS--TWRI Book 7, Chapter C3. 1981. 110 pages.

8-A1. Methods of measuring water levels in deep wells, by M. S. Garber and F. C. Koopman: USGS-TWRI Book 8, Chapter A1. 1968. 23 pages.

8-A2. Installation and service manual for U.S. Geological Survey manometers, by J. D. Craig: USGS--TWRI Book 8, Chapter A2. 1983. 57 pages.

8-B2. Calibration and maintenance of vertical-axis type current meters, by G. F. Smoot and C. E. Novak: USGS--TWRI Book 8, Chapter B2. 1968. 15 pages. 


\section{Surface-water Station Records}

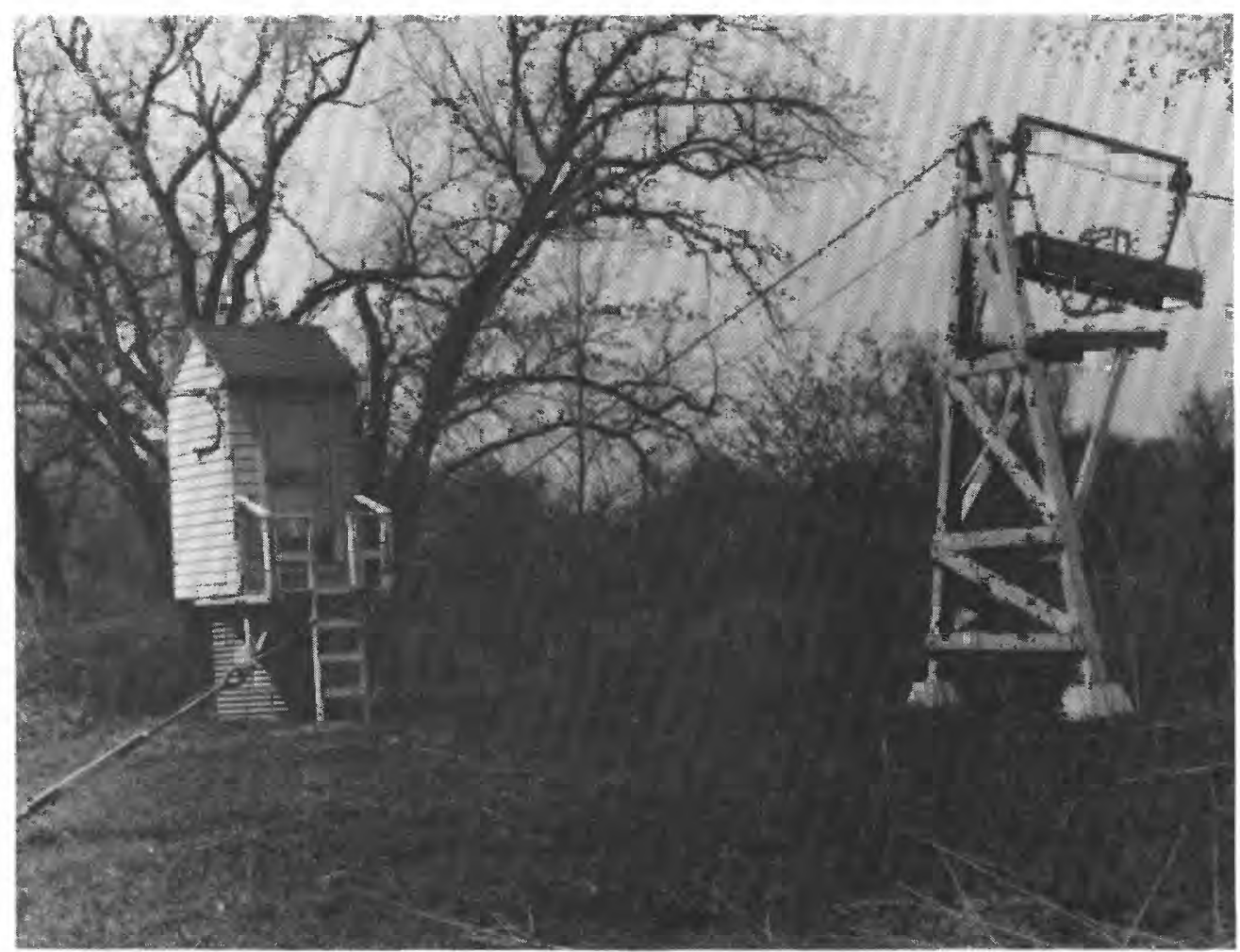

South Fork Zumbro River near Rochester May 8, 1958 


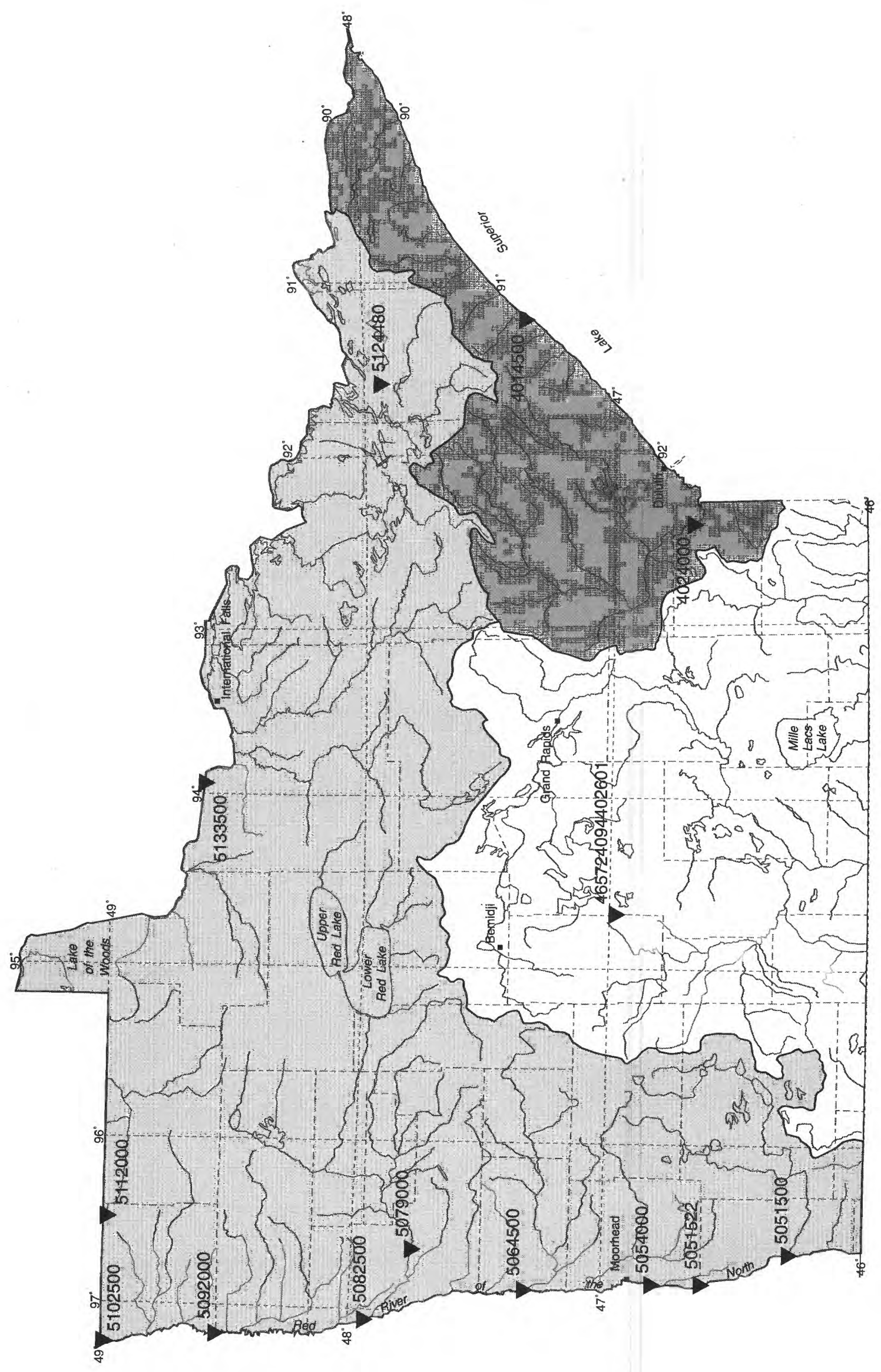



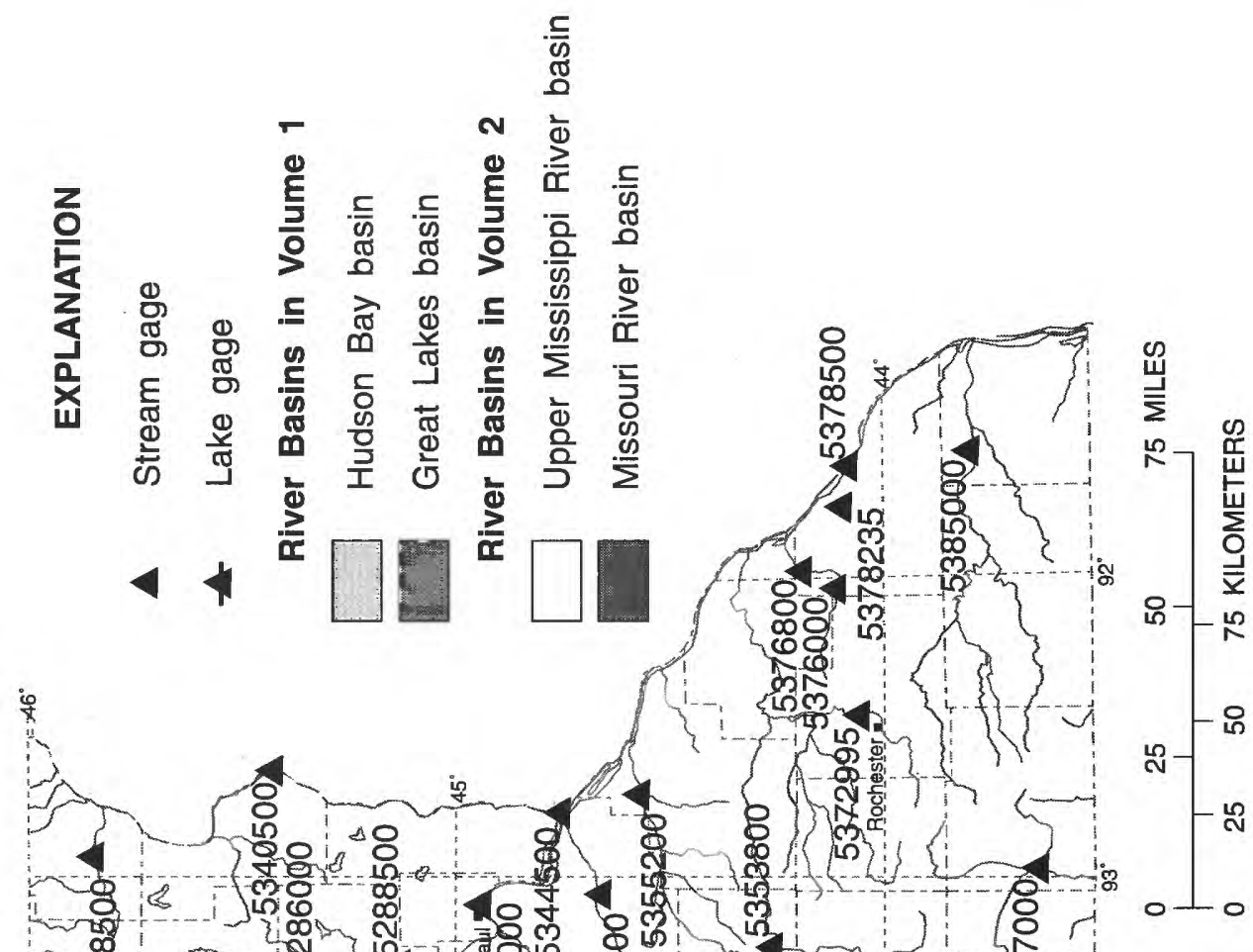

들
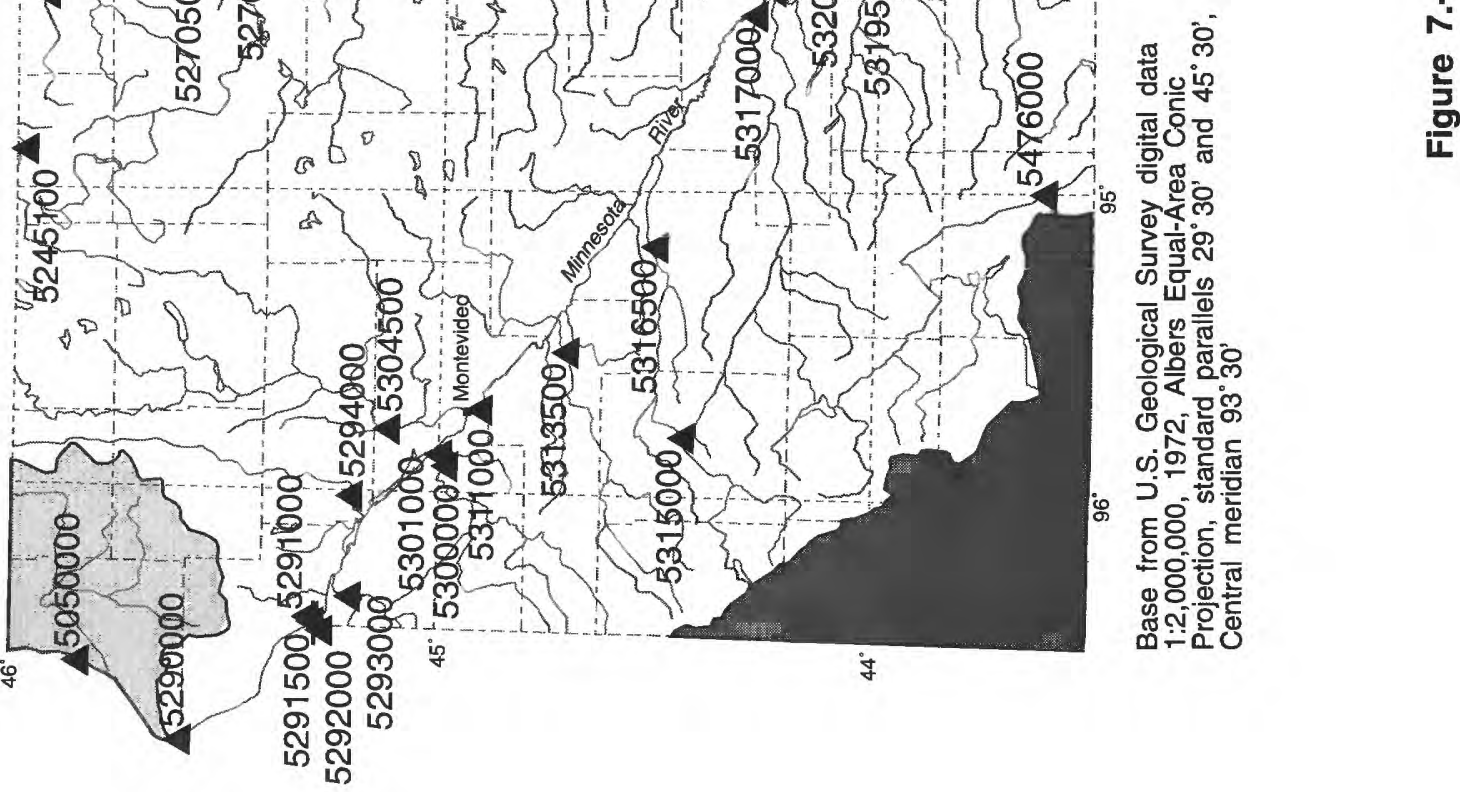


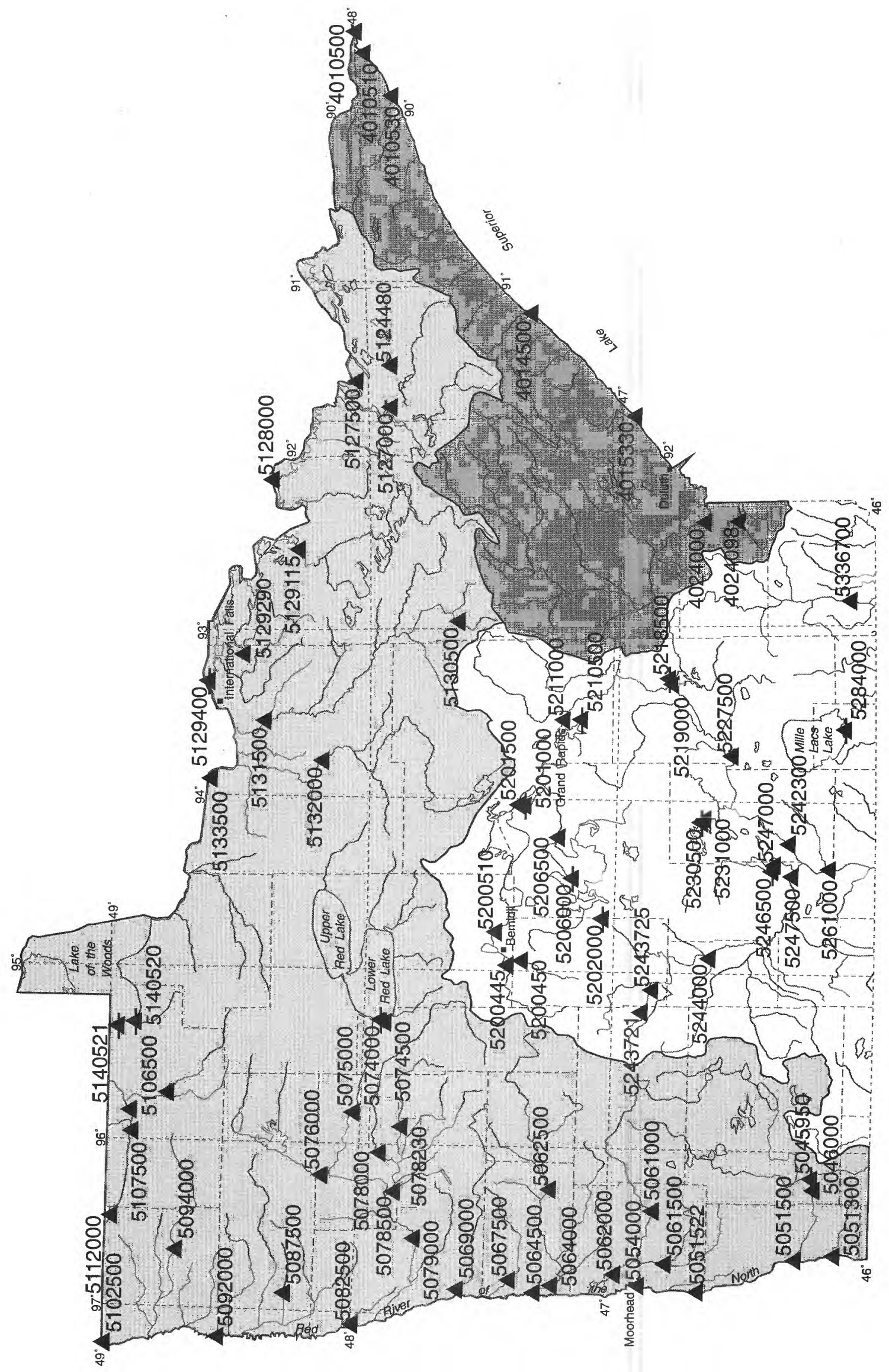



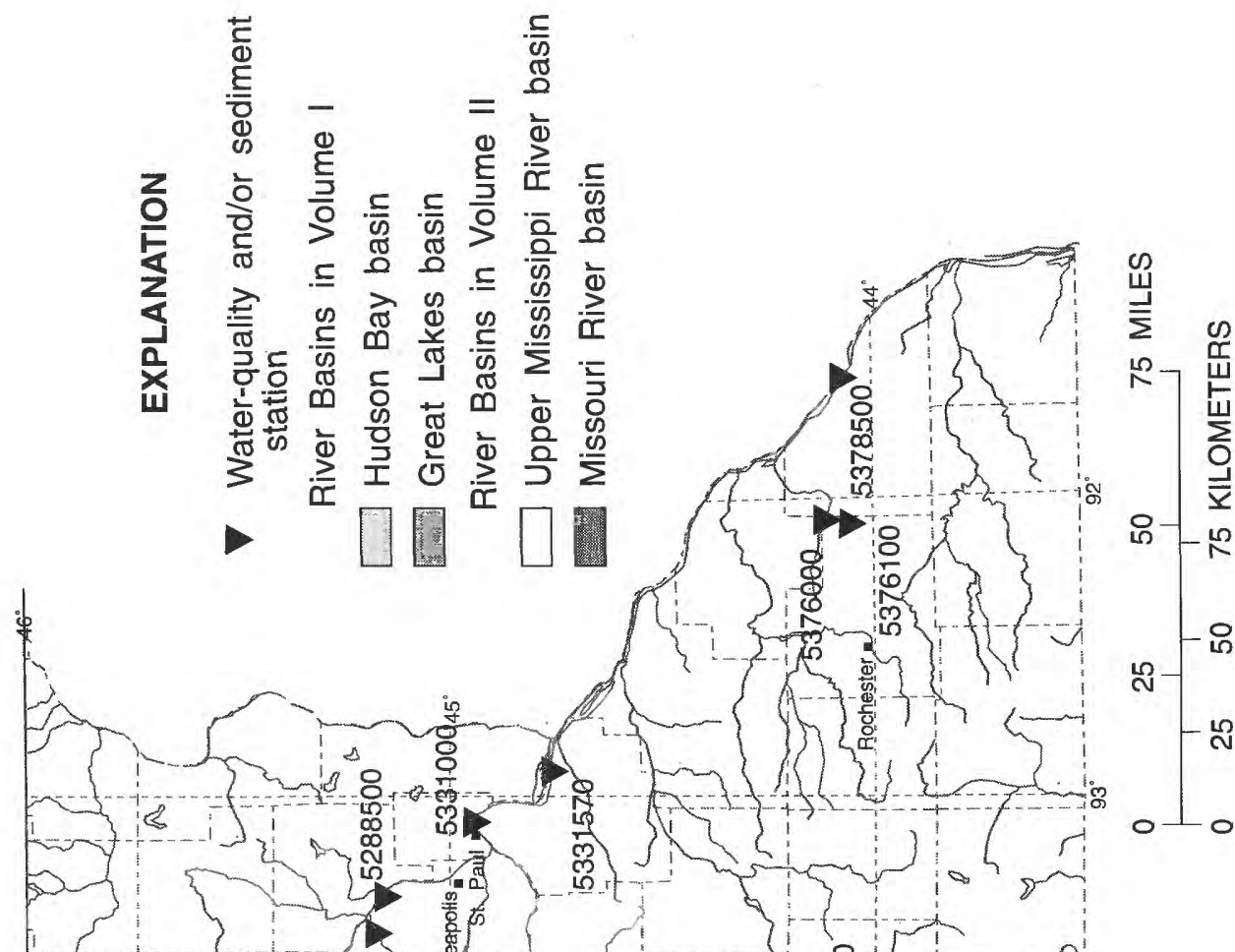

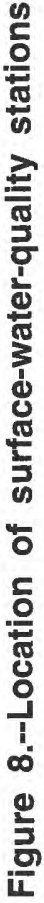

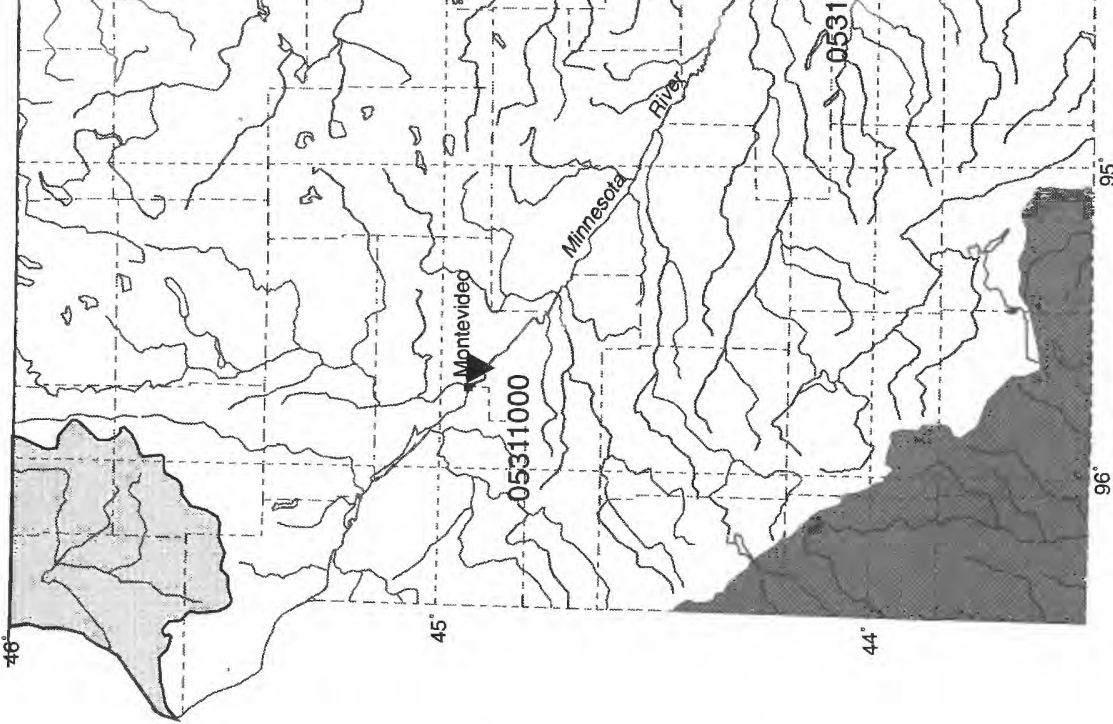

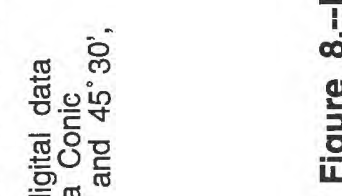


LOCATION.--Lat $47^{\circ} 29^{\prime} 00^{\prime \prime}$, long $94^{\circ} 43^{\prime} 40^{\prime \prime}$, in SEzSW sec.3, T.146 N., R. 32 W., Beltrami County, Hydrologic Unit $07010101,3.5 \mathrm{mi}$ east of Bemidji on right bank $100 \mathrm{ft}$ upstream of County Highway 12 and $400 \mathrm{ft}$ downstream from Stump Lake dam.

DRAINAGE AREA. --610 $\mathrm{mi}^{2}$, approximately.

PERIOD OF RECORD.--September 1987 to current year (no winter records).

GAGE.--Water-stage recorder. Elevation of gage is 1,315 ft above National Geodetic Vertical Datum of 1929, from topographic map.

REMARKS. --Records good except those for estimated daily discharges, which are poor. Some regulation by Stump Lake dam upstream from station.

EXTREMES FOR CURRENT YEAR.--Maximum discharge during period October, March to September, $773 \mathrm{ft}^{3} / \mathrm{s}, \mathrm{May} 17,8 \mathrm{~g}$, height, $4.58 \mathrm{ft}$; minimum, $34 \mathrm{ft}^{3} / \mathrm{s}$, Oct. 16,8 gag height, $2.27 \mathrm{ft}$.

DISCHARGE, CUBIC FEET PER SECOND, WATER YEAR OCTOBER 1990 TO SEPTEMBER 1991

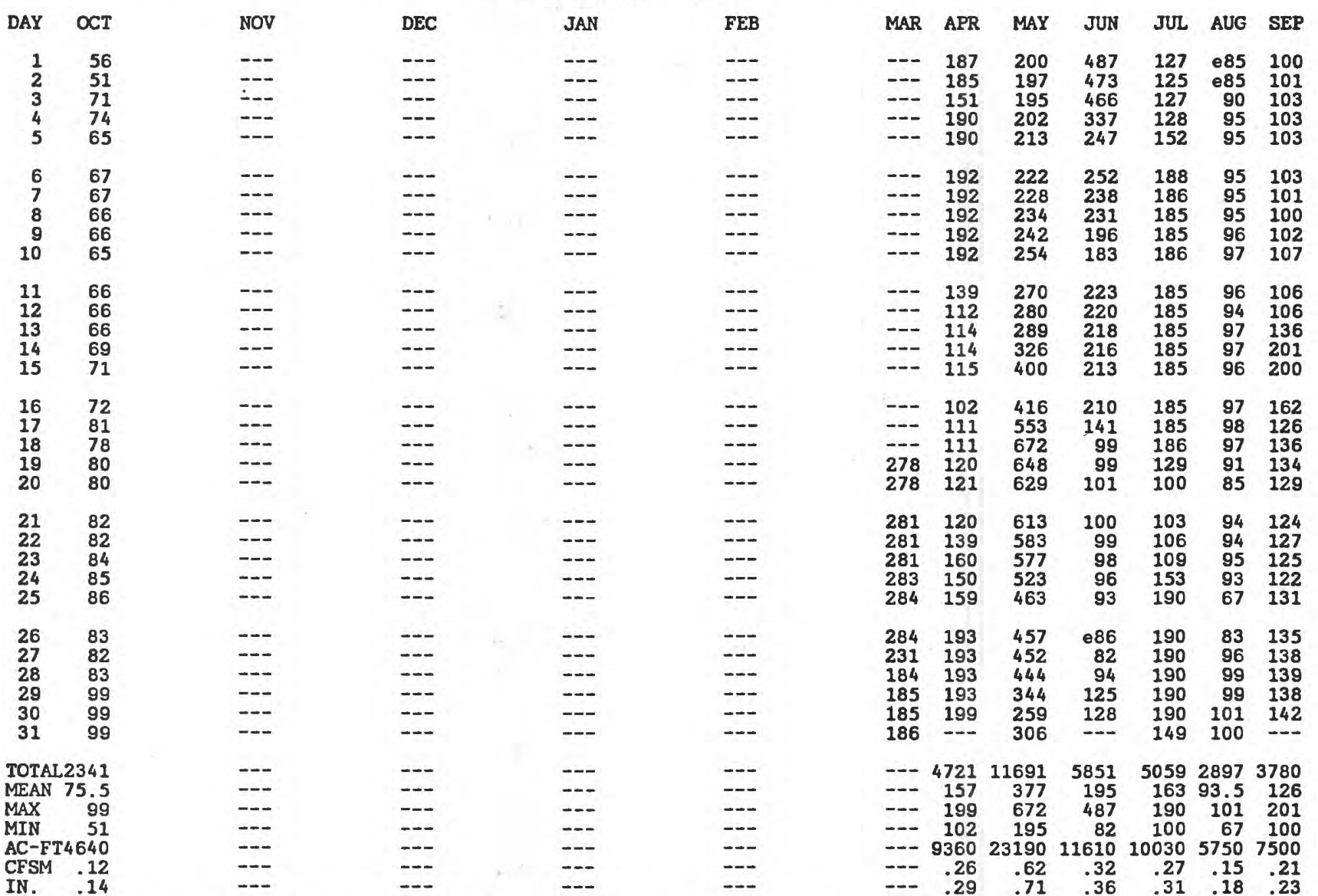

STATISTICS OF MONTHLY MEAN DATA FOR WATER YEARS 1987 - 1991, BY WATER YEAR (WY)

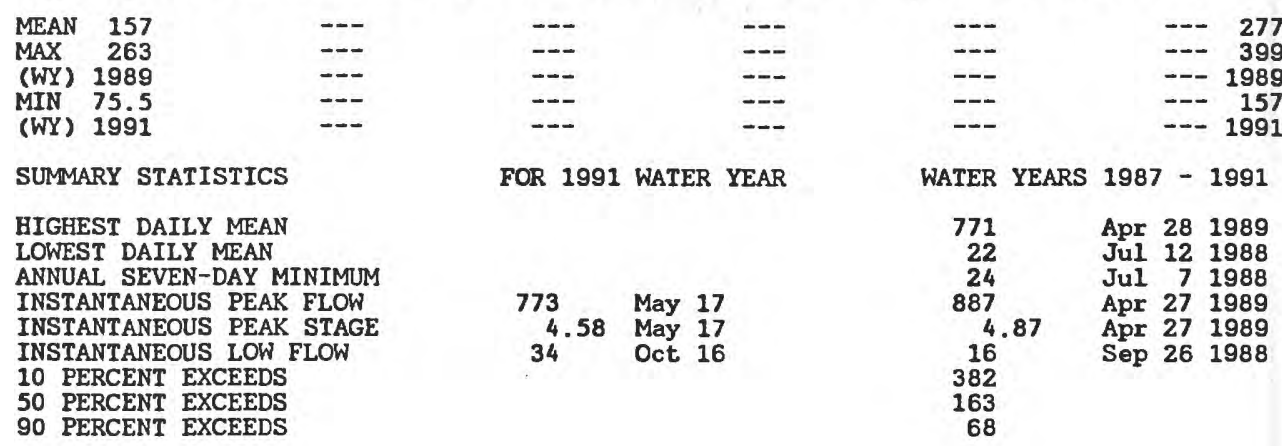

- Estimated 


\section{UPPER MISSISSIPPI RIVER BASIN}

\section{MISSISSIPPI RIVER MAIN STEM}

05201000 WINNIBIGOSHISH LAKE NEAR DEER RIVER, MN

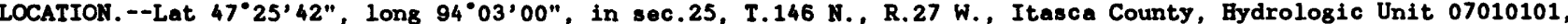
on Leech Lake Indian Reservation, at dam on Mississippi River, 1 mi northwest of Little Winnibigoshish Lake, $14 \mathrm{mi}$ northwest of city of Deer River, and at mile 1,248 upstream from Ohio River.

DRAINAGE AREA. - - 1,442 $\mathrm{mi}^{2}$.

PERIOD OF RECORD.--April 1884 to current year. Prior to October 1941 monthend contents only, published in wSP 1308. Published as Winnibigoshish Reservoir near Deer River October 1941 to September 1956.

REVISED RECORDS. --WSP 1308: 1905(M).

GAGE.--Water-stage recorder. Datum of gage is National Geodetic Vertical Datum of 1929 (levels by U.S. Army Corps of Engineers). Prior to July 8, 1949, nonrecording gage at same site, and July 9, 1949, to July 10, 1973, pater-stage recorder at same site and at datum of $1,288.94 \mathrm{ft}$ above National Geodetic Vertical Datum of 1929.

REMARKS.--Reservoir is formed by Winnibigoshish Lake and several other natural lakes controlled by a concrete and timber dam, completed in 1884; storage began in 1884. Capacity between elevations 1,294.94 $\mathrm{ft}$ and $1,303.14 \mathrm{ft}$ (maximum ailowable range) is 668,737 acre-ft of which 439,636 acre-ft is controlled storage between elevations $1,294.94 \mathrm{ft}$ and $1,300.94 \mathrm{ft}$ (normal operating range). Contents shown herein are contents above elevation $1,286.00 \mathrm{ft}$. Prior to September 1978, published contents as contents above elevation 1,288.94 ft. Water is used to benefit navigation on Mississippi River below Minneapolis.

COOPERATION. --Records were provided by U.S. Army Corps of Engineers.

EXTREMES FOR PERIOD OF RECORD,--Maximum contents observed, 996,500 acre-ft, capacity table then in use, July 30, 1905 levation, $1,303.39 \mathrm{ft}$; minimum observed, 33,680 acre-ft, below zero of capacity table then in use, Oct. 20, 1931, levation, 1,288.25 ft.

EXTREMES FOR CURRENT YEAR. --Maximum contents, 720,870 acre-ft, June 2, elevation, 1,298.51 ft; minimum, 616,510 acre-ft, Nov. 8, elevation, 1,296,92 ft.

MONTHEND ELEVATION AND CONTENTS, WATER YEAR OCTOBER 1990 TO SEPTEMBER 1991

$$
\text { Date }
$$

Sept. 30

Oct. 31

Nov. 30

Dec. 31

CAL YR 1990

Jan. 31

Feb. 28

Mar. 31

Apr. 30

May 31

June 30

July

Aug. 31

Sept. 30

WTR YR 1991

\section{Elevation (feet)}

$1,297.42$

$1,297.16$

$1,297.02$

$1,297.21$

, 297.32

$1,297.43$

$1,297.53$

$1,298.03$

$1,298.47$

$1,298.14$

$1,298.22$

$1,297.92$

$1,298.03$
Contents (acre-feet)

649,300

632,250

623,050

635,520

642,730

649,960

656,520

689,360

718,240

696,580

701,830

682,130

689,360
Change in contents (acre-feet)

$$
\begin{array}{r}
-17,050 \\
-9,190 \\
+12,470
\end{array}
$$$$
-2,590
$$$$
+7,210
$$$$
+7,220
$$$$
+6,560
$$$$
+32,830
$$$$
+28,880
$$$$
-21,660
$$$$
+5,250
$$$$
-19,690
$$$$
+7,220
$$ 
MISSISSIPPI RIVER MAIN STEM

05201500 MISSISSIPPI RIVER AT WINNIBIGOSHISH DAM NEAR DEER RIVER, MN

LOCATION, --Lat $47^{\circ} 25^{\prime} 42^{\prime \prime}$, long $94^{\circ} 03^{\prime} 00^{\prime \prime}$, in SWł sec.25, T.146 N., R.27 W. Itasca County, Hydrologic Unit 07010101 , on Leech Lake Indian Reservation, at dam $1 \mathrm{mi}$ northwest of Little Winnibigoshish Lake, $14 \mathrm{mi}$ northwest of city of Deer River, and at mile 1,248 upstream from Ohio River.

DRAINAGE AREA. $--1,442 \mathrm{mi}^{2}$.

PERIOD OF RECORD.--May 1884 to current year. Monthly discharge only for some periods, published in WSP 1308.

GAGE. - Water-stage recorder on headwater and nonrecording gage on tailwater. Datum of gage is National Geodetic Vertical Datum of 1929 (levels by U. S. Army Corps of Engineers). Prior to June 30, 1973, gages at same sites with datum at $1,289.47 \mathrm{ft}$, adjustment of 1912. Prior to July 8, 1949, nonrecording headwater gage at same site and datum in use.

REMARKS.--Daily discharge is computed on the basis of modified weir formula and corrected to conform with discharge measurements, the head being determined from readings of headwater and tailwater gages. Flow completely regulated by Winnibigoshish Lake (station 05201000).

COOPERATION. --Daily discharge computed by U. S. Army Corps of Engineers.

AVERAGE DISCHARGE (unadjusted). --107 years, $518 \mathrm{ft}^{3} / \mathrm{s}, 4.88 \mathrm{in} / \mathrm{yr}$.

EXTREMES FOR PERIOD OF RECORD.--Maximum daily discharge, 4,370 $\mathrm{ft}^{3} / \mathrm{s}$, Aug. 6, 1905; no flow at times in several years.

EXTREMES FOR CURRENT YEAR.--Maximum daily discharge, $700 \mathrm{ft}^{3} / \mathrm{s}$, Oct. 23 to Nov. 7; minimum daily, $101 \mathrm{ft}^{3} / \mathrm{s}$, Apr. 4 to 23, June 28 to July 1. DISCHARGE, CUBIC FEET PER SECOND, WATER YEAR OCTOBER 1990 TO SEPTEMBER 1991

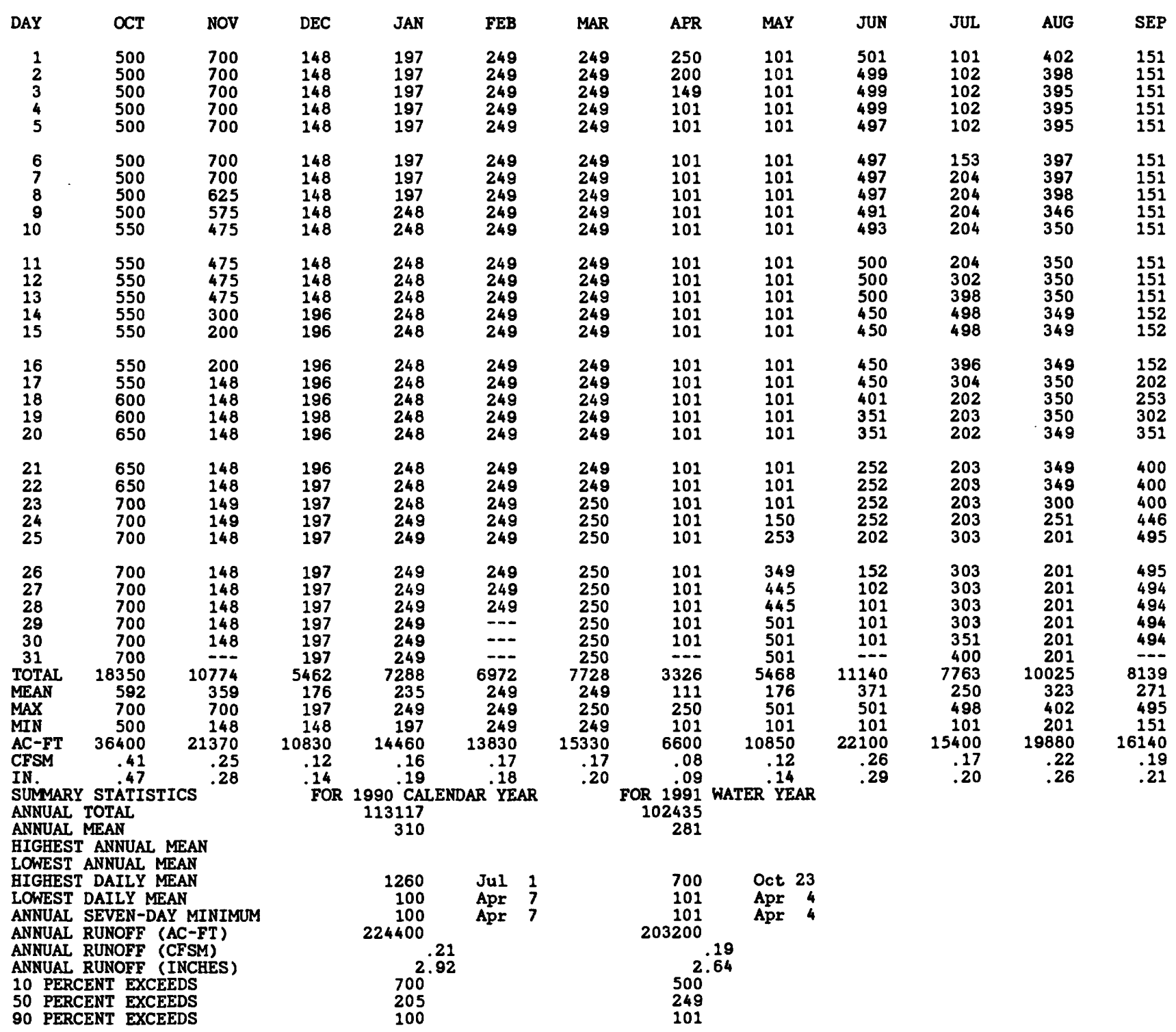


LEECH LAKE RIVER BASIN

05202000 WILLIAMS LAKE NEAR AKELEY, MN

LOCATION.--Lat $46^{\circ} 57^{\prime} 24^{\prime \prime}$, long $94^{\circ} 40^{\prime} 26^{\prime \prime}$, in SE ${ }^{\prime} \mathrm{NW} z$ sec.12, T.140 N., R.32 W., Hubbard County, Hydrologic Unit 07010102 , on northwest shore of Williams lake, $4 \mathrm{mi}$ southeast of Akeley.

DRAINAGE AREA- $-0.88 \mathrm{mi}^{2}$.

\section{GAGE-HEIGHT RECORDS}

PERIOD OF RECORD--October 1988 to current year. August 1977 to September 1988, in files of the U.S. Geological Survey's Hydrology of Lakes Section in Denver, Colorado.

GAGE--Water-stage recorder. Datum of gage is $1,379.09 \mathrm{ft}$ above National Geodetic Vertical datum of 1929 . Prior to Oct. 1, 1990, at datum $2.00 \mathrm{ft}$ higher. EXTREMES FOR PERIOD OF RECORD--Maximum gage height, $3.33 \mathrm{ft}$, present datum, June 25, $1989 ;$ minimum, $1.72 \mathrm{ft}$,
Sept. 5, 1991 .

EXTREMES FOR CURRENT YEAR--Maximum gage height, $2.53 \mathrm{ft}$, May 13, 24, 25; minimum, $1.72 \mathrm{ft}$, Sept. 5.

GAGE HEIGHT, FEET, WATER YEAR OCTOBER 1990 TO SEPTEMBER 1991

DAILY MEAN VALUES

\begin{tabular}{|c|c|c|c|c|c|c|c|c|c|c|c|c|}
\hline DAY & $\infty \mathrm{CT}$ & NOV & DEC & JAN & FEB & MAR & APR & MAY & JUN & JUL & AUG & SEP \\
\hline $\begin{array}{l}1 \\
2 \\
3 \\
4 \\
5\end{array}$ & $\begin{array}{l}2.08 \\
2.07 \\
2.18 \\
2.20 \\
2.18\end{array}$ & $\begin{array}{l}2.18 \\
2.16 \\
2.16 \\
2.15 \\
2.15\end{array}$ & $\begin{array}{l}2.04 \\
2.04 \\
2.05 \\
2.06 \\
2.05\end{array}$ & $\begin{array}{l}2.09 \\
2.09 \\
2.10 \\
2.09 \\
2.09\end{array}$ & $\begin{array}{l}2.12 \\
2.13 \\
2.14 \\
2.14 \\
2.13\end{array}$ & $\begin{array}{l}2.24 \\
2.26 \\
2.25 \\
2.26 \\
2.27\end{array}$ & $\begin{array}{l}2.23 \\
2.24 \\
2.24 \\
2.25 \\
2.24\end{array}$ & $\begin{array}{l}2.42 \\
2.42 \\
2.41 \\
2.45 \\
2.46\end{array}$ & $\begin{array}{l}2.48 \\
2.48 \\
2.47 \\
2.45 \\
2.43\end{array}$ & $\begin{array}{l}2.34 \\
2.32 \\
2.33 \\
2.35 \\
2.33\end{array}$ & $\begin{array}{l}2.06 \\
2.05 \\
2.05 \\
2.07 \\
2.05\end{array}$ & $\begin{array}{l}1.83 \\
1.81 \\
1.84 \\
1.77 \\
1.73\end{array}$ \\
\hline $\begin{array}{r}6 \\
7 \\
8 \\
9 \\
10\end{array}$ & $\begin{array}{l}2.16 \\
2.15 \\
2.14 \\
2.13 \\
2.12\end{array}$ & $\begin{array}{l}2.14 \\
2.13 \\
2.11 \\
2.12 \\
2.12\end{array}$ & $\begin{array}{l}2.04 \\
2.04 \\
2.03 \\
2.03 \\
2.04\end{array}$ & $\begin{array}{l}2.09 \\
2.09 \\
2.10 \\
2.10 \\
2.10\end{array}$ & $\begin{array}{l}2.13 \\
2.12 \\
2.11 \\
2.11 \\
2.11\end{array}$ & $\begin{array}{l}2.26 \\
2.24 \\
2.21 \\
2.20 \\
2.19\end{array}$ & $\begin{array}{l}2.24 \\
2.25 \\
2.25 \\
2.24 \\
2.24\end{array}$ & $\begin{array}{l}2.48 \\
2.49 \\
2.50 \\
2.49 \\
2.49\end{array}$ & $\begin{array}{l}2.42 \\
2.41 \\
2.39 \\
2.38 \\
2.39\end{array}$ & $\begin{array}{l}2.33 \\
2.32 \\
2.31 \\
2.29 \\
2.29\end{array}$ & $\begin{array}{l}2.04 \\
2.02 \\
2.01 \\
1.99 \\
1.98\end{array}$ & $\begin{array}{l}1.75 \\
1.74 \\
1.76 \\
1.91 \\
1.91\end{array}$ \\
\hline $\begin{array}{l}11 \\
12 \\
13 \\
14 \\
15\end{array}$ & $\begin{array}{l}2.10 \\
2.10 \\
2.10 \\
2.09 \\
2.08\end{array}$ & $\begin{array}{l}2.11 \\
2.10 \\
2.10 \\
2.10 \\
2.09\end{array}$ & $\begin{array}{l}2.05 \\
2.04 \\
2.03 \\
2.06 \\
2.06\end{array}$ & $\begin{array}{l}2.11 \\
2.11 \\
2.11 \\
2.11 \\
2.11\end{array}$ & $\begin{array}{l}2.10 \\
2.13 \\
2.18 \\
2.17 \\
2.15\end{array}$ & $\begin{array}{l}2.19 \\
2.19 \\
2.19 \\
2.20 \\
2.19\end{array}$ & $\begin{array}{l}2.24 \\
2.22 \\
2.22 \\
2.27 \\
2.31\end{array}$ & $\begin{array}{l}2.49 \\
2.49 \\
2.51 \\
2.51 \\
2.51\end{array}$ & $\begin{array}{l}2.37 \\
2.37 \\
2.37 \\
2.36 \\
2.34\end{array}$ & $\begin{array}{l}2.28 \\
2.29 \\
2.30 \\
2.28 \\
2.27\end{array}$ & $\begin{array}{l}1.98 \\
1.96 \\
1.95 \\
1.95 \\
1.92\end{array}$ & $\begin{array}{l}1.88 \\
1.88 \\
1.92 \\
1.98 \\
1.99\end{array}$ \\
\hline $\begin{array}{l}16 \\
17 \\
18 \\
19 \\
20\end{array}$ & $\begin{array}{l}2.08 \\
2.18 \\
2.24 \\
2.24 \\
2.24\end{array}$ & $\begin{array}{l}2.09 \\
2.08 \\
2.08 \\
2.08 \\
2.08\end{array}$ & $\begin{array}{l}2.04 \\
2.03 \\
2.04 \\
2.08 \\
2.13\end{array}$ & $\begin{array}{l}2.11 \\
2.11 \\
2.10 \\
2.10 \\
2.10\end{array}$ & $\begin{array}{l}2.15 \\
2.17 \\
2.18 \\
2.23 \\
2.24\end{array}$ & $\begin{array}{l}2.19 \\
2.20 \\
2.20 \\
2.20 \\
2.15\end{array}$ & $\begin{array}{l}2.32 \\
2.33 \\
2.33 \\
2.33 \\
2.32\end{array}$ & $\begin{array}{l}2.51 \\
2.49 \\
2.46 \\
2.46 \\
2.43\end{array}$ & $\begin{array}{l}2.34 \\
2.32 \\
2.30 \\
2.28 \\
2.29\end{array}$ & $\begin{array}{l}2.25 \\
2.24 \\
2.24 \\
2.23 \\
2.22\end{array}$ & $\begin{array}{l}1.91 \\
1.95 \\
1.94 \\
1.92 \\
1.91\end{array}$ & $\begin{array}{l}1.98 \\
1.96 \\
1.95 \\
1.87 \\
1.86\end{array}$ \\
\hline $\begin{array}{l}21 \\
22 \\
23 \\
24 \\
25\end{array}$ & $\begin{array}{l}2.24 \\
2.23 \\
2.22 \\
2.21 \\
2.21\end{array}$ & $\begin{array}{l}2.08 \\
2.08 \\
2.07 \\
2.07 \\
2.06\end{array}$ & $\begin{array}{l}2.09 \\
2.09 \\
2.09 \\
2.09 \\
2.09\end{array}$ & $\begin{array}{l}2.10 \\
2.11 \\
2.11 \\
2.10 \\
2.10\end{array}$ & $\begin{array}{l}2.22 \\
2.21 \\
2.19 \\
2.22 \\
2.24\end{array}$ & $\begin{array}{l}2.14 \\
2.14 \\
2.18 \\
2.21 \\
2.23\end{array}$ & $\begin{array}{l}2.32 \\
2.32 \\
2.32 \\
2.31 \\
2.31\end{array}$ & $\begin{array}{l}2.43 \\
2.43 \\
2.46 \\
2.52 \\
2.53\end{array}$ & $\begin{array}{l}2.33 \\
2.33 \\
2.31 \\
2.29 \\
2.29\end{array}$ & $\begin{array}{l}2.22 \\
2.23 \\
2.21 \\
2.18 \\
2.16\end{array}$ & $\begin{array}{l}1.90 \\
1.91 \\
1.93 \\
1.92 \\
1.96\end{array}$ & $\begin{array}{l}1.85 \\
1.82 \\
1.84 \\
1.83 \\
1.85\end{array}$ \\
\hline $\begin{array}{l}26 \\
27 \\
28 \\
29 \\
30 \\
31\end{array}$ & $\begin{array}{l}2.20 \\
2.20 \\
2.18 \\
2.19 \\
2.18 \\
2.18\end{array}$ & $\begin{array}{l}2.06 \\
2.06 \\
2.05 \\
2.04 \\
2.04\end{array}$ & $\begin{array}{l}2.09 \\
2.09 \\
2.09 \\
2.09 \\
2.09 \\
2.09\end{array}$ & $\begin{array}{l}2.11 \\
2.12 \\
2.11 \\
2.10 \\
2.10 \\
2.10\end{array}$ & $\begin{array}{r}2.24 \\
2.24 \\
2.24 \\
-.- \\
---\end{array}$ & $\begin{array}{l}2.25 \\
2.25 \\
2.23 \\
2.22 \\
2.23 \\
2.23\end{array}$ & $\begin{array}{l}2.30 \\
2.30 \\
2.32 \\
2.34 \\
2.42 \\
-\end{array}$ & $\begin{array}{l}2.50 \\
2.50 \\
2.50 \\
2.50 \\
2.51 \\
2.49\end{array}$ & $\begin{array}{r}2.27 \\
2.27 \\
2.26 \\
2.28 \\
2.26 \\
-\end{array}$ & $\begin{array}{l}2.14 \\
2.13 \\
2.13 \\
2.11 \\
2.09 \\
2.08\end{array}$ & $\begin{array}{l}1.95 \\
1.92 \\
1.90 \\
1.86 \\
1.86 \\
1.82\end{array}$ & $\begin{array}{l}1.83 \\
1.80 \\
1.79 \\
1.79 \\
1.78\end{array}$ \\
\hline $\begin{array}{l}\text { MEAN } \\
\text { MAX } \\
\text { MIN }\end{array}$ & $\begin{array}{l}2.16 \\
2.24 \\
2.07\end{array}$ & $\begin{array}{l}2.10 \\
2.18 \\
2.04\end{array}$ & $\begin{array}{l}2.06 \\
2.13 \\
2.03\end{array}$ & $\begin{array}{l}2.10 \\
2.12 \\
2.09\end{array}$ & $\begin{array}{l}2.17 \\
2.24 \\
2.10\end{array}$ & $\begin{array}{l}2.21 \\
2.27 \\
2.14\end{array}$ & $\begin{array}{l}2.29 \\
2.42 \\
2.22\end{array}$ & $\begin{array}{l}2.48 \\
2.53 \\
2.41\end{array}$ & $\begin{array}{l}2.35 \\
2.48 \\
2.26\end{array}$ & $\begin{array}{l}2.24 \\
2.35 \\
2.08\end{array}$ & $\begin{array}{l}1.96 \\
2.07 \\
1.82\end{array}$ & $\begin{array}{l}1.85 \\
1.99 \\
1.73\end{array}$ \\
\hline
\end{tabular}


LOCATIOA. --Lat '57'24", long $94^{\circ} 40^{\prime} 26^{\prime \prime}$, in SEzNEt sec.12, T.140 N., R.32 w. Hubbard County, Hydrologic Unit 07010102 . Semples are collected near the center of the lake at the deepest point.

DRAIMAGE AREA, $--0.875 \mathrm{mi}^{2}$.

PERIOD OF RECORD.--Water years 1977 to current year.

REMARKS.--Additional data are evailable by contacting the district office.

WATER QUALITY DATA, WATER YEAR OCTOBER 1990 TO SEPTEMBER 1991

$\begin{array}{ccccc}\text { SPE- } & \text { PH } & & & \\ \text { CIFIC } & \text { WATER } & & \text { MAGEE- } \\ \text { CON- } & \text { WBOLE } & \text { CALCIUM } & \text { SIUM, } & \text { SODIUM, } \\ \text { DUCT- } & \text { LAB } & \text { DIS- } & \text { DIS- } & \text { DIS- } \\ \text { ANCE } & \text { (STAND- } & \text { SOLVED } & \text { SOLVED } & \text { SOLVED } \\ \text { INAB } & \text { ARD } & \text { (MG/L } & \text { (MG/L } & \text { (MG /L } \\ (U S / G M) & \text { UNITS) } & \text { AS CA) } & \text { AS MG) } & \text { AS MMA) } \\ (90095) & (00403) & (00915) & (00925) & (00930)\end{array}$
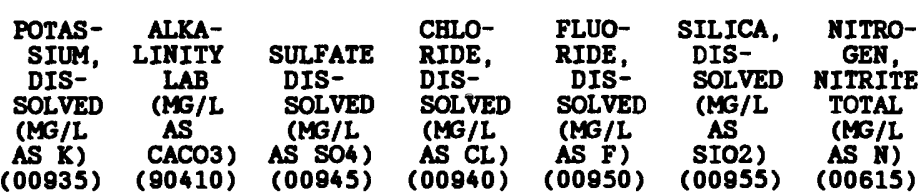

OCT
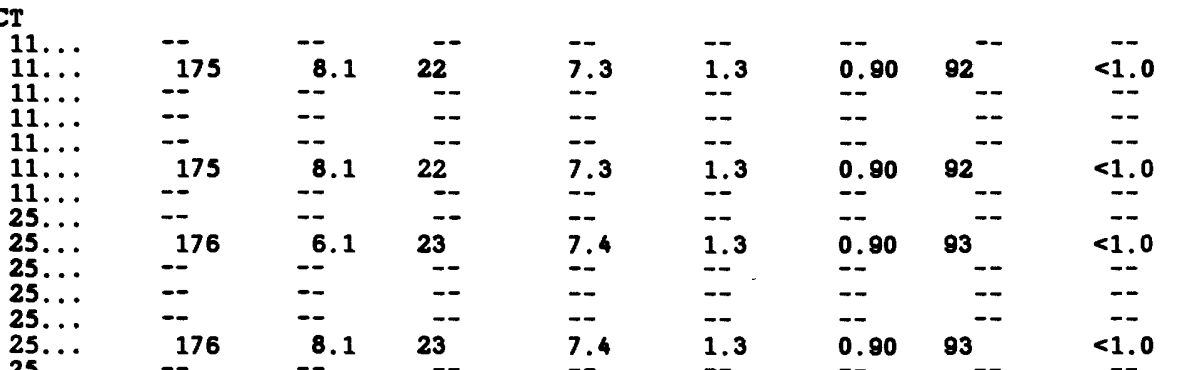

$\overline{0.70}$
$\overline{--}$
$\overline{0 .}$
$\overline{-}$
$\overline{-}$
$<0.10$
$\overline{--}$
$\overline{0.20}$

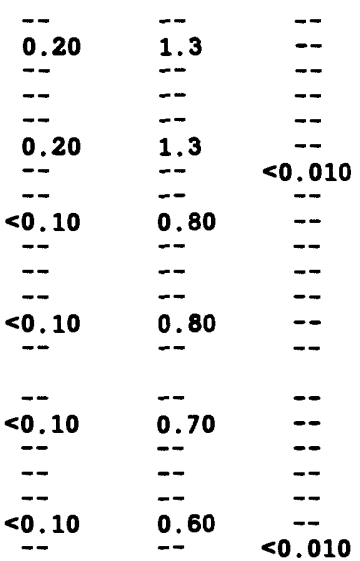

DEC

$\begin{array}{llc}-175 & -8.1 & 22 \\ -- & -- & -- \\ -- & -- & -- \\ -175 & -8.1 & 22 \\ -- & -- & -- \\ -\overline{176} & -\overline{6.1} & 23 \\ -- & -- & -- \\ -- & -- & -- \\ -- & - & -- \\ 176 & 8.1 & 23\end{array}$

NOV

$09 . . .20 .0 .178$

$09 . .--$

$09 . . . \quad-$

$09 . .$.

$09 .$.

179

$8.0 \quad 24$

$\begin{array}{ll}-- & -- \\ -- & - \\ 8.1 & 23\end{array}$

$-\overline{100}$

$13 .$.

$13 .$.

$13 .$.

$13 \ldots$

$13 . . .188$

JAH

$24 \ldots$...

$24 \ldots$.

$24 \ldots .$.

$24 \ldots$.

$24 \ldots$

202

8.127

FEB

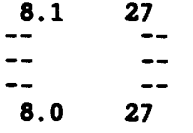

$\overline{7.5} \quad \overline{1.4}$

$0^{--} .90 \quad 94$

$\begin{array}{ll}7.5 & = \\ \overline{7.3} & \overline{-}\end{array}$

$\overline{0.80} 84$

$<\overline{1.0}$

$\overline{1.3}$

$\overline{8.0} \quad \overline{1.4}$

0.80 108

$\overline{7-} \quad \overline{7 .}$

$-$

7

$8.328^{--}$

$\begin{array}{ll}-8.3 & 28 \\ -- & -- \\ -7.9 & 28\end{array}$

$\overline{7.9} \quad 28$

$\overline{8.6} \quad \overline{1.5}$

$\begin{array}{ll}8.6 & =- \\ -- & = \\ -- & -- \\ 8.4 & 1.4\end{array}$

0.90100

$\overline{--}$

$\overline{1.0} 104^{--}$

$<\overline{-i .0}$

$\overline{--}$

0.2

$--$

-

$<1.0$

0.30

$<1.0$

0.30

$\begin{array}{lr}\overline{--} & \overline{-} \\ \overline{1.0} & 106\end{array}$

8.

$21 \ldots \quad 205$

$8.0 \quad 29$

$21 \ldots$

$21 \ldots=$

21... 210

MAR

R

219

$\begin{array}{ll}-- & =- \\ -\overline{7} & -5 \\ -5 & 31\end{array}$

205

$27 \ldots$

27 ..

27 ..

$27 \ldots$

$27 \ldots$

APR

$214 \quad 8.2 \quad 30$

$7.8 \quad 28$

$\begin{array}{ll}7.8 & 28 \\ -- & -- \\ -- & -- \\ -- & 30\end{array}$

194

$8.4 \quad 27$

$17 \ldots$

$17 \ldots$

$17 \ldots$.

$17 \ldots$.

$17 \ldots \ldots$

$\overline{--} \quad \overline{--}$

8.

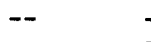

$8.5 \quad 1.5$

1.

$\begin{array}{llll}\overline{8.0} & \overline{1.4} & \overline{0.90} & 107\end{array}$

8.0 $\quad 1.4$

$\overline{8.2} \quad \overline{1.5}$

$--$

8.

$\overline{1.1} 112=$

$\overline{7.8}$

$\overline{7.8}$

$\overline{7.7} \quad \overline{1.3}$

$\begin{array}{lr}--.3 & 99 \\ --- & =- \\ -- & = \\ --0 & 101\end{array}$

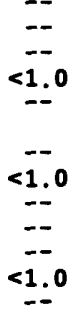

$\overline{--}$

$\overline{0.30}$

$<\overline{0.10}$

$<0.10$

$<\overline{0.10}$

0.60

$--$

$-7$

.70

$\overline{1.4}$
$\overline{--}$
$\overline{1.3}$
--

1.

$\begin{array}{ll}-- & =- \\ \overline{--} & 113 \\ -- & \end{array}$

$$
\text { }
$$

$<1.0$

$$
\begin{array}{cc}
-- & -- \\
<1.0 & 1.6 \\
-- & -- \\
-- & -- \\
<1.0 & <0.10
\end{array}
$$

\begin{tabular}{|c|c|c|c|c|}
\hline-- & -- & -- & -- & -- \\
\hline 0.40 & 1.6 & -- & 0.60 & -- \\
\hline & $\overline{-}$ & - & $\overline{--}$ & $=$ \\
\hline- & -- & - & -- & \\
\hline 0.20 & 1.8 & -- & 0.60 & - \\
\hline
\end{tabular}


LEECH LAKE RIVER BASIN

465724094402601 WILLIAMS LAKE NEAR AKELEY, MN--Continued

WATER QUALITY DATA, WATER YEAR OCTOBER 1990 TO SEPTEMBER 1991

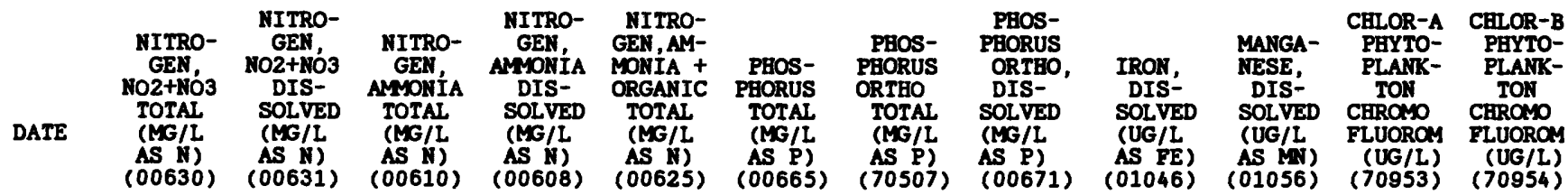

$\infty$

\begin{tabular}{|c|c|c|c|c|c|c|c|c|c|c|c|c|}
\hline $11 \ldots$ & $=$ & 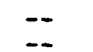 & $=$ & $=$ & -- & 0.014 & -- & -- & -- & -- & -- & -- \\
\hline $\begin{array}{l}11 \ldots \\
11 \ldots\end{array}$ & $\overline{--}$ & - & $\overline{--}$ & $=$ & $=$ & 0 & -- & -- & 9 & 3 & -- & $=$ \\
\hline $\begin{array}{l}11 . \\
11\end{array}$ & - & - & - & -- & $=-$ & 0.012 & $=$ & -- & $=$ & -- & -- & $=$ \\
\hline • & -- & -- & -- & -- & -- & 0.012 & -- & - & $=$ & $=$ & $=$ & $\overline{--}$ \\
\hline$\bullet$ & -- & $\cdots$ & -- & -- & -- & 0.012 & -- & -- & 10 & 3 & -- & -- \\
\hline & $<0.100$ & -- & $<0.010$ & -- & 1.2 & -- & $<0.010$ & -- & -- & -- & 7.60 & $<0.200$ \\
\hline • & $=$ & $=$ & $=$ & $\begin{array}{l}-- \\
--\end{array}$ & $=$ & 0.010 & $=-$ & $=$ & & -- & $\overline{--}$ & $\overline{--}$ \\
\hline . & -- & -- & $=$ & -- & -- & 0.011 & -- & - & $--^{17}$ & $--^{4}$ & $=$ & $=-$ \\
\hline & -- & -- & -- & -- & -- & 0.009 & -- & - & -- & -- & -- & -- \\
\hline & -- & $-\infty$ & -- & -- & -- & 0.005 & -- & -- & -- & -- & -- & -- \\
\hline & -- & -- & -- & -- & -- & 0.004 & -- & -- & 13 & 3 & -- & -- \\
\hline$\because$ & -- & 0.025 & -- & 0.036 & -- & 0.004 & -- & 0.004 & -- & -- & 14.0 & $<0.200$ \\
\hline 09 & -- & -- & -- & -- & -- & 0.011 & -- & -- & -- & -- & -- & -- \\
\hline . & -- & -- & -- & -- & -- & -- & -- & -- & 5 & 4 & -- & -- \\
\hline . & -- & -- & - & -- & -- & 0.035 & -- & -- & -- & - & -- & -- \\
\hline . & -- & -- & -- & -- & -- & 0.007 & -- & -- & -- & -- & -- & -- \\
\hline . & -- & -- & -- & -- & -- & 0.008 & -- & -- & -- & -- & -- & -- \\
\hline p9.... & $<0.100$ & 0.037 & 0.010 & $0 . \overline{055}$ & $\overline{0.60}$ & $\begin{array}{l}0.007 \\
0.016\end{array}$ & $\overline{0.010}$ & $0 . \overline{0} 01$ & $--^{4}$ & $--^{2}$ & $7 . \overline{60}$ & $<0 . \overline{100}$ \\
\hline 3... & - & - & -- & -- & -- & $<0.001$ & -- & -- & -- & -- & -- & -- \\
\hline 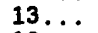 & -- & -- & -- & -- & -- & - & -- & -- & 16 & 1 & -- & -- \\
\hline $3 \ldots$ & -- & -- & -- & -- & -- & 0.001 & -- & -- & -- & -- & -- & -- \\
\hline 13. & -- & -- & -- & -- & -- & 0.02 & -- & -- & -- & -- & -- & -- \\
\hline . & -- & -- & -- & -- & -- & 0.004 & -- & -- & -- & -- & -- & -- \\
\hline 13. & -- & -- & -- & -- & -- & 0.011 & -- & -- & 5 & 3 & -- & -- \\
\hline $3 .$. & $<0.100$ & 0.040 & 0.080 & 0.042 & 0.80 & $<0.001$ & $<0.010$ & $<0.001$ & -- & -- & -- & -- \\
\hline $4 \ldots$ & -- & -- & -- & -- & -- & 0.013 & -- & -- & -- & -- & -- & -- \\
\hline & -- & -- & -- & - & -- & -- & -- & -- & 5 & $<1$ & -- & -- \\
\hline & -- & -- & -- & - & -- & 0.006 & -- & -- & -- & -- & -- & -- \\
\hline & $=$ & -- & -- & -- & -- & 0.003 & -- & -- & -- & -- & -- & -- \\
\hline & $=$ & $=$ & $=$ & $=$ & $=-$ & 0.00 & -- & $=$ & --32 & & -- & $=$ \\
\hline : & $<0.100$ & 0.041 & 0.110 & 0.103 & 0.60 & $\begin{array}{l}0.005 \\
0.009\end{array}$ & 0.010 & $<0.001$ & $--^{32}$ & $-^{13}$ & 0.500 & $<0.100$ \\
\hline & -- & -- & -- & - & -- & 0.015 & -- & -- & -- & -- & -- & -- \\
\hline & -- & -- & -- & - & -- & -- & -- & -- & 28 & 21 & -- & -- \\
\hline & -- & -- & -- & - & -- & 0.011 & -- & -- & -- & -- & -- & -- \\
\hline & -- & - & -- & -- & -- & 0.00 & -- & -- & -- & -- & -- & -- \\
\hline & - & -- & - & - & -- & 0.008 & -- & -- & -- & -- & -- & -- \\
\hline & -- & -- & - & -- & -- & 0.029 & -- & -- & 82 & 160 & -- & -- \\
\hline & $<0.100$ & 0.044 & 0.220 & 0.216 & 0.80 & 0.007 & $<0.010$ & $<0.001$ & -- & - & 0.500 & $<0.100$ \\
\hline & -- & - & -- & -- & -- & 0.001 & -- & -- & -- & -- & -- & -- \\
\hline & -- & -- & -- & -- & -- & - & -- & -- & 7 & 5 & -- & -- \\
\hline & -- & -- & -- & -- & -- & 0.004 & -- & -- & -- & -- & -- & -- \\
\hline & -- & -- & -- & -- & -- & $<0.00$ & -- & -- & -- & -- & -- & - \\
\hline & -- & -- & -- & -- & - & 0.004 & -- & -- & -- & -- & -- & -- \\
\hline & -- & -- & -- & -- & -- & 0.005 & -- & - & 13 & 5 & -- & -- \\
\hline & -- & 0.142 & -- & 0.157 & 0.80 & 0.005 & -- & $<0.001$ & -- & -- & 0.700 & $<0.100$ \\
\hline & -- & -- & -- & -- & -- & 0.007 & -- & -- & -- & -- & -- & -- \\
\hline & -- & -- & - & -- & -- & - & -- & -- & 5 & 3 & - & 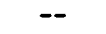 \\
\hline & -- & -- & - & -- & -- & 0.008 & -- & -- & -- & -- & -- & 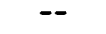 \\
\hline & -- & -- & -- & - & -- & 0.006 & -- & -- & -- & -- & -- & - \\
\hline & -- & - & -- & -- & -- & 0.006 & - & -- & -- & -- & -- & - \\
\hline & -- & - & -- & -- & -- & 0.011 & -- & -- & 4 & 4 & -- & $-\overline{300}$ \\
\hline & -- & 0.050 & -- & 0.090 & 0.60 & 0.008 & -- & $<0.001$ & -- & -- & 1.80 & $<0.100$ \\
\hline
\end{tabular}


465724094402601 WILLIAMS LAKE MEAR AKELEY, MN--Continued

WATER QUALITY DATA, WATER YEAR OCTOBER 1990 TO SEPTEMBER 1991

\begin{tabular}{|c|c|c|c|c|c|c|c|c|c|c|}
\hline & $\begin{array}{c}\text { SPE- } \\
\text { CIFIC } \\
\text { CON- } \\
\text { DUCT- } \\
\text { ANCE } \\
\text { LAB }\end{array}$ & $\begin{array}{c}\text { PH } \\
\text { WATER } \\
\text { WBOLE } \\
\text { LAB } \\
\text { (STAND- } \\
\text { ARD } \\
\text { UNITS) } \\
\text { (O0403) }\end{array}$ & $\begin{array}{l}\text { CALCIUM } \\
\text { DIS- } \\
\text { SOLVED } \\
\text { (MG/L } \\
\text { AS CA) } \\
(00915)\end{array}$ & $\begin{array}{c}\text { MAGNE- } \\
\text { SIUM, } \\
\text { DIS- } \\
\text { SOLVED } \\
\text { (MG/L } \\
\text { AS MG) } \\
\text { (00925) }\end{array}$ & $\begin{array}{c}\text { SODIUM, } \\
\text { DIS- } \\
\text { SOLVED } \\
\text { (MG/L } \\
\text { AS NAA) } \\
(00930)\end{array}$ & $\begin{array}{c}\text { POTAS- } \\
\text { SIUM, } \\
\text { DIS- } \\
\text { SOLVED } \\
\text { (MG/L } \\
\text { AS K) } \\
(00935)\end{array}$ & $\begin{array}{c}\text { ALKA- } \\
\text { LINITY } \\
\text { IAAB } \\
\text { (MG/L } \\
\text { AS } \\
\text { CACO3) } \\
\text { (90410) }\end{array}$ & $\begin{array}{c}\text { SULFATE } \\
\text { DIS- } \\
\text { SOLVED } \\
\text { (MG/L } \\
\text { AS SO4) } \\
\text { (00945) }\end{array}$ & $\begin{array}{l}\text { CHLO- } \\
\text { RIDE, } \\
\text { DIS- } \\
\text { SOLVED } \\
\text { (MG/L } \\
\text { AS CL) } \\
\text { (O0940) }\end{array}$ & $\begin{array}{l}\text { DI } \\
\text { SO } \\
\text { (M } \\
\text { SI }\end{array}$ \\
\hline
\end{tabular}

\begin{tabular}{|c|c|c|c|c|c|c|c|c|c|c|}
\hline \multicolumn{11}{|l|}{ MAY } \\
\hline \multirow{2}{*}{\multicolumn{11}{|c|}{$\begin{array}{l}02 \\
02\end{array}$}} \\
\hline $02 \ldots$ & 188 & 8.0 & 27 & 7.3 & 1.3 & 0.90 & 100 & 0.40 & 0.70 & \\
\hline $02 \ldots$ & $=$ & $=-$ & $=-$ & -- & $=$ & $=$ & -- & $=$ & $=$ & $=-$ \\
\hline $02 \ldots$ & -- & - & -- & - & - & -- & - & -- & - & - \\
\hline $02 \ldots$ & 189 & 8.1 & 28 & 7.4 & 1.3 & 1.0 & 100 & 0.50 & 0.60 & 0.30 \\
\hline $02 \ldots$ & $=$ & $=$ & $=$ & $\overline{--}$ & $=$ & $=$ & $=$ & $=$ & $\overline{-}$ & $=$ \\
\hline $16 \ldots$ & 187 & 8.4 & 27 & 7.5 & 1.3 & 0.90 & 100 & 0.40 & 0.20 & 0.20 \\
\hline $16 \ldots$ & -- & -- & -- & $\because$ & $=$ & - & -- & & -- & - \\
\hline $16 \ldots$ & -- & -- & -- & -- & -- & -- & -- & -- & - & -- \\
\hline $16 \ldots$ & - & $-\overline{7}$ & -- & -- & -- & -- & -- & -- & -- & -- \\
\hline $16 \ldots$ & 140 & 7.6 & 16 & 4.5 & 0.90 & 1.0 & 59 & 0.50 & 0.20 & 0.30 \\
\hline $16 \ldots$ & -- & -- & -- & -- & - & -- & - & -- & - & -- \\
\hline $30 \ldots$ & 183 & $\overline{8.6}$ & $26^{--}$ & $\overline{7.2}$ & 1.3 & 0.90 & $98^{--}$ & 0.20 & $\overline{0.60}$ & $0_{30}^{-30}$ \\
\hline $30 \ldots$ & - & -- & - & -- & $=$ & - & -. & -- & $\therefore$ & $-\therefore$ \\
\hline 30. & -- & - & -- & -- & -- & -- & -- & -- & - & - \\
\hline. & -- & -- & -- & -- & -- & -- & - & -- & - & -- \\
\hline $30 \ldots$ & 191 & 7.7 & 27 & 7.3 & 1.4 & 1.0 & 101 & 0.40 & 0.60 & 0.40 \\
\hline $30 \ldots$ & \multicolumn{10}{|c|}{ JUN } \\
\hline $19 .$. & -- & -- & -- & -- & -- & -- & -- & - & -- & $\cdots$ \\
\hline $19 \ldots$ & 177 & 8.7 & 25 & 7.6 & 1.4 & 1.0 & 94 & 0.50 & $<0.10$ & 1.7 \\
\hline $19 \ldots$ & - & -- & -- & -- & - & -- & -- & -- & -- & -- \\
\hline $19 \ldots$ & $=-$ & -- & -- & -- & -- & -- & -- & -- & -- & -- \\
\hline $19 .$. & $=$ & - & -- & $=-$ & $=$ & -- & -- & $=-$ & -- & -- \\
\hline $19 \ldots$ & 195 & 7.6 & 29 & 7.8 & 1.4 & 1.0 & 103 & 0.70 & $<0.10$ & 0.80 \\
\hline \multicolumn{11}{|l|}{ JuL } \\
\hline 03. & -- & -- & -- & -- & -- & -- & -- & -- & - & -- \\
\hline 03. & 174 & 8.6 & 24 & 7.4 & 1.3 & 0.90 & 92 & 0.60 & 0.70 & 0.70 \\
\hline $03 \ldots$ & -- & -- & -- & -- & -- & -- & - & -- & -- & -- \\
\hline $03 \ldots$ & $=$ & -- & - & $=$ & $\overline{--}$ & $=$ & $=$ & $\overline{--}$ & $=$ & $=$ \\
\hline & 198 & 7.5 & 28 & 7.7 & 1.3 & 1.0 & 103 & 0.70 & 0.70 & 0.90 \\
\hline 0 & -- & - & -- & -- & -- & -- & -- & -- & -- & -- \\
\hline 1 & $-\overline{166}$ & 86 & 23 & $\overline{7} 5$ & -7 & $0^{0} 80$ & $n^{--}$ & $0_{0}$ & $<010$ & 78 \\
\hline $\begin{array}{l}18 . \\
18 .\end{array}$ & $-{ }_{-}^{166}$ & -8.0 & 23 & 1.3 & 1.3 & 0.80 & 80 & $\because .40$ & -.10 & 1.8 \\
\hline 1 & -- & -- & -- & - & - & - & -- & -- & -- & -- \\
\hline & -- & -- & -- & -- & -- & -- & -- & - & -- & -- \\
\hline 1 & 201 & 7.3 & 28 & 7.6 & 1.4 & 0.90 & 107 & 0.70 & $<0.10$ & 1.5 \\
\hline 1 & -- & -- & - & - & & - & & -- & - & -- \\
\hline
\end{tabular}


LEECH LAKE RIVAR BASIN

465724094402601 WILLIAMS LAKE MEAR AKELEY, MN--Continued

WATER QUALITY DATA, WATER YEAR OCTOBER 1990 TO SEPTEMBR 1991

\begin{tabular}{|c|c|c|c|c|c|c|c|c|c|}
\hline & $\begin{array}{c}\text { NITRO- } \\
\text { GEN, } \\
\text { NO2+NO3 } \\
\text { DIS- } \\
\text { SOLVED } \\
\text { (MG/L } \\
\text { AS N) } \\
(00631)\end{array}$ & $\begin{array}{c}\text { MITRO- } \\
\text { GEN, } \\
\text { AMONIA } \\
\text { DIS- } \\
\text { SOLVED } \\
\text { (MG/L } \\
\text { AS N) } \\
(00608)\end{array}$ & $\begin{array}{l}\text { NITRO- } \\
\text { GEN,AM- } \\
\text { MONIA + } \\
\text { ORGANIC } \\
\text { TOTAL } \\
\text { (MG/L } \\
\text { AS N) } \\
(00625)\end{array}$ & $\begin{array}{c}\text { PBOS- } \\
\text { PEORUS } \\
\text { TOTAL } \\
\text { (MG/L } \\
\text { AS P) } \\
(00665)\end{array}$ & $\begin{array}{l}\text { PEOS- } \\
\text { PHORUS } \\
\text { ORTHO, } \\
\text { DIS- } \\
\text { SOLVED } \\
\text { (MG/L } \\
\text { AS P) } \\
(00671)\end{array}$ & $\begin{array}{l}\text { IRON, } \\
\text { DIS- } \\
\text { SOLVED } \\
\text { (UG/L } \\
\text { AS FE) } \\
\text { (01046) }\end{array}$ & $\begin{array}{l}\text { MANGA- } \\
\text { NESE, } \\
\text { DIS- } \\
\text { SOLVED } \\
\text { (UG/L } \\
\text { AS IN) } \\
(01056)\end{array}$ & $\begin{array}{c}\text { CHLOR-A } \\
\text { PHYTO- } \\
\text { PLANK- } \\
\text { TON } \\
\text { CEROMO } \\
\text { FLUOROM } \\
\text { (UG/L) } \\
\text { (70953) }\end{array}$ & $\begin{array}{l}\text { CHLOR-B } \\
\text { PHYTO- } \\
\text { PLANK- } \\
\text { TON } \\
\text { CHROMO } \\
\text { FLUOROM } \\
\text { (UG/L) } \\
\text { (70954) }\end{array}$ \\
\hline
\end{tabular}

\begin{tabular}{|c|c|c|c|c|c|c|c|c|c|}
\hline MAY & & & & & & & & & \\
\hline $02 \ldots$ & -- & -- & -- & 0.008 & -- & -- & -- & -- & -- \\
\hline $02 \ldots$ & -- & -- & -- & -- & -- & $<3$ & 1 & -- & -- \\
\hline $02 \ldots$ & -- & -- & -- & 0.010 & -- & -- & -- & -- & -- \\
\hline $02 \ldots$ & -- & -- & -- & 0.011 & -- & -- & -- & -- & -- \\
\hline $02 \ldots$ & -- & -- & -- & 0.010 & -- & -- & -- & -- & -- \\
\hline $02 \ldots$ & -- & -- & -- & 0.011 & -- & 5 & 2 & -- & -- \\
\hline $02 \ldots$ & 0.032 & 0.086 & 0.50 & 0.008 & $<0.001$ & -- & -- & 1.10 & $<0.200$ \\
\hline $16 \ldots$ & -- & -- & -- & 0.006 & -- & -- & -- & -- & -- \\
\hline $16 \ldots$ & -- & -- & -- & - & -- & 5 & 2 & -- & -- \\
\hline $16 \ldots$ & -- & -- & -- & 0.012 & -- & -- & - & -- & -- \\
\hline $16 \ldots$ & -- & - & - & 0.010 & -- & -- & -- & -- & -- \\
\hline $16 \ldots$ & -- & -- & -- & 0.021 & -- & -- & -- & -- & -- \\
\hline $16 \ldots$ & -- & -- & -- & 0.016 & -- & 7 & 8 & -- & -- \\
\hline $16 \ldots$ & 0.018 & 0.086 & 0.60 & 0.010 & $<0.001$ & -- & -- & -- & -- \\
\hline $30 \ldots$ & -- & -- & -- & 0.012 & -- & -- & -- & -- & -- \\
\hline $30 \ldots$ & -- & -- & -- & -- & -- & 11 & 1 & -- & -- \\
\hline $30 \ldots$ & -- & -- & -- & 0.008 & -- & -- & -- & -- & -- \\
\hline $30 \ldots$ & -- & -- & -- & 0.008 & -- & -- & -- & -- & -- \\
\hline $30 \ldots$ & -- & -- & -- & 0.010 & -- & -- & -- & -- & -- \\
\hline 30 & -- & -- & -- & 0.010 & -- & 33 & 35 & -- & -- \\
\hline 30 & 0.027 & 0.026 & 0.60 & 0.010 & $<0.001$ & -- & -- & 2.10 & $<0.200$ \\
\hline JUN & & & & 0013 & & & & & \\
\hline $19 .$. & -- & -- & -- & 0.013 & -- & -- & -- & -- & -- \\
\hline $19 \ldots$ & -- & -- & -- & $-\overline{0}$ & -- & 7 & 9 & -- & -- \\
\hline $19 \ldots$ & -- & -- & -- & 0.007 & -- & -- & -- & -- & -- \\
\hline $19 \ldots$ & -- & -- & -- & 0.010 & -- & -- & -- & -- & -- \\
\hline $19 \ldots$ & -- & -- & -- & 0.008 & -- & -- & -- & -- & -- \\
\hline 19. & -- & -- & -- & 0.011 & -- & 29 & 98 & -- & -- \\
\hline 19 & 0.006 & 0.024 & 0.70 & 0.020 & 0.005 & -- & -- & 1.30 & $<0.200$ \\
\hline JUL & & & & & & & & & \\
\hline $03 \ldots$ & -- & -- & -- & 0.003 & -- & -- & -- & -- & -- \\
\hline $03 \ldots$ & -- & -- & -- & -- & -- & 5 & 9 & -- & -- \\
\hline $03 \ldots$ & -- & -- & -- & 0.004 & -- & -- & -- & -- & -- \\
\hline $03 \ldots$ & -- & -- & -- & 0.013 & -- & -- & -- & -- & -- \\
\hline$\ldots$ & -- & -- & -- & 0.067 & -- & -- & -- & -- & -- \\
\hline 03 & 0.007 & 0.020 & 0.90 & 0.016 & $<0.001$ & -- & -- & 2.30 & 0.100 \\
\hline 18 & -- & -- & -- & 0.001 & -- & -- & -- & -- & -- \\
\hline 18 & -- & -- & -- & -- & -- & 17 & 54 & -- & -- \\
\hline $18 \ldots$ & -- & -- & -- & 0.007 & -- & -- & -- & -- & -- \\
\hline $18 \ldots$ & -- & -- & -- & 0.003 & -- & -- & -- & -- & -- \\
\hline $18 \ldots$ & -- & -- & -- & 0.005 & -- & -- & -- & -- & -- \\
\hline $\begin{array}{l}18 \ldots \\
18\end{array}$ & 0.009 & $0 . \overline{025}$ & -0 & $\begin{array}{l}0.014 \\
0.029\end{array}$ & $<0.001$ & $--^{33}$ & -280 & $\frac{--}{80}$ & $<0 . \overline{200}$ \\
\hline & & 0.020 & & 0.029 & 0.001 & & & 1.00 & $<0.200$ \\
\hline
\end{tabular}


465724094402601 WILLIAYS LAKE MEAR AKELEY, MN--Continued

WATER-QUALITY DATA, WATER YEAR OCTOBER 1990 TO SEPTEMBER 1991

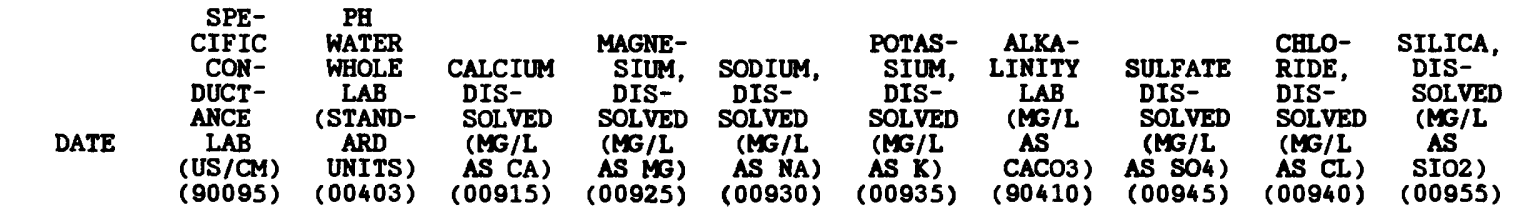

AUG

\begin{tabular}{|c|c|c|c|c|c|c|c|c|c|c|}
\hline $\begin{array}{l}01 \ldots \\
01 \ldots\end{array}$ & $=$ & $=-$ & $=$ & $=$ & -- & $\begin{array}{l}-- \\
--\end{array}$ & $=-$ & $=-$ & $=$ & $=-$ \\
\hline $01 \ldots$ & -- & -- & -- & -- & -- & -- & -- & -- & -- & -- \\
\hline $01 \ldots$ & 203 & 7.4 & 30 & 7.9 & 1.4 & 1.0 & 107 & 0.90 & 0.50 & 2.1 \\
\hline $\begin{array}{l}01 \ldots \\
07 \ldots \\
07 \ldots \\
07 \ldots \\
07 \ldots \\
07 \ldots \\
07 \ldots \\
07 \ldots\end{array}$ & $\begin{array}{l}-- \\
165 \\
166 \\
165 \\
165 \\
165 \\
165\end{array}$ & $\begin{array}{r}-- \\
8.5 \\
8.5 \\
8.6 \\
8.5 \\
8.5 \\
8.5\end{array}$ & $\begin{array}{l}22 \\
22 \\
22 \\
22 \\
22 \\
22\end{array}$ & $\begin{array}{l}7 . \\
7.8 \\
7.6 \\
7.6 \\
7.6 \\
7.6 \\
7.6\end{array}$ & $\begin{array}{l}-- \\
1.5 \\
1.5 \\
1.5 \\
1.5 \\
1.5 \\
2.6\end{array}$ & $\begin{array}{l}-- \\
0.90 \\
0.80 \\
0.80 \\
0.80 \\
0.90 \\
0.80\end{array}$ & $\begin{array}{l}89 \\
91 \\
89 \\
89 \\
89 \\
89\end{array}$ & $\begin{array}{l}-- \\
0.40 \\
0.30 \\
0.60 \\
0.40 \\
0.40 \\
0.50\end{array}$ & $\begin{array}{l}-- \\
0.20 \\
0.20 \\
0.20 \\
0.40 \\
0.40 \\
0.40\end{array}$ & $\begin{array}{l}-- \\
0.80 \\
0.70 \\
0.70 \\
0.70 \\
0.80 \\
0.80\end{array}$ \\
\hline $\begin{array}{l}15 \ldots \\
15 \ldots\end{array}$ & $-\overline{163}$ & 8.9 & $20^{--}$ & 6.9 & -7 & 10 & $87^{--}$ & $\overline{0.20}$ & $\overline{0} 10$ & 1.2 \\
\hline $15 \ldots$ & - & -- & - & $\because$ & $=-$ & $=$ & -- & -- & $=-$ & $=-2$ \\
\hline 15 & -- & -- & -- & - & -- & -- & -- & -- & -- & -- \\
\hline 15 & -- & -- & -- & -- & -- & -- & -- & -- & -- & -- \\
\hline 15. & 204 & 8.2 & 29 & 7.6 & 1.7 & 1.1 & 107 & 0.80 & 0.30 & 2.4 \\
\hline $15 \ldots$ & -- & -- & $\overline{--}$ & $\overline{-}$ & $\overline{--}$ & $=-$ & $=-$ & $=$ & $=$ & -- \\
\hline $\begin{array}{l}29 \ldots \\
29 \ldots\end{array}$ & 163 & 8.7 & 20 & 7.5 & 1.3 & $\overline{0.90}$ & $87^{--}$ & 0.50 & $<0.10$ & 0.90 \\
\hline 29. & - & -- & -- & -- & $=$ & - & -- & -- & -- & 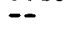 \\
\hline 29. & -- & -- & -- & -- & -- & -- & -- & -- & -- & -- \\
\hline $29 \ldots$ & 201 & 7.3 & 29 & 7.8 & 1.4 & 1.1 & 107 & 0.90 & 0.30 & 2.4 \\
\hline $29 \ldots$ & -- & -- & -1 & - & & & -- & & & \\
\hline $12 \ldots$ & -- & -- & -- & -- & -- & -- & -- & -- & -- & -- \\
\hline 12. & 167 & 8.1 & 22 & 7.7 & 1.6 & 0.90 & 90 & 0.50 & 0.50 & 0.80 \\
\hline 12. & -- & -- & 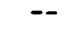 & -- & -- & -- & -- & -- & -- & \\
\hline 12. & -- & -- & -- & -- & -- & -- & -- & - & -- & -- \\
\hline & -- & -- & -- & - & -- & -- & -- & -- & -- & -- \\
\hline 12. & 208 & 7.2 & 30 & 8.2 & 1.6 & 1.1 & 109 & 0.60 & 0.60 & 3.3 \\
\hline 12 & -- & -- & -- & -- & -- & -- & -- & -- & -- & -- \\
\hline $\begin{array}{l}26 \ldots \\
26 \ldots\end{array}$ & 176 & $\overline{7.9}$ & $23^{--}$ & 7.8 & $\overline{1.4}$ & 0.90 & & 0.80 & -8 & $\overline{2.1}$ \\
\hline 26 & - & -- & 20 & .0 & 1.7 & -- & -- & -- & $=-$ & - \\
\hline & -- & -- & -- & -- & -- & -- & -- & -- & -- & -- \\
\hline & - & -- & -- & -- & -- & -- & -- & -- & -- & -- \\
\hline 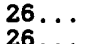 & 175 & 8.0 & 23 & 7.7 & 1.3 & 0.80 & $94 \ldots$ & 0.60 & $<0.10$ & 1.1 \\
\hline
\end{tabular}


LEECH LAKE RIVER BASIN

465724094402601 WILLIAMS LAKE NEAR AKELEY, MN--Continued

WATER-QUALITY DATA, WATER YEAR OCTOBER 1990 TO SEPTEMBER 1991

\begin{tabular}{|c|c|c|c|c|c|c|c|c|c|}
\hline DATE & $\begin{array}{c}\text { NITRO- } \\
\text { GEN, } \\
\text { MO2+NO3 } \\
\text { DIS- } \\
\text { SOLVED } \\
\text { (MG/L } \\
\text { AS IN) } \\
(00631)\end{array}$ & $\begin{array}{c}\text { NITRO- } \\
\text { GEN, } \\
\text { AMONIA } \\
\text { DIS- } \\
\text { SOLVED } \\
\text { (MG/L } \\
\text { AS N) } \\
(00608)\end{array}$ & $\begin{array}{l}\text { NITRO- } \\
\text { GEN,AM- } \\
\text { MONIA + } \\
\text { ORGANIC } \\
\text { TOTAL } \\
\text { (MG/L } \\
\text { AS N) } \\
(00625)\end{array}$ & $\begin{array}{c}\text { PEOS- } \\
\text { PHORUS } \\
\text { TOTAL } \\
\text { (MG/L } \\
\text { AS P) } \\
(00665)\end{array}$ & $\begin{array}{l}\text { PHOS- } \\
\text { PEORUS } \\
\text { ORTEO, } \\
\text { DIS- } \\
\text { SOLVED } \\
\text { (MG/L } \\
\text { AS P) } \\
\text { (00671) }\end{array}$ & $\begin{array}{l}\text { IRON, } \\
\text { DIS- } \\
\text { SOLVED } \\
\text { (UG/L } \\
\text { AS FE) } \\
\text { (01046) }\end{array}$ & $\begin{array}{l}\text { MANGA- } \\
\text { MESE, } \\
\text { DIS- } \\
\text { SOLVED } \\
\text { (UG/L } \\
\text { AS MN) } \\
\text { (01056) }\end{array}$ & $\begin{array}{c}\text { CHLOR-A } \\
\text { PHYTO- } \\
\text { PLANK- } \\
\text { TOA } \\
\text { CHRONO } \\
\text { FLUOROY } \\
\text { (UG/L) } \\
\text { (70953) }\end{array}$ & $\begin{array}{c}\text { CHLOR-B } \\
\text { PHYTO- } \\
\text { PLANK- } \\
\text { TON } \\
\text { CHROMO } \\
\text { FLUOROM } \\
\text { (UG/L) } \\
\text { (70954) }\end{array}$ \\
\hline
\end{tabular}

\begin{tabular}{|c|c|c|c|c|c|c|c|c|c|}
\hline IUG & & & & & & & & & \\
\hline $\begin{array}{l}01 \ldots \\
01 \ldots\end{array}$ & $\overline{--}$ & $=$ & $\overline{--}$ & $<0.001$ & $\overline{--}$ & -- & -- & $\overline{--}$ & $\overline{--}$ \\
\hline $01 \ldots$ & - & - & - & $<0 . \overline{001}$ & - &.$^{10}$ & $--^{20}$ & - & $\overline{--}$ \\
\hline $01 \ldots$ & -- & -- & -- & $<0.001$ & -- & -- & -- & -- & -- \\
\hline $01 \ldots$ & -- & -- & -- & 0.001 & -- & -- & -- & -- & -- \\
\hline $01 \ldots$ & -- & -- & -- & $<0.001$ & -- & 20 & 270 & -- & -- \\
\hline $\begin{array}{l}01 \ldots \\
07 \ldots\end{array}$ & $\begin{array}{r}0.009 \\
<0.005\end{array}$ & $\begin{array}{l}0.015 \\
0.005\end{array}$ & $\begin{array}{l}2.7 \\
0.60\end{array}$ & $\begin{array}{l}0.036 \\
0.011\end{array}$ & $\begin{array}{l}<0.001 \\
<0.001\end{array}$ & -- & --1 & 2.60 & $<0.200$ \\
\hline $07 \ldots$ & $\begin{array}{l}<0.005 \\
<0.005\end{array}$ & $\begin{array}{l}0.004 \\
0.002\end{array}$ & $\begin{array}{l}0.80 \\
0.70\end{array}$ & $\begin{array}{r}0.001 \\
<0.001\end{array}$ & $\begin{array}{l}<0.001 \\
<0.001\end{array}$ & $\begin{array}{l}<3 \\
<3\end{array}$ & $<1$ & $\overline{--}$ & $=$ \\
\hline $07 \ldots$ & $<0.005$ & 0.004 & 0.60 & 0.013 & $<0.001$ & $<3$ & 1 & -- & -- \\
\hline $\begin{array}{l}07 \ldots \\
07 \ldots\end{array}$ & $<0.005$ & 0.002 & 0.70 & $<0.001$ & $<0.001$ & $<3$ & $<1$ & $=$ & -- \\
\hline $15 \ldots$ & 0.00 & 0.002 & 0.80 & $\begin{array}{r}<0.001 \\
0.004\end{array}$ & $<0.001$ & $--<$ & $--^{1}$ & - & - \\
\hline $15 \ldots$ & -- & -- & -- & - & -- & $<3$ & 13 & -- & -- \\
\hline $15 \ldots$ & -- & -- & -- & 0.001 & -- & $=-$ & -- & -- & -- \\
\hline .. & -- & -- & -- & 0.005 & -- & -- & -- & -- & -- \\
\hline 15. & -- & -- & -- & 0.007 & -- & -- & $=$ & -- & -- \\
\hline & -- & -- & -- & 0.008 & -- & 11 & 190 & -- & -- \\
\hline $5 \ldots$ & 0.051 & 0.042 & 0.70 & 0.034 & $<0.001$ & -- & -- & -- & -- \\
\hline 29. & -- & -- & -- & 0.013 & -- & -- & -- & -- & -- \\
\hline 29. & -- & -- & -- & -- & -- & $<3$ & 8 & -- & -- \\
\hline 29 & -- & -- & -- & 0.005 & -- & -- & -- & -- & - \\
\hline $29 \ldots$ & -- & -- & -- & 0.010 & -- & -- & -- & -- & -- \\
\hline $29 \ldots$ & -- & -- & -- & 0.012 & -- & 5 & 150 & -- & -- \\
\hline $\begin{array}{l}29 . \\
\text { SEP }\end{array}$ & 0.059 & 0.010 & 0.60 & 0.021 & $<0.001$ & -- & -- & -- & -- \\
\hline 12. & -- & -- & - & 0.011 & -- & -- & -- & -- & -- \\
\hline 12. & -- & -- & -- & - & -- & 13 & 4 & -- & -- \\
\hline & -- & -- & -- & 0.010 & -- & -- & -- & -- & - \\
\hline 12 & -- & -- & - & 0.013 & -- & -- & -- & -- & \\
\hline 12 & -- & -- & -- & 0.011 & -- & -- & -- & -- & - \\
\hline & -- & -- & -- & 0.033 & -- & 14 & 950 & -- & -- \\
\hline 12. & 0.028 & 0.027 & 0.60 & 0.011 & $<0.001$ & -- & -- & - & \\
\hline & 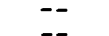 & $\overline{--}$ & $\overline{--}$ & 0.011 & 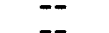 & -21 & -- & $\overline{--}$ & -- \\
\hline 26. & $=-$ & $=$ & $=$ & $\overline{0}=\overline{0}$ & $\overline{--}$ & $--^{21}$ & $--^{42}$ & $=$ & $=-$ \\
\hline 26 & -- & $=$ & - & $\begin{array}{l}0.012 \\
0.010\end{array}$ & $=$ & $=$ & - & $=$ & $=$ \\
\hline & -- & -- & -- & 0.009 & -- & -- & - & -- & -- \\
\hline & -- & -- & -- & 0.010 & -- & 17 & 20 & -- & -- \\
\hline 2 & 0.010 & 0.061 & 0.80 & 0.011 & $<0.001$ & -- & -- & -- & -- \\
\hline
\end{tabular}


LOCATION. --Lat $47^{\circ} 12^{\prime} 23^{\prime \prime}$, long $94^{\circ} 18^{\prime} 31^{\prime \prime}$, in lot 2, sec.14, T.143 N., R.29 W., Cass County, Hydrologic Unit 07010102 , on Leech Lake Indian Reservation, at head of Leech Lake' River on Waboose Bay, 5 mi southwest of town of Federal Dam.

DRAINAGE AREA. $--1,163 \mathrm{mi}^{2}$.

PERIOD OF RECORD.--April 1884 to current year. Monthend contents only for some periods, published in WSP 1308. Prior to October 1956, published as "Leech Lake Reservoir."

GAGE. - Water-stage recorder. Datum of gage is National Geodetic Vertical Datum of 1929 (levels by U.S. Army Corps of Engineers). Prior to Dec. 31, 1884, nonrecording gage 0.5 mi north of out let to Leech Lake River at datum 98.47 ft higher. Dec. 31, 1884, to May 24, 1931, nonrecording gage $0.5 \mathrm{mi}$ north of out let to Leech Lake River and May 25, 1931, to July 10, 1973, water-stage recorder at same site and at datum $92.70 \mathrm{ft}$ higher.

REMARKS .--Reservoir is formed by Leech Lake and several other natural lakes controlled by concrete and timber dam; storage began in 1884; original timber structure completed in 1884, replaced by present dam in 1902. Capacity between elevation $1,292.70 \mathrm{ft}$ and $1,297.94 \mathrm{ft}$ (maximum allowable range) is $688,985 \mathrm{acre}-\mathrm{ft}$ of which 352,637 acre-ft is controlled storage between elevations $1,292.70 \mathrm{ft}$ and $1,295.70 \mathrm{ft}$ (normal operating range). Contents shown herein are contents above elevation 1,290.00 ft. Prior to September 1978, published contents as contents above elevation 1,292.20 ft. Water is used to benefit navigation on Mississippi River below Minneapolis.

COOPERATION.--Records were provided by U.S. Army Corps of Engineers.

EXTREMES FOR PERIOD OF RECORD.--Maximum contents observed, 734,300 acre-ft, capacity table then in use, June 30 1916, levation, $1,297.88 \mathrm{ft}$; minimum, 51,380 acre-ft, capacity table then in use, Dec. 8, 24, 1976, elevation, $1,292.69 \mathrm{ft}$.

EXTREMES FOR CURRENT YEAR.--Maximum contents, 633,100 acre-ft, May 29, elevation, 1,295.09 ft; minimum, 464,810 acre-ft, Nov. 26, elevation, $1,293.73 \mathrm{ft}$.

MONTHEND ELEVATION AND CONTENTS, WATER YEAR OCTOBER 1990 to SEPTEMBER 1991

Date

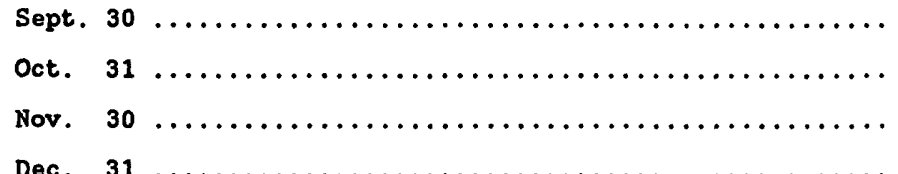

Dec. 31

CAL YR 1990

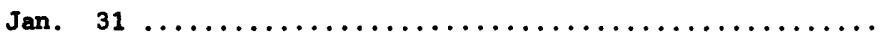

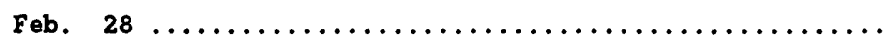

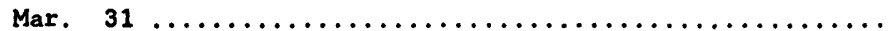

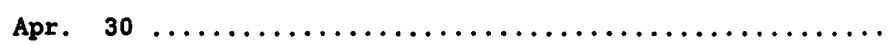

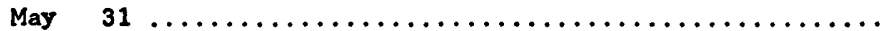

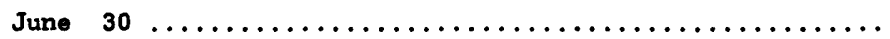

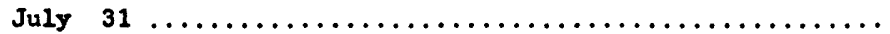

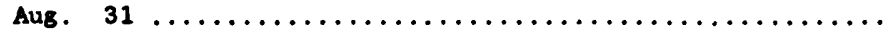

Sept. 30

WTR YR 1991

\section{Elevation (feet)}

$1,293.98$

$1,293.97$

$1,293.75$

$1,293.81$

1,293.91

$1,294.01$

$1,294.24$

$1,294.55$

$1,295.05$

$1,294.72$

$1,294.75$

$1,294.36$

$1,294.54$

\section{Content: \\ (acre-feet)}

492,880

491,680

466,990

473,520

484,520

496,540

525,620

564,830

628,050

586,310

590,110

540,790

563,560
Change in contents (acre-feet)

$-1,200$

$-24,690$

$+6,530$

$-15,720$

$+11,000$

$+12,010$

$+29,090$

$+39,210$

$+63,220$

$-41,740$

$+3,800$

$-49,320$

$+22,780$

$+70,690$ 


\section{LEECH LAKE RIVER BASIN}

05206500 LEECH LAKE RIVER AT FEDERAL DAM, MN

LOCATION. - Lat $47^{\circ} 14^{\prime} 45^{\prime \prime}$, Iong $94^{\circ} 13^{\prime} 12^{\prime \prime}$, in sec.34, T.144 N., R.28 W., Cass County, Bydrologic Unit 07010102, on Leech Lake Indian Reservation, on right bank at dam on Leech Lake River at city of Federal Dam, 2 mi downstream from natural outlet of Leech Lake.

DRAINAGE AREA. $--1,163 \mathrm{mi}^{2}$.

PERIOD OF RECORD.--May 1884 to current year. Monthly discharge only for some periods, published in wSP 1308.

GAGE.--Water-stage recorder, headwater gage, and nonrecording tailwater gage. Datum of gage is National Geodetic Vertical Datum of 1929 (ievels by U. S. Army Corps of Engineers). Prior to June 30, 1973, gages (nonrecording headwater gage prior to July 3, 1948) at same sites with datum at $1,293.23$ ft, adjustment of 1912 . May 27 to

Nov. 30, 1929, nonrecording gage at site $600 \mathrm{ft}$ downstream at different datum.

REMARKS.--Discharge computed on basis of modified weir formula, the head being obtained from readings on tailwater gage and mean gage height from recording headwater gage. Flow completely regulated by Leech Lake (station $05206000)$.

COOPERATION.--Computations of daily discharge were provided by U.S. Army Corps of Engineers.

AVERAGE DISCHARGE (unadjusted).--107 years, $370 \mathrm{ft}^{3} / \mathrm{s}, 4.32 \mathrm{in} / \mathrm{yr}$.

EXTREMES FOR PERIOD OF RECORD.--Maximum daily discharge, 2,520 $\mathrm{ft}^{3} / \mathrm{s}$, June 7, 1957 (result of dam failure); no flow at times.

EXTREMES FOR CURRENT YEAR.--Maximum daily discharge, $607 \mathrm{ft}^{3} / \mathrm{s}$, Oct. 27; minimum daily, $96 \mathrm{ft}^{3} / \mathrm{s}, \mathrm{April} 27$.

DISCHARGE, CUBIC FEET PER SECOND, WATER YEAR OCTOBER 1990 TO SEPTEMBER 1991

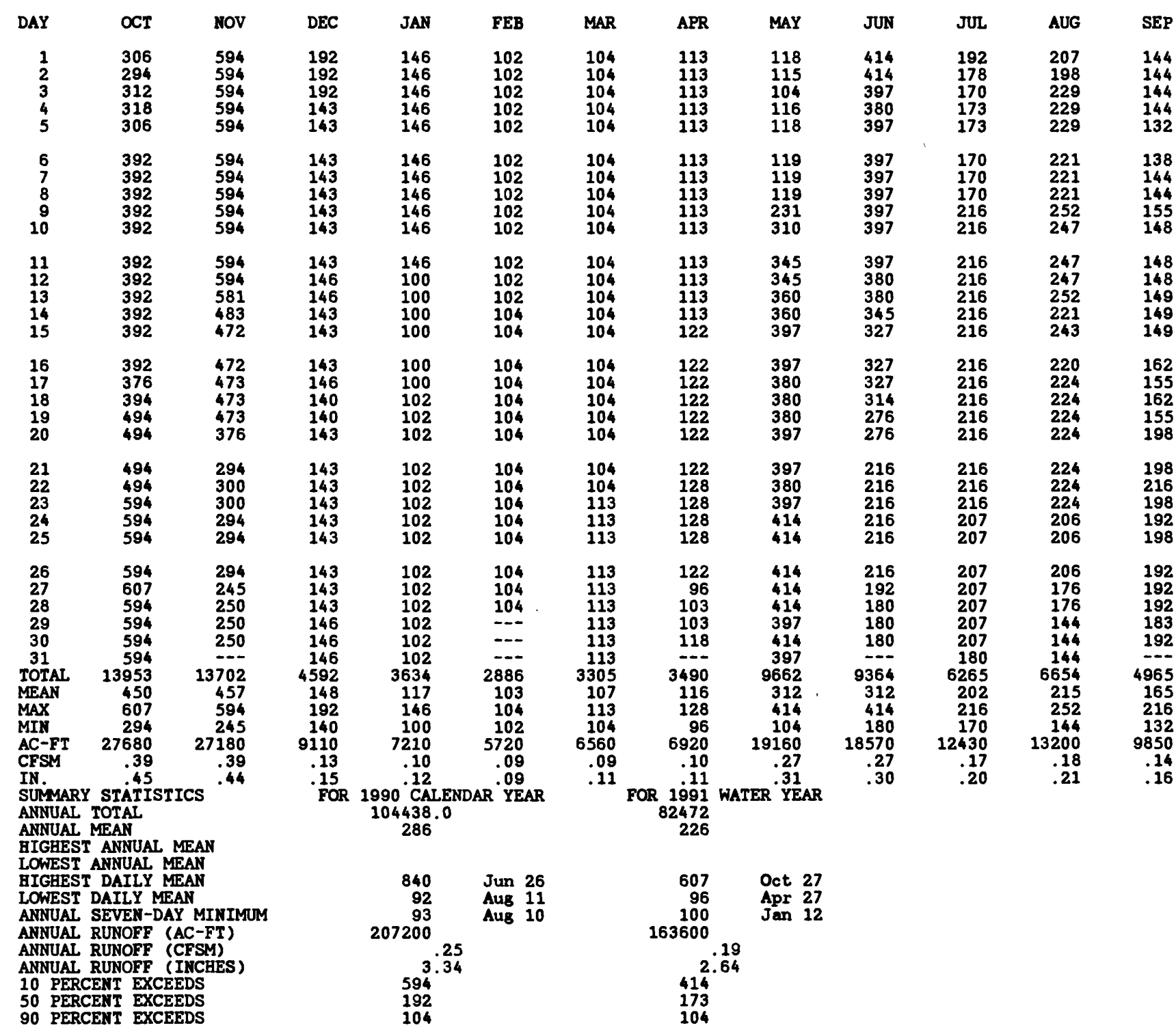




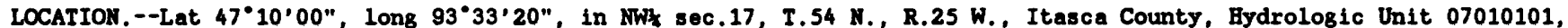
at narrows on U.S. Bighway 169, 4 mi south of Grand Rapids and at mile 1,184 upstream from Ohio River.

DRAINAGE AREA. $--3,265 \mathrm{mi}^{2}$.

PERIOD OF RECORD.--April 1884 to current year. Prior to October 1941 monthend contents only, published in WSP 1308. Published as Pokegama Reservoir near Grand Rapids, October 1941 to September 1956.

REVISED RECORDS.--WSP 1914: $1897(\mathrm{M})$.

GAGE.--Water-stage recorder. Datum of gage is National Geodetic Vertical Datum of 1929 (1evels by U.S. Army Corps of Engineers). Prior to May 30, 1949, nonrecording gage at Pooles Arm of Pokegama Lake 5 mi northwest, and May 31, 1949, to July 12, 1973, water-stage recorder at same site and at datum $64.42 \mathrm{ft}$ higher.

REMARKS. --Reservoir is formed by Pokegama Lake and several other natural lakes controlled by concrete dam; storage began in 1884; original timber dam completed in 1884, replaced by present structure in 1888-89. Capacity between elevation $1,270.42 \mathrm{ft}$ and $1,276.42 \mathrm{ft}$ (maximum allowable range) is 80,126 acre-ft of which 52,483 acre-ft is controlled storage between elevations $1,270.42 \mathrm{ft}$ and $1,274.42 \mathrm{ft}$ (normal operating range). Contents shown herein are contents above elevation 1,267.00 ft. Prior to September 1978, published contents as contents above elevation 1,268.92 ft. Water is used to benefit navigation on Mississippi River below Minneapolis.

COOPERATION.--Records were provided by U.S. Army Corps of Engineers.

EXTREMES FOR PERIOD OF RECORD.--Maximum contents, 132,160 acre-ft, May 23, 1986, elevation, 1,275.28 ft; maximum elevation, $1,277.92 \mathrm{ft}$, May 8, 1897; minimum contents observed, 4,520 acre-ft, below zero of capacity table then in use, Sept. 30, 1934, elevation, 1,268.54 ft.

EXTREMES FOR CURRENT YEAR.--Maximum contents, 103,050 acre-ft, May 7, elevation, 1,273.73 ft; minimum, 72,810 acre-ft, Dec. 28, elevation, 1,271.71 ft.

MONTHEND ELEVATION AND CONTENTS, WATER YEAR OCTOBER 1990 TO SEPTEMBER 1991

Date

Sept. $30 \ldots \ldots \ldots \ldots \ldots \ldots \ldots \ldots \ldots \ldots \ldots \ldots \ldots \ldots \ldots \ldots \ldots$

Oct. 31

Nov. 30

Dec. 31

CAL YR 1990

Jan. $\quad 31 \ldots \ldots \ldots \ldots \ldots \ldots \ldots \ldots \ldots \ldots \ldots \ldots \ldots \ldots \ldots \ldots \ldots \ldots \ldots$

Feb. $\quad 28 \ldots \ldots \ldots \ldots \ldots \ldots \ldots \ldots \ldots \ldots \ldots \ldots \ldots \ldots \ldots \ldots \ldots$

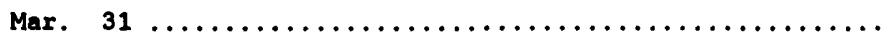

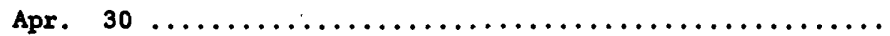

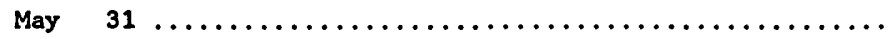

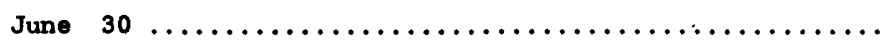

July 31

Aug. 3

Sept. 30

WIR YR 1991

\section{Elevation} (feet)

$1,273.14$

1,272.32

$1,271.80$

$1,271.71$

$1,271.75$

$1,271.80$

$1,272.56$

$1,273.53$

$1,273.53$

$1,273.32$

$1,273.28$

$1,273.35$

$1,273.36$
Contents

93,670

81,500

74,090

72,810

73,380

74,090

85,010

99,700

99,700

96,360

95,770

96,810

96,960
Change in contents (acre-feet) 


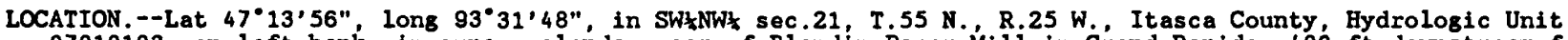
07010103 , on left bank, in super-calendar room of Blandin Paper Mili in Grand Rapids, 400 ft downstream from Blandin Dam, $400 \mathrm{ft}$ upstream from bridge on U.S. Highway 169, $2.5 \mathrm{mi}$ upstream from Prairie River, and at mile 1,182 upstream from Ohio River.

DRAINAGE AREA $--3,370 \mathrm{mi}^{2}$, approximately

PERIOD OF RECORD. --October 1883 to current year. Monthly discharge only for some periods, published in WSP 1308. Published as "at Pokegama Dam near Grand Rapids" 1942-44.

GAGE. - Water-stage recorder. Datum of gage is $1,242.03 \mathrm{ft}$ above National Geodetic Vertical Datum of 1929 . See WSP 1914 for history of changes prior to Jan. 17, 1951

REMARKS.--Records fair. Flow regulated by Winnibigoshish Lake (station 05201000), Leech Lake (station 05206000), Pokegama Lake (station 05210500 ) and occasionally at low flow by powerplant at Blandin Dam. Backwater from Prairie River occurs at times in most years.

DISCHARGE, CUBIC FEET PER SECOND, WATER YEAR OCTOBER 1990 TO SEPTEMBER 1991

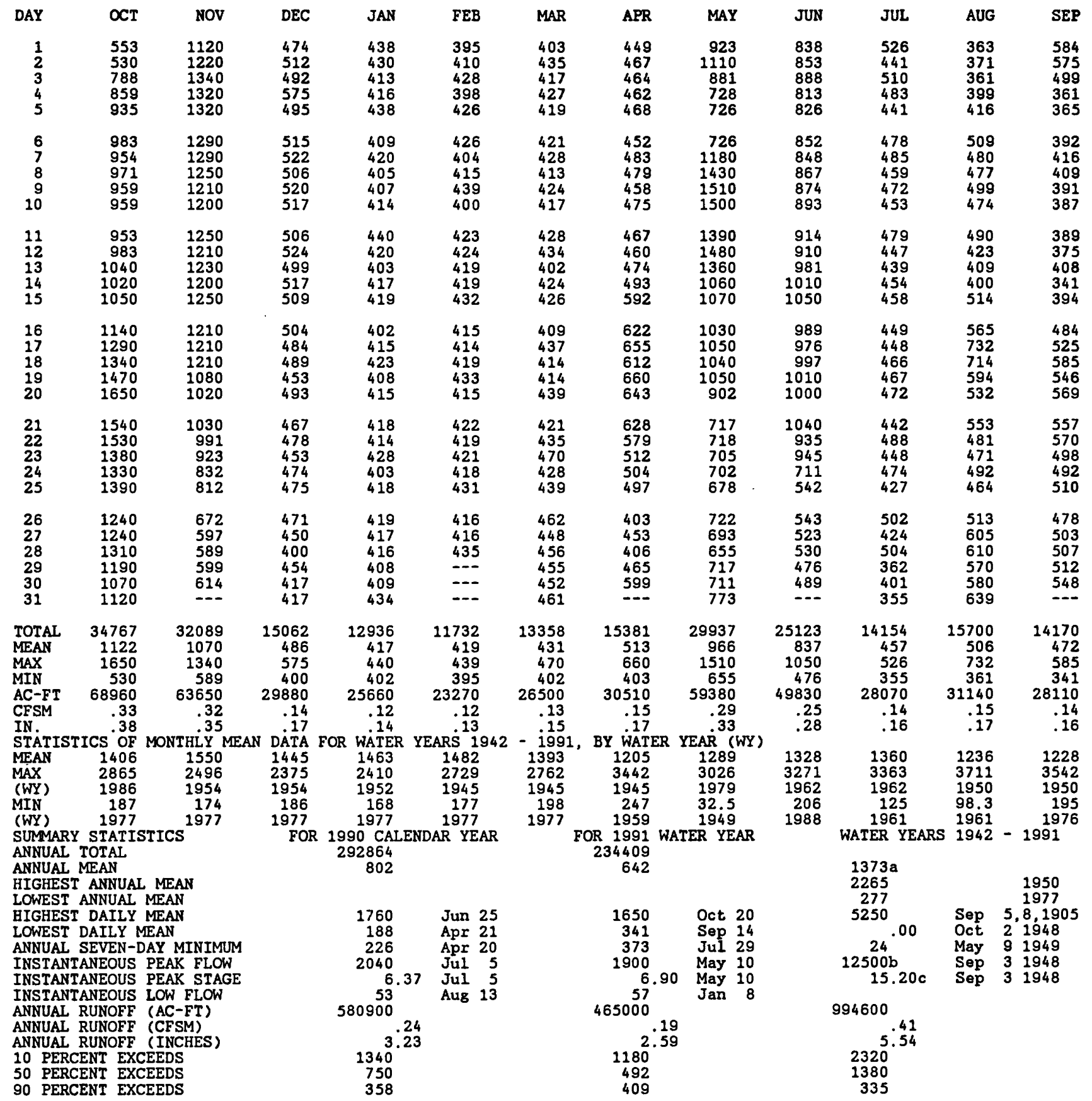

a Average based on 108 years of record is $1,185 \mathrm{ft}^{3} / \mathrm{s}$; median is $1,060 \mathrm{ft}^{3} / \mathrm{s}$.

b From rating curve extended above 4,500 $\mathrm{ft}^{3} / \mathrm{s}$.

c From floodmark, caused by dam failure. 
SANDY RIVER BASIN

05218500 SANDY LAKE AT LIBBY, MN

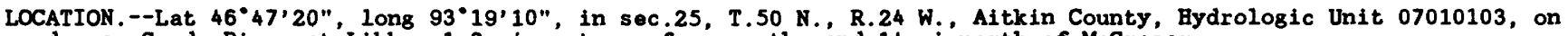
dam on Sandy River at Libby, $1.2 \mathrm{mi}$ upstream from mouth, and $14 \mathrm{mi}$ north of McGregor.

DRAINAGE AREA. $--421 \mathrm{mi}^{2}$.

PERIOD OF RECORD.--July to December 1893, October to December 1894, July 1895 to current year. Monthend contents only for some periods, published in WSP 1308. Published as Sandy Lake Reservoir at Libby, October 1941 to September 1956 .

GAGE.--Water-stage recorder. Datum of gage is National Geodetic Vertical Datum of 1929 (levels by U.S. Army Corps of Engineers). Prior to Sept. 23, 1949, nonrecording gage and Sept. 24, 1949, to Nov. 28, 1962, water-stage recorder at site $1 \mathrm{mi}$ upstream at datum 1,207.71 ft, adjustment of 1912. Nov. 29, 1962, to June 30, 1973, waterstage recorder at present site at datum 1,207.71 ft, adjustment of 1912 .

REMARKS. - Lake is formed by concrete dam which controls Sandy, Flowage, Snake, and Aitkin Lakes. Storage began in 1893; original timber crib dam completed in 1895, replaced by present structure in 1911. Capacity between elevation $1,214.31 \mathrm{ft}$ and $1,221.31 \mathrm{ft}$ (top of structure) is 73,037 acre-ft, of which 37,539 acre-ft is controlled storage between elevations $1,214.31 \mathrm{ft}$ and $1,218.31 \mathrm{ft}$ (normal operating range). Contents shown herein are contents above elevation $1,207.00 \mathrm{ft}$ Prior to September 1978 , published contents as contents above levation 1,209.03 ft. Water is used to benefit navigation on Mississippi River below Minneapolis.

COOPERATION.--Records were provided by U.S. Army Corps of Engineers.

EXTREMES FOR PERIOD OF RECORD.--Maximum contents, 167,200 acre-ft, capacity table then in use, May 19, 1950, levation, 1,224.82 ft; minimum observed, 5,950 acre-ft, below zero of capacity table then in use, Jan. 20, 1921, elevation, $1,207.96 \mathrm{ft}$.

EXTREMES FOR CURRENT YEAR.--Maximum contents, 68,940 acre-ft, May 12, elevation, 1,217.04 ft; minimum, 45,600 acre-ft, Feb. 4, elevation, 1,214.46 ft.

MONTHEND ELEVATION AND CONTENTS, WATER YEAR OCTOBER 1990 TO SEPTEMBER 1991

Date

Sept. 30 .

Oct. 31

Nov. 30

Dec. 31

CAL YR 1990

Jan $\quad 31 \ldots \ldots \ldots \ldots \ldots \ldots \ldots \ldots \ldots \ldots \ldots \ldots \ldots \ldots \ldots \ldots \ldots \ldots$

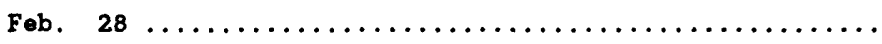

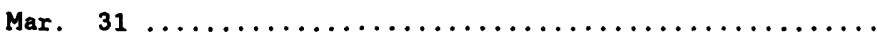

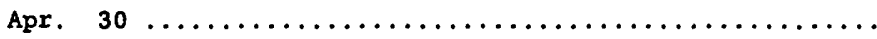

May 31

June 30

July 31

Aug. 31

Sept. 30

WTR YR 1991

\section{Elevation (feet)}

$1,216.44$

$1,216.55$

$1,215.46$

$1,214.01$

$1,214.50$

$1,214.57$

$1,215.68$

$1,216.32$

$1,216.52$

$1,216.69$

$1,216.43$

$1,216.41$

$1,216.31$
Contents (acre-feet)

63,150

64,200

54,160

49,390

45,940

46,520

56,130

62,010

63,910

65,530

63,060

62,870

61,910
Change in contents (acre-feet)

$$
\begin{array}{r}
+1,050 \\
-10,040 \\
-4,770 \\
-850 \\
-3,450 \\
+580 \\
+9,610 \\
+5,880 \\
+1,910 \\
+1,610 \\
-2,470 \\
-190 \\
-960
\end{array}
$$


SANDY RIVR BASIN

05219000 SANDY RIVER AT SANDY LAKE DAM, AT LIBBY, M

LOCATION, - Lat $46^{\circ} 47^{\prime} 20^{\prime \prime}$, long $93^{\circ} 19^{\prime} 10^{\prime \prime}$, in sec.25, T.50 N., R.24 W. Aitkin County, Hydrologic Unit 07010103, at dam at outlet of Sandy Lake, at Libby; 1.2 mi above mouth, and 14 mi north of McGregor.

DRAINAGE AREA. $--421 \mathrm{mi}^{2}$.

PERIOD OF RECORD.--July 1893 to March 1894, July 1894, November 1894 to March 1895 , August 1895 to current year. Monthly discharge only for some periods, published'in WSP 1308 . Published as "below Sandy Lake Reservoir" 1893-1916.

GAGE.--Water-stage recorders on headwater and tailwater. Datum of gages is National Geodetic Vertical Datum of 1929 (levels by U.S. Army Corps of Engineers). Prior to June 30, 1973, gages (nonrecording gages prior to June 20,1949 ) at same site with datum at $1,207.71 \mathrm{ft}$, adjustment of 1912 .

REMARKS.--Discharge computed on basis of head over dam, using modified weir formula, head being obtained from headwater and tailwater recorder records. Flow completely regulated by Sandy Lake (station 05218500 ).

COOPERATION. --Computations of daily discharge were provided by U.S. Army Corps of Engineers; discharge measurements made and records reviewed by Geological Survey.

AVERAGE DISCHARGE (unadjusted).--96 years (water years 1896-1991), $225 \mathrm{ft} / \mathrm{s}, 7.26 \mathrm{in} / \mathrm{yr}$.

EXTREMES FOR PERIOD OF RECORD.--Maximum daily discharge, 3,740 ft $3 / \mathrm{s}, \mathrm{July} 12$, 1897 ; no flow at times.

EXTREMES FOR CURRENT YEAR.--Maximum daily discharge, 1,820 ft $3 / \mathrm{s}$, Sept 14; minimum daily, $44 \mathrm{ft}^{3} / \mathrm{s}$, Mar. 28. DISCHARGE, CUBIC FEET PER SECOND, WATER YEAR OCTOBER 1990 TO SEPTEMBER 1991

\begin{tabular}{|c|c|c|c|c|c|c|c|c|c|c|c|c|}
\hline DAY & OCT & MOV & DEC & JAN & FEB & MAR & APR & MAY & JUN & JUL & AUG & SEP \\
\hline $\begin{array}{l}1 \\
2 \\
3 \\
4 \\
5\end{array}$ & $\begin{array}{l}170 \\
170 \\
170 \\
165 \\
258\end{array}$ & $\begin{array}{l}984 \\
972 \\
804 \\
804 \\
784\end{array}$ & $\begin{array}{l}250 \\
245 \\
243 \\
248 \\
250\end{array}$ & $\begin{array}{l}209 \\
209 \\
209 \\
209 \\
209\end{array}$ & $\begin{array}{l}85 \\
84 \\
84 \\
84 \\
84\end{array}$ & $\begin{array}{l}47 \\
47 \\
47 \\
47 \\
47\end{array}$ & $\begin{array}{l}112 \\
214 \\
219 \\
223 \\
320\end{array}$ & $\begin{array}{l}704 \\
680 \\
664 \\
640 \\
624\end{array}$ & $\begin{array}{l}1500 \\
1480 \\
1460 \\
1460 \\
1460\end{array}$ & $\begin{array}{r}930 \\
1330 \\
1270 \\
1270 \\
1260\end{array}$ & $\begin{array}{r}1100 \\
1090 \\
760 \\
784 \\
784\end{array}$ & $\begin{array}{l}264 \\
264 \\
264 \\
264 \\
264\end{array}$ \\
\hline $\begin{array}{r}6 \\
7 \\
8 \\
9 \\
10\end{array}$ & $\begin{array}{l}242 \\
240 \\
242 \\
245 \\
248\end{array}$ & $\begin{array}{l}514 \\
516 \\
522 \\
522 \\
522\end{array}$ & $\begin{array}{l}248 \\
248 \\
248 \\
248 \\
248\end{array}$ & $\begin{array}{l}209 \\
207 \\
207 \\
207 \\
204\end{array}$ & $\begin{array}{l}47 \\
47 \\
47 \\
47 \\
47\end{array}$ & $\begin{array}{l}47 \\
48 \\
48 \\
49 \\
49\end{array}$ & $\begin{array}{l}512 \\
506 \\
512 \\
512 \\
651\end{array}$ & $\begin{array}{r}600 \\
1420 \\
1180 \\
1150 \\
1150\end{array}$ & $\begin{array}{l}1460 \\
1110 \\
1160 \\
1160 \\
1160\end{array}$ & $\begin{array}{r}1580 \\
1400 \\
1160 \\
880 \\
744\end{array}$ & $\begin{array}{l}566 \\
515 \\
420 \\
371 \\
209\end{array}$ & $\begin{array}{l}264 \\
264 \\
256 \\
250 \\
873\end{array}$ \\
\hline $\begin{array}{l}11 \\
12 \\
13 \\
14 \\
15\end{array}$ & $\begin{array}{l}248 \\
152 \\
155 \\
155 \\
153\end{array}$ & $\begin{array}{l}522 \\
522 \\
522 \\
522 \\
522\end{array}$ & $\begin{array}{l}248 \\
248 \\
248 \\
245 \\
248\end{array}$ & $\begin{array}{l}124 \\
124 \\
124 \\
122 \\
122\end{array}$ & $\begin{array}{l}47 \\
47 \\
48 \\
47 \\
47\end{array}$ & $\begin{array}{l}48 \\
48 \\
47 \\
47 \\
47\end{array}$ & $\begin{array}{l}801 \\
792 \\
792 \\
801 \\
801\end{array}$ & $\begin{array}{l}1150 \\
1100 \\
1150 \\
1180 \\
1220\end{array}$ & $\begin{array}{l}712 \\
558 \\
570 \\
297 \\
303\end{array}$ & $\begin{array}{l}582 \\
588 \\
582 \\
582 \\
588\end{array}$ & $\begin{array}{r}114 \\
115 \\
116 \\
93 \\
94\end{array}$ & $\begin{array}{l}1420 \\
1800 \\
1800 \\
1820 \\
1780\end{array}$ \\
\hline $\begin{array}{l}16 \\
17 \\
18 \\
19 \\
20\end{array}$ & $\begin{array}{l}102 \\
102 \\
100 \\
188 \\
180\end{array}$ & $\begin{array}{l}312 \\
319 \\
319 \\
322 \\
322\end{array}$ & $\begin{array}{l}250 \\
245 \\
245 \\
245 \\
248\end{array}$ & $\begin{array}{l}122 \\
122 \\
122 \\
122 \\
122\end{array}$ & $\begin{array}{l}47 \\
48 \\
48 \\
47 \\
47\end{array}$ & $\begin{array}{l}48 \\
48 \\
48 \\
48 \\
48\end{array}$ & $\begin{array}{r}783 \\
984 \\
912 \\
1040 \\
747\end{array}$ & $\begin{array}{r}1250 \\
928 \\
992 \\
1020 \\
1040\end{array}$ & $\begin{array}{l}303 \\
303 \\
255 \\
260 \\
159\end{array}$ & $\begin{array}{l}930 \\
910 \\
713 \\
728 \\
728\end{array}$ & $\begin{array}{r}59 \\
59 \\
59 \\
59 \\
157\end{array}$ & $\begin{array}{r}1750 \\
1150 \\
1190 \\
1190 \\
744\end{array}$ \\
\hline $\begin{array}{l}21 \\
22 \\
23 \\
24 \\
25\end{array}$ & $\begin{array}{l}174 \\
170 \\
410 \\
560 \\
574\end{array}$ & $\begin{array}{l}322 \\
326 \\
326 \\
326 \\
329\end{array}$ & $\begin{array}{l}248 \\
245 \\
243 \\
240 \\
238\end{array}$ & $\begin{array}{l}122 \\
122 \\
122 \\
122 \\
121\end{array}$ & $\begin{array}{l}47 \\
47 \\
47 \\
47 \\
48\end{array}$ & $\begin{array}{l}48 \\
48 \\
47 \\
47 \\
47\end{array}$ & $\begin{array}{l}738 \\
747 \\
747 \\
747 \\
756\end{array}$ & $\begin{array}{l}592 \\
336 \\
360 \\
372 \\
376\end{array}$ & $\begin{array}{l}161 \\
510 \\
500 \\
500 \\
505\end{array}$ & $\begin{array}{r}855 \\
855 \\
940 \\
990 \\
1110\end{array}$ & $\begin{array}{l}145 \\
148 \\
148 \\
115 \\
226\end{array}$ & $\begin{array}{l}768 \\
768 \\
768 \\
408 \\
420\end{array}$ \\
\hline $\begin{array}{l}26 \\
27 \\
28 \\
29 \\
30 \\
31\end{array}$ & $\begin{array}{l}809 \\
800 \\
809 \\
819 \\
984 \\
972\end{array}$ & $\begin{array}{l}336 \\
250 \\
250 \\
250 \\
250 \\
---\end{array}$ & $\begin{array}{l}235 \\
235 \\
233 \\
233 \\
235 \\
235\end{array}$ & $\begin{array}{r}121 \\
121 \\
121 \\
83 \\
85 \\
85\end{array}$ & $\begin{array}{r}47 \\
47 \\
47 \\
--- \\
--\end{array}$ & $\begin{array}{r}45 \\
45 \\
44 \\
103 \\
106 \\
112\end{array}$ & $\begin{array}{l}609 \\
623 \\
637 \\
644 \\
644 \\
-\end{array}$ & $\begin{array}{r}744 \\
736 \\
1190 \\
1160 \\
1600 \\
1520\end{array}$ & $\begin{array}{l}510 \\
520 \\
701 \\
721 \\
960 \\
---\end{array}$ & $\begin{array}{r}594 \\
812 \\
612 \\
1130 \\
837 \\
1120\end{array}$ & $\begin{array}{l}224 \\
269 \\
269 \\
266 \\
266 \\
266\end{array}$ & $\begin{array}{l}420 \\
420 \\
424 \\
424 \\
424 \\
-\end{array}$ \\
\hline $\begin{array}{l}\text { TOTAL } \\
\text { MEAN } \\
\text { MAX } \\
\text { MIN } \\
\text { AC-FT } \\
\text { CFSM } \\
\text { IN. }\end{array}$ & $\begin{array}{r}10766 \\
347 \\
984 \\
100 \\
21350 \\
.82 \\
.95\end{array}$ & $\begin{array}{r}14113 \\
470 \\
984 \\
250 \\
27990 \\
1.12 \\
1.25\end{array}$ & $\begin{array}{r}7566 \\
244 \\
250 \\
233 \\
15010 \\
.58 \\
.67\end{array}$ & $\begin{array}{r}4530 \\
146 \\
209 \\
83 \\
8990 \\
.35 \\
.40\end{array}$ & $\begin{array}{r}1506 \\
53.8 \\
85 \\
47 \\
2990 \\
.13 \\
.13\end{array}$ & $\begin{array}{r}1645 \\
53.1 \\
112 \\
44 \\
3260 \\
.13 \\
.15\end{array}$ & $\begin{array}{r}19126 \\
638 \\
1040 \\
112 \\
37940 \\
1.51 \\
1.69\end{array}$ & $\begin{array}{r}28828 \\
930 \\
1600 \\
336 \\
57180 \\
2.21 \\
2.55\end{array}$ & $\begin{array}{r}22718 \\
757 \\
1500 \\
159 \\
45060 \\
1.80 \\
2.01\end{array}$ & $\begin{array}{r}28410 \\
916 \\
1580 \\
582 \\
56350 \\
2.18 \\
2.51\end{array}$ & $\begin{array}{r}9866 \\
318 \\
1100 \\
59 \\
19570 \\
.76 \\
.87\end{array}$ & $\begin{array}{r}23115 \\
770 \\
1820 \\
250 \\
45850 \\
1.83 \\
2.04\end{array}$ \\
\hline $\begin{array}{l}\text { SUMMARY } \\
\text { ANNUAL } \\
\text { ANNUAL } \\
\text { HIGHEST } \\
\text { LOWEST } \\
\text { HIGHEST } \\
\text { LOWEST } \\
\text { ANNUAL } \\
\text { ANNUAL } \\
\text { ANNUAL } \\
\text { ANNUAL } \\
10 \text { PERC } \\
50 \text { PERC } \\
90 \text { PERC }\end{array}$ & $\begin{array}{l}\text { STATIS } \\
\text { TOTAL } \\
\text { MEAN } \\
\text { ANNUAL } \\
\text { ANNUAL } \\
\text { DAILY } \\
\text { DAILY M } \\
\text { SEVEN-D } \\
\text { RUNOFF } \\
\text { RUNOFF } \\
\text { RUNOFF } \\
\text { ENT EXC } \\
\text { ENT EXC } \\
\text { ENT EXC }\end{array}$ & $\begin{array}{l}\text { N } \\
\text { AN } \\
\text { MINIMUM } \\
\text { (FT) } \\
\text { SM) } \\
\text { TCHES) } \\
\text { S } \\
\text { S } \\
\text { OS }\end{array}$ & FOR & $\begin{array}{r}1900 \\
15 \\
18 \\
211900 \\
9 \\
827 \\
170 \\
20\end{array}$ & $\begin{array}{ll}\text { Jun } & 3 \\
\text { Sep } & 8 \\
\text { Sep } & 5\end{array}$ & & $\begin{array}{r}1820 \\
44 \\
46 \\
341500 \\
1 \\
15 \\
1150 \\
266 \\
48\end{array}$ & $\begin{array}{l}\text { TER YEAR } \\
\text { Sep } 14 \\
\text { Mar } 28 \\
\text { Mar } 22\end{array}$ & & & & \\
\hline
\end{tabular}


LOCATION. --Lat $46^{\circ} 32^{\prime} 26^{\prime \prime}$, Long $93^{\circ} 42^{\prime} 26^{\prime \prime}$, in SWłNWk sec.24, T.47 N., R.27 W., A1tkin County, Bydrologic Unit 07010104 , on right bank upstream side' of highway bridge at north edge of Aitkin, 1 mi downstream from Ripple River and at mile $1,055.9$ upstream from Ohio River.

DRAINAGE AREA. $-6,140 \mathrm{ml}^{2}$, approximately.

PERIOD OF RECORD. - March 1945 to current year.

GAGE. - Water-stage recorder. Datum of gage is 1,182.41 ft above National Geodetic Vertical Datum of 1929 (levels by U.S. Army Corps of Engineers). Mar. 1, 1945, to Mar. 14, 1961, nonrecording 8 age, and Mar. 15, 1961 , to Sept. 30, 1967, water-stage recorder at same site at datum 3.0 ft higher. Diversion channel: Non-recording gage and crest-stage gage. Datum of gage is 1,182.02 ft above National Geodetic Vertical Datum of 1929. Apr. 9, 1955, to Apr. 10, 1956, nonrecording gage at site $4 \mathrm{mi}$ downstream at different datum. Apr. 11, 1956, to Sept. 30, 1967, non-recording gage at same site at datum $3.0 \mathrm{ft}$ higher.

REMARKS --Records good except those for estimated dally discharges, which are falr. Flow regulated by Winnibigoshish Lake (sta 05201000), Leech Lake (sta 05206000), Pokegama Lake (sta 05210500), and Sandy Lake (sta 05218500). Water diverted at medium and high stages into Aitkin diversion channel 6.5 mi above station, bypasses station and returns to river $15.5 \mathrm{mi}$ below station. Diversion began Apr. 2 , 1955 . These records include flow in diversion channel. Gage height telemeter and U.S. Army Corps of Engineers satellite telemeter at station.

EXTREMES FOR CURRENT YEAR.--River gage: Maximum discharge, 4,620 ft3/s, May 11, 12, gage height, 11.95 ft. Diversion channel: Maximum discharge, 2,890 ft $/ \mathrm{s}$, May 11, 12, gage height, $11.45 \mathrm{ft}$.

DISCHARGE, CUBIC FEET PER SECOND, WATER YEAR OCTOBER 1990 TO SEPTEMBER 1991 DAILY MEAN VALUES

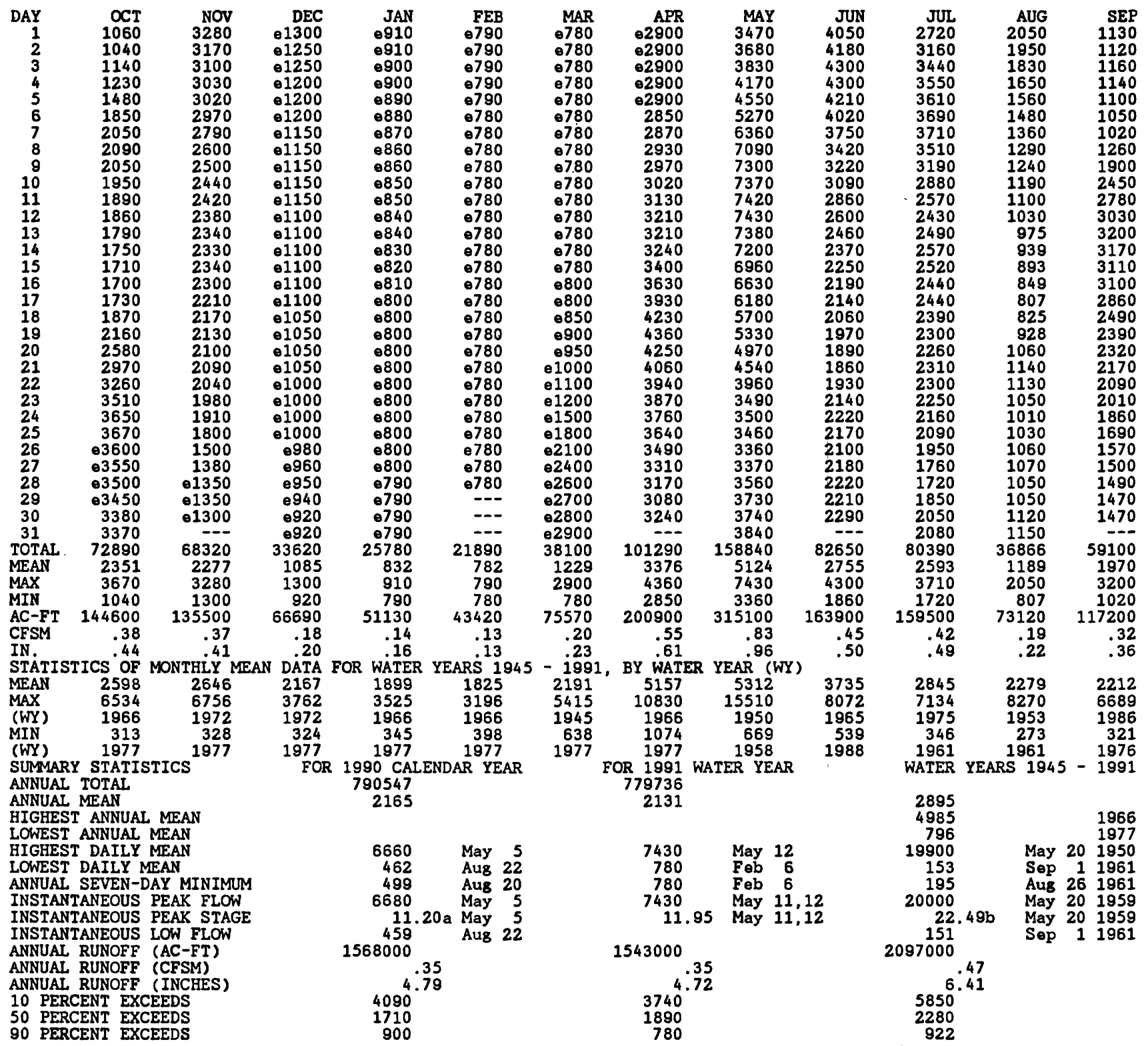

a From observer reading.

b Present datum.

- Estimated. 
PINE RIVER BASIN

05230500 PINE RIVER RESERVOIR AT CROSS LAKE, MN

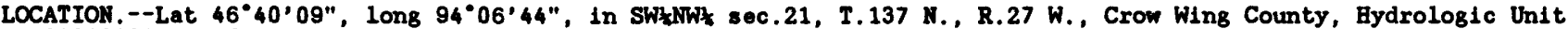
07010105, at dam on Pine River, at outlet of Cross Lake at city of Cross Lake.

DRAIMAGE AREA. $--562 \mathrm{mi}^{2}$.

PERIOD OF RECORD.--March 1886 to current year. Monthend contents only for some periods, published in WSP 1308.

GAGE.--Water-stage recorder. Datum of gage is National Geodetic Vertical Datum of 1929 (levels by U.S. Army Corps of Engineers). Prior to May 3, 1949, nonrecording gage at same site and datum.

REMARKS.--Reservoir is formed by Trout, Whitefish, Rush and Cross Lakes and several other natural lakes controlled by timber crib dams; storage began in 1886; dam completed in 1886. Capacity between elevat1ons $1,226.32 \mathrm{ft}$ and $1,234.82 \mathrm{ft}$ (maximum allowable range) is 118,703 acre-ft of which $53,272 \mathrm{acre}-\mathrm{ft}$ is controlled storage between - levations $1,226.32 \mathrm{ft}$ and $1,230.32 \mathrm{ft}$ (normal operating range). Contents shown herein are contents above an elevation 1,216.00 ft. Prior to September 1978, published contents as contents above elevation 1,218.67 ft. Water is used to benefit navigation on Mississippi River below Minneapolis.

COOPERATION.--Records were provided by U.S. Army Corps of Engineers.

EXTREMES FOR PERIOD OF RECORD.--Maximum contents observed, 173,600 acre-ft, capacity table then in use, July 10, 1916, elevation, 1,234.56 ft; minimum observed, 1,310 acre-ft, below zero of capacity table then in use, Aus. 20, 1918, elevation, 1,217.67 ft.

EXTREMES FOR CURRENT YEAR.--Maximum contents, 105,850 acre-ft, May 7, elevation, 1,229.65 ft; minimum, 93,100 acre-ft, Feb. 8, elevation, 1,228.71 ft.

MONTHEND ELEVATION AND CONTENTS, WATER YEAR OCTOBER 1990 TO SEPTEMBER 1991

Date

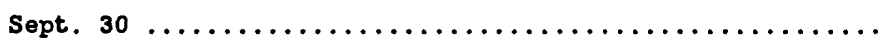

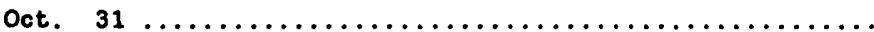

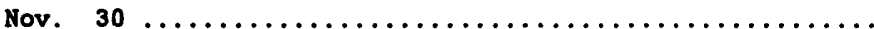

Dec. 31

CAL YR 1990

\begin{tabular}{|c|c|c|}
\hline Feb. & 28 & $\ldots \ldots \ldots \ldots \ldots \ldots \ldots \ldots \ldots \ldots \ldots \ldots \ldots \ldots \ldots \ldots$ \\
\hline Mar. & 31 & 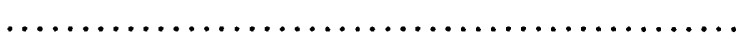 \\
\hline Apr. & 30 & ${ }_{1} \ldots \ldots \ldots \ldots \ldots \ldots \ldots \ldots \ldots \ldots \ldots \ldots \ldots \ldots \ldots \ldots \ldots \ldots \ldots \ldots$ \\
\hline May & 31 & ${ }_{\ldots} \ldots \ldots \ldots \ldots \ldots \ldots \ldots \ldots \ldots \ldots \ldots \ldots \ldots \ldots \ldots \ldots \ldots \ldots \ldots$ \\
\hline June & 30 & ${ }_{\ldots} \ldots \ldots \ldots \ldots \ldots \ldots \ldots \ldots \ldots \ldots \ldots \ldots \ldots \ldots \ldots \ldots \ldots \ldots$ \\
\hline July & 31 & $\ldots \ldots \ldots \ldots \ldots \ldots \ldots \ldots \ldots \ldots \ldots \ldots \ldots \ldots \ldots \ldots \ldots \ldots \ldots$ \\
\hline Aus. & 31 & $\ldots \ldots \ldots \ldots \ldots \ldots \ldots \ldots \ldots \ldots \ldots \ldots$ \\
\hline
\end{tabular}

Sept. 30

WIR YR 1991

\section{Elevation} ( eeet)

$1,229.13$

$1,229.11$

$1,228.72$

$1,228.79$

$1,228.73$

$1,228.83$

$1,229.20$

$1,229.57$

$1,229.33$

$1,229.45$

$1,229.38$

$1,229.27$

$1,229.25$
Content:

(acre-feet)

98,760

98,500

93,240

94,170

Change in contents (acre-feet)

\author{
orps
}




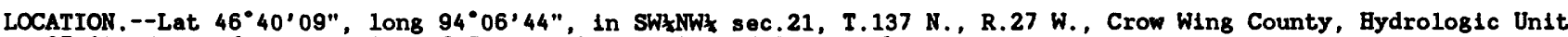
07010105 , at dam at outlet of Cross Lake at c1ty of Cross Lake.

DRAINAGE AREA. $--562 \mathrm{mi}^{2}$.

PERIOD OF RECORD. --Apr11 1886 to current year. Monthly discharge only for some periods, published in wSP 1308. Published as "below Pine River Reservolr" 1895-1916, 1929, and as "at Pine River Dam, at Cross Lake" $1941-56$.

GAGE.--Water-stage recorder, headwater gage, and nonrecording tallwater gage. Datum of gages is $1,216.32 \mathrm{ft}$ above National Geodetic Vertical Datum of 1929' (levels by U.S. Army Corps of Engineers). Mar. 26, 1886, to May 31, 1929, nonrecording gages on headwater and tall water at same sites and datum. June 1 to Nov. 30, 1929, nonrecording gage in tallwater at datum $1.60 \mathrm{ft}(0.49 \mathrm{~m})$ lower. Dec. 1, 1929, to May 2, 1949, nonrecording gage on headwater and Dec. 1, 1929, to August 1949, nonrecording gage on tailwater at present sites and datum.

REMARKS. --Discharge computed principally on basis of modified weir formula, the head being obtained from twicedally readings on tallwater gage and from headwater recorder. Flow completely regulated by Pine River Reservolr (station 05230500).

COOPERATION.--Computations of dally discharge were provided by U. S. Army Corps of Engineers.

AVERAGE DISCHARGE (unadjusted).--105 years, $220 \mathrm{ft}^{3} / \mathrm{s}, 5.32 \mathrm{ln} / \mathrm{yr}$.

EXTREMES FOR PERIOD OF RECORD.--Maximum dally discharge, 2,250 $\mathrm{ft}^{3} / \mathrm{s}$, in June 1896 (does not include flow bypassing dam through crevasse); no flow at times.

EXTREMES FOR CURRENT YEAR.--Maximum dally discharge, $900 \mathrm{ft}^{3} / \mathrm{s}$, May 8-15; minimum daily, $30 \mathrm{ft}^{3} / \mathrm{s}, \mathrm{June} 23-27$,

Aug. 7 to Sept. 19

DISCHARGE, CUBIC FEET PER SECOND, WATER YEAR OCTOBER 1990 TO SEPTEMBER 1991

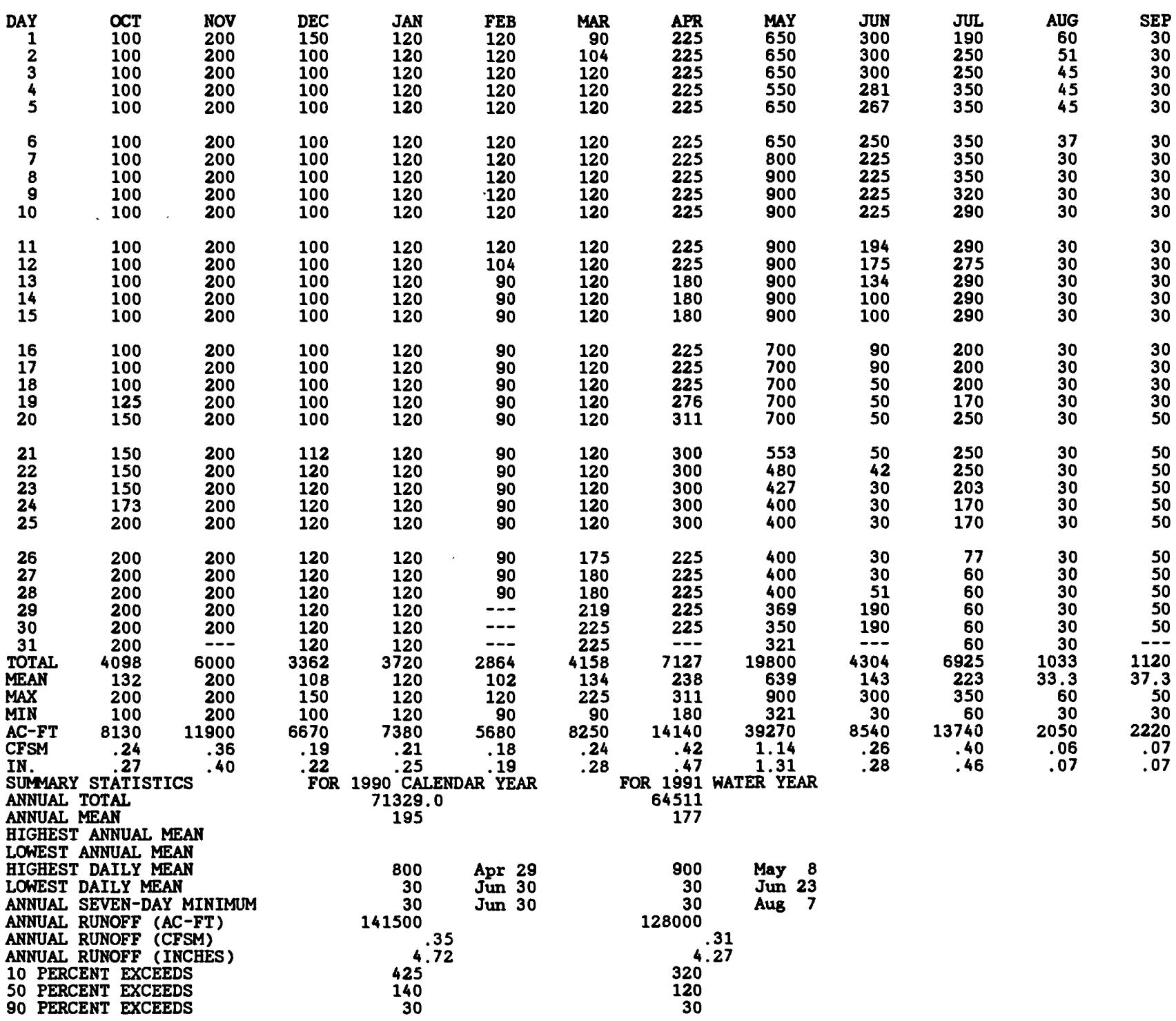


MISSISSIPPI RIVER MAIN STEM

05242300 MISSISSIPPI RIVER AT BRAINERD, M

LOCATION.--Lat $46^{\circ} 22^{\prime} 40^{\prime \prime}$, long $94^{\circ} 10^{\prime} 59^{\prime \prime}$, in SEłSWz sec. 18, T. 45 N., R. 30 W., Crow Wing County, Hydrologic Unit 07010104 , on left bank in hydro-plant of Potlach Corporation, Northwest Paper Division in Brainerd, 12.7 mi upstream from Crow Wing River, and at mile 1003.7 upstream from Ohio River.

DRAINAGE AREA. $--7,320 \mathrm{mi}^{2}$, approximately.

PERIOD OF RECORD.--October 1987 to current year.

GAGE.--Water-stage recorder. Datum of gage is $1,146.96 \mathrm{ft}$ above National Geodetic Vertical Datum of 1929.

REMARKS.--Records good except those for estimated daily discharges, which are fair. Flow regulated by Winnibigoshish Lake (sta. 05201000), Leech Lake (sta. 05206000), Pokegama Lake (sta. 05210500), Sandy Lake (sta. 05218500), and Pine River Reservoir at Cross Lake (sta 05230500).

DISCHARGE, CUBIC FEET PER SECOND, WATER YEAR $O$ CTOBER 1990 TO SEPTEMBER 1991

\begin{tabular}{|c|c|c|c|c|c|c|c|c|c|c|c|c|}
\hline DAY & $\propto T$ & NOV & DEC & JAN & FEB & MAR & APR & MAY & JUN & JUL & AUG & SEP \\
\hline $\begin{array}{r}1 \\
2 \\
3 \\
4 \\
5 \\
6 \\
7 \\
8 \\
9 \\
10 \\
11 \\
12 \\
13 \\
14 \\
15 \\
16 \\
17 \\
18 \\
19 \\
20 \\
21 \\
22 \\
23 \\
24 \\
25 \\
26 \\
27 \\
28 \\
29 \\
30 \\
31\end{array}$ & $\begin{array}{l}1160 \\
1100 \\
1460 \\
1130 \\
1710 \\
1610 \\
2090 \\
2260 \\
2310 \\
2190 \\
2180 \\
2110 \\
1940 \\
1930 \\
1950 \\
2040 \\
2260 \\
2200 \\
2160 \\
2800 \\
3270 \\
3520 \\
3820 \\
4120 \\
4150 \\
4210 \\
4040 \\
4110 \\
3910 \\
4010 \\
3930\end{array}$ & $\begin{array}{r}3700 \\
3900 \\
3660 \\
3710 \\
3720 \\
3540 \\
3710 \\
3460 \\
3200 \\
3130 \\
2740 \\
2830 \\
2840 \\
2780 \\
2800 \\
2820 \\
2720 \\
2540 \\
2610 \\
2520 \\
2460 \\
2420 \\
2400 \\
2300 \\
2090 \\
2010 \\
1570 \\
1310 \\
1300 \\
1330 \\
-\cdots\end{array}$ & $\begin{array}{r}\theta 1550 \\
\theta 1600 \\
1540 \\
1510 \\
1600 \\
1580 \\
1500 \\
1550 \\
1650 \\
1500 \\
1400 \\
1410 \\
1480 \\
1310 \\
1390 \\
\theta 1300 \\
\theta 1230 \\
\theta 1100 \\
\theta 990 \\
e 1000 \\
\theta 1100 \\
\theta 1150 \\
\theta 1200 \\
1240 \\
1260 \\
1290 \\
1390 \\
1380 \\
1340 \\
1340 \\
1350\end{array}$ & $\begin{array}{l}1330 \\
1260 \\
1150 \\
1240 \\
1230 \\
1220 \\
1210 \\
1060 \\
1110 \\
1180 \\
1190 \\
1130 \\
1140 \\
1110 \\
1060 \\
1100 \\
1110 \\
1140 \\
1050 \\
1060 \\
1090 \\
1100 \\
1130 \\
1200 \\
1130 \\
1050 \\
1040 \\
1180 \\
1130 \\
1130 \\
1080\end{array}$ & $\begin{array}{r}1090 \\
1090 \\
1080 \\
1030 \\
1010 \\
1030 \\
1090 \\
1080 \\
1050 \\
1030 \\
1040 \\
1040 \\
1040 \\
1060 \\
1090 \\
993 \\
995 \\
976 \\
981 \\
975 \\
1020 \\
1090 \\
1080 \\
1110 \\
983 \\
991 \\
1080 \\
997 \\
--2 \\
-2 \\
-2\end{array}$ & $\begin{array}{r}1020 \\
1070 \\
1020 \\
1010 \\
1090 \\
1090 \\
1010 \\
988 \\
1010 \\
995 \\
980 \\
917 \\
919 \\
1060 \\
1040 \\
1010 \\
1060 \\
1100 \\
1100 \\
1230 \\
1470 \\
1410 \\
1340 \\
1610 \\
1620 \\
2080 \\
2490 \\
2830 \\
2880 \\
2930 \\
3100\end{array}$ & $\begin{array}{l}2960 \\
3120 \\
3420 \\
3860 \\
3800 \\
3570 \\
3400 \\
3420 \\
3480 \\
3590 \\
3590 \\
3710 \\
3880 \\
3830 \\
4090 \\
4360 \\
4550 \\
4760 \\
5030 \\
5080 \\
4910 \\
4770 \\
4600 \\
4550 \\
4440 \\
4420 \\
4030 \\
3890 \\
4200 \\
3950 \\
-\cdots\end{array}$ & $\begin{array}{l}4540 \\
4960 \\
5230 \\
5460 \\
6170 \\
7020 \\
8050 \\
8750 \\
9120 \\
9360 \\
9470 \\
9430 \\
9390 \\
9450 \\
9290 \\
8950 \\
8180 \\
7520 \\
7110 \\
6740 \\
6140 \\
5490 \\
4880 \\
4630 \\
4450 \\
4420 \\
4240 \\
4480 \\
4460 \\
4800 \\
4870\end{array}$ & $\begin{array}{l}4860 \\
5010 \\
5220 \\
5150 \\
5060 \\
4760 \\
4630 \\
4200 \\
3830 \\
3730 \\
3450 \\
3330 \\
2880 \\
2890 \\
2830 \\
2400 \\
2570 \\
2480 \\
2370 \\
2410 \\
2340 \\
2290 \\
2390 \\
2580 \\
2430 \\
2390 \\
3030 \\
2850 \\
3050 \\
3070\end{array}$ & $\begin{array}{l}3690 \\
4090 \\
4500 \\
4630 \\
4640 \\
4680 \\
4700 \\
4700 \\
4580 \\
4170 \\
3560 \\
3590 \\
3650 \\
3640 \\
3640 \\
3340 \\
3190 \\
3100 \\
3270 \\
2850 \\
2790 \\
2780 \\
2840 \\
2740 \\
2580 \\
2440 \\
2350 \\
2220 \\
2210 \\
2260 \\
2480\end{array}$ & $\begin{array}{r}2640 \\
2470 \\
2370 \\
2320 \\
2040 \\
2030 \\
1780 \\
1490 \\
1530 \\
1460 \\
1260 \\
1320 \\
1320 \\
1150 \\
1070 \\
1100 \\
1150 \\
929 \\
977 \\
1240 \\
1370 \\
1340 \\
1330 \\
1270 \\
1350 \\
1300 \\
1430 \\
1200 \\
1230 \\
1200 \\
1350\end{array}$ & $\begin{array}{r}1320 \\
1180 \\
1550 \\
1280 \\
1540 \\
1210 \\
1390 \\
1550 \\
1830 \\
2360 \\
3050 \\
3290 \\
3560 \\
3520 \\
3570 \\
3490 \\
3520 \\
3150 \\
2800 \\
2700 \\
2490 \\
2410 \\
2530 \\
2220 \\
1950 \\
1910 \\
1570 \\
1570 \\
1670 \\
1570 \\
--0\end{array}$ \\
\hline $\begin{array}{l}\text { TOTAL } \\
\text { MEAN } \\
\text { MAX } \\
\text { MIN } \\
\text { AC-FT } \\
\text { CFSM } \\
\text { IN. }\end{array}$ & $\begin{array}{r}81680 \\
2635 \\
4210 \\
1100 \\
162000 \\
.36 \\
.42\end{array}$ & $\begin{array}{r}82120 \\
2737 \\
3900 \\
1300 \\
162900 \\
.37 \\
.42\end{array}$ & $\begin{array}{r}42230 \\
1362 \\
1650 \\
990 \\
83760 \\
.19 \\
.21\end{array}$ & $\begin{array}{r}35340 \\
1140 \\
1330 \\
1040 \\
70100 \\
.16 \\
.18\end{array}$ & $\begin{array}{r}29121 \\
1040 \\
1110 \\
975 \\
57760 \\
.14 \\
.15\end{array}$ & $\begin{array}{r}44479 \\
1435 \\
3100 \\
917 \\
88220 \\
.20 \\
.23\end{array}$ & $\begin{array}{r}121260 \\
4042 \\
5080 \\
2960 \\
240500 \\
.55 \\
.62\end{array}$ & $\begin{array}{r}207050 \\
6679 \\
9470 \\
4240 \\
410700 \\
.91 \\
1.05\end{array}$ & $\begin{array}{r}100480 \\
3349 \\
5220 \\
2290 \\
199300 \\
.46 \\
.51\end{array}$ & $\begin{array}{r}105900 \\
3416 \\
4700 \\
2210 \\
210100 \\
.47 \\
.54\end{array}$ & $\begin{array}{r}46016 \\
1484 \\
2640 \\
929 \\
91270 \\
.20 \\
.23\end{array}$ & $\begin{array}{r}67750 \\
2258 \\
3570 \\
1180 \\
134400 \\
.31 \\
.34\end{array}$ \\
\hline
\end{tabular}

STATISTICS OF MONTHLY MEAN DATA FOR WATER YEARS 1987 - 1991, BY WATER YEAR (WY)

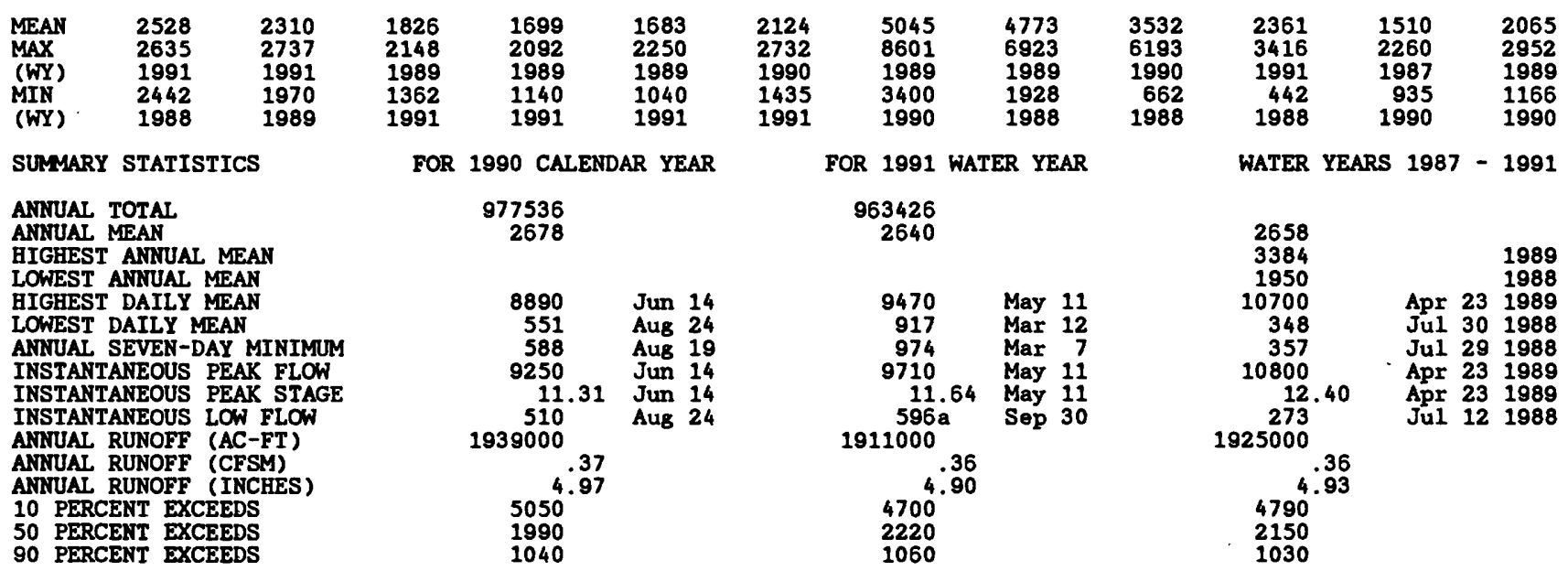

a Due to regulation.

- Estimated 
CROW WING RIVER BASIN

05243721 STRAIGHT RIVER AT COUNTY HIGHWAY 125 NEAR OSAGE, MN

LOCATION.--Lat $46^{\circ} 54^{\prime} 15^{\prime \prime}$, long $95^{\circ} 12^{\prime} 15^{\prime \prime}$, in NWł_WW sec.35, T.140 N., R. 36 W., Becker County, Hydrologic Unit 07010106 , on downstream side of culverts on County Highway 125, $2.7 \mathrm{mi}$ southest of Osage.

PERIOD OF RECORD.---October 1986 to current year (no winter records in 1987, 1990-91). Records of hourly. water temperature, available in files of the Geological Survey.

GAGE.--Water-stage recorder. Elevation of gage is 1,435 ft above National Geodetic Vertical Datum of 1929 , from topographic map.

REMARKS. - No estimated daily discharges. Records good.

DISCHARGE, CUBIC FEET PER SECOND, WATER YEAR OCTOBER 1990 TO SEPTEMBER 1991 DAILY MEAN VALUES

\begin{tabular}{|c|c|c|c|c|c|c|c|c|c|c|c|c|}
\hline DAY & $\propto T$ & Nov & DEC & JAN & FEB & MAR & APR & MAY & JUN & JUL & AUG & SEP \\
\hline $\begin{array}{l}1 \\
2 \\
3 \\
4 \\
5\end{array}$ & $\begin{array}{l}29 \\
28 \\
45 \\
45 \\
43\end{array}$ & $\begin{array}{l}--- \\
\overline{-} \\
\overline{-} \\
--\end{array}$ & $\begin{array}{l}--- \\
--- \\
--- \\
---\end{array}$ & $\begin{array}{l}--- \\
-\overline{-} \\
-- \\
--\end{array}$ & $\begin{array}{l}\cdots- \\
\cdots \\
\cdots- \\
\cdots-\end{array}$ & $\begin{array}{l}--- \\
-- \\
-- \\
--\end{array}$ & $\begin{array}{l}36 \\
36 \\
36 \\
37 \\
38\end{array}$ & $\begin{array}{l}53 \\
49 \\
48 \\
55 \\
53\end{array}$ & $\begin{array}{l}40 \\
40 \\
39 \\
36 \\
35\end{array}$ & $\begin{array}{l}42 \\
40 \\
40 \\
39 \\
38\end{array}$ & $\begin{array}{l}27 \\
27 \\
31 \\
35 \\
35\end{array}$ & $\begin{array}{l}30 \\
29 \\
34 \\
31 \\
29\end{array}$ \\
\hline $\begin{array}{r}6 \\
7 \\
8 \\
9 \\
10\end{array}$ & $\begin{array}{l}41 \\
37 \\
35 \\
33 \\
32\end{array}$ & $\begin{array}{l}--- \\
\cdots- \\
\cdots- \\
--\end{array}$ & $\begin{array}{l}--- \\
--- \\
--- \\
--\end{array}$ & $\begin{array}{l}--- \\
-- \\
-- \\
--\end{array}$ & $\begin{array}{l}-\cdots \\
\overline{-} \\
\overline{-} \\
\overline{-}\end{array}$ & $\begin{array}{l}--- \\
-\cdots \\
-\cdots \\
-\cdots\end{array}$ & $\begin{array}{l}38 \\
37 \\
36 \\
35 \\
34\end{array}$ & $\begin{array}{l}50 \\
48 \\
44 \\
41 \\
39\end{array}$ & $\begin{array}{l}34 \\
33 \\
33 \\
32 \\
34\end{array}$ & $\begin{array}{l}37 \\
36 \\
34 \\
34 \\
33\end{array}$ & $\begin{array}{l}33 \\
32 \\
31 \\
31 \\
30\end{array}$ & $\begin{array}{l}29 \\
28 \\
32 \\
36 \\
35\end{array}$ \\
\hline $\begin{array}{l}11 \\
12 \\
13 \\
14 \\
15\end{array}$ & $\begin{array}{l}31 \\
31 \\
30 \\
30 \\
30\end{array}$ & $\begin{array}{l}--- \\
--- \\
--- \\
---\end{array}$ & $\begin{array}{l}--- \\
--- \\
-\cdots \\
---\end{array}$ & $\begin{array}{l}--- \\
-\cdots \\
-- \\
--\end{array}$ & $\begin{array}{l}-\cdots \\
\cdots- \\
\cdots- \\
\cdots-\end{array}$ & $\begin{array}{l}--- \\
-\cdots \\
-\overline{-} \\
-\cdots\end{array}$ & $\begin{array}{l}34 \\
33 \\
33 \\
40 \\
46\end{array}$ & $\begin{array}{l}39 \\
38 \\
38 \\
38 \\
36\end{array}$ & $\begin{array}{l}34 \\
34 \\
32 \\
33 \\
35\end{array}$ & $\begin{array}{l}33 \\
34 \\
35 \\
33 \\
32\end{array}$ & $\begin{array}{l}30 \\
29 \\
28 \\
28 \\
28\end{array}$ & $\begin{array}{l}33 \\
32 \\
32 \\
40 \\
43\end{array}$ \\
\hline $\begin{array}{l}16 \\
17 \\
18 \\
19 \\
20\end{array}$ & $\begin{array}{l}30 \\
45 \\
47 \\
44 \\
42\end{array}$ & $\begin{array}{l}\ldots- \\
\cdots- \\
\cdots- \\
\cdots-\end{array}$ & $\begin{array}{l}\ldots- \\
\ldots \\
\cdots \\
\cdots\end{array}$ & $\begin{array}{l}--- \\
-- \\
-- \\
--\end{array}$ & 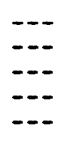 & $\begin{array}{r}--- \\
--- \\
- \\
33 \\
34\end{array}$ & $\begin{array}{l}49 \\
49 \\
47 \\
44 \\
41\end{array}$ & $\begin{array}{l}36 \\
35 \\
32 \\
30 \\
29\end{array}$ & $\begin{array}{l}34 \\
32 \\
32 \\
34 \\
37\end{array}$ & $\begin{array}{l}31 \\
31 \\
31 \\
31 \\
30\end{array}$ & $\begin{array}{l}28 \\
28 \\
28 \\
28 \\
27\end{array}$ & $\begin{array}{l}45 \\
43 \\
41 \\
39 \\
36\end{array}$ \\
\hline $\begin{array}{l}26 \\
27 \\
28 \\
29 \\
30 \\
31\end{array}$ & $\begin{array}{l}34 \\
35 \\
33 \\
32 \\
32 \\
32\end{array}$ & $\begin{array}{l}\cdots \\
\cdots \\
\cdots \\
\cdots- \\
\cdots-\end{array}$ & \begin{tabular}{l}
$\ldots-$ \\
\hdashline$\ldots$ \\
\hdashline- \\
\hdashline- \\
$-\cdots$
\end{tabular} & $\begin{array}{l}--- \\
\cdots- \\
\cdots- \\
--\end{array}$ & $\begin{array}{l}-- \\
\overline{-} \\
\overline{-} \\
\overline{-} \\
--\end{array}$ & $\begin{array}{l}43 \\
42 \\
40 \\
39 \\
37 \\
36\end{array}$ & $\begin{array}{r}34 \\
37 \\
39 \\
51 \\
61 \\
\end{array}$ & $\begin{array}{l}46 \\
43 \\
40 \\
39 \\
39 \\
40\end{array}$ & $\begin{array}{r}34 \\
35 \\
34 \\
35 \\
35 \\
-\end{array}$ & $\begin{array}{l}29 \\
28 \\
28 \\
27 \\
28 \\
27\end{array}$ & $\begin{array}{l}32 \\
31 \\
32 \\
33 \\
34 \\
33\end{array}$ & $\begin{array}{r}33 \\
31 \\
32 \\
30 \\
31 \\
\end{array}$ \\
\hline $\begin{array}{l}\text { TOTAL } \\
\text { MEAN } \\
\text { MAX } \\
\text { MIN } \\
\text { AC-FT }\end{array}$ & $\begin{array}{r}1111 \\
35.8 \\
47 \\
28 \\
2200\end{array}$ & $\begin{array}{l}--- \\
--\overline{-} \\
--- \\
--\end{array}$ & $\begin{array}{l}-\cdots \\
-\cdots \\
-\cdots \\
---\end{array}$ & $\begin{array}{l}--- \\
-\cdots \\
-\cdots \\
-\cdots\end{array}$ & $\begin{array}{l}--- \\
\cdots- \\
-\cdots \\
-\cdots\end{array}$ & $\begin{array}{l}--- \\
-\overline{-} \\
-- \\
--\end{array}$ & $\begin{array}{r}1188 \\
39.6 \\
61 \\
33 \\
2360\end{array}$ & $\begin{array}{r}1278 \\
41.2 \\
55 \\
29 \\
2530\end{array}$ & $\begin{array}{r}1052 \\
35.1 \\
40 \\
32 \\
2090\end{array}$ & $\begin{array}{r}1012 \\
32.6 \\
42 \\
27 \\
2010\end{array}$ & $\begin{array}{r}931 \\
30.0 \\
35 \\
27 \\
1850\end{array}$ & $\begin{array}{r}1016 \\
33.9 \\
45 \\
28 \\
2020\end{array}$ \\
\hline
\end{tabular}

STATISTICS OF MONTHLY MEAN DATA FOR WATER YEARS 1987 - 1991, BY WATER YEAR (WY)

\begin{tabular}{|c|c|c|c|c|c|c|c|c|c|c|c|c|}
\hline $\begin{array}{l}\text { MEAN } \\
\text { MAX } \\
\text { (WY) } \\
\text { MIN } \\
(W Y)\end{array}$ & $\begin{array}{l}38.2 \\
49.5 \\
1987 \\
31.9 \\
1989\end{array}$ & $\begin{array}{l}39.2 \\
39.7 \\
1988 \\
38.7 \\
1989\end{array}$ & $\begin{array}{l}38.9 \\
39.7 \\
1989 \\
38.2 \\
1988\end{array}$ & $\begin{array}{l}37.5 \\
37.5 \\
1988 \\
37.5 \\
1989\end{array}$ & $\begin{array}{l}35.2 \\
35.9 \\
1989 \\
34.6 \\
1988\end{array}$ & $\begin{array}{l}37.0 \\
38.2 \\
1988 \\
35.7 \\
1989\end{array}$ & $\begin{array}{l}43.7 \\
48.5 \\
1989 \\
39.6 \\
1991\end{array}$ & $\begin{array}{l}43.0 \\
46.5 \\
1989 \\
39.7 \\
1988\end{array}$ & $\begin{array}{l}37.2 \\
41.7 \\
1989 \\
33.0 \\
1988\end{array}$ & $\begin{array}{l}32.2 \\
40.2 \\
1987 \\
26.2 \\
1988\end{array}$ & $\begin{array}{l}32.7 \\
36.8 \\
1987 \\
26.8 \\
1990\end{array}$ & $\begin{array}{l}35.7 \\
41.5 \\
1989 \\
27.3 \\
1990\end{array}$ \\
\hline
\end{tabular}

SUMMARY STATISTICS

ANNUAL MEAN

HIGHEST ANNUAL MEAN

LOWEST ANNUAL MEAN

HIGHEST DAILY MEAN

LOWEST DAILY MEAN

ANNUAL SEVEN-DAY MINIMUM

INSTANTANEOUS PEAK FLOW

INSTANTANEOUS PEAK STAGE

INSTANTANEOUS LOW FLOW

ANNUAL RUNOFF (AC-FT)

10 PERCENT EXCEEDS

50 PERCENT EXCEEDS

90 PERCENT EXCEEDS

a Occurred Aug. 10, 16, Sept. 11, 15

a Occurred Aug. 10, 16, Sept. 11, 15
b Occurred July 19, 21, 22, 23, 26, 1988.

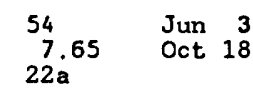

22.65
FOR 1990 CALENDAR YEAR FOR 1991 WATER YEAR

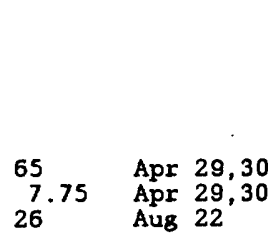

WATER YEARS 1987 - 1991

37.5

38.4

36.

6

24

7.86

$2 \mathrm{ib}$

27150

48
37

37
28

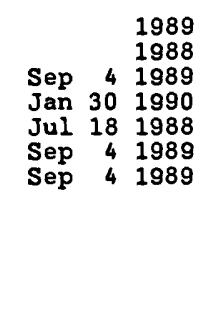


CROW WING RIVER BASIN

05243725 STRAIGHT RIVER NEAR PARK RAPIDS, MN

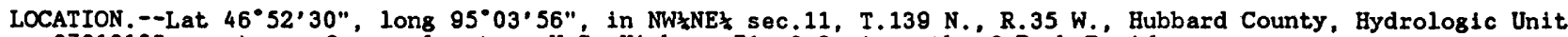
07010106 , upstream from culvert on U.S. Highway $71, \dot{3} .2$ mi south of Park Rapids.

DRAINAGE AREA. $-53.2 \mathrm{mi}^{2}$.

PERIOD OF RECORD.--Occasional low-flow measurements, water years 1970-71, 1973, 1975-76. October 1986 to current year (no winter records in 1987, 1990-91). Records of hourly water temperature, available in files of the Geological Survey.

GAGE.--Water-stage recorder. Elevation of gage is 1,400 ft above Nat1onal Geodetic Vertical Datum of 1929 , from topographic map.

REMARKS. --Records good except those for estimated daily discharges, which are fair.

EXTREMES OUTSIDE PERIOD OF RECORD. --A discharge of $35 \mathrm{ft}^{3} / \mathrm{s}$ was measured Aug. 4 , 1976 .

DISCHARGE, CUBIC FEET PER SECOND, WATER YEAR OCTOBER 1990 TO SEPTEMBER 1991 DAIIYY MEAN VALUES

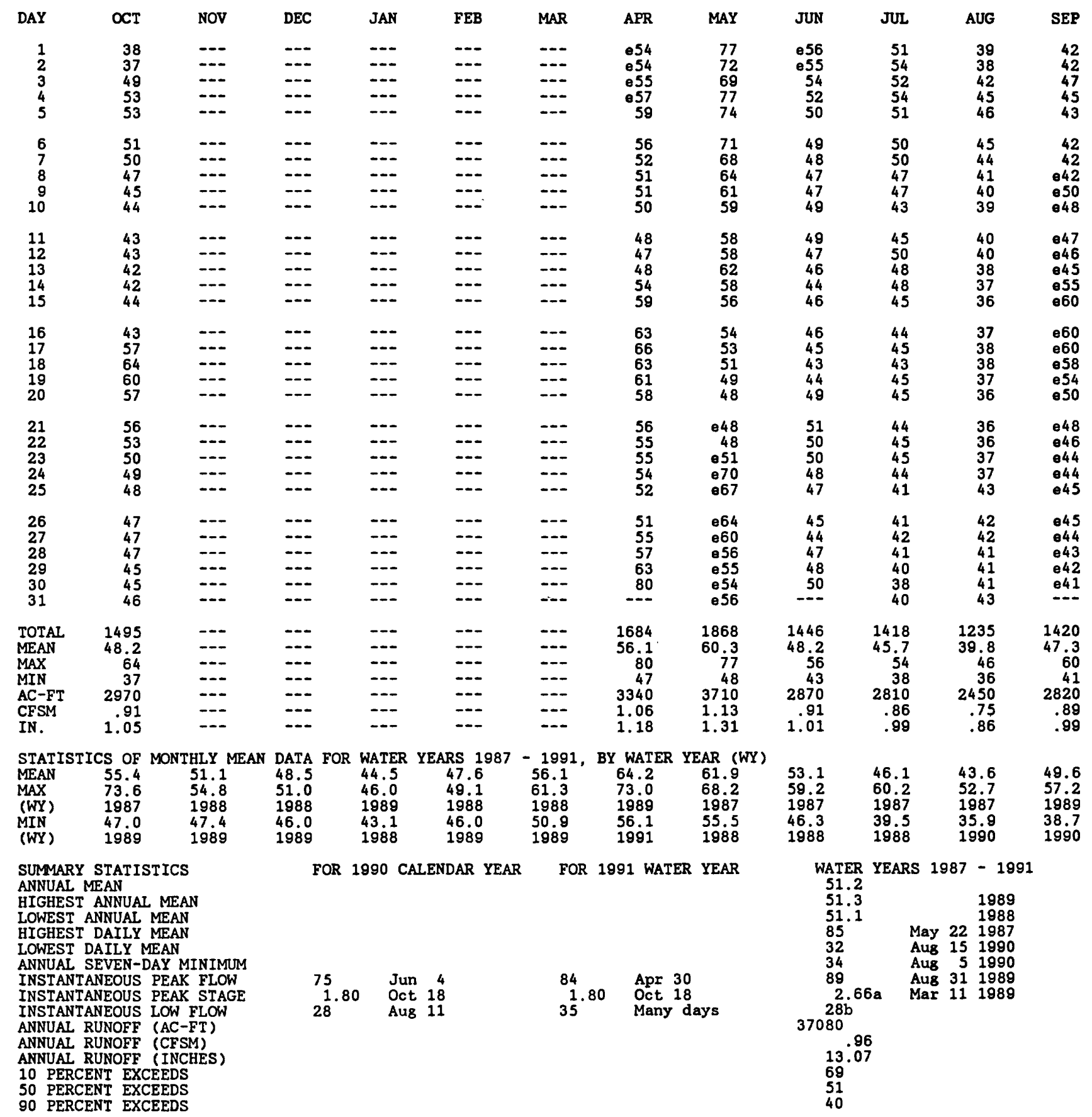

a Backwater from ice.

b Occurred July 22, 23, 1988, Aus. 11, 1990.

e Estimated. 


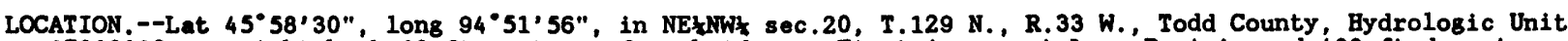
07010108, on right bank $90 \mathrm{ft}$ upstream from bridge on First Avenue at Long Prairie and $400 \mathrm{ft}$ downstream from Venewitz Creek.

DRAINAGE AREA. $--432 \mathrm{mi}^{2}$.

PERIOD OF RECORD.--October 1971 to current year.

GAGE.--Water-stage recorder. Datum of gage is $1,281.74 \mathrm{ft}$ above National Geodetic Vertical Datum of 1929.

REMARKS.--Records good except those for estimated daily discharges, which are fair.

DISCHARGE, CUBIC FEET PER SECOND, WATER YEAR OCTOBER 1990 TO SEPTEMBER 1991

\begin{tabular}{|c|c|c|c|c|c|c|c|c|c|c|c|c|}
\hline DAY & ○I & NOV & DEC & JAN & FEB & MAR & APR & MAY & JUN & JUL & AUG & SEP \\
\hline $\begin{array}{l}1 \\
2 \\
3 \\
4 \\
5\end{array}$ & $\begin{array}{r}35 \\
37 \\
101 \\
92 \\
97\end{array}$ & $\begin{array}{l}55 \\
51 \\
49 \\
47 \\
45\end{array}$ & $\begin{array}{l}e 28 \\
e 28 \\
e 27 \\
e 26 \\
e 26\end{array}$ & $\begin{array}{l}\text { e13 } \\
e 13 \\
e 12 \\
e 12 \\
e 12\end{array}$ & $\begin{array}{r}112 \\
e 12 \\
e 12 \\
e 14 \\
16\end{array}$ & $\begin{array}{l}25 \\
25 \\
25 \\
25 \\
26\end{array}$ & $\begin{array}{l}219 \\
171 \\
144 \\
139 \\
139\end{array}$ & $\begin{array}{l}373 \\
383 \\
387 \\
405 \\
410\end{array}$ & $\begin{array}{l}174 \\
183 \\
197 \\
203 \\
203\end{array}$ & $\begin{array}{l}335 \\
325 \\
306 \\
299 \\
297\end{array}$ & $\begin{array}{l}187 \\
187 \\
185 \\
180 \\
170\end{array}$ & $\begin{array}{l}92 \\
92 \\
89 \\
85 \\
82\end{array}$ \\
\hline $\begin{array}{r}6 \\
7 \\
8 \\
9 \\
10\end{array}$ & $\begin{array}{r}103 \\
92 \\
80 \\
70 \\
64\end{array}$ & $\begin{array}{l}45 \\
42 \\
36 \\
42 \\
43\end{array}$ & $\begin{array}{l}e 26 \\
e 26 \\
e 26 \\
e 27 \\
e 28\end{array}$ & $\begin{array}{r}e 12 \\
e 12 \\
e 12 \\
12 \\
12\end{array}$ & $\begin{array}{r}17 \\
19 \\
22 \\
25 \\
e 26\end{array}$ & $\begin{array}{l}28 \\
28 \\
28 \\
28 \\
31\end{array}$ & $\begin{array}{l}140 \\
141 \\
136 \\
138 \\
141\end{array}$ & $\begin{array}{l}400 \\
377 \\
357 \\
337 \\
311\end{array}$ & $\begin{array}{l}192 \\
169 \\
151 \\
148 \\
152\end{array}$ & $\begin{array}{l}311 \\
324 \\
322 \\
314 \\
300\end{array}$ & $\begin{array}{l}166 \\
162 \\
162 \\
154 \\
157\end{array}$ & $\begin{array}{r}77 \\
77 \\
117 \\
137 \\
140\end{array}$ \\
\hline $\begin{array}{l}11 \\
12 \\
13 \\
14 \\
15\end{array}$ & $\begin{array}{l}55 \\
49 \\
47 \\
44 \\
41\end{array}$ & $\begin{array}{l}43 \\
41 \\
41 \\
41 \\
39\end{array}$ & $\begin{array}{l}30 \\
30 \\
37 \\
28 \\
28\end{array}$ & $\begin{array}{l}12 \\
12 \\
12 \\
12 \\
12\end{array}$ & $\begin{array}{r}\bullet 27 \\
28 \\
28 \\
28 \\
28\end{array}$ & $\begin{array}{l}37 \\
39 \\
37 \\
37 \\
41\end{array}$ & $\begin{array}{l}135 \\
130 \\
154 \\
217 \\
266\end{array}$ & $\begin{array}{l}285 \\
264 \\
249 \\
235 \\
221\end{array}$ & $\begin{array}{l}139 \\
181 \\
191 \\
182 \\
182\end{array}$ & $\begin{array}{l}291 \\
331 \\
308 \\
282 \\
262\end{array}$ & $\begin{array}{l}147 \\
145 \\
147 \\
142 \\
145\end{array}$ & $\begin{array}{l}140 \\
143 \\
148 \\
158 \\
162\end{array}$ \\
\hline $\begin{array}{l}16 \\
17 \\
18 \\
19 \\
20\end{array}$ & $\begin{array}{l}41 \\
62 \\
77 \\
74 \\
81\end{array}$ & $\begin{array}{l}39 \\
38 \\
38 \\
38 \\
38\end{array}$ & $\begin{array}{r}28 \\
28 \\
26 \\
23 \\
e 21\end{array}$ & $\begin{array}{r}12 \\
12 \\
12 \\
+12 \\
-12\end{array}$ & $\begin{array}{l}28 \\
26 \\
26 \\
26 \\
25\end{array}$ & $\begin{array}{l}30 \\
29 \\
29 \\
50 \\
90\end{array}$ & $\begin{array}{l}300 \\
345 \\
400 \\
425 \\
426\end{array}$ & $\begin{array}{l}214 \\
203 \\
193 \\
190 \\
188\end{array}$ & $\begin{array}{l}173 \\
163 \\
161 \\
163 \\
201\end{array}$ & $\begin{array}{l}243 \\
233 \\
236 \\
235 \\
227\end{array}$ & $\begin{array}{l}146 \\
147 \\
145 \\
144 \\
145\end{array}$ & $\begin{array}{l}163 \\
162 \\
159 \\
149 \\
142\end{array}$ \\
\hline $\begin{array}{l}21 \\
22 \\
23 \\
24 \\
25\end{array}$ & $\begin{array}{l}80 \\
73 \\
70 \\
64 \\
60\end{array}$ & $\begin{array}{l}38 \\
38 \\
37 \\
32 \\
24\end{array}$ & $\begin{array}{l}e 19 \\
e 18 \\
e 17 \\
e 16 \\
e 16\end{array}$ & $\begin{array}{l}e 12 \\
e 12 \\
e 12 \\
e 12 \\
e 12\end{array}$ & $\begin{array}{l}25 \\
25 \\
25 \\
25 \\
25\end{array}$ & $\begin{array}{l}113 \\
118 \\
129 \\
128 \\
198\end{array}$ & $\begin{array}{l}417 \\
407 \\
383 \\
337 \\
290\end{array}$ & $\begin{array}{l}181 \\
174 \\
169 \\
168 \\
179\end{array}$ & $\begin{array}{l}221 \\
228 \\
237 \\
231 \\
224\end{array}$ & $\begin{array}{l}225 \\
225 \\
218 \\
216 \\
208\end{array}$ & $\begin{array}{l}143 \\
145 \\
138 \\
133 \\
141\end{array}$ & $\begin{array}{l}138 \\
135 \\
132 \\
127 \\
126\end{array}$ \\
\hline $\begin{array}{l}26 \\
27 \\
28 \\
29 \\
30 \\
31\end{array}$ & $\begin{array}{l}58 \\
54 \\
51 \\
50 \\
51 \\
59\end{array}$ & $\begin{array}{r}30 \\
35 \\
30 \\
30 \\
30 \\
--\end{array}$ & $\begin{array}{l}e 15 \\
e 15 \\
e 14 \\
e 14 \\
e 14 \\
e 13\end{array}$ & $\begin{array}{l}e 12 \\
e 12 \\
e 12 \\
e 12 \\
e 12 \\
e 12\end{array}$ & $\begin{array}{r}25 \\
25 \\
25 \\
\hdashline-- \\
\hdashline--\end{array}$ & $\begin{array}{l}294 \\
339 \\
233 \\
234 \\
255 \\
258\end{array}$ & $\begin{array}{l}259 \\
249 \\
248 \\
271 \\
340 \\
---\end{array}$ & $\begin{array}{l}177 \\
168 \\
159 \\
158 \\
158 \\
169\end{array}$ & $\begin{array}{l}225 \\
243 \\
257 \\
270 \\
283 \\
---\end{array}$ & $\begin{array}{l}200 \\
196 \\
200 \\
197 \\
194 \\
191\end{array}$ & $\begin{array}{r}139 \\
134 \\
125 \\
106 \\
102 \\
96\end{array}$ & $\begin{array}{l}121 \\
115 \\
111 \\
106 \\
107\end{array}$ \\
\hline $\begin{array}{l}\text { TOTAL } \\
\text { MEAN } \\
\text { MAX } \\
\text { MIN } \\
\text { AC-FT } \\
\text { CFSM } \\
\text { IN. }\end{array}$ & $\begin{array}{r}2012 \\
64.9 \\
103 \\
35 \\
3990 \\
.15 \\
.17\end{array}$ & $\begin{array}{r}1175 \\
39.2 \\
55 \\
24 \\
2330 \\
.09 \\
.10\end{array}$ & $\begin{array}{r}718 \\
23.2 \\
37 \\
13 \\
1420 \\
.05 \\
.06\end{array}$ & $\begin{array}{r}374 \\
12.1 \\
13 \\
12 \\
742 \\
.03 \\
.03\end{array}$ & $\begin{array}{r}645 \\
23.0 \\
28 \\
12 \\
1280 \\
.05 \\
.06\end{array}$ & $\begin{array}{r}2987 \\
96.4 \\
339 \\
25 \\
5920 \\
.22 \\
.26\end{array}$ & $\begin{array}{r}7507 \\
250 \\
426 \\
130 \\
14890 \\
.58 \\
.65\end{array}$ & $\begin{array}{r}7842 \\
253 \\
410 \\
158 \\
15550 \\
.59 \\
.68\end{array}$ & $\begin{array}{r}5927 \\
198 \\
283 \\
139 \\
11760 \\
.46 \\
.51\end{array}$ & $\begin{array}{r}8051 \\
260 \\
335 \\
191 \\
15970 \\
.60 \\
.69\end{array}$ & $\begin{array}{r}4565 \\
147 \\
187 \\
96 \\
9050 \\
.34 \\
.39\end{array}$ & $\begin{array}{r}3732 \\
124 \\
163 \\
77 \\
7400 \\
.29 \\
.32\end{array}$ \\
\hline
\end{tabular}

STATISTICS OF MONTHLY MEAN DATA FOR WATER YEARS 1972 - 1991, BY WATER YEAR (WY)

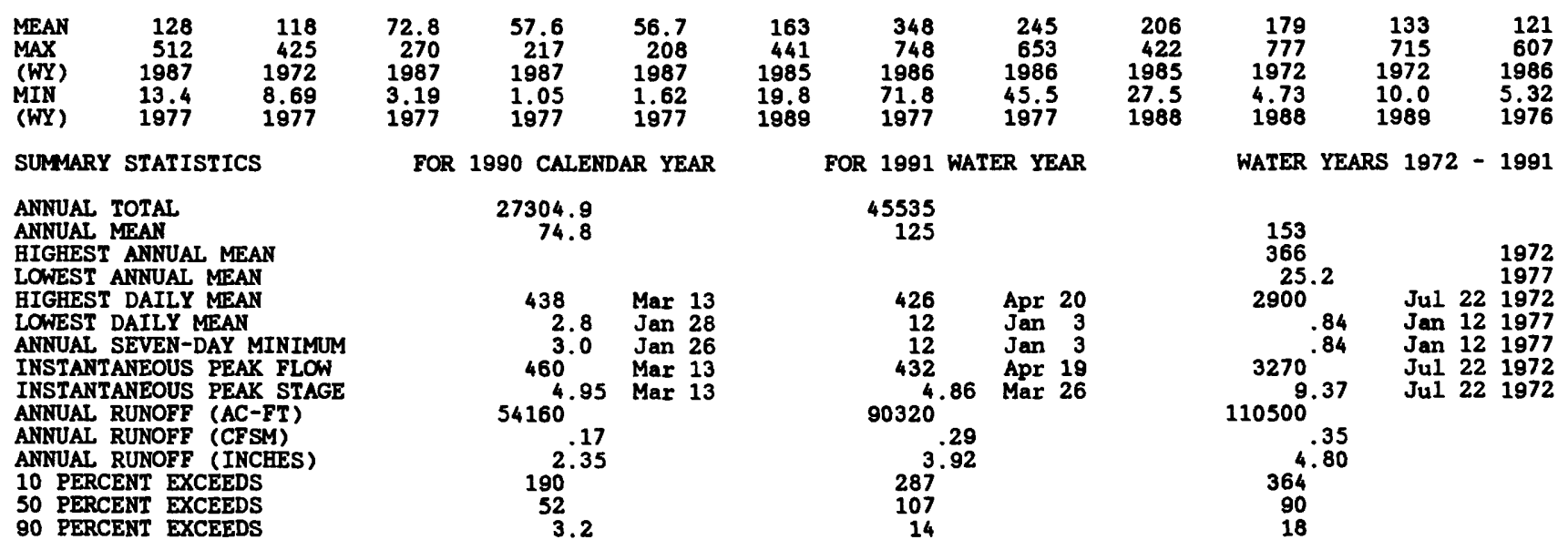


LOCATION.--Lat $46^{\circ} 24^{\prime} 40^{\prime \prime}$, long $94^{\circ} 21^{\prime} 26^{\prime \prime}$, In NF sec. 20, T.134 N., R.29 W., Cass County, Hydrologic Unit 07010106, In pool of dam on Guli River, 800 ft south of out let of Gull Lake, 0.2 ml upstream from Gull Lake Dam, and 8 mi northwest of Brainerd.

DRAINAGE AREA. $--287 \mathrm{ml}^{2}$.

PERIOD OF RECORD.--August 1911 to current year. Prior to October 1941 monthend contents only, published in WSP 1308. Published as Gull Lake Reservoir October 1941 to September 1956.

GAGE.--Water-stage recorder. Datum of gage is National Geodetic Vertical Datum of 1929 (levels by U.S. Army Corps of Engineers). Prior to Aug. 10, 1949, nonrecording gage 800 ft north of present site at same datum. Aug. 11 , 1949, to June 30, 1973, water-stage recorder at present site and at datum $1,188.14 \mathrm{ft}$, adjustment of 1912 .

REMARKS.--Reservolr is formed by Gull Lake and several other natural lakes controlled by concrete dam completed in 1913; storage began in 1912. Capacity between elevation 1,192.75 ft and 1,194.75 ft (maximum allowable range and normal operating range) is 26,008 acre-ft. Contents shown herein are contents above elevation $1,188.00 \mathrm{ft}$. Prior to September 1978, published contents as contents above elevat1on $1,188.75 \mathrm{ft}$. Water 1s used to benefit navigation on Mississippi River below Minneapolis.

COOPERATION.--Records were provided by U.S. Army Corps of Engineers.

EXTREMES FOR PERIOD OF RECORD.--Maximum contents, 74, 800 acre-ft, capacity table then in use, June 30 , 1914, elevation, 1,195.05 ft; minimum observed, 22,250 acre-ft, capacity table then in use, Mar, 20, 1924, elevation, $1,190.75 \mathrm{ft}$.

EXTREMES FOR CURRENT YEAR.--Maximum contents, 64,030 acre-ft, July 1, elevation, 1,194.23 ft; minimum, 54,260 acre-ft, Jan. 10, elevation, $1,193.48 \mathrm{ft}$.

MONTHEND ELEVATION AND CONTENTS, WATER YEAR OCTOBER 1990 TO SEPTEMBER 1991

\section{Date}

Sept. 30

Oct. 31

Nov. 30

Dec. 31

CAL YR 1990

Jan, 31

Feb. 28

Mar. 31

Apr. 30

May 31

June 30

July 31

Aug . 31

Sept. 30

WIR YR 1991

\section{Elevation} (feet)

$1,193.54$

$1,193.74$

$1,193.59$

$1,193.55$

$1,193.55$

$1,193.61$

$1,193.67$

$1,194.13$

$1,194.02$

$1,194.12$

$1,194.01$

$1,193.91$

$1,193.80$
Contents (acre-feet)

55,040

57,630

55,680

55,160

55,160

55,940

56,720

62,720

61,280

62,590

61,150

59,850

58,420
Change in contents (acre-feet) 
LOCATION.--Lat $46^{\circ} 24 \cdot 40^{\prime \prime}$, long $94^{\circ} 21^{\prime} 12^{\prime \prime}$, in sec.20, T.134 N., R.29 W., Cass County, Hydrologic Unit 07010106, in headwater and tailwater of dam at outlet of Gull Lake, $8 \mathrm{mi}$ northwest of Brainerd.

DRAINAGE AREA. $--287 \mathrm{mi}^{2}$.

PERIOD OF RECORD.--August 1911 to current year. Monthly discharge only for some periods, published in WSP 1308.

Published as "Gull Lake Reservoir" 1929.

GAGE. - Water-stage recorder on headwater and nonrecording gage on tailwater. Datum of gages is National Geodetic Vertical Datum of 1929 (levels by U.S. Army Corps of Engineers). August 1911 to May 23, 1929, and Dec. 1, 1929, to Aug 1, 1949, both gages were nonrecording gages at same site and datum in use. May 24 to Nov. 30, 1929, nonrecording gage $500 \mathrm{ft}$ downstream at different datum. Aug. 2, 1949, to June 30, 1973, at present sites with datum of gage at $1,188.14 \mathrm{ft}$, adjustment of 1912 .

REMARKS.--Discharge computed at dam on basis of modified weix formulas, the head being obtained from twice-daily readings on tailwater gage and from headwater recorder. Flow completely regulated by Gull Lake (station 05246500 ).

COOPERATION.--Computations of daily discharge were provided by U.S. Army Corps of Engineers.

AVERAGE DISCHARGE (unadjusted). --80 years, $109 \mathrm{ft}^{3} / \mathrm{s}, 5.16 \mathrm{in} / \mathrm{yr}$.

EXTREMES FOR PERIOD OF RECORD, --Maximum daily discharge, 1,120 $\mathrm{ft}^{3} / \mathrm{s}$, May 15, 1938; no flow at times.

EXTREMES FOR CURRENT YEAR.--Maximum daily discharge, $400 \mathrm{ft} 3 / \mathrm{s}$, May 8-10; minimum daily, $18 \mathrm{ft} 3 / \mathrm{s}, 0 \mathrm{ct} .1-3 ; 13-16$. DISCHARGE, CUBIC FEET PER SECOND, WATER YEAR OCTOBER 1990 TO SEPTEMBER 1991 DAILY MEAN VALUES

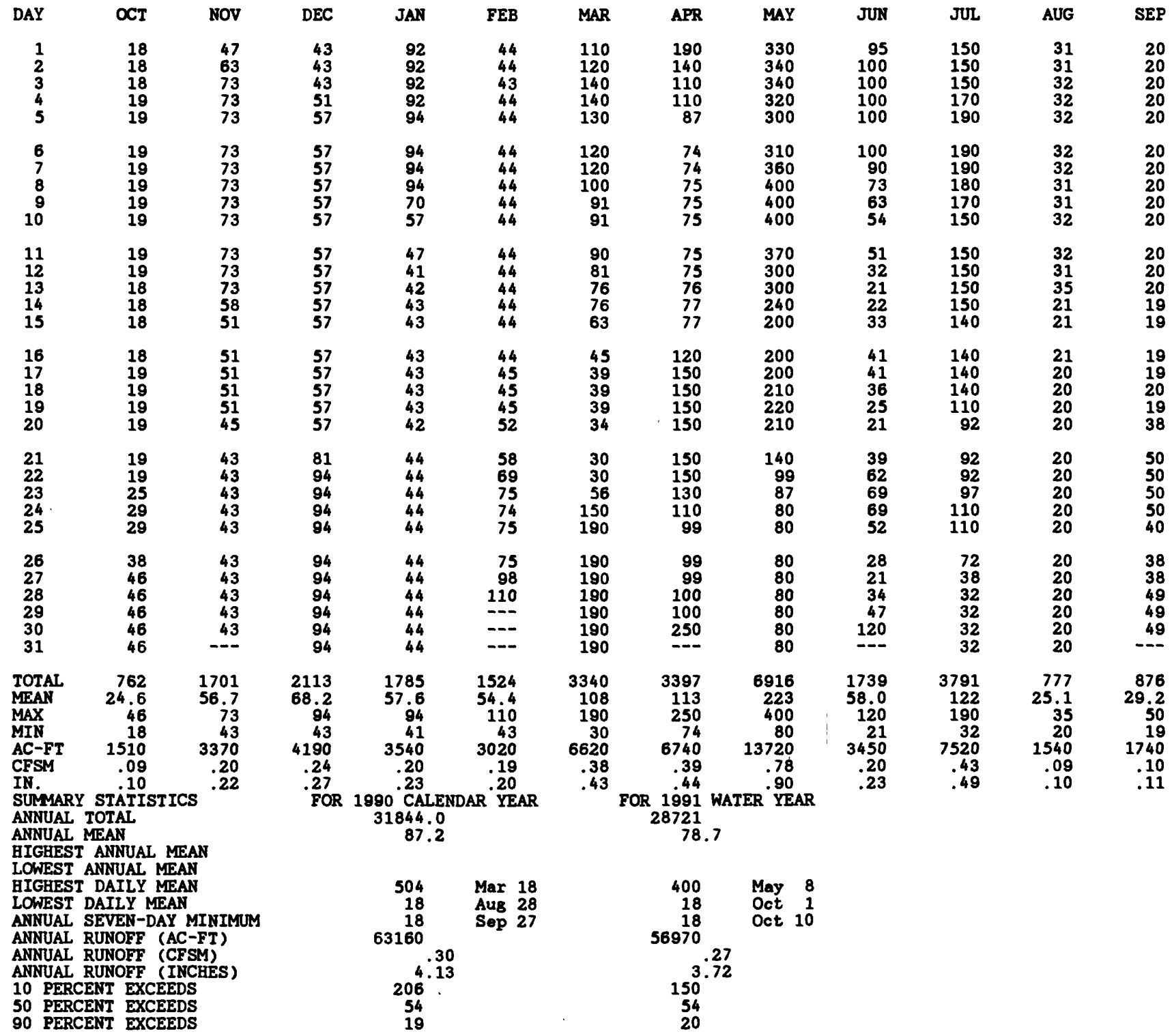


CRON WIHG RIVER BASIN

05247500 CRON WING RIVER MEAR PILIAGER, Mi

LOCATION.--Lat $46^{\circ} 18^{\prime} 18^{\prime \prime}$, Iong $94^{\circ} 22^{\prime} 38^{\prime \prime}$, in SWHEEł sec.30, T.133 N., R.29 W., Cass County, Hydrologic Unit 07010106 , at Sylvan dam powerplant of Minnesota Power Co., $3.6 \mathrm{mi}$ above mouth and 4.9 mi southeast of Pillager.

DRAINAGE AREA. $--3,520 \mathrm{mi}^{2}$, approximately.

PERIOD OF RECORD. - October 1968 to September 1986, October 1987 to current year. Records for August 1924 to September 1968 available in files of the Minnesota District Office.

GAGE. -Water stage recorder. Datum of gage is 1,151.00 ft, adjustment of 1912 . Prior to January 16 , 1991, staff gage attached to retaining wali approximately 20 ft below the turbine outlet bays. Datum of staff gage is $1,150.00 \mathrm{ft}$, adjustment of 1912 .

REMARKS.--Records for period October 1, 1990 to April 24, 1991 are poor. Records for the period of April 25, 1991 to September 30, 1991 are good except those for estimated daily discharge, which are fair.

Discharge computed on the basis of powerplant records prior to January 16, 1991 . Records for Oct. 1 , 1968 to Sept. 30, 1975, were adjusted for storage change in the Sylvan dam reservoir. Flow partly regulated by powerplants and Gull Lake (station 05246500).

COOPERATION.--Records collected by Minnesota Power Co. under general supervision of Geological Survey prior to February 1991, in connection with a Federal Power Commission project.

EXTREMES OUTSIDE PERIOD OF RECORD,-Maximum daily discharge since 1924, $18,300 \mathrm{ft} 3 / 8$, Apr. 14,1965 .

DISCHARGE, CUBIC. FEET PER SECOND, WATER YEAR OCTOBER 1990 TO SEPTEMBER 1991

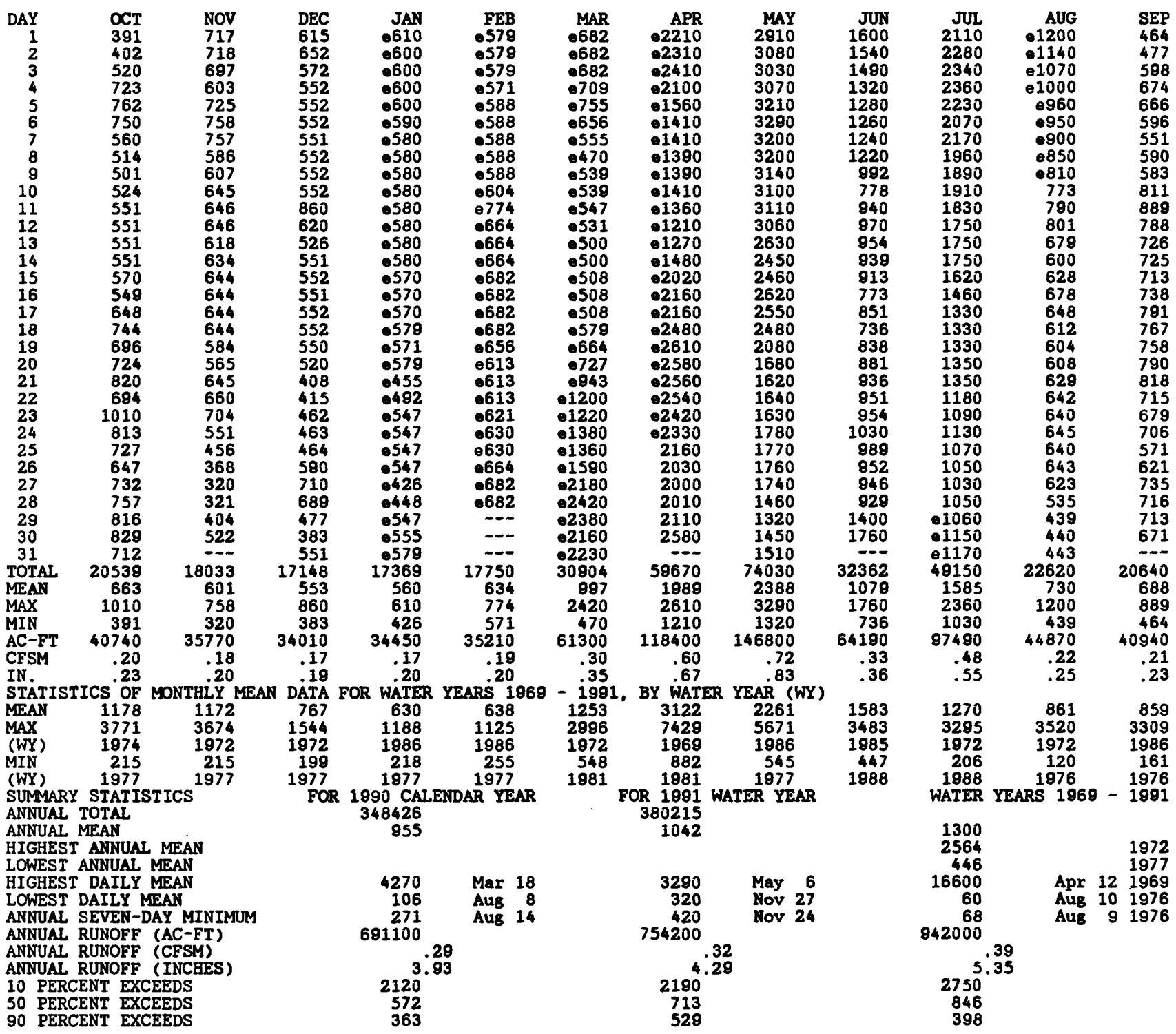

- Estimated. 
LOCATION. --Lat $46^{\circ} 10^{\prime} 50^{\prime \prime}$, long $94^{\circ} 21^{\prime} 56^{\prime \prime}$, in SEzWW sec.27, T.43 N., R.32 W., Crow Wing County, Hydrologic Unit 07010104 , on left bank 600 ft upstsream from Nokasippi River, 1.0 mile north of Fort Ripley, and at mile 982. 1 upstream from Ohio River.

DRAINAGE AREA. $--11,010 \mathrm{mi}^{2}$, approximately.

PERIOD OF RECORD.--June 1987 to current year. Operated as high-flow partial-record station October 1971 to June 1987. Prior to Oct. 1971 stage records collected by U.S. Weather Service.

GAGE.--Water-stage recorder. Datum of 8 age is 1,133.84 ft above National Geodetic Vertical Datum of 1929 . Aug. 1904 to June 1987 nonrecording gages at different datums.

REMARKS.--Records good except those for estimated daily discharges, which are fair. Flow partly regulated by powerplants and Winnibigoshish, Leech, Pokegama, Sandy, and Guli Lakes and by Pine River Reservoir (see stations 05201000, 05206000, 05210500, 05218500, 05230500, 05246500).

DISCHARGE, CUBIC FEET PER SECOND, WATER YEAR OCTOBER 1990 TO SEPTEMBER 1991

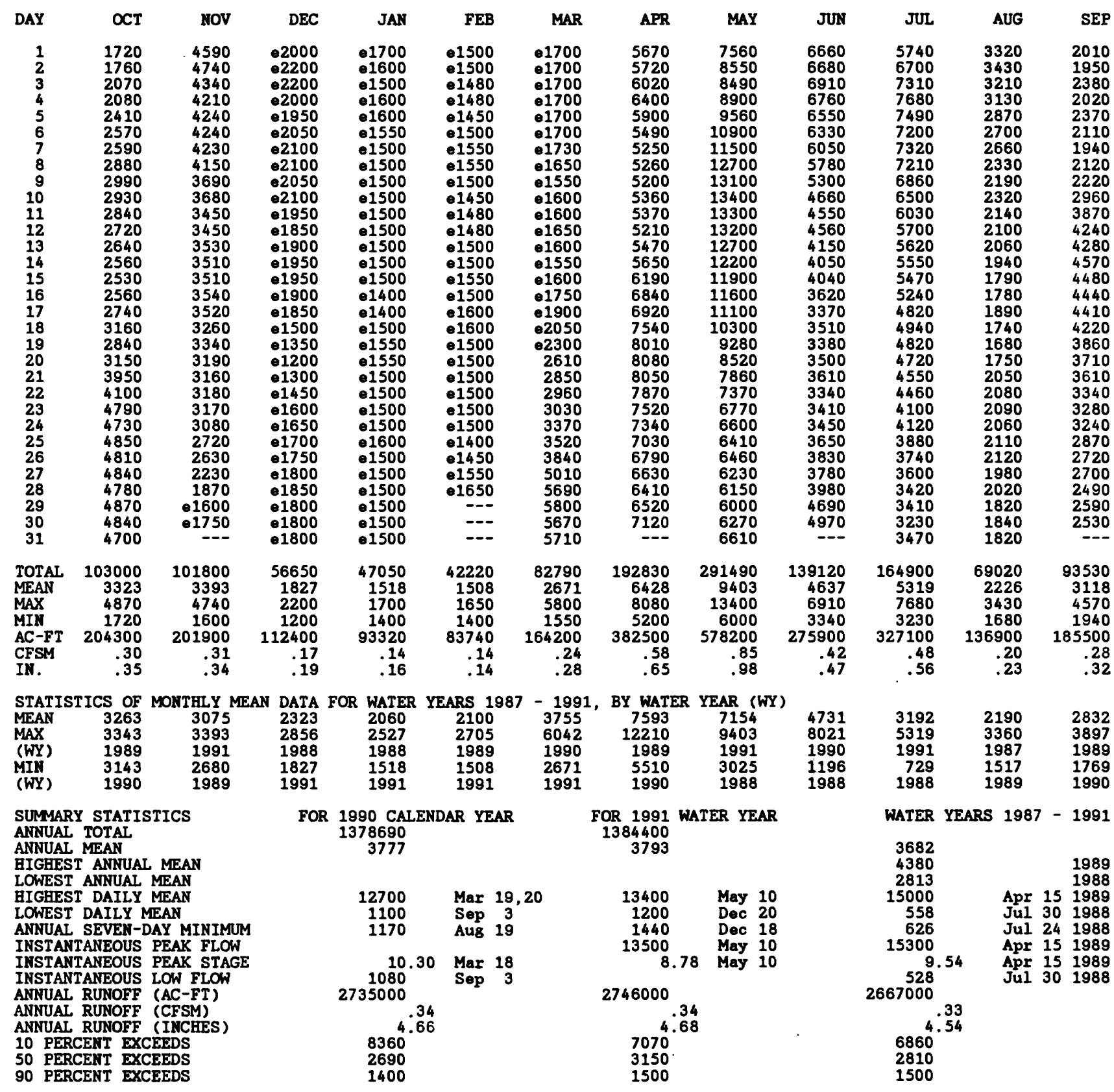


MISSISSIPPI RIVER MAIN STEM

05267000 MISSISSIPPI RIVER NEAR ROYALTON, MN

LOCATION,--Lat $45^{\circ} 51^{\prime} 41^{\prime \prime}$, long $94^{\circ} 21^{\prime} 33^{\prime \prime}$, in lot 2 , sec.20, T.39 N., R.32 W. , Morrison County, Hydrologic Un1t 07010104 at plant of Minnesota Power Co., $4 \mathrm{mi}$ northwest of Royalton, $4.5 \mathrm{mi}$ downstream from Swan River, and at mile 956 upstream from Ohio River.

DRAINAGE AREA. --11,600 $\mathrm{mi}^{2}$, approximately.

\section{WATER-DISCHARGE RECORDS}

PERIOD OF RECORD.--March 1924 to current year.

REMARKS.--No estimated daily discharges. Records poor. Discharge computed based on powerplant records. Flow partiy regulated by powerplants and Winnibigoshish, Leech, Pokegama, Sandy, and Gull Lakes and by Pine River Reservoir (see stations 05201000, 05206000, 0521050́, 05218500, 05230500, 05246500).

COOPERATION.--Records collected by Minnesota Power Co. under general supervision of Geological Survey, in connection with a Federal Power Commission project.

DISCHARGE, CUBIC FEET PER SECOND, WATER YEAR OCTOBER 1990 TO SEPTEMBER 1991

\begin{tabular}{|c|c|c|c|c|c|c|c|c|c|c|c|c|}
\hline DAY & ळт & NOV & DEC & JAN & FEB & MAR & APR & MAY & JUN & JUL & AUG & SEP \\
\hline $\begin{array}{l}1 \\
2 \\
3 \\
4 \\
5\end{array}$ & $\begin{array}{l}1620 \\
1420 \\
2000 \\
2110 \\
1830\end{array}$ & $\begin{array}{l}4380 \\
4410 \\
4300 \\
3940 \\
4030\end{array}$ & $\begin{array}{l}1860 \\
2120 \\
1970 \\
1830 \\
1750\end{array}$ & $\begin{array}{l}1970 \\
1630 \\
1590 \\
1620 \\
1710\end{array}$ & $\begin{array}{l}1600 \\
1260 \\
1460 \\
1340 \\
1420\end{array}$ & $\begin{array}{l}1500 \\
1360 \\
1580 \\
1470 \\
1400\end{array}$ & $\begin{array}{l}\mathbf{5 5 5 0} \\
5920 \\
5860 \\
6780 \\
6320\end{array}$ & $\begin{array}{r}8200 \\
9380 \\
9350 \\
10000 \\
10200\end{array}$ & $\begin{array}{l}6220 \\
6480 \\
6910 \\
6990 \\
6240\end{array}$ & $\begin{array}{l}5020 \\
6800 \\
7560 \\
7950 \\
7590\end{array}$ & $\begin{array}{l}2790 \\
3330 \\
2720 \\
2810 \\
2490\end{array}$ & $\begin{array}{l}1380 \\
1710 \\
1720 \\
1940 \\
1620\end{array}$ \\
\hline $\begin{array}{r}6 \\
7 \\
8 \\
9 \\
10\end{array}$ & $\begin{array}{l}2510 \\
2140 \\
2480 \\
2570 \\
2680\end{array}$ & $\begin{array}{l}4130 \\
3920 \\
3780 \\
3150 \\
3250\end{array}$ & $\begin{array}{l}1920 \\
2330 \\
2010 \\
2090 \\
2080\end{array}$ & $\begin{array}{l}1710 \\
1650 \\
1500 \\
1550 \\
1500\end{array}$ & $\begin{array}{l}1390 \\
1390 \\
1420 \\
1580 \\
1400\end{array}$ & $\begin{array}{l}1650 \\
1630 \\
1620 \\
1270 \\
1580\end{array}$ & $\begin{array}{l}5800 \\
4980 \\
4890 \\
4980 \\
5120\end{array}$ & $\begin{array}{l}12100 \\
12100 \\
13100 \\
13200 \\
13800\end{array}$ & $\begin{array}{l}6470 \\
5680 \\
5880 \\
5130 \\
4480\end{array}$ & $\begin{array}{l}7250 \\
7210 \\
6960 \\
6810 \\
6390\end{array}$ & $\begin{array}{l}2320 \\
2300 \\
2150 \\
1900 \\
2080\end{array}$ & $\begin{array}{l}1970 \\
1570 \\
2020 \\
2030 \\
2140\end{array}$ \\
\hline $\begin{array}{l}11 \\
12 \\
13 \\
14 \\
15\end{array}$ & $\begin{array}{l}2290 \\
2500 \\
2380 \\
2200 \\
2150\end{array}$ & $\begin{array}{l}3560 \\
3570 \\
3220 \\
3070 \\
3200\end{array}$ & $\begin{array}{l}2120 \\
1940 \\
1860 \\
1620 \\
1980\end{array}$ & $\begin{array}{l}1430 \\
1570 \\
1550 \\
1380 \\
1200\end{array}$ & $\begin{array}{l}1480 \\
1710 \\
1610 \\
1420 \\
1480\end{array}$ & $\begin{array}{l}1460 \\
1480 \\
1480 \\
1240 \\
1480\end{array}$ & $\begin{array}{l}5310 \\
4960 \\
5580 \\
6320 \\
7110\end{array}$ & $\begin{array}{l}13600 \\
13300 \\
13100 \\
12600 \\
12500\end{array}$ & $\begin{array}{l}4290 \\
4020 \\
3630 \\
3780 \\
4070\end{array}$ & $\begin{array}{l}5830 \\
5150 \\
4890 \\
4950 \\
5100\end{array}$ & $\begin{array}{l}2150 \\
1750 \\
1890 \\
1970 \\
1430\end{array}$ & $\begin{array}{l}3170 \\
3840 \\
3240 \\
3990 \\
3750\end{array}$ \\
\hline $\begin{array}{l}16 \\
17 \\
18 \\
19 \\
20\end{array}$ & $\begin{array}{l}2250 \\
2600 \\
2900 \\
2580 \\
2710\end{array}$ & $\begin{array}{l}3100 \\
3060 \\
3000 \\
2790 \\
2860\end{array}$ & $\begin{array}{l}1810 \\
1820 \\
1340 \\
1420 \\
1280\end{array}$ & $\begin{array}{l}1330 \\
1410 \\
1690 \\
1540 \\
1650\end{array}$ & $\begin{array}{l}1520 \\
1520 \\
1430 \\
1390 \\
1470\end{array}$ & $\begin{array}{l}1610 \\
1620 \\
1680 \\
1850 \\
2110\end{array}$ & $\begin{array}{l}7910 \\
8010 \\
8570 \\
8920 \\
8980\end{array}$ & $\begin{array}{r}12000 \\
11900 \\
10900 \\
9710 \\
8730\end{array}$ & $\begin{array}{l}3360 \\
3240 \\
3520 \\
3090 \\
3570\end{array}$ & $\begin{array}{l}4650 \\
4290 \\
4300 \\
3840 \\
4310\end{array}$ & $\begin{array}{l}1680 \\
1420 \\
1770 \\
1190 \\
1430\end{array}$ & $\begin{array}{l}3770 \\
3670 \\
3820 \\
3360 \\
3320\end{array}$ \\
\hline $\begin{array}{l}21 \\
22 \\
23 \\
24 \\
25\end{array}$ & $\begin{array}{l}3660 \\
3990 \\
4640 \\
4520 \\
4610\end{array}$ & $\begin{array}{l}2680 \\
2760 \\
2750 \\
2850 \\
2410\end{array}$ & $\begin{array}{l}1220 \\
1350 \\
1520 \\
1520 \\
1510\end{array}$ & $\begin{array}{l}1410 \\
1520 \\
1380 \\
1570 \\
1620\end{array}$ & $\begin{array}{l}1330 \\
1320 \\
1600 \\
1380 \\
1460\end{array}$ & $\begin{array}{l}2800 \\
3510 \\
3800 \\
3430 \\
4100\end{array}$ & $\begin{array}{l}9530 \\
8830 \\
8300 \\
7680 \\
6970\end{array}$ & $\begin{array}{l}7720 \\
7030 \\
6900 \\
6380 \\
6500\end{array}$ & $\begin{array}{l}3700 \\
3580 \\
3480 \\
3400 \\
3640\end{array}$ & $\begin{array}{l}3890 \\
3920 \\
3600 \\
3580 \\
3480\end{array}$ & $\begin{array}{l}1460 \\
1960 \\
1900 \\
1590 \\
1770\end{array}$ & $\begin{array}{l}3340 \\
2760 \\
2900 \\
3040 \\
2840\end{array}$ \\
\hline $\begin{array}{l}26 \\
27 \\
28 \\
29 \\
30 \\
31\end{array}$ & $\begin{array}{l}4550 \\
4570 \\
4390 \\
4670 \\
4550 \\
4370\end{array}$ & $\begin{array}{l}2330 \\
2120 \\
1370 \\
1270 \\
1750\end{array}$ & $\begin{array}{l}1550 \\
1700 \\
1960 \\
1960 \\
1850 \\
1710\end{array}$ & $\begin{array}{l}1470 \\
1390 \\
1330 \\
1400 \\
1520 \\
1420\end{array}$ & $\begin{array}{l}1340 \\
1430 \\
1530 \\
--- \\
--- \\
---\end{array}$ & $\begin{array}{l}4140 \\
4780 \\
5970 \\
6150 \\
5820 \\
5410\end{array}$ & $\begin{array}{l}6840 \\
7200 \\
6810 \\
6960 \\
7930\end{array}$ & $\begin{array}{l}6540 \\
6390 \\
6260 \\
6340 \\
5920 \\
6690\end{array}$ & $\begin{array}{l}3570 \\
3320 \\
4060 \\
4250 \\
4880 \\
---\end{array}$ & $\begin{array}{l}3240 \\
3290 \\
3250 \\
2890 \\
2900 \\
2820\end{array}$ & $\begin{array}{l}1930 \\
1690 \\
1490 \\
1860 \\
1310 \\
1410\end{array}$ & $\begin{array}{l}2200 \\
2420 \\
2020 \\
2190 \\
2340\end{array}$ \\
\hline $\begin{array}{l}\text { TOTAL } \\
\text { MEAN } \\
\text { MAX } \\
\text { MIN } \\
\text { AC-FT } \\
\text { CFSM } \\
\text { IN. }\end{array}$ & $\begin{array}{r}94440 \\
3046 \\
4670 \\
1420 \\
187300 \\
.26 \\
.30\end{array}$ & $\begin{array}{r}93010 \\
3100 \\
4410 \\
1270 \\
184500 \\
.27 \\
.30\end{array}$ & $\begin{array}{r}55000 \\
1774 \\
2330 \\
1220 \\
109100 \\
.15 \\
.18\end{array}$ & $\begin{array}{r}47210 \\
1523 \\
1970 \\
1200 \\
93640 \\
.13 \\
.15\end{array}$ & $\begin{array}{r}40680 \\
1453 \\
1710 \\
1260 \\
80690 \\
.13 \\
.13\end{array}$ & $\begin{array}{r}80980 \\
2612 \\
6150 \\
1240 \\
160600 \\
.23 \\
.26\end{array}$ & $\begin{array}{r}204920 \\
6831 \\
9530 \\
4890 \\
406500 \\
.59 \\
.66\end{array}$ & $\begin{array}{r}302440 \\
9756 \\
13800 \\
5920 \\
599900 \\
.84 \\
.97\end{array}$ & $\begin{array}{r}134930 \\
4498 \\
6990 \\
3090 \\
267600 \\
.39 \\
.43\end{array}$ & $\begin{array}{r}153710 \\
4958 \\
7950 \\
2820 \\
304900 \\
.43 \\
.49\end{array}$ & $\begin{array}{r}59940 \\
1934 \\
3330 \\
1190 \\
118900 \\
.17 \\
.19\end{array}$ & $\begin{array}{r}80080 \\
2669 \\
3990 \\
1380 \\
158800 \\
.23 \\
.26\end{array}$ \\
\hline $\begin{array}{l}\text { STATISI } \\
\text { MEAN } \\
\text { MAX } \\
\text { (WY) } \\
\text { MIN } \\
(W Y)\end{array}$ & $\begin{array}{r}\text { TICS OF } \\
3987 \\
12930 \\
1966 \\
632 \\
1937\end{array}$ & $\begin{array}{c}\text { MONTHLY MEAN } \\
3737 \\
14640 \\
1972 \\
618 \\
1937\end{array}$ & $\begin{array}{l}\text { W DATA } \\
2900 \\
6456 \\
1952 \\
627 \\
1935\end{array}$ & $\begin{array}{c}\text { FOR WATER } \\
2577 \\
5713 \\
1966 \\
534 \\
1935\end{array}$ & $\begin{array}{c}\text { YEARS } 1924 \\
2490 \\
5048 \\
1984 \\
758 \\
1937\end{array}$ & $\begin{array}{c}-1991 \\
3612 \\
12290 \\
1966 \\
968 \\
1940\end{array}$ & $\begin{array}{c}\text { BY WATER } \\
8890 \\
22200 \\
1966 \\
1924 \\
1931\end{array}$ & $\begin{array}{c}\text { YEAR (WY) } \\
8469 \\
24600 \\
1950 \\
1663 \\
1977\end{array}$ & $\begin{array}{r}6410 \\
18160 \\
1965 \\
1071 \\
1988\end{array}$ & $\begin{array}{r}4508 \\
12420 \\
1975 \\
648 \\
1988\end{array}$ & $\begin{array}{r}3540 \\
15230 \\
1953 \\
449 \\
1934\end{array}$ & $\begin{array}{r}3477 \\
12940 \\
1986 \\
535 \\
1934\end{array}$ \\
\hline $\begin{array}{l}\text { SUMMAR } \\
\text { ANNUAL } \\
\text { ANNUAL } \\
\text { HIGHEST } \\
\text { LOWEST } \\
\text { HIGHEST } \\
\text { LOWEST } \\
\text { ANNUAL } \\
\text { ANNUAL } \\
\text { ANNUAL } \\
\text { ANNUAL } \\
10 \text { PERR } \\
\text { 50 PERC } \\
\text { 90 PERC }\end{array}$ & $\begin{array}{l}\text { Y STATIS' } \\
\text { TOTAL } \\
\text { MEAN } \\
\text { T ANNUAL } \\
\text { ANNUAL } \\
\text { T DAILY } \\
\text { DAILY MI } \\
\text { SEVEN-D } \\
\text { RUNOFF } \\
\text { RUNOFF } \\
\text { RUNOFF } \\
\text { CENT EXCI } \\
\text { CENT EXC } \\
\text { CENT EXCI }\end{array}$ & $\begin{array}{l}\text { TICS } \\
\text { MEAN } \\
\text { MEAN } \\
\text { MEAN } \\
\text { EAN } \\
\text { AY MINIMUM } \\
\text { (AC-FT) } \\
\text { (CFSM) } \\
\text { (INCHES) } \\
\text { EEDS } \\
\text { EEDS } \\
\text { EEDS }\end{array}$ & FOR & $\begin{array}{r}11600 \\
853 \\
966 \\
2635000 \\
4.3 \\
7760 \\
2490 \\
1330\end{array}$ & $\begin{array}{lr}\text { May } & 4 \\
\text { Aus } & 21 \\
\text { Aus } & 29\end{array}$ & & $\begin{array}{r}13800 \\
1190 \\
1380 \\
2672000 \\
4 . \\
4200^{\circ} \\
2790 \\
1420\end{array}$ & $\begin{array}{ll}\text { May } & 10 \\
\text { Aus } & 19 \\
\text { Dec } & 18\end{array}$ & & $\begin{array}{r}\text { WATER } \\
4577 \\
9555 \\
1213 \\
37700 \\
254 \\
358 \\
3316000 \\
5 \\
5 \\
9520 \\
3290 \\
1250\end{array}$ & $\begin{array}{l}\text { Apr } \\
\text { Nov } \\
\text { Sep }\end{array}$ & $\begin{array}{rr}- & 1991 \\
& 1966 \\
& 19634 \\
16 & 1965 \\
25 & 1936 \\
3 & 1936\end{array}$ \\
\hline
\end{tabular}


PERIOD OF RFCORD.--Water years 1963-66, 1975 to current year.

REMARKS.--Letter $K$ indicates non-ideal colony count.

WATER QUALITY DATA, WATER YEAR OCTOBER 1990 TO SEPTEMBER 1991

\begin{tabular}{|c|c|c|c|c|c|c|c|c|c|c|c|c|}
\hline DATE & TIME & $\begin{array}{c}\text { DIS- } \\
\text { CHARGE, } \\
\text { INST. } \\
\text { CUBIC } \\
\text { FEET } \\
\text { PER } \\
\text { SECOND } \\
(00061)\end{array}$ & $\begin{array}{l}\text { SPE- } \\
\text { CIFIC } \\
\text { CON- } \\
\text { DUCT- } \\
\text { ANCE } \\
\text { (US/CY) } \\
\text { (OOO9S) }\end{array}$ & $\begin{array}{c}\text { SPE- } \\
\text { CIFIC } \\
\text { CON- } \\
\text { DUCT- } \\
\text { ANCE } \\
\text { LAB } \\
\text { (US/CY) } \\
\text { (80095) }\end{array}$ & $\begin{array}{c}\text { PH } \\
\text { (STAND- } \\
\text { ARD } \\
\text { UNITS) } \\
\text { (00400) }\end{array}$ & $\begin{array}{c}\text { PH } \\
\text { LAB } \\
\text { (STAND- } \\
\text { ARD } \\
\text { UNITS) } \\
\text { (O0403) }\end{array}$ & $\begin{array}{c}\text { TEMPER- } \\
\text { ATURE } \\
\text { WATRR } \\
\text { (DEG C) } \\
\text { (00010) }\end{array}$ & $\begin{array}{c}\text { TUR- } \\
\text { BID- } \\
\text { ITY } \\
\text { (NTU) } \\
\text { (00076) }\end{array}$ & $\begin{array}{c}\text { BARO- } \\
\text { METRIC } \\
\text { PRES- } \\
\text { SURE } \\
\text { (MM } \\
\text { OF } \\
\text { BG) } \\
(00025)\end{array}$ & $\begin{array}{c}\text { OXYGEN, } \\
\text { DIS- } \\
\text { SOLVED } \\
(M G / L) \\
(00300)\end{array}$ & $\begin{array}{l}\text { COLI- } \\
\text { FORM, } \\
\text { FECAL, } \\
0.7 \\
\text { UM-MF } \\
\text { (COLS. } \\
\text { 100 ML) } \\
\text { (31625) }\end{array}$ & $\begin{array}{c}\text { STREP- } \\
\text { TOCOCCI } \\
\text { FECAI, } \\
\text { KF AGAR } \\
\text { (COLS. } \\
\text { PER } \\
100 \mathrm{ML}) \\
(31673)\end{array}$ \\
\hline
\end{tabular}

\begin{tabular}{|c|c|c|c|c|c|c|c|c|c|c|c|c|}
\hline $\begin{array}{l}\text { DEC } \\
19 \ldots \\
\text { FEB }\end{array}$ & 1100 & 1860 & 326 & 350 & 8.3 & 8.0 & 0.0 & 1.5 & 738 & 12.5 & 36 & $\mathbf{K 8}$ \\
\hline $\begin{array}{c}05 \ldots \\
\text { APR }\end{array}$ & 1045 & 1400 & 360 & 376 & 7.4 & 7.7 & 0.5 & 2.1 & 740 & 6.0 & 95 & $\mathbf{K} 10$ \\
\hline $\mathrm{MAY}^{16 \ldots}$ & 1115 & 8170 & 222 & 260 & 8.4 & 8.2 & 5.0 & 4.1 & 740 & 12.6 & 92 . & 270 \\
\hline JuL $^{21 \ldots}$ & 0930 & 8400 & 260 & 238 & 8.1 & 7.8 & 18.0 & 3.0 & 732 & 8.5 & - & 220 \\
\hline${ }_{\text {AUG }}^{11 \ldots}$ & 1145 & 6150 & 260 & 266 & 8.0 & 8.0 & 23.0 & 4.0 & 730 & 7.8 & $\mathbf{K} 18$ & 160 \\
\hline $15 \ldots$ & 1100 & 1770 & 317 & 296 & 8.0 & 8.3 & 22.5 & 2.2 & 732 & 7.2 & $\mathbf{K} 10$ & 160 \\
\hline
\end{tabular}

\section{DATE}

CALCIUM
DIS-
SOLVED
DATE
(MG/L
AS CA)
(O0915)

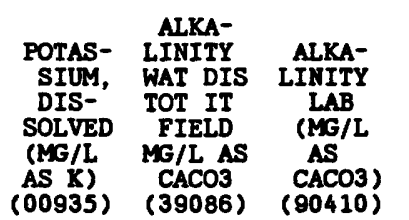

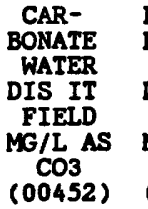

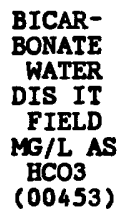

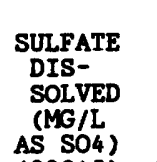

CHLO- FLUORIDE, FLUODIS- DISSOLVED SOLVED (MG/L (MG/L AS CL) AS F) $(00940)(00950)$

\begin{tabular}{|c|c|c|c|c|c|c|c|c|c|c|c|}
\hline \multirow{6}{*}{$\begin{array}{c}\text { DEC } \\
19 . \ldots \\
\text { FEB } \\
05 \ldots \\
\text { APR } \ldots \\
16 \ldots \\
\operatorname{MAY} \ldots \\
21 \ldots \\
\operatorname{JUL}_{1} \ldots \\
11 \ldots \\
\text { AUG } \\
15 \ldots\end{array}$} & 47 & 15 & 7.7 & 1.7 & 164 & 169 & 0 & 200 & 15 & 6.3 & 0.2 \\
\hline & 46 & 15 & 7.3 & 1.9 & 193 & 179 & 0 & 236 & 16 & 6.2 & 0.1 \\
\hline & 35 & 10 & 4.9 & 2.7 & -- & 116 & -- & -- & 9.9 & 6.1 & 0.1 \\
\hline & 33 & 9.7 & 4.8 & 1.5 & 123 & 113 & 0 & 150 & 6.9 & 4.7 & $<0.1$ \\
\hline & 38 & 11 & 4.1 & 1.6 & 132 & 130 & 0 & 161 & 4.5 & 3.5 & 0.1 \\
\hline & 40 & 13 & 6.3 & 1.4 & -- & 140 & -- & -- & 7.8 & 6.8 & 0.1 \\
\hline DATE & $\begin{array}{c}\text { SILICA, } \\
\text { DIS- } \\
\text { SOLVED } \\
\text { (MG/L } \\
\text { AS } \\
\text { SIO2) } \\
\text { (00955) }\end{array}$ & $\begin{array}{c}\text { SOLIDS, } \\
\text { RESIDUE } \\
\text { AT } 180 \\
\text { DEG. C } \\
\text { DIS- } \\
\text { SOLVED } \\
(M G / L) \\
(70300)\end{array}$ & $\begin{array}{c}\text { NITRO- } \\
\text { GEN, } \\
\text { NITRITE } \\
\text { DIS- } \\
\text { SOLVED } \\
\text { (MG/L } \\
\text { AS N) } \\
\text { (00613) }\end{array}$ & $\begin{array}{c}\text { NITRO- } \\
\text { GEN, } \\
\text { NO2+NO3 } \\
\text { DIS- } \\
\text { SOLVED } \\
\text { (MG/L } \\
\text { AS N) } \\
(00631)\end{array}$ & $\begin{array}{c}\text { NITRO- } \\
\text { GEN, } \\
\text { AMMONIA } \\
\text { TOTAL } \\
\text { (MG/L } \\
\text { AS N } \\
\text { (00610) }\end{array}$ & $\begin{array}{c}\text { NITRO- } \\
\text { GEN, } \\
\text { AMONIA } \\
\text { DIS- } \\
\text { SOLVED } \\
\text { (MG/L } \\
\text { AS N) } \\
\text { (00608) }\end{array}$ & $\begin{array}{l}\text { NITRO- } \\
\text { GEN,AM- } \\
\text { MONIA + } \\
\text { ORGANIC } \\
\text { TOTAL } \\
\text { (MG/L } \\
\text { AS N) } \\
(00625)\end{array}$ & $\begin{array}{c}\text { PHOS- } \\
\text { PHORUS } \\
\text { TOTAL } \\
\text { (MG/L } \\
\text { AS P) } \\
\text { (00665) }\end{array}$ & $\begin{array}{c}\text { PEOS- } \\
\text { PBORUS } \\
\text { DIS- } \\
\text { SOLVED } \\
\text { (MG/L } \\
\text { AS P) } \\
\text { (00666) }\end{array}$ & $\begin{array}{c}\text { PEOS- } \\
\text { PEORUS } \\
\text { ORTHO } \\
\text { TOTAL } \\
\text { (MG/L } \\
\text { AS P) } \\
\text { (70507) }\end{array}$ & $\begin{array}{l}\text { PHOS- } \\
\text { PEORUS } \\
\text { ORTHO, } \\
\text { DIS- } \\
\text { SOLVED } \\
\text { (MG/L } \\
\text { AS P) } \\
(00671)\end{array}$ \\
\hline $\begin{array}{l}\text { DEC } \\
19 \ldots \\
\text { FEB }\end{array}$ & 8.3 & 202 & 0.01 & 0.30 & 0.05 & 0.05 & 0.7 & 0.01 & 0.01 & $<0.01$ & $<0.01$ \\
\hline APR & 11 & 216 & 0.01 & 0.40 & 0.16 & 0.16 & 0.8 & $<0.01$ & $<0.01$ & $<0.01$ & $<0.01$ \\
\hline $\mathrm{MAY}^{16 \ldots}$ & 7.4 & 166 & $<0.01$ & 0.27 & 0.03 & $<0.01$ & 0.5 & 0.07 & 0.07 & 0.02 & $<0.01$ \\
\hline$\pi^{21} \ldots$ & 6.2 & 154 & $<0.01$ & 0.07 & 0.02 & 0.01 & 0.9 & 0.05 & 0.01 & $<0.01$ & $<0.01$ \\
\hline AUG $11 \ldots$ & 12 & 194 & $<0.01$ & 0.14 & 0.03 & 0.03 & 1.2 & 0.06 & 0.02 & 0.03 & 0.02 \\
\hline $15 \ldots$ & 9.6 & 184 & $<0.01$ & 0.05 & 0.02 & 0.01 & 0.8 & 0.06 & 0.02 & 0.01 & $<0.01$ \\
\hline
\end{tabular}


05267000 MISSISSIPPI RIVER MEAR ROYALTON, MN--Continued

(National stream-quality accounting network station)

WATER QUALITY DATA, WATER YEAR OCTOBER 1990 TO SEPTEMBER 1991

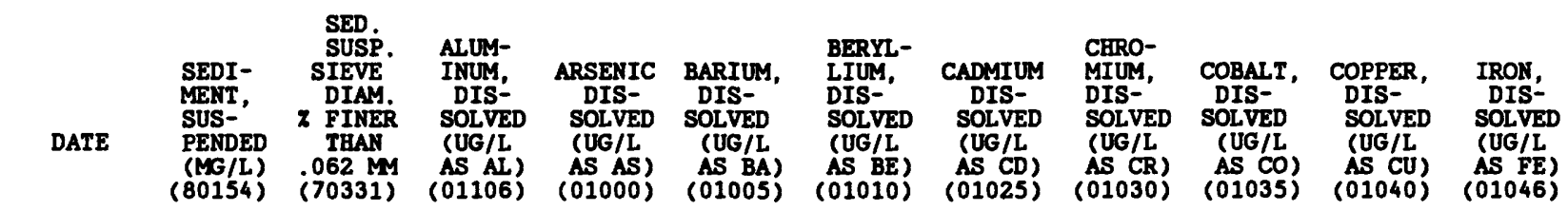

\begin{tabular}{|c|c|c|c|c|c|c|c|c|c|c|c|}
\hline $\begin{array}{l}\mathrm{DEC} \\
19 . \ldots\end{array}$ & 2 & 86 & $<10$ & $<1$ & 51 & $<0.5$ & $<1.0$ & $<1$ & $<3$ & 5 & 79 \\
\hline $\begin{array}{c}\text { FEB } \\
05 . . . \\
\text { APR }\end{array}$ & -- & -- & - & -- & -- & -- & -- & -- & -- & -- & -- \\
\hline${ }_{\mathrm{MY}}^{16} \ldots$ & 6 & - & 10 & $<1$ & 42 & $<0.5$ & $<1.0$ & 1 & $<3$ & $<1$ & 210 \\
\hline & 13 & 88 & $<10$ & 1 & 40 & $<0.5$ & $<1.0$ & $<1$ & $<3$ & 3 & 140 \\
\hline AUG $11 \ldots$ & 13 & 89 & 10 & 2 & 42 & $<0.5$ & 2.0 & $<1$ & $<3$ & 8 & 280 \\
\hline $15 \ldots$ & 3 & 100 & $<10$ & 2 & 41 & $<0.5$ & $<1.0$ & $<1$ & $<3$ & $<1$ & 52 \\
\hline
\end{tabular}

\begin{tabular}{|c|c|c|c|c|c|c|c|c|c|c|c|}
\hline DATE & $\begin{array}{l}\text { LEAD, } \\
\text { DIS- } \\
\text { SOLVED } \\
\text { (UG/L } \\
\text { AS PB) } \\
\text { (01049) }\end{array}$ & $\begin{array}{c}\text { LITHIUM } \\
\text { DIS- } \\
\text { SOLVED } \\
\text { (UG/L } \\
\text { AS LI) } \\
\text { (01130) }\end{array}$ & $\begin{array}{l}\text { MANGA- } \\
\text { NESE, } \\
\text { DIS- } \\
\text { SOLVED } \\
\text { (UG/L } \\
\text { AS MN) } \\
\text { (01056) }\end{array}$ & $\begin{array}{c}\text { MERCURY } \\
\text { DIS- } \\
\text { SOLVED } \\
\text { (UG/L } \\
\text { AS HG) } \\
\text { (71890) }\end{array}$ & $\begin{array}{c}\text { MOLYB- } \\
\text { DENUM, } \\
\text { DIS- } \\
\text { SOLVED } \\
\text { (UG/L } \\
\text { AS MO) } \\
\text { (01060) }\end{array}$ & $\begin{array}{l}\text { NICKEL, } \\
\text { DIS- } \\
\text { SOLVED } \\
\text { (UG/L } \\
\text { AS NI ) } \\
\text { (01065) }\end{array}$ & $\begin{array}{c}\text { SELE- } \\
\text { NIUM, } \\
\text { DIS- } \\
\text { SOLVED } \\
\text { (UG/L } \\
\text { AS SE) } \\
\text { (01145) }\end{array}$ & $\begin{array}{c}\text { SILVER, } \\
\text { DIS- } \\
\text { SOLVED } \\
\text { (UG/L } \\
\text { AS AG) } \\
\text { (01075) }\end{array}$ & $\begin{array}{c}\text { STRON- } \\
\text { TIUM, } \\
\text { DIS- } \\
\text { SOLVED } \\
\text { (UG/L } \\
\text { AS SR) } \\
\text { (01080) }\end{array}$ & $\begin{array}{l}\text { VANA- } \\
\text { DIUM, } \\
\text { DIS- } \\
\text { SOLVED } \\
\text { (UG/L } \\
\text { AS V) } \\
\text { (01085) }\end{array}$ & $\begin{array}{c}\text { ZINC, } \\
\text { DIS- } \\
\text { SOLVED } \\
\text { (UG/L } \\
\text { AS ZN) } \\
\text { (01090) }\end{array}$ \\
\hline
\end{tabular}

\begin{tabular}{|c|c|c|c|c|c|c|c|c|c|c|c|}
\hline $\begin{array}{l}\text { DEC } \\
19 . \ldots\end{array}$ & $<1$ & 7 & 33 & $<0.1$ & $<10$ & $<1$ & $<1$ & $<1,0$ & 95 & $<6$ & 19 \\
\hline $\begin{array}{l}\text { FEB } \\
05 . \ldots \\
A P R\end{array}$ & -- & - & -- & -- & - & -- & -- & -- & -- & -- & $\cdots$ \\
\hline${ }_{M Y}^{16} \ldots$ & $<1$ & 4 & 26 & $<0.1$ & $<10$ & $<1$ & $<1$ & $<1.0$ & 69 & $<6$ & 9 \\
\hline & $<1$ & $<4$ & 22 & $<0.1$ & $<10$ & 1 & $<1$ & $<1.0$ & 68 & $<6$ & 6 \\
\hline AUG $11 \ldots$ & 1 & $<4$ & 17 & $<0.1$ & $<10$ & 1 & $<1$ & $<1.0$ & 76 & $<6$ & 13 \\
\hline $15 \ldots$ & $<1$ & $<4$ & 2 & $<0.1$ & $<10$ & $<1$ & $<1$ & $<1.0$ & 90 & $<6$ & 13 \\
\hline
\end{tabular}


05270500 SAUK RIVER NEAR ST. CLOUD, MK

LOCATION.--Lat $45^{\circ} 33^{\prime} 35^{\prime \prime}$, long $94^{\circ} 14^{\prime} 00^{\prime \prime}$, in SWžsW sec.8, T.124 N., R. 28 W. , Stearns County, Bydrologic Unit

07010203 , on right bank $0.5 \mathrm{mi}$ northwest of Waite Park, $3 \mathrm{mi}$ west of St. Cloud, and $5 \mathrm{mi}$ upstream from mouth.

DRAINAGE AREA. $--925 \mathrm{mi}^{2}$.

PERIOD OF RECORD.--July 1909 to December 1912, April to December 1913, May to November 1929, March 1930 to

September 1931, April to November 1932, March to November 1933, March 1934 to September 1981, October 1990 to

September 30, 1991. Monthly discharge only for some periods, published in WSP 1308.

REVISED RECORDS.--WSP 895: Drainage area. WSP 1308: 1912(M), 1932(M). WSP 1508: 1937 (m).

GAGE.--Water-stage recorder. Datum of gage is $1,034.63 \mathrm{ft}$ National Geodetic Vertical Datum of 1929. Prior to Nov. 22, 1934, nonrecording gage on highway bridge $1 \mathrm{mi}$ downstream at datum $6.77 \mathrm{ft} 10 \mathrm{wer}$.

REMARKS.--Records good except those for estimated daily discharge, which are fair. Flow regulated by powerplants and reservoirs above station.

DISCHARGE, CUBIC FEET PER SECOND, WATER YEAR OCTOBER 1990 TO SEPTEMBER 1991 DAILY MEAN VALUES

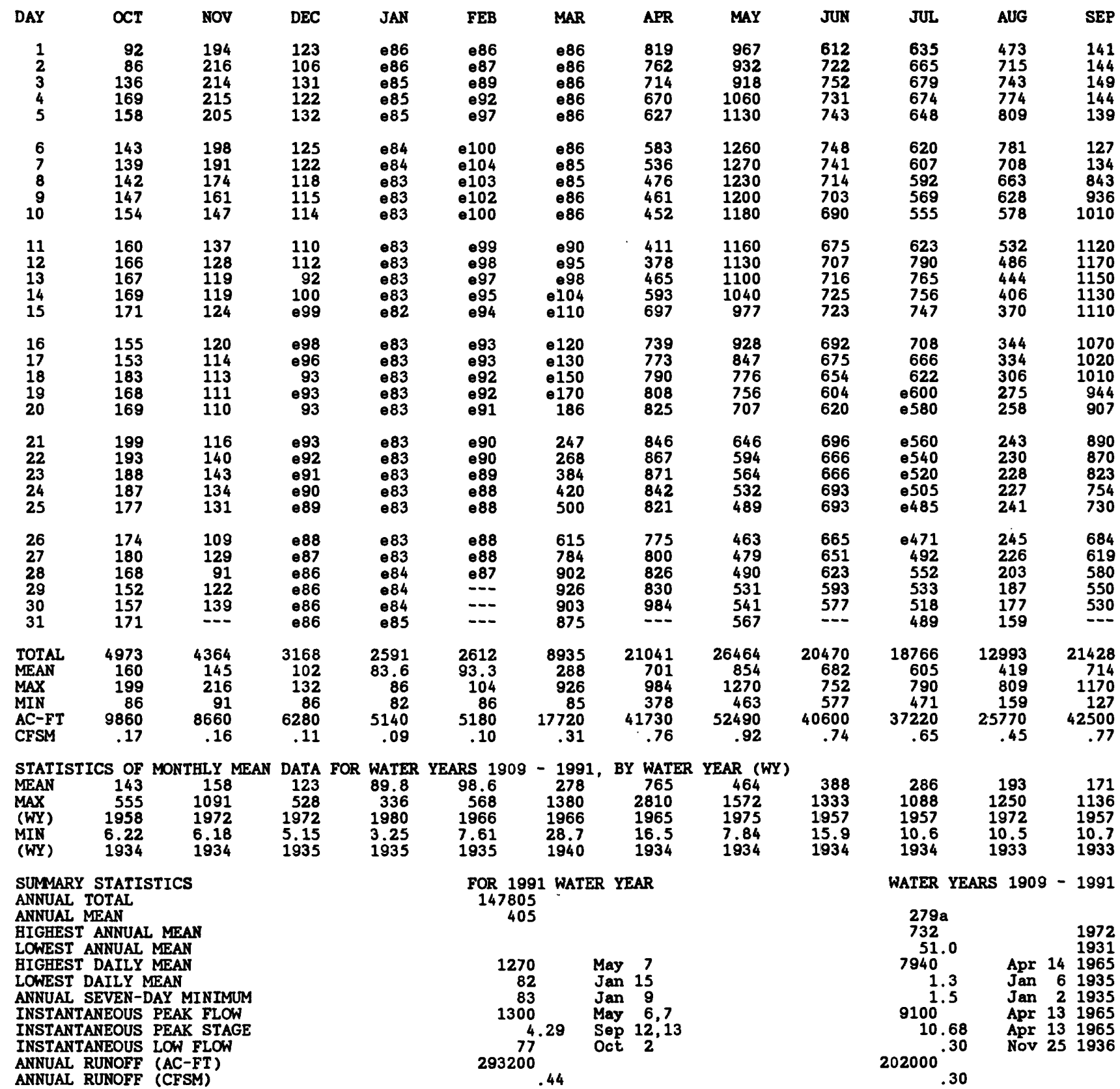

a Median of annual mean discharges is $240 \mathrm{ft}^{3} / \mathrm{s}$.

estimated. 
MISSISSIPPI RIVER MAIN STEM

05270700 MISSISSIPPI RIVER AT ST. CLOUD, MT

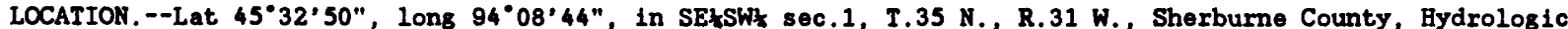
Unit 07010203 , on left bank about $250^{\circ} \mathrm{ft}$ below the left downstream end of the City of St. Cloud hydropower dam and at mile 926.3 ypstream from Ohio River.

DRAINAGE AREA. $-13,320 \mathrm{mi}^{2}$, approximately.

PERIOD OF RECORD. --October 1988 to current year.

GAGE. - Water-stage recorder. Datum of gage is $958.49 \mathrm{ft}$ above National Geodatic Vertical Datum of 1929.

REMARKS.--Records fair. Flow partly regulated by powerplants and Winnibigoshish, Leech, Pokegama, Sandy, and Gul1 Lakes and by Pine River Reservoir (see stations 05201000, 05206000, 05210500, 05218500, 05230500, 05246500 ).

DISCBARGE, CUBIC FEET PER SECOND, WATER YEAR OCTOBER 1990 TO SEPTEMBER 1991

\begin{tabular}{|c|c|c|c|c|c|c|c|c|c|c|c|c|}
\hline DAY & $\infty \mathrm{CT}$ & NOV & DEC & JAN & FEB & MAR & APR & MAY & JUN & JUL & AUG & SEP \\
\hline $\begin{array}{l}1 \\
2 \\
3 \\
4 \\
5\end{array}$ & $\begin{array}{l}2080 \\
2020 \\
3270 \\
2890 \\
2830\end{array}$ & $\begin{array}{l}\text { e5500 } \\
\text { e5500 } \\
e 5040 \\
e 4580 \\
e 4840\end{array}$ & $\begin{array}{l}e 2230 \\
e 2470 \\
e 2530 \\
e 2310 \\
e 2360\end{array}$ & $\begin{array}{l}e 2510 \\
e 2110 \\
e 1780 \\
e 2110 \\
e 2170\end{array}$ & $\begin{array}{l}e 1940 \\
e 1910 \\
e 1740 \\
e 1930 \\
e 1880\end{array}$ & $\begin{array}{l}e 2000 \\
e 1880 \\
e 1890 \\
e 1890 \\
e 1890\end{array}$ & $\begin{array}{l}8570 \\
8150 \\
8140 \\
8210 \\
8320\end{array}$ & $\begin{array}{l}10900 \\
12100 \\
11900 \\
12900 \\
13600\end{array}$ & $\begin{array}{r}9640 \\
10100 \\
10200 \\
10000 \\
9270\end{array}$ & $\begin{array}{r}8090 \\
9210 \\
10300 \\
10300 \\
10000\end{array}$ & $\begin{array}{l}4630 \\
5670 \\
5320 \\
5120 \\
4810\end{array}$ & $\begin{array}{l}2140 \\
2350 \\
2990 \\
2720 \\
2550\end{array}$ \\
\hline $\begin{array}{r}6 \\
7 \\
8 \\
9 \\
10\end{array}$ & $\begin{array}{l}3290 \\
2960 \\
3600 \\
3720 \\
3880\end{array}$ & $\begin{array}{l}\theta 4900 \\
\text { e4780 } \\
e 5040 \\
e 4180 \\
e 4010\end{array}$ & $\begin{array}{l}\mathbf{e} 2610 \\
e 2940 \\
e 2890 \\
e 2710 \\
e 3250\end{array}$ & $\begin{array}{l}e 2170 \\
e 2050 \\
e 1880 \\
e 1880 \\
e 1880\end{array}$ & $\begin{array}{l}e 1880 \\
e 1880 \\
e 1880 \\
e 1950 \\
e 2060\end{array}$ & $\begin{array}{l}e 1870 \\
e 1860 \\
e 2060 \\
e 1620 \\
e 1990\end{array}$ & $\begin{array}{l}7310 \\
7170 \\
6770 \\
6690 \\
6670\end{array}$ & $\begin{array}{l}15600 \\
16200 \\
17000 \\
17600 \\
17600\end{array}$ & $\begin{array}{l}9180 \\
8420 \\
8060 \\
8090 \\
7500\end{array}$ & $\begin{array}{l}9690 \\
9280 \\
9230 \\
8910 \\
8510\end{array}$ & $\begin{array}{l}4440 \\
4440 \\
4220 \\
3870 \\
3250\end{array}$ & $\begin{array}{l}2940 \\
2510 \\
4470 \\
3870 \\
4210\end{array}$ \\
\hline $\begin{array}{l}11 \\
12 \\
13 \\
14 \\
15\end{array}$ & $\begin{array}{l}3990 \\
\mathbf{3 6 5 0} \\
\mathbf{3 5 2 0} \\
\mathbf{3 5 8 0} \\
\mathbf{3 3 2 0}\end{array}$ & $\begin{array}{l}e 4420 \\
e 3700 \\
\theta 4260 \\
\theta 4160 \\
e 4470\end{array}$ & $\begin{array}{l}e 2710 \\
e 2460 \\
e 2120 \\
e 2410 \\
e 2460\end{array}$ & $\begin{array}{l}e 1880 \\
e 1870 \\
e 1990 \\
e 1870 \\
e 1880\end{array}$ & $\begin{array}{l}1890 \\
\oplus 2350 \\
\oplus 2180 \\
e 1940 \\
e 1900\end{array}$ & $\begin{array}{r}1940 \\
e 1880 \\
e 1880 \\
e 1620 \\
e 1920\end{array}$ & $\begin{array}{l}6780 \\
6860 \\
7020 \\
8330 \\
9770\end{array}$ & $\begin{array}{l}17300 \\
16900 \\
16700 \\
15500 \\
14500\end{array}$ & $\begin{array}{l}6950 \\
6650 \\
6400 \\
5820 \\
5910\end{array}$ & $\begin{array}{l}7920 \\
7570 \\
7060 \\
6920 \\
6770\end{array}$ & $\begin{array}{l}3810 \\
3700 \\
2870 \\
3370 \\
3020\end{array}$ & $\begin{array}{l}4910 \\
5910 \\
5730 \\
6250 \\
6560\end{array}$ \\
\hline $\begin{array}{l}16 \\
17 \\
18 \\
19 \\
20\end{array}$ & $\begin{array}{l}3370 \\
3890 \\
3870 \\
3990 \\
4030\end{array}$ & $\begin{array}{l}e 3700 \\
e 4070 \\
e 3880 \\
e 3700 \\
e 3870\end{array}$ & $\begin{array}{l}e 2290 \\
e 2350 \\
e 1940 \\
e 1630 \\
e 1500\end{array}$ & $\begin{array}{l}e 1880 \\
e 1610 \\
e 1760 \\
e 1910 \\
e 2050\end{array}$ & $\begin{array}{l}e 1900 \\
e 2180 \\
e 2070 \\
e 1880 \\
e 1880\end{array}$ & $\begin{array}{r}\mathbf{2 2 3 0} \\
\mathbf{e 2 2 2 0} \\
2750 \\
2840 \\
3260\end{array}$ & $\begin{array}{l}10600 \\
11100 \\
11500 \\
11900 \\
11900\end{array}$ & $\begin{array}{l}14100 \\
14000 \\
13000 \\
12000 \\
10900\end{array}$ & $\begin{array}{l}5770 \\
4950 \\
5100 \\
5170 \\
5730\end{array}$ & $\begin{array}{l}6730 \\
6220 \\
6280 \\
6780 \\
6730\end{array}$ & $\begin{array}{l}2690 \\
2910 \\
2570 \\
2540 \\
2330\end{array}$ & $\begin{array}{l}6270 \\
6160 \\
6070 \\
5690 \\
4880\end{array}$ \\
\hline $\begin{array}{l}21 \\
22 \\
23 \\
24 \\
25\end{array}$ & $\begin{array}{l}4160 \\
5400 \\
5730 \\
5890 \\
6110\end{array}$ & $\begin{array}{l}e 3510 \\
e 3880 \\
e 3780 \\
e 3880 \\
e 2920\end{array}$ & $\begin{array}{l}e 1490 \\
e 1880 \\
e 2020 \\
e 2060 \\
e 2060\end{array}$ & $\begin{array}{l}e 1880 \\
e 1880 \\
e 1880 \\
e 1750 \\
e 1990\end{array}$ & $\begin{array}{l}1890 \\
e 1890 \\
e 1890 \\
e 1890 \\
e 1820\end{array}$ & $\begin{array}{l}3710 \\
4700 \\
6050 \\
6160 \\
7330\end{array}$ & $\begin{array}{r}12200 \\
11400 \\
10800 \\
10300 \\
9440\end{array}$ & $\begin{array}{r}10200 \\
9360 \\
8890 \\
8810 \\
8280\end{array}$ & $\begin{array}{l}5360 \\
5170 \\
5210 \\
5170 \\
5340\end{array}$ & $\begin{array}{l}6500 \\
6430 \\
6140 \\
5420 \\
5670\end{array}$ & $\begin{array}{l}2520 \\
2570 \\
2980 \\
2650 \\
2710\end{array}$ & $\begin{array}{l}4910 \\
5470 \\
4580 \\
4440 \\
5180\end{array}$ \\
\hline $\begin{array}{l}26 \\
27 \\
28 \\
29 \\
30 \\
31\end{array}$ & $\begin{array}{r}6130 \\
5570 \\
5680 \\
e 5870 \\
e 5680 \\
e 5500\end{array}$ & $\begin{array}{l}e 2950 \\
e 2760 \\
e 2010 \\
e 1950 \\
e 2170\end{array}$ & $\begin{array}{l}e 2060 \\
e 2170 \\
e 2730 \\
e 2520 \\
e 2410 \\
e 2050\end{array}$ & $\begin{array}{l}e 1990 \\
e 1740 \\
e 1880 \\
e 1740 \\
e 1880 \\
e 1880\end{array}$ & $\begin{array}{r}e 1600 \\
e 1900 \\
e 1950 \\
\ldots \ldots \\
\ldots-\end{array}$ & $\begin{array}{r}8260 \\
9370 \\
e 9830 \\
99570 \\
9620 \\
8930\end{array}$ & $\begin{array}{r}8710 \\
9620 \\
8770 \\
9220 \\
10700\end{array}$ & $\begin{array}{l}8030 \\
8080 \\
8210 \\
8380 \\
8720 \\
9530\end{array}$ & $\begin{array}{l}5470 \\
5480 \\
5670 \\
5430 \\
6390\end{array}$ & $\begin{array}{l}5180 \\
5350 \\
5350 \\
5190 \\
4670 \\
4990\end{array}$ & $\begin{array}{l}3010 \\
2960 \\
2610 \\
2570 \\
2510 \\
1770\end{array}$ & $\begin{array}{l}3710 \\
3430 \\
3760 \\
3050 \\
4020\end{array}$ \\
\hline $\begin{array}{l}\text { TOTAL } \\
\text { MEAN } \\
\text { MAX } \\
\text { MIN } \\
\text { AC-FT } \\
\text { CFSM } \\
\text { IN. }\end{array}$ & $\begin{array}{r}129470 \\
4176 \\
6130 \\
2020 \\
256800 \\
.31 \\
.36\end{array}$ & $\begin{array}{r}118410 \\
3947 \\
5500 \\
1950 \\
234900 \\
.30 \\
.33\end{array}$ & $\begin{array}{r}71620 \\
2310 \\
3250 \\
1490 \\
142100 \\
.17 \\
.20\end{array}$ & $\begin{array}{r}59730 \\
1927 \\
2510 \\
1610 \\
118500 \\
.14 \\
.17\end{array}$ & $\begin{array}{r}54050 \\
1930 \\
2350 \\
1600 \\
107200 \\
.14 \\
.15\end{array}$ & $\begin{array}{r}125020 \\
4033 \\
9830 \\
1620 \\
248000 \\
.30 \\
.35\end{array}$ & $\begin{array}{r}272920 \\
9097 \\
12200 \\
6670 \\
541300 \\
.68 \\
.76\end{array}$ & $\begin{array}{r}386790 \\
12480 \\
17600 \\
8030 \\
767200 \\
.94 \\
1.08\end{array}$ & $\begin{array}{r}203600 \\
6787 \\
10200 \\
4950 \\
403800 \\
.51 \\
.57\end{array}$ & $\begin{array}{r}223390 \\
7206 \\
10300 \\
4670 \\
443100 \\
.54 \\
.62\end{array}$ & $\begin{array}{r}104440 \\
3369 \\
5670 \\
1770 \\
207200 \\
.25 \\
.29\end{array}$ & $\begin{array}{r}131730 \\
4391 \\
6560 \\
2140 \\
261300 \\
.33 \\
.37\end{array}$ \\
\hline
\end{tabular}

STATISTICS OF MONTHLY MEAN DATA FOR WATER YEARS 1988 - 1991, BY WATER YEAR (WY)

\begin{tabular}{|c|c|c|c|c|c|c|c|c|c|c|c|}
\hline $\begin{array}{l}\text { MEAN } \\
\text { MAX } \\
\text { (WY) } \\
\text { MIN } \\
\text { (WY) }\end{array}$ & $\begin{array}{l}3815 \\
4176 \\
1991 \\
3592 \\
1990\end{array}$ & $\begin{array}{l}3348 \\
3947 \\
1991 \\
2953 \\
1989\end{array}$ & $\begin{array}{l}2505 \\
2819 \\
1989 \\
2310 \\
1991\end{array}$ & $\begin{array}{l}2235 \\
2788 \\
1989 \\
1927 \\
1991\end{array}$ & $\begin{array}{l}2232 \\
2950 \\
1989 \\
1815 \\
1990\end{array}$ & $\begin{array}{l}5150 \\
7557 \\
1990 \\
3860 \\
1989\end{array}$ & $\begin{array}{r}10480 \\
15760 \\
1989 \\
6576 \\
1990\end{array}$ & $\begin{array}{r}10660 \\
12480 \\
1991 \\
8899 \\
1990\end{array}$ & $\begin{array}{r}7575 \\
10100 \\
1990 \\
5841 \\
1989\end{array}$ & $\begin{array}{l}5302 \\
7206 \\
1991 \\
3930 \\
1989\end{array}$ & $\begin{array}{l}2335 \\
3369 \\
1991 \\
1535 \\
1989\end{array}$ \\
\hline
\end{tabular}

SUMMARY STATISTICS

ANNUAL TOTAL

ANNUAL MEAN

HIGHEST ANNUAL MEAN

LOWEST ANNUAL MEAN

HIGHEST DAILY MEAN

LOWEST DAILY MEAN

ANNUAL SEVEN-DAY MINIMUM

INSTANTANEOUS PEAK FLOW

INSTANTANEOUS PEAK STAGE

INSTANTANEOUS LOW FLOW

ANNUAL RUNOFF (AC-FT)

ANNUAL RUNOFF (CFSM)

ANNUAL RUNOFF (INCHES)

10 PERCENT EXCEEDS

50 PERCENT EXCEEDS

90 PERCENT EXCEEDS
FOR 1990 CALENDAR YEAR

$$
\begin{gathered}
1724300 \\
4724 \\
14100 \\
1330 \\
1650 \\
16700 \mathrm{a} \\
7.27 \mathrm{a} \\
3420000 \\
.35 \\
4.82 \\
10400 \\
3640 \\
1810
\end{gathered}
$$$$
14100 \text { Mar } 17
$$$$
1650 \text { Feb } 20
$$$$
16700 a \text { Mar 16,20 }
$$$$
7.27 \text { a Mar } 16,20
$$

WATER YEARS 1988 - 1991

$$
\begin{gathered}
4950 \\
5154 \\
4615 \\
18900 \\
1010 \\
1250 \\
19700 \\
7.71 \\
792 \\
3586000 \\
.37 \\
5.05 \\
10400 \\
3610 \\
1880
\end{gathered}
$$$$
\text { Dec } 18
$$$$
\begin{array}{rrr}
1790 & \text { Dec } & 18 \\
19700 & \text { May }
\end{array}
$$$$
3731000
$$$$
\begin{aligned}
& 10000 \\
& 4030 \\
& 1880
\end{aligned}
$$

a Result of regulation.

e Estimated. 
ELK RIVER BASIN

05275000 ELK RIVER NEAR BIG LAKE, MN

LOCATION. --Lat $45^{\circ} 20^{\prime} 02^{\prime \prime}$, Iong $93^{\circ} 40^{\prime} 00^{\prime \prime}$, in NEzSW sec.23, T.22 N., R.27 W. Sherburne County, Hydrologic Unit 07010203 , on right bank at upstream side of highway bridge, 4 mi east of Big Lake and 4 mi downstream from St. Francis River.

DRAINAGE AREA. $--615 \mathrm{mi}^{2}$

PERIOD OF RECORD.--April 1911 to September 1917, April to September 1931, April to November 1932, March to November 1933, March 1934 to September 1987, October 1990 to current year.

REVISED RECORDS.-WSP 895: 1939. WSP 1308: 1912(M), 1915-17(M).

GAGE.--Water-stage recorder. Datum of gage is 899.60 ft above National Geodetic Vertical Datum of 1929 . April 1911 to Sept. 30, 1917, April 1, 1931, to July 26, 1934, nonrecording 8age at some site and datum.

REMARKS. --Records good except those for periods of estimated daily discharge, which are fair.

AVERAGE DISCBARGE. - -60 years (water years 1912-17, 1935-87, 1991), 277 ft $3 / \mathrm{s}, 6.12 \mathrm{in} / \mathrm{yr}$.

EXTREMES FOR PERIOD OF RECORD.--Maximum discharge, 7,360 $\mathrm{ft}^{3} / \mathrm{s}$, Apr. 16, 1965 , gage height, 10.86 ft; minimum, $3.6 \mathrm{ft}^{3} / \mathrm{s}, \mathrm{Ju}$ ly 31,1934

EXTREME FOR CURRENT ÝEAR. --Maximum discharge, 2,070 ft $3 / \mathrm{s}$, May 10, gage height, $5.62 \mathrm{ft}$; minimum daily discharge, $128 \mathrm{ft}^{3} / \mathrm{s}$, Jun. 22; minimum gage height, $1.06 \mathrm{ft}$, Nov. 28.

DISCBARGE, CUBIC FEET PER SECOND, WATER YEAR OCTOBER 1990 TO SEPTEMBER 1991

\begin{tabular}{|c|c|c|c|c|c|c|c|c|c|c|c|c|}
\hline DAY & OCT & NOV & DEC & JAN & FEB & MAR & APR & MAY & JUN & JUL & AUG & SEP \\
\hline $\begin{array}{r}1 \\
2 \\
3 \\
4 \\
5 \\
6 \\
7 \\
8 \\
9 \\
10 \\
11 \\
12 \\
13 \\
14 \\
15 \\
16 \\
17 \\
18 \\
19 \\
20 \\
21 \\
22 \\
23 \\
24 \\
25 \\
26 \\
27 \\
28 \\
29 \\
30 \\
31\end{array}$ & $\begin{array}{l}192 \\
188 \\
227 \\
268 \\
280 \\
278 \\
265 \\
253 \\
239 \\
228 \\
228 \\
236 \\
237 \\
231 \\
221 \\
211 \\
213 \\
234 \\
247 \\
260 \\
308 \\
354 \\
391 \\
398 \\
386 \\
376 \\
365 \\
352 \\
334 \\
316 \\
307\end{array}$ & $\begin{array}{l}302 \\
300 \\
301 \\
300 \\
299 \\
300 \\
296 \\
279 \\
253 \\
240 \\
233 \\
224 \\
218 \\
212 \\
211 \\
213 \\
211 \\
210 \\
206 \\
207 \\
210 \\
208 \\
208 \\
208 \\
210 \\
e 191 \\
e 181 \\
e 173 \\
e 191 \\
e 199 \\
---\end{array}$ & $\begin{array}{l}e 197 \\
e 192 \\
e 190 \\
e 185 \\
e 180 \\
e 180 \\
e 178 \\
e 175 \\
e 175 \\
e 175 \\
e 175 \\
e 174 \\
e 173 \\
e 168 \\
e 163 \\
e 158 \\
e 155 \\
e 152 \\
e 150 \\
e 148 \\
e 147 \\
e 146 \\
e 145 \\
e 144 \\
e 143 \\
e 142 \\
e 141 \\
e 140 \\
e 139 \\
e 138 \\
e 137\end{array}$ & $\begin{array}{l}e 136 \\
e 135 \\
e 135 \\
e 135 \\
e 135 \\
e 135 \\
e 134 \\
e 134 \\
e 133 \\
e 133 \\
e 132 \\
e 132 \\
e 132 \\
e 131 \\
e 131 \\
e 130 \\
e 130 \\
e 130 \\
e 129 \\
e 129 \\
e 129 \\
e 128 \\
e 129 \\
e 129 \\
e 129 \\
e 129 \\
e 130 \\
e 130 \\
e 130 \\
e 130 \\
e 130\end{array}$ & $\begin{array}{l}e 130 \\
e 132 \\
e 135 \\
e 137 \\
e 140 \\
e 145 \\
e 150 \\
e 153 \\
e 155 \\
e 157 \\
e 157 \\
e 156 \\
e 156 \\
e 155 \\
e 155 \\
e 154 \\
e 153 \\
e 153 \\
e 152 \\
e 152 \\
e 151 \\
e 151 \\
e 150 \\
e 150 \\
e 150 \\
e 150 \\
e 149 \\
e 149 \\
-1- \\
--- \\
---\end{array}$ & $\begin{array}{l}e 150 \\
e 155 \\
e 155 \\
e 157 \\
e 158 \\
e 160 \\
e 160 \\
e 160 \\
e 161 \\
e 162 \\
e 163 \\
e 165 \\
e 168 \\
e 170 \\
e 175 \\
e 185 \\
e 205 \\
e 230 \\
e 260 \\
e 305 \\
e 370 \\
e 460 \\
e 560 \\
e 646 \\
765 \\
831 \\
873 \\
942 \\
996 \\
1090 \\
1290\end{array}$ & $\begin{array}{r}1270 \\
1170 \\
959 \\
776 \\
649 \\
557 \\
480 \\
463 \\
428 \\
396 \\
366 \\
352 \\
441 \\
569 \\
666 \\
741 \\
837 \\
968 \\
1150 \\
1340 \\
1360 \\
1250 \\
1130 \\
1020 \\
888 \\
756 \\
667 \\
589 \\
565 \\
608 \\
---\end{array}$ & $\begin{array}{r}643 \\
660 \\
718 \\
914 \\
1120 \\
1360 \\
1470 \\
1580 \\
1880 \\
2040 \\
2030 \\
1840 \\
1650 \\
1440 \\
1280 \\
1160 \\
1030 \\
868 \\
727 \\
619 \\
534 \\
488 \\
474 \\
474 \\
480 \\
510 \\
601 \\
795 \\
1040 \\
997 \\
962\end{array}$ & $\begin{array}{r}985 \\
1080 \\
1140 \\
1100 \\
1050 \\
1080 \\
1120 \\
1040 \\
911 \\
861 \\
898 \\
916 \\
901 \\
861 \\
769 \\
674 \\
596 \\
556 \\
523 \\
528 \\
569 \\
568 \\
558 \\
547 \\
528 \\
492 \\
451 \\
418 \\
391 \\
368 \\
-2\end{array}$ & $\begin{array}{r}412 \\
414 \\
406 \\
431 \\
494 \\
564 \\
553 \\
498 \\
452 \\
416 \\
404 \\
533 \\
596 \\
592 \\
e 560 \\
e 510 \\
e 470 \\
e 450 \\
e 425 \\
e 405 \\
e 370 \\
342 \\
332 \\
325 \\
325 \\
321 \\
321 \\
360 \\
349 \\
325 \\
301\end{array}$ & $\begin{array}{r}291 \\
422 \\
478 \\
464 \\
464 \\
474 \\
499 \\
481 \\
443 \\
408 \\
374 \\
346 \\
318 \\
298 \\
281 \\
311 \\
371 \\
314 \\
e 308 \\
e 300 \\
e 285 \\
e 280 \\
e 270 \\
e 260 \\
e 250 \\
e 240 \\
e 230 \\
e 220 \\
e 215 \\
e 210 \\
202\end{array}$ & $\begin{array}{l}191 \\
191 \\
191 \\
191 \\
191 \\
188 \\
188 \\
467 \\
583 \\
546 \\
513 \\
481 \\
446 \\
425 \\
412 \\
391 \\
381 \\
374 \\
356 \\
328 \\
308 \\
288 \\
271 \\
268 \\
258 \\
248 \\
245 \\
242 \\
229 \\
219 \\
---\end{array}$ \\
\hline $\begin{array}{l}\text { TOTAL } \\
\text { MEAN } \\
\text { MAX } \\
\text { MIN } \\
\text { AC-FT } \\
\text { CFSM } \\
\text { IN. }\end{array}$ & $\begin{array}{r}8623 \\
278 \\
398 \\
188 \\
17100 \\
.45 \\
.52\end{array}$ & $\begin{array}{r}6994 \\
233 \\
302 \\
173 \\
13870 \\
.38 \\
42\end{array}$ & $\begin{array}{r}5005 \\
161 \\
197 \\
137 \\
9930 \\
.26 \\
.30\end{array}$ & $\begin{array}{r}4074 \\
131 \\
136 \\
128 \\
8080 \\
.21 \\
25\end{array}$ & $\begin{array}{r}4177 \\
149 \\
157 \\
130 \\
8290 \\
.24 \\
.25\end{array}$ & $\begin{array}{r}12427 \\
401 \\
1290 \\
150 \\
24650 \\
.65 \\
75\end{array}$ & $\begin{array}{r}23411 \\
780 \\
1360 \\
352 \\
46440 \\
1.27 \\
1.42\end{array}$ & $\begin{array}{r}32384 \\
1045 \\
2040 \\
474 \\
64230 \\
1.70 \\
1.96\end{array}$ & $\begin{array}{r}22479 \\
749 \\
1140 \\
368 \\
44590 \\
1.22 \\
1.36\end{array}$ & $\begin{array}{r}13256 \\
428 \\
596 \\
301 \\
26290 \\
.70 \\
.80\end{array}$ & $\begin{array}{r}10307 \\
332 \\
499 \\
202 \\
20440 \\
.54 \\
.62\end{array}$ & $\begin{array}{r}9610 \\
320 \\
583 \\
188 \\
19060 \\
.52 \\
.58\end{array}$ \\
\hline
\end{tabular}

STATISTICS OF MONTHLY MEAN DATA FOR WATER YEARS 1911 - 1991, BY WATER YEAR (WY)

$\begin{array}{lrrrrrrrrrrrr}\text { MEAN } & 213 & 211 & 149 & 109 & 115 & 297 & 652 & 443 & 348 & 269 & 178 & 201 \\ \text { MAX } & 778 & 794 & 410 & 290 & 392 & 1125 & 1823 & 1620 & 1647 & 1026 & 926 & 1050 \\ \text { (WY) } & 1985 & 1972 & 1966 & 1979 & 1984 & 1966 & 1969 & 1986 & 1984 & 1978 & 1972 & 1986 \\ \text { MIN } & 32.7 & 56.3 & 44.1 & 38.4 & 29.8 & 58.8 & 75.5 & 37.5 & 20.5 & 8.94 & 8.74 & 23.4 \\ \text { (WY) } & 1934 & 1935 & 1935 & 1935 & 1936 & 1934 & 1934 & 1934 & 1934 & 1934 & 1934 & 1932\end{array}$

SUMMARY STATISTICS

FOR 1991 WATER YEAR

WATER YEARS 1911 - 1991

ANNUAL TOTAL

ANNUAL MEAN

152747
418

HIGIEST ANNUAL MEAN

LOWEST ANNUAL MEAN

HIGHEST DAILY MEAN

LOWEST DAILY MEAN

ANNUAL SEVEN-DAY MINIMUM

INSTANTANEOUS PEAK FLOW

INSTANTANEOUS PEAK STAGE

INSTANTANEOUS LOW FLOW

ANNUAL RUNOFF (AC-FT)

ANNUAL RUNOFF (CFSM)

ANNUAL RUNOFF (INCHES)

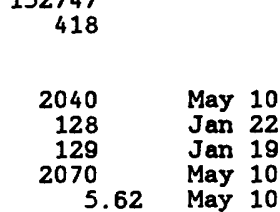

10 PERCENT EXCEEDS

50 PERCENT EXCEEDS

90 PERCENT EXCEEDS

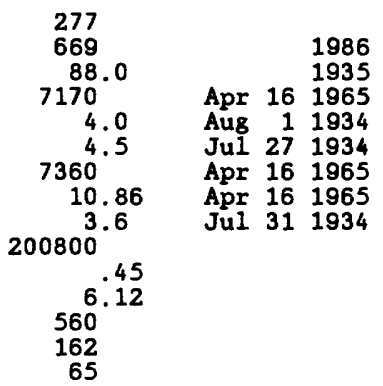


LOCATION.--Lat $45^{\circ} 05^{\prime} 12^{\prime \prime}$, long 93.44'02", in sec.29, T.119 N., R.24 W., Hennepin County, Hydrologic Unit 07010204, on right bank at Rockford, $150 \mathrm{ft}$ downstream from bridge on State Highway 55 and $1 \mathrm{mi}$ downstream from confluence of North and South Forks.

DRAINAGE AREA. $--2,520 \mathrm{mi}^{2}$ approximately.

PERIOD OF RECORD.--April to July 1906 (published as "near Dayton"), June 1909 to September 1917 , April to November 1929, March 1930 to September 1931, April to November 1932, March to November 1933, March 1934 to current year. Monthly discharge only for some periods, published in WSP 1308.

REVISED RECORDS. - WSP 1115: 1932, WSP 1508: 1933. WDR MN-77-2: 1972 (M) (m).

GAGE. - Water-stage recorder. Datum of gage is $893.08 \mathrm{ft}$ above National Geodetic Vertical Datum of 1929 . Apr. 13 to July 21, 1906 , nonrecording gage at Berning Mill $14 \mathrm{mi}$ downstream at different datum. June 4, 1909, to Sept. 30, 1917, nonrecording gage at site $600 \mathrm{ft}$ downstream at different datum. Apr. 23, 1929, to Aug. 21, 1934, nonrecording gage at site $600 \mathrm{ft}$ downstream at present datum.

REMARKS.--Records good except those for estimated daily discharges, which are fair.

DISCHARGE, CUBIC FEET PER SECOND, WATER YEAR OCTOBER 1990 TO SEPTEMBER 1991

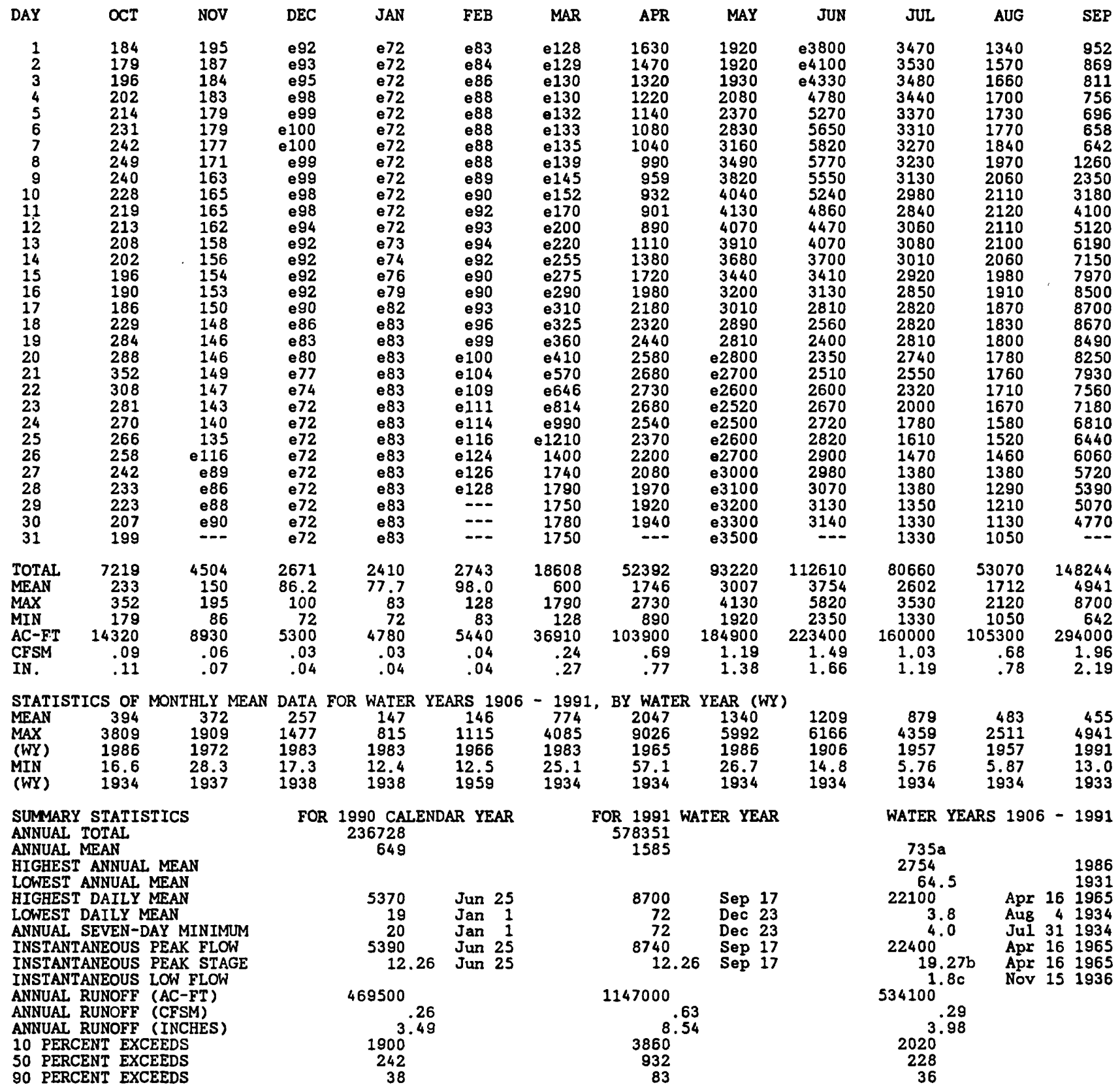

a Median of annual mean discharges is $552 \mathrm{ft}^{3} / \mathrm{s}$.

b From floodmark.

c Caused by ice jam upstream.

e Estimated. 


\section{RUM RIVER BASIN}

05284000 MILLE LACS LAKE AT COVE BAY NEAR ONAMIA, MN

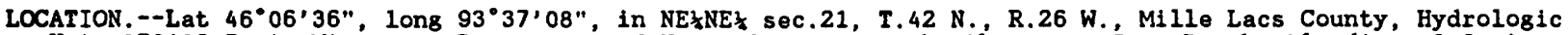
Unit 07010207 , in Minnesota Department of Natural Resources boathouse at Cove Bay boatlanding, $3.6 \mathrm{mi}$ northeast of Onamia.

PERIOD OF RECORD.--June 1931 to current year. Monthend records for the period October 1939 to September 1953 published in WSP 1278 (fragmentary 1940-4i). Published as "at Wealthwood" prior to October 1939, and as "at Garrison" October 1939 to September 1987 (gage heights collected at Wealthwood October 1939 to September 1941, but converted to gage datum at Garrison for publication).

GAGE.--Water-stage recorder. Datum of gage is $1,240.40 \mathrm{ft}$ above National Geodetic Vertical Datum of 1929 (ievels by Minnesota Department of Natural Resources). Gage readings have been reduced to elevations NGVD. Prior to Oct. 1, 1941, nonrecording gage at Wealthwood, $17 \mathrm{mi}$ north of present site, at various datums; gage readings have been reduced to elevations, adjustment of 1912 . Oct. 1, 1941, to Sept. 30, 1958, waterstage recorder at Garrison, $16 \mathrm{mi}$ northwest of present site at datum 1,240.50 ft, adjustment of 1912 . To convert these readings to National Geodetic Vertical Datum of 1929 , subtract $0.10 \mathrm{ft}$. Oct. 1,1958, to Sept. 30, 1987, water stage recorder at Garrison at present datum.

REMARKS. - Water level affected by fixed-crest spillway constructed in 1953 at outlet of Ogechie Lake, $2.7 \mathrm{mi}$ downstream from outlet of Mille Lacs Lake, with crest at elevation 1,250.50 ft. Water level subject to fluctuation caused by change in direction and velocity of wind and by seiches.

EXTREMES FOR PERIOD OF RECORD.--Maximum elevation, 1,253.87 ft, Aug. 14, 1972, affected by wind action and seiche action; maximum daily, 1,253.43 ft, Aug. 22, 1972; minimum observed, 1,245.74 ft, Oct. 16-19, 1936 .

EXTREMES FOR CURRENT YEAR. --Maximum elevation, $1,253.19 \mathrm{ft}$, July 5 , affected by wind and seiche action; maximum daily, $1,251.85 \mathrm{ft}$, Juiy 7; minimum, 1,250.04 ft, Nov. 22, affected by wind and seiche action; minimum daily, $1,250.17$ ft, Nov. 29 .

MONTHEND ELEVATION, IN FEET NGVD, WATER YEAR OCTOBER 1990 TO SEPTEMBER 1991

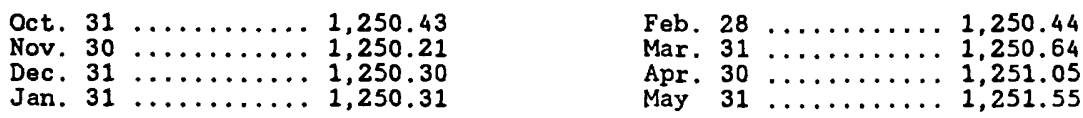


RUM RIVER BASIN

05286000 RUM RIVER MEAR ST. FRANCIS, MN

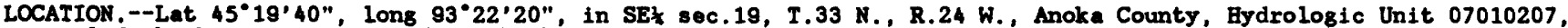
on left bank at upstrgam side or highway bridge, 4 mi south of St. Francis and 15.8 mi upstream from mouth. DRAINAGE AREA. $--1,360 \mathrm{mi}^{2}$, approximately.

PERIOD OF RECORD. -May to November 1929, March 1930 to September 1931, April to November 1932 , March 1933 to current year.

REVISED RECORDS,-WSP 1308: $1930(\mathrm{M}), 1932(\mathrm{M})$.

GAGE.- Water-stage recorder. Datum of gage is 860.74 ft above National Geodetic Vertical Datum of 1929 (levels by Anoka County Highway Department). Prior to Hov. 9, 1933, nonrecording gage at site 50 ft downstream at same datum.

REMARKS. - Records good except those for estimated daily discharges, which are fair. Occasional regulation by Ogechie (also controls Mille Lacs Lake) and Onami Lakes

AVERAGE DISCHARGE.--59 years (water years 1931, 1934-91), 626 $\mathrm{ft}^{3} / \mathrm{s}, 6.25 \mathrm{in} / \mathrm{yr}$.

EXTREMES FOR PERIOD OF RECORD.--Maximum discharge, 10,100 ft3/s, Apr. 20, 1965, Apr. 13, 1969; maximum gage height, $11.63 \mathrm{ft}$, Apr. 13, 1969; minimum discharge, $29 \mathrm{ft} / \mathrm{s}$. Aug. 18; 1934, gage height, 1.91 ft.

EXTREMES FOR CURRENT YEAR.--Maximum discharge, 4,430 $\mathrm{ft}^{3} / \mathrm{s}$, May 12, gage height, $7.28 \mathrm{ft}$; minimum daily d1scharge, $150 \mathrm{ft}^{3} / \mathrm{s}, \mathrm{Jan} .26$; minimum gage height, $2.38 \mathrm{ft}$, Nov. 28.

DISCHARGE, CUBIC FEET PER SECOND, WATER YEAR OCTOBER 1990 TO SEPTEMBER 1991

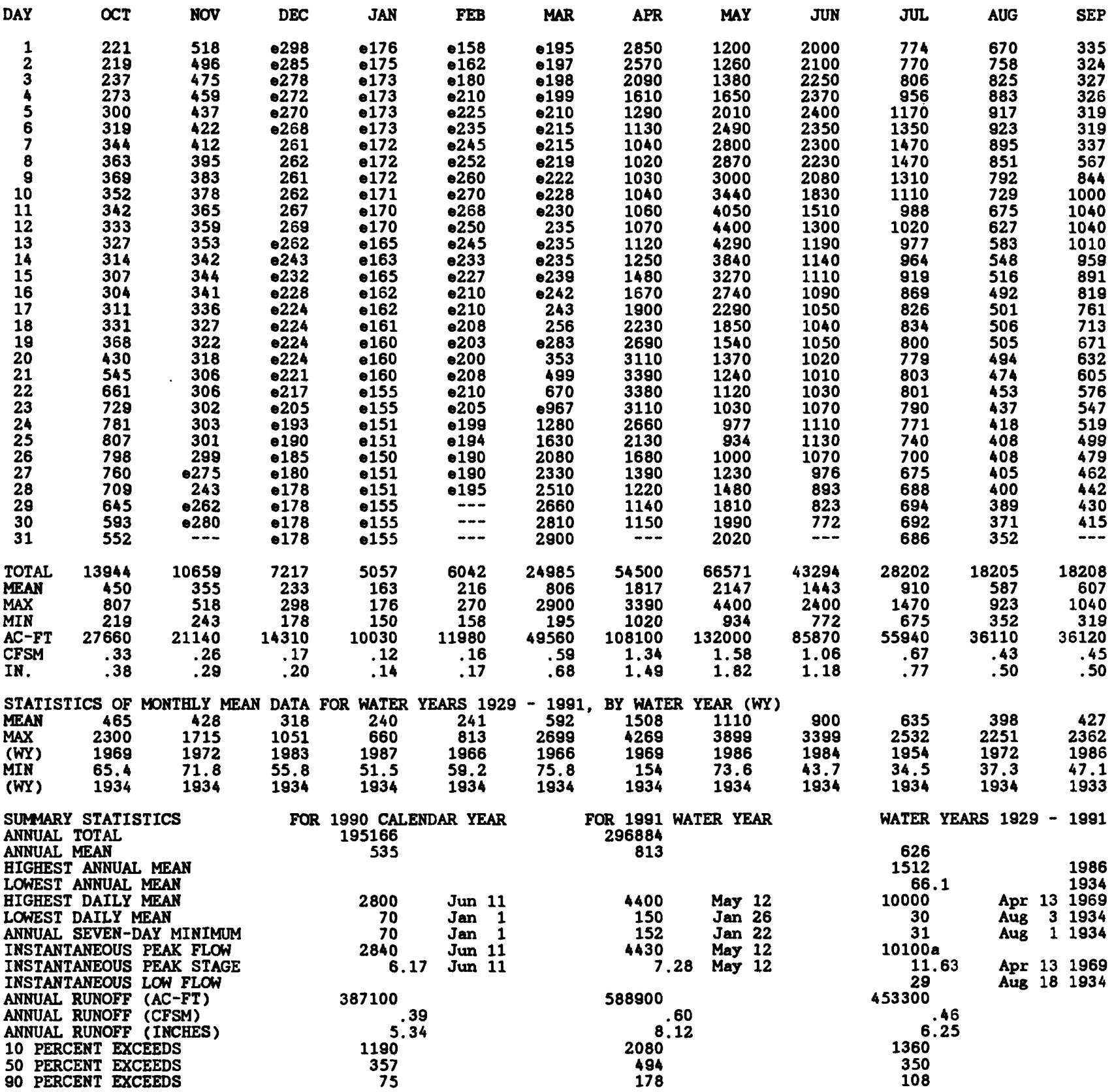

a Occurred on Apr. 20, 1965 and Apr. 13, 1969.

- Estimated. 
05287890 ELM CREEK NEAR CHAMPLIN, MA

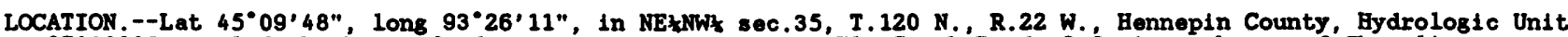

07010206 , on left bank, 33 ft downstream from bridge on Elm Creek Road, $2.5 \mathrm{mi}$ southwest of Champlin.

DRAINAGE AREA, $--84.9 \mathrm{mi}^{2}$.

WATER DISCHARGE RECORDS

PERIOD OF RECORD. --October 1978 to current year.

GAGE.--Water-stage recorder. Datum of gage is $850.71 \mathrm{ft}$ above National Geodetic Vertical Datum of 1929 . Prior to March 15, 1979, nonrecording gage at present site and datum.

REMARKS.--Records good except those for estimated dally discharges, which are fair.

DISCHARGE, CUBIC FEET PER SECOND, WATER YEAR OCTOBER 1990 TO SEPTEMBER 1991

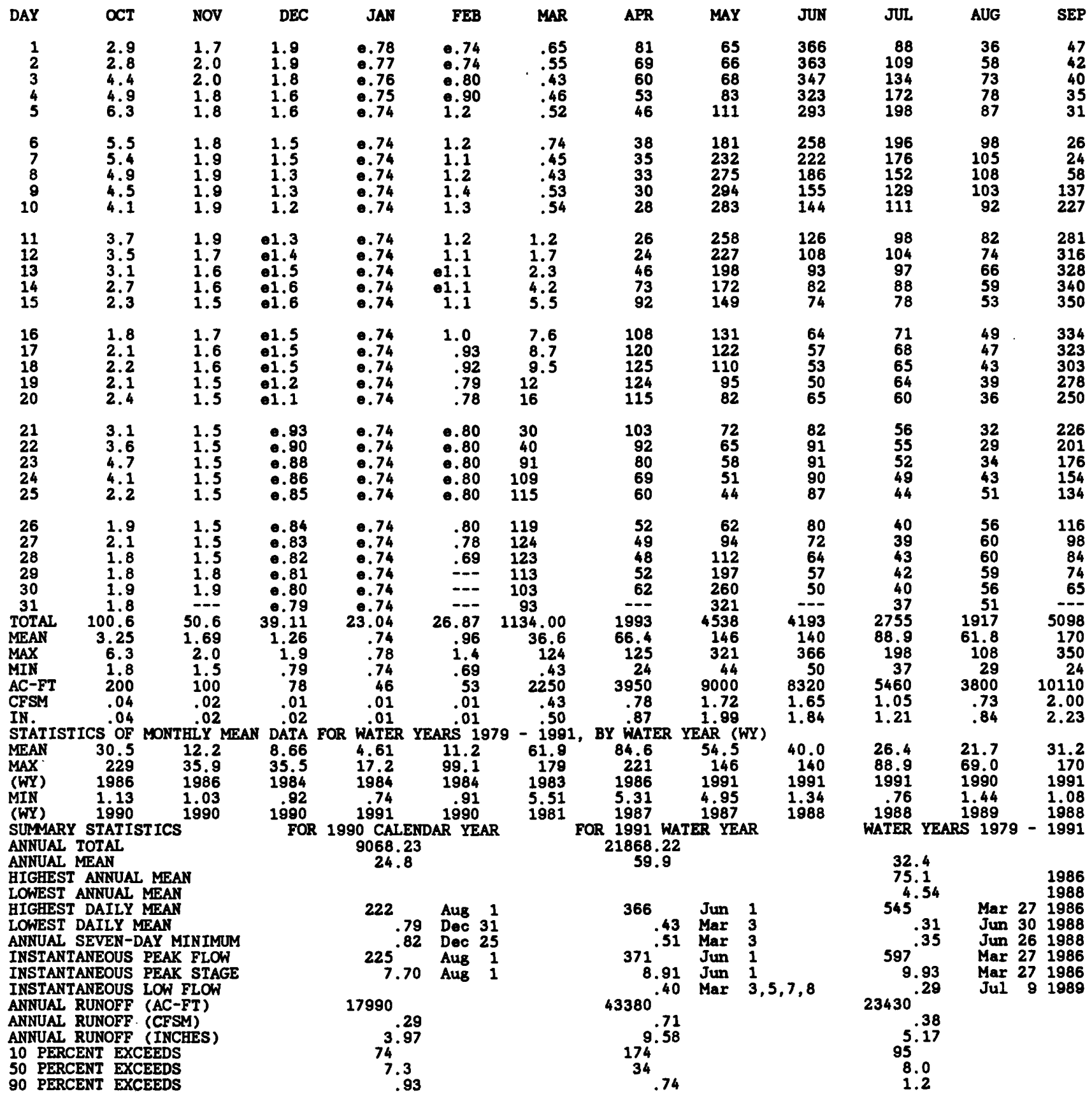


ELM CREBK BASIN

05287890 ELM CREEK NEAR CHAMPLIN, MN--Continued

WATER QUALITY RECORDS

PERIOD OF RECORD. --February 1988 to current year.

WATER QUALITY DATA, WATER YEAR OCTOBER 1990 TO SEPTEMBER 1991

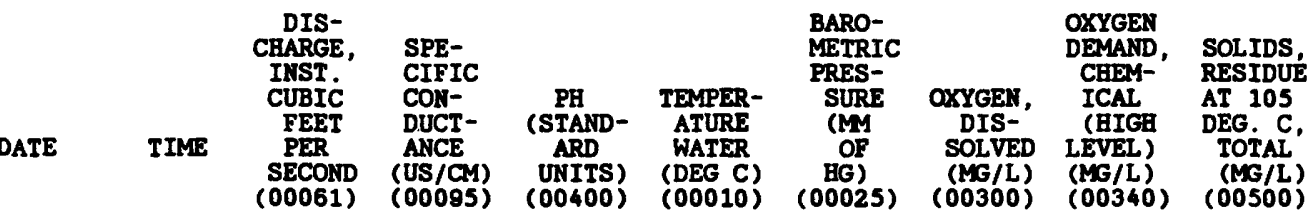

\begin{tabular}{|c|c|c|c|c|c|c|c|c|c|}
\hline${ }_{30}^{\infty} \ldots$ & 1245 & 2.0 & 581 & 8.4 & 8.0 & 744 & 7.6 & 36 & 431 \\
\hline OS... & 1200 & 1.1 & 700 & 7.7 & 2.0 & 746 & 9.5 & 17 & 418 \\
\hline$\underset{M A Y}{26} \ldots$ & 1045 & 119 & 451 & 7.5 & 3.5 & 740 & 9.2 & 58 & 305 \\
\hline $\begin{array}{r}01 \ldots \\
07 \ldots \\
22 \ldots \\
\text { JUN }\end{array}$ & $\begin{array}{l}1300 \\
1345 \\
1200\end{array}$ & $\begin{array}{r}65 \\
233 \\
63\end{array}$ & $\begin{array}{l}504 \\
463 \\
488\end{array}$ & $\begin{array}{l}7.7 \\
7.9 \\
7.9\end{array}$ & $\begin{array}{r}8.0 \\
8.0 \\
20.5\end{array}$ & $\begin{array}{l}733 \\
-- \\
737\end{array}$ & $\begin{array}{r}9.2 \\
5.7\end{array}$ & $\begin{array}{l}55 \\
47 \\
60\end{array}$ & $\begin{array}{l}320 \\
304 \\
311\end{array}$ \\
\hline $14 \ldots$ & 1230 & 82 & 417 & 7.4 & 24.0 & 728 & 4.5 & 63 & 284 \\
\hline AUG $17 \ldots$ & 1230 & 68 & 395 & 7.4 & 27.0 & 735 & 4.3 & 60 & -- \\
\hline $\operatorname{SEP}^{27} \cdots$ & 1200 & 62 & 405 & 7.7 & 28.5 & 740 & 5.5 & 64 & -- \\
\hline $10 \ldots$ & 0845 & 219 & 264 & 7.2 & 20.0 & 745 & 4.4 & 52 & -- \\
\hline
\end{tabular}

\begin{tabular}{|c|c|c|c|c|c|c|c|c|}
\hline ITE & $\begin{array}{c}\text { SOLIDS, } \\
\text { VOLA- } \\
\text { TILE ON } \\
\text { IGNI- } \\
\text { TION, } \\
\text { TOTAL } \\
\text { (MG/L) } \\
\text { (OOSO5) }\end{array}$ & $\begin{array}{l}\text { RESIDUE } \\
\text { TOTAL } \\
\text { AT 10S } \\
\text { DEG. C, } \\
\text { SUS- } \\
\text { PENDED } \\
(M G / L) \\
(00530)\end{array}$ & $\begin{array}{c}\text { RESIDUE } \\
\text { VOLA- } \\
\text { TILE, } \\
\text { SUS-' } \\
\text { PENDED } \\
(M G / L) \\
\text { (OOS35) }\end{array}$ & $\begin{array}{c}\text { NITRO- } \\
\text { GEN, } \\
\text { NO2+NO33 } \\
\text { TOTAL } \\
\text { (MG/L } \\
\text { AS N) } \\
(00630)\end{array}$ & $\begin{array}{l}\text { NITRO- } \\
\text { GEN, } \\
\text { AMMONIA } \\
\text { TOTAL } \\
\text { (MG/L } \\
\text { AS N) } \\
\text { (00610) }\end{array}$ & $\begin{array}{c}\text { NITRO- } \\
\text { GEN,AM- } \\
\text { MONIA + } \\
\text { ORGANIC } \\
\text { TOTAL } \\
\text { (MG/L } \\
\text { AS N } \\
(00625)\end{array}$ & $\begin{array}{c}\text { PHOS- } \\
\text { PHORUS } \\
\text { TOTAL } \\
\text { (MG/L } \\
\text { AS P) } \\
(00665)\end{array}$ & $\begin{array}{c}\text { PEOS- } \\
\text { PEORUS } \\
\text { DIS- } \\
\text { SOLVED } \\
\text { (MG/L } \\
\text { AS P) } \\
\text { (00666) }\end{array}$ \\
\hline
\end{tabular}

\begin{tabular}{|c|c|c|c|c|c|c|c|c|}
\hline${ }_{30}^{\mathrm{OCT}} \ldots$ & 311 & - & -- & $<0.10$ & 0.02 & 1.0 & 0.11 & 0.06 \\
\hline & 326 & -- & -- & $<0.10$ & 0.17 & 0.6 & 0.05 & 0.03 \\
\hline $\mathrm{MAY}^{26 \ldots}$ & 83 & -- & -- & 1.2 & 0.43 & 2.7 & 0.33 & 0.20 \\
\hline $\begin{array}{r}01 \ldots \\
07 \ldots \\
22 \ldots \\
\text { JUN }\end{array}$ & $\begin{array}{l}107 \\
101 \\
122\end{array}$ & $\begin{array}{r}6 \\
22 \\
22\end{array}$ & $\begin{array}{l}6 \\
9 \\
9\end{array}$ & $\begin{array}{l}0.20 \\
0.65 \\
0.13\end{array}$ & $\begin{array}{l}0.04 \\
0.04 \\
0.13\end{array}$ & $\begin{array}{l}0.9 \\
1.3 \\
1.7\end{array}$ & $\begin{array}{l}0.11 \\
0.20 \\
0.39\end{array}$ & $\begin{array}{l}0.10 \\
0.16 \\
0.39\end{array}$ \\
\hline $\operatorname{JuL}^{14 \ldots}$ & 129 & 26 & 5 & 0.15 & 0.08 & 1.2 & 0.59 & 0.39 \\
\hline AUG $^{17} \cdots$ & -- & $<1$ & $<1$ & $<0.05$ & 0.07 & 2.2 & 0.53 & 0.30 \\
\hline 27. & -- & 13 & 6 & 0.08 & 0.04 & 1.4 & 0.32 & 0.21 \\
\hline
\end{tabular}


05287890 ELM CREEK NEAR CHAMPLIN, MN--Continued

WATER QUALITY DATA, WATER YEAR OCTOBER 1990 TO SEPTEMBER 1991

SAMPLES COLLECTED BY AUTOMATIC SAMPLER

\begin{tabular}{|c|c|c|c|c|}
\hline DATE & TIME & $\begin{array}{c}\text { DIS- } \\
\text { CHARGE, } \\
\text { INST, } \\
\text { CUBIC } \\
\text { FEET } \\
\text { PER } \\
\text { SECOND } \\
\text { (00061) }\end{array}$ & $\begin{array}{c}\text { RESIDUE } \\
\text { TOTAL } \\
\text { AT 10S } \\
\text { DEG. C, } \\
\text { SUS- } \\
\text { PENDED } \\
(M G / L) \\
(00530)\end{array}$ & $\begin{array}{c}\text { PHOS- } \\
\text { PHORUS } \\
\text { TOTAL } \\
\text { (MG/L } \\
\text { AS P) } \\
(00665)\end{array}$ \\
\hline
\end{tabular}

\begin{tabular}{|c|c|c|c|c|}
\hline \multicolumn{4}{|l|}{ MAY } & \\
\hline $\begin{array}{l}01 \ldots \\
05 . . \\
06, \ldots \\
07 \ldots \\
08 \ldots \\
09 . \ldots \\
10 \ldots \\
13 . \ldots \\
15 . \ldots\end{array}$ & $\begin{array}{l}1255 \\
0552 \\
1150 \\
1748 \\
2345 \\
1444 \\
2042 \\
0837 \\
2032 \\
0827\end{array}$ & $\begin{array}{r}65 \\
86 \\
177 \\
246 \\
290 \\
298 \\
272 \\
200 \\
139 \\
106\end{array}$ & $\begin{array}{r}5 \\
3 \\
27 \\
11 \\
10 \\
12 \\
7 \\
8 \\
19 \\
11\end{array}$ & $\begin{array}{l}0.11 \\
0.10 \\
0.19 \\
0.14 \\
0.13 \\
0.14 \\
0.11 \\
0.15 \\
0.26 \\
0.38\end{array}$ \\
\hline JUN & & & & \\
\hline $\begin{array}{r}14 \ldots \\
19 \ldots \\
20 \ldots \\
20 \ldots \\
21 \ldots \\
22 \ldots \\
27 \ldots \\
30 \ldots \\
\text { JUL }\end{array}$ & $\begin{array}{l}1235 \\
0903 \\
0142 \\
1821 \\
1100 \\
2018 \\
1651 \\
1127\end{array}$ & $\begin{array}{l}77 \\
49 \\
62 \\
61 \\
82 \\
88 \\
69 \\
49\end{array}$ & $\begin{array}{l}26 \\
18 \\
47 \\
19 \\
43 \\
23 \\
22 \\
23\end{array}$ & $\begin{array}{l}0.59 \\
0.48 \\
0.51 \\
0.46 \\
0.53 \\
0.40 \\
0.49 \\
0.72\end{array}$ \\
\hline $\begin{array}{r}01 \ldots \\
02 \ldots \\
03 \ldots \\
\text { SEP }\end{array}$ & $\begin{array}{l}0406 \\
1324 \\
0603\end{array}$ & $\begin{array}{r}80 \\
108 \\
125\end{array}$ & $\begin{array}{l}81 \\
26 \\
45\end{array}$ & $\begin{array}{l}0.91 \\
0.59 \\
0.54\end{array}$ \\
\hline $\begin{array}{l}09 \ldots \\
10 \ldots \\
10 \ldots \\
11 \ldots \\
12 \ldots \\
15 \ldots \\
19 \ldots \\
24 \ldots \\
27 \ldots\end{array}$ & $\begin{array}{l}1100 \\
0339 \\
2018 \\
1257 \\
0536 \\
0012 \\
2045 \\
0039 \\
1154\end{array}$ & $\begin{array}{r}139 \\
186 \\
241 \\
267 \\
293 \\
342 \\
254 \\
153 \\
87\end{array}$ & $\begin{array}{r}76 \\
52 \\
53 \\
27 \\
106 \\
19 \\
14 \\
17 \\
17\end{array}$ & $\begin{array}{l}0.74 \\
0.44 \\
0.35 \\
0.33 \\
0.32 \\
0.39 \\
0.27 \\
0.22 \\
0.21\end{array}$ \\
\hline
\end{tabular}


MISSISSIPPI RIVER MAIN STEM

05288500 MISSISSIPPI RIVER NEAR ANOKA, MN

LOCATION, --Lat $45^{\circ} 07^{\prime} 36^{\prime \prime}$, long $93^{\circ} 17^{\prime} 48^{\prime \prime}$, in SW sec.12, T.119 N., R.21 W., Hennepin County, Hydrologic Unit

07010206 , on right bank $0.4 \mathrm{mi}$ downstream from Coon Creek, $1.3 \mathrm{mi}$ downstream from Coon Rapids dam at Coon Rapids, 6.5 mi downstrgam from Anoka, and at mile 864.8 upstream from Ohio River.

DRAINAGE AREA. $--19,100 \mathrm{mi}^{2}$, approximately.

\section{WATER-DISCHARGE RECORDS}

PERIOD OF RECORD.--June 1931 to current year. Prior to October 1931 published as "at Coon Rapids, near Anoka." GAGE.--Water-stage recorder. Datum of gage is $804.53 \mathrm{ft}$ above National Geodetic Vertical Datum of 1929 . Prior to June 14,1932 , at site $1.2 \mathrm{mi}$ upstream at different datum.

REMARKS. - No estimated daily discharges. Records good. Discharge during period of backwater from ice, Dec. 22 to Feb. 5, computed from discharge furnished by Ford Plant Dam downstream from station adjusted for time of travel, leakage through dam, and diversions to St. Paul and Minneapolis water works. Flow slightly regulated by $\mathbf{s i x}$ reservoirs on headwaters; total usable capacity, 1,640,600 acre-ft. Diurnal regulation caused by dam above station.

DISCHARGE, CUBIC FEET PER SECOND, WATER YEAR OCTOBER 1990 TO SEPTEMBER 1991

\begin{tabular}{|c|c|c|c|c|c|c|c|c|c|c|c|c|}
\hline DAY & OCT & NOV & DEC & JAN & FEB & MAR & APR & MAY & JUN & JUL & AUG & SEP \\
\hline $\begin{array}{r}1 \\
2 \\
3 \\
4 \\
5 \\
6 \\
7 \\
8 \\
9 \\
10 \\
11 \\
12 \\
13 \\
14 \\
15 \\
16 \\
17 \\
18 \\
19 \\
20 \\
21 \\
22 \\
23 \\
24 \\
25 \\
26 \\
27 \\
28 \\
29 \\
30 \\
31\end{array}$ & $\begin{array}{l}2780 \\
2640 \\
3210 \\
4070 \\
3790 \\
3730 \\
4230 \\
3820 \\
4590 \\
4620 \\
4730 \\
4320 \\
4410 \\
4200 \\
4060 \\
4030 \\
4160 \\
4720 \\
4660 \\
5200 \\
5250 \\
6120 \\
7200 \\
7320 \\
7540 \\
7580 \\
7240 \\
7250 \\
6960 \\
7050 \\
7260\end{array}$ & $\begin{array}{l}6850 \\
6620 \\
6770 \\
6540 \\
6080 \\
5830 \\
6290 \\
6010 \\
5930 \\
5820 \\
5260 \\
5370 \\
4910 \\
4970 \\
5240 \\
4930 \\
5210 \\
4840 \\
5110 \\
4700 \\
4860 \\
4550 \\
4660 \\
4840 \\
4490 \\
4580 \\
3770 \\
4000 \\
2970 \\
2810 \\
--0\end{array}$ & $\begin{array}{l}3010 \\
3280 \\
3000 \\
3350 \\
3220 \\
3300 \\
3700 \\
3740 \\
3840 \\
3380 \\
3770 \\
3480 \\
3370 \\
2520 \\
3150 \\
3180 \\
3300 \\
2790 \\
2700 \\
2430 \\
1790 \\
1700 \\
3070 \\
3240 \\
3230 \\
3110 \\
2930 \\
3510 \\
3200 \\
3180 \\
3220\end{array}$ & $\begin{array}{l}3170 \\
3100 \\
3150 \\
2940 \\
3180 \\
3070 \\
3000 \\
2990 \\
3000 \\
2960 \\
3050 \\
3050 \\
3060 \\
3210 \\
3030 \\
3120 \\
3010 \\
2870 \\
3200 \\
2640 \\
2840 \\
3000 \\
2840 \\
2800 \\
2920 \\
3170 \\
3170 \\
2830 \\
2680 \\
2640 \\
2850\end{array}$ & $\begin{array}{l}3220 \\
3060 \\
3890 \\
2960 \\
3230 \\
2550 \\
2530 \\
2510 \\
2590 \\
2540 \\
2580 \\
3000 \\
2870 \\
2540 \\
2780 \\
4430 \\
4270 \\
3420 \\
3420 \\
3610 \\
2990 \\
2620 \\
2640 \\
2930 \\
3090 \\
2760 \\
2550 \\
2420 \\
--- \\
--- \\
---\end{array}$ & $\begin{array}{r}2620 \\
2610 \\
2600 \\
2570 \\
2640 \\
2610 \\
2590 \\
2810 \\
2770 \\
2740 \\
2580 \\
2960 \\
2930 \\
2970 \\
3050 \\
2920 \\
3330 \\
3390 \\
3600 \\
4100 \\
5180 \\
6560 \\
8430 \\
10300 \\
11300 \\
12900 \\
14200 \\
15200 \\
15800 \\
15800 \\
16000\end{array}$ & $\begin{array}{r}15400 \\
14700 \\
13800 \\
12700 \\
12500 \\
11800 \\
10900 \\
10500 \\
10100 \\
10000 \\
9870 \\
9980 \\
10700 \\
11600 \\
13800 \\
15500 \\
16700 \\
17400 \\
18500 \\
19400 \\
20000 \\
20400 \\
19500 \\
18100 \\
16600 \\
15100 \\
14300 \\
14200 \\
13500 \\
14200 \\
-0-\end{array}$ & $\begin{array}{l}15600 \\
15700 \\
16900 \\
17700 \\
19900 \\
21800 \\
24700 \\
25800 \\
27200 \\
28100 \\
28800 \\
28900 \\
28100 \\
26800 \\
24800 \\
23600 \\
22300 \\
20600 \\
19000 \\
17300 \\
16400 \\
15300 \\
14300 \\
13800 \\
13200 \\
13200 \\
13300 \\
14000 \\
15400 \\
16100 \\
16500\end{array}$ & $\begin{array}{r}17900 \\
18000 \\
19200 \\
19300 \\
19500 \\
19200 \\
19200 \\
18300 \\
17900 \\
18000 \\
16000 \\
15100 \\
14300 \\
13300 \\
12400 \\
12000 \\
11300 \\
10200 \\
10200 \\
10500 \\
11300 \\
10800 \\
10700 \\
10700 \\
10600 \\
10800 \\
10700 \\
10500 \\
10900 \\
10500 \\
--0\end{array}$ & $\begin{array}{r}13000 \\
13900 \\
15100 \\
15800 \\
16100 \\
15700 \\
15600 \\
15200 \\
14900 \\
14200 \\
13400 \\
14300 \\
13300 \\
13200 \\
12500 \\
12200 \\
11800 \\
11300 \\
11500 \\
10800 \\
10800 \\
10300 \\
9790 \\
9240 \\
8440 \\
8290 \\
7920 \\
8430 \\
8090 \\
7550 \\
7200\end{array}$ & $\begin{array}{l}7480 \\
8510 \\
9680 \\
9260 \\
9030 \\
8660 \\
8390 \\
8610 \\
8190 \\
7720 \\
7170 \\
7560 \\
7190 \\
6450 \\
6610 \\
6300 \\
6230 \\
6150 \\
5790 \\
5630 \\
5300 \\
5440 \\
5660 \\
5830 \\
5460 \\
5440 \\
5500 \\
5300 \\
5020 \\
4750 \\
4510\end{array}$ & $\begin{array}{r}3770 \\
3960 \\
4120 \\
4460 \\
4280 \\
3980 \\
4360 \\
6240 \\
9980 \\
10400 \\
11400 \\
13000 \\
14500 \\
15400 \\
16800 \\
17400 \\
17400 \\
17300 \\
17000 \\
16100 \\
15200 \\
15000 \\
14700 \\
13500 \\
13100 \\
13100 \\
11300 \\
10900 \\
10600 \\
9670 \\
-0-0\end{array}$ \\
\hline $\begin{array}{l}\text { TOTAL } \\
\text { MEAN } \\
\text { MAX } \\
\text { MIN } \\
\text { AC-FT } \\
\text { CFSM } \\
\text { IN. }\end{array}$ & $\begin{array}{r}158740 \\
5121 \\
7580 \\
2640 \\
314900 \\
.27 \\
.31\end{array}$ & $\begin{array}{r}154810 \\
5160 \\
6850 \\
2810 \\
307100 \\
.27 \\
.30\end{array}$ & $\begin{array}{r}96690 \\
3119 \\
3840 \\
1700 \\
191800 \\
.16 \\
.19\end{array}$ & $\begin{array}{r}92540 \\
2985 \\
3210 \\
2640 \\
183600 \\
.16 \\
.18\end{array}$ & $\begin{array}{r}84000 \\
3000 \\
4430 \\
2420 \\
166600 \\
.16 \\
.16\end{array}$ & $\begin{array}{r}190060 \\
6131 \\
16000 \\
2570 \\
377000 \\
.32 \\
.37\end{array}$ & $\begin{array}{r}431750 \\
14390 \\
20400 \\
9870 \\
856400 \\
.75 \\
.84\end{array}$ & $\begin{array}{r}615100 \\
19840 \\
28900 \\
13200 \\
1220000 \\
1.04 \\
1.20\end{array}$ & $\begin{array}{r}419300 \\
13980 \\
19500 \\
10200 \\
831700 \\
.73 \\
.82\end{array}$ & $\begin{array}{r}369850 \\
11930 \\
16100 \\
7200 \\
733600 \\
.62 \\
.72\end{array}$ & $\begin{array}{r}208820 \\
6736 \\
9680 \\
4510 \\
414200 \\
.35 \\
.41\end{array}$ & $\begin{array}{r}338920 \\
11300 \\
17400 \\
3770 \\
672200 \\
.59 \\
.66\end{array}$ \\
\hline
\end{tabular}

STATISTICS OF MONTHLY MEAN DATA FOR WATER YEARS 1931 - 1991, BY WATER YEAR (WY)

$\begin{array}{lrrrrr}\text { MEAN } & 6203 & 5959 & 4567 & 4058 & 3977 \\ \text { MAX } & 21250 & 22800 & 10800 & 8304 & 9947 \\ \text { (WY) } & 1987 & 1972 & 1972 & 1986 & 1966 \\ \text { MIN } & 1128 & 1152 & 1006 & 935 & 1079 \\ (W Y) & 1937 & 1937 & 1935 & 1935 & 1933\end{array}$

SUMMARY STATISTICS

ANNUAL TOTAI

ANNUAL MEAN

HIGHEST ANNUAL MEAN

LOWEST ANNUAL MEAN

HIGHEST DAILY MEAN

LOWEST DAILY MEAN

ANNUAL SEVEN-DAY MINIMUM

INSTANTANEOUS PEAK FLOW

INSTANTANEOUS PEAK STAGE

INSTANTANEOUS LOW FLOW

ANNUAL RUNOFF (AC-FT)

ANNUAL RUNOFF (CFSM)

ANNUAL RUNOFF (INCHES)

10 PERCENT EXCEEDS

50 PERCENT EXCEEDS

90 PERCENT EXCEEDS
FOR 1990 CALENDAR YEAR

$\begin{array}{cc}2421040 & \\ 6633 & \\ & \\ 19800 & \text { Jun } 24 \\ 1700 & \text { Dec 22 } \\ 2270 & \text { Feb 27 } \\ 21000 & \text { Jun 24 } \\ 9.17 & \text { Jun 24 } \\ 1480 \mathrm{a} & \text { Dec 21 } \\ 4802000 & \\ .35 & \\ 4.72 & \\ 14400 & \\ 4980 & \\ 2430 & \end{array}$

$\begin{array}{rrr}6922 & 17050 & 14630 \\ 23410 & 42970 & 39760 \\ 1966 & 1969 & 1986 \\ 1602 & 3575 & 2796 \\ 1940 & 1959 & 1934\end{array}$

FOR 1991 WATER YEAR

3160580
8659

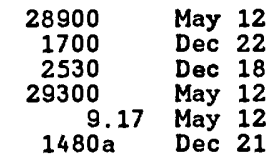

6269000

$$
\begin{aligned}
& 6.16 \\
& 17300 \\
& 6300 \\
& 2810
\end{aligned}
$$

11450
29910
1943
1646
1934

5797

22490

1972

715
1934
5720
23570

1986

888
1934

WATER YEARS 1931 - 1991

7904
17750
1603
90300
602
646
91000
19.53
$529 a$
5726000
5.41
5.62
17300
5370
1980


MISSISSIPPI RIVER MAIN STEM

05288500 MISSISSIPPI RIVER NEAR ANOKA, MN--Continued

WATER-QUALITY RECORDS

LOCATION.--Sediment samples collected at Camden Avenue bridge, in Minneapolis, 7.0 mi downstream from gage. Tritium samples collected at gage near right bank. Prior to October 1, 1978, sediment samples collected at Lowry Avenue bridge.

DRAINAGE AREA. $--19,600 \mathrm{mi}^{2}$, approximately.

PERIOD OF RECORD.--Water years 1963-67, 1975 to current year.

PERIOD OF RECORD, - -

WATER TEMPERATURES: October 1975 to current year.

SUSPENDED SEDIMENT DISCHARGE: August 1975 to current year.

REMARKS. --Sediment samples were collected approximately daily by an observer during the open-water perlod. In general, daily concentrations and loads for the open-water period are considered good. During the winter period, daily sediment concentrations and loads are based primarily on concentrations of sediment in samples that were collected monthly and on dally water-discharge records. Sediment records for the winter period are considered fair. Water temperatures were obtained by the observer approximately daily during the openwater period and monthly by U.S. Geological Survey personnel during the winter period. Many temperatures are not published because of questionable values.

EXTREMES FOR PERIOD OF DAILY RECORD

WATER TEMPERATURES (water years 1976-77, 1979-80, 1982-91): Maximum da11y, 31.0C, Aug. 25, 26, 1976, July 19, 1977; minimum daily, $0.0^{\circ} \mathrm{C}$ several days during winter period, each year.

SEDIMENT CONCENTRATIONS: Maximum daily mean, 210 mg/L Apr. 3, 1982; minimum daily mean, 1 mg/L on several days in $1978,1980,1981,1982$, and 1984 .

SEDIMENT LOADS: Maximum daily, 17,400 tons Apr. 20, 1982; minimum daily, 3.9 tons Feb. 2 , 1981.

EXTREMES FOR CURRENT YEAR --

WATER TEMPERATURES: Maximum daily, 29. $0^{\circ} \mathrm{C}$, Aug. 29, 30; minimum daily, $0.0^{\circ} \mathrm{C}$ several days during winter period. SEDIMENT CONCENTRATION: Maximum daily mean, $92 \mathrm{mg} / \mathrm{L}$, Mar, 27 ; minimum daily mean, $2 \mathrm{mg} / \mathrm{L}$, Feb. 4.

SEDIMENT LOADS: Maximum daily, 4,040 tons, May 9; minimum daily, 14 tons, Dec. $21,22$.

WATER TEMPERATURE, DEGREES CELSIUS, WATER YEAR OCTOBER 1990 TO SEPTEMBER 1991 ONCE-DAILY

\begin{tabular}{|c|c|c|c|c|c|c|c|c|c|c|c|c|}
\hline DAY & OCT & NOV & DEC & JAN & FEB & MAR & APR & MAY & JUN & JUL & AUG & SEP \\
\hline $\begin{array}{l}1 \\
2 \\
3 \\
4 \\
5\end{array}$ & $\begin{array}{r}17.0 \\
18.0 \\
16.0 \\
18.0\end{array}$ & $\begin{array}{r}12.0 \\
10.0 \\
7.0 \\
7.0 \\
6.0\end{array}$ & $\begin{array}{l}2.0 \\
\ldots- \\
\ldots- \\
\ldots-\end{array}$ & $\begin{array}{l}--- \\
--- \\
--- \\
-- \\
--\end{array}$ & $\begin{array}{l}--- \\
--- \\
-\cdots- \\
---\end{array}$ & $\begin{array}{r}-- \\
--0 \\
---\end{array}$ & $\begin{array}{r}6.0 \\
6.0 \\
9.0 \\
10.0 \\
12.0\end{array}$ & $\begin{array}{r}10.0 \\
10.0 \\
10.0 \\
10.0 \\
-\end{array}$ & $\begin{array}{l}22.0 \\
25.0 \\
25.0 \\
23.0 \\
23.0\end{array}$ & $\begin{array}{l}25.0 \\
25.0 \\
23.0 \\
24.0 \\
23.0\end{array}$ & $\begin{array}{l}25.0 \\
23.0 \\
22.0 \\
23.0 \\
23.0\end{array}$ & $\begin{array}{l}25.0 \\
22.0 \\
23.0 \\
24.0 \\
23.0\end{array}$ \\
\hline $\begin{array}{r}6 \\
7 \\
8 \\
9 \\
10\end{array}$ & $\begin{array}{r}15.0 \\
13.0 \\
14.0 \\
13.0\end{array}$ & $\begin{array}{l}5.0 \\
5.0 \\
4.0 \\
5.0 \\
5.0\end{array}$ & $\begin{array}{l}--- \\
-- \\
-- \\
--0 \\
3.0\end{array}$ & $\begin{array}{l}--- \\
--- \\
--- \\
--- \\
---\end{array}$ & $\begin{array}{l}--- \\
--- \\
--0 \\
4.0 \\
2.0\end{array}$ & $\begin{array}{l}--- \\
1.0 \\
3.0 \\
3.0 \\
4.0\end{array}$ & $\begin{array}{r}14.0 \\
15.0 \\
10.0 \\
7.0 \\
9.0\end{array}$ & $\begin{array}{r}8.0 \\
9.0 \\
9.0 \\
12.0 \\
14.0\end{array}$ & $\begin{array}{l}23.0 \\
22.0 \\
23.0 \\
24.0 \\
24.0\end{array}$ & $\begin{array}{l}25.0 \\
25.0 \\
25.0 \\
24.0 \\
25.0\end{array}$ & $\begin{array}{r}21.0 \\
21.0 \\
21.0 \\
21.0\end{array}$ & $\begin{array}{l}24.0 \\
21.0 \\
22.0 \\
23.0 \\
20.0\end{array}$ \\
\hline $\begin{array}{l}11 \\
12 \\
13 \\
14 \\
15\end{array}$ & $\begin{array}{l}13.0 \\
13.0 \\
11.0 \\
14.0 \\
13.0\end{array}$ & $\begin{array}{l}5.0 \\
4.0 \\
5.0 \\
8.0 \\
8.0\end{array}$ & $\begin{array}{l}3.0 \\
2.0 \\
1.0 \\
\ldots-\end{array}$ & $\begin{array}{l}-- \\
--- \\
--- \\
-- \\
--\end{array}$ & $\begin{array}{l}--- \\
--- \\
--- \\
---\end{array}$ & $\begin{array}{l}5.0 \\
3.0 \\
3.0 \\
4.0 \\
-\end{array}$ & $\begin{array}{l}8.0 \\
5.0 \\
5.0 \\
6.0 \\
6.0\end{array}$ & $\begin{array}{l}17.0 \\
19.0 \\
20.0 \\
22.0 \\
22.0\end{array}$ & $\begin{array}{l}25.0 \\
26.0 \\
25.0 \\
26.0 \\
24.0\end{array}$ & $\begin{array}{l}25.0 \\
23.0 \\
22.0 \\
25.0 \\
25.0\end{array}$ & $\begin{array}{l}25.0 \\
25.0 \\
24.0 \\
27.0 \\
25.0\end{array}$ & $\begin{array}{l}19.0 \\
20.0 \\
21.0 \\
21.0 \\
22.0\end{array}$ \\
\hline $\begin{array}{l}16 \\
17 \\
18 \\
19 \\
20\end{array}$ & $\begin{array}{r}12.0 \\
11.0 \\
8.0 \\
9.0 \\
\end{array}$ & $\begin{array}{l}6.0 \\
5.0 \\
6.0 \\
7.0 \\
6.0\end{array}$ & $\begin{array}{l}-- \\
-- \\
-- \\
-- \\
--\end{array}$ & $\begin{array}{l}-- \\
-- \\
-- \\
-- \\
--\end{array}$ & $=-$ & $\begin{array}{l}4.0 \\
3.0 \\
4.0 \\
7.0 \\
5.0\end{array}$ & $\begin{array}{r}6.0 \\
8.0 \\
9.0 \\
10.0 \\
7.0\end{array}$ & $\begin{array}{l}22.0 \\
18.0 \\
17.0 \\
18.0 \\
19.0\end{array}$ & $\begin{array}{l}24.0 \\
25.0 \\
25.0 \\
25.0 \\
24.0\end{array}$ & $\begin{array}{r}28.0 \\
27.0 \\
28.0 \\
-.- \\
-\ldots\end{array}$ & $\begin{array}{l}24.0 \\
23.0 \\
23.0 \\
25.0 \\
23.0\end{array}$ & $\begin{array}{l}20.0 \\
19.0 \\
15.0 \\
13.0 \\
14.0\end{array}$ \\
\hline $\begin{array}{l}21 \\
22 \\
23 \\
24 \\
25\end{array}$ & $\begin{array}{r}8.0 \\
9.0 \\
10.0 \\
9.0 \\
8.0\end{array}$ & \begin{tabular}{l}
9.0 \\
7.0 \\
4.0 \\
\hdashline-
\end{tabular} & $\begin{array}{l}-- \\
--- \\
-- \\
-- \\
--\end{array}$ & $\begin{array}{l}-- \\
-- \\
-- \\
-- \\
--\end{array}$ & $\begin{array}{l}--- \\
--- \\
--- \\
---\end{array}$ & $\begin{array}{l}4.0 \\
3.0 \\
1.0 \\
4.0 \\
5.0\end{array}$ & $\begin{array}{l}10.0 \\
10.0 \\
11.0 \\
12.0 \\
13.0\end{array}$ & $\begin{array}{l}21.0 \\
22.0 \\
22.0 \\
22.0 \\
21.0\end{array}$ & $\begin{array}{l}21.0 \\
20.0 \\
22.0 \\
23.0 \\
25.0\end{array}$ & $\begin{array}{r}--- \\
27.0 \\
27.0 \\
25.0 \\
24.0\end{array}$ & $\begin{array}{l}25.0 \\
24.0 \\
21.0 \\
23.0 \\
26.0\end{array}$ & $\begin{array}{l}14.0 \\
13.0 \\
13.0 \\
13.0 \\
13.0\end{array}$ \\
\hline $\begin{array}{l}26 \\
27 \\
28 \\
29 \\
30 \\
31\end{array}$ & $\begin{array}{r}10.0 \\
8.0 \\
8.0 \\
9.0 \\
10.0 \\
10.0\end{array}$ & $\begin{array}{l}2.0 \\
2.0 \\
2.0 \\
\cdots--\end{array}$ & $\begin{array}{l}-- \\
-- \\
-\cdots \\
-- \\
-- \\
--\end{array}$ & $\begin{array}{l}-- \\
-- \\
-\cdots \\
-- \\
-- \\
--\end{array}$ & $\begin{array}{l}--- \\
--- \\
--- \\
--- \\
---\end{array}$ & $\begin{array}{l}7.0 \\
3.0 \\
2.0 \\
1.0 \\
1.0 \\
4.0\end{array}$ & $\begin{array}{l}14.0 \\
13.0 \\
15.0 \\
15.0 \\
11.0 \\
\end{array}$ & $\begin{array}{l}20.0 \\
23.0 \\
22.0 \\
23.0 \\
23.0 \\
23.0\end{array}$ & $\begin{array}{r}26.0 \\
27.0 \\
25.0 \\
26.0 \\
26.0 \\
\end{array}$ & $\begin{array}{l}24.0 \\
23.0 \\
20.0 \\
22.0 \\
24.0 \\
25.0\end{array}$ & $\begin{array}{l}28.0 \\
28.0 \\
28.0 \\
29.0 \\
29.0 \\
26.0\end{array}$ & $\begin{array}{l}12.0 \\
12.0 \\
12.0 \\
14.0 \\
13.0\end{array}$ \\
\hline $\begin{array}{l}\text { MEAN } \\
\text { MAX } \\
\text { MIN }\end{array}$ & $\begin{array}{l}--- \\
--- \\
---\end{array}$ & $\begin{array}{l}--- \\
--- \\
---\end{array}$ & $\begin{array}{l}--- \\
--- \\
---\end{array}$ & $\begin{array}{l}--- \\
--- \\
---\end{array}$ & $=-$ & $\begin{array}{l}--- \\
--- \\
--\infty\end{array}$ & $\begin{array}{r}9.7 \\
15.0 \\
5.0\end{array}$ & $\begin{array}{l}-- \\
--- \\
---\end{array}$ & $\begin{array}{l}24.1 \\
27.0 \\
20.0\end{array}$ & $\begin{array}{l}--- \\
--- \\
---\end{array}$ & $=-$ & $\begin{array}{l}18.0 \\
25.0 \\
12.0\end{array}$ \\
\hline
\end{tabular}


MISSISSIPPI RIVER MAIN STEM

05288500 MISSISSIPPI RIVER NEAR ANOKA, MN--Continued

SUSPENDED-SEDIMENT, WATER YEAR OCTOBER 1990 TO SEPTEMBER 1991

$\begin{array}{ccc}\text { MEAN } & \\ \text { CONCEN- } & \text { LOAD } \\ \text { TRATION } & \text { (TONS/ } \\ \text { DAY } & (M G / L) & \text { DAY) }\end{array}$

MEAN

TONCEN- LOAD (MG/L)

NOVEMBER

OCTOBER

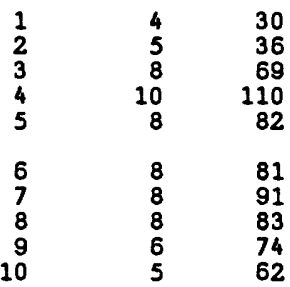

$\begin{array}{lll}11 & 5 & 64 \\ 12 & 6 & 70 \\ 13 & 6 & 71 \\ 14 & 6 & 68 \\ 15 & 7 & 77\end{array}$

$\begin{array}{lll}16 & 7 & 76 \\ 17 & 7 & 79 \\ 18 & 6 & 76 \\ 19 & 5 & 63 \\ 20 & 6 & 84\end{array}$

$\begin{array}{rrr}21 & 6 & 85 \\ 22 & 6 & 99 \\ 23 & 13 & 253 \\ 24 & 23 & 455\end{array}$

$\begin{array}{rrr}26 & 57 & 1170 \\ 27 & 41 & 801 \\ 28 & 28 & 548 \\ 29 & 17 & 319 \\ 30 & 16 & 305 \\ 31 & 19 & 372\end{array}$

TOTAL -.. 6606

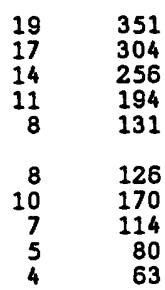

$\begin{array}{ll}5 & 71 \\ 6 & 87 \\ 7 & 93\end{array}$

$8 \quad 107$

$8 \quad 106$

$\begin{array}{ll}8 & 113 \\ 8 & 105 \\ 8 & 110\end{array}$

$9 \quad 114$

$\begin{array}{rr}10 & 131 \\ 9 & 111 \\ 7 & 88 \\ 6 & 78 \\ 5 & 61\end{array}$

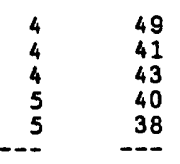

MAY

$\begin{array}{lr}25 & 1050 \\ 19 & 805 \\ 26 & 1190 \\ 26 & 1240 \\ 36 & 1930\end{array}$

$45 \quad 2650$

$\begin{array}{ll}45 & 2650 \\ 55 & 34030\end{array}$

3830
4040
4020

$49 \quad 3810$

$\begin{array}{lll}11 & 15 & 400 \\ 12 & 15 & 404 \\ 13 & 19 & 549\end{array}$

14

16
17
18
19
20

969

1210

1350

1500

2100

$\begin{array}{lll}21 & 42 & 2270 \\ 22 & 42 & 2310\end{array}$

$\begin{array}{lll}22 & 42 & 2310 \\ 23 & 37 & 1950\end{array}$

$\begin{array}{lll}24 & 27 & 1320 \\ 25 & 26 & 1170\end{array}$

$26 \quad 24 \quad 978$

$\begin{array}{lll}27 & 24 & 978 \\ 28 & 22 & 849 \\ 29 & 23 & 882 \\ & 27 & 984\end{array}$

$\begin{array}{rrr}29 & 27 & 984 \\ 30 & 26 & 997\end{array}$

TOTAL -.. 32624

YEAR $\quad 244888$

3590

3340

1940

1850

1750

2170

1740

1350

$\begin{array}{rr}23 & 1020 \\ 22 & 909 \\ 21 & 811 \\ 21 & 782 \\ 21 & 748\end{array}$

$\begin{array}{rr}23 & 820 \\ 27 & 970 \\ 43 & 1630\end{array}$

$\begin{array}{ll}43 & 1630 \\ 50 & 2080\end{array}$

2080

3560

63555 $-\cdots \quad 3488$

MEAN
CONCEN- LOAD
TRATION (TONS) (MG/L)

DECEMBER
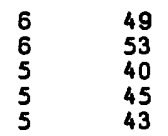

MEAN

LOAD (MG/L) (TONS/ DAY)

JANUARY

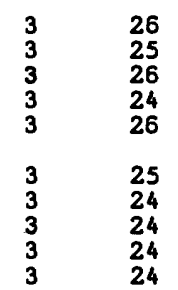

LOAD

(TONS/ MG/L)

FEBRUARY

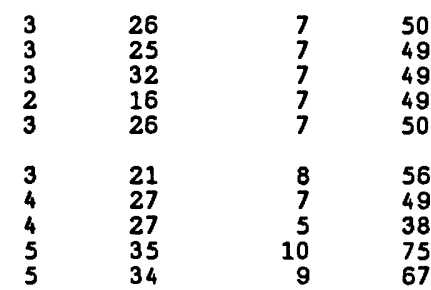

$\begin{array}{ll}5 & 35 \\ 5 & 40 \\ 7 & 54 \\ 6 & 41 \\ 6 & 45\end{array}$

$\begin{array}{ll}7 & 84 \\ 6 & 69 \\ 6 & 55 \\ 6 & 55 \\ 7 & 68\end{array}$

$\begin{array}{ll}6 & 48 \\ 6 & 42 \\ 6 & 43 \\ 7 & 55 \\ 7 & 58\end{array}$

\begin{tabular}{rr}
6 & 45 \\
6 & 41 \\
6 & 39 \\
-- & -- \\
\hline-- & --- \\
\hline--
\end{tabular}

1186

AUGUST
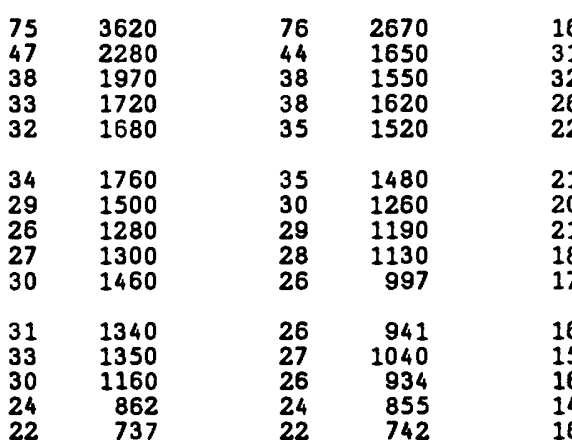

16
31
32
26
22
21
20
21
18
17
16
15
16
14
16

$$
\begin{aligned}
& 323 \\
& 712 \\
& 836
\end{aligned}
$$

650
536

491

453

488

398
354

997

310

306

311

286

323
269
249

24

198

716
822

745

729

1100

1200

867

729

612
555

524
479

215$$
504
$$

315
236

448

$\begin{array}{ll}26 & 758 \\ 29 & 838 \\ 29 & 822\end{array}$

$\begin{array}{ll}29 & 822 \\ 31 & 912\end{array}$

31
29
$--\quad 822$
-

20
19
19
14
13
15

CONCEN- LOAD

RATION (TONS

50
49
49
49
50
56
49
38
75
67
42
72
47
48
41
39
45
73
78
68
11
79
92


LOCATION, --Lat $45^{\circ} 36^{\prime} 05^{\prime \prime}$, Long $96^{\circ} 52^{\prime} 18^{\prime \prime}$, in SWl sec.13, T.125 N., R.50 W. Roberts County, Hydrologic Unit 07020001 , on Sisseton Indian Reservation, on right bank $2 \mathrm{mi}$ northwest of town of Browns Valley, MN, $5.3 \mathrm{mi}$ northeast of Peever, $7.2 \mathrm{mi}$ downstream from Jorgenson River, and $8 \mathrm{mi}$ upstream from Big Stone Lake. DRAINAGE AREA. $-447 \mathrm{mi}^{2}$.

PERIOD OF RECORD.--October 1939 to September 1981, October 1989 to current year. REVISED RECORDS.--WSP 1308: $1943(\mathrm{M})$.

GAGE.--Water-stage recorder. Datum of gage is 1,002.20 ft above National Geodetic Vertical Datum of 1929.

Oct. 1, 1939, to Mar. 20. 1940, nonrecording gage at site $4.5 \mathrm{mi}$ downstream at different datum. Mar. 21

to Apr. 12, 1940, nonrecording gage at site $100 \mathrm{ft}$ downstream at present datum. April 13 to Aug. 27 , 1940 , nonrecording gage at present site and datum.

REMARKS. --Records good except those for estimated daily discharges, which are fair.

EXTREMES FOR CURRENT YEAR.--Peak discharges greater than base discharge of $450 \mathrm{ft} / \mathrm{s}$ and maximum ( $*$ ):

\begin{tabular}{|c|c|c|c|c|c|c|c|}
\hline Date & Time & $\begin{array}{c}\text { Discharge } \\
\left(\mathrm{ft}^{3} / \mathrm{s}\right)\end{array}$ & $\begin{array}{c}\text { Gage height } \\
\text { (ft) }\end{array}$ & Date & Time & $\begin{array}{c}\text { Discharge } \\
\left(\mathrm{ft}^{3} / \mathrm{s}\right)\end{array}$ & $\begin{array}{c}\text { Gage height } \\
\text { (ft) }\end{array}$ \\
\hline $\begin{array}{l}\text { June } \\
\text { July }\end{array}$ & $\begin{array}{l}0930 \\
1300\end{array}$ & $\begin{array}{r}\star 891 \\
513\end{array}$ & $\begin{array}{r}5.77 \\
4.78\end{array}$ & $\begin{array}{ll}\text { July } & 23 \\
\text { July } 28\end{array}$ & $\begin{array}{l}1130 \\
2400\end{array}$ & $\begin{array}{l}548 \\
639\end{array}$ & $\begin{array}{l}4.87 \\
5.09\end{array}$ \\
\hline
\end{tabular}

DISCHARGE, CUBIC FEET PER SECOND, WATER YEAR OCTOBER 1990 TO SEPTEMBER 1991

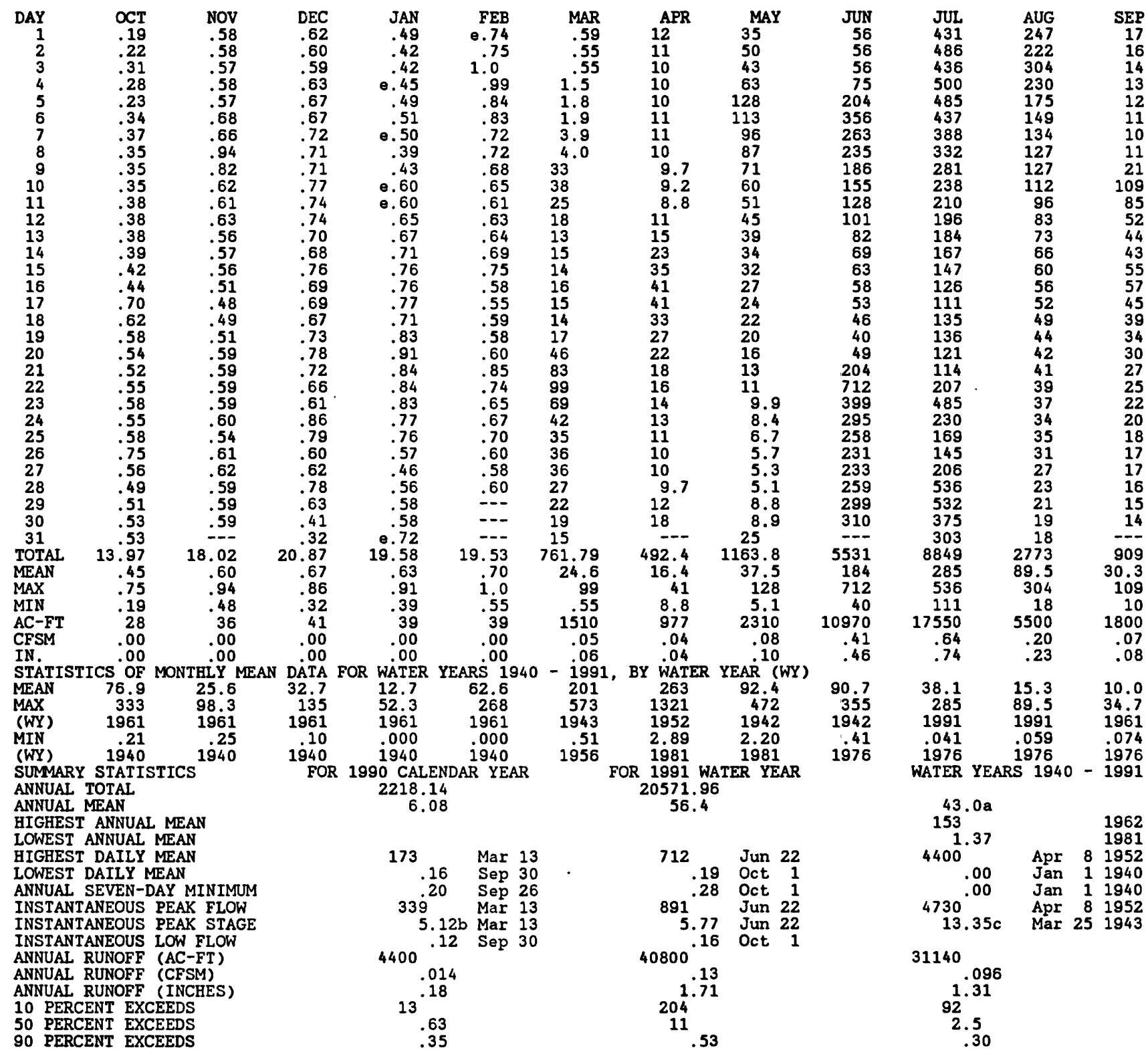

a Median of annual mean discharges is $32 \mathrm{ft}^{3} / \mathrm{s}$.

b Backwater from ice.

c From floodmark (backwater from ice).

e Estimated. 
MINNESOTA RIVER BASIN

05291000 WHETSTONE RIVER NEAR BIG STONE CITY, SD

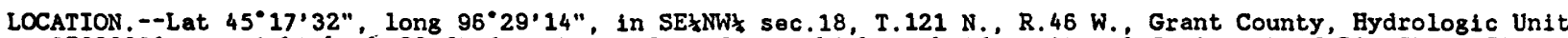
07020001 , on right bank $20 \mathrm{ft}$ downstream from former highway bridge site, $1.5 \mathrm{mi}$ west of Big Stone City, and $4.5 \mathrm{mi}$ upstream from Big Stone Lake.

DRAINAGE AREA $--389 \mathrm{mi}^{2}$.

PERIOD OF RECORD.--March 1910 to November 1912 (no winter records), and March 1931 to current year. Monthly discharge only for some periods, published in WSP 1308

REVISED RECORDS.--WSP 895: Drainage area. WSP 1308: 1932(M), 1935(M)

GAGE.--Water-stage recorder. Datum of gage is $996.96 \mathrm{ft}$ adjustment of 1912. Mar. 8, 1910, to Nov. 30, 1912, nonrecording gage $2 \mathrm{mi}$ downstream at different datum. Mar. 18, 1931, to May 3, 1939, nonrecording gage, at site $20 \mathrm{ft}$ upstream at present datum. May 4, 1939, to Nov. 8, 1952, water-stage recorder at site 80 ft downstream at present datum.

REMARKS. --Records good except those for estimated daily discharges, which are fair.

EXTREMES OUTSIDE PERIOD OF RECORD.--Maximum stage known, about 26 ft in June 1919, present site and datum, from information by local resident, discharge $29,000 \mathrm{ft}^{3} / \mathrm{s}$

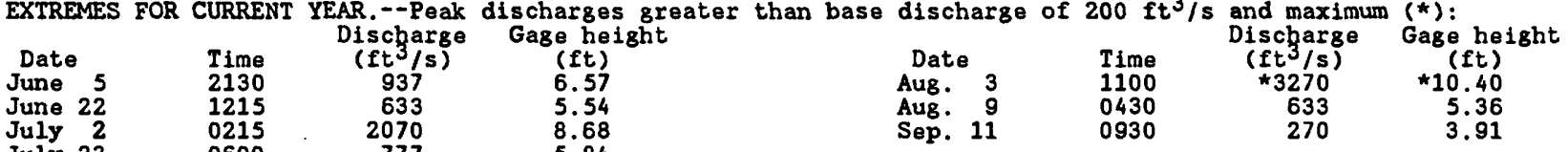

$\begin{array}{lr}\text { July } & 2 \\ \text { July } & 23\end{array}$

$0600 \quad 777 \quad 5.84$

Sep. 11

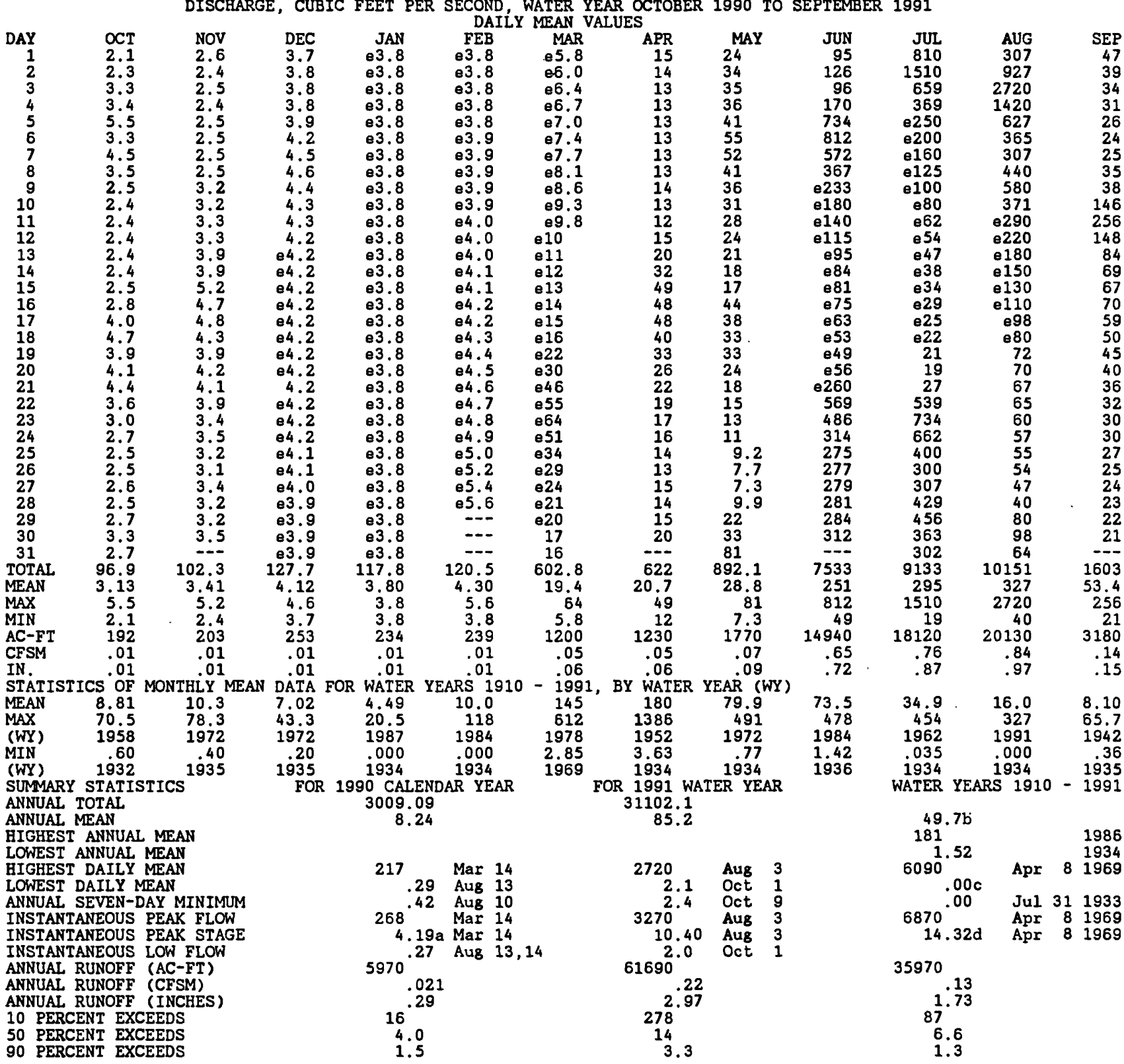

a Backwater from ice.

b Median of annual mean discharges is $35 \mathrm{ft}^{3} / \mathrm{s}$.

c No flow at times in most years.

d From floodmark.

e Estimated. 


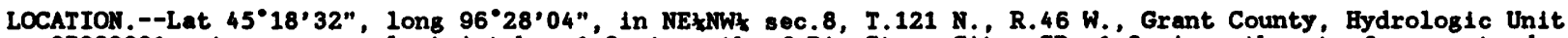
07020001 , at new powerplant intake, $1^{\prime} 2 \mathrm{mi}$ north of Big stone City, SD, $\mathrm{i}^{\prime} 2 \mathrm{mi}$ northwest of concrete dam at outlet, and $1.0 \mathrm{ml}$ west of Ortonvilie. Prior to January 1989, at old powerplant site at west edge of Ortonville.

PERIOD OF RECORD.--March 1937 to current year.

GAGE.-Nonrecording gage read once a day. Datum of gage is 957.69 ft above National Geodetic Vertical Datum of 1929. Prior to sept. 17, 1947, nonrecording gage at sito 1.2 mi southeast at same datum. Sept. 18 , 1947 , to June 30 , 1963, water-stage recorder at s1te $1.2 \mathrm{mi}$ southeast at same datum. Sept. 21 , 1959 , to June 30 , 1963, supplementary nonrecording gage read once daily, at site $0.9 \mathrm{ml}$ southeast at same datum. July 1 , 1963 to Jan. 1989 nonrecording 8 age at site $0.9 \mathrm{mi}$ southeast at same datum.

REMARKS.--Natural lake with concrete dam at outlet. Dam was rebuilt and completed in Nov. 1985, with the following changes: Elght 7 ft high by $10 \mathrm{ft}$ wide electrically operated slide gates, one 48 in. by 48 in. gate; and one $18 \mathrm{in}$. sluice gate; silis of all gates are at $3.0 \mathrm{ft}$. S1It barrier dam 700 ft upstream in outiet channel of lake completed July 7, 1958; rebuilt and completed Dec. 1986 with the new crest at 7.0 ft (previous crest was at $5.9 \mathrm{ft}$ ). Supplementary nonrecording gage readings used for stages below crest of silt barrier to June 30 , 1963. Water level subject to fluctuation caused by wind action.

EXTREMES FOR PERIOD OF RECORD.--Maximum gage height, 12.73 ft, Apr. 17, 1952; minimum observed, 3.53 ft, Mar. 2 , 1957 (strong upstream wind in channel). Minimum observations of 3.10 ft, Mar. 2, 1940, and $2.20 \mathrm{ft}$, Nov. 20, 1940, at spillway site are the result of blockage of channel to spillway by ice and snow and do not represent lake levations.

EXTREMES FOR CURRENT YEAR.--Maximum gage height observed, 8.28 ft, Aug. 4; minimum observed, 6.09 ft, Apr. 26.

GAGE BEIGET, IN FEET, OCTOBER 1990 TO SEPTEMBER 1991
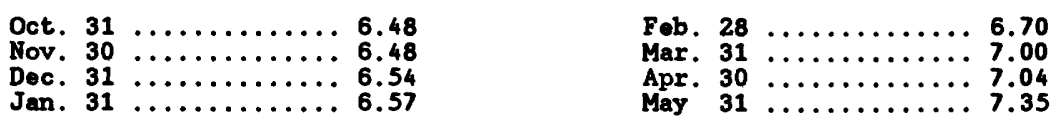

$\begin{array}{llll}\text { June } & \mathbf{3 0} & \ldots \ldots \ldots \ldots \ldots \ldots & 7.90 \\ \text { July } & \mathbf{3 1} & \ldots \ldots \ldots \ldots \ldots \ldots & \mathbf{8} .00 \\ \text { Aug. } & \mathbf{3 1} & \ldots \ldots \ldots \ldots \ldots \ldots & 7.84 \\ \text { Sept. } & \mathbf{3 0} & \ldots \ldots \ldots \ldots \ldots \ldots & 7.70\end{array}$

NOTE.--Gage-height record other than that shown above is available in the District office. 
MINNESOTA RIVER BASIN

05292000 MINNESOTA RIVER AT ORTONVILLE, MA

LOCATION. --Lat 45'17'44", long 96'26'38", In NEzWW sec.16, T.121 N., R.46 W., Big Stone County, Bydrologic Unit 07020001 , on left bank $400 \mathrm{ft}$ downstream from bridge on U.S. Highway 12 and 1,300 ft downstream from dam at outlet of Big Stone Lake, at Ortonville.

DRAINAGE AREA. --1,160 $\mathrm{mi}^{2}$, approximately.

PERIOD OF RECORD.--February 1938 to current year.

REVISED RECORDS.--WSP 895: 1939. WSP 1508: 1942 (yearly mean).

GAGE.--Water-stage recorder. Datum of gage is $956.38 \mathrm{ft}$ above National Geodetic Vertical Datum of 1929. Prior to Mar. 31, 1939, nonrecording gage on downstream side of dam 1,300 ft upstream at datum 1.31 ft higher.

REMARKS. --Records good except those for estimated daily discharges, which are fair. Some regulation by Big Stone Lake (station 05291500).

DISCHARGE, CUBIC FEET PER SECOND, WATER YEAR OCTOBER 1990 TO SEPTERBER 1991

\begin{tabular}{|c|c|c|c|c|c|c|c|c|c|c|c|c|}
\hline DAY & OCT & NOV & DEC & JAN & FEB & MAR & APR & MAY & JNN & JUL & AUG & SEP \\
\hline $\begin{array}{r}1 \\
2 \\
3 \\
4 \\
5 \\
6 \\
7 \\
8 \\
9 \\
10 \\
11 \\
12 \\
13 \\
14 \\
15 \\
16 \\
17 \\
18 \\
19 \\
20 \\
21 \\
22 \\
23 \\
24 \\
25 \\
26 \\
27 \\
28 \\
29 \\
30 \\
31\end{array}$ & $\begin{array}{l}3.4 \\
3.5 \\
4.6 \\
4.3 \\
4.4 \\
6.0 \\
5.2 \\
4.7 \\
4.6 \\
4.4 \\
3.9 \\
4.0 \\
3.7 \\
3.6 \\
3.9 \\
3.9 \\
4.4 \\
4.4 \\
4.7 \\
4.1 \\
4.0 \\
4.0 \\
3.8 \\
4.0 \\
3.7 \\
3.5 \\
3.6 \\
4.2 \\
3.9 \\
4.0 \\
4.2\end{array}$ & $\begin{array}{l}3.8 \\
3.3 \\
3.3 \\
3.1 \\
3.1 \\
2.8 \\
3.0 \\
2.8 \\
2.6 \\
2.5 \\
2.4 \\
2.5 \\
2.7 \\
2.5 \\
2.4 \\
2.8 \\
3.2 \\
3.1 \\
3.2 \\
3.4 \\
3.1 \\
2.8 \\
2.5 \\
3.1 \\
3.6 \\
3.6 \\
4.0 \\
4.0 \\
4.3 \\
4.6\end{array}$ & $\begin{array}{l}4.6 \\
4.3 \\
4.3 \\
4.3 \\
4.6 \\
4.6 \\
4.3 \\
3.6 \\
3.1 \\
2.8 \\
2.8 \\
2.5 \\
2.6 \\
2.7 \\
2.6 \\
2.7 \\
2.7 \\
3.0 \\
2.8 \\
2.7 \\
2.4 \\
e 2.5 \\
e 2.5 \\
e 2.5 \\
e 2.5 \\
e 2.5 \\
e 2.5 \\
e 2.5 \\
e 2.5 \\
e 2.5 \\
e 2.5\end{array}$ & $\begin{array}{r}e 2.5 \\
2.4 \\
2.4 \\
2.5 \\
2.3 \\
2.3 \\
e 2.3 \\
e 2.3 \\
e 2.3 \\
e 2.3 \\
e 2.3 \\
e 2.3 \\
e 2.3 \\
e 2.5 \\
e 2.6 \\
2.7 \\
2.7 \\
2.5 \\
2.2 \\
1.9 \\
2.5 \\
2.3 \\
2.2 \\
2.5 \\
2.8 \\
2.6 \\
2.4 \\
2.4 \\
2.2 \\
2.2 \\
2.2\end{array}$ & $\begin{array}{l}2.2 \\
2.2 \\
2.2 \\
2.2 \\
2.2 \\
2.2 \\
2.2 \\
2.2 \\
2.2 \\
2.2 \\
2.2 \\
2.2 \\
2.2 \\
1.7 \\
1.8 \\
1.9 \\
1.9 \\
1.9 \\
1.9 \\
1.9 \\
2.3 \\
2.2 \\
1.9 \\
2.0 \\
2.2 \\
2.2 \\
2.2 \\
2.2 \\
--- \\
--. \\
---\end{array}$ & $\begin{array}{l}2.2 \\
2.4 \\
2.5 \\
2.6 \\
3.1 \\
2.2 \\
2.2 \\
2.2 \\
2.2 \\
2.5 \\
2.7 \\
2.3 \\
2.2 \\
2.1 \\
2.2 \\
2.4 \\
2.4 \\
2.5 \\
2.6 \\
4.7 \\
7.9 \\
11 \\
17 \\
33 \\
40 \\
31 \\
29 \\
29 \\
28 \\
19 \\
16\end{array}$ & $\begin{array}{c}16 \\
15 \\
14 \\
14 \\
14 \\
14 \\
14 \\
11 \\
8.2 \\
8.0 \\
8.3 \\
9.6 \\
9.7 \\
8.7 \\
8.8 \\
8.2 \\
8.1 \\
8.1 \\
8.1 \\
17 \\
20 \\
22 \\
21 \\
15 \\
7.6 \\
7.9 \\
9.0 \\
7.7 \\
10 \\
29 \\
-.-\end{array}$ & $\begin{array}{l}49 \\
47 \\
46 \\
47 \\
48 \\
57 \\
62 \\
56 \\
38 \\
38 \\
40 \\
41 \\
42 \\
43 \\
42 \\
42 \\
38 \\
37 \\
36 \\
35 \\
37 \\
43 \\
45 \\
43 \\
37 \\
37 \\
33 \\
33 \\
31 \\
32 \\
57\end{array}$ & $\begin{array}{r}104 \\
129 \\
170 \\
301 \\
693 \\
926 \\
815 \\
792 \\
781 \\
765 \\
504 \\
144 \\
96 \\
78 \\
72 \\
68 \\
65 \\
64 \\
61 \\
130 \\
365 \\
904 \\
901 \\
784 \\
610 \\
374 \\
535 \\
721 \\
898 \\
900\end{array}$ & $\begin{array}{r}1500 \\
1790 \\
1410 \\
1050 \\
849 \\
741 \\
601 \\
572 \\
553 \\
536 \\
382 \\
318 \\
386 \\
312 \\
78 \\
64 \\
65 \\
65 \\
65 \\
65 \\
66 \\
899 \\
1280 \\
991 \\
698 \\
290 \\
470 \\
839 \\
801 \\
715 \\
707\end{array}$ & $\begin{array}{r}716 \\
1220 \\
1670 \\
1700 \\
1120 \\
838 \\
863 \\
948 \\
1030 \\
825 \\
657 \\
253 \\
185 \\
217 \\
215 \\
160 \\
92 \\
93 \\
90 \\
89 \\
90 \\
89 \\
91 \\
88 \\
90 \\
88 \\
88 \\
87 \\
100 \\
95 \\
92\end{array}$ & $\begin{array}{r}90 \\
90 \\
92 \\
60 \\
29 \\
28 \\
29 \\
29 \\
57 \\
56 \\
47 \\
63 \\
56 \\
56 \\
56 \\
57 \\
57 \\
57 \\
53 \\
51 \\
51 \\
54 \\
51 \\
51 \\
154 \\
372 \\
352 \\
299 \\
156 \\
98 \\
---\end{array}$ \\
\hline $\begin{array}{l}\text { TOTAL } \\
\text { MEAN } \\
\text { MAX } \\
\text { MIN } \\
\text { AC-FT } \\
\text { CFSM } \\
\text { IN. }\end{array}$ & $\begin{array}{r}128.6 \\
4.15 \\
6.0 \\
3.4 \\
255 \\
.00 \\
.00\end{array}$ & $\begin{array}{r}94.1 \\
3.14 \\
4.6 \\
2.4 \\
187 \\
.00 \\
.00\end{array}$ & $\begin{array}{r}95.0 \\
3.06 \\
4.6 \\
2.4 \\
188 \\
.00 \\
.00\end{array}$ & $\begin{array}{r}73.9 \\
2.38 \\
2.8 \\
1.9 \\
147 \\
.00 \\
.00\end{array}$ & $\begin{array}{r}58.8 \\
2.10 \\
2.3 \\
1.7 \\
117 \\
.00 \\
.00\end{array}$ & $\begin{array}{r}311.1 \\
10.0 \\
40 \\
2.1 \\
617 \\
.01 \\
.01\end{array}$ & $\begin{array}{r}372.0 \\
12.4 \\
29 \\
7.6 \\
738 \\
.01 \\
.01\end{array}$ & $\begin{array}{r}1312 \\
42.3 \\
62 \\
31 \\
2600 \\
.04 \\
.04\end{array}$ & $\begin{array}{r}13750 \\
458 \\
926 \\
61 \\
27270 \\
.40 \\
.44\end{array}$ & $\begin{array}{r}19158 \\
618 \\
1790 \\
64 \\
38000 \\
.53 \\
.61\end{array}$ & $\begin{array}{r}13979 \\
451 \\
1700 \\
87 \\
27730 \\
.39 \\
.45\end{array}$ & $\begin{array}{r}2751 \\
91.7 \\
372 \\
28 \\
5460 \\
.08 \\
.09\end{array}$ \\
\hline
\end{tabular}

STATISTICS OF MONTHLY MEAN DATA FOR WATER YEARS 1938 - 1991, BY WATER YEAR (WY)

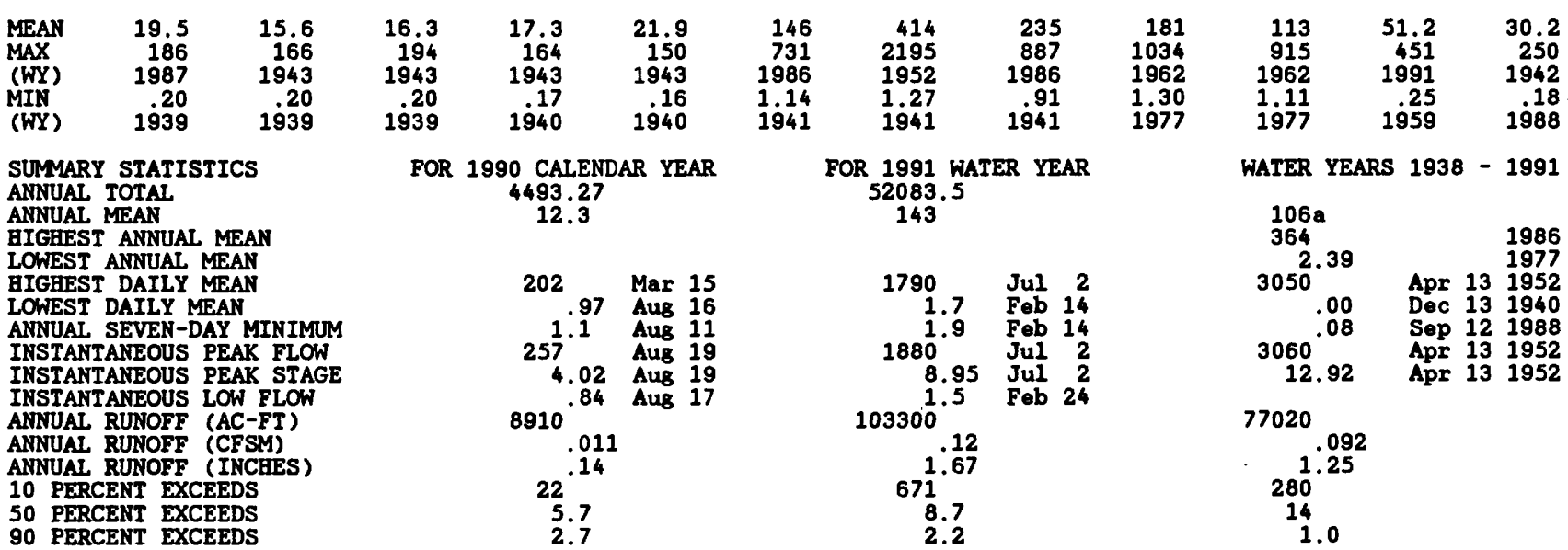

a Median of annual mean discharges is $80 \mathrm{ft}^{3} / \mathrm{s}$.

- Estimated. 
MINNESOTA RIVER BASIN

05293000 YELLOW BANK RIVER NEAR ODESSA, MN

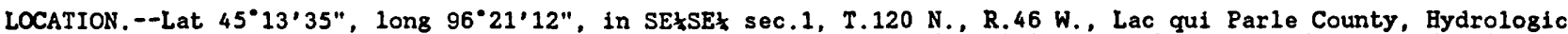
Unit 07020001 , on left bank $150 \mathrm{ft}$ downstream from highway bridge, $2.5 \mathrm{mi}$ southwest of Odessa, and $4.5 \mathrm{mi}$ upstream from mouth,

DRAINAGE AREA. $--398 \mathrm{mi}^{2}$.

PERIOD OF RECORD.--October 1939 to current year.

REVISED RECORDS.-WSP 1388: $1947(\mathrm{M}), 1950$.

GAGE - Water-stage recorder. Datum of gage is $953.34 \mathrm{ft}$ above National Geodetic Vertical Datum of 1929 (U.S. Army Corps of Engineers bench mark). Prior to Aug. 28, 1940 , nonrecording gage at site 150 ft upstream at same datum.

REMARKS. - -Records good except those for estimated daily discharges, which are fair.
EXTREMES FOR CURRENT YEAR. --Peak discharges greater than base discharge of 300 ft $/ 5$ and maximum (*):

\begin{tabular}{|c|c|c|c|c|c|c|c|}
\hline Date & Time & $\begin{array}{c}\text { Discharge } \\
\left(\mathrm{ft}^{3} / \mathrm{s}\right)\end{array}$ & $\begin{array}{c}\text { Gage height } \\
\text { (ft) }\end{array}$ & Date & Time & $\begin{array}{c}\text { Discharge } \\
\left(\mathrm{ft}^{3} / \mathrm{s}\right)\end{array}$ & $\begin{array}{c}\text { Gage height } \\
\text { (ft) }\end{array}$ \\
\hline $\begin{array}{lr}\text { June } & 5 \\
\text { June } & 23 \\
\text { July } & 2\end{array}$ & $\begin{array}{l}0200 \\
0230 \\
1600\end{array}$ & $\begin{array}{l}1190 \\
1630 \\
1070\end{array}$ & $\begin{array}{r}9.08 \\
+10.30 \\
8.28\end{array}$ & $\begin{array}{lr}\text { July } & 22 \\
\text { Aug } & 4 \\
\text { Aug } & 9\end{array}$ & $\begin{array}{l}1430 \\
0330 \\
1630\end{array}$ & $\begin{array}{r}495 \\
* 2010 \\
659\end{array}$ & $\begin{array}{r}6.07 \\
10.20 \\
6.24\end{array}$ \\
\hline
\end{tabular}

DISCHARGE, CUBIC FEET PER SECOND, WATER YEAR OCTOBER 1990 TO SEPTEMBER 1991

\begin{tabular}{|c|c|c|c|c|c|c|c|c|c|c|c|c|}
\hline DAY & OCT & DEC & JAN & FEB & MAR & APR & MAY & JUN & JUL & AUG & & SEP \\
\hline $\begin{array}{c}1 \\
2 \\
3 \\
4 \\
5 \\
6 \\
7 \\
8 \\
9 \\
10 \\
11 \\
12 \\
13 \\
14 \\
15 \\
16 \\
17 \\
18 \\
19 \\
20 \\
21 \\
22 \\
23 \\
24 \\
25 \\
26 \\
27 \\
28 \\
29 \\
30 \\
31 \\
\text { TOTAL } \\
\text { MEAN } \\
\text { MAX } \\
\text { AC-FT } \\
\text { CFSM } \\
\text { IN } \\
19\end{array}$ & $\begin{array}{l}2.1 \\
2.0 \\
1.8 \\
1.8 \\
1.9 \\
1.4 \\
1.4 \\
1.0 \\
1.2 \\
1.3 \\
1.0 \\
.98 \\
1.0 \\
1.0 \\
1.0 \\
1.0 \\
1.0 \\
1.0 \\
1.1 \\
.92 \\
1.1 \\
1.1 \\
1.0 \\
1.1 \\
e 1.0 \\
e 1.0 \\
e 1.0 \\
e 1.0 \\
e 1.0 \\
e 1.0 \\
-0 \\
36.20 \\
1.21 \\
2.1 \\
.92 \\
.72 \\
.00\end{array}$ & 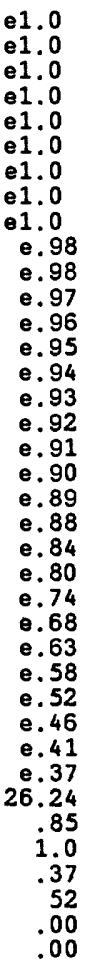 & $\begin{array}{l}\text { e. } 34 \\
\text { e.30 } \\
\text { e.28 } \\
\text { e.26 } \\
\text { e.25 } \\
\text { e.23 } \\
\text { e.22 } \\
\text { e.21 } \\
\text { e. } 20 \\
\text { e.20 } \\
\text { e.19 } \\
\text { e.18 } \\
\text { e.18 } \\
\text { e.17 } \\
\text { e.17 } \\
\text { e.17 } \\
\text { e.16 } \\
\text { e.16 } \\
\text { e.16 } \\
\text { e.16 } \\
\text { e.16 } \\
\text { e.16 } \\
\text { e. } 16 \\
\text { e.16 } \\
\text { e.15 } \\
\text { e.15 } \\
\text { e.16 } \\
\text { e.16 } \\
\text { e.16 } \\
\text { e.16 } \\
\text { e.16 } \\
5.93 \\
.19 \\
.34 \\
.15 \\
.12 \\
.00 \\
.00\end{array}$ & 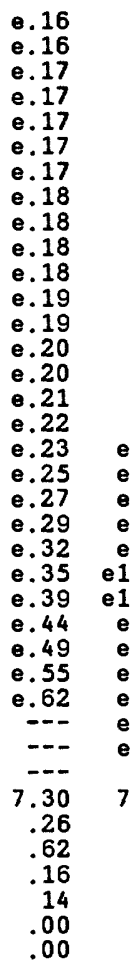 & 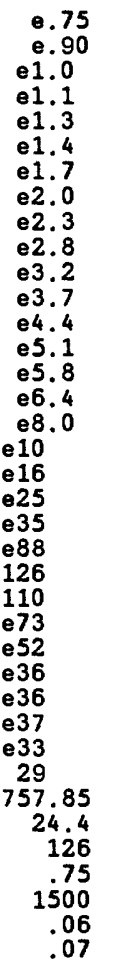 & $\begin{array}{r}25 \\
22 \\
22 \\
21 \\
19 \\
19 \\
19 \\
18 \\
16 \\
14 \\
13 \\
14 \\
18 \\
22 \\
29 \\
39 \\
40 \\
35 \\
30 \\
26 \\
21 \\
19 \\
16 \\
15 \\
13 \\
12 \\
12 \\
13 \\
14 \\
18 \\
-10 \\
614 \\
20.5 \\
40 \\
12 \\
1220 \\
.05 \\
.06\end{array}$ & $\begin{array}{r}20 \\
22 \\
30 \\
36 \\
33 \\
31 \\
37 \\
36 \\
33 \\
30 \\
28 \\
27 \\
24 \\
22 \\
21 \\
21 \\
43 \\
90 \\
72 \\
49 \\
39 \\
32 \\
27 \\
24 \\
20 \\
17 \\
16 \\
18 \\
58 \\
178 \\
276 \\
1410 \\
45.5 \\
276 \\
16 \\
2800 \\
.11 \\
.13\end{array}$ & $\begin{array}{r}295 \\
324 \\
326 \\
817 \\
974 \\
915 \\
677 \\
452 \\
324 \\
208 \\
163 \\
138 \\
112 \\
104 \\
90 \\
97 \\
92 \\
67 \\
56 \\
70 \\
230 \\
1060 \\
1450 \\
e 744 \\
e 328 \\
e 253 \\
e 266 \\
e 311 \\
462 \\
432 \\
--- \\
11837 \\
395 \\
1450 \\
536 \\
23480 \\
.99 \\
1.11\end{array}$ & $\begin{array}{c}482 \\
876 \\
587 \\
263 \\
158 \\
105 \\
72 \\
53 \\
42 \\
34 \\
29 \\
28 \\
23 \\
21 \\
17 \\
15 \\
13 \\
12 \\
11 \\
9.1 \\
9.1 \\
338 \\
242 \\
235 \\
145 \\
87 \\
104 \\
114 \\
122 \\
131 \\
102 \\
4479.2 \\
144 \\
876 \\
9.1 \\
8880 \\
.36 \\
.42\end{array}$ & $\begin{array}{r}89 \\
314 \\
1010 \\
1750 \\
937 \\
498 \\
360 \\
356 \\
569 \\
523 \\
370 \\
266 \\
204 \\
174 \\
149 \\
132 \\
120 \\
109 \\
102 \\
93 \\
85 \\
77 \\
70 \\
63 \\
57 \\
53 \\
47 \\
42 \\
40 \\
35 \\
31 \\
8725 \\
281 \\
1750 \\
31 \\
17310 \\
.71 \\
.82\end{array}$ & & $\begin{array}{r}24 \\
24 \\
23 \\
22 \\
22 \\
20 \\
19 \\
19 \\
18 \\
17 \\
16 \\
15 \\
14 \\
13 \\
13 \\
12 \\
11 \\
11 \\
11 \\
10 \\
-2- \\
571 \\
19.0 \\
30 \\
10 \\
1130 \\
.05 \\
.05\end{array}$ \\
\hline $\begin{array}{l}\text { STATIST } \\
\text { MEAN } \\
\text { MAX } \\
\text { (WY) } \\
\text { MIN } \\
\text { (WY) } \\
\text { SUMMARY } \\
\text { ANNUAL } \\
\text { ANNUAL } \\
\text { HIGHEST } \\
\text { LOWEST } \\
\text { HIGHEST } \\
\text { LOWEST } \\
\text { ANNUAL } \\
\text { INSTANT } \\
\text { INSTANT } \\
\text { ANNUAL } \\
\text { ANNUAL } \\
\text { ANNUAL } \\
\text { 10 PERCI } \\
\text { 50 PERC } \\
\text { 90 PERC }\end{array}$ & $\begin{array}{lc}\text { ICS OF } & \text { MONTHLY MEAN } \\
12.7 & 16.3 \\
104 & 201 \\
1985 & 1972 \\
.31 & .44 \\
1941 & 1977 \\
\text { STATISTICS } \\
\text { TOTAL } \\
\text { MEAN } \\
\text { ANNUAL MEAN } \\
\text { ANNUAL MEAN } \\
\text { DAILY MEAN } \\
\text { DAILY MEAN } \\
\text { SEVEN-DAY MINIMUM } \\
\text { ANEOUS PEAK FLOW } \\
\text { ANEOUS PEAK STAGE } \\
\text { RUNOFF (AC-FT) } \\
\text { RUNOFF } & \text { (CFSM) } \\
\text { RUNOFF } & \text { (INCHES) } \\
\text { ENT EXCEEDS } \\
\text { ENT EXCEEDS } \\
\text { ENT EXCEEDS }\end{array}$ & $\begin{array}{c}\text { DATA } \\
8.53 \\
66.8 \\
1972 \\
.32 \\
1977 \\
\text { FOR }\end{array}$ & $\begin{array}{c}\text { FOR WATER YEA } \\
4.67 \\
19.7 \\
1986 \\
\text { i090 } \\
1977 \\
1990 \text { CALENDA } \\
2792.05 \\
7.65\end{array}$ & $\begin{array}{c}\text { ARS } 1940 \\
10.1 \\
117 \\
1984 \\
.001 \\
1977 \\
\text { AR YEAR }\end{array}$ & $\begin{array}{c}1991 \\
150 \\
653 \\
1986 \\
1.59 \\
1965\end{array}$ & $\begin{array}{r}\text { BY WATER } \\
233 \\
1341 \\
1952 \\
9.13 \\
1981 \\
1991 \mathrm{~W} \\
28542.1 \\
78.2 \\
\\
1750 \\
.1 \\
.1 \\
2010^{\circ} \\
10.3 \\
56610 \\
.2 \\
2.6 \\
238 \\
13 \\
.2\end{array}$ & $\begin{array}{c}\text { YEAR (WY) } \\
94.3 \\
652 \\
1972 \\
2.94 \\
1981 \\
\text { ATER YEAR } \\
2\end{array}$ & $\begin{array}{r}90.7 \\
496 \\
1984 \\
1.83 \\
1976\end{array}$ & $\begin{array}{r}42.5 \\
375 \\
1962 \\
.27 \\
1976 \\
\text { WATER } \\
58 . \\
225^{\circ} \\
3 . \\
6640^{\circ} \\
. \\
6970^{\circ} \\
19 . \\
42340^{\circ} \\
2 . \\
120^{\circ} \\
8 . \\
.\end{array}$ & $\begin{array}{lr} & 25.0 \\
& 281 \\
& 1991 \\
& .088 \\
& 1976 \\
\text { YEARS } & 1940 \\
& \\
4 \mathrm{a} & \\
98 & \\
98 & \\
00 \mathrm{~b} & \mathrm{Apr} \\
00 & \mathrm{Jan} \\
& \mathrm{Apr} \\
07 \mathrm{~d} & \mathrm{Apr} \\
& \\
15 & \\
00 & \\
3 & \\
30 & \end{array}$ & $\begin{array}{r}9 \\
26 \\
9 \\
9\end{array}$ & $\begin{array}{l}1986 \\
1981 \\
1969 \\
\\
1940 \\
1969 \\
1969\end{array}$ \\
\hline
\end{tabular}

a Median of annual mean discharges is $47 \mathrm{ft}^{3} / \mathrm{s}$.

b Many days in several years.

c Backwater from ice.

d From floodmark. 
MINNESOTA RIVER BASIN

05294000 POME DE TERRE RIVER AT APPLETON, MN

LOCATION.--Lat $45^{\circ} 12^{\prime} 10^{\prime \prime}$, long 96.01'20", in SWłNWł sec.14, T.120 N., R.43 W., Swift County, Hydrologic Unit 07020002 , on left bank 60 ft upstream from bridge on U.S. Highway 59 and State Highway 119 at Appleton and $8 \mathrm{mi}$ upstream from mouth.

DRAIKAGE AREA. $--905 \mathrm{mi}^{2}$ approximately.

PERIOD OF RECORD. --March 1931 to September 1935 (no winter records), October 1935 to current year. Prior to October 1953, published as "near Appleton."

REVISED RECORDS. --WSP 1308: $1931(\mathrm{M}), 1937(\mathrm{M})$.

GAGE. - Water-stage recorder and concrete control. Datum of gage is $978.00 \mathrm{ft}$ above National Geodetic Vertical Datum of 1929. Prior to Dec. 22, 1952, nonrecording gage at site 4 mi upstream at datum 25.17 ft higher. REMARKS.--Records good. Flow affected by lakes above station. Occasional regulation at low flow by old milidam $500 \mathrm{ft}$ upstream.

EXTREMES FOR CURRENT YEAR. --Peak discharges greater than base discharge of $200 \mathrm{ft}^{3} / \mathrm{s}$ and maximum ( ${ }^{*}$ ):

\begin{tabular}{|c|c|c|c|c|c|c|c|}
\hline Date & Time & $\begin{array}{l}\text { Discharge } \\
\left(\mathrm{ft}^{3} / \mathrm{s}\right)\end{array}$ & $\begin{array}{c}\text { Gage height } \\
\text { (ft) }\end{array}$ & Date & Time & $\begin{array}{c}\text { Discharge } \\
\left(\mathrm{ft}^{3} / \mathrm{s}\right)\end{array}$ & $\begin{array}{c}\text { Gage height } \\
\text { (ft) }\end{array}$ \\
\hline Jun 2 & $\begin{array}{l}1500 \\
\text { Unknown }\end{array}$ & $\begin{array}{l}244 \\
226\end{array}$ & $\begin{array}{l}5.50 \\
5.44\end{array}$ & $\begin{array}{lr}\text { Jul } & 15 \\
\text { Aug } & 3\end{array}$ & $\begin{array}{l}\text { Unknown } \\
0815\end{array}$ & $\begin{array}{r}232 \\
* 320\end{array}$ & $\begin{array}{r}5.46 \\
\star 5.72\end{array}$ \\
\hline
\end{tabular}

DISCHARGE, CUBIC FEET PER SECOND, WATER YEAR OCTOBER 1990 TO SEPTEMBER 1991

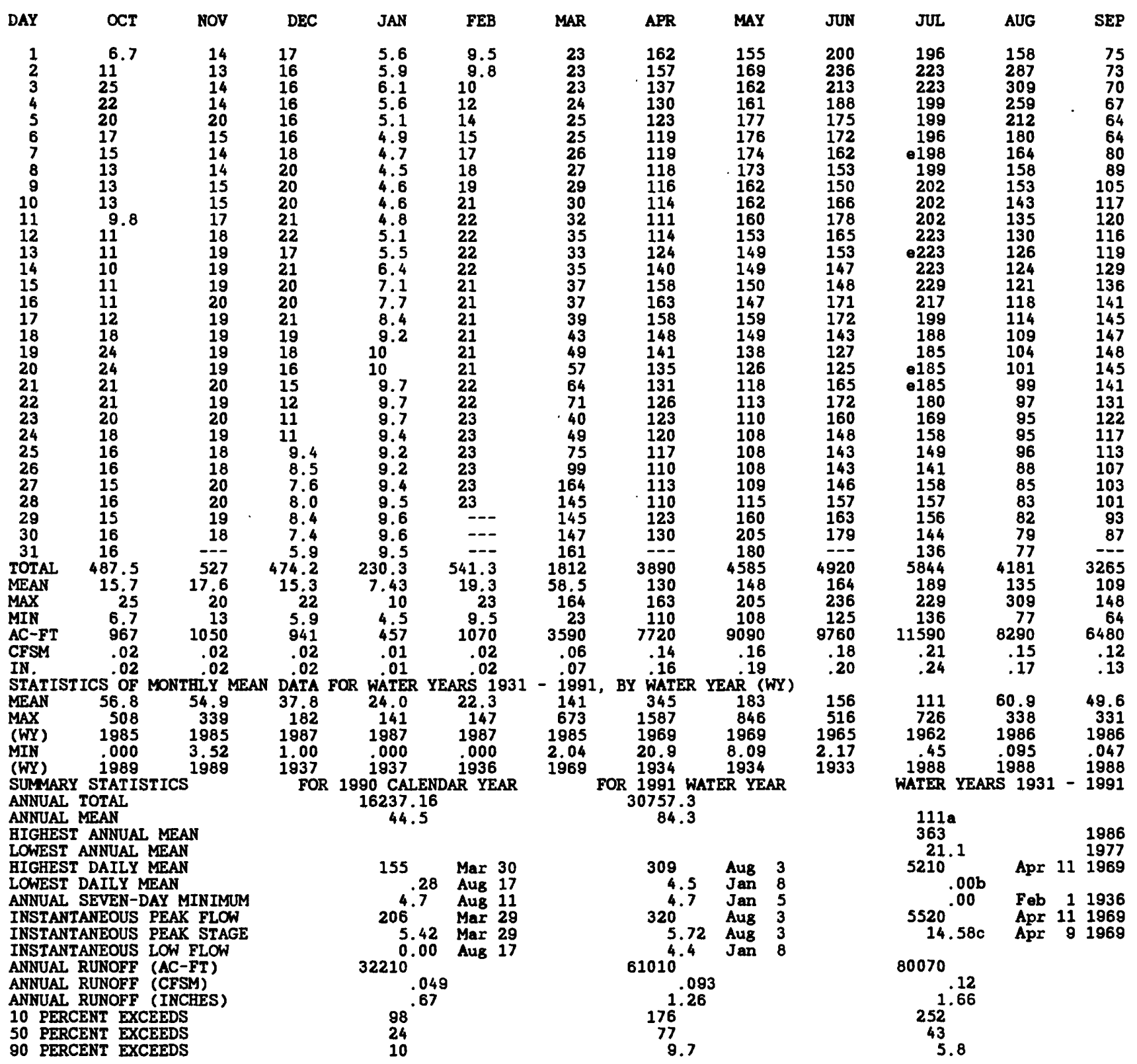

a Median of annual mean discharges is $91 \mathrm{ft}^{3} / \mathrm{s}$.

b Occurred on many days in several years.

c Backwater from ice.

estimated. 
MINNESOTA RIVER BASIN

05300000 LAC QUI PARLE RIVER NEAR LAC QUI PARLE, MN

LOCATION. --Lat $44^{\circ} 59^{\prime} 42$, long $95^{\circ} 55^{\prime} 09^{\prime \prime}$ in SW子SW sec.27, T.118 N., R.42 W., Lac qui Parle County, Hydrologic Unit 07020003 , on right bank $40 \mathrm{ft}$ downstream from highway bridge and $0.5 \mathrm{mi}$ southwest of city of Lac qui

DRAINAGE AREA. $--983 \mathrm{mi}^{2}$.

PERIOD OF RECORD.--Aprii 1910 to November 1914; March 1931 to current year (winter records incomplete prior to 1934) Published as "at Lac gui Parle" 1910-14.

REVISED RECORDS. --WSP 1308: $1912(\mathrm{M}), 1935(\mathrm{M})$.

GAGE. - Water-stage recorder. Datum of gage is $951.98 \mathrm{ft}$ above National Geodetic Vertical Datum of 1929

(Minnesota Department of Transportation benchmark) Apr. 27, 1910, to Nov. 15, 1914 , nonrecording 8 age at

upstream at present datum.

REMARKS.--Records good except those for estimated daily discharges, which are fair.

DISCHARGE, CUBIC FEET PER SECOND, WATER YEAR OCTOBER 1990 TO SEPTEMBER 1991

\begin{tabular}{|c|c|c|c|c|c|c|c|c|c|c|c|c|}
\hline DAY & $\infty \mathrm{CT}$ & Nov & DEC & JAN & FEB & MAR & APR & MAY & JUN & JUL & AUG & SEP \\
\hline $\begin{array}{r}1 \\
2 \\
3 \\
4 \\
5 \\
6 \\
7 \\
8 \\
9 \\
10 \\
11 \\
12 \\
13 \\
14 \\
15 \\
16 \\
17 \\
18 \\
19 \\
20 \\
21 \\
22 \\
23 \\
24 \\
25 \\
26 \\
27 \\
28 \\
29 \\
30 \\
31\end{array}$ & $\begin{array}{c}6.6 \\
9.9 \\
99 \\
19 \\
28 \\
23 \\
18 \\
65 \\
58 \\
54 \\
46 \\
37 \\
35 \\
32 \\
28 \\
29 \\
26 \\
30 \\
31 \\
30 \\
28 \\
35 \\
35 \\
37 \\
47 \\
46 \\
52 \\
46 \\
44 \\
34 \\
35 \\
33\end{array}$ & $\begin{array}{l}29 \\
26 \\
23 \\
21 \\
20 \\
21 \\
19 \\
19 \\
18 \\
13 \\
12 \\
16 \\
19 \\
21 \\
19 \\
18 \\
20 \\
25 \\
28 \\
27 \\
27 \\
27 \\
37 \\
29 \\
23 \\
22 \\
21 \\
23 \\
27 \\
23\end{array}$ & $\begin{array}{r}20 \\
19 \\
18 \\
17 \\
17 \\
17 \\
17 \\
19 \\
20 \\
21 \\
24 \\
27 \\
22 \\
025 \\
29 \\
28 \\
25 \\
24 \\
23 \\
23 \\
17 \\
12 \\
9.0 \\
7.3 \\
96.7 \\
6.3 \\
e 6.2 \\
06.0 \\
e 5.9 \\
95.8\end{array}$ & 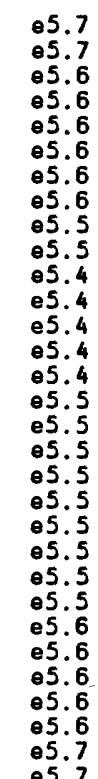 & 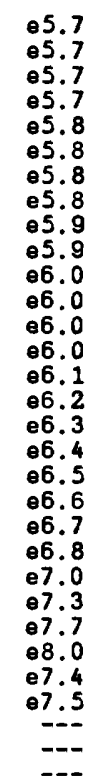 & 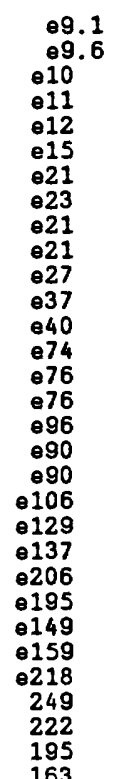 & $\begin{array}{r}140 \\
128 \\
118 \\
111 \\
106 \\
103 \\
101 \\
99 \\
98 \\
96 \\
92 \\
95 \\
112 \\
128 \\
203 \\
276 \\
279 \\
251 \\
220 \\
195 \\
176 \\
161 \\
149 \\
140 \\
133 \\
126 \\
130 \\
122 \\
130 \\
183\end{array}$ & $\begin{array}{l}208 \\
269 \\
344 \\
362 \\
346 \\
374 \\
401 \\
368 \\
324 \\
289 \\
263 \\
246 \\
227 \\
209 \\
213 \\
220 \\
219 \\
192 \\
170 \\
161 \\
155 \\
148 \\
143 \\
133 \\
130 \\
141 \\
152 \\
173 \\
227 \\
275 \\
273\end{array}$ & $\begin{array}{r}407 \\
1060 \\
973 \\
2290 \\
2480 \\
2500 \\
2510 \\
2430 \\
2200 \\
1960 \\
1680 \\
1400 \\
1150 \\
954 \\
851 \\
882 \\
952 \\
895 \\
805 \\
800 \\
1860 \\
2080 \\
2200 \\
2180 \\
2110 \\
1970 \\
1720 \\
1400 \\
1150 \\
988\end{array}$ & $\begin{array}{r}1430 \\
1380 \\
1250 \\
1090 \\
924 \\
789 \\
676 \\
583 \\
507 \\
443 \\
401 \\
366 \\
338 \\
313 \\
284 \\
254 \\
228 \\
205 \\
184 \\
167 \\
158 \\
445 \\
380 \\
271 \\
221 \\
194 \\
214 \\
293 \\
296 \\
278 \\
246\end{array}$ & $\begin{array}{r}229 \\
800 \\
1090 \\
931 \\
825 \\
626 \\
525 \\
534 \\
525 \\
516 \\
467 \\
396 \\
335 \\
289 \\
251 \\
222 \\
203 \\
187 \\
171 \\
157 \\
150 \\
139 \\
129 \\
119 \\
110 \\
100 \\
90 \\
81 \\
74 \\
68 \\
62\end{array}$ & $\begin{array}{l}56 \\
52 \\
49 \\
44 \\
41 \\
38 \\
43 \\
56 \\
57 \\
52 \\
54 \\
56 \\
58 \\
62 \\
63 \\
65 \\
65 \\
63 \\
57 \\
54 \\
51 \\
47 \\
44 \\
49 \\
46 \\
44 \\
45 \\
46\end{array}$ \\
\hline $\begin{array}{l}31 \\
\text { TOTAL } \\
\text { MEAN } \\
\text { MAX } \\
\text { MIN } \\
\text { AC-FT } \\
\text { CFSM } \\
\text { IN. }\end{array}$ & $\begin{array}{r}3077.5 \\
34.8 \\
65 \\
6.6 \\
2140 \\
.04 \\
.04\end{array}$ & $\begin{array}{r}-7 .- \\
673 \\
22.4 \\
37 \\
12 \\
1330 \\
.02 \\
.03\end{array}$ & $\begin{array}{r}05.7 \\
522.9 \\
16.9 \\
29 \\
5.7 \\
1040 \\
.02 \\
.02\end{array}$ & $\begin{array}{r}95.7 \\
171.9 \\
5.55 \\
5.7 \\
5.4 \\
341 \\
.01 \\
.01\end{array}$ & $\begin{array}{r}178.3 \\
6.37 \\
8.0 \\
5.7 \\
354 \\
.01 \\
.01\end{array}$ & $\begin{array}{r}163 \\
2886.7 \\
93.1 \\
249 \\
9.1 \\
5730 \\
.09 \\
.11\end{array}$ & $\begin{array}{r}4401 \\
147 \\
279 \\
92 \\
8730 \\
.15 \\
.17\end{array}$ & $\begin{array}{r}273 \\
7355 \\
237 \\
401 \\
130 \\
14590 \\
.24 \\
.28\end{array}$ & $\begin{array}{r}46837 \\
1561 \\
2510 \\
407 \\
92900 \\
1.59 \\
1.77\end{array}$ & $\begin{array}{r}246 \\
14808 \\
478 \\
1430 \\
158 \\
29370 \\
.49 \\
.56\end{array}$ & $\begin{array}{r}10401 \\
336 \\
1090 \\
62 \\
20630 \\
.34 \\
.39\end{array}$ & $\begin{array}{r}1551 \\
51.7 \\
65 \\
38 \\
3080 \\
.05 \\
.06\end{array}$ \\
\hline
\end{tabular}

STATISTICS OF MONTHLY MEAN DATA FOR WATER YEARS 1910 - 1991, BY WATER YEAR (WY)

\begin{tabular}{|c|c|c|c|c|c|c|c|c|}
\hline $\begin{array}{l}\text { MEAN } \\
\text { MAX } \\
(W Y) \\
\text { MIN } \\
(W Y)\end{array}$ & $\begin{array}{r}28.8 \\
482 \\
1985 \\
1000 \\
1932\end{array}$ & $\begin{array}{r}35.5 \\
345 \\
1971 \\
1000 \\
1932\end{array}$ & $\begin{array}{r}18.0 \\
112 \\
1972 \\
1000 \\
1932\end{array}$ & $\begin{array}{l}7.75 \\
43.9 \\
1987 \\
1000 \\
1932\end{array}$ & $\begin{array}{r}16.1 \\
140 \\
1984 \\
1000 \\
1934\end{array}$ & $\begin{array}{r}278 \\
1634 \\
1985 \\
.000 \\
1934\end{array}$ & $\begin{array}{r}50 \\
357 \\
196 \\
00 \\
193\end{array}$ & 1944 \\
\hline
\end{tabular}

SUMMARY STATISTICS

\section{ANNUAL TOTAL}

HIGHEST ANNUAL MEAN

LOWEST ANNUAL MEAN

HIGHEST DAILY MEAN

LOWEST DAILY MEAN

ANNUAL SEVEN-DAY MINIMUM

INSTANTANEOUS PEAK FLOW

INSTANTANEOUS PEAK STAGE

INSTANTANEOUS LOW FLOW

ANNUAL RUNOFF (AC-FT)

ANNUAL RUNOFE (CFSM)

ANNUAL RUNOFF (INCHES)

10 PERCENT EXCEEDS

50 PERCENT EXCEEDS

90 PERCENT EXCEEDS
FOR 1990 CALENDAR YEAR

$$
\begin{array}{cc}
21139.0 & \\
57.9 & \\
988 & \text { Jun } 19 \\
1.1 & \text { Sep } 17 \\
1.4 & \text { Feb } 17 \\
1050^{2} & \text { Jun } 19 \\
5.14 & \text { Jun } 19 \\
41930 & \\
.059 & \\
.80 & \\
134 & \\
24 & \\
2.6 &
\end{array}
$$

FOR 1991 WATER YEAR

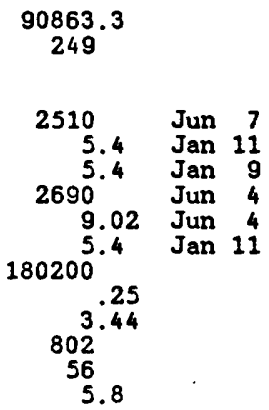

WATER YEARS 1910 - 1991

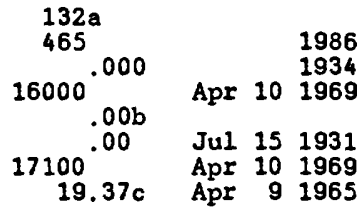

95620

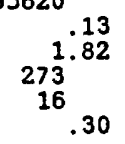

a Median of annual mean discharges is $101 \mathrm{ft}^{3} / \mathrm{s}$.

b Many days in several years.

c From floodmark (backwater from ice). 
MINNESOTA RIVER BASIN

05301000 MINNESOTA RIVER NEAR LAC QUI PARLE, MN

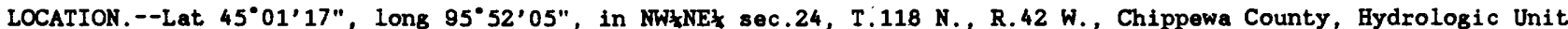
07020004 , on left bank $200 \mathrm{ft}$ downstream from dam at Lac qui Parie Outlet, 2.4 mi northeast of city of Lac qui Parle, and $3.5 \mathrm{mi}$ west of Watson.

DRAINAGE AREA. $--4,050 \mathrm{mi}^{2}$, approximately.

PERIOD OF RECORD.--October 1942 to current year.

GAGE.--Water-stage recorder. Datum of gage is $900.00 \mathrm{ft}$ above National Geodetic Vertican Datum of 1929 (levels by U.S. Army Corps of Engineers). Prior to Nov. 10, 1944, at datum $0.20 \mathrm{ft}$ lower.

REMARKS.--Records good. Part of flow from $2,050 \mathrm{mi}^{2}$, of Chippewa River basin at times diverted into Minnesota River above station. Some regulation by Big Stone Lake since Apr. 17, 1937, Lac qui Parle since January 1938, Marsh Lake since Nov. 1, 1939, and Odessa Dam since May 1974.

REVISIONS.--Revised daily discharge, in cubic feet per second, for September 13, 1979 is 69 . This figure supersedes that published in the report for 1979.

$\begin{array}{lrrrrrr} & \text { TOTAL } & \text { MEAN } & \text { MAX } & \text { MIN } & \text { (FT } 3 / 3) / M^{2} & \text { IN } \\ \text { September 1979 } & 7967.8 & 266 & 672 & 1.8 & .07 & .07 \\ \text { Wtr Yr 1979 } & 428438.8 & 1174 & 10500 & 1.8 & .29 & 3.94 \\ \text { CaI Yr 1979 } & 444901.8 & 1219 & 10500 & 1.8 & .30 & 4.09\end{array}$

DISCHARGE, CUBIC FEET PER SECOND, WATER YEAR OCTOBER 1990 TO SEPTEMBER 1991 DAILY MEAN VALUES

\begin{tabular}{|c|c|c|c|c|c|c|c|c|c|c|c|c|}
\hline DAY & Ост & NOV & DEC & JAN & FEB & MAR & APR & MAY & JUN & JUL & AUG & SEP \\
\hline $\begin{array}{r}1 \\
2 \\
3 \\
4 \\
5 \\
6 \\
7 \\
8 \\
9 \\
10 \\
11 \\
12 \\
13 \\
14 \\
15 \\
16 \\
17 \\
18 \\
19 \\
20 \\
21 \\
22 \\
23 \\
24 \\
25 \\
26 \\
27 \\
28 \\
29 \\
30 \\
31\end{array}$ & $\begin{array}{r}27 \\
28 \\
72 \\
100 \\
100 \\
102 \\
101 \\
102 \\
102 \\
102 \\
163 \\
199 \\
199 \\
199 \\
198 \\
199 \\
214 \\
201 \\
201 \\
201 \\
200 \\
202 \\
201 \\
201 \\
202 \\
200 \\
205 \\
202 \\
202 \\
202 \\
202\end{array}$ & $\begin{array}{r}202 \\
200 \\
200 \\
202 \\
135 \\
95 \\
95 \\
97 \\
61 \\
40 \\
40 \\
40 \\
40 \\
40 \\
63 \\
91 \\
91 \\
91 \\
91 \\
91 \\
93 \\
91 \\
91 \\
100 \\
88 \\
91 \\
91 \\
93 \\
91 \\
88 \\
---\end{array}$ & $\begin{array}{l}88 \\
88 \\
88 \\
91 \\
88 \\
88 \\
88 \\
88 \\
88 \\
91 \\
91 \\
88 \\
91 \\
91 \\
91 \\
91 \\
88 \\
91 \\
91 \\
91 \\
e 90 \\
e 90 \\
e 90 \\
e 90 \\
e 90 \\
e 90 \\
990 \\
988 \\
986 \\
986 \\
986\end{array}$ & $\begin{array}{l}986 \\
986 \\
978 \\
972 \\
970 \\
968 \\
966 \\
66 \\
63 \\
63 \\
63 \\
63 \\
63 \\
61 \\
63 \\
61 \\
61 \\
61 \\
61 \\
61 \\
63 \\
61 \\
61 \\
63 \\
63 \\
61 \\
61 \\
39 \\
24 \\
24 \\
24\end{array}$ & $\begin{array}{l}24 \\
24 \\
24 \\
24 \\
25 \\
24 \\
24 \\
25 \\
24 \\
24 \\
25 \\
25 \\
24 \\
23 \\
29 \\
25 \\
25 \\
25 \\
25 \\
26 \\
25 \\
25 \\
25 \\
24 \\
25 \\
38 \\
50 \\
51 \\
--- \\
--- \\
---\end{array}$ & $\begin{array}{r}81 \\
118 \\
121 \\
188 \\
226 \\
222 \\
227 \\
224 \\
226 \\
227 \\
224 \\
264 \\
330 \\
393 \\
424 \\
424 \\
422 \\
418 \\
418 \\
441 \\
415 \\
416 \\
415 \\
417 \\
495 \\
655 \\
890 \\
1060 \\
1200 \\
1280 \\
1280\end{array}$ & $\begin{array}{r}1070 \\
684 \\
553 \\
546 \\
546 \\
546 \\
543 \\
545 \\
544 \\
544 \\
541 \\
542 \\
544 \\
547 \\
654 \\
768 \\
771 \\
839 \\
938 \\
932 \\
922 \\
919 \\
917 \\
769 \\
542 \\
511 \\
472 \\
470 \\
544 \\
727 \\
---\end{array}$ & $\begin{array}{r}861 \\
861 \\
862 \\
868 \\
873 \\
919 \\
1120 \\
1220 \\
1220 \\
1210 \\
1200 \\
1190 \\
1070 \\
833 \\
826 \\
876 \\
964 \\
911 \\
908 \\
904 \\
878 \\
824 \\
801 \\
768 \\
672 \\
667 \\
664 \\
667 \\
669 \\
743 \\
895\end{array}$ & $\begin{array}{l}1000 \\
1070 \\
1160 \\
1590 \\
1630 \\
1610 \\
1650 \\
1690 \\
1820 \\
1950 \\
2030 \\
2070 \\
2070 \\
2080 \\
2150 \\
2150 \\
2160 \\
2170 \\
2130 \\
2110 \\
2400 \\
3590 \\
4960 \\
4720 \\
4140 \\
4060 \\
4000 \\
3820 \\
3600 \\
3500\end{array}$ & $\begin{array}{l}3570 \\
3640 \\
3690 \\
3640 \\
3570 \\
3500 \\
3430 \\
3330 \\
3200 \\
3080 \\
2960 \\
2870 \\
2730 \\
2580 \\
2380 \\
2060 \\
1960 \\
1940 \\
1890 \\
1820 \\
1760 \\
1830 \\
1860 \\
1850 \\
1750 \\
1580 \\
1550 \\
1580 \\
1580 \\
1590 \\
1690\end{array}$ & $\begin{array}{r}1700 \\
1890 \\
2190 \\
2700 \\
3160 \\
3290 \\
3360 \\
3430 \\
3450 \\
3430 \\
3370 \\
3320 \\
3230 \\
3120 \\
3010 \\
2920 \\
2810 \\
2680 \\
2450 \\
2190 \\
1960 \\
1850 \\
1770 \\
1670 \\
1600 \\
1570 \\
1570 \\
1550 \\
1440 \\
1030 \\
505\end{array}$ & $\begin{array}{r}485 \\
482 \\
525 \\
599 \\
590 \\
472 \\
354 \\
356 \\
420 \\
527 \\
658 \\
809 \\
1050 \\
1460 \\
1450 \\
1420 \\
1320 \\
1320 \\
1250 \\
1000 \\
455 \\
437 \\
432 \\
469 \\
534 \\
526 \\
521 \\
519 \\
515 \\
511\end{array}$ \\
\hline $\begin{array}{l}\text { TOTAL } \\
\text { MEAN } \\
\text { MAX } \\
\text { MIN } \\
\text { AC-FT } \\
\text { CFSM } \\
\text { IN. }\end{array}$ & $\begin{array}{r}5029 \\
162 \\
214 \\
27 \\
9980 \\
.04 \\
.05\end{array}$ & $\begin{array}{r}2922 \\
97.4 \\
202 \\
40 \\
5800 \\
.02 \\
.03\end{array}$ & $\begin{array}{r}2766 \\
89.2 \\
91 \\
86 \\
5490 \\
.02 \\
.03\end{array}$ & $\begin{array}{r}1880 \\
60.6 \\
86 \\
24 \\
3730 \\
.01 \\
.02\end{array}$ & $\begin{array}{r}757 \\
27.0 \\
51 \\
23 \\
1500 \\
.01 \\
.01\end{array}$ & $\begin{array}{r}14141 \\
456 \\
1280 \\
81 \\
28050 \\
.11 \\
.13\end{array}$ & $\begin{array}{r}19990 \\
666 \\
1070 \\
470 \\
39650 \\
.16 \\
.18\end{array}$ & $\begin{array}{r}27944 \\
901 \\
1220 \\
664 \\
55430 \\
.22 \\
.26\end{array}$ & $\begin{array}{r}75080 \\
2503 \\
4960 \\
1000 \\
148900 \\
.62 \\
.69\end{array}$ & $\begin{array}{r}76460 \\
2466 \\
3690 \\
1550 \\
151700 \\
.61 \\
.70\end{array}$ & $\begin{array}{r}74215 \\
2394 \\
3450 \\
505 \\
147200 \\
.59 \\
.68\end{array}$ & $\begin{array}{r}21466 \\
716 \\
1460 \\
354 \\
42580 \\
.18 \\
.20\end{array}$ \\
\hline \multicolumn{13}{|c|}{ STATISTICS OF MONTHLY MEAl } \\
\hline $\begin{array}{l}\text { MEAN } \\
\text { MAX } \\
\text { (WY) } \\
\text { MIN } \\
\text { (WY) }\end{array}$ & $\begin{array}{r}252 \\
2924 \\
1987 \\
4.16 \\
1977\end{array}$ & $\begin{array}{r}255 \\
2327 \\
1985 \\
196 \\
1977\end{array}$ & $\begin{array}{r}197 \\
1204 \\
1985 \\
197 \\
1977\end{array}$ & $\begin{array}{r}138 \\
574 \\
1987 \\
119 \\
1977\end{array}$ & $\begin{array}{r}164 \\
634 \\
1987 \\
1094 \\
1977\end{array}$ & $\begin{array}{r}762 \\
4108 \\
1985 \\
46.5 \\
1956\end{array}$ & $\begin{array}{r}2556 \\
10750 \\
1986 \\
151 \\
1961\end{array}$ & $\begin{array}{r}1285 \\
5771 \\
1986 \\
122 \\
1959\end{array}$ & $\begin{array}{l}1058 \\
4229 \\
1984 \\
29.5 \\
1988\end{array}$ & $\begin{array}{r}807 \\
3309 \\
1953 \\
14.7 \\
1988\end{array}$ & $\begin{array}{r}403 \\
2415 \\
1986 \\
11.8 \\
1974\end{array}$ & $\begin{array}{r}240 \\
2402 \\
1986 \\
5.59 \\
1967\end{array}$ \\
\hline
\end{tabular}


MINNESOTA RIVER BASIN

SUMMARY STATISTICS

ANNUAL TOTAL

ANNUAL MEAN

HIGHEST ANNUAL MEAN

LOWEST ANNUAL MEAN

HIGHEST DAILY MEAN

LOWEST DAILY MEAN

ANNUAL SEVEN-DAY MINIMUM

INSTANTANEOUS PEAK FLOW

INSTANTANEOUS PEAK STAGE

INSTANTANEOUS LOW FLOW

ANNUAL RUNOFF (AC-FT)

ANNUAL RUNOFF (CFSM)

ANNUAL RUNOFF (INCHES)

10 PERCENT EXCEEDS

50 PERCENT EXCEEDS

90 PERCENT EXCEEDS

05301000 MINNESOTA RIVER NEAR LAC QUI PARLE, MN--Continued

FOR 1990 CALENDAR YEAR

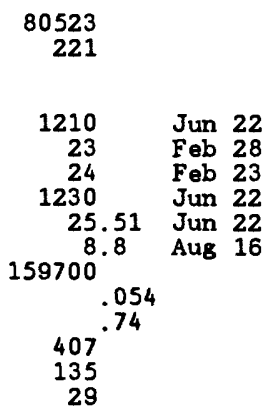

FOR 1991 WATER YEAR

$\begin{array}{cc}322650 & \\ 884 & \\ & \\ 4960 & \text { Jun } 23 \\ 23 & \text { Feb 14 } \\ 24 & \text { Jan 29 } \\ 5040 & \text { Jun 23, 24 } \\ 33.39 & \text { Jun 23,24 } \\ 21 & \text { Feb 14 } \\ 640000 & \\ .22 & \\ 2.96 & \\ 2690 & \\ 470 & \\ 40 & \end{array}$

WATER YEARS 1943 - 1991

\begin{tabular}{|c|c|c|}
\hline $\begin{array}{c}676 \\
2507 \\
75.7 \\
28700\end{array}$ & Apr & 121 \\
\hline 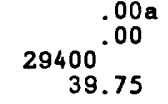 & $\begin{array}{l}\text { Oct } \\
\text { Apr } \\
\text { Apr } 1\end{array}$ & $\begin{array}{ll}19 & 1 \\
12 & 1 \\
12 & 1\end{array}$ \\
\hline $\begin{array}{c}490100 \\
.17 \\
2.27 \\
1680 \\
191 \\
25\end{array}$ & & \\
\hline
\end{tabular}

a Many days in several years.

e Estimated. 
MIMESOTA RIVER BASIN

05301000 MIMNESOTA RIVER MEAR LAC QUI PARLE, MA--Continued

WATER QUALITY RECORDS

PERIOD OF RECORD. --Water years 1963, 1967, 1989 to current year.

REMARKS, --Letter $K$ indicates non-ideal colony count.

WATER QUALITY DATA, WATER YEAR OCTOBER 1990 TO SEPTEMBER 1991

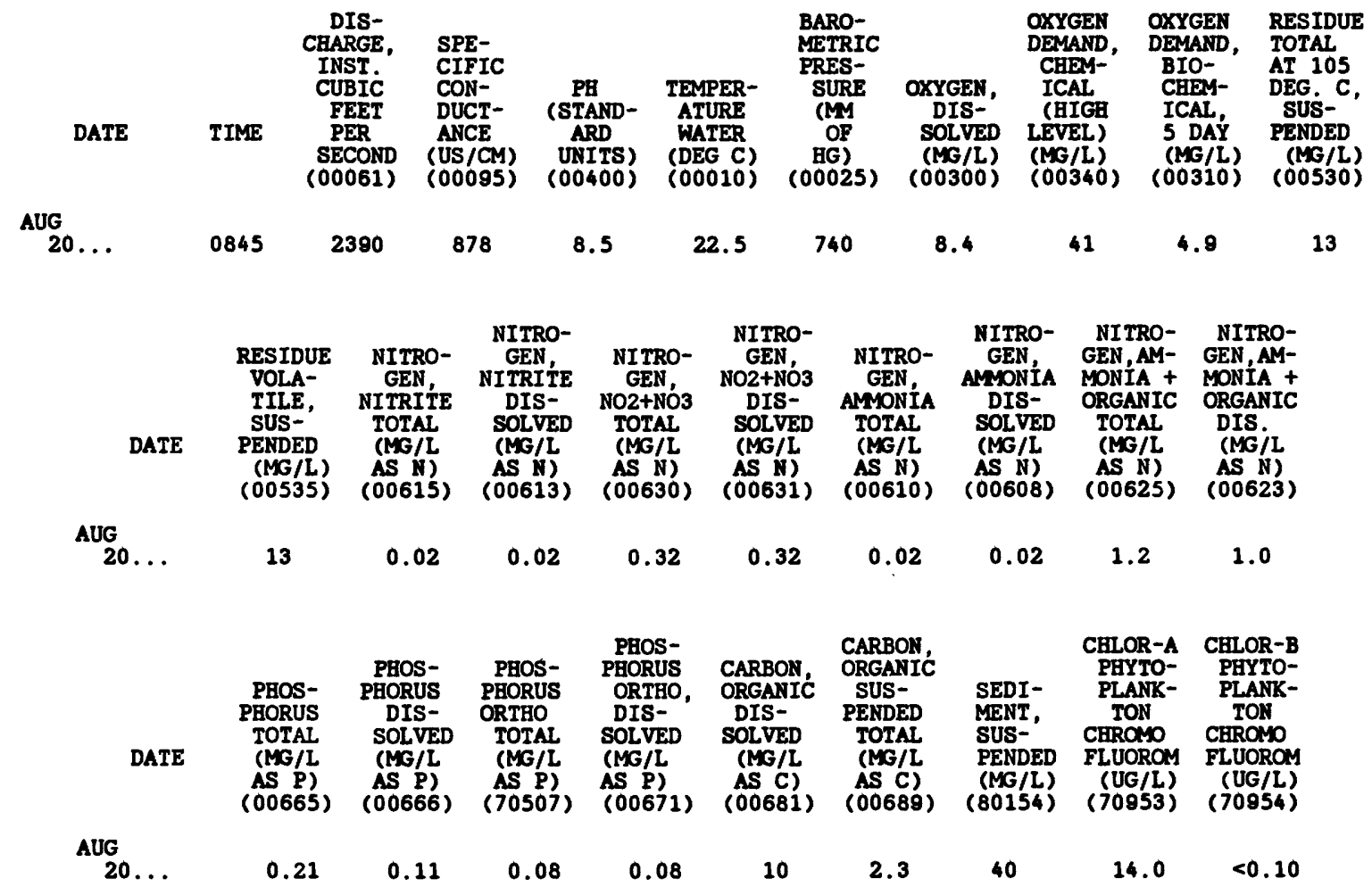




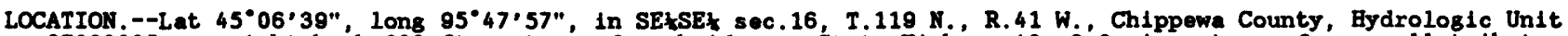

07020005 , on right bank 800 ft upstream from bridge on state Highway $40,2.0 \mathrm{mi}$ upstream from small tributary, and $5.5 \mathrm{mi}$ east of Milan.

DRAIMAGE AREA. $-1,870 \mathrm{mi}^{2}$ approximately.

PERIOD OF RECORD. - March 1937 to current year.

REVISED RECORDS. --WSP 1145: Drainage area.

GAGE.--Water-stage recorder. Datum of gage is $959.69 \mathrm{ft}$ above National Geodetic Vertical Datum of 1929 . Prior to June 15, 1942, nonrecording gage on bridge $800 \mathrm{ft}$ downstream at same datum.

REMARKS. --Records good except those for estimated daily discharges, which are fair. Flow regulated by several smali lakes upstream from gage.

EXTREMES FOR CURRENT YEAR.--Peak discharges greater than base discharge of $400 \mathrm{ft} 3 / \mathrm{s}$, and maximum (*):

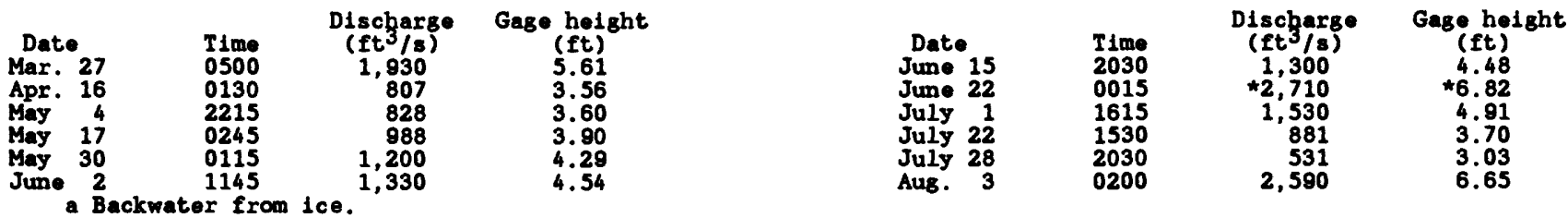

DISCHARGE, CUBIC FEET PER SECOND, WATER YEAR OCTOBER 1990 TO SEPTEMBER 1991

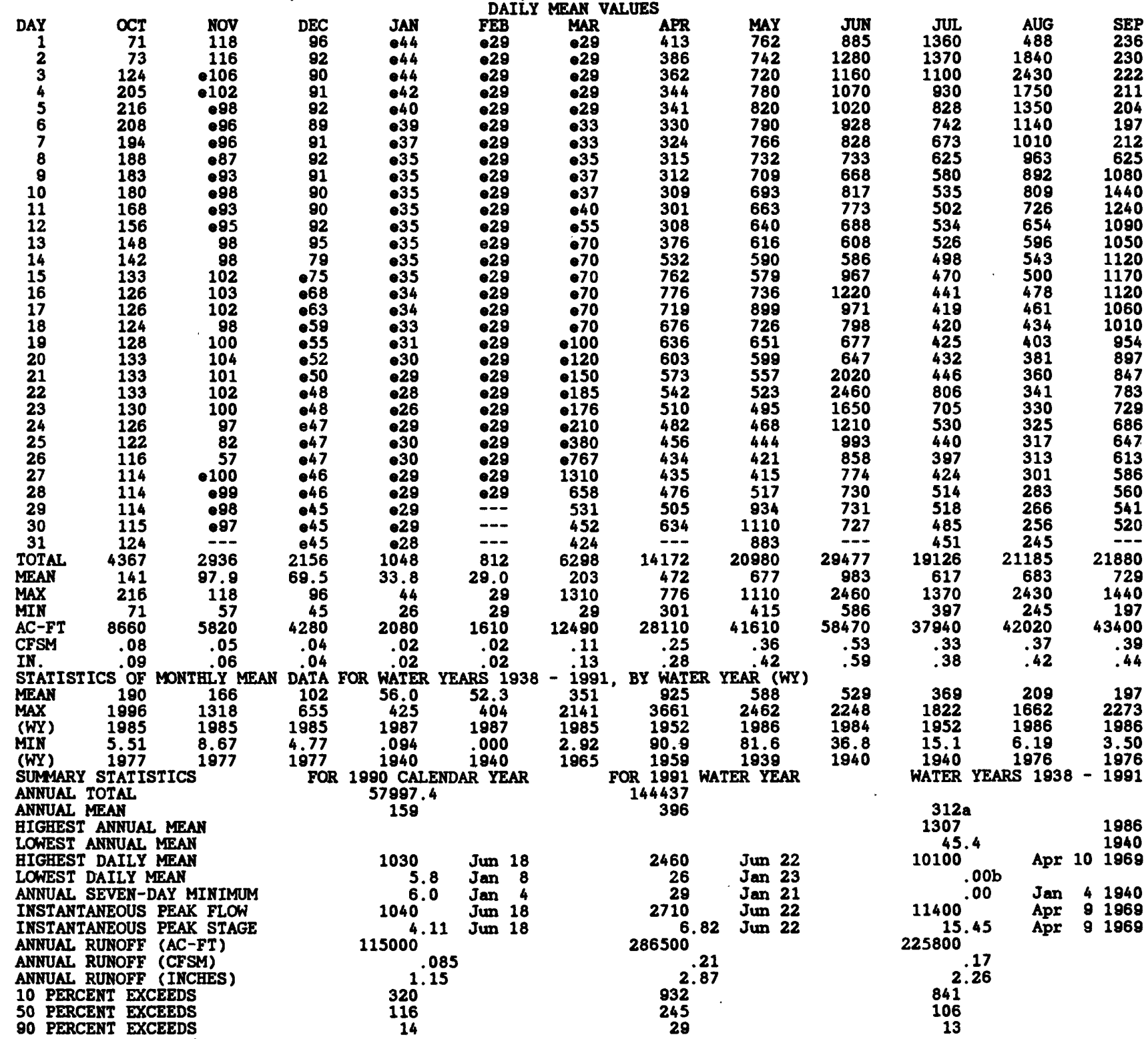

- Median of annual mean discharges is $229 \mathrm{ft}^{3} / \mathrm{s}$.

$b$ Many days during 1940

- Estimated 
MIMNESOTA RIVER BASIN

05311000 MIMNESOTA RIVER AT MONTEVIDEO, MA

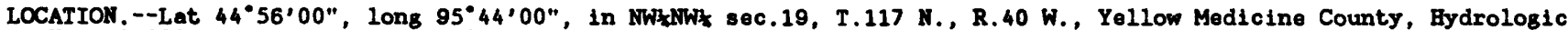
Unit 07020004 , on right bank $100 \mathrm{ft}$ upstream from bridge on U.s. Highway 212 , at Montevideo, and $400 \mathrm{ft}$ downstream from Chipperwa River.

DRAIMAGE AREA. $--6,180 \mathrm{mi}^{2}$, approximately.

\section{WATER DISCHARGE RECORDS}

PERIOD OF RECORD.--July 1909 to September 1917, October 1917 to September 1929 (no winter records), October 1929 to current year. Prior to October 1939, published as "near Montevideo." Monthly discharge only for some periods, published in WSP 1308

REVISED RECORDS.-WSP 1035: 1919(M). WSP 1085: 1935-36. WSP 1508: 1912, 1925(M), 1929(M).

GAGE - Weter-stage recorder. Datum of gage is $909.12 \mathrm{ft}$ above National Geodetic Vertical Datum of 1929. July 22 , 1909, to Feb. 4, 1932, nonrecording gage at bridge 600 ft downstream at present datum. Feb. 5 , 1932 , to Nov.' 26, 1934, nonrecording gage at bridge $100 \mathrm{ft}$ domstream at present datum.

REMARKS. - Records good except those for estimated daily discharges, which are fair. Flow regulated by Big Stone

Lake since Apr. 17, 1937, Lac qui Parle since Jan. 1938, and Marsh Lake since Hov. 1, 1939. DISCBARGE, CUBIC FEET PER SECOND, WATER YEAR OCTOBER 1990 TO SEPTEMBER 1991

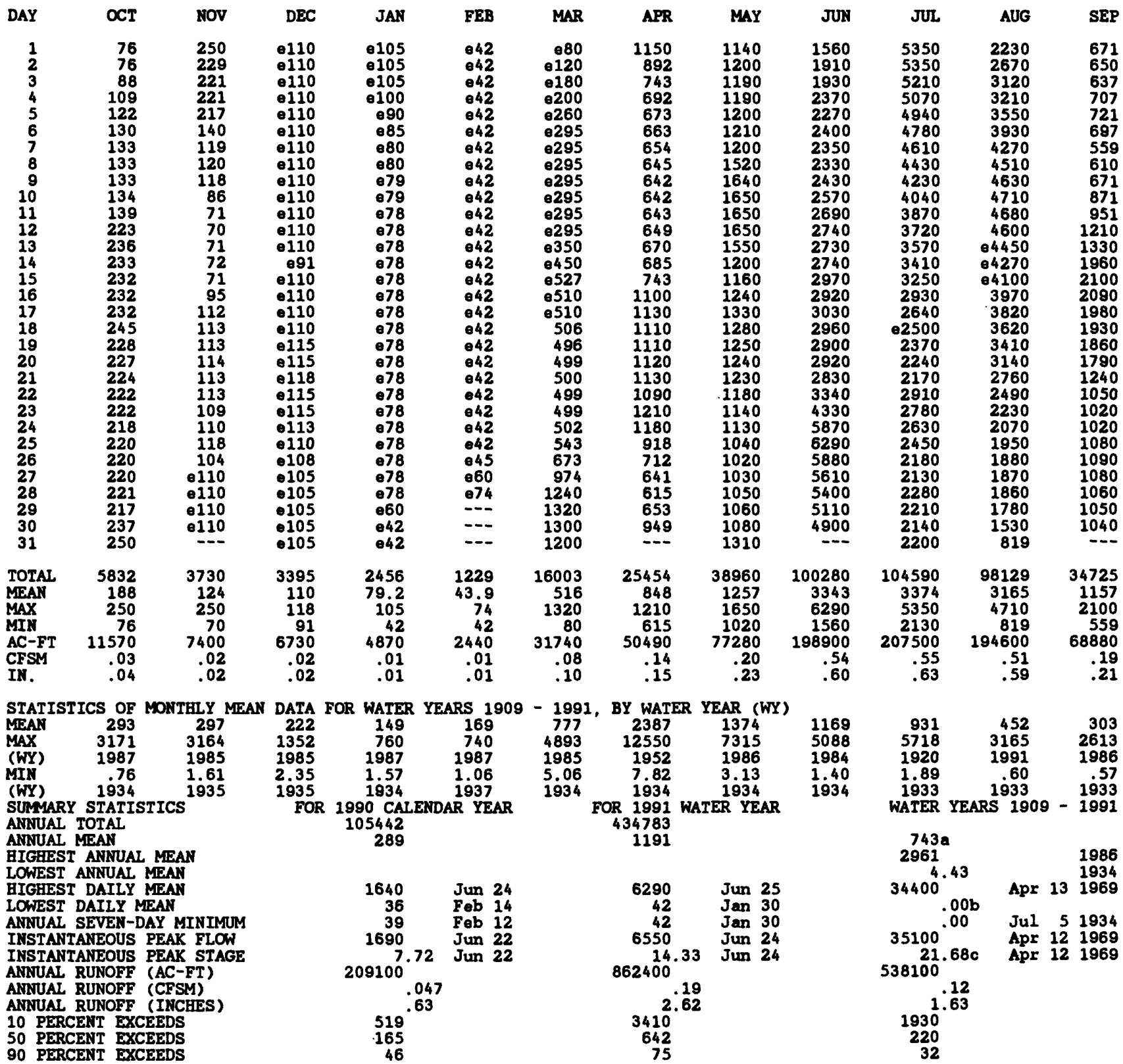

a Median of annual mean discharges is $570 \mathrm{ft}^{3} / \mathrm{s}$.

b Occurred several days in 1933, 1934, 1936 .

c From highwater mark.

- Estimated. 
MIMNESOTA RIVER BASIN

05311000 MINNESOTA RIVER AT MONTEVIDEO, MN--Continued

WATER QUALITY RECORDS

PERIOD OF RECORD.--Water years 1961-67, 1972-76, 1989 to current year.

REMARKS.--Letter $\mathrm{K}$ indicates non-ideal colony count.

WATER QUALITY DATA, WATER YEAR OCTOBER 1990 TO SEPTEMBER 1991

\begin{tabular}{|c|c|c|c|c|c|c|c|c|c|c|}
\hline DATE & TIME & $\begin{array}{c}\text { DIS- } \\
\text { CEARGE, } \\
\text { INST. } \\
\text { CUBIC } \\
\text { FEET } \\
\text { PER } \\
\text { SECOND } \\
(00061)\end{array}$ & $\begin{array}{l}\text { SPE- } \\
\text { CIFIC } \\
\text { CON- } \\
\text { DUCT- } \\
\text { ANCE } \\
\text { (US/CM) } \\
(00095)\end{array}$ & $\begin{array}{c}\text { PH } \\
\text { (STAND- } \\
\text { ARD } \\
\text { UNITS) } \\
(00400)\end{array}$ & $\begin{array}{c}\text { TEMPER- } \\
\text { ATURE } \\
\text { WATER } \\
\text { (DEG C) } \\
(00010)\end{array}$ & $\begin{array}{c}\text { BARO- } \\
\text { METRIC } \\
\text { PRES- } \\
\text { SURE } \\
\text { (MY } \\
\text { OF } \\
\text { BG) } \\
(00025)\end{array}$ & $\begin{array}{c}\text { OXYGEN, } \\
\text { DIS- } \\
\text { SOLVED } \\
(\text { MG /L) } \\
(00300)\end{array}$ & $\begin{array}{l}\text { OXYGEH } \\
\text { DEMAND, } \\
\text { CHEM- } \\
\text { ICAL } \\
\text { (HIGH } \\
\text { LEVEL) } \\
\text { (MG/L) } \\
(00340)\end{array}$ & $\begin{array}{l}\text { OXYGEA } \\
\text { DEMAND, } \\
\text { BIO- } \\
\text { CHEM- } \\
\text { ICAL, } \\
5 \text { DAY } \\
(\text { MG/L) } \\
(00310)\end{array}$ & $\begin{array}{l}\text { COLI- } \\
\text { FORM, } \\
\text { FECAI, } \\
0.7 \\
\text { UM-MF } \\
\text { (COLS.' } \\
100 \text { MI') } \\
\text { (31625) }\end{array}$ \\
\hline ov & & & & & & & & & & \\
\hline $\begin{array}{r}01 \ldots \\
29 . \ldots\end{array}$ & $\begin{array}{l}1345 \\
1045\end{array}$ & $\begin{array}{l}258 \\
154\end{array}$ & $\begin{array}{l}898 \\
929\end{array}$ & $\begin{array}{l}8.7 \\
8.4\end{array}$ & $\begin{array}{r}10.5 \\
0.5\end{array}$ & $\begin{array}{l}729 \\
746\end{array}$ & $\begin{array}{l}10.5 \\
13.1\end{array}$ & $\begin{array}{l}49 \\
30\end{array}$ & $\begin{array}{l}4.6 \\
2.1\end{array}$ & $\begin{array}{r}2500 \\
110\end{array}$ \\
\hline $\operatorname{JAN}^{27} \cdots$ & 1500 & 106 & 980 & 8.1 & 0.0 & 733 & 10.0 & 30 & 1.4 & K7300 \\
\hline$\underset{\text { FEB }}{31}$ & 1415 & 42 & 1130 & 7.8 & 0.5 & 740 & 7.6 & 26 & 1.6 & 2600 \\
\hline $\operatorname{MAR}^{28} \ldots$ & 0930 & 74 & 990 & 8.4 & 0.5 & 732 & 13.9 & 32 & 2.3 & K2900 \\
\hline$\underset{A P R}{28} \cdots$ & 1230 & 1200 & 458 & 7.9 & 2.0 & 732 & 13.8 & 51 & 5.5 & -- \\
\hline $\operatorname{MAY}^{24} \cdots$ & 1320 & 1290 & 878 & 8.6 & 11.5 & 734 & 10.0 & 36 & 2.9 & K120 \\
\hline JUN & 1115 & 1140 & 842 & 8.4 & 24.5 & 732 & 7.9 & 43 & 3.6 & 42 \\
\hline $\begin{array}{r}19 \ldots \\
22 \ldots \\
24 \ldots \\
\text { JUL }\end{array}$ & $\begin{array}{l}1125 \\
1445 \\
1715\end{array}$ & $\begin{array}{l}2910 \\
3810 \\
6310\end{array}$ & $\begin{array}{l}828 \\
507 \\
628\end{array}$ & $\begin{array}{l}8.3 \\
7.9 \\
8.2\end{array}$ & $\begin{array}{l}25.0 \\
20.5 \\
22.5\end{array}$ & $\begin{array}{l}739 \\
741 \\
738\end{array}$ & $\begin{array}{l}7.1 \\
7.4 \\
6.4\end{array}$ & $\begin{array}{l}45 \\
46 \\
36\end{array}$ & $\begin{array}{l}1.3 \\
2.8 \\
2.1\end{array}$ & $\begin{array}{c}500 \\
---\end{array}$ \\
\hline $\begin{array}{r}02 \ldots \\
30 \ldots \\
\text { AUG }\end{array}$ & $\begin{array}{l}0945 \\
1330\end{array}$ & $\begin{array}{l}5360 \\
2120\end{array}$ & $\begin{array}{l}712 \\
857\end{array}$ & $\begin{array}{l}8.4 \\
8.3\end{array}$ & $\begin{array}{l}23.5 \\
22.5\end{array}$ & $\begin{array}{l}734 \\
738\end{array}$ & $\begin{array}{l}6.7 \\
7.8\end{array}$ & $\begin{array}{l}43 \\
40\end{array}$ & $\begin{array}{l}2.1 \\
2.5\end{array}$ & $\mathrm{~K} 4200$ \\
\hline $\begin{array}{r}20 \ldots \\
28 \ldots \\
\operatorname{SEP}\end{array}$ & $\begin{array}{l}1245 \\
0730\end{array}$ & $\begin{array}{l}3150 \\
1860\end{array}$ & $\begin{array}{l}834 \\
844\end{array}$ & $\begin{array}{l}8.4 \\
8.7\end{array}$ & $\begin{array}{l}23.0 \\
25.5\end{array}$ & $\begin{array}{l}740 \\
739\end{array}$ & $\begin{array}{l}7.8 \\
7.4\end{array}$ & $\begin{array}{l}41 \\
46\end{array}$ & $\begin{array}{l}3.9 \\
4.6\end{array}$ & $\mathrm{~K} 2 \overline{4}$ \\
\hline $25 \ldots$ & 1230 & 1100 & 830 & 8.6 & 13.5 & 734 & 12.9 & 39 & -- & 69 \\
\hline
\end{tabular}

\begin{tabular}{|c|c|c|c|c|c|c|c|c|}
\hline $\begin{array}{c}\text { STREP- } \\
\text { TOCOCCI } \\
\text { FECAL } \\
\text { KF AGAR } \\
\text { (COLS. } \\
\text { PER } \\
100 \mathrm{ML}) \\
(31673)\end{array}$ & $\begin{array}{l}\text { CALCIUM } \\
\text { DIS- } \\
\text { SOLVED } \\
\text { (MG/L } \\
\text { AS CA) } \\
\text { (00915) }\end{array}$ & $\begin{array}{c}\text { MAGNE- } \\
\text { SIUM, } \\
\text { DIS- } \\
\text { SOLVED } \\
\text { (MG/L } \\
\text { AS MG) } \\
\text { (00925) }\end{array}$ & $\begin{array}{c}\text { SODIUM, } \\
\text { DIS- } \\
\text { SOLVED } \\
\text { (MG/L } \\
\text { AS KA ) } \\
(00930)\end{array}$ & $\begin{array}{c}\text { POTAS- } \\
\text { SIUM, } \\
\text { DIS- } \\
\text { SOLVED } \\
\text { (MG/L } \\
\text { AS K) } \\
(00935)\end{array}$ & $\begin{array}{l}\text { SULFATE } \\
\text { DIS- } \\
\text { SOLVED } \\
\text { (MG/L } \\
\text { AS SO4) } \\
(00945)\end{array}$ & $\begin{array}{l}\text { FLUO- } \\
\text { RIDE, } \\
\text { DIS- } \\
\text { SOLVED } \\
\text { (MG/L } \\
\text { AS F) } \\
\text { (00950) }\end{array}$ & $\begin{array}{l}\text { RESIDUE } \\
\text { TOTAL } \\
\text { AT } 105 \\
\text { DEG. C, } \\
\text { SUS- } \\
\text { PENDED } \\
\text { (MG/L) } \\
(00530)\end{array}$ & $\begin{array}{c}\text { RESIDUE } \\
\text { VOLA- } \\
\text { TILE, } \\
\text { SUS- } \\
\text { PENDED } \\
\text { (MG/L) } \\
(00535)\end{array}$ \\
\hline
\end{tabular}

NOV

Nov 29.

(00925) (00930)

(00945)

(00530) (00535)

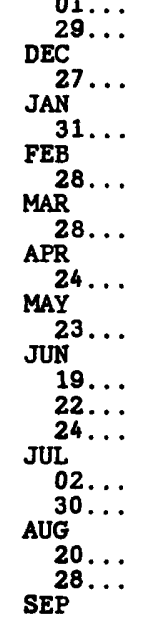

$\begin{array}{rr}160 & - \\ 120 & - \\ 2100 & - \\ 950 & - \\ 440 & - \\ - & 87 \\ 27 & - \\ 440 & - \\ 780 & - \\ -- & - \\ -- & - \\ 780 & - \\ 300 & - \\ 890 & -\end{array}$

--
--
--
--
87
--
--
--
--
--
--
--
--
--

$\begin{array}{ll}-- & - \\ -- & - \\ -- & - \\ -- & -- \\ 47 & 25 \\ -- & -- \\ -- & -- \\ -- & - \\ -- & - \\ -- & - \\ -- & - \\ -- & - \\ -- & -- \\ -- & - \\ -- & -\end{array}$

$\begin{array}{ll}-- & - \\ -- & - \\ -- & - \\ -- & \\ 25 & 9 \\ -- & - \\ -- & - \\ -- & - \\ -- & - \\ -- & - \\ -- & - \\ -- & -\end{array}$

$\begin{array}{lll}-- & - \\ -- & - \\ -- & - \\ -- & - \\ 9.2 & 240 \\ -- & - \\ -- & - \\ -- & - \\ -- & - \\ -- & - \\ -- & - \\ -- & - \\ - & - \\ -- & -\end{array}$

--
--
--
--
240
--
--
--
--
--
--
--
--

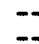

$\begin{array}{rrr}-- & <1 & <1\end{array}$

$--<1<1$

$--\quad 6$

$\begin{array}{lll}-- & 7\end{array}$

$0.2 \quad 47 \quad 11$

$\begin{array}{lll}-- & 16 & \end{array}$

$\begin{array}{lll}-- & 64 & 16\end{array}$

$\begin{array}{lrr}-- & 22 & 6 \\ -- & 137 & 26 \\ -- & 52 & 11\end{array}$

-- $24 \quad 11$

50

$\begin{array}{lll}-- & 31 & 15 \\ -- & 31 & 15\end{array}$

$48 \quad 7$ 
MINNESOTA RIVER BASIN

05311000 MINNESOTA RIVER AT MONTEVIDEO, MN--Continued

WATER QUALITY DATA, WATER YEAR OCTOBER 1990 TO SEPTEMBER 1991

\begin{tabular}{|c|c|c|c|c|c|c|c|c|c|}
\hline DATE & $\begin{array}{c}\text { BORON, } \\
\text { DIS- } \\
\text { SOLVED } \\
\text { (UG/L } \\
\text { AS B) } \\
\text { (01020) }\end{array}$ & $\begin{array}{c}\text { NITRO- } \\
\text { GEN, } \\
\text { NITRITE } \\
\text { TOTAL } \\
(\text { MG /L } \\
\text { AS N) } \\
(00615)\end{array}$ & $\begin{array}{c}\text { NITRO- } \\
\text { GEN, } \\
\text { NITRITE } \\
\text { DIS- } \\
\text { SOLVED } \\
\text { (MG/L } \\
\text { AS N) } \\
(00613)\end{array}$ & $\begin{array}{c}\text { NITRO- } \\
\text { GEN, } \\
\text { NO2+NO3 } \\
\text { TOTAL } \\
(\text { MG/L } \\
\text { AS N) } \\
(00630)\end{array}$ & $\begin{array}{c}\text { NITRO- } \\
\text { GEN, } \\
\text { NO2+NO3 } \\
\text { DIS- } \\
\text { SOLVED } \\
\text { (MG/L } \\
\text { AS N) } \\
(00631)\end{array}$ & $\begin{array}{c}\text { NITRO- } \\
\text { GEN, } \\
\text { AMNONIA } \\
\text { TOTAL } \\
\text { (MG/L } \\
\text { AS N) } \\
\text { (00610) }\end{array}$ & $\begin{array}{c}\text { NITRO- } \\
\text { GEN, } \\
\text { AMMONIA } \\
\text { DIS- } \\
\text { SOLVED } \\
(M G / L \\
\text { AS N) } \\
(00608)\end{array}$ & $\begin{array}{c}\text { NITRO- } \\
\text { GEN,AM- } \\
\text { MONIA + } \\
\text { ORGANIC } \\
\text { TOTAL } \\
(M G / L \\
\text { AS N) } \\
(00625)\end{array}$ & $\begin{array}{l}\text { NITRO- } \\
\text { GEN, AM- } \\
\text { MONIA + } \\
\text { ORGANIC } \\
\text { DIS } \\
\text { (MG/L } \\
\text { AS N }) \\
(00623)\end{array}$ \\
\hline \multicolumn{10}{|l|}{ NOV } \\
\hline $\begin{array}{r}01 \ldots \\
29 \\
\text { DEC }\end{array}$ & $\because$ & $\begin{array}{l}0.03 \\
0.02\end{array}$ & $\begin{array}{l}0.02 \\
0.02\end{array}$ & $\begin{array}{l}0.20 \\
0.30\end{array}$ & $\begin{array}{l}0.10 \\
0.30\end{array}$ & $\begin{array}{l}0.26 \\
0.44\end{array}$ & $\begin{array}{l}0.26 \\
0.44\end{array}$ & $\begin{array}{l}1.7 \\
1.9\end{array}$ & $\begin{array}{l}1.3 \\
1.4\end{array}$ \\
\hline JAN 27 & - & 0.01 & 0.01 & 0.50 & 0.50 & 0.63 & 0.63 & 1.7 & 1.7 \\
\hline $31 \ldots$ & -- & 0.02 & 0.02 & 0.80 & 0.78 & 0.74 & 0.74 & 1.9 & 1.5 \\
\hline $28 \ldots$ & -- & 0.01 & 0.01 & 0.57 & 0.57 & 0.32 & 0.32 & 0.5 & 0.4 \\
\hline APR & 120 & 0.06 & 0.05 & 1.3 & 1.3 & 0.06 & 0.06 & 1.7 & 1.1 \\
\hline $\mathrm{MAY}^{24} \ldots$ & -- & 0.02 & $<0.01$ & 0.7 & 0.70 & 0.03 & 0.02 & 1.3 & 0.9 \\
\hline JUN $23 .$. & -- & 0.08 & 0.06 & 1.1 & 1.1 & 0.16 & 0.16 & 1.4 & 1.1 \\
\hline $\begin{array}{r}19 \ldots \\
22 \ldots \\
24 \ldots\end{array}$ & $\begin{array}{l}-- \\
--\end{array}$ & $\begin{array}{l}0.11 \\
0.12 \\
0.11\end{array}$ & $\begin{array}{l}0.09 \\
0.09 \\
0.09\end{array}$ & $\begin{array}{l}2.4 \\
2.4 \\
2.1\end{array}$ & $\begin{array}{l}2.4 \\
2.3 \\
2.1\end{array}$ & $\begin{array}{l}0.12 \\
0.15 \\
0.10\end{array}$ & $\begin{array}{l}0.10 \\
0.14 \\
0.10\end{array}$ & $\begin{array}{l}1.4 \\
1.1 \\
1.3\end{array}$ & $\begin{array}{l}1.2 \\
1.0 \\
1.0\end{array}$ \\
\hline $\begin{array}{r}02 \ldots \\
30 \ldots \\
\text { AUG }\end{array}$ & $=$ & $\begin{array}{l}0.10 \\
0.04\end{array}$ & $\begin{array}{l}0.07 \\
0.04\end{array}$ & $\begin{array}{l}1.9 \\
1.6\end{array}$ & $\begin{array}{l}1.9 \\
1.7\end{array}$ & $\begin{array}{l}0.08 \\
0.09\end{array}$ & $\begin{array}{l}0.07 \\
0.09\end{array}$ & $\begin{array}{l}1.1 \\
1.6\end{array}$ & $\begin{array}{l}0.8 \\
1.2\end{array}$ \\
\hline $\begin{array}{r}20 \ldots \\
28 \ldots \\
\text { SEP }\end{array}$ & $=$ & $\begin{array}{l}0.02 \\
0.01\end{array}$ & $\begin{array}{r}0.02 \\
<0.01\end{array}$ & $\begin{array}{l}0.44 \\
0.06\end{array}$ & $\begin{array}{r}0.44 \\
<0.05\end{array}$ & $\begin{array}{l}0.01 \\
0.01\end{array}$ & $\begin{array}{l}0.01 \\
0.01\end{array}$ & $\begin{array}{l}1.5 \\
1.7\end{array}$ & $\begin{array}{l}0.8 \\
0.9\end{array}$ \\
\hline $25 \ldots$ & -- & 0.02 & 0.02 & 1.7 & 1.7 & 0.01 & 0.01 & 1.3 & 0.9 \\
\hline DATE & $\begin{array}{c}\text { PHOS- } \\
\text { PHORUS } \\
\text { TOTAL } \\
\text { (MG/L } \\
\text { AS P) } \\
(00665)\end{array}$ & $\begin{array}{c}\text { PHOS- } \\
\text { PHORUS } \\
\text { DIS- } \\
\text { SOLVED } \\
\text { (MG/L } \\
\text { AS P) } \\
(00666)\end{array}$ & $\begin{array}{c}\text { PHOS- } \\
\text { PHORUS } \\
\text { ORTHO } \\
\text { TOTAL } \\
\text { (MG/L } \\
\text { AS P) } \\
\text { (70507) }\end{array}$ & $\begin{array}{l}\text { PHOS- } \\
\text { PHORUS } \\
\text { ORTHO, } \\
\text { DIS- } \\
\text { SOLVED } \\
\text { (MG/L } \\
\text { AS P) } \\
(00671)\end{array}$ & $\begin{array}{l}\text { CARBON, } \\
\text { ORGANIC } \\
\text { DIS- } \\
\text { SOLVED } \\
(\text { MG /L } \\
\text { AS C) } \\
(00681)\end{array}$ & $\begin{array}{l}\text { CARBON, } \\
\text { ORGANIC } \\
\text { SUS- } \\
\text { PENDED } \\
\text { TOTAL } \\
\text { (MG/L } \\
\text { AS C) } \\
\text { (O0689) }\end{array}$ & $\begin{array}{l}\text { SEDI- } \\
\text { MENT, } \\
\text { SUS- } \\
\text { PENDED } \\
(\text { MG/L) } \\
(80154)\end{array}$ & $\begin{array}{c}\text { CHLOR-A } \\
\text { PHYTO- } \\
\text { PLANK- } \\
\text { TON } \\
\text { CHROMO } \\
\text { FLUOROM } \\
\text { (UG/L) } \\
\text { (70953) }\end{array}$ & $\begin{array}{c}\text { CHLOR-B } \\
\text { PHYTO- } \\
\text { PLANK- } \\
\text { TON } \\
\text { CHROMO } \\
\text { FLUOROM } \\
\text { (UG/L) } \\
(70954)\end{array}$ \\
\hline \multicolumn{10}{|l|}{ NOV } \\
\hline $\begin{array}{c}01 \ldots \\
29 .\end{array}$ & $\begin{array}{l}0.13 \\
0.15\end{array}$ & $\begin{array}{l}0.06 \\
0.13\end{array}$ & $\begin{array}{l}0.09 \\
0.09\end{array}$ & $\begin{array}{l}0.06 \\
0.09\end{array}$ & $\begin{array}{l}9.3 \\
8.5\end{array}$ & $\begin{array}{l}1.2 \\
0.6\end{array}$ & $\begin{array}{l}23 \\
18\end{array}$ & $\begin{array}{r}12.0 \\
6.2\end{array}$ & $\begin{array}{l}<0.50 \\
<0.30\end{array}$ \\
\hline $27 \ldots$ & 0.13 & 0.11 & 0.13 & 0.08 & 11 & 0.3 & 69 & 2.7 & $<0.20$ \\
\hline $31 \ldots$ & 0.18 & 0.16 & 0.18 & 0.16 & 9.0 & 0.4 & 32 & 0.7 & $<0.10$ \\
\hline${ }_{\text {MAR }}^{28} \ldots$ & 0.26 & 0.17 & 0.17 & 0.16 & 9.4 & 1.1 & 165 & 9.0 & 0.40 \\
\hline${ }_{A P R}^{28} \ldots$ & 0.22 & 0.09 & 0.10 & 0.04 & 10 & 1.5 & 120 & 12.0 & 0.90 \\
\hline $\operatorname{MY}^{24} \ldots$ & 0.06 & $<0.01$ & 0.02 & $<0.01$ & 9.4 & 1.0 & 82 & 2.8 & 0.40 \\
\hline JUN & 0.19 & 0.07 & 0.08 & 0.06 & 10 & 2.1 & 180 & 2.6 & $<1.00$ \\
\hline $\begin{array}{l}19 \ldots \\
22 \ldots \\
24 \ldots \\
\text { JUL }\end{array}$ & $\begin{array}{l}0.26 \\
0.27 \\
0.17\end{array}$ & $\begin{array}{l}0.17 \\
0.18 \\
0.17\end{array}$ & $\begin{array}{l}0.15 \\
0.20 \\
0.17\end{array}$ & $\begin{array}{l}0.14 \\
0.15 \\
0.14\end{array}$ & $\begin{array}{l}9.4 \\
7.5 \\
8.7\end{array}$ & $\begin{array}{r}>5.0 \\
4.7 \\
1.7\end{array}$ & $\begin{array}{r}104 \\
215 \\
81\end{array}$ & $\begin{array}{l}3.7 \\
2.1 \\
2.2\end{array}$ & $\begin{array}{l}<0.60 \\
<0.60 \\
<0.70\end{array}$ \\
\hline $\begin{array}{r}02 \ldots \\
30 \ldots \\
\text { AUG }\end{array}$ & $\begin{array}{l}0.30 \\
0.29\end{array}$ & $\begin{array}{l}0.17 \\
0.15\end{array}$ & $\begin{array}{l}0.19 \\
0.11\end{array}$ & $\begin{array}{l}0.15 \\
0.10\end{array}$ & 10.0 & $\begin{array}{l}2.1 \\
3.1\end{array}$ & $\begin{array}{l}89 \\
96\end{array}$ & $\begin{array}{r}3.3 \\
14.0\end{array}$ & $\begin{array}{r}<0.60 \\
0.80\end{array}$ \\
\hline $\begin{array}{r}20 \ldots \\
28 \ldots \\
\text { SEP }\end{array}$ & $\begin{array}{l}0.20 \\
0.25\end{array}$ & $\begin{array}{l}0.11 \\
0.10\end{array}$ & $\begin{array}{l}0.09 \\
0.04\end{array}$ & $\begin{array}{l}0.06 \\
0.03\end{array}$ & 10 & $\begin{array}{l}2.4 \\
2.6\end{array}$ & $\begin{array}{r}62 \\
86\end{array}$ & $\begin{array}{l}16.0 \\
18.0\end{array}$ & $\begin{array}{l}<0.10 \\
<0.10\end{array}$ \\
\hline & 0.10 & 0.10 & 0.05 & 0.05 & 9.6 & 2.8 & 142 & 15.0 & $<0.10$ \\
\hline
\end{tabular}


MIMNESOTA RIVER BASIN

05313500 YELLOW MEDICINE RIVER NEAR GRANITE FALLS, M

LOCATION, --Lat $44^{\circ} 43^{\prime} 18^{\prime \prime}$, long $95^{\circ} 31^{\prime} 07^{\prime \prime}$, in SWk sec.35, T.115 N., R.39 W., Yellow Medicine County, Hydrologic Unit 07020004 , on right bank 50 ft domstream from highway bridge, 6 mi upstream from mouth, and 8 mi south of town of Granite Falls.

DRAINAGE ARFA. $--653 \mathrm{mi}$

PERIOD OF RECORD.-March 1931 to September 1935 (no winter records), October 1935 to September 1938, October 1939 to current year. Monthly discharge only for some periods, publ1shed in WSP 1308.

REVISED RECORDS - -WSP 1508: 1931, 1934(M), $1937(\mathrm{M}), 1946(\mathrm{M}), 1950(\mathrm{M})$

GAGE.--Water-stage recorder. Datum of gage is 960.64 ft above Hational Geodetic Vertical Datum of 1929. Mar. 16, 1931, to June 13, 1938, nonrecording gage, on bridge 50 ft upstream at present datum. Oct. 12, 1939 , to Hov. 30, 1952, nonrecording gage $500 \mathrm{ft}$ downstream at present datum.

REMARKS. - Records good except those for estimated daily discharges, which are falr.

EXTREMES OUTSIDE PERIOD OF RECORD.-Flood in June 1919 reached a stage of $17.5 \mathrm{ft}$, from information by local residents, discharge, $25,200 \mathrm{ft}^{3} / \mathrm{s}$

EXIRENES FOR CURRENT YEAR. - Peak discharge greater than base discharge of $300 \mathrm{ft} 3 / \mathrm{s}$ and maximum (*):

\begin{tabular}{|c|c|c|c|c|c|c|c|}
\hline Date & Time & $\begin{array}{c}\text { D1scharge } \\
\left(\mathrm{ft}^{3} / \mathrm{s}\right)\end{array}$ & $\begin{array}{c}\text { Gage height } \\
\text { (ft) }\end{array}$ & Date & Time & $\begin{array}{c}\text { Discharge } \\
\left(\mathrm{ft}^{3} / \mathrm{s}\right)\end{array}$ & $\underset{(f t)}{\text { Gage helght }}$ \\
\hline $\begin{array}{l}\text { June } 7 \\
\text { June } 18\end{array}$ & $\begin{array}{l}2130 \\
0315\end{array}$ & $\begin{array}{r}\star 2,730 \\
2,550\end{array}$ & $\begin{array}{r}\star 6.93 \\
6.77\end{array}$ & $\begin{array}{l}\text { June } 23 \\
\text { July } 11\end{array}$ & $\begin{array}{l}1745 \\
1500\end{array}$ & $\begin{array}{r}2,510 \\
604\end{array}$ & $\begin{array}{l}6.73 \\
4.17\end{array}$ \\
\hline
\end{tabular}

DISCEARGE, CUBIC FEET PER SECOND, WATER YEAR OCTOBER 1990 TO SEPTEMER 1991

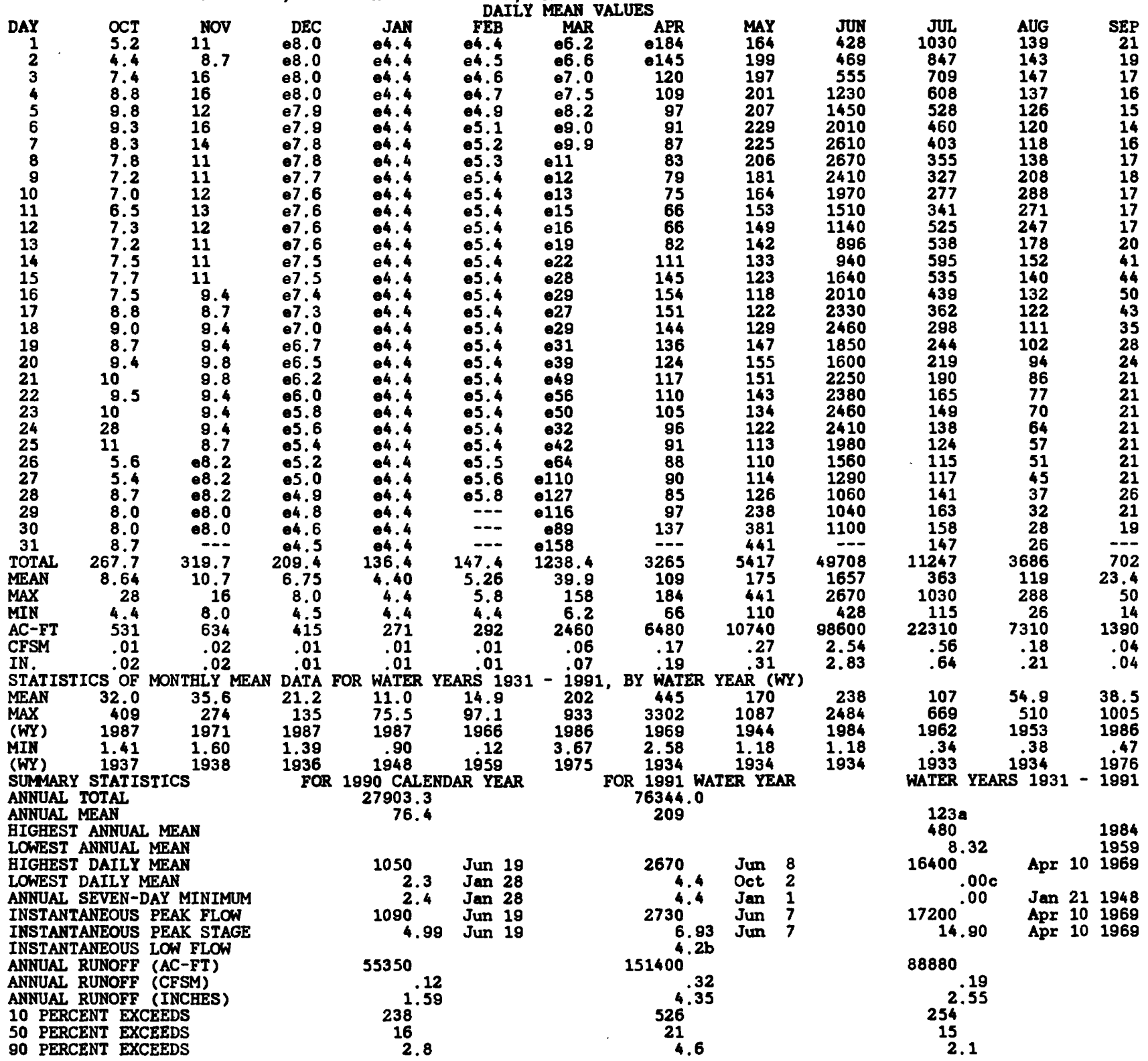

a Median of annual mean discharges is $79 \mathrm{ft}^{3} / \mathrm{s}$.

b Occurred Oct. $1,2,3,26,27$, but may have been less during perlod of 1 ce effect.

c Many days in several years.

- Estimated. 
MINNESOTA RIVER BASIN

05315000 REDWOOD RIVER NEAR MARSHALL, MN

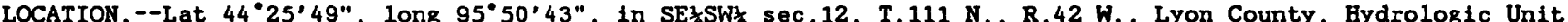
07020006 , on right bank $2.0 \mathrm{mi}$ upstream from Redwood River diversion structure on southwest edge of town of Marshall, MN. Prior to Apr. 10, 1980, at site $5 \mathrm{ml}$ downstream.

DRAINAGE AREA. --259 mi 2

PERIOD OF RECORD. --March 1940 to current year. Monthly discharge only for some periods, published in WSP 1308. REVISED RECORDS. - WDR MN-89-2: Drainage area.

GAGE. - Water-stage recorder. Datum of gage is $1,188.23 \mathrm{ft}$ above National Geodetic Vertical Datum of 1929 . March 1940 to April 9, 1980, nonrecording gage $5.0 \mathrm{mi}$ downstream from present site at datum 43.35 ft lower (crest-stage gage added June 12, 1968). Since March 1964, nonrecording gage and crest-stage gage on diversion channel $1.5 \mathrm{mi}$ downstream at datum 1,100.00 ft above National Geodetic Vertical Datum of 1929 .

REMARKS.--Records fair. Water diverted at medium and high stages into diversion channel $2.0 \mathrm{mi}$ below station. Diversion began Mar. 18, 1964. Unknown amount of natural diversion into Cottonwood River basin occurs at extremely high stages $0.8 \mathrm{mi}$ below station.

EXTREMES FOR PERIOD OF RECORD.--River only, maximum discharge, 5, $370 \mathrm{ft}^{3} / \mathrm{s}$, June 17, 1957, gage height, $10.14 \mathrm{ft}$; maximum gage height, $11.05 \mathrm{ft}, \mathrm{Apr} .6,1951$, from floodmark; no flow at times.

Diversion only, maximum discharge, $4,440 \mathrm{ft} / \mathrm{s}$, Apr. 10, 1969, gage height, $78.45 \mathrm{ft}$; no flow on many days.

Combined flow, maximum discharge, 5,590 ft $3 / \mathrm{s}, \mathrm{Apr}, 10,1969$; no flow at times.

DISCHARGE, CUBIC FEET PER SECOND, WATER YEAR OCTOBER 1990 TO SEPTEMBER 1991

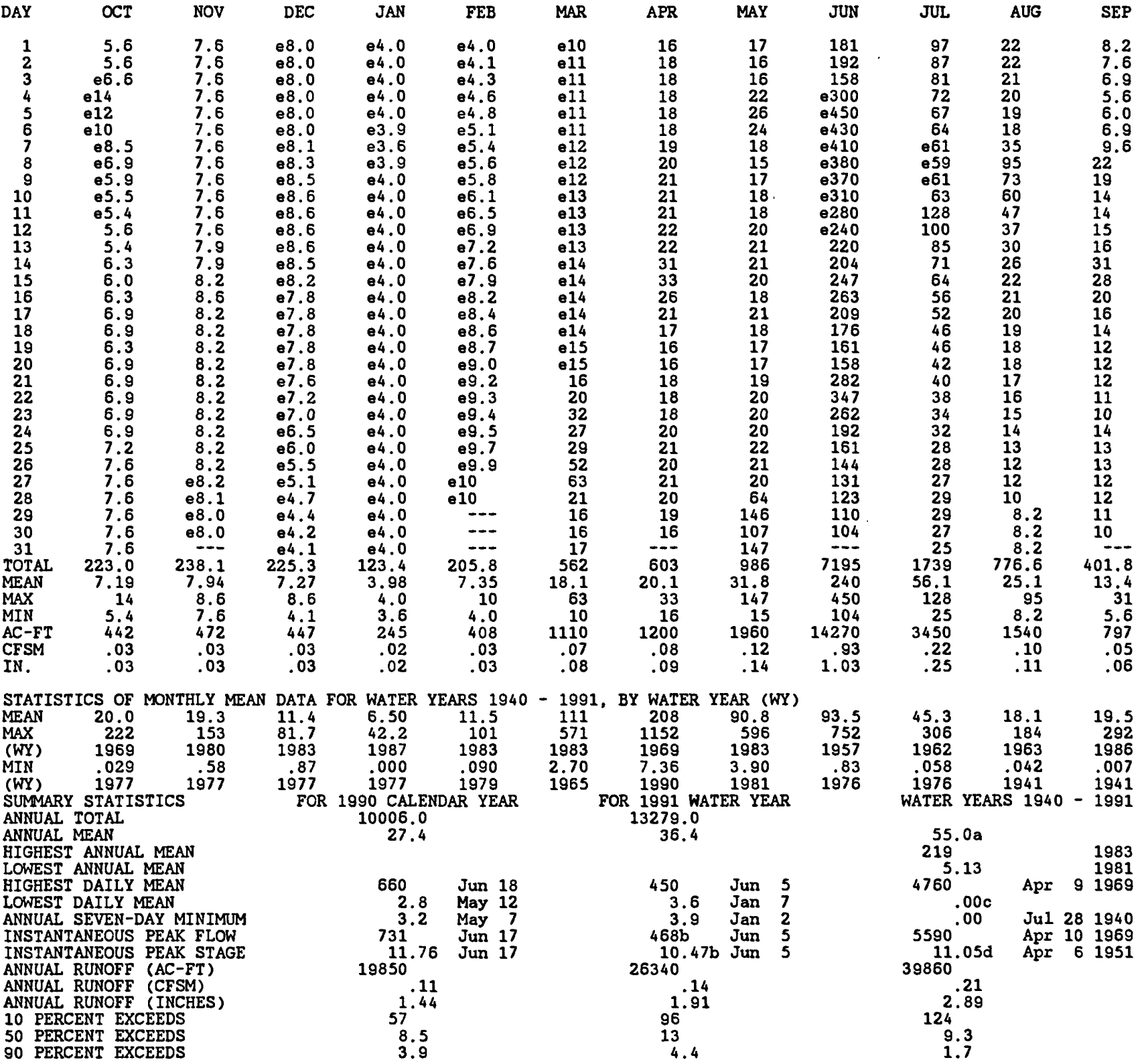

a Medium of annual mean discharges in $38 \mathrm{ft}^{3} / \mathrm{s}$.

$b$ Highest observed (no gage height record).

c Many days in several years.

d Site and datum then in use. 
LOCATION.--Iat $44^{\circ} 31^{\prime} 25^{\prime \prime}$, long $95^{\circ} 10^{\prime} 20^{\prime \prime}$, in SEzNEz sec.9, T.112 N., R. 36 W., Redwood County, Hydrologic Unit 07020006 , on right bank 4 ft upstream from highway bridge, 3 mi west of town of Redwood Falls, and 8.5 mi upstream from mouth

PERIOD OE RECORD.--July 1909 to September 1914 (no winter records except 1911-12). August 1930 to September 1935 (no winter records), October 1935 to current year.

REVISED RECORDS. - WDR MN-89-2: Drainage area.

GAGE. - Water-stage recorder. Datum of gage is $972.33 \mathrm{ft}$ above National Geodetic Vertical Datum of 1929 . July 1909 to September 1914, nonrecording gage at bridge $20 \mathrm{ft}$ downstream at datum 0.22 ft $10 w e r$. August 1930 to Oct. 25, 1949, nonrecording gage, at bridge $20 \mathrm{ft}$ downstream at present datum.

REMARKS.--Records good except those for estimated daily discharges, which are fair. Natural discharge affected by unknown amount of interbasin flow between Yellow Medicine, Redwood, and Cottonwood River basins during extreme floods.

EXTREMES EOR CURRENT YEAR.--Peak discharges greater than base discharge of $400 \mathrm{ft} 3 / \mathrm{s}$ and maximum ( $*$ ):

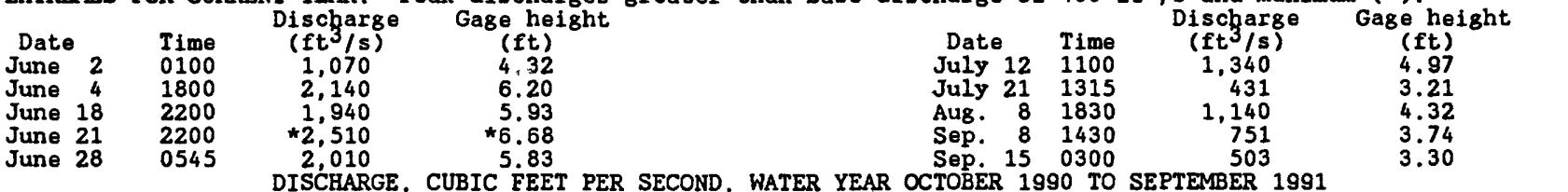

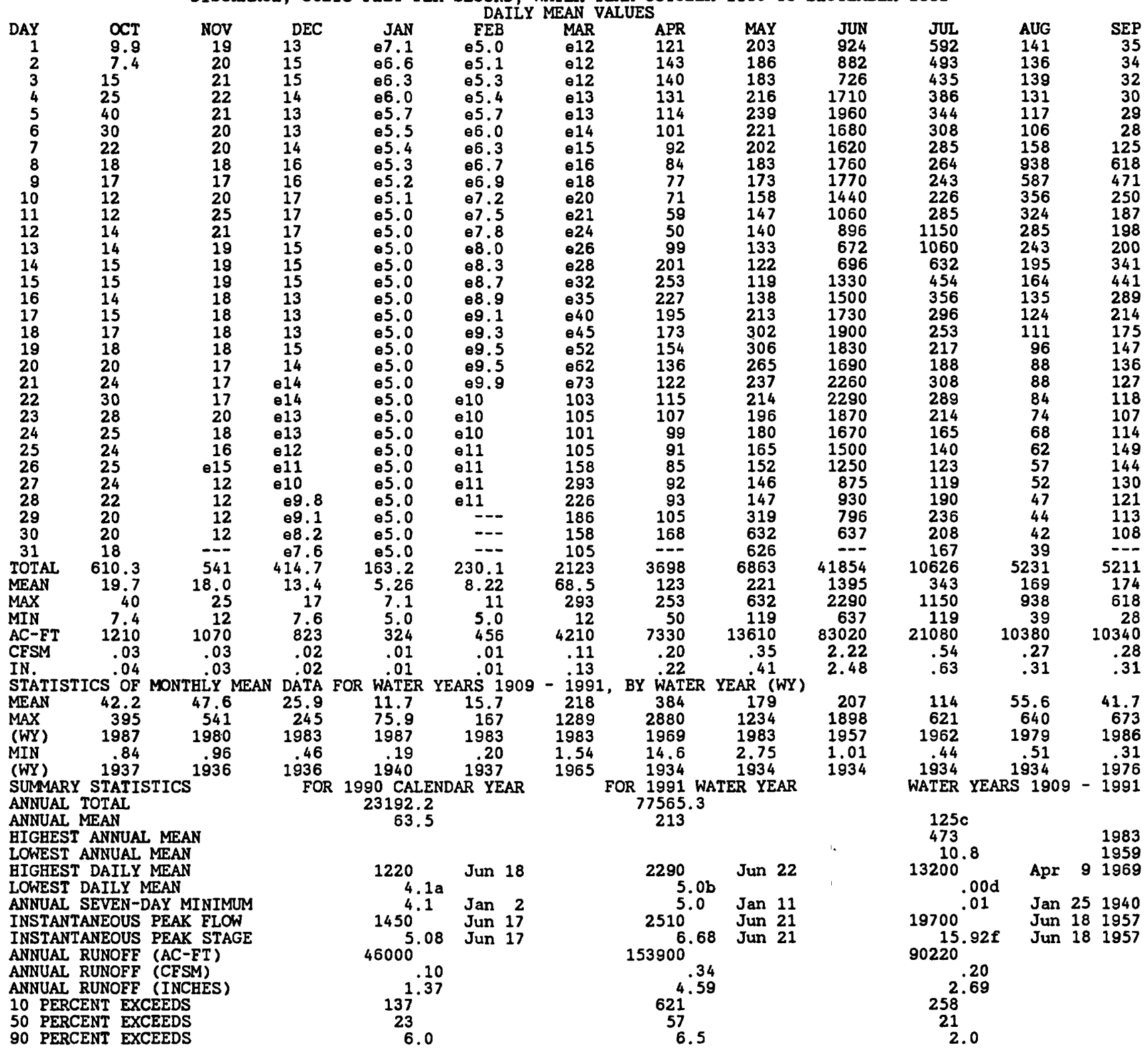

ENT EXCEEDS

a Occurred Jan. 2-Feb. 2

b Occurred Jan. 11-Feb. 1

c Median of annual mean discharges is $80 \mathrm{ft}^{3} / \mathrm{s}$.

d Several days in 1940 and 1959.

e Estimated.

$f$ From floodmark. 


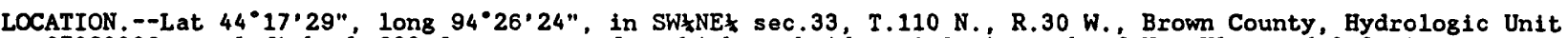
07020008 , on left bank $600 \mathrm{ft}$ upstream from highway bridge, $1.8 \mathrm{mi}$ south of New Ulm, and $3.2 \mathrm{mi}$ upstream from mouth.

DRAINAGE AREA. $-1,280 \mathrm{mi}^{2}$, approximately.

PERIOD OF RECORD.--July 1909 to December 1913, March 1931 to March 1938, August 1938 to current year (winter records incomplete prior to 1936).

REVISED RECORDS. - -WSP 355: 1912.

GAGE --Water-stage recorder, Datum of gage is $796.83 \mathrm{ft}$ above National Geodetic Vertical Datum of 1929. July 1. 1909, to Dec. 13, 1913, nonrecording gage at site 2.7 mi upstream at different datum. Mar. 15 , 1931 . to Mar. 31, 1938, nonrecording gage $2.2 \mathrm{mi}$ upstream at datum $11.41 \mathrm{ft}$ higher. Aug. 23,1938 , to June 25.1948, nonrecording gage at present site and datum.

REMARKS. --Records good except those for estimated daily discharges, which are fair

EXTREMES FOR CURRENT YEAR. --Peak discharges greater than base discharge of $1,300 \mathrm{ft} 3 / \mathrm{s}$ and maximum ( $*$ ):

\begin{tabular}{|c|c|c|c|c|c|c|c|}
\hline Date & Time & $\begin{array}{c}\text { Discharge } \\
\left(f t^{3} / \mathrm{s}\right)\end{array}$ & $\begin{array}{c}\text { Gage height } \\
\text { (ft) }\end{array}$ & Date & Time & $\begin{array}{c}\text { Discharge } \\
\left(\mathrm{ft}^{3} / \mathrm{s}\right)\end{array}$ & $\begin{array}{c}\text { Gage height } \\
\text { (ft) }\end{array}$ \\
\hline $\begin{array}{lr}\text { Apr } & 15 \\
\text { May } & 20 \\
\text { Jun } & 5 \\
\text { Jun } & 23\end{array}$ & $\begin{array}{l}2130 \\
1800 \\
2100 \\
2030\end{array}$ & $\begin{array}{r}1830 \\
1560 \\
4300 \\
+5440\end{array}$ & $\begin{array}{r}6.72 \\
6.14 \\
10.78 \\
+11.99\end{array}$ & $\begin{array}{lr}\text { Jul } & 12 \\
\text { Aug } & 9 \\
\text { Sep } & 14\end{array}$ & $\begin{array}{l}2300 \\
0330 \\
2400\end{array}$ & $\begin{array}{l}1580 \\
1490 \\
1330\end{array}$ & $\begin{array}{l}6.20 \\
6.00 \\
5.64\end{array}$ \\
\hline
\end{tabular}

DISCHARGE, CUBIC FEET PER SECOND, WATER YEAR OCTOBER 1990 TO SEPTEMBER 1991

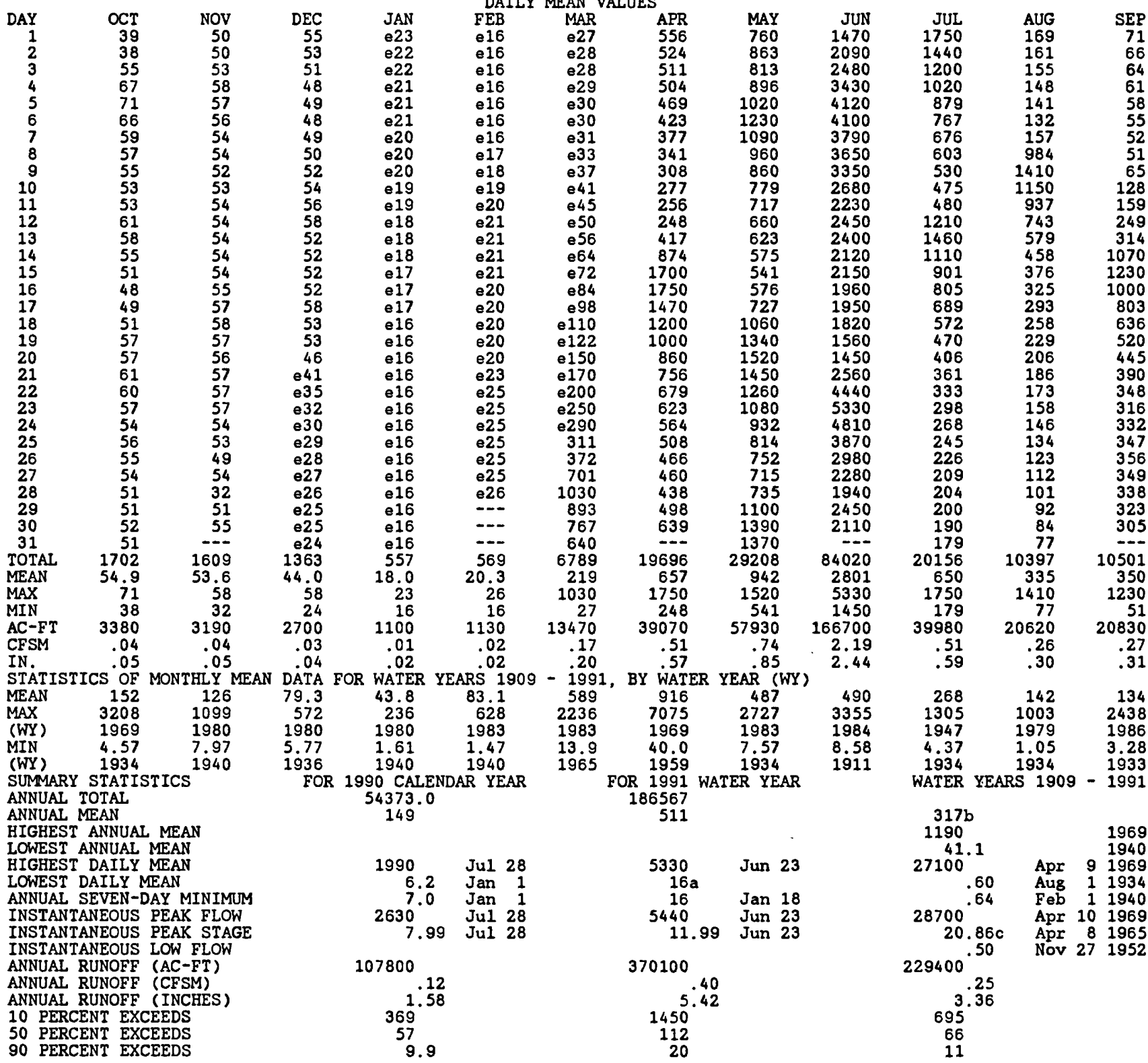

a Jan, $18-\mathrm{Feb}, 7$.

b Median of annual mean discharges is $224 \mathrm{ft}^{3} / \mathrm{s}$

c From floodmark (backwater from ice). 
PERIOD OF RECORD.--Water years 1960-68, 1970-76, 1989 to current year.

REMARKS.--Letter $K$ indicates non-ideal colony count.

WATER QUALITY DATA, WATER YEAR OCTOBER 1990 TO SEPTEMBER 1991

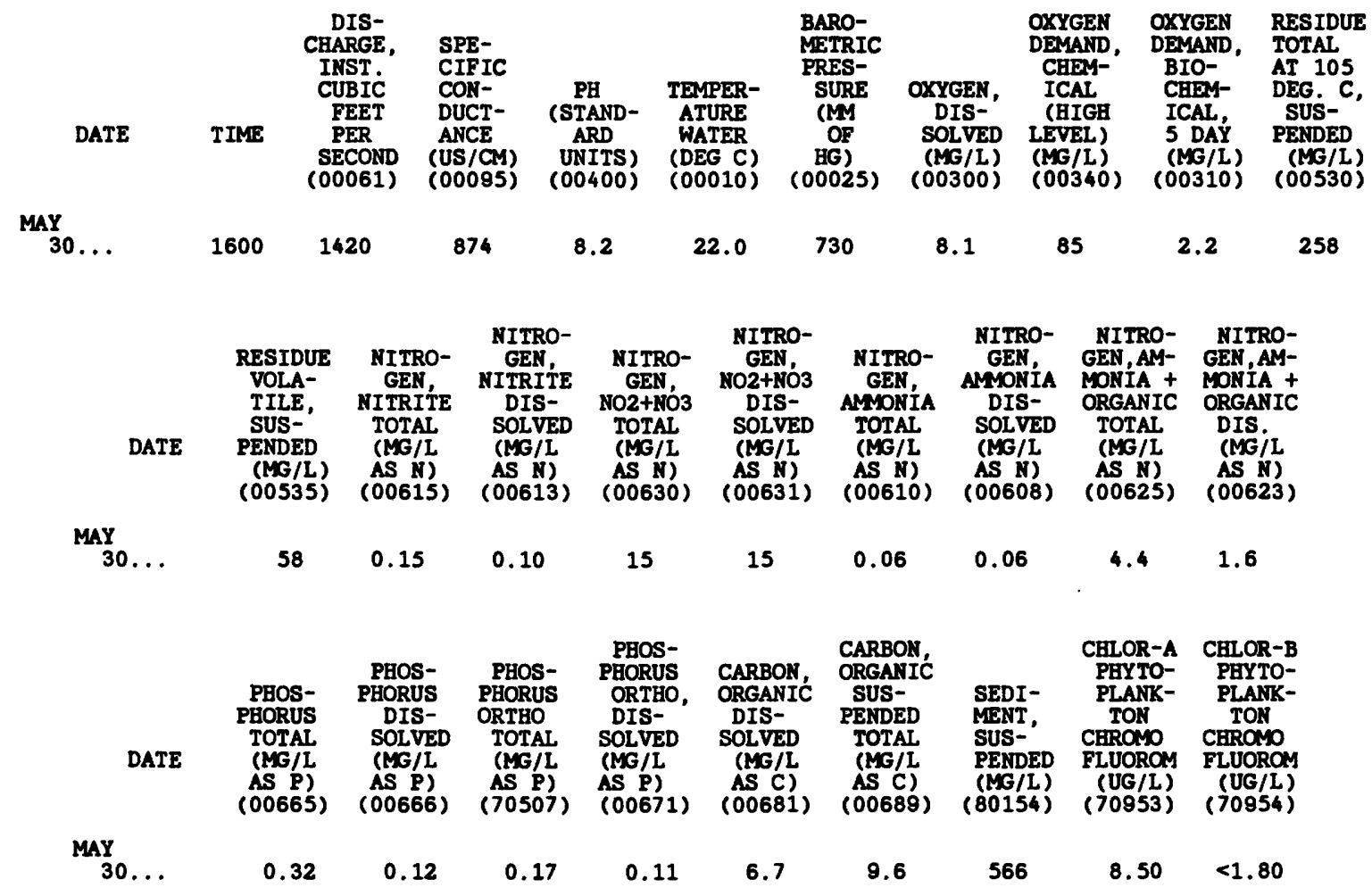


MINNESOTA RIVER BASIN

05317200 LITTLE COTTONWOOD RIVER NEAR COURTLAND, MN

LOCATION. -Lat $44^{\circ} 14^{\prime} 47^{\prime \prime}$, long $94^{\circ} 20^{\prime} 19^{\prime \prime}$, in SWzNEz sec.17, T.109 N., R.29 W., Blue Earth County, Hydrologic Unit 07020007 , on right bank $30 \mathrm{ft}$ downstream from bridge on State Highway $68,0.7 \mathrm{mi}$ above mouth, $1.5 \mathrm{mi}$ south of Courtland.

DRAINAGE AREA $--230 \mathrm{mi}^{2}$ approximately.

PERIOD OF RECORD.--October 1973 to current year. September 1969 to September 1973, operated as a low-flow station only.

GAGE.--Water-stage recorder. Datum of gage is 788.25 ft above National Geodetic Vertical Datum of 1929.

REMARKS. -Records fair.

EXTREMES FOR CURRENT YEAR. --Peak discharges greater than base discharge of $180 \mathrm{ft} 3 / \mathrm{s}$ and maximum (*):

$\begin{array}{lccc}\text { Date } & \text { Time } & \begin{array}{c}\text { Discharge } \\ \text { (ft } / \mathrm{s} \text { ) }\end{array} & \begin{array}{c}\text { Gage height } \\ \text { (ft) }\end{array} \\ \text { Mar. 25 } & 1500 & 185 & 4.05 \\ \text { Apr. } 14 & 2230 & 475 & 5.60 \\ \text { Apr. } 30 & 1500 & 219 & 4.16 \\ \text { May } 5 & 2200 & 368 & 5.05 \\ \text { May } 22 & 2000 & 195 & 3.97 \\ \text { Jun. 5 } & 0330 & \star 526 & 5.90\end{array}$

Date

Jun. 9

Jun. 14

Jun. 27

Jul. 12

Aug. 8

Sep. 14

Dis charge
(ft f $^{3}$ )
$(\mathrm{a})$
455
321
463
266
451

Gage height
(ft)
$* 5.96$
5.78
5.11
5.89
4.82
5.75

a Increasing negative shift.

Time
1830
0630
1000
1400
1300
0430

451

DISCHARGE, CUBIC FEET PER SECOND, WATER YEAR OCTOBER 1990 TO SEPTEMBER 1991

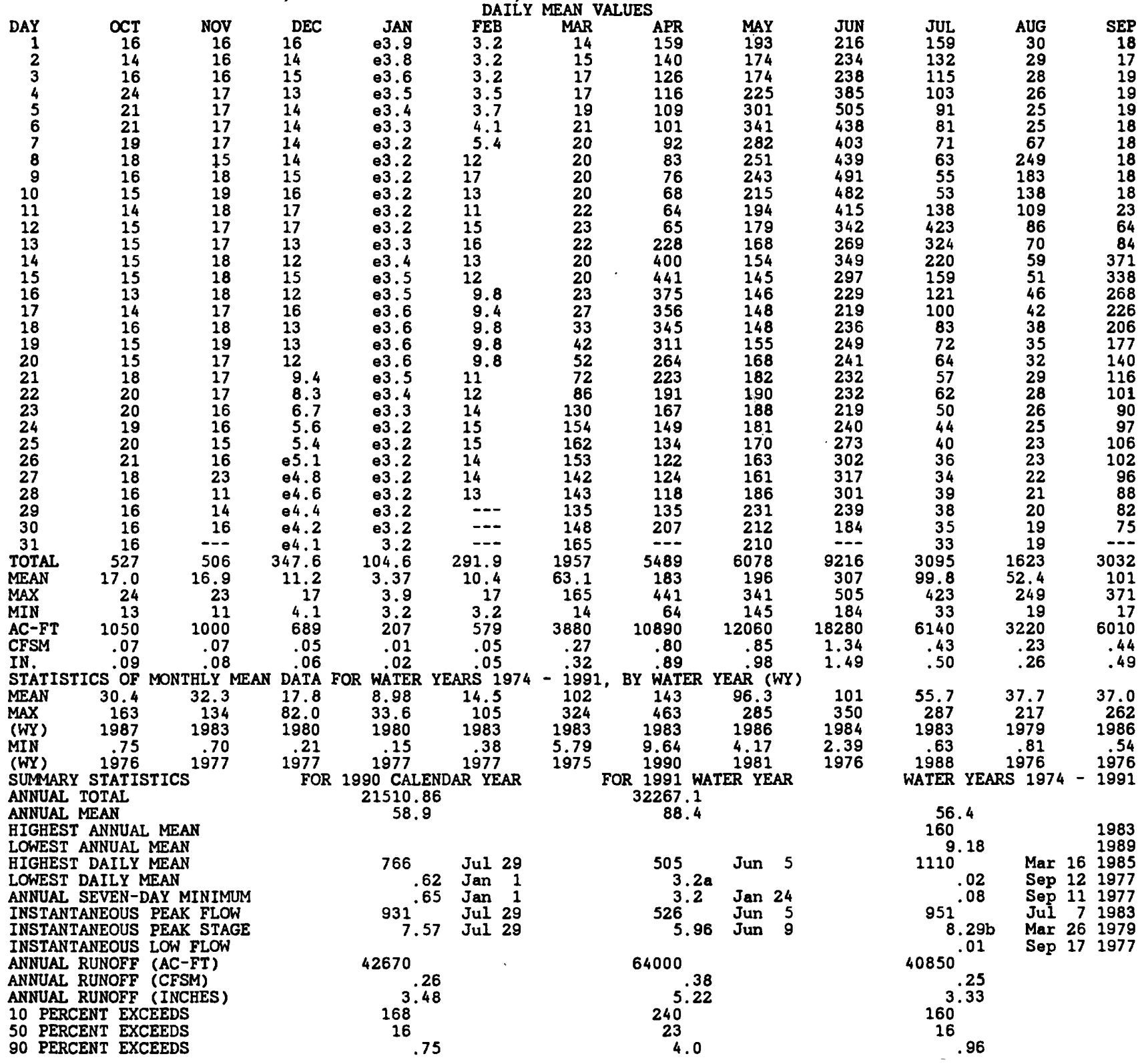

a Jan, 7-12, Jan, 24-Feb. 3.

b Backwater from ice.

e Estimated. 
LOCATION.--Lat $44^{\circ} 02^{\prime} 4^{\prime \prime}$, long $94^{\circ} 11^{\prime} 4^{\prime \prime}$, in SWzNE sec.28, T.107 N., R.28 W., Blue Earth County, Hydrologic Unit 07020010 , on left bank 25 ft downstream from bridge on County Highway 13, 1.5 miles west of Garden City, 7.3 mi upstream from mouth, and $9.2 \mathrm{mi}$ downstream from Perch Creek.

DRAINAGE AREA. - $812 \mathrm{mi} 2$.

PERIOD OF RECORD, --March 1940 to September 1945, September 1976 to current year. 1953, 1960, 1961, and 1969 (one or more discharge measurements each year).

REVISED RECORDS. - -WDR MN-78-2: 1977

GAGE. - Water-stage recorder. Datum of gage is $905.05 \mathrm{ft}$ above National Geodetic Vertical Datum of 1929. Prior to September 30, 1945, nonrecording gage at site $200 \mathrm{ft}$ upstream and at datum $0.17 \mathrm{ft}$ higher.

REMARKS. - Records good except those for the periods of estimated daily discharge, which are fair.

EXTREMES OUTSIDE PERIOD OF RECORD. -F lood of Apr. 7, 1965, reached a stage of $18.89 \mathrm{ft}$ at datum $0.17 \mathrm{ft}$ higher, from floodmarks, discharge, $19,000 \mathrm{ft}^{3} / \mathrm{s}$.

EXTREMES FOR CURRENT YEAR. --Peak discharges greater than base discharge of $900 \mathrm{ft}^{3} / \mathrm{s}$ and maximum ( $*$ ):

\begin{tabular}{|c|c|c|c|c|c|c|c|}
\hline $\begin{array}{ll}\text { Date } \\
\text { Mar. } & 28 \\
\text { Apr. } & 17 \\
\text { May } & 7 \\
\text { May } & 21 \\
\text { May } & 29\end{array}$ & $\begin{array}{l}\text { T1me } \\
0730 \\
1230 \\
1230 \\
0800 \\
0330\end{array}$ & $\begin{array}{l}\text { Discharge } \\
\left(\mathrm{ft}^{3} / \mathrm{s}\right) \\
1,400 \text { cfs } \\
2,970 \\
2,820 \\
2,530 \\
1,650\end{array}$ & $\begin{array}{c}\text { Gage height } \\
\text { (ft) } \\
4.77 \\
7.55 \\
7.31 \\
6.84 \\
5.27\end{array}$ & $\begin{array}{l}\text { Date } \\
\text { Jun. } 7 \\
\text { Jun. } 24 \\
\text { Ju1. } 1 \\
\text { Ju1. } 13 \\
\text { Sep. } 16\end{array}$ & $\begin{array}{l}\text { Time } \\
1000 \\
1830 \\
0130 \\
0830 \\
1130\end{array}$ & $\begin{array}{c}\text { Discharge } \\
\left(\mathrm{ft}^{3} / \mathrm{s}\right) \\
\star 3,410 \\
1,930 \\
2,680 \\
1,290 \\
1,490\end{array}$ & $\begin{array}{c}\text { Gage height } \\
\text { (ft) } \\
\star 8.21 \\
5.81 \\
7.09 \\
4.55 \\
4.96\end{array}$ \\
\hline
\end{tabular}

DISCHARGE, CUBIC FEET PER SECOND, WATER YEAR OCTOBER 1990 TO SEPTEMBER 1991

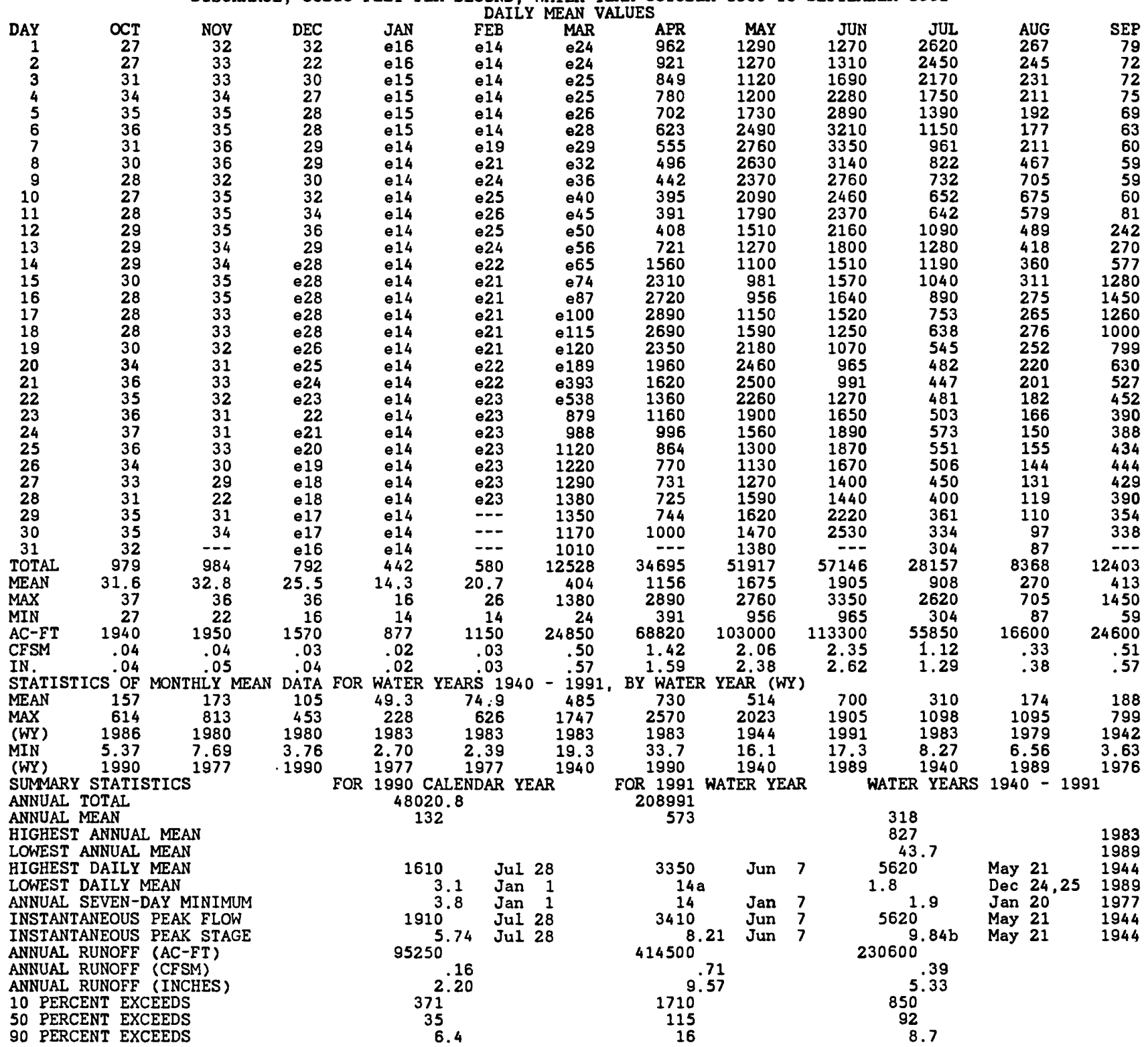

a Jan. 7 - Feb. 6.

b Datum then in use.

e Estimated. 
MIMESOTA RIVER BASIN

05319500 WATONWAN RIVIR REAR GARDEN CITY, M--Continued

WATER QUALITY RECORDS

PERIOD OF RECORD.--Water years 1960-61, 1969, 1977-78, 1980-81, 1989 to current year.

REMARKS. --Letter $\mathrm{K}$ indicates non-ideal colony count.

WATER QUALITY DATA, WATER YEAR OCTOBER 1990 TO SEPTEMBER 1991

\begin{tabular}{|c|c|c|c|c|c|c|c|c|c|c|c|c|}
\hline ATE & TIME & $\begin{array}{c}\text { DIS- } \\
\text { CBARGE, } \\
\text { INST. } \\
\text { CUBIC } \\
\text { FEET } \\
\text { PER } \\
\text { SECOND } \\
\text { (00061) }\end{array}$ & $\begin{array}{l}\text { SPE- } \\
\text { CIFIC } \\
\text { CON- } \\
\text { DUCT- } \\
\text { ANCE } \\
\text { (US/CM) } \\
\text { (00095) }\end{array}$ & $\begin{array}{c}\text { PH } \\
\text { (STAND- } \\
\text { ARD } \\
\text { UNITS) } \\
(00400)\end{array}$ & $\begin{array}{c}\text { TEMPER- } \\
\text { ATURE } \\
\text { WATER } \\
\text { (DEG C) } \\
(00010)\end{array}$ & $\begin{array}{c}\text { BARO- } \\
\text { METRIC } \\
\text { PRES- } \\
\text { SURE } \\
\text { (MM } \\
\text { OF } \\
\text { BG }) \\
(00025)\end{array}$ & $\begin{array}{c}\text { OXYGEN, } \\
\text { DIS- } \\
\text { SOLVED } \\
(M G / L) \\
(00300)\end{array}$ & $\begin{array}{l}\text { OXYGEN } \\
\text { DEMAND, } \\
\text { CGEMY- } \\
\text { ICAL } \\
\text { (HIGH } \\
\text { LEVEL) } \\
\text { (MG/L) } \\
(00340)\end{array}$ & $\begin{array}{c}\text { OXYGEN } \\
\text { DEMAND, } \\
\text { BIO-- } \\
\text { CHEM- } \\
\text { ICAL, } \\
5 \text { DAY } \\
(\mathrm{MG} / \mathrm{L}) \\
(\mathrm{O}) 310)\end{array}$ & $\begin{array}{l}\text { CALCIUM } \\
\text { DIS- } \\
\text { SOLVED } \\
\text { (MG/L } \\
\text { AS CA) } \\
\text { (00915) }\end{array}$ & $\begin{array}{c}\text { MAGNE- } \\
\text { SIUM, } \\
\text { DIS- } \\
\text { SOLVED } \\
\text { (MG/L } \\
\text { AS MG) } \\
\text { (00925) }\end{array}$ & $\begin{array}{c}\text { SODIUM, } \\
\text { DIS- } \\
\text { SOLVED } \\
\text { (MG/L } \\
\text { AS MA) } \\
(00930)\end{array}$ \\
\hline
\end{tabular}

\begin{tabular}{|c|c|c|c|c|c|c|c|c|c|c|c|c|}
\hline \multicolumn{13}{|l|}{ MAR } \\
\hline $\begin{array}{l}16 \ldots \\
19 \ldots \\
20 \ldots \\
22 \ldots \\
23 \ldots \\
26 \ldots \\
27 \ldots \\
28 \ldots\end{array}$ & $\begin{array}{l}1330 \\
1145 \\
1145 \\
1145 \\
1000 \\
0930 \\
0830 \\
0945\end{array}$ & $\begin{array}{r}87 \\
120 \\
182 \\
538 \\
819 \\
1230 \\
1260 \\
1400\end{array}$ & $\begin{array}{l}845 \\
725 \\
694 \\
547 \\
475 \\
612 \\
604 \\
547\end{array}$ & $\begin{array}{l}8.7 \\
7.6 \\
8.3 \\
8.3 \\
8.8 \\
8.1 \\
7.7 \\
8.4\end{array}$ & $\begin{array}{l}0.5 \\
1.0 \\
0.5 \\
1.0 \\
1.0 \\
3.5 \\
4.0 \\
2.0\end{array}$ & $\begin{array}{l}744 \\
738 \\
733 \\
728 \\
723 \\
726 \\
728 \\
732\end{array}$ & $\begin{array}{l}13.0 \\
14.0 \\
13.0 \\
12.9 \\
12.2 \\
12.2 \\
12.4 \\
12.6\end{array}$ & $\begin{array}{l}20 \\
49 \\
29 \\
56 \\
70 \\
53 \\
45 \\
44\end{array}$ & $\begin{array}{l}2.0 \\
4.5 \\
5.1 \\
5.3 \\
7.1 \\
5.1 \\
4.4 \\
3.5\end{array}$ & $\begin{array}{r}100 \\
91 \\
87 \\
69 \\
63 \\
80 \\
80 \\
78\end{array}$ & $\begin{array}{l}35 \\
32 \\
29 \\
22 \\
19 \\
25 \\
25 \\
24\end{array}$ & $\begin{array}{c}32 \\
30 \\
23 \\
16 \\
12 \\
9.6 \\
10 \\
8.9\end{array}$ \\
\hline $\begin{array}{r}\text { AFK } \\
03 \ldots \\
10 \ldots \\
\text { MAY } \ldots\end{array}$ & $\begin{array}{l}1315 \\
1345\end{array}$ & $\begin{array}{l}852 \\
393\end{array}$ & $\begin{array}{l}790 \\
913\end{array}$ & $\begin{array}{l}8.2 \\
8.4\end{array}$ & $\begin{array}{l}9.5 \\
9.0\end{array}$ & $\begin{array}{l}742 \\
748\end{array}$ & $\begin{array}{l}11.4 \\
11.7\end{array}$ & $\begin{array}{l}32 \\
32\end{array}$ & $\begin{array}{l}1.6 \\
2.3\end{array}$ & $=$ & $\overline{-}$ & $=-$ \\
\hline $05 \ldots$ & 1445 & 2950 & 565 & 8.3 & 19.0 & 743 & 7.8 & 37 & 1.7 & -- & -- & -- \\
\hline
\end{tabular}

\begin{tabular}{|c|c|c|c|c|c|c|c|c|c|c|c|c|}
\hline ATE & $\begin{array}{c}\text { POTAS- } \\
\text { SIUM, } \\
\text { DIS- } \\
\text { SOLVED } \\
\text { (MG/L } \\
\text { AS K) } \\
(00935)\end{array}$ & $\begin{array}{c}\text { SULFATE } \\
\text { DIS- } \\
\text { SOLVED } \\
\text { (MG/L } \\
\text { AS SO4) } \\
(00945)\end{array}$ & $\begin{array}{l}\text { CHLO- } \\
\text { RIDE, } \\
\text { DIS- } \\
\text { SOLVED } \\
\text { (MG/L } \\
\text { AS CL) } \\
\text { (00940) }\end{array}$ & $\begin{array}{l}\text { FLUO- } \\
\text { RIDE, } \\
\text { DIS- } \\
\text { SOLVED } \\
(\text { MG/L } \\
\text { AS F }) \\
(00950)\end{array}$ & $\begin{array}{l}\text { RESIDUE } \\
\text { TOTAL } \\
\text { AT 105 } \\
\text { DEG. C, } \\
\text { SUS- } \\
\text { PENDED } \\
(\mathrm{MG} / \mathrm{L}) \\
(00530)\end{array}$ & $\begin{array}{c}\text { RESIDUE } \\
\text { VOLA- } \\
\text { TILE, } \\
\text { SUS- } \\
\text { PENDED } \\
\text { (MG/L) } \\
(00535)\end{array}$ & $\begin{array}{l}\text { BORON, } \\
\text { DIS- } \\
\text { SOLVED } \\
\text { (UG/L } \\
\text { AS B } \\
(01020)\end{array}$ & $\begin{array}{c}\text { MITRO- } \\
\text { GEN, } \\
\text { NITRITE } \\
\text { TOTAL } \\
\text { (MG/L } \\
\text { AS N) } \\
\text { (00615) }\end{array}$ & $\begin{array}{c}\text { NITRO- } \\
\text { GEN, } \\
\text { NITRITE } \\
\text { DIS- } \\
\text { SOLVED } \\
\text { (MG/L } \\
\text { AS N) } \\
\text { (O0613) }\end{array}$ & $\begin{array}{c}\text { NITRO- } \\
\text { GEN, } \\
\text { NO2+NO3 } \\
\text { TOTAL } \\
\text { (MG/L } \\
\text { AS N } \\
(00630)\end{array}$ & $\begin{array}{c}\text { NITRO- } \\
\text { GEN, } \\
\text { NO2+NÓ3 } \\
\text { DIS- } \\
\text { SOLVED } \\
\text { (MG/L } \\
\text { AS N } \\
(00631)\end{array}$ & $\begin{array}{c}\text { NITRO- } \\
\text { GEN, } \\
\text { AMMONIA } \\
\text { TOTAL } \\
\text { (MG/L } \\
\text { AS N) } \\
\text { (00610) }\end{array}$ \\
\hline
\end{tabular}

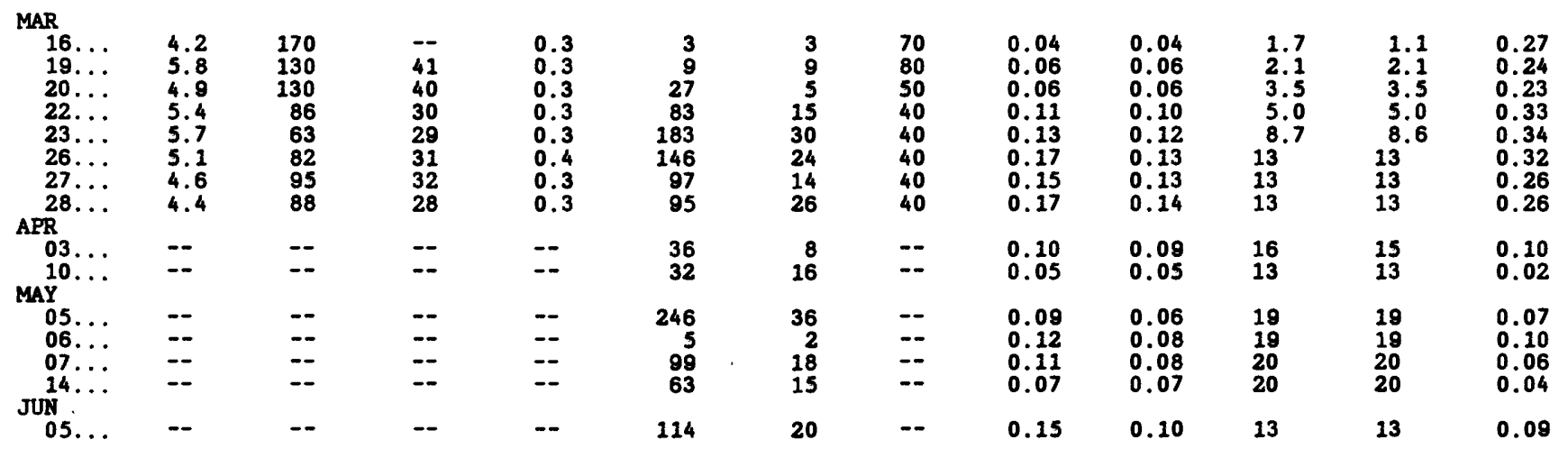


WATER QUALITY DATA, WATER YEAR OCTOBER 1990 TO SEPTDMBER 1991

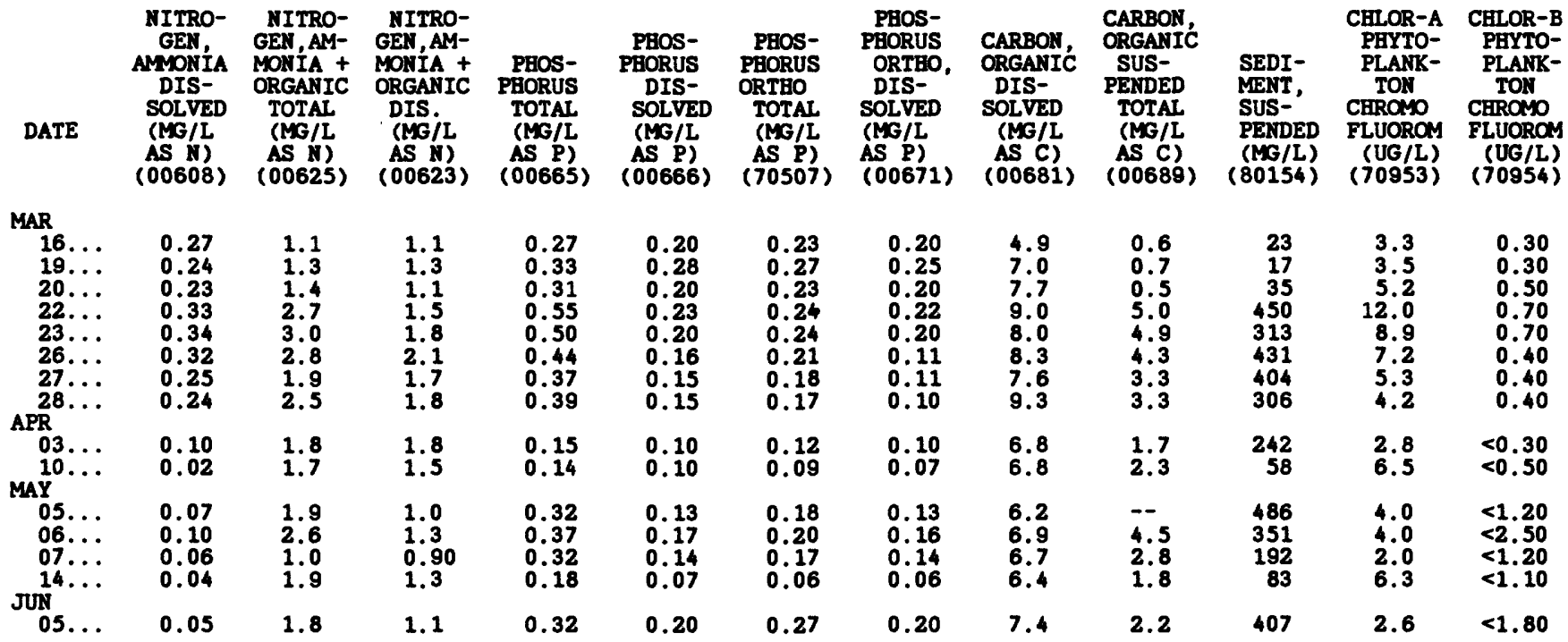


MINNESOTA RIVER BASIN

05320000 BLUE EARTH RIVER NEAR RAPIDAN, MN

LOCATION, --Lat $44^{\circ} 05^{\prime} 44^{\prime \prime}$, long $94^{\circ} 06^{\prime} 33^{\prime \prime}$, in SE $\frac{1}{4}$ SE $^{\prime}$ sec.6, T.107 N., R.27 W., Blue Earth County, Bydrologic Unit 07020009 , on left bank $0.2 \mathrm{mi}$ downstream from powerpiant (reactivated in i984) operated by Rapidan

Redevelopment Limited Partnership, $2 \mathrm{mi}$ west of Rapidan, $3.5 \mathrm{mi}$ downstream from Watonwan River, and $7.8 \mathrm{mi}$ upstream from Le Sueur River.

DRAINAGE AREA. $--2,430 \mathrm{mi}^{2}$ approximately

PERIOD OF RECORD.--July 1909 to November 1910 (published as "at Rapidan Mills," no winter records), October 1939 to September 1945, July 1949 to current year.

REVISED RECORDS. - -WSP 895: Drainage area. WSP 1508: 1910.

GAGE.--Water-stage recorder. Datum of gage is $807.83 \mathrm{ft}$ above National Geodetic Vertical Datum of 1929 . July 20, 1909, to Apr. 28, 19io, nonrecording gage at site $0.2 \mathrm{mi}$ upstream at different datum. Apr. 29 to Nov. 12 , 1910. nonrecording gage at site $800 \mathrm{ft}$ upstream at different datum. Oct. 4 to Nov. 14, 1939, nonrecording gage at present site and datum.

REMARKS.--Records good except those for estimated daily discharges, which are fair.

DISCHARGE, CUBIC FEET PER SECOND, WATER YEAR OCTOBER 1990 TO SEPTEMBER 1991

\begin{tabular}{|c|c|c|c|c|c|c|c|c|c|c|c|c|}
\hline DAY & OT & NOV & DEC & JAN & FEB & MAR & APR & MAY & JUN & JUL & AUG & SEP \\
\hline $\begin{array}{r}1 \\
2 \\
3 \\
4 \\
5 \\
6 \\
7 \\
8 \\
9 \\
10 \\
11 \\
12 \\
13 \\
14 \\
15 \\
16 \\
17 \\
18 \\
19 \\
20 \\
21 \\
22 \\
23 \\
24 \\
25 \\
26 \\
27 \\
28 \\
29 \\
30 \\
31\end{array}$ & $\begin{array}{r}159 \\
156 \\
157 \\
157 \\
161 \\
163 \\
163 \\
157 \\
151 \\
149 \\
125 \\
112 \\
126 \\
142 \\
157 \\
117 \\
146 \\
163 \\
152 \\
107 \\
89 \\
188 \\
292 \\
101 \\
97 \\
102 \\
139 \\
139 \\
138 \\
133 \\
128\end{array}$ & $\begin{array}{r}114 \\
117 \\
144 \\
144 \\
119 \\
101 \\
121 \\
138 \\
138 \\
123 \\
113 \\
116 \\
117 \\
131 \\
134 \\
103 \\
87 \\
96 \\
107 \\
134 \\
137 \\
138 \\
127 \\
84 \\
84 \\
87 \\
113 \\
87 \\
92 \\
92 \\
-9\end{array}$ & $\begin{array}{r}92 \\
92 \\
92 \\
86 \\
84 \\
86 \\
86 \\
86 \\
87 \\
86 \\
87 \\
87 \\
87 \\
87 \\
87 \\
87 \\
125 \\
145 \\
143 \\
102 \\
83 \\
84 \\
83 \\
964 \\
50 \\
58 \\
66 \\
56 \\
70 \\
50 \\
64\end{array}$ & $\begin{array}{l}45 \\
54 \\
54 \\
39 \\
52 \\
42 \\
49 \\
46 \\
47 \\
51 \\
58 \\
44 \\
61 \\
58 \\
48 \\
61 \\
63 \\
55 \\
61 \\
67 \\
66 \\
56 \\
58 \\
61 \\
47 \\
53 \\
52 \\
52 \\
52 \\
53 \\
53\end{array}$ & $\begin{array}{r}53 \\
52 \\
52 \\
52 \\
52 \\
53 \\
52 \\
68 \\
85 \\
84 \\
87 \\
100 \\
102 \\
94 \\
101 \\
84 \\
84 \\
84 \\
84 \\
82 \\
98 \\
98 \\
98 \\
99 \\
98 \\
111 \\
111 \\
113 \\
--- \\
--- \\
---\end{array}$ & $\begin{array}{r}119 \\
126 \\
129 \\
146 \\
161 \\
183 \\
175 \\
207 \\
279 \\
367 \\
433 \\
382 \\
319 \\
289 \\
356 \\
320 \\
320 \\
318 \\
331 \\
364 \\
856 \\
1880 \\
2280 \\
2720 \\
3200 \\
3400 \\
3520 \\
3570 \\
3540 \\
3700 \\
3470\end{array}$ & $\begin{array}{l}3180 \\
3100 \\
2920 \\
2660 \\
2430 \\
2250 \\
2080 \\
1920 \\
1750 \\
1600 \\
1540 \\
1530 \\
1750 \\
2980 \\
4530 \\
5660 \\
6160 \\
5930 \\
5320 \\
4820 \\
4570 \\
4300 \\
3870 \\
3500 \\
3150 \\
2870 \\
2730 \\
2660 \\
2850 \\
3280\end{array}$ & $\begin{array}{l}3710 \\
3720 \\
3530 \\
3510 \\
4720 \\
6840 \\
7730 \\
8070 \\
8580 \\
8750 \\
8130 \\
7270 \\
6480 \\
5670 \\
4960 \\
4510 \\
4490 \\
4970 \\
5830 \\
6200 \\
6410 \\
6260 \\
5830 \\
5270 \\
4860 \\
4490 \\
4680 \\
5600 \\
5930 \\
6140 \\
5890\end{array}$ & $\begin{array}{r}5360 \\
5040 \\
6270 \\
8720 \\
10200 \\
10600 \\
11500 \\
12300 \\
11800 \\
10700 \\
9780 \\
8960 \\
8210 \\
7780 \\
7620 \\
7090 \\
7120 \\
7120 \\
6990 \\
6560 \\
5890 \\
5530 \\
5800 \\
6000 \\
5850 \\
5370 \\
4880 \\
4740 \\
5670 \\
5660\end{array}$ & $\begin{array}{l}5420 \\
4950 \\
4650 \\
4060 \\
3410 \\
3000 \\
2740 \\
2280 \\
2210 \\
2210 \\
2240 \\
2740 \\
3180 \\
3590 \\
3220 \\
2570 \\
2390 \\
2060 \\
1560 \\
1680 \\
1480 \\
2470 \\
3550 \\
3350 \\
2810 \\
2380 \\
2120 \\
2050 \\
1800 \\
1950 \\
2110\end{array}$ & $\begin{array}{r}1850 \\
1670 \\
1400 \\
1370 \\
1220 \\
1020 \\
1130 \\
1670 \\
2460 \\
3170 \\
3440 \\
3240 \\
2750 \\
2170 \\
1970 \\
1670 \\
1560 \\
1460 \\
1600 \\
1480 \\
1340 \\
1080 \\
1050 \\
950 \\
966 \\
874 \\
834 \\
735 \\
672 \\
618 \\
507\end{array}$ & $\begin{array}{r}447 \\
418 \\
417 \\
419 \\
373 \\
348 \\
348 \\
289 \\
290 \\
259 \\
320 \\
473 \\
628 \\
620 \\
2210 \\
2580 \\
2490 \\
1950 \\
1690 \\
1460 \\
1150 \\
946 \\
985 \\
984 \\
818 \\
1020 \\
860 \\
733 \\
722 \\
699 \\
---\end{array}$ \\
\hline $\begin{array}{l}\text { TOTAL } \\
\text { MEAN } \\
\text { MAX } \\
\text { MIN } \\
\text { AC-FT } \\
\text { CFSM } \\
\text { IN. }\end{array}$ & $\begin{array}{r}4466 \\
144 \\
292 \\
89 \\
8860 \\
.06 \\
.07\end{array}$ & $\begin{array}{r}3438 \\
115 \\
144 \\
84 \\
6820 \\
.05 \\
.05\end{array}$ & $\begin{array}{r}2642 \\
85.2 \\
145 \\
50 \\
5240 \\
.04 \\
.04\end{array}$ & $\begin{array}{r}1658 \\
53.5 \\
67 \\
39 \\
3290 \\
.02 \\
.03\end{array}$ & $\begin{array}{r}2331 \\
83.2 \\
113 \\
52 \\
4620 \\
.03 \\
.04\end{array}$ & $\begin{array}{r}37460 \\
1208 \\
3700 \\
119 \\
74300 \\
.50 \\
.57\end{array}$ & $\begin{array}{r}97890 \\
3263 \\
6160 \\
1530 \\
194200 \\
1.34 \\
1.50\end{array}$ & $\begin{array}{r}179030 \\
5775 \\
8750 \\
3510 \\
355100 \\
2.38 \\
2.74\end{array}$ & $\begin{array}{r}225110 \\
7504 \\
12300 \\
4740 \\
446500 \\
3.09 \\
3.45\end{array}$ & $\begin{array}{r}86230 \\
2782 \\
5420 \\
1480 \\
171000 \\
1.14 \\
1.32\end{array}$ & $\begin{array}{r}47926 \\
1546 \\
3440 \\
507 \\
95060 \\
.64 \\
.73\end{array}$ & $\begin{array}{r}26946 \\
898 \\
2580 \\
259 \\
53450 \\
.37 \\
.41\end{array}$ \\
\hline
\end{tabular}

STATISTICS OF MONTHLY MEAN DATA FOR WATER YEARS 1909 - 1991, BY WATER YEAR (WY)

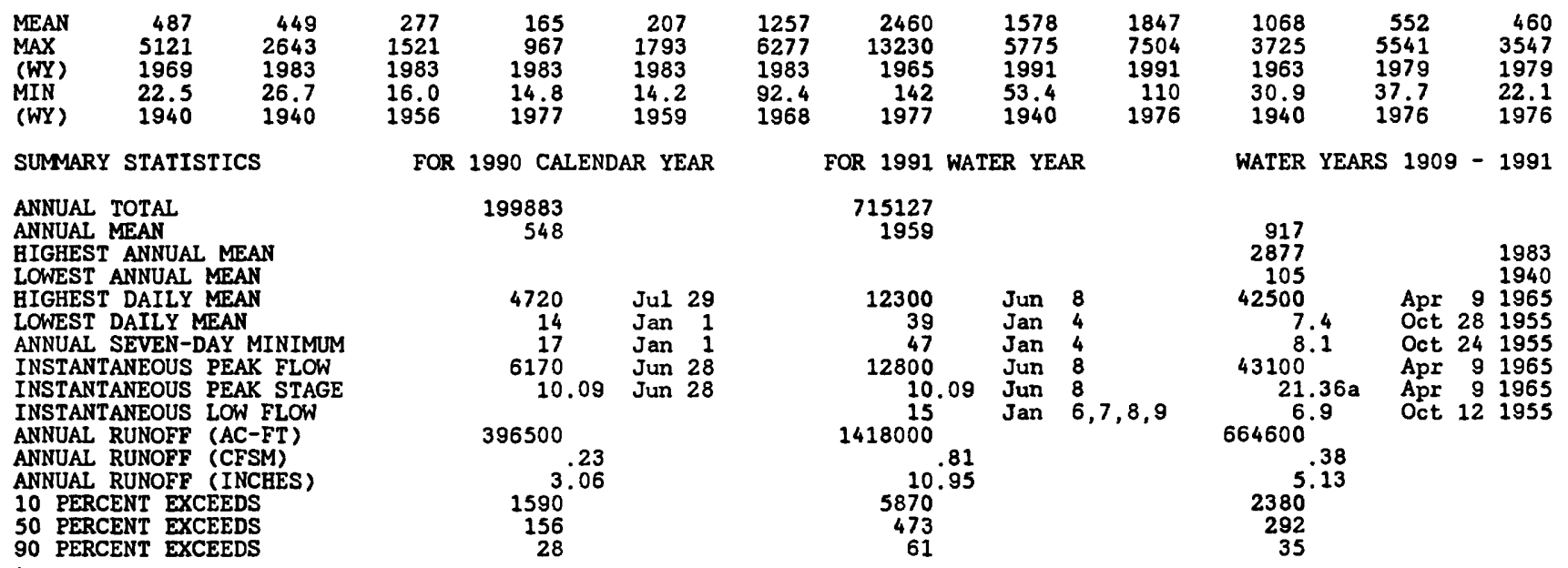

a From floodmark.

e Estimated. 


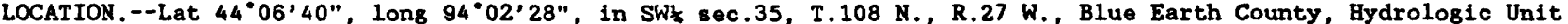
07020011 , on right bank 600 ft downstream from highway bridge, 1.8 mi northeast of Rapidan, and 2.3 mi upstream from mouth.

DRAINAGE AREA. $--1,100 \mathrm{mi}^{2}$, approximately.

PERIOD OF RECORD.--October 1939 to September 1945, July 1949 to current year.

GAGE.--Water-stage recorder. Datum of $\mathrm{gage}$ is $775.76 \mathrm{ft}$ above National Geodetic Vertical Datum of 1929 . Prior to Nov, 15, 1939, nonrecording gage at same site and datum.

REMARKS.--Records good except those for estimated daily discharges, which are falr.

EXTREMES FOR CURRENT YEAR. --Peak discharges greater than base discharge of $1,300 \mathrm{ft} 3 / \mathrm{s}$ and maximum ( $*$ ):

\begin{tabular}{|c|c|c|c|c|c|c|c|}
\hline Date & Time & $\begin{array}{c}\text { Discharge } \\
\left(\mathrm{ft}^{3} / \mathrm{s}\right)\end{array}$ & $\begin{array}{c}\text { Gage height } \\
\text { (ft) }\end{array}$ & Date & Time & $\begin{array}{c}\text { Discharge } \\
\left(\mathrm{ft}^{3} / \mathrm{s}\right)\end{array}$ & $\begin{array}{c}\text { Gage height } \\
\text { (ft) }\end{array}$ \\
\hline $\begin{array}{lr}\text { Mar } & 24 \\
\text { Apr } & 16 \\
\text { May } & 8 \\
\text { May } & 19 \\
\text { May } & 29\end{array}$ & $\begin{array}{l}1700 \\
1300 \\
2330 \\
1600 \\
0100\end{array}$ & $\begin{array}{r}1,870 \\
2,760 \\
\star 6,780 \\
3,390 \\
3,020\end{array}$ & $\begin{array}{r}4.54 \\
5.56 \\
\star 9.10 \\
6.22 \\
5.84\end{array}$ & $\begin{array}{lr}\text { June } & 6 \\
\text { June } & 15 \\
\text { July } & 12 \\
\text { July } & 24 \\
\text { Aug } & 10\end{array}$ & $\begin{array}{l}1730 \\
0700 \\
1630 \\
0330 \\
1430\end{array}$ & $\begin{array}{l}5,900 \\
3,520 \\
1,800 \\
3,250 \\
3,970\end{array}$ & $\begin{array}{l}8.42 \\
6.34 \\
4.45 \\
6.07 \\
6.77\end{array}$ \\
\hline
\end{tabular}

DISCHARGE, CUBIC FEET PER SECOND, WATER YEAR OCTOBER 1990 TO SEPTEMBER 1991

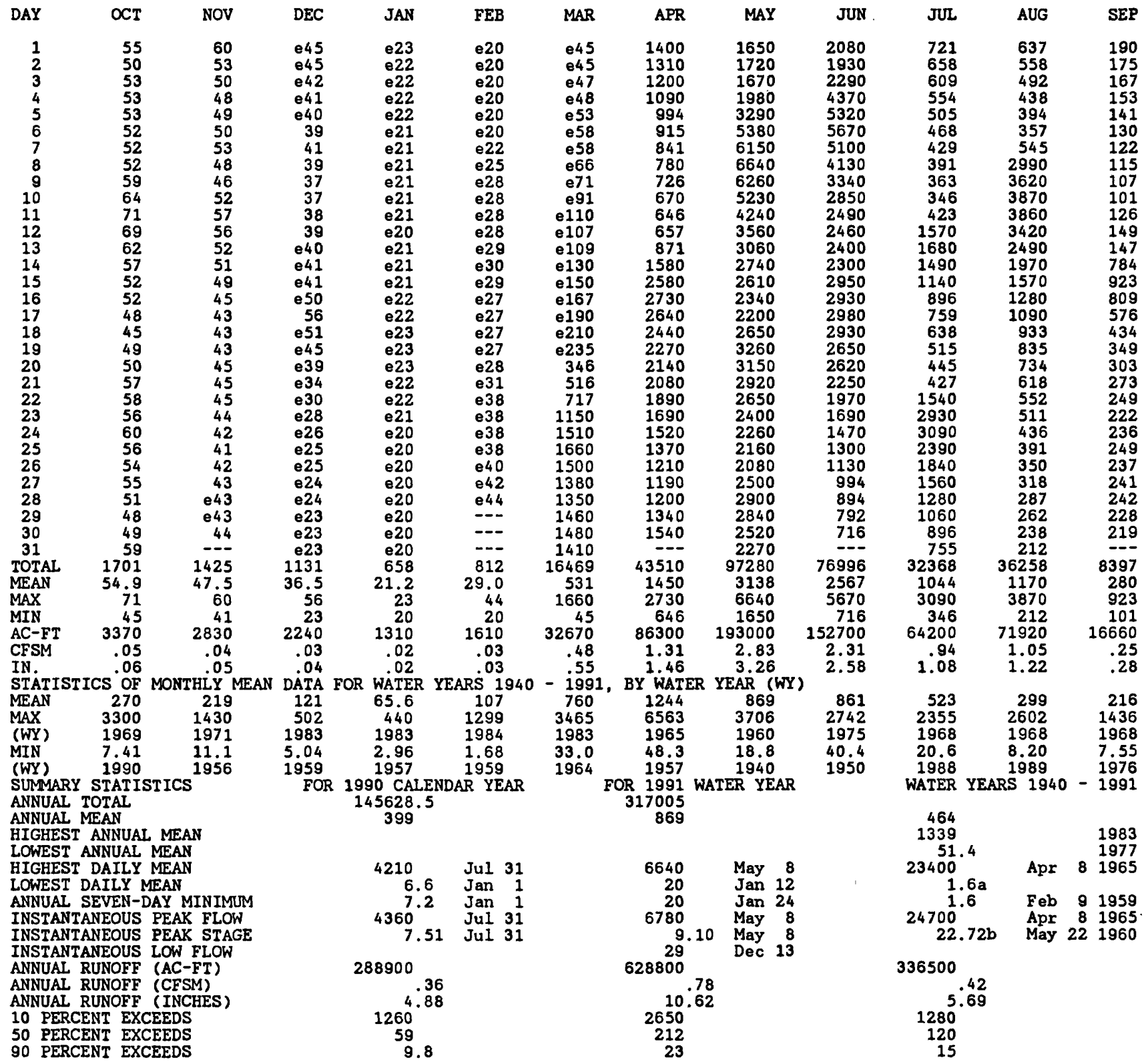

a Occurred Feb. 9-25, 1959

b From floodmark.

- Estimated. 
MINNESOTA RIVER BASIN

05320500 LE SUEUR RIVER MEAR RAPIDAN, MA--Continued

WATER QUALITY RECORDS

PERIOD OF RECORD.--Water years 1967-69, 1989 to current year.

REMARKS. - Letter $K$ indicates non-ideal colony count.

WATER QUALITY DATA, WATER YEAR OCTOBER 1990 TO SEPTEMBER 1991

\begin{tabular}{|c|c|c|c|c|c|c|c|c|c|c|c|c|}
\hline DATE & TIME & $\begin{array}{c}\text { DIS- } \\
\text { CHARGE, } \\
\text { INST } \\
\text { CUBIC } \\
\text { FEET } \\
\text { PER } \\
\text { SECOND } \\
\text { (ODO61) }\end{array}$ & $\begin{array}{l}\text { SPE- } \\
\text { CIFIC } \\
\text { CON- } \\
\text { DUCT- } \\
\text { ANCE } \\
\text { (US/CM) } \\
\text { (OOOSS) }\end{array}$ & $\begin{array}{c}\text { PH } \\
\text { (STAND- } \\
\text { ARD } \\
\text { UNITS) } \\
\text { (00400) }\end{array}$ & $\begin{array}{c}\text { TEMPER- } \\
\text { ATURE } \\
\text { WATER } \\
\text { (DEG C) } \\
(00010)\end{array}$ & $\begin{array}{c}\text { BARO- } \\
\text { METRIC } \\
\text { PRES- } \\
\text { SURE } \\
\text { (MM } \\
\text { OF } \\
\text { BG) } \\
(00025)\end{array}$ & $\begin{array}{c}\text { OXYGEN, } \\
\text { DIS- } \\
\text { SOLVED } \\
\text { (MG/L) } \\
(00300)\end{array}$ & $\begin{array}{l}\text { OXYGEN } \\
\text { DEMAND, } \\
\text { CHEM- } \\
\text { ICAL } \\
\text { (BIGH } \\
\text { LEVEL) } \\
\text { (MG/L) } \\
(00340)\end{array}$ & $\begin{array}{c}\text { OXYGEN } \\
\text { DEMAAND, } \\
\text { BIO- } \\
\text { CHEM- } \\
\text { ICAL, } \\
\text { S DAY } \\
\text { (MG/L) } \\
(00310)\end{array}$ & $\begin{array}{l}\text { CALCIUM } \\
\text { DIS- } \\
\text { SOLVED } \\
\text { (MG/L } \\
\text { AS CA) } \\
\text { (00915) }\end{array}$ & $\begin{array}{c}\text { MAGNE- } \\
\text { SIUM, } \\
\text { DIS- } \\
\text { SOLVED } \\
\text { (MG/L } \\
\text { AS MG) } \\
\text { (00925) }\end{array}$ & $\begin{array}{c}\text { SODIUM, } \\
\text { DIS- } \\
\text { SOLVED } \\
\text { (MG/L } \\
\text { AS MA) } \\
\text { (00930) }\end{array}$ \\
\hline
\end{tabular}

MAR

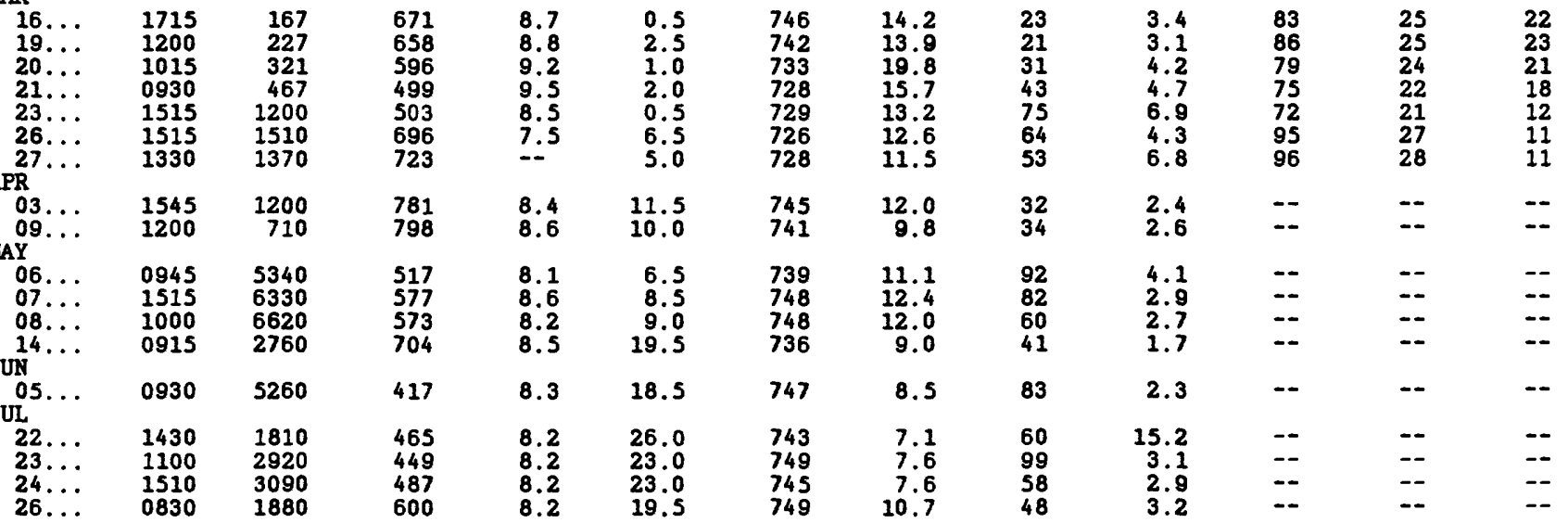

\begin{tabular}{|c|c|c|c|c|c|c|c|c|c|c|c|c|}
\hline ATE & $\begin{array}{l}\text { POTAS- } \\
\text { SIUM, } \\
\text { DIS- } \\
\text { SOLVED } \\
\text { (MG/L } \\
\text { AS K) } \\
(00935)\end{array}$ & $\begin{array}{c}\text { SULFATE } \\
\text { DIS- } \\
\text { SOLVED } \\
\text { (MG/L } \\
\text { AS SO4) } \\
\text { (00945) }\end{array}$ & $\begin{array}{l}\text { CHLO- } \\
\text { RIDE, } \\
\text { DIS- } \\
\text { SOLVED } \\
\text { (MG/L } \\
\text { AS CL) } \\
\text { (00940) }\end{array}$ & $\begin{array}{l}\text { FLUO- } \\
\text { RIDE, } \\
\text { DIS- } \\
\text { SOLVED } \\
\text { (MG/L } \\
\text { AS F) } \\
\text { (00950) }\end{array}$ & $\begin{array}{l}\text { RESIDUE } \\
\text { TOTAL } \\
\text { AT 10S } \\
\text { DEG. C, } \\
\text { SUS- } \\
\text { PENDED } \\
\text { (MG/L) } \\
\text { (OOS30) }\end{array}$ & $\begin{array}{l}\text { RESIDUE } \\
\text { VOLA- } \\
\text { TILE, } \\
\text { SUS- } \\
\text { PENDED } \\
\text { (MG/L) } \\
\text { (00535) }\end{array}$ & $\begin{array}{l}\text { BORON, } \\
\text { DIS- } \\
\text { SOLVED } \\
\text { (UG/L } \\
\text { AS B) } \\
(01020)\end{array}$ & $\begin{array}{l}\text { NITRO- } \\
\text { GEN, } \\
\text { NITRITE } \\
\text { TOTAL } \\
\text { (MG/L } \\
\text { AS N) } \\
\text { (00615) }\end{array}$ & $\begin{array}{c}\text { NITRO- } \\
\text { GEN, } \\
\text { NITRITE } \\
\text { DIS- } \\
\text { SOLVED } \\
\text { (MG/L } \\
\text { AS N) } \\
(00613)\end{array}$ & $\begin{array}{l}\text { NITRO- } \\
\text { GEN, } \\
\text { NO2+NO3 } \\
\text { TOTAL } \\
\text { (MG/L } \\
\text { AS N) } \\
\text { (00630) }\end{array}$ & $\begin{array}{c}\text { NITRO- } \\
\text { GEN, } \\
\text { NO2+NO3 } \\
\text { DIS- } \\
\text { SOLVED } \\
\text { (MG/L } \\
\text { AS N) } \\
(00631)\end{array}$ & $\begin{array}{l}\text { NITRO- } \\
\text { GEN, } \\
\text { AMMONIA } \\
\text { TOTAL } \\
\text { (MG/L } \\
\text { AS N) } \\
\text { (00610) }\end{array}$ \\
\hline
\end{tabular}

MAR

\begin{tabular}{|c|c|c|c|c|c|c|c|c|c|c|c|c|}
\hline $\begin{array}{c}16 \ldots \\
19 \ldots \\
20 \ldots \\
21 \ldots \\
23 \ldots \\
26 \ldots \\
27 \ldots \\
\end{array}$ & $\begin{array}{l}3.9 \\
3.9 \\
3.9 \\
4.1 \\
4.4 \\
4.1 \\
3.7\end{array}$ & $\begin{array}{l}68 \\
69 \\
60 \\
61 \\
47 \\
63 \\
70\end{array}$ & $\begin{array}{l}34 \\
38 \\
36 \\
32 \\
28 \\
32 \\
34\end{array}$ & $\begin{array}{l}0.3 \\
0.3 \\
0.2 \\
0.3 \\
0.3 \\
0.3 \\
0.4\end{array}$ & $\begin{array}{r}15 \\
41 \\
31 \\
110 \\
669 \\
355 \\
221\end{array}$ & $\begin{array}{r}10 \\
10 \\
7 \\
26 \\
70 \\
43 \\
26\end{array}$ & $\begin{array}{l}60 \\
60 \\
50 \\
50 \\
30 \\
40 \\
40\end{array}$ & $\begin{array}{l}0.03 \\
0.02 \\
0.03 \\
0.05 \\
0.07 \\
0.11 \\
0.10\end{array}$ & $\begin{array}{l}0.03 \\
0.02 \\
0.03 \\
0.04 \\
0.06 \\
0.07 \\
0.07\end{array}$ & $\begin{array}{l}5.9 \\
5.4 \\
5.1 \\
5.9 \\
10 \\
19 \\
19\end{array}$ & $\begin{array}{l}5.5 \\
5.4 \\
5.1 \\
5.9 \\
10 \\
19 \\
19\end{array}$ & $\begin{array}{l}0.11 \\
0.09 \\
0.11 \\
0.19 \\
0.26 \\
0.17 \\
0.15\end{array}$ \\
\hline $\begin{array}{l}03 \\
09\end{array}$ & $=$ & -2 & $=$ & -- & $\begin{array}{r}100 \\
77\end{array}$ & $\begin{array}{l}19 \\
15\end{array}$ & $\cdots$ & $\begin{array}{l}0.07 \\
0.05\end{array}$ & $\begin{array}{l}0.05 \\
0.05\end{array}$ & $\begin{array}{l}20 \\
17\end{array}$ & $\begin{array}{l}18 \\
16\end{array}$ & $\begin{array}{l}0.04 \\
0.02\end{array}$ \\
\hline $\begin{array}{l}06 \ldots \\
07 \ldots \\
08 \ldots \\
14 \ldots \\
\text { UN } \ldots\end{array}$ & $\begin{array}{l}-- \\
-- \\
--\end{array}$ & $\begin{array}{l}-- \\
-- \\
-- \\
--\end{array}$ & $\begin{array}{l}-- \\
-- \\
--\end{array}$ & $\begin{array}{l}-- \\
-- \\
--\end{array}$ & $\begin{array}{l}664 \\
602 \\
438 \\
118\end{array}$ & $\begin{array}{r}61 \\
100 \\
68 \\
23\end{array}$ & $\begin{array}{l}-- \\
--\end{array}$ & $\begin{array}{l}0.18 \\
0.20 \\
0.19 \\
0.10\end{array}$ & $\begin{array}{l}0.10 \\
0.10 \\
0.10 \\
0.10\end{array}$ & $\begin{array}{l}19 \\
22 \\
22 \\
21\end{array}$ & $\begin{array}{l}19 \\
22 \\
21 \\
20\end{array}$ & $\begin{array}{l}0.16 \\
0.15 \\
0.12 \\
0.04\end{array}$ \\
\hline $05 .$. & - & -- & - & -- & 500 & 82 & -- & 0.27 & 0.13 & 12 & 12 & 0.21 \\
\hline $\begin{array}{l}22 \ldots \\
23 \ldots \\
24 \ldots \\
26 \ldots\end{array}$ & $\begin{array}{l}-- \\
-- \\
--\end{array}$ & $\begin{array}{l}-- \\
-- \\
--\end{array}$ & $\begin{array}{l}-- \\
-- \\
--\end{array}$ & $\begin{array}{l}-- \\
-- \\
--\end{array}$ & $\begin{array}{r}1120 \\
676 \\
225 \\
182\end{array}$ & $\begin{array}{r}148 \\
108 \\
30 \\
29\end{array}$ & $\begin{array}{l}-- \\
-- \\
--\end{array}$ & $\begin{array}{l}0.03 \\
0.05 \\
0.06 \\
0.04\end{array}$ & $\begin{array}{l}0.03 \\
0.04 \\
0.04 \\
0.03\end{array}$ & $\begin{array}{l}8.9 \\
10 \\
12 \\
13\end{array}$ & $\begin{array}{l}8.8 \\
10 \\
12 \\
13\end{array}$ & $\begin{array}{r}<0.01 \\
0.03 \\
<0.01 \\
<0.01\end{array}$ \\
\hline
\end{tabular}


WATER QUALITY DATA, WATER YEAR OCTOBER 1990 TO SEPTEMBER 1991

\begin{tabular}{|c|c|c|c|c|c|c|c|c|c|c|c|c|}
\hline DATE & $\begin{array}{c}\text { MITRO- } \\
\text { GEN, } \\
\text { AMONIA } \\
\text { DIS- } \\
\text { SOLVED } \\
\text { (MG/L } \\
\text { AS N } \\
\text { (00608) }\end{array}$ & $\begin{array}{l}\text { NITRO- } \\
\text { GEN,AM- } \\
\text { MONIA + } \\
\text { ORGANIC } \\
\text { TOTAL } \\
\text { (MG/L } \\
\text { AS N } \\
(00625)\end{array}$ & $\begin{array}{l}\text { MITRO- } \\
\text { GEM,AM- } \\
\text { MONIA + } \\
\text { ORGANIC } \\
\text { DIS. } \\
\text { (MG/L } \\
\text { AS N } \\
\text { (00623) }\end{array}$ & $\begin{array}{c}\text { PBOS- } \\
\text { PEORUS } \\
\text { TOTAL } \\
\text { (MG/L } \\
\text { AS P) } \\
(00665)\end{array}$ & $\begin{array}{c}\text { PHOS- } \\
\text { PHORUS } \\
\text { DIS- } \\
\text { SOLVED } \\
\text { (MG/L } \\
\text { AS P) } \\
(00666)\end{array}$ & $\begin{array}{c}\text { PHOS- } \\
\text { PHORUS } \\
\text { ORTHO } \\
\text { TOTAL } \\
\text { (MG/L } \\
\text { AS P) } \\
(70507)\end{array}$ & $\begin{array}{c}\text { PHOS- } \\
\text { PBORUS } \\
\text { ORTHO, } \\
\text { DIS- } \\
\text { SOLVED } \\
\text { (MS /L } \\
\text { AS P) } \\
(00671)\end{array}$ & $\begin{array}{l}\text { CARBOH, } \\
\text { ORGANIC } \\
\text { DIS- } \\
\text { SOLVED } \\
(M G / L \\
\text { AS C) } \\
(00681)\end{array}$ & $\begin{array}{l}\text { CARBOH, } \\
\text { ORGANIC } \\
\text { SUS- } \\
\text { PENDED } \\
\text { TOTAL } \\
\text { (MG/L } \\
\text { AS C) } \\
\text { (00689) }\end{array}$ & $\begin{array}{l}\text { SEDI- } \\
\text { MENT, } \\
\text { SUS- } \\
\text { PENDED } \\
(M G / L) \\
(80154)\end{array}$ & $\begin{array}{c}\text { CHLOR-A } \\
\text { PHYTO- } \\
\text { PLANK- } \\
\text { TON } \\
\text { CEROMO } \\
\text { FLUOROM } \\
\text { (UG/L) } \\
\text { (70953) }\end{array}$ & $\begin{array}{c}\text { CHLOR-B } \\
\text { PHYTO- } \\
\text { PLANK- } \\
\text { TON } \\
\text { CEROMO } \\
\text { FLUOROM } \\
\text { (UG/L) } \\
\text { (70954) }\end{array}$ \\
\hline $\begin{array}{l}\text { AR } \\
16 \ldots \\
19 . \ldots \\
20 \ldots \\
21 \ldots \\
23 \ldots \\
26 \ldots \\
27 \ldots\end{array}$ & $\begin{array}{l}0.09 \\
0.09 \\
0.02 \\
0.16 \\
0.26 \\
0.17 \\
0.15\end{array}$ & $\begin{array}{l}1.4 \\
1.5 \\
1.6 \\
2.3 \\
1.9 \\
3.1 \\
2.6\end{array}$ & $\begin{array}{l}1.4 \\
1.5 \\
1.2 \\
1.3 \\
1.5 \\
2.1 \\
1.7\end{array}$ & $\begin{array}{l}0.14 \\
0.19 \\
0.16 \\
0.26 \\
0.46 \\
0.51 \\
0.39\end{array}$ & $\begin{array}{l}0.09 \\
0.10 \\
0.11 \\
0.11 \\
0.19 \\
0.16 \\
0.14\end{array}$ & $\begin{array}{l}0.10 \\
0.10 \\
0.11 \\
0.14 \\
0.20 \\
0.19 \\
0.17\end{array}$ & $\begin{array}{l}0.08 \\
0.10 \\
0.10 \\
0.11 \\
0.18 \\
0.10 \\
0.10\end{array}$ & $\begin{array}{l}5.5 \\
7.0 \\
7.2 \\
7.9 \\
9.5 \\
8.9 \\
8.3\end{array}$ & $\begin{array}{r}1.1 \\
1.1 \\
1.6 \\
1.9 \\
8.1 \\
>5.0 \\
3.5\end{array}$ & $\begin{array}{r}40 \\
140 \\
238 \\
365 \\
843 \\
862 \\
715\end{array}$ & $\begin{array}{r}4.8 \\
5.3 \\
9.7 \\
12.0 \\
16.0 \\
-8 \\
14.0\end{array}$ & $\begin{array}{r}<0.50 \\
0.40 \\
0.60 \\
0.60 \\
0.90 \\
- \\
<1.30\end{array}$ \\
\hline $\begin{array}{c}03 . \ldots \\
09 . \ldots \\
A Y\end{array}$ & $\begin{array}{r}0.04 \\
<0.01\end{array}$ & $\begin{array}{l}1.5 \\
2.0\end{array}$ & $\begin{array}{l}1.5 \\
0.8\end{array}$ & $\begin{array}{l}0.21 \\
0.15\end{array}$ & $\begin{array}{l}0.07 \\
0.06\end{array}$ & $\begin{array}{l}0.12 \\
0.06\end{array}$ & $\begin{array}{l}0.07 \\
0.03\end{array}$ & $\begin{array}{l}7.6 \\
7.8\end{array}$ & $\begin{array}{l}2.4 \\
2.5\end{array}$ & $\begin{array}{l}303 \\
156\end{array}$ & $\begin{array}{r}8.2 \\
13.0\end{array}$ & $\begin{array}{l}0.60 \\
0.70\end{array}$ \\
\hline $\begin{array}{c}06 \ldots \\
07 \ldots \\
08 \ldots \\
14 \ldots\end{array}$ & $\begin{array}{l}0.15 \\
0.11 \\
0.11 \\
0.04\end{array}$ & $\begin{array}{l}2.9 \\
3.3 \\
2.8 \\
2.4\end{array}$ & $\begin{array}{l}1.4 \\
1.2 \\
0.8 \\
1.8\end{array}$ & $\begin{array}{l}0.49 \\
0.58 \\
0.49 \\
0.14\end{array}$ & $\begin{array}{l}0.21 \\
0.20 \\
0.18 \\
0.10\end{array}$ & $\begin{array}{l}0.32 \\
0.31 \\
0.29 \\
0.11\end{array}$ & $\begin{array}{l}0.21 \\
0.20 \\
0.18 \\
0.08\end{array}$ & $\begin{array}{l}10 \\
10 \\
9.2 \\
8.9\end{array}$ & $\begin{array}{r}>10 \\
>10 \\
5.6 \\
2.4\end{array}$ & $\begin{array}{r}1770 \\
1110 \\
1040 \\
468\end{array}$ & $\begin{array}{l}3.0 \\
1.8 \\
3.0 \\
3.6\end{array}$ & $\begin{array}{l}<1.20 \\
<1.10 \\
<1.20 \\
<1.10\end{array}$ \\
\hline $\begin{array}{l}22 \ldots \\
23 \ldots \\
24 \ldots \\
26 \ldots\end{array}$ & $\begin{array}{r}0.03 \\
0.03 \\
<0.01 \\
<0.01\end{array}$ & $\begin{array}{l}4.0 \\
2.4 \\
2.0 \\
2.4\end{array}$ & $\begin{array}{l}1.7 \\
1.4 \\
1.4 \\
1.2\end{array}$ & $\begin{array}{l}0.66 \\
0.57 \\
0.56 \\
0.43\end{array}$ & $\begin{array}{l}0.21 \\
0.26 \\
0.28 \\
0.24\end{array}$ & $\begin{array}{l}0.19 \\
0.25 \\
0.30 \\
0.25\end{array}$ & $\begin{array}{l}0.19 \\
0.25 \\
0.25 \\
0.22\end{array}$ & $\begin{array}{l}7.3 \\
3.7 \\
9.0 \\
9.1\end{array}$ & $\begin{array}{r}>5.0 \\
>5.0 \\
>5.0 \\
4.3\end{array}$ & $\begin{array}{r}2440 \\
1280 \\
712 \\
460\end{array}$ & $\begin{array}{l}3.8 \\
2.8 \\
3.2 \\
3.8\end{array}$ & $\begin{array}{l}<1.40 \\
<1.40 \\
<1.20 \\
<0.80\end{array}$ \\
\hline
\end{tabular}


MINNESOTA RIVER BASIN

05325000 MINNESOTA RIVER AT MANKATO, MN

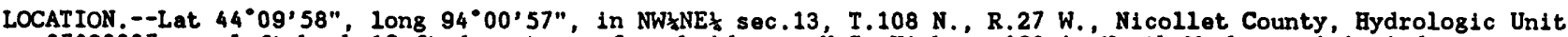
07020007, on left bank $12 \mathrm{ft}$ downstream from bridge on U.S. Highway 169 in North Mankato, $1.1 \mathrm{mi}$ downstream

from Blue Earth River and at mile 107.1 upstream from Mississippi River.

DRAINAGE AREA. $--14,900 \mathrm{mi} 2$, approximately.

WATER-DISCHARGE RECORDS

PERIOD OF RECORD.--May 1903 to current year (no winter records 1904, 1906-10, 1918-29). Monthly discharge only for some periods, published in WSP 1308. Published as "near Mankato": 1903-21.

REVISED RECORDS.--WSP 875: 1917. WSP 955: Drainage area. WSP 1085: 1929. WSP 1238: 1903, 1908, 1919. WSP 1508: $1916(M), 1918(M), 1926(M), 1928,1930,1932(M), 1938(M)$. WDR-MN-76-1: $1881(M)$.

GAGE. - Water-stage recorder. Datum of gage is $747.92 \mathrm{ft}$ above National Geodetic Vertical Datum of 1929. Prior to Oct. 19, 1921, nonrecording gage, at site $1.1 \mathrm{mi}$ upstream at datum $6.4 \mathrm{ft}$ higher. Mar. 15, 1922, to Nov. 30 , 1924, nonrecording 8age, and Dec. 1, 1924 to May 24, 1971, recorder at site $0.5 \mathrm{mi}$ downstream at present datum. May 25, 1971 to Aug. 14, 1977, recorder at site 0.2 mi downstream at present datum. Aug. 14, 1977 to July 27, 1978 , nonrecording gage at present site and datum.

REMARKS, --Records good except those for estimated daily discharges, which are fair.

EXTREMES OUTSIDE PERIOD OF RECORD.--Maximum stage known, $29.9 \mathrm{ft}$, Apr, 26, 1881, present site and datum, from

floodmark (discharge, $110,000 \mathrm{ft}^{3} / \mathrm{s}$ ).

DISCHARGE, CUBIC FEET PER SECOND, WATER YEAR OCTOBER 1990 TO SEPTEMBER 1991

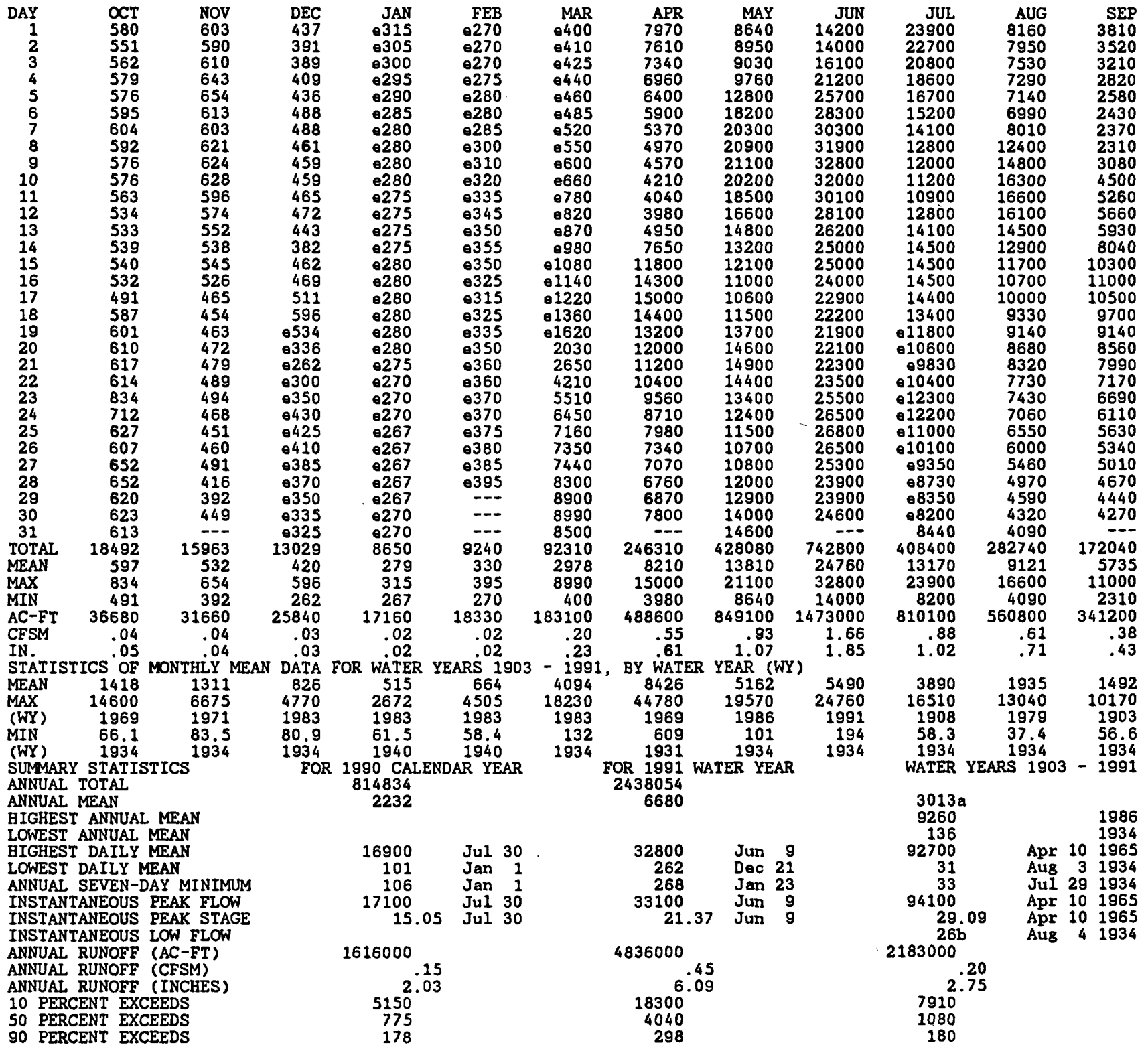

a Median of annual mean discharges is $2,550 \mathrm{ft}^{3} / \mathrm{s}$.

b Minimum observed.

- Estimated. 
PERIOD OF RECORD. --Water years 1963-66, 1968 to current year.

PERIOD OF DAILY RECORD.--

WATER TEMPERATURES: October 1967 to September 30, 1981, October 1982 to current year (fragmentary records). SUSPENDED-SEDIMENT DISCHARGE: October 1967 to current year.

REMARKS.--Sediment samples were collected approximately daily by an observer during the open-water period. In general, daily concentrations and loads for the open-water period are considered good. During the winter period, daily sediment concentrations and loads are based primarily on concentrations of sediment in samples that were collected monthly and on daily water-discharge records. Sediment records for the winter period are considered fair. Water temperatures were obtained by the observer approximately daily during the open-water period and monthly by U.S. Geological Survey personnel during the winter period. Some temperatures are not published because of questionable values.

EXTREMES FOR PERIOD OF DAILY RECORD.--

WATER TEMPERATURES: Maximum daily, $31.0^{\circ} \mathrm{C}$, July $4-9,1989$; minimum daily, $0.0^{\circ} \mathrm{C}$ on many days each year.

SEDIMENT CONCENTRATIONS: Maximum daily mean, 2,850 mg/L, Aug. 7, 1968; minimum dally mean, $9 \mathrm{mg} / \mathrm{L}$,

Jan. $15-19,1991$.

SEDIMENT LOADS: Maximum daily, 247,000 tons, Apr. 9, 1969; minimum daily, 5.2 tons, Nov. 6, 1976.

EXTREMES FOR CURRENT YEAR --

WATER TEMPERATURES: Maximum daily observed, $29.0^{\circ} \mathrm{C}$, July 21 ; minimum daily observed, $1.0^{\circ} \mathrm{C}$, Dec. $^{2}, 3,4$.

SEDIMENT CONCENTRATIONS: Maximum daily mean, $1,200 \mathrm{mg} / \mathrm{L}$, May 6 ; minimum daily mean, $9 \mathrm{mg} / \mathrm{L}, \mathrm{Jan}$. 15-19.

SEDIMENT LOADS: Maximum daily, 59,600 tons, May 6; minimum daily, 6.8 tons, Jan, 15-19.

WATER TEMPERATURE, DEGREES CELSIUS, WATER YEAR OCTOBER 1990 TO SEPTEMBER 1991 ONCE-DAILY

\begin{tabular}{|c|c|c|c|c|c|c|c|c|c|c|c|c|}
\hline DAY & $\propto T$ & nov & DEC & JAN & FEB & MAR & APR & MAY & JUN & JUL & AUG & SEP \\
\hline $\begin{array}{l}1 \\
2 \\
3 \\
4 \\
5\end{array}$ & $\begin{array}{l}18.0 \\
17.5 \\
17.0 \\
16.5 \\
18.0\end{array}$ & $\begin{array}{r}13.0 \\
10.0 \\
9.0 \\
7.0 \\
5.0\end{array}$ & $\begin{array}{l}1.0 \\
1.0 \\
1.0 \\
1.5\end{array}$ & $\begin{array}{l}--- \\
--- \\
--- \\
--- \\
--\end{array}$ & $\begin{array}{l}--- \\
--- \\
--- \\
--- \\
---\end{array}$ & \begin{tabular}{l}
2.0 \\
\hdashline 1.5 \\
1.5 \\
---
\end{tabular} & $\begin{array}{r}--- \\
10.0 \\
10.3 \\
-\overline{10.5}\end{array}$ & $\begin{array}{r}10.5 \\
10.0 \\
-.- \\
-.\end{array}$ & $\begin{array}{l}22.0 \\
23.0 \\
23.0 \\
20.5 \\
20.0\end{array}$ & $\begin{array}{l}25.5 \\
26.0 \\
23.0 \\
23.5 \\
24.5\end{array}$ & $\begin{array}{l}24.0 \\
24.0 \\
24.0 \\
23.0 \\
23.5\end{array}$ & $\begin{array}{r}25.0 \\
24.5 \\
23.5 \\
24.0 \\
--.\end{array}$ \\
\hline $\begin{array}{r}6 \\
7 \\
8 \\
9 \\
10\end{array}$ & $\begin{array}{l}14.5 \\
13.5 \\
13.0 \\
12.5 \\
13.0\end{array}$ & $\begin{array}{l}--- \\
--. \\
4.0 \\
3.0 \\
4.0\end{array}$ & $\begin{array}{l}1.5 \\
2.0 \\
2.5 \\
2.0 \\
1.5\end{array}$ & $\begin{array}{l}--- \\
--- \\
=-- \\
--- \\
---\end{array}$ & $\begin{array}{l}2.0 \\
--0 \\
2.0 \\
1.5 \\
--.\end{array}$ & $\begin{array}{l}2.0 \\
1.5 \\
2.0 \\
2.0 \\
1.5\end{array}$ & $\begin{array}{l}11.0 \\
10.5 \\
10.0 \\
10.0 \\
10.0\end{array}$ & $\begin{array}{r}7.5 \\
7.0 \\
9.5 \\
12.0 \\
16.0\end{array}$ & $\begin{array}{l}20.5 \\
19.5 \\
21.0 \\
22.0 \\
23.0\end{array}$ & $\begin{array}{l}24.0 \\
24.0 \\
24.0 \\
24.0 \\
24.5\end{array}$ & $\begin{array}{r}21.5 \\
19.5 \\
19.5 \\
20.0\end{array}$ & $\begin{array}{r}24.0 \\
--. \\
23.0 \\
23.0 \\
20.5\end{array}$ \\
\hline $\begin{array}{l}11 \\
12 \\
13 \\
14 \\
15\end{array}$ & $\begin{array}{l}13.5 \\
13.5 \\
13.0 \\
13.0 \\
12.0\end{array}$ & $\begin{array}{r}4.0 \\
4.5 \\
7.0 \\
10.5 \\
10.0\end{array}$ & $\begin{array}{l}2.0 \\
2.0 \\
--- \\
---\end{array}$ & $\begin{array}{l}--- \\
--- \\
--- \\
--- \\
---\end{array}$ & $\begin{array}{l}--- \\
--- \\
--- \\
--- \\
---\end{array}$ & $\begin{array}{l}2.0 \\
2.0 \\
1.5 \\
2.0 \\
2.0\end{array}$ & $\begin{array}{l}9.0 \\
-8.0 \\
7.0 \\
7.5\end{array}$ & $\begin{array}{l}12.0 \\
19.0 \\
20.0 \\
-- \\
20.0\end{array}$ & $\begin{array}{r}23.5 \\
-.- \\
24.0 \\
24.5 \\
22.5\end{array}$ & $\begin{array}{r}--- \\
24.0 \\
24.0 \\
24.0 \\
24.0\end{array}$ & $\begin{array}{r}22.0 \\
23.0 \\
24.0 \\
-- \\
24.0\end{array}$ & $\begin{array}{r}20.0 \\
20.0 \\
20.5 \\
-- \\
20.0\end{array}$ \\
\hline $\begin{array}{l}16 \\
17 \\
18 \\
19 \\
20\end{array}$ & $\begin{array}{r}--- \\
11.5 \\
10.0 \\
10.0 \\
10.5\end{array}$ & $\begin{array}{l}--- \\
\overline{7 .-} \\
7.0 \\
9.0\end{array}$ & $\begin{array}{l}--- \\
--- \\
--- \\
--- \\
--\end{array}$ & $\begin{array}{l}--- \\
--- \\
--- \\
--- \\
---\end{array}$ & $\begin{array}{l}--- \\
--- \\
--- \\
--- \\
---\end{array}$ & $\begin{array}{l}2.0 \\
2.0 \\
2.0 \\
3.0 \\
-.-\end{array}$ & $\begin{array}{l}8.0 \\
9.5 \\
9.0 \\
9.0 \\
9.0\end{array}$ & $\begin{array}{l}20.0 \\
14.0 \\
13.0 \\
14.0 \\
15.0\end{array}$ & $\begin{array}{l}23.0 \\
23.0 \\
24.0 \\
23.5 \\
23.0\end{array}$ & $\begin{array}{r}26.5 \\
26.5 \\
28.0 \\
--- \\
27.5\end{array}$ & $\begin{array}{r}--. \\
21.0 \\
23.0 \\
23.5 \\
22.0\end{array}$ & $\begin{array}{l}20.0 \\
17.5 \\
14.0 \\
12.5 \\
13.0\end{array}$ \\
\hline $\begin{array}{l}21 \\
22 \\
23 \\
24 \\
25\end{array}$ & $\begin{array}{l}10.5 \\
11.0 \\
10.0 \\
10.0 \\
10.0\end{array}$ & $\begin{array}{l}9.0 \\
5.0 \\
5.0 \\
5.0 \\
3.0\end{array}$ & $\begin{array}{l}--- \\
--- \\
--- \\
--- \\
--\end{array}$ & $\begin{array}{l}--- \\
--- \\
--- \\
--- \\
--\end{array}$ & $\begin{array}{l}2.0 \\
--- \\
-- \\
1.5\end{array}$ & $\begin{array}{l}--- \\
3.0 \\
2.0 \\
2.0 \\
2.5\end{array}$ & $\begin{array}{r}9.5 \\
10.0 \\
23.0 \\
12.0 \\
12.5\end{array}$ & $\begin{array}{l}17.0 \\
19.0 \\
20.5 \\
19.0 \\
18.5\end{array}$ & $\begin{array}{r}20.5 \\
-.-5 \\
22.0 \\
23.0 \\
23.5\end{array}$ & $\begin{array}{l}29.0 \\
26.5 \\
24.5 \\
24.0 \\
23.5\end{array}$ & $\begin{array}{l}23.5 \\
24.5 \\
24.0 \\
24.5 \\
24.5\end{array}$ & $\begin{array}{l}13.5 \\
13.0 \\
12.5 \\
12.0 \\
12.0\end{array}$ \\
\hline $\begin{array}{l}26 \\
27 \\
28 \\
29 \\
30 \\
31\end{array}$ & $\begin{array}{l}10.0 \\
10.0 \\
10.0 \\
10.5 \\
10.5 \\
12.0\end{array}$ & $\begin{array}{l}3.0 \\
--- \\
2.0 \\
2.0 \\
--\end{array}$ & $\begin{array}{l}--- \\
--- \\
--- \\
--- \\
---\end{array}$ & $\begin{array}{l}--- \\
--- \\
--- \\
--- \\
---\end{array}$ & $\begin{array}{l}--- \\
2.0 \\
--- \\
--- \\
---\end{array}$ & $\begin{array}{l}3.0 \\
3.0 \\
--- \\
--- \\
---\end{array}$ & $\begin{array}{r}--- \\
15.0 \\
15.0 \\
12.0 \\
-.0\end{array}$ & $\begin{array}{r}--- \\
21.5 \\
22.0 \\
22.0 \\
21.0\end{array}$ & $\begin{array}{r}25.0 \\
26.0 \\
26.5 \\
25.5 \\
27.0 \\
---\end{array}$ & $\begin{array}{r}22.0 \\
21.0 \\
21.5 \\
23.0 \\
23.5\end{array}$ & $\begin{array}{l}26.0 \\
26.5 \\
27.0 \\
28.0 \\
28.0\end{array}$ & $\begin{array}{l}12.5 \\
12.0 \\
13.5 \\
13.5 \\
14.0\end{array}$ \\
\hline $\begin{array}{l}\text { MEAN } \\
\text { MAX }\end{array}$ & --- & --- & --- & --- & --- & --- & --- & --- & --- & --- & $\overline{---}$ & $\overline{---}$ \\
\hline MIN & -- & --- & --- & -- & -- & -- & -- & -- & -- & -- & --- & - \\
\hline
\end{tabular}


MINNESOTA RIVER BASIN

05325000 MINNESOTA RIVER AT MANKATO, MN--Continued SUSPENDED-SEDIMENT, WATER YEAR OCTOBER 1990 TO SEPTEMBER 1991

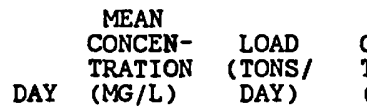

MEAN MEAN

TRATION (TONS/ (MG/L)

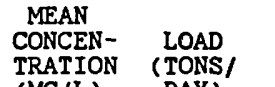
(MG/L) DAY)

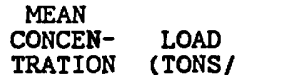
(MG/L) DAY)
MEAN

CONCEN- LOAD

TRATION (TONS/ (MG/L)

DAY
OCTOBER NOVEMBER DECEMBER

$\begin{array}{ll}39 & 61 \\ 45 & 67 \\ 65 & 99 \\ 57 & 89 \\ 45 & 70 \\ & \\ 46 & 74 \\ 47 & 77 \\ 38 & 61 \\ 34 & 53 \\ 35 & 54\end{array}$

$\begin{array}{ll}50 & 81 \\ 42 & 67 \\ 43 & 71 \\ 39 & 68 \\ 33 & 58 \\ & \\ 28 & 46 \\ 28 & 46 \\ 27 & 45 \\ 24 & 40 \\ 45 & 76\end{array}$

$\begin{array}{ll}40 & 61 \\ 49 & 71 \\ 48 & 69 \\ 56 & 81 \\ 56 & 82\end{array}$

59

1

12
14
15
1
17
1
1
20
2
22
2
2
2
2
2
2
2
3

$\begin{array}{ll}52 & 75 \\ 58 & 77 \\ 42 & 67 \\ 33 & 54 \\ 37 & 61\end{array}$

21
22
23
24
25
26
27
28
29
30
31

$\begin{array}{rr}67 & 112 \\ 47 & 78 \\ 77 & 173 \\ 50 & 96 \\ 45 & 76\end{array}$

$45 \quad 76$

TOTAL

10

10

1740

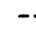

$\begin{array}{ll}30 & 38 \\ 17 & 23 \\ 11 & 18 \\ 10 & 14\end{array}$

APRIL

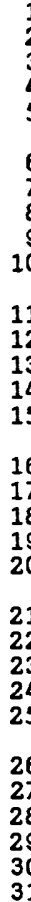

TOTA

YEAR
2398

8840

4890
3280

2690

2350

2280

2220

1630

1460

5540

1610

25600

24700

20500

16600

10800

6020

5190

4650

4140
3730

2870

2840

2740
3020

5710
7850
6620
4890

1200
910 $\quad 496000$

$---670200$

JANUARY

FEBRUARY

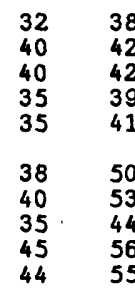

$\begin{array}{ll}36 & 45 \\ 45 & 57 \\ 50 & 60 \\ 50 & 52 \\ 45 & 56\end{array}$

$10 \quad 14$

$10 \quad 7$.

10

10

12

$\begin{array}{ll}10 & 8.5 \\ 10 & 8.2 \\ 10 & 8.1 \\ 10 & 8.0 \\ 10 & 7.8 \\ 10 & 7.7 \\ 10 & 7.6 \\ 10 & 7.6 \\ 10 & 7.6 \\ 10 & 7.6\end{array}$

8.5
8.2

8.1

8.0
7.8

7.7

7.6

7.6

7.4
7.4

7.4

7.4
6.8

6.8

6.8
6.8

6.8

9
20

15

.1

$38 \quad 28$

$41 \quad 30$

$\begin{array}{ll}227 & 5300 \\ 152 & 3670 \\ 120 & 2930\end{array}$

$\begin{array}{lr}186 & 4900 \\ 460 & 16600\end{array}$

910
636
55900

636
581
53100

$501 \quad 27300$

$\begin{array}{ll}427 & 21300 \\ 364 & 16300\end{array}$

$\begin{array}{ll}364 & 16300 \\ 334 & 13300\end{array}$

$330 \quad 13300$

$327 \quad 10700$

$\begin{array}{rr}232 & 6890 \\ 198 & 5670\end{array}$

450
430
244300

$\begin{array}{rr}430 & 15900 \\ 244 & 9620\end{array}$

1917680

1807000

225

6870

7530
5960

$180 \quad 5200$

$\begin{array}{lr}201 & 5860 \\ 360 & 11700\end{array}$

11700

12600

16900

16900 $\begin{array}{ll}10 & 11 \\ 10 & 10\end{array}$

11
10
10
9.4
9.
8.

$--\quad 937.9$
JUNE

$\begin{array}{ll}387 & 14800 \\ 353 & 13300 \\ 907 & 40200\end{array}$

926

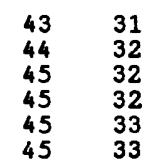
-- 479.3
JULY

$\begin{array}{ll}144 & 9290 \\ 154 & 9440 \\ 170 & 9550 \\ 165 & 8290 \\ 173 & 7800\end{array}$

AUGUST

$167 \quad 3680$

$164 \quad 3520$

$\begin{array}{ll}158 & 3210 \\ 155 & 3050\end{array}$

\begin{tabular}{l}
155 \\
159 \\
\hline
\end{tabular}

$538 \quad 41100$

506

438

389
358

41400

37700

189
194

100
195

7760

7390

7390
6910
6320

186
280
659
451
274

3510

6050

22100
18000

12100

333
312

312
289

27100

23700

20400

13700

$226 \quad 6650$

430
311

253

15000

11800

332

14900

11600

8060
7310

5970

8300

189

14800

167

$\begin{array}{ll}228 & 14800 \\ 220 & 13600\end{array}$

$251 \quad 14800$

167
154
145

145
144

6540

5990

4590

$\begin{array}{ll}251 & 14800 \\ 587 & 35000\end{array}$

144
154

4410

18

5210
4760

4520

3890
3570

153

$158 \quad 4190$

$230 \quad 13800$

$208 \quad 14300$

$187 \quad 13400$

25300

18700
10700

1723860

$177 \quad 3690$

$155-3110$

$\begin{array}{ll}150 & 2860 \\ 155 & 2740\end{array}$

$\begin{array}{ll}260 & 7720 \\ 169 & 4790\end{array}$

1423720

$151 \quad 3730$

$180 \quad 4160$

\section{$154 \quad 11000$}

$\begin{array}{ll}154 & 11000 \\ 148 & 10100\end{array}$

$\begin{array}{ll}148 & 10100 \\ 189 & 12200\end{array}$

$205 \quad 13200$

$158 \quad 10500$

228
186

160
158

158
205
183

\section{0}

4700

174

174
172

2820

2540

2540
1840
1570

3560
4540

127
147
12

1710
158
152 3410

1482670

$\begin{array}{ll}145 & 2390 \\ 122 & 1850\end{array}$ $\begin{array}{ll}128 & 1850 \\ 131 & 1770 \\ 125 & 1580 \\ 121 & 1450 \\ 115 & 1330 \\ --- & --\end{array}$

-.- 259550

$--$

176140 
110

MIMNESOTA RIVER BASIK

05325000 MINESSOTA RIVER AT MANKATO, MN--Continued

WATER QUALITY RECORDS

PERIOD OF RECORD.--Water years 1960-67, 1970-79, 1981-87, 1989 to current year.

WATER QUALITY DATA, WATER YEAR OCTOBER 1990 TO SEPTEMBER 1991

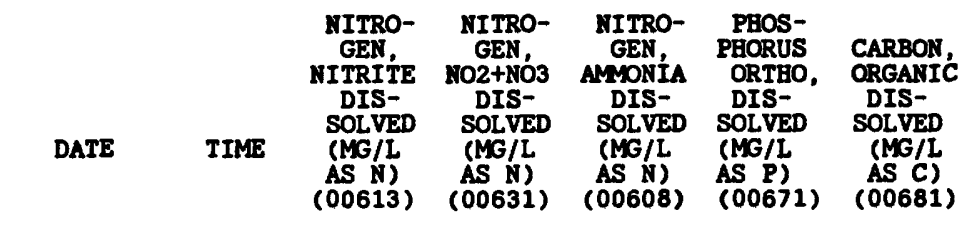

\begin{tabular}{|c|c|c|c|c|c|c|}
\hline \multicolumn{7}{|l|}{ MAY } \\
\hline $\begin{array}{r}15 \ldots \\
17 \ldots \\
19 . \ldots \\
21 . . \\
23 . \ldots \\
25 \ldots \\
28 \ldots \\
30 \ldots \\
\text { Jun }\end{array}$ & $\begin{array}{l}2000 \\
2035 \\
1820 \\
2025 \\
1925 \\
2025 \\
2030 \\
2100\end{array}$ & $\begin{array}{l}0.07 \\
0.06 \\
0.08 \\
0.08 \\
0.08 \\
0.07 \\
0.06 \\
0.08\end{array}$ & $\begin{array}{l}17 \\
16 \\
16 \\
16 \\
17 \\
17 \\
17 \\
18\end{array}$ & $\begin{array}{r}0.11 \\
0.06 \\
0.04 \\
0.06 \\
0.31 \\
<0.01 \\
<0.01 \\
0.03\end{array}$ & $\begin{array}{l}0.05 \\
0.05 \\
0.08 \\
0.05 \\
0.04 \\
0.04 \\
0.07 \\
0.11\end{array}$ & $\begin{array}{r}6.9 \\
7.5 \\
17 \\
34 \\
6.6 \\
6.7 \\
6.8 \\
7.2\end{array}$ \\
\hline $\begin{array}{l}01 \ldots \\
03 . . \\
05 \ldots \\
07 \ldots \\
09 \ldots \\
11 \ldots \\
13 \ldots \\
15 \ldots \\
17 \ldots \\
20 \ldots \\
23 \ldots \\
25 \ldots \\
27 \ldots \\
29 . \ldots\end{array}$ & $\begin{array}{l}2155 \\
1930 \\
2030 \\
2250 \\
2150 \\
2003 \\
2035 \\
2155 \\
2130 \\
1727 \\
1803 \\
2110 \\
1950 \\
2025\end{array}$ & $\begin{array}{l}0.10 \\
0.12 \\
0.13 \\
0.16 \\
0.15 \\
0.15 \\
0.12 \\
0.09 \\
0.08 \\
0.24 \\
0.08 \\
0.15 \\
0.06 \\
0.06\end{array}$ & $\begin{array}{l}17 \\
13 \\
13 \\
13 \\
11 \\
12 \\
11 \\
12 \\
12 \\
12 \\
11 \\
11 \\
10 \\
8.9\end{array}$ & $\begin{array}{l}0.04 \\
0.04 \\
0.08 \\
0.06 \\
0.06 \\
0.04 \\
0.04 \\
0.04 \\
0.05 \\
0.22 \\
0.04 \\
0.17 \\
0.03 \\
0.03\end{array}$ & $\begin{array}{l}0.11 \\
0.15 \\
0.20 \\
0.15 \\
0.14 \\
0.14 \\
0.13 \\
0.14 \\
0.16 \\
0.01 \\
0.12 \\
0.03 \\
0.15 \\
0.15\end{array}$ & $\begin{array}{c}7.6 \\
6.7 \\
7.5 \\
7.4 \\
7.2 \\
7.3 \\
7.2 \\
7.3 \\
13 \\
7.0 \\
16 \\
18 \\
>50 \\
6.7\end{array}$ \\
\hline $\begin{array}{l}\text { JUL } \\
01 . \ldots \\
09 . \ldots \\
24 \ldots \\
30 . \ldots\end{array}$ & $\begin{array}{l}2210 \\
1920 \\
2055 \\
2135\end{array}$ & $\begin{array}{l}0.06 \\
0.03 \\
0.03 \\
0.03\end{array}$ & $\begin{array}{l}8.4 \\
7.1 \\
3.1 \\
7.7\end{array}$ & $\begin{array}{l}0.04 \\
0.03 \\
0.05 \\
0.02\end{array}$ & $\begin{array}{l}0.15 \\
0.12 \\
0.14 \\
0.12\end{array}$ & $\begin{array}{r}7.2 \\
>50^{7} \\
7.2 \\
7.0\end{array}$ \\
\hline $\begin{array}{r}05 \ldots \\
13 \ldots \\
21 \ldots \\
26 \ldots \\
\text { SEP }\end{array}$ & $\begin{array}{l}2145 \\
2120 \\
2140 \\
2050\end{array}$ & $\begin{array}{r}0.03 \\
0.02 \\
<0.01 \\
<0.01\end{array}$ & $\begin{array}{l}6.9 \\
9.9 \\
2.1 \\
1.3\end{array}$ & $\begin{array}{l}<0.01 \\
0.03 \\
0.02 \\
0.02\end{array}$ & $\begin{array}{r}0.10 \\
0.11 \\
0.05 \\
<0.01\end{array}$ & $\begin{array}{r}6.8 \\
>50^{\circ} \\
7.6 \\
7.6\end{array}$ \\
\hline $\begin{array}{l}03 \ldots \\
10 \ldots \\
17 \ldots \\
24 \ldots\end{array}$ & $\begin{array}{l}2030 \\
2030 \\
2145 \\
0851\end{array}$ & $\begin{array}{l}0.01 \\
0.05 \\
0.04 \\
0.02\end{array}$ & $\begin{array}{l}1.3 \\
2.5 \\
9.2 \\
7.1\end{array}$ & $\begin{array}{l}0.02 \\
0.06 \\
0.02 \\
0.02\end{array}$ & $\begin{array}{l}<0.01 \\
0.09 \\
0.16 \\
0.08\end{array}$ & $\begin{array}{r}7.4 \\
8.7 \\
11 \\
7.7\end{array}$ \\
\hline
\end{tabular}


MINNESOTA RIVER BASIN

05327000 GIGH ISLAND CREEX NEAR GENDERSON, MN

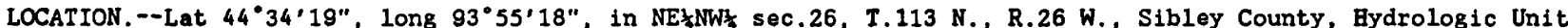
07020012 , on left bank 20 ft downstream from bridge on County Road $6,1.6$ mi upstream from mouth, and 3.1 mi north of Henderson. 2

PERIOD OF RECORD. --October 1973 to current year. May 1970 to September 1973, operated as a low-flow station only. REVISED RECORDS - -WDR-MN-80-2: 1974-75, $1977-79$

GAGE. - Water-stage recorder. Datum of gage is $728.56 \mathrm{ft}$ above National Geodetic Vertical Datum of 1929.

REMARKS. --Records good except those for estimated daily discharges, which are fair.

EXTREMES FOR CURRENT YEAR. --Peak discharges greater than base discharge of $300 \mathrm{ft} / \mathrm{s}$ and maximum ( $(*)$

\begin{tabular}{|c|c|c|c|c|c|c|c|}
\hline Date & Time & $\begin{array}{c}\text { Discharge } \\
\left(\mathrm{ft}^{3} / \mathrm{s}\right)\end{array}$ & $\begin{array}{c}\text { Gage height } \\
\text { (ft) }\end{array}$ & Date & Time & $\begin{array}{c}\text { Discharge } \\
\left(\mathrm{ft}^{3} / \mathrm{s}\right)\end{array}$ & $\begin{array}{c}\text { Gage height } \\
\text { (ft) }\end{array}$ \\
\hline $\begin{array}{lr}\text { Mar. } & 23 \\
\text { Apr. } & 20 \\
\text { May } & 5\end{array}$ & $\begin{array}{l}1230 \\
1430 \\
2000\end{array}$ & $\begin{array}{l}322 \\
636 \\
710\end{array}$ & $\begin{array}{l}2.99 \\
3.43 \\
4.90\end{array}$ & $\begin{array}{lr}\text { May } & 29 \\
\text { May } & 31 \\
\text { June } & 1 \\
\text { Sep. } & 14\end{array}$ & $\begin{array}{l}0100 \\
1100 \\
2230 \\
1430\end{array}$ & $\begin{array}{r}539 \\
1,030 \\
586 \\
\star 1,300\end{array}$ & $\begin{array}{r}4.10 \\
6.24 \\
4.50 \\
* 7.31\end{array}$ \\
\hline
\end{tabular}

DISCHARGE, CUBIC FEET PER SECOND, WATER YEAR OCTOBER 1990 TO SEPTEMBER 1991

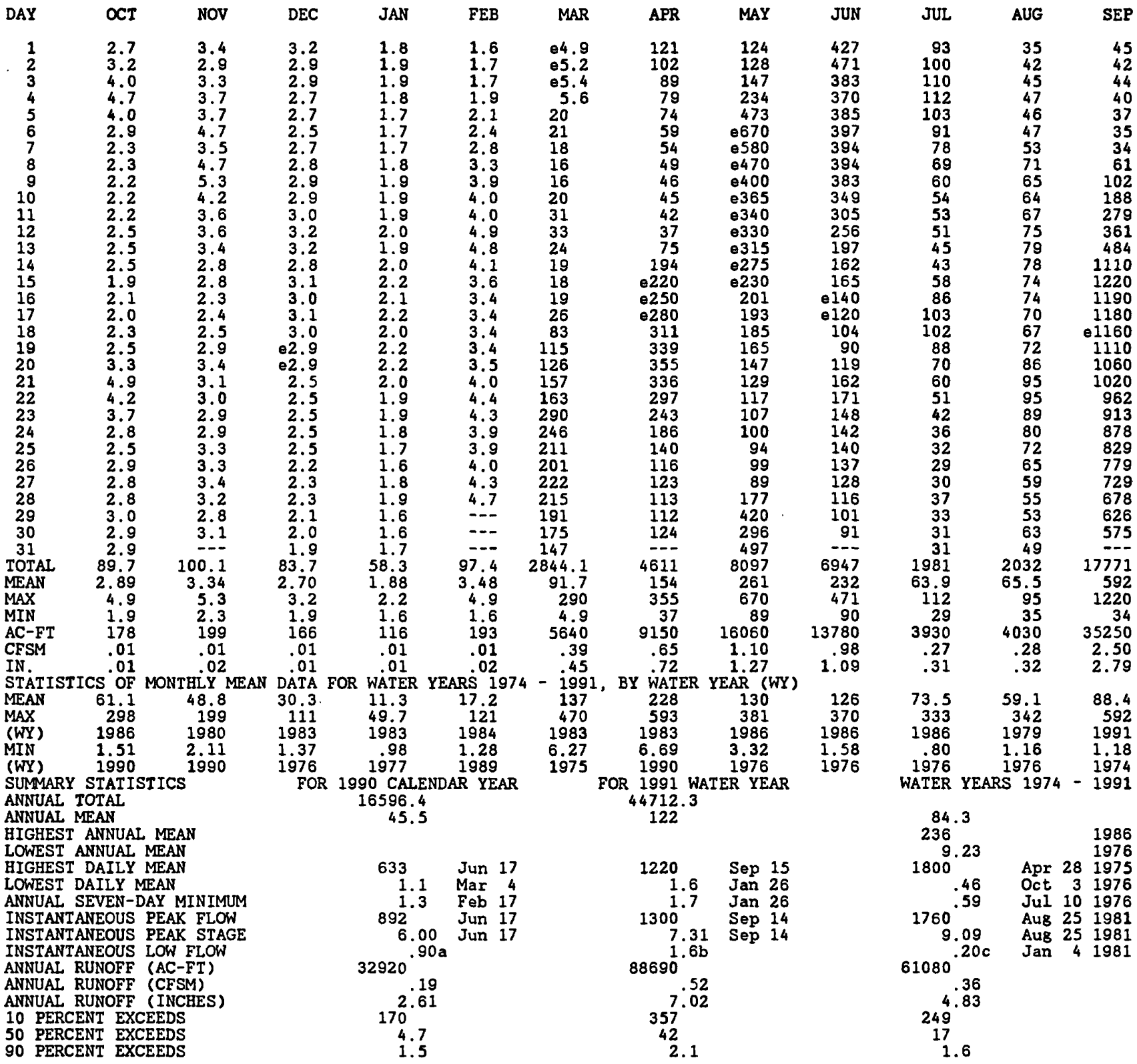

a Occurred Oct. 24 and Nov. 15 .

b Occurred all or part of each day Jan. 25, 26, 29-31, Feb. 1.

c Result of freezeup.

e Estimated. 
PERIOD OF RECORD.--Water years 1969, 1989 to current year.

WATER QUALITY DATA, WATER YEAR OCTOBER 1990 TO SEPTEMBER 1991

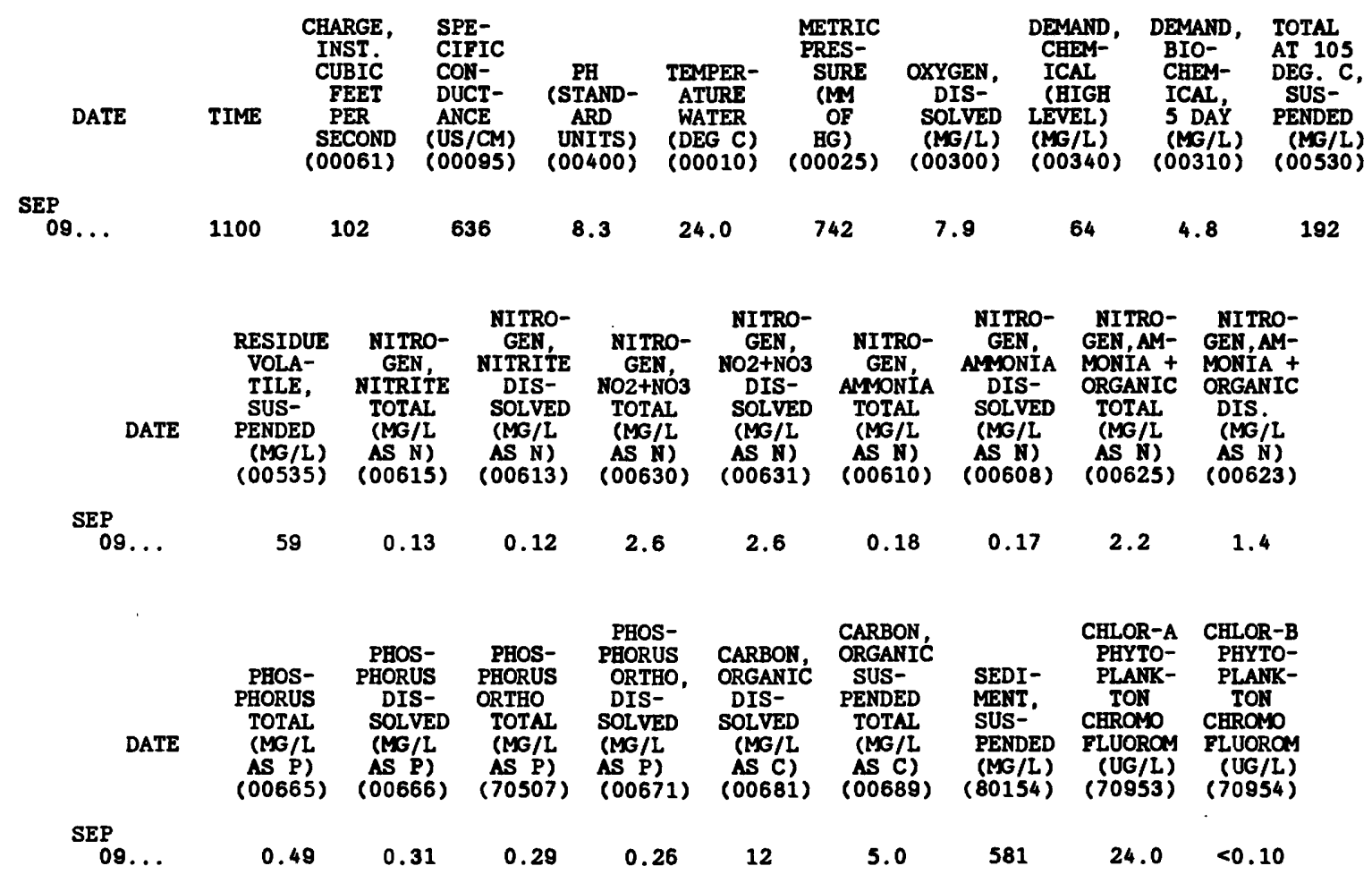


MINNESOTA RIVER BASIN

05330000 MINNESOTA RIVER NEAR JORDAN, MN

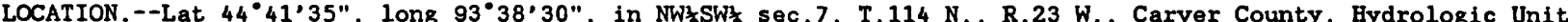
07020012 , on pier at center downstream side of bridge, 1.5 mi northwest of Jordan, and at mile 39.4 upstream from Mississippi River.

DRAINAGE AREA. $--16,200 \mathrm{mi}^{2}$, approximately.

WATER-DISCHARGE RECORDS

PERIOD OF RECORD.--September 1934 to current year. Prior to Oct. 1, 1966, published as "near Carver, Minn".

REVISED RECORDS.--WSP 955: Drainage area. WSP 1508: 1935. WDR MN-87-2: 1976 (cal. yr. summary).

GAGE.--Water-stage recorder. Datum of gage is $690.00 \mathrm{ft}$ above National Geodetic Vertical Datum of 1929. Prior to Oct. 1, 1966, water-stage recorder $2.8 \mathrm{mi}$ downstream with auxiliary nonrecording gage at present site and present datum.

REMARKS. - Records good except for those for estimated daily discharges, which are fair. DISCHARGE, CUBIC FEET PER SECOND, WATER YEAR OCTOBER 1990 TO SEPTEMBER 1991

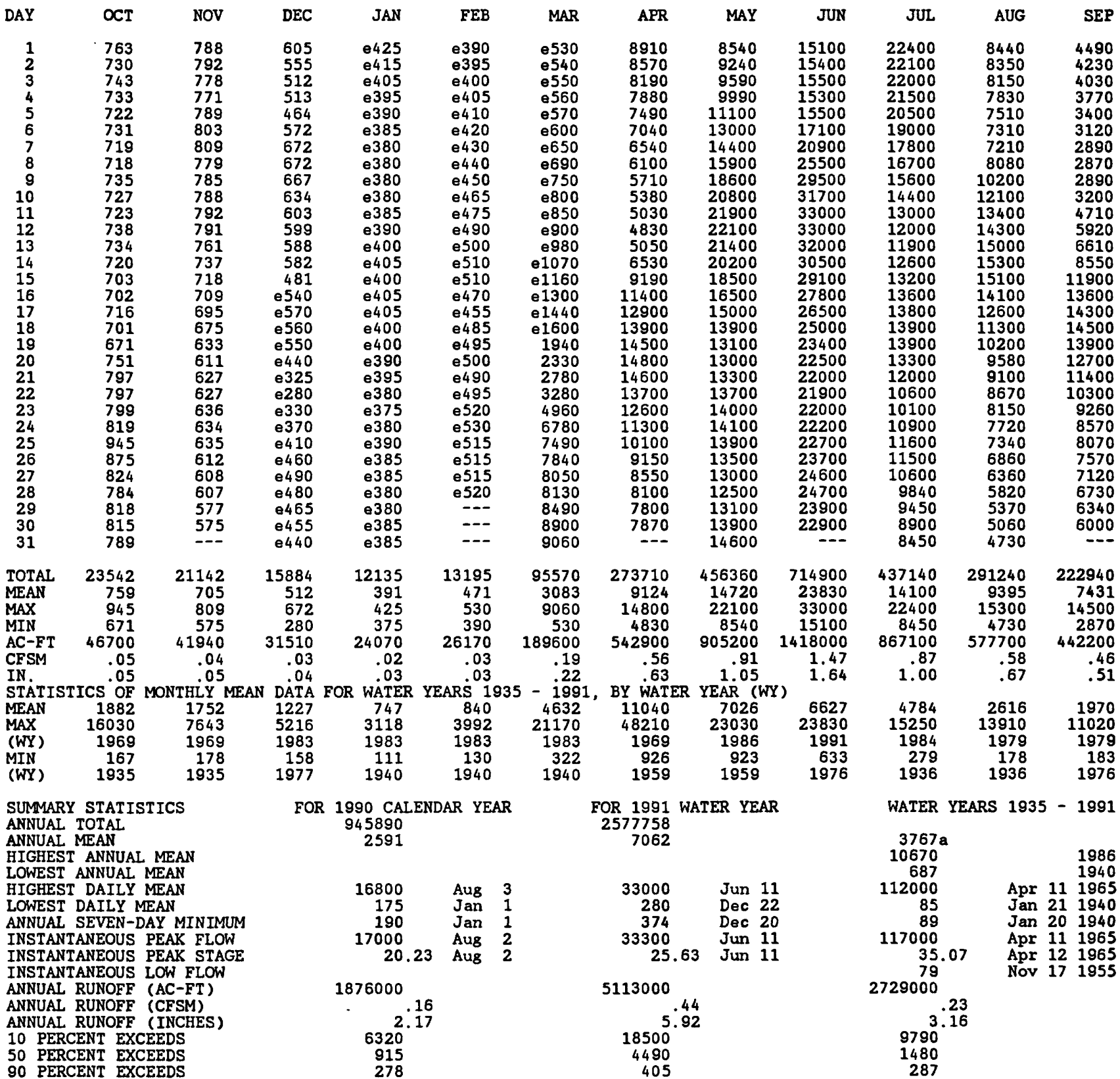

a Median of annual mean discharges is $3,230 \mathrm{ft}^{3} / \mathrm{s}$.

e Estimated. 
05330000 MIMNESOTA RIVER NEAR JORDAN, MN--Continued (National stream-quality accounting network station)

WATER-QUALITY RECORDS

PERIOD OF RECORD.--Water years 1952, 1963-69, 1972 to current year.

REMARKS.--Letter $\mathrm{K}$ indicates non-ideal colony count.

WATER QUALITY DATA, WATER YEAR OCTOBER 1990 TO SEPTEMBER 1991

\begin{tabular}{|c|c|c|c|c|c|c|c|c|c|c|c|c|}
\hline ATE & TIME & $\begin{array}{c}\text { DIS- } \\
\text { CHARGE, } \\
\text { INST } \\
\text { CUBIC } \\
\text { FEET } \\
\text { PER } \\
\text { SECOND } \\
(00061)\end{array}$ & $\begin{array}{l}\text { SPE- } \\
\text { CIFIC } \\
\text { CON- } \\
\text { DUCT- } \\
\text { ANCE } \\
\text { (US/CM) } \\
\text { (00095) }\end{array}$ & $\begin{array}{c}\text { SPE- } \\
\text { CIFIC } \\
\text { CON- } \\
\text { DUCT- } \\
\text { ANCE } \\
\text { LAB } \\
\text { (US/CM) } \\
\text { (90095) }\end{array}$ & $\begin{array}{c}\text { PA } \\
\text { (STAND- } \\
\text { ARD } \\
\text { UNITS) } \\
(00400)\end{array}$ & $\begin{array}{c}\text { PH } \\
\text { LAB } \\
\text { (STAND- } \\
\text { ARD } \\
\text { UNITS) } \\
\text { (00403) }\end{array}$ & $\begin{array}{c}\text { TEMPER- } \\
\text { ATURE } \\
\text { WATER } \\
\text { (DEG C) } \\
\text { (00010) }\end{array}$ & $\begin{array}{c}\text { TUR- } \\
\text { BID- } \\
\text { ITY } \\
\text { (NTU) } \\
(\text { OO076) }\end{array}$ & $\begin{array}{l}\text { BARO- } \\
\text { METRIC } \\
\text { PRES- } \\
\text { SURE } \\
\text { (MM } \\
\text { OF } \\
\text { BG) } \\
(00025)\end{array}$ & $\begin{array}{c}\text { OXYGEN, } \\
\text { DIS- } \\
\text { SOLVED } \\
(M G / L) \\
(00300)\end{array}$ & $\begin{array}{l}\text { COLI- } \\
\text { FORM, } \\
\text { FECAL, } \\
0.7 \\
\text { UM-MF } \\
\text { (COLS.' } \\
100 \mathrm{ML}) \\
(31625)\end{array}$ & $\begin{array}{c}\text { STREP- } \\
\text { TOCOCCI } \\
\text { FECAL } \\
\text { KF AGAR } \\
\text { (COLS. } \\
\text { PER } \\
100 \mathrm{ML}) \\
(31673)\end{array}$ \\
\hline
\end{tabular}

\begin{tabular}{|c|c|c|c|c|c|c|c|c|c|c|c|c|}
\hline $\begin{array}{l}\text { NOV } \\
27 \ldots\end{array}$ & 1120 & 608 & 931 & 968 & 8.4 & 8.0 & 3.5 & 6.5 & 732 & 14.6 & 170 & 88 \\
\hline AUG 15. & 1145 & 17900 & 810 & 839 & 8.2 & 8.2 & 20.0 & 22 & 738 & 7.5 & 58 & 91 \\
\hline $13 \ldots$ & 1300 & 15100 & 741 & 749 & 8.2 & 8.2 & 22.5 & 37 & 749 & 7.4 & 510 & 570 \\
\hline
\end{tabular}

\begin{tabular}{|c|c|c|c|c|c|c|c|c|c|c|c|}
\hline DATE & $\begin{array}{l}\text { CALCIUM } \\
\text { DIS- } \\
\text { SOL̇VD } \\
\text { (MG/L } \\
\text { AS CA) } \\
\text { (00915) }\end{array}$ & $\begin{array}{c}\text { MAGNE- } \\
\text { SIUM, } \\
\text { DIS- } \\
\text { SOLVED } \\
\text { (MG/L } \\
\text { AS MG) } \\
\text { (00925) }\end{array}$ & $\begin{array}{c}\text { SODIUM, } \\
\text { DIS- } \\
\text { SOLVED } \\
\text { (MG/L } \\
\text { AS MA) } \\
(00930)\end{array}$ & $\begin{array}{c}\text { POTAS- } \\
\text { SIUM, } \\
\text { DIS- } \\
\text { SOLVED } \\
\text { (MG/L } \\
\text { AS K) } \\
\text { (00935) }\end{array}$ & $\begin{array}{l}\text { ALKA- } \\
\text { LINIIY } \\
\text { WAT DIS } \\
\text { TOT IT } \\
\text { FIELD } \\
\text { MG/L AS } \\
\text { CACO3 } \\
\text { (39086) }\end{array}$ & $\begin{array}{c}\text { ALKA- } \\
\text { LINITY } \\
\text { IAB } \\
\text { (MG/L } \\
\text { AS } \\
\text { CACO3) } \\
(90410)\end{array}$ & $\begin{array}{c}\text { CAR- } \\
\text { BONATE } \\
\text { WATER } \\
\text { DIS IT } \\
\text { FIELD } \\
\text { MG/L AS } \\
\text { CO3 } \\
(00452)\end{array}$ & $\begin{array}{c}\text { BICAR- } \\
\text { BONATE } \\
\text { WATER } \\
\text { DIS IT } \\
\text { FIELD } \\
\text { MG/L AS } \\
\text { HCO3 } \\
(00453)\end{array}$ & $\begin{array}{l}\text { SULFATE } \\
\text { DIS- } \\
\text { SOLVED } \\
\text { (MG/L } \\
\text { AS SO4) } \\
(00945)\end{array}$ & $\begin{array}{l}\text { CHLO- } \\
\text { RIDE, } \\
\text { DIS- } \\
\text { SOLVED } \\
\text { (MG/L } \\
\text { AS CL) } \\
(00940)\end{array}$ & $\begin{array}{c}\text { FLUO- } \\
\text { RIDE, } \\
\text { DIS- } \\
\text { SOLVED } \\
\text { (MG/L } \\
\text { AS F) } \\
(00950)\end{array}$ \\
\hline & & & & & & & & & & & \\
\hline & 97 & 46 & 48 & 5.7 & - & 297 & -- & -- & 180 & 58 & 0.3 \\
\hline & 120 & 45 & 53 & 6.5 & 344 & 339 & 0 & 420 & 200 & 66 & 0.3 \\
\hline & 100 & 37 & 15 & 3.8 & 215 & 220 & 0 & 262 & 130 & 36 & 0.4 \\
\hline & 94 & 36 & 14 & 4.8 & 282 & 225 & 0 & 344 & 120 & 23 & 0.4 \\
\hline
\end{tabular}

\begin{tabular}{|c|c|c|c|c|c|c|c|c|c|c|c|}
\hline $\operatorname{ATE}$ & $\begin{array}{c}\text { SILICA, } \\
\text { DIS- } \\
\text { SOLVED } \\
\text { (MG/L } \\
\text { AS } \\
\text { SIO2) } \\
\text { (00955) }\end{array}$ & $\begin{array}{c}\text { SOLIDS, } \\
\text { RESIDUE } \\
\text { AT } 180 \\
\text { DEG. C } \\
\text { DIS- } \\
\text { SOLVED } \\
\text { (MG/L) } \\
(70300)\end{array}$ & $\begin{array}{c}\text { NITRO- } \\
\text { GEN, } \\
\text { NITRITE } \\
\text { DIS- } \\
\text { SOLVED } \\
\text { (MG/L } \\
\text { AS N) } \\
\text { (00613) }\end{array}$ & $\begin{array}{c}\text { NITRO- } \\
\text { GEN, } \\
\text { NO2+NO3 } \\
\text { DIS- } \\
\text { SOLVED } \\
\text { (MG/L } \\
\text { AS N) } \\
(00631)\end{array}$ & $\begin{array}{c}\text { MITRO- } \\
\text { GEN, } \\
\text { AMOONIA } \\
\text { TOTAL } \\
\text { (MG/L } \\
\text { AS N) } \\
(00610)\end{array}$ & $\begin{array}{c}\text { NITRO- } \\
\text { GEN, } \\
\text { AMYONIA } \\
\text { DIS- } \\
\text { SOLVED } \\
\text { (MG/L } \\
\text { AS N) } \\
\text { (00608) }\end{array}$ & $\begin{array}{l}\text { MITRO- } \\
\text { GEN,AM- } \\
\text { MONIA + } \\
\text { ORGANIC } \\
\text { TOTAL } \\
\text { (MG/L } \\
\text { AS N) } \\
(00625)\end{array}$ & $\begin{array}{c}\text { PHOS- } \\
\text { PHORUS } \\
\text { TOTAL } \\
\text { (MG/L } \\
\text { AS P) } \\
(00665)\end{array}$ & $\begin{array}{c}\text { PHOS- } \\
\text { PHORUS } \\
\text { DIS- } \\
\text { SOLVED } \\
\text { (MG/L } \\
\text { AS P) } \\
(00666)\end{array}$ & $\begin{array}{c}\text { PEOS- } \\
\text { PBORUS } \\
\text { ORTHO } \\
\text { TOTAL } \\
\text { (MG/L } \\
\text { AS P) } \\
(70507)\end{array}$ & $\begin{array}{c}\text { PHOS- } \\
\text { PHORUS } \\
\text { ORTHO, } \\
\text { DIS- } \\
\text { SOLVED } \\
\text { (MS/L } \\
\text { AS P) } \\
(00671)\end{array}$ \\
\hline
\end{tabular}

\begin{tabular}{|c|c|c|c|c|c|c|c|c|c|c|c|}
\hline $\begin{array}{l}\text { NOV } \\
27 \ldots\end{array}$ & 5.7 & 623 & 0.02 & 0.8 & 0.04 & 0.04 & 0.8 & 0.12 & $<0.01$ & 0.02 & $<0.01$ \\
\hline $25 .$. & 19 & 735 & 0.03 & 2.2 & 0.64 & 0.64 & 1.5 & 0.20 & 0.12 & 0.14 & 0.12 \\
\hline AUG $15 .$. & 16 & 563 & 0.08 & 17.0 & 0.03 & 0.03 & 1.8 & 0.10 & 0.07 & 0.06 & 0.05 \\
\hline $13 \ldots$ & 25 & 497 & 0.05 & 9.6 & 0.03 & $<0.01$ & 1.6 & 0.36 & 0.27 & 0.20 & 0.18 \\
\hline
\end{tabular}


MIMNESOTA RIVER BASIM

05330000 MINNESOTA RIVER MEAR JORDAN, MN--Continued

\begin{tabular}{|c|c|c|c|c|c|c|c|c|c|c|c|}
\hline DATE & $\begin{array}{l}\text { SEDI- } \\
\text { MENT, } \\
\text { SUS- } \\
\text { PENDED } \\
\text { (MG/L) } \\
(80154)\end{array}$ & $\begin{array}{c}\text { SED. } \\
\text { SUSP. } \\
\text { SIEVE } \\
\text { DIAM. } \\
\text { TINER } \\
\text { THAN } \\
\text { TE2 MY } \\
\text { (70331) }\end{array}$ & $\begin{array}{l}\text { ALUM- } \\
\text { INUM, } \\
\text { DIS- } \\
\text { SOLVED } \\
\text { (UG/L } \\
\text { AS AL) } \\
\text { (01106) }\end{array}$ & $\begin{array}{c}\text { ARSENIC } \\
\text { DIS- } \\
\text { SOLVED } \\
\text { (UG/L } \\
\text { AS AS) } \\
(01000)\end{array}$ & $\begin{array}{l}\text { BARIUM, } \\
\text { DIS- } \\
\text { SOLVED } \\
\text { (UG/L } \\
\text { AS BA) } \\
\text { (01005) }\end{array}$ & $\begin{array}{l}\text { BERYL- } \\
\text { LIUM, } \\
\text { DIS- } \\
\text { SOLVED } \\
\text { (UG/L } \\
\text { AS BE) } \\
\text { (01010) }\end{array}$ & $\begin{array}{c}\text { CADMIUM } \\
\text { DIS- } \\
\text { SOLVED } \\
\text { (UG/L } \\
\text { AS CD) } \\
(01025)\end{array}$ & $\begin{array}{l}\text { CERO- } \\
\text { MIUM, } \\
\text { DIS- } \\
\text { SOLVED } \\
\text { (UG/L } \\
\text { AS CR) } \\
(01030)\end{array}$ & $\begin{array}{c}\text { COBALT, } \\
\text { DIS- } \\
\text { SOLVED } \\
\text { (UG/L } \\
\text { AS CO) } \\
(01035)\end{array}$ & $\begin{array}{l}\text { COPPER, } \\
\text { DIS- } \\
\text { SOLVED } \\
\text { (UG/L } \\
\text { AS CU) } \\
(01040)\end{array}$ & $\begin{array}{c}\text { IRON, } \\
\text { DIS- } \\
\text { SOLVED } \\
\text { (UG/L } \\
\text { AS FE) } \\
\text { (01046) }\end{array}$ \\
\hline \multirow{5}{*}{$\begin{array}{c}\text { KOV } \\
27 \ldots \\
\text { FEB } \\
25 \ldots \\
\operatorname{MAY} \ldots \\
15 \ldots \\
\text { AUG } \\
13 \ldots\end{array}$} & & & & & & & & & & & \\
\hline & -- & - & 150 & 1 & 75 & $<0.5$ & $<1.0$ & $<1$ & $<3$ & 2 & 240 \\
\hline & 95 & 21 & $<10$ & 1 & 81 & $<0.5$ & 3.0 & $<1$ & $<3$ & 2 & 10 \\
\hline & 116 & $\cdots$ & $<10$ & 2 & 87 & $<0.5$ & $<1.0$ & $<1$ & $<3$ & 2 & 5 \\
\hline & 340 & 55 & $<10$ & 3 & 83 & $<0.5$ & $<1.0$ & $<1$ & $<3$ & 6 & 5 \\
\hline DATE & $\begin{array}{c}\text { LEAD, } \\
\text { DIS- } \\
\text { SOLVED } \\
\text { (UG/L } \\
\text { AS PB) } \\
(01049)\end{array}$ & $\begin{array}{c}\text { LITHIUM } \\
\text { DIS- } \\
\text { SOLVED } \\
\text { (UG/L } \\
\text { AS LI) } \\
(01130)\end{array}$ & $\begin{array}{l}\text { MANGA- } \\
\text { NESE, } \\
\text { DIS- } \\
\text { SOLVED } \\
\text { (UG/L } \\
\text { AS MN) } \\
(01056 \text { ) }\end{array}$ & $\begin{array}{c}\text { MERCURY } \\
\text { DIS- } \\
\text { SOLVED } \\
\text { (UG/L } \\
\text { AS HG) } \\
(71890)\end{array}$ & $\begin{array}{c}\text { MOLYB- } \\
\text { DENUM, } \\
\text { DIS- } \\
\text { SOLVED } \\
\text { (UG/L } \\
\text { AS MO) } \\
(01060)\end{array}$ & $\begin{array}{c}\text { NICKEL, } \\
\text { DIS- } \\
\text { SOLVED } \\
\text { (UG/L } \\
\text { AS NI) } \\
(01065)\end{array}$ & $\begin{array}{c}\text { SELE- } \\
\text { NIUM, } \\
\text { DIS- } \\
\text { SOLVED } \\
\text { (UG/L } \\
\text { AS SE) } \\
(01145)\end{array}$ & $\begin{array}{c}\text { SILVER, } \\
\text { DIS- } \\
\text { SOLVED } \\
\text { (UG/L } \\
\text { AS AG) } \\
(01075)\end{array}$ & $\begin{array}{c}\text { STRON- } \\
\text { TIUM, } \\
\text { DIS- } \\
\text { SOLVED } \\
\text { (UG/L } \\
\text { AS SR) } \\
\text { (01080) }\end{array}$ & $\begin{array}{l}\text { VANA- } \\
\text { DIUM, } \\
\text { DIS- } \\
\text { SOLVED } \\
\text { (UG/L } \\
\text { AS V) } \\
(01085)\end{array}$ & $\begin{array}{l}\text { ZINC. } \\
\text { DIS- } \\
\text { SOLVED } \\
\text { (UG/L } \\
\text { AS ZN) } \\
\text { (01090) }\end{array}$ \\
\hline Nov & & & & & & & & & & & \\
\hline $\mathrm{FEB}^{27} \cdots$ & 2 & 38 & 190 & 0.1 & $<10$ & 3 & $<1$ & $<1.0$ & 410 & $<6$ & $<3$ \\
\hline $\operatorname{MAY}^{25} \cdots$ & $<1$ & 35 & 160 & $<0.1$ & $<10$ & 2 & $<1$ & $<1.0$ & 450 & $<6$ & 4 \\
\hline AUG 15. & $<1$ & 29 & 3 & $<0.1$ & $<10$ & 2 & 4 & $<1.0$ & 330 & $<6$ & 8 \\
\hline $13 \ldots$ & $<1$ & 31 & 4 & $<0.1$ & $<10$ & 3 & 3 & $<1.0$ & 300 & $<6$ & 15 \\
\hline
\end{tabular}


MISSISSIPPI RIVER MAIN STEM

05331000 MISSISSIPPI RIVER AT ST. PAUL, MN

LOCATION.--Lat $44^{\circ} 56^{\prime} 40^{\prime \prime}$, long $93^{\circ} 05^{\prime} 20^{\prime \prime}$, in SEłNE sec.6, T.28 N., R.22 W., Ramsey County, Hydrologic Unit 07010206 , on left bank in St. Paul, 300 ft upstream from Robert Street Bridge, 6 mi downstream from Minnesota River, and at mile 839.3 upstream from Ohio River.

DRAINAGE AREA. $--36,800 \mathrm{mi}^{2}$, approximately.

WATER-DISCHARGE RECORDS

PERIOD OF RECORD.--Water year 1867-69, 1872-92 (annual maximums), March 1892 to current year (prior to 1901, fragmentary during some winters). Records prior to March 1892, published in the 19th Annual Report, Part 4, have been found to be unreliable and should not be used. Monthly discharge only for some periods, published in WSP 1308. Gage-height records (winter records incomplete) collected at same site since 1866 are contained in reports of U.S. Weather Bureau, War Department and Mississippi River Commission.

REVISED RECORDS.--WSP 285: 1892-96. WSP 715: Drainage area. WSP 875: 1938. WSP 895: 1939. WSP 1308: $1867(\mathrm{M})$. WSP 1508: $1897,1898(\mathrm{M}), 1903(\mathrm{M}), 1917-18(\mathrm{M}), 1928(\mathrm{M}), 1929$. WRD MN-74: 1973.

GAGE.--Water-stage recorder. Datum of gage is $683.62 \mathrm{ft}$ above National Geodetic Vertical Datum of 1929. Prior to Mar. 18, 1925, nonrecording gage at several sites within 300 ft of present site at present datum. Mar. 18, 1925 , to Mar. 10, 1933, water-stage recorder and Mar. 11, 1933, to Sept. 14, 1939, nonrecording gage, at present site and datum. Since September 1938, auxiliary water-stage recorder $5.6 \mathrm{mi}$ downstream.

REMARKS, --Records good. Slight regulation except during extreme floods by reservoirs on headwaters and by power plants. Beginning July 20,1938, sewage from Minneapolis and St. Paul, which formerly entered above station, was diverted to a sewage-disposal plant, thence to river below station. Figures of daily discharge do not include this diversion.

COOPERATION.--Records of Mississipp 1 River at Twin City lock and dam computed and furnished by Ford Motor Co. Diversion through sewage disposal plant furnished by Metropolitan Waste Control Commission.

DISCHARGE, CUBIC FEET PER SECOND, WATER YEAR OCTOBER 1990 TO SEPTEMBER 1991 DAILY MEAN VALUES

\begin{tabular}{|c|c|c|c|c|c|c|c|c|c|c|c|c|}
\hline DAY & OCT & NOV & DEC & JAN & FEB & MAR & APR & MAY & JUN & JUL & AUG & SEP \\
\hline $\begin{array}{l}1 \\
2 \\
3 \\
4 \\
5\end{array}$ & $\begin{array}{l}4170 \\
3600 \\
3500 \\
3960 \\
4850\end{array}$ & $\begin{array}{l}7980 \\
7570 \\
7320 \\
7500 \\
7270\end{array}$ & $\begin{array}{l}3350 \\
3560 \\
3800 \\
3570 \\
3930\end{array}$ & $\begin{array}{l}3540 \\
3480 \\
3380 \\
3470 \\
3340\end{array}$ & $\begin{array}{l}3130 \\
3490 \\
3350 \\
4180 \\
3240\end{array}$ & $\begin{array}{l}2880 \\
3100 \\
3120 \\
3120 \\
3060\end{array}$ & $\begin{array}{l}25400 \\
24600 \\
23600 \\
22300 \\
20900\end{array}$ & $\begin{array}{l}22300 \\
24400 \\
25200 \\
26800 \\
28000\end{array}$ & $\begin{array}{l}31700 \\
33700 \\
34100 \\
35400 \\
35300\end{array}$ & $\begin{array}{l}34400 \\
36300 \\
36900 \\
38000 \\
38200\end{array}$ & $\begin{array}{l}15900 \\
16100 \\
17100 \\
18100 \\
17300\end{array}$ & $\begin{array}{l}9260 \\
8280 \\
8200 \\
8140 \\
8350\end{array}$ \\
\hline $\begin{array}{r}6 \\
7 \\
8 \\
9 \\
10\end{array}$ & $\begin{array}{l}4490 \\
4390 \\
4880 \\
4460 \\
5210\end{array}$ & $\begin{array}{l}6770 \\
6530 \\
6990 \\
6690 \\
6610\end{array}$ & $\begin{array}{l}3770 \\
3790 \\
4210 \\
4240 \\
4310\end{array}$ & $\begin{array}{l}3590 \\
3470 \\
3380 \\
3360 \\
3360\end{array}$ & $\begin{array}{l}3520 \\
2860 \\
2830 \\
2820 \\
2910\end{array}$ & $\begin{array}{l}3130 \\
3110 \\
3090 \\
3320 \\
3290\end{array}$ & $\begin{array}{l}20300 \\
19100 \\
17700 \\
16800 \\
16000\end{array}$ & $\begin{array}{l}31400 \\
35300 \\
39700 \\
42400 \\
46600\end{array}$ & $\begin{array}{l}35700 \\
37000 \\
41000 \\
45000 \\
48800\end{array}$ & $\begin{array}{l}37400 \\
35400 \\
34100 \\
32500 \\
31000\end{array}$ & $\begin{array}{l}16700 \\
16100 \\
15800 \\
16900 \\
18700\end{array}$ & $\begin{array}{r}8050 \\
7350 \\
7440 \\
9110 \\
12800\end{array}$ \\
\hline $\begin{array}{l}11 \\
12 \\
13 \\
14 \\
15\end{array}$ & $\begin{array}{l}5240 \\
5360 \\
4940 \\
5030 \\
4840\end{array}$ & $\begin{array}{l}6520 \\
5970 \\
6090 \\
5590 \\
5660\end{array}$ & $\begin{array}{l}3790 \\
4190 \\
3870 \\
3920 \\
3170\end{array}$ & $\begin{array}{l}3310 \\
3390 \\
3390 \\
3390 \\
3430\end{array}$ & $\begin{array}{l}2900 \\
2950 \\
3360 \\
3200 \\
2890\end{array}$ & $\begin{array}{l}3280 \\
3140 \\
3540 \\
3490 \\
3640\end{array}$ & $\begin{array}{l}15600 \\
15100 \\
14900 \\
15900 \\
18300\end{array}$ & $\begin{array}{l}49800 \\
51700 \\
52000 \\
50500 \\
47900\end{array}$ & $\begin{array}{l}51100 \\
50500 \\
49500 \\
47700 \\
45100\end{array}$ & $\begin{array}{l}29100 \\
26800 \\
26700 \\
25600 \\
26200\end{array}$ & $\begin{array}{l}20200 \\
21100 \\
22400 \\
22700 \\
22300\end{array}$ & $\begin{array}{l}13300 \\
15400 \\
19000 \\
21300 \\
24200\end{array}$ \\
\hline $\begin{array}{l}16 \\
17 \\
18 \\
19 \\
20\end{array}$ & $\begin{array}{l}4690 \\
4650 \\
4810 \\
5370 \\
5280\end{array}$ & $\begin{array}{l}5940 \\
5620 \\
5910 \\
5500 \\
5750\end{array}$ & $\begin{array}{l}3780 \\
3810 \\
3810 \\
3350 \\
3260\end{array}$ & $\begin{array}{l}3340 \\
3420 \\
3320 \\
3180 \\
3520\end{array}$ & $\begin{array}{l}3160 \\
4810 \\
4670 \\
3820 \\
3890\end{array}$ & $\begin{array}{l}3770 \\
3730 \\
4270 \\
4460 \\
4890\end{array}$ & $\begin{array}{l}23300 \\
27300 \\
30100 \\
31800 \\
33600\end{array}$ & $\begin{array}{l}44100 \\
40700 \\
37900 \\
35000 \\
32600\end{array}$ & $\begin{array}{l}42800 \\
41000 \\
38900 \\
36200 \\
34500\end{array}$ & $\begin{array}{l}26100 \\
26200 \\
26100 \\
25700 \\
25900\end{array}$ & $\begin{array}{l}22300 \\
20900 \\
19300 \\
17800 \\
16400\end{array}$ & $\begin{array}{l}29100 \\
31500 \\
32200 \\
32400 \\
31500\end{array}$ \\
\hline $\begin{array}{l}21 \\
22 \\
23 \\
24 \\
25\end{array}$ & $\begin{array}{l}5820 \\
5870 \\
6730 \\
7790 \\
7890\end{array}$ & $\begin{array}{l}5320 \\
5480 \\
5160 \\
5260 \\
5410\end{array}$ & $\begin{array}{l}2950 \\
2260 \\
2130 \\
3570 \\
3730\end{array}$ & $\begin{array}{l}2970 \\
3160 \\
3340 \\
3170 \\
3130\end{array}$ & $\begin{array}{l}4090 \\
3480 \\
3110 \\
3120 \\
3390\end{array}$ & $\begin{array}{r}5590 \\
7290 \\
9350 \\
12600 \\
17300\end{array}$ & $\begin{array}{l}34800 \\
35200 \\
34600 \\
32600 \\
29800\end{array}$ & $\begin{array}{l}30800 \\
30200 \\
29500 \\
28800 \\
28400\end{array}$ & $\begin{array}{l}33900 \\
34200 \\
33600 \\
33600 \\
33800\end{array}$ & $\begin{array}{l}24500 \\
23200 \\
21300 \\
20200 \\
20500\end{array}$ & $\begin{array}{l}15500 \\
14600 \\
14300 \\
14100 \\
13800\end{array}$ & $\begin{array}{l}29300 \\
27000 \\
25700 \\
24300 \\
22400\end{array}$ \\
\hline $\begin{array}{l}26 \\
27 \\
28 \\
29 \\
30 \\
31\end{array}$ & $\begin{array}{l}8190 \\
8280 \\
7950 \\
7970 \\
7800 \\
7820\end{array}$ & $\begin{array}{l}5040 \\
5090 \\
4310 \\
4540 \\
3580 \\
---\end{array}$ & $\begin{array}{l}3730 \\
3600 \\
3360 \\
3880 \\
3530 \\
3510\end{array}$ & $\begin{array}{l}3260 \\
3470 \\
3480 \\
3120 \\
2960 \\
2940\end{array}$ & $\begin{array}{r}3540 \\
3240 \\
3030 \\
-\ldots- \\
--- \\
---\end{array}$ & $\begin{array}{l}19000 \\
21000 \\
22500 \\
23600 \\
24600 \\
25000\end{array}$ & $\begin{array}{c}27000 \\
24500 \\
23100 \\
22500 \\
21500 \\
---\end{array}$ & $\begin{array}{l}27600 \\
27200 \\
26900 \\
27000 \\
29100 \\
30600\end{array}$ & $\begin{array}{c}34200 \\
35500 \\
36300 \\
36200 \\
35700 \\
\end{array}$ & $\begin{array}{l}20400 \\
20100 \\
18900 \\
18600 \\
17800 \\
16700\end{array}$ & $\begin{array}{r}13100 \\
12500 \\
12000 \\
11200 \\
10500 \\
9840\end{array}$ & $\begin{array}{l}21500 \\
20900 \\
18700 \\
17800 \\
17100 \\
-0\end{array}$ \\
\hline $\begin{array}{l}\text { TOTAL } \\
\text { MEAN } \\
\text { MAX } \\
\text { MIN } \\
\text { AC-FT } \\
\text { CFSM } \\
\text { IN. } \\
\vdots \\
\text { MEAN } \\
\text { CFSM } \\
\text { IN } \$ \\
\text { CAI YR }\end{array}$ & $\begin{array}{r}175830 \\
5672 \\
8280 \\
3500 \\
348800 \\
.15 \\
18 \\
341 \\
6013 \\
0.16 \\
0.19\end{array}$ & $\begin{array}{r}178970 \\
5966 \\
7980 \\
3580 \\
355000 \\
.16 \\
.18 \\
321 \\
6287 \\
0.17 \\
0.19\end{array}$ & $\begin{array}{r}111730 \\
3604 \\
4310 \\
2130 \\
221600 \\
.10 \\
.11 \\
307 \\
3911 \\
0.11 \\
0.12\end{array}$ & $\begin{array}{r}103060 \\
3325 \\
3590 \\
2940 \\
204400 \\
.09 \\
.10 \\
308 \\
3633 \\
0.10 \\
0.11\end{array}$ & $\begin{array}{r}94980 \\
3392 \\
4810 \\
2820 \\
188400 \\
.09 \\
110 \\
319 \\
3711 \\
0.10 \\
0.11\end{array}$ & $\begin{array}{r}257260 \\
8299 \\
25000 \\
2880 \\
510300 \\
.23 \\
.26 \\
344 \\
8643 \\
0.23 \\
0.27\end{array}$ & $\begin{array}{r}718200 \\
23940 \\
35200 \\
14900 \\
1425000 \\
.65 \\
.73 \\
363 \\
24303 \\
0.66 \\
0.74\end{array}$ & $\begin{array}{r}1080400 \\
34850 \\
52000 \\
22300 \\
2143000 \\
.95 \\
1.09 \\
457 \\
35307 \\
0.96 \\
1.11\end{array}$ & $\begin{array}{r}1162000 \\
38730 \\
51100 \\
31700 \\
2305000 \\
1.05 \\
1.17 \\
408 \\
39138 \\
1.06 \\
1.19\end{array}$ & $\begin{array}{r}840800 \\
27120 \\
38200 \\
16700 \\
1668000 \\
.74 \\
.85 \\
397 \\
27517 \\
0.75 \\
0.86\end{array}$ & $\begin{array}{r}515540 \\
16630 \\
22700 \\
9840 \\
1023000 \\
.45 \\
.52 \\
392 \\
17022 \\
0.46 \\
0.53\end{array}$ & $\begin{array}{r}561580 \\
18720 \\
32400 \\
7350 \\
1114000 \\
.51 \\
.57 \\
453 \\
19173 \\
0.52 \\
0.58\end{array}$ \\
\hline
\end{tabular}


MISSISSIPPI RIVER MAIN STEM

05331000 MISSISSIPPI RIVER AT ST. PAUL, MN--Continued
STATISTICS OF MONTHLY MEAN DATA FOR WATER YEARS 1892 - 1991, BY WATER YEAR (WY)

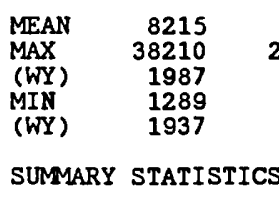

ANNUAL TOTAL

ANNUAL MEAN

HIGHEST ANNUAL MEAN

LOWEST ANNUAL MEAN

HIGHEST DAILY MEAN

LOWEST DAILY MEAN

ANNUAI SEVEN-DAY MINIMUM

INSTANTANEOUS PEAK FLOW

INSTANTANEOUS PEAK STAGE

ANNUAL RUNOFF (AC-FT)

ANNUAL RUNOFF (CFSM)

ANNUAL RUNOFF (INCHES)

10 PERCENT EXCEEDS

50 PERCENT EXCEEDS

90 PERCENT EXCEEDS

5234
16080
1983
1277
1935

4299
11500
1983

14700

1983

1097
1935

4291
14700

1966

1300

1895

10470
43240
1983

24580

1757
1940

91610

1969

1969
3421

19880

66470

1986

3085

$1895 \quad 1934$

FOR 1990 CALENDAR YEAR

FOR 1991 WATER YEAR

3362830

9213 a

$35500 \quad$ Jun 26
$2130 \quad$ Dec 23

$\begin{array}{lll}2130 & \text { Dec } & 23 \\ 2490 & \text { Feb } & 28\end{array}$

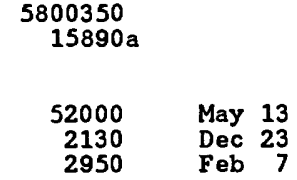

6670000

3.25
$20100^{.40}$
6690
2630

$\begin{array}{lr}\text { Dec } & 23 \\ \text { Feb } & 7\end{array}$

11500000

$35300^{5.43}$
9350
3250

17490

56530

1908

1980

1934 $\begin{array}{lll}13260 & 8259 \quad 7808\end{array}$

$43290 \quad 33380 \quad 34380$

$\begin{array}{lll}1905 & 1953 & 1986\end{array}$

$1272 \quad 864 \quad 1143$

$1934 \quad 1934 \quad 1934$

WATER YEARS 1892 - 1991

$10960 \mathrm{ab}$

29580

1935

171000

632

741

171000

7937000

4.05

25800

6690

2610

Diversion equivalent in cubic feet per second through sewage disposal plant. Adjusted for diversion.

Unadjusted.

b Adjusted mean is $11,188 \mathrm{ft}^{3} / \mathrm{s}$. Unadjusted median is $9,956 \mathrm{ft}^{3} / \mathrm{s}$. 


\section{MISSISSIPPI RIVER BASIN}

05331000 MISSISSIPPI RIVER AT ST. PAUL, MN--Continued

WATER-QUALITY RECORDS

PERIOD OF RECORD.--Water years 1956 to December 1990 (discontinued).

PERIOD OF DAILY RECORD.--

WATER TEMPERATURES: October 1956 to December 1990 (discontinued).

INSTRUMENTATION, --Temperature recorder since October 1956.

EXTREMES FOR PERIOD OF DAILY RECORD.--

WATER TEMPERATURES: Maximum, $31.0^{\circ} \mathrm{C}$, July $24-28,1964$, July 31, 1975, July 19, 21, 1977, Aug. 17, 1988; minimum, $0.0^{\circ} \mathrm{C}$ many days during winter periods.

EXTREMES FOR CURRENT PERIOD--October to December 1990:

WATER TEMPERATURES: Maximum during period, $15.0^{\circ} \mathrm{C}$, Oct. 15 ; minimum, $0.0^{\circ} \mathrm{C}$, Dec. 5.

WATER TEMPERATURE, DEGREES CELSIUS, WATER YEAR OCTOBER 1990 TO SEPTEMBER 1991

\begin{tabular}{|c|c|c|c|c|c|c|c|c|c|c|c|c|}
\hline \multirow[t]{2}{*}{ DAY } & $\operatorname{MAX}$ & MIN & MEAN & $\operatorname{MAX}$ & MIN & MEAN & $\operatorname{MAX}$ & MIN & MEAN & $\max$ & MIN & MEAN \\
\hline & \multicolumn{3}{|c|}{ OCTOBER } & \multicolumn{3}{|c|}{ NOVEMBER } & \multicolumn{3}{|c|}{ DECEMBER } & \multicolumn{3}{|c|}{ JANUARY } \\
\hline $\begin{array}{l}1 \\
2 \\
3 \\
4 \\
5\end{array}$ & $\begin{array}{l}--- \\
\cdots- \\
\cdots-- \\
---\end{array}$ & $\begin{array}{l}--- \\
-- \\
-- \\
--- \\
\end{array}$ & $\begin{array}{l}-- \\
--- \\
\cdots-- \\
---\end{array}$ & $\begin{array}{r}10.0 \\
10.5 \\
10.5 \\
9.5 \\
8.5\end{array}$ & $\begin{array}{r}9.0 \\
10.0 \\
9.5 \\
8.5 \\
7.5\end{array}$ & $\begin{array}{r}9.5 \\
10.0 \\
10.0 \\
9.0 \\
8.0\end{array}$ & $\begin{array}{l}2.5 \\
2.0 \\
2.5 \\
1.5 \\
1.5\end{array}$ & $\begin{array}{r}1.5 \\
1.5 \\
1.5 \\
.5 \\
.0\end{array}$ & $\begin{array}{l}2.0 \\
1.5 \\
1.5 \\
1.0 \\
1.0\end{array}$ & $\begin{array}{l}--- \\
--- \\
--- \\
---\end{array}$ & $\begin{array}{l}--- \\
--- \\
--- \\
--- \\
---\end{array}$ & $\begin{array}{l}--- \\
--- \\
--- \\
---\end{array}$ \\
\hline $\begin{array}{r}6 \\
7 \\
8 \\
9 \\
10\end{array}$ & $\begin{array}{l}--- \\
--- \\
--- \\
---\end{array}$ & $\begin{array}{l}--- \\
--- \\
-- \\
--- \\
--\end{array}$ & $\begin{array}{l}--- \\
--- \\
--- \\
--- \\
\end{array}$ & $\begin{array}{l}8.0 \\
7.5 \\
6.5 \\
5.5 \\
5.0\end{array}$ & $\begin{array}{l}7.0 \\
6.5 \\
5.5 \\
5.0 \\
4.5\end{array}$ & $\begin{array}{l}7.5 \\
7.0 \\
6.0 \\
5.5 \\
4.5\end{array}$ & $\begin{array}{l}1.5 \\
2.0 \\
2.5 \\
3.0 \\
3.5\end{array}$ & $\begin{array}{r}.5 \\
.5 \\
1.0 \\
2.0 \\
2.5\end{array}$ & $\begin{array}{l}1.0 \\
1.5 \\
1.5 \\
2.5 \\
3.0\end{array}$ & $\begin{array}{l}--- \\
--- \\
--- \\
---\end{array}$ & $\begin{array}{l}--- \\
--- \\
--- \\
---\end{array}$ & $\begin{array}{l}--- \\
--- \\
--- \\
---\end{array}$ \\
\hline $\begin{array}{l}11 \\
12 \\
13 \\
14 \\
15\end{array}$ & \begin{tabular}{r}
--- \\
14.5 \\
\hdashline 14.5 \\
15.0
\end{tabular} & $\begin{array}{r}14.0 \\
14.0 \\
14.0\end{array}$ & \begin{tabular}{r}
14.0 \\
\hdashline 14.5 \\
14.5
\end{tabular} & $\begin{array}{l}5.0 \\
6.0 \\
6.5 \\
7.0 \\
7.0\end{array}$ & $\begin{array}{l}4.5 \\
5.0 \\
5.5 \\
5.5 \\
5.5\end{array}$ & $\begin{array}{l}5.0 \\
5.5 \\
6.0 \\
6.0 \\
6.5\end{array}$ & $\begin{array}{l}4.0 \\
4.5 \\
3.5 \\
3.5 \\
2.0\end{array}$ & $\begin{array}{l}3.0 \\
3.0 \\
2.5 \\
2.0 \\
1.5\end{array}$ & $\begin{array}{l}3.5 \\
4.0 \\
3.0 \\
2.5 \\
1.5\end{array}$ & $\begin{array}{l}--- \\
--- \\
--- \\
---\end{array}$ & $\begin{array}{l}--- \\
--- \\
--- \\
---\end{array}$ & $\begin{array}{l}--- \\
--- \\
--- \\
--- \\
--\end{array}$ \\
\hline $\begin{array}{l}16 \\
17 \\
18 \\
19 \\
20\end{array}$ & $\begin{array}{l}14.5 \\
14.5 \\
13.0 \\
12.5 \\
11.5\end{array}$ & $\begin{array}{l}14.0 \\
13.0 \\
12.0 \\
11.0 \\
10.0\end{array}$ & $\begin{array}{l}14.5 \\
14.0 \\
12.5 \\
11.5 \\
10.5\end{array}$ & $\begin{array}{l}7.5 \\
7.5 \\
7.0 \\
6.5 \\
7.0\end{array}$ & $\begin{array}{l}6.5 \\
6.5 \\
6.5 \\
6.0 \\
6.0\end{array}$ & $\begin{array}{l}7.0 \\
7.0 \\
6.5 \\
6.5 \\
6.5\end{array}$ & $\begin{array}{l}2.0 \\
2.5 \\
3.0 \\
2.5 \\
-.-\end{array}$ & $\begin{array}{l}1.5 \\
1.0 \\
1.5 \\
1.5 \\
-.-\end{array}$ & $\begin{array}{l}1.5 \\
2.0 \\
2.0 \\
1.5 \\
--.\end{array}$ & $\begin{array}{l}--- \\
--- \\
--- \\
---\end{array}$ & $\begin{array}{l}--- \\
--- \\
--- \\
-\cdots\end{array}$ & $\begin{array}{l}--- \\
--- \\
--- \\
---\end{array}$ \\
\hline $\begin{array}{l}21 \\
22 \\
23 \\
24 \\
25\end{array}$ & $\begin{array}{l}10.0 \\
10.0 \\
10.5 \\
10.5 \\
10.5\end{array}$ & $\begin{array}{r}9.5 \\
9.5 \\
9.5 \\
10.0 \\
9.5\end{array}$ & $\begin{array}{r}10.0 \\
9.5 \\
10.0 \\
10.0 \\
10.0\end{array}$ & $\begin{array}{l}8.5 \\
8.0 \\
8.0 \\
6.0 \\
4.5\end{array}$ & $\begin{array}{l}7.0 \\
7.0 \\
6.0 \\
5.0 \\
3.0\end{array}$ & $\begin{array}{l}7.5 \\
7.5 \\
7.5 \\
5.5 \\
4.0\end{array}$ & $\begin{array}{l}\cdots- \\
\cdots- \\
\cdots- \\
\cdots-\end{array}$ & $\begin{array}{l}--- \\
=-- \\
--- \\
---\end{array}$ & $\begin{array}{l}--- \\
--- \\
--- \\
--- \\
\end{array}$ & $\begin{array}{l}--- \\
--- \\
--- \\
---\end{array}$ & $\begin{array}{l}--- \\
--- \\
--- \\
---\end{array}$ & $\begin{array}{l}--- \\
--- \\
--- \\
---\end{array}$ \\
\hline $\begin{array}{l}26 \\
27 \\
28 \\
29 \\
30 \\
31\end{array}$ & $\begin{array}{r}10.0 \\
10.0 \\
9.5 \\
9.5 \\
9.5 \\
10.0\end{array}$ & $\begin{array}{l}9.5 \\
9.0 \\
9.0 \\
8.5 \\
8.5 \\
9.5\end{array}$ & $\begin{array}{l}9.5 \\
9.5 \\
9.5 \\
9.0 \\
9.0 \\
9.5\end{array}$ & $\begin{array}{l}4.5 \\
4.5 \\
4.0 \\
3.0 \\
3.5 \\
--.\end{array}$ & $\begin{array}{l}3.0 \\
3.5 \\
2.5 \\
2.0 \\
1.5 \\
--.\end{array}$ & $\begin{array}{l}4.0 \\
4.0 \\
3.5 \\
2.5 \\
2.5 \\
---\end{array}$ & $\begin{array}{l}--- \\
--- \\
--- \\
--- \\
---\end{array}$ & $\begin{array}{l}--- \\
--- \\
--- \\
--- \\
---\end{array}$ & $\begin{array}{l}--- \\
--- \\
--- \\
--- \\
--- \\
---\end{array}$ & $\begin{array}{l}--- \\
--- \\
--- \\
--- \\
---\end{array}$ & $\begin{array}{l}--- \\
--- \\
--- \\
--- \\
--- \\
--\end{array}$ & $\begin{array}{l}--- \\
--- \\
--- \\
--- \\
---\end{array}$ \\
\hline MONTH & --- & --- & -- & 10.5 & 1.5 & 6.3 & -- & --- & -- & --- & --- & -- \\
\hline
\end{tabular}


LOCATION. --Lat $44^{\circ} 46^{\prime} 22^{\prime \prime}$, long $92^{\circ} 54^{\prime} 07^{\prime \prime}$, NEtNEk sec.18, T.115 N., R.17 W., Dakota County, Bydrologic Unit 07010206 , on right bank at the end of'Jason Avenue, and at mile 817.8 upstream erom Ohio River.

DRAINAGE AREA. $-37,000 \mathrm{ml}^{2}\left(95,000 \mathrm{~km}^{2}\right)$, approximate1y.

PERIOD OF RECORD.--January 1977 to current year.

REMARKS. -Water-discharge computed on the basis of discharge for Mississippi River at St. Paul (station 05331000) adjusted for inflow and travel time. Letter $K$ indicates non-1deal colony count. Letter $E$ indicates estimated value.

WATER QUALITY DATA, WATER YEAR OCTOBER 1990 TO SEPTEMBER 1991

\begin{tabular}{|c|c|c|c|c|c|c|c|c|c|c|c|c|}
\hline ATE & TIME & $\begin{array}{c}\text { DIS- } \\
\text { CBARGE, } \\
\text { IN } \\
\text { CUBIC } \\
\text { FEET } \\
\text { PER } \\
\text { SECOND } \\
(00060)\end{array}$ & $\begin{array}{l}\text { SPE- } \\
\text { CIFIC } \\
\text { CON- } \\
\text { DUCY- } \\
\text { ANCE } \\
\text { (US/CM) } \\
\text { (00095) }\end{array}$ & $\begin{array}{c}\text { SPE- } \\
\text { CIFIC } \\
\text { CON- } \\
\text { DUCT- } \\
\text { ANCE } \\
\text { LAB } \\
\text { (US/CM) } \\
(90095)\end{array}$ & $\begin{array}{c}\text { PH } \\
\text { (STAND- } \\
\text { ARD } \\
\text { UNITS) } \\
(00400)\end{array}$ & $\begin{array}{c}\text { PA } \\
\text { LAB } \\
\text { (STAND- } \\
\text { ARD } \\
\text { UNITS) } \\
(00403)\end{array}$ & $\begin{array}{c}\text { TEMPER- } \\
\text { ATURE } \\
\text { WATER } \\
\text { (DEG C) } \\
(00010)\end{array}$ & $\begin{array}{c}\text { TUR- } \\
\text { BID- } \\
\text { ITY } \\
\text { (NTU) } \\
\text { (00076) }\end{array}$ & $\begin{array}{c}\text { BARO- } \\
\text { METRIC } \\
\text { PRES- } \\
\text { SURE } \\
\text { (MY } \\
\text { OF } \\
\text { BG) } \\
(00025)\end{array}$ & $\begin{array}{c}\text { OXYGEA, } \\
\text { DIS- } \\
\text { SOLVED } \\
(M G / L) \\
(00300)\end{array}$ & $\begin{array}{l}\text { COLI- } \\
\text { FORM, } \\
\text { FECAI, } \\
0.7 \\
\text { UM-MF } \\
\text { (COLS ' } \\
100 \mathrm{MI}) \\
(31625)\end{array}$ & $\begin{array}{c}\text { STREP- } \\
\text { TOCOCCI } \\
\text { FECAL } \\
\text { KF AGAR } \\
\text { (COLS. } \\
\text { PER } \\
100 \mathrm{ML}) \\
(31673)\end{array}$ \\
\hline
\end{tabular}

\begin{tabular}{|c|c|c|c|c|c|c|c|c|c|c|c|c|}
\hline $\begin{array}{l}\mathrm{NOV}_{28} \ldots \\
\mathrm{FEB}\end{array}$ & 1215 & 5610 & 449 & 508 & 8.5 & 8.2 & 2.5 & 15 & 738 & 15.2 & E380 & 100 \\
\hline $27 \ldots$ & 0830 & 3980 & 683 & 721 & 7.9 & 7.8 & 1.0 & 2.0 & 743 & 12.6 & 51 & $\mathbf{K} \boldsymbol{9}$ \\
\hline${ }_{\text {AUG }}^{17} \ldots$ & 1245 & 24500 & 545 & 556 & 8.2 & 8.1 & 7.5 & 35 & 747 & 12.0 & $\mathbf{K 3 2 0}$ & 620 \\
\hline $01 \ldots$ & 1215 & 17700 & 607 & 620 & 7.8 & 8.1 & 24.0 & 25 & 745 & 7.2 & 220 & 180 \\
\hline
\end{tabular}

$\begin{array}{cccc} & & \text { MAGN- } \\ & \text { CALCIUM } & \text { SIUM, } & \text { SODIUM, } \\ \text { DIS- } & \text { DIS- } & \text { DIS- } \\ & \text { SOLVED } & \text { SOLVED } & \text { SOLVED } \\ \text { SOTE } & (M G / L & (M G / L & (M G / L \\ & \text { AS CA) } & \text { AS MG) } & \text { AS MA) } \\ & (00915) & (00925) & (00930)\end{array}$
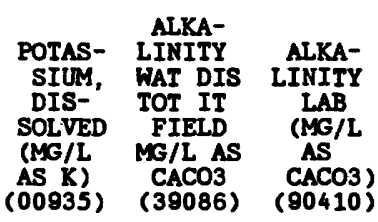

CAR-
BONATE
WATER
DIS IT
FIELD
MG/L AS
CO3
$(00452)$

BICAR-
BONATE
WATER
DIS IT
FIELD
MG $/ L$ AS
ECO3
$(00453)$

SULFATE
DIS-
SOLVED
(MG/L
AS SO4)
(00945)

$\begin{array}{ll}\text { CELO- } & \text { FLUO- } \\ \text { RIDE, } & \text { RIDE, } \\ \text { DIS- } & \text { DIS- } \\ \text { SOLVED } & \text { SOLVED } \\ \text { (MG/L } & (M G / L \\ \text { AS CL) } & \text { AS F) } \\ (00940) & (00950)\end{array}$

\begin{tabular}{|c|c|c|}
\hline $\begin{array}{c}\text { NOV } \\
28 \ldots \\
\text { FFB }\end{array}$ & 53 & 20 \\
\hline${ }_{A P R}^{27} \cdots$ & 66 & 23 \\
\hline${ }_{A U G}^{17} \cdots$ & 68 & 24 \\
\hline $01 \ldots$ & 73 & 27 \\
\hline
\end{tabular}

$\begin{array}{rr}3.2 & 174 \\ 4.3 & 218 \\ 4.3 & 166 \\ 4.0 & --\end{array}$

188
219
169
203

5
0
0

202
266
203
--

$$
\begin{aligned}
& 40 \\
& 47 \\
& 70 \\
& 90
\end{aligned}
$$

40
47
70
90

29
68
25
22

$\mathbf{0 . 3}$

0.2

0.1

0.3

$\mathbf{k g}$

180 
MISSISSIPPI RIVER MAIN STEM

05331570 MISSISSIPPI RIVER AT NININGER, MN--Continued

WATER QUALITY DATA, WATER YEAR OCTOBER 1990 TO SEPTEMBER 1991

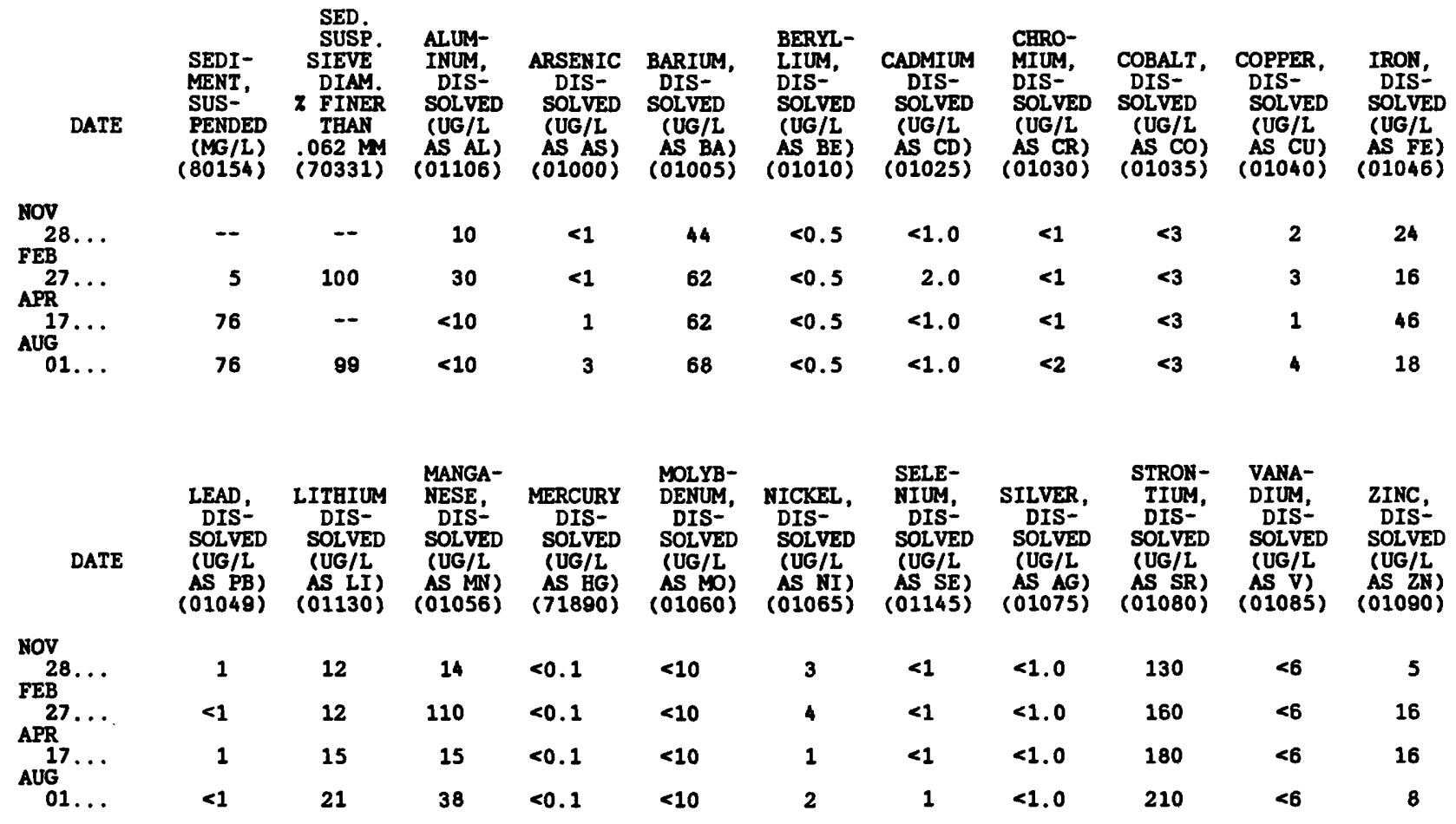




\section{ST. CROIX RIVER BASIN}

05336700 KETTLE RIVER BELOW SANDSTONE, MT

LOCATION, --Lat $46^{\circ} 06^{\prime} 20^{\prime \prime}$, long $92^{\circ} 51^{\prime} 50^{\prime \prime}$, in NWtSW sec.22, T.42 N., R.20 W., Pine County, Hydrologic Unit 07030003, on Sandstone Federal Correctional Institution property, on left bank about 900 ft downstream from abandoned powerplant dam, $1.8 \mathrm{mi}$ south of Sandstone.

DRAIMAGE AREA. $--863 \mathrm{mi}^{2}$

PERIOD OF RECORD. - October 1967 to current year.

GAGE.--Water-stage recorder and crest-stage gage. Datum of gage is 931.50 ft above National Geodetic Vertical Datum of 1929. (Minnesota Department of Transportation bench mark).

REMARKS. - Records good except those for estimated daily discharge, which are fair.

AVERAGE DISCBARGE. -24 years, $716 \mathrm{ft}^{3} / \mathrm{s}, 11.27 \mathrm{in} / \mathrm{yr}$.

EXTREMES FOR PERIOD OF RECORD. --Maximum discharge, 17, $200 \mathrm{ft} 3 / \mathrm{s}, \mathrm{July} 23,1972, \mathrm{gage}$ height, $15.38 \mathrm{ft}$; minimum, $25 \mathrm{ft}^{3} / \mathrm{s}$. Nov $11,12,1977$, gage height, $3.37 \mathrm{ft}$ result of freezeup.

EXTREMES OUTSIDE PERIOD OF RECORD.--Flood in April 1965 reached a stage of $12.96 \mathrm{ft}$, from flood marks, discharge, $13,400 \mathrm{ft}^{3} / \mathrm{s}$

EXIREMES FOR CURRENT YEAR. - Peak discharges greater than base discharge of $3,600 \mathrm{ft} 3 / \mathrm{s}$ and maximum (*)

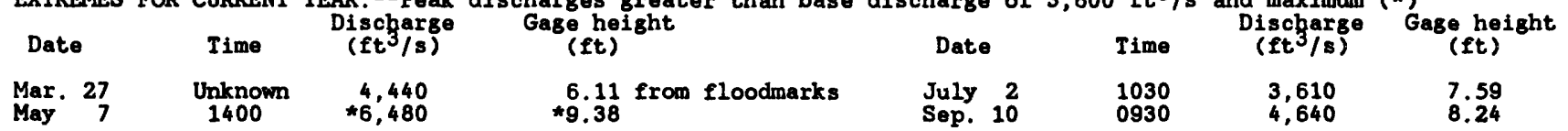

DISCAARGE, CUBIC FEET PER SECOND, WATER YEAR OCTOBER 1990 TO SEPTEMBER 1991

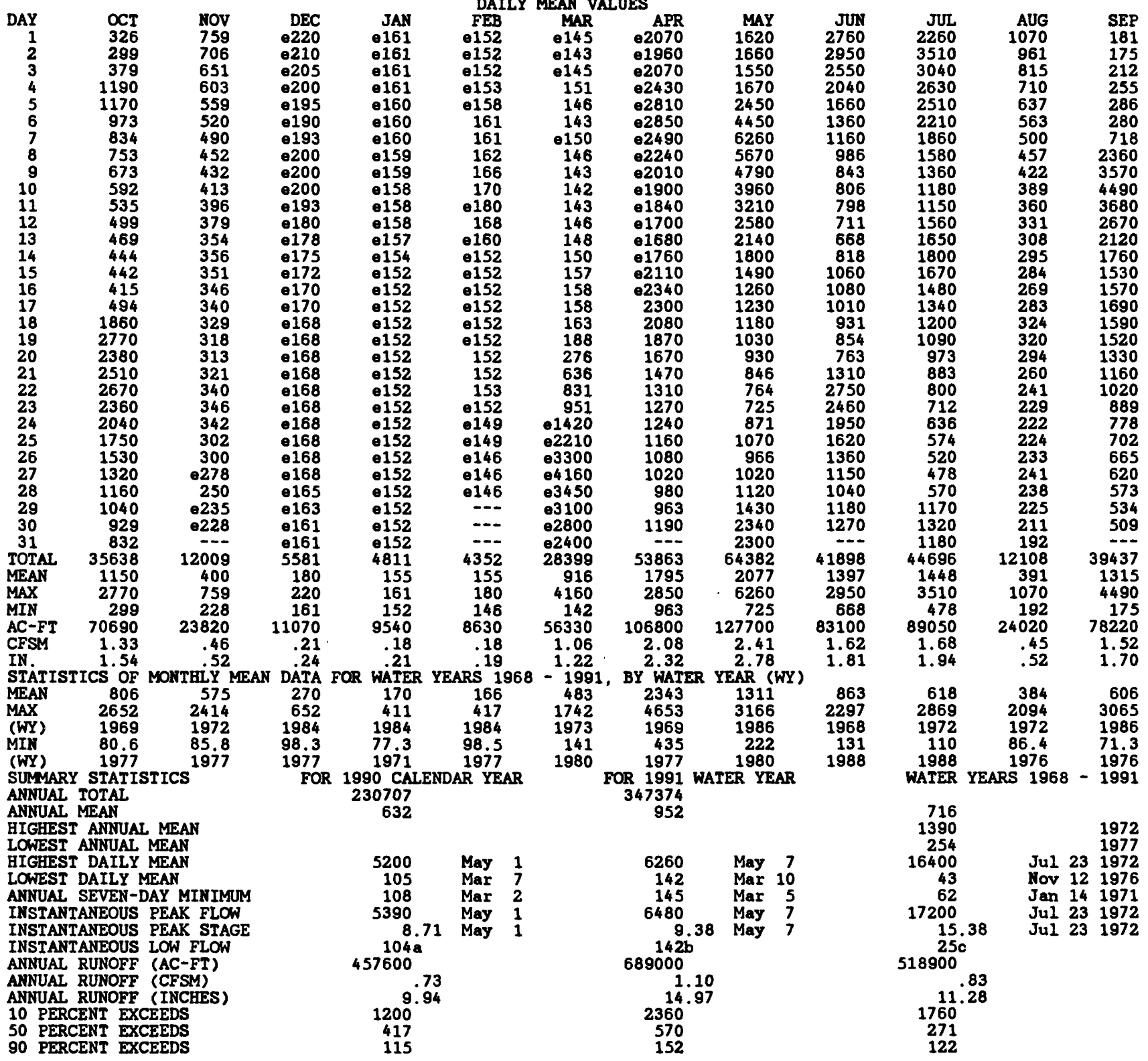

a Occurred Mar. 6, 7,8.

b Occurred Mar. 6, 7,9, 10, 11 .

c Occurred Nov. 1i, 12,1977 , result of freeze up.

Estimated. 


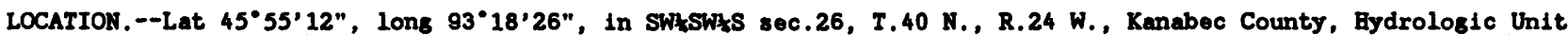
07030004 , on left bank $400 \mathrm{ft}$ upstream from bridge on County Bighway 77, 1.1 mi upstream from mouth and 2.5 mi north of Mora.

DRAIKAGE AREA.--102 $\mathrm{mi}^{2}$.

PERIOD OF RECORD.--Occasional low-flow measurements, water years 1969-74; July 1974 to current year.

GAGE. - Water-stage recorder. Datum of sage 1s $991.20 \mathrm{ft}$ above National Geodetic Vertical Datum of 1929.

(Kanabec County bench mark).

REMARKS.--Records good except those for periods of estimated daily discharges, which are fair.

EXTREMES OUTSIDE PERIOD OF RECORD. - Flood of July 26,1972 reached a stage of 14.0 ft, from information by local resident (discharse not determined). Result of dam failure and backwater from collapsed bridge.

EXTREMES FOR CURRENT YEAR. --Peak discharges greater than base dischargo of $500 \mathrm{ft}^{3} / \mathrm{s}$ and maximum ( $*$ ):

\begin{tabular}{|c|c|c|c|c|c|c|c|}
\hline Date & Time & $\begin{array}{l}\text { Dischargo } \\
\left(\mathrm{ft}^{3} / \mathrm{s}\right)\end{array}$ & $\begin{array}{c}\text { Gage height } \\
\text { (ft) }\end{array}$ & Date & Time & $\begin{array}{c}\text { Discharge } \\
\left(\mathrm{ft}^{3} / \mathrm{s}\right)\end{array}$ & $\begin{array}{c}\text { Gage height } \\
\text { (ft) }\end{array}$ \\
\hline
\end{tabular}

DISCHARGE, CUBIC FEET PER SECOND, WATER YEAR OCTOBER 1990 TO SEPTEMBER 1991

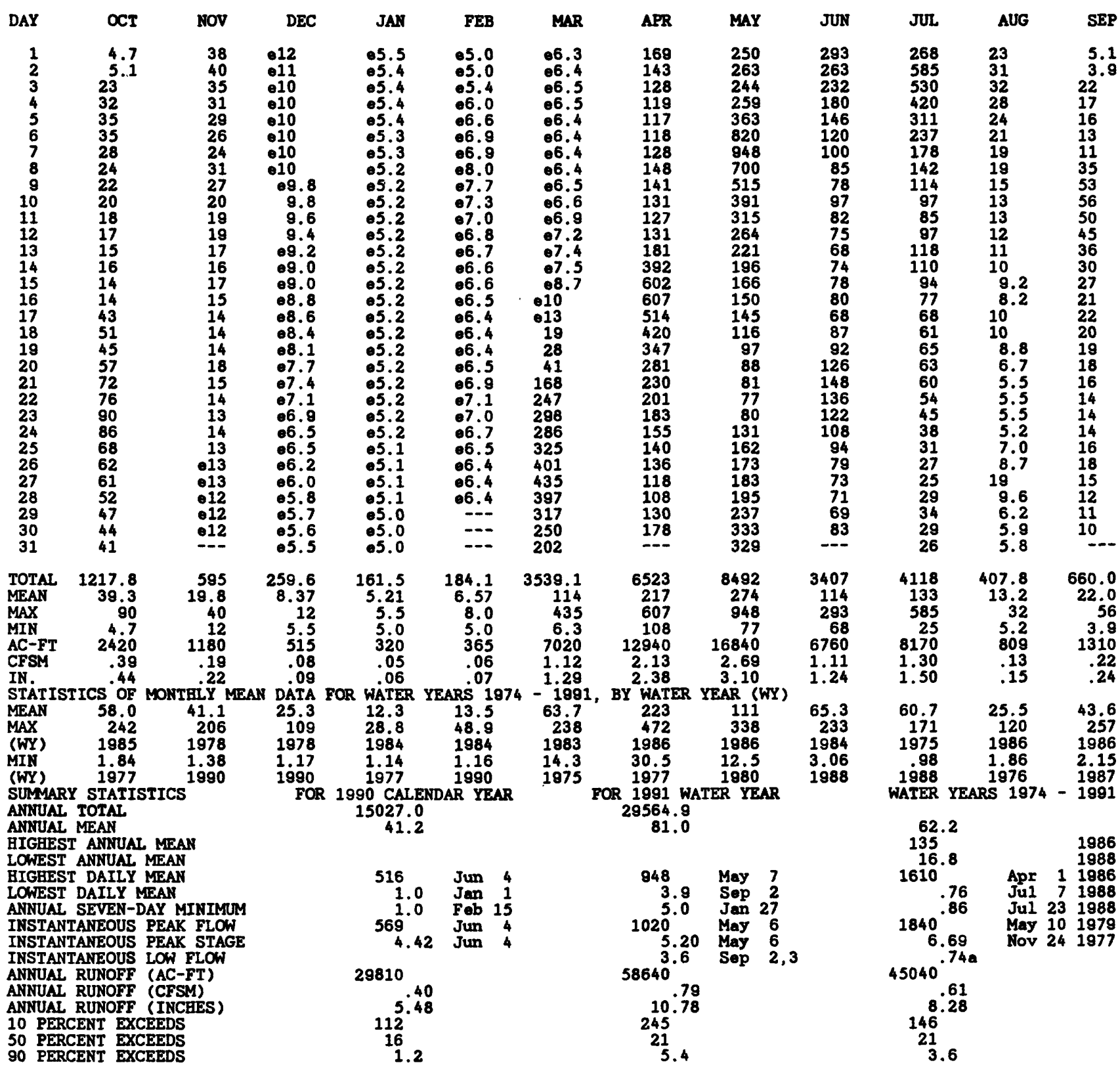

a Occurred July 6, 7, 28, 29, 1988.

- Estimated. 
ST. CROIX RIVER BASIN

05340500 ST. CROIX RIVER AT ST. CROIX FALLS, WI

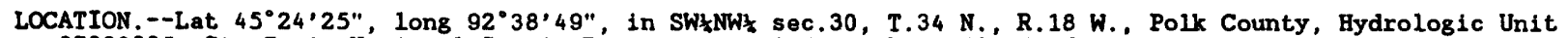
07030005 , St. Croix National Scenic Riverway, on left bank, 1,500 ft downstream from powerplant of Northern States Power Co., in St. Croix Falls, and at mile 52.2.

DRAINAGE AREA. $--6,240 \mathrm{mi}^{2}$.

PERIOD OF RECORD.--January 1902 to current year. Prior to January 1910, monthly discharge only, published in WSP 1308. Prior to October 1939, published as "near St. Croix Falls."

REVISED RECORDS.--WSP 1115: 1929. WDR WI-82-1: Drainage area.

GAGE.--Water-stage recorder and crest-stage gage. Datum of gage is $689.94 \mathrm{ft}$ above National Geodetic Vertical Datum of 1929. Prior to July 1905, gage heights and discharge measurements were used by Loweth and Wolff. consulting engineers of St. Paul, Minn., to determine the flow. July 1905 to February 1940, records were computed from power generation at the St. Croix Falls Powerplant. February 1940 to Sept. 30, 1979, waterstage recorder at site $300 \mathrm{ft}$ downstream at same datum.

REMARKS.--Estimated daily discharges: June 13-24, based on powerplant data provided by Northern States Power Company. Records good. Diurnal fluctuation caused by St. Croix Falls Powerplant 1,500 ft upstream. Datacollection platform at station.

AVERAGE DISCHARGE. --89 years, $4,312 \mathrm{ft}^{3} / \mathrm{s}, 9.38 \mathrm{in} / \mathrm{yr}$.

EXTREMES FOR PERIOD OF RECORD,--Maximum discharge, 54,900 ft3/s, May 8, 1950, gage height, $25.19 \mathrm{ft}$; minimum daily, $75 \mathrm{ft}^{3 / \mathrm{s}}$, July 17, i910.

EXTREMES FOR CURRENT YEAR.--Maximum discharge, 25,300 $\mathrm{ft}^{3} / \mathrm{s}$, May 9, gage height, $11.97 \mathrm{ft}$; minimum daily, $1,420 \mathrm{ft}^{3} / \mathrm{s}$, Dec. 4 .

RATING TABLE (gage height, in feet, and discharge, in cubic feet per second).

$\begin{array}{rrrr}2.5 & 1,400 & 6.0 & 10,700 \\ 3.0 & 2,350 & 8.0 & 15,700 \\ 4.0 & 4,950 & 12.0 & 25,400\end{array}$

DISCHARGE, CUBIC FEET PER SECOND, WATER YEAR OCTOBER 1990 TO SEPTEMBER 1991

\begin{tabular}{|c|c|c|c|c|c|c|c|c|c|c|c|c|}
\hline DAY & $\propto T$ & NOV & DEC & JAN & FEB & MAR & APR & MAY & JUN & JUL & AUG & SEP \\
\hline $\begin{array}{l}1 \\
2 \\
3 \\
4 \\
5\end{array}$ & $\begin{array}{l}2590 \\
2310 \\
2830 \\
2710 \\
3560\end{array}$ & $\begin{array}{l}4520 \\
4090 \\
4130 \\
3950 \\
3640\end{array}$ & $\begin{array}{l}2640 \\
2060 \\
2070 \\
1420 \\
1820\end{array}$ & $\begin{array}{l}1800 \\
2380 \\
1820 \\
2010 \\
1810\end{array}$ & $\begin{array}{l}2120 \\
1610 \\
1670 \\
1930 \\
1810\end{array}$ & $\begin{array}{l}1820 \\
2040 \\
2000 \\
2030 \\
1870\end{array}$ & $\begin{array}{r}11900 \\
10400 \\
9210 \\
8690 \\
8400\end{array}$ & $\begin{array}{r}7950 \\
9920 \\
10700 \\
10600 \\
11000\end{array}$ & $\begin{array}{l}12800 \\
14000 \\
16200 \\
15700 \\
12600\end{array}$ & $\begin{array}{r}7030 \\
9130 \\
10500 \\
10900 \\
10600\end{array}$ & $\begin{array}{l}6220 \\
5950 \\
5980 \\
5480 \\
4610\end{array}$ & $\begin{array}{l}2040 \\
1710 \\
3460 \\
2880 \\
2480\end{array}$ \\
\hline $\begin{array}{r}6 \\
7 \\
8 \\
9 \\
10\end{array}$ & $\begin{array}{l}4000 \\
3780 \\
3560 \\
3130 \\
3460\end{array}$ & $\begin{array}{l}4050 \\
3190 \\
3310 \\
2960 \\
3300\end{array}$ & $\begin{array}{l}2340 \\
2710 \\
2730 \\
2760 \\
2230\end{array}$ & $\begin{array}{l}1790 \\
1870 \\
1660 \\
1970 \\
1790\end{array}$ & $\begin{array}{l}1570 \\
2180 \\
1790 \\
2130 \\
1870\end{array}$ & $\begin{array}{l}2000 \\
1880 \\
2080 \\
1720 \\
1780\end{array}$ & $\begin{array}{l}8430 \\
8460 \\
8650 \\
9090 \\
9240\end{array}$ & $\begin{array}{l}15800 \\
19900 \\
24000 \\
24800 \\
23400\end{array}$ & $\begin{array}{r}11000 \\
9680 \\
8380 \\
7480 \\
7040\end{array}$ & $\begin{array}{r}10600 \\
10100 \\
9220 \\
8270 \\
7620\end{array}$ & $\begin{array}{l}4130 \\
4390 \\
3660 \\
3600 \\
3340\end{array}$ & $\begin{array}{r}2700 \\
2570 \\
4180 \\
9100 \\
11700\end{array}$ \\
\hline $\begin{array}{l}11 \\
12 \\
13 \\
14 \\
15\end{array}$ & $\begin{array}{l}2940 \\
3200 \\
2500 \\
3010 \\
2810\end{array}$ & $\begin{array}{l}3130 \\
2790 \\
2820 \\
3390 \\
3080\end{array}$ & $\begin{array}{l}2840 \\
2450 \\
2570 \\
2160 \\
2340\end{array}$ & $\begin{array}{l}2080 \\
1850 \\
1630 \\
1990 \\
1860\end{array}$ & $\begin{array}{l}2090 \\
2070 \\
1910 \\
1650 \\
2050\end{array}$ & $\begin{array}{l}2220 \\
1910 \\
2210 \\
2080 \\
2200\end{array}$ & $\begin{array}{r}9230 \\
9110 \\
9220 \\
10100 \\
11000\end{array}$ & $\begin{array}{l}20900 \\
18000 \\
15600 \\
13700 \\
11600\end{array}$ & $\begin{array}{l}6740 \\
6360 \\
5450 \\
5980 \\
5860\end{array}$ & $\begin{array}{l}7070 \\
6790 \\
7710 \\
8220 \\
7800\end{array}$ & $\begin{array}{l}3200 \\
3010 \\
3160 \\
2710 \\
2710\end{array}$ & $\begin{array}{r}13600 \\
13600 \\
11500 \\
10500 \\
9280\end{array}$ \\
\hline $\begin{array}{l}16 \\
17 \\
18 \\
19 \\
20\end{array}$ & $\begin{array}{l}2840 \\
3480 \\
3550 \\
5240 \\
7410\end{array}$ & $\begin{array}{l}2900 \\
2830 \\
2900 \\
2890 \\
2910\end{array}$ & $\begin{array}{l}1920 \\
2250 \\
2240 \\
2110 \\
1890\end{array}$ & $\begin{array}{l}1960 \\
2250 \\
1910 \\
2020 \\
2340\end{array}$ & $\begin{array}{l}2010 \\
1800 \\
1880 \\
1800 \\
1850\end{array}$ & $\begin{array}{l}2580 \\
2290 \\
2330 \\
2900 \\
3190\end{array}$ & $\begin{array}{l}11700 \\
12500 \\
12400 \\
11900 \\
11300\end{array}$ & $\begin{array}{r}10400 \\
9210 \\
8780 \\
9630 \\
7720\end{array}$ & $\begin{array}{l}7050 \\
7050 \\
7090 \\
6940 \\
6540\end{array}$ & $\begin{array}{l}7370 \\
6710 \\
6410 \\
5610 \\
5820\end{array}$ & $\begin{array}{l}2740 \\
2720 \\
2360 \\
2770 \\
2630\end{array}$ & $\begin{array}{l}7570 \\
7620 \\
7650 \\
7360 \\
7480\end{array}$ \\
\hline $\begin{array}{l}26 \\
27 \\
28 \\
29 \\
30 \\
31\end{array}$ & $\begin{array}{l}7150 \\
6550 \\
5840 \\
5470 \\
4860 \\
4710\end{array}$ & $\begin{array}{l}2230 \\
2820 \\
2270 \\
1760 \\
1820\end{array}$ & $\begin{array}{l}2030 \\
1810 \\
2030 \\
2350 \\
2070 \\
1820\end{array}$ & $\begin{array}{l}1740 \\
1710 \\
1940 \\
1700 \\
1900 \\
1950\end{array}$ & $\begin{array}{r}1830 \\
1850 \\
2010 \\
--- \\
--- \\
--\end{array}$ & $\begin{array}{l}14800 \\
16200 \\
18000 \\
17800 \\
15800 \\
13600\end{array}$ & $\begin{array}{l}7360 \\
6820 \\
6640 \\
6330 \\
6320\end{array}$ & $\begin{array}{r}7590 \\
9250 \\
11200 \\
11600 \\
11800 \\
12600\end{array}$ & $\begin{array}{l}8210 \\
7300 \\
6960 \\
5610 \\
6020\end{array}$ & $\begin{array}{l}3970 \\
3780 \\
3800 \\
3870 \\
5250 \\
6010\end{array}$ & $\begin{array}{l}2680 \\
2410 \\
2270 \\
2210 \\
2080 \\
2040\end{array}$ & $\begin{array}{l}4940 \\
5090 \\
4560 \\
4120 \\
3820\end{array}$ \\
\hline $\begin{array}{l}\text { TOTAL } \\
\text { MEAN } \\
\text { MAX } \\
\text { MIN } \\
\text { CFSM } \\
\text { IN. }\end{array}$ & $\begin{array}{r}143090 \\
4616 \\
8460 \\
2310 \\
.74 \\
.85\end{array}$ & $\begin{array}{r}91670 \\
3056 \\
4520 \\
1760 \\
.49 \\
.55\end{array}$ & $\begin{array}{r}65770 \\
2122 \\
2840 \\
1420 \\
.34 \\
.39\end{array}$ & $\begin{array}{r}59820 \\
1930 \\
2380 \\
1630 \\
.31 \\
.36\end{array}$ & $\begin{array}{r}53080 \\
1896 \\
2180 \\
1570 \\
.30 \\
.32\end{array}$ & $\begin{array}{r}183630 \\
5924 \\
18000 \\
1720 \\
.95 \\
1.09\end{array}$ & $\begin{array}{r}279510 \\
9317 \\
12500 \\
6320 \\
1.49 \\
1.67\end{array}$ & $\begin{array}{r}377070 \\
12160 \\
24800 \\
5240 \\
1.95 \\
2.25\end{array}$ & $\begin{array}{r}260260 \\
8675 \\
16200 \\
5450 \\
1.39 \\
1.55\end{array}$ & $\begin{array}{r}215960 \\
6966 \\
10900 \\
3780 \\
1.12 \\
1.29\end{array}$ & $\begin{array}{r}100950 \\
3256 \\
6220 \\
2040 \\
.52 \\
.60\end{array}$ & $\begin{array}{r}192390 \\
6413 \\
13600 \\
1710 \\
1.03 \\
1.15\end{array}$ \\
\hline
\end{tabular}

CAL YR 1990 TOTAL 1368710 MEAN 3750 MAX 15700 MIN 1200 CFSM .60 IN. 8.16 WTR YR 1991 TOTAL 2023200 MEAN 5543 MAX 24800 MIN 1420 CFSM .89 IN. 12.06 
LOCATION.--Lat $44^{\circ} 44^{\prime} 45^{\prime \prime}$, long 92*48'00", in sec.9, T.26 N., R. 20 W., Pierce County, Hydrologic Unit 07040001, on left bank at Prescott, $200 \mathrm{ft}$ downstream from St. Croix River, 300 ft south of Chicago, Burlington \& Quincy Railroad bridge, $800 \mathrm{ft}$ south of bridge on U.S. Highway 10 , and at mile 811.4 upstream from Ohio River.

DRAINAGE AREA. $-44,800 \mathrm{mi}^{2}$ approximately.

PERIOD OF RECORD. --June 1928 to current year.

REVISED RECORDS.--WSP 1508: 1941. WRD MN-74: 1973.

GAGE. - Water-stage recorder. Datum of gage is $64 \dot{9} .50 \mathrm{ft}$ above National Geodetic Vertical Datum of 1929. Prior to Aug. 2, 1932, nonrecording gage at railroad bridge 300 ft upstream at following datums: June 3 , 1928, to Sept. 30, 1929, 19.27 ft higher; Oct. 1, 1929, to Sept. 30, 1930, 17.68 ft higher; Oct. 1, 1930, to Aug. 1, 1932 , $19.28 \mathrm{ft}$ higher. Aug. 2, 1932, to Oct. 30 , 1938, water-stage recorder at present site at datum 19.28 ft higher; Nov. 1, 1938, to Sept. 7, 1971, water-stage recorder at present site at datum 50.00 ft lower.

REMARKS. - Records good. Some regulation by reservoirs, navigation dams, and powerplants at low and medium stages. Flood flow not materially affected by artificial storage.

AVERAGE DISCHARGE - -63 years, $17,182 \mathrm{ft} / \mathrm{s}, 5.21 \mathrm{in} / \mathrm{yr}$; median of yearly mean $\mathrm{discharges,} 16,000 \mathrm{ft} / \mathrm{s}, 4.85 \mathrm{in} / \mathrm{yr}$ EXTREMES FOR PERIOD OF RECQRD. - Maximum daily discharge, 228,000 ft $/ \mathrm{s}$, Apr. 18, 1965, gage height, 43.11 ft; minimum daily, $1,380 \mathrm{ft}^{3} / \mathrm{s}$, July 13,1940 ; minimum gage height, $15.08 \mathrm{ft}$, Aug. 29, 1934 , present datum.

EXTREMES FOR CURRENT YEAR. --Maximum daily discharge, 74,300 ft $/ \mathrm{s}$, May 12; maximum gage height, 33.27 ft,

May 11; minimum daily discharge, 4,320 ft $3 / \mathrm{s}$, Dec. 23; minimum gage height, $24.53 \mathrm{ft}$, Dec. 25 , result of

freeze up.

DISCHARGE, CUBIC FEET PER SECOND, WATER YEAR OCTOBER 1990 TO SEPTEMBER 1991

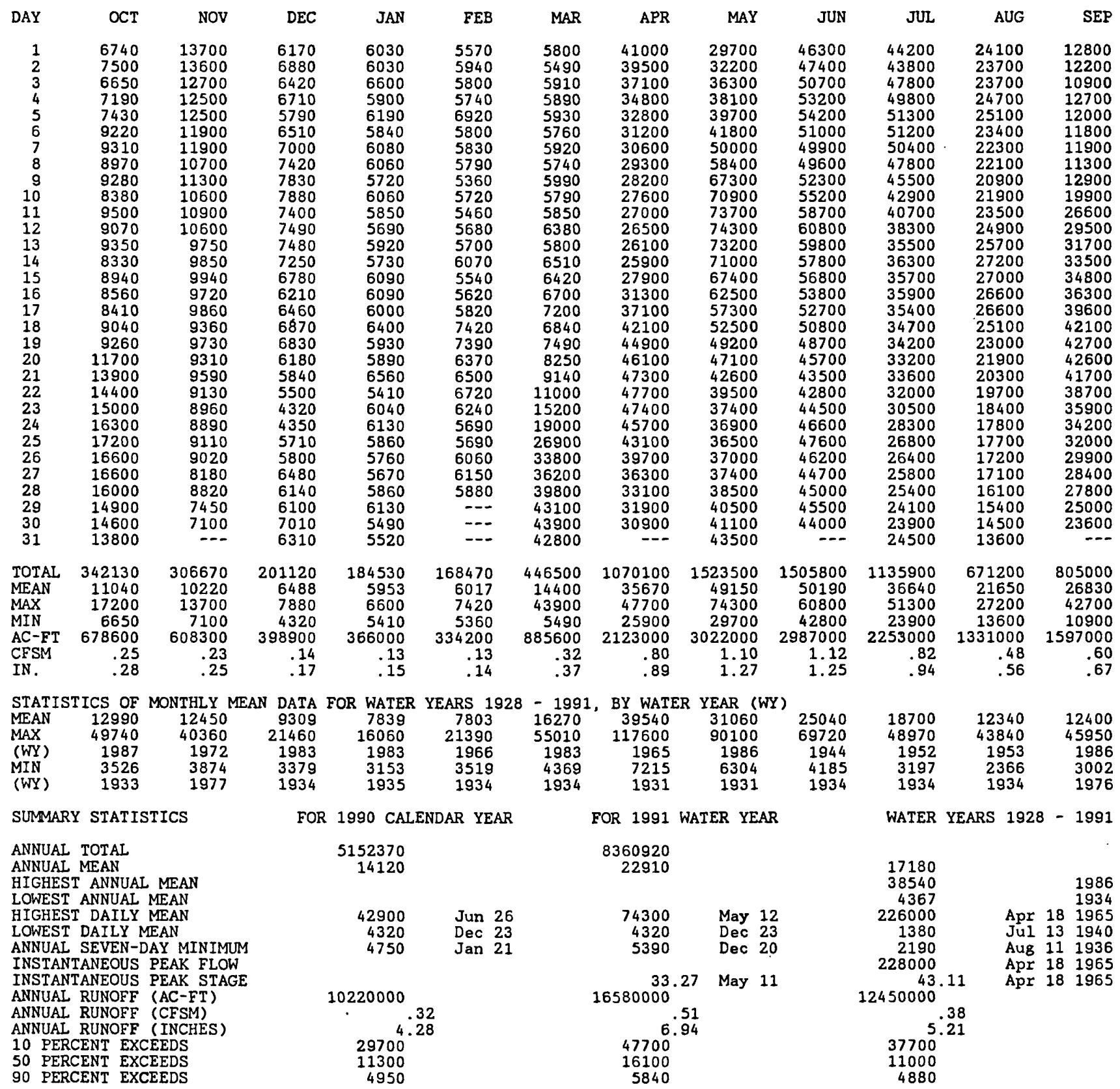


VERMILLION RIVER BASIN

05345000 VERMILLION RIVER NEAR EMPIRE, MN

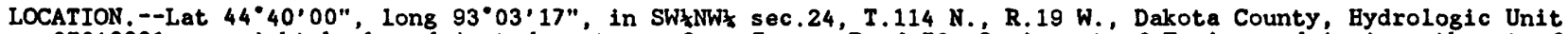
07040001 , on right bank and just downstream from County Road 79, 2 mi west of Empire and 4 mi northeast of Farmingtion.

DRAINAGE AREA. $-110 \mathrm{mi}^{2}$.

WATER-DISCHARGE RECORDS

PERIOD OF RECORD.--May 1942 to June 1945 (no record during July, August, and September 1944 ), September 1969 to September 1973 (discharge measurements only), October 1973 to current year. Prior to October 1975 published as "near Empire City".

GAGE.--Water-stage recorder. Datum of gage is $851.99 \mathrm{ft}$ above National Geodetic Vertical Datum of 1929 (levels by U.S. Army Corps of Engineers). Apri1 12, 1942, to June 30, 1944, and October 1, 1944, to July 7, 1945, nonrecording gage at same site and present datum.

REMARKS. - Records good except those for estimated daily discharges, which are fair. Some regulation at low-flow by sewage plant upstream.

EXTREMES FOR CURRENT YEAR. --Peak discharges greater than base discharge of $200 \mathrm{ft}{ }^{3} / \mathrm{s}$ and maximum (*):

\begin{tabular}{|c|c|c|c|c|c|c|c|}
\hline Date & Time & $\begin{array}{c}\text { Discharge } \\
\left(\mathrm{ft}^{3} / \mathrm{s}\right)\end{array}$ & $\begin{array}{c}\text { Gage height } \\
\text { (ft) }\end{array}$ & Date & Time & $\begin{array}{c}\text { Discharge } \\
\left(\mathrm{ft}^{3} / \mathrm{s}\right)\end{array}$ & $\begin{array}{c}\text { Gage height } \\
\text { (ft) }\end{array}$ \\
\hline May 6 & 2000 & $\star 256$ & $\star 5.49$ & May 30 & 1330 & 204 & 5.09 \\
\hline
\end{tabular}

DISCHARGE, CUBIC FEET PER SECOND, WATER YEAR OCTOBER 1990 TO SEPTEMBER 1991

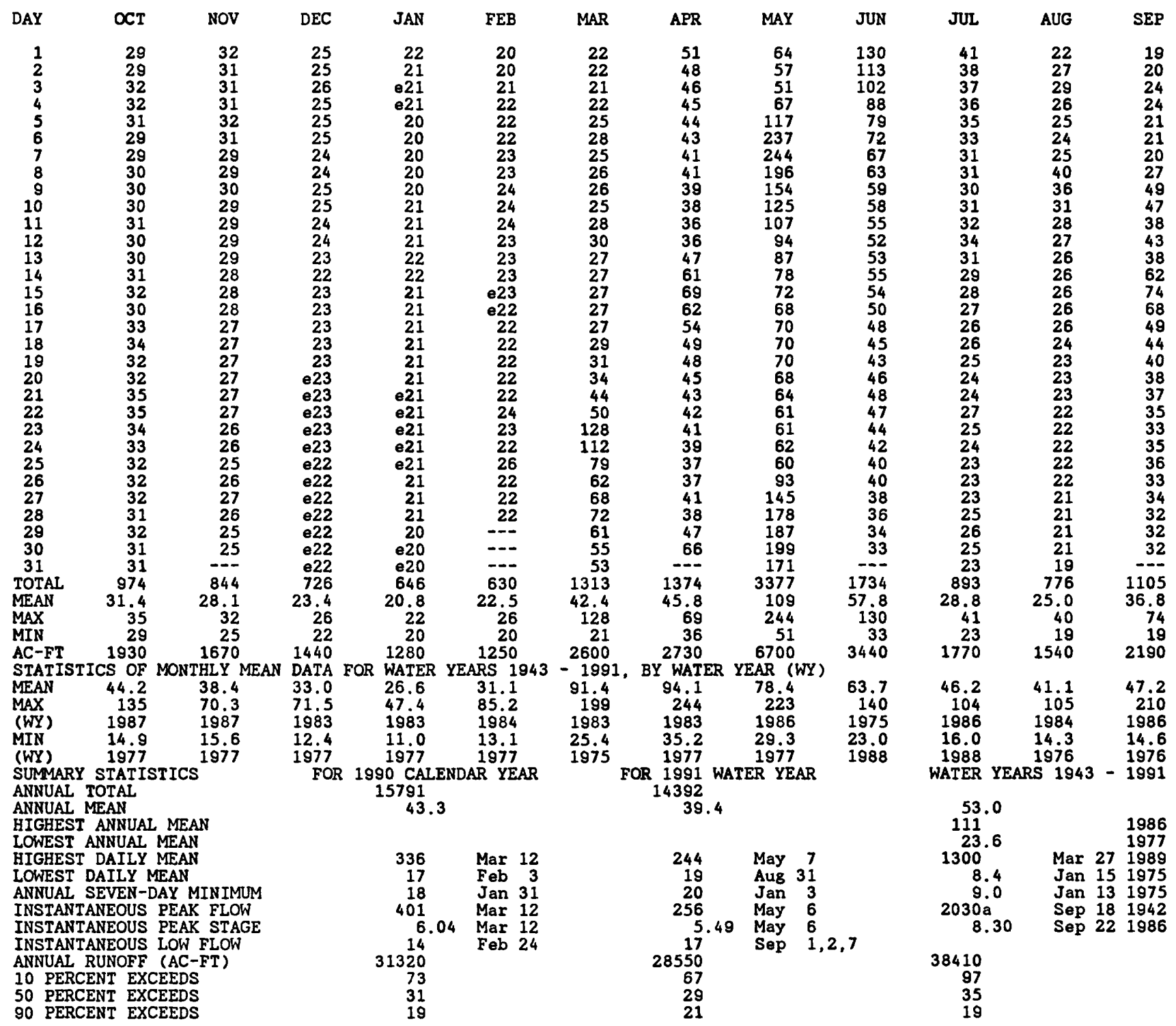

a Instantaneous peak flow outside period of record is $6,200 \mathrm{ft} 3 / \mathrm{s}$ in Apr. 1965 , from rating extended above $2,100 \mathrm{ft}^{3} / \mathrm{s}$.

estimated. 
VERMILLIOA RIVER BASIN

05345000 VERMILLION RIVER REAR EMPIRE, MH--Continued

WATER QUALITY RECORDS

PERIOD OF RECORD.--1972-76, 1980-81, 1990 to current year.

WATER QUALITY DATA, WATER YEAR OCTOBER 1990 TO SEPTEMBER 1991

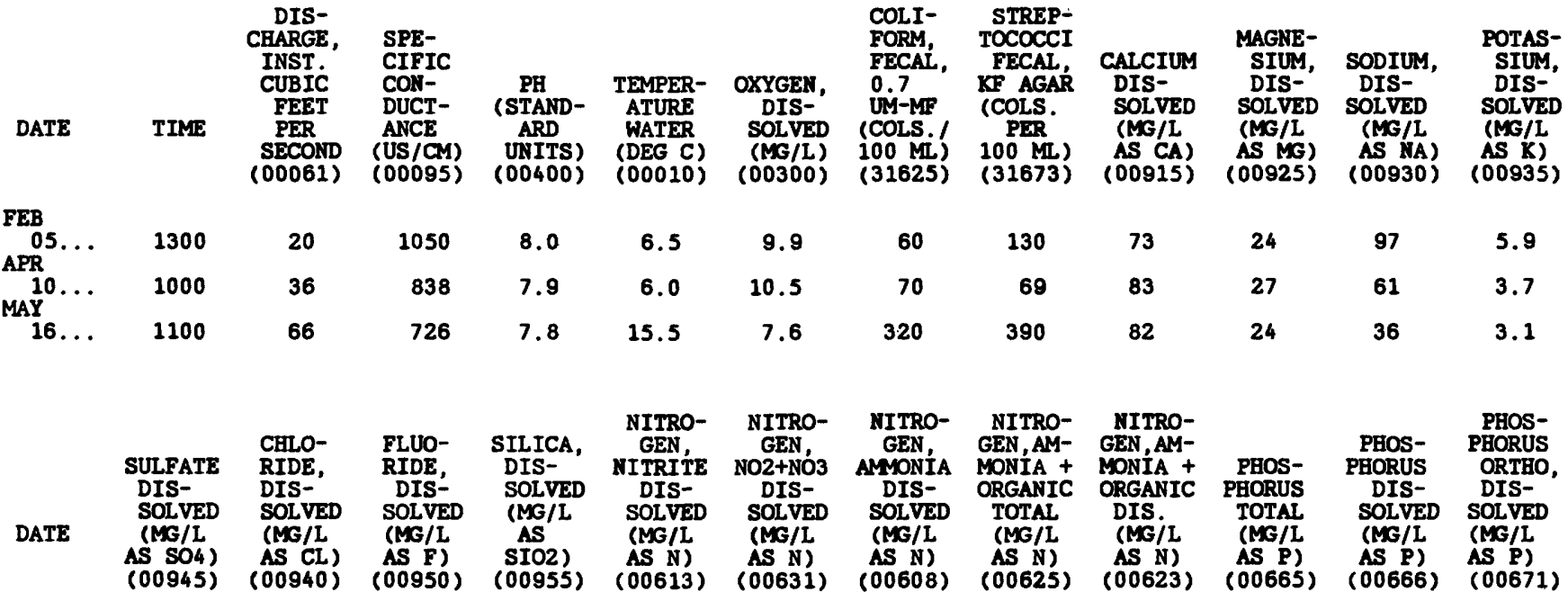

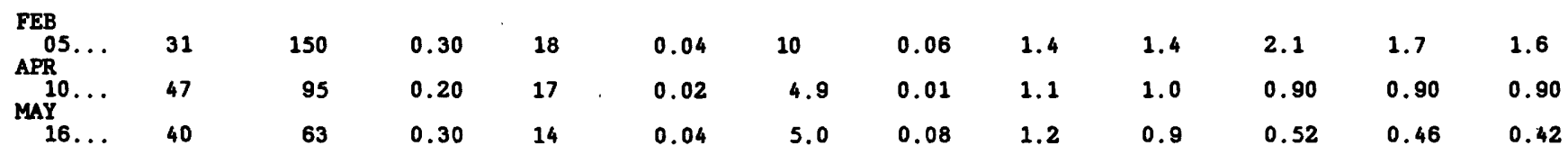

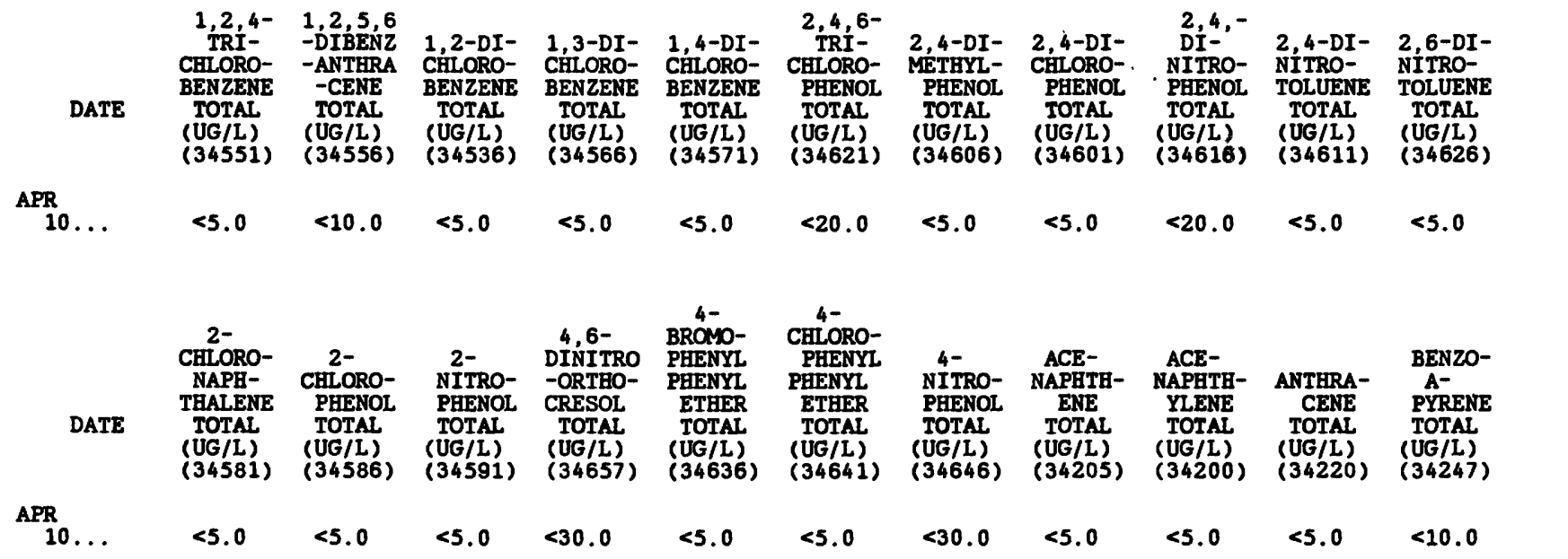


VERMILLION RIVER BASIN

05345000 VERMILLION RIVER NEAR DMPIRE, MN--Continued

WATER QUALITY DATA, WATER YEAR OCTOBER 1990 TO SEPTEMBER 1991

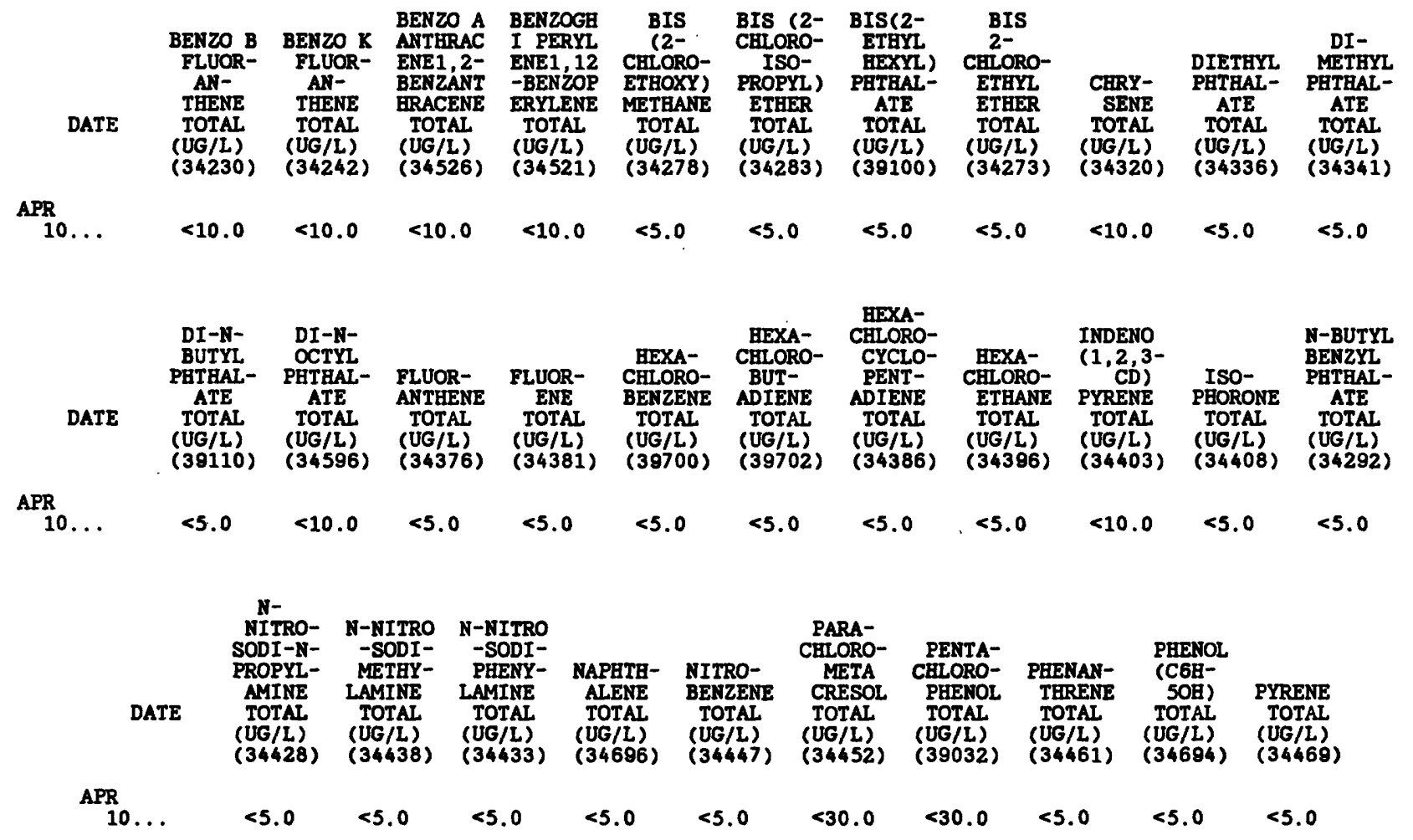


CANNON RIVER BASIN

05353800 STRAIGHT RIVER NEAR FARIBAULT, MN

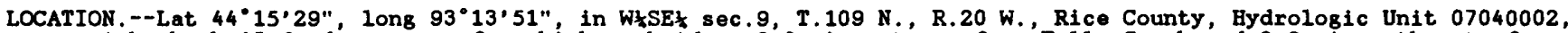
on right bank $15 \mathrm{ft}$ downstream from highway bridge, $2.8 \mathrm{mi}$ upstream from Falls Creek and $3.2 \mathrm{mi}$ southeast of Faribault.

DRAINAGE AREA. $--442 \mathrm{mi}^{2}$

PERIOD OF RECORD.--October 1965 to current year.

GAGE. - Water-stage recorder. Datum of gage is $1,034.58 \mathrm{ft}$ above National Geodetic Vertical Datum of 1929.

REMARKS. - Records good except those for estimated daily discharges, which are fair

EXTREMES FOR CURRENT YEAR. - Peak discharge greater than base discharge of $1,500 \mathrm{ft} 3 / \mathrm{s}$ and maximum (

\begin{tabular}{|c|c|c|c|c|c|c|c|}
\hline Date & Time & $\begin{array}{c}\text { Discharge } \\
\left(\mathrm{ft}^{3} / \mathrm{s}\right)\end{array}$ & $\begin{array}{c}\text { Gage height } \\
\text { (ft) }\end{array}$ & Date & Time & $\begin{array}{c}\text { Discharge } \\
\left.\text { (ft } \mathrm{ft}^{3} / \mathrm{s}\right)\end{array}$ & $\begin{array}{c}\text { Gage height } \\
\text { (ft) }\end{array}$ \\
\hline $\begin{array}{lr}\text { May } & 6 \\
\text { May } & 18 \\
\text { May } & 28\end{array}$ & $\begin{array}{l}2100 \\
2200 \\
1200\end{array}$ & $\begin{array}{l}2,760 \\
1,500 \\
1,520\end{array}$ & $\begin{array}{l}8.60 \\
6.96 \\
6.98\end{array}$ & $\begin{array}{lr}\text { Jul. } & 12 \\
\text { Jul. } & 22 \\
\text { Aug. } & 8\end{array}$ & $\begin{array}{l}0900 \\
1700 \\
1400\end{array}$ & $\begin{array}{r}2,780 \\
\star 3,720 \\
3,280\end{array}$ & $\begin{array}{r}8.62 \\
* 9.58 \\
9.17\end{array}$ \\
\hline
\end{tabular}

DISCHARGE, CUBIC FEET PER SECOND, WATER YEAR OCTOBER 1990 TO SEPTEMBER 1991

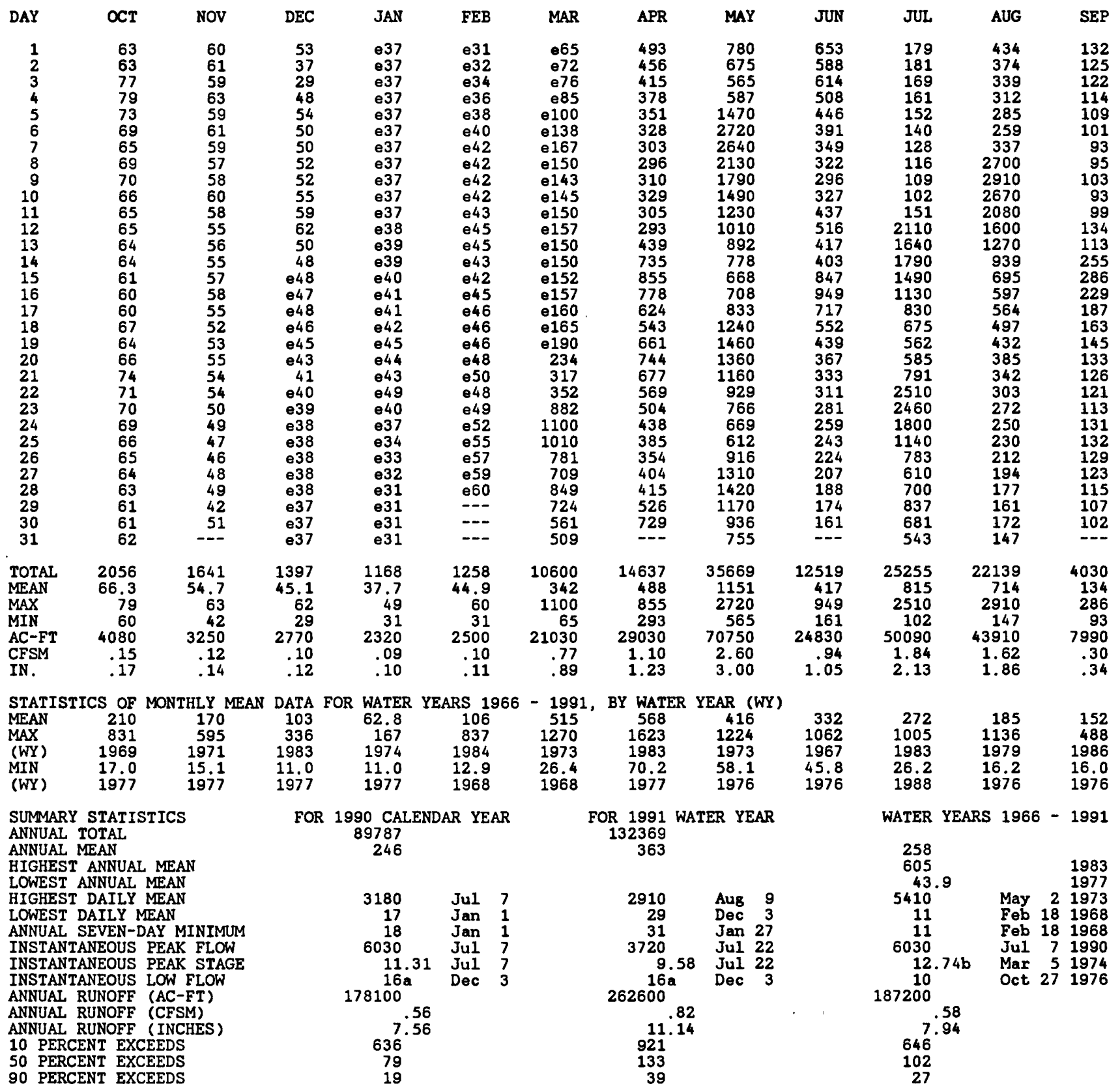

a Result of temporary storage from freeze up.

b Backwater from ice.

e Estimated. 
ZUMBRO RIVER BASIN

05372995 SOUTH FORK ZUMBRO RIVER AT ROCHESTER, MN

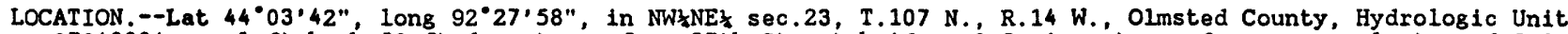
07040004 , on left bank $50 \mathrm{ft}$ downstream from 37 th Street bridge, $0.2 \mathrm{mi}$ upstream from sewer plant, and $2.0 \mathrm{mi}$ downstream from Silyer Lake Dam.

DRAINAGE AREA. $--303 \mathrm{mi} 2$.

PERIOD OF RECORD. --March 1981 to current year.

GAGE. - Water-stage recorder. Datum of gage is $950.00 \mathrm{ft}$ above National Geodetic Vertical Datum of 1929.

REMARKS.--Records good except those for estimated daily discharges, which are.poor. Slight regulation at times from Silver Lake.

EXTREMES OUTSIDE PERIOD OF RECORD. -Flood of JU1y 6, 1978, reached a stage of about $28.0 \mathrm{ft}$, on upstream side of bridge, discharge $30,500 \mathrm{ft}^{3} / \mathrm{s}$. This is the highest known stage since at least 1908.

EXTREMES FOR CURRENT YEAR. --Peak discharges greater than base discharge of $1,300 \mathrm{ft} / \mathrm{s}$ and maximum ( $*$ ):

\begin{tabular}{|c|c|c|c|c|c|c|c|}
\hline Date & Time & $\begin{array}{c}\text { Discharge } \\
\left(\mathrm{ft}^{3} / \mathrm{s}\right)\end{array}$ & $\begin{array}{c}\text { Gage height } \\
(f t)\end{array}$ & Date & Time & $\begin{array}{c}\text { Discharge } \\
\left(\mathrm{ft}^{3} / \mathrm{s}\right)\end{array}$ & $\begin{array}{c}\text { Gage height } \\
\text { (ft) }\end{array}$ \\
\hline
\end{tabular}

DISCHARGE, CUBIC FEET PER SECOND, WATER YEAR OCTOBER 1990 TO SEPTEMBER 1991

\begin{tabular}{|c|c|c|c|c|c|c|c|c|c|c|c|c|}
\hline DAY & OCT & NOV & DEC & JAN & FEB & MAR & APR & MAY & JUN & JUL & AUG & SEP \\
\hline $\begin{array}{r}1 \\
2 \\
3 \\
4 \\
5 \\
6 \\
7 \\
8 \\
9 \\
10 \\
11 \\
12 \\
13 \\
14 \\
15 \\
16 \\
17 \\
18 \\
19 \\
20 \\
21 \\
22 \\
23 \\
24 \\
25 \\
26 \\
27 \\
28 \\
29 \\
30 \\
31\end{array}$ & $\begin{array}{l}e 85 \\
e 85 \\
e 90 \\
e 93 \\
e 88 \\
e 82 \\
e 75 \\
e 78 \\
e 78 \\
e 76 \\
e 73 \\
e 74 \\
e 72 \\
e 72 \\
e 70 \\
e 69 \\
e 70 \\
e 79 \\
e 74 \\
e 75 \\
e 84 \\
e 82 \\
e 80 \\
e 78 \\
e 76 \\
e 76 \\
e 74 \\
e 73 \\
e 72 \\
e 71 \\
e 70\end{array}$ & $\begin{array}{l}68 \\
67 \\
73 \\
73 \\
72 \\
72 \\
72 \\
72 \\
70 \\
72 \\
70 \\
68 \\
67 \\
67 \\
67 \\
66 \\
66 \\
66 \\
67 \\
68 \\
66 \\
64 \\
65 \\
66 \\
65 \\
64 \\
65 \\
69 \\
60 \\
64 \\
---\end{array}$ & $\begin{array}{r}64 \\
60 \\
60 \\
59 \\
64 \\
66 \\
65 \\
65 \\
64 \\
65 \\
65 \\
67 \\
62 \\
57 \\
66 \\
64 \\
65 \\
65 \\
63 \\
62 \\
48 \\
e 48 \\
e 48 \\
847 \\
846 \\
846 \\
846 \\
45 \\
e 45 \\
845 \\
844\end{array}$ & $\begin{array}{r}e 43 \\
e 42 \\
e 41 \\
e 41 \\
41 \\
40 \\
40 \\
40 \\
41 \\
40 \\
41 \\
42 \\
42 \\
43 \\
45 \\
45 \\
45 \\
43 \\
47 \\
44 \\
42 \\
50 \\
43 \\
e 41 \\
40 \\
37 \\
36 \\
37 \\
37 \\
36 \\
35\end{array}$ & $\begin{array}{l}34 \\
36 \\
44 \\
47 \\
46 \\
47 \\
50 \\
53 \\
53 \\
53 \\
51 \\
53 \\
59 \\
57 \\
50 \\
57 \\
56 \\
56 \\
57 \\
56 \\
65 \\
60 \\
58 \\
63 \\
61 \\
68 \\
66 \\
65 \\
--- \\
-0- \\
---\end{array}$ & $\begin{array}{r}96 \\
92 \\
78 \\
89 \\
109 \\
190 \\
187 \\
187 \\
168 \\
148 \\
156 \\
180 \\
170 \\
152 \\
150 \\
162 \\
155 \\
159 \\
180 \\
225 \\
289 \\
410 \\
1000 \\
815 \\
485 \\
411 \\
447 \\
471 \\
443 \\
343 \\
300\end{array}$ & $\begin{array}{r}298 \\
268 \\
257 \\
246 \\
247 \\
234 \\
216 \\
343 \\
673 \\
487 \\
378 \\
360 \\
427 \\
620 \\
722 \\
549 \\
455 \\
438 \\
636 \\
658 \\
506 \\
450 \\
417 \\
377 \\
339 \\
381 \\
839 \\
863 \\
815 \\
1230 \\
-1-\end{array}$ & $\begin{array}{r}883 \\
675 \\
574 \\
534 \\
974 \\
2330 \\
1230 \\
913 \\
864 \\
711 \\
609 \\
548 \\
516 \\
462 \\
435 \\
522 \\
522 \\
524 \\
581 \\
514 \\
452 \\
412 \\
382 \\
350 \\
307 \\
299 \\
409 \\
467 \\
412 \\
362 \\
353\end{array}$ & $\begin{array}{l}294 \\
280 \\
245 \\
226 \\
212 \\
191 \\
222 \\
217 \\
195 \\
182 \\
205 \\
369 \\
360 \\
284 \\
242 \\
216 \\
198 \\
186 \\
175 \\
164 \\
155 \\
146 \\
136 \\
128 \\
118 \\
111 \\
107 \\
---\end{array}$ & $\begin{array}{r}140 \\
112 \\
103 \\
104 \\
101 \\
95 \\
95 \\
94 \\
88 \\
94 \\
186 \\
284 \\
229 \\
160 \\
132 \\
117 \\
185 \\
151 \\
131 \\
139 \\
126 \\
805 \\
419 \\
259 \\
218 \\
123 \\
93 \\
195 \\
199 \\
178 \\
156\end{array}$ & $\begin{array}{r}139 \\
134 \\
126 \\
119 \\
110 \\
103 \\
237 \\
1160 \\
1460 \\
632 \\
424 \\
347 \\
290 \\
251 \\
221 \\
212 \\
209 \\
190 \\
171 \\
156 \\
148 \\
137 \\
126 \\
118 \\
112 \\
108 \\
100 \\
95 \\
89 \\
86 \\
82\end{array}$ & $\begin{array}{r}80 \\
81 \\
80 \\
76 \\
73 \\
73 \\
73 \\
105 \\
86 \\
78 \\
90 \\
84 \\
82 \\
154 \\
111 \\
103 \\
90 \\
87 \\
82 \\
78 \\
76 \\
76 \\
75 \\
95 \\
82 \\
78 \\
76 \\
76 \\
74 \\
69 \\
-1-\end{array}$ \\
\hline $\begin{array}{l}\text { TOTAL } \\
\text { MEAN } \\
\text { MAX } \\
\text { MIN } \\
\text { AC-FT } \\
\text { CFSM } \\
\text { IN. }\end{array}$ & $\begin{array}{r}2394 \\
77.2 \\
93 \\
69 \\
4750 \\
.25 \\
.29\end{array}$ & $\begin{array}{r}2031 \\
67.7 \\
73 \\
60 \\
4030 \\
.22 \\
.25\end{array}$ & $\begin{array}{r}1776 \\
57.3 \\
67 \\
44 \\
3520 \\
.19 \\
.22\end{array}$ & $\begin{array}{r}1280 \\
41.3 \\
50 \\
35 \\
2540 \\
.14 \\
.16\end{array}$ & $\begin{array}{r}1521 \\
54.3 \\
68 \\
34 \\
3020 \\
.18 \\
.19\end{array}$ & $\begin{array}{r}8447 \\
272 \\
1000 \\
78 \\
16750 \\
.90 \\
1.04\end{array}$ & $\begin{array}{r}14729 \\
491 \\
1230 \\
216 \\
29210 \\
1.62 \\
1.81\end{array}$ & $\begin{array}{r}19126 \\
617 \\
2330 \\
299 \\
37940 \\
2.04 \\
2.35\end{array}$ & $\begin{array}{r}6557 \\
219 \\
369 \\
107 \\
13010 \\
.72 \\
.81\end{array}$ & $\begin{array}{r}5511 \\
178 \\
805 \\
88 \\
10930 \\
.59 \\
.68\end{array}$ & $\begin{array}{r}7892 \\
255 \\
1460 \\
82 \\
15650 \\
.84 \\
.97\end{array}$ & $\begin{array}{r}2543 \\
84.8 \\
154 \\
69 \\
5040 \\
.28 \\
.31\end{array}$ \\
\hline \multicolumn{3}{|c|}{ 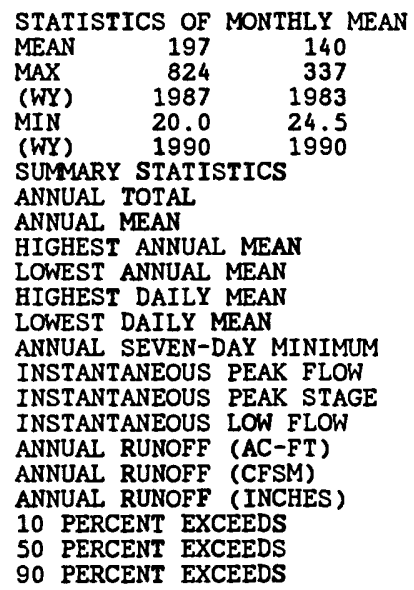 } & $\begin{array}{c}\text { DATA F } \\
114 \\
300 \\
1983 \\
21.0 \\
1990 \\
\text { FOR }\end{array}$ & $\begin{array}{c}\text { FOR WATER YE } \\
74.8 \\
167 \\
1983 \\
22.5 \\
1990 \\
1990 \text { CALEND } \\
75844 \\
208\end{array}$ & $\begin{array}{c}\text { EARS } 1981 \\
125 \\
454 \\
1984 \\
23.8 \\
1990 \\
\text { DAR YEAR }\end{array}$ & $\begin{array}{r}-1991, \\
399 \\
760 \\
1983 \\
165 \\
1987 \\
F\end{array}$ & $\begin{array}{c}2330 \\
34 \\
36 \\
2640 \\
8.49 \\
33 \\
146400 \\
9.67 \\
9.06 \\
486 \\
93 \\
46\end{array}$ & $\begin{array}{c}\text { YEAR (W } \\
317 \\
617 \\
1991 \\
88.3 \\
1989 \\
\text { TER YEA }\end{array}$ & $\begin{array}{r}178 \\
384 \\
1990 \\
49.0 \\
1989\end{array}$ & $\begin{array}{c}213 \\
546 \\
1981 \\
23.2 \\
1988 \\
\text { WATER YEA } \\
206 \\
360 \\
87.3 \\
7710 \\
12 \\
14 \\
10000 \\
20.77 \\
10 \mathrm{a} \\
149300 \\
.68 \\
9.24 \\
467 \\
112 \\
29\end{array}$ & $\begin{array}{lr}\text { Sep } & 21 \\
\text { Sep } & 12 \\
\text { Sep } & 8 \\
\text { Sep } & 21 \\
\text { Sep } & 21 \\
\text { Oct } & 23\end{array}$ & $\begin{array}{l}1983 \\
1989 \\
1986 \\
1988 \\
1988 \\
1986 \\
1986 \\
1981\end{array}$ \\
\hline
\end{tabular}

a Result of regulation.

e Estimated. 
LOCATION.--Lat $44^{\circ} 05^{\prime} 30^{\prime \prime}$, long 92.03'57", in sec.7, T.107 N., R.10 W., Winona County, Hydrologic Unit 07040003, on left bank $2.3 \mathrm{mi}$ upstream from Middle Fork, $2.4 \mathrm{mi}$ west of Elba, and $3.5 \mathrm{mi}$ upstream from confluence with South Fork.

DRAINAGE AREA. $--101 \mathrm{mi}^{2}$.

\author{
WATER-DISCHARGE RECORDS
}

PERIOD OF RECORD.--May 1939 to September 1941, July 1967 to current year.

REVISED RECORDS. --WRD MN-74: $1967(\mathrm{M}), 1969(\mathrm{M}), 1971(\mathrm{M}), 1972(\mathrm{M}), 1973(\mathrm{M}), \quad$ WRD MN-80-2: 1978.

GAGE. - Water-stage recorder. Datum of gage is $769.60 \mathrm{ft}$ above National Geodetic Vertical Datum of 1929. Prior to

Oct. 12, 1939, nonrecording gage at site $2 \mathrm{mi}$ downstream at different datum. Oct. 12, 1939, to Sept. 30, 1941,

water-stage recorder at site $600 \mathrm{ft}$ downstream at present datum. Prior to July 6, 1978, water-stage recorder at

same site and present datum (gage destroyed by flood of July 1978), July 6 to Oct. 30, 1978, nonrecording gage

at same site and present datum.

REMARKS.--Records good except those for estimated daily discharges, which are fair.

DISCHARGE, CUBIC FEET PER SECOND, WATER YEAR $\propto$ CTOBER 1990 TO SEPTEMBER 1991

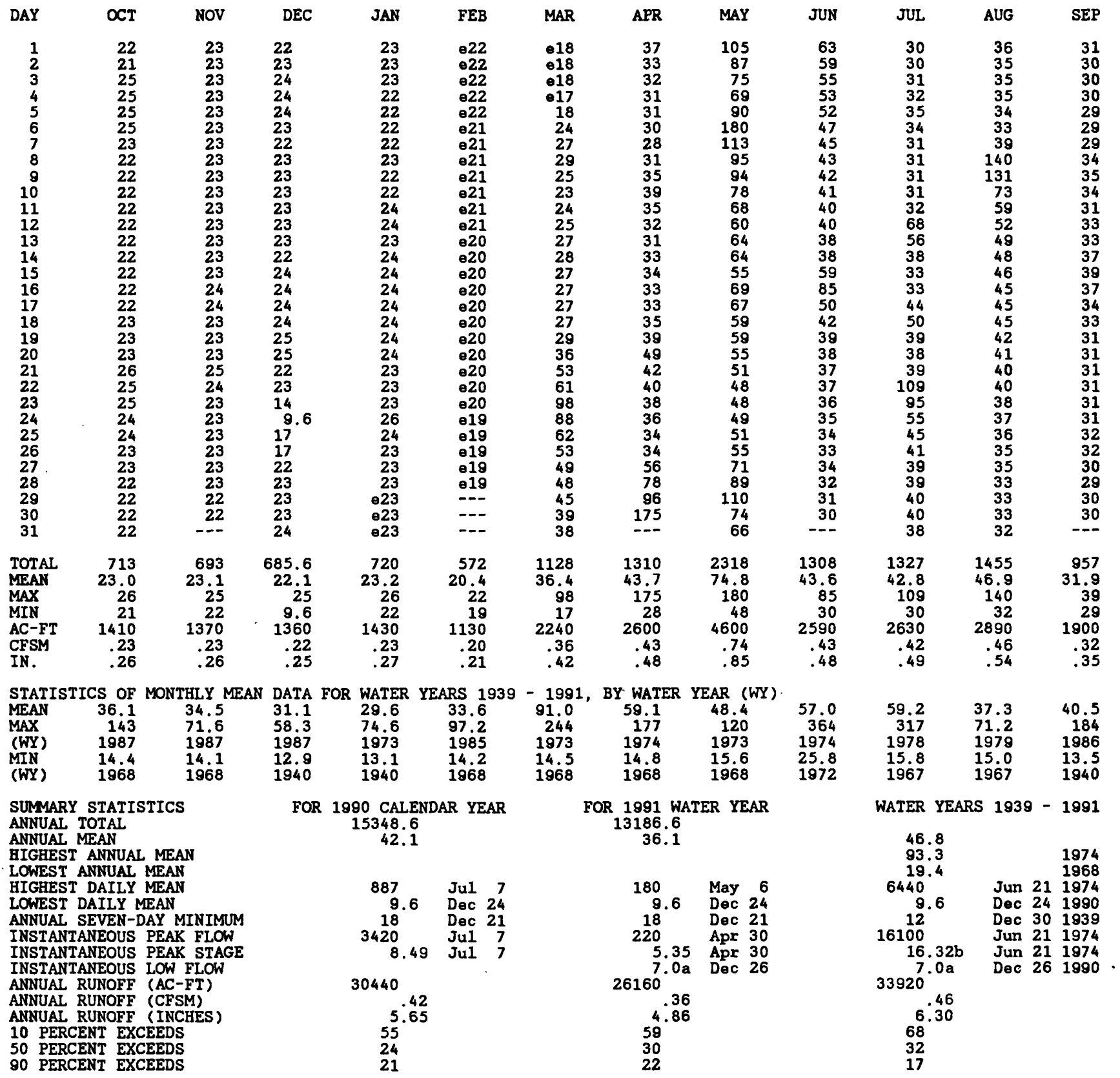

a Result of freezeup.

b From floodmark.

- Estimated. 
WHITEWATER RIVER BASIN

05376000 NORTH FORK WHITEWATER RIVER MEAR ELBA, MN--Continued (Hydrologic bench-mark station)

WATER-QUALITY RECORDS

PERIOD OF RECORD. --Water years 1967 to current year.

REMARKS.--Letter $\mathrm{X}$ indicates non-ideal colony count.

WATER QUALITY DATA, WATER YEAR OCTOBER 1990 TO SEPTEMBER 1991

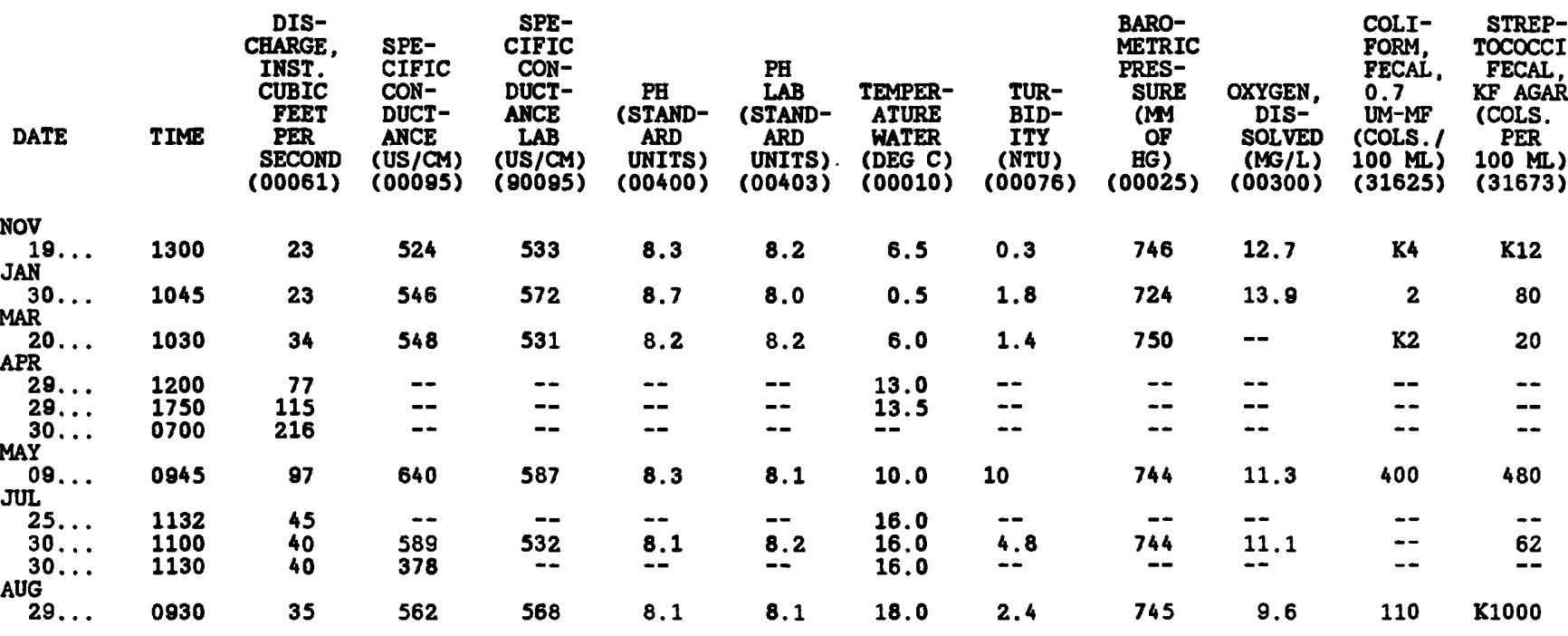

\begin{tabular}{|c|c|c|c|c|c|c|c|c|c|c|c|c|}
\hline ATE & $\begin{array}{l}\text { CALCIUM } \\
\text { DIS- } \\
\text { SOLVED } \\
\text { (MG/L } \\
\text { AS CA) } \\
(00915)\end{array}$ & $\begin{array}{c}\text { MAGNE- } \\
\text { SIUM, } \\
\text { DIS- } \\
\text { SOLVED } \\
\text { (MG/L } \\
\text { AS MG) } \\
\text { (00925) }\end{array}$ & $\begin{array}{c}\text { SODIUM, } \\
\text { DIS- } \\
\text { SOLVED } \\
\text { (MG/L } \\
\text { AS NA) } \\
(00930)\end{array}$ & $\begin{array}{c}\text { POTAS- } \\
\text { SIUM, } \\
\text { DIS- } \\
\text { SOLVED } \\
\text { (MG/L } \\
\text { AS K) } \\
\text { (00935) }\end{array}$ & $\begin{array}{c}\text { ALKA- } \\
\text { LINITY } \\
\text { WAT DIS } \\
\text { IOT IT } \\
\text { FIELD } \\
\text { MG/L AS } \\
\text { CACO3 } \\
\text { (39086) }\end{array}$ & $\begin{array}{c}\text { ALKA- } \\
\text { LINITY } \\
\text { IAB } \\
\text { (MG/L } \\
\text { AS } \\
\text { CACO3) } \\
(90410)\end{array}$ & $\begin{array}{c}\text { CAR- } \\
\text { BONATE } \\
\text { WATER } \\
\text { DIS IT } \\
\text { FIELD } \\
\text { MG/L AS } \\
\text { CO3 } \\
(00452)\end{array}$ & $\begin{array}{c}\text { BICAR- } \\
\text { BONATE } \\
\text { WATER } \\
\text { DIS IT } \\
\text { FIELD } \\
\text { MG/L AS } \\
\text { BCO3 } \\
(00453)\end{array}$ & $\begin{array}{c}\text { SULFATE } \\
\text { DIS- } \\
\text { SOLVED } \\
\text { (MG/L } \\
\text { AS SO4) } \\
(00945)\end{array}$ & $\begin{array}{l}\text { CHLO- } \\
\text { RIDE, } \\
\text { DIS-' } \\
\text { SOLVED } \\
\text { (MG/L } \\
\text { AS CL) } \\
\text { (00940) }\end{array}$ & $\begin{array}{l}\text { FLUO- } \\
\text { RIDE, } \\
\text { DIS- } \\
\text { SOLVED } \\
\text { (MG/L } \\
\text { AS F) } \\
\text { (00950) }\end{array}$ & $\begin{array}{c}\text { SILICA, } \\
\text { DIS- } \\
\text { SOLVED } \\
\text { (MG/L } \\
\text { AS } \\
\text { SIO2) } \\
\text { (00955) }\end{array}$ \\
\hline
\end{tabular}

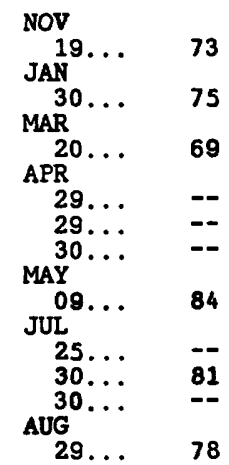

\begin{tabular}{|c|c|c|c|c|}
\hline 27 & 5.0 & 1.1 & 251 & 244 \\
\hline 26 & 4.6 & 1.0 & 276 & 280 \\
\hline 25 & 5.9 & 1.3 & 221 & 254 \\
\hline$=$ & $=$ & $\because$ & $=$ & $=$ \\
\hline-- & -- & $=$ & $=$ & - \\
\hline 22 & 7.1 & 2.4 & 256 & 257 \\
\hline-- & -- & -. & -- & -- \\
\hline 26 & 6.6 & 2.0 & 262 & 272 \\
\hline-- & -- & - & -- & -- \\
\hline 27 & 6.2 & 1.7 & 276 & 249 \\
\hline
\end{tabular}

0
0
0
--
--
-0
--
0

$\begin{array}{cc}306 & 13 \\ 336 & 15 \\ 270 & 15 \\ -- & -- \\ -- & -- \\ -- & -- \\ 312 & 18 \\ -- & -- \\ 320 & 18 \\ -- & -- \\ 337 & 17\end{array}$

$\begin{array}{ccc}9.6 & 0.1 & 13 \\ 11 & <0.1 & 16 \\ 10 & 0.1 & 12 \\ -- & -- & -- \\ -- & -- & -- \\ -- & -\therefore & -- \\ 18 & 0.1 & 14 \\ -- & -- & -- \\ 16 & 0.1 & 16 \\ -- & -- & -- \\ 16 & 0.2 & 13\end{array}$




\begin{tabular}{|c|c|c|c|c|c|c|c|c|c|c|c|}
\hline & $\begin{array}{l}\text { SOLIDS, } \\
\text { RESIDUE } \\
\text { AT } 180 \\
\text { DEG. C } \\
\text { DIS- } \\
\text { SOLVED } \\
(M G / L) \\
(70300)\end{array}$ & $\begin{array}{c}\text { NITRO- } \\
\text { GEN, } \\
\text { NITRITE } \\
\text { DIS- } \\
\text { SOLVED } \\
\text { (MG/L } \\
\text { AS N) } \\
(00613)\end{array}$ & $\begin{array}{c}\text { NITRO- } \\
\text { GEN, } \\
\text { NO2+NO33 } \\
\text { DIS- } \\
\text { SOLVED } \\
\text { (MG/L } \\
\text { AS N) } \\
(00631)\end{array}$ & $\begin{array}{c}\text { NIT } \\
\text { GE } \\
\text { AMMO } \\
\text { TOT } \\
\text { (MG } \\
\text { AS } \\
\text { COOG }\end{array}$ & $\begin{array}{c}\text { NITRO- } \\
\text { GEN, } \\
\text { AMMONIA } \\
\text { DIS- } \\
\text { SOLVED } \\
\text { (MG/L } \\
\text { AS N) } \\
(00608)\end{array}$ & $\begin{array}{c}\text { NITRO- } \\
\text { GEN, AM- } \\
\text { MONIA + } \\
\text { ORGANIC } \\
\text { TOTAL } \\
(M G / L \\
\text { AS N) } \\
(00625)\end{array}$ & $\begin{array}{c}\text { PHO } \\
\text { PHOP } \\
\text { TOI } \\
\text { (ME } \\
\text { AS } \\
1006\end{array}$ & $\begin{array}{r}\mathrm{PH} \\
\mathrm{PHO} \\
\mathrm{D} \\
\mathrm{SO} \\
\mathbf{C M} \\
\mathrm{AS} \\
\mathrm{COO}\end{array}$ & S & 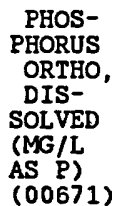 & $\begin{array}{l}\text { SED. } \\
\text { SUSP. } \\
\text { SIEVE } \\
\text { DIAM. } \\
7 \text { FINER } \\
\text { THAN } \\
.062 \mathrm{MM}\end{array}$ \\
\hline
\end{tabular}

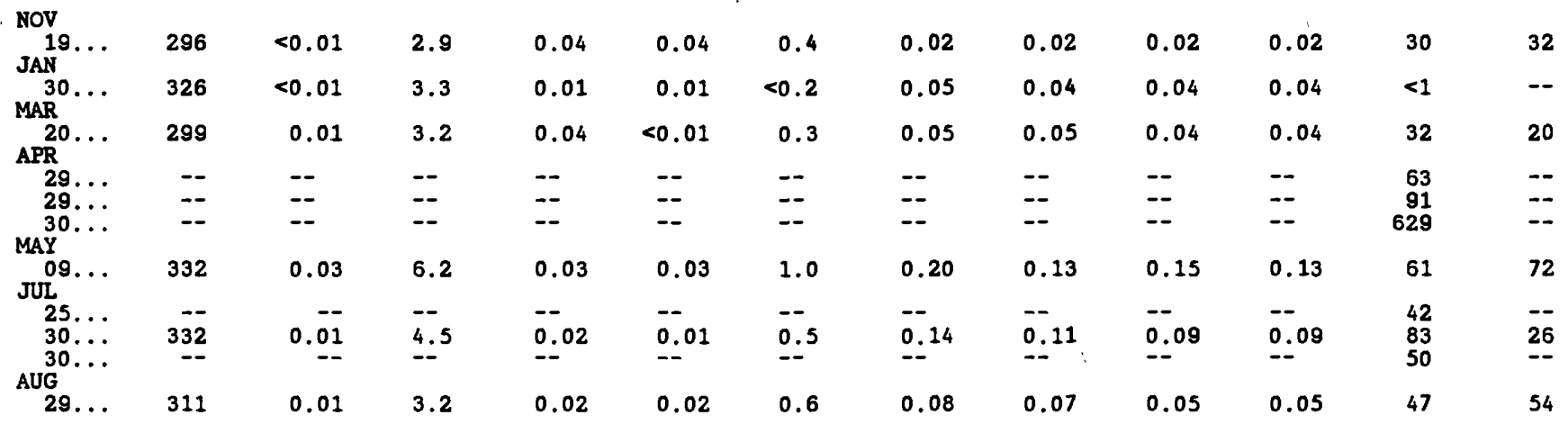

DATE TIME

Nov

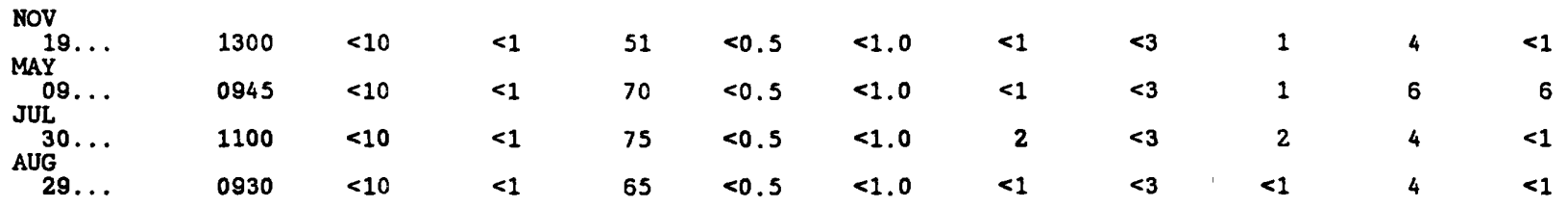

$<0.5<1.0<1$

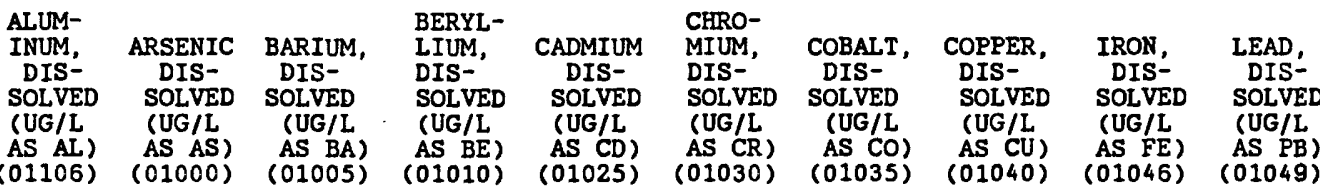

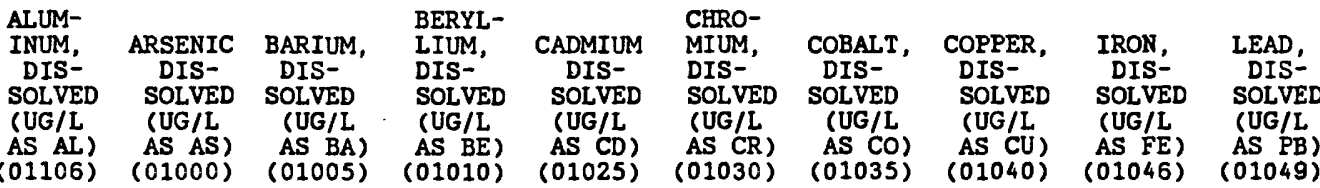

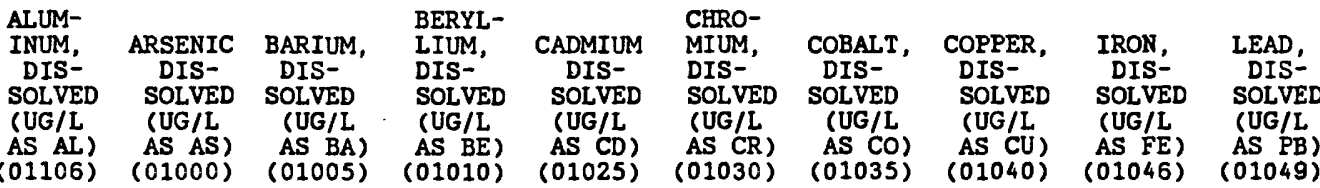

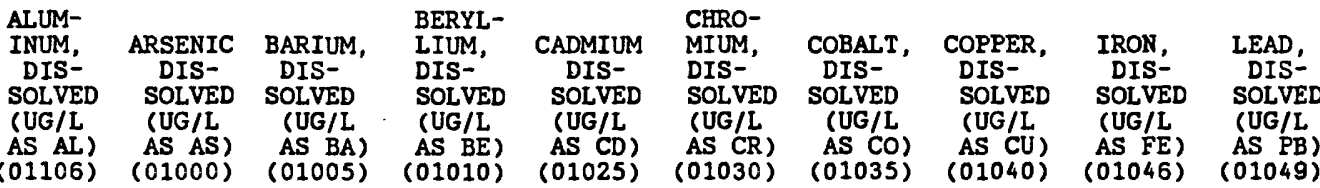

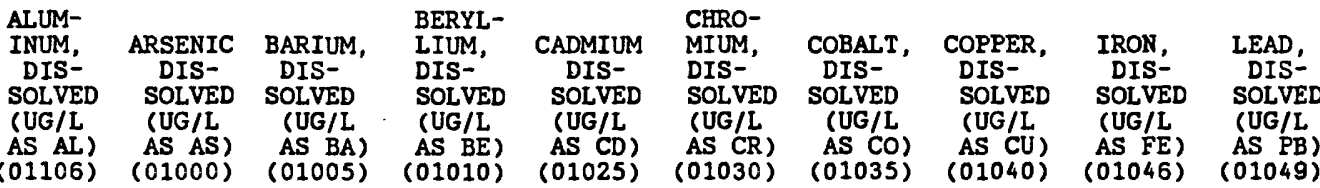

\begin{tabular}{|c|c|c|c|c|c|c|c|c|c|}
\hline $\begin{array}{c}\text { LITHIUM } \\
\text { DIS- } \\
\text { SOLVED } \\
\text { (UG/L } \\
\text { AS LI) }\end{array}$ & $\begin{array}{l}\text { MANGA- } \\
\text { NESE, } \\
\text { DIS- } \\
\text { SOLVED } \\
\text { (UG/L } \\
\text { AS MN) } \\
(01056)\end{array}$ & $\begin{array}{c}\text { MERCURY } \\
\text { DIS- } \\
\text { SOLVED } \\
\text { (UG/L } \\
\text { AS HG) } \\
\text { (71890) }\end{array}$ & $\begin{array}{l}\text { MOLYB- } \\
\text { DENUM, } \\
\text { DIS- } \\
\text { SOLVED } \\
\text { (UG/L } \\
\text { AS MO) } \\
(01060)\end{array}$ & $\begin{array}{l}\text { NICKEL, } \\
\text { DIS- } \\
\text { SOLVED } \\
\text { (UG/L } \\
\text { AS NI) } \\
\text { (01065) }\end{array}$ & $\begin{array}{l}\text { SELE- } \\
\text { NIUM, } \\
\text { DIS- } \\
\text { SOLVED } \\
\text { (UG/L } \\
\text { AS SE) } \\
(01145)\end{array}$ & $\begin{array}{c}\text { SILVER, } \\
\text { DIS- } \\
\text { SOLVED } \\
\text { (UG/L } \\
\text { AS AG) } \\
(01075 \text { ) }\end{array}$ & $\begin{array}{c}\text { STRON- } \\
\text { TIUM, } \\
\text { DIS- } \\
\text { SOLVED } \\
\text { (UG/L } \\
\text { AS SR) } \\
(01080)\end{array}$ & $\begin{array}{l}\text { VANA- } \\
\text { DIUM, } \\
\text { DIS- } \\
\text { SOLVED } \\
\text { (UG/I } \\
\text { AS V) } \\
(01085)\end{array}$ & $\begin{array}{l}\text { D } \\
\text { SOI } \\
\text { (UC } \\
\text { AS } \\
\text { Co1 }\end{array}$ \\
\hline
\end{tabular}

NOV.
MAY...
O9...
JUL
30...
AUG
$29 .$.

$\begin{array}{rrrrrrrrrr}8 & 7 & 0.1 & <10 & 2 & <1 & <1.0 & 72 & <6 & 4 \\ 5 & 21 & <0.1 & <10 & 2 & 1 & <1.0 & 97 & <6 & <3 \\ 6 & 14 & <0.1 & <10 & <1 & <1 & <1.0 & 85 & <6 & <3 \\ 8 & 12 & <0.1 & <10 & <1 & <1 & <1.0 & 81 & <6 & 9\end{array}$


WHITEWATER RIVER BASIN

05376000 NORTH FORK WHITEWATER RIVER NEAR ELBA, MN--Continued

RADIOCHEMICAL ANALYSIS, WATER YEAR OCTOBER 1990 TO SEPTEMBER 1991

\begin{tabular}{|c|c|c|c|c|c|c|c|c|c|}
\hline DATE & TIME & $\begin{array}{l}\text { GROSS } \\
\text { ALPHA, } \\
\text { DIS- } \\
\text { SOLVED } \\
\text { (UG/L } \\
\text { AS } \\
\text { U-NAT) } \\
(80030)\end{array}$ & $\begin{array}{l}\text { GROSS } \\
\text { ALPHA, } \\
\text { SUSP. } \\
\text { TOTAL } \\
\text { (UG/L } \\
\text { AS } \\
\text { U-NAT) } \\
(80040)\end{array}$ & $\begin{array}{c}\text { GROSS } \\
\text { BETA } \\
\text { DIS- } \\
\text { SOLVED } \\
\text { (PCI/L } \\
\text { AS } \\
\text { CS-137) } \\
(03515)\end{array}$ & $\begin{array}{c}\text { GROSS } \\
\text { BETA. } \\
\text { SUSP. } \\
\text { TOTAL } \\
\text { (PCI /L } \\
\text { AS } \\
\text { CS-137) } \\
(03516)\end{array}$ & $\begin{array}{l}\text { GROSS } \\
\text { BETA, } \\
\text { DIS- } \\
\text { SOLVED } \\
\text { (PCI/L } \\
\text { AS SR/ } \\
\text { YT-90) } \\
(80050)\end{array}$ & $\begin{array}{l}\text { GROSS } \\
\text { BETA, } \\
\text { SUSP } \\
\text { TOTAL } \\
\text { (PCI/L } \\
\text { AS SR/ } \\
\text { YT-90) } \\
(80060)\end{array}$ & $\begin{array}{c}\text { RADIUM } \\
226, \\
\text { DIS- } \\
\text { SOLVED, } \\
\text { RADON } \\
\text { METHOD } \\
\text { (PCI /L) } \\
(09511)\end{array}$ & $\begin{array}{c}\text { URANIUM } \\
\text { NATURAL } \\
\text { DIS- } \\
\text { SOLVED } \\
\text { (UG/L } \\
\text { AS U) } \\
(22703)\end{array}$ \\
\hline
\end{tabular}

$\begin{array}{llllllllll}\text { JAN } & & & & & & & \\ 30 \ldots & 1045 & 0.6 & <0.6 & 2.3 & <0.6 & 1.7 & <0 & 0.05 & 0.56 \\ \text { MAR } & 1030 & 0.6 & <0.6 & 2.8 & <0.6 & 2.1 & <0.6 & 0.05 & 0.62\end{array}$

SUSPENDED-SEDIMENT, WATER YEAR OCTOBER 1990 TO SEPTEMBER 1991

\begin{tabular}{|c|c|c|c|c|c|c|}
\hline \multirow[t]{2}{*}{ DAY } & $\begin{array}{l}\text { MEAN } \\
\text { CONCEN- } \\
\text { TRATION } \\
(\mathrm{MG} / \mathrm{L})\end{array}$ & $\begin{array}{c}\text { LOAD } \\
\text { (TONS/ } \\
\text { DAY) }\end{array}$ & $\begin{array}{l}\text { MEAN } \\
\text { CONCEN- } \\
\text { TRATION } \\
\text { (MG/L) }\end{array}$ & $\begin{array}{c}\text { LOAD } \\
\text { (TONS/ } \\
\text { DAY) }\end{array}$ & $\begin{array}{l}\text { MEAN } \\
\text { CONCEN- } \\
\text { TRATION } \\
\text { (MG/L) }\end{array}$ & $\begin{array}{c}\text { LOAD } \\
\text { (TONS/ } \\
\text { DAY) }\end{array}$ \\
\hline & \multicolumn{2}{|c|}{ APRIL } & \multicolumn{2}{|c|}{ JUNE } & \multicolumn{2}{|c|}{ JULY } \\
\hline 15 & --- & -- & 61 & 15 & - & -- \\
\hline 16 & -- & -- & 97 & 22 & -- &.- \\
\hline 23 & -- & $\cdots$ & --- & -- & 195 & 73 \\
\hline $\begin{array}{l}29 \\
30\end{array}$ & $\begin{array}{l}146 \\
500\end{array}$ & $\begin{array}{r}55 \\
236\end{array}$ & $=-$ & -- & $=-$ & $=-$ \\
\hline
\end{tabular}




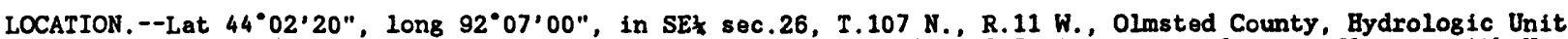
07040003 , on left downstream side of bridge on County Road 107, $8.5 \mathrm{mi}$ upstream from confluence with North Fork.

DRAINAGE AREA. --Undetermined.

\section{WATER-DISCHARGE RECORDS}

PERIOD OF RECORD.--September 1987 to June 1988 (discharge measurements only). July 1988 to current year during open-water periods only.

GAGE.--Water-stage recorder. Datum of gage is undetermined.

REMARKS.--Records good. Gage operated during open-water period only. Data for years previous to the 1991 water year are questionable and were not published.

EXTREMES FOR PERIOD OF RECORD.--Maximum discharge observed $637 \mathrm{ft}^{3} / \mathrm{s}$, Mar. 11, 1989, gage height, $13.42 \mathrm{ft}$ minimum discharge observed, $4.9 \mathrm{ft}^{3} / \mathrm{s}$, June $29,1989$.

EXTREMES FOR CURRENT PERIOD.--March to September 1991: Maximum discharge $542 \mathrm{ft}^{3} / \mathrm{s}$, July 21, gage height, 12.87 ft; minimum $6.7 \mathrm{ft}^{3} / \mathrm{s}$, Mar. $15-18$, gage height, $10.62 \mathrm{ft}$.

DISCHARGE, CUBIC FEET PER SECOND, WATER YEAR OCTOBER 1990 TO SEPTEMBER 1991

DAILY MEAN VALUES

\begin{tabular}{|c|c|c|c|c|c|c|c|c|c|c|c|c|}
\hline DAY & $\infty \mathrm{CT}$ & NOV & DEC & JAN & FEB & MAR & APR & MAY & JUN & JUL & AUG & SEP \\
\hline $\begin{array}{l}1 \\
2 \\
3 \\
4 \\
5\end{array}$ & $\begin{array}{l}--- \\
=-- \\
=-- \\
=-- \\
---\end{array}$ & $\begin{array}{l}--- \\
--- \\
--- \\
--- \\
---\end{array}$ & $\begin{array}{l}--- \\
--- \\
--- \\
--- \\
---\end{array}$ & $\begin{array}{l}--- \\
\overline{--} \\
\overline{---} \\
---\end{array}$ & $\begin{array}{l}--- \\
--- \\
--- \\
--- \\
---\end{array}$ & $\begin{array}{l}--- \\
-- \\
-- \\
--- \\
--\end{array}$ & $\begin{array}{l}14 \\
14 \\
13 \\
13 \\
13\end{array}$ & $\begin{array}{l}26 \\
24 \\
22 \\
22 \\
52\end{array}$ & $\begin{array}{l}22 \\
21 \\
19 \\
18 \\
17\end{array}$ & $\begin{array}{l}12 \\
12 \\
11 \\
14 \\
12\end{array}$ & $\begin{array}{l}18 \\
18 \\
17 \\
17 \\
16\end{array}$ & $\begin{array}{l}13 \\
13 \\
13 \\
13 \\
13\end{array}$ \\
\hline $\begin{array}{r}6 \\
7 \\
8 \\
9 \\
10\end{array}$ & $\begin{array}{l}--- \\
--- \\
--- \\
---\end{array}$ & $\begin{array}{l}--- \\
--- \\
--- \\
--- \\
---\end{array}$ & $\begin{array}{l}--- \\
--- \\
--- \\
---\end{array}$ & $\begin{array}{l}--- \\
--- \\
--- \\
---\end{array}$ & $\begin{array}{l}--- \\
\overline{--} \\
--- \\
---\end{array}$ & $\begin{array}{l}--- \\
--- \\
--- \\
---\end{array}$ & $\begin{array}{l}13 \\
13 \\
16 \\
18 \\
15\end{array}$ & $\begin{array}{l}35 \\
27 \\
29 \\
28 \\
25\end{array}$ & $\begin{array}{l}16 \\
16 \\
16 \\
15 \\
16\end{array}$ & $\begin{array}{l}11 \\
11 \\
11 \\
11 \\
11\end{array}$ & $\begin{array}{l}16 \\
18 \\
46 \\
24 \\
21\end{array}$ & $\begin{array}{l}13 \\
12 \\
14 \\
14 \\
12\end{array}$ \\
\hline $\begin{array}{l}11 \\
12 \\
13 \\
14 \\
15\end{array}$ & $\begin{array}{l}--- \\
--- \\
--- \\
---\end{array}$ & $\begin{array}{l}--- \\
--- \\
--- \\
--- \\
--\end{array}$ & $\begin{array}{l}--- \\
--- \\
--- \\
---\end{array}$ & $\begin{array}{l}--- \\
--- \\
--- \\
---\end{array}$ & $\begin{array}{l}--- \\
=- \\
-- \\
--- \\
--\end{array}$ & $\begin{array}{l}--- \\
--- \\
--- \\
--\overline{6} \\
6.7\end{array}$ & $\begin{array}{l}14 \\
15 \\
17 \\
21 \\
20\end{array}$ & $\begin{array}{l}24 \\
23 \\
28 \\
25 \\
23\end{array}$ & $\begin{array}{l}16 \\
15 \\
16 \\
16 \\
44\end{array}$ & $\begin{array}{l}12 \\
31 \\
13 \\
13 \\
13\end{array}$ & $\begin{array}{l}19 \\
19 \\
18 \\
17 \\
17\end{array}$ & $\begin{array}{l}13 \\
13 \\
13 \\
15 \\
14\end{array}$ \\
\hline $\begin{array}{l}16 \\
17 \\
18 \\
19 \\
20\end{array}$ & $\begin{array}{l}--- \\
--- \\
--- \\
---\end{array}$ & $\begin{array}{l}--- \\
--- \\
=-- \\
---\end{array}$ & $\begin{array}{l}--- \\
--- \\
--- \\
---\end{array}$ & $\begin{array}{l}--- \\
=-- \\
--- \\
---\end{array}$ & $\begin{array}{l}--- \\
--- \\
--- \\
--- \\
---\end{array}$ & $\begin{array}{r}6.7 \\
6.7 \\
6.9 \\
8.5 \\
12\end{array}$ & $\begin{array}{l}17 \\
17 \\
16 \\
23 \\
19\end{array}$ & $\begin{array}{l}36 \\
27 \\
25 \\
24 \\
23\end{array}$ & $\begin{array}{l}20 \\
17 \\
16 \\
15 \\
15\end{array}$ & $\begin{array}{l}13 \\
25 \\
16 \\
16 \\
18\end{array}$ & $\begin{array}{l}17 \\
18 \\
17 \\
16 \\
16\end{array}$ & $\begin{array}{l}13 \\
12 \\
12 \\
11 \\
11\end{array}$ \\
\hline $\begin{array}{l}21 \\
22 \\
23 \\
24 \\
25\end{array}$ & $\begin{array}{l}--- \\
--- \\
--- \\
---\end{array}$ & $\begin{array}{l}--- \\
--- \\
--- \\
---\end{array}$ & $\begin{array}{l}--- \\
--- \\
--- \\
---\end{array}$ & $\begin{array}{l}--- \\
--- \\
--- \\
---\end{array}$ & $\begin{array}{l}--- \\
--- \\
--- \\
--- \\
---\end{array}$ & $\begin{array}{l}19 \\
18 \\
36 \\
20 \\
18\end{array}$ & $\begin{array}{l}18 \\
18 \\
18 \\
17 \\
16\end{array}$ & $\begin{array}{l}22 \\
21 \\
21 \\
21 \\
20\end{array}$ & $\begin{array}{l}14 \\
14 \\
14 \\
14 \\
14\end{array}$ & $\begin{array}{l}63 \\
61 \\
26 \\
22 \\
20\end{array}$ & $\begin{array}{l}16 \\
15 \\
15 \\
15 \\
14\end{array}$ & $\begin{array}{l}11 \\
11 \\
11 \\
12 \\
12\end{array}$ \\
\hline $\begin{array}{l}26 \\
27 \\
28 \\
29 \\
30 \\
31\end{array}$ & $\begin{array}{l}--- \\
--- \\
--- \\
--- \\
--- \\
---\end{array}$ & $\begin{array}{l}--- \\
--- \\
--- \\
--- \\
--- \\
---\end{array}$ & $\begin{array}{l}--- \\
--- \\
--- \\
--- \\
---\end{array}$ & $\begin{array}{l}--- \\
--- \\
--- \\
--- \\
---\end{array}$ & $\begin{array}{l}--- \\
--- \\
--- \\
--- \\
---\end{array}$ & $\begin{array}{l}17 \\
18 \\
17 \\
16 \\
15 \\
15\end{array}$ & $\begin{array}{r}17 \\
32 \\
21 \\
53 \\
35 \\
---\end{array}$ & $\begin{array}{l}21 \\
22 \\
26 \\
21 \\
19 \\
31\end{array}$ & $\begin{array}{r}13 \\
13 \\
12 \\
12 \\
12 \\
---\end{array}$ & $\begin{array}{l}19 \\
19 \\
21 \\
20 \\
18 \\
18\end{array}$ & $\begin{array}{l}14 \\
14 \\
13 \\
13 \\
13 \\
13\end{array}$ & $\begin{array}{l}11 \\
11 \\
10 \\
11 \\
11 \\
-\end{array}$ \\
\hline $\begin{array}{l}\text { TOTAL } \\
\text { MEAN } \\
\text { MAX } \\
\text { MIN } \\
\text { AC-FT } \\
\text { CFSM } \\
\text { IN. }\end{array}$ & $\begin{array}{l}--- \\
--- \\
--- \\
--- \\
--- \\
--- \\
--\end{array}$ & $\begin{array}{l}--- \\
--- \\
--- \\
--- \\
--- \\
--- \\
--\end{array}$ & $\begin{array}{l}--- \\
--- \\
--- \\
--- \\
--- \\
--- \\
---\end{array}$ & $\begin{array}{l}--- \\
--- \\
--- \\
--- \\
--- \\
---\end{array}$ & $\begin{array}{l}--- \\
=-- \\
=-- \\
--- \\
--- \\
--- \\
--\end{array}$ & $\begin{array}{l}--- \\
--- \\
--- \\
--- \\
--- \\
--- \\
--\end{array}$ & $\begin{array}{r}566 \\
18.9 \\
53 \\
13 \\
1120 \\
.75 \\
.84\end{array}$ & $\begin{array}{r}793 \\
25.6 \\
52 \\
19 \\
1570 \\
1.02 \\
1.17\end{array}$ & $\begin{array}{r}498 \\
16.6 \\
44 \\
12 \\
988 \\
.66 \\
.74\end{array}$ & $\begin{array}{r}593 \\
19.1 \\
63 \\
11 \\
1180 \\
.76 \\
.88\end{array}$ & $\begin{array}{r}540 \\
17.4 \\
46 \\
13 \\
1070 \\
.69 \\
.80\end{array}$ & $\begin{array}{r}368 \\
12.3 \\
15 \\
10 \\
730 \\
.49 \\
.54\end{array}$ \\
\hline
\end{tabular}




\section{WHITEWATER RIVER BASIN}

05376100 MIDDLE FORK WHITEWATER RIVER NEAR ST. CHARLES, MN--Continued

\section{WATER-QUALITY RECORDS}

PERIOD OF RECORD.--September 1987 to current year during open-water periods only.

INSTRUMENTATION. --Automatic sampler since September 1987 for storm-event sampling during open-water periods. REMARKS. - Data for years previous to the 1991 water year are questionable and were not published.

WATER QUALITY DATA, WATER YEAR OCTOBER 1990 TO SEPTEMBER 1991

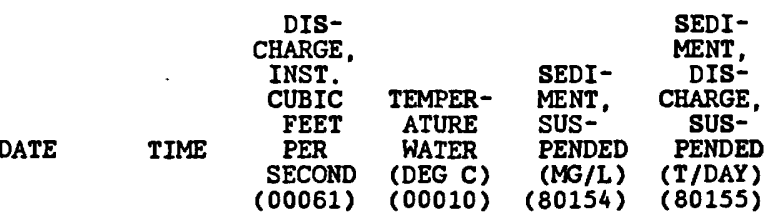

\begin{tabular}{|c|c|c|c|c|c|}
\hline \multicolumn{6}{|l|}{ MAR } \\
\hline $\begin{array}{r}19 \ldots \\
23 \ldots \\
\text { APR }\end{array}$ & $\begin{array}{l}1025 \\
1224\end{array}$ & $32^{9.0}$ & $\begin{array}{l}4.0 \\
3.0\end{array}$ & $\begin{array}{r}46 \\
351\end{array}$ & $30^{1.1}$ \\
\hline $\begin{array}{r}29 \ldots \\
39 \ldots \\
30 \ldots\end{array}$ & $\begin{array}{l}1330 \\
1720 \\
0915\end{array}$ & $\begin{array}{l}81 \\
57 \\
35\end{array}$ & $\begin{array}{l}13.5 \\
14.0 \\
--.\end{array}$ & $\begin{array}{l}539 \\
558 \\
271\end{array}$ & $\begin{array}{r}118 \\
86 \\
26\end{array}$ \\
\hline $22 \ldots$ & 1245 & 21 & 18.0 & 48 & 2.7 \\
\hline JUL $15 \ldots$ & 1324 & 33 & 19.0 & 782 & 70 \\
\hline $\begin{array}{l}11 \ldots \\
21 \ldots \\
22 \ldots \\
30 \ldots\end{array}$ & $\begin{array}{l}1145 \\
2300 \\
1330 \\
1301\end{array}$ & $\begin{array}{r}11 \\
110 \\
57 \\
18\end{array}$ & $\begin{array}{l}\overline{20.0} \\
16.0\end{array}$ & $\begin{array}{r}44 \\
1080 \\
495 \\
66\end{array}$ & $\begin{array}{c}11.3 \\
76 \\
3.2\end{array}$ \\
\hline
\end{tabular}




\section{WHITEWATER RIVER BASIN}

05376800 WHITEWATER RIVER NEAR BEAVER, MN

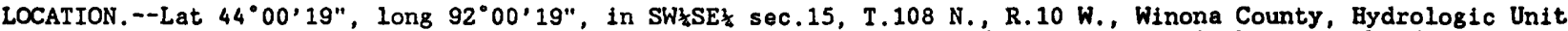
07040003 , on left bank at downstream side of bridge on County Road No. $30,0.5$ mi above mouth of Beaver Creek, and $4.7 \mathrm{mi}$ north of Elba.

DRAINAGE: $--271 \mathrm{mi}^{2}$.

\section{WATER-DISHARGE RECORDS}

PERIOD OF RECORD.--May 1975 to September 1985, May 1991 to current year.

GAGE.--Water-stage recorder. Datum of gage is $692.01 \mathrm{ft}$ above National Geodetic Vertical Datum of 1929. Prior to Oct. 1,1976 , at datum $2.00 \mathrm{ft}$ higher.

REMARKS. --Records good except those for estimated daily discharges, which are fair.

EXTREMES OUTSIDE PERIOD OF RECORD.--Maximum discharge since at least 1939, 19,200 ft $3 / \mathrm{s}, \mathrm{June} \mathrm{21,} \mathrm{1974,} \mathrm{gage} \mathrm{height,}$ $13.00 \mathrm{ft}$, present datum, determined by contracted-opening measurement.

EXTREMES FOR CURRENT PERIOD.--May to September 1991: Peak discharges greater than base discharge of 2,000 $\mathrm{ft}^{3} / \mathrm{s}$ and $\operatorname{maximum}(*)$ :

$\begin{array}{cccc}\text { Date } & \text { Time } & \begin{array}{c}\text { Discharge } \\ \left(\mathrm{ft}^{3} / \mathrm{s}\right)\end{array} & \begin{array}{c}\text { Gage height } \\ (\mathrm{ft})\end{array} \\ \text { Jul 22 } & 0100 & \star 2,040 & \star 6.95\end{array}$
Date
Time Discharge
Gage height

No other peak greater than base discharge.

DISCHARGE, CUBIC FEET PER SECOND, WATER YEAR OCTOBER 1990 TO SEPTEMBER 1991

\begin{tabular}{|c|c|c|c|c|c|c|c|c|c|c|c|c|}
\hline DAY & ○т & NOV & DEC & JAN & FEB & MAR & APR & MAY & JUN & JUL & AUG & SEP \\
\hline$\frac{1}{2}$ & 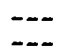 & $\ddot{---}$ & -- & $\sim-$ & $\ldots$ & - & - & $\sim-$ & 210 & 120 & 139 & 123 \\
\hline $\begin{array}{l}2 \\
3\end{array}$ & 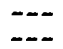 & --- & $\cdots$ & $\cdots$ & $\cdots$ & $\cdots$ & $\sim-$ & -- & e192 & 123 & 138 & 123 \\
\hline $\begin{array}{l}3 \\
4\end{array}$ & 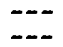 & $\ddot{---}$ & $\ldots$ & $\cdots$ & -- & $\cdots$ & $\cdots$ & $\cdots$ & 0164 & e130 & 140 & 22 \\
\hline $\begin{array}{l}4 \\
5\end{array}$ & $\ddot{-n}$ & $\ddot{--}$ & $\overline{--}$ & $\cdots$ & $\cdots$ & $\cdots$ & $\cdots$ & $\cdots$ & 155 & 132 & 139 & 11 \\
\hline 5 & $\ddot{--}$ & -- & $\ldots$ & $\cdots$ & $\cdots$ & $\cdots$ & $\cdots$ & -- & 150 & 138 & 138 & 121 \\
\hline 6 & 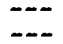 & $\cdots$ & -- & $\cdots$ & $\ldots$ & $\cdots$ & $\cdots$ & $\cdots$ & e137 & e129 & 138 & \\
\hline 7 & 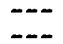 & - & -- & $\cdots$ & -- & -- & $\cdots$ & $\cdots$ & e131 & 0119 & 152 & 118 \\
\hline 8 & $\overline{---}$ & $\cdots$ & --- & $\cdots$ & - & -- & $\cdots$ & -- & 0128 & e115 & 340 & 126 \\
\hline 9 & 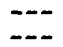 & $\cdots$ & $\ldots$ & $-\infty$ & - & $\cdots$ & $\cdots$ & -- & $e 126$ & e107 & 336 & 130 \\
\hline 10 & $\overline{-a}$ & $\cdots$ & -- & $\cdots$ & -- & - - & $\cdots$ & $\cdots$ & $e 124$ & 0117 & 247 & 122 \\
\hline 11 & $\overline{--}$ & $\cdots$ & --- & $\cdots$ & $\ldots$ & $\cdots$ & $\cdots$ & $\cdots$ & e120 & e114 & 212 & 114 \\
\hline 12 & $\ddot{--}$ & $\cdots$ & -- & $-\ldots$ & $\cdots$ & $\cdots$ & -- & -- & e117 & e182 & 195 & 119 \\
\hline 13 & 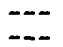 & $\cdots$ & -- & 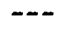 & -- & $m$ & $\ldots$ & -- & e130 & e170 & 185 & 112 \\
\hline 14 & 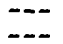 & -- & $\cdots$ & -- & $\ldots$ & -- & - & -- & 0180 & e130 & 180 & 126 \\
\hline 15 & 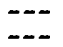 & $\cdots$ & $\ldots$ & $-\infty$ & $\ldots$ & $\cdots$ & $\cdots$ & 220 & e312 & 0124 & 175 & 127 \\
\hline 16 & $\ddot{--}$ & $\cdots$ & -- & $\cdots$ & $\cdots$ & $-\infty$ & -- & 311 & e231 & e107 & 172 & 116 \\
\hline 17 & $\ddot{--}$ & $\cdots$ & --- & $\cdots$ & $\cdots$ & $\cdots$ & $\cdots$ & 285 & e171 & 0141 & 177 & 112 \\
\hline 18 & $\ddot{-n}$ & $\cdots$ & -- & -- & $\cdots$ & $\cdots$ & $\cdots$ & e236 & e160 & e169 & 167 & 109 \\
\hline 19 & $\overline{--}$ & $\cdots$ & --- & -- & $\ldots$ & -- & $\cdots$ & e228 & e148 & e130 & 160 & 109 \\
\hline 20 & $\overline{--}$ & $\cdots$ & $\overline{--}$ & --- & -- & --- & $\cdots$ & $e 204$ & e139 & 0124 & 158 & \\
\hline 21 & $\ddot{-\infty}$ & $\cdots$ & $\overline{--}$ & $\cdots$ & $\cdots$ & $\cdots$ & $\cdots$ & e188 & 147 & e188 & 156 & 7 \\
\hline 22 & 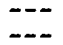 & $\cdots$ & $\cdots$ & $\cdots$ & $\cdots$ & $\cdots$ & $\cdots$ & e175 & 144 & 703 & 152 & 116 \\
\hline 23 & $\ldots$ & $\cdots$ & -- & $\cdots$ & $\cdots$ & -- & $\cdots$ & $\bullet 162$ & 141 & 280 & 151 & 0 \\
\hline 24 & $\bar{m}$ & $\cdots$ & -- & -- & $\cdots$ & -- & $\cdots$ & $e 160$ & 0130 & 191 & 148 & 1 \\
\hline 25 & $\bar{m}$ & -- & $\cdots$ & $\cdots$ & $\cdots$ & $\cdots$ & $\cdots$ & e161 & $e 130$ & 162 & 145 & 123 \\
\hline 26 & $\overline{--}$ & $\cdots$ & $\cdots$ & -- & -.- & $\cdots$ & $\cdots$ & e169 & e130 & 148 & 142 & 4 \\
\hline 27 & $\overline{--}$ & $\cdots$ & $\cdots$ & $\cdots$ & $\cdots$ & $\cdots$ & $\cdots$ & e180 & 0124 & 142 & 138 & 109 \\
\hline 28 & 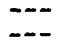 & $\cdots$ & $\ldots$ & $\cdots$ & $\ldots$ & $\cdots$ & $\cdots$ & e200 & 0118 & 153 & 132 & 116 \\
\hline 29 & $\overline{--}$ & $\cdots$ & -- & $\cdots$ & $\ldots$ & - - & $-\cdots$ & 224 & e117 & 155 & 130 & 115 \\
\hline 30 & $\overline{--}$ & 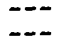 & -- & $\cdots$ & -- & $\cdots$ & $\cdots$ & 198 & e118 & 147 & 129 & 109 \\
\hline 31 & $\overline{--}$ & $\cdots$ & -- & -- & -- & $\cdots$ & -- & 209 & $\cdots$ & 144 & 127 & \\
\hline TOTAL & $\overline{-\cdots}$ & $\overline{-n}$ & -- & $\cdots$ & $\cdots$ & $\cdots$ & $\cdots$ & $\cdots$ & 4524 & 5034 & 5238 & 3528 \\
\hline $\begin{array}{l}\text { MEAN } \\
\text { MAX }\end{array}$ & 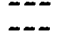 & $\overline{-1}$ & -- & $\overline{-n}$ & $\cdots$ & - & $-\infty$ & -- & 151 & 162 & 169 & 118 \\
\hline MAX & $\ldots$ & $\ldots$ & -- & $\overline{-n}$ & $\cdots$ & $\overline{-}$ & $\cdots$ & -- & 312 & 703 & 340 & 130 \\
\hline $\begin{array}{l}\text { MIN } \\
A C-F T\end{array}$ & $\ldots$ & $\ldots$ & -- & $=-$ & $\ddot{--}$ & $\overline{-m}$ & $\overline{-1}$ & -- & 117 & 107 & 127 & 109 \\
\hline $\begin{array}{l}\text { AC-FT } \\
\text { CFSM }\end{array}$ & -.- & $\ldots$ & $-\ldots$ & -- & -- & $\ldots$ & 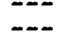 & - & 8970 & 9980 & 10390 & 7000 \\
\hline $\begin{array}{l}\text { CFSM } \\
\text { IN. }\end{array}$ & -- & -- & --- & $\ldots$ & -.- & $\ldots$ & $\ldots$ & $\cdots$ & $\begin{array}{r}.56 \\
.62\end{array}$ & $\begin{array}{l}.60 \\
.69\end{array}$ & $\begin{array}{r}.62 \\
.72\end{array}$ & 4 \\
\hline
\end{tabular}

STATISTICS OF MONTHLY MEAN DATA FOR WATER YEARS 1975 - 1991, BY WATER YEAR (WY)

\begin{tabular}{|c|c|c|c|c|c|c|c|c|c|c|c|c|}
\hline $\begin{array}{l}\text { MEAN } \\
\text { MAX } \\
\text { (WY) } \\
\text { MIN } \\
\text { (WY) }\end{array}$ & $\begin{array}{r}131 \\
187 \\
1984 \\
88.0 \\
1978\end{array}$ & $\begin{array}{r}138 \\
203 \\
1984 \\
84.8 \\
1978\end{array}$ & $\begin{array}{r}128 \\
180 \\
1984 \\
77.0 \\
1977\end{array}$ & $\begin{array}{r}120 \\
188 \\
1980 \\
80.6 \\
1978\end{array}$ & $\begin{array}{r}141 \\
236 \\
1985 \\
59.7 \\
1978\end{array}$ & $\begin{array}{r}264 \\
512 \\
1985 \\
84.2 \\
1978\end{array}$ & $\begin{array}{r}180 \\
264 \\
1983 \\
92.9 \\
1977\end{array}$ & $\begin{array}{r}174 \\
255 \\
1984 \\
89.6 \\
1977\end{array}$ & $\begin{array}{r}174 \\
240 \\
1980 \\
112 \\
1976\end{array}$ & $\begin{array}{r}237 \\
973 \\
1978 \\
92.1 \\
1977\end{array}$ & $\begin{array}{r}147 \\
205 \\
1979 \\
87.1 \\
1977\end{array}$ & $\begin{array}{r}143 \\
230 \\
1978 \\
85.5 \\
1977\end{array}$ \\
\hline
\end{tabular}

SUMMARY STATISTICS

FOR 1991 PERIOD

WATER YEARS 1975 - 1991

ANNUAL MEAN

HIGHEST ANNUAL MEAN

LOWEST ANNUAL MEAN

HIGHEST DAILY MEAN

LOWEST DAILY MEAN

ANNUAL SEVEN-DAY MINIMUM

INSTANTANEOUS PEAK FLOW

INSTANTANEOUS PEAK STAGE

INSTANTANEOUS LOW FLOW

ANNUAL RUNOFF (AC-FT)

ANNUAL RUNOFF (CFSM)

ANNUAL RUNOFF (INCHES)

$\begin{array}{cl}703 & \text { Jul } 22 \\ 107 & \text { Jul } 9,16 \\ 2040 & \text { Jul } 22 \\ 6.95 & \text { Jul } 22 \\ 93 & \text { Sep } 30\end{array}$

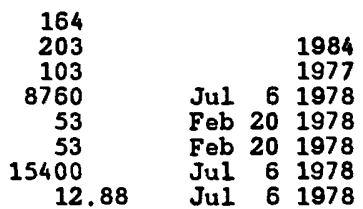

119100 
WHITEWATER RIVER BASIN

05376800 WHITEWATER RIVER NEAR BEAVER, MN--Continued

WATER QUALITY RECORDS

PERIOD OF RECORD.--Water years, 1975-1981, 1991.

WATER QUALITY DATA, WATER YEAR OCTOBER 1990 TO SEPTEMBER 1991

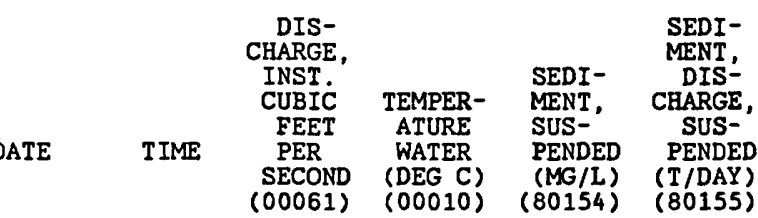

\begin{tabular}{|c|c|c|c|c|c|}
\hline \\
\hline $19 \ldots$ & $\begin{array}{l}1305 \\
1610\end{array}$ & $\begin{array}{l}120 \\
313\end{array}$ & $\begin{array}{l}7.0 \\
3.0\end{array}$ & $\begin{array}{r}40 \\
347\end{array}$ & $\begin{array}{r}13 \\
293\end{array}$ \\
\hline $\begin{array}{r}\text { AFR } \\
29 \ldots \\
29 \ldots \\
30 \ldots \\
\text { MAY }\end{array}$ & $\begin{array}{l}1345 \\
1730 \\
0930\end{array}$ & $\begin{array}{l}E 460 \\
E 435 \\
\text { E460 }\end{array}$ & $\begin{array}{r}14.0 \\
14.0 \\
8.0\end{array}$ & $\begin{array}{r}1420 \\
5900 \\
992\end{array}$ & $\begin{array}{l}=- \\
--\end{array}$ \\
\hline $\begin{array}{r}09 . . \\
16 . .\end{array}$ & $\begin{array}{l}1315 \\
2025\end{array}$ & $\begin{array}{l}304 \\
502\end{array}$ & $\begin{array}{l}12.0 \\
17.0\end{array}$ & $\begin{array}{r}227 \\
8640\end{array}$ & $\begin{array}{r}186 \\
11700\end{array}$ \\
\hline JUL $15 . .$. & 1357 & 319 & 19.0 & 2840 & 2450 \\
\hline $\begin{array}{r}10 \ldots \\
21 \ldots \\
22 \ldots \\
30 \ldots \\
\text { AUG }\end{array}$ & $\begin{array}{l}1145 \\
2318 \\
1405 \\
1206\end{array}$ & $\begin{array}{l}123 \\
980 \\
600 \\
145\end{array}$ & $\begin{array}{l}18.0 \\
21.0 \\
22.0 \\
16.0\end{array}$ & $\begin{array}{r}54 \\
8710 \\
975 \\
109\end{array}$ & $\begin{array}{r}18 \\
23000 \\
1580 \\
43\end{array}$ \\
\hline $08 \ldots$ & 1125 & 369 & 16.0 & 515 & 513 \\
\hline
\end{tabular}


GARVIN BROOK BASIN

05378235 GARVIN BROOK NEAR MIMRESOTA CITY, M

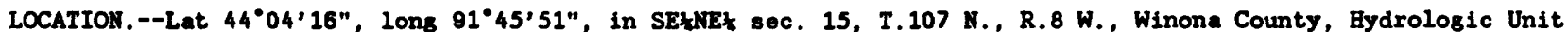
07040003 , on left bank, $20 \mathrm{ft}$ domstream from County 23 bridge, $1.8 \mathrm{mi}$ south of Minnesota City, and $2.3 \mathrm{mi}$ upstream from Rollingstone Creek.

PERIOD OF RECORD.--March 1982 to November 1983, January 1984 to current year (partial winter records in 1984), (discontinued).

GAGE.--Water stage recorder and broad-crested weir.

REMARKS.--Records good except those for estimated daily discharges, which are fair.

DISCEARGE, CUBIC FEET PER SECOND, WATER YEAR OCTOBER 1990 TO SEPTEMBER 1991

\begin{tabular}{|c|c|c|c|c|c|c|c|c|c|c|c|c|}
\hline DAY & OCT & Nov & DEC & JAN & FEB & MAR & APR & MAY & JUN & JUL & AUG & SEP \\
\hline $\begin{array}{l}1 \\
2 \\
3 \\
4 \\
5\end{array}$ & $\begin{array}{l}29 \\
29 \\
29 \\
29 \\
29\end{array}$ & $\begin{array}{l}27 \\
28 \\
28 \\
29 \\
29\end{array}$ & $\begin{array}{l}27 \\
27 \\
28 \\
37 \\
34\end{array}$ & $\begin{array}{l}\text { e30 } \\
\text { e30 } \\
\text { e30 } \\
\text { e30 } \\
\text { e30 }\end{array}$ & $\begin{array}{l}027 \\
027 \\
027 \\
027 \\
027\end{array}$ & $\begin{array}{l}24 \\
27 \\
24 \\
21 \\
22\end{array}$ & $\begin{array}{r}\text { e27 } \\
25 \\
26 \\
26 \\
27\end{array}$ & $\begin{array}{l}39 \\
37 \\
36 \\
36 \\
44\end{array}$ & $\begin{array}{l}40 \\
40 \\
34 \\
34 \\
32\end{array}$ & $\begin{array}{l}\bullet 31 \\
e 30 \\
\bullet 28 \\
\bullet 28 \\
\bullet 29\end{array}$ & $\begin{array}{l}44 \\
45 \\
41 \\
42 \\
41\end{array}$ & $\begin{array}{l}\bullet 36 \\
\bullet 35 \\
\bullet 34 \\
\bullet 33 \\
\bullet 33\end{array}$ \\
\hline $\begin{array}{r}6 \\
7 \\
8 \\
9 \\
10\end{array}$ & $\begin{array}{l}29 \\
29 \\
29 \\
29 \\
29\end{array}$ & $\begin{array}{l}28 \\
27 \\
27 \\
27 \\
27\end{array}$ & $\begin{array}{l}30 \\
29 \\
29 \\
29 \\
28\end{array}$ & $\begin{array}{l}\text { e30 } \\
\text { e30 } \\
\text { e30 } \\
\text { e30 } \\
\text { e30 }\end{array}$ & $\begin{array}{r}26 \\
226 \\
026 \\
26 \\
26\end{array}$ & $\begin{array}{l}28 \\
26 \\
24 \\
23 \\
23\end{array}$ & $\begin{array}{l}27 \\
28 \\
28 \\
29 \\
29\end{array}$ & $\begin{array}{l}41 \\
37 \\
39 \\
39 \\
39\end{array}$ & $\begin{array}{r}31 \\
37 \\
37 \\
037 \\
038\end{array}$ & $\begin{array}{l}-28 \\
-27 \\
-28 \\
029 \\
e 30\end{array}$ & $\begin{array}{l}40 \\
43 \\
70 \\
45 \\
41\end{array}$ & $\begin{array}{l}\text { e33 } \\
e 33 \\
e 33 \\
e 36 \\
e 33\end{array}$ \\
\hline $\begin{array}{l}11 \\
12 \\
13 \\
14 \\
15\end{array}$ & $\begin{array}{l}29 \\
29 \\
29 \\
29 \\
29\end{array}$ & $\begin{array}{l}27 \\
27 \\
27 \\
28 \\
28\end{array}$ & $\begin{array}{r}28 \\
29 \\
29 \\
033 \\
33\end{array}$ & $\begin{array}{r}-30 \\
e 30 \\
e 38 \\
41 \\
35\end{array}$ & $\begin{array}{r}26 \\
-26 \\
-25 \\
25 \\
24\end{array}$ & $\begin{array}{r}24 \\
25 \\
-26 \\
-26 \\
-26\end{array}$ & $\begin{array}{l}30 \\
31 \\
31 \\
32 \\
32\end{array}$ & $\begin{array}{l}40 \\
39 \\
38 \\
37 \\
38\end{array}$ & $\begin{array}{l}e 39 \\
038 \\
038 \\
038 \\
040\end{array}$ & $\begin{array}{l}e 28 \\
e 37 \\
e 35 \\
e 35 \\
e 35\end{array}$ & $\begin{array}{l}38 \\
37 \\
37 \\
37 \\
38\end{array}$ & $\begin{array}{l}e 33 \\
e 34 \\
e 33 \\
e 34 \\
035\end{array}$ \\
\hline $\begin{array}{l}16 \\
17 \\
18 \\
19 \\
20\end{array}$ & $\begin{array}{l}30 \\
30 \\
30 \\
30 \\
30\end{array}$ & $\begin{array}{l}28 \\
28 \\
27 \\
27 \\
28\end{array}$ & $\begin{array}{l}32 \\
33 \\
32 \\
32 \\
32\end{array}$ & $\begin{array}{l}32 \\
31 \\
31 \\
30 \\
30\end{array}$ & $\begin{array}{r}e 24 \\
e 24 \\
e 24 \\
24 \\
24\end{array}$ & $\begin{array}{l}e 26 \\
e 26 \\
-27 \\
e 28 \\
e 31\end{array}$ & $\begin{array}{l}32 \\
30 \\
29 \\
33 \\
31\end{array}$ & $\begin{array}{l}98 \\
56 \\
46 \\
44 \\
41\end{array}$ & $\begin{array}{l}e 38 \\
e 37 \\
e 37 \\
e 36 \\
e 35\end{array}$ & $\begin{array}{l}\text { e35 } \\
e 35 \\
e 39 \\
e 39 \\
e 39\end{array}$ & $\begin{array}{l}37 \\
38 \\
37 \\
37 \\
37\end{array}$ & $\begin{array}{l}\text { e33 } \\
e 33 \\
e 33 \\
e 33 \\
e 33\end{array}$ \\
\hline $\begin{array}{l}21 \\
22 \\
23 \\
24 \\
25\end{array}$ & $\begin{array}{l}30 \\
30 \\
30 \\
29 \\
28\end{array}$ & $\begin{array}{l}28 \\
27 \\
28 \\
28 \\
28\end{array}$ & $\begin{array}{l}e 31 \\
e 29 \\
e 28 \\
e 27 \\
e 29\end{array}$ & $\begin{array}{l}\text { e30 } \\
-29 \\
-29 \\
-29 \\
-29\end{array}$ & $\begin{array}{l}24 \\
24 \\
23 \\
23 \\
28\end{array}$ & $\begin{array}{l}-38 \\
-58 \\
e 51 \\
047 \\
043\end{array}$ & $\begin{array}{l}29 \\
26 \\
26 \\
26 \\
25\end{array}$ & $\begin{array}{l}38 \\
37 \\
37 \\
37 \\
37\end{array}$ & $\begin{array}{l}e 34 \\
e 34 \\
e 33 \\
e 32 \\
e 31\end{array}$ & $\begin{array}{r}1990 \\
0500 \\
0105 \\
058 \\
041\end{array}$ & $\begin{array}{l}37 \\
37 \\
37 \\
37 \\
37\end{array}$ & $\begin{array}{l}\text { e33 } \\
e 33 \\
033 \\
e 34 \\
e 34\end{array}$ \\
\hline $\begin{array}{l}26 \\
27 \\
28 \\
29 \\
30 \\
31\end{array}$ & $\begin{array}{l}28 \\
27 \\
27 \\
27 \\
27 \\
27\end{array}$ & $\begin{array}{r}28 \\
27 \\
25 \\
24 \\
27 \\
--\end{array}$ & $\begin{array}{l}\text { e30 } \\
e 32 \\
e 32 \\
e 31 \\
e 31 \\
e 31\end{array}$ & $\begin{array}{l}-28 \\
-28 \\
-28 \\
-28 \\
-28 \\
-27\end{array}$ & \begin{tabular}{r}
025 \\
23 \\
23 \\
-- \\
\hdashline- \\
-
\end{tabular} & $\begin{array}{l}\text { e39 } \\
e 36 \\
\text { e33 } \\
\text { e31 } \\
\text { e30 } \\
28\end{array}$ & $\begin{array}{r}28 \\
36 \\
31 \\
83 \\
46 \\
--\end{array}$ & $\begin{array}{l}37 \\
36 \\
36 \\
36 \\
35 \\
39\end{array}$ & $\begin{array}{l}\text { e30 } \\
\text { e30 } \\
\text { e30 } \\
\text { e30 } \\
030 \\
-30\end{array}$ & $\begin{array}{r}041 \\
041 \\
043 \\
046 \\
47 \\
46\end{array}$ & $\begin{array}{l}37 \\
37 \\
36 \\
36 \\
36 \\
36\end{array}$ & $\begin{array}{l}\text { e33 } \\
\text { e33 } \\
\text { e33 } \\
\text { e33 } \\
\text { e33 } \\
---\end{array}$ \\
\hline $\begin{array}{l}\text { TOTAL } \\
\text { MEAN } \\
\text { MAX } \\
\text { MIN } \\
\text { AC-FT }\end{array}$ & $\begin{array}{r}895 \\
28.9 \\
30 \\
27 \\
1780\end{array}$ & $\begin{array}{r}822 \\
27.4 \\
29 \\
24 \\
1630\end{array}$ & $\begin{array}{r}942 \\
30.4 \\
37 \\
27 \\
1870\end{array}$ & $\begin{array}{r}941 \\
30.4 \\
41 \\
27 \\
1870\end{array}$ & $\begin{array}{r}704 \\
25.1 \\
28 \\
23 \\
1400\end{array}$ & $\begin{array}{r}941 \\
30.4 \\
58 \\
21 \\
1870\end{array}$ & $\begin{array}{r}939 \\
31.3 \\
83 \\
25 \\
1860\end{array}$ & $\begin{array}{r}1269 \\
40.9 \\
98 \\
35 \\
2520\end{array}$ & $\begin{array}{r}1050 \\
35.0 \\
40 \\
30 \\
2080\end{array}$ & $\begin{array}{r}3603 \\
116 \\
1990 \\
27 \\
7150\end{array}$ & $\begin{array}{r}1228 \\
39.6 \\
70 \\
36 \\
2440\end{array}$ & $\begin{array}{r}1005 \\
33.5 \\
36 \\
33 \\
1990\end{array}$ \\
\hline
\end{tabular}

STATISTICS OF MONTHLY MEAN DATA FOR WATER YEARS 1982 - 1991, BY WATER YEAR (WY)

\begin{tabular}{|c|c|c|c|c|c|c|c|c|c|c|c|c|}
\hline $\begin{array}{l}\text { MEAN } \\
\text { MAX } \\
\text { (WY) } \\
\text { MIN } \\
\text { (WY) }\end{array}$ & $\begin{array}{l}33.5 \\
51.1 \\
1987 \\
21.4 \\
1989\end{array}$ & $\begin{array}{l}31.5 \\
38.6 \\
1985 \\
26.1 \\
1990\end{array}$ & $\begin{array}{l}31.1 \\
37.5 \\
1985 \\
25.6 \\
1990\end{array}$ & $\begin{array}{l}28.9 \\
34.1 \\
1985 \\
21.1 \\
1989\end{array}$ & $\begin{array}{l}34.3 \\
62.3 \\
1985 \\
25.1 \\
1991\end{array}$ & $\begin{array}{l}47.3 \\
80.0 \\
1989 \\
30.4 \\
1991\end{array}$ & $\begin{array}{l}40.5 \\
85.0 \\
1990 \\
31.3 \\
1989\end{array}$ & $\begin{array}{l}35.4 \\
40.9 \\
1991 \\
25.6 \\
1989\end{array}$ & $\begin{array}{l}33.4 \\
44.7 \\
1984 \\
22.5 \\
1989\end{array}$ & $\begin{array}{r}40.6 \\
116 \\
1991 \\
23.4 \\
1988\end{array}$ & $\begin{array}{l}32.1 \\
39.6 \\
1991 \\
21.9 \\
1988\end{array}$ & $\begin{array}{l}35.1 \\
67.1 \\
1986 \\
20.6 \\
1988\end{array}$ \\
\hline
\end{tabular}

SUMMARY STATISTICS

ANNUAL TOTAL

HIGHEST ANNUAL MEAN

LOWEST ANRUAL MEAN

BIGHEST DAILY MEAN

LOWEST DAILY MEAN

ANNUAL SEVEH-DAY MINIMUM

IRSTANTANEOUS PEAK FLOW

INSTANTANEOUS PEAK STAGE

INSTANTANEOUS LOW FLOW

ANNUAL RUNOFF (AC-FT)

10 PERCENT EXCEEDS

50 PERCENT EXCEEDS

90 PERCENT EXCEEDS
FOR 1990 CALENDAR YEAR

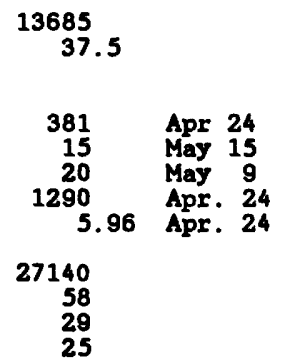

FOR 1991 WATER YEAR

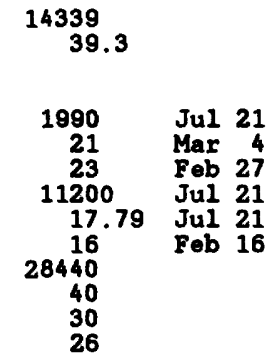

WATER YEARS 1982 - 1991

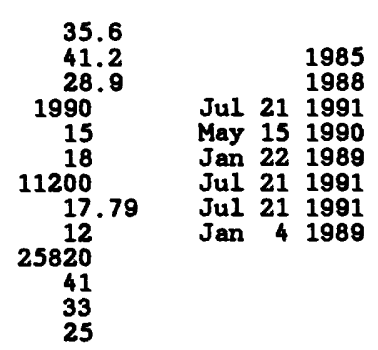


MISSISSIPPI RIVER MAIN STEM

05378500 MISSISSIPPI RIVER AT WINONA, MN

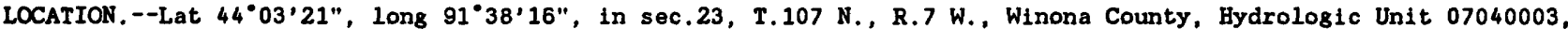
on right bank at Winona pumping station in Winona, $9.5 \mathrm{mi}$ upstream from Trempealeau River, and at mile 725.7 upstream from the Ohio River.

DRAINAGE AREA. $--59,200 \mathrm{mi}^{2}$, approximately.

\author{
WATER-DISCHARGE RECORDS
}

PERIOD OF RECORD.--June 1928 to current year. Gage-height records collected in this vicinity since 1878 are contained in reports of Mississippi River Commission.

GAGE.- Water-stage recorder. Datum of gage is $639.64 \mathrm{ft}$ above National Geodetic Vertical Datum of 1929 . June 10 , 1928, to Apr. 15, 1931, nonrecording gage at site $800 \mathrm{ft}$ upstream. Prior to Oct. 1 , 1929 , at datum $0.20 \mathrm{ft}$ higher and Oct. 1, 1929, to Apr. 15, 1931, at datum 0.12 ft lower. Apr. 16, 1931, to Nov. 12, 1934, nonrecording 8 age at present site and datum. Since Mar. 31, 1937, auxiliary water-stage recorder 2.7 mi upstream at tailwater of navigation dam $5 \mathrm{~A}$.

REMARKS. - No estimated daily discharges. Records good. Some regulation by reservoirs, navigation dams, and powerplants at low and medium stages. Flood flow not materially affected by artificial storage.

EXTREMES FOR PERIOD OF RECORD.--Minimum gage height, $-3.38 \mathrm{ft}$, Aug, 31 , 1934 (prior to dam construction in 1936); minimum gage height since 1938 , after completion of dam, 1.95 ft, Jan. $27,1944$.

EXTREMES OUTSIDE PERIOD OF RECORD,--Flood of June 18, 1880, reached an elevation of 657.14 ft, discharge, $172,000 \mathrm{ft}^{3} / \mathrm{s}$, from information by U.S. Army Corps of Engineers.

DISCHARGE, CUBIC FEET PER SECOND, WATER YEAR OCTOBER 1990 TO SEPTEMBER 1991

\begin{tabular}{|c|c|c|c|}
\hline 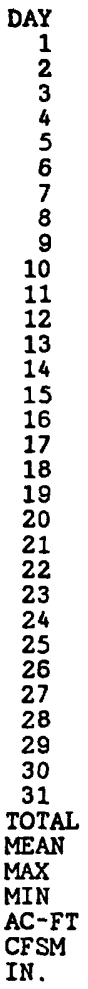 & $\begin{array}{r}\text { OCT } \\
18300 \\
17900 \\
15600 \\
15900 \\
18400 \\
19500 \\
22200 \\
23900 \\
23600 \\
22500 \\
22400 \\
21800 \\
21800 \\
21000 \\
20800 \\
20000 \\
18400 \\
17100 \\
19100 \\
20500 \\
24600 \\
26700 \\
29600 \\
35000 \\
36900 \\
35100 \\
33200 \\
31200 \\
29300 \\
26500 \\
27300 \\
736100 \\
23750 \\
36900 \\
15600 \\
1460000 \\
.40 \\
.46\end{array}$ & $\begin{array}{r}\text { NOV } \\
26800 \\
26000 \\
25200 \\
24200 \\
23100 \\
21200 \\
20500 \\
19100 \\
18400 \\
19100 \\
20300 \\
22100 \\
21600 \\
19800 \\
17700 \\
18400 \\
18100 \\
16200 \\
15100 \\
15800 \\
15500 \\
16500 \\
20000 \\
21000 \\
20400 \\
17600 \\
15100 \\
14400 \\
15200 \\
14400 \\
-0- \\
578800 \\
19290 \\
26800 \\
14400 \\
1148000 \\
.33 \\
.36\end{array}$ & $\begin{array}{r}\text { DEC } \\
15700 \\
14400 \\
14300 \\
14700 \\
14100 \\
13800 \\
13700 \\
12900 \\
12800 \\
12900 \\
12700 \\
13200 \\
15000 \\
15500 \\
14400 \\
14300 \\
14200 \\
14300 \\
14000 \\
13900 \\
12100 \\
11400 \\
9000 \\
8400 \\
8800 \\
8900 \\
9000 \\
9400 \\
10300 \\
10600 \\
12200 \\
390900 \\
12610 \\
15700 \\
8400 \\
775400 \\
121 \\
.25\end{array}$ \\
\hline
\end{tabular}

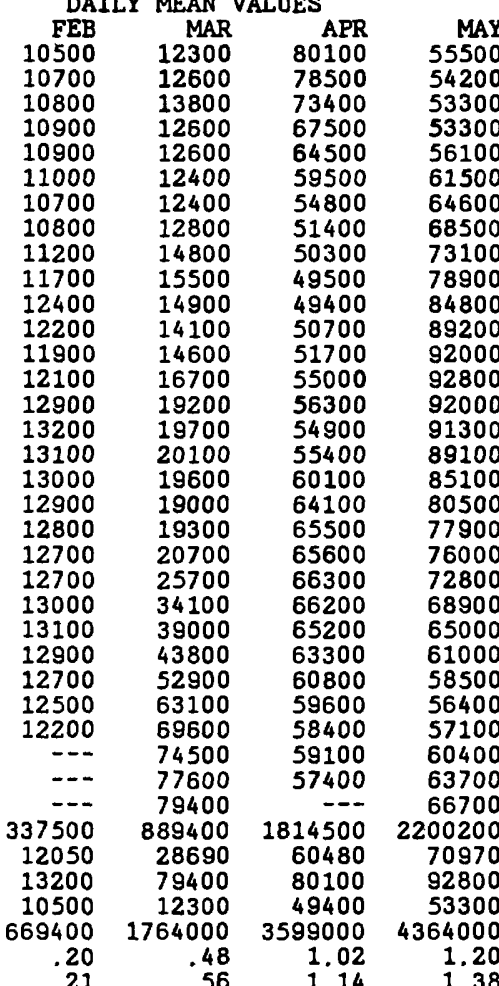

JUN
71400
75100
79500
83600
87400
89800
88400
83500
78400
74600
71400
70500
68400
66400
69600
70300
69600
67900
67000
66500
65100
63200
60200
59500
63300
64200
63000
62300
60400
58800
$-5-1$
2119300
70640
89800
58800
404000
1.19
1.33

JuL 56600

56400

57500

58500

59300

60400

61700

61800

60400

58100

56700

55500

54900

52700

50100

50200

52500

51600

50700

50700

54100

50600

47600

44600

42600

41800

40300
37900

36200

1623500

52370

61800

36200

322000

.88
1.02

AUG
35800

17900

$36000 \quad 13800$

$36500 \quad 14100$

$36100 \quad 16000$

$36100 \quad 19300$

$38900 \quad 22800$

$38200 \quad 25800$

$39100 \quad 31100$

$36800 \quad 39000$

$35300 \quad 45100$

$35500 \quad 49900$

$36400 \quad 51900$

$38200 \quad 53400$

$38200 \quad 54600$

36500

57700

$\begin{array}{ll}32200 & 57400 \\ 30500 & 56500\end{array}$

$30300-55900$

$29800 \quad 54500$

$28100 \quad 51800$

$26900 \quad 48900$

$26000 \quad 47500$

$25200 \quad 45600$

23500

$22400 \quad 37100$

22400

20700

0191001164000

38800

$39300 \quad 57700$

$20700 \quad 13800$

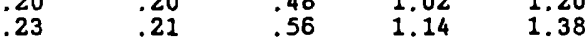

.56

.66

STATISTICS OF MONTHLY MEAN DATA FOR WATER YEARS 1928 - 1991, BY WATER YEAR (WY)

$\begin{array}{lrr}\text { MEAN } & 21750 & 21460 \\ \text { MAX } & 85950 & 50040 \\ \text { (WY) } & 1987 & 1972 \\ \text { MIN } & 6774 & 7367 \\ \text { (WY) } & 1934 & 1934\end{array}$

SUMMARY STATISTICS

ANNUAL TOTAL

ANNUAL MEAN

HIGHEST ANNUAL MEAN

LOWEST ANNUAL MEAN

HIGHEST DAILY MEAN

LOWEST DAILY MEAN

ANNUAL SEVEN-DAY MINIMUM

INSTANTANEOUS PEAK FLOW

INSTANTANEOUS PEAK STAGE

INSTANTANEOUS LOW FLOW

ANNUAL RUNOFF (AC-FT)

ANNUAL RUNOFF (CFSM)

ANNUAL RUNOFF (INCHES)

10 PERCENT EXCEEDS

50 PERCENT EXCEEDS

$16630 \quad 14500 \quad 14710$

29250

59040

47010

38240

29340

$19890 \quad 21570$

$1983 \quad 30480 \quad 3590086420$

$6282 \quad 6742 \quad 7874 \quad 9023$

111500

$12810 \quad 11930$

95980

64490

58410

69490

$1957 \quad 1972 \quad 1986$

$1934 \quad 1934 \quad 1933$

FOR 1990 CALENDAR YEAR 9827800

26930

FOR 1991 WATER YEAR

13239100

36270

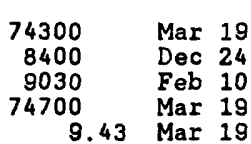

19490000

$$
\begin{array}{r}
6.45 \\
6.18 \\
47300 \\
22500 \\
9900
\end{array}
$$

26260000

$$
\begin{aligned}
& 69200^{8.32} \\
& 27300^{.61}
\end{aligned}
$$

WATER YEARS 1928 - 1991

27810

56850

9741

264000

2250

3210
268000

$20.77 a$

$1940 \mathrm{~b}$

20140000

a From floodmark.

b Result of 1 ce $j$ am. 


\section{MISSISSIPPI RIVER MAIN STEM}

05378500 MISSISSIPPI RIVER AT WINONA, MN--Continued

WATER-QUALITY RECORDS

PERIOD OF RECORD.--Water years 1963 to current year.

WATER QUALITY DATA, WATER YEAR OCTOBER 1990 TO SEPTEMBER 1991

$$
\begin{array}{cc} 
& \text { SEDI- } \\
\text { TEMPER- } & \text { MENT, } \\
\text { ATURE } & \text { SUS- } \\
\text { WATER } & \text { PENDED } \\
\text { (DEG C) } & (M G / L) \\
(00010) & (80154)
\end{array}
$$

DATE TIME WATER PENDED

$\begin{array}{cccc}\text { MAY } & 1603 & 10.0 & 56\end{array}$


ROOT RIVER BASIN

05385000 ROOT RIVER NEAR HOUSTON, MN

LOCATION, $-43^{\circ} 46^{\prime} 07^{\prime \prime}$, long $91^{\circ} 34^{\prime} 11^{\prime \prime}$, in SW INW $_{4}$ sec.33, T.104 N., R.6 W. Houston County, Hydrologic Unit 07040008 , on right bank $0.2 \mathrm{mi}$ north of Houston and $1.6 \mathrm{mi}$ upstream from South Fork and $18.2 \mathrm{mi}$ upstream from mouth.

DRAINAGE AREA. $-1,270 \mathrm{mi}^{2}$, approximately.

PERIOD OF RECORD, - May 1909 to September 1917, May to November 1929, March 1930 to 1983 , 1991. Operated as high-flow partial-record station October 1983 to September 1990. Monthly discharge only for some periods, published in WSP 1308

REVISED RECORDS.--WSP 895: Drainage area. WSP 1508: 1911-12. WSP 1628: 1948(P).

GAGE.--Water-stage recorder. Datum of gage is $667.00 \mathrm{ft}$ National Geodetic Vertical Datum of 1929 . May 28 , 1909, to Sept. 30, 1917, nonrecording gage at site $1.3 \mathrm{mi}$ downstream at different datum. May 4 , 1929 , to Sept. 27, 1933, nonrecording gage and Sept. 28, 1933 to June 26, 1980, recording gage at site 0.9 mi upstream at datum $671.86 \mathrm{ft}$

REMARKS.--Records good except those for estimated daily discharges, which are fair. Slight diurnal fluctuation at low flows caused by powerplants above station.

EXTREME FOR CURRENT YEAR. --Peak discharges above base of $5,000 \mathrm{ft}^{3} / \mathrm{s}$ and maximum (*).

$\begin{array}{llcccc} & \text { Discharge } & \text { Gage height } & \text { Discharge } & \text { Gage height } \\ \text { Date } & \text { Time } & \text { (ft } / \mathrm{s}) & \text { (ft) } & \text { Date (ft) } & \text { Time } \\ \text { May 7 } & 0130 & \star 4,940 & \star 10.04 & \text { No peak greater than base discharge. }\end{array}$

DISCHARGE, CUBIC FEET PER SECOND, WATER YEAR OCTOBER 1990 TO SEPTEMBER 1991

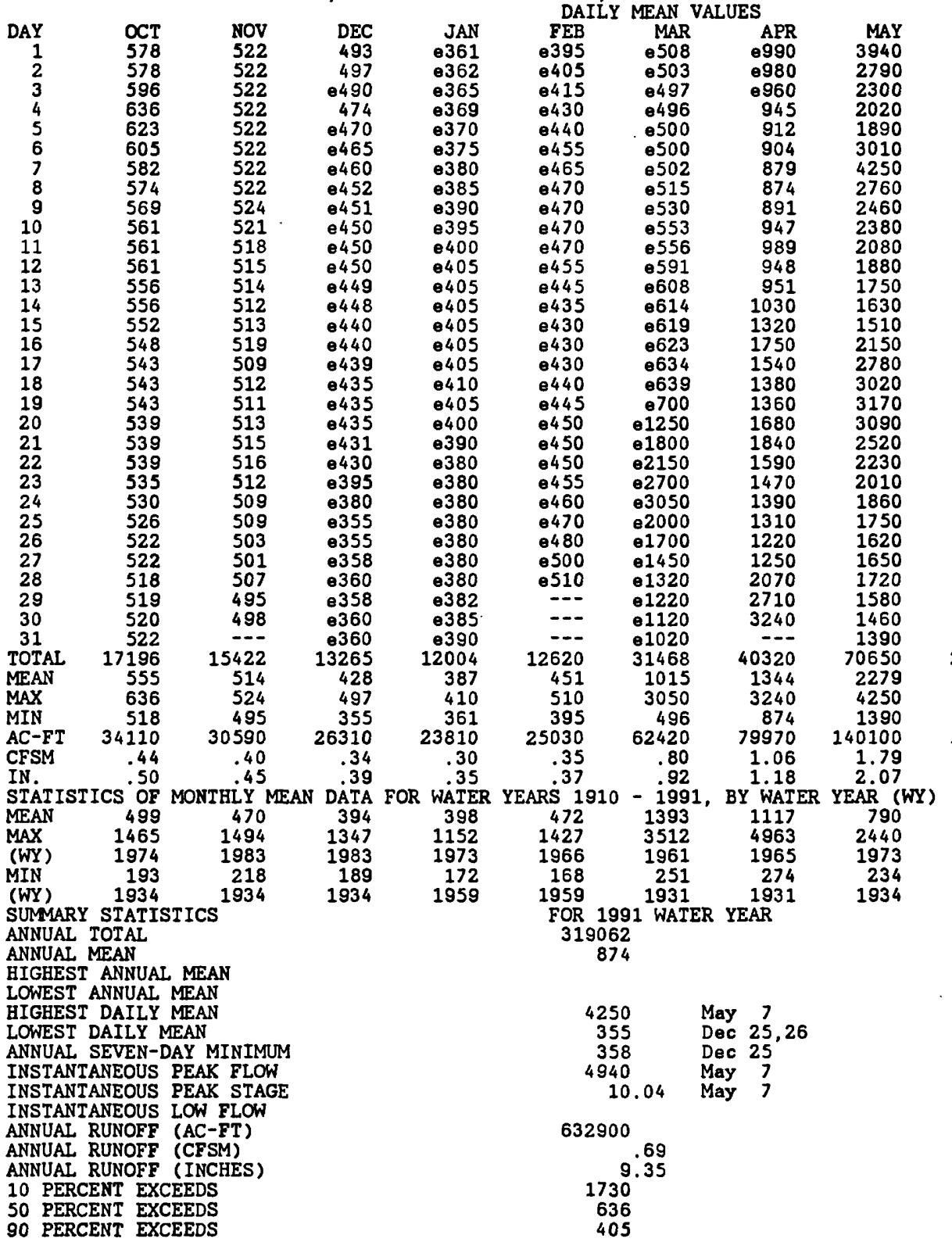

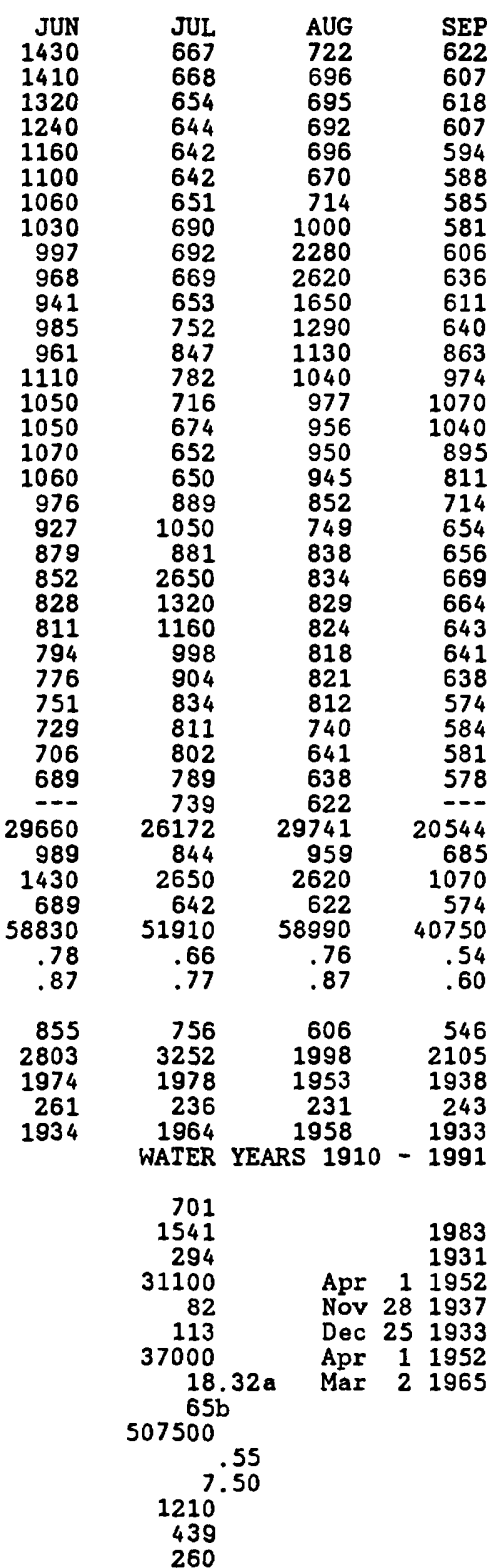

Backwater from ice

b Occurred Dec. 26, 1933 and Feb. 25, 1935.

e Estimated. 


\section{IOWA RIVER BASIN}

05457000 CEDAR RIVER NEAR AUSTIN, MN

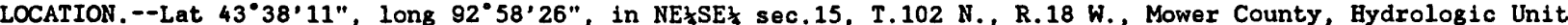
07080201 , on left bank 200 ft upstream from abandoned powerhouse, 500 ft downstrean from highway bridge, 1.1 mi downstream from Turtile Creek, and $1.1 \mathrm{mi}$ south of Austin.

DRAINAGE AREA. $--425 \mathrm{mi}^{2}$.

PERIOD OF RECORD. - May 1909 to September 1914, October 1944 to current year.

REVISED RECORDS.--WSP 1145: 1945, 1948.

GAGE.-Water-stage recorder. Datum of gage is 1,162.10 ft above National Geodetic Vertical Datum of 1929 . May 1909 to April 1912, nonrecording gage in tailwater of powerplant 200 ft downstream at datum 3.1 ft lower. May 1912 to September 1914, nonrecording 8 age on highway bridge 500 ft downstream at datum 1.1 ft lower.

REMARKS.--Records good except those for estimated daily discharges, which are fair.

EXTREMES FOR CURRENT YEAR. - Peak discharges greater than base discharge of $1,400 \mathrm{ft} 3 / \mathrm{s}$ and maximum ( $\star$ ):

\begin{tabular}{|c|c|c|c|c|c|c|c|}
\hline $\begin{array}{l}\text { Date } \\
\text { Mar. } 23 \\
\text { Apr. } 15 \\
\text { Apr. } 19 \\
\text { Apr. } 27\end{array}$ & $\begin{array}{l}\text { Time } \\
2230 \\
0100 \\
2000 \\
1630\end{array}$ & $\begin{array}{c}\text { Discharge } \\
(\text { ft } 3 / s) \\
3,000 \\
1,560 \\
1,480 \\
1,790\end{array}$ & $\begin{array}{c}\text { Gage height } \\
\text { (ft) } \\
8.87 \\
6.19 \\
6.04 \\
6.65\end{array}$ & \begin{tabular}{lr}
\multicolumn{2}{c}{ Date } \\
Apr. & 30 \\
May & 6 \\
May & 18 \\
Aug. & 8
\end{tabular} & $\begin{array}{l}\text { Time } \\
0830 \\
0630 \\
2000 \\
2400\end{array}$ & $\begin{array}{c}\text { Discharge } \\
(f t / / s) \\
1,600 \\
* 4,640 \\
3,000 \\
4,460\end{array}$ & $\begin{array}{c}\text { Gage height } \\
\text { (ft) } \\
6.28 \\
\star 11.52 \\
8.87 \\
11.24\end{array}$ \\
\hline
\end{tabular}

DISCHARGE, CUBIC FEET PER SECOND, WATER YEAR OCTOBER 1990 TO SEPTEMBER 1991

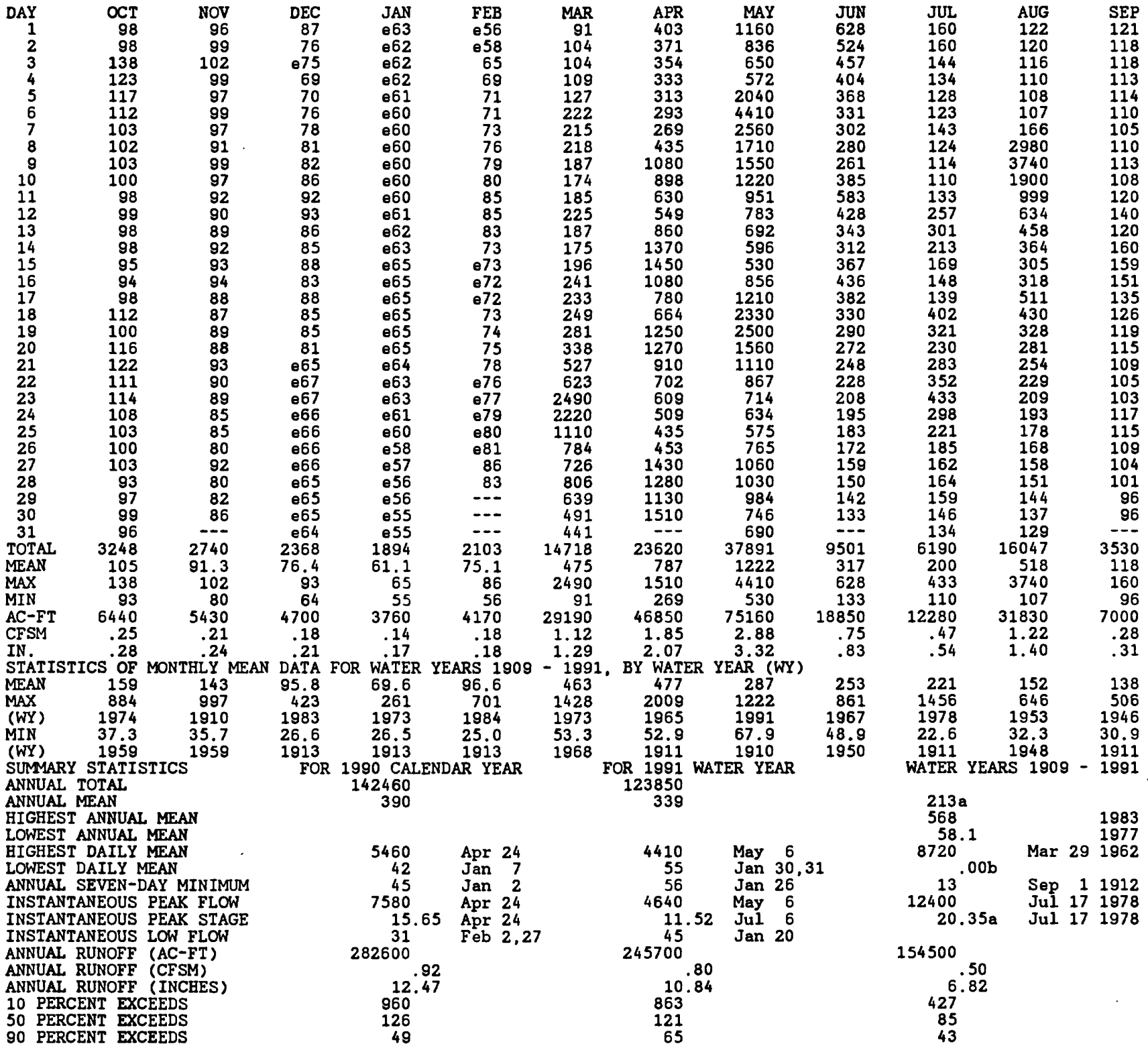

a Median of annual mean discharges is $195 \mathrm{ft}^{3} / \mathrm{s}$.

b Occurred for several days in 1911 .

c From floodmark in well.

Estimated 
DES MOINES RIVER BASIN

05476000 DES MOINES RIVER AT JACKSON, MN

LOCATION.--Lat $43^{\circ} 37^{\prime} 10^{\prime \prime}$, long $94^{\circ} 59^{\prime} 10^{\prime \prime}$, in SE $\frac{1}{2}$ SW 07100001 , on right bayk in storage room of city powerplant in Jackson.

DRAINAGE AREA. $-1,220 \mathrm{mi}^{2}$ approximately.

PERIOD OF RECORD. --May 19óg to December i913, August 1930 to current year (winter record incomplete prior to 1936). Published as Des Moines River near Jackson, 1930-35, as West Fork Des Moines River near Jackson, 1936-44 and as West Fork Des Moines River at Jackson, 1945-69.

REVISED RECORDS --WSP 1115: 1942, WSP 1175: Drainage area, WSP 1238: 1950, WSP 1308: $1938(\mathrm{M})$

GAGE.--Water-stage recorder. Datum of gage is $1,287.75 \mathrm{ft}$ above National Geodetic Vertical Datum of 1929.

May 31, 1909, to Dec, 20, 1913, nonrecording gage at site $0.6 \mathrm{mi}$ downstream at datum $0.99 \mathrm{ft} 10$ ler. Aug. 22. 1930, to Sept. 30, 1944. nonrecording gage at site 7 mi upstream at datum 17.10 ft higher. Oct. 1, 1944,

to Oct. 26, 1949, nonrecording gage at site $600 \mathrm{ft}$ upstream at datum $10.64 \mathrm{ft}$ higher. Oct. 27, 1949, to Dec. 15, 1965, water-stage recorder $200 \mathrm{ft}$ downstream at present datum.

REMARKS. --Records good except those for estimated daily discharges, which are fair. Regulation at times by Yankton, Long, Shetek, and Heron Lakes.

EXTREMES FÓR CURRENT YEAR. - Peak discharge greater than base discharge of $900 \mathrm{ft}^{3} / \mathrm{s}$ and maximum ( $*$ ):

\begin{tabular}{|c|c|c|c|c|c|c|c|}
\hline Date & Time & $\begin{array}{c}\text { Discharge } \\
\left(\mathrm{ft}^{3} / \mathrm{s}\right)\end{array}$ & $\begin{array}{c}\text { Gage height } \\
(f t)\end{array}$ & Date & Time & $\begin{array}{c}\text { Discharge } \\
(\mathrm{ft} / \mathrm{s})\end{array}$ & $\begin{array}{l}\text { Gage height } \\
\text { (ft) }\end{array}$ \\
\hline $\begin{array}{ll}\text { May } 21 \\
\text { June } & 10\end{array}$ & $\begin{array}{l}2100 \\
0615\end{array}$ & $\begin{array}{l}1,810 \\
3,400\end{array}$ & $\begin{array}{r}8.97 \\
12.20\end{array}$ & June 23 & 0400 & $\star 3,470$ & $\neq 12.32$ \\
\hline
\end{tabular}

DISCHARGE, CUBIC FEET PER SECOND, WATER YEAR OCTOBER 1990 TO SEPTEMBER 1991

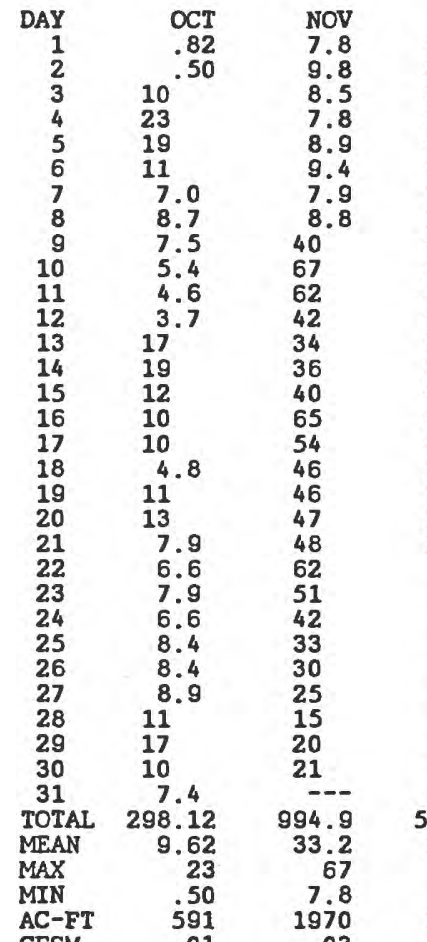

DEC
23
25
23
21
23
21
19
18
20
23
26
29
28
30
26
21
22
19
16
17
11
9.3
8.0
4.7
4.5
3.6
3.2
3.3
3.6
2.6
1.2
505.0
16.3
30
1.2
1000
1.01

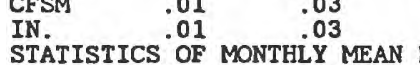

$\begin{array}{lll}\text { MEAN } & 140 & 143 \\ \text { MAX } & 1724 & 1833\end{array}$

$\begin{array}{lrr}\text { MAX } & 1724 & 1833 \\ \text { (WY) } & 1987 & 1980 \\ \text { MIN } & 000 & 000\end{array}$

$\begin{array}{lll}\text { MIN } & 000 & 000\end{array}$

SUMMARY STATISTICS

ANNUAL TOTAL

ANNUAL MEAN

HIGHEST ANNUAL MEAN

LOWEST ANNUAL MEAN

HIGHEST DAILY MEAN

LOWEST DAILY MEAN

ANNUAL SEVEN-DAY MINIMUM

INSTANTANEOUS PEAK STAGE

INSTANTANEOUS LOW FLOW

ANNUAL RUNOFF (AC-FT)

ANNUAL RUNOFF (CFSM)

ANNUAL RUNOFF (INCHES)

10 PERCENT EXCEEDS

50 PERCENT EXCEEDS

90 PERCENT EXCEEDS

a Median of annual mean discharges is $240 \mathrm{ft}^{3} / \mathrm{s}$.

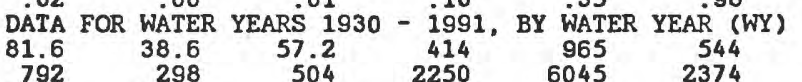

792
1980

$\begin{array}{lll}1000 & 000 & 000 \\ 1956 & 1956 & 1936\end{array}$

FOR 1990 CALENDAR YEAR

$\begin{array}{ll}11.8 & 9.37 \\ 1959 & 1959\end{array}$

e Estimated.

JAN
1.0
1.0
.86
.93
.76
e1.0
e2.2
3.7
1.4
1.4
e1.4
e1.4
e1.5
1.6
1.8
1.3

FEB
1.1
1.1
1.8
8.1
10
11
16

\section{MAR APR}

(1)

20
11

APR MAY

271

1901

144

133

124

135

135

170

149

121

166

503
760

838

753

652

590

544

511

473

446
423

423
321

321
247

386

453

476
529

529

606

11410

380
838

838

121

22630

.31

$.01 \quad 6260$

$.00 \quad .01$

$\begin{array}{rr}298 & 504 \\ 1980 & 1983\end{array}$

22247.51

61.0

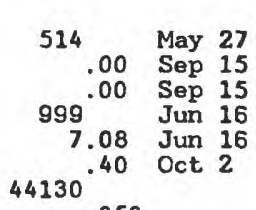

2250
1983

$1983 \quad 1969$

$\begin{array}{ll}19.37 & 2.59\end{array}$

2.59
1934

FOR 1991 WATER YEAR

158934.07

435

MAY JUN

$538 \quad 01300$

$503 \quad 1460$

$752 \quad 2050$

6932200

621

612
596

579

565

547
535
553

800

1430

1690

1760

1780

1760

1450

Q1220

-1350

- 1350

@1250

e1100

32108

1036
1780

503

63690

2410

2880

3080

3260

3020

2880

2830

2810

2770

02600
$\bullet 2400$

2400
2170

2280

3300

3280

3310

3050

2890

2730

2700

2170

77660

77660
2589

3310

1200

154000

2.12
2.37

506

2843
1984

3.76

1931

$$
150^{.68}
$$

3310 Jun 23

\begin{aligned} .50 & Oct 2 \\ $3470^{.96} &$ Dec 31 \\ 12.32 & Jun 23 \\ \hline & Jun 23\end{aligned}

$315200^{.40}$ Oct 2

$1510^{\circ}$
62

3.5

JUL
1930
1750
1590
1450
1290
1180

AUG SE

$309 \quad 32$

$295 \quad 45$

$281 \quad 43$

$245 \quad 42$

$1090 \quad 260$

$1000 \quad 251$

$873 \quad 195$

$833 \quad 136$

887

814
714

647

588

537
490

450

431

402

544

520
460

404

362

351

399
383

360

335

23985

774
1930

335

47570

.63
.73

136
128
130

130

120

104

117
286

286
272

229

213

138
89

89
79
68

68

58

46
41
39

36

5059

163
309

36

10030
.13

.13

$\begin{array}{lll}403 & 162 & 152\end{array}$

$2453 \quad 1408 \quad 2244$

$1983 \quad 1979 \quad 1942$

$\begin{array}{lll}1.04 & .13 & 000 \\ 1931 & 1955 & 1931\end{array}$

WATER YEARS 1930 - 1991

$$
\begin{aligned}
& 320 a \\
& 1199 \\
& 15.1 \\
& 15500
\end{aligned}
$$

$15700^{.00}$

19.45

$231800^{.00}$

$3^{.25}$
$863^{56}$
66
2.4
1983

Apr 111969

Ju1 191931

$\begin{array}{lll}\text { Jul } & 19 & 1931 \\ \text { Apr } & 11 & 1969\end{array}$

Apr 111969

Many years 


\section{Partial-Record Stations and Miscellaneous Sites}

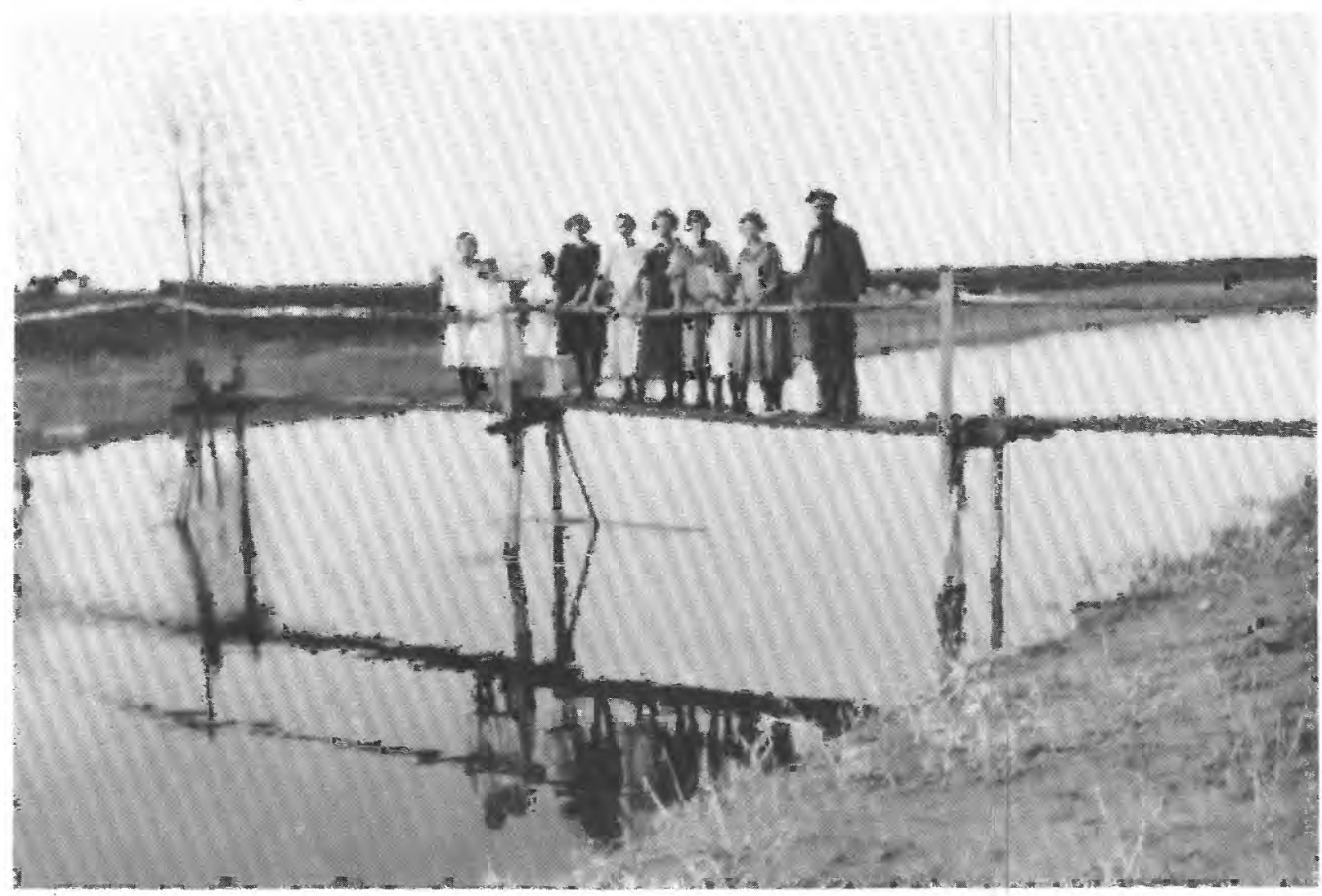

Roseau River

November, 1928 


\section{High-Flow Partial-Record Stations}

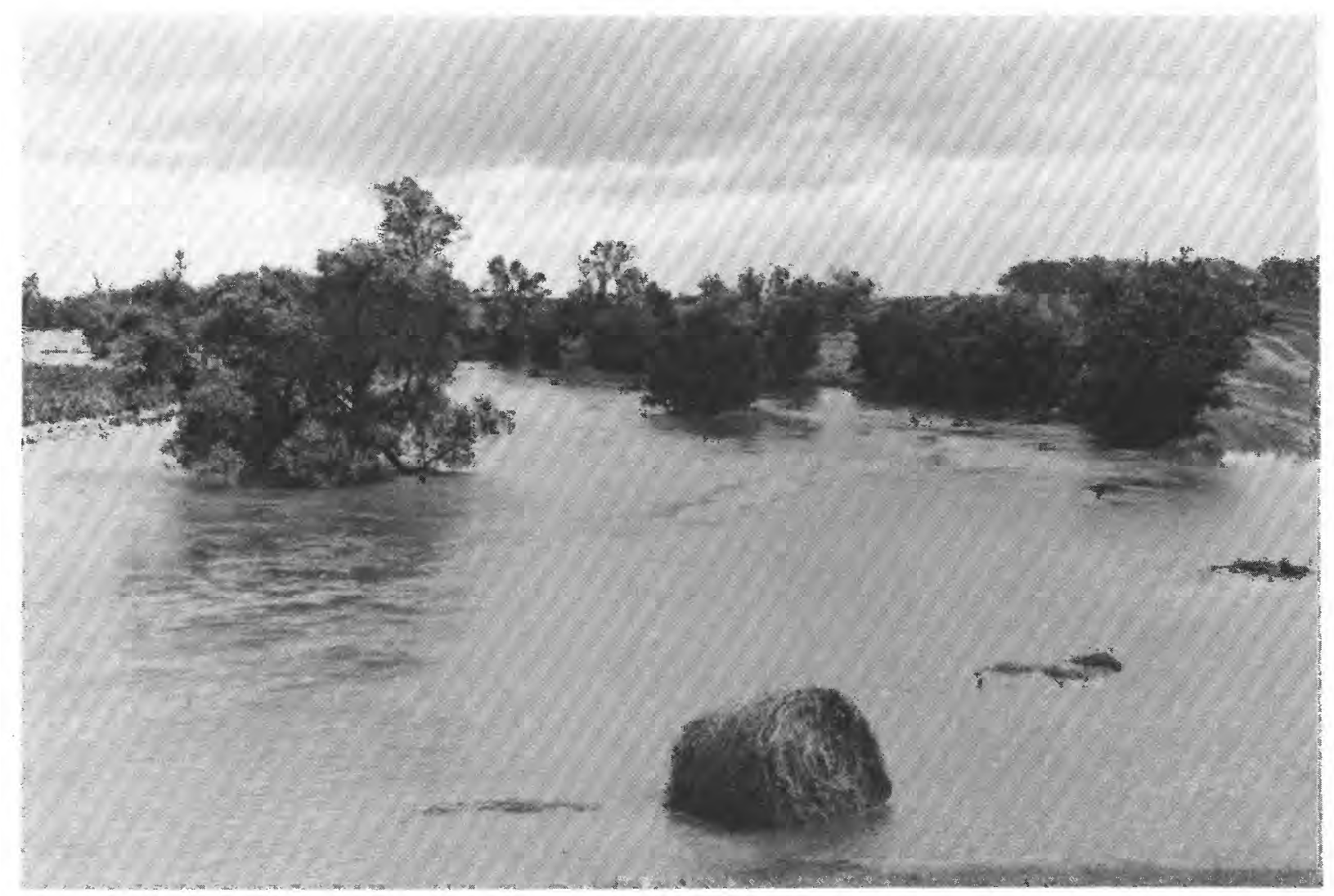

Hawk Creek near Maynard June 17, 1992 


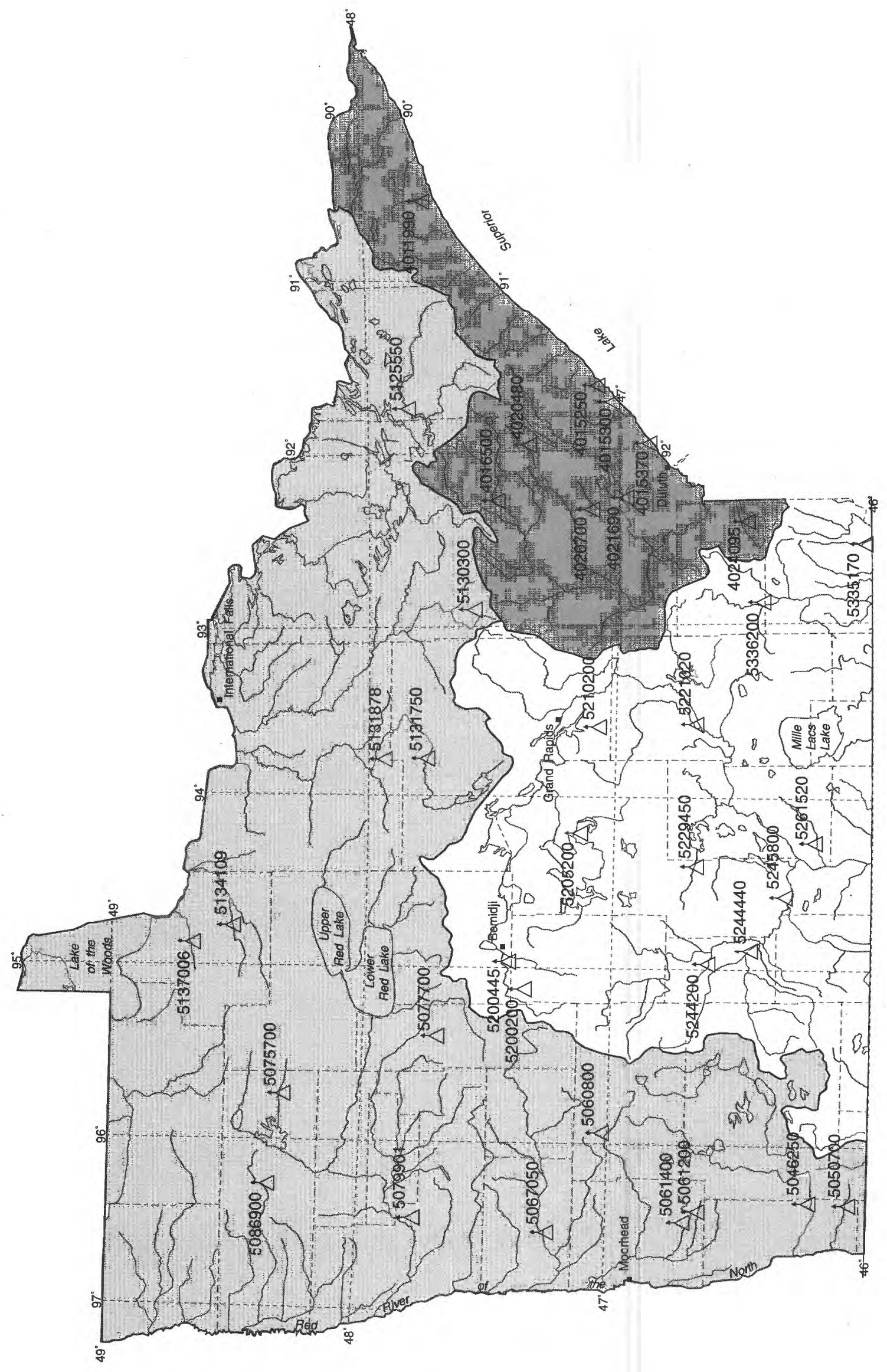



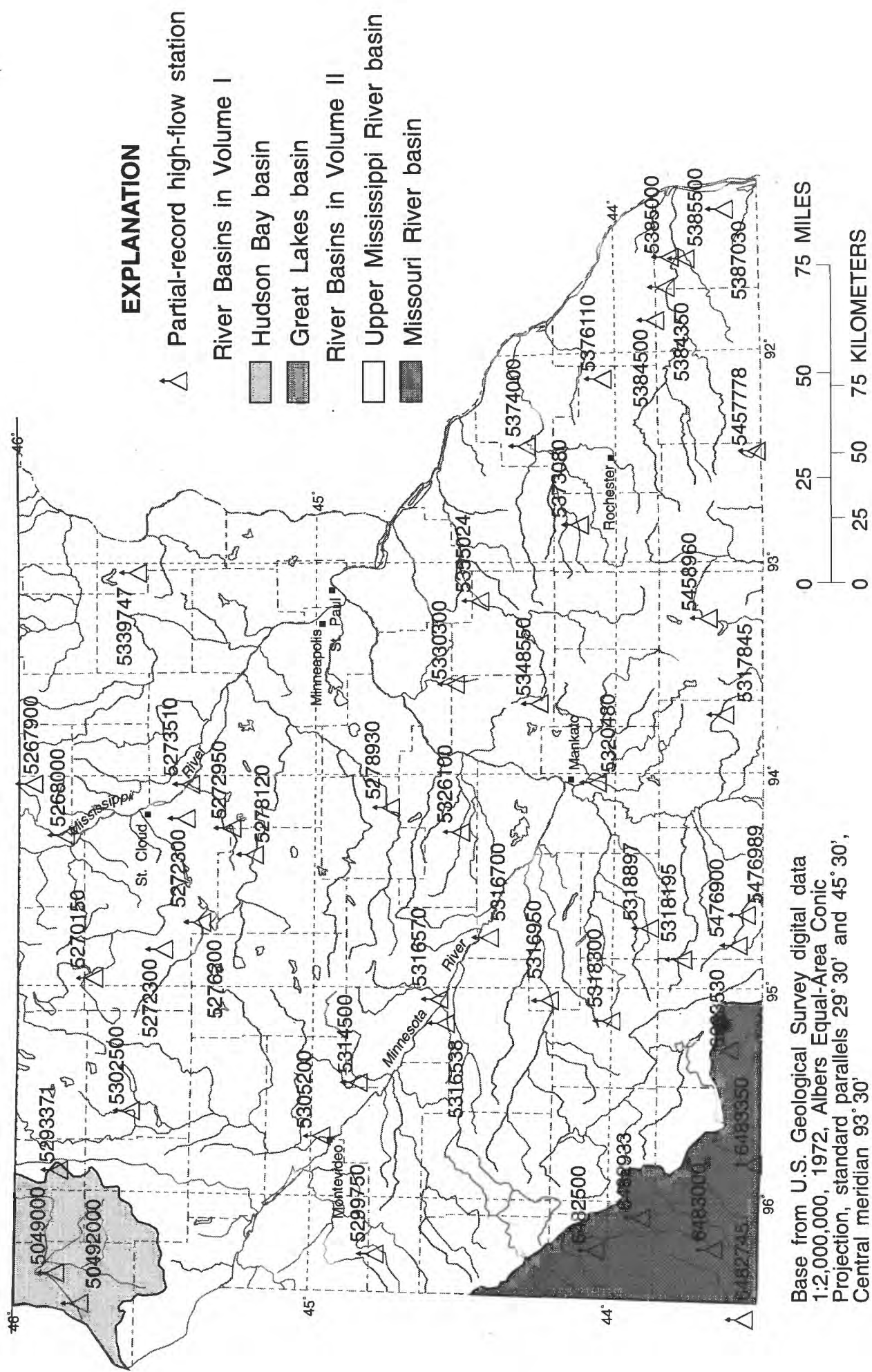

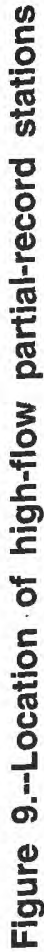


As the number of streams on which streamflow information is likely to be desired far exceeds the number of stream-gaging stations feasible to operate at one time, the Geological Survey collects limited streamflow data at sites other than stream-gaging stations. When limited streamflow data are collected on a systematic basis over a period of years for use in hydrologic analyses, the site at which the data are collected is called a partial-record station. Data collected at these partial-record stations are usable in low-flow or flood-flow analyses, depending on the type of data collected. In addition, discharge measurements are made at other sites not included in the partial-record program. These measurements are generally made in times of drought or flood to give better areal coverage to those events. Those measurements and others collected for some special reason are called measurements at miscellaneous sites.

Records collected at partial-record stations or miscellaneous sites are presented in two tables. The first is a table of discharge at high-flow partial-record stations and the second is a table of discharge measurements made at miscellaneous sites for both low flow and high flow.

\section{High-flow partial-record stations}

The following table contains annual maximum discharge for high-flow stations. A high-flow partial-record station is equipped with a crest-stage gage, a device which will register the peak stage occurring between inspections of the gage. A stage-discharge relation for each gage is developed from discharge measurements made by indirect measurements of peak flow or by current meter. The date of the maximum discharge is not always certain but is usually determined by comparison with nearby continuous-record stations, weather records, or local incuiry. Only the maximum discharge for each water year is given. Information on some lower floods may have been obtained, and discharge measurements may have been made for purposes of establishing the stage-discharge relation, but these are not published herein. The years given in the period of record represent water years for which the annual maximum has been determined.

Annual maximum discharge at high-flow partial-record stations during water year 1991

\begin{tabular}{|c|c|c|c|c|c|c|c|c|}
\hline \multirow[b]{2}{*}{$\begin{array}{c}\text { Station name } \\
\text { and } \\
\text { number }\end{array}$} & \multirow[b]{2}{*}{$\begin{array}{c}\text { Location } \\
\text { and } \\
\text { drainage area }\end{array}$} & \multirow[b]{2}{*}{$\begin{array}{l}\text { Period } \\
\text { of } \\
\text { record }\end{array}$} & \multicolumn{3}{|c|}{ Water year 1991 maximum } & \multicolumn{3}{|c|}{ Period of record maximum } \\
\hline & & & date & $\begin{array}{l}\text { gage } \\
\text { height } \\
\text { (ft) }\end{array}$ & $\begin{array}{c}\text { discharge } \\
\left(\mathrm{ft}^{3} / \mathrm{s}\right)\end{array}$ & date & $\begin{array}{c}\text { gage } \\
\text { height } \\
\text { (ft) }\end{array}$ & $\begin{array}{c}\text { discharge } \\
\left(\mathrm{ft}^{3} / \mathrm{s}\right)\end{array}$ \\
\hline \multicolumn{9}{|c|}{ Mississippi River main stem } \\
\hline $\begin{array}{l}\text { Hennepin Creek } \\
\text { near Becida, } \\
\text { MN } \\
05200200\end{array}$ & $\begin{array}{l}\text { Lat } 47^{\circ} 23^{\prime} 52^{\prime \prime}, \text { long } 95^{\circ} 05^{\prime} 12^{\prime \prime}, \\
\text { in NW'NE' sec. 11, T. } 145 \mathrm{~N} \text {. } \\
\text { R. } 35 \text { W. Hubbard County, Hydro- } \\
\text { logic Unit } 07010101 \text { at culvert } \\
\text { on Stumphges Rapids Trail approxi- } \\
\text { mately } 0.5 \text { mile west of Hubbard } \\
\text { County Road } 3,3 \text { miles north of } \\
\text { Becida, } 1.5 \text { miles upstream from } \\
\text { mouth. Drainage area } 41.4 \mathrm{mi}^{2} \text {. }\end{array}$ & 1979-91 & $5-3-91$ & a11. 78 & 41 & $5-11-85$ & 15.25 & 375 \\
\hline $\begin{array}{l}\text { Mississippi } \\
\text { River at } \\
\text { Bemidji, MN } \\
05200445\end{array}$ & $\begin{array}{l}\text { Lat } 46^{\circ} 27^{\prime} 04^{\prime \prime} \text { long } 94^{\circ} 54^{\prime} 23^{\prime \prime}, \\
\text { in NW'NW' sec. } 20 \text {, T.146 N., } \\
\text { R. } 33 \text { W. Beltrami County, Hydro- } \\
\text { logic Unit } 07010101 \text {, at bridge on } \\
\text { County Highway 11, } 1.4 \text { miles south } \\
\text { west of intersection of U.S. } \\
\text { Highway } 2 \text { and County Highway } 7 \\
\text { in Bemidji. Drainage area } 400 \mathrm{mi} \\
\text { Leech La }\end{array}$ & $\begin{array}{l}1973-87 \\
1988-89 \\
1990-91 \\
\text { h- } \\
\text { 2. } \\
\text { ke River ba }\end{array}$ & $5-9-91$ & b11.24 & 330 & $4-23-79$ & 13.04 & 1,690 \\
\hline $\begin{array}{l}\text { Boy River } \\
\text { near Remer, } \\
\text { MN } \\
05205200\end{array}$ & $\begin{array}{l}\text { Lat } 47^{\circ} 04^{\prime} 51^{\prime \prime}, \text { long } 94^{\circ} 05^{\prime} 54^{\prime \prime} \text {, } \\
\text { in SEसSE sec.28 T.142 N. } \\
\text { R. } 27 \text { W. Cass County, Hydro- } \\
\text { logic Unit } 07010102 \text {, at bridge on } \\
\text { County Highway } 53,1.9 \text { miles up- } \\
\text { stream from Boy Lake and } 9 \text { miles } \\
\text { northwest of Remer. Drainage } \\
\text { area } 310 \mathrm{mi}^{2} \text {. }\end{array}$ & $1986-91$ & $7-12-91$ & b10.4 & 340 & $7-23-87$ & 11.64 & 660 \\
\hline \multicolumn{9}{|c|}{ Smith Creek basin } \\
\hline $\begin{array}{c}\text { Smith Creek } \\
\text { near Hil1 } \\
\text { City, MN } \\
05210200\end{array}$ & 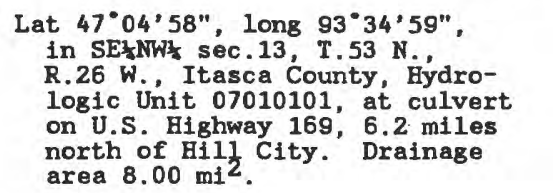 & $1961-91$ & $10-3-90$ & 4.59 & 51 & $8-5-81$ & 7.95 & 445 \\
\hline
\end{tabular}


DISCHARGE AT PARTIAL-RECORD STATIONS AND MISCELLANEOUS SITES

Annual maximum discharge at high-flow partial-record stations during water year 1991--Continued

\begin{tabular}{|c|c|c|c|c|c|c|c|c|}
\hline \multirow[b]{2}{*}{$\begin{array}{c}\text { Station name } \\
\text { and } \\
\text { number }\end{array}$} & \multirow[b]{2}{*}{$\begin{array}{c}\text { Location } \\
\text { and } \\
\text { drainage area }\end{array}$} & \multirow[b]{2}{*}{$\begin{array}{l}\text { Period } \\
\text { of } \\
\text { record }\end{array}$} & \multicolumn{3}{|c|}{ Water year 1991 maximum } & \multicolumn{3}{|c|}{ Period of record maximum } \\
\hline & & & date & $\begin{array}{c}\text { gage } \\
\text { height } \\
\text { (ft) }\end{array}$ & $\begin{array}{c}\text { discharge } \\
\left(\mathrm{ft}^{3} / \mathrm{s}\right)\end{array}$ & date & $\begin{array}{l}\text { gage } \\
\text { height } \\
\text { (ft) }\end{array}$ & $\begin{array}{c}\text { discharge } \\
\left(\mathrm{ft}^{3} / \mathrm{s}\right)\end{array}$ \\
\hline \multicolumn{9}{|c|}{ Willow River basin } \\
\hline $\begin{array}{l}\text { Willow River } \\
\text { below } \\
\text { Palisade, } \\
\text { MN } \\
05221020\end{array}$ & 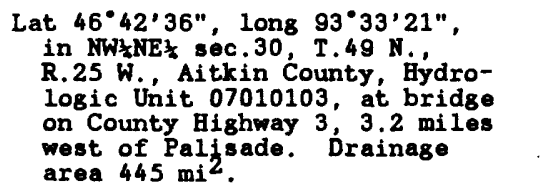 & $1972-91$ & $5-7-91$ & 11.80 & 1,180 & $4-25-79$ & 17.25 & 3,730 \\
\hline \multicolumn{9}{|c|}{ Pine River basin } \\
\hline $\begin{array}{l}\text { Pine River } \\
\text { near Pine } \\
\text { River, MN } \\
05229450\end{array}$ & 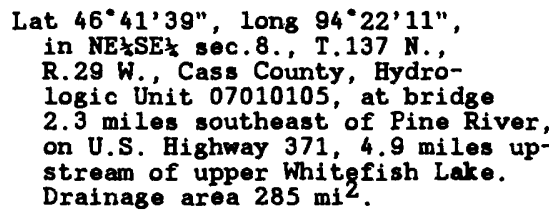 & $\begin{array}{l}1986-91 \\
-\end{array}$ & $5-8-91$ & C3. 11 & 431 & $3-28-86$ & 4.35 & 1,150 \\
\hline \multicolumn{9}{|c|}{ Crow Wing River basin } \\
\hline $\begin{array}{l}\text { Cat River near } \\
\text { Nimrod, MN } \\
05244200\end{array}$ & $\begin{array}{l}\text { Lat } 46^{\circ} 37^{\prime} 49^{\prime \prime}, \text { long } 94^{\circ} 55^{\prime} 51^{\prime \prime}, \\
\text { in SW'SW' sec. } 36 \text {, T. } 137 \text { N., } \\
\text { R.34 W. Wadena County, Hydro- } \\
\text { logic Unit o7010106, at bridge } \\
\text { on State Highway } 227,2.5 \\
\text { miles west of Nimrod, } 3.0 \text { miles } \\
\text { upstream from mouth. Drainage } \\
\text { area } 49.2 \text { mi }{ }^{2} \text {. }\end{array}$ & $1961-91$ & $7-1-91$ & $\mathrm{ab} 5.52$ & 92 & $10-12-73$ & 9.43 & 560 \\
\hline $\begin{array}{l}\text { Leaf River } \\
\text { near } \\
\text { Aldrich, MN } \\
05244440\end{array}$ & $\begin{array}{l}\text { Lat } 46^{\circ} 27^{\prime} 25^{\prime \prime}, \text { long } 94^{\circ} 50^{\prime} 29^{\prime \prime}, \\
\text { in SWzSW sec.34, T. } 135 \text { N. } \\
\text { R.33 W., Wadena County, Hydro- } \\
\text { logic Unit 07010107, at bridge } \\
\text { on County Highway } 29,3.3 \text { miles } \\
\text { upstream from mouth, } 7.0 \text { miles } \\
\text { northeast of Aldrich. Drainage } \\
\text { area } 860 \mathrm{mi}^{2} \text {. }\end{array}$ & $1972-91$ & $4-16-91$ & 11.45 & 1030 & $4-22-79$ & 16.15 & 5,170 \\
\hline $\begin{array}{l}\text { Sevenmile } \\
\text { Creek near } \\
\text { Pillager, MN } \\
05245800\end{array}$ & 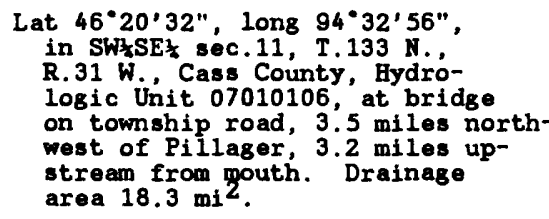 & $1979-91$ & $6-30-91$ & 11.81 & 80 & $6-14-83$ & 13.08 & 285 \\
\hline \multicolumn{9}{|c|}{ Nokasippi River basin } \\
\hline $\begin{array}{l}\text { Nokasippi } \\
\text { River near } \\
\text { Fort Ripley, } \\
\text { MN } \\
05261520\end{array}$ & $\begin{array}{l}\text { Lat } 46^{\circ} 12 \text { '02", long } 94^{\circ} 19^{\prime} 03^{\prime \prime} \\
\text { on line between secs.13 and } 24 \text {, } \\
\text { T.43 } \mathrm{N} ., \mathrm{R} .32 \mathrm{~W} \text {. Crow Wing } \\
\text { County, Hydrologic Unit } \\
\text { o7010104, at bridge on County } \\
\text { Highway } 2,3 \text { miles northeast } \\
\text { of Fort Ripley. Drainage } \\
\text { area } 178 \mathrm{mi} \text {. }\end{array}$ & $\begin{array}{c}1967-70+ \\
1974+ \\
1976+ \\
1986-91\end{array}$ & $6-30-91$ & 10.54 & 357 & $4-4-86$ & 13.90 & 828 \\
\hline & Platte & River basin & & & & & & \\
\hline $\begin{array}{l}\text { Hillman Creek } \\
\text { near Pi erz, } \\
05267900\end{array}$ & 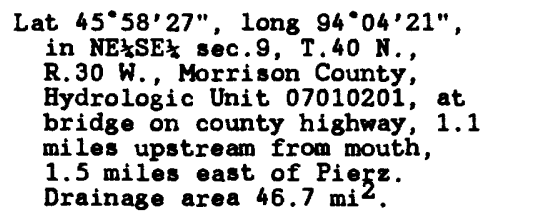 & $1964-91$ & $5-1-91$ & 13.70 & 490 & $4-9-69$ & 15.48 & 2,960 \\
\hline
\end{tabular}




\begin{tabular}{|c|c|c|c|c|c|c|c|c|}
\hline \multirow[b]{2}{*}{$\begin{array}{c}\text { Station name } \\
\text { and } \\
\text { number }\end{array}$} & \multirow[b]{2}{*}{$\begin{array}{c}\text { Location } \\
\text { and } \\
\text { drainage area }\end{array}$} & \multirow[b]{2}{*}{$\begin{array}{l}\text { Period } \\
\text { of } \\
\text { record }\end{array}$} & \multicolumn{3}{|c|}{ Water year 1991 maximum } & \multicolumn{3}{|c|}{ Period of record maximum } \\
\hline & & & dete & $\begin{array}{l}\text { gage } \\
\text { height } \\
\text { (ft) }\end{array}$ & $\begin{array}{c}\text { discharge } \\
\left(\mathrm{ft}^{3} / \mathrm{s}\right)\end{array}$ & date & $\begin{array}{l}\text { gage } \\
\text { height } \\
\text { (ft) }\end{array}$ & $\begin{array}{c}\text { discharge } \\
\left(\mathrm{ft}^{3} / \mathrm{s}\right)\end{array}$ \\
\hline \multicolumn{9}{|c|}{ Platte River basin--Continued } \\
\hline $\begin{array}{l}\text { Platte River } \\
\text { above } \\
\text { Royalton, } \\
\text { MN } \\
05266000\end{array}$ & 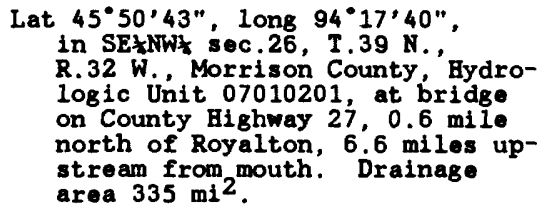 & $\begin{array}{l}1929-36 \\
1972-91\end{array}$ & $4-16-91$ & 12.47 & 2,150 & $7-26-72$ & 7.84 & 6,850 \\
\hline \multicolumn{9}{|c|}{ Sauk River basin } \\
\hline $\begin{array}{l}\text { Ashley Creek } \\
\text { near Sauk } \\
\text { Centre, MN } \\
05270150\end{array}$ & 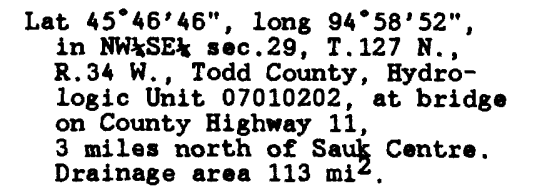 & $\begin{array}{l}1963-70+ \\
1974+ \\
1976+ \\
1986-88 \\
1989 \\
1990-91\end{array}$ & $9-9-91$ & 14.88 & 342 & $9-25-86$ & 16.52 & 600 \\
\hline $\begin{array}{l}\text { Sauk River } \\
\text { tributary } \\
\text { at Spring } \\
\text { Hi11, MN } \\
05270300\end{array}$ & 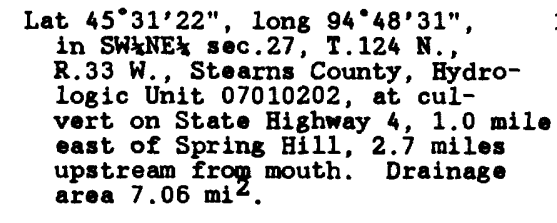 & $1960-91$ & $9-9-91$ & b10.67 & 152 & $7-6-78$ & 22.76 & 1,440 \\
\hline \multicolumn{9}{|c|}{ Johnson Creek basin } \\
\hline $\begin{array}{l}\text { Johnson Creek } \\
\text { near St. } \\
\text { Augusta, MN } \\
05272300\end{array}$ & $\begin{array}{l}\text { Lat } 45^{\circ} 27^{\prime} 49^{\prime \prime}, \text { long } 94^{\circ} 09^{\prime} 19^{\prime \prime}, \\
\text { in NW'SWt sec.13, T.123 N., } \\
\text { R.28 W. Stearns County, Hydro- } \\
\text { logic Unit 07010203, at bridge } \\
\text { on County Highway } 7 \text {, } 1.0 \text { mile } \\
\text { south of St. Augusta, } 3.3 \text { miles } \\
\text { upstream from mouth. Drainage } \\
\text { area } 46.7 \mathrm{mi}^{2} \text {. }\end{array}$ & $1964-91$ & $9-8-91$ & 12.80 & 255 & $9-9-85$ & 16.37 & 2,350 \\
\hline \multicolumn{9}{|c|}{ Clearwater River basin } \\
\hline $\begin{array}{l}\text { Clearwater } \\
\text { River near } \\
\text { South Haven, } \\
\text { MN } \\
05272950\end{array}$ & $\begin{array}{l}\text { Lat } 45^{\circ} 16^{\prime} 45^{\prime \prime}, \text { long } 94^{\circ} 15^{\prime} 04^{\prime \prime}, \\
\text { in NEtWW' sec.19, T.121 N., } \\
\text { R.28 W., Wright County, Hydro- } \\
\text { logic Unit } 07010203 \text {, at cul- } \\
\text { vert } 3.4 \text { mi les southeast of } \\
\text { Kimbali, } 0.25 \text { mile dowstream } \\
\text { of Scott Lake Outlot, } 2.0 \text { miles } \\
\text { southeast of South Haven. } \\
\text { Drainage area -. } \\
\text { Mississipp }\end{array}$ & I River mair & $9-16-91$ & 14.76 & 236 & $9-9-85$ & 17.11 & 1,040 \\
\hline $\begin{array}{l}\text { Mississippi } \\
\text { River at } \\
\text { Clearwater, } \\
\text { MN } \\
05273510\end{array}$ & $\begin{array}{l}\text { Lat } 45^{\circ} 25^{\prime} 15^{\prime \prime} \text {, long } 94^{\circ} 02^{\prime} 37^{\prime \prime}, \\
\text { in NW'sW } \\
\text { R.30 W. Sherburne County, Hydro- } \\
\text { logic Unit } 07010203 \text {, on left bank } \\
700 \text { ft upstream from bridge, on } \\
\text { State Highway } 24 \text { at Clearwater. } \\
\text { Drainage area }-. \\
\text { Crow }\end{array}$ & River basin & $5-10-91 i$ & bc 12.85 & 18,300 & $4-24-79$ & 18.75 & 33,900 \\
\hline $\begin{array}{l}\text { North Fork } \\
\text { Crow River at } \\
\text { Paynesville, } \\
\text { MN } \\
05276200\end{array}$ & 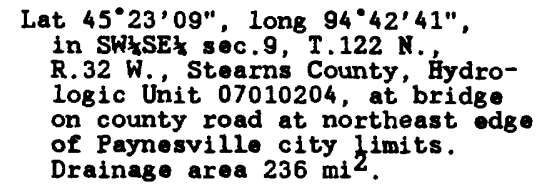 & $1973-91$ & $5-29-91$ & 24.43 & c620 & $6-21-83$ & 9.29 & 2,300 \\
\hline
\end{tabular}


DISCHARGE AT PARTIAL-RECORD STATIONS AND MISCELLANEOUS SITES

Annual maximum discharge at high-flow partial-record stations during water year 1991--Continued

\begin{tabular}{|c|c|c|c|c|c|c|c|c|}
\hline \multirow[b]{2}{*}{$\begin{array}{l}\text { Station name } \\
\text { and } \\
\text { number }\end{array}$} & \multirow[b]{2}{*}{$\begin{array}{l}\text { Location } \\
\text { and } \\
\text { drainage area }\end{array}$} & \multirow[b]{2}{*}{$\begin{array}{l}\text { Period } \\
\text { of } \\
\text { record }\end{array}$} & \multicolumn{3}{|c|}{ Water year 1991 maximum } & \multicolumn{3}{|c|}{ Period of record maximum } \\
\hline & & & date & $\begin{array}{l}\text { gage } \\
\text { height } \\
\text { (ft) }\end{array}$ & $\begin{array}{c}\text { discharge } \\
\left(\mathrm{ft}^{3} / \mathrm{s}\right)\end{array}$ & date & $\begin{array}{l}\text { gage } \\
\text { height } \\
\text { (ft) }\end{array}$ & $\begin{array}{c}\text { discharge } \\
\left(\mathrm{ft}^{3} / \mathrm{s}\right)\end{array}$ \\
\hline \multicolumn{9}{|c|}{ Crow River basin--Continued } \\
\hline $\begin{array}{l}\text { North Fork } \\
\text { Crow River } \\
\text { near } \\
\text { Kingston, MN } \\
05278120\end{array}$ & 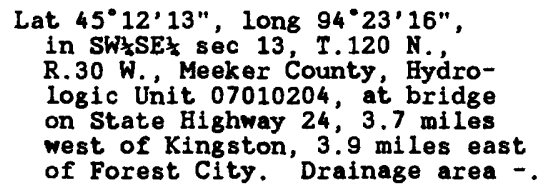 & $1986-91$ & $9-16-91$ & 15.70 & 2,750 & $5-1-86$ & 17.82 & 4,850 \\
\hline $\begin{array}{l}\text { Buffalo Creek } \\
\text { near Glencoe, } \\
\text { MN } \\
05278930\end{array}$ & $\begin{array}{l}\text { Lat } 44^{\circ} 45^{\prime} 50^{\prime \prime}, \text { long } 94^{\circ} 05^{\prime} 27^{\prime \prime}, \\
\text { in SW'SW' sec. 16, T.115 N.', } \\
\text { R. } 27 \mathrm{~W} \text {. McLeod County, Hydro- } \\
\text { logic Unit } 07010205 \text {, on right } \\
\text { bank, } 20 \text { ft ( } 6 \mathrm{~m} \text { ) downstream } \\
\text { from bridge on County Highway } 1 \text {, } \\
2.6 \mathrm{mi} \text { (4.2 km) east of Glencoe. } \\
\text { Drainage area } 374 \mathrm{mi}^{2} \text {. }\end{array}$ & $\begin{array}{l}1972 \\
1973-80 *\end{array}$ & $9-12-91$ & 11.78 & 4,300 & $9-12-91$ & 11.78 & 4,300 \\
\hline \multicolumn{9}{|c|}{ Minnesota River basin } \\
\hline $\begin{array}{l}\text { Pomme de terre } \\
\text { River near } \\
\text { Elbow Lake, } \\
\text { MN } \\
05293371\end{array}$ & 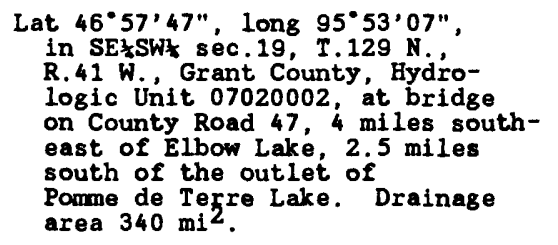 & $1986-91$ & $7-2-91$ & 4.54 & 195 & $4-6-69$ & d & e200 \\
\hline $\begin{array}{l}\text { Florida Creek } \\
\text { near Burr, MN } \\
05299750\end{array}$ & 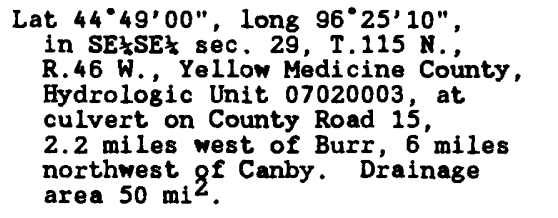 & $\begin{array}{l}1982 \\
1983-84 *\end{array}$ & $6-6-91$ & 14.47 & 81 & $3-29-84$ & 18.38 & 471 \\
\hline $\begin{array}{l}\text { Little Chippewa } \\
\text { River near } \\
\text { Starbuck, MN } \\
05302500\end{array}$ & $\begin{array}{l}\text { Lat } 45^{\circ} 36^{\prime} 52^{\prime \prime}, \text { long } 95^{\circ} 37^{\prime} 12^{\prime \prime}, \\
\text { in NW'NEt sec. } 30, T .125 \mathrm{~N} ., \\
\text { R. } 39 \text { W. Pope County, Hydro- } \\
\text { logic Unit } 07020005, \text { at cul- } \\
\text { vert on State Highway } 28,4.4 \\
\text { miles west of Starbuck. } \\
\text { Drainage area } 69.6 \mathrm{mi}^{2} \text {. }\end{array}$ & $1979-91$ & $6-30-91$ & c12.54 & 178 & $6-30-91$ & c12.54 & 178 \\
\hline $\begin{array}{l}\text { Spring Creek } \\
\text { near } \\
\text { Montevideo, } \\
\text { MN } \\
05305200\end{array}$ & $\begin{array}{l}\text { Lat } 44^{\circ} 58^{\prime} 41^{\prime \prime}, \text { long } 95^{\circ} 42^{\prime} 57^{\prime \prime}, \\
\text { in NW'NW' sec.5, T.117 N. ' } \\
\text { R. } 40 \text { W. Chippewa County, Hydro- } \\
\text { logic Unit } 07020005, \text { at culvert } \\
\text { on State Highway 29, } 1.2 \text { miles } \\
\text { upstream from mouth, } 2.0 \text { miles } \\
\text { north of Montevideo. Drainage } \\
\text { area } 16.0 \mathrm{mi}^{2} \text {. }\end{array}$ & $1959-91$ & $7-21-91$ & 18.00 & 470 & $7-19-62$ & 18.22 & 492 \\
\hline $\begin{array}{c}\text { Hawk Creek near } \\
\text { Maynard, MN } \\
05314500\end{array}$ & 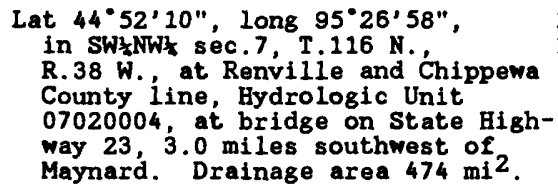 & $\begin{array}{l}1949-54 * \\
1981-91\end{array}$ & $9-9-91$ & 15.96 & 1,540 & $6-18-57$ & 16.10 & 6,970 \\
\hline $\begin{array}{l}\text { Ramsey Creek } \\
\text { near } \\
\text { Redwood Falls, } \\
\text { MN } \\
05316538\end{array}$ & 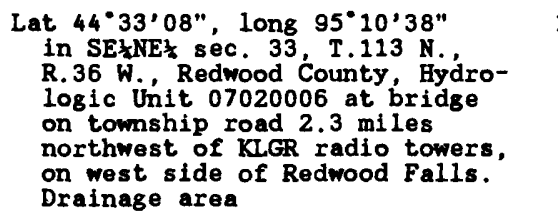 & 1991 & $6-4-91$ & 24.69 & 755 & $6-4-91$ & 24.69 & 755 \\
\hline
\end{tabular}


DISCHARGE AT PARTIAL-RECORD STATIONS AND MISCELLANEOUS SITES

Annual maximum discharge at high-flow partial-record stations during water year 1991--Continued

\begin{tabular}{|c|c|c|c|c|c|c|c|c|}
\hline \multirow[b]{2}{*}{$\begin{array}{c}\text { Station name } \\
\text { and } \\
\text { number }\end{array}$} & \multirow[b]{2}{*}{$\begin{array}{l}\text { Location } \\
\text { and } \\
\text { drainage area }\end{array}$} & \multirow[b]{2}{*}{$\begin{array}{l}\text { Period } \\
\text { of } \\
\text { record }\end{array}$} & \multicolumn{3}{|c|}{ Water year 1991 maximum } & \multicolumn{3}{|c|}{ Period of record maximum } \\
\hline & & & date & $\begin{array}{l}\text { gage } \\
\text { height } \\
\text { (ft) }\end{array}$ & $\begin{array}{c}\text { discharge } \\
\left(\mathrm{ft}^{3} / \mathrm{s}\right)\end{array}$ & date & $\begin{array}{l}\text { gage } \\
\text { height } \\
\text { (ft) }\end{array}$ & $\begin{array}{c}\text { discharge } \\
\left(\mathrm{ft}^{3} / \mathrm{s}\right)\end{array}$ \\
\hline \multicolumn{9}{|c|}{ Minnesota River basin--Continued } \\
\hline $\begin{array}{l}\text { Beaver Creek at } \\
\text { Beaver Falls, } \\
\text { MN } \\
05316570\end{array}$ & $\begin{array}{l}\text { Lat } 44^{\circ} 35^{\prime} 03^{\prime \prime}, \text { long } 95^{\circ} 02^{\prime} 49^{\prime \prime}, \\
\text { in NEkNWk sec. } 22 \text {, T. } 113 \text { H. } \\
\text { R.35 W. Renville County, Hydro- } \\
\text { logic Unit } 07020004 \text {, at bridge on } \\
\text { County Highway } 2 \text { in Beaver Falls, } \\
2.2 \text { miles upstream from mouth, } \\
3.8 \text { miles northwest } 8^{f} \text { Morton. } \\
\text { Drainage area } 194 \mathrm{mi}^{2} \text {. }\end{array}$ & $1972-91$ & $9-8-91$ & $\mathbf{a} 11.80$ & 965 & $4-23-85$ & a11.33 & 1,070 \\
\hline $\begin{array}{l}\text { Spring Creek } \\
\text { near Sleepy } \\
\text { Eye, MN } \\
05316700\end{array}$ & $\begin{array}{l}\text { Lat } 44^{\circ} 24^{\prime} 12^{\prime \prime}, \text { long } 94^{\circ} 44^{\prime} 41^{\prime \prime}, \\
\text { In NEłSEł sec.24, T. } 111 \text { H.', } \\
\text { R. } 33 \text { W. Brown County, Hydro- } \\
\text { logic Unit } 07020007 \text {, at cul- } \\
\text { vert on county highway, } 4.3 \\
\text { miles upstream from mouth, } \\
7.5 \text { miles north of Sleepy } \\
\text { Eye. Drainage area } 31.3 \mathrm{mi}^{2} \text {. }\end{array}$ & 1959-91 & $6-22-91$ & 14.54 & 510 & $4-10-65$ & 17.79 & 930 \\
\hline $\begin{array}{l}\text { Cottonwood } \\
\text { River near } \\
\text { Springfield, } \\
\text { MN } \\
05316950\end{array}$ & 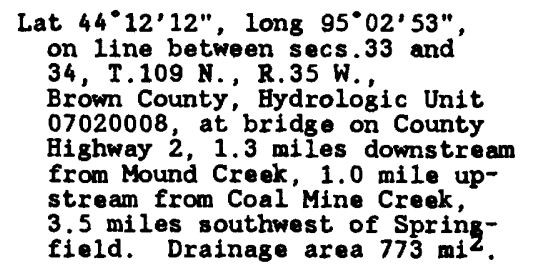 & $1973-91$ & $6-21-91$ & 223.72 & 3,400 & $6-25-84$ & 29.12 & 8,500 \\
\hline $\begin{array}{l}\text { East Branch } \\
\text { Blue Earth } \\
\text { River near } \\
\text { Walters, MN } \\
\text { 05317845 }\end{array}$ & $\begin{array}{l}\text { Lat } 43^{\circ} 37^{\prime} 58^{\prime \prime} \text {, long } 93^{\circ} 42^{\prime} 28^{\prime \prime}, \\
\text { in SEKSEt sec.16, T.102 H., } \\
\text { R. } 24 \text { W. Faribauit County, } \\
\text { Hydrologic Unit } 07020009, \text { at } \\
\text { culvert on State Highway } 22 \text {, } \\
2.5 \text { miles northwest of } \\
\text { Walters. Drainage area } 29.6 \mathrm{mi}^{2} \text {. }\end{array}$ & $1979-91$ & $6-1-91$ & 17.87 & 500 & $\begin{array}{l}4-28-81 \\
6-1-91\end{array}$ & $\begin{array}{l}18.17 \\
17.87\end{array}$ & $\begin{array}{l}500 \\
500\end{array}$ \\
\hline $\begin{array}{l}\text { Elm Creek near } \\
\text { Trimont, MN } \\
05318195\end{array}$ & $\begin{array}{l}\text { Lat } 43^{\circ} 45^{\prime} 27^{\prime \prime}, \text { long } 94^{\circ} 50^{\prime} 30^{\prime \prime}, \\
\text { in NWtNWz sec.5, T. } 103 \text { N. } \\
\text { R. } 33 \text { W. Martin County, Hydro- } \\
\text { logic Unit } 07020009, \text { at bridge } \\
\text { on County Road } 103,12.5 \text { miles } \\
\text { northeast of Jackson, } 5 \text { miles } \\
\text { west Trimont. Drainage area }\end{array}$ & 1991 & $6-4-91$ & 21.92 & 2,000 & $6-4-91$ & 21.92 & 2,000 \\
\hline $\begin{array}{l}\text { +Watonwan } \\
\text { River near } \\
\text { Delft, MN } \\
05318300\end{array}$ & $\begin{array}{l}\text { Lat } 43^{\circ} 59^{\prime} 55^{\prime \prime}, \text { long } 95^{\circ} 07^{\prime} 11^{\prime \prime}, \\
\text { in NEKSEK sec. } 11, \mathrm{~T} .106 \mathrm{~N}^{\prime}, \\
\mathrm{R} .36 \mathrm{~W} \text {. Cottonwood County', } \\
\text { Hydrologic Unit } 07020010 \text {, at } \\
\text { culvert on U.S. Highway } 71^{\prime} \\
1.7 \text { miles northwest of Delft. } \\
\text { Drainage area } 13.0 \mathrm{mi}^{2} \text {. }\end{array}$ & $1960-91$ & $6-21-91$ & 17.10 & 665 & $5-30-80$ & 17.82 & 900 \\
\hline $\begin{array}{l}\text { South Fork } \\
\text { Watonwan } \\
\text { River near } \\
\text { Ormsby, MN } \\
05318897\end{array}$ & 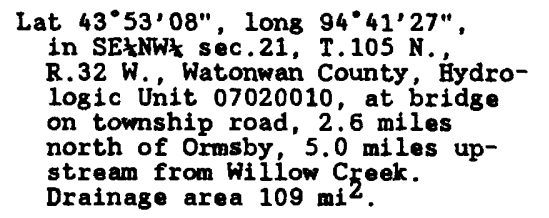 & $1979-91$ & $6-15-91$ & 14.42 & 638 & $5-31-80$ & 18.40 & 1,410 \\
\hline $\begin{array}{l}\text { Maple River } \\
\text { near } \\
\text { Rapidan, MN } \\
05320480\end{array}$ & $\begin{array}{l}\text { Lat } 44^{\circ} 03^{\prime} 54^{\prime \prime}, \text { long } 94^{\circ} \mathrm{O} 1^{\prime} 32^{\prime \prime}, \\
\text { in SWz sec.i3, T. } 107^{\mathrm{N} .}, \\
\text { R.27 W. Biue Earth County, } \\
\text { Hydrologic Unit } 07020011 \text {, at } \\
\text { bridge on County Highway } 35 \text {, } \\
3.0 \text { miles southeast of Rapidan, } \\
3.3 \text { miles upstream from mouth. } \\
\text { Drainage area } 343 \mathrm{mi}^{2} \text {. }\end{array}$ & $1972-91$ & $6-5-91$ & 12.05 & 3,520 & $3-1-83$ & 12.73 & 4,550 \\
\hline
\end{tabular}


DISCHARGE AT PARTIAL-RECORD STATIONS AND MISCELLANEOUS SITES

Annual maximum discharge at high-flow partial-record stations during water year 1991--Continued

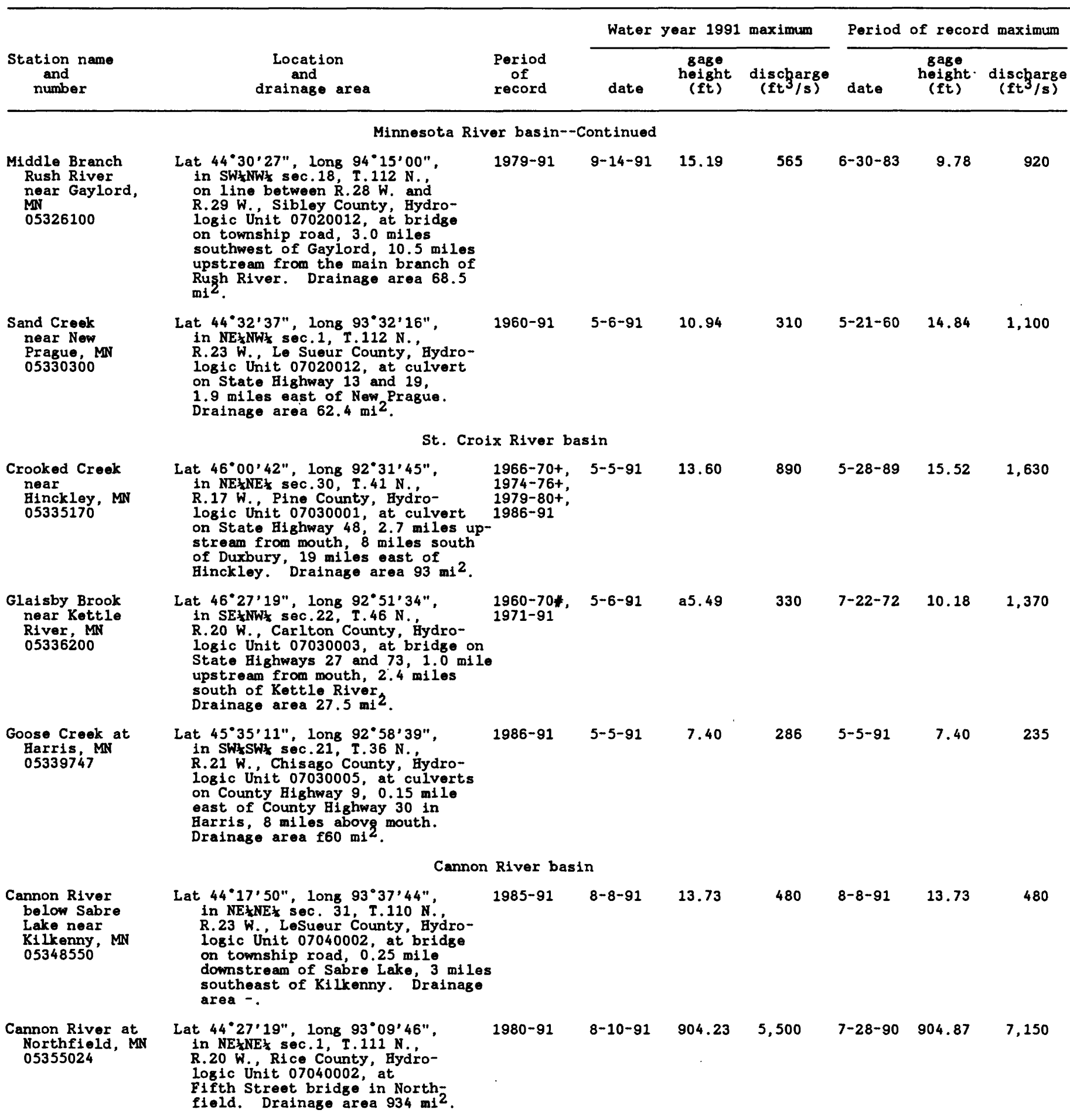

\footnotetext{
"See footnotes at end of the table."
} 
Annual maximum discharge at high-flow partial-record stations during water year 1991--Continued

\begin{tabular}{|c|c|c|c|c|c|c|c|c|}
\hline \multirow[b]{2}{*}{$\begin{array}{c}\text { Station name } \\
\text { and } \\
\text { number }\end{array}$} & \multirow[b]{2}{*}{$\begin{array}{l}\text { Location } \\
\text { and } \\
\text { drainage area }\end{array}$} & \multirow[b]{2}{*}{$\begin{array}{l}\text { Period } \\
\text { of } \\
\text { record }\end{array}$} & \multirow[t]{2}{*}{ Water } & year 1991 & maximum & \multicolumn{3}{|c|}{ Period of record maximum } \\
\hline & & & & $\begin{array}{l}\text { gage } \\
\text { height } \\
\text { (ft) }\end{array}$ & $\begin{array}{c}\text { discharge } \\
\left(\mathrm{ft}^{3} / \mathrm{s}\right)\end{array}$ & date & $\begin{array}{l}\text { gage } \\
\text { height } \\
\text { (ft) }\end{array}$ & $\begin{array}{c}\text { discharge } \\
\left(\mathrm{ft}^{3} / \mathrm{s}\right)\end{array}$ \\
\hline \multicolumn{9}{|c|}{ Zumbro River basin } \\
\hline $\begin{array}{l}\text { Milliken Creek } \\
\text { near Concord, } \\
\text { MN } \\
05373080\end{array}$ & 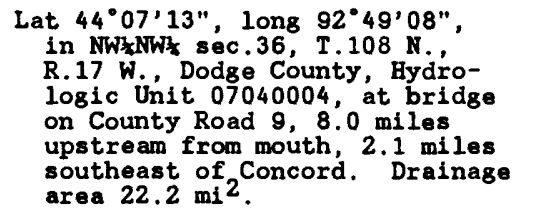 & $1979-91$ & $8-8-91$ & 13.57 & 425 & $5-31-82$ & 14.50 & 580 \\
\hline $\begin{array}{l}\text { Zumbro River } \\
\text { at Zumbro } \\
\text { Falls, MN } \\
05374000\end{array}$ & $\begin{array}{l}\text { Lat } 44^{\circ} 17^{\prime} 12^{\prime \prime} \text {, long } 92^{\circ} 25^{\prime} 56^{\prime \prime}, \\
\text { in sec.36, T. } 110 \mathrm{~N} \text {. R. } 14 \mathrm{~W}^{\prime} \text {, } \\
\text { Wabasha County, Hydrologic Unit } \\
07040004 \text {, on left bank in Zumbro } \\
\text { Falls, 1,000 ft downstsream from } \\
\text { Cold Creek, } 0.7 \text { mi upstream from } \\
\text { bridge on U.S. Highway } 63 \text {, and } \\
6.3 \text { mi downstream from North Fork } \\
\text { Drainage area f1, } 130 \mathrm{mi} \text {. }\end{array}$ & $\begin{array}{l}1909-17 \#, \\
1929-80 \#, \\
1990-91\end{array}$ & $5-6-91$ & 13.66 & 7,000 & $7-21-51$ & 30.80 & 35,900 \\
\hline \multicolumn{9}{|c|}{ Whitewater River basin } \\
\hline $\begin{array}{l}\text { Middle Fork } \\
\text { Whitewater } \\
\text { River neaer } \\
\text { State Park } \\
\text { Group Camp } \\
\text { near St. } \\
\text { Charles, MN } \\
\text { 05376110 }\end{array}$ & $\begin{array}{l}\text { Lat 03'21", long } 9203^{\prime} 13^{\prime \prime}, \\
\text { in SWl sec. } 20, T .107 \mathrm{~N} . \text {, } \\
\text { R. } 10 \text { W. Oimsted County, Hydro- } \\
\text { logic Unit } 07040003 \text {, at wooden } \\
\text { bridge near Group Camp in } \\
\text { Whitewater State Park. } \\
\text { Drainage area -.. }\end{array}$ & $1986-91$ & $7-22-91$ & 65.50 & $\uparrow$ & $4-24-90$ & 67.39 & $\dagger$ \\
\hline \multicolumn{9}{|c|}{ Root River basin } \\
\hline $\begin{array}{l}\text { Root River near } \\
\text { Lanesboro, MN } \\
05384000\end{array}$ & $\begin{array}{l}\text { Lat } 43^{\circ} 44^{\prime} 58^{\prime \prime}, \text { long } 91^{\circ} 58^{\prime} 43^{\prime \prime}, \\
\text { in sec. 1, T. } 103^{\prime} \text {. }^{\prime} \mathrm{R} .10^{\prime} \mathrm{W}, \\
\text { Filimore County, Hydrologic } \\
\text { Unit } 07040008 \text {, on left bank } \\
0.5 \mathrm{mi} \text { upstream from highway } \\
\text { bridge, } 1.2 \mathrm{mi} \text { upstream from } \\
\text { South Branch, and } 2.5 \mathrm{mi} \\
\text { northeast of Lanesboro } \\
\text { Drainage area }-615 \mathrm{mi} \text {. }\end{array}$ & $\begin{array}{l}1910-17 \# \\
1940-85 \# \\
1986 \\
1987-90 \# \\
1991\end{array}$ & $5-6-91$ & 6.07 & 3,230 & $3-29-62$ & 16.11 & 22,100 \\
\hline $\begin{array}{l}\text { Root River at } \\
\text { Rushford, MN } \\
05384350\end{array}$ & 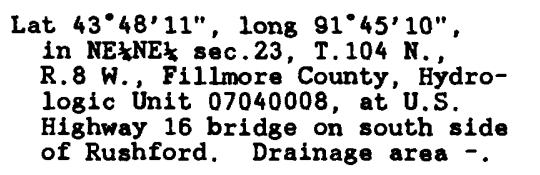 & $1985-91$ & $5-6-91$ & $8<23.93$ & 04,500 & $4-25-90$ & - & 9,000 \\
\hline $\begin{array}{l}\text { Rush Creek near } \\
\text { Rushford, MN } \\
05384500\end{array}$ & $\begin{array}{l}\text { Lat } 43^{\circ} 50^{\prime} 00^{\prime \prime}, \text { long } 91^{\circ} 46^{\prime} 40^{\prime \prime}, \\
\text { on line between secs.3 and } \\
10 \text { T. } 104 \mathrm{~N}, \text { R. } 8 \mathrm{~W} . \text {. } \\
\text { Filimore County, Hydrologic } \\
\text { Unit } 07040008, \text { at bridge, } 1.5 \\
\text { miles northwest of Rushford, } \\
3.0 \text { miles upstream from mouth. } \\
\text { Drainage area } 129 \mathrm{mi} \text {. }\end{array}$ & $\begin{array}{l}1942-79 * \\
1980-91\end{array}$ & $7-21-91$ & 7.81 & 3,480 & $3-26-50$ & 13.54 & 11,600 \\
\hline $\begin{array}{l}\text { South Fork Root } \\
\text { River near } \\
\text { Houston, MN } \\
05385500\end{array}$ & $\begin{array}{l}\text { Lat } 43^{\circ} 44^{\prime} 19^{\prime \prime} \text {, long } 91^{\circ} 33^{\prime} 50^{\prime \prime} \text {, in } \\
\text { NEłSWk sec.9, T.103 N., R. } 6^{\prime} \mathrm{W} \text {. , } \\
\text { Houston County, Hydrologic Unit } \\
07040008 \text {, on left bank, } 50 \text { feet } \\
\text { downstream from State Highway } 76 \\
\text { bridge, } 0.5 \mathrm{mile} \text { upstream from } \\
\text { Badger Creek, } 1.5 \text { mile south of } \\
\text { Houston. Drainage area } 275 \mathrm{mi} \text {. }\end{array}$ & $\begin{array}{l}1953-83 \#, \\
1985-91\end{array}$ & $4-29-91$ & 6.72 & 815 & $6-21-74$ & 13.81 & 11,000 \\
\hline
\end{tabular}


DISCHARGE AT PARTIAL-RECORD STATIONS AND MISCELLANEOUS SITES

Annual maximum discharge at high-flow partial-record stations during water year 1991--Continued

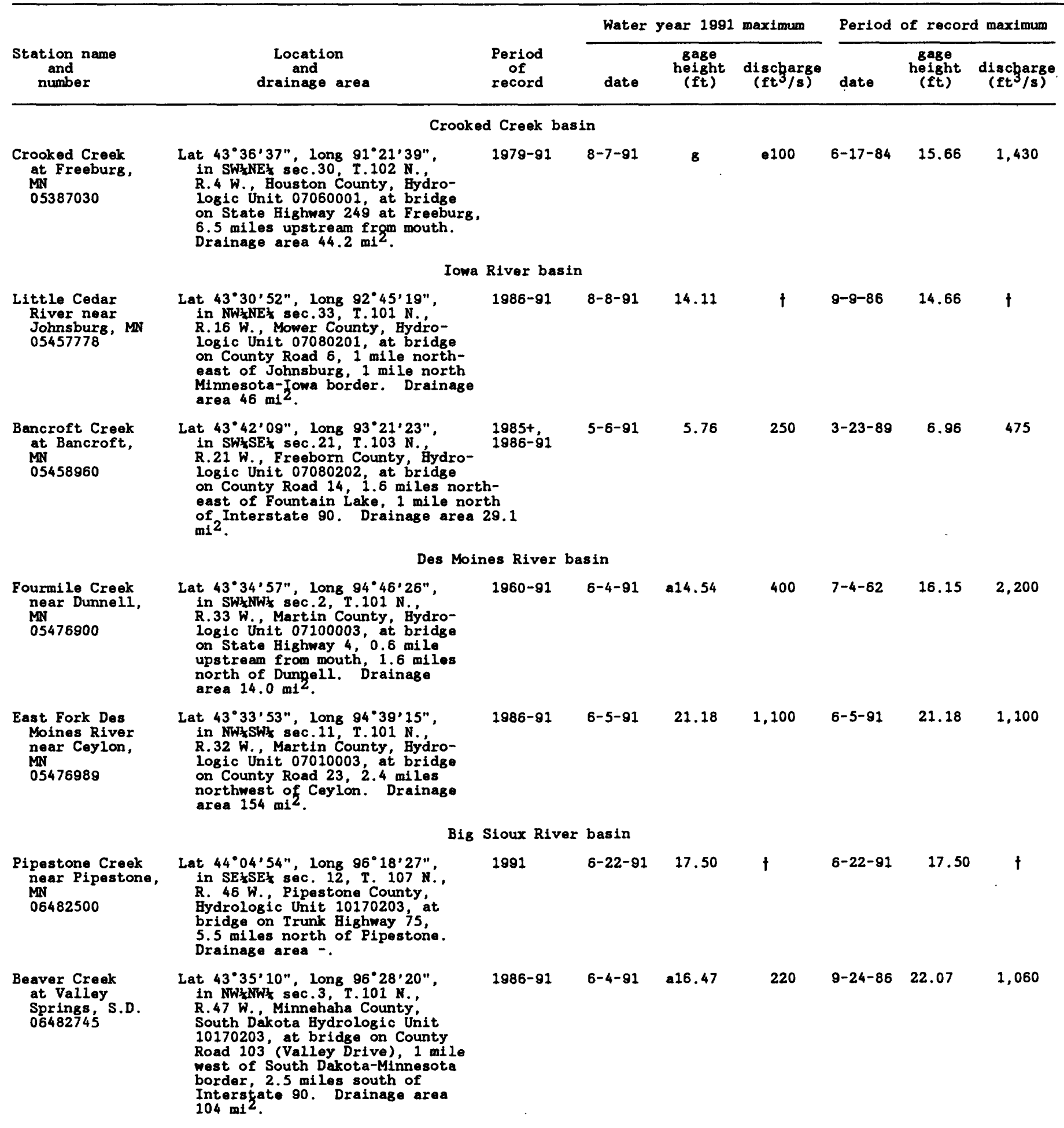


Annual maximum discharge at high-flow partial-record stations during water year 1991--Continued

\begin{tabular}{|c|c|c|c|c|c|c|c|c|}
\hline \multirow[b]{2}{*}{$\begin{array}{c}\text { Station name } \\
\text { and } \\
\text { number }\end{array}$} & \multirow[b]{2}{*}{$\begin{array}{c}\text { Location } \\
\text { and } \\
\text { drainage area }\end{array}$} & \multirow[b]{2}{*}{$\begin{array}{l}\text { Period } \\
\text { of } \\
\text { record }\end{array}$} & \multicolumn{3}{|c|}{ Water year 1991 maximum } & \multicolumn{3}{|c|}{ Period of record maximum } \\
\hline & & & date & $\begin{array}{c}\text { gage } \\
\text { height } \\
\text { (ft) }\end{array}$ & $\begin{array}{c}\text { discharge } \\
\left(\mathrm{ft}^{3} / \mathrm{s}\right)\end{array}$ & date & $\begin{array}{c}\text { gage } \\
\text { height } \\
\text { (ft) }\end{array}$ & $\begin{array}{c}\text { discharge } \\
\left(\mathrm{ft}^{3} / \mathrm{s}\right)\end{array}$ \\
\hline \multicolumn{9}{|c|}{ Big Sioux River basin--Continued } \\
\hline $\begin{array}{l}\text { Chanarambi } \\
\text { Creek near } \\
\text { Edgerton, MN } \\
06482933\end{array}$ & 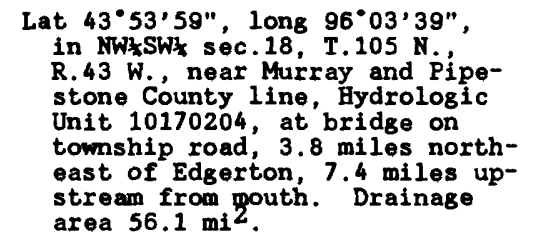 & $1979-91$ & $3-19-91$ & 10.62 & 62 & $9-19-86$ & 18.09 & 580 \\
\hline $\begin{array}{c}\text { Rock River at } \\
\text { Luverne, } \\
06483000\end{array}$ & $\begin{array}{l}\text { Lat } 43^{\circ} 39^{\prime} 15^{\prime \prime}, \text { long } 96^{\circ} 12^{\prime} 03^{\prime \prime}, \\
\text { in SW'NE sec.11, T. } 102 \text { N., } \\
\text { R.45 W. Rock Count, Hydro- } \\
\text { logic Unit 10170204, at bridge } \\
\text { on Main Street (County Highway 4) } \\
\text { in Luverne. Drainage area } 425 \\
\text { mi } 2^{2} \text {. }\end{array}$ & $\begin{array}{l}1911-14 * \\
1972-91\end{array}$ & $6-6-91 \bullet$ & 8 & $\ominus 300$ & $6-13-14$ & 13.20 & 11,600 \\
\hline $\begin{array}{l}\text { Little Rock River } \\
\text { near } \\
\text { Rushmore, MN } \\
06483350\end{array}$ & $\begin{array}{l}\text { Lat } 43^{\circ} 32^{\prime} 36^{\prime \prime} \text {, long } 95^{\circ} 48^{\prime} 58^{\prime \prime} \\
\text { in NEtNEt sec } 24, \mathrm{~T} .101 \mathrm{~N}, \\
\text { R. } 42 \mathrm{~W} \text {. Nobies County, } \\
\text { Hydrologic Unit } 10170204 \text {, at } \\
\text { bridge \#4967, on County Road } 6, \\
1.5 \text { miles west of Ransom, } \\
5.1 \text { miles south of Rushmore. } \\
\text { Drainage area }\end{array}$ & 1991 & $6-6-91$ & 24.67 & 380 & $6-6-91$ & 24.67 & 380 \\
\hline \multicolumn{9}{|c|}{ Little Sioux River basin } \\
\hline $\begin{array}{l}\text { Little Sioux } \\
\text { River near } \\
\text { Spafford, MN } \\
06603530\end{array}$ & $\begin{array}{l}\text { Lat } 43^{\circ} 36^{\prime} 08^{\prime \prime} \text {, long } 95^{\circ} 15^{\prime} 27^{\prime \prime}, \\
\text { in NEłNEt sec.34, T.102 N. , } \\
\text { R.37 W., Jackson County, Hydro- } \\
\text { logic Unit } 10230003 \text { at bridge } \\
\text { on county highway, i.6 miles } \\
\text { downstream from Jackson County } \\
\text { ditch No. 11, } 5.8 \text { miles east of } \\
\text { Spafford. Drainage area } 41.1 \text { mi }\end{array}$ & 1962-91 & $6-22-91$ & a9.82 & 600 & $6-29-69$ & 12.06 & 4.500 \\
\hline $\begin{array}{l}\text { < Less than, peak } \\
+ \text { Operated as low } \\
\text { \# Operated as a ce } \\
\text { † Discharge not d } \\
\text { a Backwater from } \\
\text { b Not annual maxin } \\
\text { c Affected by shi } \\
\text { d Peak stage unkn } \\
\text { e Discharge estim } \\
\text { f Approximately. } \\
\text { g Peak stage did } \\
\text { h At datum then ir }\end{array}$ & $\begin{array}{l}\text { stage unknown, discharge estimated. } \\
\text { flow site. } \\
\text { ntinuous-record gaging station. } \\
\text { atermined. } \\
\text { cquatic growth or debris. } \\
\text { num gage height. } \\
\text { eting control. } \\
\text { own. } \\
\text { ated. } \\
\text { at reach bottom of pipe. } \\
\text { ase, } 5.84 \text { ft higher. }\end{array}$ & & & & & & & \\
\hline
\end{tabular}




\section{Miscellaneous Sites}

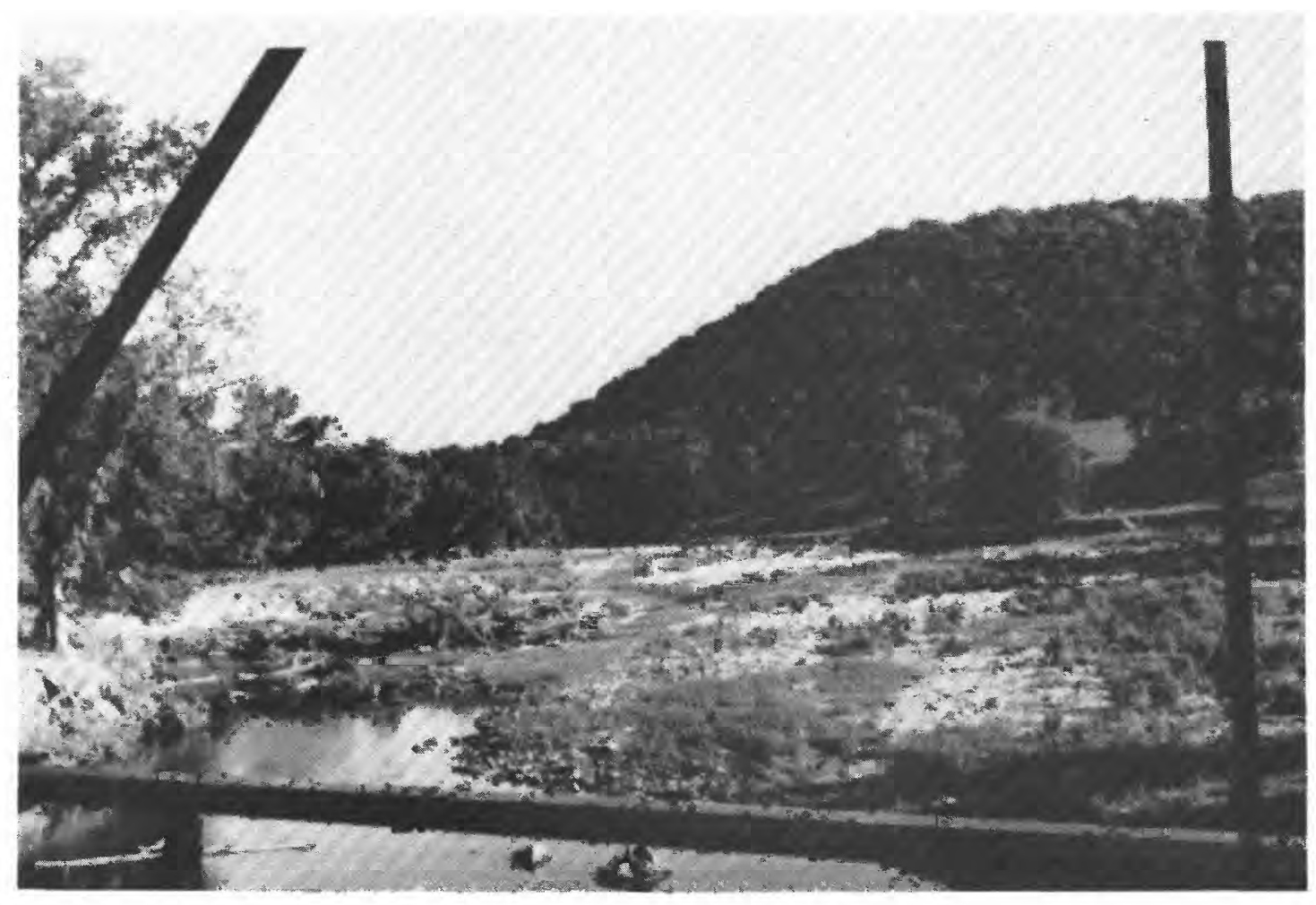

Rush Creek near Rushford

August 14, 1962 
Measurements of streamflow at points other than gaging stations are given in the following table. The measurements of base flow are designated by an asterisk ( $*$; weasurements of peak flow by a dager ( $f$ ).

Discharge measurements made at miscellaneous sites during water year 1991

\begin{tabular}{|c|c|c|c|c|c|c|}
\hline Streem & Tributary & Location & $\begin{array}{c}\text { Drainage } \\
\text { area } \\
\text { (mic) }\end{array}$ & $\begin{array}{l}\text { Period } \\
\text { of } \\
\text { record }\end{array}$ & Date & $\begin{array}{c}\text { Discharge } \\
\left(\mathrm{It}^{3} / \mathrm{s}\right)\end{array}$ \\
\hline \multicolumn{7}{|c|}{ Mississippi River main stem } \\
\hline $\begin{array}{l}\text { Mississippl } \\
\text { River }\end{array}$ & $\begin{array}{l}\text { Gulf of } \\
\text { Mexico }\end{array}$ & $\begin{array}{l}\text { Lat } 47^{\circ} 27^{\prime} 01^{\prime \prime} \text {, long } 94^{\circ} 28^{\prime} 40^{\prime \prime} \text {, in } \\
\text { SWlWW sec.22, T.146 N. R. 30 W. } \\
\text { Beltrami County, Hydrologic Unit } \\
07010101 \text {, } 1000^{\prime} \text { feet belon Knutson } \\
\text { Dam at outlet to Cass Lake, } 2.2 \\
\text { miles South of Pennington. }\end{array}$ & - & 1991 & $2-26-91$ & 230 \\
\hline $\begin{array}{l}\text { Mississippi } \\
\text { River }\end{array}$ & $\begin{array}{l}\text { Gulf of } \\
\text { Mexico }\end{array}$ & 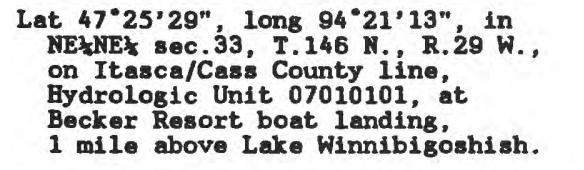 & - & 1991 & $2-26-91$ & 294 \\
\hline $\begin{array}{l}\text { Mississipp1 } \\
\text { River }\end{array}$ & $\begin{array}{l}\text { Gulf of } \\
\text { Mexico }\end{array}$ & $\begin{array}{l}\text { Lat } 47^{\circ} 25^{\prime} 42^{\prime \prime} \text {, long } 9^{\circ} 03^{\circ} 00^{\prime \prime} \text { in } \\
\text { SW' sec.25, T. } 146^{N} \text {. R. } 27 \text { W. } \\
\text { Itasca County, Hydrologic Unit } \\
\text { 07010101, on Leech Lake Indian } \\
\text { Reservation, at dam I mile } \\
\text { northwest of Little Winnibigoshish } \\
\text { Lake, } 14 \text { miles northwest of city of } \\
\text { Deer River, at mile } 1,248 \text { upstreem } \\
\text { from Ohio River (05201500). }\end{array}$ & 1,442 & $\# 1884-91$ & $2-26-91$ & 233 \\
\hline $\begin{array}{l}\text { Mississippi } \\
\text { River }\end{array}$ & $\begin{array}{l}\text { Gulf of } \\
\text { Mexico }\end{array}$ & 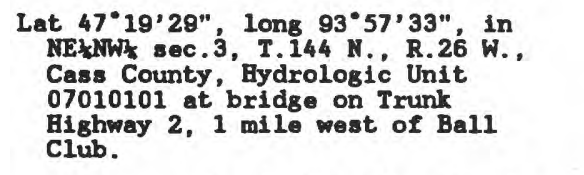 & - & $1990-91$ & $2-27-91$ & 269 \\
\hline \multicolumn{7}{|c|}{ Leech Lake River basin } \\
\hline Boy River & $\begin{array}{l}\text { Leech Lake } \\
\text { River }\end{array}$ & $\begin{array}{l}\text { Lat } 47^{\circ} 04^{\prime} 51^{\prime \prime} \text {, long } 94^{\circ} 05^{\prime} 54^{\prime \prime} \text {, in } \\
\text { SEkSEz sec.28 T. } 142 \text { N. R. } 27 \mathrm{~W} . \\
\text { Cass County, Hydrologic Unit } \\
07010102 \text {, at bridge on County } \\
\text { Highway } 53 \text {, } 1.9 \text { miles upstream } \\
\text { from Boy Lake and } 9 \text { miles } \\
\text { northwest of Remer (05205200). }\end{array}$ & 310 & $+1986-91$ & $2-25-91$ & 58 \\
\hline Boy River & $\begin{array}{l}\text { Leech Lake } \\
\text { River }\end{array}$ & $\begin{array}{l}\text { Lat } 47^{\circ} 09^{\prime} 56^{\prime \prime} \text {, long } 94^{\circ} 10^{\circ} 41^{\prime \prime} \text {, in } \\
\text { NWwWW sec.36, T.143 N. N. } 28 \mathrm{~W} ., \\
\text { Cass County, Bydrologic Unit } \\
07010102 \text {, at bridge on County } \\
\text { Road } 8,2.3 \text { miles upstream of } \\
\text { Leech Lake (Boy Bay), } 6 \text { miles } \\
\text { south of Federal Dam. }\end{array}$ & - & $1990-91$ & $1-17-90$ & 69 \\
\hline $\begin{array}{l}\text { Leech Lake } \\
\text { River }\end{array}$ & $\begin{array}{l}\text { Mississippi } \\
\text { River }\end{array}$ & 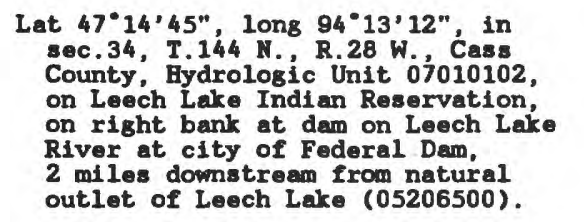 & 1,163 & *1984-91 & $2-28-91$ & 99 \\
\hline $\begin{array}{l}\text { Leech Lake } \\
\text { River }\end{array}$ & $\begin{array}{l}\text { Mississippi } \\
\text { River }\end{array}$ & 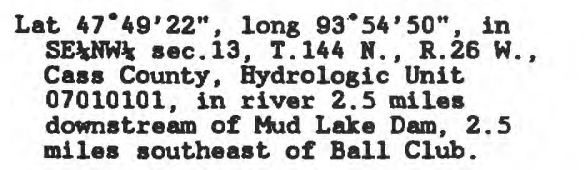 & - & 1991 & $2-28-91$ & 106 \\
\hline
\end{tabular}


Discharge measurements made at miscellaneous sites during water year 1991--Continued

\begin{tabular}{|c|c|c|c|c|c|c|}
\hline Stream & Tributary & Location & $\begin{array}{c}\text { Drainage } \\
\text { areg } \\
\left(m i^{2}\right)\end{array}$ & $\begin{array}{l}\text { Period } \\
\text { of } \\
\text { record }\end{array}$ & Date & $\begin{array}{c}\text { Discharge } \\
\left(\mathrm{ft}^{3} / \mathrm{s}\right)\end{array}$ \\
\hline \multicolumn{7}{|c|}{ Mississippi River main stem } \\
\hline $\begin{array}{l}\text { Mississippi } \\
\text { River }\end{array}$ & $\begin{array}{l}\text { Gulf of } \\
\text { Mexico }\end{array}$ & 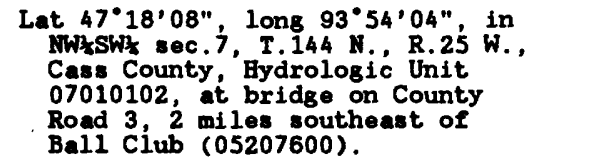 & - & $\begin{array}{c}1945-48 \\
1957 \\
1990-91\end{array}$ & $2-27-91$ & 401 \\
\hline $\begin{array}{l}\text { Mississippi } \\
\text { River }\end{array}$ & $\begin{array}{l}\text { Gulf of } \\
\text { Mexico }\end{array}$ & $\begin{array}{l}\text { Lat } 47^{\circ} 15^{\prime} 10^{\prime \prime} \text {, long } 93^{\circ} 48^{\prime} 00^{\prime \prime} \text { in } \\
\text { NELNW sec.35, T. } 144 \text { N. R. } 25 \text { W. , } \\
\text { Itasca County, Hydrologic Unit } \\
\text { 07010101, at bridge on County } \\
\text { Road 18, at Days Bigh Landing, } \\
5.6 \text { miles south of Deer River. } \\
(05210000) \text {. }\end{array}$ & $\mathbf{a}, 190$ & $\begin{array}{r}1945-50 \\
1079-91\end{array}$ & $2-28-91$ & 373 \\
\hline \multicolumn{7}{|c|}{ Crow River basin } \\
\hline $\begin{array}{c}\text { Fountain } \\
\text { Creek }\end{array}$ & $\begin{array}{l}\text { North Fork } \\
\text { Crow River }\end{array}$ & 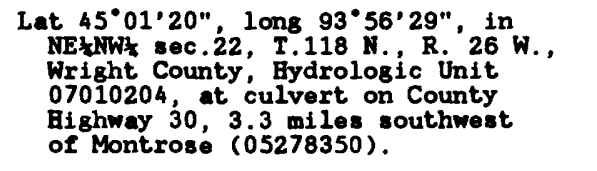 & 6.73 & $\begin{array}{l}1962-85 \\
1991\end{array}$ & $9-7-91$ & 202 \\
\hline $\begin{array}{r}\text { Otter Creek } \\
\text { tributary }\end{array}$ & $\begin{array}{l}\text { South Fork } \\
\text { Crow River }\end{array}$ & 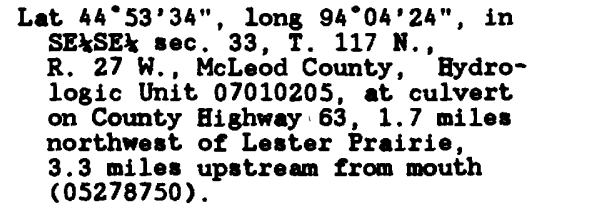 & 1.54 & $\begin{array}{l}+1962-86 \\
1991\end{array}$ & $9-8-91$ & $\uparrow 58$ \\
\hline $\begin{array}{c}\text { Buffalo Creek } \\
\text { tributary }\end{array}$ & $\begin{array}{l}\text { South Fork } \\
\text { Crow River }\end{array}$ & 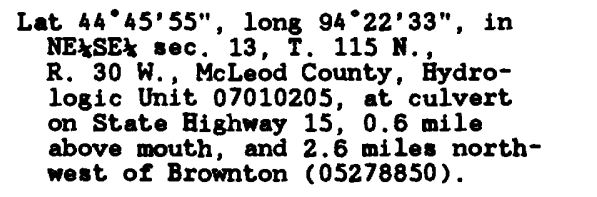 & 9.45 & $\begin{array}{l}+1961-87 \\
1991\end{array}$ & $9-8-91$ & $\uparrow 175$ \\
\hline $\begin{array}{l}\text { South Fork } \\
\text { Crow River }\end{array}$ & Crow River & 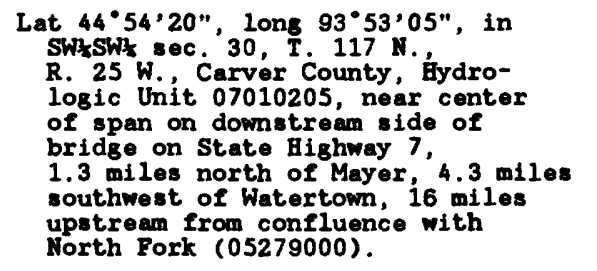 & 1,170 & $\begin{array}{l}+1934-79 \\
+1980-84 \\
+1986 \\
1991\end{array}$ & $9-13-91$ & $\$ 6760$ \\
\hline $\begin{array}{l}\text { Mississippi } \\
\text { River }\end{array}$ & $\begin{array}{l}\text { Gulf of } \\
\text { Mexico }\end{array}$ & $\begin{array}{l}\text { Lat } 44^{\circ} 58^{\prime} 46^{\prime \prime}, \text { long } 93^{\circ} 14^{\prime} 50 \text { ", in } \\
\text { SEzSEk sec.23, T.29 N. R. } 24 \text { 'W. } \\
\text { Bennepin County, Hydrologic Unit } \\
07010206 \text {, at lower St. Anthony } \\
\text { Falls lock and dam in Minneapolis } \\
\text { at River Mile } 853.3 \text { upstream from } \\
\text { Ohio River. (Discharge measurements } \\
\text { made between Hennepin Avenue and } \\
\text { Franklin Avenue bridges over the } \\
\text { Mississippi River are included). } \\
\text { (05288920). }\end{array}$ & $\mathbf{1 9}, 700$ & $\begin{array}{l}1912 \\
1938-39 \\
1941 \\
1943 \\
1953-54 \\
1957 \\
1963-85 \\
1990-91\end{array}$ & $\begin{array}{l}4-26-91 \\
8-8-91\end{array}$ & $\begin{array}{r}\uparrow 15,700 \\
9,040\end{array}$ \\
\hline \multicolumn{7}{|c|}{ Minnesota River basin } \\
\hline $\begin{array}{c}\text { Chippewa } \\
\text { River }\end{array}$ & $\underset{\text { River }}{\text { Minnesota }}$ & 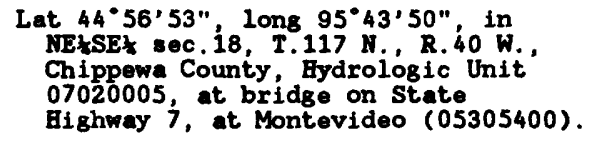 & - & $1990-91$ & $6-22-91$ & 857 \\
\hline $\begin{array}{l}\text { Chippewa } \\
\text { River } \\
\text { below } \\
\text { diversion }\end{array}$ & $\begin{array}{l}\text { Minnesota } \\
\text { River }\end{array}$ & $\begin{array}{l}\text { Lat } 45^{\circ} 01^{\prime} 10^{\prime \prime}, \text { long } 95^{\circ} 47^{\prime} 30^{\prime \prime} \text { in } \\
\text { NW sec.22, T.118 N., R. } 41^{\text {W. }} \\
\text { Chippewa County, Hydrologic Unit } \\
07020005,1.4 \text { miles northeast of } \\
\text { Watson. }\end{array}$ & - & $1945-91$ & $11-7-90$ & $\star 19$ \\
\hline
\end{tabular}




\begin{tabular}{|c|c|c|c|c|c|c|}
\hline Stream & Tributary & Location & $\begin{array}{c}\text { Drainage } \\
\text { areg } \\
\left(\mathrm{mi}^{2}\right)\end{array}$ & $\begin{array}{l}\text { Period } \\
\text { of } \\
\text { record }\end{array}$ & Date & $\begin{array}{c}\text { Discharge } \\
\left(\mathrm{ft}^{3} / \mathrm{s}\right)\end{array}$ \\
\hline \multicolumn{7}{|c|}{ Minnesota River basin--Continued } \\
\hline Hawk Creek & $\begin{array}{c}\text { Minnesota } \\
\text { River }\end{array}$ & $\begin{array}{l}\text { Lat } 44^{\circ} 44^{\prime} 11^{\prime \prime}, \text { long } 95^{\circ} 25^{\prime} 21^{\prime \prime} \text {, in } \\
\text { NEzSE sec.28, T.115 N. R. 38 W. } \\
\text { Renvilie County, Hydrologic Unit } \\
07020004 \text {, at mouth of Hawk Creek, } \\
0.25 \text { mile northwest of County } \\
\text { Highway } 10 \text { bridge on Minnesota } \\
\text { River near Sacred Heart }(05314550) \text {. }\end{array}$ & - & $1990-91$ & $7-1-91$ & 1,180 \\
\hline$\underset{\text { River }}{\text { Minnesota }}$ & $\begin{array}{l}\text { Mississippi } \\
\text { River }\end{array}$ & $\begin{array}{l}\text { Lat } 44^{\circ} 43^{\prime} 54^{\prime \prime}, \text { long } 95^{\circ} 25^{\prime} 14^{\prime \prime} \text {, in } \\
\text { SEtSE sec. } 27 \text {, T. } 115 \text { N. R. } 38 \text { W., } \\
\text { Renvilie County, Hydrologic Unit } \\
07020004 \text { at bridge on County } \\
\text { Highway 10, } 4.5 \text { miles southwest } \\
\text { Sacred Heart }(05314560) \text {. }\end{array}$ & - & $1990-91$ & $8-20-91$ & 3,890 \\
\hline$\underset{\text { River }}{\text { Minnesota }}$ & $\begin{array}{l}\text { Mississippi } \\
\text { River }\end{array}$ & $\begin{array}{l}\text { Lat } 44^{\circ} 37^{\prime} 11^{\prime \prime}, \text { long } 95^{\circ} 10^{\prime} 39^{\prime \prime} \text {, in } \\
\text { NWłSW sec.3, T.113 N., R.36 W., } \\
\text { Renville County, Hydrologic Unit } \\
\text { 07020004, at bridge on County } \\
\text { Highway } 21^{3} 3 \text { miles north of } \\
\text { Delhi }(05314740) \text {. }\end{array}$ & - & $1990-91$ & $8-20-91$ & 4.060 \\
\hline $\begin{array}{c}\text { Minnesota } \\
\text { River }\end{array}$ & $\begin{array}{l}\text { Mississippi } \\
\text { River }\end{array}$ & $\begin{array}{l}\text { Lat } 44^{\circ} 32^{\prime} 46^{\prime \prime} \text {, long } 94^{\circ} 59^{\prime} 45^{\prime \prime} \text {, in } \\
\text { NEtSE sec.36, T.113 N. R.35 W., } \\
\text { Renvilie County, Hydrologic Unit } \\
\text { 07020007, at bridge on State } \\
\text { Highway ig and Trunk Highway } 71 \\
\text { at Morton }(05316580) \text {. }\end{array}$ & - & $1990-91$ & $8-21-91$ & 4,460 \\
\hline$\underset{\text { River }}{\text { Minnesota }}$ & $\begin{array}{l}\text { Mississippi } \\
\text { River }\end{array}$ & $\begin{array}{l}\text { Lat } 44^{\circ} 26^{\prime} 01^{\prime \prime}, \text { long } 94^{\circ} 43^{\prime} 01^{\prime \prime} \text {, in } \\
\text { NEłSW sec.8, T.111 N., R.32 W., } \\
\text { Nicollet County, Hydroiogic Unit } \\
\text { 07020007, at bridge on State } \\
\text { Highway 4, } 4 \text { miles south of } \\
\text { Fairfax }(05316685) \text {. }\end{array}$ & - & $1990-91$ & $8-21-91$ & 4,590 \\
\hline$\underset{\text { River }}{\text { Minnesota }}$ & $\begin{array}{l}\text { Mississippi } \\
\text { River }\end{array}$ & 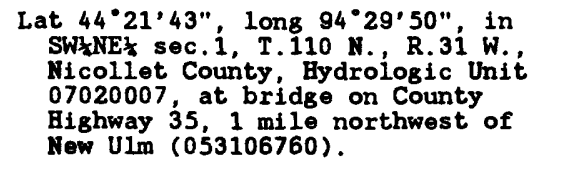 & - & $1990-91$ & $8-21-91$ & 5,240 \\
\hline $\begin{array}{c}\text { Minnesota } \\
\text { River }\end{array}$ & $\begin{array}{l}\text { Mississippi } \\
\text { River }\end{array}$ & 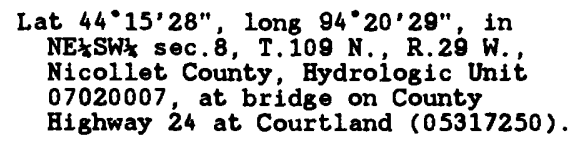 & a11,200 & $\begin{array}{l}1938-50 \\
1990-91\end{array}$ & $\begin{array}{l}8-22-91 \\
9-6-91\end{array}$ & $\begin{array}{l}5,500 \\
1,170\end{array}$ \\
\hline$\underset{\text { River }}{\text { Minnesota }}$ & $\begin{array}{l}\text { Mississippi } \\
\text { River }\end{array}$ & 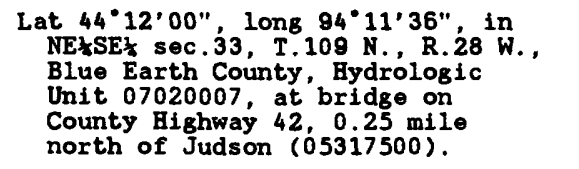 & - & $1990-91$ & $\begin{array}{l}8-22-91 \\
9-6-91\end{array}$ & $\begin{array}{l}5,540 \\
1,230\end{array}$ \\
\hline $\begin{array}{l}\text { Blue Earth } \\
\text { River }\end{array}$ & $\begin{array}{c}\text { Minnesota } \\
\text { River }\end{array}$ & 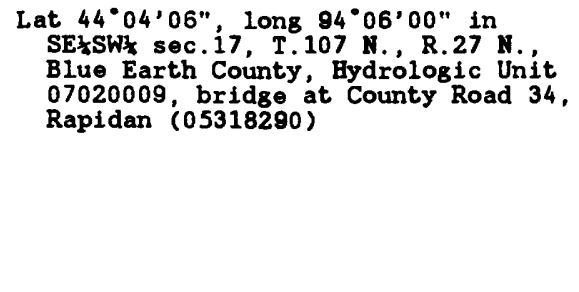 & - & & $\begin{array}{l}3-15-91 \\
3-21-91 \\
3-22-92 \\
3-26-91 \\
4-3-91 \\
4-9-91 \\
5-6-91 \\
5-7-91 \\
5-8-91 \\
5-9-91 \\
6-5-91\end{array}$ & $\begin{array}{r}278 \\
958 \\
1,570 \\
2,530 \\
2,170 \\
1,330 \\
4,490 \\
5,070 \\
5,480 \\
6,760 \\
7,820\end{array}$ \\
\hline
\end{tabular}


Discharge measurements made at miscellaneous sites during water year 1991--Continued

\begin{tabular}{|c|c|c|c|c|c|c|}
\hline Stream & Tributary & Location & $\begin{array}{l}\text { age } \\
\text { 2) }\end{array}$ & $\begin{array}{l}\text { Period } \\
\text { of } \\
\text { record }\end{array}$ & Date & $\begin{array}{c}\text { Discharge } \\
\left(\mathrm{ft}^{3} / \mathrm{s}\right)\end{array}$ \\
\hline & & Minnesota River basin--Continued & & & & \\
\hline \multirow[t]{2}{*}{$\begin{array}{l}\text { Blue Earth } \\
\text { River }\end{array}$} & $\begin{array}{c}\text { Minnesota } \\
\text { River }\end{array}$ & $\begin{array}{l}\text { Lat } 44^{\circ} 09^{\prime} 48^{\prime \prime} \text { long } 94^{\circ} 02 \cdot 12 " \text { in } \\
\text { SWhNE sec. 13, T.108 N. R.27 W. } \\
\text { Blue Earth County, Hydrologic Unit } \\
\text { 07020009, at confiuence with } \\
\text { Minnesota River in Sibley Park } \\
\text { above dam in Mankato (05322000). }\end{array}$ & - & $1990-91$ & $\begin{array}{l}10-24-90 \\
11-28-90 \\
12-18-90 \\
1-11-91 \\
2-27-91 \\
3-6-91 \\
3-14-91 \\
3-19-91 \\
3-20-91 \\
3-21-91 \\
3-25-91 \\
3-26-91 \\
4-2-91 \\
4-10-91 \\
4-17-91 \\
4-25-91 \\
4-30-91 \\
5-10-91 \\
5-15-91 \\
5-24-91 \\
5-31-91 \\
6-6-91 \\
6-13-91 \\
6-20-91 \\
6-28-91 \\
7-3-91 \\
7-10-91 \\
7-16-91 \\
7-25-91 \\
7-31-91 \\
8-30-91\end{array}$ & $\begin{array}{r}257 \\
133 \\
219 \\
\star 76 \\
\star 192 \\
344 \\
557 \\
570 \\
873 \\
1,410 \\
5,160 \\
5,180 \\
4,550 \\
2,360 \\
9,360 \\
4,470 \\
4,850 \\
114,400 \\
7,850 \\
7,980 \\
8,700 \\
16,400 \\
15,700 \\
9,480 \\
5,650 \\
5,480 \\
2,360 \\
3,660 \\
5,720 \\
2,980 \\
\star 655\end{array}$ \\
\hline & & & & & $9-26-91$ & 1,350 \\
\hline $\begin{array}{l}\text { Minnesota } \\
\text { River }\end{array}$ & $\begin{array}{l}\text { Mississippi } \\
\text { River }\end{array}$ & $\begin{array}{l}\text { Lat } 44^{\circ} 18^{\prime} 26^{\prime \prime}, \text { long } 93^{\circ} 57^{\prime} 35^{\prime \prime}, \text { in } \\
\text { SEtNWt sec.28, T.110 N, R.26 W. , } \\
\text { Nicoliet County, Hydrologic Unit } \\
\text { 07020007, at bridge on State } \\
\text { Highway } 22 \text { at St. Peter }(05325200) \text {. }\end{array}$ & - & $1990-91$ & $8-22-91$ & 7,950 \\
\hline Rush River & $\begin{array}{c}\text { Minnesota } \\
\text { River }\end{array}$ & $\begin{array}{l}\text { Lat } 44^{\circ} 29^{\prime} 57^{\prime \prime} \text {, long } 93^{\circ} 54^{\prime} 18^{\prime \prime} \text { in } \\
\text { NWHWW sec.24, T.112 N. R.26 W. . } \\
\text { Sibley County, Hydrologic Unit } \\
\text { o7020007, at bridge on State } \\
\text { Highway } 93,2 \text { miles south of } \\
\text { Henderson }(05326400) \text {. }\end{array}$ & - & $1990-91$ & $5-30-91$ & 1.820 \\
\hline \multirow[t]{2}{*}{$\begin{array}{l}\text { Minnesota } \\
\text { River }\end{array}$} & $\begin{array}{l}\text { Mississippi } \\
\text { River }\end{array}$ & $\begin{array}{l}\text { Lat } 44^{\circ} 31^{\prime} 46^{\prime \prime}, \text { long } 93^{\circ} 54^{\prime} 02^{\prime \prime} \text { in } \\
\text { SEzSW sec.1, T.112 N., R.26 W., } \\
\text { Sibley County, Hydrologic Unit } \\
\text { 07020012, at bridge on State } \\
\text { Bighway } 19^{2} \text { at Henderson (05326450). }\end{array}$ & - & $1990-91$ & $8-23-91$ & 7,940 \\
\hline & \multicolumn{6}{|c|}{ Garvin Brook basin } \\
\hline Garvin Brook & $\begin{array}{l}\text { Mississippi } \\
\text { River }\end{array}$ & $\begin{array}{l}\text { Lat } 44^{\circ} 01^{\prime} 24^{\prime \prime}, \text { long } 91^{\circ} 47^{\prime} 06^{\prime \prime} \text {, in } \\
\text { SWłSEK sec. 33, T.107 N, , R. at } 8 \mathrm{~W} ., \\
\text { Winona County, Bydrologic Unit } \\
\text { 07040003, on Chicago and North- } \\
\text { western Railroad bridge, } 1.0 \text { mile } \\
\text { west of Stockton, } 0.9 \text { mile upstream } \\
\text { from Stockton Vailey Creek (05378220). }\end{array}$ & - & $\begin{array}{c}\text { *1982-83 } \\
1991\end{array}$ & $7-21-91$ & 11,000 \\
\hline
\end{tabular}




\begin{tabular}{|c|c|c|c|c|c|c|}
\hline Stream & Tributary & Location & $\begin{array}{l}\text { Drainage } \\
\text { areg } \\
\left.(\operatorname{mi})^{2}\right)\end{array}$ & $\begin{array}{l}\text { Period } \\
\text { of } \\
\text { record }\end{array}$ & Date & $\begin{array}{l}\text { Discharg } \\
\left(\mathrm{ft}^{3} / \mathrm{s}\right)\end{array}$ \\
\hline & & \multicolumn{5}{|c|}{ Garvin Brook basin--Continued } \\
\hline $\begin{array}{l}\text { Stockton } \\
\text { Valley Creek }\end{array}$ & $\begin{array}{c}\text { Garvin } \\
\text { Brook }\end{array}$ & $\begin{array}{l}\text { Lat } 44^{\circ} 00^{\prime} 56^{\prime \prime}, \text { long } 91^{\circ} 45^{\prime} 36^{\prime \prime}, \text { in } \\
\text { SEzNEt sec. 3, T. } 106 \mathrm{~N} \text {. R. } 8 \mathrm{~W} \text {. } \\
\text { Winone County, Bydrologic Unit } \\
\text { 07040003, } 0.9^{\circ} \text { mile above mouth, } \\
\text { and } 1.0 \text { mile south of Stockton } \\
(05378230) \text {. }\end{array}$ & - & $\begin{array}{l}1982-83 \\
\text { 1984-85, } \\
1991\end{array}$ & $7-21-91$ & $\$ 42,300$ \\
\hline \multirow[t]{2}{*}{ Garvin Brook } & $\begin{array}{l}\text { Mississippi } \\
\text { River }\end{array}$ & 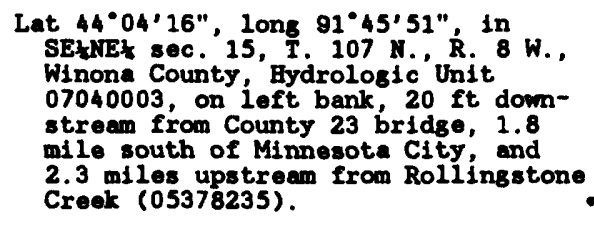 & - & $\begin{array}{l}1982-83 \\
+1984-91\end{array}$ & $7-21-91$ & $\$ 11,200$ \\
\hline & \multicolumn{6}{|c|}{ Gilmore Creek basin } \\
\hline G1 lmore Creek & $\begin{array}{l}\text { Mississippi } \\
\text { River }\end{array}$ & 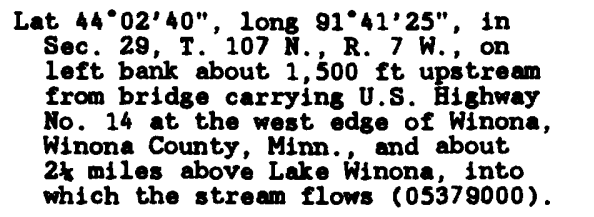 & 8.95 & $\begin{array}{l}\text { 1939-63, } \\
+1964-65 \\
1991\end{array}$ & $7-21-91$ & te4, 400 \\
\hline
\end{tabular}

b Stage only.

- Estimated. 


\section{Water-quality Partial Record Stations}

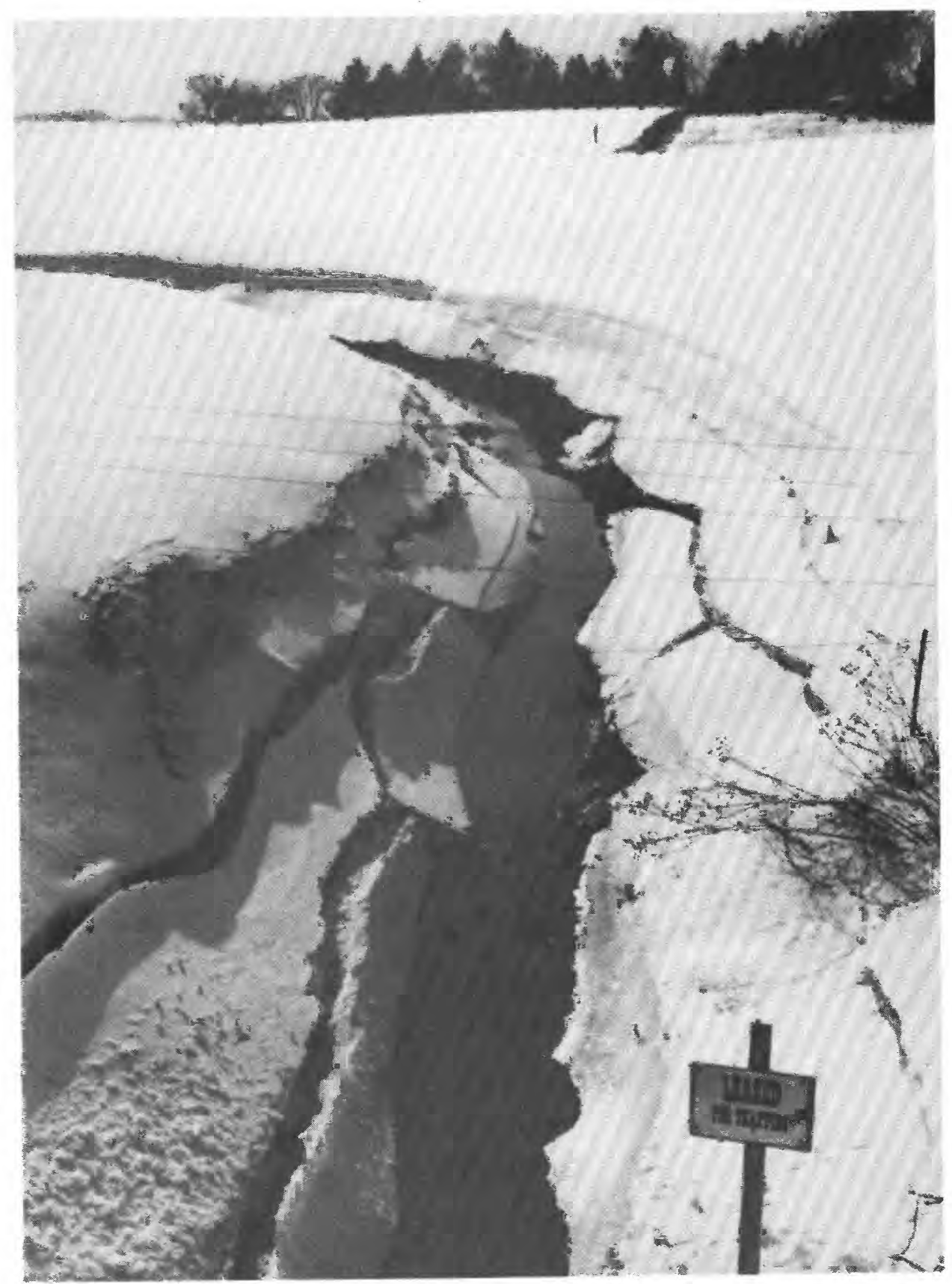


Water-quality partial-record stations are particular sites where chemical-quality, biolosical and (or) sediment data are collected systematically over a period of years for use in hydrolosic analyses. Letter $\mathrm{K}$ indicates non-ideal colony count.

05305400 CEIPPEWA RIVER AT MOKTEVIDEO

WATER QUALITY DATA, WATER YEAR OCTOBER 1990 TO SEPTEMBER 1991

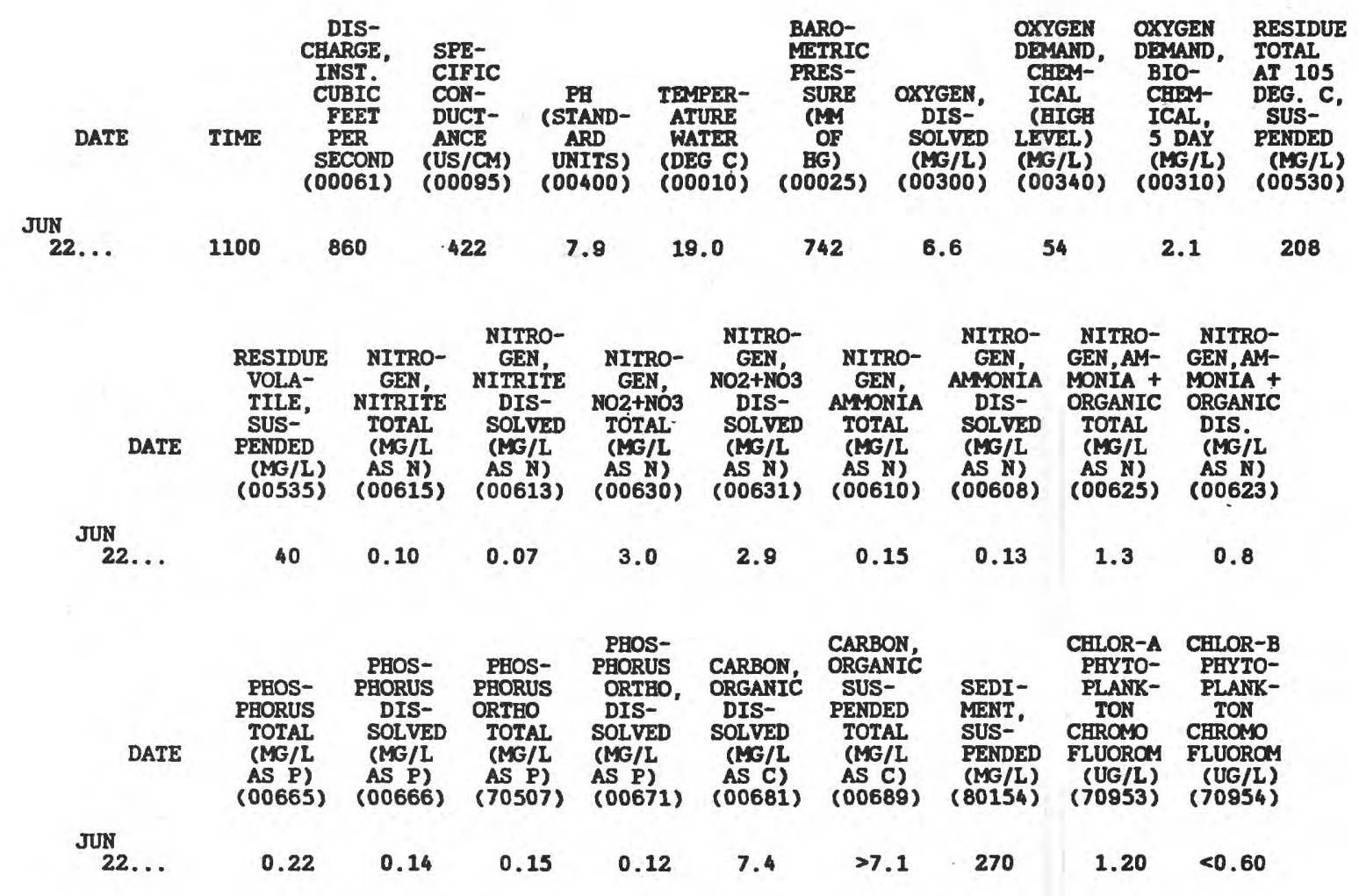


ANALYSES OF SAMPLES COLLECTED AT WATER-QUALITY PARTIAL-RECORD STATIONS

05313510 YELLOW MEDICINE RIVER ON BWY 67 MEAR GRANITE FALLS

WATER QUALITY DATA, WATER YEAR OCTOBER 1990 TO SEPTEMBER 1991

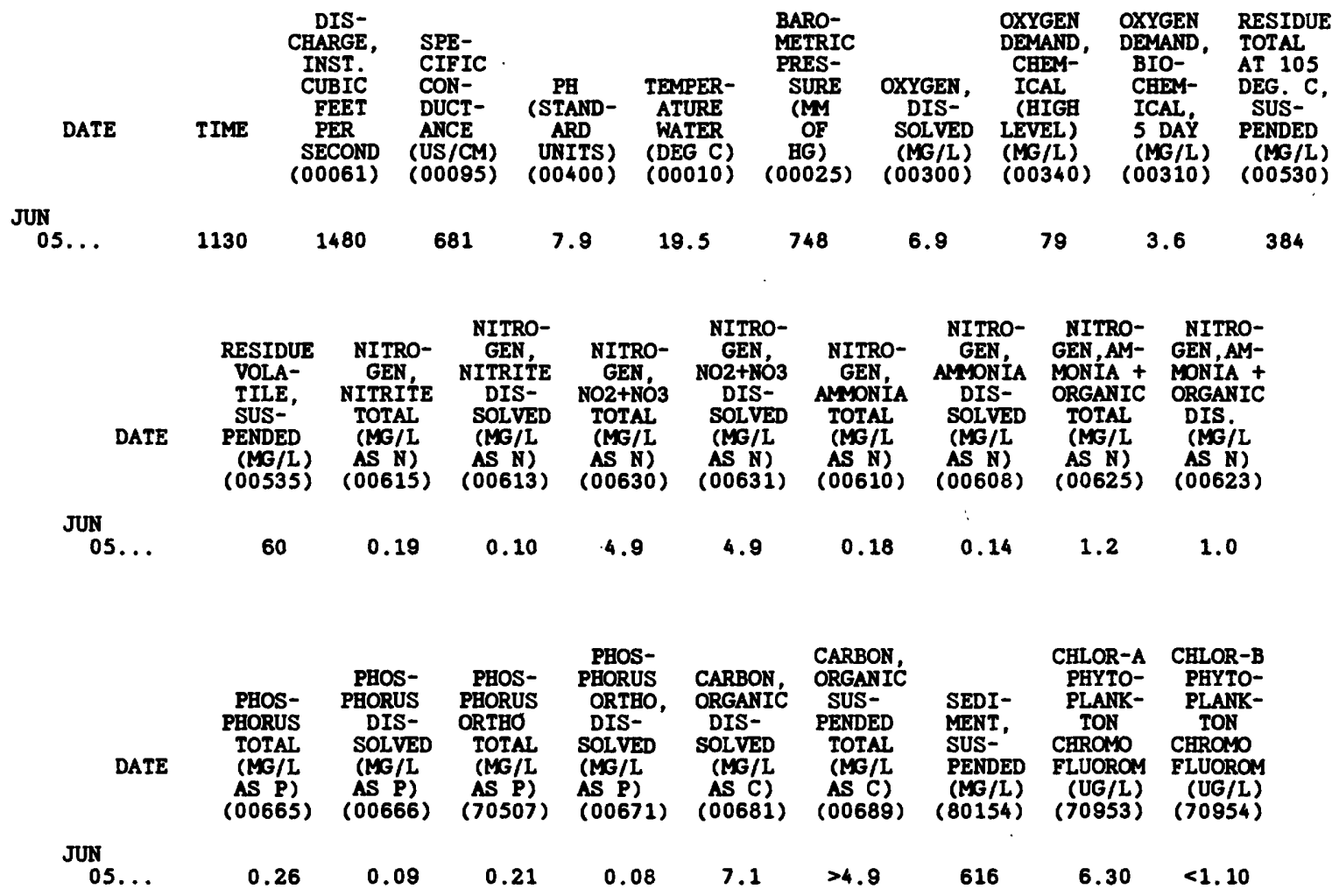


ANALYSES OF SAMPLES COLLECTED AT WATER-QUALITY PARTIAL-RECORD STATIONS

05314550 HAWK CREEK AT MOUTH NEAR SACRED HEART

WATER QUALITY DATA, WATER YEAR OCTOBER 1990 TO SEPTEMBER 1991

\begin{tabular}{|c|c|c|c|c|c|c|c|c|c|c|c|c|c|c|c|c|c|}
\hline \multirow[b]{2}{*}{$\begin{array}{l}\text { JUL } \\
01 . . .\end{array}$} & \multicolumn{2}{|c|}{$\begin{array}{c}\text { DIS- } \\
\text { CBARGE, } \\
\text { INST. } \\
\text { CUBIC } \\
\text { FEET } \\
\text { PER } \\
\text { SECOND } \\
\text { (O0061) }\end{array}$} & \multicolumn{2}{|c|}{$\begin{array}{l}\text { SPE- } \\
\text { CIFIC } \\
\text { CON- } \\
\text { DUCT- } \\
\text { ANCE } \\
\text { (US/CM) } \\
(00095)\end{array}$} & \multicolumn{2}{|c|}{$\begin{array}{c}\text { PH } \\
\text { (STAND- } \\
\text { ARD } \\
\text { UNITS) } \\
(00400)\end{array}$} & \multicolumn{2}{|c|}{$\begin{array}{l}\text { TEMPER- } \\
\text { ATURE } \\
\text { WATER } \\
\text { (DEG C) } \\
(00010)\end{array}$} & \multicolumn{2}{|c|}{$\begin{array}{c}\text { BARO- } \\
\text { METRIC } \\
\text { PRES- } \\
\text { SURE } \\
\text { (MM } \\
\text { OF } \\
\text { HG) } \\
(00025)\end{array}$} & \multicolumn{2}{|c|}{$\begin{array}{c}\text { OXYGEN, } \\
\text { DIS- } \\
\text { SOLVED } \\
(M G / L) \\
(00300)\end{array}$} & \multicolumn{2}{|c|}{$\begin{array}{l}\text { OXYGEN } \\
\text { DEYAND, } \\
\text { CHEY- } \\
\text { ICAL } \\
\text { (EIGR } \\
\text { LEVEI) } \\
\text { (MG/L) } \\
(00340)\end{array}$} & \multicolumn{2}{|c|}{$\begin{array}{c}\text { OXYGEN } \\
\text { DEYAND, } \\
\text { BIO- } \\
\text { CHEM- } \\
\text { ICAL, } \\
5 \text { DAY } \\
\text { (MG/L) } \\
(00310)\end{array}$} & $\begin{array}{l}\text { RESIDUE } \\
\text { TOTAL } \\
\text { AT } 105 \\
\text { DEG. C, } \\
\text { SUS- } \\
\text { PENDED } \\
\text { (MG/L) } \\
(00530)\end{array}$ \\
\hline & 1530 & 180 & & 70 & & & & 3.5 & & 33 & 7 & .7 & \multicolumn{2}{|c|}{120} & \multicolumn{2}{|c|}{4.2} & 552 \\
\hline DATE & $\begin{array}{c}\text { RESIDUE } \\
\text { VOLA- } \\
\text { TILE, } \\
\text { SUS- } \\
\text { PENDED } \\
(M G / L) \\
(00535)\end{array}$ & $\begin{array}{l}\text { NIT } \\
\text { GE } \\
\text { NIIR } \\
\text { TOI } \\
\text { (ME } \\
\text { AS } \\
\text { COOG }\end{array}$ & $\begin{array}{l}\text { RO- } \\
\text { ITE } \\
\text { NI } \\
\text { IL) } \\
\text { 15) }\end{array}$ & $\begin{array}{l}\mathrm{NII} \\
\mathrm{GE} \\
\mathrm{NITH} \\
\mathrm{DI} \\
\text { SOI } \\
\mathrm{CMC} \\
\mathrm{AS} \\
\mathrm{COOE}\end{array}$ & $\begin{array}{l}\text { RO- } \\
\text { M, } \\
\text { ITE } \\
\text { S- } \\
\text { VED } \\
\text { /L } \\
\text { N) } \\
\text { 13) }\end{array}$ & $\begin{array}{r}\text { NI } \\
\text { G] } \\
\text { NO2 } \\
\text { TO } \\
\text { (M } \\
\text { AS } \\
\text { COO }\end{array}$ & $\begin{array}{l}\text { RO- } \\
\text { N, } \\
\text { NÓ3 } \\
\text { AL. } \\
\text { (L } \\
\text { N) } \\
\text { 30) }\end{array}$ & $\begin{array}{l}\text { NII } \\
\text { GE } \\
\text { NO2+ } \\
\text { DI } \\
\text { SOI } \\
\text { (ME } \\
\text { AS } \\
\text { COOE }\end{array}$ & & $\begin{array}{r}\text { NI } \\
\text { GI } \\
\text { AMM } \\
\text { TO } \\
\text { (M } \\
\text { AS } \\
\text { COO }\end{array}$ & $\begin{array}{l}\text { RO- } \\
\text { NiA } \\
\text { NiL } \\
\text { AL } \\
\text { Ni) } \\
10)\end{array}$ & $\begin{array}{c}\text { NIT } \\
\text { GE } \\
\text { AMMO } \\
\text { DI } \\
\text { SOL } \\
\text { MMG } \\
\text { AS } \\
\text { CODG }\end{array}$ & $\begin{array}{l}\text { RO- } \\
\text { No } \\
\text { NIA } \\
\text { S- } \\
\text { VED } \\
\text { (L) } \\
\text { N) } \\
\text { O8) }\end{array}$ & $\begin{array}{l}\text { NIT } \\
\text { GEN, } \\
\text { MONI } \\
\text { ORGA } \\
\text { TOI } \\
\text { (ME } \\
\text { AS } \\
\text { COOE }\end{array}$ & \multicolumn{3}{|c|}{$\begin{array}{cc}\text { O- } & \text { NITRO- } \\
\text { M- } & \text { GEN,AM- } \\
+ & \text { MONIA + } \\
\text { IC } & \text { ORGANIC } \\
\text { OC } & \text { DIS. } \\
\text { L } & (M G / L \\
\text { f } & \text { AS N }) \\
5) & (00623)\end{array}$} \\
\hline$\underset{01 \ldots}{J U L}$ & 28 & \multicolumn{2}{|c|}{0.07} & \multicolumn{2}{|c|}{0.06} & \multicolumn{2}{|c|}{11} & 10 & \multicolumn{3}{|c|}{0.06} & 0. & \multicolumn{3}{|c|}{2.8} & \multicolumn{2}{|c|}{1.2} \\
\hline DATE & $\begin{array}{c}\text { PHOS- } \\
\text { PHORUS } \\
\text { TOTAL } \\
\text { (MG/L } \\
\text { AS P) } \\
(00665)\end{array}$ & $\begin{array}{l}\text { PHO } \\
\text { PHOR } \\
\text { DI } \\
\text { SOI } \\
\text { (ME } \\
\text { AS } \\
\mathrm{COO6}\end{array}$ & $\begin{array}{l}\text { US } \\
\text { U- } \\
\text { VED } \\
\text { (L } \\
\text { P) } \\
66 \text { ) }\end{array}$ & $\begin{array}{l}\text { PHC } \\
\text { PHOI } \\
\text { ORTE } \\
\text { TOI } \\
\text { (MX } \\
\text { AS } \\
\text { (70S }\end{array}$ & $\begin{array}{l}S- \\
\text { US } \\
0 \\
\text { AL } \\
(L L \\
\text { P) } \\
\text { O7) }\end{array}$ & $\begin{array}{l}\text { PHC } \\
\text { PHOI } \\
\text { OR } \\
\text { DI } \\
\text { SOL } \\
\text { (MG } \\
\text { AS } \\
\text { (OO }\end{array}$ & $\begin{array}{l}\text { S- } \\
\text { US } \\
\text { HO, } \\
\text { ED } \\
\text { L } \\
\text { 71) }\end{array}$ & $\begin{array}{l}\text { CARE } \\
\text { ORG } \\
\text { DIS } \\
\text { SOLV } \\
(M E \\
\text { AS } \\
1006\end{array}$ & & $\begin{array}{l}\text { CAR } \\
\text { ORG } \\
\text { SU: } \\
\text { PEN } \\
\text { TO } \\
\text { (M } \\
\text { AS } \\
\text { COO }\end{array}$ & $\begin{array}{l}\text { ON, } \\
\text { NIC } \\
\text { ED } \\
\text { AI } \\
\text { iL } \\
\text { C) } \\
88)\end{array}$ & $\begin{array}{l}\text { SED } \\
\text { MEN } \\
\text { SUS } \\
\text { PEN } \\
\text { (MG } \\
(801\end{array}$ & $\begin{array}{l}I- \\
T, \\
\text { DED } \\
(L) \\
54)\end{array}$ & $\begin{array}{l}\text { CHLC } \\
\text { PHY } \\
\text { PLA } \\
\text { TC } \\
\text { CHRC } \\
\text { FLUC } \\
\text { (UEC } \\
\text { T708 }\end{array}$ & $\begin{array}{l}\text { R-A } \\
\text { TO- } \\
\text { WK- } \\
\text { NK } \\
\text { MO } \\
\text { ROM } \\
(L) \\
(53)\end{array}$ & $\begin{array}{l}\text { CHLO } \\
\text { PHY } \\
\text { PLA } \\
\text { TO } \\
\text { CHRO } \\
\text { FLUO } \\
\text { (UE } \\
\text { (708 }\end{array}$ & $\begin{array}{l}\text { OR-B } \\
\text { TTO- } \\
\text { INK- } \\
\text { ONO } \\
\text { OROM } \\
(1 \mathrm{LO}) \\
354)\end{array}$ \\
\hline UL ${ }_{01 \ldots} . .$. & 0.49 & 0. & & & 25 & & 25 & 7. & & $>9$ & & 1 & & & 90 & 1. & 20 \\
\hline
\end{tabular}


ANALYSES OF SAMPLES COLLECTED AT WATER-QUALITY PARTIAL-RECORD STATIOAS

O5314560 MINAESOTA RIVER NEAR SACRED HEART

WATER QUALITY DATA, WATER YEAR OCTOBER 1990 TO SEPTEMBER 1991

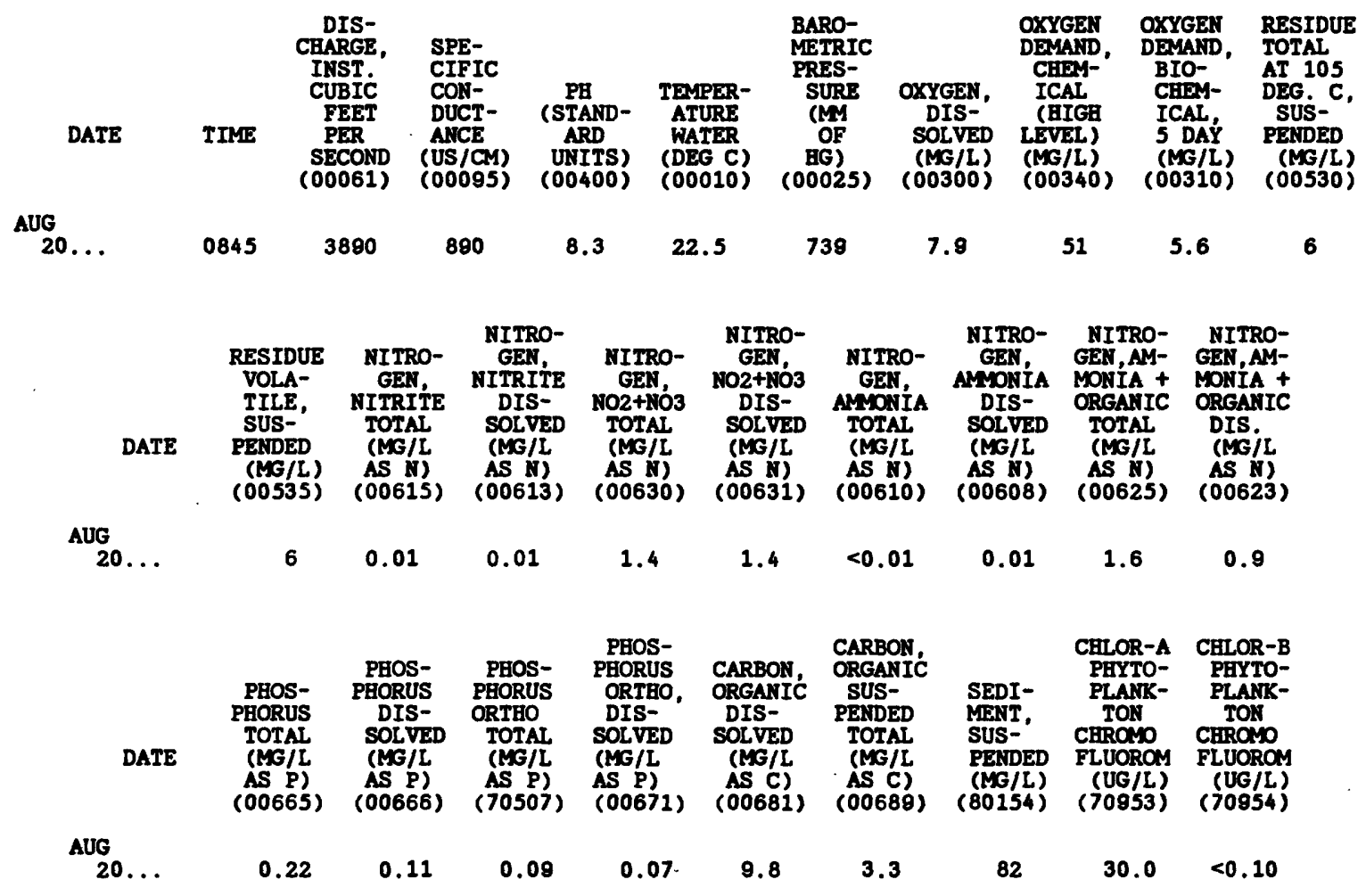


05314740 MIMNESOTA RIVER NEAR DELBI

WATER QUALITY DATA, WATER YEAR OCTOBER 1990 TO SEPTEMBER 1991

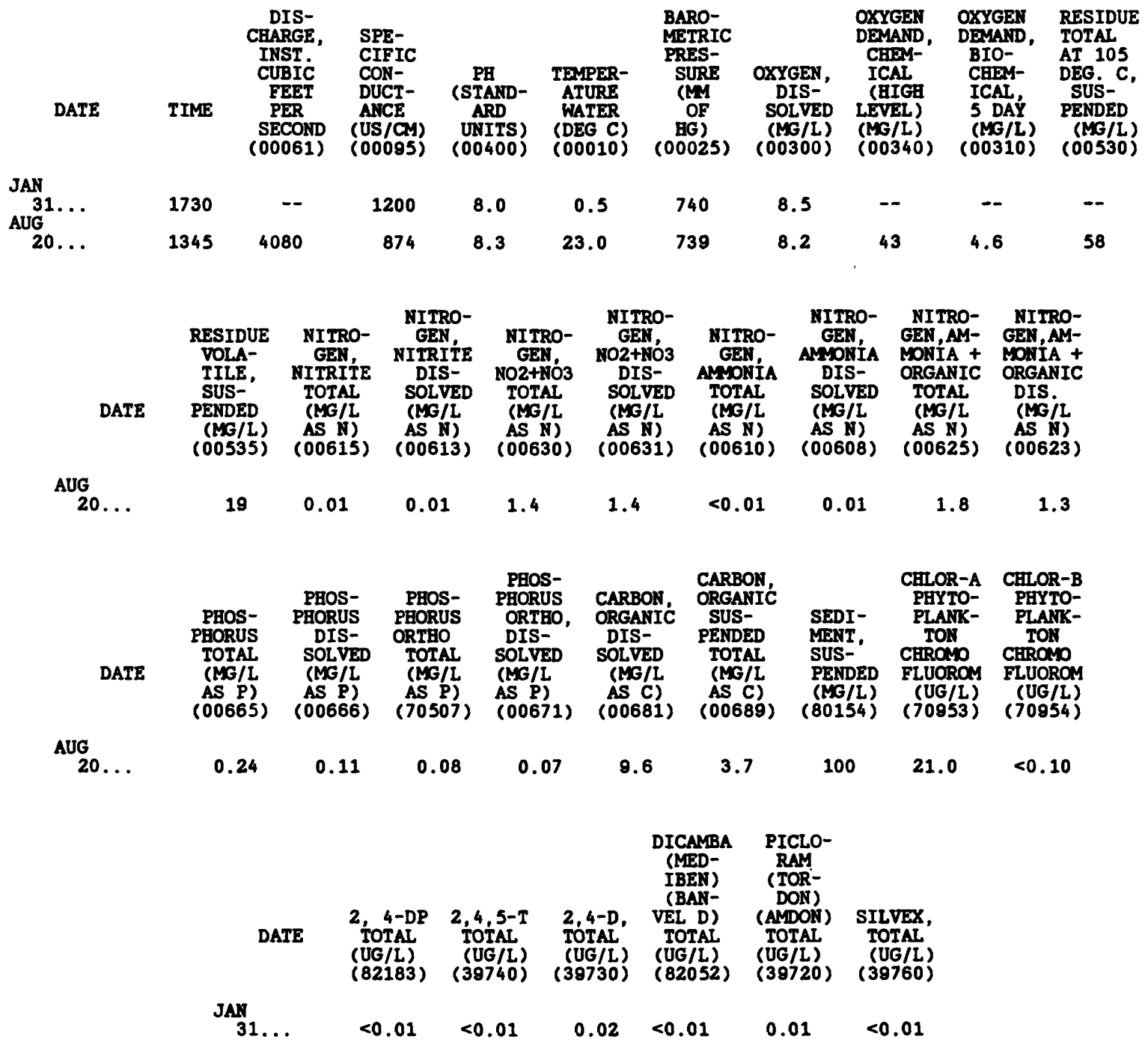


ANALYSES OF SAMPLES COLLECTED AT WATER-QUALITY PARTIAL-RECORD STATIONS

05316541 REDWOOD RIVER BELOW RAMSEY CREEK AT REDWOOD FALLS

WATER QUALITY DATA, WATER YEAR OCTOBER 1990 TO SEPTEMBER 1991

\begin{tabular}{|c|c|c|c|c|c|c|c|c|c|c|c|c|c|c|c|c|c|c|}
\hline DATE & TIME & \multicolumn{2}{|c|}{$\begin{array}{c}\text { DIS- } \\
\text { CHARGE, } \\
\text { INST. } \\
\text { CUBIC } \\
\text { FEET } \\
\text { PER } \\
\text { SECOND } \\
\text { (00061) }\end{array}$} & \multicolumn{2}{|c|}{$\begin{array}{l}\text { SPE- } \\
\text { CIFIC } \\
\text { CON- } \\
\text { DUCT- } \\
\text { ANCE } \\
\text { (US/CM) } \\
(00095)\end{array}$} & $\begin{array}{r}P \\
\text { CST } \\
\text { A } \\
\text { UN } \\
\text { OD }\end{array}$ & $\begin{array}{l}\text { AND- } \\
\text { AD } \\
\text { ITS) } \\
400 \text { ) }\end{array}$ & $\begin{array}{l}\text { TEM } \\
\text { AT } \\
\text { WA } \\
\text { CDE } \\
\text { COO }\end{array}$ & $\begin{array}{l}\text { RR- } \\
R E \\
\text { C) } \\
\text { (0) }\end{array}$ & \multicolumn{2}{|c|}{$\begin{array}{l}\text { BARO- } \\
\text { METRIC } \\
\text { PRES- } \\
\text { SURE } \\
\text { (MY } \\
\text { OF } \\
\text { HG) } \\
(00025)\end{array}$} & $\begin{array}{r}O X Y \\
D \\
\text { SO } \\
(M \\
S O D\end{array}$ & $\begin{array}{l}\text { GEN, } \\
\text { IS- } \\
\text { LVED } \\
\text { G/L) } \\
300)\end{array}$ & \multicolumn{2}{|c|}{$\begin{array}{c}\text { OXYGEN } \\
\text { DEMAND, } \\
\text { CBEM- } \\
\text { ICAL } \\
\text { (BIGH } \\
\text { LEVEL) } \\
\text { (MG/L) } \\
(00340)\end{array}$} & \multicolumn{2}{|c|}{$\begin{array}{c}\text { OXYGEN } \\
\text { DEMAND, } \\
\text { BIO- } \\
\text { CHEY- } \\
\text { ICAL, } \\
5 \text { DAY } \\
(\mathrm{MG} / \mathrm{L}) \\
(00310)\end{array}$} & $\begin{array}{l}\text { RESIDUE } \\
\text { TOTAL } \\
\text { AT } 105 \\
\text { DEG. C, } \\
\text { SUS- } \\
\text { PENDED } \\
\text { (MG/L) } \\
\text { (00530) }\end{array}$ \\
\hline $\operatorname{JN}_{05} \ldots$ & 1600 & \multicolumn{2}{|c|}{2640} & \multicolumn{2}{|c|}{515} & \multicolumn{2}{|c|}{8.0} & \multicolumn{2}{|c|}{20.0} & \multicolumn{2}{|c|}{748} & \multicolumn{2}{|c|}{8.9} & \multicolumn{4}{|c|}{2.9} & 264 \\
\hline DATE & \multicolumn{2}{|c|}{$\begin{array}{c}\text { RESIDUE } \\
\text { VOLA- } \\
\text { TILE, } \\
\text { SUS-' } \\
\text { PENDED } \\
\text { (MG/L) } \\
(00535)\end{array}$} & \multicolumn{2}{|c|}{$\begin{array}{l}\text { NITRO- } \\
\text { GEN, } \\
\text { NITRITE } \\
\text { TOTAL } \\
\text { (MG/L } \\
\text { AS N) } \\
\text { (00615) }\end{array}$} & \multicolumn{2}{|c|}{$\begin{array}{c}\text { NITRO- } \\
\text { GEN, } \\
\text { NITRITE } \\
\text { DIS- } \\
\text { SOLVED } \\
\text { (MG/L } \\
\text { AS N) } \\
(00613)\end{array}$} & \multicolumn{2}{|c|}{$\begin{array}{c}\text { NITRO- } \\
\text { GEN } \\
\text { NO2+NÓ3 } \\
\text { TOTAL } \\
(M G / L \\
\text { AS N) } \\
(00630)\end{array}$} & \multicolumn{2}{|c|}{$\begin{array}{c}\text { NITRO- } \\
\text { GEN, } \\
\text { NO2+NO3 } \\
\text { DIS- } \\
\text { SOLVED } \\
\text { (MG/L } \\
\text { AS N) } \\
\text { (00631) }\end{array}$} & \multicolumn{2}{|c|}{$\begin{array}{c}\text { NITRO- } \\
\text { GEH, } \\
\text { AMONIA } \\
\text { TOTAL } \\
\text { (MG/L } \\
\text { AS N) } \\
(00610)\end{array}$} & \multicolumn{2}{|c|}{$\begin{array}{c}\text { NITRO- } \\
\text { GEN, } \\
\text { AMYNIA } \\
\text { DIS- } \\
\text { SOLVED } \\
\text { (MG/L } \\
\text { AS N) } \\
(00608)\end{array}$} & \multicolumn{2}{|c|}{$\begin{array}{l}\text { NITRO- } \\
\text { GEN,AM- } \\
\text { MONIA + } \\
\text { ORGANIC } \\
\text { TOTAL } \\
\text { (MG/L } \\
\text { AS N) } \\
(00625)\end{array}$} & \multicolumn{2}{|c|}{$\begin{array}{l}\text { NITRO- } \\
\text { GEN,AM- } \\
\text { MONIA + } \\
\text { ORGANIC } \\
\text { DIS. } \\
\text { (MG/L } \\
\text { AS N) } \\
(00623)\end{array}$} \\
\hline$\underset{05 . . .}{\text { Jun }}$ & \multicolumn{2}{|c|}{46} & \multicolumn{2}{|c|}{0.21} & \multicolumn{2}{|c|}{0.10} & \multicolumn{2}{|c|}{6.5} & \multicolumn{2}{|c|}{6.5} & \multicolumn{4}{|c|}{0.15} & \multicolumn{2}{|c|}{1.8} & \multicolumn{2}{|c|}{1.7} \\
\hline DATE & \multicolumn{2}{|c|}{$\begin{array}{c}\text { PHOS- } \\
\text { PHORUS } \\
\text { TOTAL } \\
\text { (MG/L } \\
\text { AS P) } \\
(00665)\end{array}$} & \multicolumn{2}{|c|}{$\begin{array}{c}\text { PHOS- } \\
\text { PHORUS } \\
\text { DIS- } \\
\text { SOLVED } \\
\text { (MG/L } \\
\text { AS P) } \\
(00666)\end{array}$} & \multicolumn{2}{|c|}{$\begin{array}{c}\text { PHOS- } \\
\text { PEORUS } \\
\text { ORTHO } \\
\text { TOTAL } \\
\text { (MG/L } \\
\text { AS P) } \\
(70507)\end{array}$} & \multicolumn{2}{|c|}{$\begin{array}{l}\text { PHOS- } \\
\text { PHORUS } \\
\text { ORTHO, } \\
\text { DIS- } \\
\text { SOLVED } \\
\text { (MG/L } \\
\text { AS P) } \\
(00671)\end{array}$} & \multicolumn{2}{|c|}{$\begin{array}{l}\text { CARBON, } \\
\text { ORGANIC } \\
\text { DIS- } \\
\text { SOLVED } \\
\text { (MG/L } \\
\text { AS C }) \\
(00681)\end{array}$} & $\begin{array}{l}\text { CARB } \\
\text { ORGA } \\
\text { SUS } \\
\text { PEND } \\
\text { TOT } \\
\text { (ME } \\
\text { AS } \\
1006\end{array}$ & $\begin{array}{l}\text { ON, } \\
\text { NIC } \\
- \\
\text { ED } \\
\text { AL } \\
\text { IL } \\
\text { C) } \\
\text { 89) }\end{array}$ & $\begin{array}{c}\text { SED } \\
\text { MEN } \\
\text { SUS } \\
\text { PEN } \\
\text { CMG } \\
\text { C } 801\end{array}$ & $\begin{array}{l}I- \\
T, \\
\text { DED } \\
(L) \\
54)\end{array}$ & $\begin{array}{l}\text { CHLC } \\
\text { PHY } \\
\text { PLA } \\
\text { TC } \\
\text { CHRC } \\
\text { FLUC } \\
\text { CUC } \\
\text { C7OS }\end{array}$ & $\begin{array}{l}\mathrm{R}-\mathrm{A} \\
\mathrm{TO}- \\
\mathrm{NKK} \\
\mathrm{N} \\
\mathrm{MO} \\
\mathrm{ROM} \\
(\mathrm{L}) \\
\text { 53) }\end{array}$ & $\begin{array}{l}\text { CHLC } \\
\text { PHY } \\
\text { PLA } \\
\text { TC } \\
\text { CHRC } \\
\text { FLUC } \\
\text { (U⿺ } \\
\text { (70 }\end{array}$ & $\begin{array}{l}\text { R-B } \\
\text { TO- } \\
\text { NK- } \\
\text { N } \\
\text { Yo } \\
\text { ROM } \\
\text { (L) } \\
\text { 54) }\end{array}$ \\
\hline $\begin{array}{l}\text { UN } \\
05 . . .\end{array}$ & & & & 20 & & 33 & & 19 & 6. & & $>4$. & & 29 & & & 70 & $<1$ & \\
\hline
\end{tabular}


05316580 MINNESOTA RIVER AT MORTON

WATER QUALITY DATA, WATER YEAR OCTOBER 1990 TO SEPTEMBER 1991

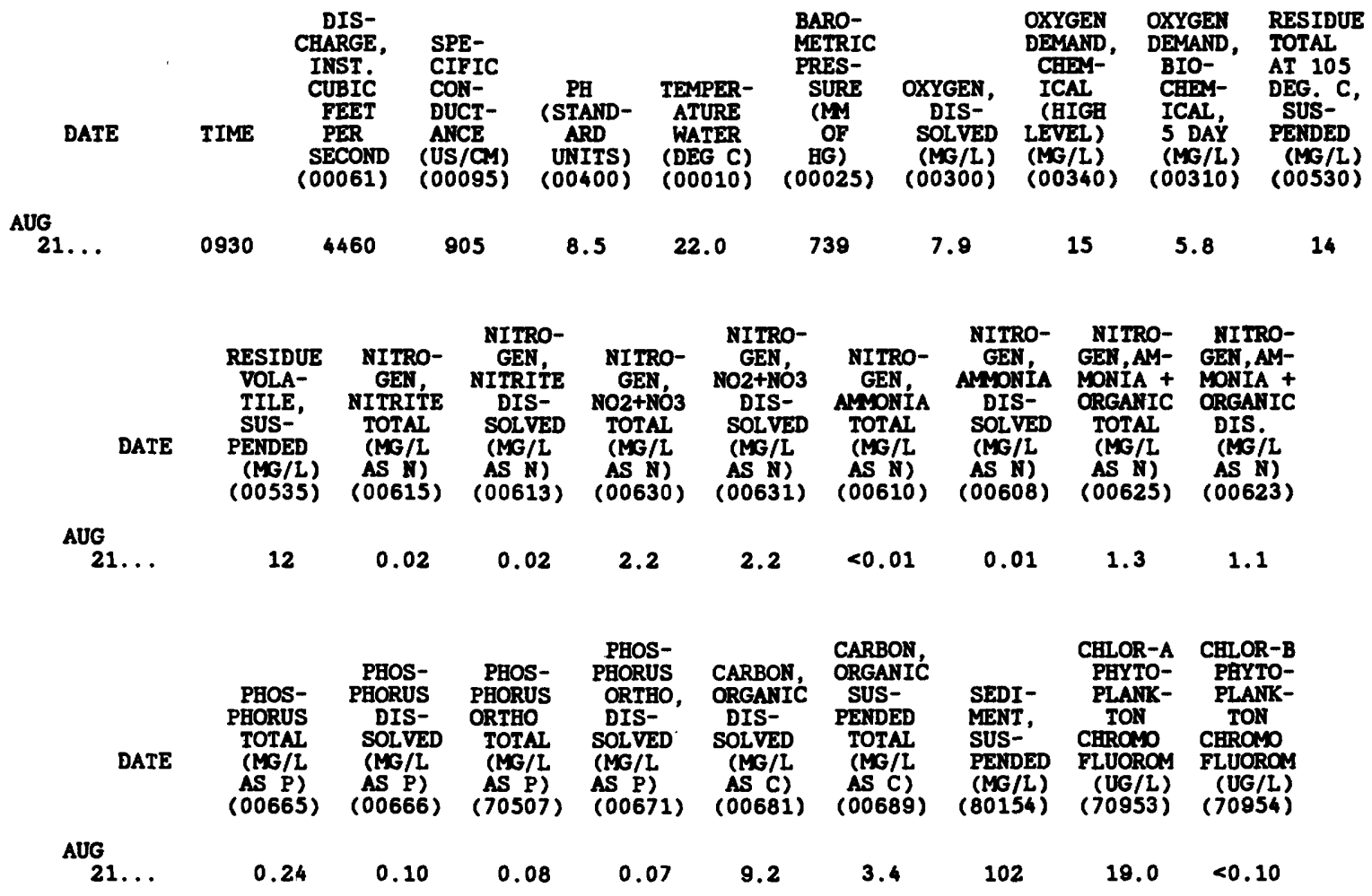


ANALYSES OF SAMLES COLLECTED AT WATER-QUALITY PARTIAL-RECORD STATIONS

05316685 MIMAESOTA RIVER MEAR FAIRFAX

WATER QUALITY DATA, WATER YEAR OCTOBER 1990 TO SEPTEMBER 1991

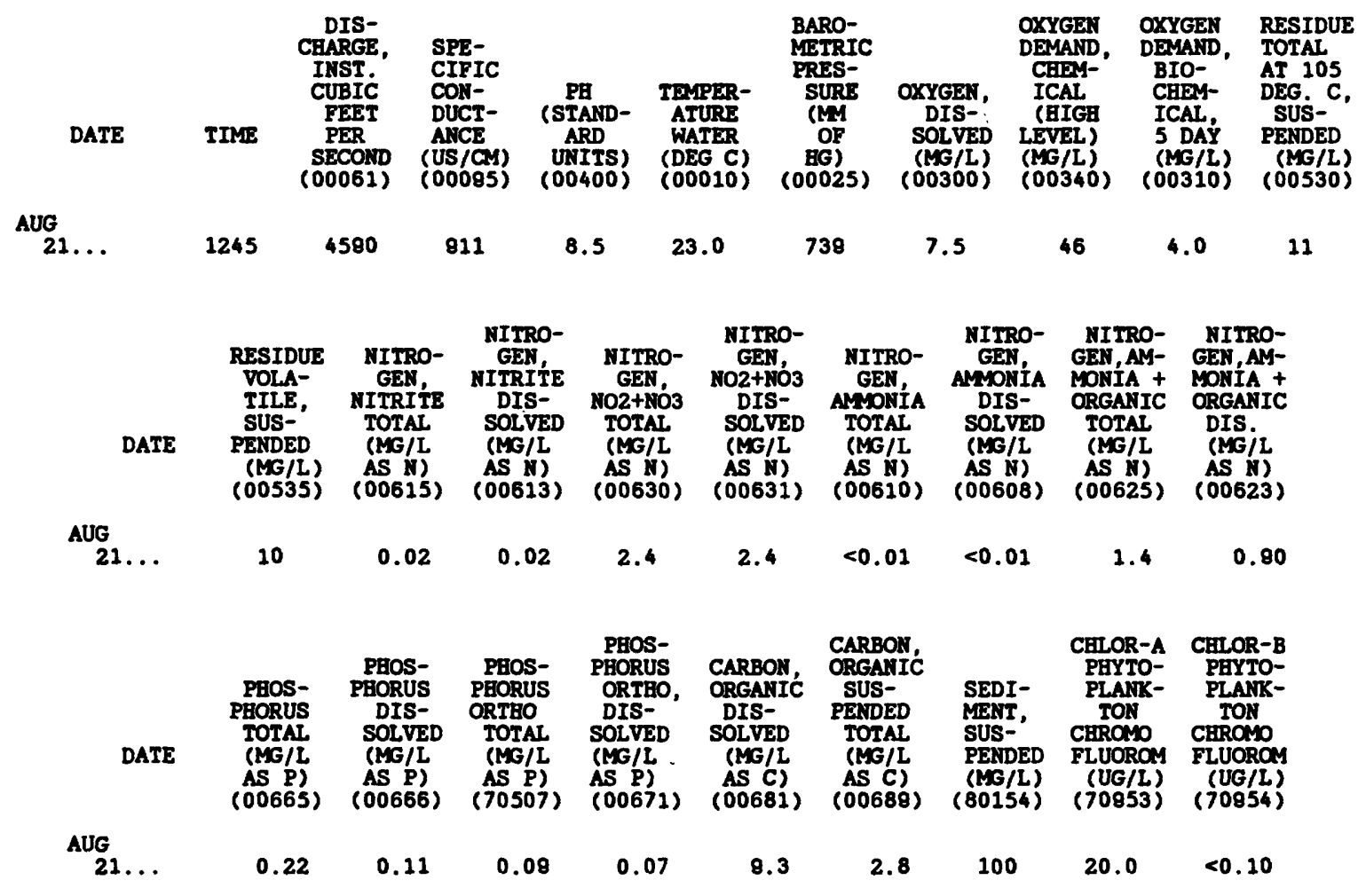


ANALYSES OF SAMPLES COLLECTED AT WATER-QUALITY PARTIAL-RECORD STATIONS

05316760 MINNESOTA RIVER NEAR NEW ULM

WATER QUALITY DATA, WATER YEAR OCTOBER 1990 TO SEPTEMBER 1991

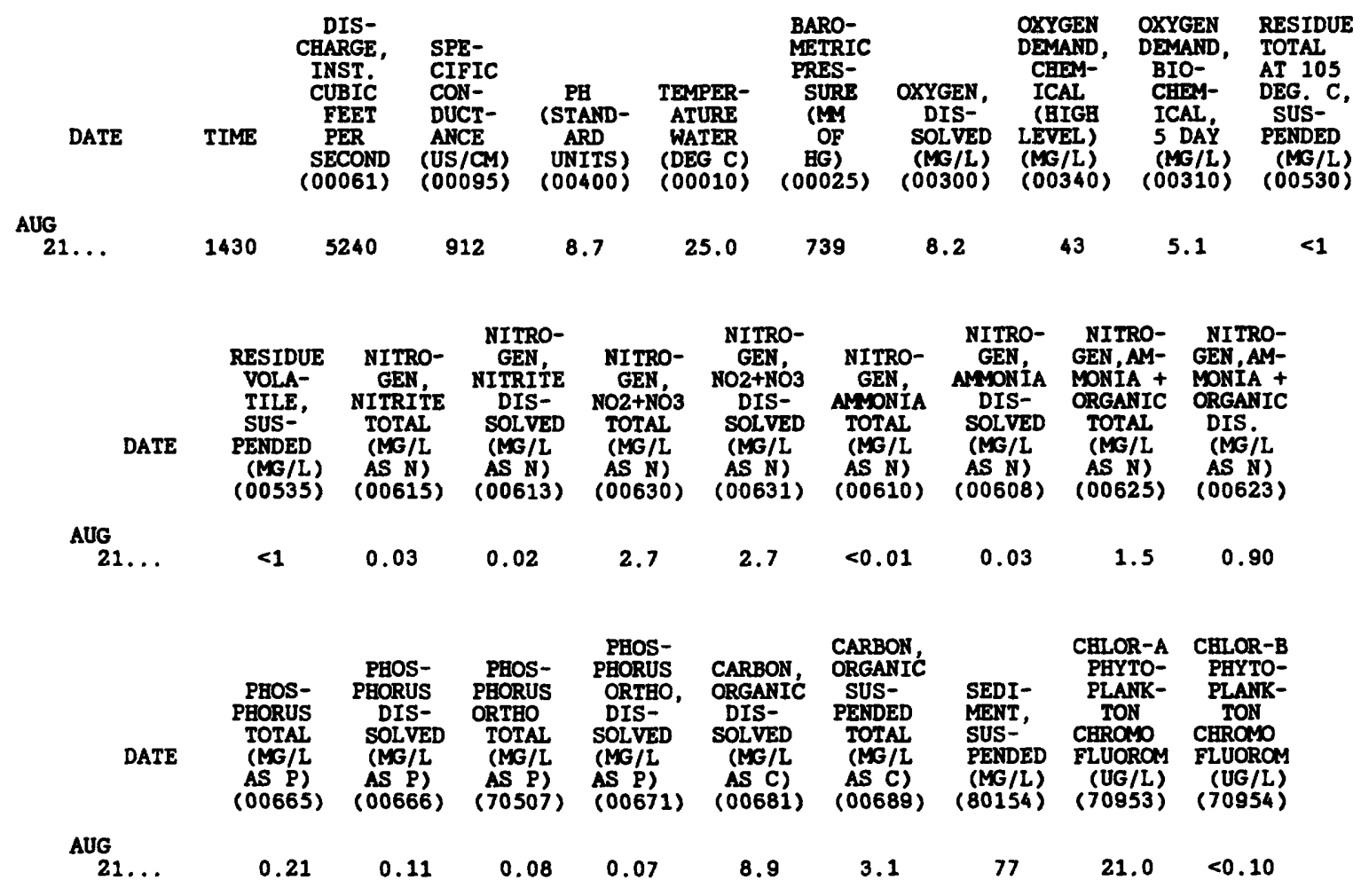


AMALYSES OF SAMPLES COLLECTED AT WATER-QUALITY PARTIAL-RECORD STATIONS

05317250 MINEESOTA RIVER AT COURTLAND

WATER QUALITY DATA, WATER YEAR OCTOBER 1990 TO SEPTEMBER 1991

\begin{tabular}{|c|c|c|c|c|c|c|c|c|c|c|c|c|c|c|c|c|c|c|}
\hline DATE & TIME & \multicolumn{2}{|c|}{$\begin{array}{l}\text { DIS- } \\
\text { CRARGE, } \\
\text { INST. } \\
\text { CUBIC } \\
\text { FEET } \\
\text { PER } \\
\text { SECOND } \\
(00061)\end{array}$} & \multicolumn{2}{|c|}{$\begin{array}{l}\text { SPE- } \\
\text { CIFIC } \\
\text { CON- } \\
\text { DUCT- } \\
\text { ANCE } \\
\text { (US/GH) } \\
(00095)\end{array}$} & $\begin{array}{c}\mathrm{PI} \\
\mathbf{S T} \\
\mathrm{AN} \\
\mathrm{UN} \\
\mathrm{COO}\end{array}$ & $\begin{array}{l}\text { IND- } \\
\text { LD } \\
(T S) \\
00)\end{array}$ & $\begin{array}{l}\text { TEM } \\
\text { ATI } \\
\text { WA } \\
\text { CDE } \\
\text { COO }\end{array}$ & $\begin{array}{l}\text { PER- } \\
\text { JRE } \\
\text { IRER } \\
\text { G C) } \\
\text { (10) }\end{array}$ & \multicolumn{2}{|c|}{$\begin{array}{l}\text { BARO- } \\
\text { MEIRIC } \\
\text { PRES- } \\
\text { SURE } \\
\text { (MY } \\
\text { OF } \\
\text { BG) } \\
(00025)\end{array}$} & $\begin{array}{r}\text { OXY } \\
D \\
\text { SO } \\
(M \\
C 00\end{array}$ & $\begin{array}{l}\text { EN, } \\
\text { S- } \\
\text { VED } \\
\text { iLs } \\
00 \text { ) }\end{array}$ & \multicolumn{2}{|c|}{$\begin{array}{l}\text { OXYGEN } \\
\text { DEMAND, } \\
\text { CEEYY- } \\
\text { ICAL } \\
\text { (EIGH } \\
\text { LEVEL) } \\
(\text { MG/L) } \\
(00340)\end{array}$} & \multicolumn{2}{|c|}{$\begin{array}{l}\text { OXYGEN } \\
\text { DEMAND, } \\
\text { BIO- } \\
\text { CEDY- } \\
\text { ICAL, } \\
5 \text { DAY } \\
(M G / L) \\
(00310)\end{array}$} & $\begin{array}{l}\text { RESIDUE } \\
\text { TOTAI } \\
\text { AT 105 } \\
\text { DEG. C, } \\
\text { SUS- } \\
\text { PENDED } \\
\text { (MG/L) } \\
(00530)\end{array}$ \\
\hline \multirow{2}{*}{$\begin{array}{l}\text { AUG } \\
22 \ldots \\
\text { SEP } \\
06 \ldots\end{array}$} & 0900 & \multicolumn{2}{|c|}{5500} & \multicolumn{2}{|c|}{866} & \multicolumn{2}{|c|}{8.4} & \multicolumn{2}{|c|}{23.0} & \multicolumn{2}{|c|}{746} & \multicolumn{2}{|c|}{7.2} & & 39 & \multicolumn{2}{|c|}{3.9} & 33 \\
\hline & 1140 & \multicolumn{2}{|c|}{1170} & \multicolumn{2}{|c|}{961} & \multicolumn{2}{|c|}{8.2} & 21 & .5 & \multicolumn{2}{|c|}{746} & \multicolumn{2}{|c|}{7.9} & \multicolumn{4}{|c|}{4.9} & 53 \\
\hline $\begin{array}{l}\text { DATE } \\
.\end{array}$ & $\begin{array}{r}\text { RES } \\
\text { VO } \\
T I \\
\text { SU } \\
\text { PEI } \\
(I-1 \\
C O O\end{array}$ & $\begin{array}{l}\text { DUE } \\
\text { A- } \\
\text { E, } \\
\text { ED } \\
\text { (L) } \\
\text { 35) }\end{array}$ & $\begin{array}{l}\text { NIT } \\
\text { GE } \\
\text { NITR } \\
\text { TOT } \\
\text { (MG } \\
\text { AS } \\
\text { CO06 }\end{array}$ & \multicolumn{5}{|c|}{$\begin{array}{cc}\text { NITRO- } & \\
\text { GEN, } & \text { NITRO- } \\
\text { NITRITE } & \text { GEN, } \\
\text { DIS- } & \text { NO2+NO3 } \\
\text { SOLVED } & \text { TOTAL } \\
\text { SOLVIL } & (\text { MG/L } \\
\text { (MG/L } & \text { AS N) } \\
\text { AS NI) } & \text { AS } \\
\text { (00613) } & (00630)\end{array}$} & \multicolumn{2}{|c|}{$\begin{array}{l}\text { MITRO- } \\
\text { GEN, } \\
\text { MO2+NÓ3 } \\
\text { DIS- } \\
\text { SOLVED } \\
\text { (MG/L } \\
\text { AS N) } \\
\text { (O0631) }\end{array}$} & $\begin{array}{c}\text { MIT } \\
\text { GE } \\
\text { AMMO } \\
\text { TOT } \\
\left(M T^{\prime}\right. \\
\text { AS } \\
\text { COOG }\end{array}$ & $\begin{array}{l}\text { RO- } \\
\text { NAIA } \\
\text { NIA } \\
\text { AL } \\
\text { in } \\
10)\end{array}$ & \multicolumn{2}{|c|}{$\begin{array}{c}\text { NITRO- } \\
\text { GEN, } \\
\text { AMONIA } \\
\text { DIS- } \\
\text { SOLVED } \\
\text { (MG/L } \\
\text { AS N) } \\
(00608)\end{array}$} & \multicolumn{2}{|c|}{$\begin{array}{l}\text { NITRO- } \\
\text { GEN,AM- } \\
\text { MONIA + } \\
\text { ORGANIC } \\
\text { TOTAL } \\
\text { (MG/L } \\
\text { AS N) } \\
(00625)\end{array}$} & \multicolumn{2}{|c|}{$\begin{array}{l}\text { MITRO- } \\
\text { GEN,AM- } \\
\text { MONIA + } \\
\text { ORGANIC } \\
\text { DIS. } \\
\text { (MG/L } \\
\text { AS IN) } \\
\text { (00623) }\end{array}$} \\
\hline $\begin{array}{l}\text { AUG } \\
22 \ldots \\
\text { SEP } \\
06 \ldots\end{array}$ & \multicolumn{2}{|c|}{14} & \multicolumn{2}{|c|}{0.03} & \multicolumn{2}{|c|}{.02} & \multicolumn{2}{|c|}{2.7} & \multicolumn{2}{|c|}{2.7} & $<0$. & 01 & \multicolumn{2}{|c|}{$<0.01$} & \multicolumn{2}{|c|}{1.0} & \multicolumn{2}{|c|}{0.70} \\
\hline DATE & $\begin{array}{r}\mathrm{PE} \\
\mathrm{PHC} \\
\mathrm{TC} \\
\mathbf{( N} \\
\mathrm{AS} \\
\mathrm{COO}\end{array}$ & & $\begin{array}{c}\text { PBO } \\
\text { PEOR } \\
\text { DI } \\
\text { SOL } \\
\text { (MG } \\
\text { AS } \\
\text { COOG }\end{array}$ & $\begin{array}{l}\text { S- } \\
\text { US } \\
\text { S- } \\
\text { VED } \\
\text { (L } \\
\text { P) } \\
66)\end{array}$ & $\begin{array}{l}\text { PHO } \\
\text { PHOR } \\
\text { ORTH } \\
\text { TOT } \\
\text { (ME } \\
\text { AS } \\
6705\end{array}$ & $\begin{array}{l}\text { S- } \\
\text { US } \\
\text { AI } \\
\text { (L } \\
\text { P) } \\
\text { 07) }\end{array}$ & $\begin{array}{r}\text { PEO } \\
\text { PBOR } \\
\text { ORT } \\
\text { DIS } \\
\text { SOLV } \\
\text { (MGI } \\
\text { AS F } \\
\text { COOG }\end{array}$ & $\begin{array}{l}\text { S- } \\
\text { US } \\
\text { BO, } \\
\text { ED } \\
\text { L } \\
\text { 71) }\end{array}$ & $\begin{array}{l}\text { CARE } \\
\text { ORGA } \\
\text { DIS } \\
\text { SOLV } \\
\text { (ME } \\
\text { AS } \\
\text { COOG }\end{array}$ & $\begin{array}{l}\text { ON, } \\
\text { NIC } \\
\text { ED } \\
\text { (L) } \\
\text { C) }\end{array}$ & $\begin{array}{l}\text { CARB } \\
\text { ORGA } \\
\text { SUS } \\
\text { PEND } \\
\text { TOT } \\
\text { (ME } \\
\text { AS } \\
\text { COOG }\end{array}$ & $\begin{array}{l}\text { ON, } \\
\text { NIC } \\
-- \\
\text { ED } \\
\text { AL } \\
\text { (L) } \\
\text { C) } \\
89 \text { ) }\end{array}$ & $\begin{array}{l}\text { SED } \\
\text { MEN } \\
\text { SUS } \\
\text { PEN } \\
\text { (MG } \\
\text { (801 }\end{array}$ & $\begin{array}{l}\text { I- } \\
\text { I, } \\
\text { DED } \\
\text { (L) } \\
\text { S4) }\end{array}$ & $\begin{array}{c}\text { CHLOI } \\
\text { PHY } \\
\text { PLAI } \\
\text { TOI } \\
\text { CERO } \\
\text { FLUOI } \\
\text { SUG } \\
\text { C709 }\end{array}$ & $\begin{array}{l}R-A \\
\text { TO- } \\
\text { NK- } \\
\text { No } \\
\text { Mo } \\
\text { ROM } \\
\text { (L) } \\
53 \text { ) }\end{array}$ & $\begin{array}{c}\text { CHLO } \\
\text { PHY } \\
\text { PLA } \\
\text { TO } \\
\text { CERO } \\
\text { FLUO } \\
\text { CUG } \\
\text { C709 }\end{array}$ & $\begin{array}{l}\text { R-B } \\
\text { TO- } \\
\text { NKK- } \\
\text { MO } \\
\text { MOM } \\
(1 \mathrm{~L}) \\
\text { (54) }\end{array}$ \\
\hline $\begin{array}{l}\text { AUG } \\
22 . \\
\text { SEP }\end{array}$ & & 21 & & & 0. & 08 & & 06 & 8. & & 2. & 5 & & 11 & 11 & .0 & $<0$. & 10 \\
\hline $10 .$. & & 17 & & 09 & & 06 & & 04 & 8. & & $>1$. & & & 22 & & .90 & $<0$. & \\
\hline
\end{tabular}


05317500 MINNESOTA RIVER AT JUDSON, MN

WATER QUALITY DATA, WATER YEAR OCTOBER 1990 TO SEPTEMBER 1991

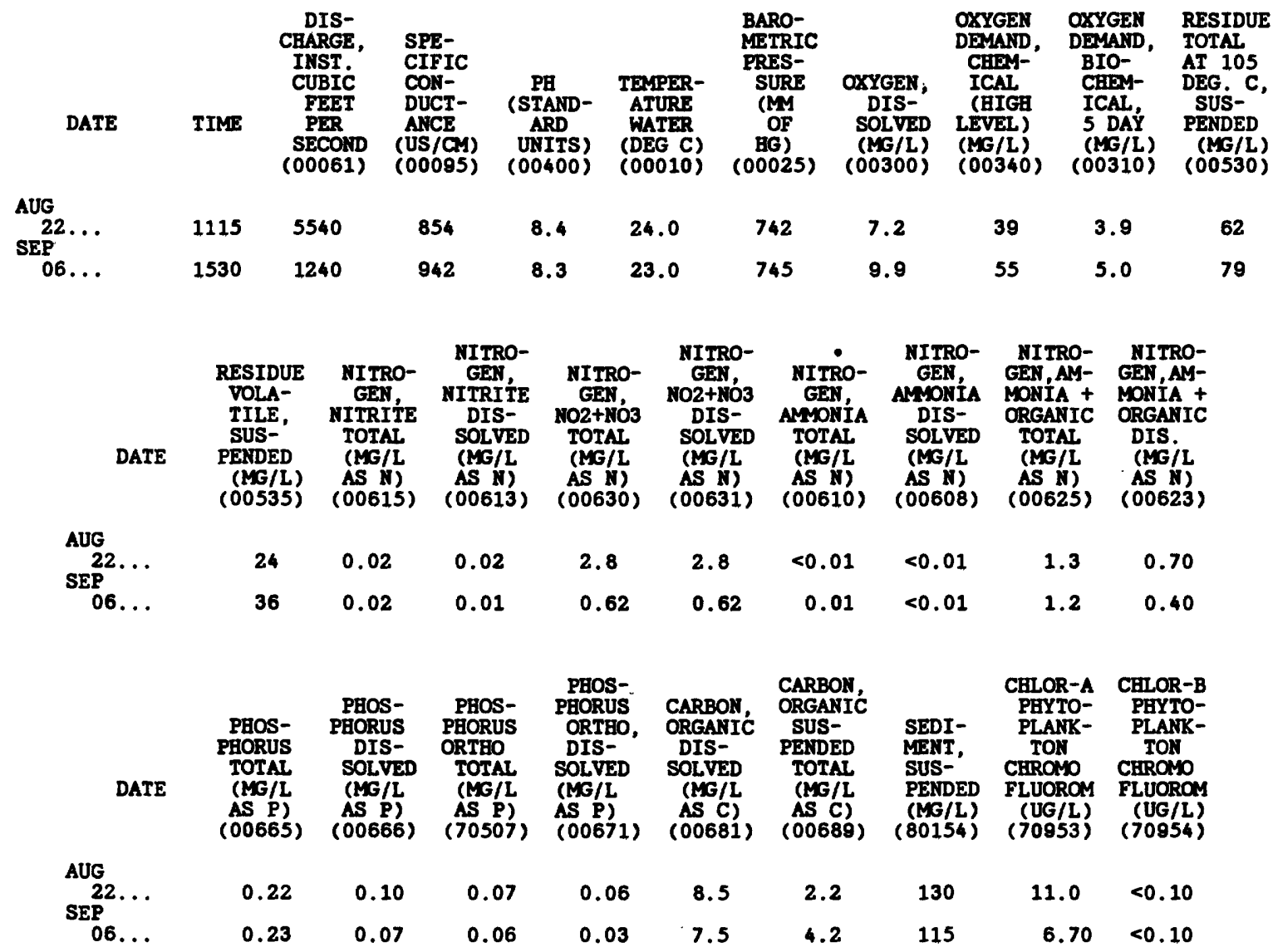


ANALYSES OF SAMPLES COLLECTED AT WATER-QUALITY PARTIAL-RECORD STATIONS

05318290 BLUE EARTH RIVER MEAR GOOD THUNDER, MIMAESOTA

WATER QUALITY DATA, WATER YEAR OCTOBER 1990 TO SEPTEMBER 1991

\begin{tabular}{|c|c|c|c|c|c|c|c|c|c|c|c|c|}
\hline DATE & TIME & $\begin{array}{c}\text { DIS- } \\
\text { CHARGE, } \\
\text { INST. } \\
\text { CUBIC } \\
\text { FEET } \\
\text { PER } \\
\text { SECOND } \\
(00061)\end{array}$ & $\begin{array}{l}\text { SPE- } \\
\text { CIFIC } \\
\text { CON- } \\
\text { DUCT- } \\
\text { ANCE } \\
\text { (US/CM) } \\
\text { (OOO95) }\end{array}$ & $\begin{array}{c}\text { PH } \\
\text { (STAND- } \\
\text { ARD } \\
\text { UNITS) } \\
(00400)\end{array}$ & $\begin{array}{l}\text { TEMPER- } \\
\text { ATURE } \\
\text { WATER } \\
\text { (DEG C) } \\
(00010)\end{array}$ & $\begin{array}{c}\text { BARO- } \\
\text { MEIRIC } \\
\text { PRES- } \\
\text { SURE } \\
\text { (MM } \\
\text { OF } \\
\text { BG) } \\
(00025)\end{array}$ & $\begin{array}{c}\text { OXYGEN, } \\
\text { DIS- } \\
\text { SOLVED } \\
(M G / L) \\
(00300)\end{array}$ & $\begin{array}{l}\text { OXYGEN } \\
\text { DEMAND, } \\
\text { CHEYY- } \\
\text { ICAL } \\
\text { (BIGB } \\
\text { LEVEL) } \\
\text { (MG/L) } \\
\text { (O0340) }\end{array}$ & $\begin{array}{l}\text { OXYGEN } \\
\text { DEMAND, } \\
\text { BIO- } \\
\text { CHEY- } \\
\text { ICAL, } \\
5 \text { DAY } \\
(\mathrm{MG} / \mathrm{L}) \\
(\mathrm{OO310})\end{array}$ & $\begin{array}{l}\text { CALCIUM } \\
\text { DIS- } \\
\text { SOLVED } \\
\text { (MG/L } \\
\text { AS CA) } \\
\text { (00915) }\end{array}$ & $\begin{array}{c}\text { MAGNE- } \\
\text { SIUM, } \\
\text { DIS- } \\
\text { SOLVED } \\
\text { (MG/L } \\
\text { AS IMG) } \\
\text { (00925) }\end{array}$ & $\begin{array}{c}\text { SODIUM, } \\
\text { DIS- } \\
\text { SOLVED } \\
\text { (MG/L } \\
\text { AS NA) } \\
(00930)\end{array}$ \\
\hline \multicolumn{13}{|l|}{ MAR } \\
\hline $\begin{array}{r}15 \ldots \\
19 \ldots \\
21 \ldots \\
22 \ldots \\
26 \ldots \\
\mathrm{APR}\end{array}$ & $\begin{array}{l}1300 \\
1615 \\
1145 \\
1545 \\
0845\end{array}$ & $\begin{array}{r}278 \\
419 \\
958 \\
1570 \\
2530\end{array}$ & $\begin{array}{l}637 \\
600 \\
606 \\
526 \\
678\end{array}$ & $\begin{array}{l}8.4 \\
8.0 \\
8.7 \\
8.3 \\
8.5\end{array}$ & $\begin{array}{l}1.0 \\
1.0 \\
0.5 \\
1.5 \\
5.0\end{array}$ & $\begin{array}{l}747 \\
736 \\
728 \\
724 \\
726\end{array}$ & $\begin{array}{l}13.5 \\
12.9 \\
13.3 \\
13.0 \\
11.3\end{array}$ & $\begin{array}{l}20 \\
23 \\
50 \\
66 \\
49\end{array}$ & $\begin{array}{l}1.1 \\
3.3 \\
3.7 \\
5.2 \\
3.9\end{array}$ & $\begin{array}{l}84 \\
81 \\
75 \\
68 \\
94\end{array}$ & $\begin{array}{l}26 \\
26 \\
23 \\
20 \\
28\end{array}$ & $\begin{array}{l}19 \\
21 \\
17 \\
12 \\
12\end{array}$ \\
\hline $\begin{array}{r}03 . . . \\
09 .\end{array}$ & $\begin{array}{l}0900 \\
.1600\end{array}$ & $\begin{array}{l}2170 \\
1330\end{array}$ & $\begin{array}{l}722 \\
761\end{array}$ & $\begin{array}{l}6.5 \\
8.7\end{array}$ & $\begin{array}{r}8.0 \\
10.5\end{array}$ & $\begin{array}{l}744 \\
738\end{array}$ & $\begin{array}{l}13.5 \\
10.4\end{array}$ & $\begin{array}{c}34 \\
38\end{array}$ & $\begin{array}{l}2.4 \\
4.5\end{array}$ & $\because$ & $\because$ & $=$ \\
\hline $\begin{array}{l}06 \ldots \\
07 \ldots \\
08 \ldots \\
09 . \ldots \\
16 \ldots\end{array}$ & $\begin{array}{l}0915 \\
0945 \\
1420 \\
1015 \\
0945\end{array}$ & $\begin{array}{l}4490 \\
5070 \\
5480 \\
6760 \\
3640\end{array}$ & $\begin{array}{l}599 \\
609 \\
607 \\
565 \\
722\end{array}$ & $\begin{array}{l}7.9 \\
8.3 \\
7.8 \\
7.8 \\
8.3\end{array}$ & $\begin{array}{r}7.5 \\
8.0 \\
9.0 \\
10.0 \\
19.0\end{array}$ & $\begin{array}{l}737 \\
745 \\
740 \\
740 \\
735\end{array}$ & $\begin{array}{r}10.8 \\
12.6 \\
10.0 \\
10.1 \\
8.9\end{array}$ & $\begin{array}{l}60 \\
45 \\
43 \\
47 \\
33\end{array}$ & $\begin{array}{l}2.8 \\
2.5 \\
2.5 \\
2.9 \\
1.6\end{array}$ & $\begin{array}{l}=- \\
=- \\
--\end{array}$ & $\begin{array}{l}-- \\
-- \\
--\end{array}$ & $\begin{array}{l}=- \\
=- \\
=-\end{array}$ \\
\hline
\end{tabular}

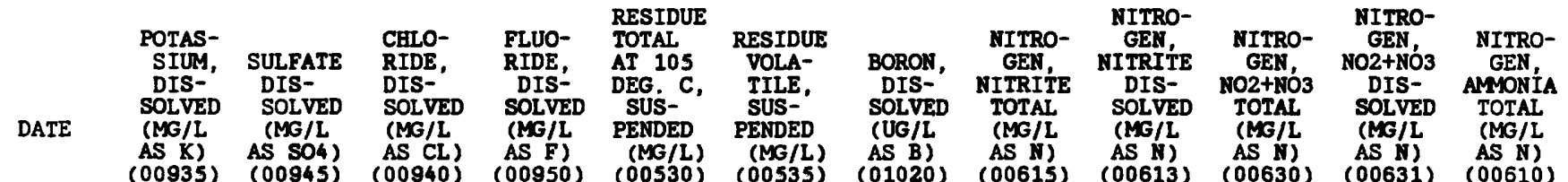

MAR

\begin{tabular}{|c|c|c|c|c|c|c|c|c|c|c|c|c|}
\hline $\begin{array}{l}15 \ldots \\
19 \ldots \\
21 \ldots \\
22 \ldots \\
26\end{array}$ & $\begin{array}{l}3.5 \\
3.2 \\
3.6 \\
3.4 \\
3.5\end{array}$ & $\begin{array}{l}70 \\
69 \\
69 \\
43 \\
56\end{array}$ & $\begin{array}{l}30 \\
30 \\
34 \\
27 \\
35\end{array}$ & $\begin{array}{l}0.3 \\
0.3 \\
0.3 \\
0.3 \\
0.4\end{array}$ & $\begin{array}{r}9 \\
128 \\
231 \\
391 \\
275\end{array}$ & $\begin{array}{r}2 \\
24 \\
25 \\
89 \\
34\end{array}$ & $\begin{array}{l}50 \\
60 \\
60 \\
40 \\
30\end{array}$ & $\begin{array}{l}0.04 \\
0.03 \\
0.04 \\
0.09 \\
0.12\end{array}$ & $\begin{array}{l}0.04 \\
0.03 \\
0.03 \\
0.07 \\
0.06\end{array}$ & $\begin{array}{r}7.0 \\
5.2 \\
5.3 \\
9.3 \\
17\end{array}$ & $\begin{array}{r}6.7 \\
5.2 \\
4.8 \\
7.5 \\
17\end{array}$ & $\begin{array}{l}0.21 \\
0.16 \\
0.19 \\
0.21 \\
0.26\end{array}$ \\
\hline $\begin{array}{l}03 \ldots \\
09 \\
{ }_{Y}\end{array}$ & -- & -- & $=-$ & $=$ & $\begin{array}{r}119 \\
99\end{array}$ & $\begin{array}{l}20 \\
17\end{array}$ & $=$ & $\begin{array}{l}0.05 \\
0.03\end{array}$ & $\begin{array}{l}0.05 \\
0.03\end{array}$ & $\begin{array}{l}16 \\
15\end{array}$ & $\begin{array}{l}15 \\
15\end{array}$ & $\begin{array}{r}0.02 \\
<0.01\end{array}$ \\
\hline $06 \ldots$ & $\begin{array}{l}-- \\
=- \\
=-\end{array}$ & $\begin{array}{l}-- \\
= \\
--\end{array}$ & $\begin{array}{l}= \\
=- \\
=\end{array}$ & $\begin{array}{l}=- \\
=- \\
--\end{array}$ & $\begin{array}{l}488 \\
363 \\
241 \\
356 \\
105\end{array}$ & $\begin{array}{l}46 \\
56 \\
34 \\
47 \\
20\end{array}$ & $\begin{array}{l}\ddot{-} \\
\ddot{-}\end{array}$ & $\begin{array}{l}0.15 \\
0.15 \\
0.16 \\
0.13 \\
0.08\end{array}$ & $\begin{array}{l}0.07 \\
0.08 \\
0.11 \\
0.13 \\
0.08\end{array}$ & $\begin{array}{l}18 \\
18 \\
22 \\
23 \\
21\end{array}$ & $\begin{array}{l}18 \\
18 \\
22 \\
23 \\
20\end{array}$ & $\begin{array}{l}0.08 \\
0.08 \\
0.09 \\
0.07 \\
0.02\end{array}$ \\
\hline $06 \ldots$ & -- & -- & -- & -- & 59 & 8 & -- & 0.21 & 0.11 & 14 & 14 & 0.14 \\
\hline $\begin{array}{l}2 \\
22 \ldots \\
23 \ldots \\
24 \ldots \\
25 \ldots\end{array}$ & $\begin{array}{l}-- \\
-- \\
--\end{array}$ & $=-$ & $\begin{array}{l}=- \\
=- \\
--\end{array}$ & $\begin{array}{l}-- \\
=- \\
--\end{array}$ & $\begin{array}{l}800 \\
616 \\
386 \\
243\end{array}$ & $\begin{array}{r}148 \\
100 \\
31 \\
30\end{array}$ & $\begin{array}{l}= \\
-- \\
--\end{array}$ & $\begin{array}{l}0.04 \\
0.03 \\
0.04 \\
0.03\end{array}$ & $\begin{array}{l}0.04 \\
0.03 \\
0.03 \\
0.02\end{array}$ & $\begin{array}{c}10 \\
9.9 \\
11 \\
13\end{array}$ & $\begin{array}{l}11 \\
9.8 \\
11 \\
13\end{array}$ & $\begin{array}{r}0.02 \\
<0.01 \\
<0.01 \\
<0.01\end{array}$ \\
\hline
\end{tabular}


05318290 BLUE EARTE RIVER MEAR GOOD THUNDER, MINNESOTA--Continued

WATER QUALITY DATA, WATER YEAR OCTOBER 1990 TO SEPTEMBER 1991

\begin{tabular}{|c|c|c|c|c|c|c|c|c|c|c|c|c|}
\hline DATE & $\begin{array}{c}\text { NIIRO- } \\
\text { GEN, } \\
\text { AMONIA } \\
\text { DIS- } \\
\text { SOLVED } \\
(\text { MG/L } \\
\text { AS N) } \\
(00608)\end{array}$ & $\begin{array}{l}\text { NITRO- } \\
\text { GEN,AM- } \\
\text { MONIA + } \\
\text { ORGANIC } \\
\text { TOTAL } \\
\text { (MG/L } \\
\text { AS N) } \\
(00625)\end{array}$ & $\begin{array}{l}\text { NITRO- } \\
\text { GEN,AM- } \\
\text { MONIA + } \\
\text { ORGANIC } \\
\text { DIS. } \\
\text { (MG/L } \\
\text { AS N }) \\
(00623)\end{array}$ & $\begin{array}{c}\text { PHOS- } \\
\text { PEORUS } \\
\text { TOTAL } \\
\text { (MG/L } \\
\text { AS P) } \\
(00665)\end{array}$ & $\begin{array}{c}\text { PEOS- } \\
\text { PEORUS } \\
\text { DIS- } \\
\text { SOLVED } \\
\text { (MG/L } \\
\text { AS P) } \\
(00666)\end{array}$ & $\begin{array}{c}\text { PHOS- } \\
\text { PBORUS } \\
\text { ORTBO } \\
\text { TOTAL } \\
\text { (MG/L } \\
\text { AS P) } \\
\text { (70507) }\end{array}$ & $\begin{array}{l}\text { PHOS- } \\
\text { PHORUS } \\
\text { ORTEO, } \\
\text { DIS- } \\
\text { SOLVED } \\
\text { (MG/L } \\
\text { AS P) } \\
(00671)\end{array}$ & $\begin{array}{l}\text { CARBOH, } \\
\text { ORGANIC } \\
\text { DIS- } \\
\text { SOLVED } \\
\text { (MG/L } \\
\text { AS C) } \\
(00681)\end{array}$ & $\begin{array}{c}\text { CARBOK, } \\
\text { ORGANIC } \\
\text { SUS- } \\
\text { PEIDED } \\
\text { TOTAL } \\
(M G / L \\
\text { AS C) } \\
(00689)\end{array}$ & $\begin{array}{l}\text { SEDI- } \\
\text { MENT, } \\
\text { SUS- } \\
\text { PENDED } \\
(M G / L) \\
(80154)\end{array}$ & $\begin{array}{c}\text { CHLOR-A } \\
\text { PHYTO- } \\
\text { PLANK- } \\
\text { TON } \\
\text { CHROMO } \\
\text { FLUOROM } \\
\text { (UG/L) } \\
\text { (70953) }\end{array}$ & $\begin{array}{c}\text { CHLOR-B } \\
\text { PHYTO- } \\
\text { PLANK- } \\
\text { TON } \\
\text { CHROMO } \\
\text { FLUOROM } \\
\text { (UG/L) } \\
\text { (70954) }\end{array}$ \\
\hline $\begin{array}{r}\text { MAR } \\
15 \ldots \\
19 \ldots \\
21 \ldots \\
22 \ldots \\
26 \ldots \\
\text { APR }\end{array}$ & $\begin{array}{l}0.21 \\
0.16 \\
0.19 \\
0.21 \\
0.26\end{array}$ & $\begin{array}{l}1.5 \\
1.5 \\
1.1 \\
2.7 \\
2.4\end{array}$ & $\begin{array}{l}1.5 \\
1.0 \\
0.90 \\
1.5 \\
1.8\end{array}$ & $\begin{array}{l}0.12 \\
0.12 \\
0.30 \\
0.17 \\
0.42\end{array}$ & $\begin{array}{l}0.09 \\
0.05 \\
0.09 \\
0.12 \\
0.12\end{array}$ & $\begin{array}{l}0.10 \\
0.08 \\
0.12 \\
0.13 \\
0.20\end{array}$ & $\begin{array}{l}0.09 \\
0.05 \\
0.09 \\
0.12 \\
0.08\end{array}$ & $\begin{array}{l}5.1 \\
6.3 \\
6.5 \\
7.4 \\
7.0\end{array}$ & $\begin{array}{r}0.8 \\
2.7 \\
>5.0 \\
6.1 \\
>5.0\end{array}$ & $\begin{array}{r}37 \\
193 \\
736 \\
785 \\
707\end{array}$ & $\begin{array}{c}6 .- \\
6.70 \\
13.0 \\
13.0 \\
6.00\end{array}$ & $\begin{array}{l}-- \\
0.60 \\
1.10 \\
0.70 \\
0.40\end{array}$ \\
\hline $03 . .$. & $\begin{array}{r}0.02 \\
<0.01\end{array}$ & $\begin{array}{l}1.8 \\
1.9\end{array}$ & $\begin{array}{l}1.4 \\
1.0\end{array}$ & $\begin{array}{l}0.17 \\
0.21\end{array}$ & $\begin{array}{l}0.04 \\
0.03\end{array}$ & $\begin{array}{l}0.06 \\
0.05\end{array}$ & $\begin{array}{r}0.04 \\
<0.01\end{array}$ & $\begin{array}{l}6.4 \\
6.1\end{array}$ & $\begin{array}{l}2.9 \\
4.8\end{array}$ & $\begin{array}{l}242 \\
270\end{array}$ & $\begin{array}{r}9.30 \\
17.0\end{array}$ & $\begin{array}{l}0.40 \\
0.90\end{array}$ \\
\hline $\begin{array}{r}06 \ldots \\
07 \ldots \\
08 . \ldots \\
09 . \ldots \\
16 . .\end{array}$ & $\begin{array}{l}0.07 \\
0.07 \\
0.08 \\
0.07 \\
0.02\end{array}$ & $\begin{array}{l}2.8 \\
2.9 \\
2.8 \\
1.9 \\
1.8\end{array}$ & $\begin{array}{l}1.1 \\
0.70 \\
0.70 \\
1.7 \\
1.2\end{array}$ & $\begin{array}{l}0.50 \\
0.44 \\
0.38 \\
0.14 \\
0.19\end{array}$ & $\begin{array}{l}0.12 \\
0.14 \\
0.13 \\
0.14 \\
0.06\end{array}$ & $\begin{array}{l}0.21 \\
0.23 \\
0.19 \\
0.12 \\
0.06\end{array}$ & $\begin{array}{l}0.11 \\
0.14 \\
0.13 \\
0.12 \\
0.05\end{array}$ & $\begin{array}{l}5.8 \\
7.2 \\
7.0 \\
9.4 \\
6.5\end{array}$ & $\begin{array}{l}8.8 \\
-. \\
5.3 \\
3.4 \\
2.2\end{array}$ & $\begin{array}{l}993 \\
856 \\
631 \\
753 \\
365\end{array}$ & $\begin{array}{l}5.90 \\
2.70 \\
3.00 \\
2.70 \\
6.30\end{array}$ & $\begin{array}{l}<2.50 \\
<1.10 \\
<1.20 \\
<1.10 \\
<1.10\end{array}$ \\
\hline
\end{tabular}


ANALYSES OF SAMPLES COLLECTED AT WATER-QUALITY PARTIAL-RECORD STATIONS

05322000 BLUE EARTH RIVER AT MOUTE AT MANKATO

WATER QUALITY DATA, WATER YEAR OCTOBER 1990 TO SEPTEMBER 1991

\begin{tabular}{|c|c|c|c|c|c|c|c|c|c|c|}
\hline DATE & TIME & $\begin{array}{c}\text { DIS- } \\
\text { CEARGE, } \\
\text { INST. } \\
\text { CUBIC } \\
\text { FEET } \\
\text { PER } \\
\text { SECOND } \\
\text { (00061) }\end{array}$ & $\begin{array}{l}\text { SPE- } \\
\text { CIFIC } \\
\text { COH- } \\
\text { DUCT- } \\
\text { AlCE } \\
\text { (US/CM) } \\
\text { (O0095) }\end{array}$ & $\begin{array}{c}\text { PH } \\
\text { (STAND- } \\
\text { ARD } \\
\text { UNITS) } \\
(00400)\end{array}$ & $\begin{array}{c}\text { TEMPER- } \\
\text { ATURE } \\
\text { WATER } \\
\text { (DEG C) } \\
(00010)\end{array}$ & $\begin{array}{c}\text { BARO- } \\
\text { MEIRIC } \\
\text { PRES- } \\
\text { SURE } \\
\text { (MM } \\
\text { OR } \\
\text { BG) } \\
(00025)\end{array}$ & $\begin{array}{c}\text { OXYGEN, } \\
\text { DIS- } \\
\text { SOLVED } \\
(M G / L) \\
(00300)\end{array}$ & $\begin{array}{l}\text { OXYGEN } \\
\text { DEMAND, } \\
\text { CHEY- } \\
\text { ICAL } \\
\text { (BIGH } \\
\text { LEVEL) } \\
\text { (MG/L) } \\
\text { (00340) }\end{array}$ & $\begin{array}{l}\text { OXYGEN } \\
\text { DEMAND, } \\
\text { BIO- } \\
\text { CEDY- } \\
\text { ICAL, } \\
\text { S DAY } \\
\text { (MG/L) } \\
(00310)\end{array}$ & $\begin{array}{l}\text { COLI- } \\
\text { FORM, } \\
\text { FECAL, } \\
\text { O.7 } \\
\text { UM-MF } \\
\text { (COLS. } \\
100 \mathrm{MI}) \\
\text { (31625) }\end{array}$ \\
\hline $\mathrm{CT}$ & & & & & & & & & & \\
\hline $24 \ldots$ & 1300 & 257 & 693 & 8.8 & 9.5 & 749 & 16.0 & 51 & 6.8 & K6 \\
\hline${ }_{\mathrm{EC}}^{28 \ldots}$ & 1340 & 133 & 840 & 8.3 & 2.0 & 746 & 13.7 & 21 & 4.6 & K8 \\
\hline JAN $18 \ldots$ & 1400 & 219 & 953 & 8.6 & 1.0 & 734 & 13.5 & 18 & 1.9 & $\mathbf{K 5}$ \\
\hline FEB $11 \ldots$ & 1400 & 76 & 1090 & 7.9 & 2.5 & 745 & 10.2 & 14 & 2.0 & $>2$ \\
\hline $\operatorname{MAR}^{27} \cdots$ & 1145 & 192 & 504 & 8.5 & 1.5 & 742 & 17.4 & 24 & 1.5 & 18 \\
\hline $\begin{array}{l}06 \ldots \\
14 \ldots \\
19 \ldots \\
20 \ldots \\
21 \ldots \\
25 \ldots \\
26 \ldots \\
A P R\end{array}$ & $\begin{array}{l}1200 \\
1115 \\
0930 \\
1500 \\
1630 \\
1515 \\
1430\end{array}$ & $\begin{array}{r}344 \\
557 \\
570 \\
873 \\
1410 \\
5160 \\
5180\end{array}$ & $\begin{array}{l}763 \\
676 \\
662 \\
633 \\
603 \\
571 \\
656\end{array}$ & $\begin{array}{l}8.0 \\
8.3 \\
-.- \\
8.9 \\
8.8 \\
8.2 \\
8.3\end{array}$ & $\begin{array}{l}3.0 \\
1.5 \\
1.5 \\
2.0 \\
1.5 \\
3.5 \\
6.0\end{array}$ & $\begin{array}{l}738 \\
749 \\
742 \\
727 \\
734 \\
735 \\
728\end{array}$ & $\begin{array}{l}11.1 \\
13.4 \\
13.3 \\
-1 \\
14.1 \\
12.5 \\
11.6\end{array}$ & $\begin{array}{l}18 \\
25 \\
17 \\
26 \\
37 \\
64 \\
51\end{array}$ & $\begin{array}{l}1.5 \\
2.6 \\
1.3 \\
3.5 \\
3.8 \\
4.2 \\
3.1\end{array}$ & $\begin{array}{c}\text { K10 } \\
=- \\
=- \\
=- \\
=-\end{array}$ \\
\hline $\begin{array}{r}02 \ldots \\
10 \ldots \\
17 \ldots \\
25 \ldots \\
30 \ldots \\
\text { MAY }\end{array}$ & $\begin{array}{l}1415 \\
0945 \\
1230 \\
0845 \\
0930\end{array}$ & $\begin{array}{l}4550 \\
2360 \\
9360 \\
4470 \\
4850\end{array}$ & $\begin{array}{l}690 \\
774 \\
726 \\
800 \\
776\end{array}$ & $\begin{array}{l}8.5 \\
8.7 \\
8.3 \\
8.3 \\
8.7\end{array}$ & $\begin{array}{r}6.5 \\
9.5 \\
9.5 \\
12.5 \\
12.5\end{array}$ & $\begin{array}{l}748 \\
748 \\
745 \\
740 \\
734\end{array}$ & $\begin{array}{l}12.9 \\
11.5 \\
12.4 \\
12.5 \\
10.5\end{array}$ & $\begin{array}{l}45 \\
34 \\
52 \\
29 \\
32\end{array}$ & $\begin{array}{l}2.7 \\
3.4 \\
1.8 \\
1.1 \\
0.9\end{array}$ & $\begin{array}{l}-- \\
-- \\
-- \\
42 \\
--\end{array}$ \\
\hline $\begin{array}{r}10 \ldots \\
15 \ldots \\
24 \ldots \\
31 \ldots \\
\text { JUN }\end{array}$ & $\begin{array}{l}1000 \\
0845 \\
0910 \\
0830\end{array}$ & $\begin{array}{r}14400 \\
7850 \\
7980 \\
8700\end{array}$ & $\begin{array}{l}601 \\
665 \\
729 \\
730\end{array}$ & $\begin{array}{l}8.1 \\
8.2 \\
8.4 \\
8.2\end{array}$ & $\begin{array}{l}13.0 \\
19.0 \\
19.5 \\
21.0\end{array}$ & $\begin{array}{l}744 \\
738 \\
740 \\
735\end{array}$ & $\begin{array}{r}11.1 \\
9.5 \\
9.7 \\
9.2\end{array}$ & $\begin{array}{l}49 \\
37 \\
34 \\
37\end{array}$ & $\begin{array}{l}2.4 \\
1.5 \\
1.7 \\
1.2\end{array}$ & K200 \\
\hline $\begin{array}{l}06 \ldots \\
13 \ldots \\
20 \ldots \\
28 \ldots \\
\text { Ju }\end{array}$ & $\begin{array}{l}1000 \\
1045 \\
0745 \\
0650\end{array}$ & $\begin{array}{r}16400 \\
15700 \\
9480 \\
5650\end{array}$ & $\begin{array}{l}534 \\
621 \\
714 \\
721\end{array}$ & $\begin{array}{l}8.1 \\
8.3 \\
8.4 \\
8.0\end{array}$ & $\begin{array}{l}20.5 \\
23.0 \\
21.5 \\
25.5\end{array}$ & $\begin{array}{l}748 \\
738 \\
742 \\
741\end{array}$ & $\begin{array}{l}8.4 \\
8.6 \\
9.1 \\
8.5\end{array}$ & $\begin{array}{l}55 \\
41 \\
51 \\
39\end{array}$ & $\begin{array}{l}2.3 \\
2.2 \\
2.0 \\
1.5\end{array}$ & K2500 \\
\hline $\begin{array}{r}03 \ldots \\
10 \ldots \\
16 \ldots \\
25 \ldots \\
31 \ldots \\
\text { AUG }\end{array}$ & $\begin{array}{l}0800 \\
0800 \\
0900 \\
0745 \\
0830\end{array}$ & $\begin{array}{l}5580 \\
2360 \\
3660 \\
5720 \\
2980\end{array}$ & $\begin{array}{l}672 \\
746 \\
720 \\
607 \\
666\end{array}$ & $\begin{array}{l}8.3 \\
8.3 \\
8.4 \\
8.4 \\
8.3\end{array}$ & $\begin{array}{l}23.5 \\
22.5 \\
24.0 \\
21.0 \\
21.5\end{array}$ & $\begin{array}{l}738 \\
742 \\
744 \\
746 \\
744\end{array}$ & $\begin{array}{r}8.1 \\
7.0 \\
10.2 \\
10.4 \\
8.6\end{array}$ & $\begin{array}{l}39 \\
25 \\
40 \\
44 \\
30\end{array}$ & $\begin{array}{l}1.7 \\
2.5 \\
1.3 \\
3.0 \\
1.8\end{array}$ & $\begin{array}{r}-- \\
-- \\
=- \\
=- \\
\mathbf{K} 420\end{array}$ \\
\hline${ }_{\text {EP }}^{30} \ldots$ & 1030 & 655 & 641 & 8.8 & 26.0 & 746 & 10.8 & 43 & 7.3 & 370 \\
\hline $26 \ldots$ & 1245 & 1350 & 819 & 8.3 & 13.0 & 741 & 12.7 & 23 & -- & 310 \\
\hline
\end{tabular}


ANALYSES OF SAMPLES COLLECTED AT WATER-QUALITY PARTIAI-RECORD STATIONS

05322000 BLUE EARTH RIVER AT MOUTH AT MANKATO--Continued

WATER QUALITY DATA, WATER YEAR OCTOBER 1990 TO SEPTEMBER 1991

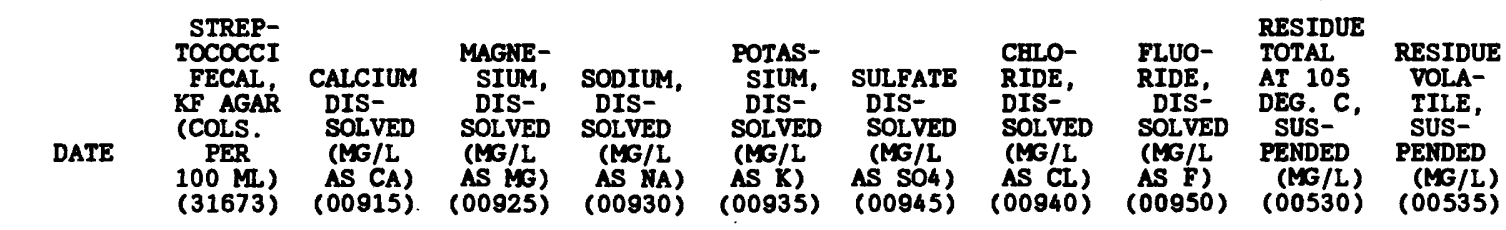

\begin{tabular}{|c|c|c|c|c|c|c|c|c|c|c|}
\hline$\infty_{24}$ & K23 & -- & -- & -- & -- & -- & -- & -- & 29 & 15 \\
\hline $\begin{array}{l}v_{28} \\
28 . .\end{array}$ & K9 & -- & -- & -- & -- & -- & -- & -- & 16 & $<1$ \\
\hline 18... & 20 & -- & -- & -- & -- & -- & -- & -- & 12 & 11 \\
\hline N11... & $>2$ & -- & -- & -- & -- & -- & -- & -. & 6 & 6 \\
\hline $27 \ldots$ & 37 & -- & -- & -- & -- & -- & -- & -- & 6 & 6 \\
\hline $\begin{array}{l}\mathrm{AR} \\
06 \ldots\end{array}$ & 7200 & -- & -- & -- & -- & -- & -- & -- & 3 & 1 \\
\hline $\begin{array}{l}14 \ldots \ldots \\
19 . \ldots\end{array}$ & $=$ & $\begin{array}{l}88 \\
87\end{array}$ & $\begin{array}{l}28 \\
27\end{array}$ & $\begin{array}{l}22 \\
22\end{array}$ & $\begin{array}{l}4.0 \\
3.8\end{array}$ & $\begin{array}{l}80 \\
81\end{array}$ & $\begin{array}{l}37 \\
13\end{array}$ & $\begin{array}{l}0.2 \\
0.3\end{array}$ & $\begin{array}{l}20 \\
16\end{array}$ & $\begin{array}{l}5 \\
9\end{array}$ \\
\hline $\begin{array}{l}20 \ldots \\
21 \ldots\end{array}$ & $\overline{-}$ & $\begin{array}{l}80 \\
76\end{array}$ & $\begin{array}{l}25 \\
24\end{array}$ & $\begin{array}{l}21 \\
19\end{array}$ & $\begin{array}{l}3.7 \\
4.8\end{array}$ & $\begin{array}{l}82 \\
79\end{array}$ & $\begin{array}{l}35 \\
33\end{array}$ & $\begin{array}{l}0.3 \\
0.3\end{array}$ & $\begin{array}{l}21 \\
31\end{array}$ & $\begin{array}{l}9 \\
9\end{array}$ \\
\hline $\begin{array}{l}25 \ldots \ldots \\
26 \ldots\end{array}$ & $=-$ & $\begin{array}{l}79 \\
87\end{array}$ & 23 & 10 & 3.8 & $\begin{array}{l}63 \\
65\end{array}$ & $\begin{array}{l}30 \\
32\end{array}$ & 0.3 & 371 & 46 \\
\hline$\underset{02}{A P R}$ & - & & & & & & & & & \\
\hline${ }_{10}^{22} \ldots$ & $=-$ & $=$ & $=$ & $=-$ & $=-$ & $=-$ & $=-$ & $=$ & 114 & 20 \\
\hline $17 \ldots$ & -- & -- & -- & -- & -- & -- & -- & -- & 243 & 33 \\
\hline $\begin{array}{l}25 \ldots \ldots \\
30 \ldots\end{array}$ & $\begin{array}{r}\text { K52 } \\
--\end{array}$ & $=-$ & $=$ & $=$ & $=$ & $=-$ & $=$ & $=$ & $\begin{array}{l}25 \\
72\end{array}$ & $\begin{array}{l}11 \\
16\end{array}$ \\
\hline $\begin{array}{l}\text { MAY } \\
10\end{array}$ & -- & -- & -- & -- & -- & -- & -- & -- & 221 & 32 \\
\hline $15 \ldots$ & - & - & - & - & - & -- & - & -- & 127 & 21 \\
\hline $\begin{array}{l}24 . \ldots \\
31 \ldots\end{array}$ & 230 & $=$ & $=$ & $=$ & $=-$ & $=$ & $=$ & $=$ & $\begin{array}{l}110 \\
111\end{array}$ & $\begin{array}{r}17 \\
5\end{array}$ \\
\hline JUN & -- & -- & $-\cdots$ & -- & -- & -- & -- & -- & 346 & 5 \\
\hline $13 \ldots$ & $=$ & -- & $=$ & - & - & $=$ & $=$ & $=$ & $\begin{array}{l}340 \\
149\end{array}$ & $\begin{array}{l}32 \\
26\end{array}$ \\
\hline $20 \ldots$ & K4600 & $=-$ & $=$ & -- & -- & $=$ & $=-$ & $=-$ & 248 & $\begin{array}{l}36 \\
22\end{array}$ \\
\hline JUL & -- & -- & -- & -- & -- & -- & -- & -- & & \\
\hline $10 \ldots$ & $=$ & $=$ & $=$ & 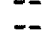 & - & $=-$ & $=-$ & $=$ & 35 & 8 \\
\hline $16 \ldots$ & -- & -- & - & -- & -- & -- & -- & -- & 117 & 21 \\
\hline & $=-$ & -- & -- & -- & -- & -- & -- & -- & 119 & 21 \\
\hline $\begin{array}{l}31 \ldots \\
\text { AUG }\end{array}$ & K260 & -- & - & - & -- & $=$ & -- & -- & & 3 \\
\hline $30 \ldots$ & 1600 & -- & -- & -- & -- & -- & -- & -- & 9 & $<1$ \\
\hline 26. & 1400 & -- & -- & -- & -- & -- & -- & -- & 3 & $<1$ \\
\hline
\end{tabular}


ANALYSES OF SAMPLES COLLECTED AT WATER-QUALITY PARTIAL-RECORD STATIONS

05322000 BLUE EARTE RIVER AT MOUTH AT MANKATO--Continued

WATER QUALITY DATA, WATER YEAR OCTOBER 1990 TO SEPTEEBER 1991

DATE

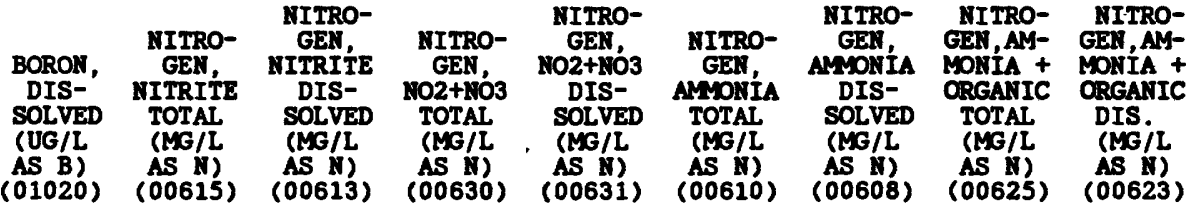

\begin{tabular}{|c|c|c|c|c|c|c|c|c|c|}
\hline $\operatorname{MOVT}_{24} \ldots$ & -- & 0.02 & 0.02 & 0.70 & 0.70 & 0.06 & 0.06 & 1.8 & 1.7 \\
\hline DEC $28 \ldots$ & -- & 0.02 & 0.02 & 2.5 & 2.5 & 0.07 & 0.07 & 1.2 & 0.50 \\
\hline JAN $18 \ldots$ & -- & 0.02 & 0.02 & 3.5 & 3.5 & 0.16 & 0.16 & 0.70 & 0.60 \\
\hline FEB $11 \ldots$ & -- & 0.04 & 0.04 & 3.1 & 3.1 & 0.67 & 0.62 & 1.4 & 0.90 \\
\hline${ }_{\text {MAR }}^{27} \cdots$ & -- & 0.03 & 0.03 & 4.2 & 4.2 & 0.55 & 0.55 & 1.0 & 0.90 \\
\hline $\begin{array}{r}06 \ldots \\
14 \ldots \\
19 \ldots \\
20 \ldots \\
21 \ldots \\
25 \ldots \\
26 \ldots \\
\text { APR }\end{array}$ & $\begin{array}{l}-- \\
60 \\
60 \\
70 \\
60 \\
40 \\
40\end{array}$ & $\begin{array}{l}0.04 \\
0.04 \\
0.04 \\
0.03 \\
0.04 \\
0.10 \\
0.11\end{array}$ & $\begin{array}{l}0.04 \\
0.04 \\
0.04 \\
0.03 \\
0.03 \\
0.08 \\
0.08\end{array}$ & $\begin{array}{r}5.1 \\
6.0 \\
5.0 \\
4.7 \\
4.9 \\
14 \\
17\end{array}$ & $\begin{array}{l}5.1 \\
6.0 \\
5.0 \\
4.3 \\
4.7 \\
14 \\
17\end{array}$ & $\begin{array}{l}0.43 \\
0.20 \\
0.14 \\
0.15 \\
0.15 \\
0.27 \\
0.24\end{array}$ & $\begin{array}{l}0.43 \\
0.20 \\
0.14 \\
0.14 \\
0.15 \\
0.27 \\
0.24\end{array}$ & $\begin{array}{l}1.3 \\
0.70 \\
1.2 \\
1.4 \\
1.9 \\
2.1 \\
1.9\end{array}$ & $\begin{array}{l}1.2 \\
0.70 \\
1.2 \\
1.3 \\
0.90 \\
2.1 \\
1.8\end{array}$ \\
\hline $\begin{array}{r}02 \ldots \\
10 \ldots \\
17 \ldots \\
25 \ldots \\
30 \ldots \\
\text { MAY }\end{array}$ & $\begin{array}{l}-- \\
-- \\
-- \\
--\end{array}$ & $\begin{array}{l}0.08 \\
0.04 \\
0.08 \\
0.04 \\
0.05\end{array}$ & $\begin{array}{l}0.06 \\
0.04 \\
0.06 \\
0.03 \\
0.04\end{array}$ & $\begin{array}{l}17 \\
15 \\
21 \\
20 \\
18\end{array}$ & $\begin{array}{l}16 \\
15 \\
21 \\
20 \\
18\end{array}$ & $\begin{array}{l}0.09 \\
0.02 \\
0.12 \\
0.02 \\
0.02\end{array}$ & $\begin{array}{r}0.08 \\
<0.01 \\
0.12 \\
0.02 \\
<0.01\end{array}$ & $\begin{array}{l}1.2 \\
1.9 \\
1.8 \\
2.0 \\
2.1\end{array}$ & $\begin{array}{l}1.2 \\
1.5 \\
1.4 \\
0.80 \\
1.4\end{array}$ \\
\hline $\begin{array}{r}10 \ldots \\
15 \ldots \\
24 \ldots \\
31 \ldots \\
\text { JUN }\end{array}$ & $\begin{array}{l}-- \\
=- \\
--\end{array}$ & $\begin{array}{l}0.11 \\
0.10 \\
0.10 \\
0.09\end{array}$ & $\begin{array}{l}0.08 \\
0.08 \\
0.06 \\
0.07\end{array}$ & $\begin{array}{l}24 \\
20 \\
19 \\
19\end{array}$ & $\begin{array}{l}24 \\
20 \\
19 \\
19\end{array}$ & $\begin{array}{l}0.06 \\
0.03 \\
0.04 \\
0.02\end{array}$ & $\begin{array}{l}0.06 \\
0.03 \\
0.04 \\
0.02\end{array}$ & $\begin{array}{l}2.3 \\
3.0 \\
1.4 \\
1.6\end{array}$ & $\begin{array}{l}1.6 \\
1.6 \\
1.4 \\
1.6\end{array}$ \\
\hline $\begin{array}{r}06 \ldots \\
13 \ldots \\
20 \ldots \\
28 \ldots \\
\text { JUL }\end{array}$ & $\begin{array}{l}-- \\
-- \\
-- \\
--\end{array}$ & $\begin{array}{l}0.22 \\
0.13 \\
0.09 \\
0.05\end{array}$ & $\begin{array}{l}0.11 \\
0.09 \\
0.05 \\
0.03\end{array}$ & $\begin{array}{l}14 \\
15 \\
17 \\
16\end{array}$ & $\begin{array}{l}14 \\
15 \\
17 \\
16\end{array}$ & $\begin{array}{l}0.14 \\
0.05 \\
0.05 \\
0.03\end{array}$ & $\begin{array}{l}0.10 \\
0.02 \\
0.02 \\
0.03\end{array}$ & $\begin{array}{l}1.9 \\
2.1 \\
1.9 \\
2.0\end{array}$ & $\begin{array}{l}1.1 \\
1.1 \\
1.9 \\
1.4\end{array}$ \\
\hline $\begin{array}{r}03 \ldots \\
10 \ldots \\
16 \ldots \\
25 \ldots \\
31 \ldots \\
\text { AUG }\end{array}$ & $\begin{array}{l}-- \\
-- \\
-- \\
--\end{array}$ & $\begin{array}{l}0.07 \\
0.02 \\
0.04 \\
0.04 \\
0.02\end{array}$ & $\begin{array}{l}0.04 \\
0.02 \\
0.03 \\
0.03 \\
0.02\end{array}$ & $\begin{array}{l}13 \\
13 \\
15 \\
12 \\
11\end{array}$ & $\begin{array}{l}13 \\
13 \\
15 \\
12 \\
11\end{array}$ & $\begin{array}{r}0.03 \\
0.01 \\
<0.01 \\
<0.01 \\
0.01\end{array}$ & $\begin{array}{l}0.02 \\
0.01 \\
0.02 \\
0.01 \\
0.01\end{array}$ & $\begin{array}{l}1.4 \\
1.5 \\
1.4 \\
1.2 \\
1.5\end{array}$ & $\begin{array}{l}1.0 \\
1.1 \\
1.5 \\
1.2 \\
1.1\end{array}$ \\
\hline $\begin{array}{l}\text { 30... } \\
\text { SEP }\end{array}$ & -- & 0.02 & 0.02 & 5.3 & 5.3 & 0.04 & 0.04 & 1.7 & 0.9 \\
\hline $26 \ldots$ & -- & 0.02 & 0.01 & 9.3 & 9.0 & 0.08 & $<0.01$ & 1.2 & 0.6 \\
\hline
\end{tabular}


ANALYSES OF SAMPES COLLECTED AT WATER-QUALITY PARTIAL-RECORD STATIONS

05322000 BLUE EARTH RIVER AT MOUTH AT MANKATO--Continued

WATER QUALITY DATA, WATER YEAR OCTOBER 1990 TO SEPTEMBER 1991
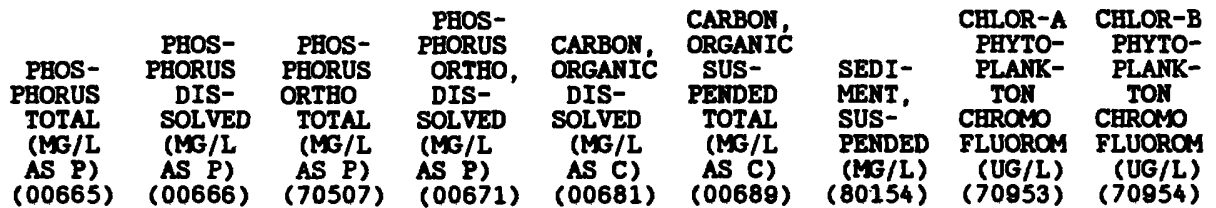

${ }_{24}$

NOV

DEC

18.

0.10

0.10

0.02

0.02

$6.4>5.0$

65

41.0

$<1.10$

JAN 11 .

0.06

0.04

0.03

$<0.01$

$4.4 \quad 1.5$

62

17.0

$<0.60$

27...

0.13

0.04

0.04

0.03

5.4

1.0

41

5.80

$<0.70$

0.13

0.05

0.06

0.01

4.8

$0.3^{\circ}$

73

1.10

$<0.30$

MAR

0.23

0.16

0.17

0.13

6.7

0.6

--

$<0.20$

$<0.20$

$06 \ldots$

0.18

0.12

0.12
0.13
0.08

0.13

0.11
$<0.06$

19.

0.13

0.11

0.08

6.8
6.2
5.6

0.11

0.13
0.09

0.09
0.14

0.13

0.16

0.10

0.09
0.10

6.6

0.1

$21 \ldots$

APR

0.31
0.42

0.15

0.16

0.10

6.9
8.2
8.0

0.1

1.6
$>5.0$

156
98
36

1. 50

4.10

2.40

11.0

112

831
554

3.00
4.30

$<0.20$

$<0.50$

$<0.40$

0.40

0.70

02 ...

0.28

0.10

0.14

0.09

0.02

\section{6.}

2.

0.07

0.06

0.12
0.06

0.07

0.04
0.03

6.9

6.4

2.1

4.0
$>5.0$

30 .

$\begin{array}{lll}0.12 & 0.04 & 0.06 \\ 0.15 & 0.05 & 0.05\end{array}$

0.03

6.1

1.5

305

7.70

18.0

6.10

$\begin{array}{lll}188 & 3.00 & <0.30\end{array}$

0.40
0.40

MAY.

0.15

0.11

0.09

0.15

$15 \ldots$

0.19
0.20

0.11

8.1

0.12

0.11
0.07
0.07

0.07

3.2
2.8

JUN 31 .

$\begin{array}{lll}0.29 & 0.25 & 0.12\end{array}$

0.10

7.3

0.20
0.15

0.14

0.34

$13 \ldots$

$20 \ldots$

JUL

0.18

0.21

0.34
0.18

0.20

0.15

0.12

7.6
6.9
6.5
6.1

1.8
1.6

2.00

2.00
6.30

$<.60$
5.10

0.60

0.80

$<0.50$

JUL

0.31

0.14
0.09

0.09
0.13

0.21

0.12
0.09

0.09
0.12

0.19

$>4$.

$>4.9$

4.9
3.5
1.9
3.0

2.70
6.20

2.90
4.00

0.40

$<1.20$

390
253

267

608

328
645
176

4.00

149

7.70

2.7

$16 . .$.

$31 \ldots$

0.44

0.120 .09

0.09

6.3

2.7
2.1
2.9

2.9
4.4

98
169
337

7.70
6.10
6.90

6.90

11.0

$<1.50$

$<1.20$

SEP..

0.40

0.01

$<0.01$

$<0.01$

7.0

$>5.0$

70

14.0

$<1.10$

$<1.30$

$<0.60$
$<0.70$

0.13

0.09

0.09

7.4

2.5

154

7.80

0.90

0.60

1.30

$<0.80$

$<0.10$

$<0.10$ 
ANALYSES OF SAMPES COLLECTED AT WATER-QUALITY PARTIAL-RECORD STATIONS

05325050 MINNESOTA RIVER AT US BWY 14 BRIDGE AT MANKATO

WATER QUALITY DATA, WATER YEAR OCTOBER 1990 TO SEPTEMBER 1991

\begin{tabular}{|c|c|c|c|c|c|c|c|c|c|c|}
\hline DATE & TIME & $\begin{array}{c}\text { DIS- } \\
\text { CEARGE, } \\
\text { INST. } \\
\text { CUBIC } \\
\text { FEET } \\
\text { PER } \\
\text { SECOND } \\
(00061)\end{array}$ & $\begin{array}{l}\text { SPE- } \\
\text { CIFIC } \\
\text { CON- } \\
\text { DUCT- } \\
\text { ANCE } \\
\text { (US/CM) } \\
(00095)\end{array}$ & $\begin{array}{c}\text { PH } \\
\text { (STAND- } \\
\text { ARD } \\
\text { UNITS) } \\
(00400)\end{array}$ & $\begin{array}{l}\text { TEMPER- } \\
\text { ATURE } \\
\text { WATER } \\
\text { (DEG C) } \\
(00010)\end{array}$ & $\begin{array}{c}\text { BARO- } \\
\text { METRIC } \\
\text { PRES- } \\
\text { SURE } \\
\text { (MM } \\
\text { OF } \\
\text { HG) } \\
(00025)\end{array}$ & $\begin{array}{c}\text { OXYGEN, } \\
\text { DIS- } \\
\text { SOLVED } \\
(\mathrm{MG} / \mathrm{L}) \\
(00300)\end{array}$ & $\begin{array}{l}\text { OXYGEN } \\
\text { DEMAND, } \\
\text { CHEM- } \\
\text { ICAL } \\
\text { (HIGH } \\
\text { LEVEL) } \\
\text { (MG/L) } \\
(00340)\end{array}$ & $\begin{array}{l}\text { OXYGEN } \\
\text { DEMAND, } \\
\text { BIO- } \\
\text { CHIMY- } \\
\text { ICAL, } \\
5 \text { DAY } \\
(M G / L) \\
(00310)\end{array}$ & $\begin{array}{l}\text { RESIDU } \\
\text { TOTAL } \\
\text { AT } 105 \\
\text { DEG. C } \\
\text { SUS- } \\
\text { PENDED } \\
\text { (MG /L } \\
\text { (00530 }\end{array}$ \\
\hline \multicolumn{11}{|l|}{ APR } \\
\hline $\begin{array}{r}17 \ldots \\
25 \ldots \\
30 \ldots \\
M A Y\end{array}$ & $\begin{array}{l}1750 \\
1500 \\
1445\end{array}$ & $\begin{array}{r}15100 \\
7760 \\
7940\end{array}$ & $\begin{array}{l}776 \\
896 \\
844\end{array}$ & $\begin{array}{l}8.3 \\
8.4 \\
8.5\end{array}$ & $\begin{array}{l}10.0 \\
13.0 \\
12.5\end{array}$ & $\begin{array}{l}743 \\
738 \\
734\end{array}$ & $\begin{array}{l}11.0 \\
10.7\end{array}$ & $\begin{array}{l}50 \\
36 \\
38\end{array}$ & $\begin{array}{l}2.2 \\
1.1 \\
0.9\end{array}$ & $\begin{array}{r}236 \\
58 \\
98\end{array}$ \\
\hline $\begin{array}{r}10 \ldots \\
15 \ldots \\
24 \ldots \\
31 \ldots \\
\text { JuN }\end{array}$ & $\begin{array}{l}1445 \\
1425 \\
1345 \\
1245\end{array}$ & $\begin{array}{l}20000 \\
11500 \\
12100 \\
14700\end{array}$ & $\begin{array}{l}690 \\
746 \\
766 \\
764\end{array}$ & $\begin{array}{l}8.3 \\
8.4 \\
8.4 \\
8.3\end{array}$ & $\begin{array}{l}14.0 \\
20.0 \\
20.0 \\
22.0\end{array}$ & $\begin{array}{l}743 \\
739 \\
740 \\
735\end{array}$ & $\begin{array}{r}10.8 \\
9.6 \\
9.3 \\
8.7\end{array}$ & $\begin{array}{l}39 \\
35 \\
34 \\
59\end{array}$ & $\begin{array}{l}1.6 \\
2.1 \\
1.8 \\
1.8\end{array}$ & $\begin{array}{l}332 \\
100 \\
102 \\
266\end{array}$ \\
\hline $\begin{array}{l}06 \ldots \\
13 \ldots \\
20 \ldots \\
28 \ldots \\
\text { JUL }\end{array}$ & $\begin{array}{l}1515 \\
1535 \\
1300 \\
1215\end{array}$ & $\begin{array}{l}27700 \\
26000 \\
22200 \\
23900\end{array}$ & $\begin{array}{l}621 \\
738 \\
784 \\
743\end{array}$ & $\begin{array}{l}8.1 \\
8.4 \\
8.2 \\
8.1\end{array}$ & $\begin{array}{l}20.0 \\
23.5 \\
23.0 \\
25.5\end{array}$ & $\begin{array}{l}748 \\
735 \\
741 \\
739\end{array}$ & $\begin{array}{l}7.8 \\
7.7 \\
8.4 \\
7.4\end{array}$ & $\begin{array}{l}43 \\
41 \\
53 \\
33\end{array}$ & $\begin{array}{l}1.8 \\
2.2 \\
1.8 \\
1.8\end{array}$ & $\begin{array}{r}228 \\
103 \\
253 \\
57\end{array}$ \\
\hline $\begin{array}{r}03 \ldots \\
10 \ldots \\
16 \ldots \\
25 \ldots \\
31 \ldots \\
\text { AUG }\end{array}$ & $\begin{array}{l}1315 \\
1415 \\
1545 \\
1345 \\
1310\end{array}$ & $\begin{array}{r}20700 \\
10400 \\
14400 \\
11000 \\
8530\end{array}$ & $\begin{array}{l}754 \\
872 \\
720 \\
749 \\
743\end{array}$ & $\begin{array}{l}8.3 \\
8.3 \\
8.3 \\
8.3 \\
8.2\end{array}$ & $\begin{array}{l}24.0 \\
25.0 \\
26.0 \\
23.0 \\
23.5\end{array}$ & $\begin{array}{l}738 \\
742 \\
744 \\
748 \\
745\end{array}$ & $\begin{array}{l}6.9 \\
7.5 \\
9.2 \\
9.6 \\
7.6\end{array}$ & $\begin{array}{l}37 \\
39 \\
34 \\
46 \\
36\end{array}$ & $\begin{array}{l}2.5 \\
2.3 \\
2.2 \\
3.4 \\
2.5\end{array}$ & $\begin{array}{r}52 \\
35 \\
88 \\
125 \\
80\end{array}$ \\
\hline $22 \ldots$ & 1300 & 7410 & 811 & 8.4 & 24.0 & 746 & 8.9 & 120 & 4.0 & 40 \\
\hline
\end{tabular}

\begin{tabular}{|c|c|c|c|c|c|c|c|c|c|}
\hline DATE & $\begin{array}{c}\text { RESIDUE } \\
\text { VOLA- } \\
\text { TILE, } \\
\text { SUS- } \\
\text { PENDED } \\
\text { (MG/L) } \\
\text { (O0535) }\end{array}$ & $\begin{array}{c}\text { MITRO- } \\
\text { GEN, } \\
\text { NITRITE } \\
\text { TOTAI } \\
\text { (MG/L } \\
\text { AS N) } \\
\text { (00615) }\end{array}$ & $\begin{array}{c}\text { NITRO- } \\
\text { GEN, } \\
\text { NITRITE } \\
\text { DIS- } \\
\text { SOLVED } \\
\text { (MG/L } \\
\text { AS N) } \\
(00613)\end{array}$ & $\begin{array}{c}\text { NITRO- } \\
\text { GEN, } \\
\text { NO2+NO3 } \\
\text { TOTAL } \\
\text { (MG/L } \\
\text { AS N) } \\
(00630)\end{array}$ & $\begin{array}{c}\text { NITRO- } \\
\text { GEN, } \\
\text { NO2+NO3 } \\
\text { DIS- } \\
\text { SOLVED } \\
\text { (MG/L } \\
\text { AS N) } \\
(00631)\end{array}$ & $\begin{array}{c}\text { NITRO- } \\
\text { GEN, } \\
\text { AMONIA } \\
\text { TOTAI } \\
\text { (MG/L } \\
\text { AS IN) } \\
(00610)\end{array}$ & $\begin{array}{c}\text { NITRO- } \\
\text { GEN, } \\
\text { AMONIA } \\
\text { DIS- } \\
\text { SOLVED } \\
\text { (MG/L } \\
\text { AS N) } \\
\text { (00608) }\end{array}$ & $\begin{array}{l}\text { NITRO- } \\
\text { GEN,AM- } \\
\text { MONIA + } \\
\text { ORGANIC } \\
\text { TOTAL } \\
(\text { MG/L } \\
\text { AS N) } \\
(00625)\end{array}$ & $\begin{array}{l}\text { NITRO- } \\
\text { GEN,AM- } \\
\text { MONIA + } \\
\text { ORGANIC } \\
\text { DIS. } \\
\text { (MG/I } \\
\text { AS N) } \\
(00623)\end{array}$ \\
\hline \multicolumn{10}{|l|}{ APR } \\
\hline $\begin{array}{r}17 \ldots \\
25 \ldots \\
30 \ldots \\
\text { MAY }\end{array}$ & $\begin{array}{l}29 \\
12 \\
20\end{array}$ & $\begin{array}{l}0.08 \\
0.04 \\
0.05\end{array}$ & $\begin{array}{l}0.06 \\
0.03 \\
0.04\end{array}$ & $\begin{array}{l}20 \\
15 \\
14\end{array}$ & $\begin{array}{l}19 \\
14 \\
13\end{array}$ & $\begin{array}{l}0.10 \\
0.02 \\
0.14\end{array}$ & $\begin{array}{r}0.10 \\
<0.01 \\
0.04\end{array}$ & $\begin{array}{l}1.7 \\
1.5 \\
1.6\end{array}$ & $\begin{array}{l}0.90 \\
1.1 \\
1.2\end{array}$ \\
\hline $\begin{array}{r}10 \ldots \\
15 \ldots \\
24 \ldots \\
31 \ldots\end{array}$ & $\begin{array}{l}28 \\
18 \\
19 \\
48\end{array}$ & $\begin{array}{l}0.08 \\
0.08 \\
0.10 \\
0.17\end{array}$ & $\begin{array}{l}0.07 \\
0.07 \\
0.07 \\
0.10\end{array}$ & $\begin{array}{l}20 \\
16 \\
16 \\
16\end{array}$ & $\begin{array}{l}20 \\
16 \\
16 \\
16\end{array}$ & $\begin{array}{l}0.04 \\
0.02 \\
0.03 \\
0.05\end{array}$ & $\begin{array}{l}0.04 \\
0.02 \\
0.03 \\
0.05\end{array}$ & $\begin{array}{l}2.8 \\
1.7 \\
1.5 \\
2.2\end{array}$ & $\begin{array}{l}2.0 \\
1.4 \\
1.5 \\
1.4\end{array}$ \\
\hline $\begin{array}{l}06 \ldots \\
13 \ldots \\
20 \ldots \\
28 \ldots \\
\text { JUL }\end{array}$ & $\begin{array}{r}38 \\
19 \\
38 \\
9\end{array}$ & $\begin{array}{l}0.20 \\
0.16 \\
0.13 \\
0.09\end{array}$ & $\begin{array}{l}0.15 \\
0.14 \\
0.07 \\
0.06\end{array}$ & $\begin{array}{c}12 \\
10 \\
10 \\
8.9\end{array}$ & $\begin{array}{l}12 \\
10 \\
10 \\
9.1\end{array}$ & $\begin{array}{l}0.12 \\
0.03 \\
0.08 \\
0.03\end{array}$ & $\begin{array}{l}0.08 \\
0.03 \\
0.04 \\
0.03\end{array}$ & $\begin{array}{l}1.7 \\
1.7 \\
2.1 \\
1.5\end{array}$ & $\begin{array}{l}1.2 \\
1.2 \\
1.4 \\
1.1\end{array}$ \\
\hline $\begin{array}{l}03 \ldots \\
10 \ldots \\
16 \ldots \\
25 \ldots \\
31 \ldots \\
\text { QUG }\end{array}$ & $\begin{array}{r}28 \\
9 \\
18 \\
24 \\
13\end{array}$ & $\begin{array}{l}0.09 \\
0.03 \\
0.07 \\
0.04 \\
0.04\end{array}$ & $\begin{array}{l}0.06 \\
0.02 \\
0.04 \\
0.03 \\
0.04\end{array}$ & $\begin{array}{l}7.6 \\
6.9 \\
7.0 \\
8.0 \\
7.7\end{array}$ & $\begin{array}{l}7.6 \\
6.8 \\
6.9 \\
8.0 \\
7.7\end{array}$ & $\begin{array}{l}0.04 \\
0.01 \\
0.06 \\
0.01 \\
0.02\end{array}$ & $\begin{array}{r}0.04 \\
0.05 \\
0.05 \\
<0.02 \\
<0.01\end{array}$ & $\begin{array}{l}1.1 \\
1.3 \\
1.2 \\
1.8 \\
1.7\end{array}$ & $\begin{array}{l}1.1 \\
0.90 \\
1.3 \\
1.3 \\
1.2\end{array}$ \\
\hline 22. & 21 & 0.03 & 0.02 & 4.6 & 4.6 & 0.01 & $<0.01$ & 1.4 & 1.3 \\
\hline
\end{tabular}


ANALYSES OF SAMPLES COLLECTED AT WATER-QUALITY PARTIAL-RECORD STATIONS

05325050 MIMNESOTA RIVER AT US BWY 14 BRIDGE AT MANKATO--Continued

WATER QUALITY DATA, WATER YEAR OCTOBER 1990 TO SEPTEMBER 1991

\begin{tabular}{|c|c|c|c|c|c|c|c|c|c|}
\hline DATE & $\begin{array}{c}\text { PEOS- } \\
\text { PEORUS } \\
\text { TOTAL } \\
\text { (MG/L } \\
\text { AS P) } \\
(00665)\end{array}$ & $\begin{array}{c}\text { PHOS- } \\
\text { PBORUS } \\
\text { DIS- } \\
\text { SOLVED } \\
\text { (MG/L } \\
\text { AS P) } \\
(00666)\end{array}$ & $\begin{array}{c}\text { PEOS- } \\
\text { PEORUS } \\
\text { ORTHO } \\
\text { TOTAL } \\
\text { (MG/L } \\
\text { AS P) } \\
(70507)\end{array}$ & $\begin{array}{l}\text { PHOS- } \\
\text { PEORUS } \\
\text { ORTHO, } \\
\text { DIS- } \\
\text { SOLVED } \\
\text { (MG/L } \\
\text { AS P) } \\
(00671)\end{array}$ & $\begin{array}{l}\text { CARBOH, } \\
\text { ORGANIC } \\
\text { DIS- } \\
\text { SOLVED } \\
\text { (MG/L } \\
\text { AS C) } \\
(00681)\end{array}$ & $\begin{array}{c}\text { CARBOH, } \\
\text { ORGANIC } \\
\text { SUS- } \\
\text { PENDED } \\
\text { TOTAI } \\
\text { (MG/L } \\
\text { AS C) } \\
(00689)\end{array}$ & $\begin{array}{l}\text { SEDI- } \\
\text { MIENIT, } \\
\text { SUS- } \\
\text { PENDED } \\
(\text { MG/L) } \\
(80154)\end{array}$ & $\begin{array}{c}\text { CELOR-A } \\
\text { PHYTO- } \\
\text { PLANK- } \\
\text { TOL } \\
\text { CEROYO } \\
\text { FLUOROM } \\
\text { (UG/L) } \\
\text { (70953) }\end{array}$ & $\begin{array}{l}\text { CELOR-B } \\
\text { PHYTO- } \\
\text { PLANK- } \\
\text { TOR } \\
\text { CEROMO } \\
\text { FLUOROM } \\
\text { (UG/L) } \\
\text { (70954) }\end{array}$ \\
\hline $\begin{array}{r}\text { APR } \\
17 \ldots \\
25 \ldots \\
30 \ldots \\
\text { MY }^{2} \ldots\end{array}$ & $\begin{array}{l}0.23 \\
0.12 \\
0.18\end{array}$ & $\begin{array}{l}0.07 \\
0.03 \\
0.05\end{array}$ & $\begin{array}{l}0.12 \\
0.04 \\
0.05\end{array}$ & $\begin{array}{l}0.06 \\
0.03 \\
0.02\end{array}$ & $\begin{array}{l}7.3 \\
6.6 \\
6.4\end{array}$ & $\begin{array}{l}4.5 \\
1.8 \\
3.2\end{array}$ & $\begin{array}{l}594 \\
172 \\
328\end{array}$ & $\begin{array}{r}4.50 \\
6.70 \\
19.0\end{array}$ & $\begin{array}{r}<0.50 \\
0.50 \\
0.80\end{array}$ \\
\hline $\begin{array}{r}10 \ldots \\
15 \ldots \\
24 \ldots \\
31 \ldots \\
\text { Jun }\end{array}$ & $\begin{array}{l}0.18 \\
0.13 \\
0.18 \\
0.36\end{array}$ & $\begin{array}{l}0.09 \\
0.06 \\
0.07 \\
0.12\end{array}$ & $\begin{array}{l}0.12 \\
0.07 \\
0.10 \\
0.18\end{array}$ & $\begin{array}{l}0.09 \\
0.04 \\
0.07 \\
0.11\end{array}$ & $\begin{array}{r}11 \\
7.3 \\
6.9 \\
7.1\end{array}$ & $\begin{array}{l}3.2 \\
2.7 \\
1.9 \\
4.5\end{array}$ & $\begin{array}{l}482 \\
332 \\
232 \\
473\end{array}$ & $\begin{array}{l}1.00 \\
8.90 \\
2.60 \\
6.00\end{array}$ & $\begin{array}{r}<1.20 \\
1.10 \\
<1.50 \\
<1.20\end{array}$ \\
\hline $\begin{array}{r}06 \ldots \\
13 \ldots \\
20 \ldots \\
28 \ldots\end{array}$ & $\begin{array}{l}0.36 \\
0.17 \\
0.24 \\
0.31\end{array}$ & $\begin{array}{l}0.18 \\
0.15 \\
0.17 \\
0.18\end{array}$ & $\begin{array}{l}0.27 \\
0.17 \\
0.19 \\
0.19\end{array}$ & $\begin{array}{l}0.18 \\
0.14 \\
0.15 \\
0.15\end{array}$ & $\begin{array}{l}7.6 \\
7.2 \\
6.8 \\
7.2\end{array}$ & $\begin{array}{r}>4.9 \\
2.8 \\
>5.0 \\
2.0\end{array}$ & $\begin{array}{l}512 \\
297 \\
415 \\
126\end{array}$ & $\begin{array}{l}1.70 \\
7.20 \\
3.30 \\
1.80\end{array}$ & $\begin{array}{r}<1.10 \\
1.30 \\
<0.60 \\
0.80\end{array}$ \\
\hline $\begin{array}{r}03 \ldots \\
10 \ldots \\
16 \ldots \\
25 \ldots \\
31 \ldots \\
\text { AUG }\end{array}$ & $\begin{array}{l}0.33 \\
0.18 \\
0.29 \\
0.42 \\
0.31\end{array}$ & $\begin{array}{l}0.18 \\
0.10 \\
0.16 \\
0.17 \\
0.12\end{array}$ & $\begin{array}{l}0.21 \\
0.14 \\
0.20 \\
0.17 \\
0.11\end{array}$ & $\begin{array}{l}0.16 \\
0.14 \\
0.16 \\
0.14 \\
0.09\end{array}$ & $\begin{array}{l}8.1 \\
8.0 \\
7.7 \\
8.1 \\
7.2\end{array}$ & $\begin{array}{l}1.5 \\
2.5 \\
2.3 \\
4.4 \\
3.4\end{array}$ & $\begin{array}{l}152 \\
188 \\
166 \\
244 \\
168\end{array}$ & $\begin{array}{r}3.10 \\
0.80 \\
7.50 \\
17.0 \\
14.0\end{array}$ & $\begin{array}{r}<0.90 \\
0.10 \\
0.80 \\
2.10 \\
1.10\end{array}$ \\
\hline $22 \ldots$ & 0.22 & 0.08 & 0.07 & 0.06 & 8.0 & 3.0 & 154 & 13.0 & $<0.10$ \\
\hline
\end{tabular}


ANALYSES OF SAMPLES COLLECTED AT WATER-QUALITY PARTIAL-RECORD STATIONS

05325200 MIMNESOTA RIVER AT ST. PETER

WATER QUALITY DATA, WATER YEAR OCTOBER 1990 TO SEPTEMBER 1991

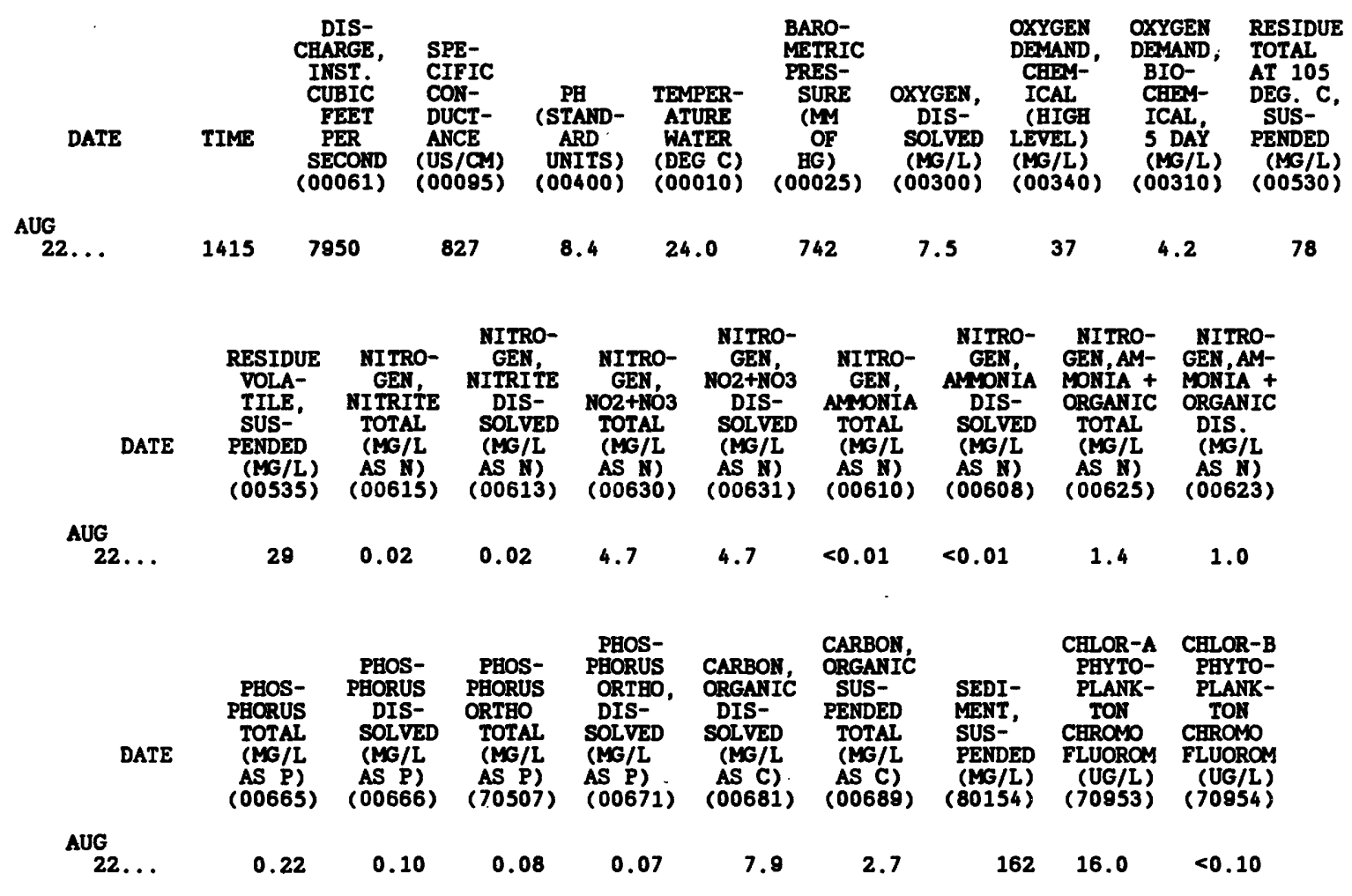


05326400 RUSA RIVER NEAR HENDERSON, MN

WATER QUALITY DATA, WATER YEAR OCTOBER 1990 TO SEPTEMBER 1991

\begin{tabular}{|c|c|c|c|c|c|c|c|c|c|c|c|c|c|c|c|c|c|}
\hline \multirow[b]{2}{*}{$\stackrel{\text { MAY }}{30 \ldots}$} & \multicolumn{2}{|c|}{$\begin{array}{c}\text { DIS- } \\
\text { CRARGE, } \\
\text { INST. } \\
\text { CUBIC } \\
\text { FEET } \\
\text { PER } \\
\text { SECOND } \\
\text { (00061) }\end{array}$} & \multicolumn{2}{|c|}{$\begin{array}{l}\text { SPE- } \\
\text { CIFIC } \\
\text { CON- } \\
\text { DUCT- } \\
\text { ANCE } \\
\text { (US/CM) } \\
(00095)\end{array}$} & \multicolumn{6}{|c|}{$\begin{array}{l}\text { BARO- } \\
\text { METRIC } \\
\text { PRES- } \\
\text { SURE } \\
\text { (MM } \\
\text { OF } \\
\text { HG) } \\
(00025)\end{array}$} & \multicolumn{2}{|c|}{$\begin{array}{c}\text { OXYGEN, } \\
\text { DIS- } \\
\text { SOLVED } \\
(\text { MG/L) } \\
(00300)\end{array}$} & \multicolumn{2}{|c|}{$\begin{array}{c}\text { OXYGEN } \\
\text { DEMAND, } \\
\text { CHEM- } \\
\text { ICAL } \\
\text { (BIGH } \\
\text { LEVEL) } \\
\text { (MG/L) } \\
(00340)\end{array}$} & \multicolumn{2}{|c|}{$\begin{array}{l}\text { OXYGEN } \\
\text { DDMAND, } \\
\text { BIO- } \\
\text { CEIMY- } \\
\text { ICAL, } \\
5 \mathrm{DAY} \\
(\mathrm{MG} / \mathrm{L}) \\
(00310)\end{array}$} & $\begin{array}{l}\text { RESIDUE } \\
\text { TOTAL } \\
\text { AT 105 } \\
\text { DEG. C, } \\
\text { SUS- } \\
\text { PENDED } \\
\text { (MG/L) } \\
\text { (O0530) }\end{array}$ \\
\hline & 1030 & 1820 & \multicolumn{2}{|c|}{504} & \multicolumn{4}{|c|}{19.0} & \multicolumn{2}{|c|}{734} & \multicolumn{2}{|c|}{7.0} & \multicolumn{4}{|c|}{3.5} & 436 \\
\hline DATE & $\begin{array}{l}\text { RESIDUE } \\
\text { VOLA- } \\
\text { TILE, } \\
\text { SUS- } \\
\text { PENDED } \\
(\text { MG /L) } \\
(00535)\end{array}$ & $\begin{array}{l}\text { NIT } \\
\text { GE } \\
\text { NITI } \\
\text { TOI } \\
\text { (ME } \\
\text { AS } \\
\text { (OOG }\end{array}$ & \multicolumn{3}{|c|}{$\begin{array}{lc}\text { NITRO- } \\
\text { GEN, } \\
\text { NITRITE } \\
\text { DIS- } \\
\text { SOLVBD } \\
\text { (MG/L } \\
\text { AS N) } \\
\text { 5) }(00613)\end{array}$} & \multicolumn{4}{|c|}{$\begin{array}{c}\text { NITRO- } \\
\text { GEN, } \\
\text { NO2+NO3 } \\
\text { DIS- } \\
\text { SOLVED } \\
\text { (MG/L } \\
\text { AS N) } \\
(00631)\end{array}$} & \multicolumn{4}{|c|}{$\begin{array}{c}\text { NITRO- } \\
\text { GEN, } \\
\text { AMONIA } \\
\text { DIS- } \\
\text { SOLVID } \\
(M G / L \\
\text { AS N) } \\
(00608)\end{array}$} & \multicolumn{2}{|c|}{$\begin{array}{l}\text { NITRO- } \\
\text { GEN,AM- } \\
\text { MONIA + } \\
\text { ORGANIC } \\
\text { TOTAL } \\
\text { (MG/I } \\
\text { AS N) } \\
(00625)\end{array}$} & \multicolumn{2}{|c|}{$\begin{array}{l}\text { NITRO- } \\
\text { GEN,AM- } \\
\text { MONIA + } \\
\text { ORGANIC } \\
\text { DIS. } \\
(M G / L \\
\text { AS N) } \\
(00623)\end{array}$} \\
\hline $\begin{array}{l}\text { MAY } \\
\quad 30 \ldots\end{array}$ & 58 & \multicolumn{2}{|c|}{0.30} & \multicolumn{2}{|c|}{0.19} & \multicolumn{2}{|c|}{20} & \multicolumn{2}{|c|}{19} & \multicolumn{4}{|c|}{0.21} & \multicolumn{2}{|c|}{2.1} & \multicolumn{2}{|c|}{2.1} \\
\hline DATE & $\begin{array}{c}\text { PEOS- } \\
\text { PBORUS } \\
\text { TOTAL } \\
\text { (MG/L } \\
\text { AS P) } \\
(00665)\end{array}$ & $\begin{array}{l}\text { PHC } \\
\text { PEOI } \\
\text { DI } \\
\text { SOI } \\
\text { (MC } \\
\text { AS } \\
\text { (OOG }\end{array}$ & $\begin{array}{l}\text { S- } \\
\text { JS } \\
\text { - } \\
\text { JED } \\
\text { (L } \\
\text { ?) } \\
\text { (6) }\end{array}$ & $\begin{array}{l}\text { PHO } \\
\text { PHOR } \\
\text { ORTE } \\
\text { TOT } \\
\text { (ME } \\
\text { AS } \\
6705\end{array}$ & $\begin{array}{l}\text { S- } \\
\text { US } \\
0 \\
\text { AL } \\
\text { /L } \\
\text { P) } \\
07 \text {, }\end{array}$ & $\begin{array}{l}\text { PEO } \\
\text { PEOR } \\
\text { ORT } \\
\text { DIS } \\
\text { SOLV } \\
\text { (MG/ } \\
\text { AS P } \\
\text { (O06 }\end{array}$ & 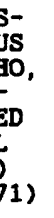 & $\begin{array}{l}\text { CARB } \\
\text { ORGA } \\
\text { DIS } \\
\text { SOLV } \\
\text { (MG } \\
\text { AS } \\
\text { COO6 }\end{array}$ & $\begin{array}{l}\text { ON, } \\
\text { IIC } \\
\text { OD } \\
\text { (L } \\
\text { () } \\
\text { II) }\end{array}$ & $\begin{array}{l}\text { CARI } \\
\text { ORG! } \\
\text { SUS } \\
\text { PENI } \\
\text { TO! } \\
\text { (ME } \\
\text { AS } \\
\text { COOE }\end{array}$ & $\begin{array}{l}\text { ON } \\
\text { NIC } \\
\text { ED } \\
\text { AL } \\
(12 \\
C) \\
89)\end{array}$ & $\begin{array}{l}\text { SED } \\
\text { MEN } \\
\text { SUS } \\
\text { PEN } \\
\text { (MG } \\
\text { (801 }\end{array}$ & $\begin{array}{l}I- \\
T, \\
\text { DED } \\
(L) \\
54)\end{array}$ & $\begin{array}{l}\text { CHLC } \\
\text { PHY } \\
\text { PLA } \\
\text { TC } \\
\text { CRRC } \\
\text { FLUC } \\
\text { (UC } \\
\text { C70S }\end{array}$ & $\begin{array}{l}\mathrm{R}-\mathrm{A} \\
\mathrm{TO}- \\
\mathrm{NK}- \\
\mathrm{N} \\
\mathrm{YO} \\
\mathrm{ROM} \\
\text { (1) } \\
53 \text { ) }\end{array}$ & $\begin{array}{r}\text { CHLO } \\
\text { PHY } \\
\text { PLA } \\
\text { TO } \\
\text { CHRO } \\
\text { FLUO } \\
\text { (UG } \\
\text { (709 }\end{array}$ & $\begin{array}{l}R-B \\
T O- \\
N K- \\
N \\
M O \\
R O M \\
(L) \\
154)\end{array}$ \\
\hline$\frac{\text { MAY }}{\mathbf{3 0}}$ & 0.55 & & & & 36 & & & 10 & & & & 10 & 70 & & 10 & $<1$. & 20 \\
\hline
\end{tabular}


AHALYSES OF SAMPLES COLLECTED AT WATER-QuALITY PARTIAL-RECORD STATIONS

05326450 MIMNESOTA RIVER AT HENDERSOA

WATER QUALITY DATA, WATER YEAR OCTOBER 1990 TO SEPTPMBER 1991

\begin{tabular}{|c|c|c|c|c|c|c|c|c|c|c|c|c|c|c|c|c|c|c|}
\hline DATE & TIME & \multicolumn{2}{|c|}{$\begin{array}{c}\text { DIS- } \\
\text { CHARGE, } \\
\text { INST. } \\
\text { CUBIC } \\
\text { FEET } \\
\text { PER } \\
\text { SECOND } \\
\text { (00061) }\end{array}$} & \multicolumn{2}{|c|}{$\begin{array}{l}\text { SPE- } \\
\text { CIFIC } \\
\text { CON- } \\
\text { DUCT- } \\
\text { ANCE } \\
\text { (US/GM) } \\
(00095)\end{array}$} & $\begin{array}{r}\text { P } \\
\mathbf{S T} \\
\text { UN } \\
\mathbf{W N}\end{array}$ & $\begin{array}{l}\text { AND- } \\
\text { AND } \\
\text { ITS ) } \\
400 \text { ) }\end{array}$ & $\begin{array}{l}\text { TEM } \\
\text { ATI } \\
\text { WA } \\
\text { CDE } \\
\text { COO }\end{array}$ & $\begin{array}{l}\text { PER- } \\
\text { JRE } \\
\text { TER } \\
\text { (C) } \\
\text { O10) }\end{array}$ & \multicolumn{2}{|c|}{$\begin{array}{l}\text { BARO- } \\
\text { METRIC } \\
\text { PRES- } \\
\text { SURE } \\
\text { (MM } \\
\text { OF } \\
\text { HG) } \\
(00025)\end{array}$} & $\begin{array}{r}\text { OXY } \\
\text { D } \\
\text { SOI } \\
(M X \\
C 00\end{array}$ & $\begin{array}{l}\text { GEN, } \\
\text { IS- } \\
\text { LVED } \\
\text { G/L) } \\
300 \text { ) }\end{array}$ & \multicolumn{2}{|c|}{$\begin{array}{l}\text { OXYGEN } \\
\text { DEMAND, } \\
\text { CEMM- } \\
\text { ICAL } \\
\text { (BIGA } \\
\text { LEVEL) } \\
(\text { MG/L) } \\
(00340)\end{array}$} & \multicolumn{2}{|c|}{$\begin{array}{l}\text { OXYGER } \\
\text { DEMAND, } \\
\text { BIO- } \\
\text { CHIIM- } \\
\text { ICAL } \\
5 \text { DAY } \\
\text { (MG/L) } \\
(00310)\end{array}$} & $\begin{array}{l}\text { RESIDUE } \\
\text { TOTAL } \\
\text { AT 105 } \\
\text { DEG. C, } \\
\text { SUS- } \\
\text { PENDED } \\
\text { (MG/L) } \\
(00530)\end{array}$ \\
\hline UG & 1000 & \multicolumn{2}{|c|}{7940} & \multicolumn{2}{|c|}{874} & \multicolumn{2}{|c|}{8.4} & \multicolumn{2}{|c|}{23.5} & \multicolumn{2}{|c|}{747} & \multicolumn{2}{|c|}{7.8} & \multicolumn{2}{|c|}{140} & \multicolumn{2}{|c|}{4.1} & 59 \\
\hline DATE & $\begin{array}{r}\text { RES } \\
\text { VO } \\
\text { TI } \\
\text { SU } \\
\text { PEN } \\
\text { (N } \\
\text { CO }\end{array}$ & & $\begin{array}{l}\text { NI: } \\
\text { GI } \\
\text { NITI } \\
\text { TO' } \\
(M \\
\text { AS } \\
\text { COO }\end{array}$ & \multicolumn{3}{|c|}{ 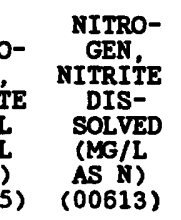 } & \multicolumn{2}{|c|}{$\begin{array}{c}\text { NITRO- } \\
\text { GEN, } \\
\text { NO2+NOB } \\
\text { TOTAI } \\
\text { (MG/L } \\
\text { AS N) } \\
(00630)\end{array}$} & \multicolumn{2}{|c|}{$\begin{array}{c}\text { NITRO- } \\
\text { GEN, } \\
\text { NO2+NÓ3 } \\
\text { DIS- } \\
\text { SOLVED } \\
\text { (MG/L } \\
\text { AS N) } \\
\text { (O0631) }\end{array}$} & \multicolumn{2}{|c|}{$\begin{array}{c}\text { NITRO- } \\
\text { GEN, } \\
\text { AMYONIA } \\
\text { TOTAI } \\
\text { (MG/L } \\
\text { AS N) } \\
\text { (00610) }\end{array}$} & \multicolumn{2}{|c|}{$\begin{array}{c}\text { KITRO- } \\
\text { GEN, } \\
\text { AMONIA } \\
\text { DIS- } \\
\text { SOLVED } \\
\text { (MG/L } \\
\text { AS N } \\
(00608)\end{array}$} & \multicolumn{2}{|c|}{$\begin{array}{l}\text { NITRO- } \\
\text { GEN,AM- } \\
\text { MONIA + } \\
\text { ORGANIC } \\
\text { TOTAL } \\
\text { (MG/L } \\
\text { AS N) } \\
\text { (O0625) }\end{array}$} & \multicolumn{2}{|c|}{$\begin{array}{l}\text { MITRO- } \\
\text { GEN,AM- } \\
\text { MONIA + } \\
\text { ORGANIC } \\
\text { DIS. } \\
\text { (MG/L } \\
\text { AS N) } \\
(00623)\end{array}$} \\
\hline $\begin{array}{l}\text { AUG } \\
\quad 23 \ldots\end{array}$ & \multicolumn{2}{|c|}{22} & \multicolumn{2}{|c|}{0.03} & \multicolumn{2}{|c|}{0.02} & \multicolumn{2}{|c|}{4.8} & \multicolumn{2}{|c|}{4.8} & \multicolumn{2}{|c|}{0.01} & \multicolumn{4}{|l|}{0.} & \multicolumn{2}{|c|}{1.0} \\
\hline \multicolumn{3}{|c|}{$\begin{array}{c}\text { PHOS- } \\
\text { PHORUS } \\
\text { TOTAL } \\
\text { (MG/L } \\
\text { AS P) } \\
(00665)\end{array}$} & $\begin{array}{r}\mathrm{PBC} \\
\mathrm{PBO} \\
\mathrm{D} \\
\mathrm{SO} \\
\mathbf{C M} \\
\mathrm{AS} \\
\mathrm{COO}\end{array}$ & $\begin{array}{l}S- \\
\text { US } \\
S- \\
\text { VED } \\
\text { /L } \\
\text { P) } \\
66)\end{array}$ & $\begin{array}{l}\text { PEC } \\
\text { PHOI } \\
\text { ORTI } \\
\text { TOI } \\
\text { (MX } \\
\text { AS } \\
670\end{array}$ & $\begin{array}{l}S- \\
\text { US } \\
0 \\
\text { AL } \\
\text { (L) } \\
\text { P) } \\
07)\end{array}$ & \multicolumn{2}{|c|}{$\begin{array}{l}\text { PHOS- } \\
\text { PHORUS } \\
\text { ORTHO, } \\
\text { DIS- } \\
\text { SOLVED } \\
\text { (MG/L } \\
\text { AS P) } \\
(00671)\end{array}$} & $\begin{array}{l}\text { CARE } \\
\text { ORGA } \\
\text { DIS } \\
\text { SOLV } \\
\text { (ME } \\
\text { AS } \\
\text { COOE }\end{array}$ & $\begin{array}{l}\text { SN } \\
\text { NIĆ } \\
\text { ED } \\
\text { (L) } \\
\text { Bi) }\end{array}$ & \multicolumn{2}{|c|}{$\begin{array}{l}\text { CARBON, } \\
\text { ORGANIC } \\
\text { SUS- } \\
\text { PENDED } \\
\text { TOTAL } \\
\text { (MG/L } \\
\text { AS C) } \\
\text { (O0689) }\end{array}$} & $\begin{array}{l}\text { SEI } \\
\text { MEI } \\
\text { SUS } \\
\text { PE } \\
(M \\
\text { (80) }\end{array}$ & $\begin{array}{l}I- \\
\text { IT, } \\
\text { IDED } \\
(\mathrm{L}) \\
\text { (54) }\end{array}$ & \multicolumn{2}{|c|}{$\begin{array}{c}\text { CHLOR-A } \\
\text { PHYTO- } \\
\text { PLANK- } \\
\text { TON } \\
\text { CEROMO } \\
\text { FLUOROM } \\
\text { (UG/L) } \\
(70953)\end{array}$} & \multicolumn{2}{|c|}{$\begin{array}{c}\text { CHLOR-B } \\
\text { PHYTO- } \\
\text { PLANK- } \\
\text { TON } \\
\text { CHROMO } \\
\text { FLUOROM } \\
\text { (UG/L) } \\
(70954)\end{array}$} \\
\hline $\begin{array}{l}\text { UG } \\
23 \ldots\end{array}$ & \multicolumn{2}{|c|}{0.20} & & 11 & & 08 & & 07 & 7. & & & & 17 & & & .0 & $<0$. & 10 \\
\hline
\end{tabular}




\section{ANALYSES OF SAMPLES COLLECTED AT WATER-QUALITY PARTIAL-RECORD STATIONS}

05344995 VERMILLION RIVER TRIBUTARY MEAR FARMINGTON, M

WATER QUALITY DATA, WATER YEAR OCTOBER 1990 TO SEPTEEBER 1991

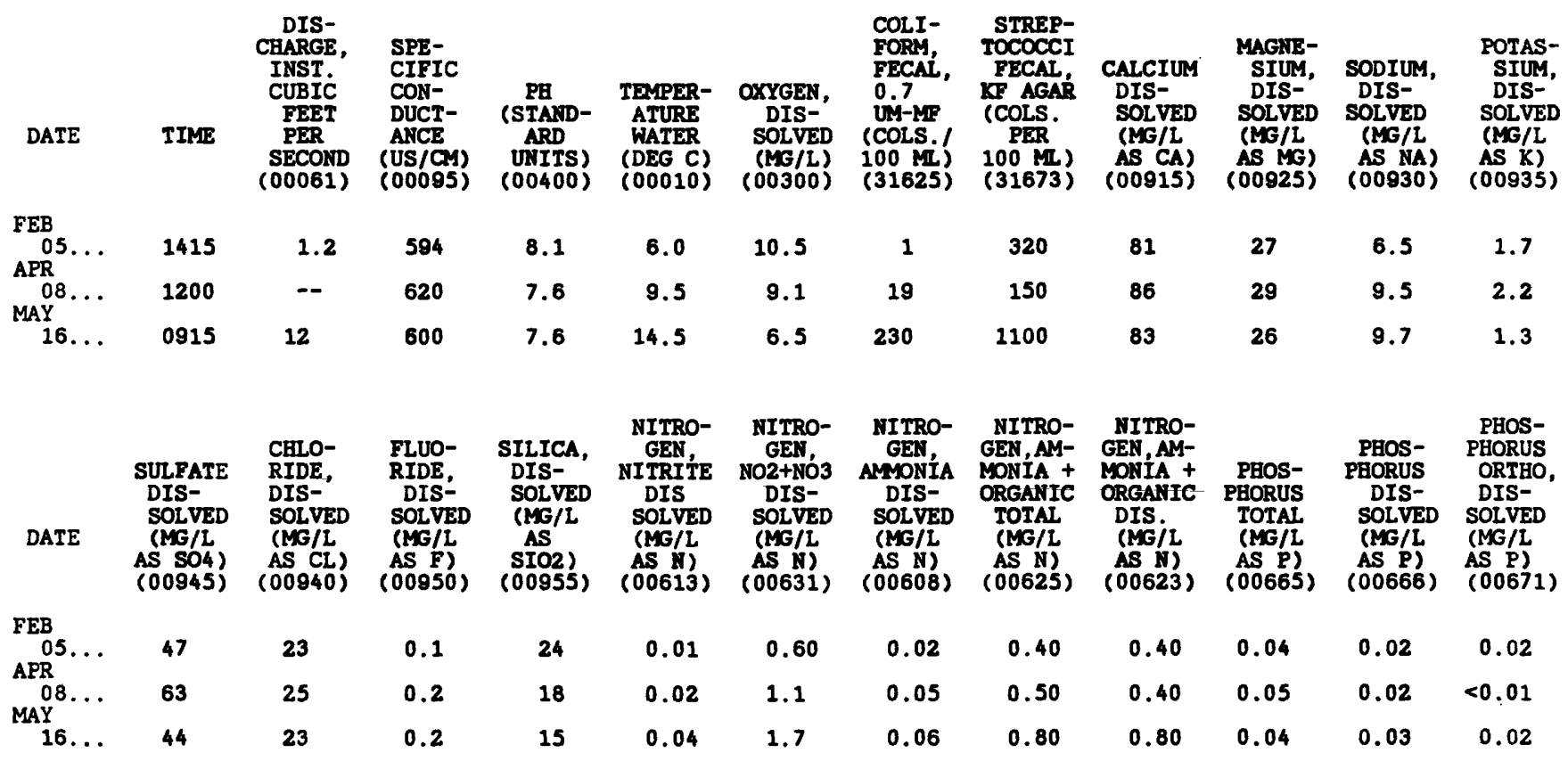




\section{ANALYSES OF SAMPLES COLLECTED AT WATER-QUALITY PARTIAL-RECORD STATIONS}

05345200 VERMILLION RIVER TRIBUTARY NEAR EMPIRE, MN

WATER QUALITY DATA, WATER YEAR OCTOBER 1990 TO SEPTEMBER 1991

\begin{tabular}{|c|c|c|c|c|c|c|c|c|c|c|c|c|}
\hline DATE & TIME & $\begin{array}{c}\text { DIS- } \\
\text { CHARGE, } \\
\text { INST. } \\
\text { CUBIC } \\
\text { FEET } \\
\text { PER } \\
\text { SECOND } \\
\text { (00061) }\end{array}$ & $\begin{array}{l}\text { SPE- } \\
\text { CIFIC } \\
\text { CON- } \\
\text { DUCI- } \\
\text { ANCE } \\
\text { (US/CM) } \\
(00095 \text { ) }\end{array}$ & $\begin{array}{c}\text { PH } \\
\text { (STAND- } \\
\text { ARD } \\
\text { UNITS) } \\
\text { (00400) }\end{array}$ & $\begin{array}{l}\text { TEMPER- } \\
\text { ATURE } \\
\text { WATER } \\
\text { (DEG C) } \\
(00010)\end{array}$ & $\begin{array}{c}\text { OXYGEN, } \\
\text { DIS- } \\
\text { SOLVED } \\
(M G / L) \\
(00300)\end{array}$ & $\begin{array}{l}\text { COLI- } \\
\text { FORM, } \\
\text { FECAL, } \\
0.7 \\
\text { UM-MF } \\
\text { (COLS./ } \\
\text { 100 ML) } \\
(31625)\end{array}$ & $\begin{array}{c}\text { STREP- } \\
\text { TOCOCCI } \\
\text { FECAL, } \\
\text { KF AGAR } \\
\text { (COLS. } \\
\text { PER } \\
100 \mathrm{ML}) \\
(31673)\end{array}$ & $\begin{array}{l}\text { CALCIUM } \\
\text { DIS- } \\
\text { SOLVED } \\
\text { (MG/L } \\
\text { AS CA) } \\
(00915)\end{array}$ & $\begin{array}{c}\text { MAGNE- } \\
\text { SIUM, } \\
\text { DIS- } \\
\text { SOLVED } \\
\text { (MG/L } \\
\text { AS MG) } \\
(00925)\end{array}$ & $\begin{array}{l}\text { SODIUM, } \\
\text { DIS- } \\
\text { SOLVED } \\
\text { (MG/L } \\
\text { AS NA) } \\
(00930)\end{array}$ & $\begin{array}{c}\text { POTAS- } \\
\text { SIUM, } \\
\text { DIS- } \\
\text { SOLVED } \\
\text { (MG/L } \\
\text { AS K) } \\
(00935)\end{array}$ \\
\hline $\begin{array}{l}\mathrm{AY} \\
16 \ldots\end{array}$ & 1230 & 0.01 & 694 & 7.5 & 20.0 & 2.1 & 160 & 980 & 100 & 30 & 6.0 & 7.0 \\
\hline DATE & $\begin{array}{c}\text { SULFATE } \\
\text { DIS- } \\
\text { SOLVED } \\
\text { (MG/L } \\
\text { AS SO4) } \\
(00945)\end{array}$ & $\begin{array}{l}\text { CHLO- } \\
\text { RIDE, } \\
\text { DIS- } \\
\text { SOLVED } \\
\text { (MG/L } \\
\text { AS CL) } \\
(00940)\end{array}$ & $\begin{array}{l}\text { FLUO- } \\
\text { RIDE, } \\
\text { DIS- } \\
\text { SOLVED } \\
\text { (MG/L } \\
\text { AS F) } \\
(00950)\end{array}$ & $\begin{array}{c}\text { SILICA, } \\
\text { DIS- } \\
\text { SOLVED } \\
\text { (MG/L } \\
\text { AS } \\
\text { SIO2) } \\
(00955)\end{array}$ & $\begin{array}{c}\text { NITRO- } \\
\text { GEN, } \\
\text { MITRITE } \\
\text { DIS- } \\
\text { SOLVED } \\
\text { (MG /L } \\
\text { AS N) } \\
(00613)\end{array}$ & $\begin{array}{c}\text { NITRO- } \\
\text { GEN, } \\
\text { NO2+NO3 } \\
\text { DIS- } \\
\text { SOLVED } \\
\text { (MG /L } \\
\text { AS N) } \\
(00631)\end{array}$ & $\begin{array}{c}\text { NITRO- } \\
\text { GEN, } \\
\text { AMMONIA } \\
\text { DIS- } \\
\text { SOLVED } \\
\text { (MG/L } \\
\text { AS N } \\
(00608)\end{array}$ & $\begin{array}{l}\text { NITRO- } \\
\text { GEN, AM- } \\
\text { MONIA + } \\
\text { ORGANIC } \\
\text { TOTAL } \\
\text { (MG/L } \\
\text { AS N }) \\
(00625 \text { ) }\end{array}$ & $\begin{array}{l}\text { NITRO- } \\
\text { GEN,AM- } \\
\text { MONIA + } \\
\text { ORGANIC } \\
\text { DIS. } \\
(M G / L \\
\text { AS N }) \\
(00623)\end{array}$ & $\begin{array}{c}\text { PHOS- } \\
\text { PEORUS } \\
\text { TOTAL } \\
\text { (MG/L } \\
\text { AS P) } \\
(00665)\end{array}$ & $\begin{array}{c}\text { PHOS- } \\
\text { PHORUS } \\
\text { DIS- } \\
\text { SOLVED } \\
\text { (MG/L } \\
\text { AS P) } \\
(00666)\end{array}$ & $\begin{array}{l}\text { PHOS- } \\
\text { PHORUS } \\
\text { ORTHO, } \\
\text { DIS- } \\
\text { SOLVED } \\
\text { (MG /L } \\
\text { AS P) } \\
(00671)\end{array}$ \\
\hline
\end{tabular}

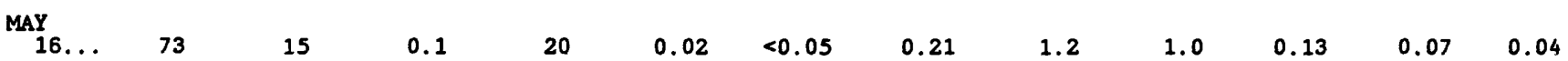


443933093002801 - SOUTH BRANCE VERMILLION RIVER AT DMPIRE, MI

WATER QUALITY DATA, WATER YEAR OCTOBER 1990 TO SEPTEMBER 1991

\begin{tabular}{|c|c|c|c|c|c|c|c|c|c|c|c|c|}
\hline DATE & TIME & $\begin{array}{c}\text { DIS- } \\
\text { CEARGE, } \\
\text { INST. } \\
\text { CUBIC } \\
\text { FEET } \\
\text { PER } \\
\text { SECOND } \\
\text { (00061) }\end{array}$ & $\begin{array}{l}\text { SPE- } \\
\text { CIFIC } \\
\text { CON- } \\
\text { DUCT- } \\
\text { ANCE } \\
\text { (US/CM) } \\
\text { (O0D95) }\end{array}$ & $\begin{array}{c}\text { PH } \\
\text { (STAND- } \\
\text { ARD } \\
\text { UNITS) } \\
\text { (O0400) }\end{array}$ & $\begin{array}{c}\text { TEMPER- } \\
\text { ATURE } \\
\text { WATER } \\
\text { (DEG C) } \\
\text { (DO010) }\end{array}$ & $\begin{array}{c}\text { OXYGEN, } \\
\text { DIS- } \\
\text { SOLVED } \\
(M G / L) \\
(00300)\end{array}$ & $\begin{array}{l}\text { COLI- } \\
\text { FORM, } \\
\text { FECAL, } \\
0.7 \\
\text { UM-MF } \\
\text { (COLS.' } \\
100 \mathrm{ML}) \\
(31625)\end{array}$ & $\begin{array}{c}\text { STREP- } \\
\text { TOCOCCI } \\
\text { FECAL, } \\
\text { KF AGAR } \\
\text { (COLS. } \\
\text { PER } \\
100 \mathrm{ML}) \\
(31673)\end{array}$ & $\begin{array}{l}\text { CALCIUM } \\
\text { DIS- } \\
\text { SOLVED } \\
\text { (MG/L } \\
\text { AS CA) } \\
\text { (00915) }\end{array}$ & $\begin{array}{c}\text { MAGNE- } \\
\text { SIUM, } \\
\text { DIS- } \\
\text { SOLVED } \\
\text { (MG/L } \\
\text { AS MG) } \\
\text { (D0925) }\end{array}$ & $\begin{array}{l}\text { SODIUM, } \\
\text { DIS- } \\
\text { SOLVED } \\
\text { (MG/L } \\
\text { AS NA) } \\
(00930)\end{array}$ & $\begin{array}{c}\text { POTAS } \\
\text { SIUM } \\
\text { DIS- } \\
\text { SOLVE } \\
\text { (MG/I } \\
\text { AS K }) \\
\text { (O0935 }\end{array}$ \\
\hline & 1015 & 8.7 & 463 & 8.1 & 2.0 & 11.0 & 400 & 2400 & 57 & 22 & 4.6 & 4.0 \\
\hline 11 . & 0930 & -- & 514 & 8.0 & 6.5 & 10.3 & 42 & 84 & 70 & 24 & 5.4 & 1.4 \\
\hline & 045 & 15 & 491 & 7.8 & 13.5 & 3.5 & 170 & 350 & 68 & 23 & 5.0 & \\
\hline
\end{tabular}

DATE

$\begin{array}{clll} & \text { CHLO- } & \text { FLUO- } & \text { SILICA, } \\ \text { SULFATE } & \text { RIDE, } & \text { RIDE, } & \text { DIS- } \\ \text { DIS- } & \text { DIS- } & \text { DIS- } & \text { SOLVED } \\ \text { SOLVED } & \text { SOLVED } & \text { SOLVED } & \text { (MG/L } \\ \text { (MG/L } & \text { (MG/L } & \text { (MG/L } & \text { AS } \\ \text { AS SO4) } & \text { AS CL) } & \text { AS F) } & \text { SIO2) } \\ (00945) & (00940) & (00950) & (00955)\end{array}$

NITRO- NITRO- NITRO- NITRO- NITRO-

GEN, GEN, GEN, GEN,AM- GEN,AMNITRITE $\mathrm{NO}^{2}+\mathrm{NO} 3$ DIS-

AMONIA SOLVED SOLVED (WG/L SOLVED DISORGANIC MORGANIC PHOSSOLVED TOTAL DIS. (MG/L (MG/L (MG/L (MG/L (00613)

(00631)

(00608)

(00625) (00623) AS P)

$\begin{array}{cc}\text { PHOS- } & \text { PHOS- } \\ \text { PHORUS } \\ \text { PHORUS } & \text { ORTHO, } \\ \text { DIS- } & \text { DIS- } \\ \text { SOLVED } & \text { SOLVED } \\ \text { (MG/L } & \text { (MG/L } \\ \text { AS P) } & \text { AS P) } \\ (00666) & (00671)\end{array}$

FEB
$05 \ldots$
APR $\ldots . . .27$
$\underset{11}{M A Y}$
$17 \ldots$

$\begin{array}{ll}27 & 14 \\ 48 & 16 \\ 28 & 18\end{array}$

$\begin{array}{ll}0.2 & 13 \\ 0.2 & 11 \\ 0.2 & 9.9\end{array}$

0.02

5.4

0.27

1.2

1.1

0.21

0.14

0.14

18

0.02

4.7

$<0.01$

0.60

0.60

0.03

$0.02<0.01$

0.05

4.7

0.08

1.1

0.70

0.06

0.04

$<0.01$ 
ANALYSES OF SAMPLES COLLECTED AT WATER-QUALITY PARTIAL-RECORD STATIONS

05346000 VERMILLION RIVER AT HASTINGS, MN

WATER QUALITY DATA, WATER YEAR OCTOBER 1990 TO SEPTEMBER 1991

\begin{tabular}{|c|c|c|c|c|c|c|c|c|c|c|c|c|}
\hline ATE & TIME & $\begin{array}{c}\text { DIS- } \\
\text { CHARGE, } \\
\text { INST. } \\
\text { CUBIC } \\
\text { FEET } \\
\text { PER } \\
\text { SECOND } \\
(00061)\end{array}$ & $\begin{array}{l}\text { SPE- } \\
\text { CIFIC } \\
\text { CON- } \\
\text { DUCT- } \\
\text { ANCE } \\
\text { (US/CM) } \\
(00095)\end{array}$ & $\begin{array}{c}\text { PH } \\
\text { (STAND- } \\
\text { ARD } \\
\text { UNITS) } \\
(00400)\end{array}$ & $\begin{array}{c}\text { TEMPER- } \\
\text { ATURE } \\
\text { WATER } \\
\text { (DEG C) } \\
\text { (00010) }\end{array}$ & $\begin{array}{c}\text { OXYGEN, } \\
\text { DIS- } \\
\text { SOLVED } \\
(M G / L) \\
(00300)\end{array}$ & $\begin{array}{l}\text { COLI- } \\
\text { FORM, } \\
\text { FECAL, } \\
0.7 \\
\text { UM-MF } \\
\text { (COLS.' } \\
\text { 100 Mi) } \\
\text { (31625) }\end{array}$ & $\begin{array}{c}\text { STREP- } \\
\text { TOCOCCI } \\
\text { FECAL, } \\
\text { KF AGAR } \\
\text { (COLS. } \\
\text { PER } \\
100 \mathrm{ML}) \\
(31673)\end{array}$ & $\begin{array}{l}\text { CALCIUM } \\
\text { DIS- } \\
\text { SOLVED } \\
\text { (MG/L } \\
\text { AS CA) } \\
\text { (00915) }\end{array}$ & $\begin{array}{c}\text { MAGNE- } \\
\text { SIUM, } \\
\text { DIS- } \\
\text { SOLVED } \\
\text { (MG/L } \\
\text { AS MG) } \\
\text { (00925) }\end{array}$ & $\begin{array}{l}\text { SODIUM, } \\
\text { DIS- } \\
\text { SOLVED } \\
\text { (MG/L } \\
\text { AS NA) } \\
\text { (00930) }\end{array}$ & $\begin{array}{c}\text { POTAS- } \\
\text { SIUM, } \\
\text { DIS- } \\
\text { SOLVED } \\
\text { (MG/L } \\
\text { AS K }) \\
(00935)\end{array}$ \\
\hline
\end{tabular}

\begin{tabular}{|c|c|c|c|c|c|c|c|c|c|c|c|}
\hline $\begin{array}{c}\text { FEB } \\
07 \ldots \\
\text { APR }\end{array}$ & 1400 & -- & 709 & 8.0 & 0.0 & 14.3 & 62 & -- & 68 & 23 & 51 \\
\hline${ }_{\operatorname{MAY}}^{11} \ldots$ & 1230 & - & 742 & 8.4 & 7.5 & 12.7 & 90 & 100 & 80 & 27 & 44 \\
\hline $17 \ldots$ & 1315 & 93 & 650 & 8.1 & 14.0 & 9.2 & 1700 & 1500 & 77 & 25 & 30 \\
\hline
\end{tabular}

\begin{tabular}{|c|c|c|c|c|c|c|c|c|c|c|c|c|}
\hline 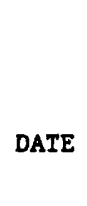 & $\begin{array}{c}\text { SULFATE } \\
\text { DIS- } \\
\text { SOLVED } \\
\text { (MG/L } \\
\text { AS SO4) } \\
(00945)\end{array}$ & $\begin{array}{l}\text { CHLO- } \\
\text { RIDE, } \\
\text { DIS- } \\
\text { SOLVED } \\
\text { (MG/L } \\
\text { AS CL) } \\
\text { (00940) }\end{array}$ & $\begin{array}{l}\text { FLUO- } \\
\text { RIDE, } \\
\text { DIS- } \\
\text { SOLVED } \\
\text { (MG/L } \\
\text { AS F) } \\
\text { (00950) }\end{array}$ & $\begin{array}{c}\text { SILICA, } \\
\text { DIS- } \\
\text { SOLVED } \\
\text { (MG/L } \\
\text { AS } \\
\text { SIO2) } \\
(00955)\end{array}$ & $\begin{array}{c}\text { NITRO- } \\
\text { GEN, } \\
\text { NITRITE } \\
\text { DIS- } \\
\text { SOLVED } \\
\text { (MG/L } \\
\text { AS N) } \\
\text { (00613) }\end{array}$ & $\begin{array}{c}\text { NITRO- } \\
\text { GEN, } \\
\text { NO2+NÓ3 } \\
\text { DIS- } \\
\text { SOLVED } \\
\text { (MG/L } \\
\text { AS N) } \\
\text { (00631) }\end{array}$ & $\begin{array}{c}\text { NITRO- } \\
\text { GEN, } \\
\text { AMMONIA } \\
\text { DIS- } \\
\text { SOLVED } \\
\text { (MG/L } \\
\text { AS N } \\
(00608)\end{array}$ & $\begin{array}{c}\text { NITRO- } \\
\text { GEN,AM- } \\
\text { MONIA + } \\
\text { ORGANIC } \\
\text { TOTAL } \\
\text { (MG/L } \\
\text { ASN) } \\
(00625)\end{array}$ & $\begin{array}{l}\text { NITRO- } \\
\text { GEN,AM- } \\
\text { MONIA + } \\
\text { ORGANIC } \\
\text { DIS. } \\
\text { (MG/L } \\
\text { AS N) } \\
(00623)\end{array}$ & $\begin{array}{c}\text { PHOS- } \\
\text { PHORUS } \\
\text { TOTAL } \\
\text { (MG/L } \\
\text { AS P) } \\
(00665)\end{array}$ & $\begin{array}{c}\text { PHOS- } \\
\text { PHORUS } \\
\text { DIS- } \\
\text { SOLVED } \\
\text { (MG/L } \\
\text { AS P) } \\
\text { (00666) }\end{array}$ & $\begin{array}{l}\text { PHOS- } \\
\text { PHORUS } \\
\text { ORTHO, } \\
\text { DIS- } \\
\text { SOLVED } \\
\text { (MG/L } \\
\text { AS P) } \\
(00671)\end{array}$ \\
\hline
\end{tabular}

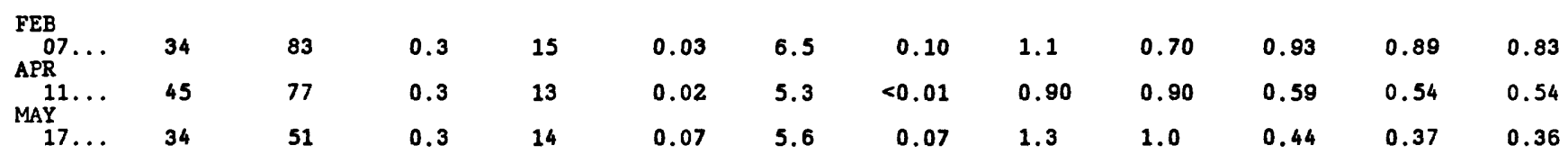


ANALYSES OF SAMPLES COLLECTED AT WATER QUALITY PARTIAL RECORD STATIONS

05376500 SOUTH FORK WHITEWATER RIVER NEAR ALTURA, MN

WATER QUALITY DATA, WATER YEAR OCTOBER 1990 TO SEPTEMBER 1991

\begin{tabular}{|c|c|c|c|}
\hline DATE & TIME & $\begin{array}{c}\text { TEMPER- } \\
\text { ATURE } \\
\text { WATER } \\
\text { (DEG C) } \\
\text { (00010) }\end{array}$ & $\begin{array}{l}\text { SEDI- } \\
\text { MENT, } \\
\text { SUS- } \\
\text { PENDED } \\
\text { (MG/L) } \\
\text { ( } 80154)\end{array}$ \\
\hline $\begin{array}{l}\text { MAR } \\
23 \ldots \\
23 \ldots \\
\text { APR }\end{array}$ & $\begin{array}{l}1211 \\
1721\end{array}$ & $\begin{array}{l}4.0 \\
3.5\end{array}$ & $\begin{array}{l}582 \\
757\end{array}$ \\
\hline $\begin{array}{r}29 \ldots \\
29 \ldots \\
30 \ldots \\
\text { MAY }\end{array}$ & $\begin{array}{l}1120 \\
1710 \\
0900\end{array}$ & $\begin{array}{l}13.0 \\
15.0 \\
11.5\end{array}$ & $\begin{array}{r}1880 \\
1670 \\
733\end{array}$ \\
\hline $\begin{array}{r}16 \ldots \\
16 \ldots \\
\text { JUN }\end{array}$ & $\begin{array}{l}1815 \\
1938\end{array}$ & $\begin{array}{l}17.0 \\
17.0\end{array}$ & $\begin{array}{l}4530 \\
1250\end{array}$ \\
\hline JUL $15 \ldots$ & 1253 & -- & 2080 \\
\hline $\begin{array}{l}21 \ldots \ldots \\
30 \ldots\end{array}$ & $\begin{array}{l}2235 \\
1331\end{array}$ & $\begin{array}{l}21.0 \\
18.0\end{array}$ & $\begin{array}{r}3870 \\
89\end{array}$ \\
\hline
\end{tabular}




\section{Ground-water Levels}

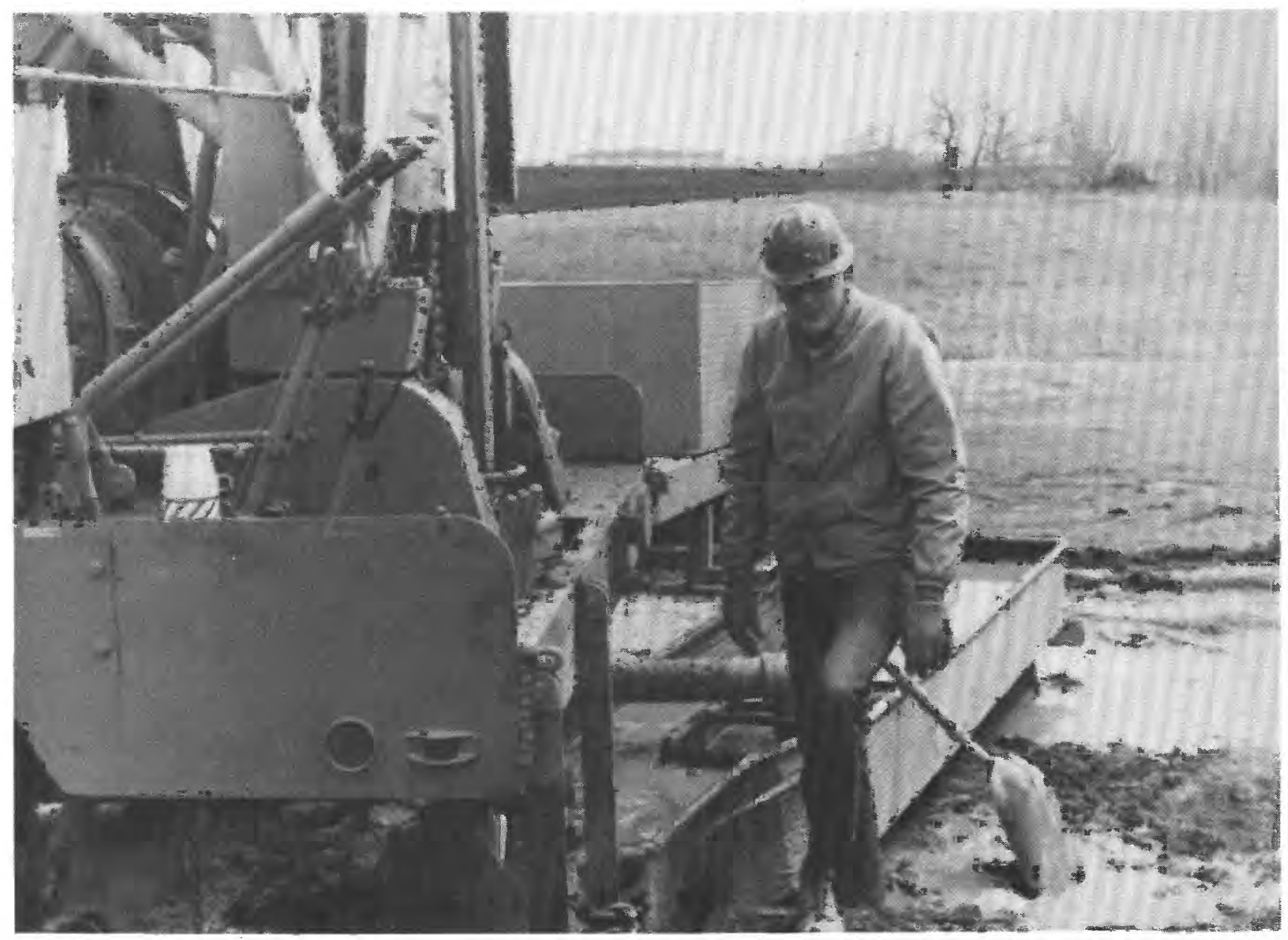

May, 1967 


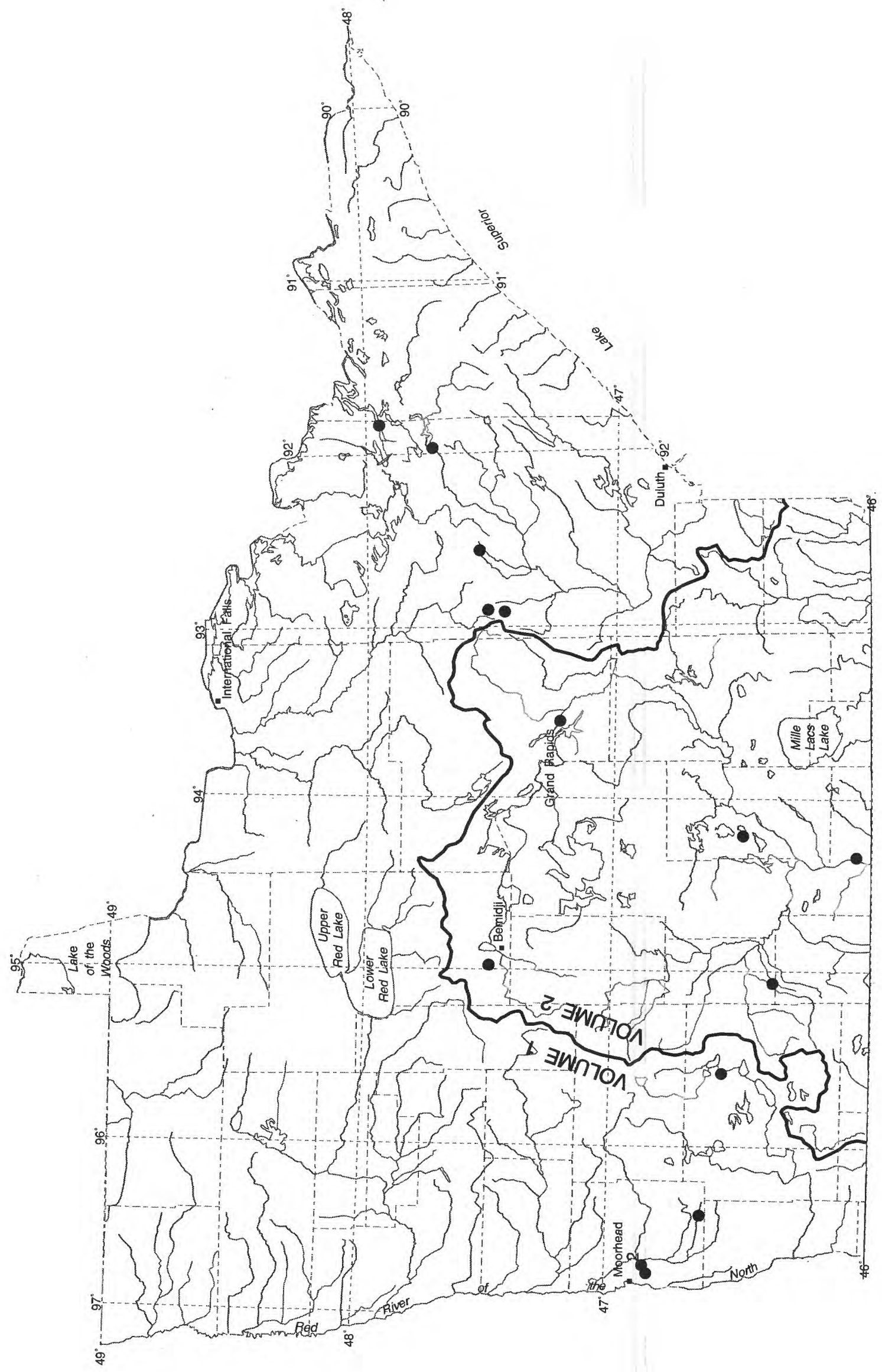



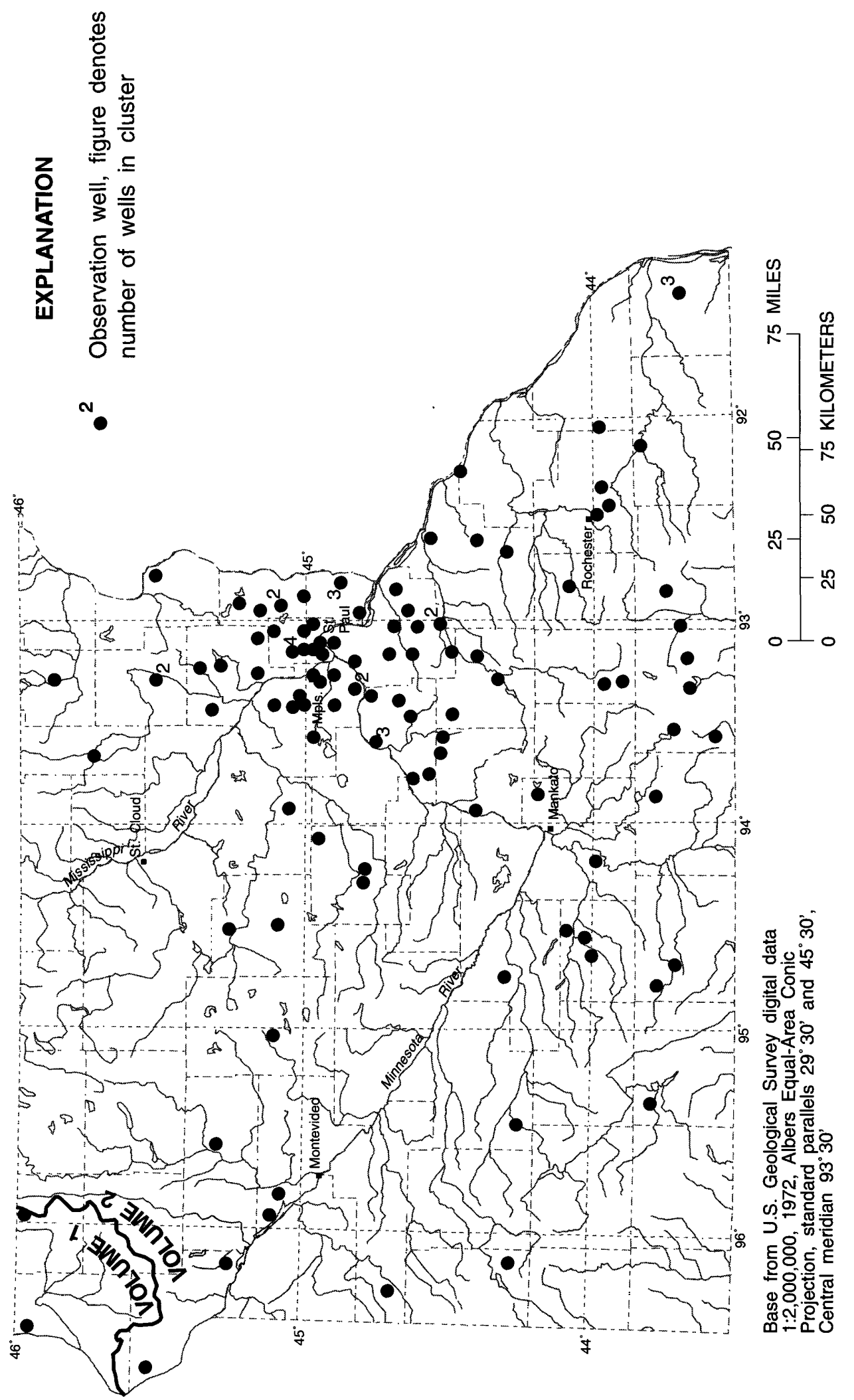

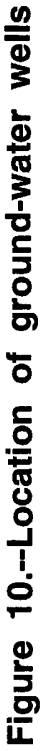


450927093033802. Local number, 031H22W23CBC02.

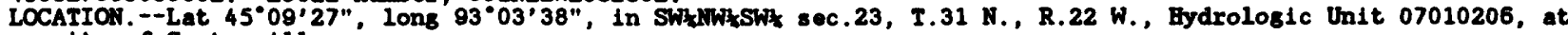
city of Centerville. Omer: U.S. Geological Survey.

AQUIFER. --Jordan Sandstone of Late Cembrian Age.

WELL CBARACTERISTICS. --Drilled observation artesian well, diameter 4 in., depth $277 \mathrm{ft}$, screened 272 to $277 \mathrm{ft}$.

DATUM. --Land-surface datum $18901.6 \mathrm{ft}$ National Geodetic Vertical Datum of 1929 . Measuring point: Top of recorder platform, $2.20 \mathrm{ft}$ above land-surface datum.

REMARKS. - Water level affected by nearby flowing wells.

PERIOD OF RECORD. - Fobruary 1971 to current year.

EXTREYES FOR PERIOD OF RECORD. --Highest water leve1, $8.10 \mathrm{ft}$ below land-surface datum, July 5, 1975; 10west, $18.57 \mathrm{ft}$ below land-surface datum, Oct. 2 , 1989.

WATER LEVEL, IN FEET BELON LAND-SURFACE DATAUM, WATER YEAR OCTOBER 1990 TO SEPTEMER 1991

\begin{tabular}{|c|c|c|c|c|c|c|c|c|c|c|c|c|c|c|c|c|c|}
\hline \multicolumn{2}{|c|}{ DATE } & $\begin{array}{l}\text { WATER } \\
\text { LEVEL }\end{array}$ & \multicolumn{2}{|c|}{ DATE } & $\begin{array}{l}\text { WATER } \\
\text { LEVEL }\end{array}$ & \multicolumn{2}{|c|}{ DATE } & $\begin{array}{l}\text { WATERR } \\
\text { LEVEL }\end{array}$ & \multicolumn{2}{|c|}{ DATE } & $\begin{array}{l}\text { KATER } \\
\text { LEVEL }\end{array}$ & \multicolumn{2}{|c|}{ DATE } & $\begin{array}{l}\text { WATER } \\
\text { LEVEL }\end{array}$ & \multicolumn{2}{|c|}{ DATE } & $\begin{array}{l}\text { WATER } \\
\text { LEVEL }\end{array}$ \\
\hline Hov & $\begin{array}{r}5 \\
10 \\
15 \\
20 \\
25 \\
31 \\
5 \\
10 \\
15 \\
20 \\
25 \\
30\end{array}$ & $\begin{array}{l}12.32 \\
12.40 \\
12.33 \\
12.22 \\
12.40 \\
12.30 \\
12.19 \\
12.26 \\
12.26 \\
12.26 \\
12.45 \\
12.38\end{array}$ & Dec & $\begin{array}{r}5 \\
10 \\
15 \\
20 \\
25 \\
31 \\
5 \\
10 \\
15 \\
20 \\
25 \\
31\end{array}$ & $\begin{array}{l}12.31 \\
12.38 \\
12.56 \\
12.52 \\
12.40 \\
12.49 \\
12.64 \\
12.54 \\
12.48 \\
12.60 \\
12.59 \\
12.65\end{array}$ & Feb & $\begin{array}{r}5 \\
10 \\
15 \\
20 \\
25 \\
28 \\
5 \\
10 \\
15 \\
20 \\
25 \\
31\end{array}$ & $\begin{array}{l}12.58 \\
12.64 \\
12.57 \\
12.62 \\
12.62 \\
14.49 \\
14.96 \\
15.09 \\
15.17 \\
14.95 \\
14.75 \\
14.68\end{array}$ & Apr & $\begin{array}{r}5 \\
10 \\
15 \\
20 \\
25 \\
30 \\
5 \\
10 \\
15 \\
20 \\
25 \\
31\end{array}$ & $\begin{array}{l}12.35 \\
11.96 \\
11.67 \\
11.78 \\
11.74 \\
11.85 \\
11.46 \\
11.30 \\
11.29 \\
11.27 \\
11.24 \\
10.94\end{array}$ & Jun & $\begin{array}{r}5 \\
10 \\
15 \\
20 \\
25 \\
30 \\
5 \\
10 \\
15 \\
20 \\
25 \\
31\end{array}$ & $\begin{array}{l}11.11 \\
11.18 \\
11.05 \\
11.23 \\
11.24 \\
11.48 \\
11.27 \\
11.31 \\
11.44 \\
11.50 \\
11.47 \\
11.40\end{array}$ & Aus & $\begin{array}{l}5 \\
10 \\
15 \\
20 \\
25 \\
31 \\
5 \\
10 \\
15 \\
20 \\
25 \\
30\end{array}$ & 11 \\
\hline
\end{tabular}

451210093170201. Local number, 031N24W01CBBO1

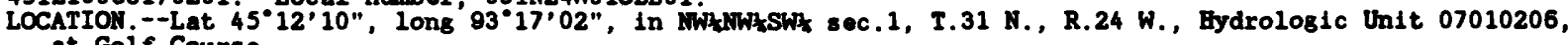
at Golf Course.

Omer: City of Coon Rapids.

AOUIFER. --Buried sand of Plei stocene Ase.

WELL CBARACTERISTICS.--Drilled Irrigation artesion well, diameter $12 \mathrm{in}$. depth 193 ft, screened 163 to $193 \mathrm{ft}$.

DATUM, -Aititude of land-surface datum 1s $897 \mathrm{ft}$. Measuring point: Top of breather pipe, 2.00 ft above 1and-surface datum.

PERIOD OF RECORD. --December 1980 to current year.

EXTREMES FOR PERIOD OF RECORD. --Highest water level, 18.43 ft below land-surface datum, May 13, 1986

lowest, $41.10 \mathrm{ft}$ below land-surface datum, Sept. $21,1989$.

WATER LEVEL, IN FEET BELON LAND-SURFACE DATUM, WATER YEAR OCTOBER 1990 TO SEPTEMBER 1991

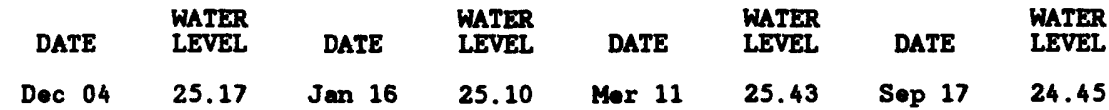

451742093122102. Local number, 032N23W04AADO2.

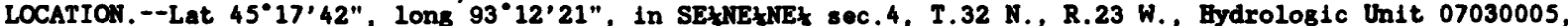
$1.5 \mathrm{mi}$ east of Soderville

Omer: U.S. Geologicai Survey.

AQUIFER. --Surficial outwash sand of Pleistocene Age.

WELL CBARACTERISTICS. --Bored observation water-table well, diemeter 2 in., depth 21 ft, screened 19 to $21 \mathrm{ft}$.

DATUM. --Altitude of land-surface datum is $916 \mathrm{ft}$. Measuring point: Top of casing, $3.50 \mathrm{ft}$ above landsurface datum.

PERIOD OF RECORD. --August 1969 to current year

EXTREMES FOR PERIOD OF RECORD,--Highest water level, $5.82 \mathrm{ft}$ below land-surface datum, May 13, 1986;

lowest, $13.22 \mathrm{ft}$ below land-surface datum, Mar. 5-9, 1977.

WATER LEVEL, IN FEET BELOW LAND-SURFACE DATUM, WATER YEAR OCTOBER 1990 TO SEPTEMBER 1991

$\begin{array}{cccccc}\text { DATE } & \begin{array}{c}\text { WATER } \\ \text { LEVEL }\end{array} & \text { DATE } & \begin{array}{r}\text { WATER } \\ \text { LEVEL }\end{array} & \text { DATE } & \begin{array}{r}\text { WATER } \\ \text { LEVEL }\end{array} \\ \text { Dec 04 } & 10.03 & \text { Mar 11 } & \mathbf{1 0 . 6 8} & \text { Jul 22 } & \mathbf{7 . 6 3} \\ \text { Jan 16 } & \mathbf{1 0 . 4 5} & \text { May 16 } & \mathbf{7 . 3 4} & \text { Sop } 17 & \mathbf{7 . 4 4}\end{array}$




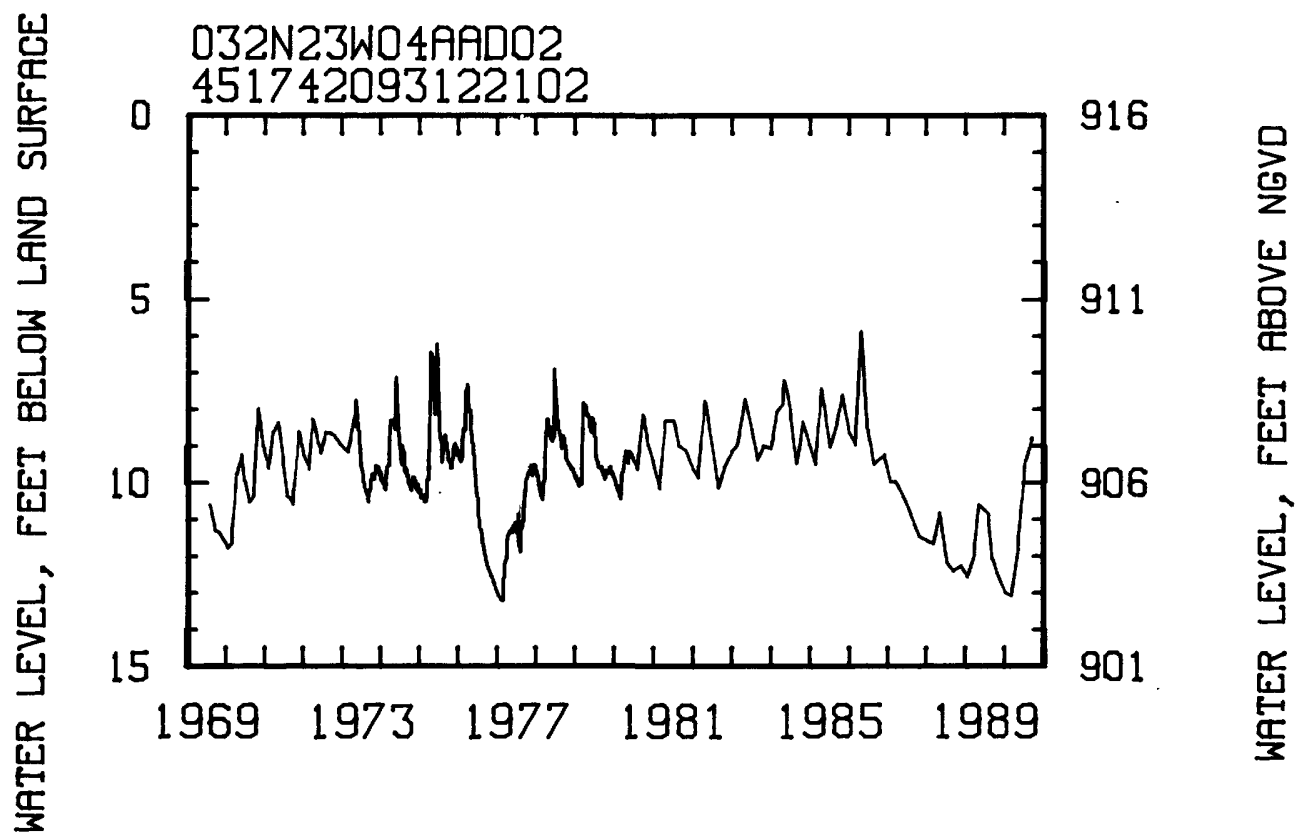

452305093141501 , Local number, 033N23W05BAB01.

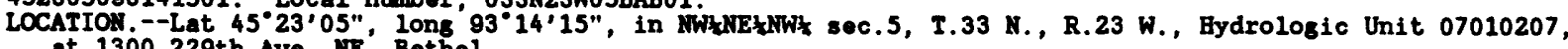
at 1300 229.th Ave. ME', Bethel. Owner: Friendship Baptist Church.

AOUIFER. --Franconian Sandstone of Late Cambrian Ase.

WELL CBARACTERISTICS. --Drilled domestic artesian weil, diemeter 4 in., depth $141 \mathrm{ft}$, cased to $126 \mathrm{ft}$. DATUS. --Altitude of land-surface datum is $923 \mathrm{ft}$. Measuring point: Top of well cap, $0.80 \mathrm{ft}$ above land-surface datum.

PERIOD OF RECORD. --April 1980 to current year.

EXTRRareS FOR PERIOD OF RECORD. --Highest water level, $19.45 \mathrm{ft}$ below land-surface datum, July 10,1986

lowest, $23.11 \mathrm{ft}$ below land-surface datum, Mar. 13, 1990 .

WATER LEVEL, IN FEET BELOW LAND-SURFACE DATUM, WATER YEAR OCTOBER 1990 TO SEPTEMBER 1991

$\begin{array}{cccccc}\text { DATE } & \begin{array}{c}\text { WATER } \\ \text { LEVEL }\end{array} & \text { DATE } & \begin{array}{c}\text { WATER } \\ \text { LEVEL }\end{array} & \text { DATE } & \begin{array}{c}\text { WATER } \\ \text { LEVEL }\end{array} \\ \text { Dec 04 } & 21.87 & \text { Mer 11 } & 22.39 & \text { Jul 22 } & 20.22 \\ \text { Jan 16 } & 22.10 & \text { Mey 16 } & 21.58 & \text { Sop } 17 & 19.62\end{array}$

451938093223101. Local number, 033N24W30ABB01.

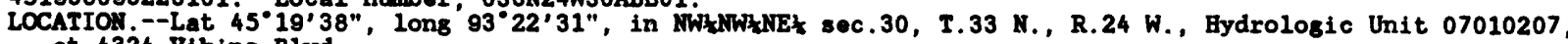
at 4324 Viking Blvd.

Omer: Rorthwestern Bell Telephone Co.

AOUIFER. --Ironton-Galesville Sandstone of Late Cambrian Ase.

WELL CHARACTERISTICS.--Drilled domestic artesian well, diemeter 4 in. depth $280 \mathrm{ft}$, cased to $223 \mathrm{ft}$

DATUM. - -Altitude of iand-surface datum is $900 \mathrm{ft}$. Measuring point: 'Top of casing, $1.50 \mathrm{ft}$ above landsurface datum.

PERIOD OF RECORD:--April 1977 to current year.

EXTRRES FOR PERIOD OF RECORD. --Highest water level, 26.64 ft below land-surface datum, Mey 13, 1986 ;

lowest, $32.93 \mathrm{ft}$ below land-surface datum, Oct. 31, 1989.

WATER LEVEL, IN FEET BELON LAND-SURFACE DATUM, WATER YEAR OCTOBER 1990 TO SEPTEMBER 1991

$\begin{array}{cccccc}\text { DATE } & \begin{array}{c}\text { WATER } \\ \text { LEVEI }\end{array} & \text { DATE } & \begin{array}{c}\text { WATER } \\ \text { LEVEL }\end{array} & \text { DATE } & \begin{array}{c}\text { WATER } \\ \text { LEVEI }\end{array} \\ \text { Dec 04 } & 30.75 & \text { Mar 11 } & 31.05 & \text { Ju1 22 } & 28.83 \\ \text { Jan 16 } & 30.77 & \text { May 16 } & \mathbf{2 8 . 6 7} & \text { Søp 17 } & 28.86\end{array}$


473023094570901. Local number, 147N34W35ADC01.

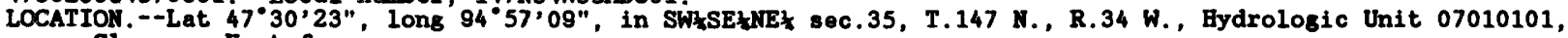
on Clarence Hart farm.

Omer: U.S. Geological Survey.

AQUIFER. --Surficial outwash sand of Pleistocene Ase.

WELL CHARACTERISTICS.--Bored observation water-table well, diameter $1 \frac{1}{\mathrm{t}}$ in., depth $20 \mathrm{ft}$, screened 18 to $20 \mathrm{ft}$.

DATUM.--Altitude of land-surface datum is 1,383 ftm. Measuring point: Top of casing, $3.00 \mathrm{ft}$ above landsurface datum.

PERIOD OF RECORD.--October 1970 to current year.

EXTRERES FOR PERIOD OF RECORD.--Highest water level, $6.17 \mathrm{ft}$ below land-surface datum, Aug. 1, 1975; lowest, $12.11 \mathrm{ft}$ below land-surface datum, Mar. 11, 1991 .

WATER LEVEL, IN FEET BELON LAND-SURFACE DATUM, WATER YEAR OCTOBER 1990 TO SEPTEMBER 1991

\begin{tabular}{|c|c|c|c|c|c|c|c|c|c|}
\hline DATE & $\begin{array}{l}\text { WATER } \\
\text { LEVEL }\end{array}$ & DATE & $\begin{array}{l}\text { WATER } \\
\text { LEVEL }\end{array}$ & DATE & $\begin{array}{l}\text { WATER } \\
\text { LEVEL }\end{array}$ & DATE & $\begin{array}{l}\text { WATER } \\
\text { LEVEL }\end{array}$ & DATE & $\begin{array}{l}\text { WATER } \\
\text { LEVEL }\end{array}$ \\
\hline t $\begin{array}{l}01 \\
29\end{array}$ & $\begin{array}{l}11.28 \\
11.39\end{array}$ & $\begin{array}{ll}\text { Dec } & 11 \\
\text { Jan } 28\end{array}$ & $\begin{array}{l}11.59 \\
12.02\end{array}$ & $\begin{array}{ll}\text { Mar } & 11 \\
\text { May } 10\end{array}$ & $\begin{array}{l}12.11 \\
11.69\end{array}$ & $\begin{array}{l}\text { Jun } 27 \\
\text { Aug } 07\end{array}$ & $\begin{array}{l}10.79 \\
10.50\end{array}$ & Sep 17 & 10.87 \\
\hline
\end{tabular}

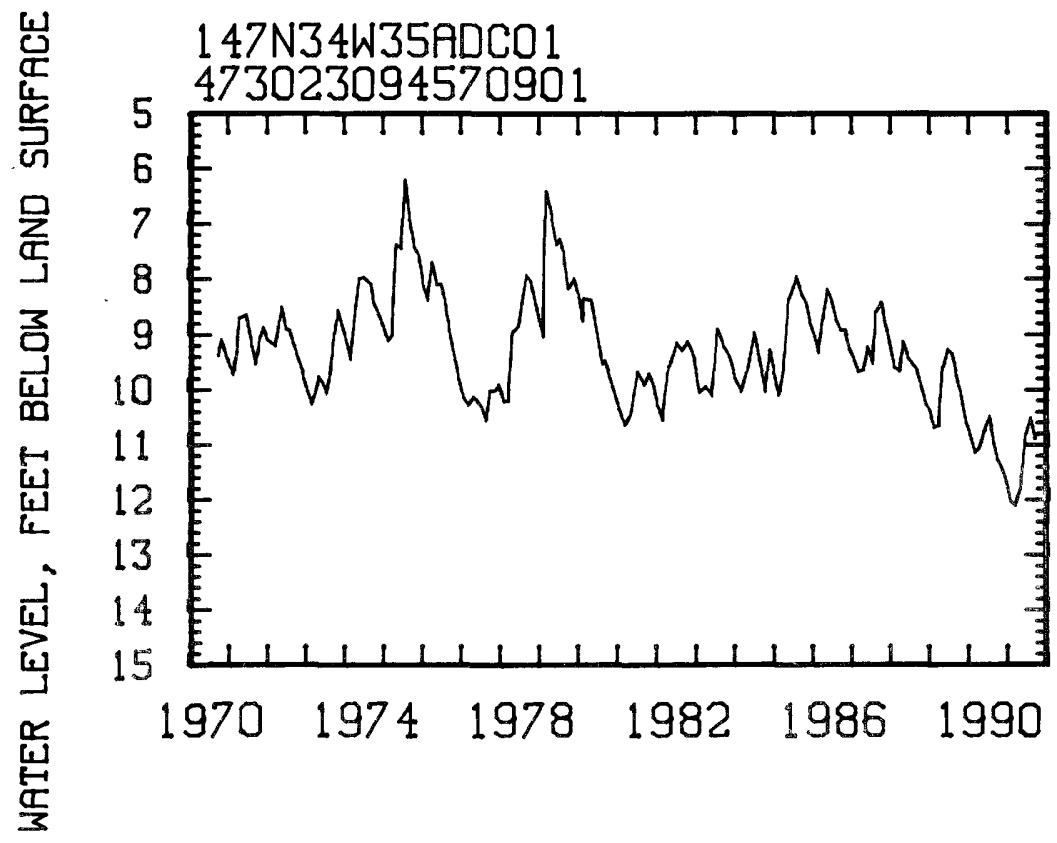

451517096104501, Local number, 121N44W27CCC01.

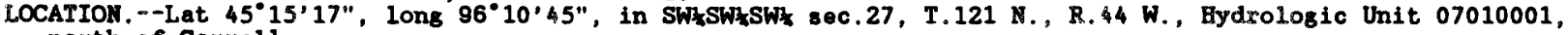
north of Correll.

Omer: U.S. Geological Survey

AQUIFER. - - Surficial sand and gravel of Pleistocene Age.

WELL CBARACTERISTICS.--Bored observation water-table well, diemeter 1 it in., depth 16 ft, screened 14 to $16 \mathrm{ft}$.

DATUM.--Aititude of land-surface datum is 1,018 ft. Measuring point: Top of casing, $3.10 \mathrm{ft}$ above landsurface datum.

PERIOD OF RECORD. --September 1972 to February 1974, August 1976 to current year.

EXTREMES FOR PERIOD OF RECORD. --Highest water level, 2.16 ft below land-surface datum, June 12, 1986

lowest, 8.99 ft below land-surface datum, Feb. 8, 1977.

WATER LEYEL, IN FEET BELOW LAAN-SURFACE DATUM, WATER FILAR OCTOBER 1990 TO SEFTEMBER 1991

$\begin{array}{crcrcrrr}\text { DATE } & \begin{array}{r}\text { WATER } \\ \text { LEVEI }\end{array} & \text { DATE } & \begin{array}{r}\text { WATER } \\ \text { LEVEL }\end{array} & \text { DATE } & \begin{array}{c}\text { WATER } \\ \text { LEVEL }\end{array} & \text { DATE } & \begin{array}{r}\text { WATEVR } \\ \text { LEVEL }\end{array} \\ \text { Nov 1.6 } & 7.78 & \text { Jan 18 } & 8.07 & \text { Apr 12 } & 8.10 & \text { Jul } 24 & \mathbf{6 . 2 2}\end{array}$


GROUID-WATER LEVELS

BIG STONE COUNTY--Continued

453330096420201. Local number, 124N48W17MMA01.

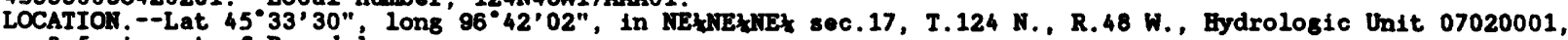
$0.5 \mathrm{mi}$ east of Beardsiey.

Owner: U.S. Geolosical Survey.

AQUIFER. --Buried sand of Pleistocene Age.

WELI CHARACTERISTICS.--Drilled observation artesian well, diameter 2 in., depth 282 ft, screened 242 to $282 \mathrm{ft}$.

DATUY.--Aititude of land-surface datum is 1,086.8 ft National Geodetic Vertical Datum of 1929. Measuring point: Top of casing, $3.60 \mathrm{ft}$ above land-surface datum.

PERIOD OF RECORD. --November 1978 to current year.

EXTRERES FOR PERIOD OF RECORD.--Highest water leve1, 9.77 ft below land-surface datum, Mar. 23, 1987; lowest, $22.10 \mathrm{ft}$ below land-surface datum, July $21,1988$.

WATER LEVEL, IN FEET BELON LAND-SURFACE DATUM, WATER YEAR OCTOBER 1990 TO SEPTEMBER 1991

$\begin{array}{cccccc}\text { DATE } & \begin{array}{c}\text { WATER } \\ \text { LEVEL }\end{array} & \text { DATE } & \begin{array}{c}\text { WATER } \\ \text { LEVEL }\end{array} & \text { DATE } & \begin{array}{c}\text { WATER } \\ \text { LEVEL }\end{array} \\ \text { Oct 15 } & 16.23 & \text { Feb 12 } & 16.23 & \text { Jul 24 } & 20.45 \\ \text { Dec 11 } & 16.13 & \text { May 29 } & 16.61 & \text { Sep 10 } & 16.80\end{array}$

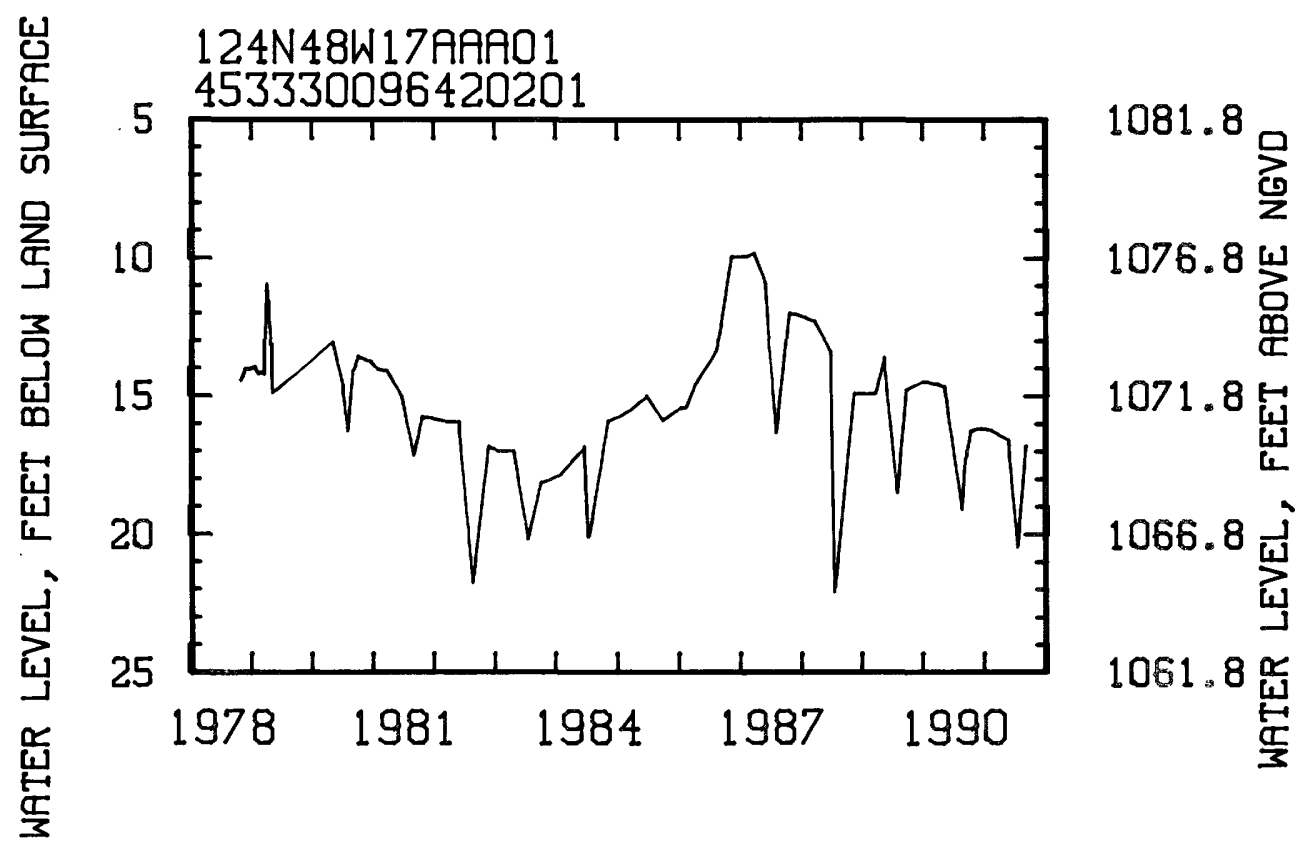

BLUE EARTH COUNTY

440050094102801. Local number, 106N28W03DBA01.

LOCATION. - Lat $44^{\circ} 00^{\prime} 50^{\prime \prime}$, long $94^{\circ} 10^{\prime} 28^{\prime \prime}$, In NEłWW

at Farmland Industries Ammonia Plant, 3.2 mi north of Vernon Center.

Omer: Farmland Industries.

AQUIFER. - - ronton-Galesville Sandstones of Late Cambrian Age.

WELI CAARACTERISTICS.--Drilled unused artesian well, diemeter 16 in., dopth $390 \mathrm{ft}$, cased to $150 \mathrm{ft}$

DATUM.--Altitude of land-surface datum is 1,005 ft. Measuring point: Top of recorder floor, $2.00 \mathrm{ft}$ above land-surface datum.

PERIOD OF RECORD.--October 1973 to current year.

EXTREMES FOR PERIOD OF RECORD.--Highest water level, 71.81 ft below land-surface datum, Apr. 26, 1983;

lowest, $76.73 \mathrm{ft}$ below land-surface datum, Oct. 18, 1989.

WATER LEVEL, IN FEET BELON LAND-SURFACE DATAUM, WATER YEAR OCTOBER 1990 TO SEPTEMBER 1991

$\begin{array}{rrrrrrrr}\text { DATE } & \begin{array}{c}\text { WATER } \\ \text { LEVEL }\end{array} & \text { DATE } & \begin{array}{c}\text { WATER } \\ \text { LEVEL }\end{array} & \text { DATE } & \begin{array}{l}\text { WATER } \\ \text { LEVEL }\end{array} & \text { DATE } & \begin{array}{r}\text { WATER } \\ \text { LEVEL }\end{array} \\ \text { Nov 20 } & 75.46 & \text { Mar } 05 & 75.37 & \text { Aus } 05 & 74.37 & \text { Sep } 05 & 74.31 \\ \text { Jan 31 } & 75.88 & 10 & 75.72 & 10 & 74.18 & 10 & 74.43 \\ \text { Feb } 05 & 75.88 & 15 & 75.76 & 15 & 74.01 & 15 & 74.13 \\ 10 & 75.91 & 20 & 75.49 & 20 & 74.06 & 20 & 74.48 \\ 15 & 75.93 & 25 & 75.43 & 25 & 74.14 & 25 & 74.27 \\ 20 & 75.73 & \text { May 29 } & 74.29 & 31 & 74.32 & 30 & 74.40 \\ 25 & 75.96 & \text { Jul 25 } & 74.10 & & & & \end{array}$


441134093505301. Local number, 108N25W04BBC01.

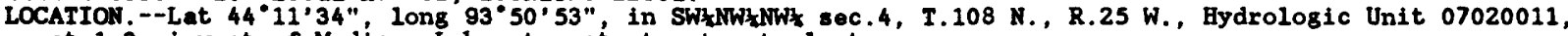
at $1.3 \mathrm{mi}$ west of Madison Lake at waste treatment plant.

Omer: City of Madison Lake.

AQUIFER.--Prairie du Chien Group of Early Ordovician Age.

WELL CHARACTERISTICS.--Drilled domestic artesian well, diameter $6 \mathrm{in}$, depth $313 \mathrm{ft}$, cased to $296 \mathrm{ft}$.

DATUM.--Altitude of iand-surface datum is 1,036 ft. Measuring point: Top of casing, $1.60 \mathrm{ft}$ above land-surface datum.

PERIOD OF RECORD.--May 1979 to current year.

EXIREMES FOR PERIOD OF RECORD.--Highest water level, $92.52 \mathrm{ft}$ below land-surface datum, July 17, 1986 ;

lowest, $95.62 \mathrm{ft}$ below land-surface datum, July 25, 1990.

WATER LEVEL, IN FEET BELOW LAND-SURFACE DATUM, WATER YEAR OCTOBER 1990 TO SEPTEMBER 1991

$\begin{array}{cccccc}\text { DATE } & \begin{array}{c}\text { WATER } \\ \text { LEVEL }\end{array} & \text { DATE } & \begin{array}{c}\text { WATER } \\ \text { LEVEL }\end{array} & \text { DATE } & \begin{array}{c}\text { WATER } \\ \text { LEVEL }\end{array} \\ \text { Nov 20 } & 95.55 & \text { May 29 } & 95.51 & \text { Sep 25 } & 94.94 \\ \text { Mar 26 } & 95.43 & \text { JuI 24 } & 95.34 & & \end{array}$

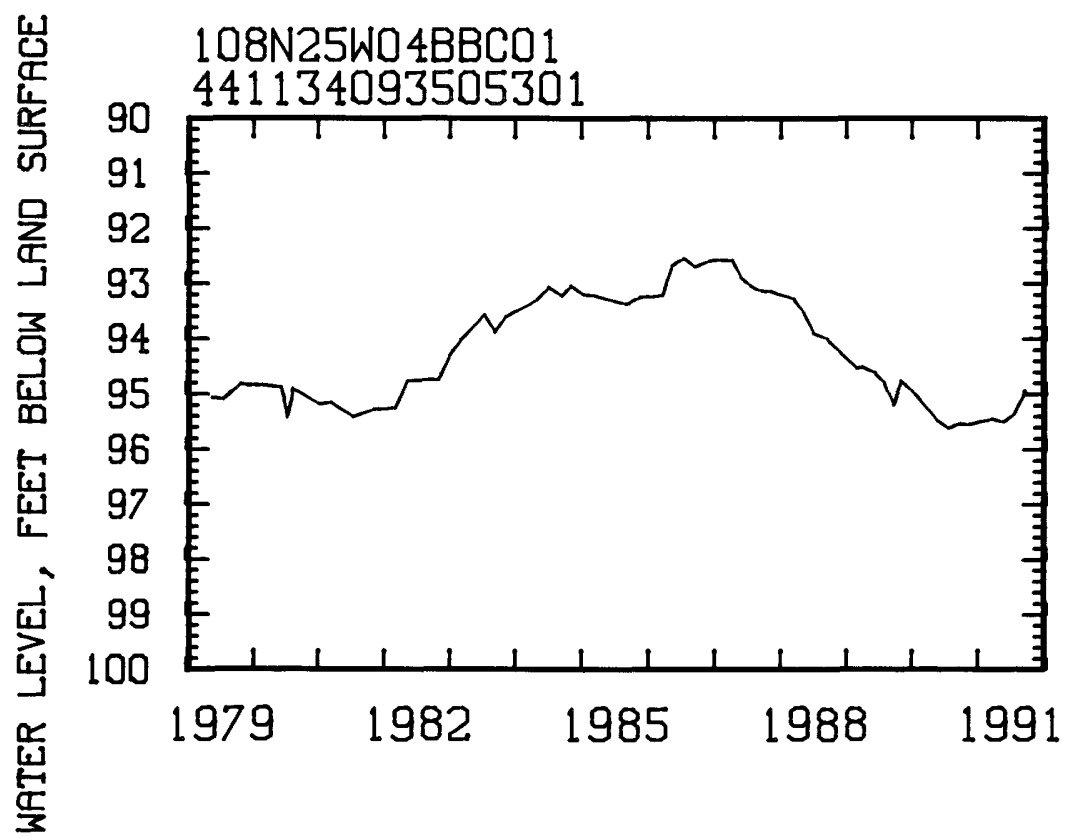

946 
GROUND-WATER LEVELS

BROWN COUNTY

441800094434301 . Local number, 110N32W30DDB01.

LOCATION, - Lat $44^{\circ} 18^{\prime} 00^{\prime \prime}$, long $94^{\circ} 43^{\prime} 43^{\prime \prime}$, in NWł SE $\}_{4}$ SE sec.30, T.110 N., R.32 W., Hydrologic Unit 07020008 ,

in Sleepy Eye at hospital.

Owner: City of Sleepy Eye.

AQUIFER.--Buried sand and gravel of Pleistocene Age.

WELI CHARACTERISTICS.--Drilled observation artesian well, diameter 2 in., depth $176 \mathrm{ft}$.

DATUM.--Altitude of iand-surface datum is $1,030 \mathrm{ft}$. Measuring point: Top of casing, $i .30 \mathrm{ft}$ above landsurface datum.

REMARKS. - Water level affected by pumping from nearby wells.

PERIOD OF RECORD.--August 1976 to current year.

EXTREMES FOR PERIOD OF RECORD.--Highest water ievel, $46.80 \mathrm{ft}$ below land-surface datum, Apr. 1, 1987;

lowest, $118.1 \mathrm{ft}$ below land-surface datum, Sept.' 15, 1976.

WATER LEVEL, IN FEET BELOW LAND-SURFACE DATUM, WATER YEAR OCTOBER 1990 TO SEPTEMBER 1991

$\begin{array}{cccccc}\text { DATE } & \begin{array}{c}\text { WATER } \\ \text { LEVEL }\end{array} & \text { DATE } & \begin{array}{c}\text { WATER } \\ \text { LEVEL }\end{array} & \text { DATE } & \begin{array}{c}\text { WATER } \\ \text { LEVEL }\end{array} \\ \text { Oct 01 } & 62.20 & \text { Feb 04 } & 52.90 & \text { May 01 } & 50.90 \\ \text { Nov 01 } & 57.70 & \text { Mar 01 } & 52.00 & \text { Jun 06 } & 52.10 \\ \text { Dec 01 } & 54.70 & 26 & 51.46 & \text { Jul 01 } & 52.70 \\ \text { Jan 02 } & 54.70 & \text { Apr 01 } & 51.70 & \text { Aug 01 } & 56.10 \\ & & & & \text { Sep 03 } & 59.70\end{array}$

CHIPPEWA COUNTY

450447095490101 . Local number, 119N41W29DDD01.

LOCATION, - Lat $45^{\circ} 04^{\prime} 47^{\prime \prime}$, long $95^{\circ} 40^{\prime} 01^{\prime \prime}$, in SE $\Varangle$ SE $\frac{1}{2}$ SE sec.29, T.119 N., R.41 W., Hydrologic Unit 07020005 , $5 \mathrm{mi}$ north of Watson.

Omer: U.S. Geological Survey.

AQUIFER. - Surficial sand and gravel of Pleistocene Age.

WELL CHARACTERISTICS. --Bored observation water-table well, diameter $1 \frac{1}{\mathrm{l}} \mathrm{in} .$, depth $19 \mathrm{ft}$, screened 17 to

DATUM. - Altitude of land-surface datum is $992 \mathrm{ft}$. Measuring point: Top of casing, $3.75 \mathrm{ft}$ above landsurface datum.

PERIOD OF RECORD. --September 1972 to February 1974 January 1977 to current year.

EXTREMES FOR PERIOD OF RECORD.--Highest water levei, 3.43 ft below land-surface datum, Apr. 10, 1984; lowest, $9.06 \mathrm{ft}$ below land-surface datum, Feb. 8, 1977.

WATER LEVEL, IN FEET BELOW LAND-SURFACE DATUM, WATER YEAR OCTOBER 1990 TO SEPTEMBER 1991

$\begin{array}{crcrcrrr}\text { DATE } & \begin{array}{r}\text { WATER } \\ \text { LEVEL }\end{array} & \text { DATE } & \begin{array}{r}\text { WATER } \\ \text { LEVEL }\end{array} & \text { DATE } & \begin{array}{r}\text { WATER } \\ \text { LEVEL }\end{array} & \text { DATE } & \begin{array}{r}\text { WATER } \\ \text { LEVEL }\end{array} \\ \text { Oct } 11 & 4.90 & \text { Apr } 12 & 3.85 & \text { Jun } 21 & 3.98 & \text { Sep } 04 & 4.74\end{array}$

450631095562201 Local number, 119N42W17DDD01.

LOCATION. --Lat $45^{\circ} 06^{\prime} 31^{\prime \prime}$, long $95^{\circ} 56^{\prime} 22^{\prime \prime}$, in SEłSE SE $_{4}$ sec.17, T.119 N., R.42 W., Hydrologic Unit 07020001 , west of Milan.

Omer: U.S. Geological Survey.

AQUIFER. --Surficial silt of Pleistocene Age.

WELL CHARACTERISTICS. --Bored observation water-table well, diameter $1 \frac{1}{4}$ in., depth 19 ft, screened 17 to $19 \mathrm{ft}$.

DATUM.--Altitude of land-surface datum is 1,027 ft. Measuring point: Top of casing, 4.50 ft above landsurface datum.

PERIOD OF RECORD. --September 1972 to October 1973, April 1977 to current year.

EXTREMES FOR PERIOD OF RECORD.--Highest water level, $1.50 \mathrm{ft}$ below land-surface datum, May 7, 1973; lowest, $17.46 \mathrm{ft}$ below land-surface datum, Apr. $i$, 1977.

WATER LEVEL, IN FEET BELOW LAND-SURFACE DATUM, WATER YEAR OCTOBER 1990 TO SEPTEMBER 1991

$\begin{array}{crcrcr}\text { DATE } & \begin{array}{r}\text { WATER } \\ \text { LEVEL }\end{array} & \text { DATE } & \begin{array}{c}\text { WATER } \\ \text { LEVEL }\end{array} & \text { DATE } & \text { WATER } \\ \text { Oct 11 } & 5.80 & \text { Jan 18 } & 8.72 & \text { Jun 28 } & 2.58 \\ \text { Nov 07 } & 9.70 & \text { Apr 12 } & 4.97 & \text { Sep 04 } & 4.45\end{array}$


453125092445401. Local number, 035N19W17BDB01.

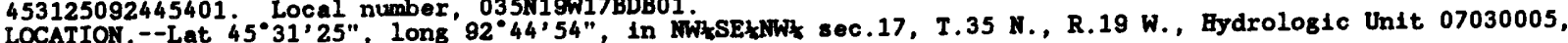

at Wild River State Park.

Omer: State of Minnesota.

AQUIFER. - Mount SImon Sandstone of Late Cambrian Age.

WFII CHARACTERISTICS, --Drilled domestic artesian meli, diameter $6 \mathrm{ln}$., depth $270 \mathrm{ft}$, cased $230 \mathrm{ft}$.

DATUM - - Altitude of iand-surface datum is $820 \mathrm{ft}$. Measuring point: Top of casing, 0.70 ft above land-

surface datum.

PERIOD OF RECORD:--October 1980 to current year.

EXTRRAES FOR PERIOD OP RECORD.--Highest water lovel, $40.06 \mathrm{ft}$ below land-surface datum, Oct. 20, 1986;

lowest, $44.19 \mathrm{ft}$ below land-surface datum, June 8, 1983.

WATER LEVEL, IN FEET BELON LAND-SURFACE DATUM, WATER YEAR OCTOBER 1990 TO SEPTEMBER 1991

$\begin{array}{cccc}\text { DATE } & \begin{array}{c}\text { WATER } \\ \text { LEVEL }\end{array} & \text { DATE } & \begin{array}{c}\text { WATER } \\ \text { LEVEL }\end{array} \\ \text { Aug 02 } & 41.75 & \text { Sep } 30 & 41.80\end{array}$

CROW WIMG COUNTY

463006094131201. Local number, 135N28W16CCD01.

LOCATION, --Lat $46^{\circ} 30^{\prime} 06^{\prime \prime}$, Long $94^{\circ} 13^{\prime} 12^{\prime \prime}$, in SEłSWłSWł sec.16, T.135 N., R.28 W., Hydrologic Unit 07010106, northwest of Merrifield.

Omer: U.S. Geological Survey.

AQUIFER. --Surficial sand of Pleistocene Age.

WELL CHARACTERISTICS. --Bored observation water-table well, diemeter 1t in., depth 18 ft, screened 16 to

DATUM.--Altitude of land-surface datum is 1,212 ft. Measuring point: Top of casing, $2.00 \mathrm{ft}$ above 1 andsurface datum.

PERIOD OF RECORD:--October 1970 to current year.

DXTREMES FOR PERIOD OF RECORD. --Highest mater level, 7.20 ft below land-surface datum, May 1, 1982

lowest. 11.38 ft below land-surface datum, Oct. 16, 1970, Feb. 11, 1977, Mar. 11, 1977.

WATER LEVEL, IN FEET BELOW LAND-SURFACE DATUM, WATER YEAR OCTOBER 1990 TO SEPTEMBER 1991

$\begin{array}{crrrrr}\text { DATE } & \begin{array}{r}\text { WATER } \\ \text { LEVEL }\end{array} & \text { DATE } & \begin{array}{r}\text { WATER } \\ \text { LEVEL }\end{array} & \text { DATE } & \begin{array}{r}\text { WATER } \\ \text { LEVEL }\end{array} \\ \text { Jan 31 } & 10.38 & \text { May } 03 & 9.89 & \text { Aug } 01 & 9.29 \\ \text { Feb 04 } & 10.40 & 06 & 9.78 & 06 & 9.33 \\ \text { Apr 03 } & 10.32 & 14 & 9.54 & 22 & 9.55 \\ 15 & 10.19 & \text { Jul } 02 & 9.48 & 26 & 9.59 \\ 22 & 10.08 & 11 & 9.34 & \text { Sep } 06 & 9.70 \\ & & 17 & 9.32 & 12 & 9.68 \\ & & 26 & 9.30 & 20 & 9.67 \\ & & & & 25 & 9.68\end{array}$

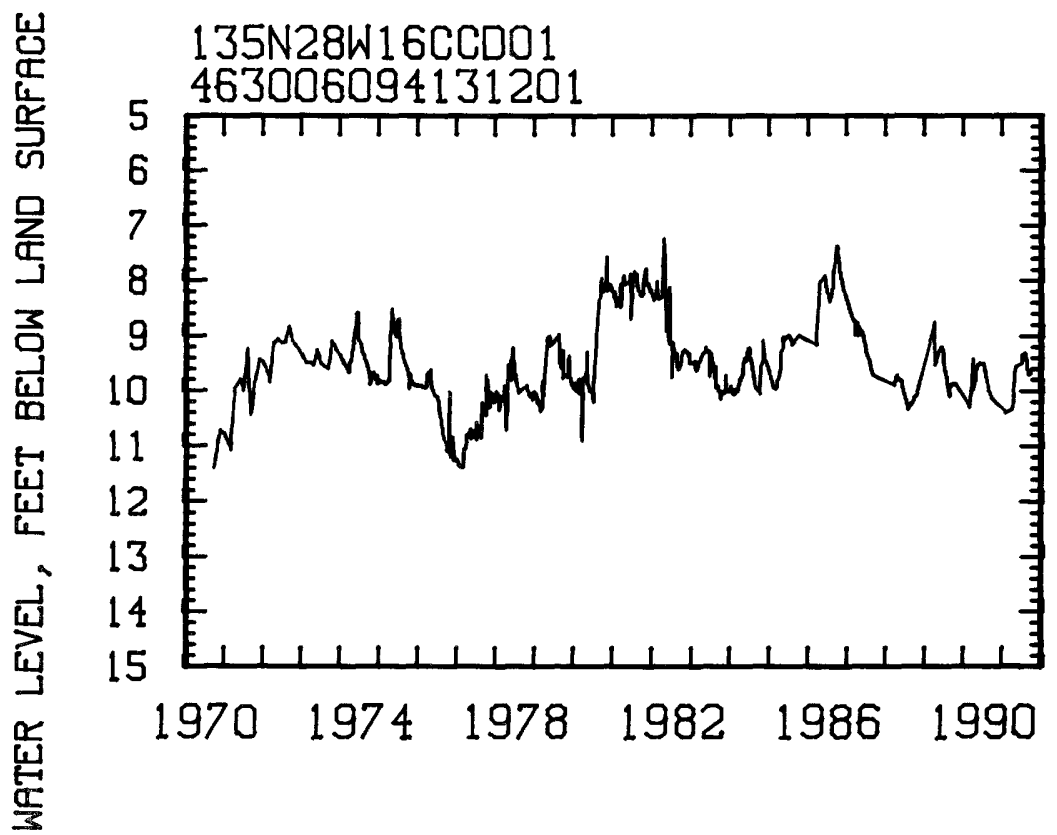


GROUND-WATER LEVELS

DAKOTA COUITY

445044093102401. Local number, 027N23W09ABD01.

LOCATION. --Lat $44^{\circ} 50^{\prime} 44^{\prime \prime}$, Long $93^{\circ} 10^{\prime} 24^{\prime \prime}$, In SEłWWZNEz sec.9, T.27 N., R.23 W., Hydrologic Unit 07020012 , at Eagan.

Ower: City of Eagan, Timberline Addition.

AQUIFER. - Jordan Sandstone of Late Cambrian Age.

WELL CBARACTERISTICS. - Drilled public-supply artesian we11, diameter $10 \mathrm{ln}$, depth 503 ft, cased to $401 \mathrm{ft}$.

DATUM.--Aititude of land-surface datum is $900 \mathrm{ft}$. Measuring point: Bole in well cap, $2.60 \mathrm{ft}$ above land-surface datum.

REMARKS. - Water-level affected by pumping.

PERIOD OF RECORD.--December 1965, Apri1 1966, December 1966, March 1967, December 1970, August 1971. August 1977 to current year.

EXTREMES FOR PERIOD OF RECORD.--Highest water level, $141.40 \mathrm{ft}$ below land-surface datum, Apr. 5, 1966; lowest, 173.87 ft below land-surface datum, July 17, 1991 .

WATER LEVEL, IN FEET BELOW LAND-SURFACE DATUM, WATER YEAR OCTOBER 1990 TO SEPTEMBER 1991

\begin{tabular}{|c|c|c|c|c|c|}
\hline DATE & $\begin{array}{l}\text { WATBR } \\
\text { LEVEL }\end{array}$ & DATE & $\begin{array}{l}\text { WATER } \\
\text { LEVEL }\end{array}$ & DATE & $\begin{array}{l}\text { WATER } \\
\text { LEVEL }\end{array}$ \\
\hline $\begin{array}{l}\text { Kov } 13 \\
\text { Jan } 08\end{array}$ & $\begin{array}{l}165.38 \\
161.80\end{array}$ & $\begin{array}{l}\text { Mar } 06 \\
\text { May } 08\end{array}$ & $\begin{array}{l}164.57 \\
166.80\end{array}$ & $\begin{array}{ll}\text { Jul } & 17 \\
\text { Sep } & 13\end{array}$ & $\begin{array}{l}173.87 \\
162.76\end{array}$ \\
\hline
\end{tabular}

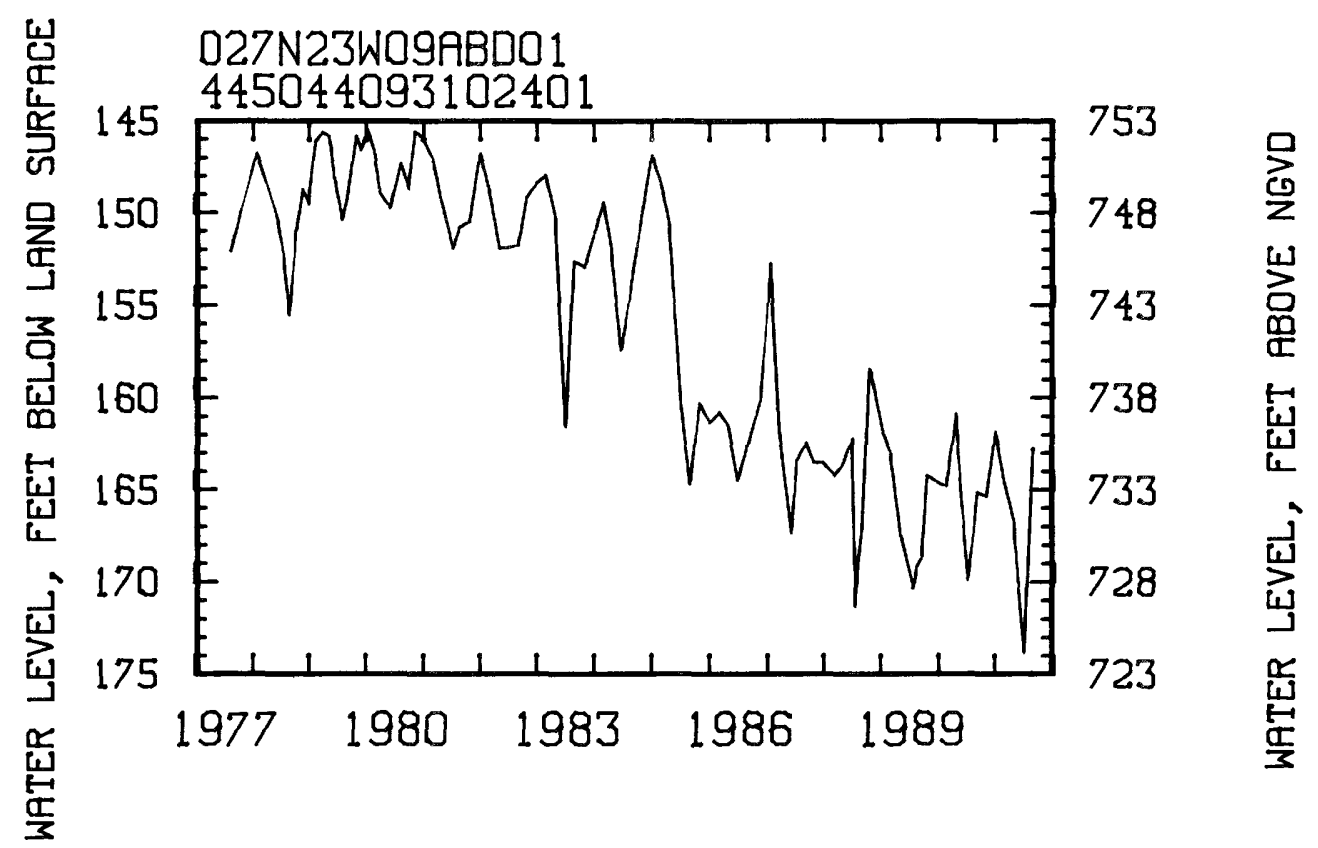


GROUHD-WATER LEVELS

DAKOTA COUNTY--Continued

445330093054301. Local number, 028N22W19DCC02.

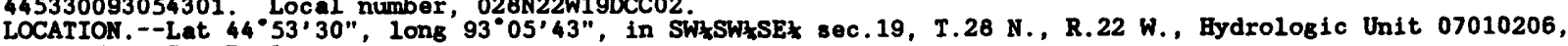

in West St. Paul.

Omer: U.S. Geological Survey, 2-N.

AOUTFRR --Prairie du Chien Group of Early Ordovician Ase.

WELI CBARACTERISTICS. -Drilled observation artesian mell, diameter 6 in., depth 539 ft, cased to 407 ft.

DATUM.--Land-surface datum is 1,036 ft National Geodetic vertical Datum of 1929 . Measuring point: Top of

casing, $2.60 \mathrm{ft}$ above land-surface datum.

REMARKS. - Water-level affected by regional pumping.

PERIOD OF RECORD.--January 1971 to current year.

EXIREMES FOR PERIOD OF RECORD.--Highest mater level, 309.13 ft below land-surface datum, Apr. 4, 1988; 10west,

328.0 ft below land-surface datum, July 31,1975 .

WATER LEVEL, IN FEET BELON LAND-SURFACE DATUM, WATER YEAR OCTOBER 1990 TO SEPTEMBER 1991

\begin{tabular}{|c|c|c|c|c|c|c|c|c|c|c|c|c|c|c|c|c|}
\hline DATE & $\begin{array}{l}\text { HATER } \\
\text { LEVEL }\end{array}$ & DA & & $\begin{array}{l}\text { WATER } \\
\text { LEVEL }\end{array}$ & DA & & $\begin{array}{l}\text { WATER } \\
\text { LEVEL }\end{array}$ & DA & & $\begin{array}{l}\text { WATXR } \\
\text { LEVEL }\end{array}$ & DA & & $\begin{array}{l}\text { WATRR } \\
\text { LEVIL }\end{array}$ & $D A$ & & $\begin{array}{l}\text { WATER } \\
\text { LEVEL }\end{array}$ \\
\hline $\begin{array}{r}05 \\
\text { Oct } \\
10 \\
15 \\
20 \\
25 \\
31 \\
\text { Nov } 05 \\
10 \\
15 \\
20 \\
25 \\
30\end{array}$ & $\begin{array}{l}313.61 \\
313.24 \\
312.50 \\
311.43 \\
312.13 \\
311.64 \\
311.19 \\
310.99 \\
311.10 \\
310.94 \\
310.75 \\
310.92\end{array}$ & Jan & $\begin{array}{l}05 \\
10 \\
15 \\
20 \\
25 \\
31 \\
05 \\
10 \\
15 \\
20\end{array}$ & $\begin{array}{l}310.86 \\
310.76 \\
310.84 \\
310.75 \\
310.85 \\
310.51 \\
310.44 \\
311.10 \\
310.28 \\
310.19\end{array}$ & $\begin{array}{l}\text { Mar } \\
\text { Apr }\end{array}$ & $\begin{array}{l}10 \\
15 \\
20 \\
25 \\
31 \\
05 \\
10 \\
15 \\
20 \\
25 \\
30\end{array}$ & $\begin{array}{l}310.54 \\
310.84 \\
310.46 \\
309.99 \\
310.53 \\
311.64 \\
312.75 \\
310.65 \\
310.92 \\
312.46 \\
311.36\end{array}$ & $\begin{array}{l}\text { May } \\
\text { Jun }\end{array}$ & $\begin{array}{l}05 \\
10 \\
15 \\
20 \\
25 \\
31 \\
05\end{array}$ & $\begin{array}{l}310.32 \\
311.62 \\
313.41 \\
312.31 \\
313.95 \\
314.39 \\
315.87\end{array}$ & $\begin{array}{l}\text { Jul } \\
\text { Aus }\end{array}$ & $\begin{array}{l}20 \\
25 \\
31 \\
05 \\
10 \\
15 \\
20 \\
25 \\
31\end{array}$ & $\begin{array}{l}316.32 \\
317.73 \\
315.71 \\
314.12 \\
314.40 \\
318.21 \\
316.86 \\
315.25 \\
318.03\end{array}$ & Sep & $\begin{array}{l}05 \\
10 \\
15 \\
20 \\
25 \\
30\end{array}$ & $\begin{array}{l}317.24 \\
314.67 \\
313.80 \\
312.36 \\
311.53 \\
312.05\end{array}$ \\
\hline
\end{tabular}

443146093002201, Local number, 112N18W08ABA01.

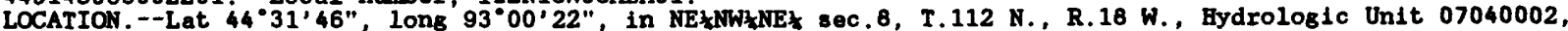
northeast of Randolph.

Omer: U.S. Geological Survey

AQUIFMR. - Surficial outwash sand and gravel of Pleistocene Ase.

WELL CHARACTERISTICS.--Bored observation water-table well, diameter 1 in., depth 44 ft, screened 42 to $44 \mathrm{ft}$.

DATUM.--Altitude of land-surface datum is $880 \mathrm{ft}$, Measuring point: Top of casing, $3.40 \mathrm{ft}$ above 1 andsurface datum.

PERIOD OF RECORD.--April 1977 to current year.

EXTREMES FOR PERIOD OF RECORD, - Highest pater level, 18,13 ft below land-surface datum, May 3 , 1983;

lowest, 23.80 ft below land-surface datum, Feb. 21, 1979.

WATER LEVEL, IN FEET BELOW LAND-SURFACE DATUM, WATER YEAR OCTOBER 1990 TO SEPTEMBER 1991

$\begin{array}{cccccc}\text { DATE } & \begin{array}{c}\text { WATER } \\ \text { LEVEL }\end{array} & \text { DATE } & \begin{array}{l}\text { WATER } \\ \text { LEVEL }\end{array} & \text { DATE } & \begin{array}{c}\text { WATER } \\ \text { LEVEL }\end{array} \\ \text { Nov 09 } & 20.59 & \text { Mar 12 } & 21.51 & \text { Ju1 26 } & 20.00 \\ \text { Jan 09 } & 21.24 & \text { May 09 } & 21.08 & \text { Sep 12 } & 20.43\end{array}$

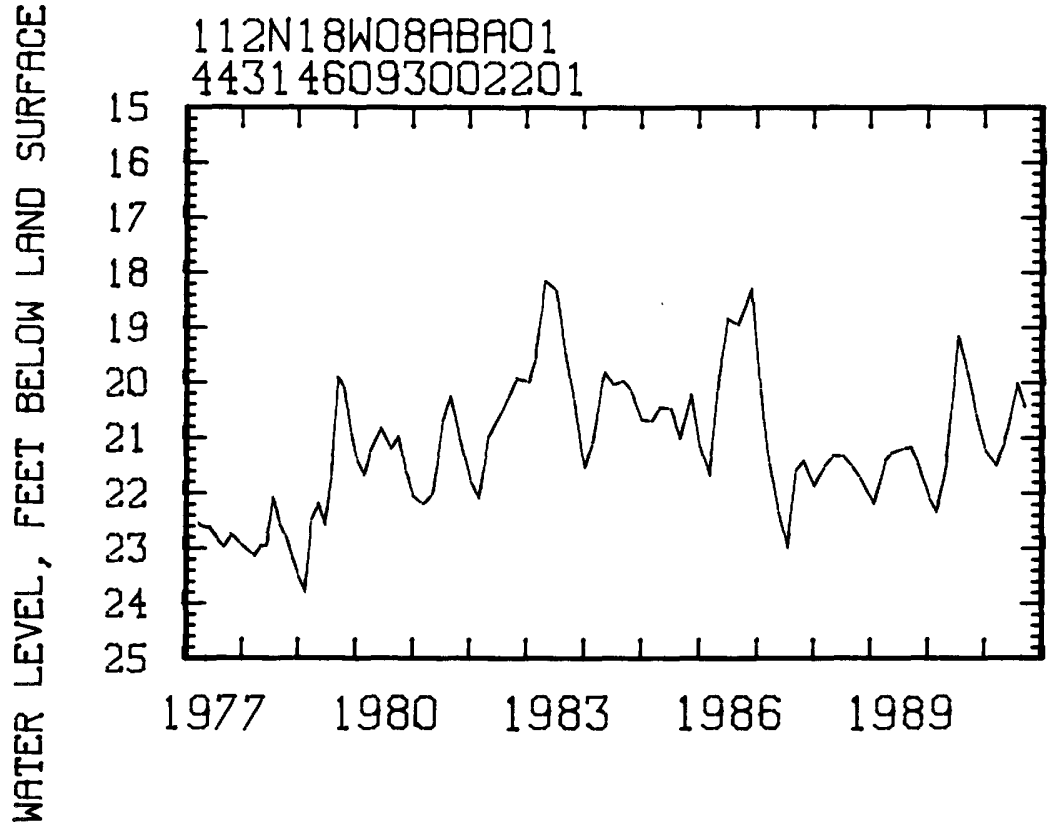

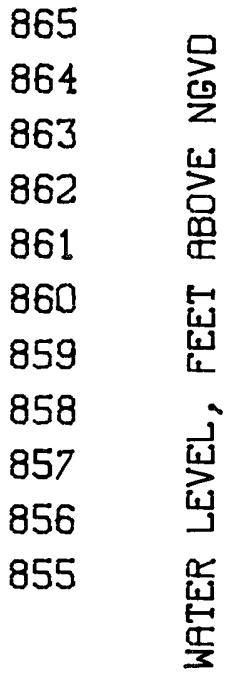


443134093010601. Local number, 112N18N08BBC01.

LOCATION. - Lat $44^{\circ} 31^{\prime} 34^{\prime \prime}$, long $93^{\circ} 01^{\prime} 06^{\prime \prime}$, in sirtwWtwW sec.8, T.112 N., R.18 W., Hydrologic Unit 07040002 , at Randolph Fire Station

Omer: City of Randoliph.

AQUIFER. - Prairie du Chien Group of Early Ordovician Age.

WELL CBARACTERISTICS.--Drilled fire protection artesian we11, diameter 10 in., depth 150 ft, cased to $64 \mathrm{ft}$.

DATUM. --Altitude of land-surface datum $18883 \mathrm{ft}$. Measurins point: Top of 3/4-1nch breather pipe, $2.20 \mathrm{ft}$ above land-surface datum.

PERIOD OF RECORD. - July 1873 to current year.

EXTREMES FOR PERIOD OF RECORD.--Highest water level, 10.47 ft below land-surface datum, May 3, 1983;

lowest, $19.70 \mathrm{ft}$ below land-surface datum, Aus. 11, 1977.

WATER LEVEL, IN FEET BELON LAND-SURFACE DATUM, WATER YEAR OCTOBER 1990 TO SEPTEMBER 1991

$\begin{array}{cccccc}\text { DATE } & \begin{array}{c}\text { WATER } \\ \text { LEVEL }\end{array} & \text { DATE } & \begin{array}{c}\text { WATER } \\ \text { LEVEL }\end{array} & \text { DATE } & \text { WATER } \\ \text { LEVEL }\end{array}$

442830093085201. Local number, 112N19N30DBD01.

LOCATION. - Lat $44^{\circ} 28^{\prime} 30^{\prime \prime}$, Long $93^{\circ} 08^{\prime} 52^{\prime \prime}$, in SEłWWzSEł sec.30, T.112 N., R.19 N., Hydrologic Unit 07040002, at Northfield waste treatment plant. Omer: City of Northfield.

AQUIFER. --Jordan Sandstone of Late Cambrian Age.

WELl CEARACTERISTICS.--Drilled domestic artesian well, diameter 6 in., depth 275 ft, cased to 212 ft.

DATUY.--Altitude of land-surface datum is $890 \mathrm{ft}$. Measuring point: Center of pressure guage, 2.05 ft above land-surface datum.

PERIOD OF RECORD.--May 1979 to current year.

EXIREMES FOR PERIOD OF RECORD.--Highest water level, 18.54 ft above land-surface datum, July 12, 1983; lowest, $6.66 \mathrm{ft}$ above land-surface datum, Mar. 12, 1991.

WATER LEVEL, IN FEET ABOVE LAND-SURFACE DATUM, WATER YEAR OCTOBER 1990 TO SEPTEMBER 1991

$\begin{array}{cccccc}\text { DATE } & \begin{array}{c}\text { WATER } \\ \text { LEVEL }\end{array} & \text { DATE } & \begin{array}{c}\text { WATER } \\ \text { LEVEL }\end{array} & \text { DATE } & \begin{array}{c}\text { WATER } \\ \text { LEVEL }\end{array} \\ \text { Nov 09 } & +7.81 & \text { Mar 12 } & +6.66 & \text { Jul } 26 & +8.38 \\ \text { Jan 15 } & +7.12 & \text { May 09 } & +8.50 & \text { Sep 18 } & +8.04\end{array}$

443645093014701. Local number, 113N18\%07BAC01.

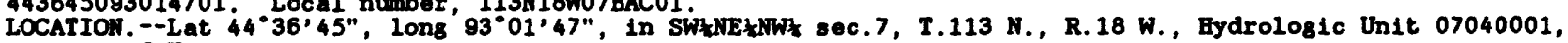
west of Hempton.

Omer: Eugene Dohmen.

AQUIFER. - Prairie du Chien Group of Early Ordovictan Age and Jordan Sandstone of Late Cambrian Age.

WELL CEARACTERISTICS.--Drilled irrigation artesian wel1, dianeter 16 in., depth $325 \mathrm{ft}$, cased to $65 \mathrm{ft}$.

DATUM. --Altitude of land-surface datum is $915 \mathrm{ft}$. Measuring point: Hole in pump base, 1.60 ft above land-surface datum.

PERIOD OF RECORD.--April 1977 to August 1977, January 1978, June 1979 to current year.

EXTRRMES FOR PERIOD OF RECORD.--Highest water level, 23.15 ft below land-surface datum, Dec. 4, 1986; lowest, $33.19 \mathrm{ft}$ below land-surface datum, Aug. 12, 1977 .

WATER LEVEL, IN FEET BELOW LAND-SURFACE DATUM, WATER YEAR OCTOBER 1990 TO SEPTEMER 1991

$\begin{array}{cccccc}\text { DATE } & \begin{array}{c}\text { WATER } \\ \text { LEVEL }\end{array} & \text { DATE } & \begin{array}{l}\text { WATER } \\ \text { LEVEL }\end{array} & \text { DATE } & \begin{array}{l}\text { WATER } \\ \text { LEVEL }\end{array} \\ \text { Mov 09 } & \mathbf{3 0 . 4 2} & \text { Mar 12 } & \mathbf{3 1 . 0 6} & \text { Sep 12 } & \mathbf{3 1 . 7 8} \\ \text { Jan 15 } & \mathbf{3 0 . 8 2} & \text { May 09 } & \mathbf{3 0 . 8 5} & & \end{array}$


205092500001. Local number, 114N17W10AM01.

LOCATION.-Lat "42'05", long 92"50'00", in NEłNEłNEł sec.10, T.114 N., R.17 W., Hydrologic Unit 07040001, southeast of Hastings.

Omer: John Conzenius.

AOUIFER. - Prairie du Chien Group of Early Ordovician Aga.

WELL CBARACTERISTICS, -Drilled unused artesian well, diameter 4 in., depth $151 \mathrm{ft}$, depth of casing unknown.

DATUA.--Altitude of land-surface datum is 827 ft. Measuring point: Top of platform, 2.50 ft above landsurface datum.

PERIOD OF RECORD. --Apr 11976 to current year.

EXTRERES FOR PERIOD OF RECORD. - Highest water leve1, 94.10 ft below land-surlace datum, Mar. 31, 1987; 10west, 107. It below land-surface datum, Mar. 12, 1978.

WATER LEVEL, IN FEET BELON LAND-SURFACE DATAUM, WATER YEAR OCTOBER 1990 TO SEPTEMBR 1981

\begin{tabular}{|c|c|c|c|c|c|c|c|c|c|c|c|c|c|c|c|c|c|}
\hline DAT & TE & $\begin{array}{l}\text { WATLR } \\
\text { LEVAL }\end{array}$ & DAI & & $\begin{array}{l}\text { WATAR } \\
\text { LEVEL }\end{array}$ & DAI & & $\begin{array}{l}\text { WATAR } \\
\text { LEVEL }\end{array}$ & DAT & & $\begin{array}{l}\text { WATER } \\
\text { LEVRL }\end{array}$ & DAT & & $\begin{array}{l}\text { WATER } \\
\text { LEVIR }\end{array}$ & DAI & & $\begin{array}{l}\text { WATER } \\
\text { LEVEL }\end{array}$ \\
\hline Nov & $\begin{array}{l}05 \\
10 \\
15 \\
20 \\
25 \\
31 \\
05 \\
10 \\
15 \\
20 \\
25 \\
30\end{array}$ & $\begin{array}{l}101.87 \\
101.94 \\
102.00 \\
101.88 \\
102.03 \\
101.90 \\
101.86 \\
102.00 \\
101.89 \\
101.88 \\
102.05 \\
101.92\end{array}$ & $\operatorname{Jan}$ & $\begin{array}{l}05 \\
10 \\
15 \\
20 \\
25 \\
31 \\
05 \\
10 \\
15 \\
20 \\
25 \\
31\end{array}$ & $\begin{array}{l}101.90 \\
102.03 \\
101.99 \\
102.09 \\
102.17 \\
102.06 \\
102.07 \\
102.11 \\
102.05 \\
102.23 \\
102.27 \\
102.24\end{array}$ & Mar & $\begin{array}{l}05 \\
10 \\
15 \\
20 \\
25 \\
28 \\
05 \\
10 \\
15 \\
20 \\
25 \\
31\end{array}$ & $\begin{array}{l}102.18 \\
102.17 \\
102.31 \\
102.17 \\
102.30 \\
102.04 \\
101.95 \\
102.35 \\
102.33 \\
102.09 \\
102.32 \\
102.31\end{array}$ & Apr & $\begin{array}{l}05 \\
10 \\
15 \\
20 \\
25 \\
30 \\
05 \\
10 \\
15 \\
20 \\
25 \\
31\end{array}$ & $\begin{array}{l}102.15 \\
102.36 \\
102.19 \\
102.34 \\
102.26 \\
102.33 \\
102.33 \\
102.40 \\
102.32 \\
102.36 \\
102.28 \\
102.29\end{array}$ & Jul & $\begin{array}{l}05 \\
10 \\
15 \\
20 \\
25 \\
30 \\
05 \\
10 \\
15 \\
20\end{array}$ & $\begin{array}{l}102.51 \\
102.28 \\
102.41 \\
102.52 \\
102.38 \\
103.07 \\
102.76 \\
103.18 \\
103.06 \\
103.30\end{array}$ & Sep & $\begin{array}{l}15 \\
20 \\
25 \\
30\end{array}$ & $\begin{array}{l}103.28 \\
103.47 \\
103.28 \\
103.38\end{array}$ \\
\hline
\end{tabular}

404092521801 LOCS1 numbar, 114N17W16CBBO1.

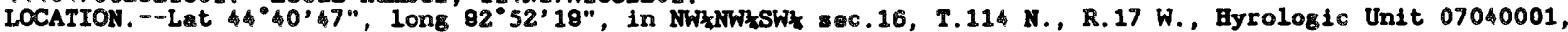
Kirby Avanu*, $0.5 \mathrm{mi}(0.8 \mathrm{~km})$ north of $180 \mathrm{th}$ Street. Omer: Jim Bunek. Construction Company.

AQUIFER - -Surficis sand and gravel of Pleistocons Aso

WELI CHARACTERISTICS.--Drilled domest1c water-table wal1, diametar in., depth 170 ft, screaned 164 to $170 \mathrm{ft}$.

DATUM,--Aititude of land-suxface datum 1s $823 \mathrm{ft}$. Measuring point: Top of casing, 1.10 ft above 1 andsur Iacs datum.

PERIOD OF RECORD.--March 1976, March 1977 to currant yaar.

EXTREDS FOR PERIOD OF RECORD, - - B1 ghest water lave1, 73.32 ft balow land-surface datum, Mar. 31, 1987; lowest, 87.75 ft below land-surface datum, Jun: 27,1878 .

WATER LEVEL, IN FEET BELOW LAND-SURFACE DATUM, WATER YEAR OCTOBER 1990 TO SEPTEMBER 1981

$\begin{array}{cccccc}\text { DATE } & \begin{array}{c}\text { WATER } \\ \text { LEVEL }\end{array} & \text { DATE } & \begin{array}{c}\text { WATER } \\ \text { LEVEL }\end{array} & \text { DATE } & \begin{array}{c}\text { WATER } \\ \text { LEVEL }\end{array} \\ \text { Nov 07 } & \mathbf{8 2 . 7 5} & \text { Mar 05 } & \mathbf{8 2 . 1 0} & \text { Ju1 26 } & \mathbf{8 4 . 0 5} \\ \text { Jan 09 } & \mathbf{8 2 . 2 5} & \text { May 07 } & \mathbf{8 2 . 5} & \text { Sep 12 } & \mathbf{8 4 . 8 2}\end{array}$

4\$3827092521801. Loc number, 114N17W33BBCO1.

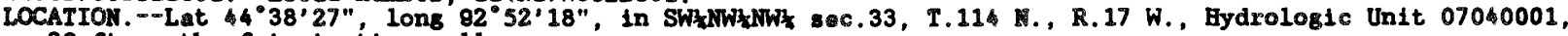
39 ft south of irrigation well.

Omer: Rainer Kimmes.

AQUIFER. - Prairie du Chien Group of Early Ordovician Age

WELL CHARACTERISTICS.-Drillad unused water-table we11, diametar 16 in. depth $290 \mathrm{ft}$, cased to $25 \mathrm{ft}$.

DATU. - -Altitude of land-surface datum is $862 \mathrm{ft}$. Measuring point: Hols in plate over well, 2.00 ft above land-surface datum.

PERIOD OF RECORD.--August 1977 to current year.

EXTRENES FOR PERIOD OF RECORD.--Bighest water leve1, 6.14 ft belon land-surface datum, Dec. 1, 1986;

lowest, 79.20 ft bolow land-surface datum, July i1, 1985 .

WATER LEVEL, IA FEET BELON LAND-SURFACE DATUM, WATER YEAR OCTOBER 1990 TO SEPTEMBER 1991

$\begin{array}{ccccccc}\text { DATE } & \begin{array}{c}\text { WATER } \\ \text { LEVEL }\end{array} & \text { DATE } & \begin{array}{l}\text { WATER } \\ \text { LEVEL }\end{array} & \text { DATE } & \begin{array}{l}\text { WATER } \\ \text { LEVEL }\end{array} \\ \text { Mov 09 } & 66.19 & \text { Mar 05 } & 66.14 & \text { Jul 26 } & \mathbf{7 2 . 2 2} \\ \text { Jan 09 } & 66.27 & \text { May } 07 & 67.35 & \text { Sag 12 } & \mathbf{7 1 . 9 2}\end{array}$


GROUND-WATER LEVELS

DAKOTA COUNTY--Continued

444117092595701. Local number, 114N18W17AMB01.

LOCATIOA.--Lat $44^{\circ} 41^{\prime} 17^{\prime \prime}$, Long $92^{\circ} 59^{\prime} 57^{\prime \prime}$, in NWHEWHEk sec.17, T.114 N., R.18 W., Hydrologic Unit 07040001, 180 th Street, $0.25 \mathrm{mi}$ west of Emery Avenue. Omer: Joe Ries.

AQUIFER.--Prairie du Chien Group of Early Ordovician Ase and Jordan Sandstone of Late Cambrian Age. WELL CHARACTERISTICS.--Drilled irrigation artesian well, diameter 16 in., depth 280 ft, cased to 39 ft. DATUM.--Altitude of land-surface datum is $905 \mathrm{ft}$. Measuring point: Edge of vent pipe, $1.40 \mathrm{ft}$ above land-surface datum.

PERIOD OF RECORD.--June 1979 to current year.

EXTREMA FOR PERIOD OF RECORD.--Hishest water level, 57.30 ft below land-surface datum, Dec, 1 , 1986 ;

lowest, $74.15 \mathrm{ft}$ below land-surface datum, Sept. 13, 1988.

WATER LEVEL, IN FEET BELON LAND-SURFACE DATUM, WATER YEAR OCTOBER 1990 TO SEPTEMBER 1991

$\begin{array}{cccccccr}\text { DATE } & \begin{array}{c}\text { WATER } \\ \text { LEVEL }\end{array} & \text { DATE } & \begin{array}{l}\text { WATER } \\ \text { LEVEL }\end{array} & \text { DATE } & \begin{array}{c}\text { WATER } \\ \text { LEVEL }\end{array} & \text { DATE } & \text { WATER } \\ \text { LEVEL }\end{array}$

443801092571301. Local number, 114N18W35CCB01.

LOCATIOA, - Lat $44^{\circ} 38^{\prime} 01^{\prime \prime}$, long $92^{\circ} 57^{\prime} 13^{\prime \prime}$, in NWSWhSWk sec.35, T.114 H., R.18 W., Hydrologic Unit 07040001, Goodwin Avenue, $1.1 \mathrm{mi}(1.8 \mathrm{~km})$ south of Northfield Boulevard. Omer: Al Wagner, Jr.

AQUIFER. - Buried gravel of P1eistocene Age.

WELL CBARACTERISTICS.-Driled irrigation artesian we11, diameter 12 in., depth 203 ft, screened 173 to 203 ft.

DATUM.--Aititude of land-surface datum is $898 \mathrm{ft}$. Measuring point: Hole in pump base, $1.25 \mathrm{ft}$ above land-surface datum.

PERIOD OF RECORD. - June 1979 to current year.

EXIREMES FOR PERIOD OF RECORD,-Highest water level, 16.83 ft below land-surface datum, Dec. 1, 1986; lowest, $38.28 \mathrm{ft}$ below land-surface datum, Sept. 13, 1982.

WATER LEVEL, IN FEET BELON LAND-SURFACE DATUM, WATER YEAR OCTOBER 1990 TO SEPTEMBER 1991

$\begin{array}{cccccc}\text { DATE } & \begin{array}{c}\text { WATER } \\ \text { LEVEL }\end{array} & \text { DATE } & \begin{array}{c}\text { WATER } \\ \text { LEVEL }\end{array} & \text { DATE } & \begin{array}{c}\text { WATER } \\ \text { LEVEL }\end{array} \\ \text { Nov 09 } & 32.18 & \text { Mar 05 } & 32.34 & \text { Jul } 26 & 36.81 \\ \text { Jan 09 } & 32.41 & \text { May 09 } & 33.12 & \text { Sep 12 } & 35.67\end{array}$

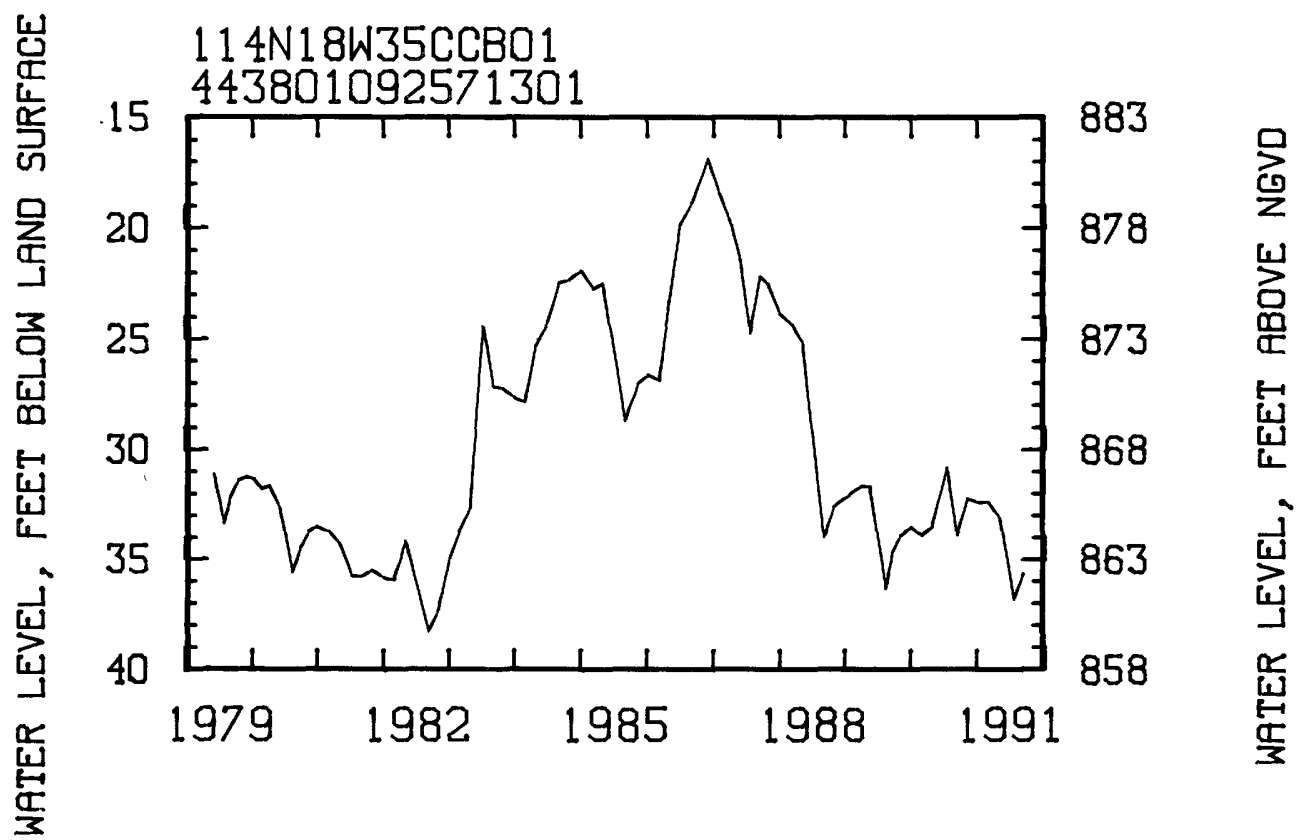


444220093055001. Local number, 114R19W04DAC01.

LOCATIOH. - Lat $44^{\circ} 42^{\prime} 20^{\prime \prime}$, long $93^{\circ} 05^{\prime} 50^{\prime \prime}$, in SWHEEtSEt sec.4, T.114 N., R.19 W., Bydrologic Unit 07040001 , $2.1 \mathrm{mi}$ southeast of Rosemount.

Omer: University of Minnesota Agricultural Experiment Station (Plant Patholosy).

AOUIFER. --Jordan Sandstone of Late Cambrian Ase.

WELI CRARACTERISTICS.--Drilled Irrigation artesian woll, diameter $6 \mathrm{in}$., depth $415 \mathrm{ft}$, cased to $355 \mathrm{ft}$. DATUM. --Altitude of land-surface datum is $947 \mathrm{ft}$. Measuring point: Top of 1-inch breather pipe, $2.10 \mathrm{ft}$ above land-surface datum.

PERIOD OF RECORD. - August 1970 to current year.

EXIRDAES FOR PERIOD OF RECORD. --Highest water level, $55.36 \mathrm{ft}$ below land-surface datum, Dec. 1, 1986 ;

lowest, $65.23 \mathrm{ft}$ below land-surface datum, Nov, 27,1970 .

WATER LEVEL, IN FEET BELOW LAND-SURFACE DATUM, WATER YEAR OCTOBER 1990 TO SEPTDMBER 1991

\begin{tabular}{|c|c|c|c|c|c|}
\hline DATE & $\begin{array}{l}\text { WATER } \\
\text { LEVEL }\end{array}$ & DATE & $\begin{array}{l}\text { WATER } \\
\text { LEVEL }\end{array}$ & DATE & $\begin{array}{l}\text { WATER } \\
\text { LEVEL }\end{array}$ \\
\hline $\begin{array}{l}\text { Nov } 09 \\
\text { Jan } 15\end{array}$ & $\begin{array}{l}60.71 \\
61.02\end{array}$ & $\begin{array}{l}\text { Mar } 06 \\
\text { May } 09\end{array}$ & $\begin{array}{l}61.32 \\
61.27\end{array}$ & $\begin{array}{ll}\text { Jul } & 26 \\
\text { Sop } & 19\end{array}$ & $\begin{array}{l}61.97 \\
61.78\end{array}$ \\
\hline
\end{tabular}

DODGE COUNTY

440448092485501. Local number, 107\$17W13BBA01.

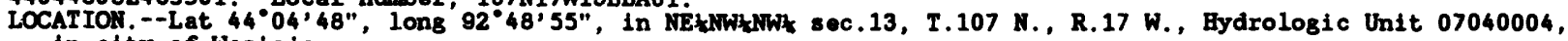
in city of Wasioja. Ower: Wasioja Township Garage.

AQUIFER. --Galena Formation of Middle Ordovician Ase.

WELL CHARACTERISTICS,--Drilled maintenance artesian well, diameter 6 in., depth $100 \mathrm{ft}$ cased to $52 \mathrm{ft}$.

DATUM. - Altitude of iand-surface datum is 1,185 ft. Measuring point: Top of casing, $1.60 \mathrm{ft}$ above land-surface datum.

PERIOD OF RECORD. --January 1975 to current year.

EXTREMES FOR PERIOD OF RECORD.--Highest water level, 12.94 ft below land-surface datum, May 23, 1983

lowest, $26.88 \mathrm{ft}$ below land-surface datum, Jan. 5, 1978.

WATER LEVEL, IN FEET BELOW LAND-SURFACE DATUM, WATER YEAR OCTOBER 1990 TO SEPTEMBER 1991

\begin{tabular}{|c|c|c|c|c|c|c|c|}
\hline DATE & $\begin{array}{l}\text { WATER } \\
\text { LEVEL }\end{array}$ & DATE & $\begin{array}{l}\text { WATER } \\
\text { LEVEL }\end{array}$ & DATE & $\begin{array}{l}\text { WATER } \\
\text { LEVEL }\end{array}$ & DATE & $\begin{array}{l}\text { WATER } \\
\text { LEVEL }\end{array}$ \\
\hline $\begin{array}{l}\text { Oct } 30 \\
\text { Dec } 13\end{array}$ & $\begin{array}{l}19.64 \\
21.87\end{array}$ & $\begin{array}{l}\text { Mar } 04 \\
\text { Apr } 17\end{array}$ & $\begin{array}{l}22.68 \\
21.53\end{array}$ & $\begin{array}{l}\text { May } 29 \\
\text { Jul } 08\end{array}$ & $\begin{array}{l}17.97 \\
18.10\end{array}$ & Aus 19 & 15.64 \\
\hline
\end{tabular}

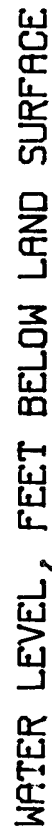

107N17W13BBAD 1

440448092485501

10

$11+1111$

3

25

20

5

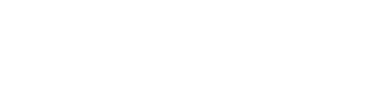

(1)

4.

$\frac{1}{4}$

30

1975

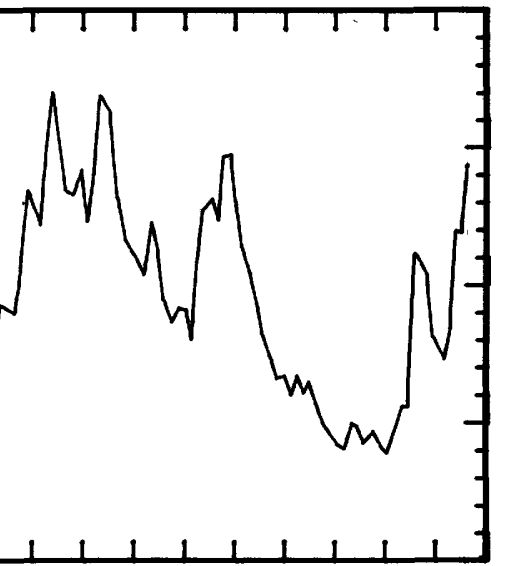

1175

$\stackrel{D}{20}_{2}$

峞

1165

㫐

1160

1)

1155

$\frac{\alpha}{6}$ 
GROUND-WATER LEVELS

FARIBAULT COUNTY

434558093540001 . Local number, 104N26W36CACO1.

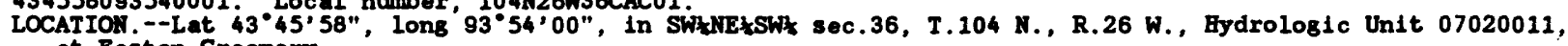
at Easton Creamery.

Omer: City of Easton.

AQUIFER. --Piattevilie Formation of Middle Ordovician Age.

WELI CBARACTERISTICS. --Drilled public supply artesian weil, diameter $6 \mathrm{ln}$, depth $145 \mathrm{ft}$, cased to $120 \mathrm{ft}$. DATUM. --Altitude of iand-surface datum is 1,060 ft. Measuring point: Top of well cap, $1.20 \mathrm{ft}$ above land-surface datum.

PERIOD OF RECORD.--August 1979, April 1980, May 1981 to current year.

EXTREMES FOR PERIOD OF RECORD. --Highest water leve1, 29.97 ft below iand-surface datum, May 10, 1983 ; lowest, $36.36 \mathrm{ft}$ below land-surface datum, Aug. 2, 1989.

WATER LEVEL, IN FEET BELOW LAND-SURFACE DATUM, WATER YEAR OCTOBER 1990 TO SEPTEMBER 1991

$\begin{array}{cccccc}\text { DATE } & \begin{array}{c}\text { WATER } \\ \text { LEVEL }\end{array} & \text { DATE } & \begin{array}{c}\text { WATER } \\ \text { LEVEL }\end{array} & \text { DATE } & \begin{array}{c}\text { WATER } \\ \text { LEVEL }\end{array} \\ \text { Nov 19 } & 34.65 & \text { Mar 25 } & 34.00 & \text { Sep 24 } & 31.30 \\ \text { Jan 30 } & 32.22 & \text { Jul 23 } & 32.08 & & \end{array}$

\section{FILIMORE COUNTY}

434936092102201. Local number, 104N11W08ADC01.

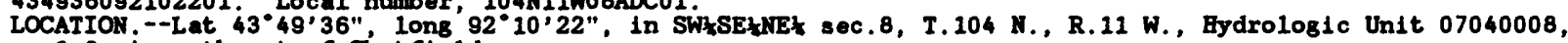
$0.8 \mathrm{mi}$ southeast of Chatfleld.

Omer: Fillmore County Highway Department.

AQUIFER. - Pralrie du Chien group of early Ordovician age and Jordan sandstone of late Cambrian age.

WELL CBARACTERISTICS. --Drilied domestic water table weil, diameter 4 in., depth $284 \mathrm{ft}$, cased to $128 \mathrm{ft}$.

DATUM. - Altitude of iand-surface datum $1 \mathrm{~s} 981 \mathrm{ft}$. Measuring point: Top of casing, $0.50 \mathrm{ft}$ above landsurface datum.

PERIOD OF RECORD. - November 1989 to September 1990

EXTREAES FOR PERIOD OF RECORD. --Highest water levei, 28.73 ft below land-surface datum, May 7, 1991;

lowest, $38.51 \mathrm{ft}$ below land-surface date, Feb. 6, 1990.

WATER LEVEL, IN FEET BELOW LAND-SURFACE DATUM, WATER YEAR OCTOBER 1990 TO SEPTEMBER 1991

$\begin{array}{cccccc}\text { DATE } & \begin{array}{c}\text { WATER } \\ \text { LEVEL }\end{array} & \text { DATE } & \begin{array}{c}\text { WATER } \\ \text { LEVEL }\end{array} & \text { DATE } & \begin{array}{c}\text { WATER } \\ \text { LEVEL }\end{array} \\ \text { Dec 05 } & 33.64 & \text { Mar 05 } & 35.40 & \text { Aug 01 } & 31.14 \\ \text { Jan 09 } & \mathbf{3 4 . 8 1} & \text { May 07 } & \mathbf{2 8 . 7 3} & \text { Sep 12 } & 32.44\end{array}$

FREEBORN COUNTY

433434093331201. Local number, 101N23W02DAC01.

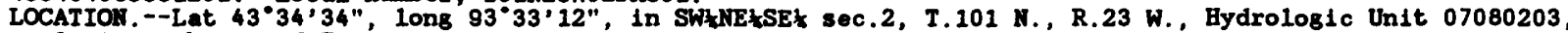

$3 \mathrm{mi}$ southmest of Conger.

Owner: Richard Steele.

AQUIFER. - Upper Carbonates of Devonian and Ordovician ABe.

WELL CHARACTERISTICS.--Drilled irrigation artesian weli, diameter $16 \mathrm{in}$., depth $373 \mathrm{ft}$ (114 m), cased to 156 ft.

DATUM.--Altitude of land-surface datum is 1,280 ft. Measuring point: Vent plpe, 1.50 ft above landsurface datum.

PERIOD OF RECORD:- -July 1981 to current year.

EXTREAES FOR PERIOD OF RECORD.--Highest water level, 65.58 ft below land-surface datum, Mar. 8, 1983;

lowest, $75.45 \mathrm{ft}$ below land-surface datum. Aug. 2, 1989.

WATER LEVEL, IN FEET BELOW LAND-SURFACE DATUM, WATER YEAR OCTOBER 1990 TO SEPTEMBER 1991

$\begin{array}{cccccc}\text { DATE } & \begin{array}{c}\text { WATER } \\ \text { LEVEL }\end{array} & \text { DATE } & \begin{array}{c}\text { WATER } \\ \text { LEVEI }\end{array} & \text { DATE } & \begin{array}{c}\text { WATER } \\ \text { LEVEL }\end{array} \\ \text { Nov 19 } & 67.55 & \text { Mar 25 } & 68.25 & \text { Jul 23 } & 70.16 \\ \text { Jan 30 } & 68.22 & \text { May 28 } & 67.52 & \text { Sep 24 } & 68.52\end{array}$


433846093220601 . Local number, 102N21W09CCB01.

LOCATION.--Lat $43^{\circ} 38^{\prime} 46^{\prime \prime}$, long $93^{\circ} 22^{\prime} 06^{\prime \prime}$, in NWZSW SW sec.9, T.102 N., R.21 W., Hydrologic Unit 07080202 at Freeborn County Courthouse.

Owner: Freeborn County.

AQUIFER. --Cedar Valley Formation of Middle Devonian Age.

WELL CRARACTERISTICS.--Drilled public supply artesian well, diemeter 5 in,, depth $150 \mathrm{ft}$, cased to $138 \mathrm{ft}$

DATUM.--Aititude of land-surface datum is 1,240 ft. Measuring point: Top of casing, $1.00 \mathrm{ft}$ above land-surface datum.

PERIOD OF RECORD. --November 1981 to current year.

EXTREMES FOR PERIOD OF RECORD.--Highest water level, $37.50 \mathrm{ft}$ below land-surface datum, Nov. 19,

1990; lowest, $49.32 \mathrm{ft}$ below land-surface datum, Aug. 2, 1989.

WATER LEVEL, IN FEET BELOW LAND-SURFACE DATUM, WATER YEAR OCTOBER 1990 TO SEPTEMBER 1991

$\begin{array}{cccccc}\text { DATE } & \begin{array}{c}\text { WATER } \\ \text { LEVEL }\end{array} & \text { DATE } & \begin{array}{c}\text { WATER } \\ \text { LEVEL }\end{array} & \text { DATE } & \begin{array}{c}\text { WATER } \\ \text { LEVEL }\end{array} \\ \text { Nov 19 } & 37.50 & \text { May 28 } & 42.30 & \text { Sep 24 } & 43.05 \\ \text { Mar 25 } & 40.51 & \text { Jul 23 } & 44.59 & & \end{array}$

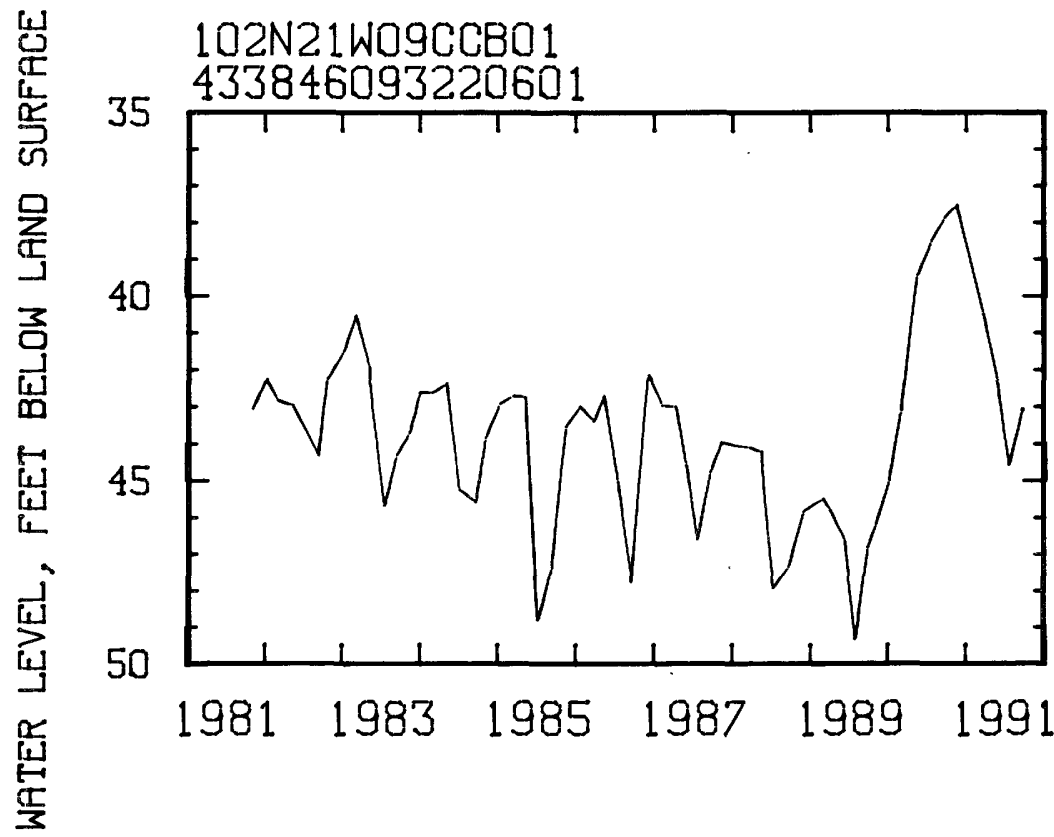

1205

200

1195

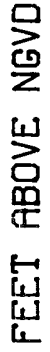

प]

1190

$\stackrel{\frac{1}{6}}{\frac{1}{3}}$

434032093111801 . Local number, 103N20W36CCB01.

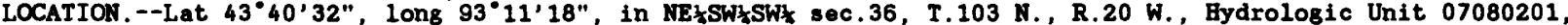
at Pillsbury Grain Station. Omer: Pillsbury Co.

AQUIFER. --Cedar Valley Formation of Middle Devonian Age.

WELI CHARACTERISTICS.--Drilled domestic artesian well, diameter $5 \mathrm{in}$., depth $231 \mathrm{ft}$, cased to $136 \mathrm{ft}$. DATUM.--Altitude of land-surface datum is $1,255 \mathrm{ft}$. Measuring point: Top of casing, $1.80 \mathrm{ft}$ above land-surface datum.

PERIOD OF RECORD. --Juily 1981 to current year.

EXTREMES FOR PERIOD OF RECORD.--Highest water level, $48.40 \mathrm{ft}$ below land-surface datum, May 10, 1984 ; lowest, $55.95 \mathrm{ft}$ below land-surface datum, July 13, 1988.

WATER LEVEL, IN FEET BELOW LAND-SURFACE DATUM, WATER YEAR OCTOBER 1990 TO SEPTEMBER 1991

$\begin{array}{cccccc}\text { DATE } & \begin{array}{c}\text { WATER } \\ \text { LEVEL }\end{array} & \text { DATE } & \begin{array}{l}\text { WATER } \\ \text { LEVEL }\end{array} & \text { DATE } & \begin{array}{c}\text { WATER } \\ \text { LEVEL }\end{array} \\ \text { Nov 19 } & 49.62 & \text { Mar 25 } & 50.37 & \text { Jul 23 } & 50.84 \\ \text { Jan 30 } & 50.32 & \text { May 28 } & 48.96 & \text { Sep 24 } & 50.60\end{array}$


GROUND-WATER LEVELS

FREEBORH COUNTY--Continued

434308093322001 . Local number, 103N23W13CDA01.

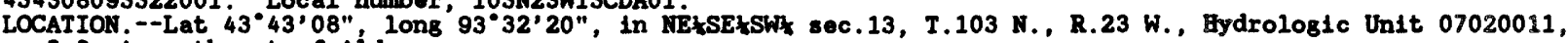
$3.3 \mathrm{mi}$ northeast of Alden.

Omer: Oakview Golf Course

AQUIFER. --Galena Formation of Middie Ordovician Age.

WELL CHARACTERISTICS.-Drilled irrigation artesian mell, diemeter 6 in., depth $270 \mathrm{ft}$, cased to $158 \mathrm{ft}$.

DATUM. - Altitude of land-surface datum is 1,250 ft. Measuring point: Hole in sell cap, $1.90 \mathrm{ft}$ above land-surface datum.

PERIOD OF RECORD.--July 1981 to current year.

EXTRFAES FOR PERIOD OF RECORD,--Bighest water level, 42.00 ft below land-surface datum, May 10, 1983;

lowest, 48.77 ft below land-surface datum, Aug. 2, 1989

WATER LEVEL, IN FEET BELON LAND-SURFACE DATUM, WATER YEAR OCTOBER 1990 TO SEFTEMER 1991

\begin{tabular}{|c|c|c|c|c|c|}
\hline DATE & $\begin{array}{l}\text { WATXR } \\
\text { LEVEL }\end{array}$ & DATE & $\begin{array}{l}\text { WATDR } \\
\text { LEVEL }\end{array}$ & DATE & $\begin{array}{l}\text { WAIDR } \\
\text { LEVDL }\end{array}$ \\
\hline $\begin{array}{l}\text { Nov } 19 \\
\text { Jan } 30\end{array}$ & $\begin{array}{l}42.33 \\
43.13\end{array}$ & $\begin{array}{ll}\text { Mar } 25 \\
\text { May } 28\end{array}$ & $\begin{array}{l}43.50 \\
42.90\end{array}$ & $\begin{array}{ll}\text { Jul } & 23 \\
\text { Sep } & 24\end{array}$ & $\begin{array}{l}45.03 \\
44.45\end{array}$ \\
\hline
\end{tabular}

GOODHuE COUNTY

441737092400501. Local number, 110N15W31BBD01.

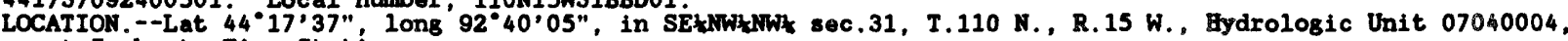
at Zumbrota Fire Station.

Omer: City of Zumbrota, well 3.

AQUIFER. - - Prairie du Chien Group of Early Ordovician ABe.

WELl CHARACTERISTICS. -Drilled unused artesian well, diameter 12 in., depth $210 \mathrm{ft}$, cased to $50 \mathrm{ft}$.

DATUM. - -Altitude of land-surface datum is 1,000 ft. Measuring point: Bole in pump base, $2.20 \mathrm{ft}$ above land-surface datum.

PERIOD OF RECORD.--June 1973 to current year.

EXTREMES FOR PERIOD OF RECORD.--Highest water level, $17.38 \mathrm{ft}$ below land-surface datum, Jan 7 , 1987;

lowest, $27.00 \mathrm{ft}$ below land-surface datum, Jan. 5, 1978.

WATER LEVEL, IN FEET BELON LAND-SURFACE DATUM, WATER YEAR OCTOBER 1990 TO SEPTEMBER 1991

$\begin{array}{cccccccr}\text { DATE } & \begin{array}{c}\text { WATER } \\ \text { LEVEL }\end{array} & \text { DATE } & \begin{array}{l}\text { WATER } \\ \text { LEVEL }\end{array} & \text { DATE } & \begin{array}{c}\text { WATER } \\ \text { LEVEI. }\end{array} & \text { DATE } & \begin{array}{c}\text { WATER } \\ \text { LEVEL }\end{array} \\ \text { Oct } 22 & 23.35 & \text { Jan } 14 & 22.90 & \text { Apr 15 } & 23.52 & \text { Aus } 12 & 21.39 \\ \text { Dec } 04 & 23.04 & \text { Feb 25 } & 23.89 & \text { May 20 } & 22.06 & & \end{array}$

442401092372501 Local number 111\%15W21CDAO1.

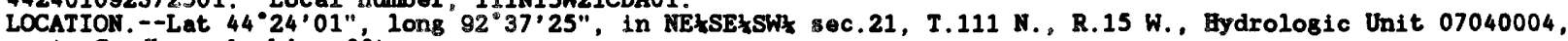
in Goodhue clerk's office.

Omer: City of Goodhue, creamery well.

AQUIFER. - Prairie du Chien Group of Early Ordovician Age and Jordan Sandstone of Late Cambrian age.

WELI CHARACTERISTICS. - Drilled public-supply artesian well, diameter 12 in., depth 310 ft, cased to

DATUM.-Aititude of land-surface datum is 1,125 ft. Measuring point: Top of 1k in elbow, $1.50 \mathrm{ft}$ above land-surface datum.

PERIOD OF RECCORD.--June 1973 to current year.

EXIROMES FOR PERIOD OF RECORD. - - Highest pater leve1, 119.00 ft below land-surface datum, Feb. 26,

1987; lowest, 156.5 it below land-surface datum, May 26, 1983.

WATER LEVEL, IN FEET BELON LAND-SURFACE DATUM, WATER YEAR OCTOBER 1990 TO SEPTEMBER 1991

$\begin{array}{crcrrr}\text { DATE } & \begin{array}{c}\text { WATER } \\ \text { LEVEL }\end{array} & \text { DATE } & \begin{array}{c}\text { WATER } \\ \text { LEVEL }\end{array} & \text { DATE } & \begin{array}{r}\text { WATER } \\ \text { LEVEL }\end{array} \\ \text { Oct 22 } & 131.02 & \text { Jan } 14 & 134.63 & \text { Apr } 15 & 135.52 \\ \text { Dec 04 } & 130.47 & \text { Feb 25 } & 135.82 & & \end{array}$


443012092362201. Local number, 113N15W27BAB01.

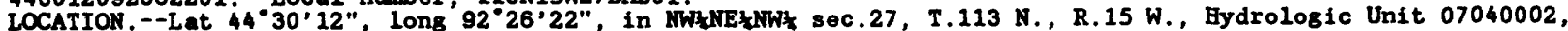
at Red Wing.

Omer: City of Red Wing, Anderson Park.

AQUIFER. - Eau Claire-Mount Simon Sandstones of Late Cambrian Age.

WELL CHARACTERISTISC. --Drilled unused artesian well, diameter $12 \mathrm{in}$, depth $560 \mathrm{ft}$, cased to $243 \mathrm{ft}$.

DATUM,--Altitude of land-surface datum is $800 \mathrm{ft}$. Measuring point: Edge of casing, $2.70 \mathrm{ft}$ above land-surface datum.

PERIOD OF RECORD.--April 1976, June 1978 to current year.

EXTREMES FOR PERIOD OF RECORD. -- Highest water level, 100.50 ft below land-surface datum, Apr. 20, 1983; lowest, $109.62 \mathrm{ft}$ below land-surface datum, Sept. 10, 1990.

WATER LEVEL, IN FEET BELOW LAND-SURFACE DATUM, WATER YEAR OCTOBER 1990 TO SEPTEMBER 1991

\begin{tabular}{|c|c|c|c|c|c|c|c|c|c|}
\hline ATE & $\begin{array}{l}\text { WATER } \\
\text { LEVEL }\end{array}$ & DATE & $\begin{array}{l}\text { WATER } \\
\text { LEVEI }\end{array}$ & DATE & $\begin{array}{l}\text { WATER } \\
\text { LEVEL }\end{array}$ & DATE & $\begin{array}{l}\text { WATER } \\
\text { LEVEL }\end{array}$ & DATE & $\begin{array}{l}\text { WATER } \\
\text { LEVEL }\end{array}$ \\
\hline $\begin{array}{ll}\text { ct } & 22 \\
\text { oc } & 04\end{array}$ & $\begin{array}{l}108.37 \\
107.56\end{array}$ & $\begin{array}{l}\text { Jan } 14 \\
\text { Feb } 25\end{array}$ & $\begin{array}{l}106.59 \\
106.54\end{array}$ & $\begin{array}{ll}\text { Apr } & 15 \\
\text { May } & 20\end{array}$ & $\begin{array}{l}106.29 \\
106.13\end{array}$ & $\begin{array}{ll}\text { Aug } & 12 \\
\text { Sep } 27\end{array}$ & $\begin{array}{l}107.40 \\
107.05\end{array}$ & Sep 30 & 106.95 \\
\hline
\end{tabular}

HENNEPIN COUNTY

444815093194901. Local number, 027N24W30MM01

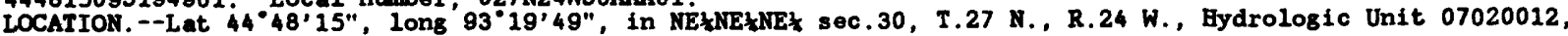
at 4001 West 110th Street, Bloomington. Owner: Transfiguration Church.

AOUIFER. - Buried Sand of Pleistocene Ase.

WELL CHARACTERISTICS.--Drilled domestic artesian well, diameter in., depth $139 \mathrm{ft}$, screened 135 to

DATUM.--Aititude of land-surface datum is $832 \mathrm{ft}$. Measuring point: Top of casing, $0.50 \mathrm{ft}$ above landsurface datum.

PERIOD OF RECORD. --March 1979 to current year.

EXTREMES FOR PERIOD OF RECORD, --Highest water level, $63.97 \mathrm{ft}$ below land-surface datum, Mar. 2, 1979; lowest, $73.84 \mathrm{ft}$ below land-surface datum, July 19, 1991.

WATER LEVEL, IN FEET BELOW LAND SURFACE DATUM, WATER YEAR OCTOBER 1990 TO SEPTEMBER 1991

$\begin{array}{cccccc}\text { DATE } & \begin{array}{c}\text { WATER } \\ \text { LEVEL }\end{array} & \text { DATE } & \begin{array}{c}\text { WATER } \\ \text { LEVEL }\end{array} & \text { DATE } & \begin{array}{c}\text { WATER } \\ \text { LEVEL }\end{array} \\ \text { Nov 14 } & \mathbf{7 2 . 8 5} & \text { Mar 07 } & 73.23 & \text { Ju1 19 } & 73.84 \\ \text { Jan 08 } & 73.00 & \text { May 08 } & 73.46 & \text { Sep 19 } & 73.74\end{array}$

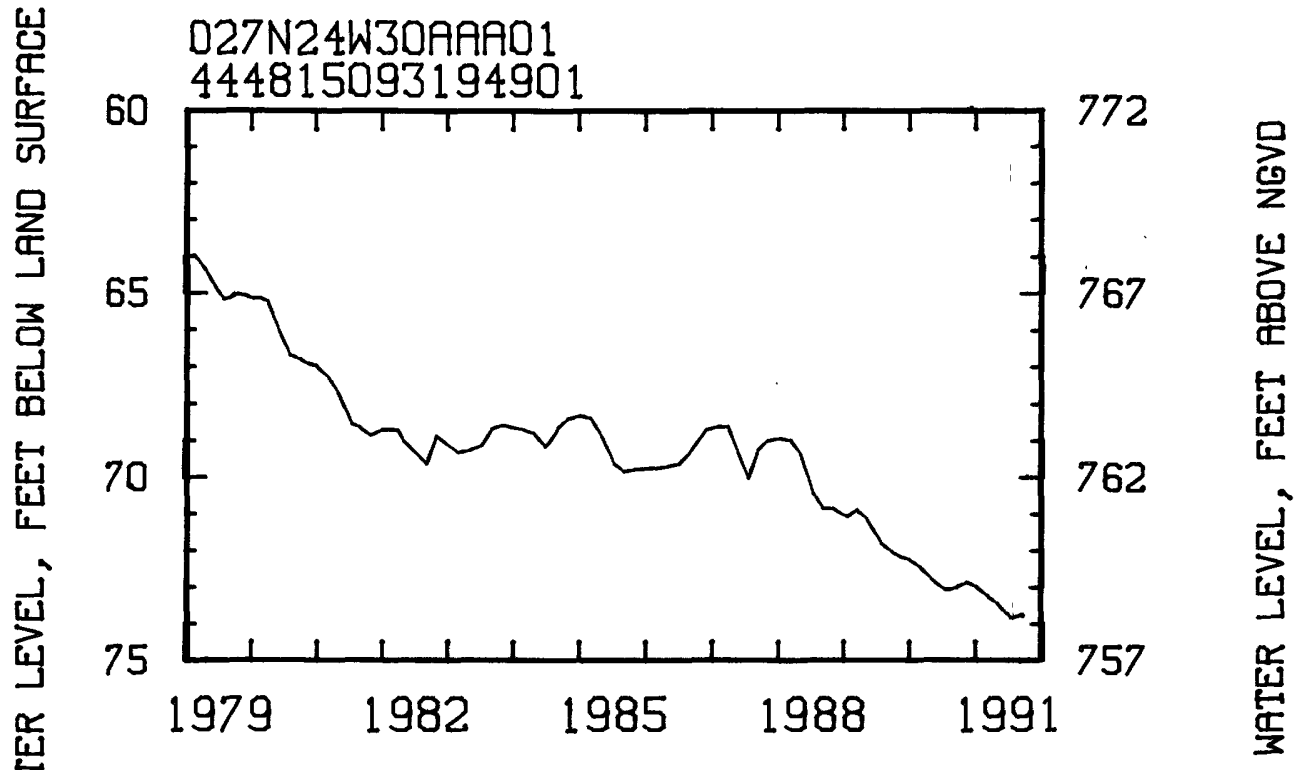


GROUND-WATER LEVELS

HENAEPIN COUNTY--ContInued

444801093202801. Local number, 027N24W30BDA01.

年 Bloomington.

Owner: City of Bloomington, at Southwood Terrace.

AQUIFER. --Jordan Sandstone of Late Cambrian Age.

WRI CBARACTERISTICS, --Drilled unused artesion well, diameter $12 \mathrm{in}$., depth $330 \mathrm{ft}$, cased to $269 \mathrm{ft}$.

DATU: - Altitude of iand-surface datum is $815 \mathrm{ft}$. Measuring point: Top of recorder platform, $2.20 \mathrm{ft}$ above land-surface datum.

REMARKS. - Water level affected by pumping.

PERIOD OF RECORD. --March 1969 to current year.

EXTRETS FOR PERIOD OF RECORD, --Bighest water level, 63.05 ft below land-surface datum, Apr. 15, 1969; 10west, 84.86 ft below land-surface datum, July 1, 1988.

WATER LEVEL, IN FEET BELOW IAND-SURFACE DATAUM, WATER YEAR OCTOBER 1990 TO SEPTEMBER 1991

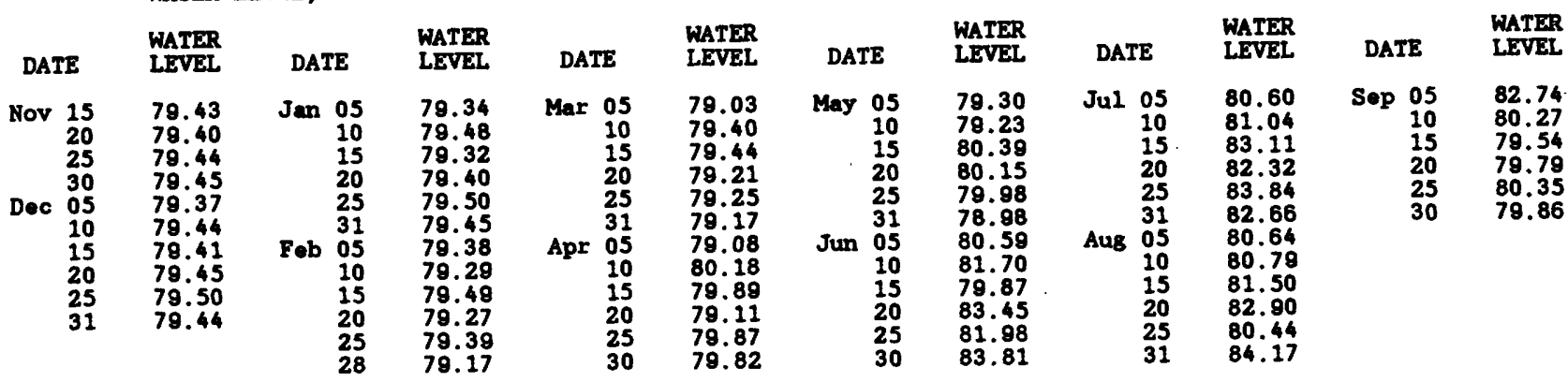

445356093145301. Local number, 028N24W23ADD01.

LOCATIOH. --Lat $4^{\circ} 53^{\prime} 56^{\prime \prime}$, Iong $93^{\circ} 14^{\prime} 53^{\prime \prime}$, in SEłsEłNEł sec.23, T.28 N., R.24 W., Hydrologic Unit 07010206,

at 5728 Cedar Avenue, Minneapolis.

Omer: Hope Lutheran Church.

AOUIFER. - Prairie du Chien Group of Early Ordovician Age.

WRI CBARACTERISTICS.-Drilled irrigation artesian weli, diameter 6 in., depth $245 \mathrm{ft}$, cased to $172 \mathrm{ft}$. DATUM, - - Altitude of iand-surface datum is $835 \mathrm{ft}$. Measuring point: Top of casing, $0.30 \mathrm{ft}$ above landsurface datum.

PERIOD OF RECORD.--APril 1979 to current year. Iowest, 80.17 ft below land-surface datum, June 21, 1988 .

WATER LEVEL, IN FEET BELOW LAND SURFACE DATUM, WATER YEAR OCTOBER 1990 TO SEPTEMBER 1991

\begin{tabular}{|c|c|c|c|c|}
\hline DATE & $\begin{array}{l}\text { WATER } \\
\text { LEVEL }\end{array}$ & DATE & $\begin{array}{l}\text { WATER } \\
\text { LEVEL }\end{array}$ & DATE \\
\hline $\begin{array}{l}\text { Nov } 14 \\
\text { Jan } 08\end{array}$ & $\begin{array}{l}39.95 \\
39.35\end{array}$ & $\begin{array}{l}\text { Mar } 07 \\
\text { May } 08\end{array}$ & $\begin{array}{l}39.97 \\
40.03\end{array}$ & $\begin{array}{ll}\text { Jul } & 18 \\
\text { Sep } & 26\end{array}$ \\
\hline
\end{tabular}

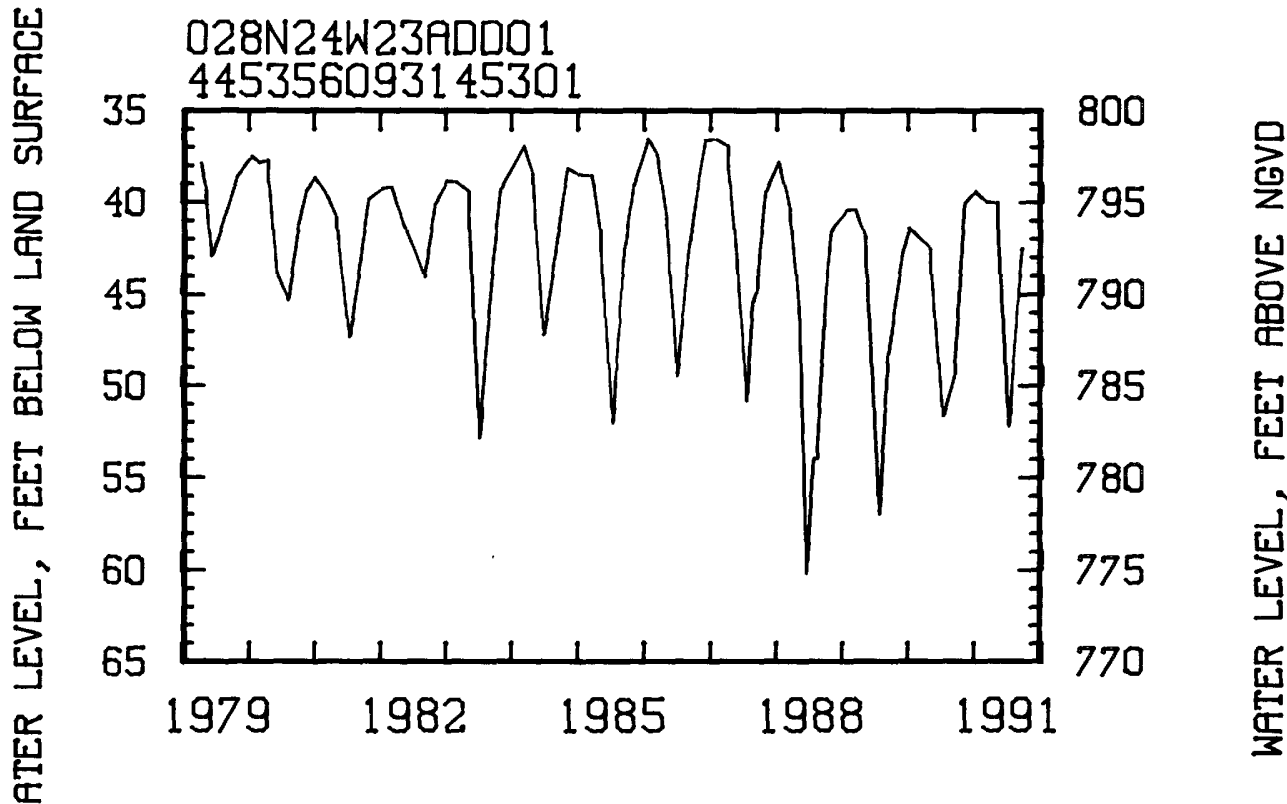


450116093205301 . Local number, 029N24W06CCC01

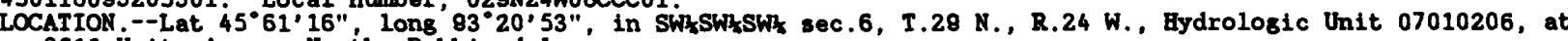
3610 Unity Avenue North, Robbinsdale.

Omer: Minnesota Department of Transportation.

AQUIFER. - St. Peter Sandstone of Middle Ordovician Age.

WELL CHARACTERISTICS.-Drilled unused artesian mell, diameter $5 \mathrm{in}$, depth $200 \mathrm{ft}$, cased to $152 \mathrm{ft}$.

DATUM. --Altitude of land-surface datum is $870 \mathrm{ft}$. Measuring point: Top of casing, 3.50 ft above land-surface datum.

REMARKS.--Water level affected by pumping.

PERIOD OF RECORD. --March 1973 to current year.

EXTREMES FOR PERIOD OF RECORD.--Highest water leve1, 24.54 ft below land-surface datum, Dec. 28-29, 1975; 1owest, 53.03 ft below land-surface datum, June 15, 1988.

WATER LEVEL, IN FEET BELOW LAND-SURFACE DATAUM, WATER YEAR OCTOBER 1990 TO SEPTEMBER 1991

\begin{tabular}{|c|c|c|c|c|c|c|c|c|c|c|c|c|c|c|}
\hline DATE & $\begin{array}{l}\text { WATER } \\
\text { LEVEL }\end{array}$ & DA: & & $\begin{array}{l}\text { WATER } \\
\text { LEVEI }\end{array}$ & DA! & & $\begin{array}{l}\text { WATER } \\
\text { LEVEL }\end{array}$ & DAT & & $\begin{array}{l}\text { WATER } \\
\text { LEVEL }\end{array}$ & DAT & $\begin{array}{l}\text { WATERR } \\
\text { LEVEEL }\end{array}$ & DATE & $\begin{array}{l}\text { WATER } \\
\text { LEVEEL }\end{array}$ \\
\hline $\begin{array}{r}15 \\
20 \\
25 \\
30 \\
\text { Dec } 05 \\
10 \\
15 \\
20\end{array}$ & $\begin{array}{l}33.72 \\
32.90 \\
31.35 \\
33.40 \\
33.07 \\
33.03 \\
33.00 \\
33.45\end{array}$ & Jan & $\begin{array}{l}10 \\
15 \\
20 \\
25 \\
31 \\
05 \\
10 \\
15 \\
20 \\
25 \\
28\end{array}$ & $\begin{array}{l}32.79 \\
32.72 \\
32.42 \\
32.87 \\
33.04 \\
33.13 \\
32.42 \\
32.86 \\
32.82 \\
32.40 \\
32.30\end{array}$ & Apr & $\begin{array}{l}05 \\
10 \\
15 \\
20 \\
25 \\
31 \\
05 \\
10 \\
15 \\
20 \\
25 \\
30\end{array}$ & $\begin{array}{l}31.97 \\
31.61 \\
32.40 \\
32.02 \\
32.02 \\
31.51 \\
33.52 \\
33.64 \\
32.65 \\
32.49 \\
35.59 \\
36.44\end{array}$ & Jun & $\begin{array}{l}05 \\
10 \\
15 \\
20 \\
25 \\
31 \\
05 \\
10 \\
15 \\
20 \\
25 \\
30\end{array}$ & $\begin{array}{l}31.67 \\
35.44 \\
37.69 \\
35.04 \\
34.68 \\
35.43 \\
35.16 \\
35.78 \\
40.32 \\
40.33 \\
38.44 \\
42.95\end{array}$ & $\begin{array}{l}\text { Jul } \\
\text { Aug }\end{array}$ & $\begin{array}{l}36.04 \\
37.58 \\
39.62 \\
39.48 \\
39.59 \\
36.89 \\
35.05 \\
34.51 \\
36.98 \\
35.93 \\
38.71 \\
41.22\end{array}$ & $\begin{aligned} & \text { Sep } 05 \\
& 10 \\
& 15 \\
& 20 \\
& 25 \\
& 30\end{aligned}$ & $\begin{array}{l}38.89 \\
36.41 \\
34.42 \\
32.77 \\
32.47 \\
33.55\end{array}$ \\
\hline
\end{tabular}

445833093154301. Local number, 029N24W26BAB01.

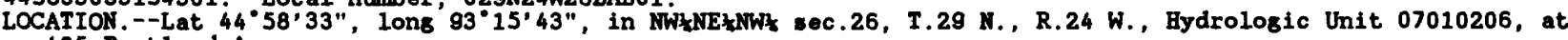
425 Portland Avenue.

Omer: Minneapolis Star and Tribune.

AOUIFER.--Prairie du Chien Group of Early Ordovician Ase and Jordan Sandstone of Late Cambrian Age.

WELL CHARACTERISTICS.--Drilled unused artesian well, diameter $16 \mathrm{in}$., depth $445 \mathrm{ft}$, cased to $252 \mathrm{ft}$

DATUM. - - Altitude of land-surface datum is $835 \mathrm{ft}$. Measuring point: 'Top of steel cover, $7.60 \mathrm{ft}$ below landsurface datum.

RDMARKS. - Water level affected by pumping.

PERIOD OF RECORD. --June 1981 to current year.

EXTREMES FOR PERIOD OF RECORD.--Highest water level, 56.45 ft below land-surface datum, Jan. 10, 1983; lowest, 149.36 ft below land-surface datum, Aug. 16, 1988

WATER LEVEL, IN FEET BELOW LAND-SURFACE DATUM, WATER YEAR OCTOBER 1990 TO SEPTEMBER 1991

\begin{tabular}{|c|c|c|c|c|c|c|c|c|c|c|c|}
\hline DATE & $\begin{array}{l}\text { WATER } \\
\text { LEVEL }\end{array}$ & DATE & $\begin{array}{l}\text { WATER } \\
\text { LEVEL }\end{array}$ & DATE & $\begin{array}{l}\text { WATER } \\
\text { LEVEL }\end{array}$ & DATE & $\begin{array}{l}\text { WATER } \\
\text { LEVEL }\end{array}$ & DATE & $\begin{array}{l}\text { WATER } \\
\text { LEVEL }\end{array}$ & DATE & $\begin{array}{l}\text { WATER } \\
\text { LEVEL }\end{array}$ \\
\hline $\begin{array}{l}29 \\
21\end{array}$ & $\begin{array}{l}69.50 \\
75.80\end{array}$ & $\begin{array}{l}\text { Dec } 26 \\
\text { Jan } 31\end{array}$ & $\begin{array}{l}64.65 \\
67.25\end{array}$ & $\begin{array}{l}\text { Feb } 26 \\
\text { Max } 27\end{array}$ & $\begin{array}{l}67.85 \\
69.70\end{array}$ & $\begin{array}{l}\text { Apr } 26 \\
\text { May } 30\end{array}$ & $\begin{array}{r}72.99 \\
109.67\end{array}$ & $\begin{array}{l}\text { Jun } 26 \\
\text { Jul } 29\end{array}$ & $\begin{array}{r}116.30 \\
93.64\end{array}$ & $\begin{array}{ll}\text { Aug } & 27 \\
\text { Sep } & 20\end{array}$ & $\begin{array}{r}118.46 \\
74.10\end{array}$ \\
\hline
\end{tabular}

445829093162901 . Local number, 029\$24W27ABD01.

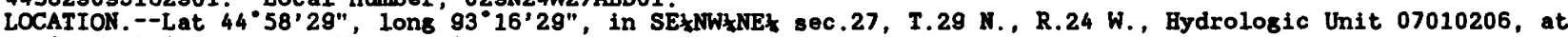

911 Lasalle Avenue, Minneapolis.

Omer: American Linen Supply Co.

AOUIFER. - Mount Simon Sandstone of Late Cambrian Age and Hinckley Sandstone of Late Precambrian Age.

WELL CHARACTERISTICS.--Drilled unused artestan wel1, diameter 16 in., depth $1,094 \mathrm{ft}$, cased to $812 \mathrm{ft}$.

DATUM. --Altitude of iand-surface datum is $850 \mathrm{ft}$. Measuring point: Hole in pump base, 22.00 ft below landsurface datum.

REMARKS. - Water level affected by regional pumping.

PERIOD OF RECORD. --July 1970 to current year.

EXTREMES FOR PERIOD OF RECORD.--H1ghest water leve1, 169.8 ft below land-surface datum, Apr. 15, 1980; 10west, $269.92 \mathrm{ft}$ below land-surface datum, Aug. 28, 1989.

WATER LEVEL, IN FEET BELOW LAND SURFACE DATUM, WATER YEAR OCTOBER 1990 TO SEPTEMBER 1991

\begin{tabular}{|c|c|c|c|c|c|c|c|c|c|c|c|}
\hline DATE & $\begin{array}{l}\text { WATER } \\
\text { LEVEL }\end{array}$ & DATE & $\begin{array}{l}\text { WATER } \\
\text { LEVEL }\end{array}$ & DATE & $\begin{array}{l}\text { WATER } \\
\text { LEVEL }\end{array}$ & DATE & $\begin{array}{l}\text { WATER } \\
\text { LEVEL }\end{array}$ & DATE & $\begin{array}{l}\text { WATER } \\
\text { LEVEL }\end{array}$ & DATE & $\begin{array}{l}\text { WATER } \\
\text { LEVEL }\end{array}$ \\
\hline $\begin{array}{l}29 \\
21\end{array}$ & $\begin{array}{l}245.76 \\
241.28\end{array}$ & $\begin{array}{l}\text { Dec } 26 \\
\text { Jan } 31\end{array}$ & $\begin{array}{l}233.19 \\
228.92\end{array}$ & $\begin{array}{l}\text { Feb } 26 \\
\text { Mar } 27\end{array}$ & $\begin{array}{l}227.95 \\
230.00\end{array}$ & $\begin{array}{l}\text { Apr } 26 \\
\text { May } 30\end{array}$ & $\begin{array}{l}231.54 \\
241.35\end{array}$ & $\begin{array}{l}\text { Jun } 26 \\
\text { JuI } 29\end{array}$ & $\begin{array}{l}240.73 \\
248.85\end{array}$ & $\begin{array}{l}\text { Aug } 27 \\
\text { Sep } 20\end{array}$ & $\begin{array}{l}247.37 \\
250.08\end{array}$ \\
\hline
\end{tabular}


GROUND-WATER LEVELS

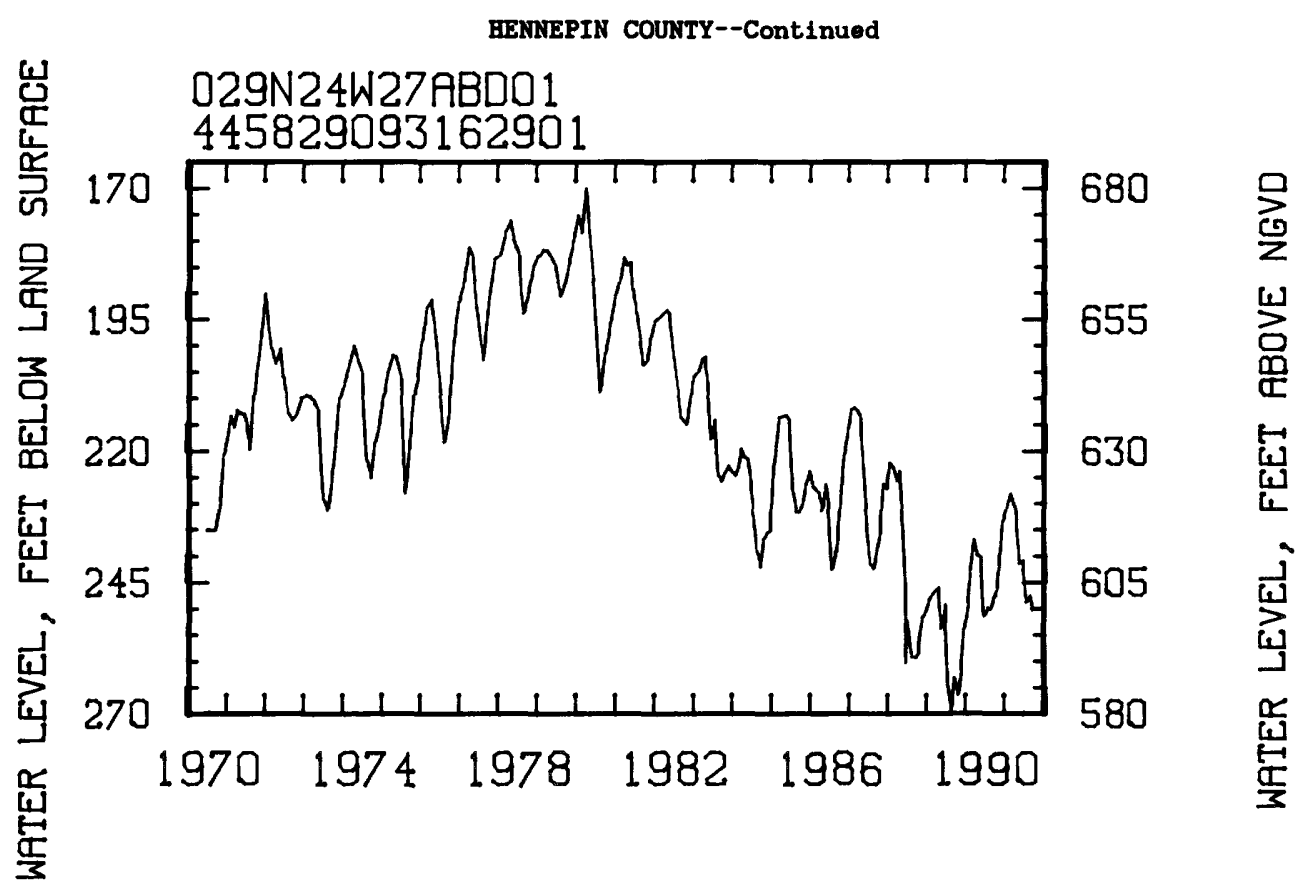

445618093211801 . Local number, 117N21W16CDB01.

LOCATIOA. --Lat $44^{\circ} 56^{\prime} 18^{\prime \prime}$, long $93^{\circ} 21^{\prime} 18^{\prime \prime}$, In NW'sE $\}_{4}$ SW sec.16, T.117 N., R.21 W., Hydrologic Unit 07010206 , at 2565 Wooddale Avenue South, St. Louis Park.

Omer: D-A Lubricant Co.

AQUIFER. - - Ironton-Galesville Sandstones of Late Cambrian Age.

WELL CBARACTERISTICS. --Drilled observation artesian well, diameter $4 \mathrm{ln}$, depth $691 \mathrm{ft}$, screened 651 to $661 \mathrm{ft}$.

DATUM.--Aititude of land-surface datum is $917.2 \mathrm{ft}$, National Geodetic Vertical Datum of 1929. Measuring point: Hole in well seal, $3.60 \mathrm{ft}$ above land-surface datum.

REMARKS. - Water level affected by pumping.

PERIOD OF RECORD,--April 1980 to current year.

EXTRPAES FOR PERIOD OF RECORD. --Highest water level, 130.25 ft below land-surface datum, Feb. 6, 1987;

lowest, $155.46 \mathrm{ft}$ below land-surface datum, Sept. 20, 1988.

WATER LEVEL, IN FEET BELOW LAND SURFACE DATUM, WATER YEAR OCTOBER 1990 TO SEPTEMBER 1991

$\begin{array}{crcrrr}\text { DATE } & \begin{array}{r}\text { WATER } \\ \text { LEVEL }\end{array} & \text { DATE } & \begin{array}{r}\text { WATER } \\ \text { LEVEL }\end{array} & \text { DATE } & \begin{array}{r}\text { WATER } \\ \text { LEVEL }\end{array} \\ \text { Nov 14 } & 144.34 & \text { Mar 07 } & 137.01 & \text { Jul 19 } & 141.30 \\ \text { Jan 10 } & 140.32 & \text { May 13 } & 134.72 & \text { S } 19 \text { 19 } & 145.70\end{array}$

445646093395301. Local number, 117N24W13BBCO4.

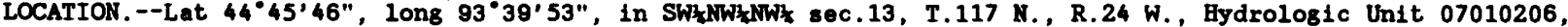
at 3-Point Road.

Omer: City of Mound, well 4.

AQUIFER. - Mount Simon Sandstone of Late Cambrian Age.

WELI CHARACTERISTICS.--Drilled unused artesian mell, diameter $10 \mathrm{in}$, depth $729 \mathrm{ft}$, cased to $600 \mathrm{ft}$.

DATUM.--Altitude of land-surface datum is $945 \mathrm{ft}$ : Measuring point: Top of breather pipe, $2.35 \mathrm{ft}$

above land-surface datum.

PERIOD OF RECORD. - November 1985 to current year.

EXTREAES FOR PERIOD OF RECORD. --Highest water level, 65.30 ft below land-surface datum, Mar. 4, 1980

lowest, $72.80 \mathrm{ft}$ below-1and surface datum, Nov. 2, 1989.

WATER LEVEL, IN FEET BELOW LAND SURFACE DATUM, WATER YEAR OCTOBER 1990 TO SEPTEMBER 1991

$\begin{array}{cccccc}\text { DATE } & \begin{array}{c}\text { WATER } \\ \text { LEVEL }\end{array} & \text { DATE } & \begin{array}{c}\text { WATER } \\ \text { LEVEL }\end{array} & \text { DATE } & \begin{array}{c}\text { WATER } \\ \text { LEVEL }\end{array} \\ \text { Nov 11 } & 66.45 & \text { Mar 07 } & 66.32 & \text { Jul 19 } & 66.57 \\ \text { Jan 10 } & 66.59 & \text { May 13 } & 66.01 & \text { Sep 19 } & 66.95\end{array}$


445740093333001. Local number, 117N23W11BBD01

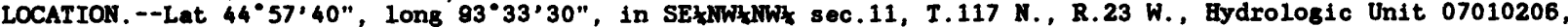

2 mi southwest of Wayzata, at Lake Minnetonk

Owner: Minnetonka Boat Works, Inc.. Orono.

AQUIFER. - Prairie du Chien Group of Early' Ordovician Age and Jordan Sandstone of Late Cambrian Age.

WELI CBARACTERISTICS. --Drilled observation artesian well, diameter 6 in. depth $437 \mathrm{ft}$, cased to $270 \mathrm{ft}$.

DATUM. --Altitude of land-surface datum is $930.8 \mathrm{ft}$ National Geodetic Vertical Datum of 1929 . Measuring point:

Wood floor of instrument shelter, $3.30 \mathrm{ft}$ above land-surface datum.

REMARKS. - Water level affected by pumping.

PERIOD OF RECORD. - August 1942 to current year.

EXTREMES FOR PERIOD OF RECORD.--Highest water level, $14.05 \mathrm{ft}$ below land-surface datum, Apr. 30, 1954; 10west,

$44.77 \mathrm{ft}$ below land-surface datum, June $28,1988$.

WATER LEVEL, IN FEET BELOW LAND-SURFACE DATUM, WATER YEAR OCTOBER 1980 TO SEPTERBER 1981

\begin{tabular}{|c|c|c|c|c|c|c|c|c|c|c|c|c|c|c|c|c|}
\hline DATE & $\begin{array}{l}\text { HATDR } \\
\text { LEVILL }\end{array}$ & DAI & & $\begin{array}{l}\text { HATIR } \\
\text { LEVIL }\end{array}$ & DAI & & $\begin{array}{l}\text { WATDR } \\
\text { LEVDL }\end{array}$ & DAI & & $\begin{array}{l}\text { WATDR } \\
\text { LEVEL }\end{array}$ & DAI & & $\begin{array}{l}\text { WATDR } \\
\text { LEVIL }\end{array}$ & DA: & & $\begin{array}{l}\text { WAIDR } \\
\text { LEVDL }\end{array}$ \\
\hline $\begin{array}{r}05 \\
05 \\
10 \\
15 \\
20 \\
25 \\
31 \\
\text { Hov } 05 \\
10 \\
15 \\
20 \\
25 \\
30\end{array}$ & $\begin{array}{l}35.20 \\
35.14 \\
34.48 \\
33.88 \\
34.10 \\
34.67 \\
33.40 \\
32.93 \\
33.00 \\
32.88 \\
32.35 \\
32.48\end{array}$ & Jan & $\begin{array}{l}05 \\
10 \\
15 \\
20 \\
25 \\
31 \\
05 \\
10 \\
15 \\
20 \\
25 \\
31\end{array}$ & $\begin{array}{l}32.47 \\
32.53 \\
33.04 \\
33.00 \\
33.01 \\
32.79 \\
32.52 \\
32.62 \\
32.04 \\
32.44 \\
32.91 \\
33.26\end{array}$ & Mar & $\begin{array}{l}05 \\
10 \\
15 \\
20 \\
25 \\
28 \\
05 \\
10 \\
15 \\
20 \\
25 \\
31\end{array}$ & $\begin{array}{l}33.07 \\
32.69 \\
32.48 \\
32.10 \\
32.28 \\
32.16 \\
32.40 \\
32.88 \\
32.89 \\
32.38 \\
32.32 \\
32.72\end{array}$ & Apr & $\begin{array}{l}05 \\
10 \\
15 \\
20 \\
25 \\
30 \\
05 \\
10 \\
15 \\
20 \\
25 \\
31\end{array}$ & $\begin{array}{l}33.28 \\
33.62 \\
33.33 \\
33.01 \\
33.14 \\
33.00 \\
33.21 \\
33.51 \\
34.33 \\
33.95 \\
34.49 \\
32.80\end{array}$ & Jul & $\begin{array}{l}05 \\
10 \\
15 \\
20 \\
25 \\
30 \\
05 \\
10 \\
15 \\
20 \\
25 \\
31\end{array}$ & $\begin{array}{l}34.32 \\
32.96 \\
35.94 \\
35.55 \\
37.44 \\
38.23 \\
35.42 \\
35.60 \\
34.26 \\
36.46 \\
37.51 \\
35.48\end{array}$ & Aus & $\begin{array}{l}05 \\
10 \\
15 \\
20 \\
25 \\
31 \\
05 \\
10 \\
15 \\
20 \\
25\end{array}$ & $\begin{array}{l}34.36 \\
33.13 \\
34.34 \\
34.22 \\
35.05 \\
37.30 \\
37.37 \\
36.67 \\
35.45 \\
35.10 \\
34.42\end{array}$ \\
\hline
\end{tabular}

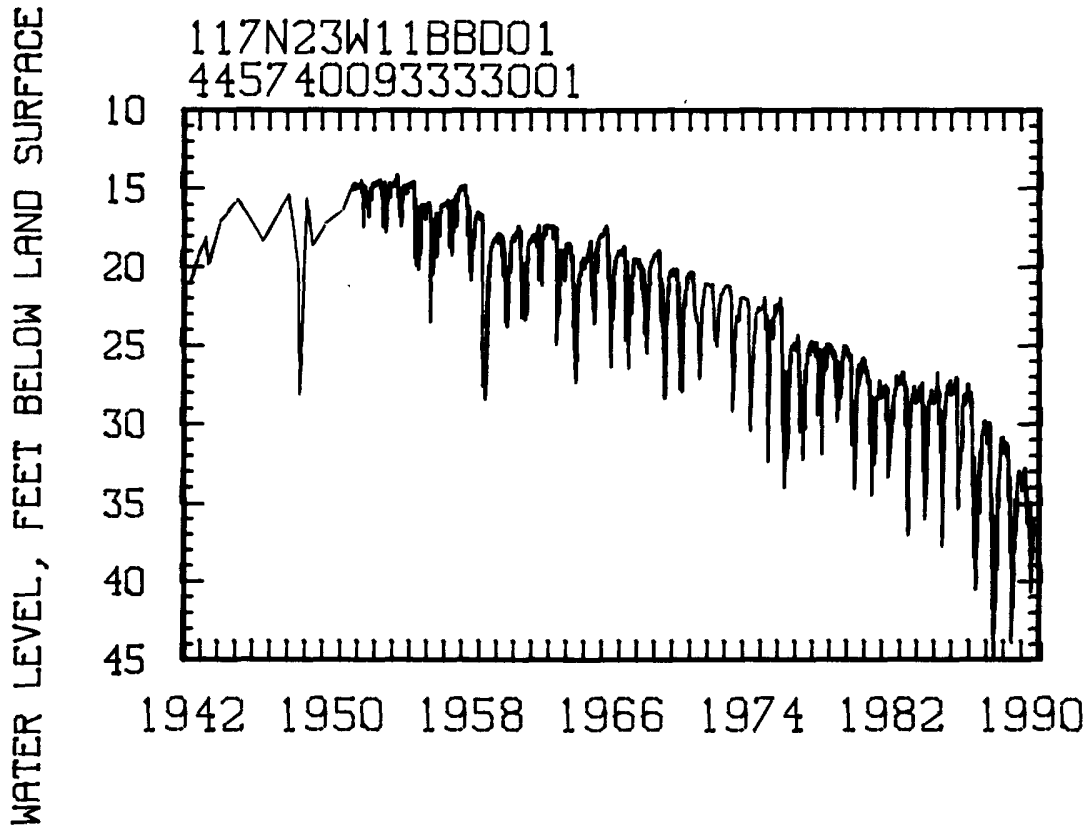

920.81

915.81 을

910.81

905.81

900.81 㞾

895.81

890.81 峞

885.81 年

息 
GROUND-WATER LEVELS

HENNEPIN COUNTY--Continued

450223093231801. Local number, 118N21W07DCB01.

LOCATION.--Lat $45^{\circ} 02^{\prime} 23^{\prime \prime}$, long $93^{\circ} 23^{\prime} 18^{\prime \prime}$, in NWłSWłSEz sec.7, T.118 N., R.21 W., Hydrologic Unit 07010206, at

47 th Avenue North and Aquila Avenue. Owner: City of Now Hope.

AQUIFER. --Jordan Sands tone of Late Cambrian Age.

WELL CHARACTERISTICS. --Drilled unused artesian well, diameter $16 \mathrm{in}$, depth $422 \mathrm{ft}$, cased to $339 \mathrm{ft}$

DATUM.--Altitude of land-surface datum is $933 \mathrm{ft}$. Measuring point: Top of wood platform, $3.00 \mathrm{ft}$ above landsurface datum.

REMARKS. - Water level affected by pumping.

PERIOD OF RECORD. --October 1965 to current year.

EXTREMES FOR PERIOD OF RECORD.--Highest water leve1, $60.46 \mathrm{ft}$ below land-surface datum, Dec. 17, 1967; 10west, $77.56 \mathrm{ft}$ below land-surface datum, July 11, 1985 .

WATER LEVEL, IN FEET BELOW LAND-SURFACE DATAUM, WATER YEAR OCTOBER 1990 TO SEPTEMBER 1991

\begin{tabular}{|c|c|c|c|c|c|c|c|c|c|c|c|c|c|c|c|}
\hline DATE & $\begin{array}{l}\text { WATER } \\
\text { LEVEL }\end{array}$ & DA? & & $\begin{array}{l}\text { WATER } \\
\text { LEVEL }\end{array}$ & DAI & & $\begin{array}{l}\text { WATER } \\
\text { LEVEL }\end{array}$ & DAI & & $\begin{array}{l}\text { WATER } \\
\text { LEVEL }\end{array}$ & DATE & $\begin{array}{l}\text { WATER } \\
\text { LEVEL }\end{array}$ & DAI & & $\begin{array}{l}\text { WATER } \\
\text { LEVEL }\end{array}$ \\
\hline $\begin{array}{r}05 \\
\text { Oct } \\
10 \\
15 \\
20 \\
25 \\
31 \\
\text { Nov } 05 \\
10 \\
15 \\
20 \\
25 \\
30\end{array}$ & $\begin{array}{l}71.41 \\
70.84 \\
70.00 \\
69.74 \\
68.75 \\
69.79 \\
69.19 \\
69.07 \\
69.53 \\
69.10 \\
69.04 \\
69.31\end{array}$ & Dec & $\begin{array}{l}05 \\
10 \\
15 \\
20 \\
25 \\
31 \\
05 \\
10 \\
15 \\
20 \\
25 \\
31\end{array}$ & $\begin{array}{l}69.43 \\
69.26 \\
69.62 \\
69.81 \\
68.78 \\
68.90 \\
69.32 \\
69.65 \\
69.12 \\
69.23 \\
69.89 \\
69.87\end{array}$ & Mar & $\begin{array}{l}05 \\
10 \\
15 \\
20 \\
25 \\
28 \\
05 \\
10 \\
15 \\
20 \\
25 \\
31\end{array}$ & $\begin{array}{l}69.86 \\
69.42 \\
70.06 \\
69.56 \\
69.34 \\
69.31 \\
69.09 \\
69.58 \\
69.79 \\
69.43 \\
69.46 \\
69.43\end{array}$ & Apr & $\begin{array}{l}05 \\
10 \\
15 \\
20 \\
25 \\
30 \\
05 \\
10 \\
15 \\
20 \\
25 \\
31\end{array}$ & $\begin{array}{l}69.88 \\
70.50 \\
68.81 \\
69.68 \\
70.48 \\
70.25 \\
70.05 \\
70.37 \\
71.95 \\
71.35 \\
70.79 \\
70.42\end{array}$ & $\begin{aligned} & \text { Jun } 0 \\
& 1 \\
& 1 \\
& 1 \\
& 2 \\
& 2 \\
& 3 \\
& \text { Jul } 0\end{aligned}$ & $\begin{array}{l}70.65 \\
71.82 \\
72.17 \\
72.17 \\
71.70 \\
74.25 \\
70.78 \\
71.97 \\
72.41 \\
72.77 \\
73.51 \\
71.99\end{array}$ & Aug & $\begin{array}{l}05 \\
10 \\
15 \\
20 \\
25 \\
31 \\
05 \\
10 \\
15 \\
20 \\
25 \\
30\end{array}$ & $\begin{array}{l}71.18 \\
70.71 \\
71.50 \\
71.37 \\
71.40 \\
73.97 \\
72.65 \\
71.62 \\
70.13 \\
69.97 \\
69.61 \\
69.53\end{array}$ \\
\hline
\end{tabular}

445905093224401 . Local number, 118N21W32CBB01.

LOCATION.--Lat $44^{\circ} 59^{\prime} 05^{\prime \prime}$, Iong $93^{\circ} 22^{\prime} 44^{\prime \prime}$, in NW'NW at Winnetka Avenue and Highway 55, Goiden Valley.

Owner: Red Owl Store.

AQUIFER. - Surficial sand and gravel of Ploistocene Age.

WELL CHARACTERISTICS.--Drilled unused water-table woli, diameter $6 \mathrm{in.,} \mathrm{depth} 95 \mathrm{ft}$, screened 87 to $95 \mathrm{ft}$.

DATUM. --iltitude of land-surface datum is $895 \mathrm{ft}$. Measuring point: Top of well cap, $0.80 \mathrm{ft}$ above land-surface datum.

PERIOD OF RECORD. - -May 1979 to current year.

EXTREMES FOR PERIOD OF RECORD.--Highest water lovel, $17.72 \mathrm{ft}$ below land-surface datum, May 12, 1986 ; lowest, $22.43 \mathrm{ft}$ below land-surface datum, Jan. 18,1990 .

WATER LEVEL, IN FEET BELOW LAND-SURFACE DATUM, WATER YEAR OCTOBER 1990 TO SEPTEMBER 1991

$\begin{array}{cccccc}\text { DATE } & \begin{array}{c}\text { WATER } \\ \text { LEVEL }\end{array} & \text { DATE } & \begin{array}{c}\text { WATER } \\ \text { LEVEL }\end{array} & \text { DATE } & \begin{array}{c}\text { WATER } \\ \text { LEVEL }\end{array} \\ \text { Nov 14 } & 21.14 & \text { Mar 07 } & 21.82 & \text { Jul 19 } & 19.97 \\ \text { Jan 10 } & 21.53 & \text { May 13 } & 20.99 & \text { Søp 19 } & 19.29\end{array}$

445857093223101. Local number, 118N21W32CBD01.

LOCATION.--Lat $44^{\circ} 58^{\prime} 57^{\prime \prime}$, long $93^{\circ} 22^{\prime} 3^{\prime \prime}$, in SEzWWZSWK sec.32, T.118 N., R.21 W., Bydrologic Unit 07010206 , at 760 Harold Avenue, Golden Valley.

Owner: Golden Vailey Methodist Church.

AQUIFER. - - St. Peter Sandstone of Middle Ordovician Age

WELI CHARACTERISTICS.-Drilled unused artesian well, diameter 6 in., depth 265 ft, cased to $200 \mathrm{ft}$.

DATUM.--Altitude of iand-surface datum is $890 \mathrm{ft}$. Measuring point: Top of well cap, $0.70 \mathrm{ft}$ above

land-surface datum.

PERIOD OF RECORD. --February 1971 to current year.

EXTREMES FOR PERIOD OF RECORD.--Highest water level, 31.33 ft below land-surface datum, May 12, 1986 ;

lowest, $40.62 \mathrm{ft}$ below land-surface datum, Sept. 14, 1990.

WATER LEVEL, IN FEET BELOW LAAND-SURFACE DATUM, WATER YEAR OCTOBER 1990 TO SEPTEMBER 1991

$\begin{array}{cccccc}\begin{array}{l}\text { WATER } \\ \text { DATE }\end{array} & \text { LEVEL } & \begin{array}{c}\text { WATER } \\ \text { DATE }\end{array} & \text { LEVEL } & \begin{array}{c}\text { WATER } \\ \text { DATE }\end{array} & \text { LEVEL } \\ \text { Nov 14 } & \mathbf{3 8 . 3 5} & \text { Mar 07 } & \mathbf{3 8 . 6 3} & \text { Jul 19 } & \mathbf{3 9 . 2 0} \\ \text { Jan 10 } & \mathbf{3 8 . 7 1} & \text { May 13 } & \mathbf{3 8 . 4 0} & \text { Søp 19 } & \mathbf{3 7 . 1 7}\end{array}$




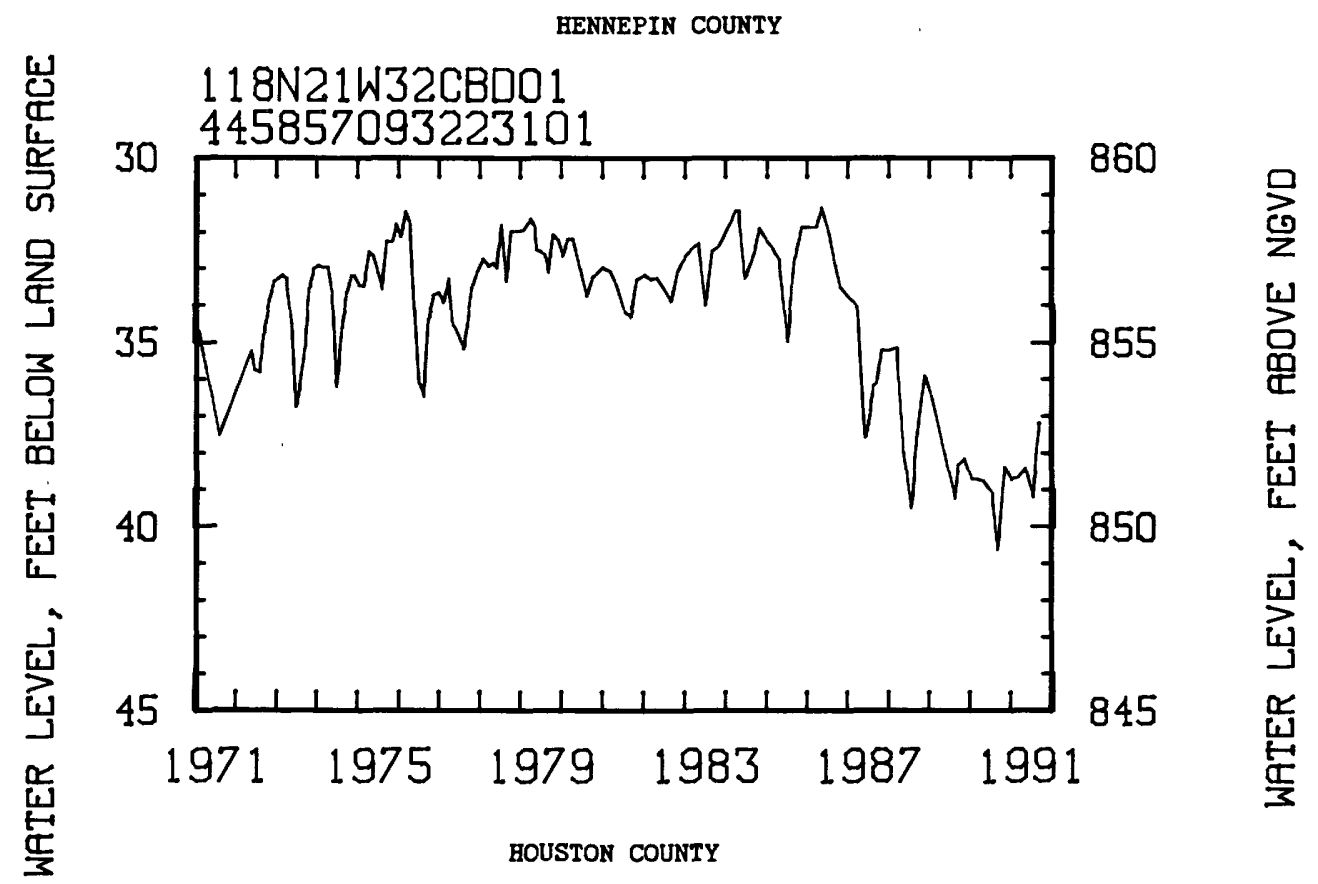

433935091251801. Local number, 102N05W03DCC01.

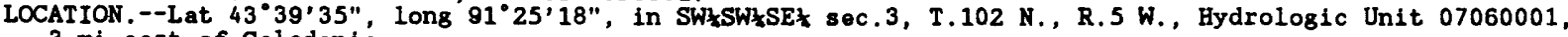
3 mi east of Caledonia.

Owner: U.S Geological Survey.

AQUIFER. - Jordan Sandstone of Late Cambrian Age.

WELL CHARACTERISTICS.--Drilled observation water-table well, diameter 4 in,, depth $360 \mathrm{ft}$, cased to $309 \mathrm{ft}$.

DATUM.--Aititude of land-surface datum is 1,210 ft. Measuring point: Top of casing, $2.50 \mathrm{ft}$ above land-surface datum.

PERIOD OF RECORD. --June 1981 to current year.

EXTREMES FOR PERIOD OF RECORD.--Highest water level, $213.76 \mathrm{ft}$ below land-surface datum, July 17 ,

1985; lowest, 246.64 ft below land-surface datum, Sept. 20, 1991.

WATER LEVEL, IN FEET BELOW LAND-SURFACE DATUM, WATER YEAR OCTOBER 1990 TO SEPTEMBER 1991

\begin{tabular}{rrrrrr} 
DATE & $\begin{array}{r}\text { WATER } \\
\text { LEVEL }\end{array}$ & \multicolumn{1}{c}{ DATE } & $\begin{array}{r}\text { WATER } \\
\text { LEVEL }\end{array}$ & DATE & $\begin{array}{r}\text { WATER } \\
\text { LEVEL }\end{array}$ \\
Nov 10 & 242.82 & Mar 08 & 244.16 & Sep 05 & 246.20 \\
15 & 242.66 & Aug 15 & 245.96 & 10 & 246.33 \\
20 & 242.84 & 20 & 246.13 & 15 & 246.07 \\
25 & 242.82 & 25 & 246.25 & 20 & 246.64 \\
30 & 243.36 & 31 & 246.34 & 25 & 246.19 \\
& & & & 30 & 246.35
\end{tabular}

433935091252001 . Local number, 102N05W03DCC02.

LOCATION, - Lat $43^{\circ} 39^{\prime} 35^{\prime \prime}$, Iong $91^{\circ} 25^{\prime} 2^{\prime \prime}$, in SWł SWZSEz sec.3, T. 102 N., R.5 W., Hydrologic Unit 07060001 ,

$3 \mathrm{mi}$ east of Caledonia.

Owner: U.S. Geological Survey.

AQUIFER. --Ironton-Galesville Sandstones of Late Cambrian Age.

WELI CBARACTERISTICS.--Drilled observation artesian we11, diameter $4 \mathrm{in}$, depth $650 \mathrm{ft}$, cased to $614 \mathrm{ft}$.

DATUM.--Altitude of land-surface datum is 1,210 ft. Measuring point: Top of casing, $2.50 \mathrm{ft}$ above land-surface datum.

PERIOD OF RECORD. --November 1980 to current year.

EXTREMES FOR PERIOD OF RECORD, --Highest water level, $441.50 \mathrm{ft}$ below land-surface datum, June 4 , 1981; lowest, $452.21 \mathrm{ft}$ below land-surface datum, July $31,1990$.

WATER LEVEL, IN FEET BELOW LAND-SURFACE DATUM, WATER YEAR OCTOBER 1990 TO SEPTEMBER 1991

$\begin{array}{crcrcrrr}\text { DATE } & \begin{array}{r}\text { WATER } \\ \text { LEVEL }\end{array} & \text { DATE } & \begin{array}{r}\text { WATER } \\ \text { LEVEL }\end{array} & \text { DATE } & \begin{array}{r}\text { WATER } \\ \text { LEVEL }\end{array} & \text { DATE } & \begin{array}{r}\text { WATER } \\ \text { LEVEL }\end{array} \\ \text { Nov 08 } & 451.40 & \text { Mar 08 } & 451.76 & \text { Aug } 14 & 451.40 & \text { Sep 27 } & 451.43\end{array}$


GROUND-WATER LEVELS

HOUSTON COUNTY

443935091252901. Local number, 102N05W03DCC03.

LOCATION. - Lat $44^{\circ} 39^{\prime} 35^{\prime \prime}$, long $91^{\circ} 25^{\prime} 1^{\prime \prime}$, in SWzSWzEł sec.3, T.102 N., R.5 W., Hydrologic Unit 07060001, $3 \mathrm{mi}$ east of Caledonia.

Owner: U.S. Geological Survey

AQUIFER. - Mount Simon Sandstone of Late Cambrian Age.

WELL CBARACTERISTICS.--Drilled observation artesian well, diameter $4 \mathrm{in}$., depth $888 \mathrm{ft}$, cased to $858 \mathrm{ft}$.

DATUM.--Altitude of land-surface datum is 1,210 ft. Measuring point: Top of casing, $2.00 \mathrm{ft}$ above landsurface datum.

PERIOD OF RECORD.--July 1982 to current year.

EXTREMES FOR PERIOD OF RECORD.--Highest water level, 522.00 ft below land-surface datum, Nov. 10, 1983;

lowest, $526.00 \mathrm{ft}$ below land-surface datum, Sept. 29, 1989.

WATER LEVEL, IN FEET BELOW LAND-SURFACE DATUM, WATER YEAR OCTOBER 1990 TO SEPTEMBER 1991

$\begin{array}{crcrcr}\text { DATE } & \begin{array}{r}\text { WATER } \\ \text { LEVEL }\end{array} & \text { DATE } & \begin{array}{r}\text { WATER } \\ \text { LEVEL }\end{array} & \text { DATE } & \begin{array}{r}\text { WATER } \\ \text { LEVEL }\end{array} \\ \text { Nov } 08 & 525.68 & \text { Mar 08 } & 525.46 & \text { Sep } 27 & 525.92\end{array}$

HUBBARD COUNTY

465142094433201. Local number, 139N32W16AAA01.

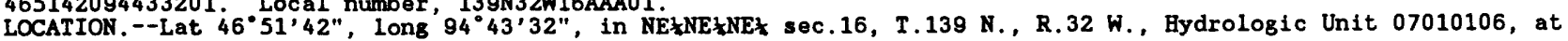
Badoura Nursery.

Omer: U.S. Geological Survey.

AQUIFER. --Surficial outwash sand of Pleistocene Age.

WELI CHARACTERISTICS.--Bored observation water-table well, diameter 1 in., depth $21 \mathrm{ft}$, screened 19 to $21 \mathrm{ft}$. DATUM. --Altitude of iand-surface datum is 1,419 ft. Measuring point: Top of casing, $2.00 \mathrm{ft}$ above land-surface datum.

RFMARKS --Measured weekly by Archie Hakala.

PERIOD OF RECORD. --September 1970 to current year.

EXTREMES FOR PERIOD OF RECORD. -- Highest water level, $10.63 \mathrm{ft}$ below land-surface datum, Sept. 24, 1985; lowest, $16.15 \mathrm{ft}$ below land-surface datum, Mar. 19, 1991.

WATER LEVEL, IN FEET BELOW LAND-SURFACE DATUM, WATER YEAR OCTOBER 1990 TO SEPTEMBER 1991

\begin{tabular}{|c|c|c|c|c|c|c|c|c|c|c|c|c|c|c|c|c|}
\hline DATE & $\begin{array}{l}\text { WATER } \\
\text { LEVEL }\end{array}$ & DAT & TE & $\begin{array}{l}\text { WATER } \\
\text { LEVEL }\end{array}$ & $\mathrm{DA}^{\prime}$ & & $\begin{array}{l}\text { WATER } \\
\text { LEVEL }\end{array}$ & DA & & $\begin{array}{l}\text { WATER } \\
\text { LEVEL }\end{array}$ & $D A^{\prime}$ & & $\begin{array}{l}\text { WATER } \\
\text { LEVEL }\end{array}$ & $D A^{\prime}$ & & $\begin{array}{l}\text { WATER } \\
\text { LEVEL }\end{array}$ \\
\hline $\begin{array}{ll}0 c t & 02 \\
09 \\
16 \\
22 \\
30 \\
\text { Nov } 07 \\
13 \\
20 \\
27\end{array}$ & $\begin{array}{l}15.50 \\
15.51 \\
15.55 \\
15.56 \\
15.59 \\
15.60 \\
15.60 \\
15.61 \\
15.63\end{array}$ & $\begin{array}{l}\text { Dec } \\
\text { Jan }\end{array}$ & $\begin{array}{l}18 \\
25 \\
01 \\
08 \\
15 \\
22 \\
29\end{array}$ & $\begin{array}{l}15.72 \\
15.75 \\
15.78 \\
15.81 \\
15.86 \\
15.89 \\
15.93\end{array}$ & $\begin{array}{l}\text { Feb } \\
\text { Mar }\end{array}$ & $\begin{array}{l}05 \\
12 \\
19 \\
26 \\
05 \\
12 \\
19 \\
26\end{array}$ & $\begin{array}{l}15.96 \\
16.00 \\
16.03 \\
16.05 \\
16.10 \\
16.12 \\
16.15 \\
16.10\end{array}$ & $\begin{array}{l}\text { Apr } \\
\text { May }\end{array}$ & $\begin{array}{l}02 \\
09 \\
16 \\
23 \\
30 \\
07 \\
14 \\
21 \\
28\end{array}$ & $\begin{array}{l}16.10 \\
16.12 \\
16.14 \\
16.14 \\
16.10 \\
15.97 \\
15.78 \\
15.63 \\
15.42\end{array}$ & $\begin{array}{l}\text { Jun } \\
\text { Jul }\end{array}$ & $\begin{array}{l}04 \\
11 \\
18 \\
25 \\
02 \\
09 \\
16 \\
23 \\
30\end{array}$ & $\begin{array}{l}15.29 \\
15.22 \\
15.20 \\
15.15 \\
15.11 \\
15.07 \\
15.05 \\
15.03 \\
15.02\end{array}$ & $\begin{array}{l}\text { Aug } \\
\text { Sep }\end{array}$ & $\begin{array}{l}06 \\
13 \\
20 \\
27 \\
03 \\
10 \\
17 \\
24\end{array}$ & $\begin{array}{l}15.05 \\
15.16 \\
15.23 \\
15.39 \\
15.46 \\
15.44 \\
15.43 \\
15.44\end{array}$ \\
\hline
\end{tabular}

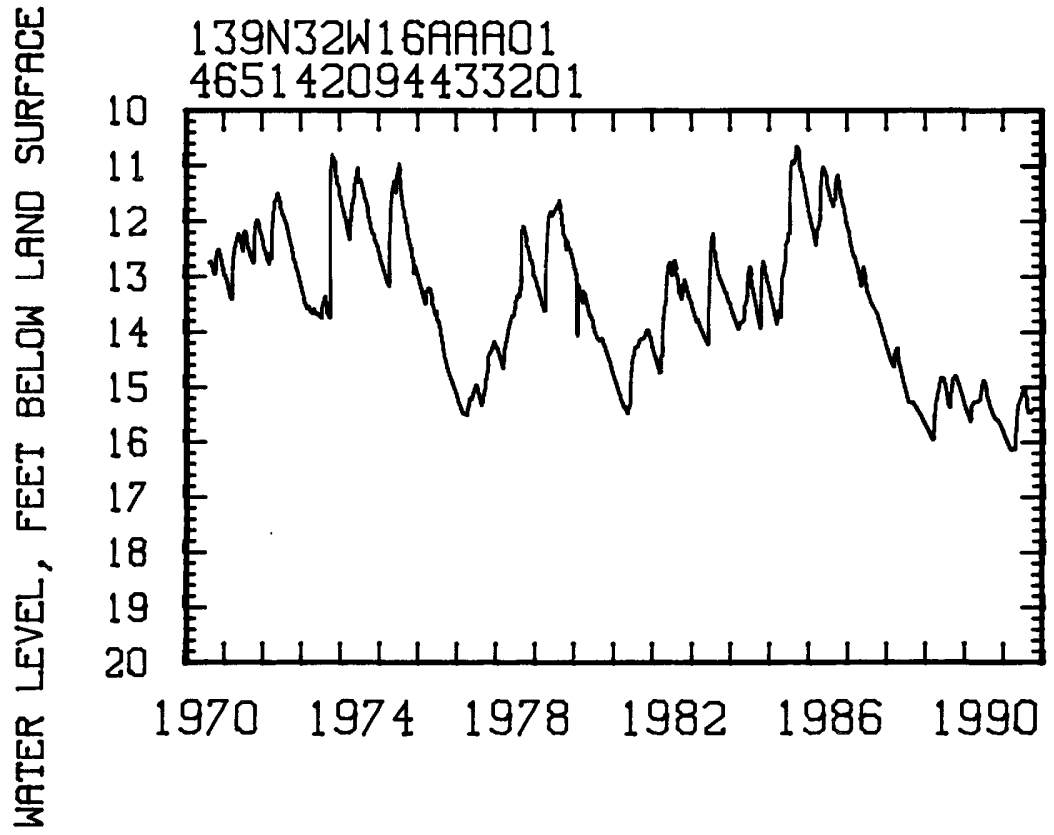

1409 1408 
453125093181101 Local number, 035N24W14BCDO1

LOCATION, --Lat $45^{\circ} 31^{\prime} 25^{\prime \prime}$, Long $93^{\circ} 18^{\prime} 11^{\prime \prime}$, in SEłSW\}WW sec.14, T.35 N., R.24 W., Hydrologic Unit 07010207, northmest of Isenti.

Owner: Allen Kluck.

AQUIFER.--Eeu Claire - Mount Simon Formations of Late Cambrian Age.

WELL CBARACTERISTICS.--Drilled irrigation artesian vell, diameter $12 \mathrm{in}$, depth $300 \mathrm{ft}$, cased to $105 \mathrm{ft}$

DATUM.--Altitude of land-surface datum is $940 \mathrm{ft}$. Measuring point: Bole in pump base, $0.10 \mathrm{ft}$ above

land-surface datum.

PERIOD OF RECORD. - February 1968 to current year.

EXTREAIES FOR PERIOD OF RECORD. - Highest water level, $11.18 \mathrm{ft}$ below land-surface datum, June 24, 1986 ;

lowest, $16.16 \mathrm{ft}$ below land-surface datum, Oct. 31 , 1989 .

WATER LEVEL, IN FEET BELOW LAND-SURFACE DATUM, WATER YEAR OCTOBER 1990 TO SEPTEMBER 1981

$\begin{array}{cccc}\text { DATE } & \begin{array}{c}\text { WATER } \\ \text { LEVEL }\end{array} & \text { DATE } & \begin{array}{c}\text { WATER } \\ \text { LEVEL }\end{array} \\ \text { Aug 02 } & 12.43 & \text { Sep 30 } & 12.38\end{array}$

453058093175901. Local number, 035N24W14CDC01.

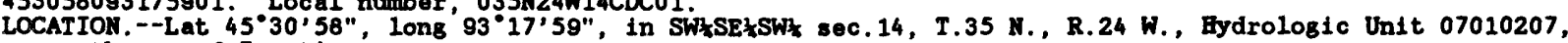
northwest of Isant1.

Omer: Ernest Kiuck.

AQUIFER. - - Surficial outwash sand of Pleistocene Age.

WELL CHARACTERISTICS. - Driven unused water-table weil, diemeter $1 *$ in., depth 17 ft, screen information not available.

DATUM.--Altitude of land-surface datum is $930 \mathrm{ft}$. Measuring point: Top of casing, $3.00 \mathrm{ft}$ above landsurface datum.

PERIOD OF RECORD. --March 1968 to current year.

EXTREMES FOR PERIOD OF RECORD. --Highest water level, 2.50 ft belon land-surface datum, June 24, 1986 ; lowest, $10.87 \mathrm{ft}$ below land-surface datum, Oct. 31, 1989.

WATER LEVEL, IN FEET BELON LAND-SURFACE DATUA, WATER YEAR OCTOBER 1990 TO SEPTEMBER 1991

$\begin{array}{crcr}\text { DATE } & \begin{array}{r}\text { WATER } \\ \text { LEVEL }\end{array} & \text { DATE } & \begin{array}{r}\text { WATER } \\ \text { LEVEL }\end{array} \\ \text { Aug 02 } & 7.09 & \text { Sep } 30 & 6.90\end{array}$

ITASCA COUNTY

471448093322001. Local number, 055N25W17ACD01.

LOCATION. - Lat 47 $14^{\prime} 46^{\prime \prime}$, long $93^{\circ} 32^{\prime} 20^{\prime \prime}$, in SEłSWłNEz sec.17, T.55 N., R.25 W., Bydrologic Unit 07010103 , at west end of 13th Street NW, Grand Rapids.

owner: U.S. Geological Survey.

AQUIFER - - Buried sand and gravel of Pleistocene Age.

WELL CHARACTERISTICS. --Drilled observation water-table well, diemeter $4 \mathrm{in}$, depth $147 \mathrm{ft}$, screened 143 to $147 \mathrm{ft}$.

DATUM.--Altitude of land-surface datum is 1,318 ft. Measuring point: Top of platform, $1.60 \mathrm{ft}$ above land-surface datum.

PERIOD OF RECORD --April 1962 to current year.

EXTREMES FOR PERIOD OF RECORD. -- Highest water level, 29.04 ft below land-surface datum, June 1, 1966; lowest, 33.92 ft below land-surface datum, May 17, 1977 .

WATER LEVEL, IN FEET BELOW LAND-SURFACE DATUA, WATER YEAR OCTOBER 1990 TO SEPTEMBER 1991

DATE WATER WATER WATER DATE WATER WATER

$\begin{array}{llllllllllll}\text { Oct } 10 & 31.88 & \text { Nov } 23 & 31.72 & \text { Feb } 14 & 32.06 & \text { May } 09 & 32.28 & \text { Ju1 } 30 & 32.18\end{array}$

$\begin{array}{llllllllll}12 & 31.87 & \text { Jan } 04 & 31.98 & \text { Mar 25 } & 32.18 & \text { Jun } 12 & 31.72 & \text { Sep } 06 & 31.39\end{array}$


GROUID-WATER LEVELS

JACKSOA COUNTY

434742095191501. Local number, 104N37W19DBD01.

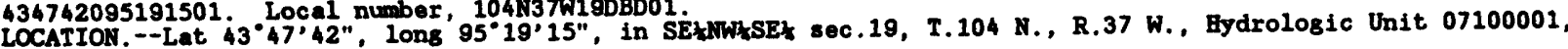
at Beron Lake.

Omer: City of Beron Lake, old railroad well.

AQUIFER --Sioux Quartzite of Late Precambrian Ase.

205 to $225 \mathrm{ft}$.
DATUy.--Altitude of land-surface datum is 1,420 ft. Measuring point: Edge of breather pipe, 2.60 ft above land-surface datum.

PERIOD OP RECORD.-August 1972, July 1973, September 1976, July 1978 to current year.

EXIRDYES FOR PERIOD OF RECORD.--Highest water leve1, 59.16 ft below land-surface datum, Aus. 11, 1972 ;

lowest, $66.10 \mathrm{ft}$ below land-surface datum, July i4, 1981.

WATER LEVEL, IN FEET BELON LAND-SURFACE DATUY, WATER YEAR OCTOBER 1990 TO SEPTEMBER 1991

$\begin{array}{cccccc}\text { DATE } & \begin{array}{c}\text { WATER } \\ \text { LEVEL }\end{array} & \text { DATE } & \begin{array}{l}\text { WATER } \\ \text { LEVEI }\end{array} & \text { DATE } & \begin{array}{c}\text { WATER } \\ \text { LEVEL }\end{array} \\ \text { Oct 23 } & 64.78 & \text { Feb 01 } & 63.85 & \text { Jun 19 } & 61.44 \\ \text { Hov 28 } & 64.43 & \text { Apr 17 } & 62.40 & \text { Aug 19 } & 62.06\end{array}$

KANABDC COUNTY

455236093172301. Local number, 039N24W11DDC01.

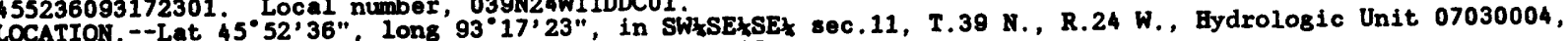

intersection of Forest Avenue and U.S. Highway 65

Omer: City of Mora, well 3.

AQUIFER.-Buried sand and gravel of Pleistocene Ase.

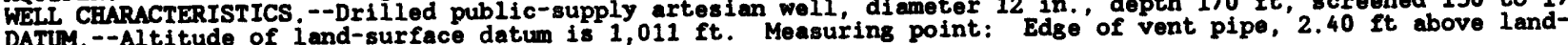
surface datum.

PERIOD OF RECORD.--March 1968 to current year.

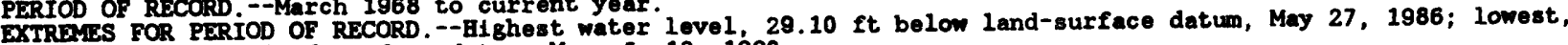
46.90 ft below land-surface datum, Mar. 5, 12, 1990 .

WATER LEVEL, IN FEET BELON LAND-SURFACE DATUM, WATER YEAR OCTOBER 1990 TO SEPTEMBER 1991

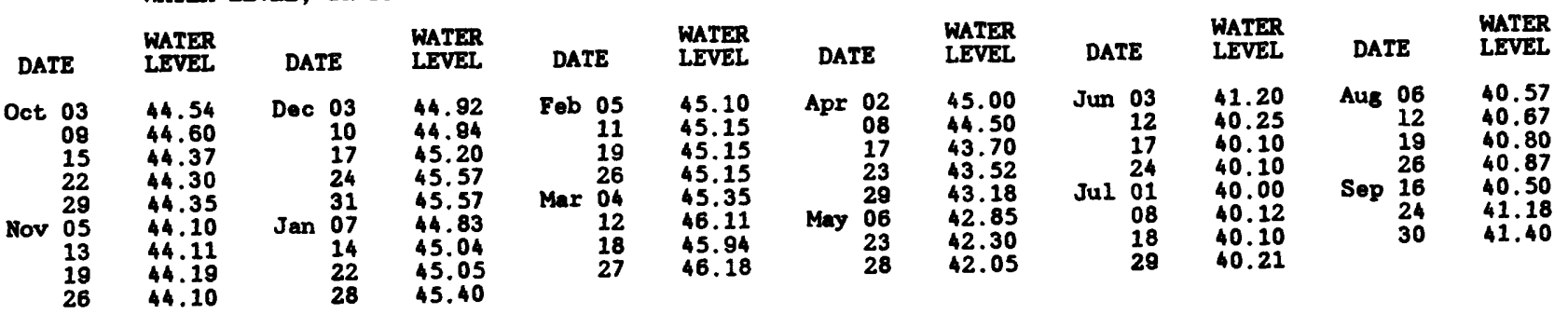

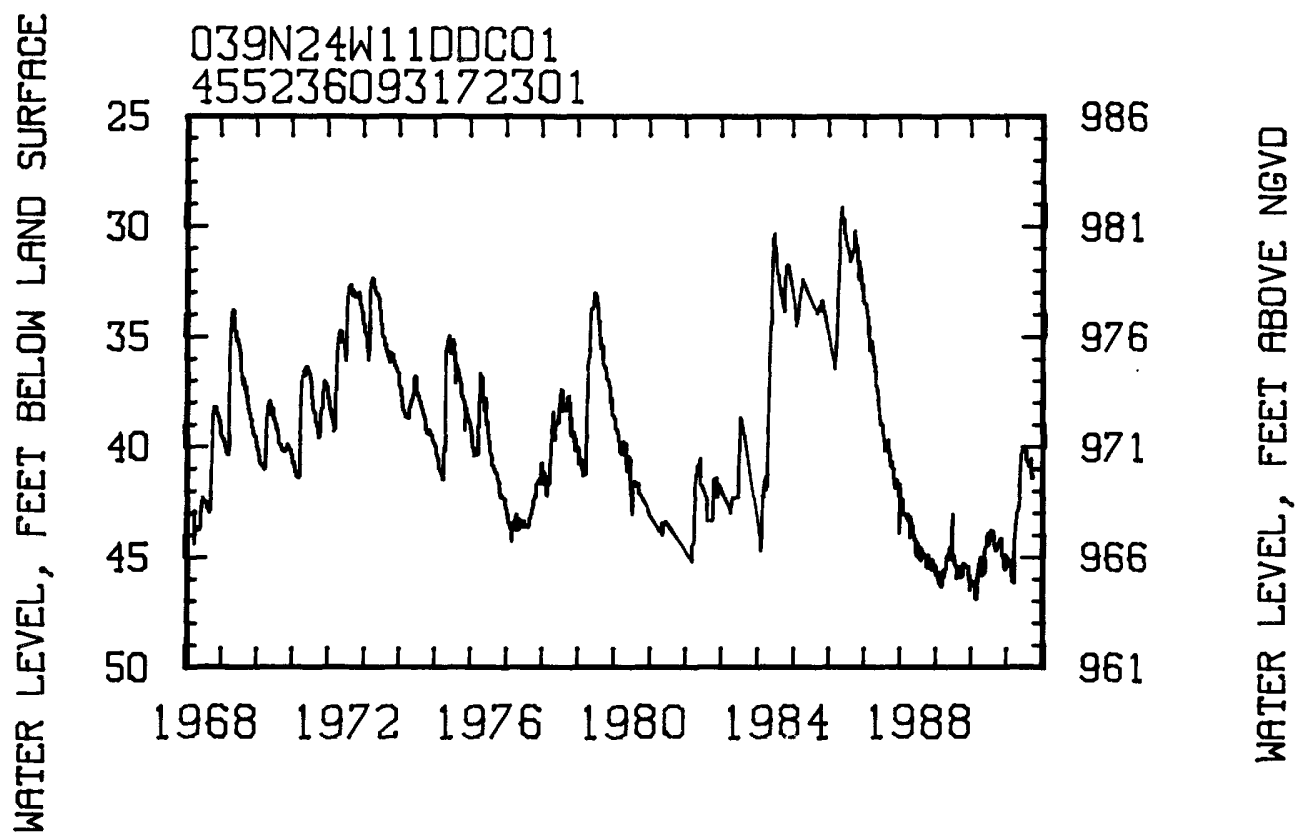


442522093543901. Local number, 111N26W14ADA01.

LOCATION. - Lat $44^{\circ} 25^{\prime} 22^{\prime \prime}$, Iong $93^{\circ} 54^{\prime} 39^{\prime \prime}$, in NEkSEkNEk sec.14, T.111 N., R.26 W., Hydrologic Unit 07020012 , 0.85 mi south of te sueur.

Owner: Merle Moser.

AQUIFGR.--Buried gravel of Pleistocene Age and Jordan Sandstone of Late Cambrian Age.

WELL CHARACTERISTICS. --Drilled Irrigation artesian well, diameter 16 in., depth 242 ft, screened 212 to $242 \mathrm{ft}$

DATUM. --Altitude of land-surface datum is $855 \mathrm{ft}$. Measuring point: Edge of vent pipe, $1.20 \mathrm{ft}$ above land-surface datum.

PERIOD OF RECORD. --January 1980 to current year.

EXTREMIES FOR PERIOD OF RECORD.--H1ghest water level, $71.82 \mathrm{ft}$ below land-surface datum, Feb. 11, 1987

lowest, $84.55 \mathrm{ft}$ below land-surface datum, Mar. 9, 1982.

WATER LEVEL, IN FEET BELON LAND-SURFACE DATUM, WATER YEAR OCTOBER 1990 TO SEPTEMBER 1991

$\begin{array}{cccccccc}\text { DATE } & \begin{array}{c}\text { WATER } \\ \text { LEVEL }\end{array} & \text { DATE } & \begin{array}{c}\text { WATER } \\ \text { LEVEI }\end{array} & \text { DATE } & \begin{array}{c}\text { WATER } \\ \text { LEVEL }\end{array} & \text { DATE } & \begin{array}{c}\text { WATER } \\ \text { LEVEL }\end{array} \\ \text { Nov 20 } & \mathbf{8 2 . 7 8} & \text { Mar 26 } & 83.00 & \text { May 29 } & 83.03 & \text { Sep 25 } & \mathbf{8 2 . 0 2}\end{array}$

443234093333501 Local number, 112\$23W02BAB01.

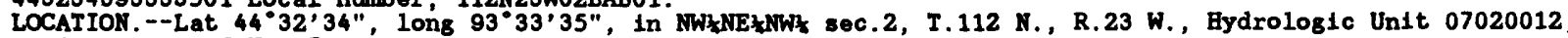
just east of New Prague.

Omer: Holy Trinity Lutheran Church.

AQUIFER. - - St. Lawrence Formation of Late Cambrian Age.

WELL CBARACTERISTICS.--Drilled domestic artesian weli, diameter 4 in., depth $180 \mathrm{ft}$, cased to $155 \mathrm{ft}$. DATUM. - Altitude of land-surface datum is 1,005 ft. Measuring point: Top of casing, $1.00 \mathrm{ft}$ above 1and-surface datum.

PERIOD OF RECORD. --April 1979 to current year.

EXTREMES FOR PERIOD OF RECORD. --Bighest water level, $93.56 \mathrm{ft}$ below land-surface datum, Feb. 3, 1987; lowest, $99.42 \mathrm{ft}$ below land-surface datum, July 26, 1979.

WATER LEVEL, IN FEET BELOW LAND-SURFACE DATUM, WATER YEAR OCTOBER 1990 TO SEPTEMBER 1991

$\begin{array}{cccccc}\text { DATE } & \begin{array}{c}\text { WATER } \\ \text { LEVEL }\end{array} & \text { DATE } & \begin{array}{c}\text { WATER } \\ \text { LEVEL }\end{array} & \text { DATE } & \begin{array}{c}\text { WATER } \\ \text { LEVEL }\end{array} \\ \text { Nov 20 } & 96.73 & \text { May 10 } & 96.15 & \text { Sep } 18 & 97.00\end{array}$

443147093374501. Local number, 112N23W06DDD01.

LOCATION. - Lat $44^{\circ} 31^{\prime} 47^{\prime \prime}$, Iong $93^{\circ} 37^{\prime} 45^{\prime \prime}$, in SEłSEłSEk sec.6, T.112 N., R. 23 W., Hydrologic Un1t 07020012 3 mi southwest of New' Prague.

Oner: Friedens Lutheran Church.

AQUIFER. - - St. Lawrence Formation of Late Cambrian Age.

WELL CEARACTERISTICS.--Drilled domestic artesian well, diameter $5 \mathrm{in}$, depth $265 \mathrm{ft}$, cased to $209 \mathrm{ft}$.

DATUM. - Altitude of iand-surface datum is $1,019 \mathrm{ft}$. Measuring point: Top of casing, $1.70 \mathrm{ft}$ above land-surface datum.

PERIOD OF RECORD.--April 1979 to current year.

EXTREMES FOR PERIOD OF RECORD. -- Highest water level, 150.85 ft below land-surface datum, Mar. 18, 1981;

lowest, 153.58 ft below land-surface datum, July $19,1988$.

WATER LEVEL, IN FEET BELON LAND-SURFACE DATUM, WATER YEAR OCTOBER 1990 TO SEPTEMBER 1991

$\begin{array}{crcrcccr}\text { DATE } & \begin{array}{r}\text { WATER } \\ \text { LEVEL }\end{array} & \text { DATE } & \begin{array}{r}\text { WATER } \\ \text { LEVEL }\end{array} & \text { DATE } & \begin{array}{r}\text { WATER } \\ \text { LEVEL }\end{array} & \text { DATE } & \begin{array}{r}\text { WATER } \\ \text { LEVEL }\end{array} \\ \text { Nov 20 } & 151.70 & \text { Mar 14 } & 151.82 & \text { May 10 } & 151.78 & \text { Sep } 18 & 152.52\end{array}$


GROUID-WATER LEVELS

LINCOLN COUNTY

441705096084501. Local number, 110N44W33DCD01.

LOCATION --Lat $44^{\circ} 17^{\prime} 05^{\prime \prime}$, long $96^{\circ} 08^{\prime} 45^{\prime \prime}$, in SEzSWzSEz sec.33, T.110 N., R.44 W., Bydrologic Unit 07020006 , at Tylor.

Omer: U.S. Geological Survey.

AQUIFER. --Dakota Sandstone of Early Cretaceous Age.

WELL CHARACTERISTICS.--Drilled observation artesian well, diameter $8 \mathrm{in.,} \mathrm{depth} 967$ ft, screened 890 to $900 \mathrm{ft}$.

DATUM.--Aititude of land-surface datum is 1,738 ft. Measuring point: Top of recorder platform, $3.50 \mathrm{ft}$ above land-surface datum

PERIOD OF RECORD.--llovember 1969 to current year.

EXTRERES FOR PERIOD OF RECORD. --Highest water level, 247.41 ft below land-surface datum, Mar. 23, 1987; lowest, $250.82 \mathrm{ft}$ below land-surface datum, Nov. 12, 1976.

WATER LEVEL, IN FEET BELOW LAND-SURFACE DATUM, WATER YEAR OCTOBER 1990 TO SEPTEMBER 1991

$\begin{array}{crcccccr}\text { DATE } & \begin{array}{r}\text { WATER } \\ \text { LEVEI }\end{array} & \text { DATE } & \begin{array}{r}\text { WATER } \\ \text { LEVEL }\end{array} & \text { DATE } & \begin{array}{r}\text { WATER } \\ \text { LEVEI }\end{array} & \text { DATE } & \begin{array}{r}\text { WATER } \\ \text { LEVEL }\end{array} \\ \text { Oct } 23 & 249.14 & \text { Fob 21 } & 249.17 & \text { Apr 08 } & 249.12 & \text { Jul } 10 & 249.17\end{array}$

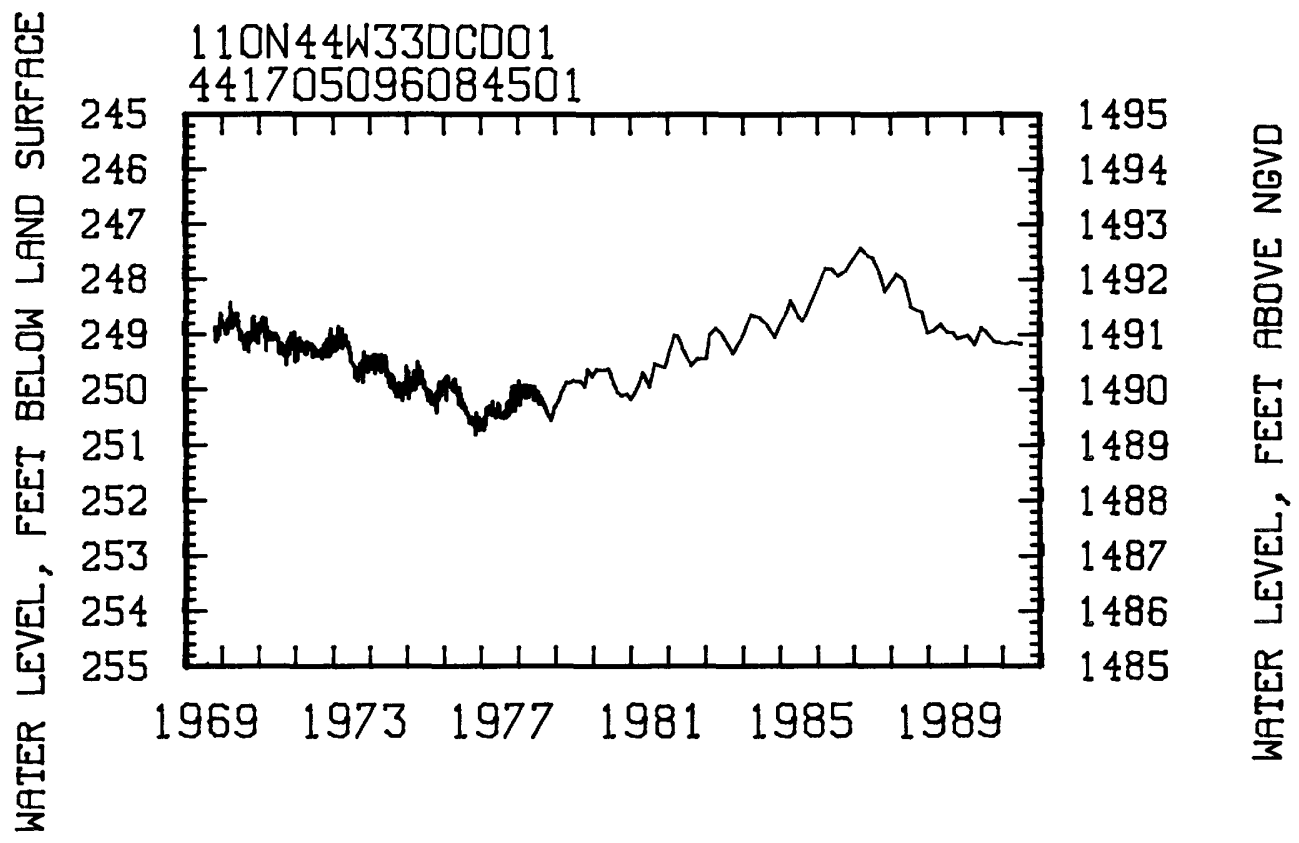

MARTIN COUNTY

434359094422201. Local number, 103\$32W08CCD01.

LOCATION. - Lat $43^{\circ} 43^{\prime} 59^{\prime \prime}$, Long $94^{\circ} 42^{\prime} 22^{\prime \prime}$, in SEłSW3 SW sec.8, T.103 N., R.32 W., Hydrologic Unit 07020009,

1.5 mi south of Trimont.

Omer: Robert Olson.

AQUIFER.--Sandstone of Cretaceous Ase

WELL CHARACTERISTICS.--Drilled irrigation artesian ve11, diameter $16 \mathrm{in.,} \mathrm{depth} 412 \mathrm{ft}$, screened 372 to $412 \mathrm{ft}$.

DATUM.--Altitude of land-surface datum is 1,242 ft. Measuring point: Vent pipe, $0.50 \mathrm{ft}$ above landsurface datum.

PERIOD OF RECORD. - July 1981 to current year.

EXTREMES FOR PERIOD OF RECORD.--Highest water level, 90.50 ft below land-surface datum, Apr. 14, 1987 lowest, $96.22 \mathrm{ft}$ below land-surface datum, July $21,1987$.

WATER LEVEL, IN FEET BELOW LAND-SURFACE DATUM, WATER YEAR OCTOBER 1990 TO SEPTEMBER 1991

$\begin{array}{cccccc}\text { DATE } & \begin{array}{c}\text { WATER } \\ \text { LEVEL }\end{array} & \text { DATE } & \begin{array}{c}\text { WATER } \\ \text { LEVEL }\end{array} & \text { DATE } & \begin{array}{c}\text { WATER } \\ \text { LEVEL }\end{array} \\ \text { Nov 19 } & \mathbf{9 2 . 7 1} & \text { Mar 25 } & \mathbf{9 2 . 0 0} & \text { Ju1 23 } & \mathbf{9 2 . 1 2} \\ \text { Jan 29 } & \mathbf{9 2 . 5 0} & \text { May 28 } & \mathbf{9 2 . 0 2} & \text { Sep 24 } & \mathbf{9 2 . 5 4}\end{array}$


434725094483001 . Local number, 104H33W28BAB01.

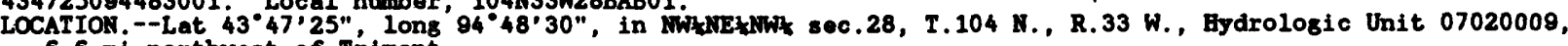
$6.6 \mathrm{mi}$ northwest of Trimont.

Omer: Kenneth Schafer.

AQUIFER.--Sioux Quartzite of Late Precambrian Age.

WELL CHARACTERISTICS.--Drilled unused artesian woil, diameter $5 \mathrm{in}$., depth $178 \mathrm{ft}$, cased to $121 \mathrm{ft}$

DATUM. --Altitude of iand-surface datum is 1,290 ft. Measuring point: Iop of casing, $1.30 \mathrm{ft}$ above land-surface datum.

PERIOD OF RECORD. --September 1981 to current year.

EXTREMES FOR PERIOD OF RECORD. --Highest water leve1, 82.99 ft below land-surface datum, Apr. 14, 1987;

lowest, $86.37 \mathrm{ft}$ below land-surface datum, Mar. 25, 1991.

WATER LEVEL, IN FEET BELON LAND-SURFACE DATUM, WATER YEAR OCTOBER 1990 TO SEPTEMBER 1991

$\begin{array}{cccccc}\text { DATE } & \begin{array}{c}\text { WATER } \\ \text { LEVEL }\end{array} & \text { DATE } & \begin{array}{c}\text { WATER } \\ \text { LEVEL }\end{array} & \text { DATE } & \begin{array}{c}\text { WATER } \\ \text { LEVEL }\end{array} \\ \text { Nov 19 } & \mathbf{8 5 . 7 8} & \text { Mar 25 } & \mathbf{8 6 . 3 7} & \text { Jul 23 } & \mathbf{8 5 . 5 6} \\ \text { Jan 29 } & \mathbf{8 6 . 0 2} & \text { May 28 } & \mathbf{8 5 . 5 0} & \text { Sop 24 } & \mathbf{8 5 . 2 5}\end{array}$

MC LEOD COUNTY

444758094132101. Local number, 115N28w05ACC01.

LOCATION. --Lat $44^{\circ} 47^{\prime} 58^{\prime \prime}$, Long $94^{\circ} 13^{\prime} 21^{\prime \prime}$, in S'WkSWkNE sec.5, T.115 N., R.28 W., Bydrologic Unit 07010205, northwest of Glencoe.

Omer: Graupmann Farms, Inc.

AOUIFER.--Mount Simon Sandstone of Late Cambrian Age.

WELI CHARACTERISTICS.--Drilled irrigation artesian woll, diameter 12 in., depth 472 ft, screened 432 to $472 \mathrm{ft}$.

DATUM. --Altitude of land-surface datum is 1,036 ft. Measuring point: Edge of vent pipe, $2.00 \mathrm{ft}$ above land-surface datum.

PERIOD OF RECORD. --September 1978 to current year.

EXTREMES FOR PERIOD OF RECORD.--Highest water level, 80.50 ft below land-surface datum, Aug. 20, 1979 lowest, $109.65 \mathrm{ft}$ below land-surface datum, Oct.' 1, 1979 .

WATER LEVEL, IN FEET BELOW LAND-SURFACE DATUM, WATER YEAR OCTOBER 1990 TO SEPTEMBER 1991

$\begin{array}{cccccc}\text { DATE } & \begin{array}{c}\text { WATER } \\ \text { LEVEL }\end{array} & \text { DATE } & \begin{array}{c}\text { WATER } \\ \text { LEVEL }\end{array} & \text { DATE } & \begin{array}{c}\text { WATER } \\ \text { LEVEL }\end{array} \\ \text { Nov 16 } & 91.83 & \text { May 14 } & \mathbf{8 5 . 8 8} & \text { Sop 23 } & \mathbf{9 8 . 4 5} \\ \text { Mar 13 } & \mathbf{8 6 . 9 5} & \text { Jul 24 } & \mathbf{9 1 . 9 2} & & \end{array}$

444819094164701. Local number, 116N29W35DDC01.

LOCATION. --Lat $44^{\circ} 48^{\prime} 19^{\prime \prime}$, long $94^{\circ} 16^{\prime} 47^{\prime \prime}$, in SWłSEłSEł sec.35, T.116 N., R.29 W., Hydrologic Unit 07010205, 1.3 mi south of Biscay.

Owner: Charles Johnson.

AOUIFER. --Buried sand of Pleistocene Ase.

WELL CHARACTERISTICS.--Drilled irrigation artesian well, diameter 12 in., depth $269 \mathrm{ft}$, screoned 229 to $269 \mathrm{ft}$.

DATUM.--Altitude of land-surface datum is $1,050 \mathrm{ft}$. Measuring point: Edge of vent pipe, $1.00 \mathrm{ft}$ above land-surface datum.

PERIOD OF RECORD. --September 1978 to current year.

EXTREMES FOR PERIOD OF RECORD. --Highest water level, $24,21 \mathrm{ft}$ below land-surface datum, Jan. 23, 1986; lowest, $34.80 \mathrm{ft}$ below land-surface datum, Aug. 26, 1989.

WATER LEVEL, IN FEET BELON LAND-SURFACE DATUM, WATER YEAR OCTOBER 1990 TO SEPTEMBER 1991

$\begin{array}{cccccc}\text { DATE } & \begin{array}{c}\text { WATER } \\ \text { LEVEL }\end{array} & \text { DATE } & \begin{array}{c}\text { WATER } \\ \text { LEVEL }\end{array} & \text { DATE } & \begin{array}{c}\text { WATER } \\ \text { LEVEL }\end{array} \\ \text { Nov 16 } & 30.18 & \text { May 14 } & 28.73 & \text { Sep 23 } & 28.55 \\ \text { Mar 13 } & 29.45 & \text { Jul 25 } & 28.60 & & \end{array}$


GROUND-WATER LEVELS

MC LEOD COUNTY--Continued

445721094031201. Local number 117N27W10DAA01.

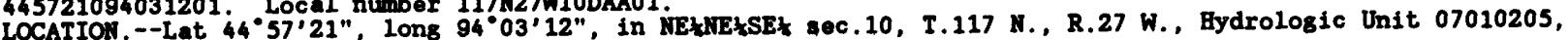

$0.1 \mathrm{mi}$ south of Winsted.

Omer: Winsted Farmers Coop.

AQUIFER. - Buried sand of Pleistocene Age.

WELI CHARACTERISTICS.-Drilled industrial artesian well, diameter 4 in., depth 129 ft, screened 125 to $129 \mathrm{ft}$.

DATUM. - Altitude of land-surface datum is 1,015 ft. Measuring point: Top of casing, 1.40 ft above land-surface datum.

PERIOD OF RECORD. - November 1977 to current year.

EXIRDMES FOR PERIOD of RECORD. - -Highest water level, $30.63 \mathrm{ft}$ below land-surface datum, Dec. 10, 1986 ;

lowest, $45.50 \mathrm{ft}$ below land-surface datum, Oct. 7, 1987.

WATER LEVEL, IN FEET BELON LAND-SURFACE DATUM, WATER YEAR OCTOBER 1990 TO SEPTEMBER 1991

$\begin{array}{cccccc}\text { DATE } & \begin{array}{c}\text { WATER } \\ \text { LEVEL }\end{array} & \text { DATE } & \begin{array}{c}\text { WATER } \\ \text { LEVEL }\end{array} & \text { DATE } & \text { WATER } \\ \text { LEVEL }\end{array}$

MEEKER COUNTY

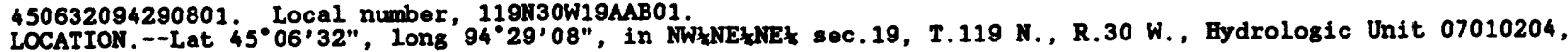
on Ted Carlson farm.

Omer: U.S. Geological Survey.

AOUIFER - Surficial sand of Pleistocene Ase.

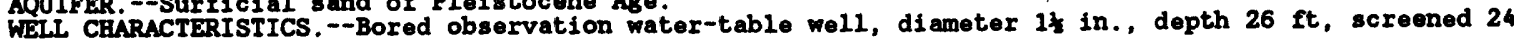

to 26 ft. land-surface datum.

PERIOD OF RECORD. -November 1977 to current year.

EXIRPMES FOR PERIOD OF RECORD,--Highest water level, 2.47 ft below land-surface datum, June 14, 1983; lowest $8.09 \mathrm{ft}$ below land-surface datum, Feb. 13, 1989.

WATER LEVEL, IN FEET BELOW LAND-SURFACE DATUM, WATER YEAR OCTOBER 1990 TO SEPTEMBER 1981

$\begin{array}{crcrcrrr}\text { DATE } & \begin{array}{r}\text { WATER } \\ \text { LEVEL }\end{array} & \text { DATE } & \begin{array}{c}\text { WATER } \\ \text { LEVEL }\end{array} & \text { DATE } & \begin{array}{c}\text { WATER } \\ \text { LEVEL }\end{array} & \text { DATE } & \begin{array}{r}\text { WATER } \\ \text { LEVEL }\end{array} \\ \text { Nov } 08 & 6.75 & \text { Feb 08 } & 7.17 & \text { Jul } 18 & 5.40 & \text { Sep } 18 & 4.70\end{array}$

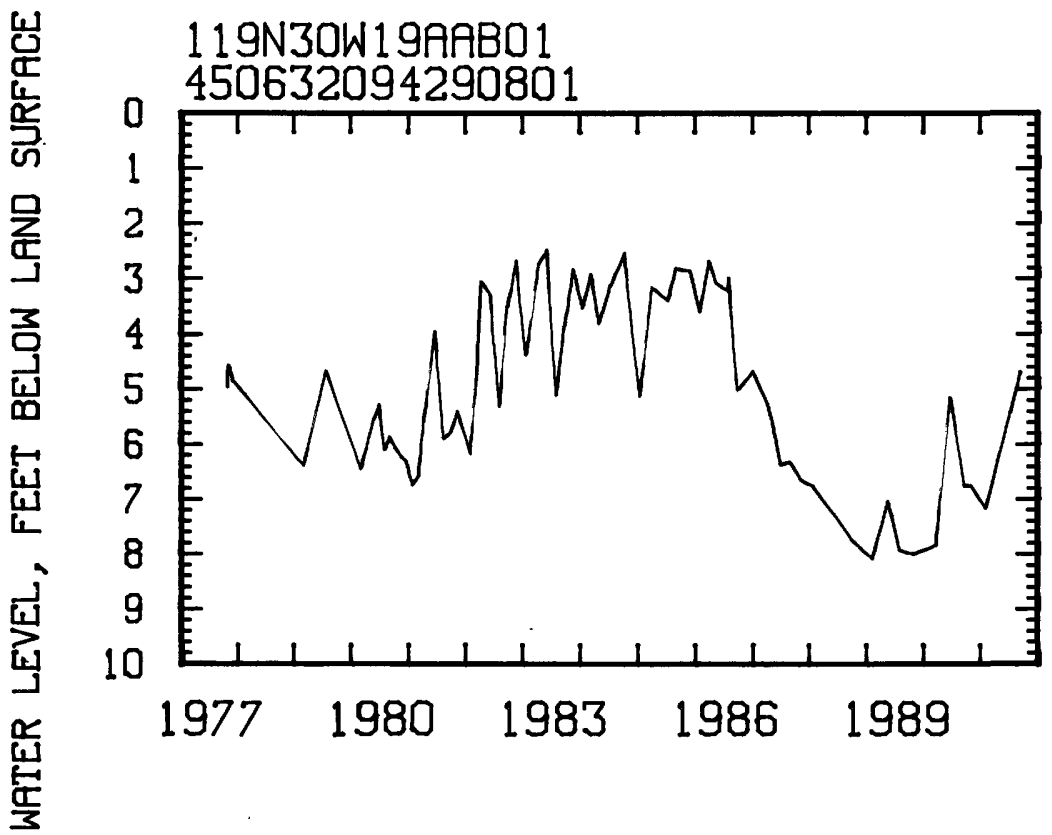

1123

1122

1121

荘 
451542094322301. Local number, 121K31W26BDC01.

LOCATION, - Lat $45^{\circ} 15^{\prime} 42^{\prime \prime}$, Iong $94^{\circ} 32^{\prime} 23^{\prime \prime}$, in SWzSEłWk sec.26, T.121 N., R.31 W., Hydrologic Unit 07010204, on Keith Langmo farm.

Ower: U.S. Geological Survey.

AQUIFER. - Surficial sand of Pleistocene ABe.

WEL CEARACTERISTICS.-Bored observation water-table we11, diameter 1 in., depth 16 ft, screened 14 to $16 \mathrm{ft}$.

DATUM.--Altitude of land-surface datum is 1,112 ft. Measuring point: Top of casing, 3.00 ft above land-surface datum.

PERIOD OF RECORD. - November 1977 to current year.

EXTREMES FOR PERIOD OF RECORD.--Highest water level, 2.70 ft below land-surface datum, Aus. 18, 1986;

lowest, 9.19 ft below land-surface datum, Mar. 30, 1990.

WATER LEVEL, IN FEET BELON LAND-SURFACE DATUM, WATER YEAR OCTOBER 1990 TO SEPTEMBER 1991

$\begin{array}{crcrcrrr}\text { DATE } & \begin{array}{c}\text { WATER } \\ \text { LEVEL }\end{array} & \text { DATE } & \begin{array}{c}\text { WATER } \\ \text { LEVEL }\end{array} & \text { DATE } & \begin{array}{l}\text { WATER } \\ \text { LEVEL }\end{array} & \text { DATE } & \begin{array}{r}\text { WATER } \\ \text { LEVEL }\end{array} \\ \text { Hov } 08 & 6.15 & \text { Feb } 08 & 6.98 & \text { Jul } 18 & 4.08 & \text { Sep } 18 & 3.46\end{array}$

MILLE LACS COUNTY

454450093395701. Local number, 038N27W35ABC01.

LOCATION, -Lat $45^{\circ} 44^{\prime} 50^{\prime \prime}$, long $93^{\circ} 39^{\prime} 57^{\prime \prime}$, in SWkWWwEk sec.35, T.38 R., R.27 W., Hydrologic Unit 07010207,

in Milaca.

Owner: City of Milaca, creamery well.

AQUIFER. - Buried sand and gravel of Pleistocene Ase.

WELL CEARACTERISTICS,--Drilled unused artesian well, diameter 12 in., depth $82 \mathrm{ft}$, screened 67 to $82 \mathrm{ft}$ DATUM.--Land-surface datum is $1,082.2$ ft National Geodetic Vertical Datum of 1929. Measuring point:

Top of breather pipe, 4.00 ft above land-surface datum.

REMARKS. - Water level affected by pumping.

PERIOD OF RECORD.--September 1967 to current year.

EXTREMES FOR PERIOD OF RECORD.--Highest water level, 35.72 ft below land-surface datum, July 20, 1984 ;

lowest, 42.81 ft below land-surface datum, Aug. 27, 1977.

WATER LEVEL, IN FEET BELON LAND-SURFACE DATUM, WATER YEAR OCTOBER 1990 TO SEPTEMBER 1991

$\begin{array}{cccc}\text { DATE } & \begin{array}{c}\text { WATER } \\ \text { LEVEL }\end{array} & \text { DATE } & \begin{array}{c}\text { WATER } \\ \text { LEVEL }\end{array} \\ \text { Aug 02 } & 38.60 & \text { Sep 30 } & 39.30\end{array}$

MORRISOA COUNTY

460444094212501. Local number, 130N29W08DCC01.

LOCATION. - Lat $46^{\circ} 04^{\circ} 44^{\prime \prime}$, Iong $94^{\circ} 21^{\prime} 25^{\prime \prime}$, in SWkSWSEk sec.8, T.130 N., R.29 W., Hydrologic Unit 07010104, at

Camp Ripley.

Omer: U.s. Geological Survey.

AQUIFER. - Surficial outwash sand and gravel of Pleistocene Ase.

WELL CHARACTERISTICS.--Drilled observation water-table well, diameter 2 in., depth 59 ft, screened 56 to 59 ft. DATUM-Land-surface datum is 1,149.0 ft National Geodetic Vertical Datum of 1929. Measuring point: Top of

casing, 2.10 ft above land-surface datum.

RFMARKS - Water levels used in monthly Water Resources Revien.

PERIOD OF RECORD.--Apri! 1949 to current year.

EXTREMES FOR PERIOD OF RECORD. - - Highest water level, $7.35 \mathrm{ft}$ below land-surface datum, July 28 , 1972 ; lowest,

18.75 ft below land-surface datum, Aug. 4, 1861.

WATER LEVEL, IN FEET BELON LAND-SURFACE DATUM, WATER YEAR OCTOBER 1990 TO SEPTEMBER 1991

\begin{tabular}{|c|c|c|c|c|c|c|c|c|c|c|c|c|c|c|c|}
\hline ATE & $\begin{array}{l}\text { WATER } \\
\text { LEVEL }\end{array}$ & DATE & $\begin{array}{l}\text { WATER } \\
\text { LEVEL }\end{array}$ & DAT & $\mathbf{I E}$ & $\begin{array}{l}\text { WATER } \\
\text { LEVEL }\end{array}$ & DAT & & $\begin{array}{l}\text { WATER } \\
\text { LEVIXL }\end{array}$ & DAT & & $\begin{array}{l}\text { WATER } \\
\text { LEVEL }\end{array}$ & DAT & TE & $\begin{array}{l}\text { WATER } \\
\text { LEVEL }\end{array}$ \\
\hline $\begin{array}{l}05 \\
12 \\
19 \\
26 \\
02 \\
09 \\
16 \\
23 \\
30\end{array}$ & $\begin{array}{l}14.67 \\
14.74 \\
14.58 \\
14.58 \\
14.61 \\
14.74 \\
14.64 \\
14.67 \\
14.68\end{array}$ & $\begin{array}{rr}\text { Dec } & 07 \\
14 \\
21 \\
28 \\
28 \\
\operatorname{Jan} 04 \\
11 \\
18 \\
25\end{array}$ & $\begin{array}{l}14.70 \\
14.85 \\
14.93 \\
15.12 \\
14.98 \\
15.03 \\
15.41 \\
15.33\end{array}$ & Mar & $\begin{array}{l}01 \\
08 \\
15 \\
22 \\
01 \\
08 \\
15 \\
22\end{array}$ & $\begin{array}{l}15.21 \\
15.29 \\
15.68 \\
15.53 \\
15.68 \\
15.81 \\
15.55 \\
15.79\end{array}$ & $\begin{array}{l}\text { Apr } \\
\text { May }\end{array}$ & $\begin{array}{l}03 \\
05 \\
19 \\
26 \\
03 \\
10 \\
18 \\
24\end{array}$ & $\begin{array}{l}15.49 \\
15.36 \\
15.52 \\
15.14 \\
15.13 \\
15.12 \\
14.99 \\
14.47\end{array}$ & Jul & $\begin{array}{l}01 \\
07 \\
14 \\
22 \\
28 \\
05 \\
12 \\
19 \\
26\end{array}$ & $\begin{array}{l}14.08 \\
13.85 \\
14.78 \\
14.52 \\
14.22 \\
14.02 \\
13.78 \\
13.66 \\
14.00\end{array}$ & $\begin{array}{l}\text { Aug } \\
\text { Sep }\end{array}$ & $\begin{array}{l}02 \\
09 \\
16 \\
23 \\
06 \\
13 \\
20 \\
27\end{array}$ & $\begin{array}{l}13.85 \\
13.36 \\
14.02 \\
14.05 \\
14.14 \\
14.00 \\
13.84 \\
13.81\end{array}$ \\
\hline
\end{tabular}


GROUND-WATER LEVELS

MOWER COUNTY

434010093010801. Local number, 102N18W05ACB01.

LOCATION. - Lat $43^{\circ} 40^{\prime} 10^{\prime \prime}$, long $93^{\circ} 01^{\prime} 08^{\prime \prime}$, in NWkSWkNEk sec.5, T.102 N., R.18 W., Hydrologic Unit 07080201, in Austin.

Owner: Church of Latter Day Saints.

AQUIFER. - Cedar Valley Formation of Middle Devonian Age.

WELL CHARACTERISTICS,-Drilled domestic artesian well, diameter 5 in., depth 100 ft, cased to 77 ft DATUM. --Altitude of land-surface datum is 1,230 It. Measuring point: Top of casing, $0.80 \mathrm{It}$ above land-surface datum.

PERIOD OF RECORD, --July 1981 to current year.

EXTREMES FOR PERIOD OF RECORD.--Highest water level, $33.69 \mathrm{ft}$ below land-surface datum, May 10, 1984 ; lowest, $38.44 \mathrm{ft}$ below land-surface datum, July $10,1985$.

WATER LEVEL, IN FEET BELON LAND-SURFACE DATUM, WATER YEAR OCTOBER 1990 TO SEPTEMBER 1991

$\begin{array}{cccccc}\text { DATE } & \begin{array}{c}\text { WATER } \\ \text { LEVEL }\end{array} & \text { DATE } & \begin{array}{c}\text { WATER } \\ \text { LEVEL }\end{array} & \text { DATE } & \begin{array}{l}\text { WATER } \\ \text { LEVEL }\end{array} \\ \text { Nov 19 } & 36.09 & \text { May 28 } & 33.98 & \text { Sep 24 } & 35.89 \\ \text { Mar 25 } & 35.87 & \text { Jul 23 } & 35.82 & & \end{array}$

434417093521001 . Local number, 103N17W09DAA01.

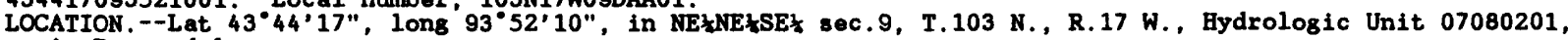
in Brownsdale.

Owner: Land O'Lakes, creamery well.

AQUIFER, --Cedar Valley Formation of Middle Devonian Age.

WELL CHARACTERISTICS.--Drilled unused artesian well, diameter 4 in., depth 130 ft, casing information not available.

DATUM.--Altitude of land-surface datum is 1,280 It. Measuring point: Top of well cap, 0.40 et above land-surface datum.

RFMARKS. - Water level affected by pumping.

PERIOD OF RECORD. - February 1976 to current year.

EXTREMES FOR PERIOD OF RECORD.--Highest water level, 35.97 ft below land-surface datum, May 2, 1984; lowest, 45.20 ft below land-surface datum, Mar. 30, 1978.

WATER LEVEL, IN FEET BELON LAND-SURFACE DATUM, WATER YEAR OCTOBER 1990 TO SEPTEMBER 1991

$\begin{array}{cccccc}\text { DATE } & \begin{array}{c}\text { WATER } \\ \text { LEVEL }\end{array} & \text { DATE } & \begin{array}{l}\text { WATER } \\ \text { LEVEL }\end{array} & \text { DATE } & \begin{array}{l}\text { WATER } \\ \text { LEVEL }\end{array} \\ \text { Nov 01 } & 38.83 & \text { Mar 05 } & 41.52 & \text { May 29 } & 37.67 \\ \text { Dec 12 } & 40.64 & \text { Apr 17 } & 40.76 & \text { Jul 09 } & 37.37\end{array}$

OLMSTED COUNTY

445538092232601. Local number, 105N13W04CAM01.

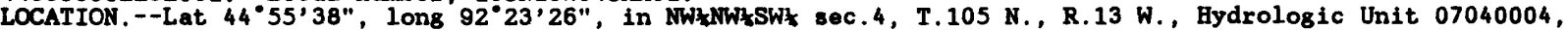

1 mile east of Simpson. Owner: Robert Sheehan

AQUIFER - -Galena Formation of Middle Ordovician Age.

WELL CHARACTERISTICS.-Drilled unused artesian well, diameter 4 in., depth 75 ft, cased to 23 ft.

DATUM.--Altitude of land-surface datum is 1,270 ft: Measuring point: Top of weil cap, $1.20 \mathrm{ft}$ above

land-surface datum.

PERIOD OF RECORD.--March 1987 to current year.

EXTREMES FOR PERIOD OF RECORD, - - Highest water leve1, 15.16 ft below land-surface datum, May 7, 1991; lowest, $33.30 \mathrm{ft}$ below land-surface datum, Feb. 6, 1990.

WATER LEVEL, IN FEET BELOW LAND-SURFACE DATUM, WATER YEAR OCTOBER 1990 TO SEPTEMBER 1991

$\begin{array}{cccccc}\text { DATE } & \begin{array}{c}\text { WATER } \\ \text { LEVEL }\end{array} & \text { DATE } & \begin{array}{l}\text { WATER } \\ \text { LEVEL }\end{array} & \text { DATE } & \text { WATER } \\ \text { LEVEL }\end{array}$


435757092224201 Local number, 106N13W22CCB01.

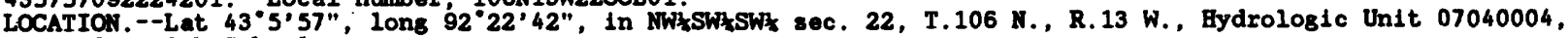
at. Burr Oak School.

Omer: Independent School District 535.

AQUIFER. --Jordan Sandstone of Late Cambrian Age.

WEL CBARACTERISTICS,-Drilled domestic artesian well diameter 6 in. depth $510 \mathrm{ft}$, cased to $430 \mathrm{ft}$

DATUM. --Altitude of iand-surface datum $181,090 \mathrm{ft}$ : Measuring point: Top of well cap, 1.00 ft above land-surface datum.

PERIOD OF RECORD.--February 1987 to current year.

EXTRDMIES FOR PERIOD OF RECORD,--H1ghest water level, $28.01 \mathrm{ft}$ below land-surface datum, Feb. 25, 1987;

lowest, $32.63 \mathrm{ft}$ below land-surface datum, Feb. 6, 1990.

WATER LEVEL, IN FEET BELON LAND-SURFACE DATUM, WATER YEAR OCTOBER 1990 TO SEPTEMBER 1991

$\begin{array}{cccccc}\text { DATE } & \begin{array}{c}\text { WATER } \\ \text { LEVEL }\end{array} & \text { DATE } & \begin{array}{c}\text { WATER } \\ \text { LEVEL }\end{array} & \text { DATE } & \begin{array}{c}\text { WATER } \\ \text { LEVEL }\end{array} \\ \text { Dec 05 } & 29.60 & \text { Mar 04 } & 30.18 & \text { Aug 01 } & 28.88 \\ \text { Jan 09 } & 30.53 & \text { May 07 } & 29.68 & \text { Sep 12 } & 29.15\end{array}$

435920092273801. Local number, 106N14W14ADB01.

LOCATION. - Lat 43'59'20", Iong 92'27'38", in NWzSEłNEk sec.14, T.106 N., R.14 W., Hydrologic Unit 07040004, in Rochester.

Omer: Golden Hill School Dist. \#1371

AQUIFER. --Jordan Sandstone of Late Cambrian Age.

WEIL CBARACTERISTICS.--Drilled unused artesian well, diameter 6 in., depth $478 \mathrm{ft}$, cased to 397 ft.

DATUM.--Altitude of land-surface datum is 1,065 ft. Measuring point: Edge of weil cap, $1.80 \mathrm{ft}$ above land-surface datum.

RDMARKS. - Water level affected by pumping.

PERIOD OF RECORD.--August 1974 to current year.

EXIRDYIES FOR PERIOD OF RECORD,--H1ghest water level, 50.58 ft below land-surface datum, Apr. 12, 1983 ;

lowest, $71.86 \mathrm{ft}$ below land-surface datum, July 26, 1989.

WATER LEVEL, IN FEET BELON LAND-SURFACE DATUM, WATER YEAR OCTOBER 1990 TO SEPTEMBER 1991

$\begin{array}{cccccc}\text { DATE } & \begin{array}{c}\text { WATER } \\ \text { LEVEL }\end{array} & \text { DATE } & \begin{array}{l}\text { WATER } \\ \text { LEVEL }\end{array} & \text { DATE } & \begin{array}{c}\text { WATER } \\ \text { LEVEL }\end{array} \\ \text { Dec 05 } & 58.30 & \text { Mar 05 } & 63.65 & \text { Aus 01 } & 64.82 \\ \text { Jan 09 } & 64.29 & \text { May 07 } & 54.35 & \text { Sep 12 } & 58.04\end{array}$

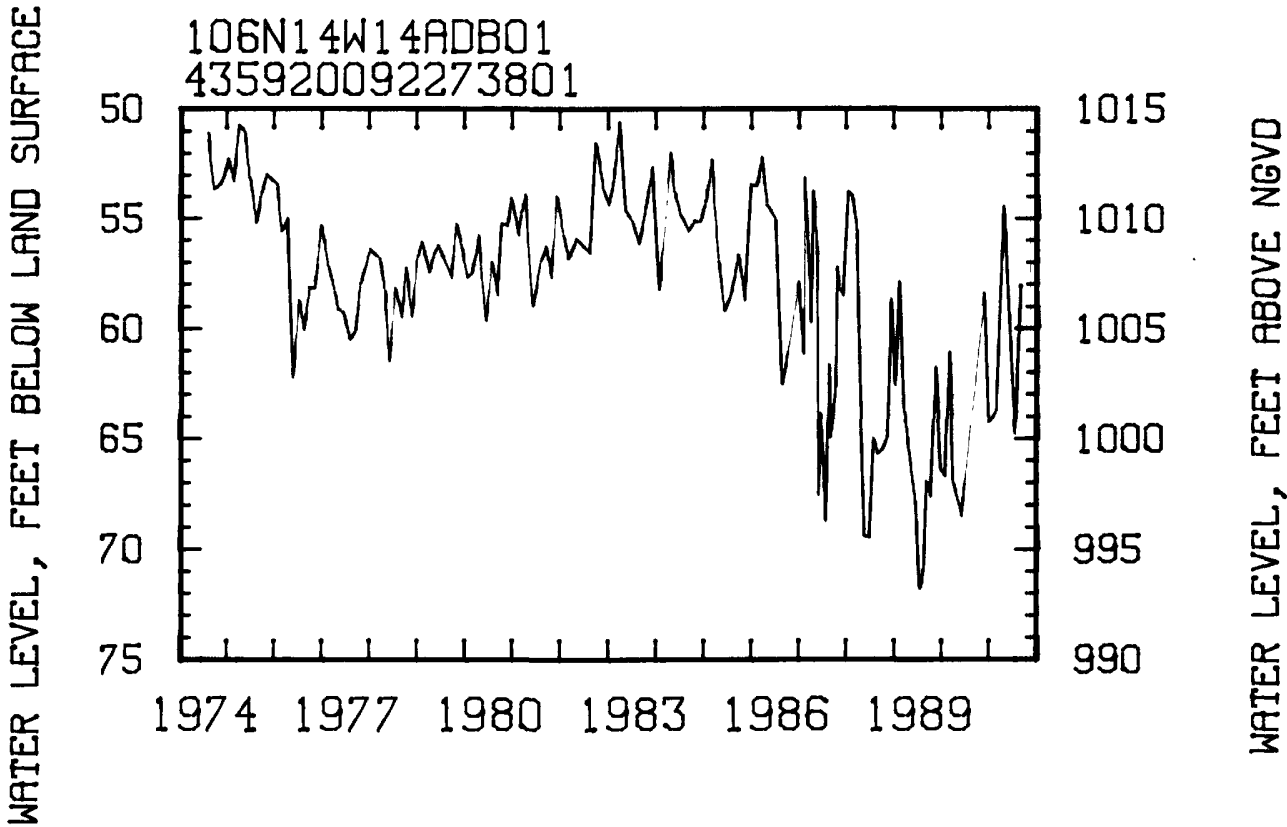


GROUND-WATER LEVELS

RAMSEY COUNTY

445955093011001. LoCal number, 029N22W14CABO1.

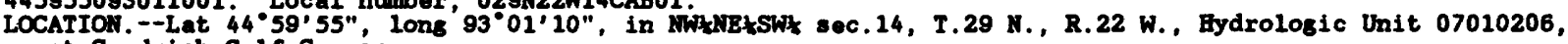
at Goodrich Golf Course.

Onner: Ramsey County.

AQUIFER. - Prairie du Chien Group of Early Ordovician Age and Jordan Sandstone of Late Cambrian Age.

WELL CBARACTERISTICS.--Drilled irrigation artesian well, diemeter $12 \mathrm{in}$., depth $523 \mathrm{ft}$, cased to $303 \mathrm{ft}$

DATUM. - Altitude of iand-surface datum is $970 \mathrm{ft}$. Measuring point: Edge of vent pipe, $2.50 \mathrm{ft}$ above land-surface datum.

PERIOD OF RECORD.--Mey 1965, April 1966 to August 1966, August 1971, May 1980 to current year.

EXTREMES FOR PERIOD OF RECORD.--Highest water level, 124.62 ft below land-surface datum, Feb. 6, 1987; lowest, 140.60 ft below land-surface datum, Apr. 6, 1966.

WATER LEVEL, IN FEET BELOW LAND-SURFACE DATUM, WATER YEAR OCTOBER 1990 TO SEPTEMBER 1991

\begin{tabular}{|c|c|c|c|c|c|}
\hline DATE & $\begin{array}{l}\text { WATER } \\
\text { LEVEL }\end{array}$ & DATE & $\begin{array}{l}\text { WATER } \\
\text { LEVEL }\end{array}$ & DATE & $\begin{array}{l}\text { WATER } \\
\text { LEVEL }\end{array}$ \\
\hline $\begin{array}{l}\text { Nov } 06 \\
\text { Jan } 07\end{array}$ & $\begin{array}{l}131.50 \\
132.00\end{array}$ & $\begin{array}{l}\text { May } 16 \\
\text { Jul } 16\end{array}$ & $\begin{array}{l}134.23 \\
138.67\end{array}$ & Sep 17 & 130 \\
\hline
\end{tabular}

445955093011002. Local number, 029K22W14CAB02.

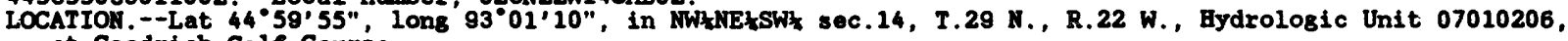
at Goodrich Golf Course.

Omer: U.S. Geological Survey.

AQUIFER. --Buried gravel of Pleistocene Age.

WELL CBARACTERISTICS.--Bored observation artesian well, diameter 2 in., depth 81 ft, screened 78 to $81 \mathrm{ft}$.

DATUM. --Altitude of land-surface datum is $970 \mathrm{ft}$. Measuring point: Top of casing, 1.30 ft above land-surface datum.

PERIOD OF RECORD.--October 1966 to August 1971, August 1977, June 1980 to current year.

EXTREMES FOR PERIOD OF RECORD.--Highest water level, $30.80 \mathrm{ft}$ belon land-surface datum, Oct. 28 ,

1986; lowest, 45.36 ft below land-surface datum, June $3,1968$.

WATER LEVEL, IN FEET BELON LAND-SURFACE DATUM, WATER YEAR OCTOBER 1990 TO SEPTEMBER 1991

$\begin{array}{cccccc}\text { DATE } & \begin{array}{c}\text { WATER } \\ \text { LEVEL }\end{array} & \text { DATE } & \begin{array}{c}\text { WATER } \\ \text { LEVEI }\end{array} & \text { DATE } & \begin{array}{c}\text { WATER } \\ \text { LEVEL }\end{array} \\ \text { Nov 06 } & \mathbf{3 7 . 8 9} & \text { May 16 } & \mathbf{3 8 . 9 0} & \text { Sep 17 } & 35.86 \\ \text { Jan 07 } & \mathbf{3 8 . 7 3} & \text { Jul 16 } & \mathbf{3 6 . 4 7} & & \end{array}$

445955093011003, Local number, 029N22W14CAB03.

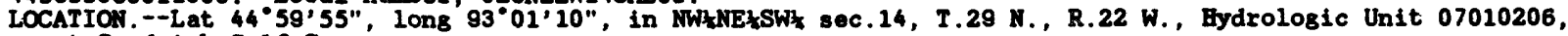
at Goodrich Golf Course.

Owner: U.S. Geological Survey.

AQUIFER. --Buried gravel of Pleistocene Age.

WELL CHARACTERISTICS. --Bored observation water-table well, diemeter 2 in., depth 52 ft, screened 49 to $52 \mathrm{ft}$

DATUM. --Altitude of land-surface detum is $970 \mathrm{ft}$. Measuring point: Top of casing, $1.80 \mathrm{ft}$ above land-surface datum.

PERIOD OF RECORD.--October 1966 to August 1971, June 1980 to current year.

EXTRRMES FOR PERIOD OF RECORD. --Highest water level, 8.97 ft belon land-surface datum, Oct. 28, 1986; lowest, 25.43 ft below land-surface datum, June 3, 1968.

WATER LEVEL, IN FEET BELOW LAND-SURFACE DATUM, WATER YEAR OCTOBER 1990 TO SEPTEMBER 1991

$\begin{array}{cccccc}\text { DATE } & \begin{array}{c}\text { WATER } \\ \text { LEVEL }\end{array} & \text { DATE } & \begin{array}{c}\text { WATER } \\ \text { LEVEI }\end{array} & \text { DATE } & \begin{array}{c}\text { WATER } \\ \text { LEVEL }\end{array} \\ \text { Nov 06 } & \mathbf{1 9 . 8 5} & \text { May 16 } & \mathbf{2 1 . 9 2} & \text { Sop } 17 & 17.23 \\ \text { Jan 07 } & \mathbf{2 1 . 0 4} & \text { Jul 16 } & \mathbf{1 8 . 5 2} & & \end{array}$


450001093024701. Local number, 029N22W16ADD01.

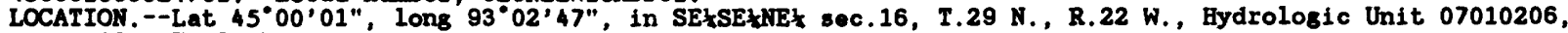
at 1955 English St. Omer: Maplewood Bowl.

AQUIFER. --Buried sand of Pleistocene Age.

WELL CHARACTERISTICS.--Drilled unused artesian well, diameter 4 in., depth $163 \mathrm{ft}$, screened 158 to $163 \mathrm{ft}$ DATUM.--Altitude of iand-surface datum is $900 \mathrm{ft}$. Measuring point: Top of well cap, $1.00 \mathrm{ft}$ above landsurface datum.

PERIOD OF RECORD:--January 1981 to current year.

EXTREMIES FOR PERIOD OF RECORD.--Highest water level, $65.99 \mathrm{ft}$ below land-surface datum, Fob. 6, 1987;

lowest, $73.83 \mathrm{ft}$ below land-surface datum, Apr. 30, 1990 .

WATER LEVEL, IN FEET BELOW LAND-SURFACE DATUM, WATER YEAR OCTOBER 1990 TO SEPTEMBER 1991

$\begin{array}{cccccc}\text { DATE } & \begin{array}{c}\text { WATER } \\ \text { LEVEL }\end{array} & \text { DATE } & \begin{array}{c}\text { WATER } \\ \text { LEVEL }\end{array} & \text { DATE } & \begin{array}{c}\text { WATER } \\ \text { LEVEL }\end{array} \\ \text { Hov 06 } & \mathbf{7 3 . 2 2} & \text { Mar 04 } & \mathbf{7 3 . 1 1} & \text { Aug 02 } & \mathbf{7 2 . 8 5} \\ \text { Jan 07 } & \mathbf{7 3 . 0 8} & \text { May 16 } & \mathbf{7 3 . 1 6} & \text { Søp 17 } & \mathbf{7 2 . 8 4}\end{array}$

445918092590901 . Local number, 029:22W24ADA01.

LOCATION. --Lat 44 "59'18", long 92 59'09", in NEłSEłNEł sec.24, T.29 N., R.22 W., Hydrologic Unit 07010206, at 1555 Century Avenue.

Omer: Northern States Power Co., Maplewood Gas Plant.

AQUIFER. --Jordan Sandstone of Late Cambrian ABe.

WELL CHARACTERISTICS.--Drilled fire protection artesian well, diameter 12 in., depth 523 ft, cased to $420 \mathrm{ft}$.

DATUM. --Land-surface datum is $996.5 \mathrm{ft}$ National Geodetic Vertical Datum of 1929 . Measuring point:

Edge of 2 in. breather pipe, $2.40 \mathrm{ft}$ above land-surface datum.

REMARKS. - Water level affected by pumping.

PERIOD OF RECORD. --August 1970 to current year

EXTREMES FOR PERIOD OF RECORD. -- Highest water level, $131.90 \mathrm{ft}$ below land-surface datum, Mar. 9, 1987

lowest, $151.0 \mathrm{ft}$ below land-surface datum, May $14,1981$.

WATER LEVEL, IN FEET BELOW LAND-SURFACE DATUM, WATER YEAR OCTOBER 1990 TO SEPTEMBER 1991

$\begin{array}{crcr}\text { DATE } & \begin{array}{r}\text { WATER } \\ \text { LEVEL }\end{array} & \text { DATE } & \begin{array}{r}\text { WATER } \\ \text { LEVEL }\end{array} \\ \text { Nov } 06 & 138.80 & \text { Jun } 11 & 144.70\end{array}$

445700093051001. Local number, 029*22W31DDD01

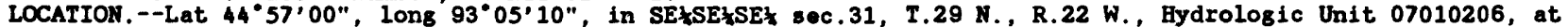
261 East 5th Street, St. Paul.

Owner: Control Data Corp.

AQUIFER.--Prairie du Chien Group of Early Ordovician Age and Jordan Sandstone of Late Cambrian Age.

WELL CHARACTERISTICS.--Drilled unused artesian well, diameter 12 in., depth $298 \mathrm{ft}$, cased to $151 \mathrm{ft}$.

DATUM. - Altitude of land-surface datum is $750 \mathrm{ft}$. Measuring point: Top of recorder platform, $9.00 \mathrm{ft}$ belon land-surface datum.

REMARKS. - Water level affected by pumping of nearby wells.

PERIOD OF RECORD. --December 1971 to current year.

EXTREMES FOR PERIOD OF RECORD.--Highest water leve1, $27.85 \mathrm{ft}$ below land-surface datum, Apr. 1, 1991; 10west, $83.28 \mathrm{ft}$ below land-surface datum, Aug. 4, 1989.

WATER LEVEL, IN FEET BELOW LAND-SURFACE DATUM, WATER YEAR OCTOBER 1990 TO SEPTEMBER 1991

\begin{tabular}{|c|c|c|c|c|c|c|c|c|c|c|c|c|}
\hline DATE & $\begin{array}{l}\text { WATER } \\
\text { LEVEL }\end{array}$ & DATE & $\begin{array}{l}\text { WATER } \\
\text { LEVEI }\end{array}$ & DAT & & $\begin{array}{l}\text { WATER } \\
\text { LEVEL }\end{array}$ & DATE & $\begin{array}{l}\text { WATER } \\
\text { LEVEL }\end{array}$ & DATE & $\begin{array}{l}\text { WATER } \\
\text { LEVEL }\end{array}$ & DATE & $\begin{array}{l}\text { WATER } \\
\text { LEVEI }\end{array}$ \\
\hline $\begin{array}{r}05 \\
\text { Oct } \\
10 \\
15 \\
20 \\
25 \\
31 \\
31 \\
\text { Nov } 05 \\
10 \\
15 \\
20 \\
25 \\
30\end{array}$ & $\begin{array}{l}62.96 \\
40.33 \\
43.80 \\
36.65 \\
40.83 \\
49.91 \\
35.66 \\
34.94 \\
39.92 \\
35.64 \\
34.36 \\
35.69\end{array}$ & $\begin{array}{rr}\text { Dec } & 05 \\
10 \\
15 \\
20 \\
25 \\
25 \\
31 \\
\text { Jan } 05 \\
10 \\
15 \\
20 \\
25 \\
31 \\
31\end{array}$ & $\begin{array}{l}35.64 \\
35.71 \\
35.50 \\
34.32 \\
34.26 \\
33.62 \\
33.85 \\
33.84 \\
33.16 \\
33.80 \\
35.45 \\
33.11\end{array}$ & $\operatorname{Mar}$ & $\begin{array}{l}05 \\
10 \\
15 \\
20 \\
25 \\
28 \\
05 \\
10 \\
15 \\
20 \\
25 \\
31\end{array}$ & $\begin{array}{l}32.68 \\
30.92 \\
34.26 \\
33.41 \\
31.99 \\
33.13 \\
32.63 \\
29.54 \\
32.93 \\
33.35 \\
33.57 \\
29.42\end{array}$ & $\begin{array}{rr}\text { Apr } & 05 \\
10 \\
15 \\
20 \\
25 \\
25 \\
30 \\
\text { May } 05 \\
10 \\
15 \\
20 \\
25 \\
31\end{array}$ & $\begin{array}{l}57.43 \\
35.49 \\
31.45 \\
36.16 \\
54.90 \\
41.08 \\
30.11 \\
59.53 \\
67.41 \\
59.72 \\
60.11 \\
68.23\end{array}$ & $\begin{array}{rr}\text { Jun } & 0 \\
1 \\
1 \\
1 \\
2 \\
2 \\
3 \\
\text { Jul } \\
0 \\
1 \\
1 \\
1\end{array}$ & $\begin{array}{r}65.68 \\
68.38 \\
63.61 \\
70.20 \\
72.28 \\
66.53 \\
68.58 \\
70.69 \\
71.83 \\
-1-- \\
72.90\end{array}$ & $\begin{array}{rr}\text { Aug } & 05 \\
10 \\
15 \\
20 \\
20 \\
25 \\
31 \\
\text { Sep } 05 \\
10 \\
15 \\
20 \\
25 \\
30\end{array}$ & $\begin{array}{l}68.52 \\
63.88 \\
72.62 \\
67.94 \\
67.05 \\
69.53 \\
68.88 \\
68.05 \\
65.89 \\
45.10 \\
48.66 \\
51.80\end{array}$ \\
\hline
\end{tabular}


GROUND-WATER LEVELS

RAMSEY COUNTY--Continued

450026093084201. Local number, 029N23W11CCCO1.

LOCATION. - Lat $45^{\circ} 00^{\circ} 26^{\prime \prime}$, long $93^{\circ} 08^{\prime} 42^{\prime \prime}$, in SWłSWłSW sec.11, T.29 N., R.23 W., Hydrologic Unit 07010206, at 2204 North Lexington Avenue, Roseville. Owner: Lexington Court Apartments.

AQUIFER. --St. Peter Sandstone of Middle Ordovician Ase.

WELL CHARACTERISTICS.--Drilled unused artesian well, diemeter 6 in., depth $325 \mathrm{ft}$, cased to $192 \mathrm{ft}$.

DATUM. - Altitude of land-surface datum is $945 \mathrm{ft}$. Measuring point: Top of well cap, $1.40 \mathrm{ft}$ above 1 andsurface datum.

PERIOD OF RECORD.---January 1971 to current year.

EXTREMES FOR PERIOD OF RECORD.--Highest water level, $104.77 \mathrm{ft}$ below land-surface datum, Mar. 30, 1987;

lowest, $111.30 \mathrm{ft}$ below land-surface datum, Sept. 12, 1990.

WATER LEVEL, IN FEET BELOW LAND-SURFACE DATUM, WATER YEAR OCTOBER 1990 TO SEPTEMBER 1991

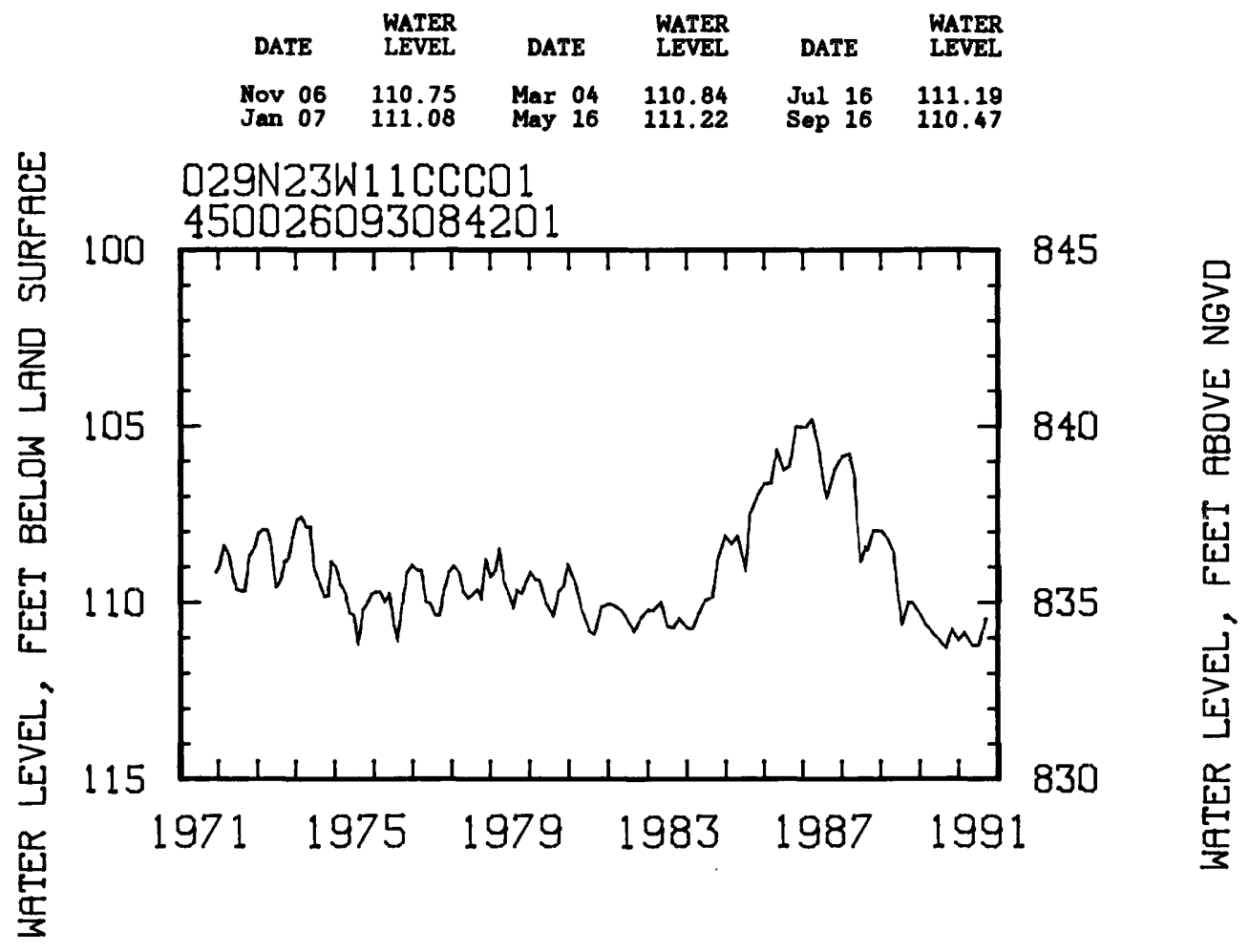

445751093072301. Local number, 029N23W25CCD01.

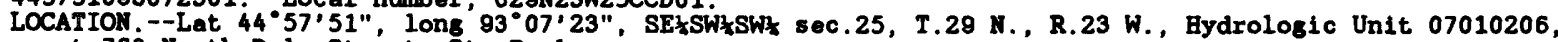
at 760 North Dale Street, St. Paul.

Owner: Burlington Northern, Inc., Dale Street Shops.

AQUIFER, - - Hinckley Sands tone of Late Precambrian Age.

WELL CHARACTERISTICS.--Drilled unused artesian well, diameter $8 \mathrm{in.,} \mathrm{depth} 999 \mathrm{ft}$, cased to $955 \mathrm{ft}$ DATUM.--Land-surface datum is $859.5 \mathrm{ft}$ National Geodetic Vertical Datum of 1929 . Measuring point:

Top of recorder floor, $4.60 \mathrm{ft}$ above land-surface datum.

REMARKS. - Water level affected by pumping.

PERIOD OF RECORD.--December 1970, November 1976 to current year.

EXTRPMES FOR PERIOD OF RECORD. -- Highest water level, 161.05 ft below land-surface datum, May 10, 1980 ;

lowest, $226.05 \mathrm{ft}$ below land-surface datum, Sept. 18, 1989.

WATER LEVEL, IN FEET BELOW LAND-SURFACE DATUM, WATER YEAR OCTOBER 1990 TO SEPTEMBER 1991

\begin{tabular}{|c|c|c|c|c|c|c|c|c|c|c|c|c|c|c|c|c|c|}
\hline DA2 & & $\begin{array}{l}\text { WATER } \\
\text { LEVIL }\end{array}$ & DAI & & $\begin{array}{l}\text { WATDR } \\
\text { LEVEL }\end{array}$ & DA: & & $\begin{array}{l}\text { WATER } \\
\text { LEVEL }\end{array}$ & DA? & & $\begin{array}{l}\text { WATER } \\
\text { LEVEL }\end{array}$ & DA? & & $\begin{array}{l}\text { WATER } \\
\text { LEVEL }\end{array}$ & DA: & & $\begin{array}{l}\text { WATER } \\
\text { LEVEL }\end{array}$ \\
\hline Hov & $\begin{array}{l}10 \\
15 \\
20 \\
25 \\
30 \\
05 \\
10 \\
15 \\
20 \\
25 \\
31\end{array}$ & $\begin{array}{l}212.14 \\
211.74 \\
211.50 \\
211.11 \\
210.83 \\
210.67 \\
210.33 \\
209.92 \\
209.61 \\
209.20 \\
208.73\end{array}$ & $F \mathrm{eb}$ & $\begin{array}{l}05 \\
10 \\
15 \\
20 \\
25 \\
31 \\
05 \\
10 \\
15 \\
20 \\
25 \\
28\end{array}$ & $\begin{array}{l}208.19 \\
208.00 \\
207.24 \\
206.84 \\
206.52 \\
205.77 \\
205.29 \\
204.96 \\
204.59 \\
203.98 \\
203.94 \\
203.36\end{array}$ & Apr & $\begin{array}{l}05 \\
10 \\
15 \\
20 \\
25 \\
31 \\
05 \\
10 \\
15 \\
20 \\
25 \\
30\end{array}$ & $\begin{array}{l}202.80 \\
203.08 \\
203.09 \\
202.62 \\
202.59 \\
202.45 \\
202.21 \\
202.27 \\
201.71 \\
201.76 \\
201.34 \\
200.98\end{array}$ & $\begin{array}{l}\text { May } \\
\text { Jun }\end{array}$ & $\begin{array}{l}05 \\
10 \\
15 \\
20 \\
25 \\
31 \\
05 \\
10 \\
15 \\
20 \\
25 \\
30\end{array}$ & $\begin{array}{l}200.98 \\
200.85 \\
200.57 \\
200.75 \\
200.72 \\
200.61 \\
201.01 \\
200.95 \\
200.90 \\
201.27 \\
201.55 \\
201.66\end{array}$ & Aus & $\begin{array}{l}05 \\
10 \\
15 \\
20 \\
25 \\
31 \\
05 \\
10 \\
15 \\
20 \\
25 \\
31\end{array}$ & $\begin{array}{l}201.88 \\
202.36 \\
202.67 \\
202.83 \\
203.39 \\
203.57 \\
204.02 \\
204.32 \\
204.36 \\
204.84 \\
205.21 \\
205.79\end{array}$ & Sep & $\begin{array}{l}05 \\
10 \\
15 \\
20 \\
25 \\
30\end{array}$ & $\begin{array}{l}206.04 \\
206.53 \\
206.63 \\
207.50 \\
207.45 \\
207.75\end{array}$ \\
\hline
\end{tabular}


445739093081201. Local number, 029N23W35BAD01.

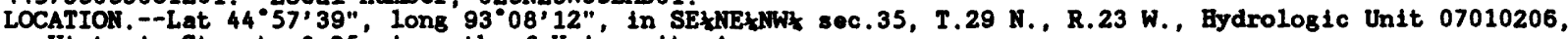
Victoria Streot, $0.35 \mathrm{mi}$ north of University Avenue. Omer: City of St. Paul.

AOUIFER --St. Peter Sandstone of Middle Ordovician Ase.

WELL CHARACTERISTICS.--Drilled unused water-table well, diemeter $16 \mathrm{in}$, dopth $234 \mathrm{ft}$, screened 174 to $234 \mathrm{ft}$.

DATUM.--Altitude of land-surface datum is $888 \mathrm{ft}$. Measuring point: Top of coupling, $0.50 \mathrm{ft}$ above land-surface datum.

PERIOD OF RECORD. --May 1981 to current year.

EXTREMIES FOR PERIOD OF RECORD.--Highest water level, $118.83 \mathrm{ft}$ below land-surface datum, Fob. 2, 1987, lowest, $133.03 \mathrm{ft}$ below land-surface datum, May 5, 1981.

WATER LEVEL, IN FEET BELOW LAND-SURFACE DATUM, WATER YEAR OCTOBER 1990 TO SEPTEMBER 1991

\begin{tabular}{|c|c|c|c|c|c|}
\hline DATE & $\begin{array}{l}\text { WATER } \\
\text { LEVEL }\end{array}$ & DATE & $\begin{array}{l}\text { WATER } \\
\text { LEVEL }\end{array}$ & DATE & $\begin{array}{l}\text { WATER } \\
\text { LEVEL }\end{array}$ \\
\hline $\begin{array}{l}\text { Nov } 06 \\
\text { Jan } 07\end{array}$ & $\begin{array}{l}120.42 \\
120.08\end{array}$ & $\begin{array}{ll}\text { Mar } & 04 \\
\text { May } & 15\end{array}$ & $\begin{array}{l}118.95 \\
119.46\end{array}$ & $\begin{array}{ll}\text { Jul } & 16 \\
\text { Sop } & 16\end{array}$ & $\begin{array}{l}120.54 \\
120.46\end{array}$ \\
\hline
\end{tabular}

450414093012701. Local number, 030N22W23CBB01.

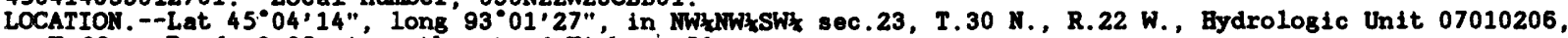
Hoffman Road, 0.85 mi southwest of Highway 61 . Omer: Whit Bear Tom Hall.

AQUIFER. --Burled sand of Pleistocene Age.

WELL CHARACTERISTICS.--Drilled domestic artesian well, diemeter $4 \mathrm{in}$. depth $96 \mathrm{ft}$, screened 91 to $96 \mathrm{ft}$

DATUM. - Altitude of land-surface datum is $928 \mathrm{ft}$. Measuring point: Top of casing, $1.00 \mathrm{ft}$ above landsurface datum.

PERIOD OF RECORD. --April 1980 to current year.

EXTREMES FOR PERIOD OF RECORD. --Highest water leve1, $17.54 \mathrm{ft}$ below land-surface datum, Oct. 28, 1986 ;

lowest, $28.08 \mathrm{ft}$ below land-surface datum, May 15, 1990.

WATER LEVEL, IN FEET BELOW LAND-SURFACE DATUM, WATER YEAR OCTOBER 1990 TO SEPTEMBER 1991

$\begin{array}{cccccc}\text { DATE } & \begin{array}{c}\text { WATER } \\ \text { LEVEL }\end{array} & \text { DATE } & \begin{array}{c}\text { WATER } \\ \text { LEVEI }\end{array} & \text { DATE } & \begin{array}{c}\text { WATER } \\ \text { LEVEL }\end{array} \\ \text { Dec 04 } & 27.07 & \text { Mar 11 } & 27.89 & \text { Jul 22 } & 27.24 \\ \text { Jan 16 } & 27.52 & \text { May 16 } & 27.62 & \text { Sep 17 } & 25.97\end{array}$

450238093082501. Local number, 030N23W35BDC01.

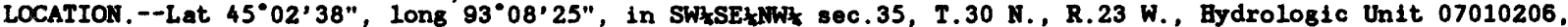
southeast corner of Arbogast Street and Richmond Avenue. Omer: City of Shoreview.

AQUIFER. - Jordan Sandstone of Late Cambrian Age.

WELL CHARACTERISTICS.--Drilled unused artesian well, diameter $12 \mathrm{in}$, depth $510 \mathrm{ft}$, cased to $465 \mathrm{ft}$.

DATUM.--Altitude of land-surface datum is $960 \mathrm{ft}$. Measuring point: Hole in shelter floor, $1.50 \mathrm{ft}$ above landsurface datum.

PERIOD OF RECORD.--April 1979 to current year.

EXTREMES FOR PERIOD OF RECORD.--Highest water level, $129.26 \mathrm{ft}$ below land-surface datum, Mar. 1, 1987; lowest, $146.01 \mathrm{ft}$ below land-surface datum, July 28,1989

WATER LEVEL, IN FEET BELOW LAND-SURFACE DATAUM, WATER YEAR OCTOBER 1990 TO SEPTEMBER 1991

\begin{tabular}{|c|c|c|c|c|c|c|c|c|c|c|c|c|c|c|}
\hline DATE & $\begin{array}{l}\text { WATER } \\
\text { LEVEL }\end{array}$ & DATE & $\begin{array}{l}\text { WATER } \\
\text { LEVEL }\end{array}$ & DAT & & $\begin{array}{l}\text { WATER } \\
\text { LEVEL }\end{array}$ & $\mathbf{D A}^{\prime}$ & & $\begin{array}{l}\text { WATER } \\
\text { LEVEL }\end{array}$ & $\mathrm{DA}^{\prime}$ & & $\begin{array}{l}\text { WATER } \\
\text { LEVEL }\end{array}$ & DATE & $\begin{array}{l}\text { WATER } \\
\text { LEVEL }\end{array}$ \\
\hline $\begin{array}{l}\text { t } 05 \\
10 \\
15 \\
20 \\
25 \\
31 \\
v 05 \\
10 \\
15 \\
20 \\
25 \\
30\end{array}$ & $\begin{array}{l}140.81 \\
140.29 \\
139.99 \\
140.01 \\
140.07 \\
139.82 \\
141.02 \\
141.50 \\
141.67 \\
141.45 \\
141.53 \\
141.91\end{array}$ & $\begin{array}{rr}\text { Dec } & 05 \\
10 \\
15 \\
20 \\
25 \\
25 \\
31 \\
\text { Jan } 05 \\
10 \\
15 \\
20 \\
25 \\
31\end{array}$ & $\begin{array}{l}141.72 \\
141.74 \\
141.79 \\
142.00 \\
141.66 \\
141.57 \\
141.62 \\
141.65 \\
141.35 \\
141.94 \\
142.30 \\
142.15\end{array}$ & Feb & $\begin{array}{l}05 \\
10 \\
15 \\
20 \\
25 \\
28 \\
05 \\
10 \\
15 \\
20 \\
25 \\
31\end{array}$ & $\begin{array}{l}142.08 \\
142.07 \\
142.36 \\
142.06 \\
142.25 \\
141.98 \\
141.67 \\
142.42 \\
142.50 \\
142.09 \\
140.18 \\
139.29\end{array}$ & Apr & $\begin{array}{l}05 \\
10 \\
15 \\
20 \\
25 \\
30 \\
05 \\
10 \\
15 \\
20 \\
25 \\
31\end{array}$ & $\begin{array}{l}139.30 \\
140.18 \\
139.18 \\
139.52 \\
139.45 \\
139.80 \\
138.46 \\
137.96 \\
139.05 \\
138.62 \\
139.64 \\
139.43\end{array}$ & Jun & $\begin{array}{l}05 \\
10 \\
15 \\
20 \\
25 \\
30 \\
05 \\
10 \\
15 \\
20 \\
25 \\
31\end{array}$ & $\begin{array}{l}139.61 \\
141.15 \\
142.43 \\
142.40 \\
141.84 \\
143.31 \\
142.21 \\
142.12 \\
142.12 \\
143.17 \\
143.16 \\
142.39\end{array}$ & $\begin{array}{r}\text { Aug } 05 \\
10 \\
15 \\
20 \\
25 \\
31 \\
31 \\
\text { Sep } 05 \\
10 \\
15 \\
20 \\
25 \\
30\end{array}$ & $\begin{array}{l}140.17 \\
140.05 \\
140.28 \\
139.91 \\
140.40 \\
141.50 \\
139.97 \\
139.80 \\
139.21 \\
138.84 \\
137.79 \\
137.46\end{array}$ \\
\hline
\end{tabular}


GROUND-WATER LEVELS

REDWOOD COUNTY

441323095280701. Local number, 109\%38W30BBD01.

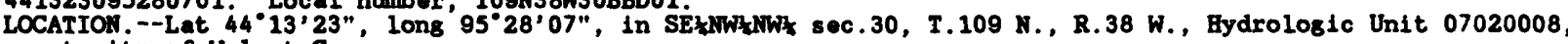
at city of Walnut Grove.

Owner: Plum Creek Cheese Co.

AQUIFER. --Sandstone of Cretaceous Age.

WELL CHARACTERISTICS. - Drilled unused artesian well, diameter 5 in., depth 240 ft, casing depth not available.

DATUM. - Altitude of land-surface datum is 1,218 ft. Measuring point: Top of well seal, $0.55 \mathrm{ft}$ above land-surface datum.

PERIOD OF RECORD. - August 1976 to current year.

EXTREMES FOR PERIOD OF RECORD.--Highest water leve1, $24.20 \mathrm{ft}$ below land-surface datum, April 3, 1984 ; lowest, $26.80 \mathrm{ft}$ below land-surface datum, Sept. 26, 1984 .

WATER LEVEL, IN FEET BELOW LAND-SURFACE DATUM, WATER YEAR OCTOBER 1990 TO SEPTEMBER 1991

\begin{tabular}{|c|c|c|c|c|c|}
\hline DATE & $\begin{array}{l}\text { WATER } \\
\text { LEVEL }\end{array}$ & DATE & $\begin{array}{l}\text { WATER } \\
\text { LEVEL }\end{array}$ & DATE & $\begin{array}{l}\text { WATER } \\
\text { LEVEL }\end{array}$ \\
\hline $\begin{array}{l}\text { Oct } 11 \\
\text { Nov } 28\end{array}$ & $\begin{array}{l}26.02 \\
25.93\end{array}$ & $\begin{array}{ll}\text { Fob } & 01 \\
\text { Jun } & 19\end{array}$ & $\begin{array}{l}25.95 \\
25.51\end{array}$ & Aug 19 & 25.88 \\
\hline
\end{tabular}

RICE COUNTY

441912093162901. Local number, 110स20W19BDC01.

LOCATIOH. - Lat $44^{\circ} 19^{\prime} 12^{\prime \prime}$, Iong $93^{\circ} 16^{\prime} 29^{\prime \prime}$, In SW'SE 4 WW sec.19, T.110 N., R.20 W., Bydrologic Unit 07040002 , just north of Faribault.

Omer: St. Lawrence Cemetery Assn.

AQUIFRR. - Jordan Sandstone of Late Cambrian Ase.

WELI CBARACTERISTICS. - Drilled domestic artesian well, diameter $4 \mathrm{in}$., depth $400 \mathrm{ft}$, cased to $357 \mathrm{ft}$. DATUM. - Altitude of land-surface datum is $985 \mathrm{ft}$. Measuring point: Top of casing, $1.60 \mathrm{ft}$ above landsurface datum.

PERIOD OF RECORD. --June 1979 to current year.

EXTRPMES FOR PERIOD OF RECORD. --Highest water level, 6.02 ft below land-surface datum, May 2, 1984 ;

lowest: $10.84 \mathrm{ft}$ below land-surface datum, July 10,1981 .

WATER LEVEL, IN FEET BELOW LAND-SURFACE DATUM, WATER YEAR OCTOBER 1990 TO SEPTEMBER 1991

$\begin{array}{lllll}\text { WATER } & \text { WATER } & \text { WATER } \\ \text { LEVEL } & \text { DATE } & \text { LEVEL } & \text { DATE } & \text { LEVEL }\end{array}$

Nov $09 \quad 9.94 \quad$ Mar $12 \quad 10.07 \quad$ Jul $23 \quad 9.44$

Jan $15 \quad 10.01$ May $09 \quad 10.42$ Sep $18 \quad 9.70$

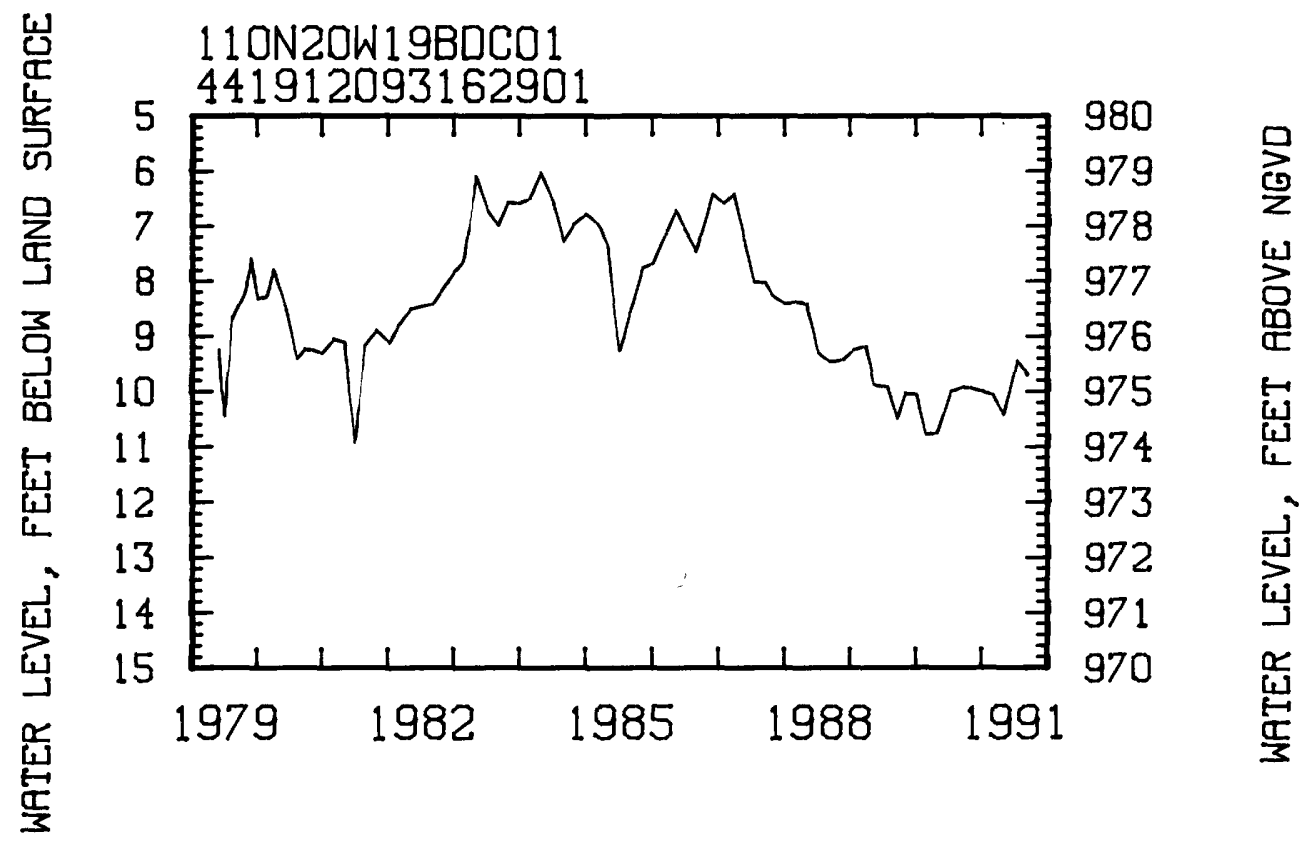


442543093113701. Local number, 111\$20W11CDC01.

LOCATION, - Lat $44^{\circ} 25^{\prime} 43^{\prime \prime}$, Iong $93^{\circ} 11^{\prime} 37^{\prime \prime}$, in SWłSEłSWł sec.11, T.111 N., R.20 W., Hydrologic Unit 07040002 , Highway 218 at Dundas.

Omer: Rollie Green.

AQUIFER. - Prairie du Chien Group of Early Ordovician Age.

WELI CBARACTERISTICS. --Drilled commercial artesian well, diameter 4 in., depth $158 \mathrm{ft}$, cased to $101 \mathrm{ft}$

DATUM.--Altitude of land-surface datum is $950 \mathrm{ft}$. Measuring point: Top of casing, $2.00 \mathrm{ft}$ above landsurface datum.

PERIOD OF RECORD. - - October 1980 to current year.

EXTREMES FOR PERIOD OF RECORD. --甘ighest water leve1, 21.72 ft below land-surface datum, May 14, 1986;

lowest, $27.44 \mathrm{ft}$ below land-surface datum, Nov. 9, 1990.

WATER LEVEL, IN FEET BELOW LAND-SURFACE DATUM, WATER YEAR OCTOBER 1990 TO SEPTEMBER 1991

$\begin{array}{cccccc}\text { DATE } & \begin{array}{c}\text { WATER } \\ \text { LEVEL }\end{array} & \text { DATE } & \begin{array}{c}\text { WATER } \\ \text { LEVEL }\end{array} & \text { DATE } & \begin{array}{c}\text { WATER } \\ \text { LEVEI }\end{array} \\ \text { Nov 09 } & 27.44 & \text { Mar 12 } & 26.56 & \text { Jul } 23 & 25.13 \\ \text { Jan 15 } & 26.89 & \text { May 09 } & 24.93 & \text { Sop 18 } & 25.56\end{array}$

442751093240701. Local number, 112N21W31CBB01.

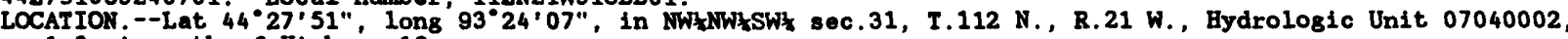

$1.0 \mathrm{mi}$ south of Highway 19 .

Owner: Trondhiem Church.

AQUIFER. - - Prairie du Chien Group of Early Ordovician Age

WELL CHARACTERISTICS.--Drilled domestic artesian well, diameter $4 \mathrm{in}$, depth $276 \mathrm{ft}$, cased to $232 \mathrm{ft}$.

DATUM.--Altitude of iand-surface datum is 1,130 ft. Measuring point: Top of casing, $1.10 \mathrm{ft}$ above

land-surface datum.

PERIOD OF RECORD. - June 1980 to current year.

EXTREMES FOR PERIOD OF RECORD.--Highest water level, $136.06 \mathrm{ft}$ below land-surface datum, Sept. 21, 1987; lowest, $141.8 \mathrm{ft}$ below land-surface datum, Oct. 30, 1981.

WATER LEVEL, IN FEET BELOW LAND-SURFACE DATUM, WATER YEAR OCTOBER 1990 TO SEPTEMBER 1991

$\begin{array}{crcrcr}\text { DATE } & \begin{array}{r}\text { WATER } \\ \text { LEVEL }\end{array} & \text { DATE } & \begin{array}{r}\text { WATER } \\ \text { LEVEL }\end{array} & \text { DATE } & \begin{array}{r}\text { WATER } \\ \text { LEVEL }\end{array} \\ \text { Nov 09 } & \begin{array}{l}140.53 \\ \text { Jan 15 }\end{array} & \begin{array}{c}\text { May 09 } \\ 140.65\end{array} & \begin{array}{l}140.82 \\ \text { Jul 26 }\end{array} & \begin{array}{l}140.93 \\ \text { Sep 18 }\end{array} & 140.76 \\ & & & & & \end{array}$

SCOTT COUNTY

443732093460301 . Local number, 113N24W06BCB01.

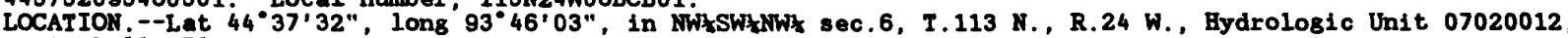

in Belle Plaine.

Omer: Creative Tool and Engineering. Formerly Belle Plaine Coop Creamery.

AQUIFER. - - Buried sand and gravel of Pleistocene Age.

WELI CHARACTERISTICS.--Drilled unused water-table well, diameter 12 in., depth 272 ft, screen information not available.

DATUM.--Altitude of land-surface datum is $840 \mathrm{ft}$. Measuring point: Top of well cap, $2.30 \mathrm{ft}$ above landsurface datum.

EXTREMES FOR PERIOD OF RECORD.--Highest water level, $116.77 \mathrm{ft}$ below land-surface datum, July 11, 1983 ; lowest, $143.96 \mathrm{ft}$ below land-surface datum, July' $9,1981$.

WATER LEVEL, IN FEET BELOW LAND-SURFACE DATUM, WATER YEAR OCTOBER 1990 TO SEPTEMBER 1991

\begin{tabular}{|c|c|c|c|c|c|}
\hline DATE & $\begin{array}{l}\text { WATER } \\
\text { LEVEL }\end{array}$ & DATE & $\begin{array}{l}\text { WATER } \\
\text { LEVEI }\end{array}$ & DATE & $\begin{array}{l}\text { WATER } \\
\text { LEVEL }\end{array}$ \\
\hline $\begin{array}{l}\text { Nov } 20 \\
\text { Jan } 15\end{array}$ & $\begin{array}{l}120.50 \\
120.87\end{array}$ & $\begin{array}{l}\text { Mar } 26 \\
\text { May } 10\end{array}$ & $\begin{array}{l}119.87 \\
118.68\end{array}$ & $\begin{array}{l}\text { Jul } 25 \\
\text { Sep } 25\end{array}$ & $\begin{array}{l}119 . \\
118.2\end{array}$ \\
\hline
\end{tabular}


GROUND-WATER LEVELS

SCOTT COUNTY--Continued

443352093423001. Local number, 113N24W280AA01.

LOCATION.--Lat $44^{\circ} 33^{\prime} 52^{\prime \prime}$, Long $93^{\circ} 42^{\prime} 30^{\prime \prime}$, in NEłNEłSEk sec.28, T.113 N., R.24 W., Hydrologic Un1t 07020012, at Michelle Wildilfe Area,

Omer: U.S. Geological Survey.

AQUIFER. - - r ronton-Galesville Sandstones of Late Cambrian Age.

WELL CAARACTERISTICS.-Drilled observation artesian well, diameter 6 in., depth 450 ft, cased to 219 ft. DATUy. --Altitude of land-surface datum is $990 \mathrm{ft}$. Measuring point: Top of pel1 seal, $2.30 \mathrm{ft}$ above landsurface datum.

PXRIOD OF RECORD,--August 1981 to current year.

EXTREMES FOR PERIOD OF RECORD.--Highest water leve1, 131.70 ft below land-surface datum, May 2, 1984;

lowest, 137.07 ft below land-surface datum, Aug. 15, 1989.

WATER LEVEL, IA FEET BELON LAND-SURFACE DATUM, MATER YEAR OCTOBER 1990 TO SEPTEMBER 1991

\begin{tabular}{|c|c|c|c|c|c|c|c|}
\hline DA & $\begin{array}{l}\text { WATER } \\
\text { LEVEL }\end{array}$ & DATE & $\begin{array}{l}\text { WATER } \\
\text { LEVEL }\end{array}$ & DATE & $\begin{array}{l}\text { WATER } \\
\text { LEVEL }\end{array}$ & DATE & $\begin{array}{l}\text { WATER } \\
\text { LEVEL }\end{array}$ \\
\hline & & $\mathrm{Jar}$ & 35 & & & Sen & \\
\hline
\end{tabular}

444025093220801. Local number, 114K21W20BAA01.

LOCATION. --Lat $44^{\circ} 40^{\prime} 25^{\prime \prime}$, Long $93^{\circ} 22^{\prime} 08^{\prime \prime}$, in NEłNEłNW sec.20, T.114 N., R.21 W., Hydrologic Unit 07020012, $0.5 \mathrm{ml}$ east of Credit River.

Omer: Credit River Town Hall.

AQUIFER. --Burted sand of Pleistocene Age.

WELL CAARACTERISTICS.-Drilled domestic artesian mell, diameter 4 in., depth $98 \mathrm{ft}$, screened 93 to $98 \mathrm{ft}$. DATU. --Altitude of land-surface datum is $946 \mathrm{ft}$. Measuring polnt: Top of casins, 1.10 ft above landsurface datum.

PERIOD OF RECORD.--September 1979 to current year.

EXTRFMES FOR PERIOD OF RECORD.--Highest pater level, 34.00 ft below land-surface datum, Feb. 3, 1987; lowest, $40.88 \mathrm{ft}$ below land-surface datum, July 26, 1991.

WATER LEVEL, IN FEET BELON LAND-SURFACE DATUM, WATER YEAR OCTOBER 1990 TO SEPTEMBER 1991

$\begin{array}{lllll}\text { WATER } & & \text { WATER } & \text { WATER } \\ \text { DATE } & \text { LEVEL } & \text { DATE } & \text { LEVEL } & \text { DATE } \\ \text { LEVEL }\end{array}$

$\begin{array}{lllllll}\text { Nov } 09 & 39.72 & \text { Mar } 12 & 39.74 & \text { Jul } 26 & 40.88\end{array}$

$\begin{array}{llllll}\text { Jan } 15 & 39.54 & \text { May } 09 & 40.00 & \text { Sep } 18 & 40.09\end{array}$

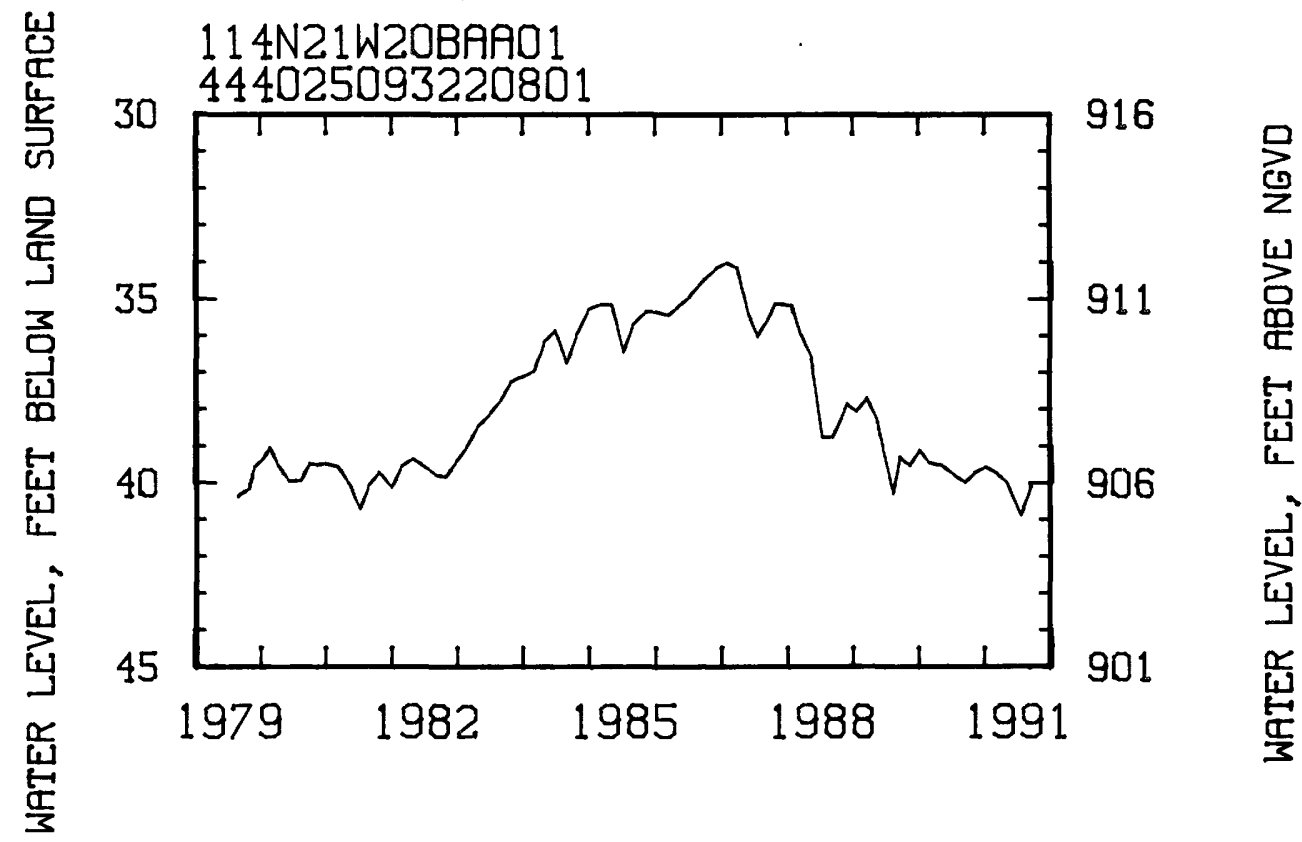


443752093254401. Local number, 114N22W35DCC01.

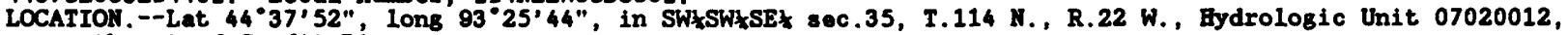
southwest of Credit River.

Owner: St. Catherine's Church.

AQUIFER. --Prairie du Chien Group of Early Ordovician Age.

WELL CBARACTERISTICS, --Drilled domestic artesian weIl, diameter 4 in., depth $235 \mathrm{ft}$, cased to $194 \mathrm{ft}$.

DATUM.--Altitude of iand-surface datum is $1,015 \mathrm{ft}$. Measuring point: Top of casing, $1.20 \mathrm{ft}$ above

land-surface datum.

PERIOD OF RECORD. --September 1979 to current year.

EXIREMIES FOR PERIOD OF RECORD.--Highest water level, $83.27 \mathrm{ft}$ below land-surface datum, Dec. 4, 1986 ;

lowest, $90.30 \mathrm{ft}$ below land-surface datum, Sept. 6 , 1979.

WATER LEVEL, IN FEET BELON LAND-SURFACE DATUM, WATER YEAR OCTOBER 1990 TO SEPTEMBER 1991

$\begin{array}{cccccc}\text { DATE } & \begin{array}{c}\text { WATER } \\ \text { LEVEL }\end{array} & \text { DATE } & \begin{array}{c}\text { WATER } \\ \text { LEVEL }\end{array} & \text { DATE } & \begin{array}{c}\text { WATER } \\ \text { LEVEL }\end{array} \\ \text { Nov 09 } & \mathbf{8 7 . 8 5} & \text { May 09 } & \mathbf{8 8 . 1 7} & \text { Sop 18 } & \mathbf{8 7 . 8 3} \\ \text { Mar 12 } & \mathbf{8 8 . 1 0} & \text { Jul 26 } & \mathbf{8 7 . 8 6} & & \end{array}$

444633093212901. Local number, 115N21w09ccC01.

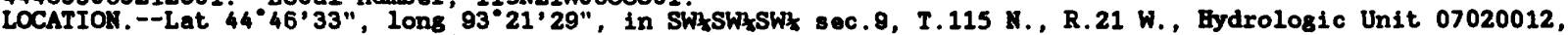
at Savage waste treatment plant.

Omer: City of Savase, well 2

AQUIFER. - Mount Simon Sandstone of Late Cambrian Age and Hinckley Sandstone of Late Precambrian Age. WELL CHARACTERISTICS.--Drilled public-supply artesian well, depth $846 \mathrm{ft}$, 16 in, casing $0 \mathrm{ft}$ to $280 \mathrm{ft}$, 10 in. casing $250 \mathrm{ft}$ to $660 \mathrm{ft}$.

DATUM. --Land-surface datum is $730 \mathrm{ft}$. Measuring point: Edge of vent pipe $0.75 \mathrm{ft}$ above land-surface datum.

PERIOD OF RECORD.--February 1971 to current year.

EXIRENISS FOR PERIOD OF RECORD.--Highest water level, 18.98 ft below land-surface datum, Aug. 9 , 1979 ; lowest, 83.57 ft below land-surface datum, Sept. 19, 1991.

WATER LEVEL, IN FEET BELOW LAND-SURFACE DATUM, WATER YEAR OCTOBER 1990 TO SEPTEMBER 1991

$\begin{array}{cccccc}\text { DATE } & \begin{array}{c}\text { WATER } \\ \text { LEVEL }\end{array} & \text { DATE } & \begin{array}{c}\text { WATER } \\ \text { LEVEL }\end{array} & \text { DATE } & \begin{array}{c}\text { WATER } \\ \text { LEVEL }\end{array} \\ \text { Nov 13 } & \mathbf{7 7 . 5 5} & \text { Mar 06 } & \mathbf{7 0 . 2 1} & \text { JuI 17 } & \mathbf{7 5 . 1 1} \\ \text { Jan 08 } & \mathbf{7 2 . 2 0} & \text { May 10 } & \mathbf{7 0 . 1 9} & \text { Sep 19 } & \mathbf{8 3 . 5 7}\end{array}$

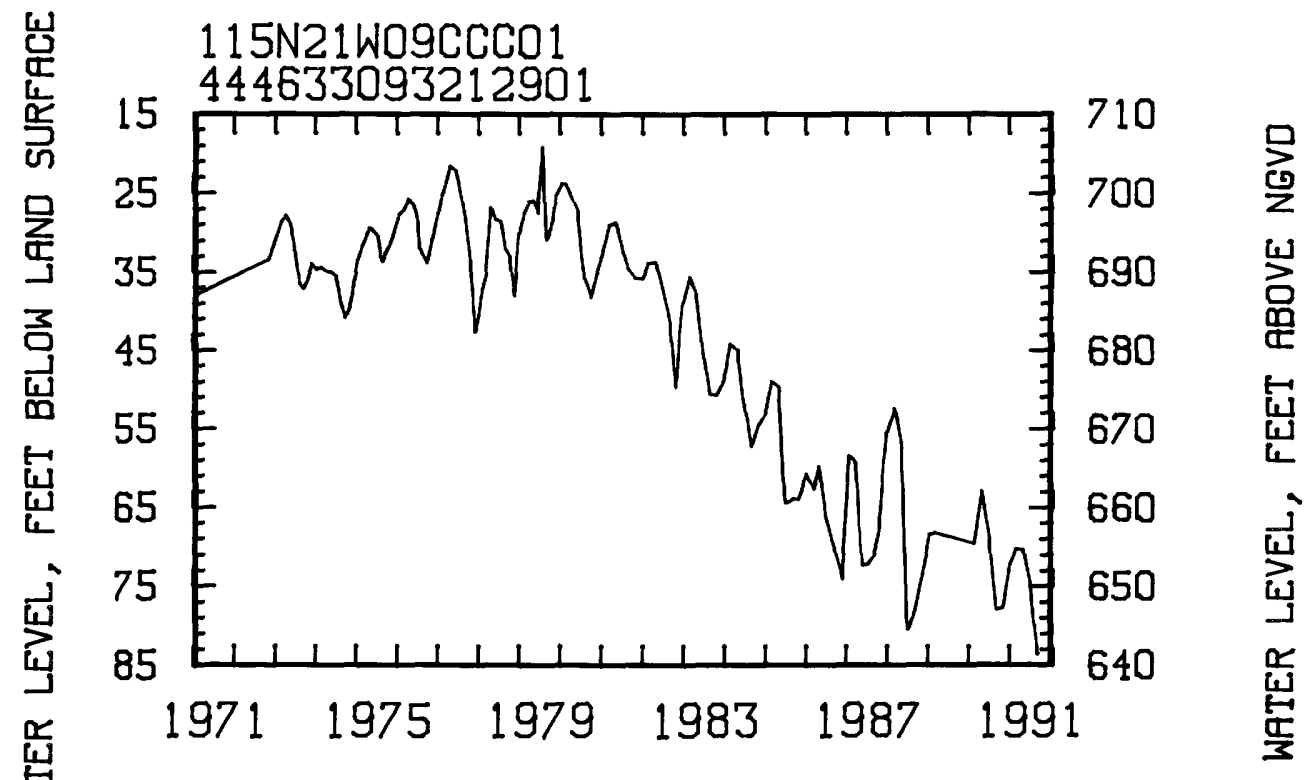


444427093353901, Local number, 115N23W28BDD01.

LOCATION. - Lat $44^{\circ} 44^{\circ} 27^{\prime \prime}$, long $93^{\circ} 43^{\prime} 53^{\prime \prime}$, in SEkSEłNW sec.28, T.115 N., R.23 W., Bydrologic Un1t 07020012, Merriam Jumction.

Omer: Chicago and Northwestern Transportation Company.

AOUIFER. --Jordan Sandstone of Late Cambrian Age.

WELL CHARACTERISTICS.--Drilled observation artesian well, diameter 16 in., depth 140 ft, cased to $75 \mathrm{ft}$.

DATUM.--Altitude of land-surface datum $18758 \mathrm{ft}$. Measuring point: Top of casing, 0.90 ft above land-surface datum.

PERIOD OF RECORD. - November 1984 to current year.

EXTREMES FOR PERIOD OF RFCORD, - Highest water leve1, 25.75 ft belom land-surface datum, Mar. 8,

1985; lowest, $43.20 \mathrm{ft}$ below land-surface datum, Feb. 28, 1990 .

WATER LEVEL, IN FEET BELOW LAND-SURFACE DATUM, WATER YEAR OCTOBER 1990 TO SEPTEMBER 1991

\begin{tabular}{|c|c|c|c|c|c|}
\hline DATE & $\begin{array}{l}\text { HATER } \\
\text { LEVEL }\end{array}$ & DATE & $\begin{array}{l}\text { WATER } \\
\text { LEVDL }\end{array}$ & DATE & $\begin{array}{l}\text { WAIDR } \\
\text { LEVDL }\end{array}$ \\
\hline $\begin{array}{ll}\text { Jov } 13 \\
\text { Jan } 08\end{array}$ & $\begin{array}{l}41.43 \\
41.50\end{array}$ & $\begin{array}{ll}\text { Mar } 06 \\
\text { May } 10\end{array}$ & $\begin{array}{l}42.39 \\
41.92\end{array}$ & $\begin{array}{ll}\text { Jul } & 17 \\
\text { Sep } & 13\end{array}$ & $\begin{array}{l}40.50 \\
40.25\end{array}$ \\
\hline
\end{tabular}

444427093353902. Local number, 115N23W28BDD02.

LOCATION, - Lat $44^{\circ} 44^{\prime} 27^{\prime \prime}$, Long $93^{\circ} 35^{\prime} 39^{\prime \prime}$, in SEłSEłWW sec.28, T.115 N., R.23 W., Bydrologic Unit 07020012, Merrian Junction.

Omer: Chicago and Northwestern Transportation Company. AOUIFER. - Ironton-Galesville Sandstone of Late Cambrian Age.

WELL CEARACTERISTICS,-Drilled observation artesian well, diameter 4 in., depth 355 ft, screened 350 to 355 ft. DATUM. - Altitude of land-surface datum is $758 \mathrm{ft}$. Measuring point: Top' of casing, $1.00 \mathrm{ft}$ above land-surface datum.

PERIOD OF RECORD. - November 1984 to current year.

EXTREMES FOR PERIOD OF RECORD.--Highest water level, 20.52 ft below land-surface datum, Mar. 21, 1986; 1owest, 45.28 ft below land-surface datum, July 29, 1991 .

WATER LEVEL, IN FEET BELOW LAND-SURFACE DATAUM, WATER YEAR OCTOBER 1990 TO SEPTEMBER 1991

\begin{tabular}{|c|c|c|c|c|c|c|c|c|c|c|c|c|}
\hline DATE & $\begin{array}{l}\text { WATER } \\
\text { LEVEL }\end{array}$ & DA: & & $\begin{array}{l}\text { WATER } \\
\text { LEVEL }\end{array}$ & DA: & & $\begin{array}{l}\text { WATER } \\
\text { LEVIL }\end{array}$ & DAI & & $\begin{array}{l}\text { WATER } \\
\text { LEVEL }\end{array}$ & DATE & $\begin{array}{l}\text { WATWR } \\
\text { LEVEL }\end{array}$ \\
\hline $\begin{aligned} & \text { Oct } 05 \\
& 10 \\
& 15 \\
& 20 \\
& 25 \\
& 31 \\
& 31 \\
& \text { Nov } 15 \\
& 20 \\
& 25 \\
& 30\end{aligned}$ & $\begin{array}{l}34.12 \\
34.37 \\
31.96 \\
29.84 \\
33.48 \\
34.23 \\
33.53 \\
30.76 \\
27.44 \\
28.25\end{array}$ & Jan & $\begin{array}{l}05 \\
10 \\
15 \\
20 \\
25 \\
31 \\
05 \\
10 \\
15 \\
20 \\
25 \\
31\end{array}$ & $\begin{array}{l}25.85 \\
25.06 \\
24.71 \\
24.40 \\
24.23 \\
24.03 \\
23.92 \\
23.94 \\
23.62 \\
23.72 \\
23.77 \\
23.62\end{array}$ & Mar & $\begin{array}{l}05 \\
10 \\
15 \\
20 \\
25 \\
28 \\
05 \\
10 \\
15 \\
20 \\
25 \\
31\end{array}$ & $\begin{array}{l}23.53 \\
23.49 \\
23.42 \\
23.16 \\
23.42 \\
23.15 \\
22.85 \\
23.27 \\
23.26 \\
22.98 \\
22.92 \\
33.90\end{array}$ & May & $\begin{array}{l}05 \\
10 \\
15 \\
20 \\
25 \\
30 \\
05 \\
10 \\
15 \\
20 \\
25 \\
31\end{array}$ & $\begin{array}{l}32.23 \\
29.90 \\
27.57 \\
30.01 \\
31.70 \\
30.86 \\
29.04 \\
30.87 \\
31.00 \\
29.25 \\
28.57 \\
29.81\end{array}$ & $\begin{aligned} & \text { Jul } 20 \\
& 25 \\
& \text { Sep } \\
& 20 \\
& 25 \\
& 30\end{aligned}$ & $\begin{array}{l}39.87 \\
39.97 \\
32.52 \\
32.31 \\
32.00\end{array}$ \\
\hline
\end{tabular}

444427093353903, Local number, 115\%23W28BDD03.

LOCATION, - Lat $44^{\circ} 44^{\prime} 27^{\prime \prime}$, long $93^{\circ} 35^{\prime} 39^{\prime \prime}$, in SEzSEłNW sec.28, T.115 N., R.23 W., Bydrologic Unit 07020012, Merrian Junction.

Owner: Chicago and Northwestern Transportation Company.

AQUIFER. - Mount Simon Sandstone of Late Cambrian Ase.

WELL CBARACTERISTICS,--Drilled observation artesian well, diameter 4 in., depth 525 ft, screened 520 to $525 \mathrm{ft}$.

DATUM.--Altitude of land-surface datum is $758 \mathrm{ft}$. Measuring point: Top of casing, $1.00 \mathrm{ft}$ above landsurface datum.

PERIOD OF RECORD.--November 1984 to current year.

EXIRDMES FOR PERIOD OR RECORD,--Highest water level, $33.85 \mathrm{ft}$ below land-surface datum, Mar. 8, 1985;

lowest, $55.12 \mathrm{ft}$ below land-surface datum, Aus. $1,1988$.

WATER LEVEL, IN FEET BELON LAND-SURFACE DATAUY, WATER YEAR OCTOBER 1990 TO SEPTEMBER 1991

\begin{tabular}{|c|c|c|c|c|c|c|c|c|c|c|c|c|}
\hline DATE & $\begin{array}{l}\text { WATER } \\
\text { LEVEL }\end{array}$ & DATE & $\begin{array}{l}\text { WATER } \\
\text { LEVEL }\end{array}$ & DATE & & $\begin{array}{l}\text { WATRR } \\
\text { LEVEL }\end{array}$ & DATE & & $\begin{array}{l}\text { WATER } \\
\text { LEVEL }\end{array}$ & DAI & & $\begin{array}{l}\text { WATER } \\
\text { LEVEL }\end{array}$ \\
\hline $\begin{array}{r}\text { Oct } 05 \\
10 \\
15 \\
20 \\
25 \\
31 \\
\text { Nov } 15 \\
20 \\
25 \\
30\end{array}$ & $\begin{array}{l}48.66 \\
48.71 \\
48.31 \\
47.79 \\
47.94 \\
47.63 \\
47.16 \\
47.06 \\
46.91 \\
46.80\end{array}$ & $\begin{array}{r}\text { Dec } 05 \\
10 \\
15 \\
20 \\
25 \\
31 \\
\text { Jan } 05 \\
10 \\
15 \\
20 \\
25 \\
31\end{array}$ & $\begin{array}{l}46.63 \\
46.26 \\
46.03 \\
46.05 \\
46.30 \\
46.12 \\
46.11 \\
46.16 \\
45.57 \\
45.32 \\
45.26 \\
45.39\end{array}$ & $\begin{array}{l}\text { Feb } \\
\text { Mar }\end{array}$ & $\begin{array}{l}05 \\
10 \\
15 \\
20 \\
25 \\
28 \\
05 \\
10 \\
15 \\
20 \\
25 \\
31\end{array}$ & $\begin{array}{l}45.61 \\
45.35 \\
45.12 \\
45.00 \\
45.20 \\
44.65 \\
44.57 \\
44.86 \\
44.52 \\
44.39 \\
44.34 \\
44.84\end{array}$ & May & $\begin{array}{l}05 \\
10 \\
15 \\
20 \\
25 \\
30 \\
05 \\
10 \\
15 \\
20 \\
25 \\
31\end{array}$ & $\begin{array}{l}45.16 \\
45.75 \\
45.23 \\
45.06 \\
44.73 \\
44.20 \\
44.21 \\
43.89 \\
43.79 \\
44.40 \\
44.38 \\
44.46\end{array}$ & $\begin{array}{l}\text { Jul } \\
\text { Sep }\end{array}$ & $\begin{array}{l}05 \\
10 \\
15 \\
20 \\
25 \\
30 \\
05 \\
10 \\
15 \\
20 \\
15 \\
20 \\
25 \\
30\end{array}$ & $\begin{array}{l}44.92 \\
44.69 \\
45.50 \\
46.01 \\
46.18 \\
46.97 \\
48.24 \\
48.87 \\
49.12 \\
49.56 \\
52.26 \\
52.65 \\
52.17 \\
51.79\end{array}$ \\
\hline
\end{tabular}


435742093164001. LOCal number, 106N20W30BAD01.

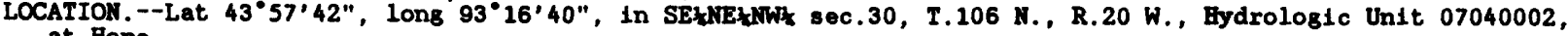
at Hope.

AOUIFER

-Galena Formation of Middle Ordovician Age.

ELL CHARACTERISTICS.--Drilled commerical artesian well, diameter $5 \mathrm{in}$, depth 215 ft, cased to $108 \mathrm{ft}$

ATUM.--Altitude of land-surface datum is 1,198 ft. Measuring point: Top of casing, $1.00 \mathrm{ft}$ above landsurface datum.

PERIOD OF RECORD. --November 1981 to current year.

EXTREMES FOR PERIOD OF RECORD.--Highest water level, $29.72 \mathrm{ft}$ below land-surface datum, May 28, 1991; lowest, $34.48 \mathrm{ft}$ below land-surface datum, July $10,1985$.

WATER LEVEL, IN FEET BELOW LAND-SURFACE DATUM, WATER YEAR OCTOBER 1990 TO SEPTEMBER 1991

$\begin{array}{cccccc}\text { DATE } & \begin{array}{c}\text { WATER } \\ \text { LEVEL }\end{array} & \text { DATE } & \begin{array}{c}\text { WATER } \\ \text { LEVEI }\end{array} & \text { DATE } & \begin{array}{c}\text { WATER } \\ \text { LEVEL }\end{array} \\ \text { Nov 19 } & 32.36 & \text { Mar 25 } & 31.87 & \text { Jul 23 } & 29.88 \\ \text { Jan 30 } & 32.02 & \text { May 28 } & 29.72 & \text { Sep 24 } & 31.12\end{array}$

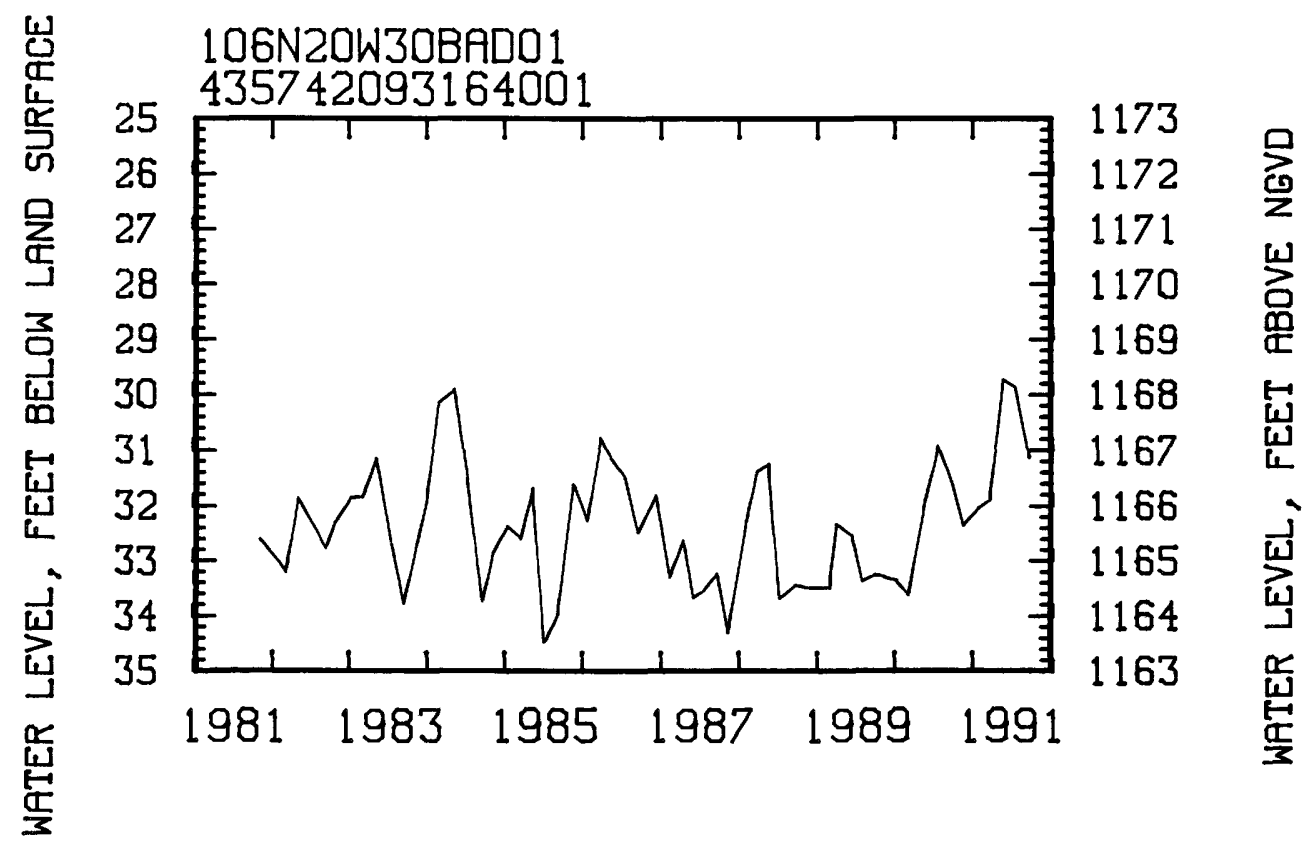

435611093163001. Local number 106N20w31DCC01.

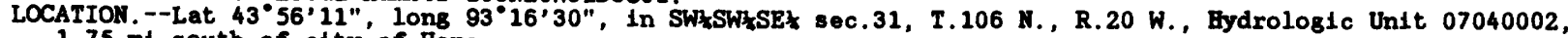

$1.75 \mathrm{mi}$ south of city of Hope.

Omer: Owatonna Gun Club.

AQUIFER. - Galena formation of Middle Ordovician age.

WELL CHARACTERISTICS.--Drilled domestic artesian well, diameter $4 \mathrm{ln}$., depth $168 \mathrm{ft}$, cased to $97 \mathrm{ft}$

DATUM.--Altitude of iand-surface datum is $1,180 \mathrm{ft}$. Measuring point: Top of casing, $1.40 \mathrm{ft}$ above

PERIOD OF RECORD, - November 1989 to current year.

EXTREMES FOR PERIOD OF RECORD. --Highest water level, 0.66 ft below land-surface datum, May 10, 1984 ;

lowest, $4.97 \mathrm{ft}$ below land-surface datum, Jan. 9, 1990.

WATER LEVEL, IN FEET BELOW LAND-SURFACE DATAUM, WATER YEAR OCTOBER 1990 TO SEPTEMBER 1991

$\begin{array}{crrrrr}\text { DATE } & \begin{array}{r}\text { WATER } \\ \text { LEVEL }\end{array} & \text { DATE } & \begin{array}{r}\text { WATER } \\ \text { LEVEL }\end{array} & \text { DATE } & \begin{array}{r}\text { WATER } \\ \text { LEVEL }\end{array} \\ \text { Nov 19 } & 3.76 & \text { May 25 } & 3.53 & \text { Jul 23 } & 1.64 \\ \text { Jan 30 } & 3.92 & 28 & 1.63 & \text { Sep 24 } & 2.87\end{array}$


GROUND-WATER LEVELS

SWIFT COUNTY

451913095370201. Local number, 121N39W06BDB01.

LOCATION.--Lat $45^{\circ} 19^{\prime} 13^{\prime \prime}$, Long $95^{\circ} 37^{\prime} 02^{\prime \prime}$, in NWzSEłNWz sec.6, T.121 N., R.39 W., Hydrologic Unit 07020005

in Ambush Park.

Omer: City of Benson.

AQUIFER. --Buried sand of Pleistocene Age.

WELL CHARACTERISTICS. --Drilled observation artesian we11, diameter 3 in., depth 143 ft, screened 123 to $143 \mathrm{ft}$.

DATUM.--Altitude of land-surface datum is 1,030 ft. Measuring point: Top of casing $3.00 \mathrm{ft}$ above land-surface datum.

REMARKS. - Water level affected by pumping.

PERIOD OF RECORD.-May 1979 to current year.

EXTRDAES FOR PERIOD OF RECORD.--Highest water level, 3.85 ft below land-surface datum, Oct. 25, 1984 ;

lowest, 19.90 ft below land-surface datum, July $24,1980$.

WATER LEVEL, IN FEET BELOW LAND-SURFACE DATUM, WATER YEAR OCTOBER 1990 TO SEPTEMBER 1991

$\begin{array}{lllll}\text { WATER } & \text { WATER } & \text { WATER } \\ \text { DATE } & \text { LEVEL } & \text { DATE } & \text { LEVEL } & \text { DATE } \\ \text { LEVEL }\end{array}$

$\begin{array}{lllrrr}\text { Nov 08 } & 8.72 & \text { Apr } 11 & 8.81 & \text { Sep } 18 & 6.52 \\ \text { Feb 08 } & 9.99 & \text { Ju1 } 17 & 11.59 & & \end{array}$

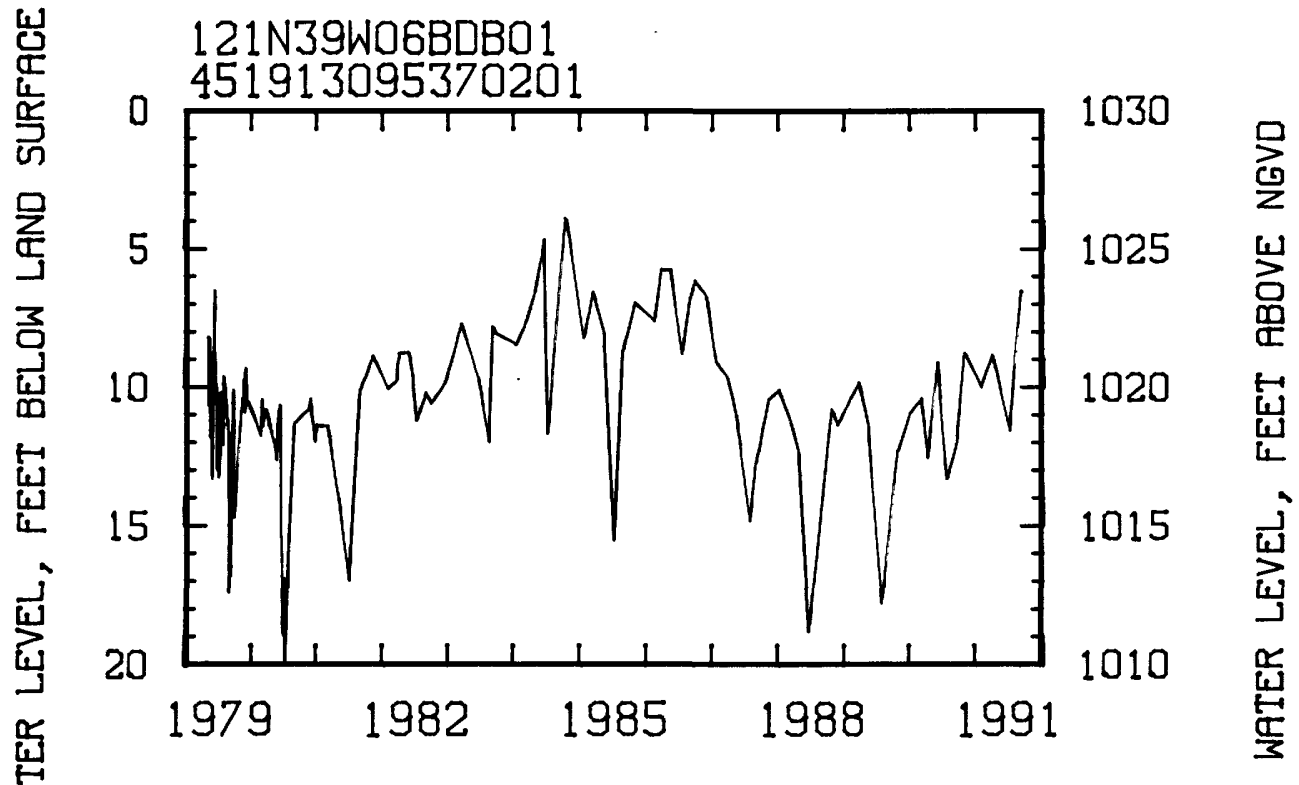


442708092155401. Local number, 111N12WO4BBDO1.

LOCATION, --Lat $44^{\circ} 27^{\circ} 08^{\prime \prime}$, long $92^{\circ} 15^{\prime} 54^{\prime \prime}$, in SEZWWHNW sec.04, T.111 N., R.12 W., Hydrologic Unit 07040001, at Lake City.

Omer: City of Lake City, mell 3

AQUIFER.--Mount Simon Sandstone of Late Cambrian Age.

WELL CBARACTERISTICS.--Drilled unused artesian weli, diameter 16 in., depth 430 ft, cased to $258 \mathrm{ft}$.

DATUM.--Altitude of land-surface datum is $685 \mathrm{ft}$. Measuring point: Top of casing, $1.00 \mathrm{ft}$ above land-surface datum.

REMARKS. --Measured weekly by David Finley.

PERIOD OF RECORD. --August 1974 to current year

EXTREMES FOR PERIOD OF RECORD. --Highest water level, 3.63 ft below land-surface datum, May 5, 1975; lowest,

$11.50 \mathrm{ft}$ below land-surface datum, Jan. 31,1977 .

WATER LEVEL, IN FEET BELOW LAND-SURFACE DATUM, WATER YEAR OCTOBER 1990 TO SEPTEMBER 1991

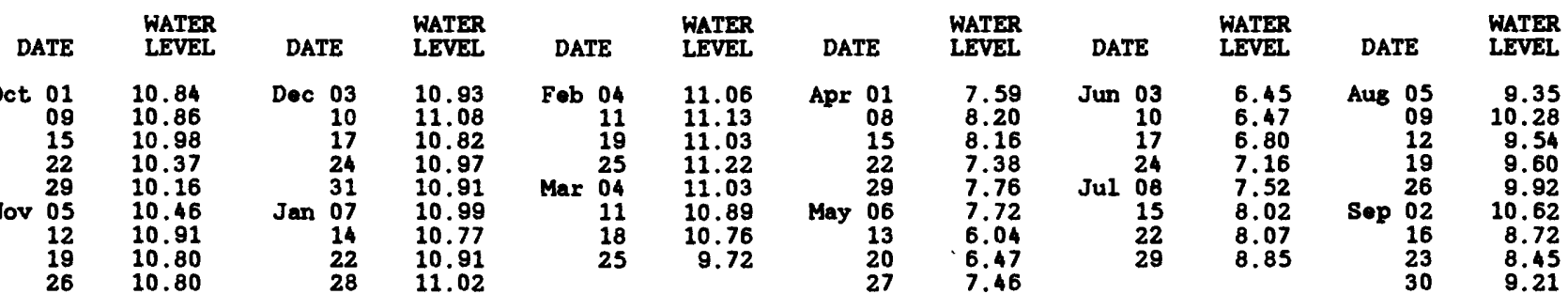

WADENA COUNTY

462415095003001. Local number, 134N34W19ADD01

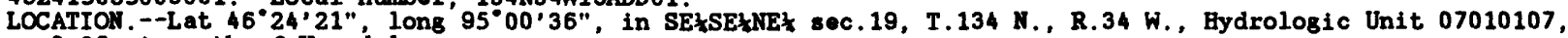
$0.05 \mathrm{mi}$ north of Verndale.

Owner: U.S. Geologicai Survey.

AOUIFER. --Surficial outwash sand and gravel of Pleistocene Age.

WELL CBARACTERISTICS.--Bored observation water-table well, diameter 2 in. depth $37 \mathrm{ft}$ screened 34 to $37 \mathrm{ft}$

DATUM.--Altitude of iand-surface datum is $1,342 \mathrm{ft}$. Measuring point: Top of casing, $1.00 \mathrm{ft}$ above land-surface datum.

PERIOD OF RECORD.--Soptember 1966 to current year.

EXTREMES FOR PERIOD OF RECORD.--Highest mater level, $9.18 \mathrm{ft}$ below land-surface datum, May 23, 1986; lowest, $15.33 \mathrm{ft}$ below land-surface datum, Mar. 10-11, 1977

WATER LEVEL, IN FEET BELOW LAND-SURFACE DATUM, WATER YEAR OCTOBER 1990 TO SEPTEMBER 1991

$\begin{array}{cccccccc}\text { DATE } & \begin{array}{c}\text { WATER } \\ \text { LEVEL }\end{array} & \text { DATE } & \begin{array}{c}\text { WATER } \\ \text { LEVEL }\end{array} & \text { DATE } & \begin{array}{c}\text { WATER } \\ \text { LEVEL }\end{array} & \text { DATE } & \begin{array}{r}\text { WATER } \\ \text { LEVEL }\end{array} \\ \text { Jan 31 } & 14.58 & \text { Apr 16 } & 14.46 & \text { Jun 17 } & 13.39 & \text { Aug 20 } & 13.59 \\ \text { Mar 16 } & 14.76 & \text { May 17 } & 13.67 & \text { Jul 16 } & 13.15 & \text { Sop 16 } & 13.80\end{array}$

WASHINGTON COUNTY

445125092464001. Local number, 027M20W02BCCO1.

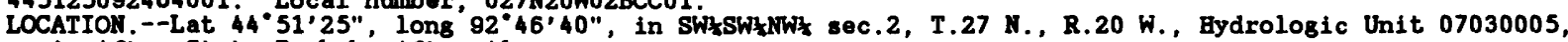
in Afton State Park by Afton Alps.

Amer: U.S. Goological Survey.

AOUIFER. - - St. Lawrence Formation and Franconian Sandstone of Late Cambrian Ase.

WELL CHARACTERISTICS.--Drilled observation artesian well, diameter 6 in. depth $285 \mathrm{ft}$, cased to $105 \mathrm{ft}$

DATUM. --Altitude of iand-surface datum is $695 \mathrm{ft}$. Measuring point: Center of pressure guage, $3.80 \mathrm{ft}$ above land-surface datum.

PERIOD OF RECORD. --March 1980 to current year.

EXTREMES FOR PERIOD OF RECORD.--Highest water level, $33.94 \mathrm{ft}$ above land-surface datum, May 2, 1980;

lowest, $19.67 \mathrm{ft}$ above land-surface datum, Jan.8, 1985.

WATER LEVEL, IN FEET ABOVE LAND-SURFACE DATUM, WATER YEAR OCTOBER 1990 TO SEPTEMBER 1991

$\begin{array}{crcrcr}\text { DATE } & \begin{array}{r}\text { WATER } \\ \text { LEVEL }\end{array} & \text { DATE } & \begin{array}{r}\text { WATER } \\ \text { LEVEL }\end{array} & \text { DATE } & \begin{array}{r}\text { WATER } \\ \text { LEVEL }\end{array} \\ \text { Jun } 19 & +26.64 & \text { Jul } 18 & +25.26 & \text { Sop } 11 & +25.26\end{array}$


GROUND-WATER LEVELS

WASHINGTON COUNTY--Continued

445125092464002. Local number, 027N20W02BCC02.

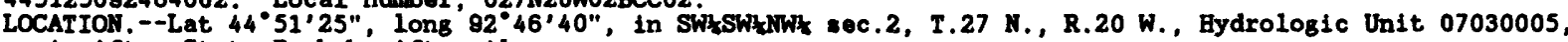
in Afton State Park by Afton Alps.

Omer: U.S. Geological Survoy.

AOUIFER. --Ironton-Galeaville Sandstones of Late Cambrian Age.

WELL CHARACTERISTICS. --Drilled observation artesian well, diameter 4 in., depth $385 \mathrm{ft}$, cased to $365 \mathrm{ft}$. DATUM. --Altitude of iand-surface datum is $695 \mathrm{ft}$. Measuring point: Center of pressure guage, $3.80 \mathrm{ft}$ above land-surface datum.

PERIOD OF RECORD. - March 1980 to current yoar.

EXTREMES FOR PERIOD OF RECORD. --Highest water leve1, 42.35 ft above land-surface datum, May 2, 1980,

lowest, $23.81 \mathrm{ft}$ above land-surface datum, Jan. $8,1985$.

WATER LEVEL, IN FEET ABOVE LAND-SURFACE DATUM, WATER YEAR OCTOBER 1990 TO SEPTEMBER 1991

$\begin{array}{crcrcr}\text { DATE } & \begin{array}{r}\text { WATER } \\ \text { LEVEL }\end{array} & \text { DATE } & \begin{array}{r}\text { WATER } \\ \text { LEVEL }\end{array} & \text { DATE } & \begin{array}{r}\text { WATER } \\ \text { LEVEL }\end{array} \\ \text { Jun } 19 & \mathbf{+ 3 2 . 0 0} & \text { Jul } 18 & \mathbf{+ 3 0 . 6 2} & \text { Sep } 11 & \mathbf{+ 3 0 . 6 2}\end{array}$

445125092464003. Local number, 027H20W02BCC03.

LOCATIOA. --Lat $44^{\circ} 51^{\prime} 2^{\prime \prime}$, long $92^{\circ} 46^{\prime} 40^{\prime \prime}$, in SWz'SWZNWZ sec.2, T.27 N., R.20 W., Hydrologic Unit 07030005, in Afton State Park by Afton Alps. Owner: U.S. Geological Survey.

AQUIFER.--Mount Simon Sandstone of Late Cambrian Age.

WELI. CHARACTERISTICS.--Drilled observation artesian vell, diameter it in., depth 535 ft, screened 530 to $535 \mathrm{ft}$.

DATUM.--Altitude of land-surface datum is $695 \mathrm{ft}$. Measuring point: Center of pressure guage, $3.40 \mathrm{ft}$ above land-surface datum.

PERIOD OF RECORD. - March 1980 to current year.

EXTREMES FOR PERIOD OF RECORD. --Highest water level, 22.05 ft above land-surface datum, May 2, 1980,

lowest, 3.40 ft above land-surface datum, Nov. $14,1989$.

WATER LEVEL, IN FEET ABOVE LAND-SURFACE DATUM, WATER YEAR OCTOBER 1990 TO SEPTEMBER 1991

$\begin{array}{cccccc}\text { DATE } & \begin{array}{c}\text { WATER } \\ \text { LEVEL }\end{array} & \text { DATE } & \begin{array}{c}\text { WATER } \\ \text { LEVEL }\end{array} & \text { DATE } & \begin{array}{c}\text { WATER } \\ \text { LEVEL }\end{array} \\ \text { Jun } 19 & +6.10 & \text { Jul } 19 & +6.04 & \text { SAp } 12 & +5.26\end{array}$

444751092563101. Local number, 027N21W28BCC01.

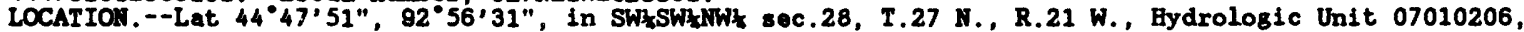

$0.1 \mathrm{mi}$ east of Ideal Avenue South. Omer: Eugene Smalilige.

AQUIFER.--Prairie du Chien Group of Early Ordovician Age and Jordan Sandstone of Late Cambrian Age.

WELL CBARACTERISTICS. --Drilled irrigation water-table well, diameter $16 \mathrm{in}$, depth $345 \mathrm{ft}$, cased to $60 \mathrm{ft}$.

DATUM. --Altitude of land-surface datum is $807 \mathrm{ft}$. Measuring point: Hole in pump base, $2.10 \mathrm{ft}$ above

land-surface datum.

PERIOD OR RECORD. --August 1977, January 1978, December 1979 to current year.

EXTREMES FOR PERIOD OF RECORD:- - Highest water level, $60.32 \mathrm{ft}$ below land-surface datum, Oct. 28, 1986 ;

lowest, $81.87 \mathrm{ft}$ below land-surface datum, Aug. 3, 1977.

WATER LEVEL, IN FEET BELOW LAND-SURFACE DATUM, WATER YEAR OCTOBER 1990 TO SEPTEMBER 1991

$\begin{array}{cccccc}\text { DATE } & \begin{array}{c}\text { WATER } \\ \text { LEVEL }\end{array} & \text { DATE } & \begin{array}{c}\text { WATER } \\ \text { LEVEL }\end{array} & \text { DATE } & \begin{array}{c}\text { WATER } \\ \text { LEVEL }\end{array} \\ \text { Nov 07 } & \mathbf{6 7 . 5 3} & \text { May 09 } & 70.99 & \text { SAp } 18 & \mathbf{7 2 . 9 6} \\ \text { Mar 25 } & \mathbf{7 0 . 5 3} & \text { Jul 26 } & \mathbf{7 1 . 4 3} & & \end{array}$


445536092462401. Local number, 028N20w11CAA01.

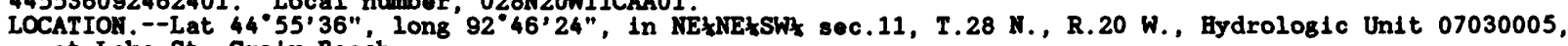

at Lake St. Croix Beach.

Omer: Lower st. Croix Valley Fire Department.

AQUIFER. - Franconian Sandstone of Late Cambrian Age.

WELL CBARACTERISTICS. -Drilled domestic water-table woll, diameter $4 \mathrm{in}$., depth $94 \mathrm{ft}$, cased to $78 \mathrm{ft}$. DATUM. - Altitude of land-surface datum is $720 \mathrm{ft}$. Measuring point: Top of electrical housing, $1.70 \mathrm{ft}$ above land-surface datum.

PERIOD OF RECORD. --June 1978 to current year.

EXTREMES FOR PERIOD OF RECORD.--Highest water level, $31.27 \mathrm{ft}, 5$ belon land-surface datum, May 1, 1986 ; lowest, $38.86 \mathrm{ft}$ below land-surface datum, Mar. 11,1991 .

WATER LEVEL, IN FEET BELOW LAND-SURFACE DATUM, WATER YEAR OCTOBER 1990 TO SEPTEMBER 1991

\begin{tabular}{|c|c|c|c|c|c|}
\hline DATE & $\begin{array}{l}\text { WATER } \\
\text { LEVEL }\end{array}$ & DATE & $\begin{array}{l}\text { WATER } \\
\text { LEVEL }\end{array}$ & DATE & $\begin{array}{l}\text { WATER } \\
\text { LEVEL }\end{array}$ \\
\hline $\begin{array}{l}\text { Nov } 06 \\
\text { Jan } 11\end{array}$ & $\begin{array}{l}38.24 \\
38.73\end{array}$ & $\begin{array}{ll}\text { Mar } & 11 \\
\text { May } & 13\end{array}$ & $\begin{array}{l}38.86 \\
34.96\end{array}$ & $\begin{array}{ll}\text { Jul } & 16 \\
\text { Sep } & 17\end{array}$ & $\begin{array}{l}33.29 \\
36.50\end{array}$ \\
\hline
\end{tabular}

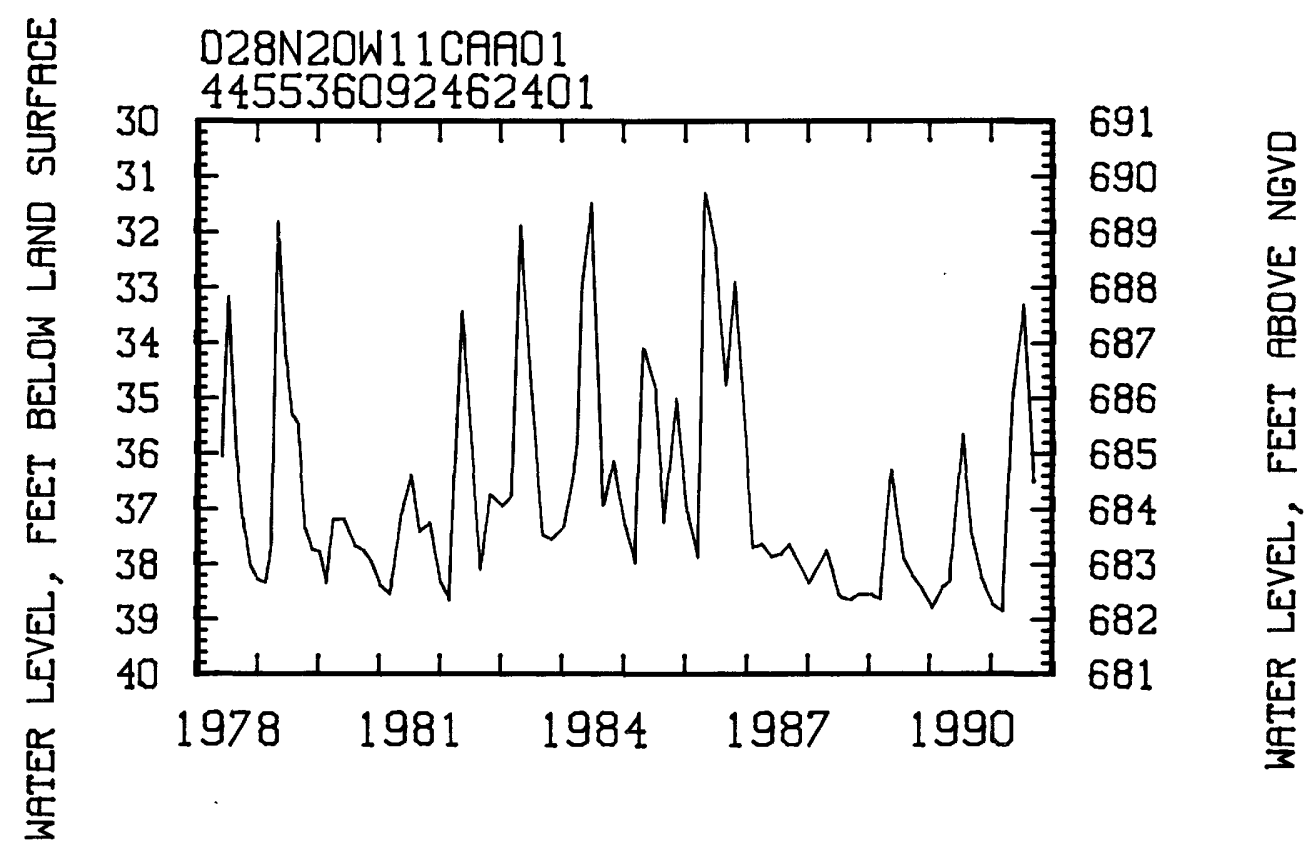

450134092583101. Local number, 029k21W06CAD01.

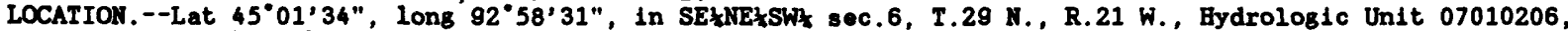
at 6488 North Bighway 36 Boulevard.

Omer: Twenty Mine Pines Irailer Park.

AQUIFER. - -st. Peter Sandstone of Middle Ordovician Age.

WELL CEARACTERISTICS.--Drilled unused artesian well, diameter 6 in., depth $210 \mathrm{ft}$, cased to $141 \mathrm{ft}$.

DATUM. --Altitude of land-surface datum is $980 \mathrm{ft}$. Measuring point: Hole in pump base, $2.20 \mathrm{ft}$ above land-surface datum.

PERIOD OF RECORD.--April 1974 to current year.

EXTREMES FOR PERIOD OF RECORD. --Highest water level, 71.07 ft belom land-surface datum, Feb. 6, 1987; lowest, $78.40 \mathrm{ft}$ below land-surface datum, Sep. 12, 1990 .

WATER LEVEL, IN FEET BELOW LAND-SURFACE DATUM, WATER YEAR OCTOBER 1990 TO SEPTEMER 1991

\begin{tabular}{|c|c|c|c|c|c|}
\hline DATE & $\begin{array}{l}\text { WATER } \\
\text { LEVEL }\end{array}$ & DATE & $\begin{array}{l}\text { WATER } \\
\text { LEVEI }\end{array}$ & DATE & $\begin{array}{l}\text { WATER } \\
\text { LEVEL }\end{array}$ \\
\hline $\begin{array}{l}\text { ov } 06 \\
\text { an } 11\end{array}$ & $\begin{array}{l}78.36 \\
78.12\end{array}$ & $\begin{array}{ll}\text { Mar } & 04 \\
\text { May } & 13\end{array}$ & $\begin{array}{l}77.47 \\
77.67\end{array}$ & $\begin{array}{ll}\text { Jul } & 16 \\
\text { Sep } & 17\end{array}$ & $\begin{array}{l}78.33 \\
78.12\end{array}$ \\
\hline
\end{tabular}


450027092552101 . Local number, 029N21W10CCC01.

LOCATION, --Lat $45^{\circ} 00^{\prime} 27^{\prime \prime}$, long $95^{\circ} 55^{\prime} 21^{\prime \prime}$, in SWł SW Lake Jane Road, $0.7 \mathrm{mi}(1.1 \mathrm{~km})$ north of Highway 212 .

Omer: City of Lake Eimo.

AQUIFER. --Jordan Sandstone of Late Cambrian Age.

WELL CEARACTERISTICS. --Drilled unused artesian we11, diameter 6 in., depth $348 \mathrm{ft}$, cased to $280 \mathrm{ft}$. DATUM. - Altitude of land-surface datum is $935 \mathrm{ft}$. Measuring point: Top of well cap, $1.20 \mathrm{ft}$ above land-surface datum.

PERIOD OF RECORD. - - September 1977, February 1978, February 1980 to current year.

EXTREMES FOR PERIOD OF RECORD. --Highest water leve1, 31.92 ft below land-surface datum, Oct. 28, 1986; lowest, $45.65 \mathrm{ft}$ below land-surface datum, Sept. $28,1977$.

WATER LEVEL, IN FEET BELOW LAND-SURFACE DATUM, WATER YEAR OCTOBER 1990 TO SEPTEMBER 1991

$\begin{array}{cccccc}\text { DATE } & \begin{array}{c}\text { WATER } \\ \text { LEVEL }\end{array} & \text { DATE } & \begin{array}{c}\text { WATER } \\ \text { LEVEL }\end{array} & \text { DATE } & \begin{array}{c}\text { WATER } \\ \text { LEVEL }\end{array} \\ \text { Nov 06 } & 44.28 & \text { Mer 04 } & 44.77 & \text { Ju1 16 } & 44.43 \\ \text { Jan 11 } & 44.50 & \text { May 13 } & 44.82 & \text { Sep 17 } & 44.15\end{array}$

450858092575001. Local number, 031N21W28ABD01

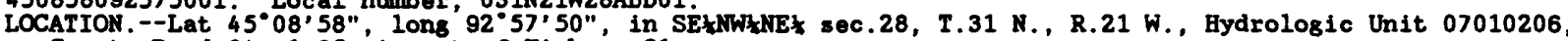
County Road 8A, 1.65 mi east of Highway 61.

Omer: White Bear Gun Club.

AQUIFER. - Prairie du Chien Group of Early Ordovician Age.

WELL CBARACTERISTICS,--Drilled domestic artesian we11, diameter 4 in, depth $142 \mathrm{ft}$, cased to $94 \mathrm{ft}$. DATUM. --Altitude of land-surface datum is $939 \mathrm{ft}$. Measuring point: Top of well cap, 1.30 ft above land-surface datum.

PERIOD OF RECORD.--September 1977, February 1978, February 1980 to current year.

EXTREMES FOR PERIOD OF RECORD.--Highest water level, 7.57 ft below land-surface datum, Oct. 28, 1986; lowest, $14.17 \mathrm{ft}$ below land-surface datum, Mar. 13, 1990.

WATER LEVEL, IN FEET BELOW LAAND-SURFACE DATUM, WATER YEAR OCTOBER 1990 TO SEPTEMBER 1991

$\begin{array}{cccccc}\text { DATE } & \begin{array}{c}\text { WATER } \\ \text { LEVEL }\end{array} & \text { DATE } & \begin{array}{c}\text { WATER } \\ \text { LEVEL }\end{array} & \text { DATE } & \begin{array}{c}\text { WATER } \\ \text { LEVEL }\end{array} \\ \text { Dec 04 } & 13.18 & \text { Mar 11 } & 13.47 & \text { Ju1 22 } & 11.59 \\ \text { Jan 16 } & 13.32 & \text { May 16 } & 12.07 & \text { Sep 17 } & 10.66\end{array}$

451355092532601 . Local number, 032N20W30BCD01.

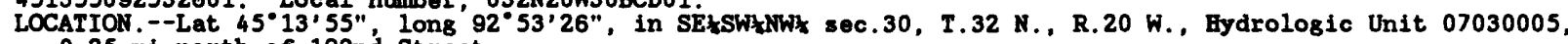
0.25 mi north of 192nd Street.

Owner: Arno Birr.

AQUIFER. - Prairie du Chien Group of Early Ordovician Age and Jordan Sandstone of Late Cambrian Age.

WELL CBARACTERISTICS. --Drilled irrigation artesian we11, diameter 12 in., depth $260 \mathrm{ft}$ cased to $141 \mathrm{ft}$

DATUM.--Altitude of land-surface datum is $990 \mathrm{ft}$. Measuring point: Vent pipe, 1.00 ft above land-surface datum.

PERIOD OF RECORD. --March 1981 to current year.

EXTREMES FOR PERIOD OF RECORD. --Highest water level, $51.56 \mathrm{ft}$ below land-surface datum, Oct. 28, 1986

lowest, $58.53 \mathrm{ft}$ below land-surface datum, Sept. 15, 1988.

WATER LEVEL, IN FEET BELON LAND-SURFACE DATUM, WATER YEAR OCTOBER 1990 TO SEPTEMBER 1991

$\begin{array}{cccccccc}\text { DATE } & \begin{array}{c}\text { WATER } \\ \text { LEVEL }\end{array} & \text { DATE } & \begin{array}{c}\text { WATER } \\ \text { LEVEI }\end{array} & \text { DATE } & \begin{array}{c}\text { WATER } \\ \text { LEVEL }\end{array} & \text { DATE } & \begin{array}{c}\text { WATER } \\ \text { LEVEL }\end{array} \\ \text { Dec 04 } & 57.30 & \text { May 16 } & 56.84 & \text { Ju1 } 22 & 56.24 & \text { Sep 17 } & 55.93\end{array}$


GROUND-WATER LEVELS

WATONWAN COUNTY

440037094372601 . Local number, 106N32W01DDB01.

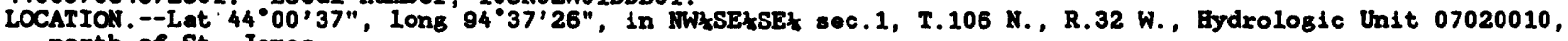
north of St. James.

Ower: U.S. Geological Survey.

AQUIFER. --Surficial outwash sand and gravel of Pleistocene Age.

WELI CHARACTERISTICS.--Bored observation water-table well, diameter $2 \mathrm{in}$, depth $22 \mathrm{ft}$, screened 19 to $22 \mathrm{ft}$.

DATUM. - -Altitude of land-surface datum is 1,056.2 et National Geodetic Vertical Datum of 1929 . Measuring point: Top of casing, $4.80 \mathrm{ft}$ above land-surface datum.

FERIOD OF RECORD. - November 1965 to current year.

EXTREMES FOR PERIOD OF RECORD.--Highest water level, 4.11 ft below land-surface datum, Apr. 27, 1969;

lowest, $16.22 \mathrm{ft}$ below land-surface datum, Mar. 7 , 1990 .

WATER LEVEL, IN FEET BELOW LAND-SURFACE DATUM, WATER YEAR OCTOBER 1990 TO SEPTEMBER 1991

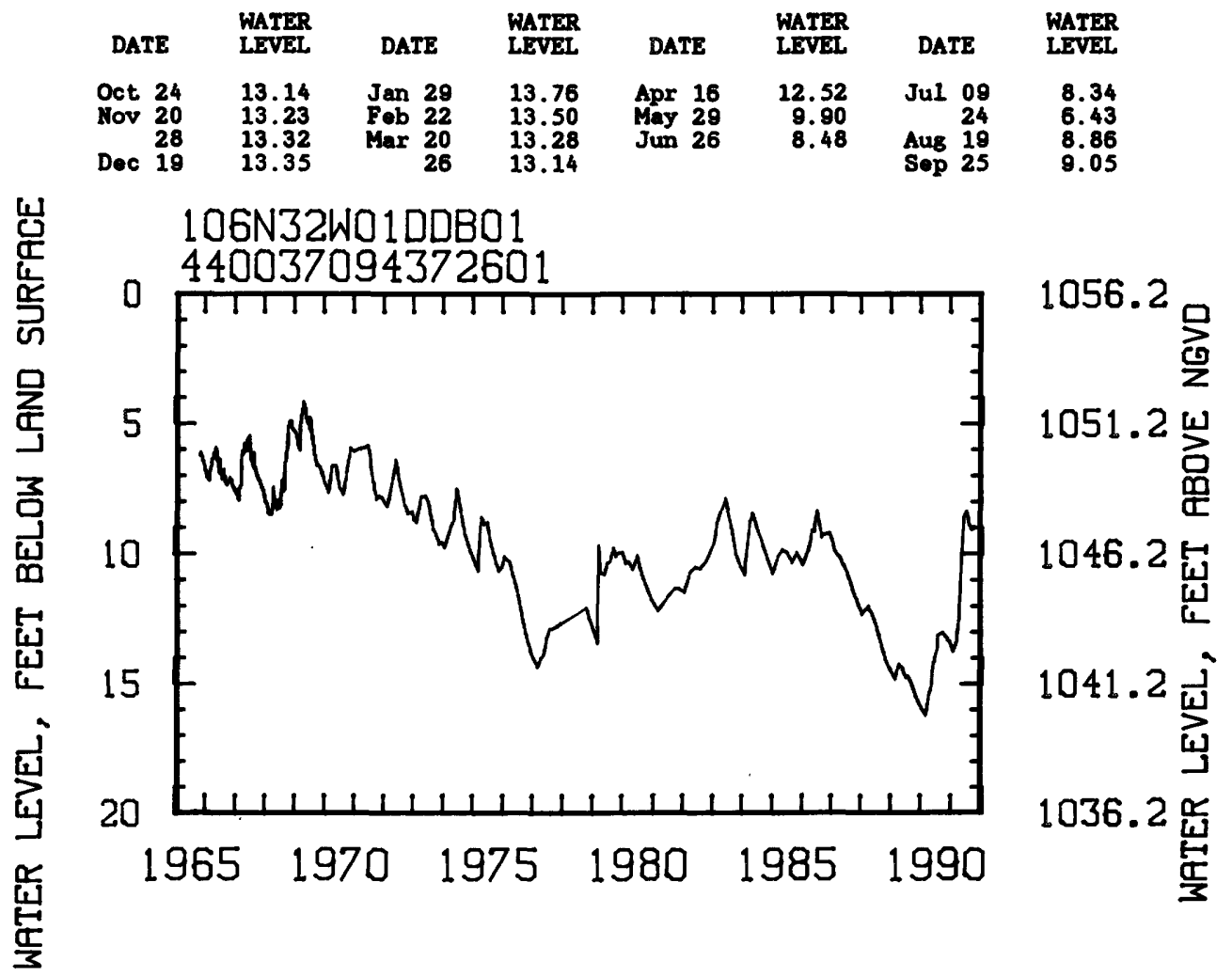

440409094304901. Local number, 107N31W14DAC01.

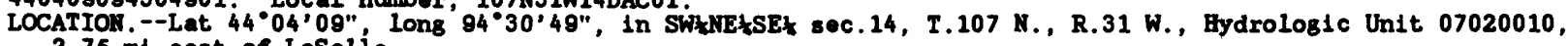
$2.75 \mathrm{mi}$ east of Lasalie.

Owner: William Lassas.

AQUIFER.--Sandstone of Cretaceous Ase.

WELL CHARACTERISTICS.--Drilled 1rrigation artesian well, diameter $12 \mathrm{ln}$., depth $150 \mathrm{ft}$, screened 100 to $135 \mathrm{ft}$

DATUM. --Altitude of land-surface datum is 1,008 ft. Measuring point: Vent pipe, $1.80 \mathrm{ft}$ above landsurface datum.

PERIOD OF RECORD. --Sept ember 1981 to current year.

EXTREMES FOR FERIOD OF RECORD. --Highest mater level, $10.44 \mathrm{ft}$ below land-surface datum, May 9, 1983 ;

lowest, 16.29 ft below land-surface datum, July 12, 1988.

WATER LEVEL, IN FEET BELOW LAND-SURFACE DATUM, WATER YEAR OCTOBER 1990 TO SEPTEMBER 1991

$\begin{array}{cccccc}\text { DATE } & \begin{array}{c}\text { WATER } \\ \text { LEVEL }\end{array} & \text { DATE } & \begin{array}{c}\text { WATER } \\ \text { LEVEL }\end{array} & \text { DATE } & \begin{array}{c}\text { WATER } \\ \text { LEVEI }\end{array} \\ \text { Mov 20 } & 13.56 & \text { May 29 } & 11.02 & \text { Sep 25 } & 11.88 \\ \text { Mar 26 } & 13.07 & \text { Jul 24 } & 11.71 & & \end{array}$


GROUND-WATER LEVELS

WATONWAN COUNTY--Continued

440133094312501. Local number, 107N31W35CAC01.

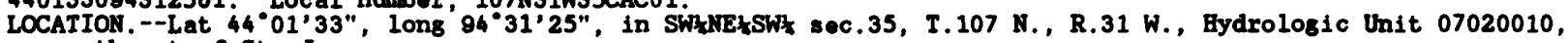
northeast of St. James.

Omer: Al Guyer.

AQUIFER. - Mount Simon Sands tone of Late Cambrian Age.

WELL CHARACTERISTICS.--Drilled irrigation artesian we1l, diameter $10 \mathrm{in.,}$ depth $350 \mathrm{ft}$, screened 310 to $350 \mathrm{ft}$.

DArus.--Altitude of land-surface datum is 1,055 ft. Measuring point: Vent pipe, $1.00 \mathrm{ft}$ above landsurface datum.

PERIOD OF RECORD.--September 1981 to current year.

EXTRDAS FOR PERIOD OF RECORD. --Highest water level, 29.83 ft below land-surface datum, May 9, 1983 ; lowest, $41.64 \mathrm{ft}$ below land-surface datum, July 12,1988 .

WATER LEVEL, IN FEET BELOW LAND-SURFACE DATUM, WATER YEAR OCTOBER 1990 TO SEPTEMBER 1991

$\begin{array}{cccccc}\text { DATE } & \begin{array}{c}\text { WATER } \\ \text { LEVEL }\end{array} & \text { DATE } & \begin{array}{c}\text { WATER } \\ \text { LEVEL }\end{array} & \text { DATE } & \begin{array}{c}\text { WATER } \\ \text { LEVEL }\end{array} \\ \text { Nov 20 } & \mathbf{3 3 . 9 3} & \text { May 29 } & 31.93 & \text { Søp 25 } & 32.12 \\ \text { Max 26 } & 33.72 & \text { Jul 24 } & 31.95 & & \end{array}$

WINONA COUNTY

435746092034202. Local number, 106N10W19DDA02.

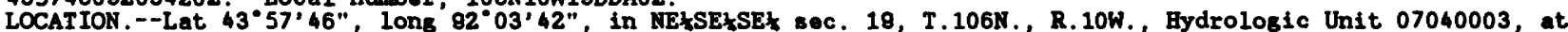
St. Charles.

Omer: City of St. Charles, Well 5.

AQUIFER. --Ironton-Galesville Sandstones of Late Cambrian Age

WELL CHARACTERISTICS, --Drilled public supply artesian well, diameter $12 \mathrm{in}$, dopth $702 \mathrm{ft}$, cased to $645 \mathrm{ft}$.

DATUM.--Altitude of land-surface datum is 1,160 ft; Measuring point: Edge of vent pipe, $1.00 \mathrm{ft}$ above landsurface datum.

REMARKS. - Water level affected by pumping.

PERIOD OF RECORD. - May 1984 to current year.

EXTREMES FOR PERIOD OF RECORD.--Highest water level, $218.80 \mathrm{ft}$ below land-surface datum, Aug. 26, 1987; 10west, 269.10 ft below land-surface datum, Oct. $28,1988$.

WATER LEVEL, IN FEET BELOW LAND SURFACE DATUM, WATER YEAR OCTOBER 1990 TO SEPTEMBER 1991

\begin{tabular}{|c|c|c|c|c|c|c|c|c|c|c|c|c|c|c|}
\hline DATE & $\begin{array}{l}\text { WATER } \\
\text { LEVEL }\end{array}$ & DAT & & $\begin{array}{l}\text { WATER } \\
\text { LEVEL }\end{array}$ & DATI & & $\begin{array}{l}\text { WATER } \\
\text { LEVEI }\end{array}$ & DATE & $\begin{array}{l}\text { WATER } \\
\text { LEVEL }\end{array}$ & DAT & & $\begin{array}{l}\text { WATERR } \\
\text { LEVEL }\end{array}$ & DATE & $\begin{array}{l}\text { WATER } \\
\text { LEVEL }\end{array}$ \\
\hline $\begin{aligned} & \text { Oct } 02 \\
& 15 \\
& 22 \\
& 31 \\
& 31 \\
& \text { Nov } 06 \\
& 20 \\
& 27 \\
& 27\end{aligned}$ & $\begin{array}{l}241.40 \\
238.88 \\
234.52 \\
237.22 \\
236.90 \\
236.35 \\
242.88\end{array}$ & $\begin{array}{l}\text { Dec } \\
\text { Jan }\end{array}$ & $\begin{array}{l}04 \\
11 \\
18 \\
26 \\
02 \\
10 \\
17 \\
24\end{array}$ & $\begin{array}{l}240.60 \\
241.70 \\
240.60 \\
239.20 \\
239.52 \\
239.86 \\
239.90 \\
243.30\end{array}$ & Feb & $\begin{array}{l}05 \\
14 \\
21 \\
28 \\
07 \\
13 \\
20 \\
27\end{array}$ & $\begin{array}{l}242.53 \\
257.80 \\
248.42 \\
244.05 \\
243.30 \\
241.55 \\
244.80 \\
244.20\end{array}$ & $\begin{array}{rr}\text { Apr } & 0 \\
1 \\
1 \\
1 \\
\text { May } & 0 \\
& 1\end{array}$ & $\begin{array}{l}244.80 \\
244.10 \\
245.50 \\
243.30 \\
243.80 \\
243.10\end{array}$ & $\begin{array}{l}\text { Jun } \\
\text { Jul }\end{array}$ & $\begin{array}{l}05 \\
12 \\
19 \\
25 \\
15 \\
22 \\
31\end{array}$ & $\begin{array}{l}247.88 \\
250.06 \\
249.80 \\
250.30 \\
268.14 \\
249.82 \\
254.62\end{array}$ & $\begin{aligned} & \text { Aug } 06 \\
& 13 \\
& 20 \\
& 26 \\
& 26 \\
& \text { Sep } 10 \\
& 17 \\
& 24\end{aligned}$ & $\begin{array}{l}240.52 \\
237.06 \\
240.00 \\
234.90 \\
234.12 \\
235.17 \\
235.40\end{array}$ \\
\hline
\end{tabular}

WRIGHT COUNTY

450403093544501 . Local number, 119N26W35DDA01.

LOCATION.--Lat 45.04'03", Long 93'54'45", in NEłSEłSEł sec.35, T.119 N., R.26 W., Hydrologic Unit 07010204,

at Montrose.

Owner: City of Montrose, well 1.

AQUIFER. -- Mount Simon Sandstone of Late Cambrian Age and Binckley Sandstone of Late Precambrian Age.

WELL CHARACTERISTICS, --Drilled unused artesian woli, diameter 10 in, depth $693 \mathrm{ft}$, cased to $526 \mathrm{ft}$

DATUM. - Altitude of iand-surface datum 1s 1,000 ft. Measuring point: Edge of breather pipe, $1.50 \mathrm{ft}$ above land-surface datum.

PERIOD OF RECORD. --September 1976 to current year.

EXTRENES FOR PERIOD OF RECORD. --Highest water level, 73.54 ft below land-surface datum, Sept. 28, 1981; lowest, 78.38 ft below land-surface datum, Nov. 3, 1977 .

WATER LEVEL, IN FEET BELOW LAND-SURFACE DATUM, WATER YEAR OCTOBER 1990 TO SEPTEMBER 1991

$\begin{array}{cccccc}\text { DATE } & \begin{array}{c}\text { WATER } \\ \text { LEVEL }\end{array} & \text { DATE } & \begin{array}{c}\text { WATER } \\ \text { LEVEL }\end{array} & \text { DATE } & \begin{array}{c}\text { WATER } \\ \text { LEVEL }\end{array} \\ \text { Nov 16 } & 77.80 & \text { Mar 13 } & 77.70 & \text { JuI 25 } & 77.03 \\ \text { Jan 17 } & 77.71 & \text { May 14 } & 77.21 & \text { Sop 23 } & 76.69\end{array}$


444219096165501. Local number, 114N45W04DCD01.

LOCATION.--Lat 44.42'19", long $96^{\circ} 16^{\prime} 55^{\prime \prime}$, in SEłSWłSE sec.4, T.114 N., R.45 W., Bydrologic Unit 07020003 , at Canby City Park.

Owner: City of Canby, well 6.

AOUIFER. --Surficial sand and gravel of Pleistocene Ase.

WELL CHARACTERISTICS.--Drilled unused water-table weil, diameter $12 \mathrm{in.,} \mathrm{depth} 62 \mathrm{ft}$, screened 44 to $68 \mathrm{ft}$.

DATUM.--Altitude of land-surface datum is 1,255 ft. Measuring point: Top of casing, 2.90 ft above land-surface datum.

PERIOD OF RECORD. --January 1964 to current year.

EXTREMES FOR PERIOD OF RECORD.--Highest water level, 4.67 ft below land-surface datum, June 5, 1965; lowest, $11.32 \mathrm{ft}$ below land-surface datum, Oct. 7,1976 .

WATER LEVEL, IN FEET BELOW LAND-SURFACE DATUM, WATER YEAR OCTOBER 1990 TO SEPTEMBER 1991

$\begin{array}{crcrcr}\text { DATE } & \begin{array}{r}\text { WATER } \\ \text { LEVEL }\end{array} & \text { DATE } & \begin{array}{c}\text { WATER } \\ \text { LEVEL }\end{array} & \text { DATE } & \begin{array}{r}\text { WATER } \\ \text { LEVEL }\end{array} \\ \text { Oct 23 } & \mathbf{8 . 5 2} & \text { Apr 04 } & \mathbf{7 . 2 0} & \text { Jul 12 } & \mathbf{7 . 8 0} \\ \text { Feb 21 } & \mathbf{8 . 4 6} & \text { Jun 06 } & \mathbf{4 . 8 7} & & \end{array}$




\section{Quality of Ground Water}

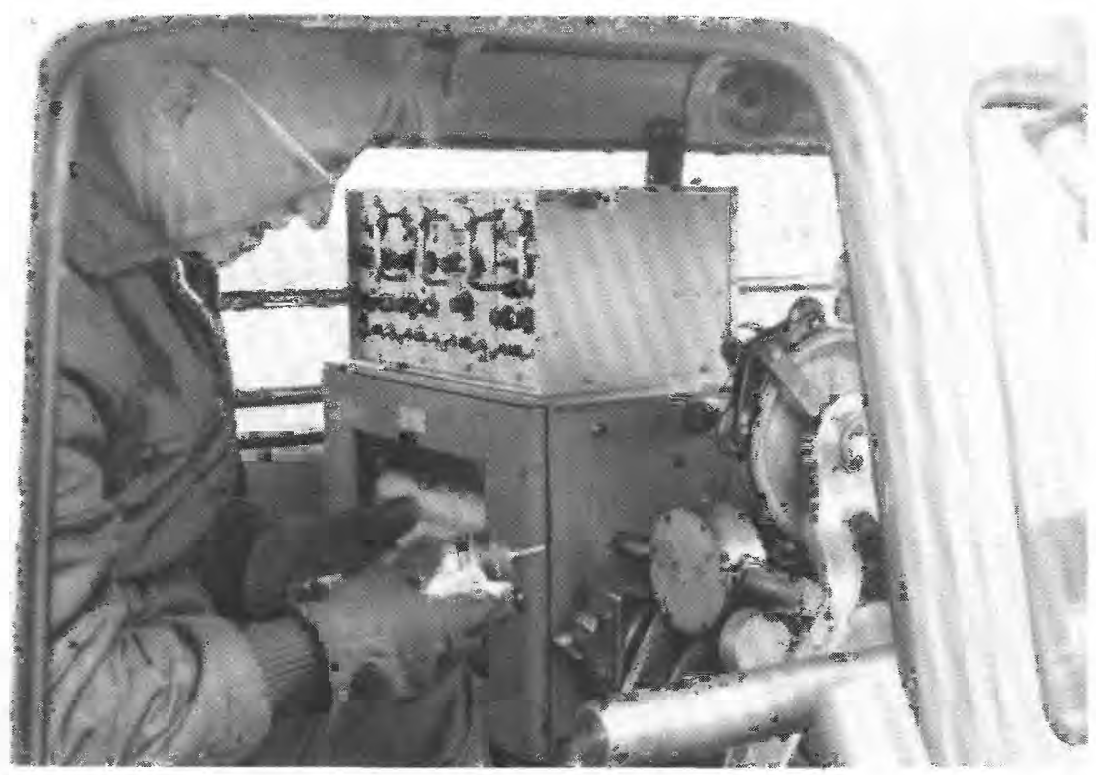

May, 1967 
QUALITY OF GROUND WATER

WATER QUALITY DATA, WATER YEAR OCTOBER 1990 TO SEPTEMBER 1991 BLUE EARTH COUNTY

\begin{tabular}{|c|c|c|c|c|c|c|}
\hline STATION & NUMBER & $\begin{array}{l}\text { LOCAL } \\
\text { IDENT- } \\
\text { I- } \\
\text { FIBR }\end{array}$ & DATE & TIME & $\begin{array}{l}\text { DEPTH } \\
\text { BELOW } \\
\text { LAND } \\
\text { SURFACE } \\
\text { (WATER } \\
\text { LEVEL) } \\
\text { (FEET) } \\
\text { (72019) }\end{array}$ & $\begin{array}{l}\text { DEPTH } \\
\text { OF } \\
\text { WELL, } \\
\text { TOTAĹ } \\
\text { (FEET) } \\
\text { (72008) }\end{array}$ \\
\hline 44090709 & 514401 & $108 \mathrm{~N}-25 W-20$ BBAAAD & $\begin{array}{l}03-25-91 \\
07-22-91\end{array}$ & $\begin{array}{l}1550 \\
1535\end{array}$ & 55.00 & $\begin{array}{l}117 \\
117\end{array}$ \\
\hline
\end{tabular}

\begin{tabular}{|c|c|c|c|c|c|c|c|c|c|c|}
\hline TATION & NUMBER & $\begin{array}{c}\text { PUMP } \\
\text { OR FLOW } \\
\text { PERIOD } \\
\text { PRIOR } \\
\text { TO SAM- } \\
\text { PLING } \\
\text { (MIN) } \\
\text { (72004) }\end{array}$ & $\begin{array}{l}\text { SPE- } \\
\text { CIFIC } \\
\text { CON- } \\
\text { DUCT- } \\
\text { ANCE } \\
\text { (US/CM) } \\
(00095)\end{array}$ & $\begin{array}{c}\text { PH } \\
\text { (STAND- } \\
\text { ARD } \\
\text { UNITS) } \\
\text { (00400) }\end{array}$ & $\begin{array}{c}\text { TEMPER- } \\
\text { ATURE } \\
\text { WATER } \\
\text { (DEG C) } \\
(00010)\end{array}$ & $\begin{array}{c}\text { OXYGEN, } \\
\text { DIS- } \\
\text { SOLVED } \\
(M G / L) \\
(00300)\end{array}$ & $\begin{array}{c}\text { NITRO- } \\
\text { GEN, } \\
\text { NITRITE } \\
\text { DIS- } \\
\text { SOLVED } \\
\text { (MG/L } \\
\text { AS NA) } \\
\text { (00613) }\end{array}$ & $\begin{array}{c}\text { MIIRO- } \\
\text { GEN, } \\
\text { NO2+NO3 } \\
\text { DIS- } \\
\text { SOLVED } \\
\text { (MG/L } \\
\text { AS N } \\
\text { (00631) }\end{array}$ & $\begin{array}{l}\text { NITRO- } \\
\text { GEN, } \\
\text { AMONIA } \\
\text { DIS- } \\
\text { SOLVED } \\
\text { (MG/L } \\
\text { AS N } \\
(00608)\end{array}$ & $\begin{array}{c}\text { PBOS- } \\
\text { PHORUS } \\
\text { ORTHO, } \\
\text { DIS- } \\
\text { SOLVED } \\
\text { (MG/L } \\
\text { AS P) } \\
(00671)\end{array}$ \\
\hline
\end{tabular}

$\begin{array}{llllllllll}440907093514401 & \overline{-} & \overline{-} & \overline{-} & \overline{-} & -\overline{0} & <0.01 & 0.06 & 1.2 & <0.01 \\ & 26 & 695 & 7.3 & 14.0 & 0.0 & <0.01 & <0.05 & 0.99 & <0.01\end{array}$


QUALITY OF GROUND WATER

WATER QUALITY DATA, WATER YEAR OCTOBER 1990 TO SEPTEMBER 1991

DAKOTA COUNTY

\begin{abstract}
LOCAL

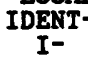

STATION NUMBER

FIER

443422093064701 113N19W21CCDCAC UN207502 S 443422093064901 113H19W21CCCDAD 207503 ors 443449093060401 113N19W21ADCCCA 207699 BON 443511092594201 113N18W20AAAAB $426961 \mathrm{COO}$ 443528092590701 113N18W16CADDBA 179135 IST

443603093020301 113N18W18BBBBCD 104113 POM 443607092565601 113N18W11CCD 1 PEINE WELL 443748093035901 113N19W02BAAABB UN418684 B 443748093085001 113N19W6BAA1 DAKOTA CO. $443848093381501114 \mathbb{N}-19 W-30$ DDDABC
\end{abstract}

443856092572101 114M18W27DAD1 STEVEN REC 443909093020401 114N18W30BCCCCB 196606 PIL $443915093081102114 N 019 W$ - SHALLOW WELL AT

$443915093081103114 N 019$ - DEEP WELL AT F

443924093010001 114N18W30AADBCB 101038 HAR

443926093010001 114N18W30AADBAA UN216439 G 443927093010101 114N18W30AAACCC 236603 KAF 443930093004801 114N18W29BBBADD 194283 NAP 443932093031601 114N19W25BBBBBD 198342 HAW 443933092533501 114N17W2OCCCCBD UN435233 P

$443933093002802114 N 018 W$ - SITE 3 SHALLOW

443933093002803 114N018W - SITE 3 DEEP WEL

443936092533701 114N17W20CCCBCC $240142 \mathrm{BAU}$ 443939092533801 114N17W19DDADDD UN207656 B $44400009303170211411019 W$ - SITE 2 SHALLOW

$44400009303170311421019 W-$ SITE 2 DEEP WEL 444024092501901 114N17W15DCD1 PAUL MABER

444025092501201 114N17W15DDCDCB UN170898 M 444026092511701 114N17W16DDDCDC 145881 LAT 444038092511401 114N17W14DADDAB $104125 \mathrm{MOL}$ $44404909302040211411018 W$ - SITE 4 SHALLOW

444049093020403 114N018W - SITE 4 DEEP WE

444056092522101 114N17W18BCC1 CBARLES BA

444058092461901 114N16W17BCB1 PAT SAGER

444109092581701 114N18W15BBDBBB $121076 \mathrm{LAR}$ 371JRDN $444147092491601114 N-17 W-11$ ACCACB

444147093182001 114N21W11ACC1 KROOK WELL

$$
\text { LEO- }
$$

367 PRDC 367 PRDC 371 RRDI 371JRDN 367PRDC

367PRDC 371JRDN

371JRDN

112PLSC 112PLSC

112PLSC

112PLSC

112PLSC

112PLSC

367PRDC

367PRDC

371 JRD

367PRDC

371 JRDN

112PLSC

112PLSC

112PLSC

112PLSC

112PLSC

112PLSC

367PRDC

367PRDC

112PLSC

112PLSC

112PLSC

$112 P L S C$

112PLSC

112PLSC

110QRNR

371JRDN

371 JRDN

112PLSC

112PLSC

112PLSC

$112 \mathrm{PLSC}$

112PLSC

\begin{tabular}{|c|c|c|c|c|}
\hline DATE & TIME & $\begin{array}{l}\text { DEPTH } \\
\text { BELOW } \\
\text { LAND } \\
\text { SURFACE } \\
\text { (WATER } \\
\text { LEVEL) } \\
\text { (FEET) } \\
\text { (72019) }\end{array}$ & $\begin{array}{c}\text { DEPTH } \\
\text { OF } \\
\text { WELL, } \\
\text { TOTAL } \\
\text { (FEET) } \\
\text { (72008) }\end{array}$ & $\begin{array}{c}\text { PUMP } \\
\text { OR FLOW } \\
\text { PERIOD } \\
\text { PRIOR } \\
\text { TO SAM- } \\
\text { PLING } \\
\text { (MIN) } \\
(72004)\end{array}$ \\
\hline $\begin{array}{l}08-01-91 \\
08-01-91 \\
08-01-91 \\
08-09-91 \\
08-09-91\end{array}$ & $\begin{array}{l}1100 \\
1200 \\
1400 \\
1100 \\
1215\end{array}$ & $\begin{array}{l}=- \\
=- \\
=- \\
=\end{array}$ & $\begin{array}{r}90 \\
90 \\
420 \\
350 \\
240\end{array}$ & $\begin{array}{l}21 \\
36 \\
24 \\
21 \\
24\end{array}$ \\
\hline $\begin{array}{l}08-02-91 \\
05-02-91 \\
08-02-91 \\
05-22-91 \\
03-08-91\end{array}$ & $\begin{array}{l}1530 \\
1045 \\
1230 \\
1020 \\
1200\end{array}$ & $\begin{array}{l}=- \\
=- \\
=- \\
=\end{array}$ & $\begin{array}{r}190 \\
-- \\
300 \\
-- \\
60\end{array}$ & $\begin{array}{l}24 \\
-- \\
14 \\
-- \\
--\end{array}$ \\
\hline $\begin{array}{l}07-16-91 \\
05-02-91 \\
08-05-91 \\
02-05-91 \\
04-08-91\end{array}$ & $\begin{array}{l}1630 \\
1130 \\
1400 \\
1445 \\
1330\end{array}$ & $\begin{array}{l}9.00 \\
=- \\
=- \\
--\end{array}$ & $\begin{array}{r}60 \\
-- \\
257 \\
9.7 \\
9.7\end{array}$ & $\begin{array}{l}25 \\
-- \\
25 \\
-- \\
--\end{array}$ \\
\hline $\begin{array}{l}05-15-91 \\
02-05-91 \\
04-08-91 \\
05-16-91 \\
08-09-91\end{array}$ & $\begin{array}{r}1110 \\
1515 \\
1240 \\
1000 \\
--\end{array}$ & $\begin{array}{l}=- \\
=- \\
=- \\
=-\end{array}$ & $\begin{array}{c}9.7 \\
21 \\
21 \\
21 \\
128\end{array}$ & $\begin{array}{l}=- \\
=- \\
=- \\
24\end{array}$ \\
\hline $\begin{array}{l}08-02-91 \\
08-09-91 \\
08-14-91 \\
08-05-91 \\
08-07-91\end{array}$ & $\begin{array}{l}1145 \\
1345 \\
1200 \\
1315 \\
1230\end{array}$ & $\begin{array}{l}=- \\
=- \\
=- \\
-\end{array}$ & $\begin{array}{l}137 \\
147 \\
234 \\
110 \\
340\end{array}$ & $\begin{array}{l}24 \\
24 \\
35 \\
19 \\
21\end{array}$ \\
\hline $\begin{array}{l}02-05-91 \\
04-11-91 \\
05-17-91 \\
02-05-91 \\
04-11-91\end{array}$ & $\begin{array}{l}1045 \\
0950 \\
1100 \\
1105 \\
1040\end{array}$ & $\begin{array}{l}=- \\
=- \\
=- \\
=-\end{array}$ & $\begin{array}{l}21 \\
21 \\
21 \\
52 \\
52\end{array}$ & $\begin{array}{l}=- \\
=- \\
=- \\
=-\end{array}$ \\
\hline $\begin{array}{l}05-17-91 \\
08-07-91 \\
08-05-91 \\
02-05-91 \\
04-10-91\end{array}$ & $\begin{array}{l}1150 \\
1345 \\
1230 \\
1250 \\
1030\end{array}$ & $\begin{array}{l}=- \\
=- \\
=- \\
--\end{array}$ & $\begin{array}{r}52 \\
125 \\
125 \\
10 \\
10\end{array}$ & $\begin{array}{l}-- \\
26 \\
28 \\
-- \\
--\end{array}$ \\
\hline $\begin{array}{l}05-15-91 \\
02-05-91 \\
04-10-91 \\
05-16-91 \\
05-01-91\end{array}$ & $\begin{array}{l}1215 \\
1320 \\
1130 \\
1150 \\
1410\end{array}$ & $\begin{array}{l}=- \\
=- \\
-- \\
--\end{array}$ & $\begin{array}{l}10 \\
42.4 \\
42.4 \\
42.4 \\
--\end{array}$ & $\begin{array}{l}=- \\
=- \\
=- \\
--\end{array}$ \\
\hline $\begin{array}{l}07-30-91 \\
07-30-91 \\
08-14-91 \\
02-07-91 \\
04-10-91\end{array}$ & $\begin{array}{l}1430 \\
1100 \\
1000 \\
1130 \\
1245\end{array}$ & $\begin{array}{l}=- \\
=- \\
=- \\
--\end{array}$ & $\begin{array}{r}174 \\
185 \\
318 \\
11 \\
11\end{array}$ & $\begin{array}{l}21 \\
44 \\
21 \\
-- \\
--\end{array}$ \\
\hline $\begin{array}{l}05-15-91 \\
02-07-91 \\
04-10-91 \\
05-16-91 \\
05-01-91\end{array}$ & $\begin{array}{l}1325 \\
1215 \\
1310 \\
1315 \\
1310\end{array}$ & $\begin{array}{l}-- \\
=- \\
-- \\
--\end{array}$ & $\begin{array}{l}11 \\
23.7 \\
23.7 \\
23.7 \\
--\end{array}$ & $\begin{array}{l}= \\
=- \\
=- \\
--\end{array}$ \\
\hline $\begin{array}{l}05-01-91 \\
08-14-91 \\
03-11-91 \\
07-16-91 \\
05-22-91\end{array}$ & $\begin{array}{l}1145 \\
1315 \\
1135 \\
1415 \\
1355\end{array}$ & $\begin{array}{l}\overline{--} \\
\overline{--} \\
80.00\end{array}$ & $\begin{array}{l}-\overline{-} \\
235 \\
160 \\
160\end{array}$ & $\begin{array}{l}\overline{14} \\
\overline{30}\end{array}$ \\
\hline
\end{tabular}

112 PLSC 
QUALITY OF GROUND WATER

WATER QUALITY DATA, WATER YEAR̆ OCTOBER 1990 TO SEPTEMBER 1991

DAKOTA COUNTY--Continued

\begin{tabular}{|c|c|c|c|c|c|c|c|c|c|c|}
\hline STATIOM NUMBER & $\begin{array}{l}\text { SPE- } \\
\text { CIFIC } \\
\text { CON- } \\
\text { DUCT- } \\
\text { ANCE } \\
\text { (USSCM) } \\
\text { (00095) }\end{array}$ & $\begin{array}{c}\text { SPE- } \\
\text { CIFIC } \\
\text { CON- } \\
\text { DUCT- } \\
\text { ANCE } \\
\text { IAB } \\
\text { (US CM) } \\
(90095)\end{array}$ & $\begin{array}{c}\text { PH } \\
\text { (STAND- } \\
\text { ARD } \\
\text { UNITS) } \\
(00400)\end{array}$ & $\begin{array}{c}\text { PH } \\
\text { LAB } \\
\text { (STAND- } \\
\text { ARD } \\
\text { UNITS) } \\
\text { (00403) }\end{array}$ & $\begin{array}{l}\text { OXID- } \\
\text { ATION } \\
\text { RED- } \\
\text { UCTION } \\
\text { POTEN- } \\
\text { TIAI } \\
\text { (MV) } \\
(00090)\end{array}$ & $\begin{array}{l}\text { TEMPER- } \\
\text { ATURE } \\
\text { WATER } \\
\text { (DEG C) } \\
(00010)\end{array}$ & $\begin{array}{c}\text { OXYGEN, } \\
\text { DIS- } \\
\text { SOLVED } \\
\text { (MG/L) } \\
(00300)\end{array}$ & $\begin{array}{l}\text { CALCIUM } \\
\text { DIS- } \\
\text { SOLVED } \\
\text { (MG/L } \\
\text { AS CA) } \\
\text { (00915) }\end{array}$ & $\begin{array}{c}\text { MAGEE- } \\
\text { SIUM, } \\
\text { DIS- } \\
\text { SOLVED } \\
\text { CMG/L } \\
\text { AS MG) } \\
(00925)\end{array}$ & $\begin{array}{l}\text { SODIUM, } \\
\text { DIS- } \\
\text { SOLVED } \\
\text { (MG/L } \\
\text { AS NA }) \\
(00930)\end{array}$ \\
\hline $\begin{array}{l}443422093064701 \\
443422093064901 \\
443449093060401 \\
44351109254201 \\
443528092590701\end{array}$ & $\begin{array}{l}436 \\
408 \\
209 \\
420 \\
433\end{array}$ & $\begin{array}{l}551 \\
505 \\
298 \\
428 \\
449\end{array}$ & $\begin{array}{l}7.5 \\
7.6 \\
7.6 \\
7.9 \\
7.8\end{array}$ & $\begin{array}{l}7.7 \\
8.1 \\
8.0 \\
8.0 \\
8.0\end{array}$ & $\begin{array}{r}0.2 \\
0.1 \\
-0.2 \\
0.1 \\
0.1\end{array}$ & $\begin{array}{l}14.5 \\
14.0 \\
17.0 \\
12.0 \\
11.5\end{array}$ & $\begin{array}{r}6.6 \\
6.5 \\
<0.1 \\
7.0 \\
1.7\end{array}$ & $\begin{array}{l}71 \\
68 \\
59 \\
50 \\
55\end{array}$ & $\begin{array}{l}24 \\
24 \\
21 \\
21 \\
22\end{array}$ & $\begin{array}{l}4.8 \\
4.1 \\
2.8 \\
4.5 \\
4.1\end{array}$ \\
\hline $\begin{array}{l}443603093020301 \\
443607092565601 \\
443748093035901 \\
443748093085001 \\
443848093381501\end{array}$ & $\begin{array}{l}484 \\
311 \\
520 \\
501 \\
--\end{array}$ & $\begin{array}{r}545 \\
316 \\
542 \\
512 \\
--\end{array}$ & $\begin{array}{l}7.4 \\
7.8 \\
7.5 \\
7.3 \\
--\end{array}$ & $\begin{array}{r}7.6 \\
7.8 \\
7.8 \\
7.4 \\
--\end{array}$ & $\begin{array}{l}0 \\
--0.1 \\
-- \\
--\end{array}$ & $\begin{array}{l}10.5 \\
10.5 \\
11.0 \\
.10 .0 \\
-.\end{array}$ & $\begin{array}{l}0.1 \\
-0.1 \\
<-. \\
--\end{array}$ & $\begin{array}{l}66 \\
47 \\
76 \\
76 \\
-\end{array}$ & $\begin{array}{l}36 \\
12 \\
26 \\
20 \\
--\end{array}$ & $\begin{array}{l}2.3 \\
4.0 \\
5.3 \\
3.4 \\
--.\end{array}$ \\
\hline $\begin{array}{l}443856092572101 \\
44390093020401 \\
443915093081102\end{array}$ & $\begin{array}{l}690 \\
483 \\
498 \\
793 \\
875\end{array}$ & $\begin{array}{l}-- \\
-496 \\
-- \\
792 \\
905\end{array}$ & $\begin{array}{l}6.9 \\
7.7 \\
7.4 \\
7.4 \\
7.2\end{array}$ & $\begin{array}{r}7.8 \\
7.8 \\
7.5 \\
7.4\end{array}$ & $\begin{array}{l}-- \\
=- \\
-0.1 \\
--\end{array}$ & $\begin{array}{r}16.0 \\
10.0 \\
11.0 \\
8.0 \\
6.5\end{array}$ & \begin{tabular}{l}
3.2 \\
\hdashline$<0.1$ \\
-- \\
--
\end{tabular} & $\begin{array}{l}-1- \\
75 \\
73 \\
82 \\
99\end{array}$ & $\begin{array}{l}21 \\
24 \\
23 \\
29\end{array}$ & $\begin{array}{l}-- \\
4.4 \\
4.2 \\
56 \\
53\end{array}$ \\
\hline $\begin{array}{l}443915093081103 \\
443924093010001\end{array}$ & $\begin{array}{l}787 \\
601 \\
575 \\
567 \\
604\end{array}$ & $\begin{array}{l}823 \\
605 \\
592 \\
586 \\
620\end{array}$ & $\begin{array}{l}7.1 \\
7.5 \\
7.4 \\
7.3 \\
7.5\end{array}$ & $\begin{array}{l}7.5 \\
7.6 \\
7.6 \\
7.6 \\
7.6\end{array}$ & $\begin{array}{l}-- \\
=- \\
-- \\
0.1\end{array}$ & $\begin{array}{r}9.0 \\
9.5 \\
9.0 \\
9.5 \\
11.5\end{array}$ & $\begin{array}{l}-- \\
0 \\
0 \\
0.1 \\
4.6\end{array}$ & $\begin{array}{l}91 \\
79 \\
78 \\
76 \\
77\end{array}$ & $\begin{array}{l}26 \\
31 \\
30 \\
28 \\
29\end{array}$ & $\begin{array}{l}40 \\
4.8 \\
4.7 \\
3.9 \\
4.4\end{array}$ \\
\hline $\begin{array}{l}443926093010001 \\
443927093010101 \\
443930093004801 \\
443932093031601 \\
443933092533501\end{array}$ & $\begin{array}{l}587 \\
567 \\
477 \\
489 \\
517\end{array}$ & $\begin{array}{l}-- \\
592 \\
462 \\
503 \\
530\end{array}$ & $\begin{array}{l}7.5 \\
7.5 \\
7.5 \\
7.6 \\
7.7\end{array}$ & $\begin{array}{r}-7 \\
7.7 \\
7.8 \\
7.8 \\
7.9\end{array}$ & $\begin{array}{r}0.2 \\
0.1 \\
-0.1 \\
-0.1 \\
0.2\end{array}$ & $\begin{array}{l}12.0 \\
11.0 \\
11.5 \\
10.5 \\
12.5\end{array}$ & $\begin{array}{r}4.3 \\
4.7 \\
<0.1 \\
<0.1 \\
4.4\end{array}$ & $\begin{array}{l}75 \\
64 \\
71 \\
65\end{array}$ & $\begin{array}{l}28 \\
23 \\
24 \\
27\end{array}$ & $\begin{array}{l}-- \\
4.4 \\
4.2 \\
5.0 \\
3.3\end{array}$ \\
\hline $\begin{array}{l}443933093002802 \\
443933093002803\end{array}$ & $\begin{array}{l}585 \\
568 \\
543 \\
504 \\
491\end{array}$ & $\begin{array}{l}595 \\
593 \\
586 \\
514 \\
509\end{array}$ & $\begin{array}{l}7.5 \\
7.4 \\
7.3 \\
7.7 \\
7.5\end{array}$ & $\begin{array}{l}7.6 \\
7.6 \\
7.7 \\
7.7 \\
7.7\end{array}$ & $\begin{array}{l}=- \\
=- \\
=-\end{array}$ & $\begin{array}{l}7.0 \\
5.5 \\
6.5 \\
9.0 \\
8.5\end{array}$ & $\begin{array}{r}0 \\
<0.1 \\
0.3 \\
0.3 \\
<0.1\end{array}$ & $\begin{array}{l}74 \\
78 \\
75 \\
68 \\
69\end{array}$ & $\begin{array}{l}27 \\
28 \\
29 \\
24 \\
24\end{array}$ & $\begin{array}{l}3.9 \\
4.0 \\
4.2 \\
4.6 \\
4.6\end{array}$ \\
\hline $\begin{array}{l}443936092533701 \\
443939092533801 \\
444000093031702\end{array}$ & $\begin{array}{r}473 \\
1400 \\
865 \\
707 \\
705\end{array}$ & $\begin{array}{r}502 \\
1440 \\
864 \\
712 \\
733\end{array}$ & $\begin{array}{l}7.4 \\
7.3 \\
7.2 \\
7.5 \\
7.2\end{array}$ & $\begin{array}{l}7.7 \\
7.7 \\
7.4 \\
7.5 \\
7.5\end{array}$ & $\begin{array}{l}-- \\
0.2 \\
0.2 \\
--\end{array}$ & $\begin{array}{r}9.0 \\
13.0 \\
11.5 \\
7.5 \\
6.0\end{array}$ & $\begin{array}{r}0.1 \\
11.0 \\
9.2 \\
2.5 \\
0.8\end{array}$ & $\begin{array}{r}67 \\
140 \\
110 \\
95 \\
100\end{array}$ & $\begin{array}{l}25 \\
45 \\
41 \\
29 \\
32\end{array}$ & $\begin{array}{c}4.8 \\
79 \\
12 \\
4.7 \\
5.2\end{array}$ \\
\hline $\begin{array}{l}444000093031703 \\
444024092501901\end{array}$ & $\begin{array}{l}749 \\
506 \\
490 \\
480 \\
626\end{array}$ & $\begin{array}{l}770 \\
518 \\
515 \\
507 \\
652\end{array}$ & $\begin{array}{l}7.1 \\
7.7 \\
7.6 \\
7.5 \\
7.5\end{array}$ & $\begin{array}{l}7.4 \\
7.7 \\
7.7 \\
7.7 \\
7.7\end{array}$ & $\begin{array}{l}=- \\
=- \\
=- \\
--\end{array}$ & $\begin{array}{r}7.5 \\
9.0 \\
8.5 \\
10.0 \\
9.5\end{array}$ & $\begin{array}{l}0.4 \\
0 \\
0.6 \\
0.1 \\
--\end{array}$ & $\begin{array}{r}110 \\
66 \\
68 \\
68 \\
79\end{array}$ & $\begin{array}{l}32 \\
27 \\
26 \\
23 \\
32\end{array}$ & $\begin{array}{l}4.9 \\
5.0 \\
5.0 \\
4.9 \\
4.0\end{array}$ \\
\hline $\begin{array}{l}444025092501201 \\
444026092511701 \\
44038092511401 \\
444049093020402\end{array}$ & $\begin{array}{l}454 \\
435 \\
567 \\
538 \\
617\end{array}$ & $\begin{array}{l}-- \\
511 \\
551 \\
542 \\
648\end{array}$ & $\begin{array}{l}7.5 \\
7.5 \\
7.8 \\
7.2 \\
7.0\end{array}$ & $\begin{array}{l}-7.7 \\
7.9 \\
7.2 \\
7.2\end{array}$ & $\begin{array}{l}0.2 \\
0.3 \\
0.2 \\
-- \\
--\end{array}$ & $\begin{array}{r}12.0 \\
12.0 \\
10.5 \\
6.5 \\
7.0\end{array}$ & $\begin{array}{l}9.3 \\
8.1 \\
8.8 \\
-- \\
--\end{array}$ & $\begin{array}{l}71 \\
71 \\
68 \\
58 \\
66\end{array}$ & $\begin{array}{l}31 \\
28 \\
20 \\
23\end{array}$ & $\begin{array}{l}-- \\
3.7 \\
3.5 \\
20 \\
28\end{array}$ \\
\hline 444056092522101 & $\begin{array}{l}652 \\
395 \\
367 \\
370 \\
558\end{array}$ & $\begin{array}{l}671 \\
398 \\
386 \\
368 \\
579\end{array}$ & $\begin{array}{l}6.9 \\
7.9 \\
7.7 \\
7.6 \\
7.6\end{array}$ & $\begin{array}{l}7.3 \\
7.8 \\
7.9 \\
7.8 \\
7.7\end{array}$ & $\begin{array}{l}=- \\
=- \\
=- \\
=\end{array}$ & $\begin{array}{r}8.5 \\
9.0 \\
9.0 \\
9.0 \\
10.5\end{array}$ & $\begin{array}{l}-\overline{8.0} \\
-8.0\end{array}$ & $\begin{array}{l}68 \\
50 \\
49 \\
48 \\
67\end{array}$ & $\begin{array}{l}22 \\
18 \\
18 \\
16 \\
28\end{array}$ & $\begin{array}{l}30 \\
2.9 \\
3.0 \\
2.9 \\
3.5\end{array}$ \\
\hline $\begin{array}{l}444058092461901 \\
44409092581701 \\
444147092491601 \\
444147093182001\end{array}$ & $\begin{array}{r}359 \\
489 \\
-373 \\
570\end{array}$ & $\begin{array}{l}365 \\
477 \\
-- \\
-597\end{array}$ & $\begin{array}{r}7.8 \\
7.7 \\
-7.5 \\
7.5\end{array}$ & \begin{tabular}{l}
8.0 \\
8.0 \\
\hdashline- \\
7.7
\end{tabular} & $\begin{array}{l}-- \\
0.1 \\
-- \\
--\end{array}$ & $\begin{array}{l}10.0 \\
10.0 \\
16.0 \\
11.0\end{array}$ & $\begin{array}{l}-- \\
-- \\
-\overline{7.6}\end{array}$ & $\begin{array}{c}49 \\
60 \\
-- \\
75\end{array}$ & $\begin{array}{c}14 \\
25 \\
-- \\
31\end{array}$ & $\begin{array}{l}2.1 \\
3.2 \\
-- \\
-- \\
5.7\end{array}$ \\
\hline
\end{tabular}


QUALITY OF GROUND WATER

WATER QUALITY DATA, WATER YEAR OCTOBER 1990 TO SEPTEMBER 1991

DAKOTA COUNTY--Continued

\begin{tabular}{|c|c|c|c|c|c|c|c|c|c|c|}
\hline STATION NUMBER & $\begin{array}{l}\text { POTAS- } \\
\text { SIUM, } \\
\text { DIS- } \\
\text { SOLVED } \\
\text { (MG/L } \\
\text { (SS K) } \\
(00935)\end{array}$ & $\begin{array}{c}\text { BICAR- } \\
\text { BONAAE } \\
\text { WATER } \\
\text { WIIIT } \\
\text { FIELD } \\
\text { MG/L AS } \\
\text { HCO3 } \\
(00450)\end{array}$ & $\begin{array}{c}\text { CAR- } \\
\text { BONATE } \\
\text { WATER } \\
\text { WEIT } \\
\text { FIILD } \\
\text { MG/L AS } \\
\text { COO3 } \\
(00447)\end{array}$ & $\begin{array}{l}\text { AIKA- } \\
\text { IIKITY } \\
\text { WAT WH } \\
\text { TOT II } \\
\text { FIIID } \\
\text { MG/L AS } \\
\text { CACO3 } \\
\text { (O0419) }\end{array}$ & $\begin{array}{l}\text { ALKA- } \\
\text { LINITY } \\
\text { WAT WH } \\
\text { TOT FET } \\
\text { FIED } \\
\text { MG/LAS } \\
\text { CACOB } \\
(00410)\end{array}$ & $\begin{array}{c}\text { ALKA- } \\
\text { LINITY } \\
\text { IAB } \\
\text { (MAG/L } \\
\text { AS } \\
\text { CACO3) } \\
\text { (90410) }\end{array}$ & $\begin{array}{c}\text { SULFATE } \\
\text { DIS- } \\
\text { SOLVED } \\
\text { (MG/L } \\
\text { AS SO4) } \\
(00945)\end{array}$ & $\begin{array}{l}\text { CHLO- } \\
\text { RIDE, } \\
\text { DIS- } \\
\text { SOLVED } \\
\text { (MG/L } \\
\text { AS CL) } \\
(00940)\end{array}$ & $\begin{array}{l}\text { FLUO- } \\
\text { RIDE, } \\
\text { DIS- } \\
\text { SOLVED } \\
\text { (MG/L } \\
\text { AS F) } \\
(00950)\end{array}$ & $\begin{array}{l}\text { SILICA, } \\
\text { DIS-, } \\
\text { SOLVED } \\
\text { (MG/L } \\
\text { AS } \\
\text { SIO2) } \\
\text { (00955) }\end{array}$ \\
\hline $\begin{array}{l}443422093064701 \\
443422093064901 \\
44344909060401 \\
44351092594201 \\
443528092590701\end{array}$ & $\begin{array}{l}1.0 \\
1.0 \\
0.9 \\
2.5 \\
0.9\end{array}$ & $\begin{array}{l}246 \\
222 \\
232 \\
184 \\
165\end{array}$ & $\begin{array}{l}-- \\
=- \\
=- \\
--\end{array}$ & $\begin{array}{l}204 \\
183 \\
191 \\
152 \\
135\end{array}$ & $\begin{array}{l}202 \\
182 \\
190 \\
151 \\
135\end{array}$ & $\begin{array}{l}197 \\
195 \\
120 \\
155 \\
142\end{array}$ & $\begin{array}{l}33 \\
32 \\
44 \\
23 \\
61\end{array}$ & $\begin{array}{l}19 \\
14 \\
0.9 \\
13 \\
15\end{array}$ & $\begin{array}{l}0.2 \\
0.2 \\
0.2 \\
0.1 \\
0.2\end{array}$ & $\begin{array}{l}15 \\
14 \\
12 \\
11 \\
11\end{array}$ \\
\hline $\begin{array}{l}443603093020301 \\
443607092565601 \\
443748093035901 \\
44374093085001 \\
443848093381501\end{array}$ & $\begin{array}{l}1.3 \\
0.5 \\
1.5 \\
2.3 \\
--\end{array}$ & $\begin{array}{l}322 \\
-346 \\
-- \\
--\end{array}$ & $\begin{array}{l}-- \\
-- \\
-- \\
--\end{array}$ & $\begin{array}{l}268 \\
-2 \\
-- \\
--\end{array}$ & $\begin{array}{l}264 \\
-284 \\
-- \\
--\end{array}$ & $\begin{array}{l}264 \\
153 \\
249 \\
247 \\
--\end{array}$ & $\begin{array}{l}36 \\
18 \\
26 \\
11 \\
--\end{array}$ & $\begin{array}{c}0.6 \\
2.4 \\
1.1 \\
18 \\
--\end{array}$ & $\begin{array}{l}0.2 \\
0.2 \\
0.2 \\
0.1 \\
-.\end{array}$ & $\begin{array}{r}11 \\
15 \\
13 \\
15 \\
--\end{array}$ \\
\hline $\begin{array}{l}443856092572101 \\
443909093020401 \\
443915093081102\end{array}$ & $\begin{array}{l}-- \\
0.7 \\
1.4 \\
5.6 \\
4.7\end{array}$ & $\begin{array}{l}-- \\
-- \\
-- \\
--\end{array}$ & $\begin{array}{l}=- \\
=- \\
=- \\
=\end{array}$ & $\begin{array}{l}-- \\
-- \\
-- \\
--\end{array}$ & $\begin{array}{l}-- \\
-- \\
-- \\
--\end{array}$ & $\begin{array}{l}165^{--} \\
291 \\
311\end{array}$ & $\begin{array}{l}24 \\
21 \\
70 \\
65\end{array}$ & $\begin{array}{l}14 \\
0- \\
0.1 \\
45 \\
80\end{array}$ & $\begin{array}{l}-- \\
0.1 \\
0.2 \\
0.2 \\
0.1\end{array}$ & $\begin{array}{l}16 \\
16 \\
13 \\
18 \\
18\end{array}$ \\
\hline $\begin{array}{l}443915093081103 \\
443924093010001\end{array}$ & $\begin{array}{l}3.9 \\
1.7 \\
1.7 \\
1.4 \\
1.1\end{array}$ & $\begin{array}{l}=- \\
=- \\
=- \\
285\end{array}$ & $\begin{array}{l}=- \\
=- \\
=- \\
=\end{array}$ & $\begin{array}{l}-- \\
-- \\
-- \\
-- \\
235\end{array}$ & $\begin{array}{l}-- \\
=- \\
-- \\
234\end{array}$ & $\begin{array}{l}294 \\
265 \\
264 \\
265 \\
241\end{array}$ & $\begin{array}{l}67 \\
58 \\
61 \\
53 \\
27\end{array}$ & $\begin{array}{l}63 \\
12 \\
12 \\
10 \\
15\end{array}$ & $\begin{array}{l}0.2 \\
0.2 \\
0.2 \\
0.1 \\
0.2\end{array}$ & $\begin{array}{l}18 \\
26 \\
25 \\
24 \\
14\end{array}$ \\
\hline $\begin{array}{l}443926093010001 \\
443267093010101 \\
443930093004801 \\
443932093031601 \\
443933092533501\end{array}$ & $\begin{array}{l}-- \\
1.1 \\
1.3 \\
1.4 \\
0.9\end{array}$ & $\begin{array}{l}-- \\
292 \\
278 \\
327 \\
235\end{array}$ & $\begin{array}{l}=- \\
=- \\
=- \\
--\end{array}$ & $\begin{array}{l}-- \\
239 \\
225 \\
272 \\
196\end{array}$ & $\begin{array}{l}- \\
-239 \\
228 \\
268 \\
193\end{array}$ & $\begin{array}{l}241^{--} \\
209 \\
264 \\
201\end{array}$ & $\begin{array}{l}28 \\
17 \\
15 \\
24\end{array}$ & $\begin{array}{c}12 \\
13 \\
0.9 \\
1.4 \\
11\end{array}$ & $\begin{array}{r}-- \\
0.1 \\
0.1 \\
0.2 \\
<0.1\end{array}$ & $\begin{array}{l}14 \\
14 \\
13 \\
21 \\
13\end{array}$ \\
\hline 443933093002802 & $\begin{array}{l}1.2 \\
1.1 \\
1.2 \\
1.4 \\
1.4\end{array}$ & $\begin{array}{l}-- \\
=- \\
=-\end{array}$ & $\begin{array}{l}-- \\
=- \\
=- \\
--\end{array}$ & $\begin{array}{l}=- \\
=- \\
--\end{array}$ & $\begin{array}{l}=- \\
=- \\
=-\end{array}$ & $\begin{array}{l}242 \\
247 \\
241 \\
269 \\
268\end{array}$ & $\begin{array}{l}28 \\
28 \\
23 \\
22 \\
18\end{array}$ & $\begin{array}{l}14 \\
15 \\
17 \\
3.0 \\
3.2\end{array}$ & $\begin{array}{l}<0.1 \\
0.2 \\
0.2 \\
0.1 \\
0.2\end{array}$ & $\begin{array}{l}14 \\
13 \\
13 \\
20 \\
20\end{array}$ \\
\hline $\begin{array}{l}443936092533701 \\
443939092533801 \\
444000093031702\end{array}$ & $\begin{array}{r}1.4 \\
16 \\
2.6 \\
1.0 \\
0.9\end{array}$ & $\begin{array}{l}-- \\
376 \\
303 \\
-- \\
--\end{array}$ & $\begin{array}{l}=- \\
=- \\
=- \\
--\end{array}$ & $\begin{array}{l}-- \\
308 \\
250 \\
-- \\
--\end{array}$ & $\begin{array}{l}-- \\
308 \\
248 \\
-- \\
--\end{array}$ & $\begin{array}{l}268 \\
316 \\
257 \\
213 \\
231\end{array}$ & $\begin{array}{l}17 \\
34 \\
37 \\
33 \\
85\end{array}$ & $\begin{array}{c}0.4 \\
160 \\
50 \\
14 \\
17\end{array}$ & $\begin{array}{r}0.2 \\
0.1 \\
0.1 \\
<0.1 \\
0.1\end{array}$ & $\begin{array}{l}18 \\
18 \\
15 \\
21 \\
20\end{array}$ \\
\hline 444024092501901 & $\begin{array}{l}0.8 \\
2.0 \\
1.9 \\
1.6 \\
0.7\end{array}$ & $\begin{array}{l}=- \\
=- \\
=-\end{array}$ & $\begin{array}{l}=- \\
=- \\
=- \\
=-\end{array}$ & $\begin{array}{l}-- \\
=- \\
-- \\
--\end{array}$ & $\begin{array}{l}=- \\
=- \\
=- \\
=-\end{array}$ & $\begin{array}{l}280 \\
281 \\
280 \\
280 \\
230\end{array}$ & $\begin{array}{c}100 \\
16 \\
11 \\
9.7 \\
29\end{array}$ & $\begin{array}{c}16 \\
3.0 \\
2.3 \\
1.9 \\
19\end{array}$ & $\begin{array}{r}<0.1 \\
0.1 \\
0.2 \\
0.2 \\
0.1\end{array}$ & $\begin{array}{l}20 \\
25 \\
26 \\
24 \\
17\end{array}$ \\
\hline $\begin{array}{l}444025092501201 \\
444026092511701 \\
444038092511401 \\
444049093020402\end{array}$ & $\begin{array}{l}-- \\
0.9 \\
1.2 \\
3.0 \\
3.2\end{array}$ & $\begin{array}{l}-- \\
252 \\
242 \\
-- \\
--\end{array}$ & $\begin{array}{l}=- \\
=- \\
=- \\
--\end{array}$ & $\begin{array}{l}-- \\
203 \\
198 \\
-- \\
--\end{array}$ & $\begin{array}{l}-- \\
206 \\
198 \\
-- \\
--\end{array}$ & $\begin{array}{l}210^{--} \\
191 \\
183 \\
190\end{array}$ & $\begin{array}{l}23 \\
57 \\
24 \\
24\end{array}$ & $\begin{array}{l}16 \\
13 \\
14 \\
24 \\
67\end{array}$ & $\begin{array}{r}-- \\
0.1 \\
0.1 \\
<0.1 \\
0.1\end{array}$ & $\begin{array}{l}-- \\
15 \\
11 \\
14 \\
14\end{array}$ \\
\hline 444056092522101 & $\begin{array}{l}3.1 \\
0.9 \\
0.9 \\
0.7 \\
0.8\end{array}$ & $\begin{array}{l}=- \\
=- \\
=- \\
--\end{array}$ & $\begin{array}{l}-- \\
=- \\
=- \\
--\end{array}$ & $\begin{array}{l}=- \\
=- \\
=- \\
--\end{array}$ & $\begin{array}{l}=- \\
=- \\
=- \\
--\end{array}$ & $\begin{array}{l}174 \\
167 \\
164 \\
160 \\
199\end{array}$ & $\begin{array}{l}24 \\
24 \\
22 \\
19 \\
22\end{array}$ & $\begin{array}{r}87 \\
4.9 \\
5.3 \\
3.8 \\
20\end{array}$ & $\begin{array}{c}<0.1 \\
0.1 \\
0.2 \\
0.1 \\
0.2\end{array}$ & $\begin{array}{l}13 \\
17 \\
18 \\
17 \\
14\end{array}$ \\
\hline $\begin{array}{l}444058092461901 \\
444109092581701 \\
444147092491601\end{array}$ & $\begin{array}{l}0.7 \\
3.7 \\
-.\end{array}$ & $--\overline{270}$ & $\begin{array}{l}=- \\
=-\end{array}$ & $--\overline{221}$ & $\begin{array}{l}-- \\
--\end{array}$ & $\begin{array}{l}132 \\
227\end{array}$ & $\begin{array}{l}14 \\
11\end{array}$ & $\begin{array}{l}8.9 \\
6.0 \\
--\end{array}$ & $\begin{array}{l}0.1 \\
0.2\end{array}$ & $\begin{array}{l}17 \\
14\end{array}$ \\
\hline 09318200 & 1.9 & -- & -- & -- & -- & 307 & 14 & 14 & 0.2 & 24 \\
\hline
\end{tabular}


QUALITY OF GROUND WATER

WATER QUALITY DATA, WATER YEAR OCTOBER 1990 TO SEPTEMBER 1991

DAKOTA COUNTY--Continued

\begin{tabular}{|c|c|c|c|c|c|c|c|c|c|c|}
\hline STATION NUMBER & $\begin{array}{c}\text { SOLIDS, } \\
\text { RESIDUE } \\
\text { AT } 180 \\
\text { DEG. C } \\
\text { DIS- } \\
\text { SOLVED } \\
\text { (MG/L) } \\
(70300)\end{array}$ & $\begin{array}{l}\text { MITRO- } \\
\text { GEN, } \\
\text { NITRITE } \\
\text { DIS- } \\
\text { SOIVED } \\
\text { (MG/L } \\
\text { AS N N } \\
(00613)\end{array}$ & $\begin{array}{c}\text { NITRO- } \\
\text { GEN, } \\
\text { NO2+NO3 } \\
\text { DIS- } \\
\text { SOLVED } \\
\text { (MG/L } \\
\text { AS N) } \\
(00631)\end{array}$ & $\begin{array}{c}\text { NITRO- } \\
\text { GEN, } \\
\text { AMONIA } \\
\text { DIS- } \\
\text { SIVIVD } \\
\text { CMG/L } \\
\text { AS N } \\
\text { A00608) } \\
(00608)\end{array}$ & $\begin{array}{l}\text { NITRO- } \\
\text { GEN,AM- } \\
\text { MONIA + } \\
\text { ORGANIC } \\
\text { DIS } \\
\text { (MG/L } \\
\text { AS N) } \\
(00623)\end{array}$ & $\begin{array}{l}\text { PHOS- } \\
\text { PEORUS } \\
\text { DIS- } \\
\text { SOLVED } \\
\text { (MG/L } \\
\text { AS P) } \\
(00666)\end{array}$ & $\begin{array}{l}\text { PHOS- } \\
\text { PBORUS } \\
\text { ORTOO, } \\
\text { DIS- } \\
\text { SOLED } \\
\text { SOG/L } \\
\text { AS P) } \\
\text { (O0671) }\end{array}$ & $\begin{array}{l}\text { IRON, } \\
\text { DIS- } \\
\text { SOLVED } \\
\text { (UG/L } \\
\text { AS FE) } \\
(01046)\end{array}$ & $\begin{array}{l}\text { MANGA- } \\
\text { NESE, } \\
\text { DIS- } \\
\text { SOLVED } \\
\text { (UG/L } \\
\text { AS MN } \\
(01056)\end{array}$ & $\begin{array}{l}\text { TRITIUM } \\
\text { IN } \\
\text { WATER } \\
\text { MOLE- } \\
\text { CULES } \\
\text { (TU) } \\
(07012)\end{array}$ \\
\hline $\begin{array}{l}443422093064701 \\
443422093064901 \\
443449093060401 \\
443511092594201 \\
443528092590701\end{array}$ & $\begin{array}{l}321 \\
327 \\
243 \\
255 \\
298\end{array}$ & $\begin{array}{l}<0.01 \\
<0.01 \\
<0.01 \\
<0.01 \\
<0.01\end{array}$ & $\begin{array}{c}8.5 \\
7.2 \\
<0.05 \\
8.1 \\
3.7\end{array}$ & $\begin{array}{l}<0.01 \\
<0.01 \\
0.03 \\
<0.01 \\
<0.01\end{array}$ & $\begin{array}{l}= \\
= \\
= \\
=\end{array}$ & $\begin{array}{r}0.02 \\
0.02 \\
<0.01 \\
<0.01 \\
<0.01\end{array}$ & $\begin{array}{l}<0.01 \\
<0.01 \\
<0.01 \\
<0.01 \\
<0.01\end{array}$ & $\begin{array}{r}10 \\
10 \\
1700 \\
7 \\
11\end{array}$ & $\begin{array}{l}<1 \\
<1 \\
23 \\
3 \\
<1\end{array}$ & $\begin{array}{l}30.1 \\
34.4 \\
1.9 \\
53.4 \\
26.4\end{array}$ \\
\hline $\begin{array}{l}443603093020301 \\
443607092565601 \\
443748093035901 \\
443748093085001 \\
443848093381501\end{array}$ & $\begin{array}{l}293 \\
-324 \\
--\end{array}$ & $\begin{array}{l}<0.01 \\
<0.01 \\
<0.01 \\
<0.01 \\
<0.01\end{array}$ & $\begin{array}{r}0.12 \\
0.05 \\
<0.05 \\
<0.05 \\
4.1\end{array}$ & $\begin{array}{r}0.01 \\
0.10 \\
0.07 \\
0.05 \\
<0.01\end{array}$ & $\begin{array}{l}<- \\
<0.20 \\
0.40\end{array}$ & $\begin{array}{r}<0.01 \\
0.04 \\
<0.01 \\
0.02 \\
--\end{array}$ & $\begin{array}{r}<0.01 \\
0.01 \\
<0.01 \\
0.02 \\
0.02\end{array}$ & $\begin{array}{l}120 \\
-- \\
2700 \\
--\end{array}$ & $\begin{array}{l}-- \\
-- \\
--\end{array}$ & $\begin{array}{l}9.9 \\
\because- \\
--\end{array}$ \\
\hline $\begin{array}{l}443856092572101 \\
443909093020401 \\
443915093081102\end{array}$ & $\begin{array}{l}-- \\
-\overline{2} \\
-- \\
--\end{array}$ & $\begin{array}{r}<0.01 \\
<0.01 \\
<0.01 \\
0.02 \\
0.01\end{array}$ & $\begin{array}{r}4.4 \\
13 \\
<0.05 \\
0.60 \\
0.12\end{array}$ & $\begin{array}{r}<0.01 \\
<0.01 \\
0.08 \\
<0.01 \\
0.07\end{array}$ & $\begin{array}{l}-- \\
0.40 \\
0.30 \\
0.20\end{array}$ & $\begin{array}{r}-- \\
0.03 \\
0.01 \\
<0.01 \\
0.02\end{array}$ & $\begin{array}{r}<0.01 \\
0.02 \\
<0.01 \\
<0.01 \\
<0.01\end{array}$ & $\begin{array}{l}-- \\
1300 \\
=-\end{array}$ & $\begin{array}{l}-- \\
--52 \\
--\end{array}$ & $\begin{array}{l}-- \\
<0.8 \\
=-\end{array}$ \\
\hline 443924093010001 & $\begin{array}{l}= \\
=- \\
\overline{346}\end{array}$ & $\begin{array}{r}0.02 \\
<0.01 \\
<0.01 \\
0.01 \\
<0.01\end{array}$ & $\begin{array}{l}0.24 \\
<0.10 \\
<0.05 \\
<0.05 \\
14\end{array}$ & $\begin{array}{r}0.09 \\
0.13 \\
0.14 \\
0.14 \\
<0.01\end{array}$ & $\begin{array}{l}0.60 \\
0.30 \\
0.40 \\
0.30 \\
. .\end{array}$ & $\begin{array}{l}<0.01 \\
0.03 \\
0.02 \\
0.02 \\
0.01\end{array}$ & $\begin{array}{r}<0.01 \\
0.03 \\
0.01 \\
0.02 \\
<0.01\end{array}$ & $\begin{array}{l}=- \\
=- \\
=\end{array}$ & $\begin{array}{l}= \\
=- \\
-- \\
<1\end{array}$ & $\begin{array}{l}=- \\
=- \\
=- \\
18.4\end{array}$ \\
\hline $\begin{array}{l}443926093010001 \\
443927093010101 \\
443930093004801 \\
443932093031601 \\
443933092533501\end{array}$ & $\begin{array}{l}-- \\
327 \\
267 \\
309 \\
314\end{array}$ & $\begin{array}{l}<0.01 \\
<0.01 \\
<0.01 \\
<0.01\end{array}$ & $\begin{array}{l}2.0 \\
11 \\
<0.05 \\
<0.05 \\
10\end{array}$ & $\begin{array}{r}<0.01 \\
0.10 \\
0.18 \\
<0.01\end{array}$ & $\begin{array}{l}= \\
\overline{-} \\
=-\end{array}$ & $\begin{array}{r}0.01 \\
0.01 \\
0.01 \\
<0.01\end{array}$ & $\begin{aligned} &-- \\
& 0.01 \\
&<0.01 \\
&<0.01 \\
&<0.01\end{aligned}$ & $\begin{array}{r}-- \\
1300 \\
670 \\
11\end{array}$ & $\begin{array}{r}- \\
<1 \\
80 \\
430 \\
5\end{array}$ & $\begin{array}{l}-\overline{17.1} \\
<0.8 \\
1.0 \\
25.9\end{array}$ \\
\hline $\begin{array}{l}443933093002802 \\
443933093002803\end{array}$ & $\begin{array}{l}=- \\
= \\
=\end{array}$ & $\begin{array}{r}0.03 \\
0.02 \\
0.03 \\
<0.01 \\
<0.01\end{array}$ & $\begin{array}{r}<7.8 \\
7.3 \\
9.3 \\
<0.10 \\
<0.05\end{array}$ & $\begin{array}{rl}<0 & 0.01 \\
<0.01 \\
0.03 \\
0.07 \\
0.06\end{array}$ & $\begin{array}{r}1.2 \\
0.30 \\
0.90 \\
0.90 \\
<0.20\end{array}$ & $\begin{array}{l}0.02 \\
0.02 \\
0.02 \\
0.03 \\
0.01\end{array}$ & $\begin{array}{r}0.02 \\
<0.01 \\
<0.01 \\
0.02 \\
<0.01\end{array}$ & $\begin{array}{l}= \\
=- \\
=\end{array}$ & $\begin{array}{l}= \\
= \\
= \\
=\end{array}$ & $\begin{array}{l}=- \\
=- \\
=-\end{array}$ \\
\hline $\begin{array}{l}443936092533701 \\
443939092533801 \\
444000093031702\end{array}$ & $\begin{array}{l}-- \\
908 \\
496 \\
--\end{array}$ & $\begin{array}{l}<0.01 \\
<0.01 \\
<0.01 \\
<0.01 \\
<0.01\end{array}$ & $\begin{array}{l}<0.05 \\
40 \\
22 \\
25 \\
13\end{array}$ & $\begin{array}{r}0.09 \\
<0.01 \\
<0.01 \\
<0.01 \\
<0.01\end{array}$ & \begin{tabular}{l}
0.50 \\
\hdashline- \\
1.2 \\
0.50
\end{tabular} & $\begin{array}{r}<0.01 \\
0.60 \\
<0.01 \\
0.01 \\
0.02\end{array}$ & $\begin{array}{r}<0.01 \\
0.57 \\
<0.01 \\
0.01 \\
<0.01\end{array}$ & $\begin{array}{l}-- \\
11 \\
-- \\
--\end{array}$ & $\begin{array}{l}- \\
<1 \\
- \\
--\end{array}$ & $\begin{array}{l}-\overline{<30.7} \\
37.3 \\
--\end{array}$ \\
\hline $\begin{array}{l}444000093031703 \\
444024092501901\end{array}$ & $=$ & $\begin{array}{r}0.01 \\
<0.01 \\
<0.01 \\
<0.01 \\
0.01\end{array}$ & $\begin{array}{l}5.5 \\
<0.10 \\
<0.05 \\
<0.05 \\
18.0\end{array}$ & $\begin{array}{l}0.02 \\
0.17 \\
0.16 \\
0.21 \\
0.02\end{array}$ & $\begin{array}{r}0.40 \\
0.60 \\
<0.20 \\
0.30 \\
0.70\end{array}$ & $\begin{array}{l}0.02 \\
0.01 \\
0.02 \\
0.03 \\
0.02\end{array}$ & $\begin{array}{r}<0.01 \\
0.01 \\
<0.01 \\
0.02 \\
<0.01\end{array}$ & $\begin{array}{l}= \\
=- \\
=\end{array}$ & $\begin{array}{l}=- \\
=- \\
= \\
=\end{array}$ & $\begin{array}{l}= \\
=- \\
=\end{array}$ \\
\hline $\begin{array}{l}444025092501201 \\
444026092511701 \\
444038092511401 \\
444049093020402\end{array}$ & $\begin{array}{l}-- \\
324 \\
335 \\
-- \\
--\end{array}$ & $\begin{array}{r}<0.01 \\
0.63 \\
0.35 \\
0.18\end{array}$ & $\begin{array}{r}18 \\
2.8 \\
6.0 \\
11.2 \\
4.2\end{array}$ & $\begin{array}{l}<\overline{-01} \\
<0.01 \\
<0.01 \\
<0.01\end{array}$ & $\begin{array}{l}-- \\
\overline{-} \\
0.80 \\
0.90\end{array}$ & $\begin{array}{r}-- \\
<0.01 \\
<0.01 \\
0.05 \\
0.06\end{array}$ & $\begin{aligned}<-- \\
<0.01 \\
<0.01 \\
0.04 \\
0.03\end{aligned}$ & $\begin{array}{r}5 \\
-- \\
--\end{array}$ & $\begin{array}{l}-- \\
-73 \\
--\end{array}$ & $\begin{array}{l}-- \\
22.8 \\
17.5 \\
--\end{array}$ \\
\hline 444049093020403 & $=$ & $\begin{array}{r}0.22 \\
<0.01 \\
<0.01 \\
0.02 \\
<0.01\end{array}$ & $\begin{array}{l}5.1 \\
4.6 \\
4.2 \\
4.0 \\
16\end{array}$ & $\begin{array}{r}0.03 \\
0.02 \\
<0.01 \\
0.02 \\
0.01\end{array}$ & $\begin{array}{l}0.70 \\
0.40 \\
0.30 \\
1.3 \\
0.60\end{array}$ & $\begin{array}{l}0.04 \\
0.05 \\
0.04 \\
0.04 \\
0.01\end{array}$ & $\begin{array}{r}0.04 \\
0.04 \\
0.02 \\
0.03 \\
<0.01\end{array}$ & $\begin{array}{l}= \\
= \\
=\end{array}$ & $\begin{array}{l}=- \\
=- \\
=\end{array}$ & $\begin{array}{l}=- \\
=- \\
=-\end{array}$ \\
\hline $\begin{array}{l}444058092461901 \\
444109092581701 \\
444147092491601 \\
444147093182001\end{array}$ & $\begin{array}{l}-\overline{286} \\
-- \\
--\end{array}$ & $\begin{array}{r}0.03 \\
<0.01 \\
0.01 \\
<0.01 \\
<0.01\end{array}$ & $\begin{array}{l}9.0 \\
5.2 \\
5.7 \\
5.4 \\
0.27\end{array}$ & $\begin{array}{r}0.04 \\
0.01 \\
<0.01 \\
0.01 \\
0.13\end{array}$ & $\begin{array}{l}0.40 \\
\because- \\
=0.20\end{array}$ & $\begin{array}{l}0.04 \\
0.02 \\
\overline{0.02}\end{array}$ & $\begin{array}{r}0.03 \\
0.01 \\
0.01 \\
<0.01 \\
0.02\end{array}$ & $\begin{array}{l}-- \\
-- \\
-- \\
--\end{array}$ & $\begin{array}{l}-- \\
--1 \\
--\end{array}$ & $\begin{array}{l}-\overline{21.3} \\
-- \\
--\end{array}$ \\
\hline
\end{tabular}


QUALITY OF GROUND MATER

WATER QUALITY DATA, WATER YEAR OCTOBER 1990 TO SEPTEMBER 1991

DAKOTA COUNTY--Continued

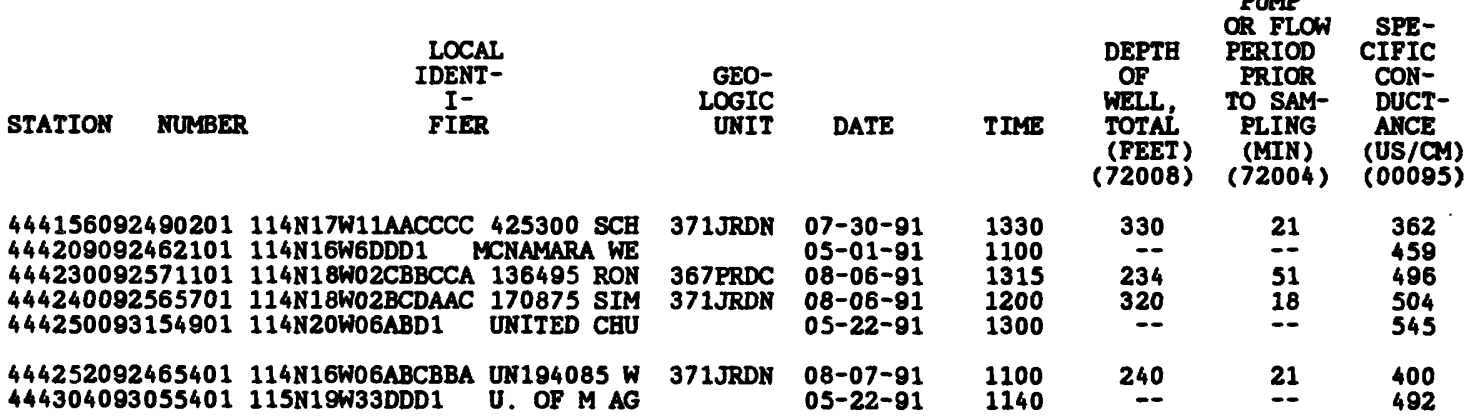

\begin{tabular}{|c|c|c|c|c|c|c|c|c|c|c|}
\hline NUMBER & $\begin{array}{c}\text { SPE- } \\
\text { CIFIC } \\
\text { CON- } \\
\text { DUCT- } \\
\text { ANCE } \\
\text { LAB } \\
\text { (US/CM) } \\
(90095)\end{array}$ & $\begin{array}{c}\text { PH } \\
\text { (STAND- } \\
\text { ARD } \\
\text { UNITS) } \\
(00400)\end{array}$ & $\begin{array}{c}\text { PH } \\
\text { LAB } \\
\text { (STAND- } \\
\text { ARD } \\
\text { UNITS) } \\
\text { (00403) }\end{array}$ & $\begin{array}{c}\text { OXID- } \\
\text { ATION } \\
\text { RED- } \\
\text { UCTION } \\
\text { POTEN- } \\
\text { TIAL } \\
\text { (MV) } \\
(00090)\end{array}$ & $\begin{array}{l}\text { TEMPER- } \\
\text { ATURE } \\
\text { WATER } \\
\text { (DEG C) } \\
(00010)\end{array}$ & $\begin{array}{c}\text { OXYGEN, } \\
\text { DIS- } \\
\text { SOLVED } \\
(M G / L) \\
(00300)\end{array}$ & $\begin{array}{c}\text { CALCIUM } \\
\text { DIS- } \\
\text { SOLVED } \\
\text { (MG/L } \\
\text { AS CA) } \\
\text { (00915) }\end{array}$ & $\begin{array}{c}\text { MAGNE- } \\
\text { SIUM, } \\
\text { DIS- } \\
\text { SOLVED } \\
\text { (MG/L } \\
\text { AS MG) } \\
\text { (00925) }\end{array}$ & $\begin{array}{c}\text { SODIUM, } \\
\text { DIS- } \\
\text { SOLVED } \\
\text { (MG/L } \\
\text { AS IA) } \\
(00930)\end{array}$ & $\begin{array}{c}\text { POTAS- } \\
\text { SIUM, } \\
\text { DIS- } \\
\text { SOLVED } \\
\text { (MG/L } \\
\text { AS K) } \\
(00935)\end{array}$ \\
\hline $\begin{array}{l}444156092490201 \\
444209092462101 \\
444230092571101 \\
444240092565701 \\
444250093154901\end{array}$ & $\begin{array}{l}433 \\
478 \\
506 \\
516 \\
564\end{array}$ & $\begin{array}{l}7.6 \\
7.6 \\
7.6 \\
7.7 \\
7.3\end{array}$ & $\begin{array}{l}7.8 \\
7.9 \\
7.8 \\
7.9 \\
7.5\end{array}$ & $\begin{array}{l}-0.1 \\
-- \\
0.2 \\
-0.2 \\
--\end{array}$ & $\begin{array}{l}12.0 \\
10.0 \\
11.5 \\
11.0 \\
14.0\end{array}$ & $\begin{array}{l}<0.1 \\
-- \\
6.6 \\
<0.1\end{array}$ & $\begin{array}{l}58 \\
59 \\
68 \\
64 \\
79\end{array}$ & $\begin{array}{l}23 \\
21 \\
23 \\
31 \\
26\end{array}$ & $\begin{array}{l}2.8 \\
4.0 \\
3.2 \\
3.0 \\
2.7\end{array}$ & $\begin{array}{l}1.2 \\
0.6 \\
1.0 \\
1.3 \\
2.1\end{array}$ \\
\hline $\begin{array}{l}4252092465401 \\
4304093055401\end{array}$ & $\begin{array}{l}411 \\
515\end{array}$ & $\begin{array}{l}7.6 \\
7.4\end{array}$ & $\begin{array}{l}8.0 \\
7.6\end{array}$ & -0.1 & $\begin{array}{r}10.5 \\
8.5\end{array}$ & $<0.1$ & $\begin{array}{l}54 \\
71\end{array}$ & $\begin{array}{l}21 \\
23\end{array}$ & $\begin{array}{l}3.1 \\
3.4\end{array}$ & $\begin{array}{l}1.0 \\
1.5\end{array}$ \\
\hline
\end{tabular}

\begin{tabular}{|c|c|c|c|c|c|c|c|c|c|c|}
\hline STATION & NUMBER & $\begin{array}{l}\text { BICAR- } \\
\text { BONATE } \\
\text { WATER } \\
\text { WH IT } \\
\text { FIELD } \\
\text { MG/L AS } \\
\text { HCO3 } \\
(00450)\end{array}$ & $\begin{array}{c}\text { ALKA- } \\
\text { LINITY } \\
\text { WAT WH } \\
\text { TOT IT } \\
\text { FIELD } \\
\text { MG/L AS } \\
\text { CACO3 } \\
(00419)\end{array}$ & $\begin{array}{l}\text { ALKA- } \\
\text { LINITY } \\
\text { WAT WH } \\
\text { TOT FET } \\
\text { FIELD } \\
\text { MG/L AS } \\
\text { CACO3 } \\
(00410)\end{array}$ & $\begin{array}{c}\text { ALKA- } \\
\text { LINITY } \\
\text { LAB } \\
\text { (MG/L } \\
\text { AS } \\
\text { CACO3) } \\
\text { (90410) }\end{array}$ & $\begin{array}{c}\text { SULFATE } \\
\text { DIS- } \\
\text { SOLVED } \\
\text { (MG/L } \\
\text { AS SO4) } \\
(00945)\end{array}$ & $\begin{array}{l}\text { CHLO- } \\
\text { RIDE, } \\
\text { DIS- } \\
\text { SOLVED } \\
\text { (MG/L } \\
\text { AS CL) } \\
\text { (00940) }\end{array}$ & $\begin{array}{l}\text { FLUO- } \\
\text { RIDE, } \\
\text { DIS- } \\
\text { SOLVED } \\
\text { (MG/L } \\
\text { AS F) } \\
\text { (00950) }\end{array}$ & $\begin{array}{c}\text { SILICA, } \\
\text { DIS- } \\
\text { SOLVED } \\
\text { (MG/L } \\
\text { AS } \\
\text { SIO2) } \\
\text { (0095S) }\end{array}$ & $\begin{array}{l}\text { SOLIDS, } \\
\text { RESIDUE } \\
\text { AT } 180 \\
\text { DEG. C } \\
\text { DIS- } \\
\text { SOLVED } \\
(M G / L) \\
(70300)\end{array}$ \\
\hline \multicolumn{2}{|c|}{$\begin{array}{l}444156092490201 \\
444209092462101 \\
444230092571101 \\
444240092565701 \\
444250093154901\end{array}$} & $\begin{array}{l}244 \\
-- \\
266 \\
332 \\
--\end{array}$ & $\begin{array}{l}202 \\
-- \\
218 \\
272 \\
--\end{array}$ & $\begin{array}{l}200 \\
-- \\
218 \\
272 \\
--\end{array}$ & $\begin{array}{l}194 \\
188 \\
225 \\
258 \\
282\end{array}$ & $\begin{array}{l}40 \\
17 \\
23 \\
16 \\
31\end{array}$ & $\begin{array}{l}4.0 \\
8.7 \\
8.3 \\
1.8 \\
2.7\end{array}$ & $\begin{array}{l}0.2 \\
0.1 \\
0.1 \\
0.1 \\
0.2\end{array}$ & $\begin{array}{l}13 \\
16 \\
16 \\
11 \\
18\end{array}$ & $\begin{array}{r}247 \\
-- \\
272 \\
302 \\
--\end{array}$ \\
\hline \multicolumn{2}{|c|}{$\begin{array}{l}444252092465401 \\
444304093055401\end{array}$} & 254 & 210 & 208 & $\begin{array}{l}186 \\
256\end{array}$ & $\begin{array}{l}17 \\
20\end{array}$ & $\begin{array}{l}1.4 \\
6.0\end{array}$ & $\begin{array}{l}0.1 \\
0.1\end{array}$ & $\begin{array}{l}14 \\
16\end{array}$ & 251 \\
\hline STATION & NUMBER & $\begin{array}{c}\text { NITRO- } \\
\text { GEN, } \\
\text { NITRITE } \\
\text { DIS- } \\
\text { SOLVED } \\
\text { (MG/L } \\
\text { AS N) } \\
\text { (00613) }\end{array}$ & $\begin{array}{c}\text { NITRO- } \\
\text { GEN, } \\
\text { NO2+NO3 } \\
\text { DIS- } \\
\text { SOLVED } \\
\text { (MG/L } \\
\text { AS N) } \\
\text { (00631) }\end{array}$ & $\begin{array}{c}\text { NITRO- } \\
\text { GEN, } \\
\text { AMONIA } \\
\text { DIS- } \\
\text { SOLVED } \\
\text { (MG/L } \\
\text { AS N) } \\
\text { (00608) }\end{array}$ & $\begin{array}{l}\text { NITRO- } \\
\text { GEN,AM- } \\
\text { MONIA + } \\
\text { ORGANIC } \\
\text { DIS. } \\
\text { (MG/L } \\
\text { AS N) } \\
(00623)\end{array}$ & $\begin{array}{c}\text { PHOS- } \\
\text { PHORUS } \\
\text { DIS- } \\
\text { SOLVED } \\
\text { (MG/L } \\
\text { AS P) } \\
(00666)\end{array}$ & $\begin{array}{l}\text { PHOS- } \\
\text { PHORUS } \\
\text { ORTHO, } \\
\text { DIS- } \\
\text { SOLVED } \\
\text { (MG /L } \\
\text { AS P) } \\
\text { (OO671) }\end{array}$ & $\begin{array}{l}\text { IRON, } \\
\text { DIS- } \\
\text { SOLVED } \\
\text { (UG/L } \\
\text { AS FE) } \\
\text { (01046) }\end{array}$ & $\begin{array}{l}\text { MANGA- } \\
\text { NESE, } \\
\text { DIS- } \\
\text { SOLVED } \\
\text { (UG/L } \\
\text { AS MN) } \\
\text { (01056) }\end{array}$ & $\begin{array}{l}\text { TRITIUM } \\
\text { IN } \\
\text { WATER } \\
\text { MOLE- } \\
\text { CULES } \\
\text { (TU) } \\
(07012)\end{array}$ \\
\hline \multicolumn{2}{|c|}{$\begin{array}{l}444156092490201 \\
444209092462101 \\
444230092571101 \\
444240092565701 \\
444250093154901\end{array}$} & $\begin{array}{l}<0.01 \\
<0.01 \\
<0.01 \\
<0.01 \\
<0.01\end{array}$ & $\begin{array}{l}<0.05 \\
11 \\
6.2 \\
<0.05 \\
<0.05\end{array}$ & $\begin{array}{r}0.01 \\
<0.01 \\
<0.01 \\
<0.01 \\
0.09\end{array}$ & $\begin{array}{l}-- \\
0.50 \\
-- \\
0.30\end{array}$ & $\begin{array}{l}<0.01 \\
0.02 \\
0.02 \\
0.01 \\
0.01\end{array}$ & $\begin{array}{r}<0.01 \\
0.01 \\
0.02 \\
<0.01 \\
0.01\end{array}$ & $\begin{array}{r}900 \\
-- \\
4 \\
1800 \\
--\end{array}$ & $\begin{array}{l}32 \\
-- \\
<1 \\
42 \\
--\end{array}$ & $\begin{array}{r}1.4 \\
15.4 \\
<0.8\end{array}$ \\
\hline $\begin{array}{l}444252092 \\
444304093\end{array}$ & 055401 & $\begin{array}{l}<0.01 \\
<0.01\end{array}$ & $\begin{array}{c}<0.05 \\
1.9\end{array}$ & $\begin{array}{r}<0.01 \\
0.02\end{array}$ & $\overline{0.60}$ & $\begin{array}{r}<0.01 \\
0.03\end{array}$ & $\begin{array}{r}<0.01 \\
0.01\end{array}$ & 620 & 47 & 0.8 \\
\hline
\end{tabular}


QUALITY OF GROUND WATER

WATER QUALITY DATA, WATER YEAR OCTOBER 1990 TO SEPTEMBER 1991

DAKOTA COUNTY--Continued

\begin{tabular}{|c|c|c|c|c|c|c|c|}
\hline STATION & $\begin{array}{l}\text { LOCAL } \\
\text { IDENT- } \\
\text { I- } \\
\text { FIDR }\end{array}$ & $\begin{array}{l}\text { GEO- } \\
\text { LOGIC } \\
\text { UNIT }\end{array}$ & DATE & TIME & $\begin{array}{l}\text { DEPTH } \\
\text { OF } \\
\text { WELL, } \\
\text { TOTAL } \\
\text { (FEET) } \\
\text { (72008) }\end{array}$ & $\begin{array}{l}\text { OR FLOW } \\
\text { PERIOD } \\
\text { PRIOR } \\
\text { TO SAM- } \\
\text { PLING } \\
\text { (MIN) } \\
(72004)\end{array}$ & $\begin{array}{l}\text { SPE- } \\
\text { CIFIC } \\
\text { CON- } \\
\text { DUCT- } \\
\text { ANCE } \\
\text { (US/CY) } \\
\text { (00095) }\end{array}$ \\
\hline $\begin{array}{l}444312092515702 \\
444333092593401 \\
444513093185401\end{array}$ & $\begin{array}{l}\text { 115N017W - SITE } 5 \text { SHALLOW } \\
\begin{array}{ll}\text { 115N18W33BCC1 } & \text { SWOBODA WE } \\
115 N 21 W 23 B C D C C D & 206037 \text { LHA }\end{array}\end{array}$ & $\begin{array}{l}\text { 112PLSC } \\
112 \text { PLSC } \\
\text { 112PLSC } \\
\text { 367PRDC }\end{array}$ & $\begin{array}{l}02-07-91 \\
04-10-91 \\
05-17-91 \\
05-02-91 \\
08-13-91\end{array}$ & $\begin{array}{l}1445 \\
1425 \\
1400 \\
1230 \\
1300\end{array}$ & $\begin{array}{r}59 \\
59 \\
59 \\
-- \\
193\end{array}$ & $\begin{array}{l}m \\
-- \\
36\end{array}$ & $\begin{array}{l}907 \\
722 \\
654 \\
751 \\
589\end{array}$ \\
\hline $\begin{array}{l}444552093080001 \\
444601093082901 \\
444634093041401 \\
444638093034401 \\
444843093045801\end{array}$ & $\begin{array}{lc}\text { 115N19W18DCA1 } & \text { DON GRUNTH } \\
\text { 115N19W18DAA1 } & \text { MARVIN KA } \\
\text { 27N22W32DCC1 } & \text { PHILIIP BRA } \\
\text { 27N22W33CCA1 } & \text { BOB PLAN WE } \\
\text { 27N22W20CBB1 } & \text { KOWSKI WELL }\end{array}$ & & $\begin{array}{l}05-23-91 \\
05-23-91 \\
06-05-91 \\
05-02-91 \\
05-23-91\end{array}$ & $\begin{array}{l}1020 \\
1115 \\
1100 \\
1425 \\
1245\end{array}$ & $\begin{array}{l}-= \\
\therefore \\
\therefore \\
=\end{array}$ & $\begin{array}{l}-- \\
= \\
= \\
-\end{array}$ & $\begin{array}{l}477 \\
531 \\
556 \\
568 \\
613\end{array}$ \\
\hline $\begin{array}{l}445000093055701 \\
445053093055001 \\
445134093082001\end{array}$ & $\begin{array}{l}\text { RABUSE WELL } \\
\text { MIKE TAURIN } \\
\text { JIM BANSON } W\end{array}$ & & $\begin{array}{l}06-04-91 \\
06-05-91 \\
06-04-91\end{array}$ & $\begin{array}{l}1015 \\
1000 \\
1115\end{array}$ & $\begin{array}{l}-- \\
--\end{array}$ & $\begin{array}{l}-- \\
--\end{array}$ & $\begin{array}{l}483 \\
822 \\
635\end{array}$ \\
\hline
\end{tabular}

\begin{tabular}{|c|c|c|c|c|c|c|c|c|c|c|}
\hline STATION & $\begin{array}{c}\text { SPE- } \\
\text { CIFIC } \\
\text { CON- } \\
\text { DUCT- } \\
\text { ANCE } \\
\text { LAB } \\
\text { (US/CY) } \\
\text { ( } 90095 \text { ) }\end{array}$ & $\begin{array}{c}\text { PH } \\
\text { (STAND- } \\
\text { ARD } \\
\text { UNITS) } \\
(00400)\end{array}$ & $\begin{array}{c}\text { PH } \\
\text { LAB } \\
\text { (STAND- } \\
\text { ARD } \\
\text { UNITS) } \\
(00403)\end{array}$ & $\begin{array}{c}\text { OXID- } \\
\text { ATION } \\
\text { RED- } \\
\text { UCTION } \\
\text { POIEN- } \\
\text { TIAL } \\
\text { (MV) } \\
(00090)\end{array}$ & $\begin{array}{l}\text { TEMPER- } \\
\text { ATURE } \\
\text { WATER } \\
\text { (DEG C) } \\
(00010)\end{array}$ & $\begin{array}{c}\text { OXYGEN, } \\
\text { DIS- } \\
\text { SOLVED } \\
(M G / L) \\
(00300)\end{array}$ & $\begin{array}{l}\text { CALCIUM } \\
\text { DIS- } \\
\text { SOLVED } \\
\text { (MG/L } \\
\text { AS CA) } \\
\text { (00915) }\end{array}$ & $\begin{array}{c}\text { MAGNE- } \\
\text { SIUM, } \\
\text { DIS- } \\
\text { SOLVED } \\
\text { (MG/L } \\
\text { AS MG) } \\
\text { (00925) }\end{array}$ & $\begin{array}{l}\text { SODIUM, } \\
\text { DIS- } \\
\text { SOLVED } \\
\text { (MG/L } \\
\text { AS NA) } \\
(00930)\end{array}$ & $\begin{array}{c}\text { POTAS- } \\
\text { SIUM, } \\
\text { DIS- } \\
\text { SOLVED } \\
\text { (MG/L } \\
\text { AS K }) \\
(00935)\end{array}$ \\
\hline $\begin{array}{l}444312092515702 \\
444333092593401 \\
444513093185401\end{array}$ & $\begin{array}{l}903 \\
752 \\
706 \\
778 \\
598\end{array}$ & $\begin{array}{l}7.5 \\
7.4 \\
7.4 \\
7.3 \\
7.6\end{array}$ & $\begin{array}{l}7.6 \\
7.7 \\
7.7 \\
7.5 \\
7.8\end{array}$ & $\begin{array}{l}-- \\
-- \\
-0 \\
-0.1\end{array}$ & $\begin{array}{r}13.0 \\
8.5 \\
6.0 \\
9.0 \\
11.5\end{array}$ & $\begin{array}{r}7.7 \\
8.3 \\
9.2 \\
-- \\
<0.1\end{array}$ & $\begin{array}{r}83 \\
69 \\
71 \\
100 \\
81\end{array}$ & $\begin{array}{l}27 \\
23 \\
25 \\
34 \\
27\end{array}$ & $\begin{array}{l}55 \\
53 \\
40 \\
5.7 \\
6.9\end{array}$ & $\begin{array}{l}4.3 \\
3.0 \\
2.8 \\
2.1 \\
3.8\end{array}$ \\
\hline $\begin{array}{l}444552093080001 \\
444601093082901 \\
444634093041401 \\
444638093034401 \\
444843093045801\end{array}$ & $\begin{array}{l}497 \\
556 \\
557 \\
595 \\
646\end{array}$ & $\begin{array}{l}7.7 \\
7.6 \\
7.4 \\
7.4 \\
7.6\end{array}$ & $\begin{array}{l}7.7 \\
7.7 \\
7.6 \\
7.6 \\
7.8\end{array}$ & $\begin{array}{l}-- \\
=- \\
=- \\
--\end{array}$ & $\begin{array}{r}10.0 \\
9.5 \\
11.0 \\
9.5 \\
10.5\end{array}$ & $\begin{array}{l}-- \\
0.6 \\
--\end{array}$ & $\begin{array}{l}64 \\
72 \\
77 \\
80 \\
82\end{array}$ & $\begin{array}{l}25 \\
28 \\
29 \\
27 \\
34\end{array}$ & $\begin{array}{l}3.4 \\
3.4 \\
3.7 \\
5.3 \\
3.7\end{array}$ & $\begin{array}{l}1.9 \\
1.6 \\
1.7 \\
1.6 \\
2.2\end{array}$ \\
\hline $\begin{array}{l}445000093055701 \\
445053093055001 \\
445134093082001\end{array}$ & $\begin{array}{l}531 \\
827 \\
743\end{array}$ & $\begin{array}{l}7.5 \\
7.3 \\
7.4\end{array}$ & $\begin{array}{l}7.7 \\
7.6 \\
7.7\end{array}$ & $=$ & $\begin{array}{l}10.5 \\
11.5 \\
10.5\end{array}$ & $\begin{array}{c}4.3 \\
10.6 \\
0\end{array}$ & $\begin{array}{l}74 \\
99 \\
89\end{array}$ & $\begin{array}{l}24 \\
40 \\
38\end{array}$ & $\begin{array}{l}3.7 \\
13 \\
12\end{array}$ & $\begin{array}{l}1.6 \\
1.9 \\
1.9\end{array}$ \\
\hline
\end{tabular}

\begin{tabular}{|c|c|c|c|c|c|c|c|c|c|}
\hline STATION & $\begin{array}{c}\text { BICAR- } \\
\text { BONATE } \\
\text { WATER } \\
\text { WH IT } \\
\text { FIELD } \\
\text { MG/L AS } \\
\text { HCO3 } \\
(00450)\end{array}$ & $\begin{array}{l}\text { ALKA- } \\
\text { LINITY } \\
\text { WAT WH } \\
\text { TOT IT } \\
\text { FIELD } \\
\text { MG/L AS } \\
\text { CACO3 } \\
\text { (00419) }\end{array}$ & $\begin{array}{l}\text { ALKA- } \\
\text { LINITY } \\
\text { WAT WH } \\
\text { TOT FET } \\
\text { FIELD } \\
\text { MG/L AS } \\
\text { CACO3 } \\
(00410)\end{array}$ & $\begin{array}{c}\text { ALKA- } \\
\text { LINITY } \\
\text { IAAB } \\
\text { (MG/L } \\
\text { AS } \\
\text { CACO3) } \\
(90410)\end{array}$ & $\begin{array}{l}\text { SULFATE } \\
\text { DIS- } \\
\text { SOLVED } \\
\text { (MG/L } \\
\text { AS SO4) } \\
\text { (00945) }\end{array}$ & $\begin{array}{l}\text { CHLO- } \\
\text { RIDE, } \\
\text { DIS- } \\
\text { SOLVED } \\
\text { (MG/L } \\
\text { AS CL) } \\
(00940)\end{array}$ & $\begin{array}{l}\text { FLUO- } \\
\text { RIDE, } \\
\text { DIS- } \\
\text { SOLVED } \\
\text { (MG/L } \\
\text { AS F) } \\
(00950)\end{array}$ & $\begin{array}{l}\text { SILICA, } \\
\text { DIS- } \\
\text { SOLVED } \\
\text { (MG/L } \\
\text { AS } \\
\text { SIO2) } \\
(00955)\end{array}$ & $\begin{array}{c}\text { SOLIDS, } \\
\text { RESIDUE } \\
\text { AT } 180 \\
\text { DEG.C } \\
\text { DIS- } \\
\text { SOLVED } \\
(M G / L) \\
(70300)\end{array}$ \\
\hline $\begin{array}{l}444333092593401 \\
444513093185401\end{array}$ & $\begin{array}{l}-- \\
-- \\
-- \\
333\end{array}$ & $\begin{array}{l}-- \\
-- \\
-- \\
273\end{array}$ & $\begin{array}{l}=- \\
=- \\
=- \\
273\end{array}$ & $\begin{array}{l}238 \\
210 \\
222 \\
246 \\
278\end{array}$ & $\begin{array}{l}35 \\
39 \\
38 \\
33 \\
39\end{array}$ & $\begin{array}{r}110 \\
74 \\
57 \\
28 \\
11\end{array}$ & $\begin{array}{l}0.2 \\
0.2 \\
0.3 \\
0.2 \\
0.1\end{array}$ & $\begin{array}{l}13 \\
13 \\
15 \\
15 \\
27\end{array}$ & $\begin{array}{l}-- \\
-- \\
340\end{array}$ \\
\hline $\begin{array}{l}444552093080001 \\
444601093082901 \\
444634093041401 \\
444638093034401 \\
444843093045801\end{array}$ & $\begin{array}{l}-- \\
=- \\
=- \\
=-\end{array}$ & $\begin{array}{l}-- \\
\overline{-} \\
\overline{--}\end{array}$ & $\begin{array}{l}-- \\
= \\
z- \\
-\end{array}$ & $\begin{array}{l}244 \\
253 \\
256 \\
259 \\
283\end{array}$ & $\begin{array}{l}31 \\
33 \\
29 \\
29 \\
30\end{array}$ & $\begin{array}{l}5.6 \\
12 \\
9.2 \\
13 \\
35\end{array}$ & $\begin{aligned}<0.1 \\
<0.1 \\
<0.1 \\
0.2 \\
<0.1\end{aligned}$ & $\begin{array}{l}16 \\
17 \\
17 \\
20 \\
23\end{array}$ & $\begin{array}{l}-- \\
-- \\
--\end{array}$ \\
\hline $\begin{array}{l}445000093055701 \\
445053093055001 \\
445134093082001\end{array}$ & $\overline{--}$ & $=-$ & $=$ & $\begin{array}{l}244 \\
297 \\
299\end{array}$ & $\begin{array}{l}43 \\
30 \\
44\end{array}$ & $\begin{array}{l}5.1 \\
75 \\
48\end{array}$ & $\begin{array}{l}<0.1 \\
<0.1 \\
<0.1\end{array}$ & $\begin{array}{l}20 \\
21 \\
18\end{array}$ & $\ddot{--}$ \\
\hline
\end{tabular}


QUALITY OF GROUND WATER

WATER QUALITY DATA, WATER YEAR OCTOBER 1990 TO SEPTEMBER 1991

DAKOTA COUNTY--Continued

\begin{tabular}{|c|c|c|c|c|c|c|c|c|c|}
\hline STATION & $\begin{array}{c}\text { NITRO- } \\
\text { GEN, } \\
\text { NITRITE } \\
\text { DIS- } \\
\text { SOLVED } \\
\text { (MG/L } \\
\text { AS N) } \\
\text { (00613) }\end{array}$ & $\begin{array}{c}\text { NITRO- } \\
\text { GEN, } \\
\text { NO2+NO3 } \\
\text { DIS- } \\
\text { SOLVED } \\
\text { (MG/L } \\
\text { AS N) } \\
\text { (00631) }\end{array}$ & $\begin{array}{c}\text { NITRO- } \\
\text { GEN, } \\
\text { AMONIA } \\
\text { DIS- } \\
\text { SOLVED } \\
\text { (MG/L } \\
\text { AS N) } \\
\text { (00608) }\end{array}$ & $\begin{array}{l}\text { NITRO- } \\
\text { GEN,AM- } \\
\text { MONIA + } \\
\text { ORGANIC } \\
\text { DIS. } \\
\text { (MG/L } \\
\text { AS N) } \\
(00623)\end{array}$ & $\begin{array}{c}\text { PEOS- } \\
\text { PHORUS } \\
\text { DIS- } \\
\text { SOLVED } \\
\text { (MG/L } \\
\text { AS P) } \\
(00666)\end{array}$ & $\begin{array}{c}\text { PBOS- } \\
\text { PBORUS } \\
\text { ORTHO, } \\
\text { DIS- } \\
\text { SOLVED } \\
\text { (MG/L } \\
\text { AS P) } \\
\text { (00671) }\end{array}$ & $\begin{array}{l}\text { IRON, } \\
\text { DIS- } \\
\text { SOLVED } \\
\text { (UG/L } \\
\text { AS FE) } \\
(01046)\end{array}$ & $\begin{array}{l}\text { MANGA- } \\
\text { NESE, } \\
\text { DIS- } \\
\text { SOLVED } \\
\text { (UG/L } \\
\text { AS MN) } \\
\text { (01056) }\end{array}$ & $\begin{array}{l}\text { TRITIUM } \\
\text { IN } \\
\text { WATER } \\
\text { MOLE- } \\
\text { CULES } \\
\text { (TU) } \\
(07012)\end{array}$ \\
\hline $\begin{array}{l}444312092515702 \\
444333092593401 \\
444513093185401\end{array}$ & $\begin{aligned}<0.01 \\
<0.01 \\
0.01 \\
0.01 \\
<0.01\end{aligned}$ & $\begin{array}{c}9.6 \\
6.9 \\
4.8 \\
26 \\
<0.05\end{array}$ & $\begin{array}{r}<0.01 \\
<0.01 \\
0.03 \\
0.02 \\
0.19\end{array}$ & $\begin{array}{l}1.2 \\
0.60 \\
0.80 \\
0.60 \\
--\end{array}$ & $\begin{array}{l}0.54 \\
0.43 \\
0.48 \\
0.02 \\
0.02\end{array}$ & $\begin{array}{r}0.50 \\
0.43 \\
0.48 \\
<0.01 \\
<0.01\end{array}$ & $\begin{array}{l}-- \\
-- \\
\overline{--} \\
910\end{array}$ & $\begin{array}{c}-- \\
\overline{--} \\
120\end{array}$ & $\begin{array}{c}-- \\
-- \\
22.5\end{array}$ \\
\hline $\begin{array}{l}444552093080001 \\
444601093082901 \\
444634093041401 \\
444638093034401 \\
444843093045801\end{array}$ & $\begin{aligned}<0.01 \\
<0.01 \\
<0.01 \\
0.01 \\
<0.01\end{aligned}$ & $\begin{array}{l}0.08 \\
3.3 \\
3.3 \\
5.4 \\
0.06\end{array}$ & $\begin{array}{l}0.04 \\
0.02 \\
0.01 \\
0.02 \\
0.13\end{array}$ & $\begin{array}{r}<0.20 \\
0.50 \\
0.30 \\
0.40 \\
0.30\end{array}$ & $\begin{array}{r}0.02 \\
0.01 \\
<0.01 \\
0.01 \\
0.03\end{array}$ & $\begin{array}{l}<0.01 \\
<0.01 \\
<0.01 \\
<0.01 \\
<0.01\end{array}$ & $\begin{array}{l}-- \\
-- \\
-- \\
--\end{array}$ & $\begin{array}{l}-- \\
-- \\
-- \\
--\end{array}$ & $\begin{array}{l}=- \\
\overline{-} \\
\overline{-} \\
\overline{-}\end{array}$ \\
\hline $\begin{array}{l}445000093055701 \\
445053093055001 \\
445134093082001\end{array}$ & $\begin{array}{r}0.02 \\
<0.01 \\
<0.01\end{array}$ & $\begin{array}{c}1.1 \\
3.7 \\
<0.05\end{array}$ & $\begin{array}{r}<0.01 \\
0.02 \\
0.04\end{array}$ & $\begin{array}{l}<0.20 \\
2.3 \\
<0.20\end{array}$ & $\begin{array}{r}<0.01 \\
0.03 \\
0.02\end{array}$ & $\begin{array}{r}<0.01 \\
0.02 \\
0.02\end{array}$ & $\begin{array}{l}-- \\
--\end{array}$ & $\begin{array}{l}-- \\
--\end{array}$ & $\begin{array}{l}-- \\
--\end{array}$ \\
\hline
\end{tabular}

ANALYSIS FOR ORGANIC CHEMICALS

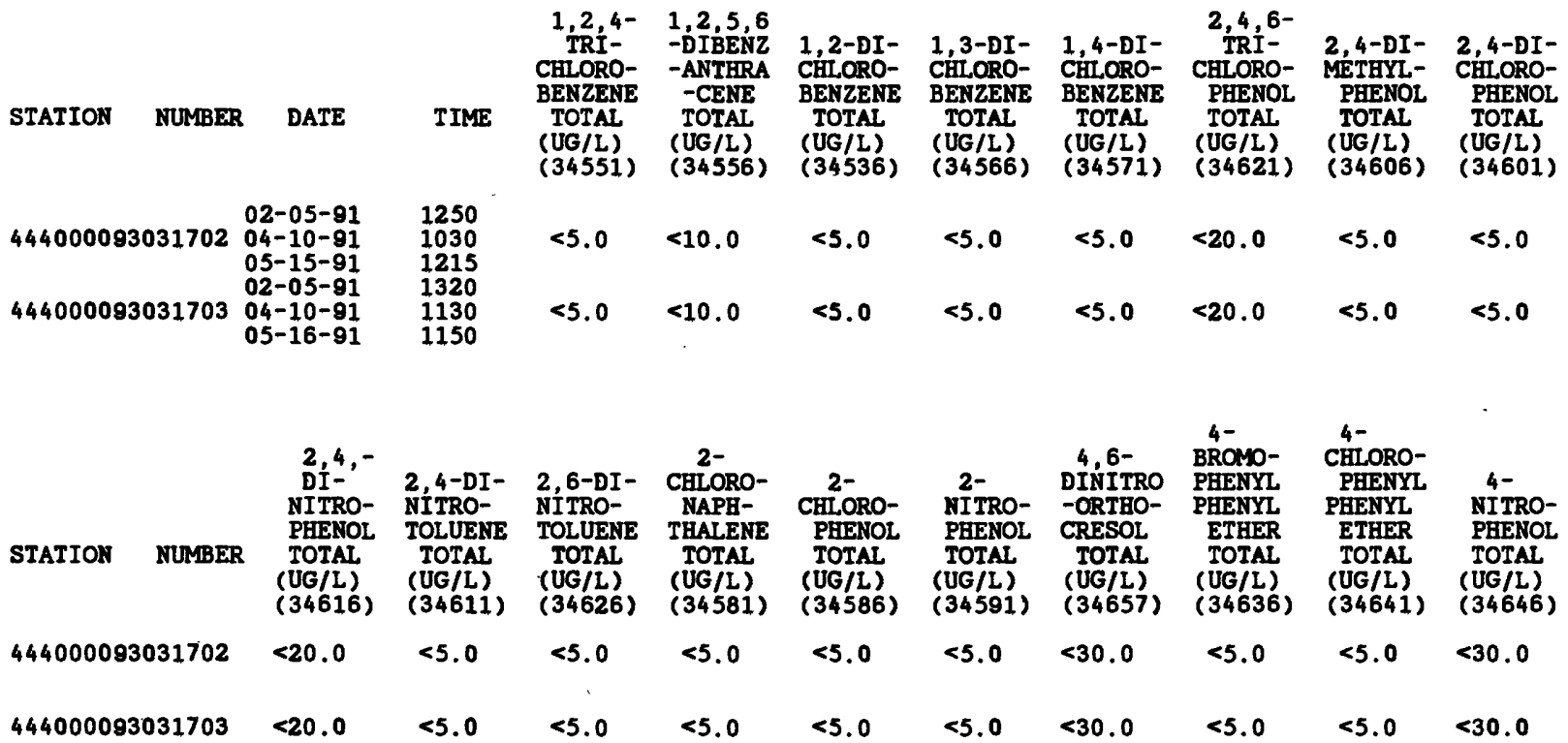


WATER QUALITY DATA, WATER YEAR OCTOBER 1990 TO SEPTEQBER 1991

DAKOTA COUNTY--Continued

\begin{tabular}{|c|c|c|c|c|c|c|c|c|c|c|}
\hline STATION & NUMBER & $\begin{array}{c}\text { ACE- } \\
\text { NAPHTH- } \\
\text { ENE } \\
\text { TOTAL } \\
\text { (UG/L) } \\
(34205)\end{array}$ & $\begin{array}{l}\text { ACE- } \\
\text { NAPBTH- } \\
\text { YLENE } \\
\text { TOTAL } \\
\text { (UG/L) } \\
(34200)\end{array}$ & $\begin{array}{c}\text { ANTERA- } \\
\text { CENE } \\
\text { TOTAL } \\
\text { (UG/L) } \\
(34220)\end{array}$ & $\begin{array}{l}\text { BENZO- } \\
\text { A- } \\
\text { PYRENE } \\
\text { TOTAL } \\
\text { (UG/L) } \\
(34247)\end{array}$ & $\begin{array}{c}\text { BENZO B } \\
\text { FLUOR- } \\
\text { AN- } \\
\text { THENE } \\
\text { TOTAL } \\
\text { (UG/L) } \\
\text { (34230) }\end{array}$ & $\begin{array}{c}\text { BENZO K } \\
\text { FLUOR- } \\
\text { AN- } \\
\text { THENE } \\
\text { TOTAL } \\
\text { (UG/L) } \\
\text { (34242) }\end{array}$ & $\begin{array}{l}\text { BENZO A } \\
\text { ANTHRAC } \\
\text { ENE1 } 2- \\
\text { BENZANT } \\
\text { BRACENB } \\
\text { TOTAL } \\
\text { (UG/L) } \\
(34526)\end{array}$ & $\begin{array}{l}\text { BENZOGH } \\
\text { I PERYL } \\
\text { ENE1,12 } \\
\text { - BENZOP } \\
\text { ERYLENE } \\
\text { TOTAL } \\
\text { (UG/L) } \\
(34521)\end{array}$ & $\begin{array}{c}\text { BIS } \\
\text { (2- } \\
\text { CHLORO- } \\
\text { ETHOXY) } \\
\text { METBANE } \\
\text { TOTAL } \\
\text { (UG/L) } \\
\text { ( } 34278)\end{array}$ \\
\hline 444000093 & 031702 & $<5.0$ & $<5.0$ & $<5.0$ & $<10.0$ & $<10.0$ & $<10.0$ & $<10.0$ & $<10.0$ & $<5.0$ \\
\hline 444000093 & 031703 & $<5.0$ & $<5.0$ & $<5.0$ & $<10.0$ & $<10.0$ & $<10.0$ & $<10.0$ & $<10.0$ & $<5.0$ \\
\hline STATION & NUMBER & $\begin{array}{c}\text { BIS (2- } \\
\text { CHLORO- } \\
\text { ISO- } \\
\text { PROPYL) } \\
\text { ETHER } \\
\text { TOTAL } \\
\text { (UG/L) } \\
\text { (34283) }\end{array}$ & $\begin{array}{c}\text { BIS (2- } \\
\text { ETHYL } \\
\text { HEXYL) } \\
\text { PHTHAL- } \\
\text { ATE } \\
\text { TOTAL } \\
\text { (UG/L) } \\
\text { (39100) }\end{array}$ & $\begin{array}{c}\text { BIS } \\
2- \\
\text { CHLORO- } \\
\text { ETHYL } \\
\text { ETHER } \\
\text { TOTAL } \\
\text { (UG/L) } \\
(34273)\end{array}$ & $\begin{array}{c}\text { CHRY- } \\
\text { SENE } \\
\text { TOTAL } \\
\text { (UG/L) } \\
(34320)\end{array}$ & $\begin{array}{l}\text { DIETHYL } \\
\text { PETHAL- } \\
\text { ATE } \\
\text { TOTAL } \\
\text { (UG/L) } \\
(34336)\end{array}$ & $\begin{array}{c}\text { DI- } \\
\text { METHYL } \\
\text { PHTHAL- } \\
\text { ATE } \\
\text { TOTAL } \\
\text { (UG/L) } \\
(34341)\end{array}$ & $\begin{array}{c}\text { DI-N- } \\
\text { BUTYL } \\
\text { PHTHAL- } \\
\text { ATE } \\
\text { TOTAL } \\
\text { (UG/L) } \\
\text { (39110) }\end{array}$ & $\begin{array}{c}\text { DI-N- } \\
\text { OCTYL } \\
\text { PHTHAL- } \\
\text { ATE } \\
\text { TOTAL } \\
\text { (UG/L) } \\
(34596)\end{array}$ & $\begin{array}{l}\text { FLUOR- } \\
\text { ANTHENE } \\
\text { TOTAL } \\
\text { (UG/L) } \\
\text { ( } 34376 \text { ) }\end{array}$ \\
\hline 444000093 & 031702 & $<5.0$ & $<5.0$ & $<5.0$ & $<10.0$ & $<5.0$ & $<5.0$ & $<5.0$ & $<10.0$ & $<5.0$ \\
\hline 444000093 & 031703 & $<5.0$ & $<5.0$ & $<5.0$ & $<10.0$ & $<5.0$ & $<5.0$ & $<5.0$ & $<10.0$ & $<5.0$ \\
\hline STATION & NUMBER & $\begin{array}{c}\text { FLUOR- } \\
\text { ENE } \\
\text { TOTAL } \\
\text { (UG/L) } \\
(34381)\end{array}$ & $\begin{array}{l}\text { HEXA- } \\
\text { CHLORO- } \\
\text { BENZENE } \\
\text { TOTAL } \\
\text { (UG/L) } \\
(39700)\end{array}$ & $\begin{array}{c}\text { HEXA- } \\
\text { CHLORO- } \\
\text { BUT- } \\
\text { ADIEME } \\
\text { TOTAL } \\
\text { (UG/L) } \\
(39702)\end{array}$ & $\begin{array}{l}\text { HEXA- } \\
\text { CHLORO- } \\
\text { CYCLO- } \\
\text { PENT- } \\
\text { ADIENE } \\
\text { TOTAL } \\
\text { (UG/L) } \\
\text { (34386) }\end{array}$ & $\begin{array}{l}\text { HEXA- } \\
\text { CHLORO- } \\
\text { ETHANE } \\
\text { TOTAL } \\
\text { (UG/L) } \\
(34396)\end{array}$ & $\begin{array}{l}\text { INDENO } \\
(1,2,3- \\
\text { CD) } \\
\text { PYRENE } \\
\text { TOTAL } \\
\text { (UG/L) } \\
(34403)\end{array}$ & $\begin{array}{l}\text { ISO- } \\
\text { PHORONE } \\
\text { TOTAL } \\
\text { (UG/L) } \\
(34408)\end{array}$ & $\begin{array}{c}\text { N-BUTYL } \\
\text { BENZYL } \\
\text { PHTHAL- } \\
\text { ATE } \\
\text { TOTAL } \\
\text { (UG/L) } \\
(34292)\end{array}$ & $\begin{array}{c}\text { N- } \\
\text { NITRO- } \\
\text { SODI-N- } \\
\text { PROPYL- } \\
\text { AMINE } \\
\text { TOTAL } \\
\text { (UG/L) } \\
(34428)\end{array}$ \\
\hline 444000093 & 031702 & $<5.0$ & $<5.0$ & $<5.0$ & $<5.0$ & $<5.0$ & $<10.0$ & $<5.0$ & $<5.0$ & $<5.0$ \\
\hline 444000093 & 031703 & $<5.0$ & $<5.0$ & $<5.0$ & $<5.0$ & $<5.0$ & $<10.0$ & $<5.0$ & $<5.0$ & $<5.0$ \\
\hline STATION & NUMBER & $\begin{array}{l}\text { N-NITRO } \\
\text { - SODI- } \\
\text { METHY- } \\
\text { LAMINE } \\
\text { TOTAL } \\
\text { (UG/L) } \\
(34438)\end{array}$ & $\begin{array}{c}\text { N-NITRO } \\
\text {-SODI- } \\
\text { PHENY- } \\
\text { LAMINE } \\
\text { TOTAL } \\
\text { (UG/L) } \\
\text { (34433) }\end{array}$ & $\begin{array}{l}\text { NAPHTH- } \\
\text { ALENE } \\
\text { TOTAL } \\
\text { (UG/L) } \\
\text { (34696) }\end{array}$ & $\begin{array}{l}\text { NITRO- } \\
\text { BENZENE } \\
\text { TOTAL } \\
\text { (UG/L) } \\
(34447)\end{array}$ & $\begin{array}{c}\text { PARA- } \\
\text { CHLORO- } \\
\text { META } \\
\text { CRESOL } \\
\text { TOTAL } \\
\text { (UG/L) } \\
(34452)\end{array}$ & $\begin{array}{l}\text { PENTA- } \\
\text { CHLORO- } \\
\text { PHENOL } \\
\text { TOTAL } \\
\text { (UG/L) } \\
(39032)\end{array}$ & $\begin{array}{l}\text { PHENAN- } \\
\text { THRENE } \\
\text { TOTAL } \\
\text { (UG/L) } \\
(34461)\end{array}$ & $\begin{array}{l}\text { PHENOL } \\
\text { (C6B- } \\
\text { 5OH) } \\
\text { TOTAL } \\
\text { (UG/L) } \\
(34694)\end{array}$ & $\begin{array}{c}\text { PYRENE } \\
\text { TOTAL } \\
\text { (UG/L) } \\
(34469)\end{array}$ \\
\hline 444000093 & 031702 & $<5.0$ & $<5.0$ & $<5.0$ & $<5.0$ & $<30.0$ & $<30.0$ & $<5.0$ & $<5.0$ & $<5.0$ \\
\hline 444000093 & 031703 & $<5.0$ & $<5.0$ & $<5.0$ & $<5.0$ & $<30.0$ & $<30.0$ & $<5.0$ & $<5.0$ & $<5.0$ \\
\hline
\end{tabular}


QUALITY OF GROURD WATER

WATER QUALITY DATA, WATER YEAR OCTOBER 1990 TO SEPTEMBER 1991

GOODHUE COUNTY

STATION NUMBER $\begin{gathered}\text { LOCAL } \\ \text { IDENT- } \\ \text { I- } \\ \text { FIER }\end{gathered}$

442003092423501

442503092434801

110N-16W-14 BCCDBA

$111 \mathrm{~N}-16 \mathrm{~W}-15 \mathrm{CCCDCC}$

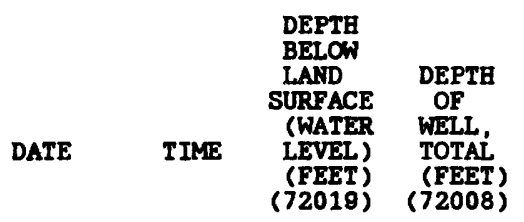

$\begin{array}{rrrr}03-13-91 & 1305 & -- & 200 \\ 07-17-91 & 1730 & 85.00 & 200 \\ 03-13-91 & 1515 & --0 & 350 \\ 07-17-91 & 1630 & 210.00 & 350\end{array}$

\begin{tabular}{|c|c|c|c|c|c|c|c|c|c|c|}
\hline ATION & NUMBER & $\begin{array}{c}\text { PUMP } \\
\text { OR FLOW } \\
\text { PERIOD } \\
\text { PRIOR } \\
\text { TO SAM- } \\
\text { PLING } \\
\text { (MIN) } \\
\text { (72004) }\end{array}$ & $\begin{array}{l}\text { SPE- } \\
\text { CIFIC } \\
\text { CON- } \\
\text { DUCT- } \\
\text { ANCE } \\
\text { (US/CM) } \\
\text { (O0095) }\end{array}$ & $\begin{array}{c}\text { PH } \\
\text { (STAND- } \\
\text { ARD } \\
\text { UNITS) } \\
(00400)\end{array}$ & $\begin{array}{c}\text { TEMPER- } \\
\text { ATURE } \\
\text { WATER } \\
\text { (DEG C) } \\
(00010)\end{array}$ & $\begin{array}{c}\text { OXYGEN, } \\
\text { DIS-' } \\
\text { SOLVED } \\
(\text { MG } / L) \\
(00300)\end{array}$ & $\begin{array}{c}\text { NITRO- } \\
\text { GEN, } \\
\text { NITRITE } \\
\text { DIS- } \\
\text { SOLVED } \\
\text { (MG/L } \\
\text { AS N } \\
\text { (O0613) }\end{array}$ & $\begin{array}{c}\text { NITRO- } \\
\text { GEN, } \\
\text { NO2+NO3 } \\
\text { DIS- } \\
\text { SOLVED } \\
\text { (MG/L } \\
\text { AS N ) } \\
\text { (00631) }\end{array}$ & $\begin{array}{c}\text { NITRO- } \\
\text { GEN, } \\
\text { AMMONIA } \\
\text { DIS- } \\
\text { SOLVED } \\
\text { (MG/L } \\
\text { AS N) } \\
\text { (00608) }\end{array}$ & $\begin{array}{l}\text { PHOS- } \\
\text { PHORUS } \\
\text { ORTHO, } \\
\text { DIS- } \\
\text { SOLVED } \\
\text { (MG/L } \\
\text { AS P) } \\
(00671)\end{array}$ \\
\hline
\end{tabular}

442003092423501

$\overline{27} \quad 3 \overline{--}$

$7 . \overline{-}$

13.0

$0 . \overline{1}$

$<0.01$

$<0.01$

$<0.01$

0.07
$<0.05$
0.18

$<0.01$

0.02

$<0.01$

$<0.01$

$<0.01$

$0.01<0.01$

BOUSTON COUNTY

$\begin{array}{cc} & \text { LOCAL } \\ \text { IDENT- } \\ \text { STATION NUMBER }\end{array}$

434613091350501

104N-06W-32 BCABC

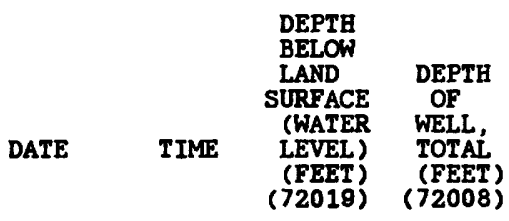

$\begin{array}{llcl}03-12-91 & 1250 & -- & 175 \\ 07-18-91 & 1500 & 135.0 & 175\end{array}$

\begin{tabular}{|c|c|c|c|c|c|c|c|c|c|c|}
\hline STATION & IUMBER & $\begin{array}{c}\text { PUMP } \\
\text { OR FLON } \\
\text { PERIOD } \\
\text { PRIOR } \\
\text { TO SAM- } \\
\text { PLING } \\
\text { (MIN) } \\
\text { (72004) }\end{array}$ & $\begin{array}{l}\text { SPE- } \\
\text { CIFIC } \\
\text { CON- } \\
\text { DUCT- } \\
\text { ANCE } \\
\text { (US/CM) } \\
(00095 \text { ) }\end{array}$ & $\begin{array}{c}\text { PH } \\
\text { (STAND- } \\
\text { ARD } \\
\text { UNITS) } \\
(00400)\end{array}$ & $\begin{array}{c}\text { TEMPER- } \\
\text { ATURE } \\
\text { WATER } \\
\text { (DEG C) } \\
(00010)\end{array}$ & $\begin{array}{c}\text { OXYGEN, } \\
\text { DIS- } \\
\text { SOLVED } \\
(\mathrm{MG} / \mathrm{L}) \\
(00300)\end{array}$ & $\begin{array}{c}\text { NITRO- } \\
\text { GEN, } \\
\text { NITRITE } \\
\text { DIS- } \\
\text { SOLVED } \\
\text { (MG /L } \\
\text { AS N) } \\
\text { (OOE13) }\end{array}$ & $\begin{array}{c}\text { NITRO- } \\
\text { GEN, } \\
\text { NO2+NO3 } \\
\text { DIS- } \\
\text { SOLVED } \\
\text { (MG/L } \\
\text { AS N) } \\
\text { (00631) }\end{array}$ & $\begin{array}{c}\text { NITRO- } \\
\text { GEN, } \\
\text { AMMNIA } \\
\text { DIS- } \\
\text { SOLVED } \\
\text { (MG/L } \\
\text { AS N) } \\
(00608)\end{array}$ & $\begin{array}{c}\text { PHOS- } \\
\text { PHORUS } \\
\text { ORTHO, } \\
\text { DIS- } \\
\text { SOLVED } \\
\text { (MG/L } \\
\text { AS P) } \\
\text { (OOG71) }\end{array}$ \\
\hline 4346130 & 350501 & $\overline{27}$ & 530 & $7 .-$ & $14 . \overline{0}$ & $13 . \overline{-}$ & $\begin{array}{r}0.01 \\
<0.01\end{array}$ & $\begin{array}{l}3.5 \\
3.7\end{array}$ & $\begin{array}{l}<0.01 \\
<0.01\end{array}$ & $\begin{array}{r}0.04 \\
<0.01\end{array}$ \\
\hline
\end{tabular}


QUALITY OF GROUND WATER

WATER QUALITY DATA, WATER YEAR OCTOBER 1990 TO SEPTEMBER 1991 JACKSON COUNTY

$\begin{array}{lc}\text { LOCAL } \\ \text { STATION NUMBER } & \begin{array}{c}\text { IDENT- } \\ \text { I- } \\ \text { FIER }\end{array} \\ 433755095113201 & 102 N-36 W-17 \text { CCAB } \\ 434028094590801 & 103 N-34 W-30 \text { CDDCAC }\end{array}$

PH
(STAND-
ARD
UNITS)
(00400)

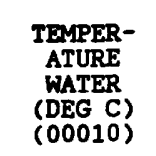

$\begin{array}{cccc}\text { DATE } & \text { TIME } & \begin{array}{c}\text { DEPTH } \\ \text { BELOW } \\ \text { LAND } \\ \text { SURFACE } \\ \text { (WATER } \\ \text { LEVEL) } \\ \text { (FEET) } \\ (72019)\end{array} & \begin{array}{c}\text { DEPTH } \\ \text { OFL } \\ \text { WELL, } \\ \text { TOTAL } \\ \text { (FEET) } \\ (72008)\end{array} \\ 03-26-91 & 0930 & -- & 26 \\ 03-26-91 & 0935 & -- & 26 \\ 07-23-91 & 1317 & 9.32 & 26 \\ 03-22-91 & 1805 & -- & 80 \\ 07-23-91 & 1015 & 45.00 & 80\end{array}$

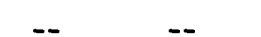

$\begin{array}{llr}433755095113201 & -- & -- \\ 434028094590801 & 32 & 473 \\ & -- & -- \\ 23 & 2080\end{array}$
$\overline{--}$
7.3
6.7

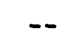
$--$
0.4
$0 . \overline{0.1}$

OXYGEN,
DIS-
SOLVED
(MG/L)
$(00300)$
NITROGEN, IITRITE DISSOLVED (ME/L

AS $N$ ) (00613)

$<0.01$

$<.01$

0.01

$<0.01$
NITROGEN $102+\mathrm{NO} 3$ DISSOLVED (MG /L

AS N) (00631)

\begin{tabular}{lll}
2.4 & $<0.01$ & 0.02 \\
\hdashline 6.7 & 0.02 & 0.03 \\
$<0.05$ & 2.1 & $<0.01$ \\
$<0.05$ & 1.9 & $<0.01$
\end{tabular}

MURRAY COUNTY

\begin{tabular}{|c|c|c|c|c|c|c|c|c|c|}
\hline STATION & & $\begin{array}{c}\text { LOCAL } \\
\text { IDENT- } \\
\text { I- } \\
\text { FIER }\end{array}$ & & & DATE & TIME & $\begin{array}{l}\text { DEPTH } \\
\text { BELOW } \\
\text { LAND } \\
\text { SURFACE } \\
\text { (WATER } \\
\text { LEVEL) } \\
\text { (FEET) } \\
\text { (72019) }\end{array}$ & $\begin{array}{l}\text { DEPTH } \\
\text { OF } \\
\text { WELL, } \\
\text { TOTAL } \\
\text { (FEET) } \\
\text { (72008) }\end{array}$ & $\begin{array}{l}\text { ELEV. } \\
\text { OF LAND } \\
\text { SURFACE } \\
\text { DATUM } \\
\text { (FT. } \\
\text { ABOVE } \\
\text { NGVD) } \\
\text { (72000) }\end{array}$ \\
\hline $\begin{array}{l}435133095360801 \\
435625095540301\end{array}$ & $\begin{array}{l}105 N \\
106 N\end{array}$ & $\begin{array}{l}-40 w-36 \\
-42 w-33\end{array}$ & $\begin{array}{l}\mathrm{BCCDC} \\
\mathrm{BCCAA}\end{array}$ & & $\begin{array}{l}03-28-91 \\
07-23-91 \\
03-21-91 \\
07-23-91\end{array}$ & $\begin{array}{l}1320 \\
1815 \\
1235 \\
1615\end{array}$ & $\begin{array}{l}3 \overline{--} \\
15.00\end{array}$ & $\begin{array}{l}37.00 \\
37.00 \\
42.00 \\
42.00\end{array}$ & $\begin{array}{l}1515 \\
1515 \\
1695 \\
1695\end{array}$ \\
\hline STATION & $\begin{array}{c}\text { PUMP } \\
\text { OR FLOW } \\
\text { PERIOD } \\
\text { PRIOR } \\
\text { TO SAM- } \\
\text { PLING } \\
\text { (MIN) } \\
\text { (72004) }\end{array}$ & $\begin{array}{l}\text { SPE- } \\
\text { CIFIC } \\
\text { CON- } \\
\text { DUCT- } \\
\text { ANCE } \\
\text { (US/CM) } \\
\text { (00095) }\end{array}$ & $\begin{array}{c}\text { PH } \\
\text { (STAND- } \\
\text { ARD } \\
\text { UNITS) } \\
(00400)\end{array}$ & $\begin{array}{l}\text { TEMPER- } \\
\text { ATURE } \\
\text { WATER } \\
\text { (DEG C) } \\
(00010)\end{array}$ & $\begin{array}{c}\text { OXYGEN, } \\
\text { DIS- } \\
\text { SOLVED } \\
(M G / L) \\
(00300)\end{array}$ & $\begin{array}{c}\text { NITRO- } \\
\text { GEN, } \\
\text { NITRITE } \\
\text { DIS- } \\
\text { SOLVED } \\
\text { (MG/L } \\
\text { AS N) } \\
\text { (00613) }\end{array}$ & $\begin{array}{c}\text { NITRO- } \\
\text { GEN, } \\
\text { NO2+NO3 } \\
\text { DIS- } \\
\text { SOLVED } \\
\text { (MG/L } \\
\text { AS N) } \\
(00631)\end{array}$ & $\begin{array}{c}\text { NITRO- } \\
\text { GEN, } \\
\text { AMMONIA } \\
\text { DIS- } \\
\text { SOLVED } \\
\text { (MG/L } \\
\text { AS N) } \\
(00608)\end{array}$ & $\begin{array}{l}\text { PHOS- } \\
\text { PEORUS } \\
\text { ORTHO, } \\
\text { DIS- } \\
\text { SOLVED } \\
\text { (MG/L } \\
\text { AS P) } \\
(00671)\end{array}$ \\
\hline $\begin{array}{l}435133095360801 \\
435625095540301\end{array}$ & $\frac{--}{15}$ & $\begin{array}{r}1030 \\
1120\end{array}$ & $\begin{array}{l}7.1 \\
6.9\end{array}$ & $\begin{array}{r}13.0 \\
10.5\end{array}$ & 3.7 & $\begin{array}{r}<0.01 \\
<0.01 \\
0.05 \\
<0.01\end{array}$ & $\begin{array}{l}0.94 \\
1.4 \\
2.1 \\
5.2\end{array}$ & $\begin{array}{l}0.09 \\
0.01 \\
0.13 \\
0.20\end{array}$ & $\begin{array}{l}<0.01 \\
<0.01 \\
<0.01 \\
<0.01\end{array}$ \\
\hline
\end{tabular}


QUALITY OF GROUND WATER

WATER QUALITY DATA, WATER YEAR OCTOBER 1980 TO SEPTEMBER 1981

OLMSTED COUNTY

\begin{tabular}{|c|c|c|c|c|c|c|c|}
\hline STATION & $\begin{array}{l}\text { LOCAL } \\
\text { IDENT- } \\
\text { I- } \\
\text { FIER }\end{array}$ & $\begin{array}{l}\text { GEO- } \\
\text { LOGIC } \\
\text { UNIT }\end{array}$ & DATE & TIME & $\begin{array}{l}\text { DEPTH } \\
\text { BELOW } \\
\text { LAND } \\
\text { SURFACE } \\
\text { (WATER } \\
\text { LEVEL) } \\
\text { (FEET) } \\
\text { (72019) }\end{array}$ & $\begin{array}{l}\text { DEPTH } \\
\text { OF } \\
\text { WELL, } \\
\text { TOTAL } \\
\text { (FEEI) } \\
\text { (72008) }\end{array}$ & $\begin{array}{c}\text { PUMP } \\
\text { OR FLOW } \\
\text { PERIOD } \\
\text { PRIOR } \\
\text { TO SAM- } \\
\text { PLING } \\
\text { (MIN) } \\
\text { ( } 72004 \text { ) }\end{array}$ \\
\hline \multicolumn{3}{|c|}{$440233092115101107 \mathrm{~N}-11 \mathrm{~W}-31$ AAAAAC } & $03-12-91$ & \multirow{2}{*}{$\begin{array}{l}1535 \\
1050 \\
1430 \\
1515 \\
1130\end{array}$} & \multirow{2}{*}{$16 \dot{5} .00$} & \multirow{2}{*}{$\begin{array}{l}262 \\
262 \\
250 \\
395 \\
220\end{array}$} & \multirow{2}{*}{$\begin{array}{l}25 \\
25 \\
24 \\
36 \\
27\end{array}$} \\
\hline $\begin{array}{l}440305092 \\
440330092 \\
440335092\end{array}$ & 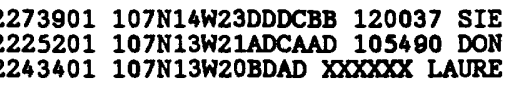 & $\begin{array}{l}367 \mathrm{PRDC} \\
367 \mathrm{PRDC}\end{array}$ & $\begin{array}{l}08-22-91 \\
08-21-91 \\
08-22-81\end{array}$ & & & & \\
\hline $\begin{array}{l}440336092 \\
440343092 \\
440346092 \\
440347092 \\
440350092\end{array}$ & 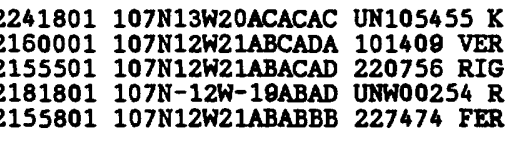 & $\begin{array}{l}\text { 367PRDC } \\
371 \mathrm{JRDN} \\
367 \mathrm{PRDC} \\
364 \mathrm{GLEN} \\
367 \mathrm{PRDC}\end{array}$ & $\begin{array}{l}08-22-81 \\
08-18-81 \\
08-20-81 \\
08-20-91 \\
08-18-81\end{array}$ & $\begin{array}{l}1245 \\
1730 \\
1230 \\
1530 \\
1245\end{array}$ & $\begin{array}{l}=- \\
=- \\
--\end{array}$ & $\begin{array}{r}282 \\
500 \\
296 \\
70 \\
181\end{array}$ & $\begin{array}{l}21 \\
30 \\
21 \\
21 \\
21\end{array}$ \\
\hline $\begin{array}{l}440352092 \\
440353092 \\
440354092 \\
440411092 \\
440412092\end{array}$ & 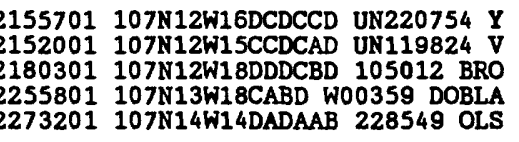 & $\begin{array}{l}\text { 367PRDC } \\
\text { 371JRDN } \\
367 \mathrm{PRDC} \\
367 \mathrm{PRDC} \\
\text { 367PRDC }\end{array}$ & $\begin{array}{l}08-19-91 \\
08-19-91 \\
08-20-91 \\
08-23-91 \\
08-21-91\end{array}$ & $\begin{array}{l}1415 \\
1545 \\
1400 \\
1745 \\
1045\end{array}$ & $\begin{array}{l}-- \\
-- \\
--\end{array}$ & $\begin{array}{l}295 \\
600 \\
350 \\
100 \\
156\end{array}$ & $\begin{array}{l}21 \\
21 \\
24 \\
21 \\
21\end{array}$ \\
\hline $\begin{array}{l}440415092 \\
440417092 \\
440433092 \\
440455092\end{array}$ & $\begin{array}{ll}2231901 & 107 N 13 W 16 C A A A D A \\
2255701 & 107 N 136145 \text { OLN } \\
252301 & 107 N 13 W 18 A B B A A C \text { UN228616 L } \\
2120601 & 107 N-12 W-12 \text { DDBDBB }\end{array}$ & $\begin{array}{l}\text { 371JRDN } \\
\text { 367PRDC } \\
\text { 367PRDC }\end{array}$ & $\begin{array}{l}08-21-91 \\
08-20-91 \\
08-23-91 \\
03-11-91 \\
07-18-91\end{array}$ & $\begin{array}{l}1815 \\
1645 \\
1215 \\
1815 \\
1705\end{array}$ & $\begin{array}{c}-- \\
-\overline{-} \\
125.00\end{array}$ & $\begin{array}{r}560 \\
140 \\
60 \\
520 \\
520\end{array}$ & $\begin{array}{l}21 \\
21 \\
27 \\
-- \\
20\end{array}$ \\
\hline $\begin{array}{l}440534092 \\
440535092 \\
440545092\end{array}$ & $\begin{array}{ll}2261701 & 107 \mathrm{~N} 13 \text { W07BBBBCD } 228650 \mathrm{KEI} \\
2233501 & 107 \mathrm{~N} 13 \text { WO9BABB } 421071 \mathrm{KIELY} \\
2264801 & 107 \mathrm{~N} 14 \text { WO1DCCABC } 228620 \mathrm{LAN}\end{array}$ & $\begin{array}{l}\text { 367PRDC } \\
371 \mathrm{JRDN} \\
\text { 371JRDN }\end{array}$ & $\begin{array}{l}08-21-91 \\
08-20-91 \\
08-21-91\end{array}$ & $\begin{array}{l}1330 \\
1115 \\
1200\end{array}$ & $\begin{array}{ll}-- \\
--\end{array}$ & $\begin{array}{l}290 \\
620 \\
384\end{array}$ & $\begin{array}{l}21 \\
21 \\
21\end{array}$ \\
\hline
\end{tabular}

\begin{tabular}{|c|c|c|c|c|c|c|c|c|c|c|}
\hline STATION & $\begin{array}{l}\text { SPE- } \\
\text { CIFIC } \\
\text { CON- } \\
\text { DUCT- } \\
\text { ANCE } \\
\text { (US/CM) } \\
(00095)\end{array}$ & $\begin{array}{c}\text { SPE- } \\
\text { CIFIC } \\
\text { CON- } \\
\text { DUCT- } \\
\text { ANCE } \\
\text { LAB } \\
\text { (US/CM) } \\
(90095)\end{array}$ & $\begin{array}{c}\text { PH } \\
\text { (STAND- } \\
\text { ARD } \\
\text { UNITS) } \\
(00400)\end{array}$ & $\begin{array}{c}\text { PH } \\
\text { LAB } \\
\text { (STAND- } \\
\text { ARD } \\
\text { UNITS) } \\
\text { (00403) }\end{array}$ & $\begin{array}{l}\text { OXID- } \\
\text { ATION } \\
\text { RED- } \\
\text { UCTION } \\
\text { POTEN- } \\
\text { TIAL } \\
\text { (MV) } \\
\text { (OOO9O) }\end{array}$ & $\begin{array}{l}\text { TEMPER- } \\
\text { ATURE } \\
\text { WATER } \\
\text { (DEG C) } \\
(00010)\end{array}$ & $\begin{array}{c}\text { OXYGEN, } \\
\text { DIS- } \\
\text { SOLVED } \\
(M G / L) \\
(00300)\end{array}$ & $\begin{array}{l}\text { CALCIUM } \\
\text { DIS- } \\
\text { SOLVED } \\
\text { (MG/L } \\
\text { AS CA) } \\
\text { (00915) }\end{array}$ & $\begin{array}{c}\text { MAGNE- } \\
\text { SIUM, } \\
\text { DIS- } \\
\text { SOLVED } \\
\text { (MG/L } \\
\text { AS MG) } \\
\text { (00925) }\end{array}$ & $\begin{array}{c}\text { SODIUM, } \\
\text { DIS- } \\
\text { SOLVED } \\
\text { (MG/L } \\
\text { AS NA) } \\
\text { (O0930) }\end{array}$ \\
\hline $\begin{array}{l}440233092115101 \\
440305092273901 \\
44033009225201 \\
440335092243401\end{array}$ & $\begin{array}{l}-- \\
627 \\
704 \\
494 \\
755\end{array}$ & $\begin{array}{l}-- \\
\overline{--} \\
502 \\
791\end{array}$ & $\begin{array}{l}7 .- \\
7.1 \\
7.2 \\
7.2\end{array}$ & $\begin{array}{l}-- \\
-- \\
7.6 \\
7.7 \\
7.6\end{array}$ & $\begin{array}{r}-- \\
-\overline{0} \\
0.4 \\
-0.1 \\
0.2\end{array}$ & $\begin{array}{l}13.0 \\
12.5 \\
11.0 \\
12.0\end{array}$ & $\begin{array}{r}-- \\
9.5 \\
7.9 \\
<0.1 \\
10.3\end{array}$ & $\begin{array}{r}-- \\
110 \\
73 \\
120\end{array}$ & $\begin{array}{l}-- \\
\overline{24} \\
21 \\
14\end{array}$ & $\begin{array}{l}-- \\
-- \\
5.2 \\
2.7 \\
8.1\end{array}$ \\
\hline $\begin{array}{l}440336092241801 \\
440343092160001 \\
440346092155501 \\
440347092181801 \\
440350092155801\end{array}$ & $\begin{array}{l}603 \\
614 \\
602 \\
611 \\
767\end{array}$ & $\begin{array}{l}600 \\
618 \\
585 \\
597 \\
780\end{array}$ & $\begin{array}{l}7.2 \\
7.3 \\
7.3 \\
7.2 \\
7.3\end{array}$ & $\begin{array}{l}8.0 \\
7.7 \\
7.7 \\
8.0 \\
7.6\end{array}$ & $\begin{array}{r}0.7 \\
-0.1 \\
-0.1 \\
0.2 \\
0.2\end{array}$ & $\begin{array}{r}12.0 \\
10.0 \\
16.0 \\
9.5 \\
11.5\end{array}$ & $\begin{array}{r}<0.1 \\
3.1 \\
<0.1 \\
7.6 \\
8.4\end{array}$ & $\begin{array}{r}90 \\
95 \\
92 \\
90 \\
120\end{array}$ & $\begin{array}{l}29 \\
21 \\
21 \\
21 \\
25\end{array}$ & $\begin{array}{l}3.5 \\
4.6 \\
3.7 \\
5.9 \\
15\end{array}$ \\
\hline $\begin{array}{l}440352092155701 \\
440353092152001 \\
440354092180301 \\
440411092255801 \\
440412092273201\end{array}$ & $\begin{array}{l}679 \\
482 \\
490 \\
572 \\
525\end{array}$ & $\begin{array}{r}678 \\
480 \\
487 \\
-- \\
531\end{array}$ & $\begin{array}{l}7.3 \\
7.4 \\
7.4 \\
7.4 \\
7.4\end{array}$ & $\begin{array}{l}7.9 \\
8.1 \\
7.9 \\
=- \\
8.0\end{array}$ & $\begin{array}{l}0.2 \\
0.0 \\
0.1 \\
0.3 \\
0.2\end{array}$ & $\begin{array}{l}10.0 \\
10.5 \\
11.5 \\
11.0 \\
11.5\end{array}$ & $\begin{array}{r}10.6 \\
6.2 \\
7.8 \\
9.5 \\
7.2\end{array}$ & $\begin{array}{r}110 \\
75 \\
70 \\
-- \\
70\end{array}$ & $\begin{array}{l}22 \\
19 \\
22 \\
- \\
26\end{array}$ & $\begin{array}{l}6.6 \\
2.2 \\
2.3 \\
-- \\
2.4\end{array}$ \\
\hline $\begin{array}{l}440415092231901 \\
440417092255701 \\
440433092252301 \\
440455092120601\end{array}$ & $\begin{array}{l}479 \\
622 \\
806 \\
-- \\
480\end{array}$ & $\begin{array}{r}462 \\
613 \\
811 \\
-- \\
--\end{array}$ & $\begin{array}{l}7.5 \\
7.4 \\
7.1 \\
7.4\end{array}$ & $\begin{array}{r}7.9 \\
7.8 \\
7.5 \\
-.- \\
--\end{array}$ & $\begin{array}{r}-0.2 \\
0.2 \\
0.3 \\
-- \\
--\end{array}$ & $\begin{array}{r}11.5 \\
11.0 \\
12.0 \\
15.5\end{array}$ & $\begin{array}{l}0.3 \\
8.8 \\
9.5 \\
-.- \\
0.2\end{array}$ & $\begin{array}{r}70 \\
94 \\
100 \\
-- \\
--\end{array}$ & $\begin{array}{l}21 \\
21 \\
32 \\
-- \\
--\end{array}$ & $\begin{array}{l}2.5 \\
3.6 \\
7.9 \\
-- \\
--\end{array}$ \\
\hline $\begin{array}{l}440534092261701 \\
440535092233501 \\
440545092264801\end{array}$ & $\begin{array}{l}568 \\
525 \\
502\end{array}$ & $\begin{array}{l}575 \\
502 \\
508\end{array}$ & $\begin{array}{l}7.3 \\
7.5 \\
7.3\end{array}$ & $\begin{array}{l}8.0 \\
7.8 \\
7.8\end{array}$ & $\begin{array}{r}0.2 \\
-0.2 \\
0.1\end{array}$ & $\begin{array}{l}12.5 \\
11.5 \\
12.0\end{array}$ & $\begin{array}{r}10.7 \\
<0.1 \\
7.3\end{array}$ & $\begin{array}{l}79 \\
70 \\
74\end{array}$ & $\begin{array}{l}27 \\
23 \\
22\end{array}$ & $\begin{array}{l}2.7 \\
2.5 \\
2.4\end{array}$ \\
\hline
\end{tabular}


QUALITY OF GROUND WATER

WATER QUALITY DATA, WATER YEAR OCTOBER 1990 TO SEPTEMBER 1991

OLMSTED COUNTY--Continued

\begin{tabular}{|c|c|c|c|c|c|c|c|c|c|c|}
\hline NUMBER & $\begin{array}{c}\text { POTAS- } \\
\text { SIUM, } \\
\text { DIS- } \\
\text { SOLVED } \\
\text { (MG/L } \\
\text { AS K } \\
\text { (D0935) }\end{array}$ & $\begin{array}{c}\text { BICAR- } \\
\text { BOLATE } \\
\text { WATER } \\
\text { WH IT } \\
\text { FIELD } \\
\text { MG/L AS } \\
\text { HCO3 } \\
(00450)\end{array}$ & $\begin{array}{c}\text { ALKA- } \\
\text { LINITY } \\
\text { WAT WI } \\
\text { TOT IT } \\
\text { FIELD } \\
\text { MG/L AS } \\
\text { CACO3 } \\
(00419)\end{array}$ & $\begin{array}{c}\text { ALKA- } \\
\text { LINITY } \\
\text { WAT WH } \\
\text { TOT FET } \\
\text { FIELD } \\
\text { MG/L AS } \\
\text { CACO3 } \\
(00410)\end{array}$ & $\begin{array}{c}\text { ALKA- } \\
\text { LINITY } \\
\text { IAAB } \\
\text { (MG/L } \\
\text { AS } \\
\text { CACO3) } \\
(90410)\end{array}$ & $\begin{array}{c}\text { SULFATE } \\
\text { DIS- } \\
\text { SOLVED } \\
\text { (MG/L } \\
\text { AS SO4) } \\
(00945)\end{array}$ & $\begin{array}{l}\text { CHIO- } \\
\text { RIDE, } \\
\text { DIS- } \\
\text { SOIVED } \\
\text { (MG/I } \\
\text { AS CL) } \\
\text { (00940) }\end{array}$ & $\begin{array}{l}\text { FLUO- } \\
\text { RIDE. } \\
\text { DIS- } \\
\text { SOIVED } \\
(M G / L \\
\text { AS F) } \\
(00950)\end{array}$ & $\begin{array}{c}\text { SILICA, } \\
\text { DIS- } \\
\text { SOLVED } \\
\text { (MG/L } \\
\text { AS } \\
\text { SIO2) } \\
(00955)\end{array}$ & $\begin{array}{c}\text { SOLIDS, } \\
\text { RESIDUE } \\
\text { AT } 180 \\
\text { DEG. C } \\
\text { DIS- } \\
\text { SOLVED } \\
\text { (MG/L) } \\
(70300)\end{array}$ \\
\hline 440233092115101 & -- & -- & -- & -- & -- & -- & -- & -- & -- & -- \\
\hline $\begin{array}{l}440305092273901 \\
440330092225201 \\
440335092243401\end{array}$ & $\begin{array}{l}1.6 \\
12.6\end{array}$ & $\begin{array}{l}373 \\
321 \\
370\end{array}$ & $\begin{array}{l}306 \\
264 \\
297\end{array}$ & $\begin{array}{l}306 \\
263 \\
303\end{array}$ & $\begin{array}{l}276 \\
306\end{array}$ & $\begin{array}{l}\overline{54} \\
22 \\
38\end{array}$ & $\begin{array}{l}14 \\
0.60 \\
31\end{array}$ & $\begin{array}{l}-- \\
0.1 \\
0.2 \\
0.2\end{array}$ & $\underset{9.7}{13}$ & $\begin{array}{l}450 \\
270 \\
462\end{array}$ \\
\hline $\begin{array}{l}440336092241801 \\
44034309216001 \\
440346092155501 \\
440347092181801 \\
440350092155801\end{array}$ & $\begin{array}{l}1.7 \\
0.6 \\
0.6 \\
1.3 \\
1.8\end{array}$ & $\begin{array}{r}354 \\
339 \\
337 \\
-- \\
374\end{array}$ & $\begin{array}{l}286 \\
278 \\
276 \\
-- \\
311\end{array}$ & $\begin{array}{l}290 \\
278 \\
276 \\
-- \\
307\end{array}$ & $\begin{array}{r}-- \\
253 \\
275 \\
317\end{array}$ & $\begin{array}{l}48 \\
64 \\
44 \\
19 \\
35\end{array}$ & $\begin{array}{l}4.4 \\
8.9 \\
6.2 \\
15 \\
41\end{array}$ & $\begin{array}{l}0.2 \\
0.1 \\
0.2 \\
0.2 \\
0.1\end{array}$ & $\begin{array}{l}11 \\
14 \\
14 \\
20 \\
17\end{array}$ & $\begin{array}{l}341 \\
344 \\
351 \\
349 \\
459\end{array}$ \\
\hline $\begin{array}{l}440352092155701 \\
440353092152001 \\
440354092180301 \\
440411092255801 \\
440412092273201\end{array}$ & \begin{tabular}{l}
1.5 \\
0.5 \\
0.7 \\
\hdashline-.1
\end{tabular} & $\begin{array}{r}352 \\
316 \\
316 \\
-- \\
295\end{array}$ & $\begin{array}{l}293 \\
261 \\
257 \\
-- \\
237\end{array}$ & $\begin{array}{r}289 \\
259 \\
259 \\
-- \\
242\end{array}$ & $\begin{array}{r}-- \\
249 \\
--\end{array}$ & $\begin{array}{l}27 \\
17 \\
16 \\
-- \\
20\end{array}$ & $\begin{array}{l}23 \\
0.80 \\
1.0 \\
13.0 \\
8,4\end{array}$ & $\begin{array}{l}0.1 \\
0.2 \\
0.1 \\
-.1 \\
0.1\end{array}$ & $\begin{array}{l}16 \\
15 \\
13 \\
-- \\
12\end{array}$ & $\begin{array}{l}393 \\
271 \\
259 \\
-- \\
305\end{array}$ \\
\hline $\begin{array}{l}440415092231901 \\
440417092255701 \\
440433092252301 \\
440455092120601\end{array}$ & $\begin{array}{l}1.1 \\
1.4 \\
24 \\
--\end{array}$ & $\begin{array}{r}332 \\
322 \\
411 \\
-- \\
--\end{array}$ & $\begin{array}{r}273 \\
266 \\
339 \\
-- \\
--\end{array}$ & $\begin{array}{r}272 \\
264 \\
337 \\
-- \\
--\end{array}$ & $\begin{array}{r}-- \\
345 \\
-- \\
--\end{array}$ & $\begin{array}{l}13 \\
20 \\
32 \\
-- \\
--\end{array}$ & $\begin{array}{l}0.70 \\
13 \\
30 \\
-- \\
--\end{array}$ & $\begin{array}{l}0.2 \\
0.1 \\
0.2 \\
-- \\
--\end{array}$ & $\begin{array}{l}10 \\
13 \\
20 \\
-- \\
--\end{array}$ & $\begin{array}{r}267 \\
359 \\
491 \\
-- \\
--\end{array}$ \\
\hline $\begin{array}{l}440534092261701 \\
440535092233501 \\
440545092264801\end{array}$ & $\begin{array}{l}1.2 \\
1.1 \\
1.2\end{array}$ & $\begin{array}{l}344 \\
340 \\
328\end{array}$ & $\begin{array}{l}284 \\
283 \\
271\end{array}$ & $\begin{array}{l}282 \\
279 \\
269\end{array}$ & $\begin{array}{l}=- \\
--\end{array}$ & $\begin{array}{l}24 \\
14 \\
19\end{array}$ & $\begin{array}{l}7.5 \\
1.3 \\
0.80\end{array}$ & $\begin{array}{l}0.1 \\
0.2 \\
0.2\end{array}$ & $\begin{array}{l}13 \\
11 \\
11\end{array}$ & $\begin{array}{l}327 \\
277 \\
276\end{array}$ \\
\hline
\end{tabular}

\begin{tabular}{|c|c|c|c|c|c|c|c|c|c|}
\hline NUMBER & $\begin{array}{c}\text { NITRO- } \\
\text { GEN, } \\
\text { MITRITE } \\
\text { DIS- } \\
\text { SOLVED } \\
\text { (MG/L } \\
\text { AS IN) } \\
\text { (00613) }\end{array}$ & $\begin{array}{c}\text { NITRO- } \\
\text { GEN, } \\
\text { MO2+NO3 } \\
\text { DIS- } \\
\text { SOLVED } \\
\text { (MG/L } \\
\text { AS NI) } \\
(00631)\end{array}$ & $\begin{array}{c}\text { NITRO- } \\
\text { GER, } \\
\text { AMONIA } \\
\text { DIS- } \\
\text { SOLVED } \\
\text { (MG/L } \\
\text { AS H) } \\
\text { (00608) }\end{array}$ & $\begin{array}{c}\text { PHOS- } \\
\text { PHORUS } \\
\text { DIS- } \\
\text { SOLVED } \\
\text { (MG/L } \\
\text { AS P) } \\
(00666)\end{array}$ & $\begin{array}{l}\text { PHOS- } \\
\text { PHORUS } \\
\text { ORTHO, } \\
\text { DIS- } \\
\text { SOLVED } \\
\text { (MG/L } \\
\text { AS P) } \\
(00671)\end{array}$ & $\begin{array}{l}\text { IRON, } \\
\text { DIS- } \\
\text { SOLVED } \\
\text { (UG/L } \\
\text { AS FE) } \\
\text { (01046) }\end{array}$ & $\begin{array}{l}\text { MANGA- } \\
\text { NESE, } \\
\text { DIS- } \\
\text { SOLVED } \\
\text { (UG/L } \\
\text { AS MN) } \\
\text { (01056) }\end{array}$ & $\begin{array}{l}\text { TRITIUM } \\
\text { TOTAL } \\
(\text { PCI/L) } \\
(07000)\end{array}$ & $\begin{array}{l}\text { TRITIUM } \\
\text { IN } \\
\text { WATER } \\
\text { MOLE- } \\
\text { CULES } \\
\text { (TU) } \\
\text { (07012) }\end{array}$ \\
\hline $\begin{array}{l}440233092115101 \\
440305092273901 \\
440330092225201 \\
440335092243401\end{array}$ & $\begin{array}{r}0.01 \\
<0.01 \\
<0.01 \\
<0.01 \\
<0.01\end{array}$ & $\begin{aligned} 4.4 \\
4.3 \\
3.5 \\
<0.05 \\
10.0\end{aligned}$ & $\begin{array}{r}<0.01 \\
0.01 \\
<0.01 \\
0.12 \\
0.02\end{array}$ & $\begin{array}{r}-- \\
-\overline{0} \\
0.02 \\
<0.01 \\
0.04\end{array}$ & $\begin{array}{r}0.02 \\
<0.01 \\
0.02 \\
<0.01 \\
0.01\end{array}$ & $\begin{array}{r}-- \\
\overline{14} \\
2000 \\
10\end{array}$ & $\begin{array}{l}-- \\
-- \\
<1 \\
33 \\
<1\end{array}$ & $\begin{array}{l}=- \\
=- \\
=- \\
=-\end{array}$ & $\begin{array}{r}-- \\
\overline{22.8} \\
0.8 \\
15.3\end{array}$ \\
\hline $\begin{array}{l}440336092241801 \\
440343092160001 \\
44034609215501 \\
440347092181801 \\
440350092155801\end{array}$ & $\begin{array}{l}<0.01 \\
<0.01 \\
<0.01 \\
<0.01 \\
-.-\end{array}$ & $\begin{array}{l}<0.05 \\
<0.05 \\
<0.05 \\
7.0 \\
-.\end{array}$ & $\begin{array}{r}<0.01 \\
0.02 \\
<0.01 \\
0.02 \\
--\end{array}$ & $\begin{array}{c}<0.01 \\
<0.01 \\
<0.01 \\
0.03 \\
-.\end{array}$ & $\begin{array}{l}<0.01 \\
<0.01 \\
<0.01 \\
<0.01\end{array}$ & $\begin{array}{r}5 \\
510 \\
2700 \\
<3 \\
9\end{array}$ & $\begin{array}{l}<1 \\
36 \\
29 \\
<1 \\
<1\end{array}$ & $\begin{array}{l}=- \\
-- \\
=- \\
--\end{array}$ & $\begin{array}{r}<0.8 \\
8.4 \\
<0.8 \\
25.7\end{array}$ \\
\hline $\begin{array}{l}440352092155701 \\
440353092152001 \\
440354092180301 \\
440411092255801 \\
440412092273201\end{array}$ & $\begin{array}{l}<0.01 \\
<0.01 \\
<0.01 \\
<0.01\end{array}$ & $\begin{array}{r}6.70 \\
<0.05 \\
<0.05 \\
6.5 \\
4.1\end{array}$ & $\begin{array}{l}<0.01 \\
<0.01 \\
<0.01 \\
-.01 \\
0.01\end{array}$ & $\begin{array}{r}0.03 \\
<0.01 \\
<0.01 \\
--0 \\
<0.01\end{array}$ & $\begin{array}{r}0.01 \\
<0.01 \\
<0.01 \\
<-0 \\
<0.01\end{array}$ & $\begin{array}{r}9 \\
280 \\
220 \\
11\end{array}$ & $\begin{array}{r}<1 \\
22 \\
6 \\
--\frac{1}{<1}\end{array}$ & $\begin{array}{l}67 \\
-- \\
-- \\
=-\end{array}$ & $\begin{array}{l}22.9 \\
<0.8 \\
<0.8 \\
-\overline{15.8}\end{array}$ \\
\hline $\begin{array}{l}440415092231901 \\
440417092255701 \\
440433092252301 \\
440455092120601\end{array}$ & $\begin{array}{l}<0.01 \\
<0.01 \\
<0.01 \\
0.01 \\
<0.01\end{array}$ & $\begin{array}{c}<0.05 \\
7.6 \\
5.2 \\
<0.05 \\
<0.05\end{array}$ & $\begin{array}{r}0.06 \\
<0.01 \\
0.03 \\
0.02 \\
0.05\end{array}$ & $\begin{array}{c}<0.01 \\
0.02 \\
0.29 \\
-- \\
--\end{array}$ & $\begin{array}{r}<0.01 \\
<0.01 \\
0.11 \\
<0.01 \\
<0.01\end{array}$ & $\begin{array}{r}4100 \\
4 \\
3 \\
-- \\
--\end{array}$ & $\begin{array}{l}69 \\
<1 \\
<1 \\
-- \\
--\end{array}$ & $\begin{array}{l}-- \\
74 \\
-- \\
--\end{array}$ & $\begin{array}{l}<0.8 \\
25.2 \\
20.1 \\
=- \\
=-\end{array}$ \\
\hline $\begin{array}{l}440534092261701 \\
440535092233501 \\
440545092264801\end{array}$ & $\begin{array}{r}<0.01 \\
<0.01 \\
0.01\end{array}$ & $\begin{array}{l}3.0 \\
<0.05 \\
<0.05\end{array}$ & $\begin{array}{l}0.02 \\
0.05 \\
0.02\end{array}$ & $\begin{array}{l}<0.01 \\
<0.01 \\
<0.01\end{array}$ & $\begin{array}{l}<0.01 \\
<0.01 \\
<0.01\end{array}$ & $\begin{array}{r}10 \\
5500 \\
79\end{array}$ & $\begin{array}{l}<1 \\
59 \\
11\end{array}$ & $\begin{array}{l}-- \\
--\end{array}$ & $\begin{array}{l}13.2 \\
<0.8 \\
<0.8\end{array}$ \\
\hline
\end{tabular}


QUALITY OF GROUND WATER

WATER QUALITY DATA, WATER YEAR OCTOBER 1990 TO SEPTEMBER 1991

REDWOOD COUNTY

\begin{tabular}{|c|c|c|c|c|c|c|c|c|c|c|}
\hline STATIOA & NUMBER & & $\begin{array}{l}\text { LOCAL } \\
\text { IDENT- } \\
\text { I- } \\
\text { FIER }\end{array}$ & & & DATE & TIME & $\begin{array}{l}\text { DEPTH } \\
\text { BELON } \\
\text { LAND } \\
\text { SURFACE } \\
\text { (WATER } \\
\text { LEVEL) } \\
\text { (FEET) } \\
\text { (72019) }\end{array}$ & $\begin{array}{l}\text { DEPTH } \\
\text { OF } \\
\text { WELL, } \\
\text { TOTAL } \\
\text { (FEET) } \\
(72008)\end{array}$ & \\
\hline \multicolumn{2}{|c|}{441753095160801} & \multicolumn{2}{|c|}{$110 \mathrm{~N}-37 \mathrm{~W}-27$} & \multicolumn{2}{|l|}{ DADDCD } & $\begin{array}{l}03-22-91 \\
08-08-91\end{array}$ & $\begin{array}{l}1420 \\
1210\end{array}$ & 20.00 & 44 & \\
\hline STATIOA & NUMBER & $\begin{array}{c}\text { PUMP } \\
\text { OR FLOW } \\
\text { PERIOD } \\
\text { PRIOR } \\
\text { TO SAM- } \\
\text { PLING } \\
\text { (MIN) } \\
\text { (72004) }\end{array}$ & $\begin{array}{l}\text { SPE- } \\
\text { CIFIC } \\
\text { CON- } \\
\text { DUCT- } \\
\text { ANCE } \\
\text { (US/CM) } \\
\text { (00095) }\end{array}$ & $\begin{array}{c}\text { PH } \\
\text { (STAND- } \\
\text { ARD } \\
\text { UNITS) } \\
(00400)\end{array}$ & $\begin{array}{c}\text { TEMPER- } \\
\text { ATURE } \\
\text { WATER } \\
\text { (DEG C) } \\
(00010)\end{array}$ & $\begin{array}{c}\text { OXYGEN, } \\
\text { DIS- } \\
\text { SOLVED } \\
(M G / L) \\
(00300)\end{array}$ & $\begin{array}{c}\text { NITRO- } \\
\text { GEN } \\
\text { NITRITE } \\
\text { DIS- } \\
\text { SOLVED } \\
\text { (MG/L } \\
\text { AS N ) } \\
\text { (00613) }\end{array}$ & $\begin{array}{c}\text { NITRO- } \\
\text { GEN, } \\
\text { NO2+NO3 } \\
\text { DIS- } \\
\text { SOLVED } \\
(\text { MG/L } \\
\text { AS N) } \\
\text { (00631) }\end{array}$ & $\begin{array}{c}\text { NITRO- } \\
\text { GEN, } \\
\text { AMONIA } \\
\text { DIS- } \\
\text { SOLVED } \\
\text { (MG/L } \\
\text { AS N) } \\
(00608)\end{array}$ & $\begin{array}{c}\text { PHOS- } \\
\text { PHORUS } \\
\text { ORTHO, } \\
\text { DIS- } \\
\text { SOLVED } \\
(M G / L \\
\text { AS P) } \\
(00671)\end{array}$ \\
\hline 441753095 & 160801 & $\overline{30}$ & 1020 & $6 . \overline{7}$ & 12.5 & $0 . \overline{1}$ & $\begin{array}{r}<0.01 \\
0.15\end{array}$ & $14^{6.9}$ & $\begin{array}{l}0.04 \\
0.07\end{array}$ & $\begin{array}{r}0.01 \\
<0.01\end{array}$ \\
\hline
\end{tabular}

WABASHA COUNTY

\begin{tabular}{|c|c|c|c|c|c|c|c|}
\hline STATIOA & NUMBER & $\begin{array}{l}\text { LOCAL } \\
\text { IDENT- } \\
\text { I- } \\
\text { FIER }\end{array}$ & DATE & TIME & $\begin{array}{l}\text { BELOW } \\
\text { LAND } \\
\text { SURFACE } \\
\text { (WATER } \\
\text { LEVEL) } \\
\text { (FEET) } \\
\text { (72019) }\end{array}$ & $\begin{array}{l}\text { DEPTH } \\
\text { OF } \\
\text { WELL, } \\
\text { TOTAL } \\
\text { (FEET) } \\
\text { (72008) }\end{array}$ & $\begin{array}{c}\text { OR FLOW } \\
\text { PERIOD } \\
\text { PRIOR } \\
\text { TO SAM- } \\
\text { PLING } \\
\text { (MIN) } \\
(72004)\end{array}$ \\
\hline 380 & 42401 & 109N-11W-01 AACBAD & $\begin{array}{l}03-11-91 \\
07-18-91\end{array}$ & $\begin{array}{l}1620 \\
1240\end{array}$ & 72.00 & $\begin{array}{l}98 \\
98\end{array}$ & $-\overline{5}$ \\
\hline
\end{tabular}

\begin{tabular}{|c|c|c|c|c|c|c|c|c|c|}
\hline STATION & NUMBER & $\begin{array}{l}\text { SPE- } \\
\text { CIFIC } \\
\text { CON- } \\
\text { DUCT- } \\
\text { ANCE } \\
\text { (US/CM) } \\
\text { (O0095) }\end{array}$ & $\begin{array}{c}\text { PH } \\
\text { (STAND- } \\
\text { ARD } \\
\text { UNITS) } \\
(00400)\end{array}$ & $\begin{array}{c}\text { TEMPER- } \\
\text { ATURE } \\
\text { WATER } \\
\text { (DEG C) } \\
(00010)\end{array}$ & $\begin{array}{c}\text { OXYGEN, } \\
\text { DIS- } \\
\text { SOLVED } \\
\text { (MG/L) } \\
(00300)\end{array}$ & $\begin{array}{c}\text { NITRO- } \\
\text { GEN, } \\
\text { NITRITE } \\
\text { DIS- } \\
\text { SOLVED } \\
\text { (MG/L } \\
\text { AS N) } \\
\text { (00613) }\end{array}$ & $\begin{array}{c}\text { NITRO- } \\
\text { GEN, } \\
\text { NO2+NO3 } \\
\text { DIS- } \\
\text { SOLVED } \\
\text { (MG/L } \\
\text { AS N) } \\
(00631)\end{array}$ & $\begin{array}{c}\text { NITRO- } \\
\text { GEN, } \\
\text { AMONIA } \\
\text { DIS- } \\
\text { SOLVED } \\
\text { (MG/L } \\
\text { AS N ) } \\
\text { (00608) }\end{array}$ & $\begin{array}{c}\text { PHOS- } \\
\text { PHORUS } \\
\text { ORTHO, } \\
\text { DIS- } \\
\text { SOLVED } \\
\text { (MG/L } \\
\text { AS P) } \\
(00671)\end{array}$ \\
\hline 44163808 & 42401 & $\overline{558}$ & $\overline{7.3}$ & 15.0 & $\overline{7.6}$ & $\begin{array}{r}0.01 \\
<0.01\end{array}$ & $\begin{array}{l}2.4 \\
2.3\end{array}$ & $\begin{array}{r}<0.01 \\
0.02\end{array}$ & $\begin{array}{r}0.02 \\
<0.01\end{array}$ \\
\hline
\end{tabular}


QUALITY OF GROUND WATER

WATER QUALITY DATA, WATER YEAR OCTOBER 1990 TO SEPTEMBER 1991

WATONWAN COUNTY

\begin{tabular}{|c|c|c|c|c|c|c|c|c|c|c|}
\hline STATION & NUMBER & & $\begin{array}{l}\text { LOCAL } \\
\text { IDENT- } \\
\text { I- } \\
\text { FIER }\end{array}$ & & & DATE & TIME & $\begin{array}{c}\text { DEPTH } \\
\text { BELOW } \\
\text { LAND } \\
\text { SURFACEE } \\
\text { (WATER } \\
\text { LEVBL) } \\
\text { (FEET) } \\
\text { (72019) }\end{array}$ & $\begin{array}{l}\text { DEPTH } \\
\text { OF } \\
\text { WELL, } \\
\text { TOTAL } \\
\text { (FEET) } \\
\text { (72008) }\end{array}$ & \\
\hline 440037094 & 372601 & $106 \mathrm{~N}$ & 32W01DDBO & & & $\begin{array}{l}03-26-91 \\
07-22-91\end{array}$ & $\begin{array}{l}1225 \\
1800\end{array}$ & $\overline{12.86}$ & $\begin{array}{l}22 \\
22\end{array}$ & \\
\hline STATION & NUMBER & $\begin{array}{c}\text { PUMP } \\
\text { OR FLOW } \\
\text { PERIOD } \\
\text { PRIOR } \\
\text { TO SAM- } \\
\text { PLING } \\
\text { (MIN) } \\
\text { (72004) }\end{array}$ & $\begin{array}{l}\text { SPE- } \\
\text { CIFIC } \\
\text { CON- } \\
\text { DUCT- } \\
\text { ANCE } \\
\text { (US/CM) } \\
(00095)\end{array}$ & $\begin{array}{c}\text { PH } \\
\text { (STAND- } \\
\text { ARD } \\
\text { UNITS) } \\
(00400)\end{array}$ & $\begin{array}{c}\text { TEMPER- } \\
\text { ATURE } \\
\text { WATER } \\
\text { (DEG C) } \\
(00010)\end{array}$ & $\begin{array}{c}\text { OXYGEN, } \\
\text { DIS- } \\
\text { SOLVED } \\
\text { (MG } / L) \\
(00300)\end{array}$ & $\begin{array}{c}\text { NITRO- } \\
\text { GEN, } \\
\text { NITRITE } \\
\text { DIS- } \\
\text { SOIVED } \\
\text { (MG/L } \\
\text { AS N) } \\
\text { (00613): }\end{array}$ & $\begin{array}{c}\text { NITRO- } \\
\text { GEN, } \\
\text { NO2+HO3 } \\
\text { DIS- } \\
\text { SOLVED } \\
\text { (MG/L } \\
\text { AS N) } \\
(00631)\end{array}$ & $\begin{array}{c}\text { NITRO- } \\
\text { GEH, } \\
\text { AMONIA } \\
\text { DIS- } \\
\text { SOLVED } \\
\text { (MG/L } \\
\text { AS H) } \\
\text { (00608) }\end{array}$ & $\begin{array}{c}\text { PHOS- } \\
\text { PHORUS } \\
\text { ORTHO, } \\
\text { DIS- } \\
\text { SOLVED } \\
(\text { MG /I } \\
\text { AS P) } \\
(00671)\end{array}$ \\
\hline 440037094 & 372601 & $\overline{23}$ & $19 \overline{30}$ & $\overline{6 .} \overline{6}$ & $12 . \overline{0}$ & 3.0 & $\begin{array}{l}<0.01 \\
<0.01\end{array}$ & $\begin{array}{l}<0.05 \\
<0.05\end{array}$ & $\begin{array}{l}0.03 \\
0.04\end{array}$ & $\begin{array}{l}<0.01 \\
<0.01\end{array}$ \\
\hline
\end{tabular}

YELLOW MEDICINE COUNTY

\begin{tabular}{|c|c|c|c|c|c|c|}
\hline STATION & NUMBER & $\begin{array}{c}\text { IOCAL } \\
\text { IDENT- } \\
\text { I- } \\
\text { FIER }\end{array}$ & DATE & TIME & $\begin{array}{c}\text { LAND } \\
\text { SURFACE } \\
\text { (WATER } \\
\text { LEVEL) } \\
\text { (FEET) } \\
\text { (72019) }\end{array}$ & $\begin{array}{l}\text { DEPTH } \\
\text { OF } \\
\text { WELL, } \\
\text { TOTAL } \\
\text { (FEET) } \\
\text { (72008) }\end{array}$ \\
\hline 44430709 & 245801 & $115 N-46 \mathrm{~W}-33 \mathrm{CCDDAA}$ & $\begin{array}{l}03-27-91 \\
08-07-91\end{array}$ & $\begin{array}{l}1650 \\
1725\end{array}$ & 10.00 & $\begin{array}{l}25 \\
25\end{array}$ \\
\hline
\end{tabular}

\begin{tabular}{|c|c|c|c|c|c|c|c|c|c|c|}
\hline STATION & NUMBER & $\begin{array}{c}\text { PUMP } \\
\text { OR FLOW } \\
\text { PERIOD } \\
\text { PRIOR } \\
\text { TO SAM- } \\
\text { PLING } \\
\text { (MIN) } \\
(72004)\end{array}$ & $\begin{array}{l}\text { SPE- } \\
\text { CIFIC } \\
\text { CON- } \\
\text { DUCT- } \\
\text { ANCE } \\
\text { (US/CY) } \\
\text { (00095) }\end{array}$ & $\begin{array}{c}\text { PH } \\
\text { (STAND- } \\
\text { ARD } \\
\text { UNITS) } \\
(00400)\end{array}$ & $\begin{array}{c}\text { TEMPER- } \\
\text { ATURE } \\
\text { WATER } \\
\text { (DEG C) } \\
(00010)\end{array}$ & $\begin{array}{c}\text { OXYGEN, } \\
\text { DIS- } \\
\text { SOLVED } \\
(M G / L) \\
(00300)\end{array}$ & $\begin{array}{c}\text { NITRO- } \\
\text { GEN, } \\
\text { NITRITE } \\
\text { DIS- } \\
\text { SOLVED } \\
\text { (MG/L } \\
\text { AS N) } \\
(00613)\end{array}$ & $\begin{array}{c}\text { NITRO- } \\
\text { GEN, } \\
\text { NO2+NO3 } \\
\text { DIS- } \\
\text { SOLVED } \\
\text { (MG/L } \\
\text { AS N }) \\
(00631)\end{array}$ & $\begin{array}{c}\text { NITRO- } \\
\text { GEN, } \\
\text { AMMONIA } \\
\text { DIS- } \\
\text { SOLVED } \\
\text { (MG/L } \\
\text { AS N) } \\
(00608)\end{array}$ & $\begin{array}{l}\text { PHOS- } \\
\text { PHORUS } \\
\text { ORTHO, } \\
\text { DIS- } \\
\text { SOLVED } \\
\text { (MG/L } \\
\text { AS P) } \\
(00671)\end{array}$ \\
\hline 44430709 & 45801 & $\overline{26}$ & 570 & 6.9 & 12.5 & 0.1 & $\begin{array}{l}<0.01 \\
<0.01\end{array}$ & $\begin{array}{l}<0.05 \\
<0.05\end{array}$ & $\begin{array}{l}0.14 \\
0.16\end{array}$ & $\begin{array}{l}<0.01 \\
<0.01\end{array}$ \\
\hline
\end{tabular}


CHEMICAL QUALITY OF PRECIPITATION

461458094295000 PRECIPITATION STATION AT CAMP RIPLEY, MN

WATER QUALITY DATA, WATER YEAR OCTOBER 1990 TO SEPTEMBER 1991

LOCATION. - Lat $46^{\circ} 14^{\prime} 58^{\prime \prime}$, Long $94^{\circ} 29^{\prime} 50^{\prime \prime}$, in NEx sec.18, T.132 N., R.30 W., Morrison County, Hydrologic Unit 07010104 , approximately $500 \mathrm{ft}$ southwest of the abandoned Gilgal Church and approximately 5 miles south of the town of Pillager.

PERIOD OF RECORD.--October 1983 to current year (weekly composite).

INSTRUMENTATION.--Samples are collected in a polyethylene bucket by an electrically operated wet/dry collector. A recording rain gage and a standard U.S. Weather Service bulk rain gage measure rainfall quantity.

REMARKS. - An observer collects only the wetfall bucket and services the rain gages every Tuesday around 0900 hours. The observer weighs the bucket and if there is enough wetfall, determines specific conductance and pH. The bucket with its remaining contents is then sent to the Illinois State Water Survey Laboratory for analysis.

CHEMICAL ANALYSIS, WATER YEAR OCTOBER 1990 TO SEPTEMBER 1991

WEEKLY COMPOSITE

GREEN-
WICH
MEAN
TIME

$\begin{array}{ccccccc}\text { TOTAL } & \text { SPEC } & \text { SPEC } & & & & \\ \text { PRECIP- } & \text { CONDUC- } & \text { CONDUC- } & & & & \\ \text { ITATION } & \text { TANCE } & \text { TANCE } & \text { PH } & \text { PH } & & \text { MAG- } \\ \text { FOR } & \text { FIELD } & \text { LAB } & \text { FIELD } & \text { LAB } & \text { CALCIUM } & \text { NESIUM } \\ \text { DEFINED } & \text { ATM DEP } & \text { ATM DEP } & \text { ATM DEP } & \text { ATM DEP } & \text { ATM DEP } & \text { ATM DEP } \\ \text { PERIOD } & \text { WET TOT } & \text { WET TOT } & \text { WET T } & \text { WET T } & \text { WET DIS } & \text { WET DIS } \\ \text { (IN) } & (U S / C M) & (U S / C M) & (U N I T S) & (U N I T S) & (M G / L) & (M G / L) \\ (00193) & (83154) & (83156) & (83106) & (83107) & (82932) & (83002)\end{array}$

OCT

OCT

09-16

OCT

${ }^{16-23}$

23-30

OCT $30-$

NOV 06

NOV

06-13

NOV

$13-20$

NOV

20-27

NOV $27-$

DEC 04

DEC

04-11

DEC

11-18

DEC

18-25

DEC 251990 -

JAN 011991

JAN

01-08

JAN

08-15

JAN

15-22

JAN

22-29

JAN 29-

FEB 05

FEB

05-12

FEB

12-19

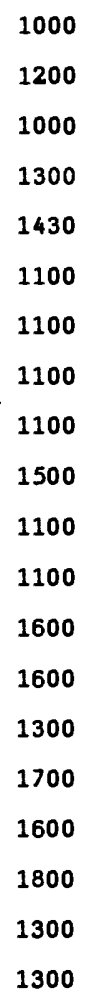

1.95

8.7

$9.7 \quad 5.25$

6.53

0.620

0.100

0.0

$-$

19.3

$-$

6.79

0.700

0.170

1.58

14.7

11.0

4.70

5.07

0.310

0.033

$-$

0.01

$-$

$-$

$-$

$-$

--

-

$$
0.0
$$

19.

6.43

0.830

0.172

0.03

$-$

--

16.6

5.09

0.300

0.067

0.20

54.7

46.7

4.60

4.62

0.340

0.046

0.30

7.1

5.8

4.70

5.63

0.110

0.026

0.0

$-$

$--$

$--$

$--$

--

$-$

0.01 --

8.3

5.71

0.100

0.023

0.75

11.4

$10.0 \quad 4.90$

4.76

0.190

0.024

0.0

$\cdots$

-

0.02

$-$

$-$

$-$

$-$

$-$

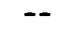

$$
0.24
$$

10.9

9.5

4.70

4.75

0.050

0.008

0.11

11.2

9.1

4.90

5. 78

0.330

0.061

0.15

4.4

3.3

5.10

5.80

0.090

0.015

0.0

-.

1.3 -.

$5.78<0.010$

$<0.003$

0.0

$-$

1.7

$-$

5.80

$<0.010$

$<0.003$

$0.58 \quad 10.7$

$9.1 \quad 4.80$

4.89

0.100

0.017 
461458094295000 - PRECIPITATION STATION AT CAMP RIPLEY, MN--Continued

CHEMICAL ANALYSIS, WATER YEAR OCTOBLR 1990 TO SEPTEMBER 1991

WEEKLY COMPOSITE

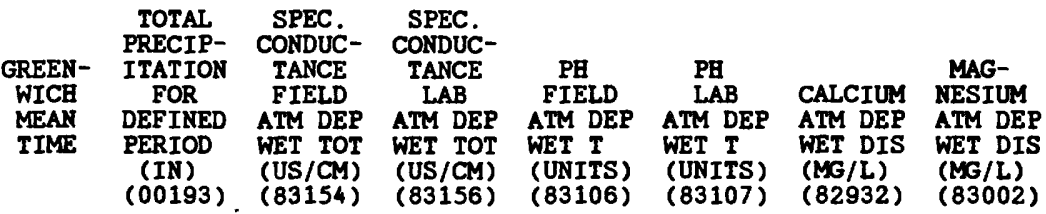

FEB

$19-26$

FEB 26-

MAR

05-12

MAR

12-19

MAR

19-26

MAR 26-

APR 02

APR

02-09

APR

09-16

APR

16-23

APR

23-30

APR 30-

MAY 07

MAY

07-14

MAY

14-21

MAY $21-28$

$21-28$
$28-$

JUN 04

JUN

04-11

JUN

11-18

JUN

JUN 25-

JUL 02

JUL.

02-09

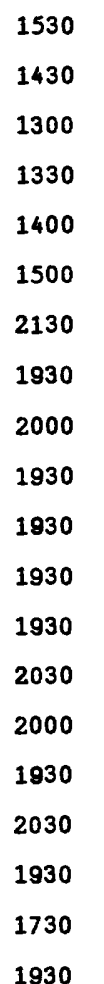

4.9

3.7

5.10

5.67

0.100

0.012

0.07

$--$

$-$

$--$

$-$

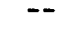

$--$

$\begin{array}{lllllll}0.0 & - & 1.7 & 5 & 5.85 & 0.090<0.003\end{array}$

0.0

$3.4--$

5.96

$<0.010$

0.003

1.94

11.2

$14.7 \quad 4.50$

4.67

0.210

0.019

0.03

$-$

25.0 --

6.82

0.820

0.237

0.25

17.8

$3.8 \quad 4.40$

5.38

0.100

0.016

2.22

12.0

$13.1 \quad 4.60$

4.62

0.100

0.015

0.06

27.1

$14.7 \quad 4.60$

6.44

0.220

0.041

1.97

11.2

9.3

4.80

5.05

0.160

0.024

1.25

8.4

$5.9 \quad 5.10$

5.08

0.070

0.006

0.46

17.1

$10.6 \quad 5.40$

6.42

0.400

0.056

0.10

13.5

$12.4 \quad 5.65$

6.49

0.380

0.088

0.95

6.5

$5.9 \quad 5.40$

6.13

0.150

0.030

1.04

7.8

7.2

5.50

5.47

0.190

0.019

0.05

35.6

$--$

6.14

1.17

0.164

2. 37

7.0

$6.5 \quad 5.40$

5.96

0.090

0.013

1.65

9.6

8.8

5.00

5.95

0.400

0.043

5.08

8.4

8.8

5.40

6.18

0.460

0.069

0.14

8.8

7.6

5.10

$5.80 \quad 0.280$

0.047 
CBEMICAL QUALITY OF PRECIPITATION

461458094295000 - PRECIPITATION STATION AT CAMP RIPLEY, MN--Continued

WATER QUALITY DATA, WATER YEAR OCTOBER 1990 TO SEPTEMBER 1991

WEEKLY COMPOSITE

\begin{tabular}{|c|c|c|c|c|c|c|c|c|}
\hline DATE & $\begin{array}{l}\text { GREEN- } \\
\text { WICH } \\
\text { MEAN } \\
\text { TIME }\end{array}$ & $\begin{array}{l}\text { TOTAL } \\
\text { PRECIP- } \\
\text { ITATION } \\
\text { FOR } \\
\text { DEFINED } \\
\text { PERIOD } \\
\text { (IN) } \\
\text { (O0193) }\end{array}$ & $\begin{array}{l}\text { SPEC. } \\
\text { CONDUC- } \\
\text { TANCE } \\
\text { FIELD } \\
\text { ATM DEP } \\
\text { WET TOT } \\
\text { (US/CM) } \\
\text { (83154) }\end{array}$ & $\begin{array}{l}\text { SPEC. } \\
\text { CONDUC- } \\
\text { TANCE } \\
\text { LAB } \\
\text { ATM DEP } \\
\text { WET TOT } \\
\text { (US/CM) } \\
\text { (83156) }\end{array}$ & $\begin{array}{c}\text { PH } \\
\text { FIELD } \\
\text { ATM DEP } \\
\text { WET T } \\
\text { (UNITS) } \\
\text { (83106) }\end{array}$ & $\begin{array}{l}\text { PH } \\
\text { LAB } \\
\text { ATM DEP } \\
\text { WET T } \\
\text { (UNITS) } \\
\text { (83107) }\end{array}$ & $\begin{array}{l}\text { CALCIUM } \\
\text { ATM DEP } \\
\text { WET DIS } \\
\text { (MG/L) } \\
(82932)\end{array}$ & $\begin{array}{l}\text { MAG- } \\
\text { NESIUM } \\
\text { ATM DEP } \\
\text { WET DIS } \\
(M G / L) \\
(83002)\end{array}$ \\
\hline $\begin{array}{l}\text { JUL } \\
\text { JUL-16 }\end{array}$ & 1930 & 0.60 & 7.7 & 7.1 & 5.10 & 5.17 & 0.120 & 0.041 \\
\hline JUL & 1430 & 0.42 & 17.4 & 15.7 & -- & 6.50 & 0.830 & 0.150 \\
\hline $\begin{array}{r}23-30 \\
\text { JUL } 30-\end{array}$ & 1930 & 0.45 & 10.0 & 8.3 & -- & 5.19 & 0.120 & 0.017 \\
\hline $\begin{array}{l}\text { AUG } 06 \\
\text { AUG }\end{array}$ & 1930 & 0.30 & 6.3 & 4.4 & -- & 5.32 & 0.080 & 0.014 \\
\hline AUG $^{06-13}$ & 1600 & 0.01 & -- & 99.1 & -- & 3.81 & -- & -- \\
\hline AUG ${ }^{13-20}$ & 1930 & 0.18 & 10.2 & 9.1 & -- & 6.01 & 0.340 & 0.077 \\
\hline $\begin{array}{r}20-27 \\
\text { AUG } 27-\end{array}$ & 1930 & 0.99 & 12.3 & 11.7 & 4.80 & 5.58 & 0.280 & 0.029 \\
\hline $\begin{array}{l}\text { SEP } 03 \\
\text { SEP }\end{array}$ & 1400 & 1.39 & 10.9 & 7.1 & -- & 5.88 & 0.170 & 0.026 \\
\hline $\operatorname{SEP}^{03-10}$ & 1930 & 1.05 & 8.7 & 7.8 & -- & 4.99 & 0.040 & 0.003 \\
\hline SEP & 2000 & 0.26 & 9.4 & 9.1 & -- & 5.58 & 0.140 & 0.022 \\
\hline $\begin{array}{r}17-24 \\
\operatorname{SEP} 24-\end{array}$ & 1930 & 0.08 & 6.0 & 4.8 & -- & 5.97 & 0.080 & 0.017 \\
\hline OCT 01 & 2000 & 0.54 & 22.7 & 24.1 & -- & 6.72 & 1.55 & 0.252 \\
\hline
\end{tabular}


461458094295000 - PRECIPITATION STATION AT CAMP RIPLEY, MN--Continued

CHEMICAL ANALYSIS, WATER YEAR OCTOBER 1990 TO SEPTEMBER 1991

WEEKLY COMPOSITE

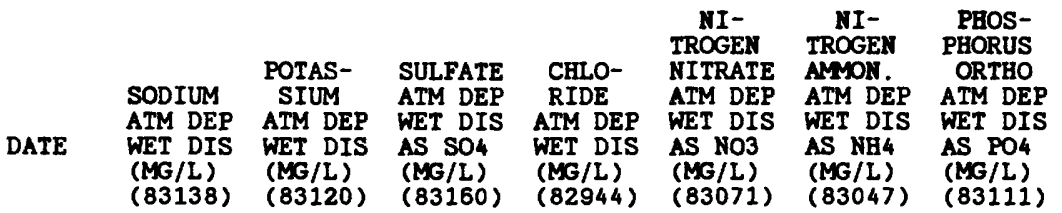

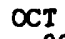

O2-09

09-16

OCT

OCT

$23-30$

NOV 06

NOV

$\begin{array}{lllllll}0.048 & 0.057 & 1.01 & 0.05 & 0.78 & 0.500 & <0.020 \\ 0.478 & 0.203 & 1.23 & 0.47 & 1.51 & 0.800 & <0.090 \\ 0.021 & 0.023 & 1.50 & 0.07 & 1.32 & 0.590 & <0.020 \\ -- & -- & -- & -- & -- & -- & -- \\ 0.693 & 0.068 & 1.54 & 0.32 & 3.05 & 0.720 & <0.070 \\ -- & -- & -- & -- & -- & -- & - \\ 0.239 & 0.035 & 2.35 & 0.15 & 3.46 & 0.750 & <0.020 \\ 0.102 & 0.042 & 7.12 & 0.38 & 7.20 & 2.44 & <0.020 \\ 0.067 & 0.044 & 0.49 & 0.07 & 1.10 & 0.510 & <0.020 \\ -- & -- & -- & -- & -- & -- & -- \\ 0.057 & 0.064 & 0.57 & 0.23 & 1.59 & 0.610 & <0.080 \\ 0.031 & 0.018 & 0.29 & 0.05 & 1.78 & 0.100 & <0.020 \\ -- & -- & -- & -- & -- & -- & -- \\ -- & -- & -- & -- & -- & -- & - \\ 0.043 & 0.010 & 0.15 & 0.08 & 1.54 & 0.080 & <0.020 \\ 0.146 & 0.031 & 0.25 & 0.18 & 2.64 & 0.560 & <0.020 \\ 0.044 & 0.013 & 0.11 & 0.07 & 0.63 & 0.130 & <0.020 \\ 0.022 & 0.006 & <0.03 & 0.08 & 0.05 & 0.020 & <0.020 \\ 0.051 & 0.005 & <0.03 & 0.08 & 0.04 & 0.260 & <0.020 \\ 0.027 & 0.007 & 0.32 & 0.06 & 1.68 & 0.200 & <0.020\end{array}$

nov $06-13$

$13-20$

20-27

MOV $27-$

DEC 04

DEC

04-11

DEC

11-18

18-25

DEC 251990

JAN 011991

JAN

01-08

08-15

JAN

15-22

22-29

JAN 29-

FEB 05

FEB

05-12

FEB

12-19

0.027

0.007

0.32

0.06

1.68

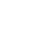


CHEMICAL QUALITY OF PRECIPITATION

461458094295000 - PRECIPITATION STATION AT CAMP RIPLEY, MN--Continued

CHEMICAL ANALYSIS, WATER YEAR OCTOBER 1990 TO SEPTEMBER 1991

WEEKIY COMPOSITE

\begin{tabular}{|c|c|c|c|c|c|c|c|}
\hline DATE & $\begin{array}{l}\text { SODIUM } \\
\text { ATM DEP } \\
\text { WET DIS } \\
\text { (MG/L) } \\
(83138)\end{array}$ & $\begin{array}{l}\text { POTAS- } \\
\text { SIUM } \\
\text { ATM DEP } \\
\text { WET DIS } \\
(M G / L) \\
(83120)\end{array}$ & $\begin{array}{l}\text { SULFATE } \\
\text { ATM DEP } \\
\text { WET DIS } \\
\text { AS SO4 } \\
(M G / L) \\
(83160)\end{array}$ & $\begin{array}{l}\text { CHLO- } \\
\text { RIDE } \\
\text { ATM DEP } \\
\text { WET DIS } \\
(\text { MG/L) } \\
(82944)\end{array}$ & \begin{tabular}{l}
\multicolumn{1}{c}{ NI- } \\
TROGEN \\
NITRATE \\
ATM DEP \\
WET DIS \\
AS NO3 \\
(MG/L) \\
$(83071)$
\end{tabular} & $\begin{array}{l}\quad \text { NI- } \\
\text { TROGEN } \\
\text { AMMON. } \\
\text { ATM DEP } \\
\text { WET DIS } \\
\text { AS NH4 } \\
\text { (MG/L) } \\
\text { ( } 83047)\end{array}$ & $\begin{array}{l}\text { PHOS- } \\
\text { PHORUS } \\
\text { ORTHO } \\
\text { ATM DEP } \\
\text { WET DIS } \\
\text { AS PO4 } \\
\text { (MG/L) } \\
\text { (83111) }\end{array}$ \\
\hline $19-20$ & 0.060 & 0.014 & 0.18 & 0.10 & 0.64 & 0.170 & $<0.020$ \\
\hline $\begin{array}{l}E B \text { 26- } \\
\text { AAR } \text { O5 } \\
A R\end{array}$ & -- & -- & -- & - & -- & -- & -- \\
\hline O5-12 & 0.033 & $<0.003$ & 0.04 & 0.05 & 0.08 & $<0.020$ & $<0.020$ \\
\hline $\mathbb{R}_{R}^{12-19}$ & 0.014 & 0.005 & 0.03 & $<0.03$ & 0.05 & $<0.020$ & $<0.020$ \\
\hline $\begin{array}{l}19-26 \\
R \quad 26-\end{array}$ & 0.034 & 0.011 & 1.81 & 0.07 & 1.14 & 0.360 & $<0.020$ \\
\hline $\begin{array}{l}\text { APR } \\
\text { PR }\end{array}$ & 0.573 & 0.035 & 2.37 & 0.17 & 2.13 & 1.54 & $<0.020$ \\
\hline $\begin{array}{l}02-09 \\
R\end{array}$ & 0.024 & $<0.003$ & 0.26 & 0.05 & 0.51 & 0.030 & $<0.020$ \\
\hline 16 & 0.038 & 0.015 & 1.08 & 0.05 & 0.86 & 0.090 & $<0.020$ \\
\hline 23 & 0.038 & 0.034 & 1.58 & 0.08 & 2.39 & 1.43 & $<0.020$ \\
\hline $\begin{array}{r}23-30 \\
\text { PR } 30-\end{array}$ & 0.035 & 0.028 & 1.22 & 0.05 & 0.93 & 0.360 & $<0.020$ \\
\hline $\begin{array}{l}\text { MAY } 07 \\
\text { AY }\end{array}$ & 0.015 & 0.007 & 0.53 & 0.03 & 0.59 & 0.120 & $<0.020$ \\
\hline $\begin{array}{l}07-14 \\
Y Y\end{array}$ & 0.117 & 0.054 & 0.94 & .14 & 1.00 & 0.780 & $<0.020$ \\
\hline 21 & 0.066 & 0.025 & 0.65 & 0.11 & 1.67 & 1.08 & $<0.020$ \\
\hline $\begin{array}{r}21-28 \\
\text { MAY } 28-\end{array}$ & 0.012 & 0.018 & 0.45 & 0.04 & 0.92 & 0.510 & $<0.020$ \\
\hline JUN 04 & 0.030 & 0.015 & 0.83 & 7 & 1.15 & 0.400 & $<0.020$ \\
\hline JUN & 0.293 & 0.328 & 3.86 & 0.64 & 4.22 & 1.00 & $<0.120$ \\
\hline JUN $^{11-18}$ & 0.013 & 0.013 & 0.66 & 0.06 & 1.03 & 0.590 & $<0.020$ \\
\hline $\begin{array}{r}18-25 \\
\text { JUN } 25-\end{array}$ & 0.040 & 0.021 & 1.22 & 0.09 & 1.45 & 0.550 & $<0.020$ \\
\hline JUL 02 & 0 . & 37 & 0.82 & 9 & 1.01 & 0.520 & $<0.020$ \\
\hline 9 & 0.053 & 0.146 & 0.56 & 0.21 & 1.01 & 0.210 & $<0.020$ \\
\hline
\end{tabular}


461458094295000 - PRECIPITATION STATION AT CAMP RIPLEY, MN--Continued

CHEMICAL ANALYSIS, WATER YEAR OCTOBER 1990 TO SEPTEMBER 1991

WEEKIY COMPOSITE

DATE

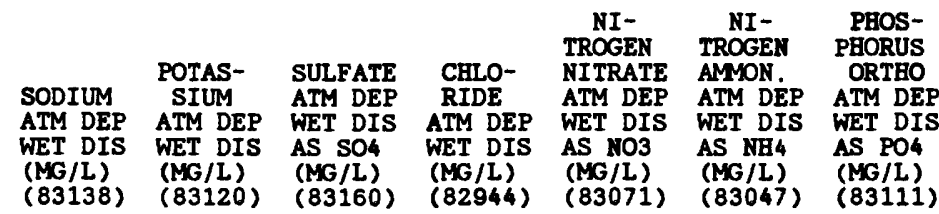

JUL

JUL

JUL $16-23$

0.025

0.020

0.69

0.06

0.99

0.190

$<0.020$

JUL

0.070

0.091

1.53

0.16

2.39

1.09

$<0.020$

0.013

$<0.003$

1.13

0.04

0.93

$0.490<0.020$

JUL 30-

0.020

0.005

0.35

0.04

0.56

0.140

0.090

06-13

AUG

13-20

AUG

20-27

AUG 27-

SEP 03

SEP

03-10

SEP

10-17

SEP $17-24$

SEP 24-

OCT 01

0.103

0.074

1.25

0.13

1.41

$0.500<0.020$

0.022

0.048

1.64

0.08

1.99

1.00

$<0.020$

0.027

0.020

1.28

0.04

0.69

$0.540<0.020$

0.016

0.003

0.94

0.04

0.63

0.310

$<0.020$

0.036

0.029

1.15

0.07

1.51

0.700

$<0.020$

0.078

0.040

0.51

0.13

0.37

0.200

0.080

0.134

0.198

2.28

0.11

2.31

1.46

$<0.020$ 


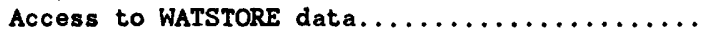

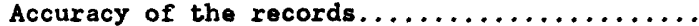

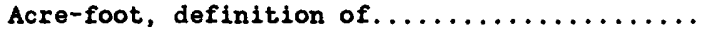
Adenosine triphosphate, definition of..........

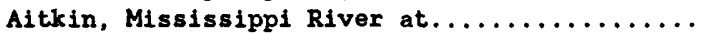

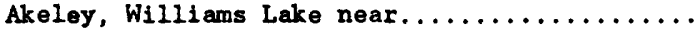

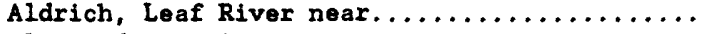

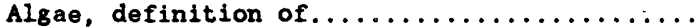
Algal growth potential, definition of.......... Analysis of samples collected at waterquality partial-record stations.........

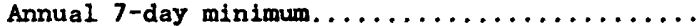

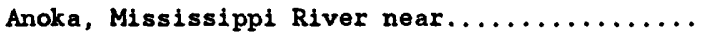

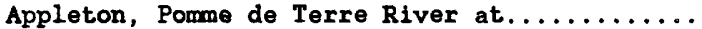

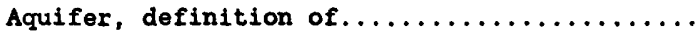

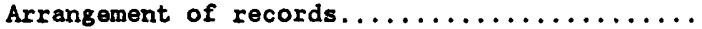

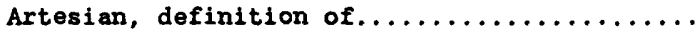
Artificial substrate, definition of..........

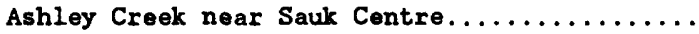

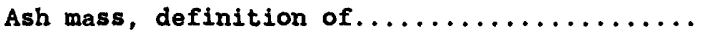

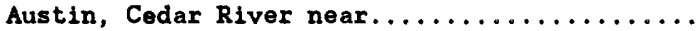

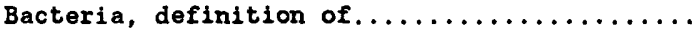

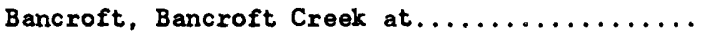

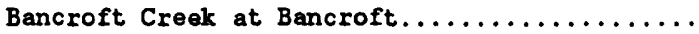
Beaver Creek at Beaver Falls...............

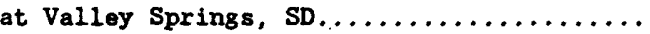

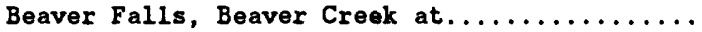

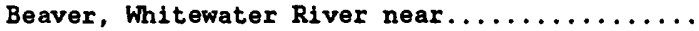

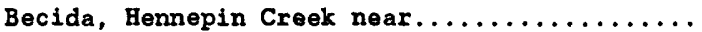

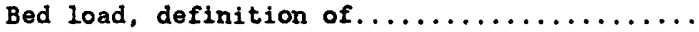

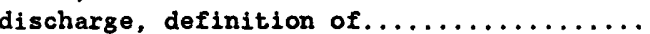

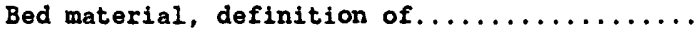
Bemidji, Mississippi River at..............

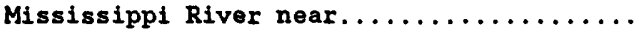

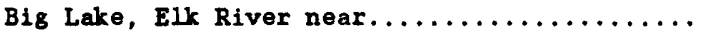

Big Sioux River basin, high-flow partial-

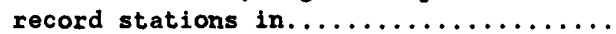

Big Stone City, SD, Big Stone Lake near.......

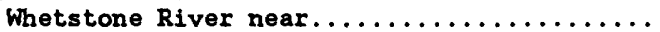

Big Stone Lake near Big Stone City, SD....... Biochemical oxygen demand, definition of......

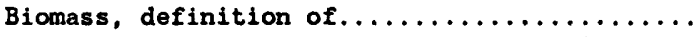
Blue Earth River, East Branch, near Walters...

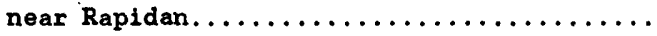

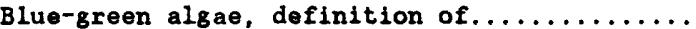

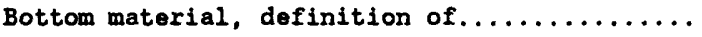

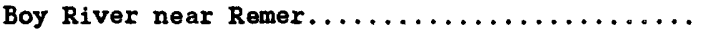

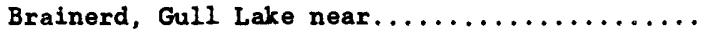
Gull River at Gull Lake Dam near.......... Mississippi River at.................

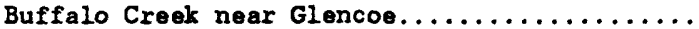

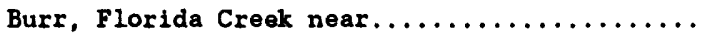

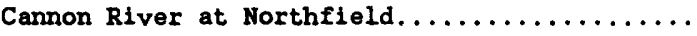
Cannon River basin, gaging-station records in. high-flow partial-record stations in...... Cannon River below Sabre Lake near Kilkenny...

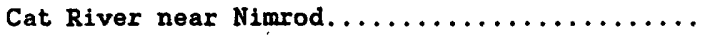

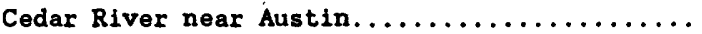

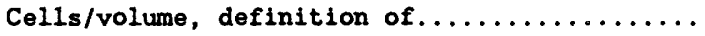
Ceylon, East Fork Des Moines River at........
77-79

85
Cfs/day, definition of $\ldots \ldots \ldots \ldots \ldots \ldots \ldots$

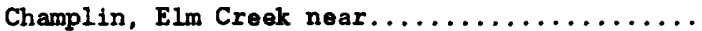

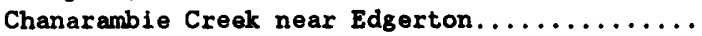
Chemical oxygen demand, definition of........ Chemical quality of precipitation.............

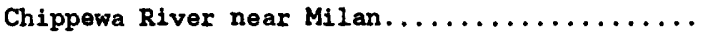

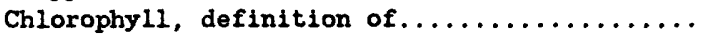

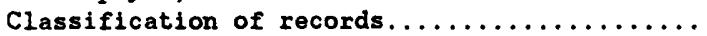

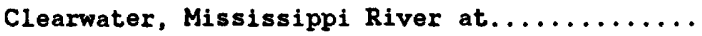
Clearwater River basin high-flow partial-

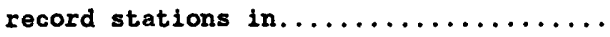

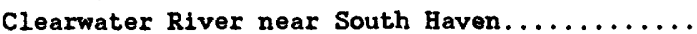
Collection and computation of ground-water

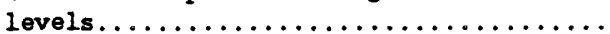

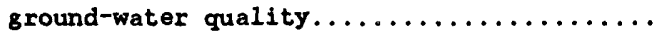
stage and water discharge...............

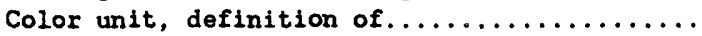

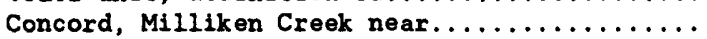

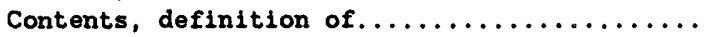

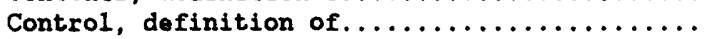

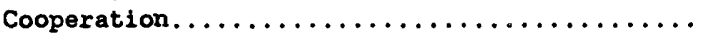

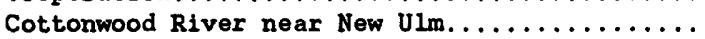

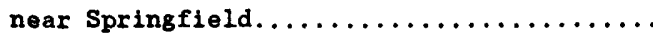
Courtland, Little Cottonwood River near...... Crooked Creek at Freeburg................. Crooked Creek basin, high-flow partial-record

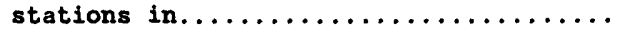

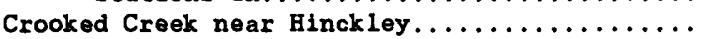
Cross Lake, Pine River at Cross Lake Dam, at.. Pine River Reservoir at...............

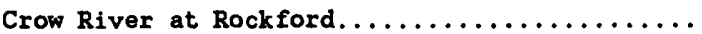

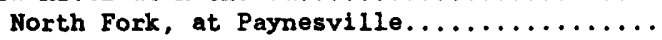

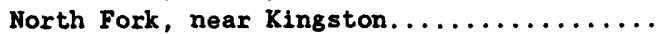
Crow River basin, gaging-station records in... high-flow partial-record stations in...... Crow Wing River basin, gaging-station

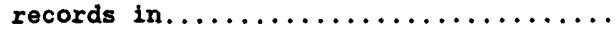

high-flow partial-record stations in......

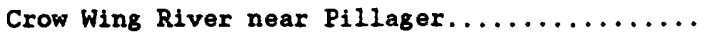
Cubic foot per second, definition of......... Cubic feet per second per square mile,

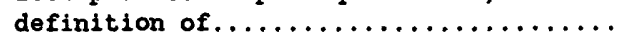
24

$74-76$

Data collection and computation,

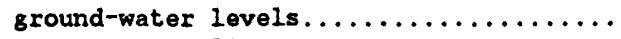

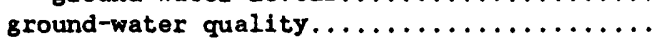
stage and water discharge...............

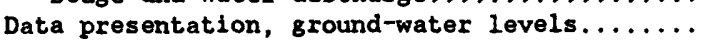

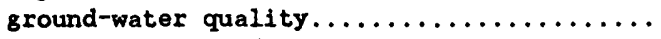

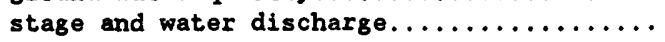

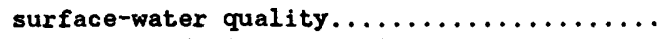
Data table of daily mean values............ Deer River, Mississippi River at

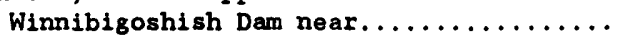

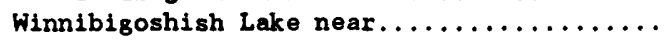

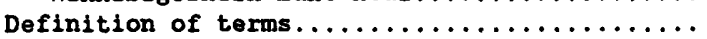

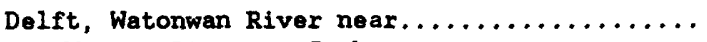
Des Moines River at Jackson............... Des Moines River basin, gaging-station

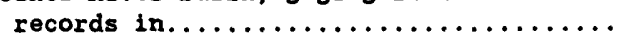

high-flow partial-record stations in......

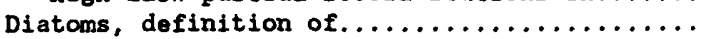


Discharge at partial-record stations and

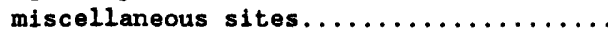
high-flow partial-record stations.........

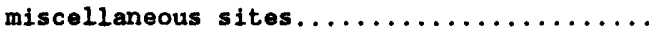

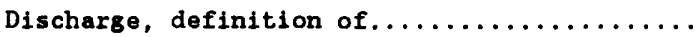

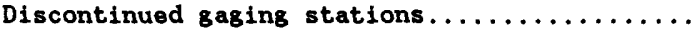

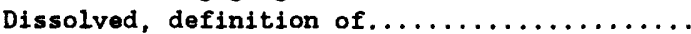

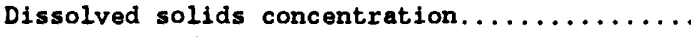

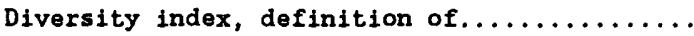
Downstream order system and station number,

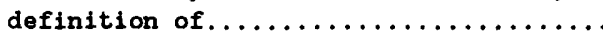

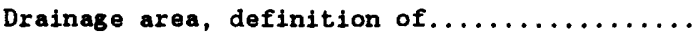

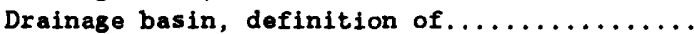

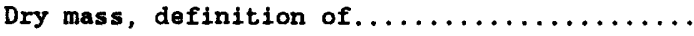

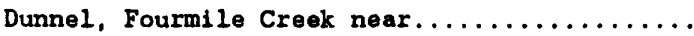

East Branch Blue Earth River near Walters.... East Fork Des Moines River near Celyon........

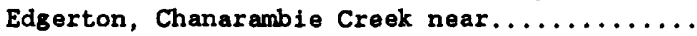
Elba, North Fork Whitewater River near.......

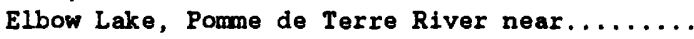
Elk River near Big Lake.................. Elm Creek basin, gaging-station records in...

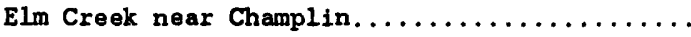
near Trimont. . . . . . . . . . . . . . .

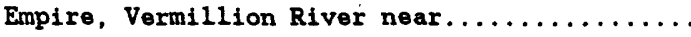

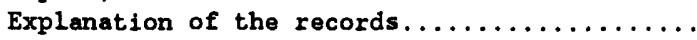

144-162 145-156

157-162 25
$x i-x i v$ 25 25 25 17 25 25 24 155

152 155 156

130-133 151 70

$74-76$

$74-76$ 152

125-127 15-23

Factors for converting Inch-Pound Units to International System (SI) Units.................... Inside back cover Faribault, Straight River near........... 128 Fecal coliform bacteria, definition of....... Fecal streptococcal bacteria, definition of...

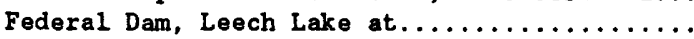
Leech Lake River at..................

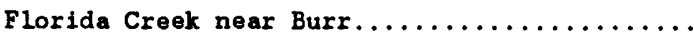
Fort Ripley, Mississippi River near.........

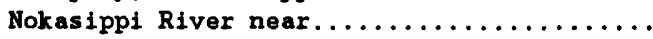

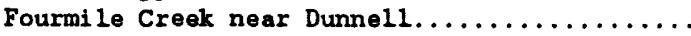

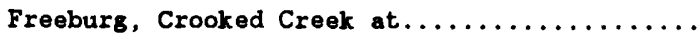

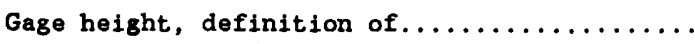
Gaging station, definition of...............

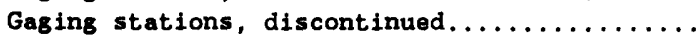

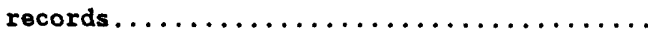

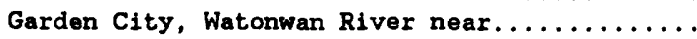
Garvin Brook near Minnesota City............ Garvin Brook basin, gaging-station

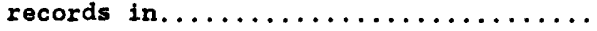
Gaylord, Middle Branch Rush River near.......

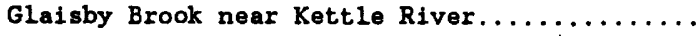

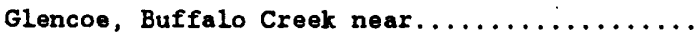

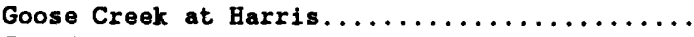
Grand Rapids, Mississippi River at..........

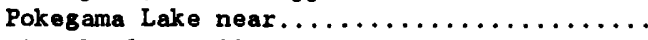
Granite Falls, Yellow Medicine River near.... Graph showing comparison of dissolved solids concentrations.................... discharge at three long-term gaging

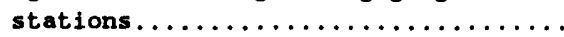

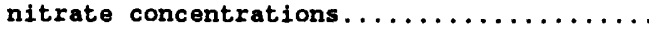

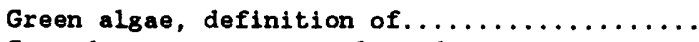
Ground water, water quality data............

in summary of hydrologic conditions....... 10-16 Ground-water records, by county, quality..... 245-260

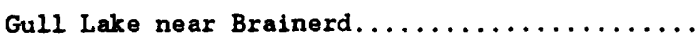
Gull River at Gull Lake Dam, near Brainerd...

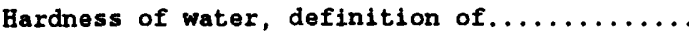

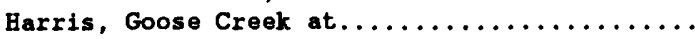

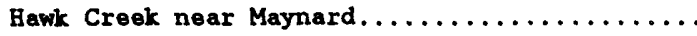

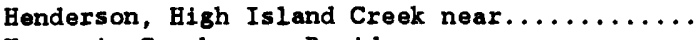

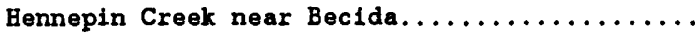

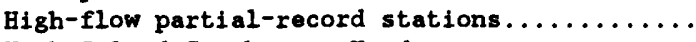

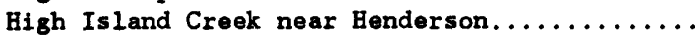

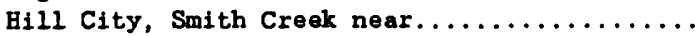

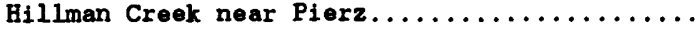

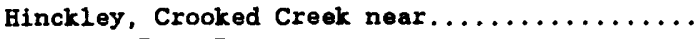

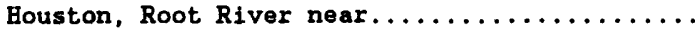

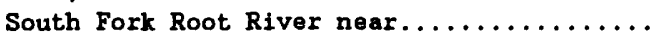
Hydrographs showing comparison of water levels in 1991 to long-term levels in different aquifers............... 11-14,16 Bydrologic bench-mark network, definition of.. 15 Hydrologic conditions, graphs or maps of.3,6-9,11-14,16

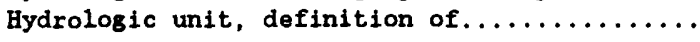

Identifying estimated daily discharge........ Introduction. . . . . . . . . . . . . . . . . . Instantaneous discharge, definition of....... Iowa River basin, gaging-station records in...

high-flow partial-record stations in......

Jackson, Des Moines River at.............

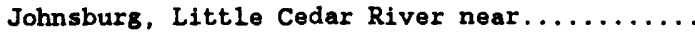
Johnson Creek basin, high-flow partial-record

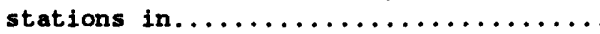
Johnson Creek near St. Augusta............ Jordan, Minnesota River near..............

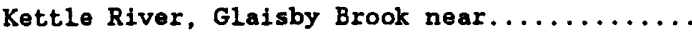

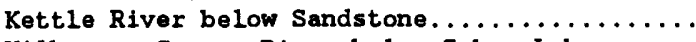
Kilkenny, Cannon River below Sabre Lake near.. Kingston, North Fork Crow River near.........

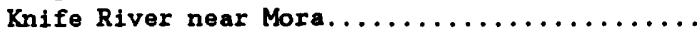

Laboratory measurements, surface-water quality Lac qui Parle, Lac qui Parle River near.......

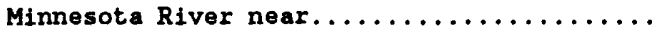
Lac qui Parle River near Lac qui Parle....... Lakes and Reservoirs:

Big Stone Lake at Ortonville near

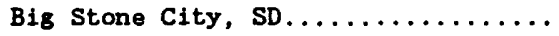

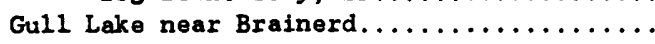
Leech Lake at Federal Dam.............. Mille Lacs Lake at Cove Bay near Onamia.... Pokegama Lake near Grand Rapids........... Pine River Reservoir at Cross Lake........

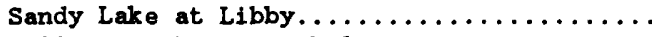

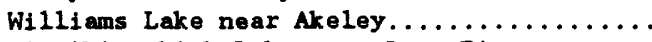
Winnibigoshish Lake near Deer River.......

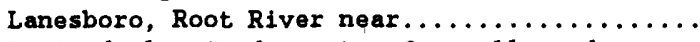
Latitude-longitude system for wells and

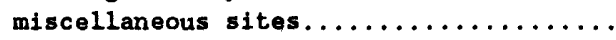

Leaf River near Aldrich. . . . . . . . . . .

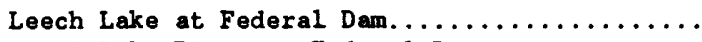
Leech Lake River at Federal Dam............ Leech Lake River basin, gaging-station records in . . . . . . . . . . . . . . . .

high-flow partial-record stations in..... measurements made at miscellaneous sites in Le Sueur River near Rapidan...............

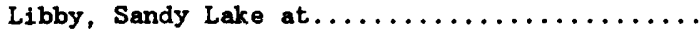


Sandy River at Sandy Lake Dam at......... List of counties for which ground-water-

level records are published...........

for which ground-water-quality records are published.................. List of gaging stations, in downstream order, for which records are published......... Little Cedar River near Johnsburg............ Little Chippewa River near Starbuck.......... Little Cottonwood River near Courtland........ Little Minnesota River near Peever, SD....... Little Rock River near Rushmore............. Little Sioux River basin, high-flow

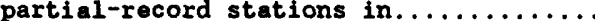

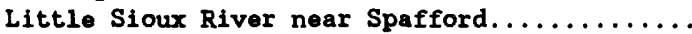
Long Prairie, Long Prairie River at.......... Long Prairie River at Long Prairie...........

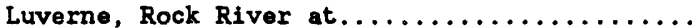

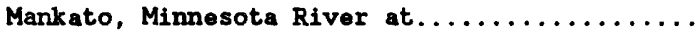
Map of Minnesota, ground-water observation

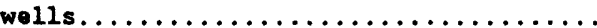

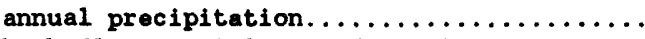
high-flow partial record stations......... lake and stream-gaging stations.......... surface water-quality stations........... Maple River near Rapidan.................

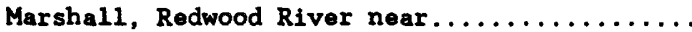

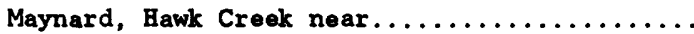
Mean concentration, definition of .............

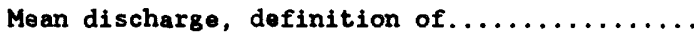
Methylene blue active substance,

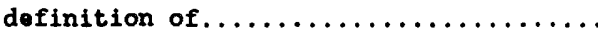

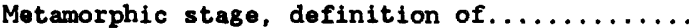

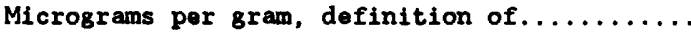
Micrograms per kilogram, definition of.........

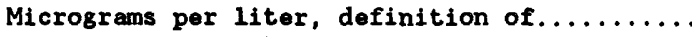
Middle Branch Rush River near Gaylord......... Middle Fork Whitewater River near State Park Group Camp near St. Charles.......

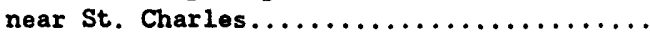

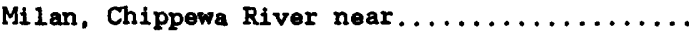
Mille Lacs Lake at Cove Bay near Onamia....... Milligrams per liter, definition of........... Milligrams of carbon per area or volume per unit time for periphyton, macrophytes,

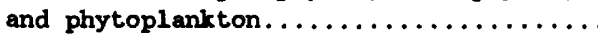

Milligrams of oxygen per area or volume per unit time for periphyton, macrophytes,

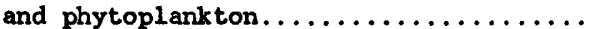

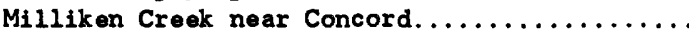

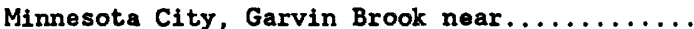

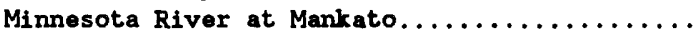

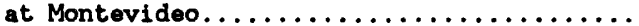

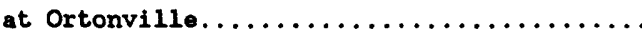

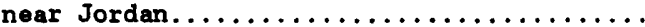

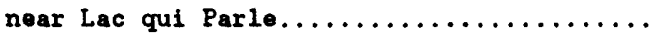
Minnesota River basin, gaging-station

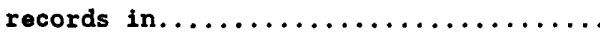
high-flow partial-record stations in...... measurements made at miscellaneous sites in Miscellaneous sites, discharge

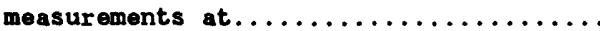

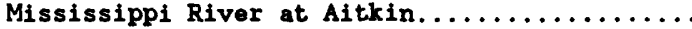

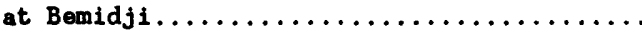

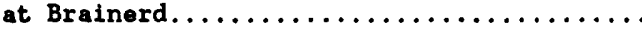

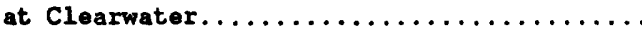

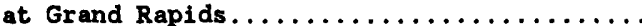

viii-x

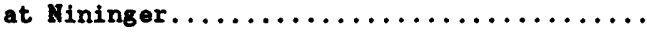

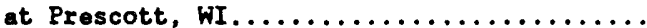

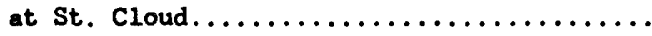

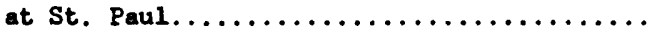
at Winnibigoshish Dam, near Deer River.....

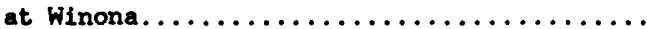

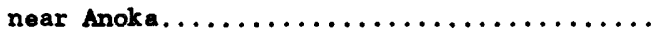

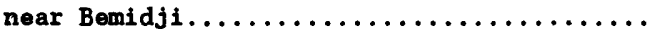
near Fort Ripley...................

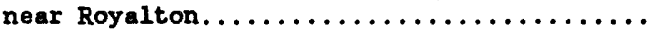

Mississippi River main stem, measurements

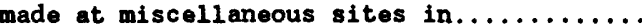
Montevideo, Minnesota River at..............

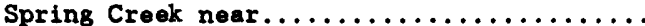

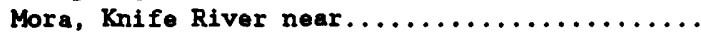

National Geodetic Vertical Datum of 1929

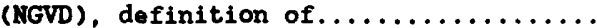
National stream-quality accounting network

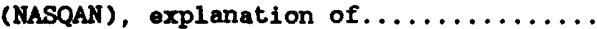
National trends network (HTN), explanation of.

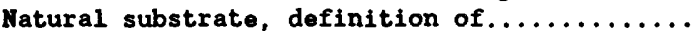

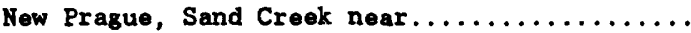

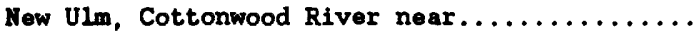

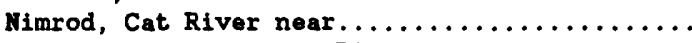
Nininger, Mississippi River at............. Nokasippi River basin, high-flow partial-

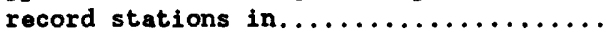
Nokasippi River near Fort Ripley........... North Fork Crow River at Paynesville.........

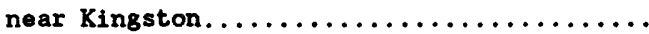
North Fork Whitewater River near Elba........

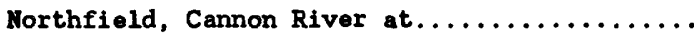
Numbering system for wells and

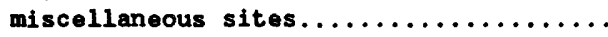

$119-120$

124

69

$116-118$

40

$139-140$

$77-79$

38

64

65-67

158

91-93

151

122

$25-26$

15,26

15,26

27

153

97-98

149

$119-120$

149

149

150

151

$130-133$

153

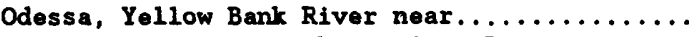
Onamia, Mille Lacs Lake at Cove Bay near...... Onsite measurement and collection........... Organic mass, definition of ................ Organism, definition of $\ldots \ldots \ldots \ldots \ldots \ldots \ldots$ count/area, definition of...............

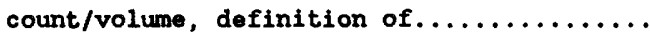
Ormsby, South Fork Watonwan River near........

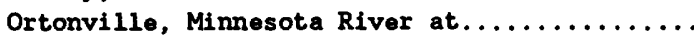
Osage, Straight River at County Highway 125

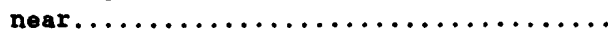
other records available.................

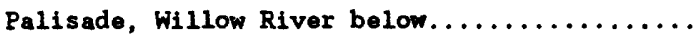

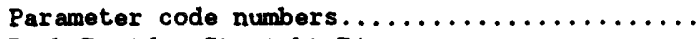
Park Rapids, Straight River near............ Partial-record station, definition of........

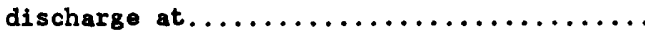
Particle size, definition of............... Particle-size classification, definition of... Paynesville, North Fork Crow River at........ Peever, SD, Little Minnesota River near.......

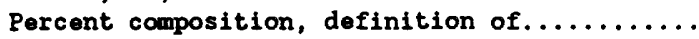

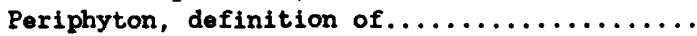

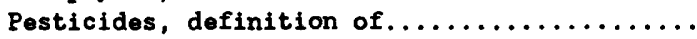

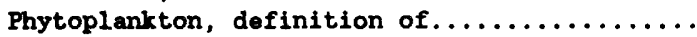

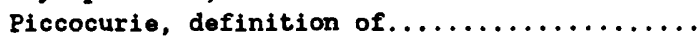

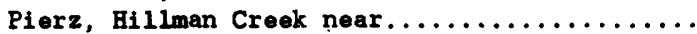

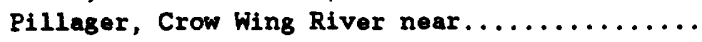

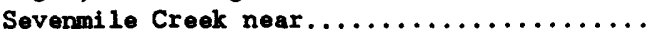
Pine River at Cross Lake Dam, at Cross Lake... near Pine River....................
20

149

26

59

26

$144-156$

26

26

150

80

26

26

26

26

26

149

63

149

56
149 
Pine River basin, gaging-station records in... high-flow partial-record stations in......

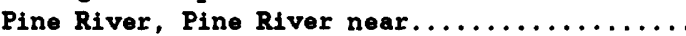
Pine River Reservoir at Cross Lake........... Pipestone Creek near Pipestone..............

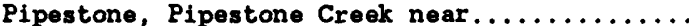

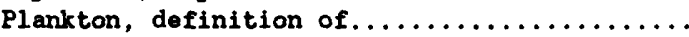

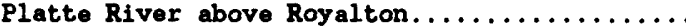
Platte River basin, high-flow partial-

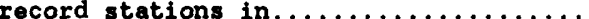

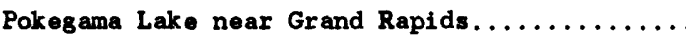
Polychlorinated biphenyls, definition of...... Pomme de Terre River at Appleton...........

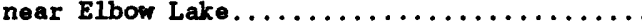

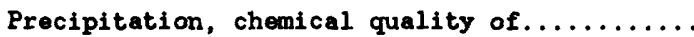
in summary of hydrologic conditions........

Prescott, WI, Mississippi River at............ Primary productivity, definition of............ Publication on techniques of water-resources

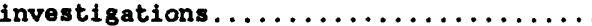

Quality of ground-water, by county............

Radiochemical program, definition of..........

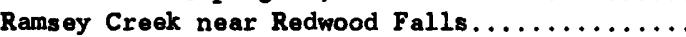

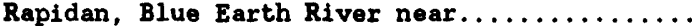

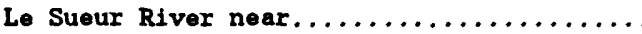

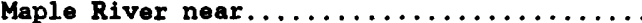

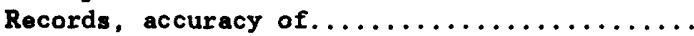

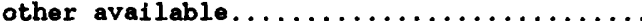

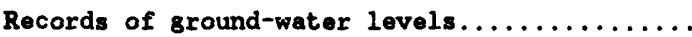

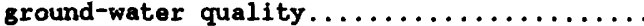
stage and water discharge..............

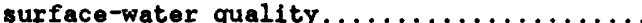

Recoverable from bottom material, definition

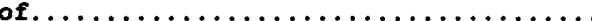

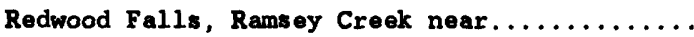
Redwood River near. . . . . . . . . . . . . . . .

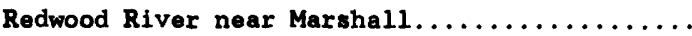

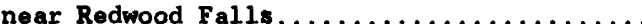

Remark codes, surface-water quality..........

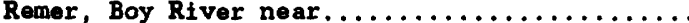
Reservoirs (see lakes and reservoirs)

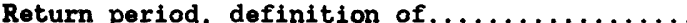

Rochester, South Fork Zumbro River at.........

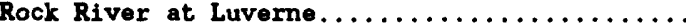

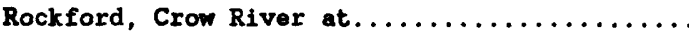

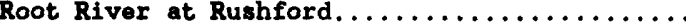

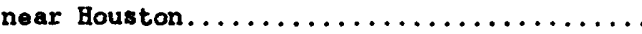

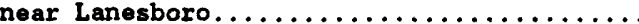

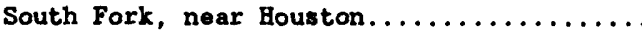

Root River basin, gaging station records in.. high-flow partial-record stations in......

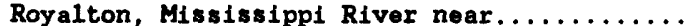

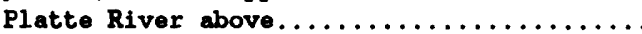
Rum River basin, gaging-station records in....

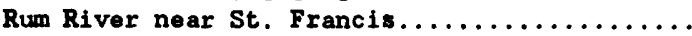
Runoff at streamflow tations (table 1).......

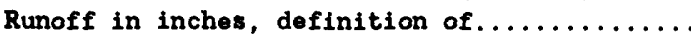

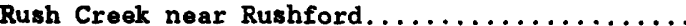

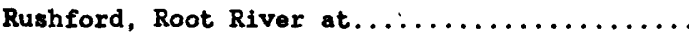

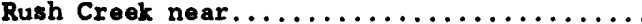

Rushmore, Little Rock River near............ Rush River, Middle Branch near Gaylord........

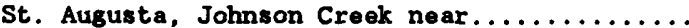
St. Charles, Middle Fork Whitewater River

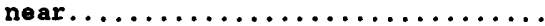
Middle Fork Whitewater River near State Park Group Camp near. ...............
55-56

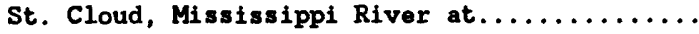

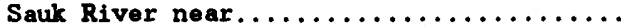

St. Croix Falls, wI, st. Croix River at...... St. Croix River at St. Croix Falls, WI........ St. Croix River basin, gaging-station

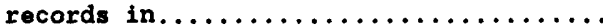

high-flow partial-record stations in.....

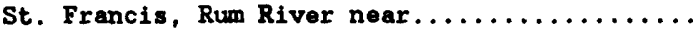

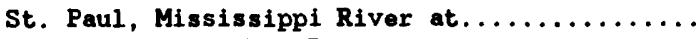

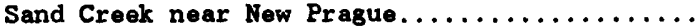

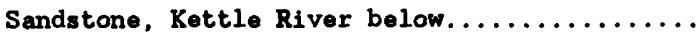

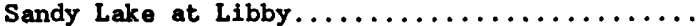
Sandy River at Sandy Lake Dam, at Libby....... Sandy River basin, gaging-station records in.. Sauk Centre, Ashley Creek near............. Sauk River basin, high-flow partial-record

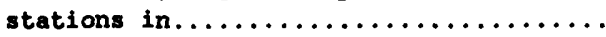

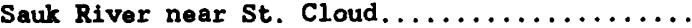
tributary at Spring Hill................

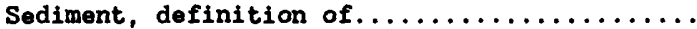

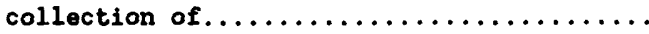
Seven-day 10-year low flow, definition of.... Sevenmile Creek near Pillager..............

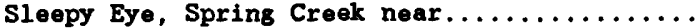
Smith Creek basin, high-flow partial-record

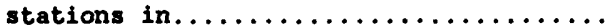

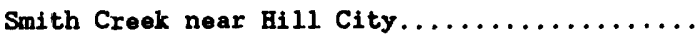
Sodium-adsorption ratio, definition of........

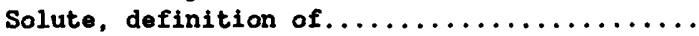

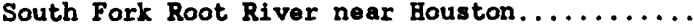

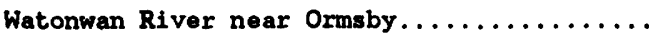

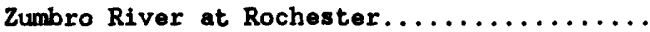

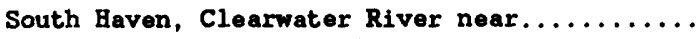
Spafford, Little Sioux River near........... Special networks and programs............. Specific conductance, definition of.......... Spring Creek near Montevideo..............

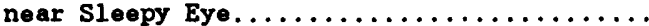
Springfield, Cottonwood River near.......... Spring Bill, Sauk River tributary at......... Stage-discharge relation, definition of....... Starbuck, Little Chippewa River near.........

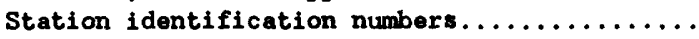

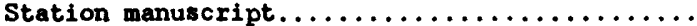
Statistics of monthly mean data............ Straight River at County Highway 125 near

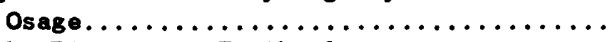

Straight River near Faribault............

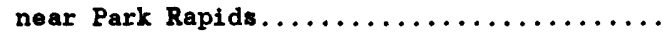

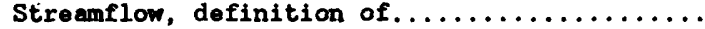
in summary of hydrologic conditions........

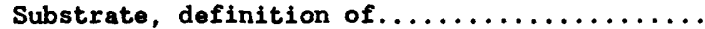

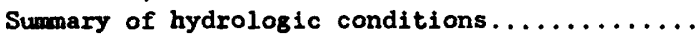

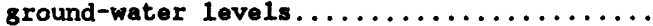

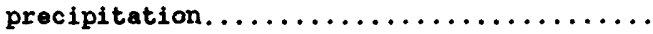

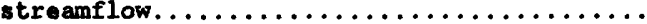

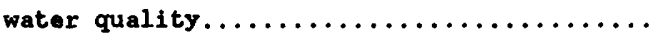

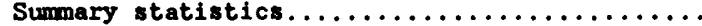

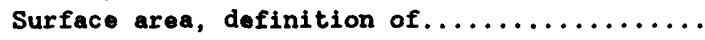
Surficial bed material, definition of.........

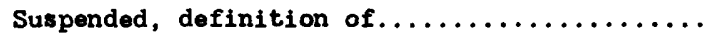

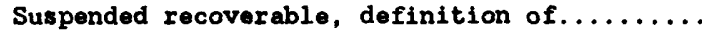
Suspended sediment, definition of........... Suspended-sediment concentration,

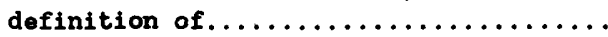
Suspended-sediment discharge, definition of... Suspended-sediment load, definition of........

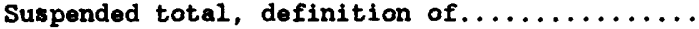


Page

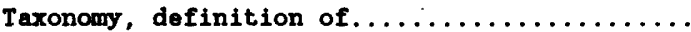

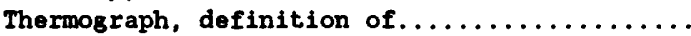
Time-weighted average, definition of..........

Tons per acre-foot, definition of............

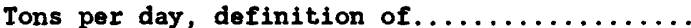

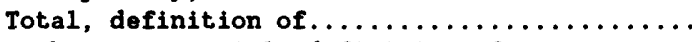

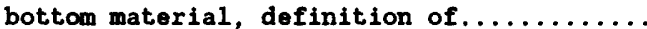
coliform bacteria, definition of..........

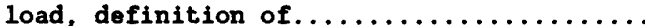
organism count, definition of............

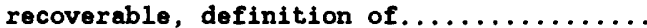
sediment discharge, definition of......... sediment load, definition of.............

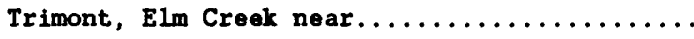

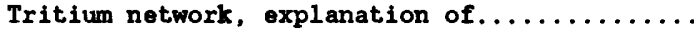

Valley Springs, SD., Beaver Creek near........ Vermillion River basin, gaging-station

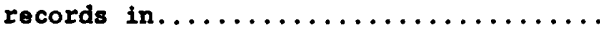

Vermillion River near Empire.............

Walters, Blue Earth River East Branch near.... Water quality, in summary of hydrologic

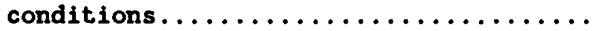

Water-quality records, analyses of samples collected at ground-water wells........ collected at partial-record stations...... Water temperature, surface-water quality......

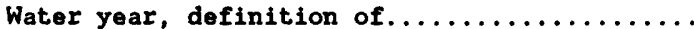

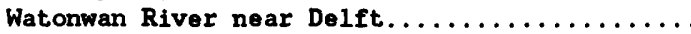
near Garden City.

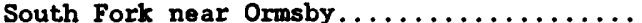
Weighted average, definition of ..........

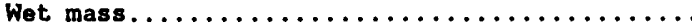
Whetstone River near Big Stone City, SD..... Whitewater River basin, gaging-station

records in....................

high-flow partial-record stations in...... Whitewater River, Middle Fork, near

St. Charles....................

Middle Fork near State Park Group

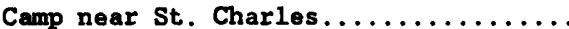

North Fork, near Elba............... Williams Lake near Akeley................ Willow River basin, high-flow partial-record

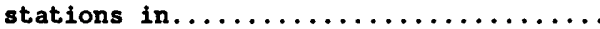

Willow River below Palis ade............. Winnibigoshish Lake near Deer River......... Winona, Mississippi River at............

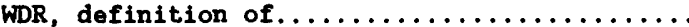

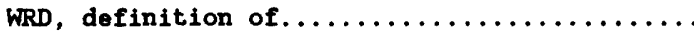
WSP, definition of $\ldots \ldots \ldots \ldots \ldots \ldots \ldots \ldots \ldots$

Yellow Bank River near Odessa.............. Yellow Medicine River near Granite Falls.....

Zooplankton, definition of............. Zumbro Falls, Zumbro River at............ Zumbro River at Zumbro Falls............ South Fork, at Rochester............. Zumbro River basin, gaging-station records in ........................... 129-131 high-flow partial-record stations in...... 153-154 


\section{FACTORS FOR CONVERTING INCH-POUND UNITS TO INTERNATIONAL SYSTEM UNITS (SI)}

The following factors may be used to convert the inch-pound units published herein to the International System of Units (SI).

Multiply inch-pound units

inches (in)
feet ( $\mathrm{ft}$ )
miles (mi)
acres
square miles (mi ${ }^{2}$ )
gallons (gal)
million gallons
cubic feet ( $\mathrm{ft}^{3}$ )
cfs-days
acre-feet (acre-ft)
cubic feet per second ( $\mathrm{ft}^{3} / \mathrm{s}$ )

tons (short)
By

To obtain SI units

\section{Length}

$2.54 \times 10^{1}$

$2.54 \times 10^{-2}$

$3.048 \times 10^{-1}$

$1.609 \times 10^{0}$

Area

$$
\begin{aligned}
& 4.047 \times 10^{3} \\
& 4.047 \times 10^{-1} \\
& 4.047 \times 10^{-3} \\
& 2.590 \times 10^{0}
\end{aligned}
$$

\section{Volume}

$3.785 \times 10^{0}$

$3.785 \times 10^{\circ}$

$3.785 \times 10^{-3}$

$3.785 \times 10^{3}$

$3.785 \times 10^{-3}$

$2.832 \times 10^{1}$

$2.832 \times 10^{-2}$

$2.447 \times 10^{3}$

$2.447 \times 10^{-3}$

$1.233 \times 10^{3}$

$1.233 \times 10^{-3}$

$1.233 \times 10^{-6}$

Flow

$2.832 \times 10^{1}$

$2.832 \times 10^{1}$

$2.832 \times 10^{-2}$

$6.309 \times 10^{-2}$

$6.309 \times 10^{-2}$

$6.309 \times 10^{-5}$

$4.381 \times 10^{1}$

$4.381 \times 10^{-2}$

Mass

$9.072 \times 10^{-1}$ millimeters $(\mathrm{mm})$

meters (m)

meters (m)

kilometers (km)

square meters $\left(\mathrm{m}^{2}\right)$

square hectometers $\left(\mathrm{hm}^{2}\right)$

square kilometers $\left(\mathrm{km}^{2}\right)$

square kilometers $\left(\mathrm{km}^{2}\right)$

liters (L)

cubic decimeters $\left(\mathrm{dm}^{3}\right)$

cubic meters $\left(\mathrm{m}^{3}\right)$

cubic meters $\left(\mathrm{m}^{3}\right)$

cubic hectometers $\left(\mathrm{hm}^{3}\right)$

cubic decimeters $\left(\mathrm{dm}^{3}\right)$

cubic meters $\left(\mathrm{m}^{3}\right)$

cubic meters $\left(\mathrm{m}^{3}\right)$

cubic hectometers $\left(\mathrm{hm}^{3}\right)$

cubic meters $\left(\mathrm{m}^{3}\right)$

cubic hectometers $\left(\mathrm{hm}^{3}\right)$

cubic kilometers $\left(\mathrm{km}^{3}\right)$

liters per second $(\mathrm{L} / \mathrm{s})$

cubic decimeters per second $\left(\mathrm{dm}^{3} / \mathrm{s}\right)$

cubic meters per second $\left(\mathrm{m}^{3} / \mathrm{s}\right)$

liters per second $(\mathrm{L} / \mathrm{s})$

cubic decimeters per second $\left(\mathrm{dm}^{3} / \mathrm{s}\right)$

cubic meters per second $\left(\mathrm{m}^{3} / \mathrm{s}\right)$

cubic decimeters per second $\left(\mathrm{dm}^{3} / \mathrm{s}\right)$

cubic meters per second $\left(\mathrm{m}^{3} / \mathrm{s}\right)$

megagrams (Mg) or metric tons 
U.S. DEPARTMENT OF THE INTERIOR

U.S. Geological Survey

702 Post Office Building

St. Paul, MN 55101

OFFICIAL BUSINESS

PENALTY FOR PRIVATE USE $\$ 300$

SPECIAL 4th CLASS BOOK RATE 